\title{
U.S. Linear Collider Technology Options Study
}

\author{
U.S. Linear Collider Steering Group \\ Accelerator Sub-committee \\ Linear Collider Option Task Forces
}

March 4, 2004

SLAC-R-827

Prepared for the Department of Energy

under contract number DE-AC02-76SF00515

Printed in the United States of America. Available from the National Technical Information Service, U.S. Department of Commerce, 5285 Port Royal Road, Springfield, VA 22161. 
This document, and the material and data contained therein, was developed under sponsorship of the United States Government. Neither the United States nor the Department of Energy, nor the Leland Stanford Junior University, nor their employees, nor their respective contractors, subcontractors, or their employees, makes an warranty, express or implied, or assumes any liability of responsibility for accuracy, completeness or usefulness of any information, apparatus, product or process disclosed, or represents that its use will not infringe privately owned rights. Mention of any product, its manufacturer, or suppliers shall not, nor is it intended to, imply approval, disapproval, or fitness of any particular use. A royalty-free, nonexclusive right to use and disseminate same of whatsoever, is expressly reserved to the United States and the University. 


\section{U.S. Linear Collider Technology Options Study}

\section{U.S. Linear Collider Steering Group Accelerator Sub-committee Linear Collider Option Task Forces}

March 4, 2004 


\section{Contents}

1 Introduction and Overview 1

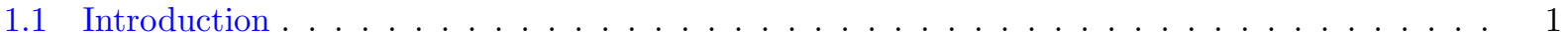

1.1.1 Initiation and purpose of this study $\ldots \ldots \ldots \ldots \ldots \ldots \ldots \ldots \ldots \ldots$

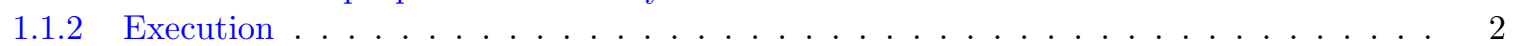

1.1.3 Presentations to Linear Collider collaborations . . . . . . . . . . . . . . . . . . . . . 4

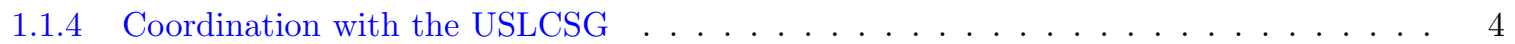

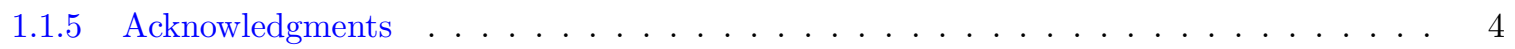

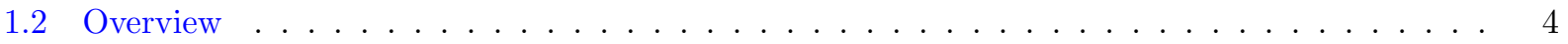

2 Executive Summary 6

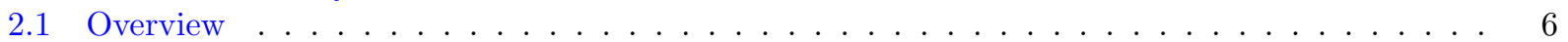

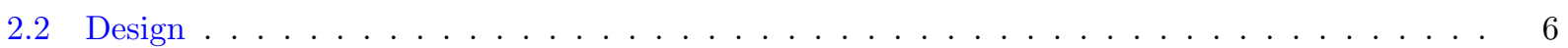

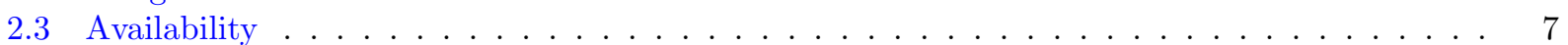

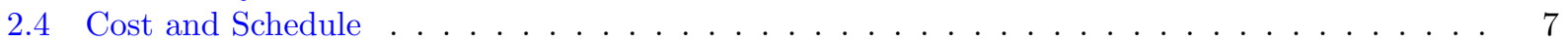

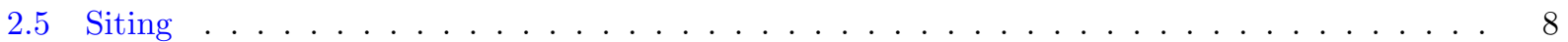

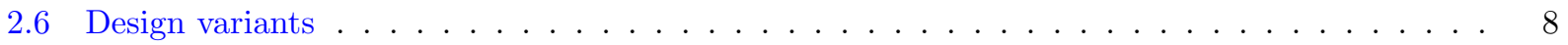

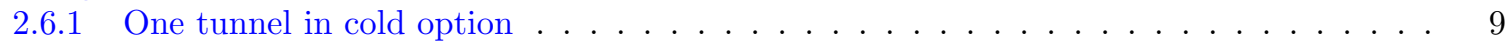

$2.6 .235 \mathrm{MV} / \mathrm{m}$ initial design gradient in cold option . . . . . . . . . . . . . . . . 9

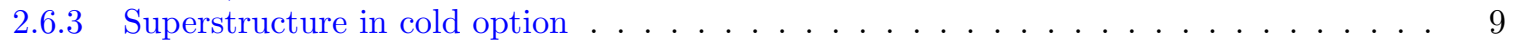

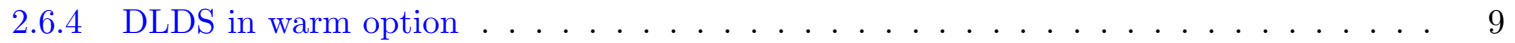

2.6.5 Conventional positron sources in both options . . . . . . . . . . . . . . . . 10

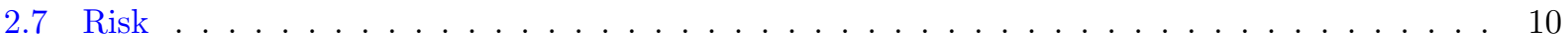

3 Reference Design Configurations $\quad 12$

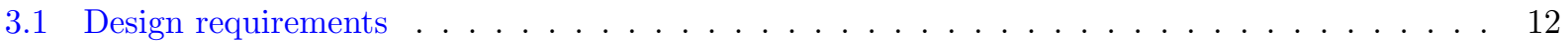

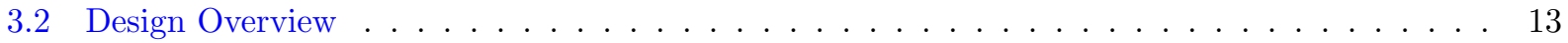

$3.2 .1500 \mathrm{GeV}$ reference designs . . . . . . . . . . . . . . . . . . 13

3.2 .2 Upgrade features of the reference designs $\ldots \ldots \ldots \ldots \ldots \ldots \ldots \ldots$

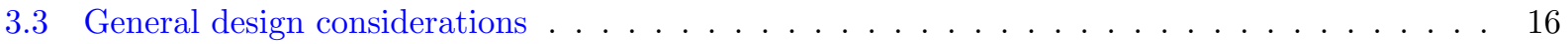

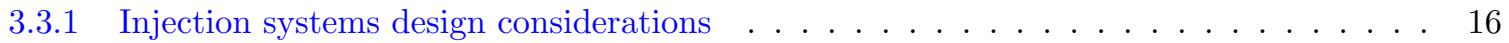

3.3.1.1 Electron Source . . . . . . . . . . . . . . . . . . . . . 16

3.3.1.1.1 Polarized electron source gun system . . . . . . . . . . . . 16

3.3.1.1.2 Buncher system . . . . . . . . . . . . . . . . . . 17

3.3.1.1.3 Pre-accelerator system _. . . . . . . . . . . . . . . 17

3.3.1.1.4 Diagnostics section . . . . . . . . . . . . . . 17

3.3.1.1.5 The cold option unpolarized injector . . . . . . . . . . . . 17

3.3.1.1.6 Electron injection linac . . . . . . . . . . . . . 18

3.3.1.1.7 Diagnostics, bunch compression, and spin rotation prior to Damping

Ring Injection . . . . . . . . . . . . . . . . 18

3.3 .1 .2 Positron Source . . . . . . . . . . . . . . . . . . . . . 18

3.3.1.2.1 Undulator positron source system . . . . . . . . . . . . 18 
3.3.1.2.1.1 The electron linac undulator bypass insertion . . . . . . . . . 19

3.3.1.2.1.2 Undulator . . . . . . . . . . . . . . . . . . . . . . . 19

3.3.1.2.2 Conventional positron source driver . . . . . . . . . . . . . . . 19

3.3.1.2.3 Positron Production Target, Capture region and Positron Pre Accelerator ....................... 20

3.3.1.2.4 Positron Production Target . . . . . . . . . . . . . . . . . 20

3.3.1.2.5 Capture and matching system . . . . . . . . . . . . . . 21

3.3.1.2.6 Positron Pre-accelerator $(\mathrm{PPA}) \ldots \ldots \ldots . \ldots \ldots 22$

3.3.1.2.7 Positron Injector Linac (PIL) . . . . . . . . . . . . . . . . . . 22

3.3.1.2.8 Positron Transfer line . . . . . . . . . . . . . . . . . . . . 22

3.3.1.2.9 Diagnostics, energy compression, and spin rotation prior to Damping Ring Injection . . . . . . . . . . . . . . . . . 23

3.3.1.2.10 Conclusions . . . . . . . . . . . . . . . . . . . . 23

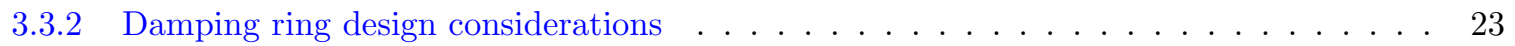

3.3.2.1 Bunch train length and ring circumference . . . . . . . . . . . . 24

3.3.2.2 Horizontal emittance and ring energy . . . . . . . . . . . . . . . 25

3.3 .2 .3 Vertical emittance and beam jitter . . . . . . . . . . . . . . 26

3.3.2.4 Longitudinal emittance (bunch length and energy spread) . . . . . . . . . . 27

3.3.2.5 Machine rep rate, damping time and damping wiggler . . . . . . . . . . . 27

3.3 .2 .6 Acceptance . . . . . . . . . . . . . . . . . . . . 28

3.3 .2 .7 Vacuum System . . . . . . . . . . . . . . . . . . . . . . . . . . 28

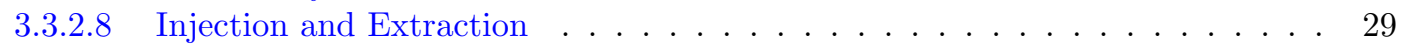

3.3.3 Main Linac RF Technology: Design Considerations and R\&D Status . . . . . . . . . . 29

3.3.3.1 Introduction . . . . . . . . . . . . . . . . . . . 29

3.3.3.2 RF Frequency Choice . . . . . . . . . . . . . . . . . . 30

3.3.3.3 Cavities and Structures . . . . . . . . . . . . . . . . . . . 31

3.3.3.4 Wakefield Suppression . . . . . . . . . . . . . . . . . . . . . 32

3.3.3.5 Modulators . . . . . . . . . . . . . . . . . . . 33

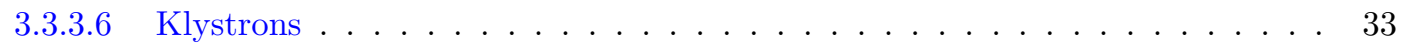

3.3 .3 .7 RF Distribution . . . . . . . . . . . . . . . . . 34

3.3 .3 .8 Low Level RF . . . . . . . . . . . . . . . . . . . . 35

3.3.4 Low emittance transport system design considerations . . . . . . . . . . . . . . 35

3.3 .5 Beam delivery system design considerations . . . . . . . . . . . . . . . . . 40

3.4 Normal-conducting X-Band Linear Collider . . . . . . . . . . . . . . . . . . . . . . 42

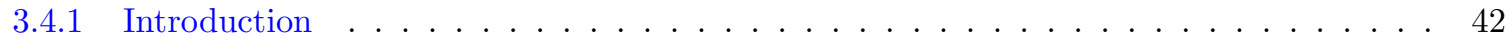

3.4.1.1 Overall Parameters . . . . . . . . . . . . . . . . . 43

3.4.1.2 Accelerator System Reference Designs . . . . . . . . . . . . . . . . . . 45

3.4.1.2.1 Electron Source . . . . . . . . . . . . . . . . . 45

3.4.1.2.2 Positron Source . . . . . . . . . . . . . . . . . 45

3.4.1.2.3 Damping Rings and Bunch Compressors . . . . . . . . . . . . 46

3.4.1.2.4 Main Linac . . . . . . . . . . . . . . . . . . . . 46

3.4.1.2.5 Beam delivery system . . . . . . . . . . . . . . 47

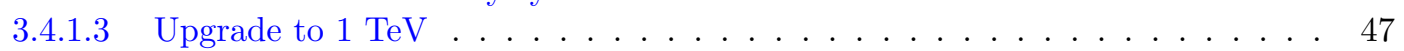

3.4 .2 Injection Systems . . . . . . . . . . . . . . . . . . . . . . 47

3.4.2.1 Electron Injection System . . . . . . . . . . . . . . . . . . . 47

3.4.2.1.1 Electron Sources . . . . . . . . . . . . . . . . . . 48

3.4.2.1.1 Laser system . . . . . . . . . . . . . . . . . 48

3.4.2.1.1.2 High voltage photocathode gun . . . . . . . . . . . 48

3.4.2.1.3 Beam Bunching System . . . . . . . . . . . . . . . . 51

3.4.2.1.1.4 S-band Electron Booster Linac . . . . . . . . . . . . . . 51

3.4.2.1.1.5 Instrumentation . . . . . . . . . . . . . . 52

3.4.2.2 Positron Injection System . . . . . . . . . . . . . . . . . . . . 52 
3.4.2.2.1 Positron Source Layout . . . . . . . . . . . . . . . . . . . . . . . 54

3.4.2.2.2 Undulator Insertion . . . . . . . . . . . . . . . . . . . 55

3.4 .2 .2 .3 Undulator . . . . . . . . . . . . . . . . . . . . . 58

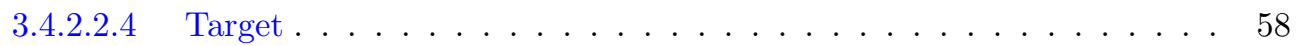

3.4.2.2.5 Positron Yield . . . . . . . . . . . . . . . . . . . . 59

3.4.2.2.6 Positron Capture Region and Pre-accelerator . . . . . . . . . . . 59

3.4.2.2.7 Positron Booster Linac (PBL) . . . . . . . . . . . . . . . . 61

3.4.2.2.8 High Energy Positron Transfer Line . . . . . . . . . . . . . . . . 62

3.4.2.2.9 Polarization control, Spin rotation, Energy compressor and Damping ring injection . . . . . . . . . . . . . . . . . 62

3.4 .3 Damping Rings . . . . . . . . . . . . . . . . . . . . . . . . . . 62

3.4.3.1 Functional and Layout Description . . . . . . . . . . . . . . . . . 62

3.4.3.1.1 Electron Damping Ring System . . . . . . . . . . . . . . . . 63

3.4.3.1.2 Positron Damping Ring System _. . . . . . . . . . . . . . . 64

3.4.3.2 Main Damping Rings . . . . . . . . . . . . . . . . . . . 65

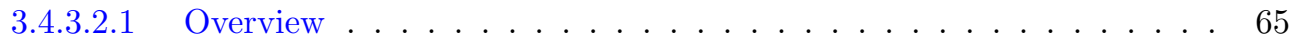

3.4.3.2.2 Lattice . . . . . . . . . . . . . . . . . . 66

3.4.3.2.2.1 Design margins . . . . . . . . . . . . . 66

3.4.3.2.3 Technical components . . . . . . . . . . . . . . . . 66

3.4.3.2.3.1 RF System . . . . . . . . . . . . . . 66

3.4.3.2.3.2 Magnet System . . . . . . . . . . . . . . . . . . . . . . . . . . . 71

3.4.3.2.3.3 Wiggler . . . . . . . . . . . . . . . . 71

3.4.3.2.3.4 Vacuum System . . . . . . . . . . . . . . . 72

3.4.3.2.3.5 Injection and Extraction . . . . . . . . . . . . . . 72

3.4.3.2.3.6 Circumference Correction Chicane . . . . . . . . . . . . 73

3.4.3.2.3.7 Diagnostics and Correction . . . . . . . . . . . . . . 73

3.4.3.2.3.8 Installation . . . . . . . . . . . . . . . . . . 74

3.4.3.3 Positron Pre-damping ring . . . . . . . . . . . . . . . . . . . 74

3.4.3.3.1 Overview . . . . . . . . . . . . . . . . . . 74

3.4 .3 .3 .2 Lattice . . . . . . . . . . . . . . . . . . . . 74

$3.4 .3 .3 .3 \quad$ RF cell . . . . . . . . . . . . . . . . . . . 75

3.4 .3 .3 .4 Chicanes . . . . . . . . . . . . . . . . 75

3.4.3.3.5 Injection/Extraction Systems . . . . . . . . . . . . . . 75

3.4.3.3.6 Technical components . . . . . . . . . . . . . . . . 78

3.4.3.3.6.1 RF System . . . . . . . . . . . . . . 78

3.4.3.3.6.2 Magnet System . . . . . . . . . . . . . . . 78

3.4.3.3.6.3 Wiggler . . . . . . . . . . . . . . . . 78

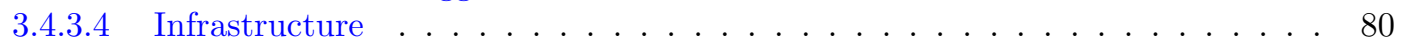

3.4.4 Bunch Compressor and pre-Linac Collimation . . . . . . . . . . . . . . . . . 81

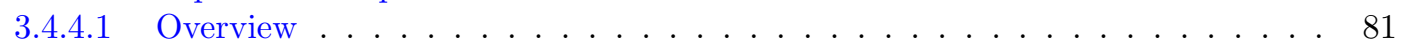

3.4.4.2 DRx: transfer line from damping ring to $\mathrm{BC} 1 \ldots \ldots \ldots$. . . . . . . . 84

3.4.4.2.1 Diagnostic section . . . . . . . . . . . . . . . . . . 84

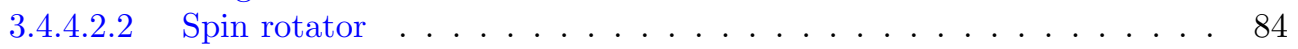

3.4.4.2.3 Coupling correction section _ . . . . . . . . . . . . 87

3.4.4.3 Bunch Compressor BC-1 . . . . . . . . . . . . . . . . . 87

3.4.4.3.1 Layout and Functional Description . . . . . . . . . . . . . . . 87

3.4.4.3.2 Magnet system . . . . . . . . . . . . . . . . . . . . . . 89

3.4.4.3.3 Vacuum System . . . . . . . . . . . . . . . . . . . . . 89

3.4.4.3.4 Instrument System . . . . . . . . . . . . . . . . . . . . . . . 89

3.4.4.3.5 Infrastructure . . . . . . . . . . . . . . . . . 89

3.4.4.4 Pre-linacs . . . . . . . . . . . . . . . . . . . . . . . . 89

3.4.4.4.1 Layout and Functional Description . . . . . . . . . . . . . 90 
3.4.4.4.2 RF system . . . . . . . . . . . . . . . . . . . 91

3.4.4.4.3 Magnet system . . . . . . . . . . . . . . . . . . . 91

3.4.4.4.4 Vacuum System . . . . . . . . . . . . . . . . . . . 91

3.4.4.4.5 Instrument System . . . . . . . . . . . . . . . . . . . 91

3.4.4.4.6 Infrastructure . . . . . . . . . . . . . . . . . 91

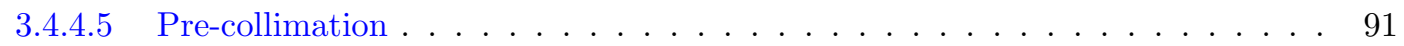

3.4.4.5.1 Layout and Functional Description . . . . . . . . . . . . . . . . 92

3.4.4.5.2 Magnet system . . . . . . . . . . . . . . . . . . . 93

3.4.4.5.3 Vacuum System . . . . . . . . . . . . . . . . . . . . . . 93

3.4.4.5.4 Instrument System . . . . . . . . . . . . . . . . . . . . . 93

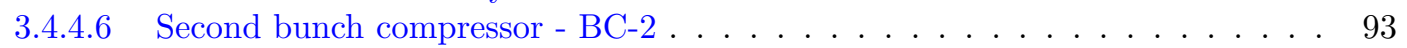

3.4.4.6.1 Layout and Functional Description . . . . . . . . . . . . . . . . 95

3.4.4.6.2 Magnet system . . . . . . . . . . . . . . . . . . . . 96

3.4.4.6.3 Vacuum System . . . . . . . . . . . . . . . . . 96

3.4.4.6.4 Instrument System . . . . . . . . . . . . . . . . . . . 96

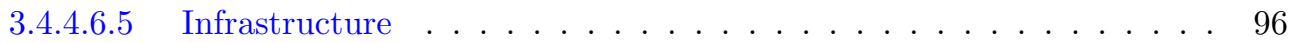

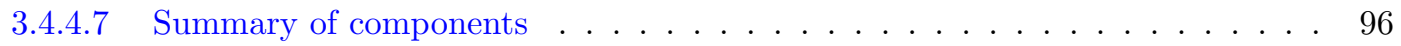

3.4 .5 Main $\operatorname{Linac} \ldots \ldots \ldots \ldots$

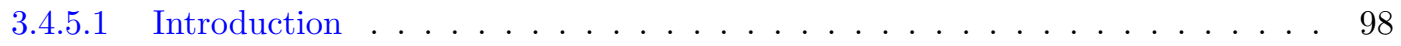

3.4 .5 .2 RF System Overview . . . . . . . . . . . . . . . . . . . 100

3.4.5.3 RFunits . . . . . . . . . . . . . . . . . . . . 100

3.4.5.3.1 Modulators and Klystrons . . . . . . . . . . . . . . 101

3.4.5.3.2 RF Distribution System . . . . . . . . . . . . . . . . . 101

3.4.5.3.3 X-Band Structures . . . . . . . . . . . . . . . . . . . . . . . 103

3.4.5.4 Optics . . . . . . . . . . . . . . . . . . . . 104

3.4.5.5 Instrumentation and feedback . . . . . . . . . . . . . . . 107

3.4.5.6 Bypass lines . . . . . . . . . . . . . . . . . . . 107

3.4.5.7 Overheads . . . . . . . . . . . . . . . . . . . . 109

3.4.6 Beam Delivery Systems . . . . . . . . . . . . . . . . . . . . . . . . . . . . . 109

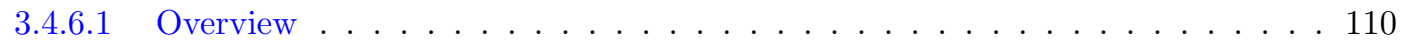

3.4 .6 .2 Layout . . . . . . . . . . . . . . . . . . . . . 110

3.4.6.3 Magnet Lattice, Optics and Subsystems . . . . . . . . . . . . . . . . . . 111

3.4.6.3.1 Second IR Design . . . . . . . . . . . . . . . . . . . 113

3.4.6.3.2 Beam Switchyard . . . . . . . . . . . . . . . . . . 113

3.4.6.3.3 Tuneup Dump Line . . . . . . . . . . . . . . . . . . . . . . . 114

3.4.6.3.4 Big Bend for IR2 . . . . . . . . . . . . . . . . . . . 116

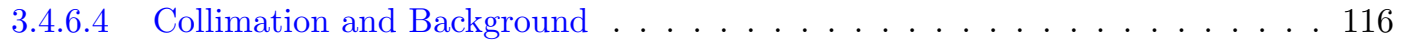

3.4.6.4.1 Design considerations . . . . . . . . . . . . . . . 118

3.4.6.5 Luminosity Performance and Stability . . . . . . . . . . . . . . . . 120

3.4.6.5.1 IP Collision Feedback . . . . . . . . . . . . . . . . . . . . 120

3.4.6.5.2 Instrumentation and Correction . . . . . . . . . . . . . . . 121

3.4.6.6 Machine Detector Interface . . . . . . . . . . . . . . . . . . . . . . . 122

3.4.6.6.1 Superconducting Doublets . . . . . . . . . . . . . . 123

3.4.6.6.2 Crab Cavities . . . . . . . . . . . . . . . . . 125

3.4.6.6.3 Forward Masking . . . . . . . . . . . . . . . . . . 125

3.4.6.6.4 Spent Beam Transport . . . . . . . . . . . . . . . . 125

3.4.6.6.5 Beam Dumps . . . . . . . . . . . . . . . . 127

3.4.6.6.6 Vacuum System . . . . . . . . . . . . . . . . 127

3.5 Superconducting L-band Linear Collider . . . . . . . . . . . . . . . . . . . . 127

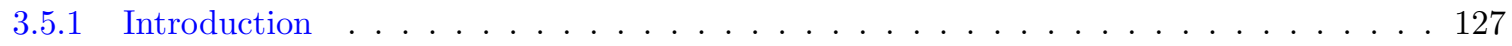

3.5.1.1 Overall Parameters . . . . . . . . . . . . . . . . 127

3.5.1.2 Accelerator System Reference Designs . . . . . . . . . . . . . . . . . 130 
3.5.1.2.1 Electron Source . . . . . . . . . . . . . . . . . . . . . . 130

3.5.1.2.2 Positron Source . . . . . . . . . . . . . . . . . . . 130

3.5.1.2.3 Damping Rings and Bunch Compressors . . . . . . . . . . . . . 130

3.5.1.2.4 Main Linac . . . . . . . . . . . . . . . . . . . . . 131

3.5.1.2.5 Beam delivery system . . . . . . . . . . . . . . . . . . . 131

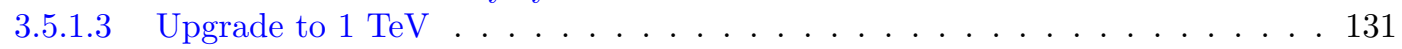

3.5 .2 Injection Systems . . . . . . . . . . . . . . . . . . . . . . . . 132

3.5.2.1 Electron Injection System . . . . . . . . . . . . . . . . . . . 132

3.5.2.1.1 Electron Sources . . . . . . . . . . . . . . . . . . . . . 132

3.5.2.1.1.1 Unpolarized source and pre-accelerator . . . . . . . . . . . 132

3.5.2.1.1.2 Polarized source and pre-accelerator . . . . . . . . . . . 133

3.5.2.1.2 Electron injector linac . . . . . . . . . . . . . . . . . . . . . . 134

3.5.2.2 Positron Injection System . . . . . . . . . . . . . . . . . . . . . 135

3.5.2.2.1 Positron Source Layout . . . . . . . . . . . . . . . . . 136

3.5.2.2.2 Undulator Insertion . . . . . . . . . . . . . . . . . . . . 138

3.5.2.2.3 Undulator . . . . . . . . . . . . . . . . . . . . . . . . . . 139

3.5.2.2.4 Target. . . . . . . . . . . . . . . . . . . 142

3.5.2.2.5 Positron Yield . . . . . . . . . . . . . . . . . . . . . 143

3.5.2.2.6 Positron Capture Region and Pre-accelerator . . . . . . . . . . . . . 144

3.5.2.2.7 Positron Injector Linac (PIL) . . . . . . . . . . . . . . . . . 145

3.5.2.2.8 High energy positron Transfer line . . . . . . . . . . . . . . . . 145

3.5.2.2.9 Polarization control, Spin rotation, Energy compressor and Damp-

ing ring injection . . . . . . . . . . . . . . 146

3.5.3 Damping Rings . . . . . . . . . . . . . . . . . . . . . . . . . 146

3.5.3.1 Functional and Layout Description . . . . . . . . . . . . . . . . . 146

3.5.3.2 Lattice and Magnet Systems . . . . . . . . . . . . . . . . . . 149

3.5.3.2.1 Lattice . . . . . . . . . . . . . . . . . . . . . . . 149

3.5.3.2.1.1 Arc lattice . . . . . . . . . . . . . . . . . . 149

3.5.3.2.1.2 Wiggler sections . . . . . . . . . . . . . . . . . 149

3.5.3.2.1.3 Long straight sections . . . . . . . . . . . . . . . 150

3.5.3.2.2 Magnets and power supplies . . . . . . . . . . . . . 150

3.5.3.2.2.1 Magnets . . . . . . . . . . . . . . . . 150

3.5.3.2.2.2 Magnet power supplies _. . . . . . . . . . . 150

3.5.3.2.3 Wigglers . . . . . . . . . . . . . . . . . 150

3.5.3.3 Injection and Extraction Systems _ . . . . . . . . . . . . . 151

3.5.3.4 RF Systems . . . . . . . . . . . . . . . . . . . . . . . 152

3.5.3.5 Vacuum Systems . . . . . . . . . . . . . . . . . . . . . 152

3.5.3.6 Infrastructure . . . . . . . . . . . . . . . . . . . 153

3.5.4 Bunch Compressor and Transfer to Main Linac . . . . . . . . . . . . . . . . . . 155

3.5.4.1 Introduction . . . . . . . . . . . . . . . . . . . 155

3.5.4.2 Bunch Compressor . . . . . . . . . . . . . . . . . 156

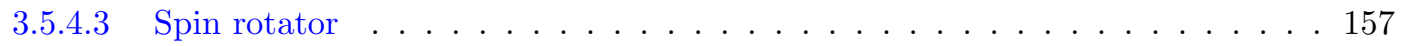

3.5.4.4 Coupling correction section . . . . . . . . . . . . . . . 158

3.5.4.5 Diagnostic and collimation section . . . . . . . . . . . . . . 159

3.5.4.6 Beamline Geometry . . . . . . . . . . . . . . . . . . 159

3.5.4.7 Summary of components . . . . . . . . . . . . . . . . 159

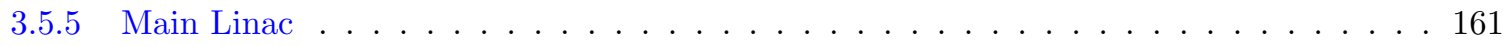

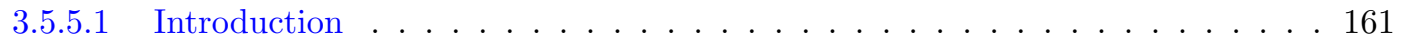

3.5.5.2 Cryomodules . . . . . . . . . . . . . . . . . . . . 162

3.5.5.2.1 Superconducting RF Cavities . . . . . . . . . . . . . . . 162

3.5.5.2.2 Cryostat . . . . . . . . . . . . . . . 162

3.5.5.2.3 Quadrupoles and Correction Magnets . . . . . . . . . . . . . 165 
3.5.5.2.4 Cryomodule layout . . . . . . . . . . . . . . 166

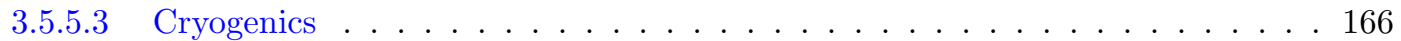

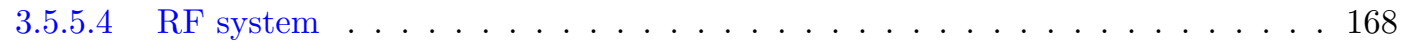

3.5.5.4.1 Klystrons and modulators . . . . . . . . . . . . . 168

3.5.5.4.2 Waveguide distribution system . . . . . . . . . . . . . . . 169

3.5.5.4.3 Low level RF . . . . . . . . . . . . . . . . . . . . . . 169

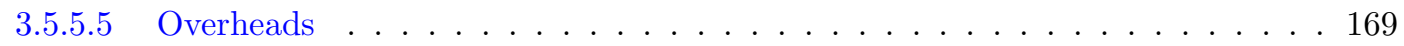

3.5.5.6 Instrumentation and feedback . . . . . . . . . . . . . . . 171

3.5.5.7 Upgrade to a beam energy of $500 \mathrm{GeV} \ldots \ldots \ldots \ldots$. . . . . . . . . 171

3.5.5.7.1 Cryomodules . . . . . . . . . . . . . . . . 171

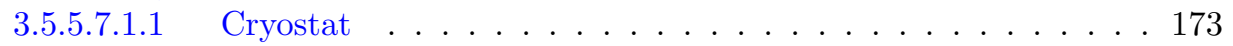

3.5.5.7.1.2 Cryomodule layout . . . . . . . . . . . . . . . 173

3.5.5.7.2 Cryogenics . . . . . . . . . . . . . . . . . 174

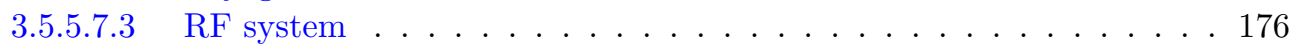

3.5.5.7.4 Waveguide distribution system . . . . . . . . . . . 176

3.5 .6 Beam Delivery Systems … . . . . . . . . . . . . . . . . . . 178

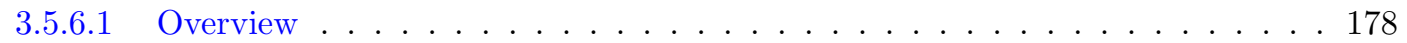

3.5.6.2 Magnet Lattice and Optics . . . . . . . . . . . . . . . . . . . . 178

3.5.6.3 Collimation Systems . . . . . . . . . . . . . . . . . . . . . . . . 179

3.5.6.4 Tuneup and Fast Extraction Beam Line . . . . . . . . . . . . . . . . . . 180

3.5.6.4.1 Fast Extraction . . . . . . . . . . . . . . . . . . 182

3.5.6.4.2 Tuneup . . . . . . . . . . . . . . . . . . . . 182

3.5.6.5 Final Focus . . . . . . . . . . . . . . . . . . . . . 182

3.5.6.5.1 Superconducting Doublets . . . . . . . . . . . . . . 182

3.5.6.5.2 Crab Cavities . . . . . . . . . . . . . . . . . 183

3.5.6.5.3 Detector Interface . . . . . . . . . . . . . . . . . . . . . . . 183

3.5.6.6 IP Feedback Systems ． . . . . . . . . . . . . . . . . . . . . . 184

3.5.6.7 Spent Beam Transport . . . . . . . . . . . . . . . . . . . . . . . . . . . . 184

3.5.6.8 Beam Dumps . . . . . . . . . . . . . . . . . . . . . . . 184

3.5.6.9 Vacuum System . . . . . . . . . . . . . . . . . . . . . 185

3.5.7 Infrastructure and Auxiliary Systems . . . . . . . . . . . . . . . . . . 185

3.5.7.1 Site layout and civil construction . . . . . . . . . . . . . . . 185

3.5.7.1.1 Overall layout . . . . . . . . . . . . . . . . 185

3.5.7.1.2 Underground construction . . . . . . . . . . . . . 186

3.5.7.1.2.1 Main Linac tunnel layout . . . . . . . . . . . . . . . 186

3.5.7.1.3 Surface buildings . . . . . . . . . . . . . . . . . 187

3.5.7.2 Radiation safety . . . . . . . . . . . . . . . . . . 187

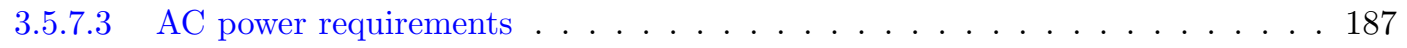

3.5.7.4 Water and HVAC . . . . . . . . . . . . . . . . . 187

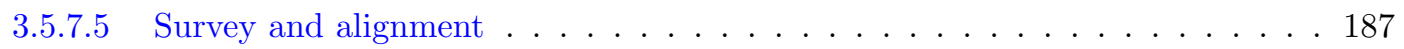

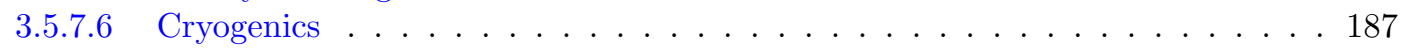

3.5.7.6.1 Cryogenic heat loads and cryogenic plant requirements . . . . . 187

3.5.7.6.1.1 $500 \mathrm{GeV} \mathrm{cm} \mathrm{operation} \mathrm{.} \mathrm{.} \mathrm{.} \mathrm{.} \mathrm{.} \mathrm{.} \mathrm{.} \mathrm{.} \mathrm{.} \mathrm{.} \mathrm{.} \mathrm{.} 187$

3.5.7.6.1.2 $1 \mathrm{TeV}$ cm operation . . . . . . . . . . . . 187

3.5.7.6.2 Cryogenic plant design specifications . . . . . . . . . . . . . . . 189

3.5.7.6.3 Cryogenic plant optimization . . . . . . . . . . . . . . . 194

3.5.7.7 Accelerator module test facility . . . . . . . . . . . . . . . . . . . 198

3.5.7.8 Global control system . . . . . . . . . . . . . . . . . . 198

4 Availability Design $\quad 200$

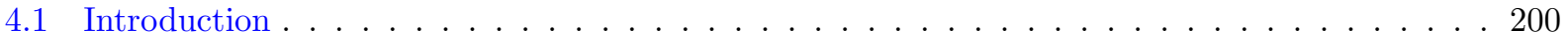

4.2 Method . . . . . . . . . . . . . . . . . . . . . . . 201 


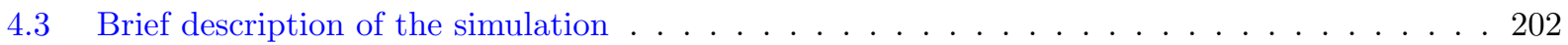

4.3.1 Handling Complex Redundancies . . . . . . . . . . . . . . . . . . . . 202

4.3 .2 Downtime planning . . . . . . . . . . . . . . . . . . . . . 203

4.3 .3 Recovery times . . . . . . . . . . . . . . . . . . . . . 203

4.3 .4 Lumped Systems . . . . . . . . . . . . . . . . . . . . . . . . 204

4.3.5 Machine Development . . . . . . . . . . . . . . . . . . . . 204

4.3.6 Important assumptions that effect availability . . . . . . . . . . . . . . . . 206

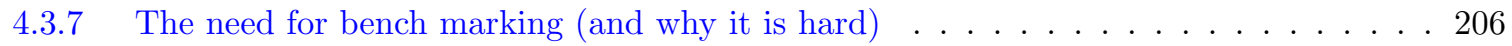

4.4 Results . . . . . . . . . . . . . . . . . . . . . . . . 207

4.4.1 Simulations of the warm and cold reference designs, the cold 1 tunnel variant, and the conventional positron source variant . . . . . . . . . . . . . . . 208

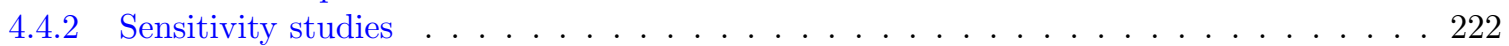

4.4 .3 Estimates of repair personnel . . . . . . . . . . . . . . . . . . . . . 224

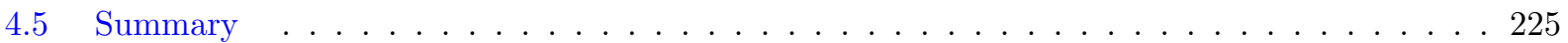

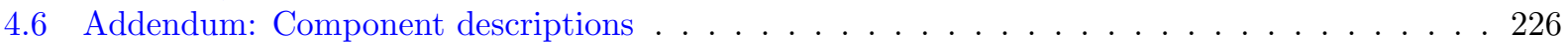

5 Cost and Schedule 233

5.1 Goals, Strategy, and Overview _. . . . . . . . . . . . . . . . 233

5.2 Technical Models and Cost Estimation . . . . . . . . . . . . . . . . . . 234

5.2 .1 Work Breakdown Structure . . . . . . . . . . . . . . . . . . . . . . . . 234

5.2.1.1 General Considerations . . . . . . . . . . . . . . . . . 234

5.2 .1 .2 Structuring the WBS . . . . . . . . . . . . . . . . . . . 234

5.2 .1 .3 Populating the WBS . . . . . . . . . . . . . . . . . . 235

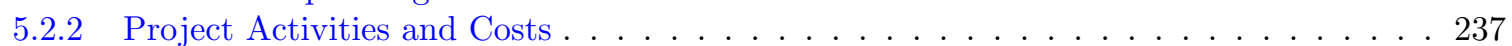

5.2.2.1 Management Services . . . . . . . . . . . . . . . . . . . . . . 237

5.2 .2 .2 Technical Services . . . . . . . . . . . . . . . . . . . . . 238

5.2.2.3 Composite Site-Wide Civil Construction . . . . . . . . . . . . . . . . . 239

5.2.2.4 Accelerator Physics and Pre-Operations . . . . . . . . . . . . . . . 239

5.2 .3 Injector Complex - Sources . . . . . . . . . . . . . . . . . . . . . . 239

5.2 .4 Beam Delivery . . . . . . . . . . . . . . . . . . . . . . . 239

5.2 .5 Damping Rings . . . . . . . . . . . . . . . . . . . . . . . . 240

5.2.5.1 Magnets (excluding wigglers) . . . . . . . . . . . . . . . 241

5.2 .5 .2 Magnet Power Supplies . . . . . . . . . . . . . . . . . . . . . . . 241

5.2 .5 .3 Damping wigglers . . . . . . . . . . . . . . . . . . . . . 242

5.2 .5 .4 Vacuum System . . . . . . . . . . . . . . . . . . . . 243

$5.2 .5 .5 \quad$ RF System . . . . . . . . . . . . . . . . . . . . . . . 243

5.2 .5 .6 Diagnostics and Controls . . . . . . . . . . . . . . . 243

5.2.5.7 Assembly and Installation . . . . . . . . . . . . . . . . . . . . 243

$5.2 .6 \quad$ Main Linac . . . . . . . . . . . . . . . . . . . . . . . . . . . . . 244

5.2.6.1 RF Sources (Including Distribution) . . . . . . . . . . . . . . . . . . 244

5.2.6.1.1 Low Level RF (LLRF) System . . . . . . . . . . . . . . . . . . 244

5.2 .6 .1 .2 Klystron . . . . . . . . . . . . . . . . . . . . . . . 244

5.2.6.1.3 Klystron Drivers . . . . . . . . . . . . . . . . . . . . . . 245

5.2.6.1.4 Modulator . . . . . . . . . . . . . . . . . 245

5.2.6.2 RF Distribution and Cavities . . . . . . . . . . . . . . . . 245

$5.2 .6 .2 .1 \quad$ RF Distribution . . . . . . . . . . . . . . . . . . 245

5.2.6.2.2 Accelerator Modules . . . . . . . . . . . . . . . . . . 246

5.2.6.2.2.1 Accelerator Structure Metal . . . . . . . . . . . . . . 246

5.2.6.2.2.2 Accelerator Structure Cavities . . . . . . . . . . . . . 246

5.2.6.2.2.3 Accelerator Structures Couplers . . . . . . . . . . . . . 247

5.2.6.2.2.4 Cavity String Assembly . . . . . . . . . . . . . . . . 247

5.2.6.2.2.5 Cryomodule/Girder Assembly . . . . . . . . . . . . 247 
5.2.6.3 Cryogenic Systems . . . . . . . . . . . . . . . . . . . . . 247

5.2 .7 Conventional Construction . . . . . . . . . . . . . . . . . . . . 253

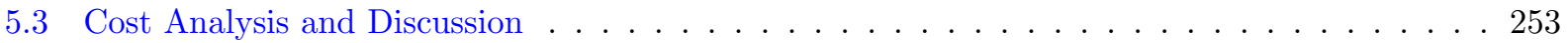

5.3 .1 Analysis of Cost Compilation . . . . . . . . . . . . . . . . . 253

5.3 .2 Discussion . . . . . . . . . . . . . . . . . . . . . . . . 258

5.4 Project Models and Schedules . . . . . . . . . . . . . . . . . . . . . 262

5.4 .1 Preconstruction Activities . . . . . . . . . . . . . . . . . . . 262

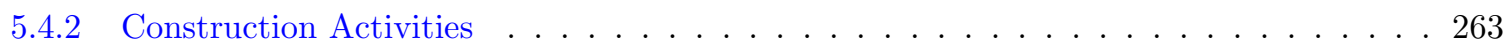

5.4 .3 Commissioning Activities . . . . . . . . . . . . . . . . . . . . . 267

5.4 .4 Summary of Schedule-related Risks . . . . . . . . . . . . . . . . . . . . . . . 268

5.5 Cost and Schedule for Upgrade and Variants . . . . . . . . . . . . . . . . . . 274

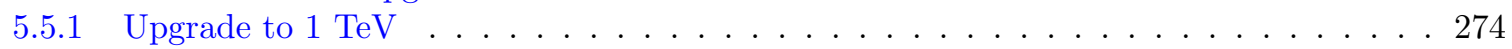

5.5 .2 One Tunnel for the Cold Option . . . . . . . . . . . . . . . . . . . . . . . 274

5.5.3 Initial Gradient of $35 \mathrm{MV} / \mathrm{m}$ for Cold Option . . . . . . . . . . . . . . . . . . . 274

5.5 .4 Superstructure for Cold Option . . . . . . . . . . . . . . . . . . . . 274

5.5 .5 DLDS for Warm Option . . . . . . . . . . . . . . . . . . . 275

5.5.6 Conventional Positron Source . . . . . . . . . . . . . . . . . . . . 275

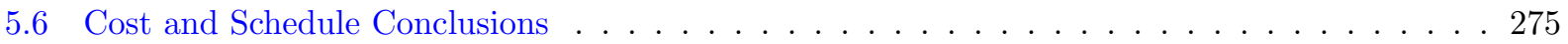

6 Civil Construction and Siting $\quad \mathbf{2 7 7}$

6.1 Introduction and overview . . . . . . . . . . . . . . . . . 277

6.2 Conventional Facilities Design Summaries and Drawings for the Normal-conducting X-band

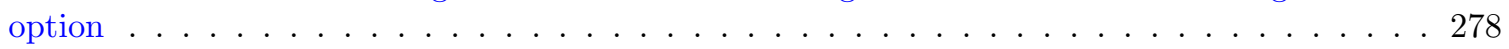

6.2.1 Conventional Facilities Reference Design Summary for a Normal-conducting X-band

Linear Collider in California . . . . . . . . . . . . . . . . . . 278

6.2.1.1 Configuration and Design Features . . . . . . . . . . . . . . . 278

6.2.1.1.1 Purpose . . . . . . . . . . . . . . . . . . 278

6.2 .1 .1 .2 Configuration . . . . . . . . . . . . . . . 279

6.2.1.1.3 Design Features . . . . . . . . . . . . . . . . . . 280

6.2.1.2 Major Components . . . . . . . . . . . . . . . . . . 280

6.2.1.2.1 Local Campus . . . . . . . . . . . . . . . . . . . . 280

6.2 .1 .2 .2 Injectors . . . . . . . . . . . . . . . . . . . . . 280

$6.2 .1 .2 .3 \quad$ Main Linac . . . . . . . . . . . . . . . . . . . . . . . 280

6.2 .1 .2 .4 Beam Delivery . . . . . . . . . . . . . . . . . . 280

6.2 .1 .2 .5 Interaction Regions . . . . . . . . . . . . . . . . . 280

6.2.1.2.6 Conventional Utilities . . . . . . . . . . . . . . . . . . . . 281

6.2.1.3 Electrical Power: Availability, Configuration and Demand . . . . . . . . . . . 281

6.2.1.4 Heat Rejection . . . . . . . . . . . . . . . . . . . . . . . . . 282

6.2.1.4.1 Overall System Description . . . . . . . . . . . . . . . . . . 282

6.2.1.4.2 LCW for Klystrons, Modulators, Magnets and Racks . . . . . . . . 282

6.2.1.4.3 LCW for Accelerator Sections and RF Pulse Compression . . . . . . 282

6.2.1.4.4 Beam Tunnel Operating States . . . . . . . . . . . . . . . . . . 283

6.2.1.4.5 Beam Tunnel Ventilation . . . . . . . . . . . . . . . . . . . . 283

6.2.1.4.6 Support Tunnel Ventilation . . . . . . . . . . . . . . . . . . . 283

6.2.1.4.7 Tunnel Drainage . . . . . . . . . . . . . . . . . . . 283

6.2 .1 .5 Vibration . . . . . . . . . . . . . . . . . . . 283

6.2.1.5.1 Mechanical Noise . . . . . . . . . . . . . . . . . . . 283

6.2.1.5.2 Experimental Region Noise and Vibration Isolation . . . . . . . . 283

6.2.1.5.3 Near Field Vibration . . . . . . . . . . . . . . . . . . . . . 284

6.2.1.5.4 Far Field Vibration Measurements . . . . . . . . . . . . . . . . . 284

6.2.1.5.5 Alignment Smoothing Interval . . . . . . . . . . . . . . . . . 284

6.2.1.6 Geology and Tunneling Means and Methods . . . . . . . . . . . . . . . . . . 284 


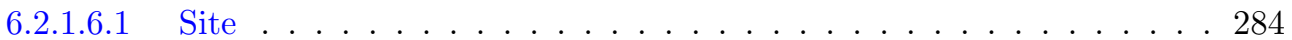

6.2.1.6.2 Rock Spoils . . . . . . . . . . . . . . . . . . . . . . 285

6.2.1.6.3 Construction Means and Methods . . . . . . . . . . . . . . . 285

6.2.2 Conventional Facilities Reference Design Summary for a Normal-conducting X-band

Linear Collider in Illinois . . . . . . . . . . . . . . . . . . . . 285

6.2.2.1 Configuration and Salient Design Features . . . . . . . . . . . . . . . 285

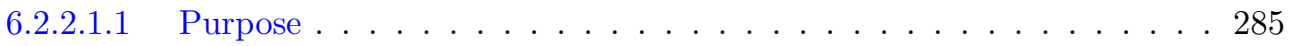

$6.2 .2 .1 .2 \quad$ Proposed Facilities . . . . . . . . . . . . . . . . . . 286

6.2.2.1.3 Siting . . . . . . . . . . . . . . . . 287

6.2.2.1.4 Salient Design Features . . . . . . . . . . . . . . . . . 287

6.2.2.2 Description of Major Components . . . . . . . . . . . . . . . . . 287

6.2.2.2.1 Site Development . . . . . . . . . . . . . . . . . . . . 287

6.2.2.2.2 Surface Buildings . . . . . . . . . . . . . . . . . . . 287

6.2.2.2.2.1 Central Campus . . . . . . . . . . . . . . 287

6.2.2.2.2.2 LCW Surface Plant Buildings . . . . . . . . . . . . . . . 288

6.2.2.2.3 Underground Structures . . . . . . . . . . . . . . . . . . . . . 288

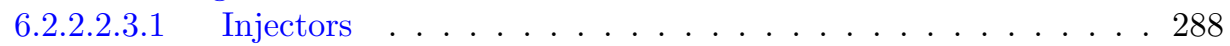

6.2.2.2.3.2 Damping Rings . . . . . . . . . . . . . . . . . 288

$6.2 .2 .2 .3 .3 \quad$ Main Linac . . . . . . . . . . . . . . . . . . . . . 288

6.2.2.2.3.4 Beam Delivery . . . . . . . . . . . . . . . 288

6.2.2.2.3.5 Interaction Regions . . . . . . . . . . . . . . . . . 289

6.2.2.3 Electrical Power . . . . . . . . . . . . . . . . . . . . . . . . . . . . 289

$6.2 .2 .3 .1 \quad$ Availability . . . . . . . . . . . . . . . . . . . 289

6.2.2.3.2 Description . . . . . . . . . . . . . . . . . . . . . 289

6.2.2.3.3 Configuration and Demand . . . . . . . . . . . . . . . 289

6.2.2.4 Heat Rejection . . . . . . . . . . . . . . . . . . . . . . . . 290

6.2.2.4.1 Project Requirements . . . . . . . . . . . . . . . . . . . . . . 290

6.2.2.4.2 LCW (Low Conductivity Water) System . . . . . . . . . . . . . . 290

6.2.2.4.3 Design variations . . . . . . . . . . . . . . . . . . . . . 292

6.2.2.4.4 Ventilation (Beam Tunnel) . . . . . . . . . . . . . . . . . 292

6.2.2.4.5 Ventilation (Service Tunnel) . . . . . . . . . . . . . . . . 292

6.2.2.4.6 Drainage . . . . . . . . . . . . . . . . . . . . 292

6.2.2.5 Geology, Siting and Tunneling Means and Methods . . . . . . . . . . . . . 292

6.2 .2 .5 .1 Site . . . . . . . . . . . . . . . . . 292

$6.2 .2 .5 .2 \quad$ Spoils . . . . . . . . . . . . . . . . . 293

6.2.2.5.3 Construction Means and Methods . . . . . . . . . . . . . 293

6.2 .2 .6 Vibration . . . . . . . . . . . . . . . . . . . . . 293

6.2.2.6.1 Mechanical Noise as an Issue in site Selection . . . . . . . . . . . . 293

6.2.2.6.2 Vibration Studies . . . . . . . . . . . . . . . . . . . . 294

6.2.3 Selected Conventional Facilities Design Drawings for the Normal Conducting X-band option . . . . . . . . . . . . . . . . . . . . . . 294

6.3 Conventional Facilities Design Summaries and Drawings for the Superconducting L-band option299

6.3.1 Conventional Facilities Reference Design Summary for a Superconducting L-band Lin-

ear Collider in California . . . . . . . . . . . . . . . . . . . 299

6.3.1.1 Configuration and Design Features . . . . . . . . . . . . . . . . . . . 299

6.3.1.1.1 Purpose . . . . . . . . . . . . . . . . . . . . 299

6.3.1.1.2 Configuration . . . . . . . . . . . . . 300

6.3.1.1.3 Design Features . . . . . . . . . . . . . . . . . 300

6.3 .1 .2 Major Components . . . . . . . . . . . . . . . . . . 301

6.3.1.2.1 Local Campus . . . . . . . . . . . . . . . . . . . 301

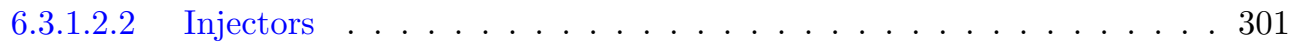

6.3 .1 .2 .3 Main Linac . . . . . . . . . . . . . . . . . . . . . . 301 
6.3.1.2.4 Beam Delivery . . . . . . . . . . . . . . . . . . . . . 301

6.3.1.2.5 Interaction Regions . . . . . . . . . . . . . . . . . . 301

6.3.1.2.6 Conventional Utilities . . . . . . . . . . . . . . . . . . . . 301

6.3.1.3 Electrical Power: Availability, Configuration and Demand . . . . . . . . . . . 302

6.3.1.4 Heat Rejection . . . . . . . . . . . . . . . . . . . . . . . 302

6.3.1.4.1 Overall System Description . . . . . . . . . . . . . . . . . 302

6.3.1.4.2 LCW for Klystrons, Modulators, Magnets and Racks . . . . . . . . 303

6.3.1.4.3 Beam Tunnel Operating States . . . . . . . . . . . . . . . . . . 303

6.3.1.4.4 Beam Tunnel Ventilation . . . . . . . . . . . . . . . . . . . 303

6.3.1.4.5 Support Tunnel Ventilation . . . . . . . . . . . . . . . . . . . . . 304

6.3.1.4.6 Tunnel Drainage . . . . . . . . . . . . . . . . . . . . . . . . 304

6.3.1.5 Vibration . . . . . . . . . . . . . . . . . . . 304

6.3.1.5.1 Mechanical Noise . . . . . . . . . . . . . . . . . . . . . 304

6.3.1.5.2 Experimental Region Noise and Vibration Isolation . . . . . . . . . 304

6.3.1.5.3 Near Field Vibration . . . . . . . . . . . . . . . . . . . . . 304

6.3.1.5.4 Far Field Vibration Measurements . . . . . . . . . . . . . . . . . . 304

6.3.1.5.5 Alignment Smoothing Interval . . . . . . . . . . . . . . . . 305

6.3.1.6 Geology and Tunneling Means and Methods . . . . . . . . . . . . . 305

6.3.1.6.1 Site . . . . . . . . . . . . . . . . . 305

6.3.1.6.2 Rock Spoils . . . . . . . . . . . . . . . . . . 306

6.3.1.6.3 Construction Means and Methods . . . . . . . . . . . . . . 306

6.3.2 Conventional Facilities Reference Design Summary for a Superconducting X-band Lin-

ear Collider in Illinois . . . . . . . . . . . . . . . . . . . . 306

6.3.2.1 Configuration and Salient Design Features . . . . . . . . . . . . . 306

6.3.2.1.1 Purpose . . . . . . . . . . . . . . . . . . . 306

6.3.2.1.2 Proposed Facilities . . . . . . . . . . . . . . . . . . . 306

6.3.2.1.3 Siting . . . . . . . . . . . . . . . . 307

6.3.2.1.4 Salient Design Features . . . . . . . . . . . . . . . . . . 307

6.3.2.2 Description of Major Components . . . . . . . . . . . . . . . . . . . . 308

6.3.2.2.1 Site Development . . . . . . . . . . . . . . . . . 308

6.3.2.2.2 Surface Buildings . . . . . . . . . . . . . . . . . . 308

6.3.2.2.2.1 Central Campus . . . . . . . . . . . . . . . 308

6.3.2.2.2.2 Cryo/LCW Surface Plant Buildings . . . . . . . . . . . . 308

6.3.2.2.3 Underground Structures . . . . . . . . . . . . . . . . . . . . 308

6.3 .2 .2 .3 .1 Injectors . . . . . . . . . . . . . . . . 308

6.3.2.2.3.2 Damping Rings . . . . . . . . . . . . . . . . . 308

6.3.2.2.3.3 Main Linac . . . . . . . . . . . . . . . . . . . . . . . 309

6.3.2.2.3.4 Beam Delivery . . . . . . . . . . . . . . . . . . . 309

6.3.2.2.3.5 Interaction Regions . . . . . . . . . . . . . . . 309

6.3.2.3 Electrical Power . . . . . . . . . . . . . . . . . . . . . . . . . 309

6.3.2.3.1 Availability . . . . . . . . . . . . . . . . . . . . 309

6.3.2.3.2 Description . . . . . . . . . . . . . . . . . . . . 310

6.3.2.3.3 Configuration and Demand . . . . . . . . . . . . . . . . . . 310

6.3.2.4 Heat Rejection . . . . . . . . . . . . . . . . . . . . . . . 310

6.3.2.4.1 Project Requirements . . . . . . . . . . . . . . . . . . . 310

6.3.2.4.2 LCW (Low Conductivity Water) System . . . . . . . . . . . . . 311

6.3.2.4.3 Design variations . . . . . . . . . . . . . . . . . . 311

6.3.2.4.4 Ventilation (Beam Tunnel) . . . . . . . . . . . . . . . 311

6.3.2.4.5 Ventilation (Service Tunnel) _ . . . . . . . . . . . . . . 313

6.3.2.4.6 Drainage . . . . . . . . . . . . . . . . . . 313

6.3.2.4.7 Cryogenic System Housing . . . . . . . . . . . . . . . . . 313

6.3.2.5 Geology, Siting and Tunneling Means and Methods . . . . . . . . . . . . . 313 


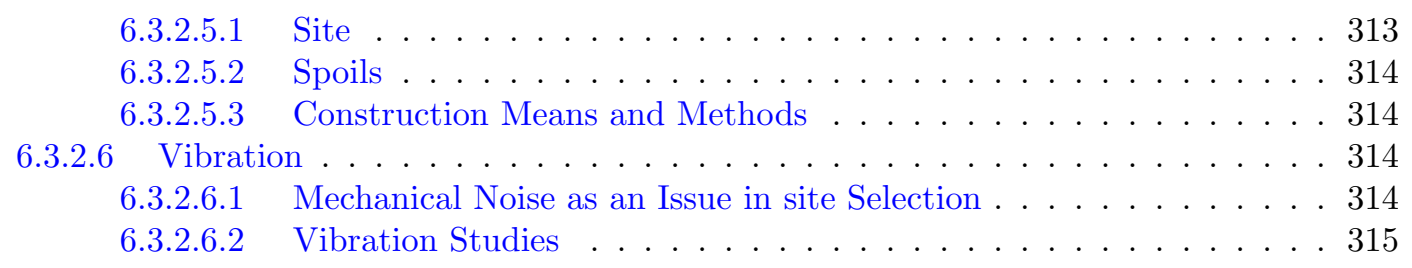

6.3.3 Selected Conventional Facilities Design Drawings for the Superconducting L-band option315

6.4 Discussion and Conclusions . . . . . . . . . . . . . . . . . . . 323

7 Design Configuration Variants $\quad 324$

7.1 Introduction . . . . . . . . . . . . . . . . . . . . . . . . . 324

7.2 Cold Option: Single tunnel architecture for the main linac . . . . . . . . . . . . . . . . 324

7.2 .1 Introduction . . . . . . . . . . . . . . . . . . . . 324

7.2 .2 Main Linac tunnel layout and discussion of assumptions . . . . . . . . . . . . . 325

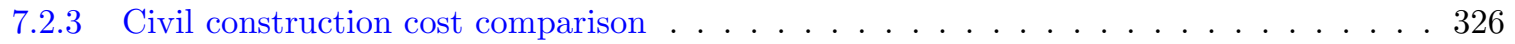

7.2 .4 Availability Considerations . . . . . . . . . . . . . . . . . . 328

7.2 .5 Main Linac infrastructure engineering . . . . . . . . . . . . . . . . . . . . 329

7.2 .6 Schedule implications . . . . . . . . . . . . . . . . . . . . . 330

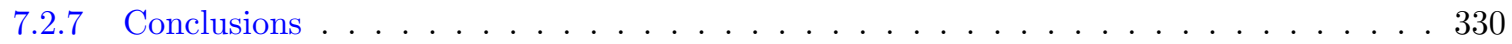

7.3 Cold Option: Initial Operation at $35 \mathrm{MV} / \mathrm{m} \ldots \ldots \ldots \ldots \ldots$

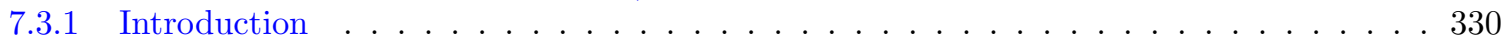

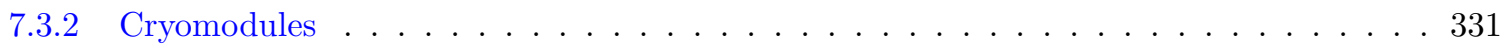

7.3 .3 Superconducting RF Cavities . . . . . . . . . . . . . . . . . . . . 331

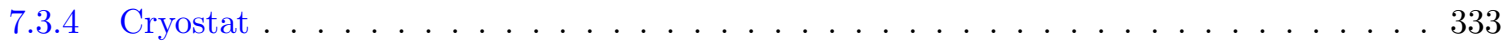

7.3.4.1 Cryomodule layout . . . . . . . . . . . . . . . . . . 333

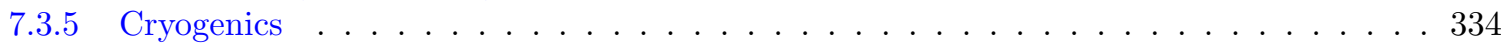

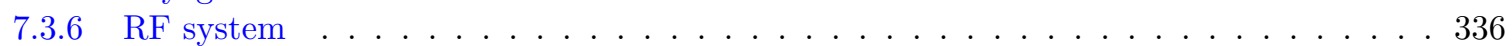

7.3.6.1 Waveguide distribution system . . . . . . . . . . . . . . . 336

7.3.6.2 Low level RF . . . . . . . . . . . . . . . . . . . . . . . 337

7.3 .7 Overheads . . . . . . . . . . . . . . . . . . . . . . . 337

7.3 .8 Overall layout . . . . . . . . . . . . . . . . . . . . . . . . . 339

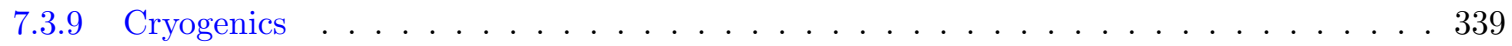

7.3.9.1 Cryogenic heat loads and cryogenic plant requirements . . . . . . . . . . 339

7.3.9.2 Cryogenic plant design specifications . . . . . . . . . . . . . . . . 339

7.3 .10 Cost implications . . . . . . . . . . . . . . . . . . . . . . 342

7.4 Warm Option: Delay Line Distribution System . . . . . . . . . . . . . . . . . . . 342

7.4 .1 Cost implications . . . . . . . . . . . . . . . . . . . . 343

7.5 Cold Option: Superstructures . . . . . . . . . . . . . . . . . . . 343

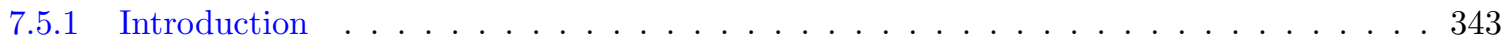

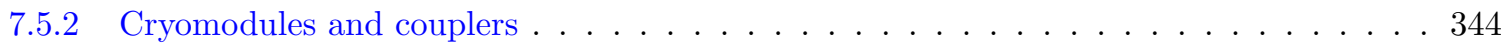

7.5 .3 Linac and site lengths . . . . . . . . . . . . . . . . . 345

7.5 .4 Superstructure R\&D . . . . . . . . . . . . . . . . . . . . 347

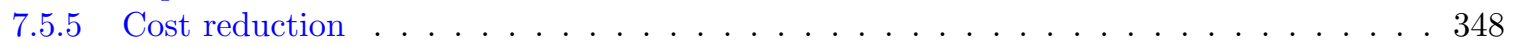

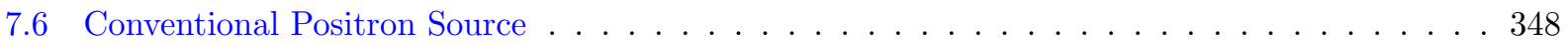

7.6 .1 Warm option . . . . . . . . . . . . . . . . . . . . 348

7.6 .2 Cold option . . . . . . . . . . . . . . . . . . . . . . . . 349

7.6 .3 Cost implications . . . . . . . . . . . . . . . . . . . . 351

7.6 .4 Availability considerations . . . . . . . . . . . . . . . . . . 351

7.6 .5 Schedule implications . . . . . . . . . . . . . . . . . . . 351

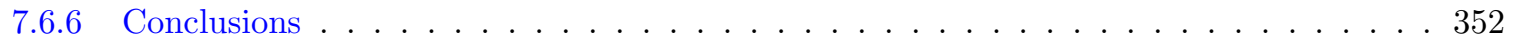

8 Risk Assessments $\quad 353$ 


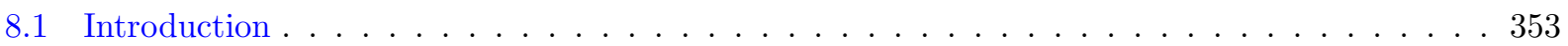

8.2 Electron and Positron Sources . . . . . . . . . . . . . . . . . . . . 355

8.2.1 Beam loading in the electron capture region limits available electron charge . . . . . 355

8.2.2 Source laser stability problems feed directly into electron beam instabilities . . . . . . 355

8.2.3 A reduced strength undulator results in reduced positron intensity for a fixed length

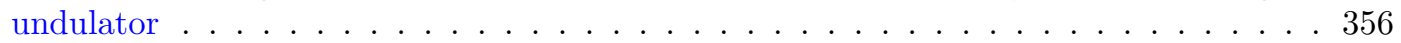

8.2.4 Low AMD performance will result in reduced positron capture efficiency . . . . . . . 356

8.2.5 Failure to achieve RF gradients in the positron capture sections will reduce positron

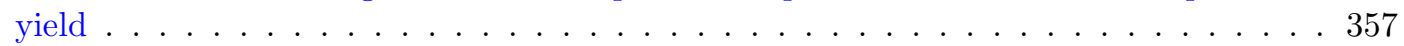

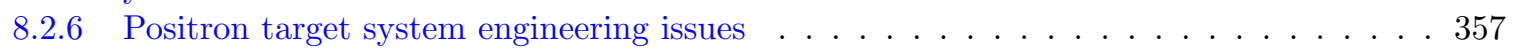

8.3 Damping Rings . . . . . . . . . . . . . . . . . . . . . . . . . 358

8.3.1 Beam acceptance limitations cause poor injection efficiency . . . . . . . . . . . 358

8.3.2 The coupling correction fails to achieve the specified vertical emittance . . . . . . . 359

8.3.3 The extracted vertical beam has large jitter . . . . . . . . . . . . . . . 360

8.3.4 The kickers fail to meet the specified performance . . . . . . . . . . . . 360

8.3.5 Stray magnetic fields cause emittance growth . . . . . . . . . . . . . . . . 361

8.3.6 Electron cloud causes beam instability in the positron damping rings . . . . . . . . . 362

8.3.7 Ion effects in the electron damping rings cause beam instabilities . . . . . . . . . . 363

8.3.8 The vacuum chamber impedance drives beam instabilities . . . . . . . . . . . . . 363

8.4 Compressors and Main Linacs: Emittance growth and beam jitter . . . . . . . . . . . . 364

8.4.1 There is excessive emittance growth in the bunch compressor/spin rotator systems. . . 364

8.4.2 There is excessive emittance growth in the main linacs . . . . . . . . . . . . 365

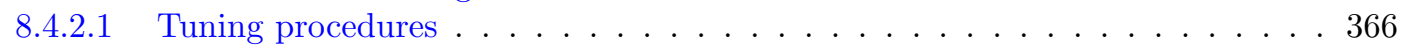

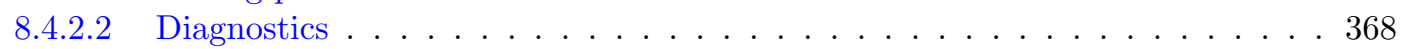

8.4 .2 .3 Main linac structures . . . . . . . . . . . . . . . . . . . . . 369

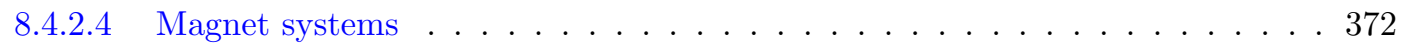

8.4.2.5 Main linac dark current wakes . . . . . . . . . . . . . . . . . . . . 373

8.4.2.6 Site characterization and civil engineering . . . . . . . . . . . . . . 374

8.4.3 There is excessive beam jitter in the main linacs . . . . . . . . . . . . . . . 375

8.4.3.1 Cultural and technical noise . . . . . . . . . . . . . . 375

8.4.3.2 Cold option main linac intratrain feedback systems . . . . . . . . . . . 376

8.5 Main Linacs: RF technology . . . . . . . . . . . . . . . . . . . . . . . . . . . 377

8.5 .1 Klystron Lifetime . . . . . . . . . . . . . . . . . . . 378

$8.5 .2 \mathrm{RF}$ distribution systems . . . . . . . . . . . . . . . . . . . 379

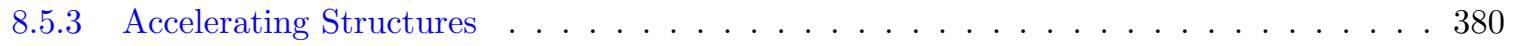

8.5 .4 Cold coupler production . . . . . . . . . . . . . . . . . . . . . 382

8.5.5 Cryogenic Heat Load capacity exceeded . . . . . . . . . . . . . . . . . . . . . . . . . . . . . . . . . . . . . . . .

8.5.6 Loss of Vacuum Integrity . . . . . . . . . . . . . . . . . . . . 383

8.5.7 Pulse-to-Pulse and Bunch-to-Bunch Energy Variations too large . . . . . . . . . . 385

8.6 Beam Delivery System . . . . . . . . . . . . . . . . . . . . . . 386

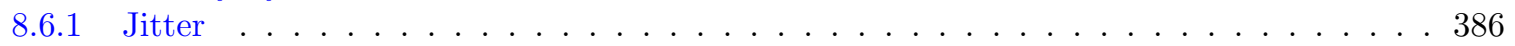

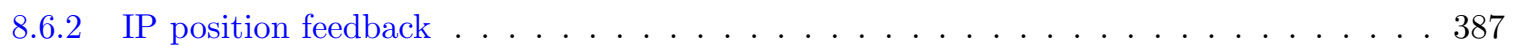

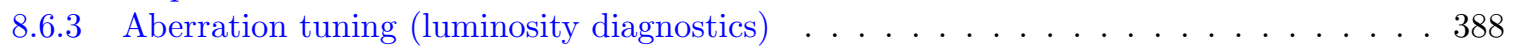

8.6.4 BDS component motion (IP jitter and aberration tuning) . . . . . . . . . . . . 389

8.6.5 High Power beams (collective/beam physics) . . . . . . . . . . . . . . . . 390

8.6.6 High Power beams (collimation, equipment damage survival and machine protection) 390

8.6 .7 High power beams (disrupted beam) . . . . . . . . . . . . . . . . . 392

8.6 .8 High power beams (detector backgrounds) . . . . . . . . . . . . . . . . 392

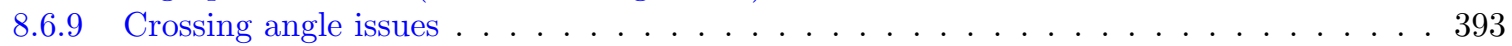

8.7 Operations and machine protection . . . . . . . . . . . . . . . . 394

8.7.1 The operation of the linear collider requires too much manual, non-automated, intervention to maintain subsystem optimization . . . . . . . . . . . . . . . . 394 
8.7.2 The machine protection system does not allow the production of steady high power

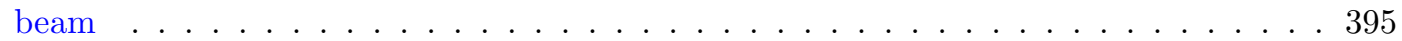

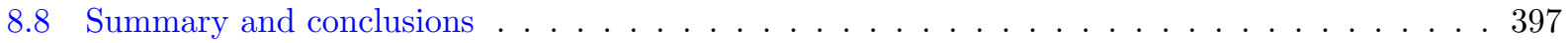

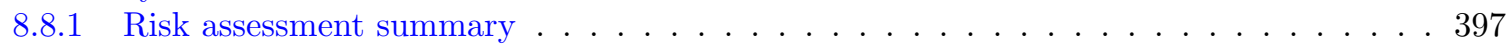

8.8 .2 Conclusions . . . . . . . . . . . . . . . . . . . . . . . . . . 398

8.8.2.1 Machine protection systems and control systems . . . . . . . . . . 398

8.8 .2 .2 Beam delivery systems . . . . . . . . . . . . . . . . . . . 399

8.8 .2 .3 Main linacs . . . . . . . . . . . . . . . . . . . . . . . . . . . 399

8.8 .2 .4 Damping Rings . . . . . . . . . . . . . . . . . . . . . . . . . . . . 400

8.8.2.5 Electron and positron sources . . . . . . . . . . . . . . . . 401

8.8.2.6 Near-term R\&D challenges . . . . . . . . . . . . . . . . . . . . . 401

9 Conclusions 403

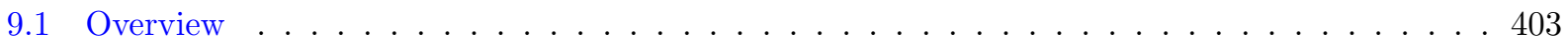

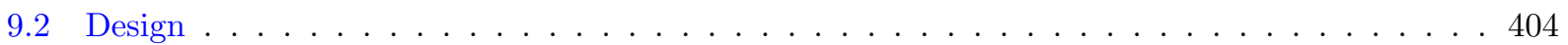

$9.2 .1500 \mathrm{GeV}$ reference designs . . . . . . . . . . . . . . . . . . . 404

9.2.2 Upgrade features of the reference designs . . . . . . . . . . . . . . . . . . 405

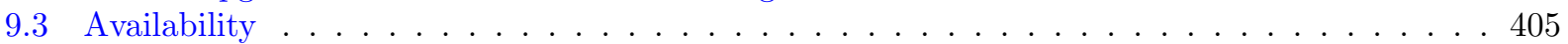

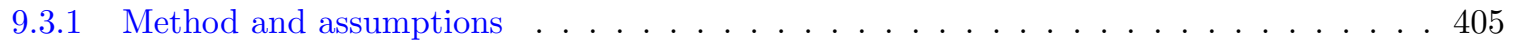

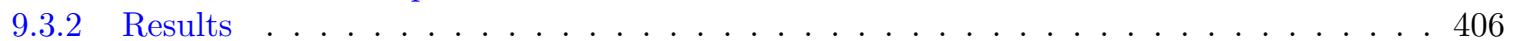

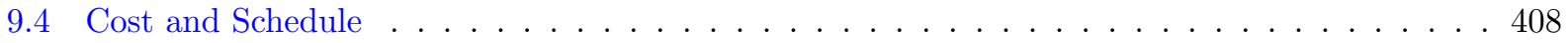

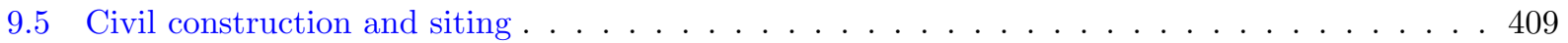

9.6 Design variants . . . . . . . . . . . . . . . . . . . . . . . 410

9.6 .1 One tunnel in cold option . . . . . . . . . . . . . . . . . . . . . 410

$9.6 .235 \mathrm{MV} / \mathrm{m}$ initial design gradient in cold option . . . . . . . . . . . . . . . 411

9.6 .3 Superstructure in cold option . . . . . . . . . . . . . . . . . . . . . 411

9.6 .4 DLDS in warm option . . . . . . . . . . . . . . . . . . . 412

9.6.5 Conventional positron sources in both options . . . . . . . . . . . . . . . . . . . . 412

9.7 Risk . . . . . . . . . . . . . . . . . . . . . . . . . . 413

9.7.1 Machine Protection . . . . . . . . . . . . . . . . . . . . . . . 414

9.7 .2 Controls . . . . . . . . . . . . . . . . . . . . . . . . . . 414

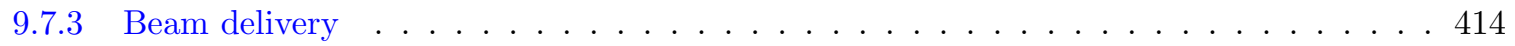

9.7 .4 Main linacs . . . . . . . . . . . . . . . . . . . . . . . . . . . . . 415

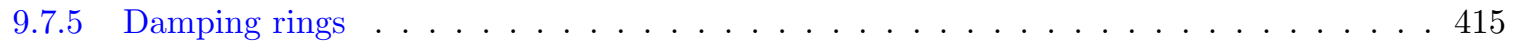

9.7 .6 Sources . . . . . . . . . . . . . . . . . . . . . . 415

9.7 .7 Near-term R\&D objectives . . . . . . . . . . . . . . . . . . . . 416

$\begin{array}{ll}\text { A Comparison of linear collider physics requirements } & 417\end{array}$

$\begin{array}{ll}\text { Bibliography } & 419\end{array}$ 


\section{List of Figures}

3.2.1.1 Luminosity roll-off with $\mathrm{cm}$ energy for the reference designs. . . . . . . . . . . . . 15

3.2.2.1 Energy versus luminosity for the upgraded $\mathrm{X}$-band collider. . . . . . . . . . . . 16

3.3.2.1 Conceptual layout of the cold positron damping ring. . . . . . . . . . . . . . . 25

3.4.1.1 Overall Machine Layout, $500 \mathrm{GeV} \mathrm{c.m.} \mathrm{.} \mathrm{.} \mathrm{.} \mathrm{.} \mathrm{.} \mathrm{.} \mathrm{.} \mathrm{.} \mathrm{.} \mathrm{.} \mathrm{.} \mathrm{.} \mathrm{.} \mathrm{.} \mathrm{.} \mathrm{.} \mathrm{.} \mathrm{.} \mathrm{.} 44$

3.4.1.2 Energy versus luminosity for the Stage II X-band collider. . . . . . . . . . . . . . . . 46

3.4.2.1 Electron Injection System layout . . . . . . . . . . . . . . . . . . . 47

3.4.2.2 Beta Functions of Electron Injection System from Gun to Damping Ring . . . . . . . . . 49

3.4.2.3 Dispersion Functions of Electron Injection System from Gun to Damping Ring . . . . . 50

3.4.2.4 Undulator-based positron generation scheme . . . . . . . . . . . . . . . . 54

3.4.2.5 Horizontal orbit through the undulator bypass. . . . . . . . . . . . . . . . 55

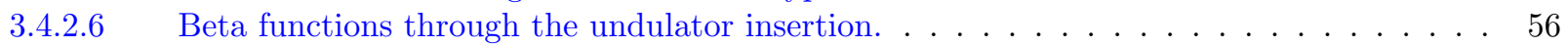

3.4.2.7 Horizontal dispersion function through the undulator insertion. . . . . . . . . . . . 56

3.4.2.8 Horizontal and vertical bandpass at the end of the undulation insertion arc. . . . . . . 57

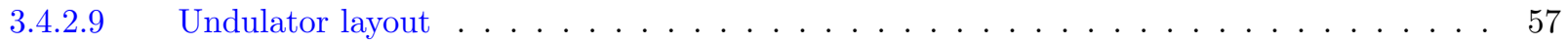

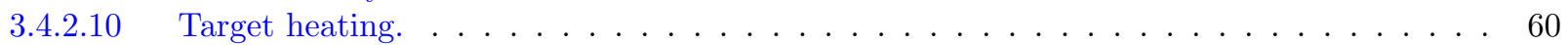

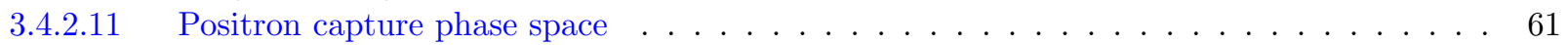

3.4.3.1 Layout of the electron damping ring complex. . . . . . . . . . . . . . . . 64

3.4.3.2 Layout of the positron damping ring complex. . . . . . . . . . . . . . . 65

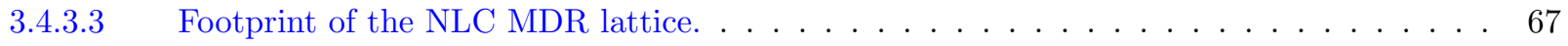

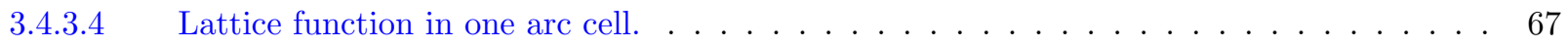

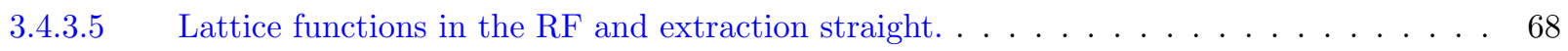

3.4.3.6 Lattice functions in the injection and chicane straight. . . . . . . . . . . . 68

3.4.3.7 Lattice functions in the wiggler straight. . . . . . . . . . . . . . . . . . 69

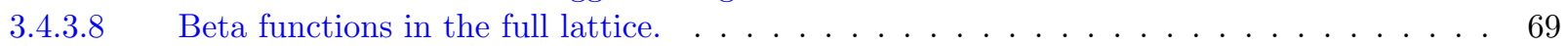

3.4.3.9 Dispersion function in the full lattice. . . . . . . . . . . . . . . . 70

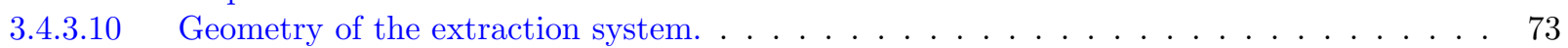

3.4.3.11 Lattice functions in one wiggler cell. . . . . . . . . . . . . . . . . . . . . . 75

3.4.3.12 Lattice functions in the RF cell. . . . . . . . . . . . . . . . . . . . . . 76

3.4.3.13 Lattice functions in the chicane cell. . . . . . . . . . . . . . . . . 76

3.4.3.14 Lattice functions in the injection cell. . . . . . . . . . . . . . . . . . 77

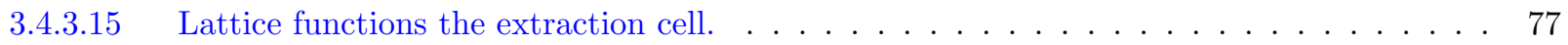

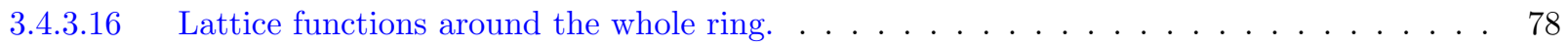

3.4.4.1 Schematic of two-stage bunch compressor layout. . . . . . . . . . . . . . 81

3.4.4.2 Optics from the ring through the second bunch compressor. . . . . . . . . . . . . . 82

3.4.4.3 Optics from the ring through the diagnostics section. . . . . . . . . . . . . . 85

3.4.4.4 Optics from the spin rotation and coupling correction sections. . . . . . . . . . . 86

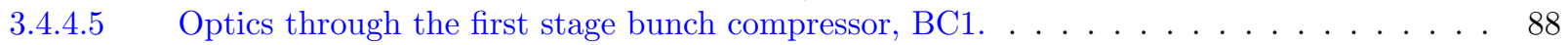

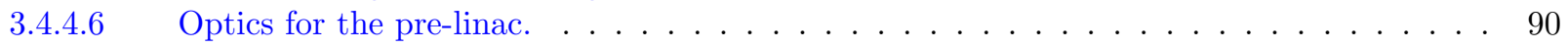

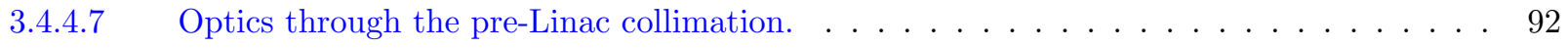

3.4.4.8 Optics through the $180^{\circ}$ arc of the second bunch compressor $\mathrm{BC} 2 \ldots \ldots \ldots$. . . . . . 94 
3.4.4.9 Optics through the second half of $\mathrm{BC} 2 \ldots \ldots \ldots \ldots \ldots \ldots$

3.4.5.1 Schematic of the X-band positron linac layout. . . . . . . . . . . . . . . 98

3.4.5.2 Schematic of an X-band linac RF unit. . . . . . . . . . . . . . . . . . . . 100

3.4.5.3 Experimental accelerator structure and measured performance. . . . . . . . . . . . 104

3.4.5.4 Schematic of the quadrupole layout for the main linac . . . . . . . . . . . . . 105

3.4.5.5 Main linac optics at $500 \mathrm{GeV}$ cms . . . . . . . . . . . . . . . . . . 105

3.4.5.6 Main linac optics at $100 \mathrm{GeV}$ cms, including bypass line. . . . . . . . . . . . . 106

3.4.5.7 Main linac optics at $300 \mathrm{GeV}$ cms, including bypass line . . . . . . . . . . . . . 106

3.4.5.8 Correlated ("BNS") component and total energy spread profile. . . . . . . . . . . . . 107

3.4.5.9 Final Focus Test Beam magnet mover schematic and performance. . . . . . . . . . . 108

3.4.5.10 Main linac diagnostic/extraction section layout. . . . . . . . . . . . . . . . 108

3.4.5.11 Main linac extraction and reinjection section layout. . . . . . . . . . . . . . . . 109

3.4.6.1 Beam delivery system layout. . . . . . . . . . . . . . . . . . . 111

3.4.6.2 Optics of the NLC collimation and final focus systems for the first IR. . . . . . . . . 113

3.4.6.3 Energy reach of the NLC Beam Delivery. . . . . . . . . . . . . . . . . . . . . 114

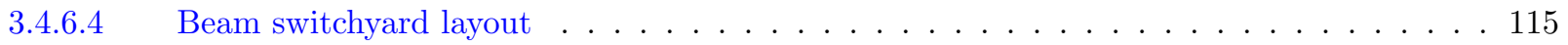

3.4.6.5 Optics for end of linac diagnostic section and tuneup line. . . . . . . . . . . . . 115

3.4.6.6 Second order dispersion in the tuneup line. . . . . . . . . . . . . . . . 116

3.4.6.7 Tuneup dump beam size versus energy offset. . . . . . . . . . . . . . . . . . . 117

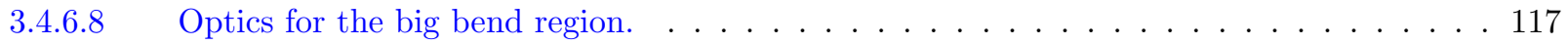

3.4.6.9 Collimation-system performance as evaluated by the TRC Collimation Task Force. . . . 118

3.4.6.10 Comparison of NLC collimation-system performance without octupoles and with oc-

tupoles and looser collimation. . . . . . . . . . . . . . . . . . . . 119

3.4.6.11 Consumable spoiler mechanical prototype. . . . . . . . . . . . . . . . . . . . . 120

3.4.6.12 Luminosity versus vertical offset between beams at the IP. . . . . . . . . . . . . . . . 121

3.4.6.13 Degradation of alignment under ATL ground motion. . . . . . . . . . . . . . . . . . 122

3.4.6.14 Average reconstructed vertical position of $\mathrm{Z}^{0} \rightarrow$ hadron. . . . . . . . . . . . . . . 123

3.4.6.15 Concept of the QD0 compact SC quad which is being prototyped by BNL. . . . . . . . . 124

3.4.6.16 Superconducting Final Focus Magnet Layout Schematic . . . . . . . . . . . . . . . . . 124

3.4.6.17 Beamline masking, forward tracking and forward calorimeters for the Silicon Detector. . 125

3.4.6.18 Optics for the spent beam extraction line . . . . . . . . . . . . . . . 126

3.5.1.1 Overall Machine Layout, $500 \mathrm{GeV}$ c.m. . . . . . . . . . . . . . . . . . . . . 128

3.5.2.1 Electron Injection System layout _ . . . . . . . . . . . . . . . . . . . 133

3.5.2.2 Undulator-based positron generation scheme . . . . . . . . . . . . . . . 136

3.5.2.3 Horizontal orbit through the undulator bypass. . . . . . . . . . . . . . . . . . 139

3.5.2.4 Beta functions through the undulator insertion . . . . . . . . . . . . . . 140

3.5.2.5 Horizontal dispersion function through the undulator insertion. . . . . . . . . . . . 140

3.5.2.6 Horizontal and vertical bandpass at the end of the undulation insertion arc. . . . . . . 141

3.5 .2 .7 Undulator layout . . . . . . . . . . . . . . . . . . . . . . . 141

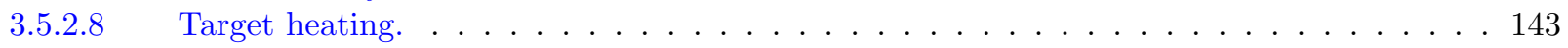

3.5.2.9 Positron capture phase space . . . . . . . . . . . . . . . . . . . . 144

3.5.3.1 Conceptual layout of the positron damping ring. . . . . . . . . . . . . . . . . . . 147

3.5.3.2 Arc cell lattice functions. . . . . . . . . . . . . . . . . . . . . . . . . . 149

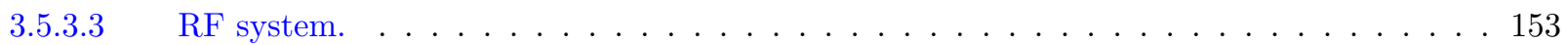

3.5.4.1 Damping ring arc, spin rotator and bunch compressor layout . . . . . . . . . . 156

3.5.4.2 Optical functions and floor plan of the wiggler bunch compressor. . . . . . . . . . . 158

3.5.4.3 Floor plan for the complete bunch compressor beamline. . . . . . . . . . . . . . 161

3.5.5.1 $Q_{0}$ vs. gradient for an electropolished 9-cell cavity. . . . . . . . . . . . . . 162

3.5.5.2 Nine-cell superconducting cavity . . . . . . . . . . . . . . . . . . . 164

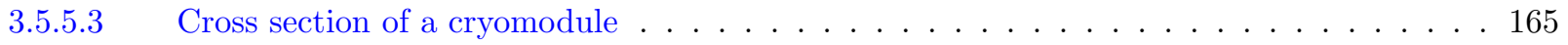

3.5.5.4 Electron main linac layout, $250 \mathrm{GeV}$ beam energy . . . . . . . . . . . . . . . 167

3.5.5.5 Positron main linac layout, $250 \mathrm{GeV}$ beam energy . . . . . . . . . . . . . . . . 167 
3.5.5.6 Waveguide distribution, two tunnels, $250 \mathrm{GeV}$ beam energy . . . . . . . . . . . . 170

3.5.5.7 Electron main linac layout, $500 \mathrm{GeV}$ beam energy . . . . . . . . . . . . . . . . . 175

3.5.5.8 Positron main linac layout, $500 \mathrm{GeV}$ beam energy . . . . . . . . . . . . . . . . 175

3.5.5.9 Waveguide distribution, two tunnels, $500 \mathrm{GeV}$ beam energy . . . . . . . . . . 177

3.5.6.1 NLC-TESLA Hybrid Optics ．.. . . . . . . . . . . . . . . . . . . . 179

3.5.6.2 Fast Extraction and Tuneup Optics . . . . . . . . . . . . . . . . . . . . 181

3.5.6.3 Superconducting Final Focus Magnet Layout Schematic . . . . . . . . . . . . . . . . 182

3.5.6.4 Superconducting QD0 Design Schematic . . . . . . . . . . . . . . . . . . . 183

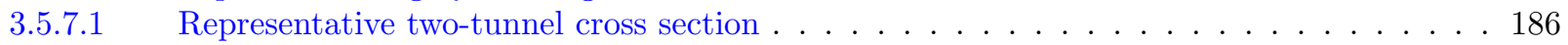

3.5.7.2 Cryounit gas return pipe temperature profile . . . . . . . . . . . . . . . . 195

3.5.7.3 Simplified cryogenic plant schematic . . . . . . . . . . . . . . . . 196

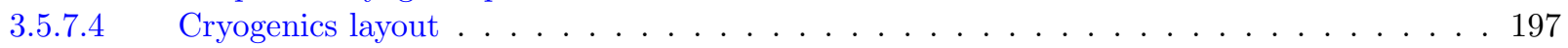

4.4.1.1 Downtime summary for $\operatorname{run} \mathrm{Warm} 2 \ldots \ldots \ldots \ldots \ldots \ldots \ldots$

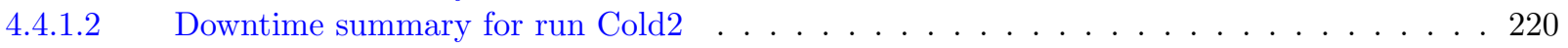

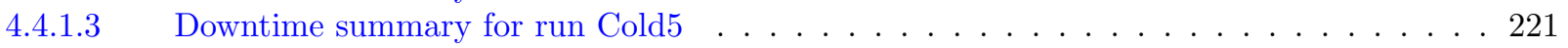

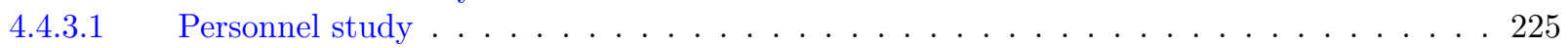

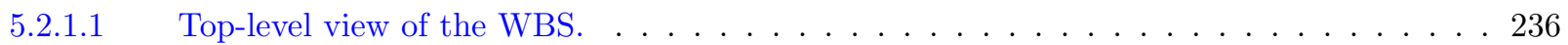

5.2.5.1 Contribution of each subsystem to the cost of hardware in the warm LC damping rings. 241

5.2.5.2 Contribution of each subsystem to the cost of hardware in the cold LC damping rings. . 242

5.3.1.1 The Total Project Cost of the Warm LC as a function of Technical Expertise . . . . . . 255

5.3.1.2 The Total Project Cost of the Cold LC as a function of Technical Expertise . . . . . . . 255

5.3.1.3 The Total Project Cost of the Warm LC broken down by the type of cost. . . . . . . . 256

5.3.1.4 The Total Project Cost of the Cold LC broken down by the type of cost. . . . . . . . 256

5.3.1.5 The Total Project Cost of the Warm LC broken down by area . . . . . . . . . . . . . . 257

5.3.1.6 The Total Project Cost of the Cold LC broken down by area. . . . . . . . . . . . . . . 257

5.3.2.1 The estimated total cost of $500 \mathrm{GeV}$ c.m. linear colliders. . . . . . . . . . . . . . 259

5.3.2.2 Costs for each technology option normalized to the total of the warm option shown by technical subsystem. . . . . . . . . . . . . . . . . . . . 260

5.3.2.3 Costs for each technology option normalized to the total of the warm option shown by machine region . . . . . . . . . . . . . . . . . . . 261

5.4.2.1 International LC Project Systems Engineering Model Schedule for Warm Linac with Undulator Positron Source (Option W) . . . . . . . . . . . . . . . . . . 269

5.4.2.2 International LC Project Systems Engineering Model Schedule for Cold Linac (Option C) 270

5.4.2.3 International LC Project Systems Engineering Model Schedule for Warm Linac with Conventional Positron Source (Option W-Variant 1) . . . . . . . . . . . . . . 271

5.4.2.4 Illustrative sample tunneling scenarios . . . . . . . . . . . . . . . . . . . . 272

5.4.3.1 Cumulative Build-up of Commissioning Time . . . . . . . . . . . . . . . 273

6.2.3.1 Normal Conducting Linear Collider Beamline Configuration . . . . . . . . . . . . . . 295

6.2.3.2 Normal Conducting Linear Collider, Support Housing Functional Criteria . . . . . . . 296

6.2.3.3 Normal Conducting Linear Collider, Main Linac layout . . . . . . . . . . . . . . . . . . 297

6.2.3.4 Normal Conducting Linear Collider, Illinois site, beam delivery and interaction region . 298

6.3.3.1 Superconducting Linear Collider Beamline Configuration . . . . . . . . . . . . . . . 316

6.3.3.2 Superconducting Linear Collider, Support Housing Functional Criteria . . . . . . . . . . 317

6.3.3.3 Superconducting Linear Collider, Positron Damping ring enclosure . . . . . . . . . . . 318

6.3.3.4 Superconducting Linear Collider, Mid-linac enclosure plans . . . . . . . . . . . . . . 319

6.3.3.5 Superconducting Linear Collider, Illinois site, beam delivery and interaction region . . . 320

6.3.3.6 Superconducting Linear Collider, California site, detector hall, elevation view . . . . . . 321

6.3.3.7 Superconducting Linear Collider, Illinois site, detector hall, elevation view . . . . . . . . 322

7.2.2.1 Site cross-section comparison for the one and two tunnel SC LC, Illinois site . . . . . . 326 
7.2.2.2 Close up cross section of the one and two tunnel accelerator enclosure tunnel. . . . . . 327

7.3.4.1 Electron main linac layout . . . . . . . . . . . . . . . . . . . 335

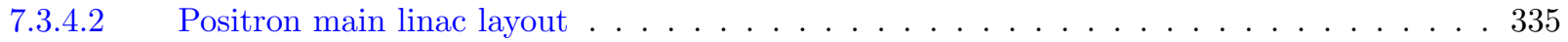

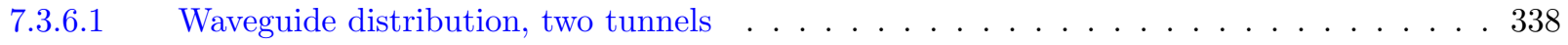

7.4.0.1 A schematic of a linac RF unit incorporating dual-moded DLDS . . . . . . . . . 343

7.5.1.1 Superstructure consisting of two 9-cell resonators joined by a $114 \mathrm{~mm}$ diameter beam

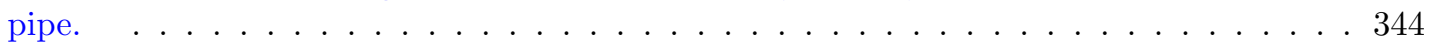

7.5.2.1 Cavity RF feed layouts with and without superstructures . . . . . . . . . . . 345

7.6.1.1 Schematic of the conventional $e^{+}$production system. . . . . . . . . . . . . . . 349

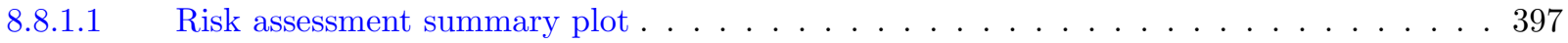




\section{List of Tables}

1.1.1.1 U. S. Linear Collider Steering Group, Executive Committee . . . . . . . . . . . . . . 1

1.1.2.1 U. S. Linear Collider Steering Group, Accelerator Subcommittee . . . . . . . . . . . . . 2

1.1.2.2 U.S. Linear Collider Option Evaluation Task Forces . . . . . . . . . . . . . . . . . . . 3

1.1.2.3 U.S. Linear Collider Option Evaluation Task Force joint meetings . . . . . . . . . . . . 4

3.2.0.1 US Linear Collider: overall parameters . . . . . . . . . . . . . . . . . . . . . . 14

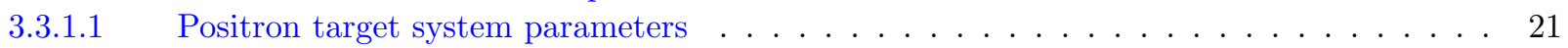

3.3.2.1 Comparison of damping ring parameters with existing storage rings . . . . . . . . . 25

3.3.2.2 Alignment sensitivities in the damping rings compared with some operating rings. . . . 26

3.3.4.1 Importance of emittance dilution sources in different regions of the LC . . . . . . . . . 36

3.3.4.2 Examples of trajectory and emittance correction techniques for each LC region . . . . . 37

3.3.4.3 Emittance and jitter simulation studies for the warm and cold linacs with LIAR . . . . . 39

3.3.4.4 Emittance and jitter sensitivities for the warm and cold BC systems . . . . . . . . 40

3.3.4.5 Emittance and jitter budgets for warm and cold LC designs . . . . . . . . . . . . 40

3.3.5.1 Comparison of SLC, FFTB, and US warm and US cold beam delivery systems . . . . . . 41

3.4.1.1 US X-band Linear Collider: overall parameters . . . . . . . . . . . . . . . 45

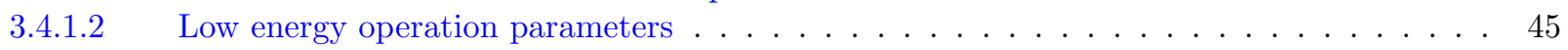

3.4.2.1 Beam parameters as delivered by the electron source system. . . . . . . . . . . . 48

3.4.2.2 Laser parameters . . . . . . . . . . . . . . . . . . . . . . 49

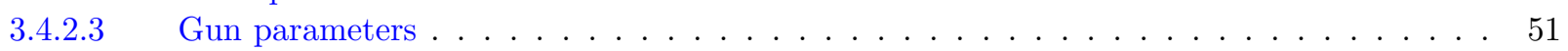

3.4.2.4 Major Technical Components: Electron Bunchers and Injector Linac . . . . . . . . . . 51

3.4.2.5 Warm Option Positron Source Parameters . . . . . . . . . . . . . . . . . 53

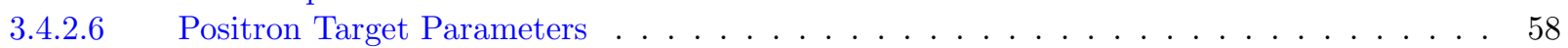

3.4.3.1 Parameters for main damping rings and the pre-damping ring. . . . . . . . . . . . 63

3.4.3.2 Summary of margins between nominal and design parameter values. . . . . . . . . . 70

3.4.3.3 Parameters of main magnets in the NLC MDR lattice. . . . . . . . . . . . . . . 71

3.4.3.4 Wiggler parameters for main damping rings. . . . . . . . . . . . . . . . . 72

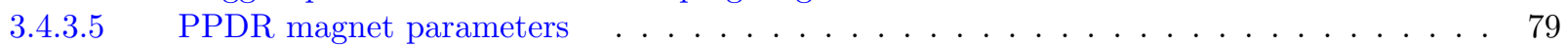

3.4.4.1 Subsystem lengths for the two stage bunch compressor . . . . . . . . . . . . . . 81

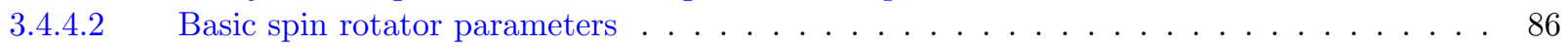

3.4.4.3 Beam parameters before and after the first bunch compressor, BC-1 . . . . . . . . 89

3.4.4.4 Beam parameters before and after the pre-Linac . . . . . . . . . . . . . . 90

3.4.4.5 Beam parameters before and after the second bunch compressor . . . . . . . . . . 95

3.4.4.6 Major system components . . . . . . . . . . . . . . . . . . . 97

3.4.5.1 Design linac beam parameters for $500 \mathrm{GeV}$ cms operation. . . . . . . . . . . . . . 99

3.4.5.2 Overview of components in the positron main linac for $500 \mathrm{GeV}$ and $1 \mathrm{TeV}$ cms. . . . . 99

3.4.5.3 Overview of components in the electron main linac for $500 \mathrm{GeV}$ and $1 \mathrm{TeV}$ cms. . . . . 99

3.4.5.4 Comparison of NLC 2003 positron main linac parameters with TRC values. . . . . . . . 102

3.4.5.5 Comparison of NLC 2003 positron main linac parameters with TRC values (Part II). . 103

3.4.5.6 Overheads assumed . . . . . . . . . . . . . . . . . . . 109 
3.4.6.1 Beam delivery system elements. . . . . . . . . . . . . . . . . . . . . 112

3.4.6.2 Tuneup dump line elements. . . . . . . . . . . . . . . . . . . . . . . . . . . . . 114

3.4.6.3 Spent beam transport line elements. . . . . . . . . . . . . . . . . . . . 126

3.5.1.1 US Superconducting Linear Collider: overall parameters . . . . . . . . . . . . . . . . 129

3.5.2.1 Major Technical Components: Unpolarized Electron Source and Pre-Accelerator _. . . 133

3.5.2.2 Performance specifications: Unpolarized Electron Source and Pre-Accelerator . . . . . . 133

3.5.2.3 Major Technical Components: Polarized Electron Source and pre-Accelerator . . . . . . 134

3.5.2.4 Performance specifications: Polarized Electron Source and Pre-Accelerator . . . . . . . . 135

3.5.2.5 Major Technical Components: Electron Injector Linac . . . . . . . . . . . . . . . . 135

3.5.2.6 Performance specifications: Electron Injector Linac . . . . . . . . . . . . . . . . . 135

3.5.2.7 Cold Option Positron Source Parameters . . . . . . . . . . . . . . . . . . . . . 137

3.5.2.8 Positron Target Parameters . . . . . . . . . . . . . . . . . . . . . . . . . . . . 142

3.5.3.1 Damping ring parameters. . . . . . . . . . . . . . . . . . . . . . 148

3.5.3.2 Damping ring magnets $($ Part 1$) \ldots \ldots \ldots \ldots \ldots \ldots \ldots$

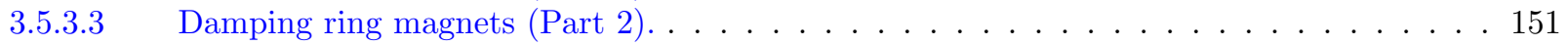

3.5.3.4 Magnet power supplies (excluding steering magnets) . . . . . . . . . . . . . . 151

3.5.3.5 Wiggler magnets. . . . . . . . . . . . . . . . . . . . . . . 151

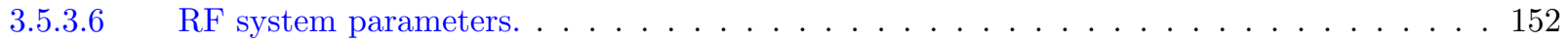

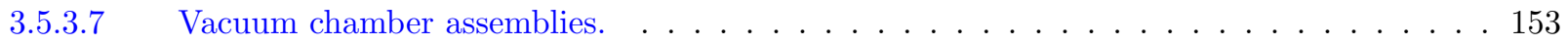

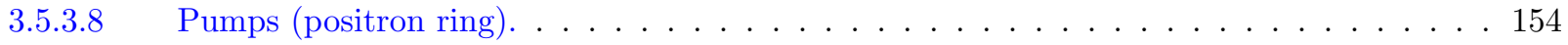

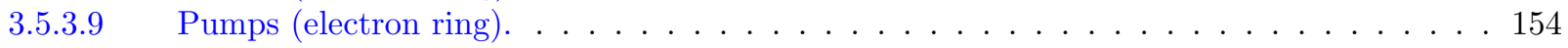

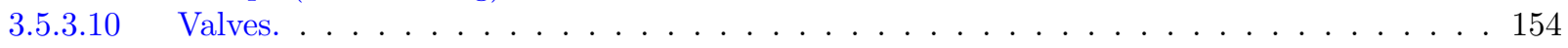

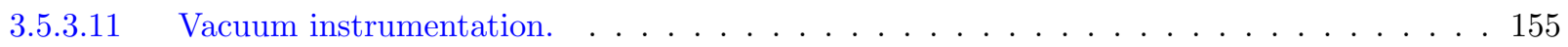

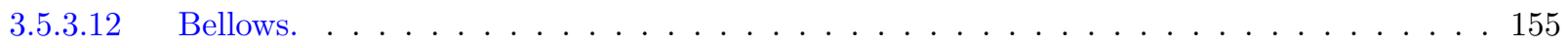

3.5.3.13 Electrical power requirements of damping ring systems. . . . . . . . . . . . . . . . 155

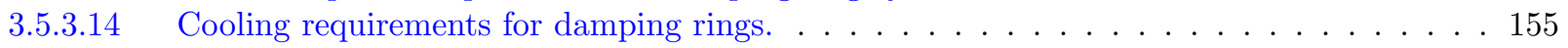

3.5.4.1 Beam parameters before and after the bunch compressor . . . . . . . . . . . . 157

3.5.4.2 Parameters of the wiggler bunch compressor . . . . . . . . . . . . . . . 157

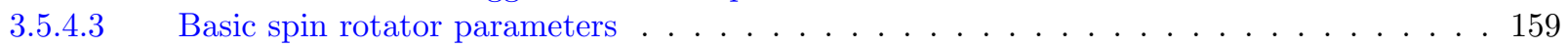

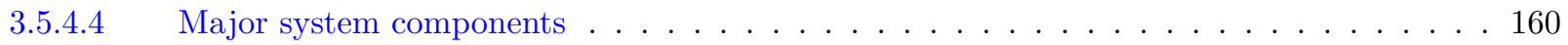

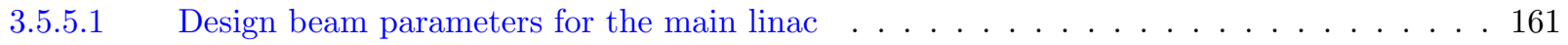

3.5.5.2 Overview of components in the electron main linac. . . . . . . . . . . . . . 163

3.5.5.3 Overview of components in the positron main linac. . . . . . . . . . . . . . 163

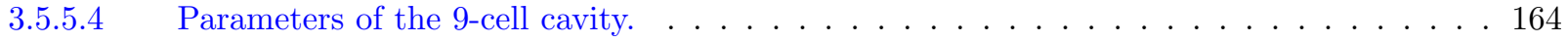

3.5.5.5 Calculated heat loads for $17 \mathrm{~m}$ long cryomodule with quadrupole. . . . . . . . . . 165

3.5.5.6 Segmentation of the electron main linac, $250 \mathrm{GeV}$ beam energy . . . . . . . . . . . 166

3.5.5.7 Segmentation of the positron main linac, $250 \mathrm{GeV}$ beam energy . . . . . . . . . . 167

3.5.5.8 Cryogenic requirements of the main linacs . . . . . . . . . . . . . . . 168

3.5.5.9 Total cryogenic operating power requirements, $250 \mathrm{GeV}$ beam energy . . . . . . . . 168

3.5.5.10 Efficiencies and power requirements for the RF system . . . . . . . . . . . . . . 169

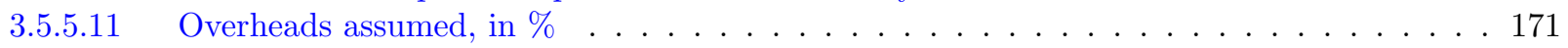

3.5.5.12 Design beam parameters for the main linac at $500 \mathrm{GeV}$ beam energy . . . . . . . . 171

3.5.5.13 Overview of components in the electron main linac for $500 \mathrm{GeV}$ beam energy. . . . . . . 172

3.5.5.14 Overview of components in the positron main linac for $500 \mathrm{GeV}$ beam energy. . . . . . . 172

3.5.5.15 Parameters of the 9-cell cavity for use at $500 \mathrm{GeV}$ beam energy. . . . . . . . . . . . 173

3.5.5.16 Calculated heat loads for $17 \mathrm{~m}$ long cryomodule with quadrupole, at $35 \mathrm{MV} / \mathrm{m}$ gradient. 173

3.5.5.17 Segmentation of the electron main linac at $500 \mathrm{GeV}$ beam energy . . . . . . . . . . 174

3.5.5.18 Segmentation of the positron main linac at $500 \mathrm{GeV}$ beam energy . . . . . . . . . . . 174

3.5.5.19 Cryogenic requirements of the main linacs at $500 \mathrm{GeV}$ beam energy . . . . . . . . 175

3.5.5.20 Total cryogenic operating power requirements, $500 \mathrm{GeV}$ beam energy . . . . . . . . 176

3.5.5.21 Efficiencies and power requirements for the RF system at $500 \mathrm{GeV}$ beam energy . . . . 176

3.5.6.1 Major Technical Components: Electron Beam Delivery Line (IR1) . . . . . . . . . . . 180 


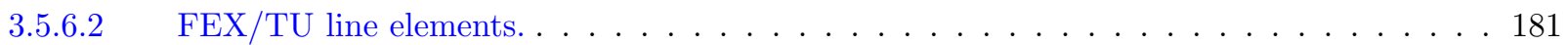

3.5.6.3 Spent beam transport line elements. . . . . . . . . . . . . . . . . . . . 185

3.5.7.1 Tabulation of technical component lengths. . . . . . . . . . . . . . . . 185

3.5.7.2 Cryounit Heat Loads for a $500 \mathrm{GeV}$ Collider . . . . . . . . . . . . . . . . . . . . 188

3.5.7.3 $500 \mathrm{GeV}$ operation cryogenic plant requirements . . . . . . . . . . . . . . . . . 189

3.5.7.4 Cryounit Heat Loads for a $1 \mathrm{TeV}$ Collider, electron linac side . . . . . . . . . . . . . . 190

3.5.7.5 Cryounit Heat Loads for a $1 \mathrm{TeV}$ Collider, positron linac side . . . . . . . . . . . . . 191

3.5.7.6 $1 \mathrm{TeV}$ operation cryogenic plant requirements . . . . . . . . . . . . . . . . . 192

3.5.7.7 Cryogenic Plant Nominal and Design, $500 \mathrm{GeV} \ldots \ldots \ldots$. . . . . . . . . 193

3.5.7.8 Cryogenic Plant Nominal and Design, $1 \mathrm{TeV} \ldots \ldots \ldots$. . . . . . . . . . . . . . 193

3.5.7.9 Cryogenic cycle comparisons. . . . . . . . . . . . . . . . . . . 199

4.3.3.1 Regions of the machine with allocated tuning time and MD . . . . . . . . . . 205

4.3.4.1 Details on how the lumped systems were modeled. . . . . . . . . . . . . . . . 205

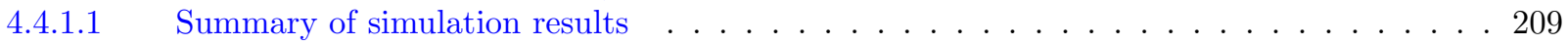

$4.4 .1 .2 \quad$ MTBFs for Warm LC . . . . . . . . . . . . . . . . . . . 210

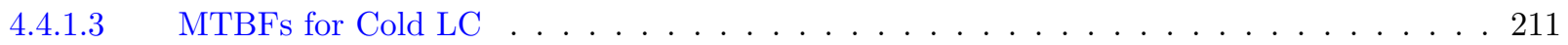

4.4.1.4 MTBFs for Cold One Tunnel LC . . . . . . . . . . . . . . . . . . . . . . . . . 213

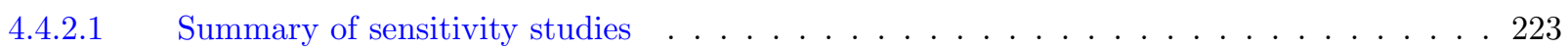

4.4.3.1 Required maintenance personnel . . . . . . . . . . . . . . . . . . . . . 224

5.2.6.1 Main Linac R\&D Component Sources: X-band (Warm) Technologies . . . . . . . . . . . 249

5.2.6.2 Main Linac R\&D Component Sources: Superconducting (Cold) Technologies . . . . . . . 250

5.2.6.3 Main Linac Basis of Cost Estimates: X-band (Warm) Technologies . . . . . . . . . . . 251

5.2.6.4 Main Linac Basis of Cost Estimates: Superconducting (Cold) Technologies . . . . . . . . 252

5.4.2.1 Activity Durations in the Model Schedule-I . . . . . . . . . . . . . . . . . . 264

5.4 .2 Activity Durations in the Model Schedule-II . . . . . . . . . . . . . . . . . . 265

5.4.2.3 Milestone Dates in the Model Schedules . . . . . . . . . . . . . . . . . . . 266

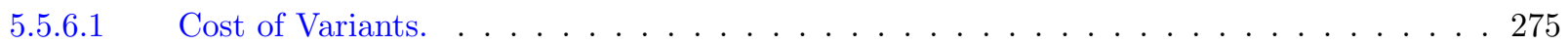

6.2.1.1 Normal-conducting Reference Design Summary Parameters . . . . . . . . . . . . . 278

6.2.1.2 Local Campus Space . . . . . . . . . . . . . . . . . . . . . . . . 281

6.2.1.3 Machine Area Power Load Summary in Megawatts, X-band collider, CA site. . . . . . . 282

6.2.2.1 Fermilab FY03 NS-NC Orientation Central Campus Facilities Assumptions . . . . . . . 288

6.2.2.2 Machine Area Power Load Summary in Megawatts, X-band collider, IL site. . . . . . 290

6.2.2.3 X-band collider, IL site, LCW Plant estimates . . . . . . . . . . . . . . . . . . . . . 291

6.3.1.1 Superconducting Reference Design Summary Parameters . . . . . . . . . . . . . . . . . 299

6.3.1.2 Local Campus Space . . . . . . . . . . . . . . . . . . . . . . 302

6.3.1.3 Machine Area Power Load Summary in Megawatts, L-band collider, CA site. . . . . . . 303

6.3.2.1 Fermilab FY03 NS-SC Orientation Central Campus Facilities Assumptions . . . . . . . . 309

6.3.2.2 Machine Area Power Load Summary in Megawatts, L-band collider, IL site. . . . . . . 311

6.3.2.3 L-band collider, IL site, LCW plant estimates . . . . . . . . . . . . . . . . . . . 312

7.2.3.1 Single tunnel civil construction and utility cost percentage breakdown summary . . . . . 328

7.2.3.2 Double tunnel civil construction and utility cost percentage breakdown . . . . . . . . . 329

7.2.3.3 Percent cost difference due to civil construction - One / Two tunnel Cold IL LC, at 500

GeV c.m. . . . . . . . . . . . . . . . . . . . . 329

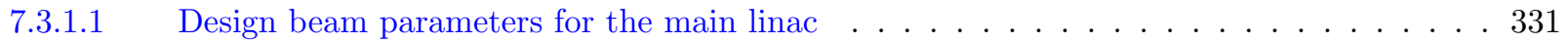

7.3.1.2 Overview of components in the electron main linac. . . . . . . . . . . . . . . . 331

7.3.1.3 Overview of components in the positron main linac. . . . . . . . . . . . . . . . 332

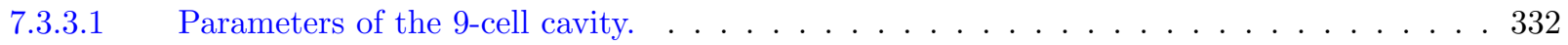

7.3.4.1 Calculated heat loads for $17 \mathrm{~m}$ long cryomodule with quadrupole. . . . . . . . . . 333

7.3.4.2 Segmentation of the electron main linac . . . . . . . . . . . . . . 334 
7.3.4.3 Segmentation of the positron main linac . . . . . . . . . . . . . . . 334

7.3.5.1 Cryogenic requirements of the main linacs . . . . . . . . . . . . . 336

7.3.5.2 Total cryogenic operating power requirements, $250 \mathrm{GeV}$ beam energy . . . . . . . 336

7.3.6.1 Efficiencies and power requirements for the RF system . . . . . . . . . . . . 337

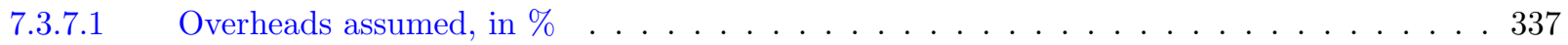

7.3.8.1 Tabulation of technical component lengths. All lengths are in km. . . . . . . . . . 339

7.3.9.1 Cryounit Heat Loads for a $500 \mathrm{GeV}$ Collider . . . . . . . . . . . . . . . . . . . . . 340

7.3.9.2 $500 \mathrm{GeV}$ operation cryogenic plant requirements . . . . . . . . . . . . . . . 341

7.3.9.3 Cryogenic Plant Nominal and Design, $500 \mathrm{GeV} \ldots \ldots \ldots$. . . . . . . . . . . 341

7.5.1.1 Parameters of the $2 \times 9$-cell superstructure . . . . . . . . . . . . . . . 344

7.5.3.1 Electron main linac, using superstructures, $250 \mathrm{GeV}$ beam energy . . . . . . . . . 346

7.5.3.2 Positron main linac, using superstructures, $250 \mathrm{GeV}$ beam energy . . . . . . . . . . . 346

7.5.3.3 Electron main linac, using superstructures, $500 \mathrm{GeV}$ beam energy . . . . . . . . . . . 346

7.5.3.4 Positron main linac, using superstructures, $500 \mathrm{GeV}$ beam energy . . . . . . . . . . . 347

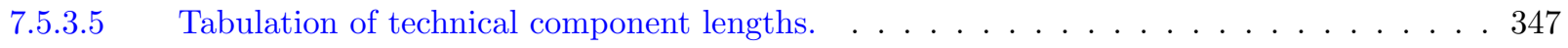

8.1.0.1 Table of Reasons . . . . . . . . . . . . . . . . . . . . . . . . 354

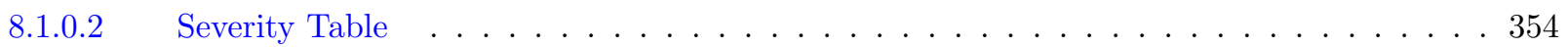

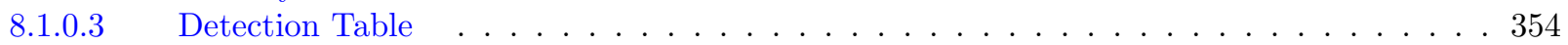

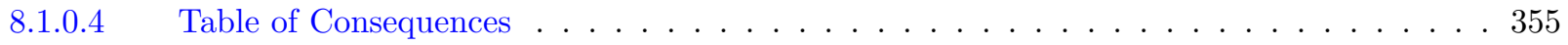

8.3.8.1 Estimated impedance and instability thresholds in the warm and cold LC damping rings 364

8.4.2.1 Rms alignment sensitivities corresponding to $1 \mathrm{~nm}$-rad of vertical emittance growth in the main linacs. . . . . . . . . . . . . . . . . . . 366

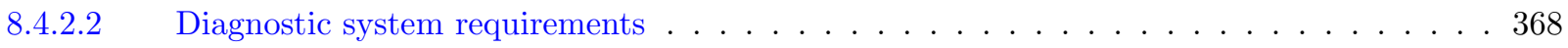

8.4.2.3 Rms initial alignment tolerances for main linac RF cavities and girders/ cryomodules . . 371

8.8.2.1 Warm option near-term R\&D challenges . . . . . . . . . . . . . . . . . . 402

8.8.2.2 Cold option near-term R\&D challenges . . . . . . . . . . . . . . . . . . . . 402

A.0.0.1 Comparison of USLCSG and ILCSC linear collider physics requirements . . . . . . . . 417 


\section{Chapter 1}

\section{Introduction and Overview}

\section{$1.1 \quad$ Introduction}

\subsubsection{Initiation and purpose of this study}

This study was carried out at the request of the United States Linear Collider Steering Group (USLCSG). The establishment of such a body was recommended by the HEPAP Subpanel on Long Range Planning [1].

The USLCSG is led by an Executive Committee, chaired by Jonathan Dorfan of SLAC, and consisting currently of the members shown in Table 1.1.1.1. Three subcommittees report to the Executive Committee: an Accelerator Subcommittee, a Physics/Detector Subcommittee, and an International Affairs Subcommittee.

Table 1.1.1.1: U. S. Linear Collider Steering Group, Executive Committee

\begin{tabular}{|c|c|}
\hline Member & Institution \\
\hline Jonathan Bagger & Johns Hopkins University \\
\hline Jim Brau & University of Oregon \\
\hline David Burke & Stanford Linear Accelerator Center \\
\hline Sally Dawson & Brookhaven National Laboratory \\
\hline Jonathan Dorfan ${ }^{a}$ & Stanford Linear Accelerator Center \\
\hline Gerald Dugan & Cornell University \\
\hline Jerome Friedman & Massachusetts Institute of Technology \\
\hline Jim Gates & University of Maryland \\
\hline Steve Holmes & Fermi National Accelerator Laboratory \\
\hline Young-Kee Kim & University of Chicago \\
\hline Harvey Lynch ${ }^{b}$ & Stanford Linear Accelerator Center \\
\hline Dan Marlow & Princeton University \\
\hline Mark Oreglia & University of Chicago \\
\hline Maury Tigner & Cornell University \\
\hline Michael Witherell & Fermi National Accelerator Laboratory \\
\hline
\end{tabular}

${ }^{a}$ Chairman

${ }^{b}$ Executive Secretary

While the functions of the Steering Group are expected to evolve with time, the initial charge includes the following items: 
- Provide an evaluation of options for building the linear collider involving factors such as scientific requirements, technical feasibility, risk, cost, initial facility parameters, upgradeability of alternate technologies, and the implications of different sites;

- Prepare the elements of a U.S. bid to host the linear collider

To address these items in its charge, the USLCSG Executive Committee asked the Accelerator Subcommittee to carry out an evaluation of two options for a US-sited linear collider. The two options were to be based on the normal conducting X-band RF technology developed by the GLC/NLC collaboration[ZDR, NLC01],[TRC, Chapter 3], and the superconducting L-band RF technology developed by the TESLA collaboration[TDR]. The physics design requirements which both technical options must meet were established [2] by the Physics and Detector Subcommittee of the USLCSG.

\subsubsection{Execution}

The charge to carry out this option evaluation was delivered to the Accelerator Subcommittee in January, 2003. The current membership of the Accelerator Subcommittee is shown in Table 1.1.2.1.

Table 1.1.2.1: U. S. Linear Collider Steering Group, Accelerator Subcommittee

\begin{tabular}{|l|l|}
\hline Member & Institution \\
\hline David Burke & Stanford Linear Accelerator Center \\
David Finley & Fermi National Accelerator Laboratory \\
Gerald Dugan & ${ }^{a}$ \\
Mike Harrison & Cornell University \\
Steve Holmes & Brookhaven National Laboratory \\
Jay Marx & Fermi National Accelerator Laboratory \\
Hasan Padamsee & Lawrence Berkeley National Laboratory \\
Tor Raubenheimer & Cornell University \\
\hline
\end{tabular}

${ }^{a}$ Chairman

This group discussed the charge and decided to form several task forces to carry out the required work. Three task forces were formed initially:

- Accelerator physics and technology design,

- Cost and schedule,

- Civil construction and siting.

A fourth task force on availability design was formed about a month later. The fifth major effort, the assessment of risk, was carried out by an ad-hoc group composed of members from each of the four task forces. The membership of the task forces is given in Table 1.1.2.2.

Initially, the accelerator physics and technology design task force set the design parameters for the two options, so that the other groups would have a firm reference to work from. This was particularly needed for the cold option, as a number of significant changes were made from the TESLA TDR [TDR] design. The design task force met in several audio conferences in February, and together as a group at Fermilab on February 5, 2003, to establish the essential features of the cold reference design used in this study. 
Table 1.1.2.2: U.S. Linear Collider Option Evaluation Task Forces

\begin{tabular}{|c|c|}
\hline Member & Institution \\
\hline \multicolumn{2}{|c|}{ Accelerator Physics and Technology Design ${ }^{a}$} \\
\hline Chris Adolphsen & Stanford Linear Accelerator Center \\
\hline Gerald Dugan $^{b}$ & Cornell University \\
\hline Helen Edwards & Fermi National Accelerator Laboratory \\
\hline Mike Harrison & Brookhaven National Laboratory \\
\hline Hasan Padamsee & Cornell University \\
\hline Tor Raubenheimer & Stanford Linear Accelerator Center \\
\hline \multicolumn{2}{|r|}{ Cost and Schedule } \\
\hline David Burke ${ }^{b}$ & Stanford Linear Accelerator Center \\
\hline John Cornuelle & Stanford Linear Accelerator Center \\
\hline David Finley & Fermi National Accelerator Laboratory \\
\hline Warren Funk & Thomas Jefferson National Accelerator Facility \\
\hline Peter Garbincius & Fermi National Accelerator Laboratory \\
\hline Mike Harrison & Brookhaven National Laboratory \\
\hline Steve Holmes & Fermi National Accelerator Laboratory \\
\hline Ray Larsen & Stanford Linear Accelerator Center \\
\hline Theodore Lavine & Stanford Linear Accelerator Center \\
\hline Cindy Lowe & Stanford Linear Accelerator Center \\
\hline Tom Markiewicz & Stanford Linear Accelerator Center \\
\hline Hasan Padamsee & Cornell University \\
\hline Brett Parker & Brookhaven National Laboratory \\
\hline Kem Robinson & Lawrence Berkeley National Laboratory \\
\hline John Sheppard & Stanford Linear Accelerator Center \\
\hline Russ Wells & Lawrence Berkeley National Laboratory \\
\hline Andy Wolski & Lawrence Berkeley National Laboratory \\
\hline \multicolumn{2}{|r|}{ Siting and Civil Construction } \\
\hline David Burke & Stanford Linear Accelerator Center \\
\hline Clay Corvin & Stanford Linear Accelerator Center \\
\hline David Finley & Fermi National Accelerator Laboratory \\
\hline Steve Holmes ${ }^{b}$ & Fermi National Accelerator Laboratory \\
\hline Vic Kuchler & Fermi National Accelerator Laboratory \\
\hline Marc Ross & Stanford Linear Accelerator Center \\
\hline \multicolumn{2}{|c|}{ Availability Design and Specification } \\
\hline Paul Czarapata & Fermi National Accelerator Laboratory \\
\hline Helen Edwards & Fermi National Accelerator Laboratory \\
\hline Tom Himel ${ }^{b}$ & Stanford Linear Accelerator Center \\
\hline Marcus Huening & Fermi National Accelerator Laboratory \\
\hline Nan Phinney & Stanford Linear Accelerator Center \\
\hline Marc Ross & Stanford Linear Accelerator Center \\
\hline
\end{tabular}

${ }^{a}$ Significant contributions in this area were also made by Brett Parker (BNL), Nan Phinney (SLAC), John Sheppard (SLAC), and Andy Wolski (LBNL)

${ }^{b}$ Primary liaison to USLCSG Accelerator Subcommittee

Throughout the duration of this study (February-November, 2003), there were numerous audio conference meetings of each of the individual task forces, through which they planned, coordinated, and executed the work described in this document.

Coordination between task forces, and overall review and planning for the study, was carried out in four 
in-person meetings, which were open to all the members of the task forces. These meetings are listed in Table 1.1.2.3. In addition, there was a 4 hour meeting of some members of the task forces at Cornell University on July 15, 2003, to discuss risk assessment.

Table 1.1.2.3: U.S. Linear Collider Option Evaluation Task Force joint meetings

\begin{tabular}{|l|l|}
\hline Meeting Date & Location \\
\hline April 14, 2003 & Fermi National Accelerator Laboratory \\
June 15-16, 2003 & Stanford Linear Accelerator Center \\
August 27-28, 2003 & Fermi National Accelerator Laboratory \\
October 13-14, 2003 & Fermi National Accelerator Laboratory \\
\hline
\end{tabular}

\subsubsection{Presentations to Linear Collider collaborations}

Presentations on the goals, methods, and progress of this study were made to the TESLA collaboration at their collaboration meeting at Frascati on May 26, 2003, and at their collaboration meeting in Hamburg on September 15, 2003. A presentation on this study was made to the GLC/NLC collaboration at the meeting of the SLAC-KEK Tenth International Study Group on Linear Colliders at SLAC on June 17, 2003.

\subsubsection{Coordination with the USLCSG}

During the course of the study, interim reports were provided to the USLCSG, and additional guidance was received and acted upon as necessary. The final draft of this study was presented to the USLCSG on December 11, 2003, and their comments and suggestions have been incorporated.

\subsubsection{Acknowledgments}

The Accelerator Subcommittee of the USLCSG wishes to thank all the members of the LC option task forces for their dedicated work on this study. There are also a number of individuals who were not officially members of the task forces, but who nonetheless made important contributions to this study. These individuals include Gerald Aarons (SLAC), Fred Assiri (SLAC), Emil Huedem (Fermilab), Arkaidy Klebaner (Fermilab), Tom Lackowski (Fermilab), Mel Magnuson (Fermilab), Fulvia Pilat (BNL), Joe Rogers (Cornell), Andrei Seryi (SLAC), Javier Sevilla (SLAC), Jeff Sims (Fermilab), Jay Theilacker (Fermilab), and Peter Tenenbaum (SLAC).

Critical cost and technical information was provided to us by the TESLA collaboration, in documents and in several meetings. Discussions were also held with members of the GLC group at KEK. We would like to thank all those individuals who provided this information, and in particular Stefan Choroba and Franz Peters of DESY, who served as the official liaisons to the TESLA collaboration. The way in which this information was incorporated into the study is entirely the responsibility of the authors of this document: the USLCSG Accelerator Subcommittee and the members of the Linear Collider Option Evaluation Task Forces.

\subsection{Overview}

This report is divided into nine chapters. A brief outline of the contents of these chapters follows. 


\section{- Chapter 1: Introduction and Overview}

This chapter introduces the study, describes how it was done, and presents an overview of the report.

\section{- Chapter 2: Executive Summary}

This chapter presents a summary of the findings of the study, in the form of bullets which highlight the results detailed in subsequent chapters.

\section{- Chapter 3: Reference Design Configurations}

This chapter presents the top-level physics design requirements for the linear collider, followed by a discussion of the design considerations for the key accelerator systems of the linear collider. Detailed reference design descriptions are then provided for two realizations of the linear collider, based on normal conducting X-band main linac RF structures, and superconducting L-band main linac RF structures. These designs rely heavily on the work of the GLC/NLC collaboration, and the TESLA collaboration.

\section{- Chapter 4: Availability Design}

This chapter presents design requirements and evaluations for the hardware availability of the linear collider.

\section{- Chapter 5: Cost and Schedule}

This chapter presents a comparison of expected costs and schedules for completion of a linear collider based on the reference designs described in Chapter 3. Absolute costs are not presented in this report, but relative costs between different accelerator systems of the collider, and between the two different technology options, are provided.

\section{- Chapter 6: Civil Construction and Siting}

This chapter describes civil design studies that have been completed for two representative U.S. sites, one situated in Illinois and the other in California. For each site, two designs are outlined, one for each technology option. Civil design criteria in all instances are based on the requirements described in Chapter 3 of this document.

\section{- Chapter 7: Design Configuration Variants}

In this chapter, five variants on the reference design were investigated. These variants were considered because they were felt to have the potential for cost reduction, performance enhancement, and/or significant positive schedule impact. For each design variant, this chapter specifies the differences from the reference design, and provides an estimate of the impact that adoption of the variant would have on cost and schedule, siting, and availability.

\section{- Chapter 8: Risk Assessment}

This chapter reports our assessment of the risks posed to successful completion of the physics mission of the linear collider. This assessment has been done at the highest levels of function of the collider - major technical systems and machine areas. Risks are assessed against the threat they pose to the highest performance parameters - beam collision energy and integrated luminosity within the time frame specified in the project mission. We assess risk as it stands today, based on the existing body of work on accelerator design and technology R\&D.

\section{- Chapter 9: Conclusions}

This chapter brings together the findings and conclusions from the previous six chapters, in a form similar to that of Chapter 2, but with more detail and elaboration. 


\section{Chapter 2}

\section{Executive Summary}

\section{$2.1 \quad$ Overview}

- The Accelerator Subcommittee of the US Linear Collider Steering Group (USLCSG) has carried out the evaluation of two options for the siting of a linear collider in the US. The two options were a warm option, following the design of the GLC/NLC Collaboration, and a cold option, similar to the TESLA design at DESY. The reference designs for both options satisfy the physics-based machine requirements specified in the USLCSG Scope Document[2].

- These reference designs also satisfy the requirements for an international linear collider, as specified by the Parameters Subcommittee of the International Linear Collider Steering Committee [3]. The linear collider requirements presented in the USLCSG Scope Document, and those specified by the Parameters Subcommittee of the International Linear Collider Steering Committee, are compared in Appendix A.

- Both options were developed in concert, using, as much as possible, similar approaches in technical design for similar accelerator systems, and a common approach to cost and schedule estimation, reliability design and evaluation, and to project risk assessments.

- The two technology options examined in this study have different challenges, advantages, and disadvantages, and differ in many details.

- We found that, within relative factors of $30 \%$ or less, the two approaches would provide similar technical performance at roughly equivalent cost.

- The two options can ${ }^{1}$ have similar levels of availability, with comparable overall levels of risk, and can be realized on roughly the same schedule.

- These two options are at comparable levels of development, and both have the potential to provide a viable route to a linear collider which meets the requirements of the USLCSG.

\subsection{Design}

- Feasible designs have been established for both technology options which meet the physics mission requirements of the USLCSG. These designs draw heavily on the work of the GLC/NLC collabora-

\footnotetext{
${ }^{1}$ See Section 2.3 , third bullet
} 
tion [ZDR, NLC01],[TRC, Chapter 3] for the warm technology option, and the work of the TESLA collaboration [TDR] for the cold technology option.

- Both $500 \mathrm{GeV}$ reference designs have an energy reach, at reduced luminosity, of up to about $625 \mathrm{GeV}$. The cold option has a design luminosity at $500 \mathrm{GeV}$ which is about $25 \%$ higher than that of the warm option.

- The warm (cold) option reference design has a main linac loaded gradient of $52(28) \mathrm{MV} / \mathrm{m}$.

- The length of the conventional construction footprint for the cold option is about $42 \%$ greater than for the warm option.

- Both options can be upgraded to $1 \mathrm{TeV}$ without additional underground civil construction. The warm (cold) option upgrade design has a main linac loaded gradient of $52(35) \mathrm{MV} / \mathrm{m}$. The upgraded warm option has an energy reach of up to about $1.3 \mathrm{TeV}$. The upgraded cold option has a design luminosity which is about $25 \%$ higher than that of the warm option at $1 \mathrm{TeV}$, but cannot reach energies above 1 $\mathrm{TeV}$.

- The $\mathrm{AC}$ power required to run the $0.5 \mathrm{TeV}$ cold reference design is about $30 \%$ less than that of the warm reference design.

\section{$2.3 \quad$ Availability}

- A hardware unavailability of $25 \%$ has been established and evaluated for both options. Of the $25 \%$ hardware-related downtime, only $15 \%$ was explicitly budgeted to specific devices and systems for hardware problems and recovery from them; the other $10 \%$ was held as contingency.

- Using a simulation, the $15 \%$ budget was allocated to collider subsystems, and compared with expectations based on estimates of component mean time between failures (MTBF) and mean time to repair (MTTR).

- Neither of the options was able to reach the required availability using the original estimates for component MTBF and MTTR. In both cases, it was necessary to increase the MTBF's of selected components to fit within the desired $15 \%$ hardware unavailability budget. A crude estimate of the cost associated with this reliability upgrade is $2 \%$ of the total project cost, for either option.

- In terms of the reliability improvements needed, there is not a great difference between warm and cold reference designs. Both are very large and complex accelerators where significant effort and expense will be needed to make them reliable enough.

\subsection{Cost and Schedule}

- The available cost estimates for both technologies are based on experience with existing accelerator facilities, technology specific R\&D prototypes and test facilities, and industrial models of large-scale production.

- The maturity and confidence in the cost estimates for both technologies are appropriate for a major U.S. project in the conceptual design stage. Extrapolated reductions in unit costs from present-day R\&D to the high-volumes needed to build the collider are a source of risk in the cost estimates. Extrapolations of up to factors of three to five were made for the components of the cold crymodules, and as high as six for some of the warm copper components. The overall extrapolation is somewhat larger for the warm technology because of the larger number of small repetitive components involved. 
- More than two thirds of the warm costs are independent of the accelerator technology, and the corresponding cold costs are the same. The cold-warm difference arises because the costs that are specific to the cold technology choice are estimated to be about twice as large as those that are specific to the warm option. The differences in the specific costs result in an estimated total project cost for the cold machine that is $25 \% \pm 10 \%$ greater than for the warm machine.

- The quoted uncertainty assumes a $\pm 15 \%$ (rms) uncertainty in the specific costs, and ignores correlations between the warm and cold cost estimates. We have not done a complete analysis of the uncertainties in these costs, which would have to include any correlations. The presence of correlations would lower the uncertainty.

- We found it necessary to re-estimate the cost of the damping rings for the cold technology to account for differences in the technical specifications from those in the TESLA TDR.

- The industrial technologies and experience needed to produce the components and systems of each machine choice are well-defined and available in world-wide industries. Industrial capacities needed to produce the quantities of the components for the main linac do not exist today for either the warm or cold technology.

- Both technologies are ready for substantial value engineering (VE) and design-for-manufacture (DFM). We expect the cost and confidence in the acquisition of the components needed for each technology to respond favorably to continued design analysis, technology R\&D, and VE and DFM.

- Provided appropriate funding during construction, the time needed to build and commission a collider can be independent of the choice of linac technology.

- With the initial configurations that we have assumed, the estimated cost to upgrade the $500 \mathrm{GeV}$ reference design to $1 \mathrm{TeV}$ is approximately the same for each technology. The cost to upgrade will depend significantly on when and how it is done.

\subsection{Siting}

- The civil construction and siting criteria for both technology options are well understood. The siting criteria for the two technology options are very similar, except for length.

- Representative sites studied in IL and CA meet the criteria for both technologies. While no general survey was undertaken to identify sites outside of these regions meeting the criteria, we would expect such sites exist.

- Desirable site characteristics from the civil construction point of view are stable, consistent, and dry geology, and access to sufficient power and water. Regarding ground motion requirements, in the absence of intra-train feedback, the sensitivity of the collision luminosity to beam jitter is approximately the same in the two machines. Intra-train feedback at the IP can be implemented in either machine, but is more effective with the bunch train format of the cold machine than with that of the warm machine.

- The civil construction cost differences between the two options are driven by the difference in the lengths of the tunnels and the differences in the cryogenic requirements.

\subsection{Design variants}

The design variants refer to modifications to the reference designs that have the potential for cost reduction, performance enhancement, and/or significant positive schedule impact. 


\subsubsection{One tunnel in cold option}

- The single tunnel cold option linear collider is similar to the design described in the TESLA Technical Design Report [TDR].

- We estimate that the tunnel and infrastructure costs will be reduced by $5.1 \%$ of the total project cost.

- Use of the one tunnel solution has an impact on the overall reliability of the collider. If all components had the same reliability, the cold 1 tunnel design would have a hardware unavailability of $25 \%$ instead of the $15 \%$ of the 2 tunnel (reference) design. To fit within the required $15 \%$ unavailability budget, the MTBF of many linac and damping ring components must be improved substantially, and the linac energy overhead also must be increased from $3 \%$ to $8 \%$. A very crude estimate of the required cost increase associated with these component reliability improvements was of the order of $3 \%$ of the total project cost.

- The net reduction in total project cost is thus about $2 \%$.

- The increased complexity of installation, caused by the sharing of a single tunnel by the damping ring, main linac accelerator components, and main linac power components, increases the risk of delay in the completion of the construction and commissioning of the collider.

\subsection{2 $35 \mathrm{MV} / \mathrm{m}$ initial design gradient in cold option}

- This variant takes $35 \mathrm{MV} / \mathrm{m}$, the maximum gradient at which the cold option cavities are qualified, as the design gradient for the $500 \mathrm{GeV}$ machine. Since the installed linac is operating at its maximum gradient, this variant will limit the energy reach of the cold option reference design to $500 \mathrm{GeV}$, unless more of the tunnel is initially filled with accelerator components, at higher cost.

- The cost savings associated with designing for an initial gradient of $35 \mathrm{MV} / \mathrm{m}$ is about $3 \%$ of the total project cost.

\subsubsection{Superstructure in cold option}

- For the cold option, the use of a superstructure (two 9-cell resonators joined by a large diameter beam pipe) allows a more efficient packing of the cavities in the cryomodules, resulting in a $\sim 6 \%$ reduction in the length of each cryomodule. It also allows a reduction of a factor of two in the number of input couplers, albeit with a doubling of the input power per coupler.

- The cost reduction associated with the superstructure variant is due to the reduction in the number of input couplers and the reduction in the length of the linac tunnel housings. The overall cost reduction is estimated to be $3 \%$ of the total project cost.

- Beam tests at TTF have confirmed the performance expectations of two prototype superstructures at low gradients. Additional $\mathrm{R} \& \mathrm{D}$ is needed to establish the reliability metrics of input couplers at power levels twice that of the reference design.

\subsubsection{DLDS in warm option}

- For the warm machine, the multi-moded Delay Line Distribution System (DLDS) is a more efficient pulse compression scheme than the SLED-II scheme used in the reference design.

- The cost savings associated with the use of the more efficient DLDS and the longer klystron pulse for the warm option is about $8 \%$ of total project cost. 
- Since the DLDS system is not as developed as SLED-II, additional R\&D, including high-power tests of the system, would be required before the DLDS could be qualified as a technically viable alternative to the SLED-II scheme.

\subsubsection{Conventional positron sources in both options}

- In contrast to the undulator-based source in the reference design, a conventional positron source will not allow the production of polarized positrons.

- Our simulations indicate that the integrated luminosity of the collider built with an undulator-based positron source could be reduced by $20 \%$ or so relative to one built with a reliable conventional source during stable operation, and by as much as a factor of two during commissioning. We have not, however, factored in any differences between the availabilities of the undulator and conventional positron sources themselves. The use of an undulator-based positron source will certainly have a significant impact on commissioning the positron source, damping ring, linac, and ultimately beam collisions.

- The use of a conventional positron source reduces the complexity of the construction, installation, and initial commissioning of the injectors and damping rings, and could result in up to a year's reduction in the construction and commissioning of the collider.

- Replacing the undulator-based source with a conventional source results in a cost savings of about $2 \%$ of the total project cost, but a later upgrade to include a polarized positron source would be difficult. If the initial configuration includes the space required in the linac to later install the components of the undulator-based source, the energy make-up in the linac, and the tunnel needed for the positron transfer line, then the cost would be approximately the same as starting with an undulator source with no conventional source.

- The option to build the collider with a conventional positron source and the vacant space needed to later implement a polarized undulator source should be more vigorously considered.

\section{$2.7 \quad$ Risk}

- Risk assessments for the major collider subsystems have been carried out. In these assessments, the risks have been evaluated in terms of the highest level collider parameters, energy and luminosity, and relative to the primary mission of the collider, as specified by the physics design requirements: the delivery of $500 \mathrm{fb}^{-1}$ of integrated luminosity at $500 \mathrm{GeV}$ in the first 4 years of operation.

- Several factors have been included in our assessment of risk. These include the origin and severity of potential modes of failure to carry out the physics mission, the method and time at which such failures will be identified, and the consequences that would occur if such a failure happens. We have not done an iteration of design and planning aimed at mitigation of these risks, but we have identified important areas of $R \& D$ that need to be carried out early in the collider project.

- The highest potential risks to the mission for both options are in the areas of the beam delivery systems, the controls systems, and the machine protection systems. The conclusion that the highest potential risks do not lie in the area of high gradient RF cavity development came as a surprise to us. This is due primarily to the intensive past and current technology R\&D programs in this area.

- The high risks in the beam delivery systems, the controls systems, and the machine protection systems are related to the fact that problems here generally are not encountered until the pre-operations phase of the project, which limits the possible response time. The risks associated with the machine protection systems need further elaboration and study. 
- The cold option has higher risk than the warm option in the damping rings. It is possible that the design of the cold damping ring could be improved, but there are a number of difficult features inherent in the design of any damping ring for the cold option.

- The warm option has higher risk than the cold option in the areas of emittance control in the main linacs, and the main linac RF technology.

- There are important objectives for $\mathrm{R} \& \mathrm{D}$ that should be addressed early in the project to mitigate risk for each choice of main linac technology. Many of these are not dependent on the linac technology choice at all. 


\section{Chapter 3}

\section{Reference Design Configurations}

\subsection{Design requirements}

The Accelerator Subcommittee of the U.S. Linear Collider Steering Group (USLCSG) has been charged with the preparation of options for the siting of a linear collider in the U.S. Two options have been developed: a warm option, following the design[ZDR, NLC01],[TRC, Chapter 3] of the GLC/NLC Collaboration, and a cold option, similar to the TESLA design[TDR] at DESY. Both options have been developed in concert, using, as much as possible, similar approaches in technical design for similar accelerator systems, and a common approach to cost and schedule estimation methodology, and to risk/reliability assessments.

For each option, the accelerator design task force has prepared a reference design configuration description. The reference designs for both options satisfy the physics-based machine requirements specified in the USLCSG Scope Document[2]. These reference designs also satisfy the requirements for an international linear collider, as specified by the Parameters Subcommittee of the International Linear Collider Steering Committee [3]. The linear collider requirements presented in the USLCSG Scope Document, and those specified by the Parameters Subcommittee of the International Linear Collider Steering Committee, are compared in Appendix A.

The requirements are:

- initial energy $\sqrt{s}=500 \mathrm{GeV}$

- upgrade energy: at least $\sqrt{s}=1000 \mathrm{GeV}$

- integrated luminosity $500 \mathrm{fb}^{-1}$ within the first 4 years of physics running, corresponding to a design luminosity of $2 \times 10^{34} \mathrm{~cm}^{-2} \mathrm{~s}^{-1}$

- A beamstrahlung-induced energy spread comparable to initial state radiation

- Ability to run at $\sqrt{s}=90-500 \mathrm{GeV}$ at luminosities commensurate with $\sqrt{s}$-scaling of the luminosity at $500 \mathrm{GeV}$

- electron beam polarization $80 \%$

- an upgrade option for positron polarization

- crossing angle at the collision point

- site consistent with two interaction regions, with one capable of $\gamma \gamma$ and $e^{-} \gamma$ collisions 
- capability for periodic running at the $Z$ resonance, with an option for high statistics running at the $Z$ or at $W^{+} W^{-}$threshold.

- capability for $e^{-} e^{-}$collisions

In Section 3.3, a design summary and overview is given, and general design considerations are presented for each of the major accelerator systems comprising the linear collider. The reference design descriptions for the two options then follow in Section 3.4 and Section 3.5.

\subsection{Design Overview}

This section presents a summary and overview of the principal parameters of the two reference designs described in detail in Section 3.4 and Section 3.5. The principal design parameters are summarized in Table 3.2.0.1.

\subsection{1 $500 \mathrm{GeV}$ reference designs}

The cold option has a design luminosity at $500 \mathrm{GeV}$ which is about $25 \%$ higher than than of the warm option. The geometric luminosity (i.e., the luminosity computed in the absence of beam-beam effects) is roughly the same for both options, despite the radically different bunch formats arising from the needs of the different RF technologies. However, the cold option, with its longer bunch length, has a higher value of the disruption parameter, leading both to a higher value of the luminosity, and to a greater sensitivity of the luminosity to beam offsets at the collision point, and to correlations in the emittance distribution at the IP.

The normal-conducting, traveling-wave accelerating structures of the warm option will be qualified to the design unloaded gradient of $65 \mathrm{MV} / \mathrm{m}$. The loaded gradient at nominal luminosity corresponds to $52 \mathrm{MV} / \mathrm{m}$, and the warm linac achieves $500 \mathrm{GeV}$ (c.m.) energy with this loaded gradient. Higher energies can be achieved with the same installed RF power, if the current (and luminosity) is reduced, corresponding to an increase in the loaded gradient, up to a maximum of $65 \mathrm{MV} / \mathrm{m}$, which yields an energy of $\frac{65}{52} \times 500 \mathrm{GeV}=625 \mathrm{GeV}$.

The design gradient for the cold option is chosen to provide roughly the same energy reach as the warm option. For the superconducting standing-wave structures of the cold option, the loaded and unloaded gradients are the same. If the cold cavities are qualified to a maximum design gradient of $35 \mathrm{MV} / \mathrm{m}$, at which the machine's energy reach should be $625 \mathrm{GeV}$ to match the warm option, then the gradient for 500 $\mathrm{GeV}$ operation is $\frac{500}{625} \times 35 \mathrm{MV} / \mathrm{m}=28 \mathrm{MV} / \mathrm{m}$. Since the installed $\mathrm{RF}$ power and cryogenics is fixed, as the gradient is increased, the current must be decreased to maintain constant RF power, and at some point the linac cycle rate must be decreased also to maintain constant cryogenic losses.

The roll-off of luminosity with energy for the two options is shown in Fig. 3.2.1.1. For the cold option, the break in the curve corresponds to the point at which the linac cycle rate must be decreased due to limited cryogenic capacity. The cycle rate at the maximum energy is about $2.5 \mathrm{~Hz}$.

As shown in Table 3.2.0.1, the efficiency of the cold option reference design, in terms of conversion of AC linac power to beam power, is about $\times 2.5$ higher than the warm option reference design. The major effect of this is that the $\mathrm{AC}$ power required to run the $0.5 \mathrm{TeV}$ cold reference design is about $30 \%$ less than that of the warm reference design. 
Table 3.2.0.1: US Linear Collider: overall parameters

\begin{tabular}{|c|c|c|c|c|}
\hline Parameter & $\begin{array}{c}\text { X-band } \\
\text { Reference design }\end{array}$ & $\begin{array}{c}\text { L-band } \\
\text { Reference design }\end{array}$ & $\begin{array}{l}\text { X-band } \\
\text { upgrade }\end{array}$ & $\begin{array}{l}\text { L-band } \\
\text { upgrade }\end{array}$ \\
\hline Beam Energy $[\mathrm{GeV}]$ & 250 & 250 & 500 & 500 \\
\hline Loaded RF gradient $[\mathrm{MV} / \mathrm{m}]$ & 52 & 28 & 52 & 35 \\
\hline Two-Linac total length ${ }^{a}[\mathrm{~km}]$ & 15.94 & 27.00 & 29.36 & 42.54 \\
\hline Bunches/pulse & 192 & 2820 & 192 & 2820 \\
\hline Electrons/bunch $\left[10^{10}\right]$ & 0.75 & 2 & 0.75 & 2 \\
\hline Pulse/s[Hz] & 120 & 5 & 120 & 5 \\
\hline$\gamma \varepsilon_{x}(\mathrm{IP})[\mu \mathrm{m}-\mathrm{rad}]$ & 3.6 & 9.6 & 3.6 & 9.6 \\
\hline$\gamma \varepsilon_{y}(\mathrm{IP})[\mu \mathrm{m}-\mathrm{rad}]$ & 0.04 & 0.04 & 0.04 & 0.04 \\
\hline$\beta_{x}(\mathrm{IP})[\mathrm{mm}]$ & 8 & 15 & 13 & 24.4 \\
\hline$\beta_{y}(\mathrm{IP})[\mathrm{mm}]$ & 0.11 & 0.4 & 0.11 & 0.4 \\
\hline$\sigma_{x}(\mathrm{IP})[\mathrm{nm}]$ & 243 & 543 & 219 & 489 \\
\hline$\sigma_{y}(\mathrm{IP})[\mathrm{nm}]$ & 3.0 & 5.7 & 2.1 & 4.0 \\
\hline$\sigma_{z}(\mathrm{IP})[\mathrm{mm}]$ & 0.11 & 0.3 & 0.11 & 0.3 \\
\hline$D_{y}$ & 12.9 & 22.0 & 10.1 & 17.3 \\
\hline$H_{D}^{b}$ & 1.46 & 1.77 & 1.41 & 1.68 \\
\hline $\mathcal{L}_{g}{ }^{c}\left[10^{33} \mathrm{~cm}^{-2} \mathrm{~s}^{-1}\right]$ & 14.2 & 14.5 & 22.2 & 22.7 \\
\hline $\mathcal{L}\left[10^{33} \mathrm{~cm}^{-2} \mathrm{~s}^{-1}\right]$ & 20.8 & 25.6 & 31.3 & 38.1 \\
\hline$N_{\gamma}$ & 1.19 & 1.48 & 1.24 & 1.58 \\
\hline$\delta_{E}[\%]$ & 4.6 & 3.0 & 8.2 & 5.9 \\
\hline Average power per beam [MW] & 6.9 & 11.3 & 13.8 & 22.6 \\
\hline Peak beam current during pulse $[\mathrm{mA}]$ & 855 & 9.51 & 855 & 9.51 \\
\hline Beam pulse length $[\mu \mathrm{s}]$ & 0.270 & 950 & 0.270 & 950 \\
\hline Total number of klystrons & 4520 & 603 & 8984 & 1211 \\
\hline Peak RF power per klystron [MW] & 75 & 10.0 & 75 & 9.7 \\
\hline Total number of structures & 18080 & 18096 & 35936 & 29064 \\
\hline Peak RF power per structure $[\mathrm{MW}]$ & 56 & 0.276 & 56 & 0.345 \\
\hline Linac $\mathrm{AC}$ power ${ }^{d}[\mathrm{MW}]^{2}$ & 207.6 & 132.7 & 389.9 & 295.9 \\
\hline Linac $\mathrm{AC}^{d}$ to beam efficiency $[\%]$ & 6.6 & 17.0 & 7.1 & 15.3 \\
\hline
\end{tabular}

$a_{\text {including overheads and insertions }}$

${ }^{b}$ Vertical waist assumed to be at the IP

${ }^{c}$ Geometric luminosity

${ }^{d} \mathrm{RF}$-related, cooling, and auxiliary power in main linacs

\subsubsection{Upgrade features of the reference designs}

Both options use a helical-undulator-based positron source, driven by a $150 \mathrm{GeV}$ electron beam. For the 500 $\mathrm{GeV}$ reference design, a short $(150 \mathrm{~m})$ undulator is used, which provides enough unpolarized positron flux to meet the design requirements. Sufficient length in the lattice is provided for extending the length of the undulator (to $200 \mathrm{~m}$ ), which allows an adiabatic upgrade path to a polarized positron source.

One of the requirements of the linear collider reference design is the ability to upgrade the machine energy from $500 \mathrm{GeV}$ to $1 \mathrm{TeV}$. We have chosen to satisfy this requirement in a way that allows an adiabatic upgrade to the higher energy, by placing both technology options initially in tunnels that are long enough to accommodate an extension of the linac to $1 \mathrm{TeV}$ at the maximum loaded gradient. At $500 \mathrm{GeV}$, the tunnels will be partially filled with linac structures; the remainder of the tunnel will contain a beam transport line. Consequently, by installing linac structures in the rest of the tunnel, both options can be upgraded to $1 \mathrm{TeV}$ without additional underground civil construction. Because of the difference in loaded gradient for the two 


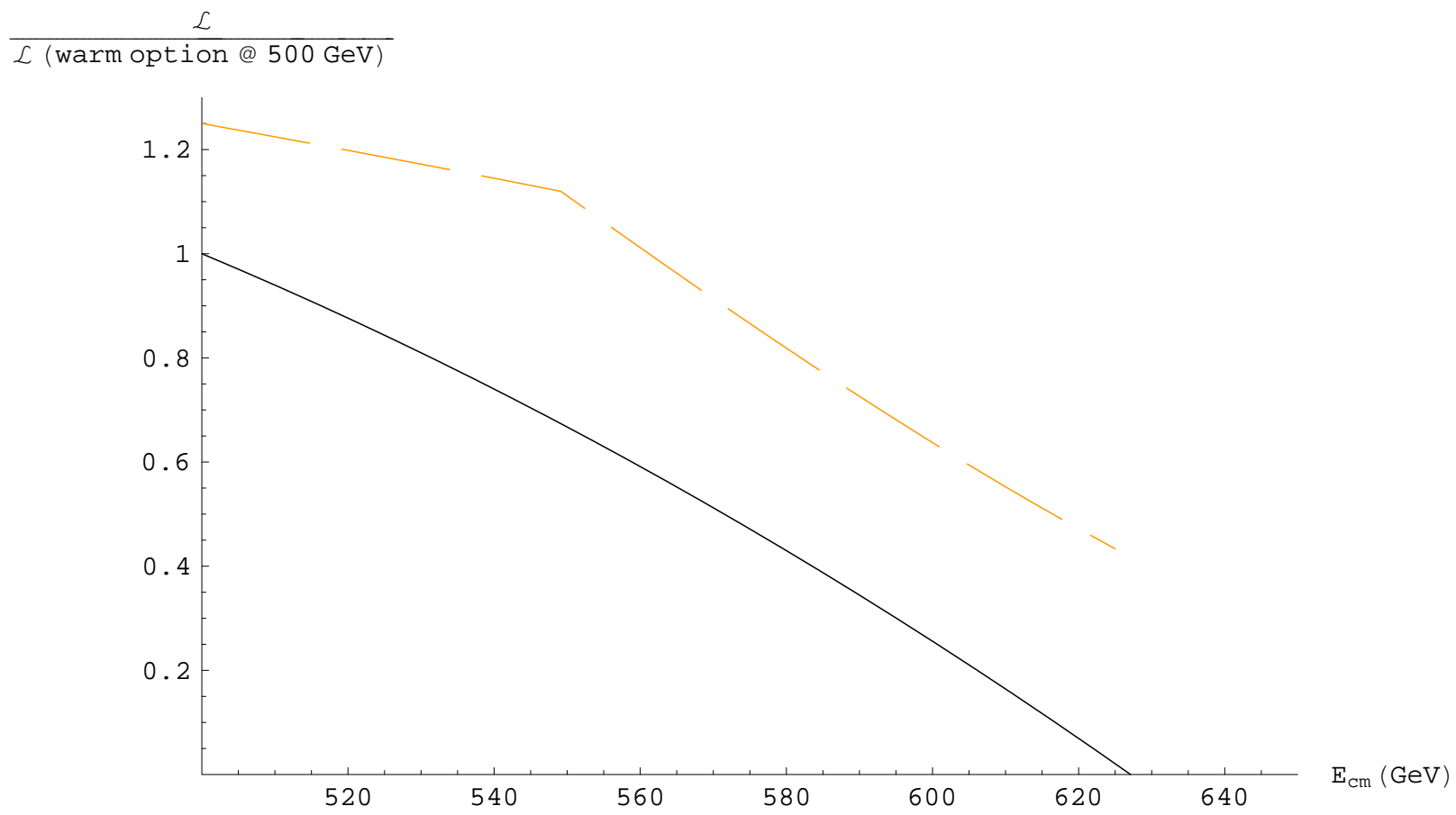

Figure 3.2.1.1: Luminosity roll-off with c.m. energy for the reference designs, normalized to the luminosity of the warm option at $500 \mathrm{GeV}$ c.m. Red (dashes): cold option. Black (solid): warm option. The endpoints of the curves correspond to the maximum gradients of $35 \mathrm{MV} / \mathrm{m}$ (loaded) and $65 \mathrm{MV} / \mathrm{m}$ (unloaded) for the two technologies. The $28 \mathrm{MV} / \mathrm{m}$ cold option design gradient was chosen to make the energy reach of the two machines approximately equal. With this gradient, the cold option reaches an energy of $610 \mathrm{GeV}$ at a luminosity of $10^{34} \mathrm{~cm}^{-2} \mathrm{~s}^{-1}$, while the warm only reaches $570 \mathrm{GeV}$ at the same luminosity.

technologies, the length of the conventional construction footprint for the cold option is about $42 \%$ greater than for the warm option.

It would also be possible to build the reference design with tunnels only long enough to accommodate 500 $\mathrm{GeV}$ operation at the maximum loaded gradient. In this case, an upgrade to higher energy would require additional underground civil construction to extend the main linac tunnels, and to extend the transfer lines from the damping rings to the new injection point. This approach was felt to be much more disruptive to an ongoing $500 \mathrm{GeV}$ physics program than the adiabatic approach adopted here, and it has not been studied further.

Table 3.2.0.1 includes a comparison of the design parameters for each option when upgraded to $1 \mathrm{TeV}$. As at $500 \mathrm{GeV}$, the upgraded cold option and warm option have similar geometric luminosities, but because of the higher disruption parameter, the cold option has a design luminosity which is about $25 \%$ higher than that of the warm option at $1 \mathrm{TeV}$.

The upgraded warm option has an energy reach of up to about $1.3 \mathrm{TeV}$, as shown in Fig. 3.2.2.1. As for the $500 \mathrm{GeV}$ case, this energy reach is available by operation with lower current, and increased gradient, up to the unloaded gradient of $65 \mathrm{MV} / \mathrm{m}$. For the cold option, since the cavities must operate at their maximum gradient of $35 \mathrm{MV} / \mathrm{m}$ to reach $1 \mathrm{TeV}$, operation above $1 \mathrm{TeV}$ is not possible. 


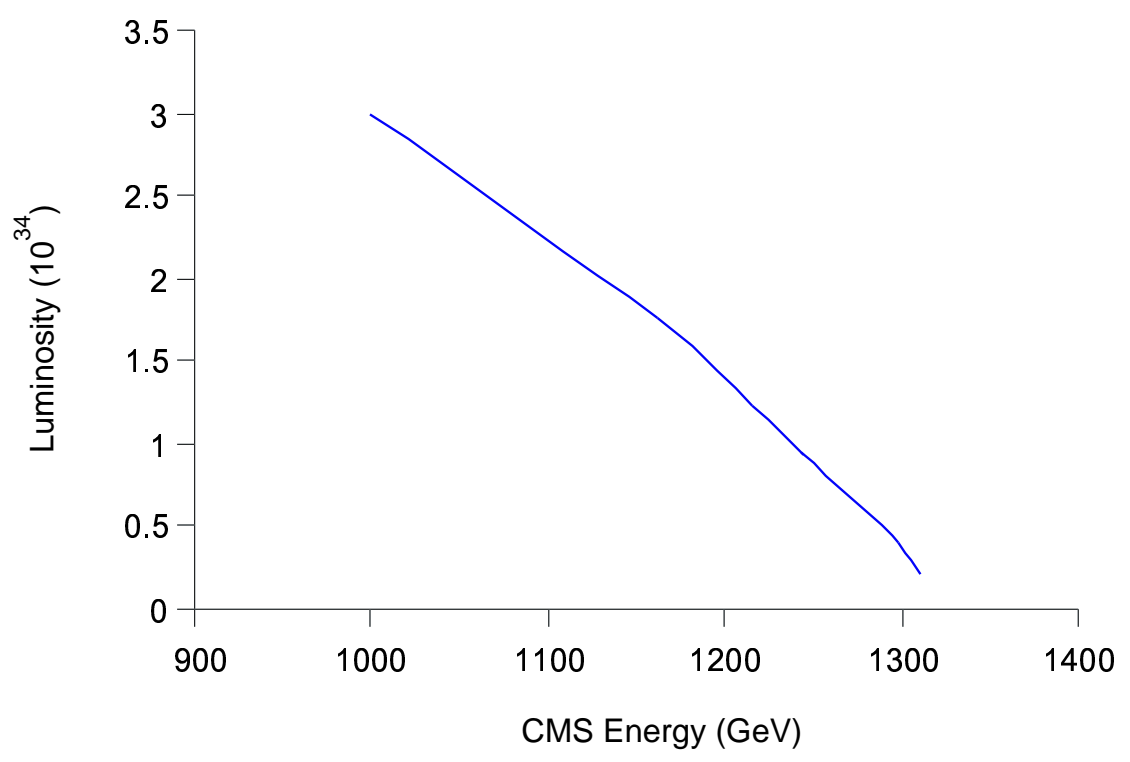

Figure 3.2.2.1: Energy versus luminosity for the upgraded X-band collider.

It should be noted that the upgrade energy is limited by the maximum gradient achievable in the linac structures installed for the upgrade. If, by the time the upgrade is implemented, R\&D has developed structures with higher maximum gradients, an upgrade energy in excess of $1 \mathrm{TeV}$ would be possible.

\subsection{General design considerations}

\subsubsection{Injection systems design considerations}

\subsubsection{Electron Source}

General performance specifications for the warm and cold $e^{-}$sources are given in Tables 3.4.2.1.1 and 3.5.2.1.1.2.

The main requirements of the sources are for bunch charge, charge jitter and bunch spacing that fulfill the linac requirements, as well as energy, energy spread, bunch length and emittance requirements set by the damping rings. A fundamental requirement is that of polarized electrons.

The electron source consists of these major subsystems: electron source gun system, buncher system (not needed for the unpolarized gun), pre accelerator, diagnostics section, $e^{-}$injector linac, injector final diagnostics systems, and spin rotation systems.

3.3.1.1.1 Polarized electron source gun system The polarized electron source gun system consists of a DC electron gun, with a GaAs cathode, and a laser to excite electrons from the cathode.

At the present state of the art, polarized sources are only possible with GaAs cathodes and dc guns. The GaAs cathodes require extremely low vacuum pressure $\left(10^{-11}-10^{-12}\right.$ Torr $)$ in order that cathode quantum efficiency does not deteriorate quickly. RF guns that would provide better beam emittances and result in simpler and better emittance injector systems have not yet been shown to work for polarized electrons. (This is an area of further R\&D.) 
Polarized GaAs cathodes also require drive lasers with the appropriate photon wavelength $(\sim 800 \mathrm{~nm})$, intensity, bunch spacing, and stability (phase and amplitude) to meet the electron beam requirements. There is a trade off between optimum quantum efficiency and polarization depending on the laser wavelength used. Thus one can compromise between more polarization or more charge if necessary. These lasers are not "off the shelf" and will require R\&D. One of the major challenges is intensity stability. The warm option requires $<0.5 \%$ jitter. The cold option is more tolerant because of the large stored energy in the superconducting cavities. Laser systems have been shown to be a reliability risk that take a concerted effort to solve.

Quantum efficiency has been an issue for high charge extraction per unit time, as for the warm option. Recent work has shown that this should no longer be a problem. However, because of the high charge extraction rate, the warm option gun operates at higher voltage and produces $175 \mathrm{kV}$ beam, whereas the cold option produces $120 \mathrm{kV}$ beam.

Because of their inherent reliability risk, there will be two polarized source gun systems available in either design. The cold option will have an additional unpolarized source.

3.3.1.1.2 Buncher system The choice of a low energy DC gun with its inherent space charge problems leads to the need for buncher systems to shorten the bunch length and capture the electrons to prepare them for efficient capture and acceleration in higher frequency higher gradient RF systems. Buncher cavities operate at sub harmonic frequencies of the final frequency. For the warm option, this is $714 \mathrm{MHz}$, followed by S-band buncher accelerator. In the cold option, three systems are used: a $108 \mathrm{MHz}$ prebuncher, followed by a $433 \mathrm{MHz}$ buncher, followed by a $1.3 \mathrm{GHz}$ buncher-accelerator.

Buncher systems are surrounded by solenoids to provide strong focusing of the divergent low energy beam. Because of the necessity of these solenoid fields, the buncher cavity systems must be normal conducting.

The beam energy coming out of the buncher systems is $\sim 175 \mathrm{kV}$ for the warm option and $12 \mathrm{MeV}$ for the cold option.

3.3.1.1.3 Pre-accelerator system The pre-accelerators accelerate the beam to an energy sufficient for its injection into the standard linac acceleration modules of the electron injection linac.

In the case of the cold option, this is accomplished by two pairs of normal conducting cavities that accelerate the beam to $76 \mathrm{MeV}$, followed by two standard superconducting modules that accelerate to $500 \mathrm{MeV}$. For the warm option, the pre-accelerator consists of a 4 meter S-band structure, and the resulting energy is 80 $\mathrm{MeV}$.

Optics focusing and matching is necessary between the sections.

3.3.1.1.4 Diagnostics section After the pre-accelerator, there is a major diagnostics section in which to analyze the beam properties, and low energy beam dumps.

Up through this point, there are two parallel fully redundant systems that can be easily switched from one to another in case of difficulty with one or the other. The systems can be run and tuned independently.

3.3.1.1.5 The cold option unpolarized injector In the case of the cold option, there is a third unpolarized electron source. This is used for commissioning, tune up, and unpolarized beam operation. The unpolarized source is much less complicated and supplies low emittance beam more easily. It consists of a CsTe cathode RF photogun operating at $1.3 \mathrm{GHz}$ and a laser system. CsTe does not have the stringent vacuum requirements of GaAs cathodes. This is followed by a superconducting booster cavity to provide 20 $\mathrm{MeV}$ beam. The beam then is accelerated in a pre-accelerator, composed of three standard superconducting 
modules, to $500 \mathrm{MeV}$, where it can be switched into operation as with the polarized sources. This system is basically the same as that used at TTF and has no unknowns.

3.3.1.1.6 Electron injection linac The electron injection linac receives beam from one of the redundant source pre-accelerator systems and accelerates it to the damping ring energy. For the warm option, thirtysix normal-conducting $4 \mathrm{~m} \mathrm{~S}$-band structures are used to provide $1.98 \mathrm{GeV}$ beam. In the cold option, 18 standard $1.3 \mathrm{GHz}$ superconducting modules are used to accelerate the beam to $5 \mathrm{GeV}$.

3.3.1.1.7 Diagnostics, bunch compression, and spin rotation prior to Damping Ring Injection The requirements for $e^{-}$and $e^{+}$beams are similar and both are discussed in the positron source section below.

\subsubsection{Positron Source}

General specifications for the warm and cold undulator and conventional based $e^{+}$sources are given in Tables 3.4.2.5 and 3.5.2.7. For this study the undulator source has been chosen as the base reference design. The conventional $e^{+}$source variant specifications are discussed in Section 7.6.

The main requirements of the $e^{+}$sources are that they provide bunch charge, charge jitter and bunch spacing that fulfill the linac requirements, as well as energy spread, bunch length and emittance requirements set by the damping rings.

Polarized positrons are not a base design requirement; however, a main advantage of the undulator based source is its ability to provide polarized $e^{+}$beams. Another advantage is that target heating and $e^{+}$emittance can be lower, because photons are generated in the undulator and only need to be pair converted in the target. In the conventional source, a separate linac system must provide the electron beam that is then converted to photons and pairs in a thicker target. Target heating and the emittance of the captured $e^{+}$become more of an issue. The former may put limits on the amount of beam that can be reasonably produced. The latter may lead to the necessity of larger acceptance damping rings, or pre-damping rings.

On the other hand, conventional positron sources are operationally much easier to deal with. The $e^{+}$and $e^{-}$ accelerators are completely decoupled. One does not need to have essentially all of the $e^{-}$accelerator working in order to begin to provide $e^{+}$beams to the $e^{+}$accelerator chain. This has benefit during construction, commissioning, and operation. Reliability and recovery from repair and beam down times are expected to be substantially better, as the bringing up of the two accelerator chains can be in parallel, not in series.

The undulator based positron source consists of these major subsystems: undulator system, production target, capture and matching section, positron pre-accelerator (PPA), diagnostics section, positron injector linac (PIL), transport, injector final diagnostics, spin rotation system (optional), and a longitudinal energy compressor system.

The conventional positron source consists of these major subsystems: a drive linac system and a production target system, followed by systems as discussed above for capture and matching, acceleration, etc. The conventional system can be located in the vicinity of the $e^{+}$damping ring and does not require the multi-km long transport line needed for the undulator source.

To a large extent the systems for warm and cold options are very similar. The general undulator source parameters are given in Tables 3.4.2.5 and 3.5.2.7.

3.3.1.2.1 Undulator positron source system The undulator system consists of the $e^{-}$linac undulator bypass insertion, and the undulator. 
3.3.1.2.1.1 The $e^{-}$linac undulator bypass insertion The undulator based positron source uses the main $e^{-}$linac beam to drive the undulator photon source. The $e^{-}$beam is diverted through a chicane bypass off-axis $2.5 \mathrm{~m}$ from the main linac line. This is to allow for the straight ahead photon, pair production and capture in an offset high radiation region which can be shielded from the main linac. The overall length of this transport is $850 \mathrm{~m}$ to allow for sufficiently low deflecting bends so as not to increase the $e^{-}$energy spread and emittance appreciably through incoherent synchrotron radiation. The bypass consists of about $250 \mathrm{~m}$ of FODO bend at each end, and about $350 \mathrm{~m}$ of straight offset line in which to match to the undulator and provide $200 \mathrm{~m}$ space for the undulator itself. The bypass has an energy bandpass of $\pm 3 \%$ to allow for operational and tuning variations of the $e^{-}$beam energy at the undulator location. Spoilers will be needed to protect against large energy errors.

The main design considerations associated with the location (energy) of the undulator in the $e^{-}$linac are discussed in Sections 3.4.2.2.1 and 3.5.2.2.1. This location gives the possibility of flexibility in the $e^{-}$collision energy without changing the beam energy at the undulator and disrupting the tune conditions at this point. In the TESLA TDR, the undulator was placed at the end of the linac, and necessitated operation of the linac in two sections for very low collision energy. An earlier variant with the undulator after the IR was found to be impractical because the large disruption caused by collisions made it difficult to capture the bean into an undulator channel.

Beam energy extracted by the undulator must be made up in the linac. This requires 95 (170) meters more linac in the warm (cold) unpolarized variant and 126 (240) in the polarized variant. By comparison, the conventional source would require about $230 \mathrm{~m}$ for the warm (cold) drive linac, in which S-band (L-band superconducting) technology would be used.

3.3.1.2.1.2 Undulator The undulator parameters are selected to provide sufficient photons to the target and $e^{+}$capture section so as to exceed the requirements for $e^{+}$charge. The specified yield per $e^{-}$is 1.5 . Thus the undulator period, field strength, and length must be optimized for photons of the desired critical energy and quantity. The critical energy is chosen to be $\sim 11 \mathrm{MeV}$ in order to be as high as possible without getting above the giant neutron resonance in order to minimize neutron radiation. With reasonable undulator parameters (undulator period $\sim 1 \mathrm{~cm}$, field strength $1.1 \mathrm{~T}$ ), a $150 \mathrm{GeV}$ drive beam energy is required to achieve $11 \mathrm{MeV}$ photon energy. For unpolarized $e^{+}$beam, the undulator could be a planar conventional permanent magnet design. However, a helical superconducting undulator has been selected for the base design in this study. It has two advantages: it can provide polarized photons in order to make polarized $e^{+}$beams for collisions as a future upgrade, and for unpolarized production it produces about twice the photon flux per unit length of a planar undulator. Thus, in the initial installation, a short undulator can be used. Later, the length can be increased from 140 to $200 \mathrm{~m}$ to provide sufficient yield for polarized $e^{+}$ operation. The longitudinal space in the undulator bypass is sufficient for a future upgrade or extension of the undulator if more flux would be required.

The undulators will be built in $2 \mathrm{~m}$ sections separated by quadrupole focusing. Helical superconducting undulators with bifilar windings are not at this time standard. Care must be taken with conductor positioning tolerances. Development of helical undulator prototypes should be part of the on-going R\&D program. The helical undulator does not allow for polarization polarity switching; this would need to be implemented downstream in the transport prior to injection into the damping rings.

3.3.1.2.2 Conventional $e^{+}$source driver A drive $e^{-}$linac is needed for a conventional source. This is a standard photoinjector linac with the pulse train structure required for the cold and warm variants, respectively. The energy of the drive beam is chosen to be $6.2 \mathrm{GeV}$, based on NLC design considerations. This energy and charge of the drive linac are optimized to yield the required positron flux. The charge per bunch is the same as that of the electron linac $e^{-}$source. The energy of the drive beam is then set to yield under optimum conditions about 1.5 times the required flux delivered to the damping ring. The target thickness is adjusted to near the shower maximum. A trade off between drive beam charge and energy is 
possible and optimization may result in a lower energy beam with more charge. These studies have not yet been carried out.

Charge fluctuations in the drive beam must be minimized, as with the $e^{-}$source. This puts requirements of $2 \%$ and $5 \%$ on the bunch-to-bunch charge fluctuations for the warm and cold drive beams respectively and tighter tolerances for train to train stability.

The warm variant drive beam consists of an RF gun injector and an S-band linac. The cold variant uses a superconducting L-band linac. The overall length of either linac is about $230 \mathrm{~m}$.

The linac beam must be separated or split to parallel target systems for either conventional $e^{+}$source variant. An RF separator deflects the beam to three (plus one spare) target system for the warm variant, and to two (plus one spare) for the cold variant. This is necessary in order to limit the target pulse heating. (See Fig. 7.6.1.1)

3.3.1.2.3 Positron Production Target, Capture region and Positron Pre Accelerator For either the undulator based source or the conventional source, the production target area is a very high radiation region, and it includes a number of challenging components. For this reason, it is configured with redundant systems.

For the undulator source, two redundant systems are aligned in series along the photon beam. The layout can also incorporate an $e^{-}$injector and any of the three systems can be switched to feed the Positron Injector Linac. (See Figures 3.4.2.4, 3.5.2.2)

The conventional source would have similar elements, but the targets would be arranged in parallel so the drive beam could be multiplexed on a bunch by bunch basic to alternative target systems. (See Fig. 7.6.1.1) Three such systems are needed for the warm variant and two for the cold. Each has one spare system and an $e^{-}$source could be incorporated as well.

3.3.1.2.4 Positron Production Target The undulator positron production target converts photons from the undulator to pairs. It is located about $220 \mathrm{~m}$ downstream from the undulator exit, in order to produce a suitable spot size. This drift is a compromise governed by the need to have a sufficiently large spot to limit target heating to a tolerable level vs. the desire to have a small spot in order to produce the smallest possible positron emittance for large angular acceptance.

The conventional target systems convert electrons from the drive linac. For this reason, the targets need to be thicker and have more of a thermal heating problem.

The target itself is one of the most critical mechanical devices of the collider system. The demands on it are not as severe for the undulator $e^{+}$production as for conventional $e^{+}$, but they are similar and will be discussed together.

The target material of preference for the undulator source is Ti alloy. This material, with a 0.4 r.l. thickness, gives optimum performance in terms of yield relative to heat capacity and thermal strength, as discussed in Sections 3.4.2.2.4 and 3.5.2.2.4. The target thickness can be relatively thin because the conversion is from photons not electrons as with the conventional source. The transverse momentum given to the $e^{+}$particles is less, and this, along with a smaller target spot, provides better emittance and higher efficiency capture of the positrons. The thin target also provides more efficient escape of the low energy particles.

For the conventional source, a 4 r.l. tungsten-rhenium target is needed in order to provide sufficient conversion from the $e^{-}$beam. One is driven to tungsten in order to have a sufficiently short target, so the emerging source spot can be kept reasonably small. A titanium target would be prohibitively long. However, the spot size on target is larger than for the undulator source, and leads to a larger emittance beam to the damping ring. For the cold option this may result in the need for a larger acceptance damping ring. Based on SLC 
Table 3.3.1.1: Positron target system parameters

\begin{tabular}{|c|c|c|c|c|}
\hline & $\begin{array}{c}\text { Warm } \\
\text { undulator }\end{array}$ & $\begin{array}{c}\text { Cold } \\
\text { undulator }\end{array}$ & $\begin{array}{c}\text { Warm } \\
\text { conventional }\end{array}$ & $\begin{array}{c}\text { Cold } \\
\text { conventional }\end{array}$ \\
\hline \# targets/spares & $1 / 1$ & $1 / 1$ & $3 / 1$ & $2($ or 1$) / 1$ \\
Energy on target/train [kJ] & 1.1 & 44.3 & 0.5 & $28.0(56.0)$ \\
Spot size (rms) [mm] & 0.75 & 0.75 & 1.6 & 2.5 \\
Target material & Ti alloy & Ti alloy & WRe & WRe \\
Target thickness [r.l.] & 0.4 & 0.4 & 4.0 & 4.0 \\
Energy absorbed [\%] & 9 & 9 & 14 & 14 \\
Target average power absorbed [kW] & 12 & 19 & 8 & $20(39)$ \\
Pulsed temp rise [ $\left.{ }^{\circ} \mathrm{C}\right]$ & 422 & 410 & 189 & 256 \\
Peak energy deposition [J/g] & 224 & 217 & 28 & 38 \\
Target radius [m] & 0.125 & 0.8 & 0.125 & 1.0 \\
Target rev rate [rpm] & 46 & 1200 & 46 & $1200(2400)$ \\
\hline
\end{tabular}

experience, the peak energy deposition in the target must be kept to $<50 \mathrm{~J} / \mathrm{g}$. The temperature rise is $\sim 200$ $\mathrm{C}$ for the parameters chosen.

The critical issues are heating and radiation damage. The target disks are rotating, in order to spread the heat from different beam pulse trains on to different areas of the target, and limit the thermal shock to $\sim 400 \mathrm{C}$ for the $\mathrm{Ti}$ undulator target and $\sim 200 \mathrm{C}$ for the tungsten conventional target. This is a factor of two below the stress limit of undamaged target material. Though it may be possible to cool the targets by radiation, one has to consider the material strength at the temperature needed to radiate the absorbed energy. Water cooling seems the more conservative solution, though the water must flow to the rotating disk. If water cooling is required, then water-to-vacuum feedthroughs based on differential pumping would need to be developed. This is clearly an area for R\&D.

The different pulse train parameters of the warm and cold options, the different target material and thickness parameters, and the different power on target for the undulator and conventional production led to a variety of target criteria. These criteria are dominated by heating, heat removal, thermal stress, and radiation damage issues. The radiation damage thresholds are not well understood and more investigation will be needed as discussed in Sections 3.4.2.2.4 and 3.5.2.2.4.

Pulse train duration and repetition rates result in different operating conditions for the rotating targets. In the warm option, the pulse train is of very short ( $270 \mathrm{~ns}$ ) duration and essentially all the drive beam for one pulse train will hit at a specific location on the target, independent of its rotational speed at beam impact. This results in the need for 3 parallel target systems to limit heating for the conventional system. But it also means that target rotation at high tangential speeds is not as critical as for the cold option, resulting in smaller target diameters and lower rotation speeds that must only move to a new target area before the next pulse. For the cold option, the pulse train duration is long enough so that it can be distributed along a bigger area if the target has sufficient velocity at the radius at which the beam hits. As the power ratio per pulse between warm and cold is of the order of the relative repetition rates $(5 \mathrm{~Hz} / 120 \mathrm{~Hz})$ it is necessary for the cold target to be of the order of $1 \mathrm{~m}$ radius, revolving at $\sim 1200 \mathrm{rpm}$ in order to effectively spread the beam pulse. Thus it is essential to understand if cooling by radiation is sufficient, or if water cooling using a vacuum feedthrough with differential pumping is required.

The positron target system parameters are listed in Table 3.3.1.1.

3.3.1.2.5 Capture and matching system The capture and matching section consists of a tapered solenoid field (magnetic matching section, MMS) to best capture as many positrons as possible and match them transversely into the down stream positron pre-accelerator (PPA) section. It basically converts beam angular divergence to a spot with parallel trajectories. This magnetic device must be able to survive in the 
very high radiation field just downstream of the production target. It must produce high magnetic fields and in the case of the cold option sustain these fields over the length of the $1 \mathrm{~ms}$ beam pulse.

For the warm option, the MMS consists of a dc tapered-field solenoid with a peak field of $1.2 \mathrm{~T}$ and a pulsed flux concentrator that acts as a phase space transformer in which the magnetic field varies adiabatically from $7 \mathrm{~T}$ to $0.5 \mathrm{~T}$. The entrance aperture is $4.5 \mathrm{~mm}$ id. The operating parameters are close to those of SLC.

The cold option, has an Adiabatic Matching Section (AMS) based on the TDR. The peak field is 6 T, tapering to $0.16 \mathrm{~T}$. Long pulse flux concentrators of about the same parameters have been built. However it would be important to construct a prototype that meets the cold design pulse and heating requirements.

It is important to realize that the flux concentrator provides about a factor of two in positron yield, so as a risk item it is not as critical as it would be if the positron production had to rely completely on it.

The length scale of the flux concentrator is of order $10 \mathrm{~cm}$ and it is located directly ( $\sim$ few $\mathrm{mm}$ ) downstream from the rotating target. It is clear that development of the target flux concentrator unit that meets design requirements for either warm or cold options is an important $R \& D$ activity.

3.3.1.2.6 Positron Pre-accelerator (PPA) The positron pre-accelerator follows the capture and matching section and accelerates the beam to $250 \mathrm{MeV}$. It is by necessity normal conducting, both because of the very high radiation field behind the target and because of the need to supply strong solenoid focusing to capture the positron beam. After the pre-accelerators, beamlines from the various target-capture systems are combined by switches in the case of the undulator source, or RF separators in the case of the conventional source. Positrons are separated from electrons and photons, and the positron beam is injected into positron injector linac (PIL).

The positron pre-accelerator and the positron injector linac both are L-band linac structures for both the warm and cold options. This frequency choice increases the capture yield of the positrons by a factor of 30 compared with S-band.

The warm option positron pre-linac consists of two $5 \mathrm{~m}$ accelerating structures operation at $24 \mathrm{MV} / \mathrm{m}$ loaded gradient. The structures are encased in a $0.5 \mathrm{~T}$ solenoid field.

The cold option positron pre-linac consists of two $1.1 \mathrm{~m}$ sections operating at $14.5 \mathrm{MV} / \mathrm{m}$, followed by seven $4 \mathrm{~m}$ structures operating at $8.5 \mathrm{MV} / \mathrm{m}$. These are standing wave structures with magnetic coupling slots. They are designed with consideration for the long RF pulse length and high resultant structure heating. Prototypes will be developed to check their operation at the required gradient and pulse duty factor.

Up to this point there are redundant targets and capture systems. At this point positrons must be separated from electrons and photons, and diagnostics supplied to measure the beam properties. The parallel or redundant systems are merged and directed into the positron injector linac.

3.3.1.2.7 Positron Injector Linac (PIL) The positron injector linac accelerates the positron beam to $1.98 \mathrm{GeV}$ for the warm option and $5 \mathrm{GeV}$ for the cold option. In the warm option, normal conducting traveling-wave L-band structures, as in the positron pre linac, will be used. Thirty-six $5 \mathrm{~m}$ RF structures with a loaded gradient of $13.2 \mathrm{MV} / \mathrm{m}$ will be used.

For the cold option, superconducting linac modules will be used. Because of the large transverse emittance of the beam, additional superconducting quadrupole doublets are used in the lower energy modules. The first 8 modules (CM-1) will have 4 cavities and 4 doublets; the following $\sim 20$ modules (CM-2) will have 8 cavities and one doublet.

3.3.1.2.8 Positron Transfer line The undulator positron source needs a very long transport from the positron injector linac at the $150 \mathrm{GeV}$ point of the electron linac, along the beam delivery line bypassing the 
IR region to the injection point of the positron damping ring. This line is 25 to $30 \mathrm{~km} \mathrm{long}$, depending on the warm/cold variant.

The conventional positron source does not require such a transport, as the drive linac can be located near the positron damping ring.

3.3.1.2.9 Diagnostics, energy compression, and spin rotation prior to Damping Ring Injection The following description applies to both $e^{-}$and $e^{+}$systems. After the injection linac and transfer line (for $e^{+}$ undulator system) beam must be transported to the damping ring. This transport must support diagnostics to quantify the beam properties, it must support necessary manipulations of the polarization, and it must provide energy compression if necessary. Beam properties to be measured include emittance, energy, energy spread, bunch length, and timing jitter. It is likely that collimation and scrapers will be necessary as well.

The polarization manipulations include first a rotation from the longitudinal to the horizontal in an arc section then from horizontal to vertical in a solenoid section. For $e^{-}$beams, the sign of the polarization can be controlled and switched by changing the laser polarization on the injector gun photocathode. This makes it rather easy to alternate between polarizations during experimental operation.

For the $e^{+}$polarization from a helical undulator, it is only feasible to generate one polarization. In this case, the spin rotation section would need to include two parallel solenoid sections after the spin has been rotated from longitudinal to horizontal. A switch system would divert the beam to one or the other leg to provide up or down polarization.

An energy compressor is desirable especially in the case of the $e^{+}$beam to better match the energy spread from the linac to the acceptance of the ring. This compressor could use the spin rotating arc to generate energy time correlation and an RF system to reduce the correlated energy spread.

From here the beam proceeds to the damping ring.

3.3.1.2.10 Conclusions The major design consideration for the sources is the choice of an undulator or conventional based positron source. The conventional source has considerable operational advantages, as is addressed in the Availability Chapter(4). However, it cannot provide polarized positron beams.

The heating and radiation issues of the positron production target and flux concentrator are a significant engineering challenge, especially for the cold option and for the conventional source. This is clearly an area of R\&D. It is possible that other target ideas (such as those being developed for neutron sources or neutrino factories) deserve study as well. The emittance from a conventional target is not as good as from the undulator target. This may affect damping ring designs.

A superconducting helical undulator is not at present standard available magnet technology. R\&D is necessary here to meet the requirements, especially those of field quality.

The polarized electron sources are based on existing technology and are expected to meet the performance specifications. However, the laser systems for these sources do require considerable engineering development. For the $e^{-}$source, a polarized RF gun might reduce the requirements and cost of the $e^{-}$damping ring. This is an area of long term $\mathrm{R} \& \mathrm{D}$ effort.

\subsubsection{Damping ring design considerations}

Damping rings in a linear collider have three purposes:

1. The damping rings must accept electron and positron beams with large transverse and longitudinal emittances, and produce beams with the small emittances needed to produce high luminosity in a time 
consistent with the collider repetition rate.

2. The damping rings must damp incoming beam jitter (transverse and longitudinal) and provide highly stable beams for systems downstream.

3. The damping rings delay bunches from the source so that feed forward systems may be used to tune downstream systems to compensate for charge variations.

Each bunch train is stored in a damping ring for one or more machine cycles. During this time, radiation damping reduces the bunch emittances close to the equilibrium values determined by the ring design. Thus, the designs of the damping rings are partly driven by the parameters required for the extracted beam; the designs must also meet specifications set by the dimensions of the injected beams (the ring acceptance) and the repetition rate of the collider (the damping time). Below, we discuss in a little more detail the general considerations that determine the ring design, comparing the warm and cold technology choices. Note that in a storage ring, nearly all the parameters are coupled to some extent. For example, the length of the damping wiggler affects not just the damping time, but also the horizontal emittance, bunch length and energy spread, and the acceptance. The fact that each design choice affects many beam parameters makes the design of a damping ring an iterative and rather convoluted process.

For both warm and cold technologies, there are separate damping rings for the electron and positron beams, with single damping rings for the electron beam. The cold technology uses a single damping ring for the positron beam, while the warm technology uses a positron pre-damping ring in addition to the positron main damping ring. Although electron sources are reaching the stage where the emittances reached in the damping rings could be produced directly, it is not presently possible to generate low emittance polarized electron beams. Also, in eliminating the electron damping rings one would lose the advantages of reducing jitter and delaying bunches to allow operation of feed-forward systems.

At the SLC, the damping rings were one of the most problematic subsystems. This was because the downstream systems are extremely sensitive to small changes in the beams extracted from the rings, and because the beams are stored in the rings for a relatively long time, which makes them more sensitive to subtle accelerator physics effects. Although the principal physical processes occurring in damping rings are well understood, the damping rings for a future linear collider will operate in regimes where effects are expected for which there are presently limited data available, or for which the models are not fully understood or are difficult to apply. These effects include the electron cloud instability (in the positron damping rings) and the fast ion instability (in the electron damping rings). Also, there are particular components (for example, the injection and extraction kickers) where the specified parameters are beyond those presently demonstrated. The risks associated with the damping rings are discussed more fully in Section 8.3.

Some of the principal parameters of the damping rings for the warm and cold technologies are given in Table 3.3.2.1, together with parameters for some existing storage rings. The damping rings for the warm technology are closer to existing rings than the damping rings for the cold technology.

\subsubsection{Bunch train length and ring circumference}

A damping ring must be capable of storing at least one full bunch train. In the case of the warm technology, the relatively short bunch train (approximately $80 \mathrm{~m}$ ) suggests a convenient ring circumference. To ease engineering challenges by providing more space, the rings are designed to store two (predamping ring) or three (main damping rings) bunch trains simultaneously. Including gaps between bunch trains for the injection and extraction kickers to turn on and off, still allows for rings of circumference around $300 \mathrm{~m}$, which can reasonably be accommodated in tunnels separated from the main linac.

For the cold technology, the fully extended bunch train would be nearly $285 \mathrm{~km}$, which is clearly impractical for a storage ring. Therefore, each bunch train is "folded over" on itself by injecting and extracting bunches individually, reducing the bunch spacing from $337 \mathrm{~ns}$ to a little under $20 \mathrm{~ns}$. This reduces the circumference 
Table 3.3.2.1: Comparison of damping ring parameters with existing storage rings

\begin{tabular}{|c|c|c|c|c|c|c|}
\hline Parameter & $\begin{array}{c}\text { Warm Main } \\
\text { Damping } \\
\text { Ring }\end{array}$ & $\begin{array}{c}\text { Warm Pre- } \\
\text { Damping } \\
\text { Ring }\end{array}$ & $\begin{array}{c}\text { Cold } \\
\text { Positron } \\
\text { Damping } \\
\text { Ring }\end{array}$ & $\begin{array}{c}\text { KEK } \\
\text { ATF }\end{array}$ & $\begin{array}{c}\text { LBNL } \\
\text { ALS }\end{array}$ & $\begin{array}{c}\text { SLAC } \\
\text { PEP-II } \\
\text { LER }\end{array}$ \\
\hline \hline Circumference $[\mathrm{m}]$ & 300 & 231 & 17000 & 139 & 197 & 2200 \\
Energy [GeV] & 1.98 & 1.98 & 5.0 & 1.28 & 1.9 & 3.1 \\
Bunch charge $\left[10^{10}\right]$ & 0.8 & 0.8 & 2.0 & 1.0 & 0.6 & 7.0 \\
Bunch trains $\times$ Bunches & $3 \times 192$ & $2 \times 192$ & $1 \times 2820$ & 20 & 272 & 939 \\
Current $[\mathrm{mA}]$ & 738 & 639 & 160 & 70 & 400 & 1400 \\
Normalized horizontal & & & & & & \\
emittance $\left(\gamma \epsilon_{x}\right)[\mu \mathrm{m}]$ & 3.0 & 46 & 8.0 & 2.6 & 25 & 150 \\
Normalized vertical & & & & & & \\
emittance $\left(\gamma \epsilon_{y}\right)[\mu \mathrm{m}]$ & 0.019 & 5 & 0.014 & 0.012 & 0.02 & 6 \\
Bunch length $[\mathrm{mm}]$ & 5.5 & 5.2 & 6.0 & 3.1 & 7.0 & 11 \\
Energy spread [\%] & 0.1 & 0.09 & 0.13 & 0.055 & 0.1 & 0.07 \\
Vertical damping time [ms] & 4.1 & 3.8 & 28 & 29 & 15 & 70 \\
Energy loss/turn from & & & & & & \\
wiggler $[\mathrm{keV}]$ & 834 & 519 & 18700 & 0 & 20 & 0 \\
Energy loss/turn total $[\mathrm{keV}]$ & 970 & 803 & 2300 & 41 & 250 & 653 \\
RF frequency [MHz] & 714 & 714 & 500 & 714 & 50 & 476 \\
RF voltage [MV] & 2.0 & 1.8 & 50 & 0.77 & 1.0 & 3.2 \\
\hline
\end{tabular}

to around $17 \mathrm{~km}$. To minimize the amount of tunnel that needs to be dug, the rings include two parallel straight sections that have a total length of $15 \mathrm{~km}$, and are placed in the same tunnel as the main linac. The straights are joined at either end by arcs of roughly $1 \mathrm{~km}$ circumference. See Fig. 3.3.2.1

Irrespective of the technology, it is desirable to make the circumference no longer than strictly necessary, since a short circumference helps achieve more rapid damping.

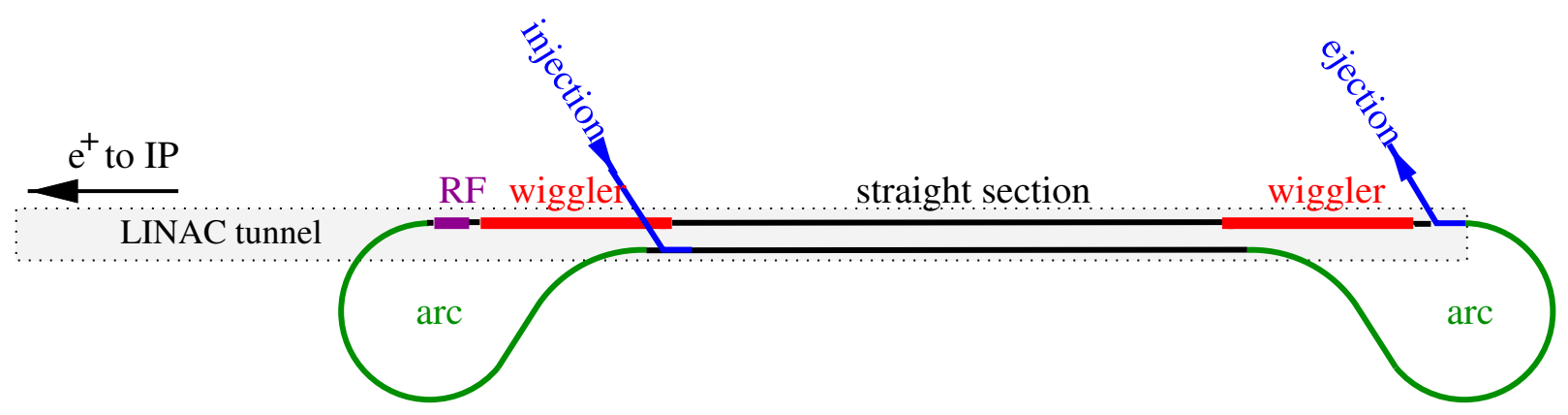

Figure 3.3.2.1: Conceptual layout of the cold positron damping ring. The electron ring is similar with the exception that the injection point is located close to the indicated ejection position at the beginning of the linac.

\subsubsection{Horizontal emittance and ring energy}

The horizontal emittance of the beam extracted from the damping ring is important for the beam size at the IP. Low beam energy helps to achieve a low horizontal (and longitudinal) emittance. However, low beam 
energy also results in long damping times, which means that a longer wiggler is needed to provide the damping required by the repetition rate. Also, low energy beams are vulnerable to a number of collective effects that disrupt beam quality or limit the stored current. The final choice of beam energy is a compromise between the competing effects of emittance against damping time, and must also take into account the presence of spin depolarization resonances at well-defined energies.

For the warm technology, the beam energy chosen for the damping rings is $1.98 \mathrm{GeV}$. In the main damping rings, something over 30 arc cells are then needed to provide the specified emittance, and these must be highly compact to allow space for other essential systems without leading to a larger circumference than necessary.

For the damping rings for the cold technology, the beam energy chosen is $5 \mathrm{GeV}$. The total arc length of 2 $\mathrm{km}$ provides sufficient space for the number of arc cells needed to achieve the low emittance required.

\subsubsection{Vertical emittance and beam jitter}

Producing a beam with very low vertical emittance from the damping ring is important for the final luminosity of the collider. In contrast to the horizontal emittance, the vertical emittance is primarily a question of alignment and tuning of the ring during operation. The sensitivity of the lattice to various misalignments can be influenced to some extent by details of the lattice design, but this is just one of many competing considerations at the design stage.

The two main sources of residual vertical emittance in a storage ring are vertical beam offsets with respect to the sextupole centers, and rotations of the quadrupoles around the beam axis. Coupling correction (using skew quadrupoles) and orbit correction are both needed to minimize the vertical emittance. The sensitivities of different lattices to sextupole and quadrupole misalignments may be quantified by the amount of misalignment generating a specified vertical emittance. For operating storage rings, we may specify the smallest vertical emittance achieved in that ring; for the damping rings, the vertical emittance is specified by the luminosity. Sensitivity values for a number of operating rings and the damping rings are given in Table 3.3.2.2; these values may be interpreted as the amount of motion that will double the vertical emittance in a lattice tuned for the emittance specified in the table.

Table 3.3.2.2: Alignment sensitivities in the damping rings compared with some operating rings.

\begin{tabular}{|c||c|c|c|c||c|c|}
\hline Parameter & APS & SLS & ALS & ATF & Warm MDR & Cold DR \\
\hline \hline Energy $[\mathrm{GeV}]$ & 7 & 2.4 & 1.9 & 1.3 & 1.98 & 5 \\
Circumference $[\mathrm{m}]$ & 1000 & 288 & 200 & 140 & 300 & 17000 \\
$\left(\gamma \epsilon_{x}\right)[\mu \mathrm{m}]$ & 34 & 23 & 24 & 2.8 & 3 & 8 \\
$\left(\gamma \epsilon_{y}\right)[\mathrm{nm}]$ & 140 & 70 & 20 & 11 & 19 & 14 \\
Sextupole vertical alignment $[\mu \mathrm{m}]$ & 74 & 71 & 30 & 52 & 53 & 11 \\
Quadrupole roll $[\mu \mathrm{rad}]$ & 240 & 374 & 205 & 873 & 511 & 38 \\
Quadrupole jitter $[\mathrm{nm}]$ & 280 & 230 & 231 & 218 & 264 & 76 \\
\hline
\end{tabular}

Also shown in Table 3.3.2.2 is the quadrupole jitter that will result in vertical beam motion equal to the beam size. This quantity is significant for the stability of the extracted beam, and is again a function of the lattice design.

In the warm LC, the specified maximum jitter is $10 \%$ of the beam size, or of the order $1 \mu \mathrm{m}$. The jitter tolerance is set by

1. experience of emittance tuning at the SLC, which became problematic when the jitter exceeded $30 \%$ of the beam size, and 
2. beam dynamical limitations at the IP, beam-delivery system collimators, etc., which again require jitter less than $30 \%$ of the beam size.

Specifying $10 \%$ jitter for the damping rings allows some margin for jitter introduced by downstream systems.

For the cold LC, there are similar considerations, though the details have not been studied to the same extent as for the warm technology. It is likely that a somewhat larger jitter could be tolerated at the collimators and IP, but the experience from emittance tuning at the SLC is still be relevant, which suggests that the budget on jitter from the damping rings will not be much larger than $10 \%$.

Fast orbit feedback systems can be used to stabilize the beam jitter, but careful attention must also be paid to the engineering of the magnet supports, and sources of ground vibration.

Orbit and coupling correction to achieve the specified low vertical emittances in the damping rings places demanding specifications on the diagnostics systems. For example, the required resolution from the beam position monitors is expected to be of order $1 \mu \mathrm{m}$.

\subsubsection{Longitudinal emittance (bunch length and energy spread)}

The bunch length is a function of the lattice design, the beam energy and the RF voltage. Although it is desirable for downstream systems to have as short a bunch as possible extracted from the damping rings, within the rings themselves it is necessary to have a relatively long bunch to reduce the charge density and minimize the effects of collective instabilities. In practice, this must be achieved through the lattice design, in particular using arc dipoles with low magnetic field. The circumference of the lattice is also a factor, so for the $17 \mathrm{~km}$ damping rings for the cold technology, the arc dipoles are $9 \mathrm{~m}$ long with a field of $1.94 \mathrm{~T}$. For the much shorter warm technology damping rings, the arc dipoles are $2 \mathrm{~m}$ long and have a field of $0.658 \mathrm{~T}$. In both cases, the rms bunch length is around $5 \mathrm{~mm}$.

The energy spread is a function only of the lattice design and the beam energy. Both the lattice design and the choice of beam energy are more strongly driven by other considerations, but since a low energy spread is desirable, this places some limitations on the lattice design.

\subsubsection{Machine rep rate, damping time and damping wiggler}

Fast damping is needed to reduce the injected beam dimensions to the required extracted values within the time the bunch trains are stored in the damping rings. Reducing the circumference gives more rapid damping, but the length of the bunch train limits the minimum circumference. Similarly, increasing the dipole field gives more rapid damping, but strong dipole fields tend to result in short bunches with a high charge density. Therefore, to achieve the necessary damping times, damping rings for both the warm and cold technology linear colliders use wigglers to increase the field seen by the beam.

In principle, the positron rings need more damping than the electron rings, since the positron sources produce much larger beams than the electron sources. This is indeed the case in the damping rings for the cold technology, where the positron ring uses approximately $430 \mathrm{~m}$ of wiggler, while the electron ring uses just under $250 \mathrm{~m}$ (peak field in each case is $1.6 \mathrm{~T}$ ).

For the warm technology, the additional damping for the positron beam is provided by a pre-damping ring, which reduces the positron beam from the source to roughly the size of the electron beam. This allows two identical main damping rings to be used to provide the electron and positron beams for the rest of the machine. The pre-damping ring stores two bunch trains and thus provides damping during an additional two machine cycles compared with the electron beam. Compared to the main damping rings, the pre-damping ring has relatively relaxed requirements for the beam emittance. The fact that the beams injected into the main damping rings are small compared to the positron beam direct from the source means that a relatively 
narrow aperture high-field wiggler may be used in the main rings, keeping the wiggler length down. Presently, the main damping rings need $62 \mathrm{~m}$ of wiggler with peak field $2.15 \mathrm{~T}$, and the pre-damping ring needs $50 \mathrm{~m}$ of wiggler with peak field $1.4 \mathrm{~T}$.

For both warm and cold technologies, the damping wigglers will affect the beam dynamics in the damping rings to a much greater extent than is typical in existing synchrotron storage rings (light sources or colliders). This is indicated by the fact that in the damping rings, of the order $90 \%$ of the radiation energy loss occurs in the wigglers, compared to typically less than $25 \%$ in third generation synchrotron light sources.

\subsubsection{Acceptance}

The high average injected beam power in the damping rings $(60 \mathrm{~kW}$ in the warm machine, and $226 \mathrm{~kW}$ in the cold) means that to avoid radiation damage to critical components, the injection efficiency must be close to $100 \%$. The injection efficiency is limited by the physical aperture and dynamic aperture. Generally, the physical aperture is limited by the required magnet strengths, with the narrowest aperture in the wiggler. The dynamic aperture is limited by the nonlinear dynamics driven by the sextupoles and the wiggler. Optimization of the dynamic aperture is a difficult problem in the design and operation of low emittance storage rings.

In linear colliders, the acceptance specifications for the damping rings are driven by the positron beam, which is much larger than the electron beam. The damping rings for the warm technology address this issue by using a pre-damping ring for the positron beam. The pre-damping ring has a larger physical aperture than the main damping ring (which is identical to the electron main damping ring), and the relatively relaxed requirement on the emittance allows a larger dynamic aperture to be achieved.

For the cold technology, the large circumference of the damping rings makes it possible to improve the physical aperture where necessary by replacing short, strong magnets by longer, weaker ones. The greater amount of space also makes it easier to tune the lattice design to provide a reasonable dynamic aperture.

\subsubsection{Vacuum System}

In the electron damping rings, instabilities can be driven by ions generated from the action of the beam on the residual gas. Of particular concern are the tune shifts resulting from the focusing effect of the ions, and coupled-bunch oscillations that can grow along the bunch train (fast ion instability). The need to avoid these effects drives the specification on the vacuum pressure in the rings to $10^{-9}$ Torr or less.

In the positron damping rings, electron cloud is a concern. Electrons generated by a variety of processes can be accelerated by the positron beam, to strike the wall of the chamber with sufficient energy to release a large number of secondary electrons. Depending on a number of parameters (principally the bunch charge and spacing, and vacuum chamber material) the number of electrons inside the chamber can increase rapidly to a level where the positron beam becomes unstable. In the B factories, solenoids were successfully used to mitigate the electron cloud by trapping low energy electrons near the wall, where they can be reabsorbed with the release of few secondaries. This solution might be possible in the cold technology positron damping ring. For the warm technology, the pre-damping and main damping rings are already packed with magnets, and solenoids would not be effective. Attention is therefore focused on developing low secondary yield coatings that can be applied to the vacuum chamber.

The design and construction of the vacuum chamber must be completed carefully to avoid impedance sources that can drive microwave instability. Long range wake fields, for example resulting from the resistive wall effect, can drive coupled-bunch instabilities that may need to be suppressed with feedback systems.

The large amounts of synchrotron radiation power generated by the dipoles and the damping wigglers must be extracted from the beam pipe through antechambers and absorbed on photon stops. 


\subsubsection{Injection and Extraction}

On-axis injection is achieved using fast kickers combined with septum magnets. In the case of the warm technology, the kickers turn on in the gap following one bunch train, and deflect an incoming bunch train onto the correct orbit in the damping ring. Extraction is the reverse of injection. For the cold technology, bunches are injected and extracted individually to allow the bunch train to be "folded over" on itself, so as to reduce the overall length of the damping ring.

As a result of the different schemes, the kickers in the case of warm technology have very different specifications from those in the cold technology. The kickers for the cold technology need faster rise/fall times (20 ns as opposed to $65 \mathrm{~ns}$ ) but the flat top needs to be only the length of a single bunch, rather than an entire bunch train.

\subsubsection{Main Linac RF Technology: Design Considerations and R\&D Status}

\subsubsection{Introduction}

The proposed warm and cold RF technologies are very different. Accordingly, the choices and challenges faced in developing a linear collider design have differed greatly as well. Brief summaries of the main RF technology issues are given in this section, followed by more detailed discussions in subsequent sections including R\&D status.

The choice of operating frequency has a major influence on almost every aspect of linear collider design. The warm machine X-band operating frequency $(11.4 \mathrm{GHz})$ is believed to provide the major cost benefits of a high frequency RF system (high gradient with good efficiency and low RF energy per pulse) while still having achievable wakefield related alignment tolerances. A similar tradeoff occurs for a superconducting linac, but the strong dependence of the cavity surface resistance on frequency limits the frequency choice to much lower values. When all issues were considered, an L-band frequency $(1.3 \mathrm{GHz})$ was chosen for the cold machine design.

These choices of frequencies are closely tied with specific gradient and wakefield performance expectations for the accelerator structures. In particular, both designs assume that stable operation can be achieved at gradients higher than previously demonstrated in comparable structures, and that the long-range wakefields generated in the structures can be suppressed to levels that will produce acceptable multiple-bunch emittance growth.

The requirement of higher gradients result from cost optimizations that consider the relative contributions of power source costs (which increase with gradient) and beam line costs (which decreases with gradient). The warm machine unloaded gradient of $65 \mathrm{MV} / \mathrm{m}$ and the cold machine maximum gradient of $35 \mathrm{MV} / \mathrm{m}$ are both close to optimal (the costs at these gradients are only a few percent above the minimum). However, achieving stable operation at these gradients has been a major $R \& D$ challenge for both groups. Long-range wakefield suppression has likewise required significant R\&D, more so for the warm machine where a larger suppression is required.

The gradient limiting mechanisms differ considerably in warm structures and cold cavities. Gradients in the latter are limited mainly by heat loss mechanisms, such as field emission (dark currents) and enhanced resistance due to sub-mm size imperfections. As a result, $\mathrm{R} \& \mathrm{D}$ has focused on achieving smooth, contaminate-free surfaces. Ultimately, the gradient is limited by the breakdown of superconductivity at the fundamental critical magnetic field, which is expected to occur at about $50 \mathrm{MV} / \mathrm{m}$ for the proposed cavity design. Gradients in the warm structures are limited by RF breakdown and the surface damage it causes. While the breakdown rate is strongly dependent on surface field, the damage that results appears to depend on the input power level. At high power, this damage leads to breakdown on subsequent pulses, which has limited the gradients in the structures being developed. The structure design efforts have focused on reducing the input power, 
which is difficult due to efficiency and wakefield constraints.

The long-range wakefields in both machines will be suppressed using a combination of damping and detuning. For the warm structures, the detuning is achieved by tailoring the cell dipole frequencies to produce a Gaussian-like distribution, and damping is provided by four manifolds that run parallel to the structures and couple to each cell. The detuning requires that both the fundamental and dipole mode frequencies be carefully controlled during cell production. For the cold machine, the cavity dipole frequencies are "naturally" detuned by normal machining variations, and HOM loads will be located between cavities to provide damping.

The relatively strong short-range wakefields in the warm structures require that the average beam offset in the four structure girders be kept to $<5 \mu \mathrm{m}$ rms. For this purpose, the dipole signals from the damping manifolds will be measured and the information used to adjust the girder positions via remote-controlled movers. No beam-based cavity alignment is required in the cold machine, where the fairly loose tolerances $(300 \mu \mathrm{m})$ are achievable by mechanical means, although not trivially given other mechanical constraints in the cryomodules.

The choices of frequency and technology also strongly influence the power source requirements. The $Q \mathrm{~s}$ of the cells in warm structures set the scale of the structure fill times $(\sim 100 \mathrm{~ns})$ and bunch train lengths $(\sim 3$ times the fill time) for good RF-to-beam energy transfer efficiency. The very high $Q \mathrm{~s}$ of the superconducting cavities allow much more freedom to choose the RF and beam parameters without loss of efficiency. For example, the linac bunch spacing could be increased to lower the cavity input power in a tradeoff that would increase the cryogenic load due to the longer RF pulses.

The peak power requirement for the warm structures is $\sim 100 \mathrm{MW} / \mathrm{m}$ at a pulse length of $400 \mathrm{~ns}$, whereas for the cold cavities, the peak power is much lower, $276 \mathrm{~kW} / \mathrm{m}(345 \mathrm{~kW} / \mathrm{m}$ for the upgrade), and the pulse length much longer, $1.5 \mathrm{~ms}$. The higher peak power for the warm machine is technically harder to produce with high efficiency, and for overall cost savings, a pulse compression is required to convert the relatively long klystron pulses $(1.6 \mu \mathrm{s})$ to those required for the structures. As a result, the cost per Joule of RF energy is significantly lower in the cold machine. However, this cost difference is offset by the added cost of cryogenic cooling $(\sim 1 \mathrm{~W} / \mathrm{m}$ at $2 \mathrm{~K})$ and the relatively high costs to produce the cavities and cryomodules compared to the warm structures and girders.

Because of small RF losses in the cavities, the steady-state RF-to-beam efficiency in the cold machine is nearly $100 \%$, compared to about $60 \%$ for the warm machine. The power source efficiency of the warm machine is also about $40 \%$ smaller, due in large part to the inefficiency of the pulse compression system. Overall, when including power for cooling and filling the structures, 133 (208) MW of AC wall plug power is required for the main linacs to generate two 11.3 (6.9) MW beams in the cold (warm) machine for $500 \mathrm{GeV}$ c.m. operation.

\subsubsection{RF Frequency Choice}

The frequency $(f)$ choice for the cold option was a trade-off among structure costs, which prefer high frequency, the superconductor surface resistance, which scales as $f^{2}$, and wakefield considerations, which are more favorable at low frequency. Wakefields scale with the second to third power of the frequency $\left(W_{\text {long }} \sim f^{2}, W_{\text {trans }} \sim f^{3}\right)$. At the low frequency end, cavities in the 350 to $500 \mathrm{MHz}$ regime are in use in electron-positron storage rings. However, for a linac of several tens of km length, the niobium and cryostat costs for these bulky cavities would be prohibitive. At the high frequency end, the BCS theory of superconductivity shows that surface resistance increases as $f^{2}$, so the dynamic heat load does as well. Above $2 \mathrm{GHz}$, the $f^{2}$ dependence of the BCS resistance drives a global thermal instability which sets in below the fundamental critical magnetic field. For example, at $3 \mathrm{GHz}$ the thermal limit is about $30 \mathrm{MV} / \mathrm{m}$. To allow future upgrades to $35-40 \mathrm{MV} / \mathrm{m}$, the frequency must be safely below $2 \mathrm{GHz}$. Finally, the particular choice of $1.3 \mathrm{GHz}$ was motivated by the availability of high power klystrons. 
For the warm machine, the $11.4 \mathrm{GHz}$ RF frequency is considered a reasonable choice in the general tradeoff of decreasing costs versus increasing operational difficulty with increasing frequency. On the plus side, higher frequency affords shorter structure fill times, shorter bunch train lengths (typically several times the fill time) and higher structure shunt impedances. With higher impedance, reasonably good RF-to-beam efficiencies are achievable at higher gradients, which makes the linac shorter. With a shorter linac and bunch train length, less RF energy per pulse is required, which decreases the number of power sources. Opposing this trend is the increasing cost of RF energy with frequency. Also, higher peak power and gradients are required which impose operational limitations due to breakdown and breakdown related damage, although in general, higher gradients have been achieved at higher frequency. Finally, the transverse wakefields in the structures increase with frequency, making structure alignment tolerances tighter and the required suppression of the long-range wakefield greater. To offset the stronger wakefield effects, beam currents that yield less than optimal RF-to-beam efficiency are typically chosen. The particular frequency of $11.4 \mathrm{GHz}$ was chosen to be a multiple of the SLAC Linac frequency.

\subsubsection{Cavities and Structures}

The TESLA collaboration has made significant advances during the past decade to improve niobium purity and surface processing, allowing gradients to rise from $5-10 \mathrm{MV} / \mathrm{m}$ typical in the 1990 s to $25-35 \mathrm{MV} / \mathrm{m}$ in 2003. Experience shows that the thermal breakdown (quench) limit in superconducting cavities can be increased by using high purity niobium and by pre-scanning the material for inclusions using an eddy-current method. The quench field limits in cavities have not been observed to degrade with time. Cleanliness in superconducting cavity surface preparation is essential to achieve acceptable field emission currents. Another benefit of dust-free surfaces is the suppression of voltage breakdown after isolated emitters are initially conditioned, a procedure that usually takes a few hours per cavity. If dust enters a cavity during final assembly or installation in the accelerator, the emitters can be eliminated by pulsed high power processing (also known as conditioning). Extensive tests of high power conditioning field emission sites in multi-cell 1.3 GHz cavities have been successfully carried out.

Seventy 9-cell cavities have been produced by industry. Most have operated CW at $25 \mathrm{MV} / \mathrm{m}$ and higher with acceptable power losses. Over the last two years, the use of electropolishing and mild baking $\left(100{ }^{\circ} \mathrm{C}\right)$ techniques for preparing 9-cell structures has yielded CW gradients between $35-37 \mathrm{MV} / \mathrm{m}$ in four units. Electropolishing eliminates micron-size steps at grain boundaries believed responsible for a $Q$-drop above 20 $\mathrm{MV} / \mathrm{m}$. Electropolishing and baking techniques must be applied to all cavities before installation in a future linear collider to achieve the required $35 \mathrm{MV} / \mathrm{m}$ gradient. Qualifying the structures at $35 \mathrm{MV} / \mathrm{m}$ should provide a healthy performance margin for commissioning the linear collider for $500 \mathrm{GeV}$ c.m. operation, where the structures need to operate at $28 \mathrm{MV} / \mathrm{m}$.

Seven cryomodules have been assembled between 1997 and 2003 and tested in TTF-I and TTF-II. The first three modules were beam tested in TTF-I. All cavities for these modules were prepared by standard chemical etching, since electropolishing was under investigation at the time. As assembly techniques and cavity gradients improved, cryomodule performance rose from $15 \mathrm{MV} / \mathrm{m}$ at the start of TTF-I in 1997 to 29 $\mathrm{MV} / \mathrm{m}$ in 2003. Six cavities in the most recently tested module reached $30 \mathrm{MV} / \mathrm{m}$.

For the electropolished cavities, a 9-cell cavity equipped with input couplers, higher order mode (HOM) couplers and tuners has been operated inside a single-cavity test cryomodule with a high power klystron to reach gradients between $35-36 \mathrm{MV} / \mathrm{m}$. The cavity operated stably for more than 1100 hours at $35 \mathrm{MV} / \mathrm{m}$ at a $Q$ value of $7 \times 10^{9}$. Operation was without quench or trips originating from the cavity-coupler system. There was no field emission below $35 \mathrm{MV} / \mathrm{m}$ as judged from the absence of X-rays. Tests of a second cavity has started and it has reached $35 \mathrm{MV} / \mathrm{m}$ at a $Q$ of $6 \times 10^{9}$ with no X-rays detected up to $34 \mathrm{MV} / \mathrm{m}$. For operation at $35 \mathrm{MV} / \mathrm{m}$, Lorenz force detuning needs to be compensated by fast piezo tuners. This has been demonstrated with a 9-cell cavity operating at $35 \mathrm{MV} / \mathrm{m}$.

Beam tests have been carried out in TTF-I. Among three modules completed, the best accelerated beam at 
$22.7 \mathrm{MV} / \mathrm{m}$. In total, 13,000 hours of beam time have been logged, mostly at $1 \mathrm{~Hz}$. During the next year (2004), the plan is to test several more fully equipped cavities in their horizontal test cryomodule, and to accelerate beam through one or more cavities operating at $35 \mathrm{MV} / \mathrm{m}$.

The high power input coupler for the cavities has posed several challenges and considerable coupler R\&D has been done. This has included the use of computational tools for analysis of multipacting and has resulted in the implementation of a dc bias between the antenna and the outer coax. Also, design changes have been made to improve vacuum pumping and minimize the warm surface area exposed to RF. About 60 coaxial-style couplers have been fabricated during four design iterations. All couplers have been tested to one MW (some higher), with $1.3 \mathrm{~ms}$ pulses in a warm, high power test stand. The most recent (Style III) couplers (22 built, 40 under construction) have performed the best.

Couplers need to be reprocessed after installation in the cavity. Conditioning times for couplers are a few days, and drop to one day for couplers pre-conditioned with high power and baked before cool down. Present processing techniques and installation procedures need to be improved to speed up processing time. Likely improvements will come from the baking of cavities and couplers to remove water.

The early warm linac designs aimed for relatively high gradients, encouraged by tests showing that unloaded gradients greater than $100 \mathrm{MV} / \mathrm{m}$ are possible and that the gradient limit increased with RF frequency. However, these tests were done with standing wave or low group velocity structures because they were the only structures that could reach high gradients with the limited peak power available at that time. Later, higher group velocity structures $(12 \% c$ in the first cell) were chosen for their lower cost and lower wakefields. However, these failed to reach the same high gradients and incurred significant breakdown related damage near their upstream ends at unloaded gradients of 45-50 MV/m with $240 \mathrm{~ns}$ pulses.

Since then, there has been an aggressive R\&D program to reach the unloaded gradient goal of $65 \mathrm{MV} / \mathrm{m}$ with $400 \mathrm{~ns}$ pulses. During the last few years, 25 prototype structures were tested $(>15,000$ hours operation at $60 \mathrm{~Hz}$ ) with the conclusion that low group velocity (3\%-4\% c initially), $60 \mathrm{~cm}$ long, high phase advance $\left(150^{\circ}\right)$, traveling-wave structures are the best candidates for the linear collider. Eight of the structures tested have the appropriate iris radii for acceptable short-range wakefields. Some of these structures include slots in the cells for long-range wakefield damping: all are detuned to suppress the long-range wakefield on the time scale of the bunch spacing (1.4 ns). Of the eight structures, the best performance achieved was a breakdown rate of 0.2 per hour, close to the requirement of $<0.1$ per hour (at the $60 \mathrm{~Hz}$ test rate). To improve the performance, a design with a somewhat smaller iris has been adopted. It has $10 \%$ lower surface fields and requires $10 \%$ less power. Testing of a structure of this design with slots will begin in November, 2003.

\subsubsection{Wakefield Suppression}

One of the main tasks in the design of the cold linac is to insure that the dipole modes which couple strongly to the beam are sufficiently damped via HOM couplers at the ends of the cavities. Beam-based measurements of mode properties have shown that this is indeed the case for the strongest bands. HOM modes that strongly couple to the beam are damped to the desired quality factors of $10^{5}$. A trapped mode at $2.58 \mathrm{GHz}$ proved an exception. This effect is now well understood and good damping has been achieved by re-orienting one of the two HOM couplers. The overall HOM damping is expected to result in satisfactory long range wakefield suppression.

For the warm structures, long range wakefield suppression is more of a challenge due the relatively strong wakefields. These fields need to be reduced by about two orders of magnitude during the $1.4 \mathrm{~ns}$ period between bunches. The approach has been to use a combination of dipole mode detuning and damping. Detuning requires that each cell of the structure have a slightly different dipole frequency, such that the wakefields from the different modes destructively add by the time of the second bunch. The frequency is varied systematically along the structure to produce a Gaussian distribution in the product of the mode density and the mode coupling strength to the beam. This detuning produces an approximately Gaussian 
falloff in the net wakefield generated by each bunch, and works well to suppress the wakefield for about $30 \mathrm{ns,}$ after which the amplitude increases due to a partial re-coherence of the mode excitations. To offset this rise, weak mode damping was introduced by coupling each cell through longitudinal slots to four $\mathrm{TE}_{11}$ circular waveguides (manifolds) that run parallel to the structure. This reduces the dipole mode quality $(Q)$ factors from about 6000 to 1000, limited by the propagation time of the dipole-mode energy through the cells.

Several damped and detuned structures have been built and the wakefields have been measured in three of them at the ASSET facility in SLAC Linac. Here a drive beam (positron bunch) is used to excite wakefields, and a witness beam (electron bunch) is used to measure them by the deflection they cause. The wakefields in these structures come close to meeting design requirements, and the reasons for the shortcomings have been identified (e.g., fabrication errors) and are considered correctable. To realize the wakefield suppression afforded by the detuning also requires that the structure straightness be maintained (up to $100 \mu \mathrm{m}$ peak-topeak excursions are allowed). Such straightness has been routinely achieved in prototype structures.

Although the structure manifolds were nominally added to damp the dipole modes, the dipole signals from them also provide a measure of the beam's transverse position in the structure. Moreover, the beam coupling to the modes is fairly localized ( 2 to 10 cells), so filtering the signals by frequency yields beam offset information at different regions along the structure. As part of the wakefield measurement program, beam centering tests of the type required for the linear collider were done using the dipole signals at two frequencies as a guide to position the drive beam. Measurements of the resulting short-range wakefield $(<300 \mathrm{ps})$ indicated that the drive beam had been centered to the $11 \mu \mathrm{m}$ rms measurement accuracy, which was limited by the position jitter in the incoming beam. As noted earlier, the NLC/GLC goal is $<5 \mu \mathrm{m}$ rms.

\subsubsection{Modulators}

For the warm machine, solid state induction modulators are being developed to lower cost and to obtain higher efficiency (70\%) and reliability compared with the PFN-style modulators traditionally used. A fullscale prototype (4-pack) has generated $500 \mathrm{kV}, 300 \mathrm{MW}$ pulses when driving a water load, and $400 \mathrm{kV}$, $500 \mathrm{MW}$ pulses when driving four $50 \mathrm{MW}$ klystrons (in each case with $1.5 \mu$ s pulse widths). However, this modulator has only run a few hundred hours at a low repetition rate $(30 \mathrm{~Hz})$. Current R\&D is focused on improving its performance (local heating problems are limiting higher rate operation) and developing a smaller version (a "Two-Pack") that will drive two $75 \mathrm{MW}$ klystrons (500 kV, $270 \mathrm{MW}$ ). The main tasks that remain are to demonstrate reliable, nominal repetition rate operation and good efficiency.

The cold machines will use a more conventional style modulator to deliver the $120 \mathrm{kV}, 15 \mathrm{MW}$ pulses to each of the $10 \mathrm{MW}$ klystrons. The design being considered has solid state switching and a bouncer-type circuit to compensate the voltage droop on the storage capacitors. Fermilab has built three modulators and industry has built five units (five modulator/transformer/klystron systems have been operated). Two of the Fermilab modulators are IGBT switched and have operated about 13,000 hours each at $1 \mathrm{~Hz}$.

The basic modulator design has been proved viable. There will be more engineering evaluation and design evolution. In particular, the switch technology is evolving rapidly and packaging for the most efficient layout will need development. The design of a control/interlock module for the modulator is evolving to include more information and remote diagnostics. The next phase of development aims for a modular layout to further enhance reliability and maintainability.

\subsubsection{Klystrons}

Both machines employ start-of-the-art klystrons with relatively low perveances to improve efficiency. The cold machine requires about 600, $10 \mathrm{MW}$ klystrons (1.5 ms pulse length) and the warm machine requires about 4500, $75 \mathrm{MW}$ klystrons (1.6 $\mu$ s pulse length). 
For the cold machine, multiple (7) beam klystrons (MBKs) are used to achieve both a low perveance and low voltage. Prototypes have been built with industry that meet efficiency $(65 \%)$ and pulse power requirements. Some of these tubes have been used in TTF. Thales has produced three klystrons, and five are on order to be delivered in 2004. All three Thales klystrons met their acceptance specifications, but after 2 to 3 months of operation, developed gun arc problems or symptoms. Thales is conducting a study to isolate the problem. The first rebuild is expected early next year. Both CPI and Toshiba are working on prototype 10 MW multi-beam tubes as well (Toshiba is also designing a $5 \mathrm{MW}$ version). These tubes have lower cathode current densities than the Thales tube and should be less prone to arcing. The CPI tube should be ready at the beginning of next year and the Toshiba tube by fall of next year. The Toshiba efficiency goal is $70 \%$.

For the warm machine, periodic permanent magnets (PPM) are used for focusing instead of solenoidal electromagnets, to halve the average power consumption. Several PPM klystrons have been made by KEK/Toshiba and SLAC. One of SLAC tubes has met the full specifications $(75 \mathrm{MW}, 1.6 \mu \mathrm{s}, 120 \mathrm{~Hz}$ pulses have produced at $55 \%$ efficiency). Another tube at KEK has met all peak power specs but at a $60 \mathrm{~Hz}$ rep rate. Nonetheless, the cathode current density is fairly high $\left(7.2 \mathrm{~A} / \mathrm{cm}^{2}\right)$ in these designs, and the tubes will likely be modified in the engineering and design phase of the project to reduce it. SLAC and KEK/Toshiba will continue to develop PPM klystrons to improve manufacturability and cost, and to achieve reliable operation at the design pulse repetition rate.

\subsubsection{RF Distribution}

The function of the RF distribution system is to transport the klystron output power to the accelerator structures. This is relatively straightforward in the cold machine. The $5 \mathrm{MW}$ of power from each klystron arm is distributed in non-evacuated waveguide. Along these lines, hybrids "tap-off" $275 \mathrm{~kW}(345 \mathrm{~kW})$ of power to 15 (12) cavities for $500 \mathrm{GeV}(1 \mathrm{TeV})$ c.m. operation. Between each hybrid and cavity is a circulator/load to absorb reflected power and a motorized three-stub tuner to allow adjustments of the cavity phase and coupling.

At TTF, the power from a single klystron has been distributed to as many as 16 cavities. Individual components have been tested at nominal operating power and higher: circulators to $400 \mathrm{KW}$, couplers and hybrids to $5 \mathrm{MW}$, and three-stub tuners to $1.5 \mathrm{MW}$. However, there has been no test of a integrated system at full power because fewer than the nominal number of cavities have been powered. No significant R\&D is planned for the distribution system, which is built from standard components. However, DESY is working with industry to develop integrated waveguide systems to reduce cost and the installation/testing effort.

For the warm machine, the RF distribution system is complicated by the requirement of pulse compression (it is optimal cost-wise to produce longer klystron pulses than required for the structures). In early warm machine designs and at the NLCTA, SLED II pulse compression was used, which is a delay line version of the SLAC Linac Energy Doubler (SLED). It consists of a $3 \mathrm{~dB}$ hybrid divider that routes the klystron output power equally to two delay lines made of circular waveguide. These lines are shorted at the far end and have irises at the near end that partially reflect the RF. During operation, the lines are resonantly filled and then effectively discharged by a $180^{\circ}$ change in the klystron RF phase. Although three SLED-II systems have worked well at NLCTA, with over 20 thousand hours of operation at $60 \mathrm{~Hz}$, they are not particularly efficient (65\%) due to the RF transmitted from the delay lines as they are being filled.

While there are more efficient compression methods, SLED II was chosen for the warm machine because of the experience with it and because it is much easier to demonstrate than the alternatives (a Delay Line Distribution System alternative is described in Section 7.4). The SLED II version proposed has been modified from the NLCTA design to lower cost and improve efficiency and power handling capability. The changes include larger diameter $(17.1 \mathrm{~cm})$ delay lines to lower power loss, dual-moded $\left(\mathrm{TE}_{01}\right.$ and $\left.\mathrm{TE}_{02}\right)$ delay line transport to halve the delay line length, a lower compression ratio (4 instead of 6 ) to improve efficiency, and the use of over-height planar components for the $3 \mathrm{~dB}$ hybrid to lower surface fields. The design goal is to deliver $450 \mathrm{MW}, 400 \mathrm{~ns}$ pulses to the structures (including transport losses), which corresponds to an overall 
efficiency of $75 \%$. A prototype of such a system has been built. In December, 2003, it was tested successfully at the NLC 8-Pack, producing a peak power output of $580 \mathrm{MW}$ and a pulse length of $400 \mathrm{~ns}$.

\subsubsection{Low Level RF}

During acceleration of the beams in either machine, the bunch energy gains must be tightly regulated both within a train and between trains to limit the loss in luminosity due to dispersion. The bunch-to-bunch and pulse-to-pulse energy variations are required to be $<0.05 \% \mathrm{rms}$ in the cold machine and $<0.3 \% \mathrm{rms}$ in the warm machine. The regulation is tighter in the cold machine to allow $300 \mu \mathrm{m}$ quadrupole magnet alignment tolerances in the cryomodules, typical for cold cryostats (in the warm machine, the larger intra-bunch energy spread required for BNS damping does permit such a tradeoff).

The main factors that affect the beam energy are the RF amplitude, RF phase, beam current and cavity tuning. The higher beam loading in the cold machine makes it more sensitive to current variations: for example, a $1 \%$ change in the average current would change the gradient by $1 \%$ in the cold machine if the $\mathrm{RF}$ is not compensated, while the change in the warm machine would be $0.25 \%$. Also, the higher loaded $Q \mathrm{~s}$ of the cavities in the cold machine makes their tuning much more critical than in the warm machine (the effective warm structure $Q \mathrm{~s}$ are several orders of magnitude smaller). Specifically, the cold cavities have a narrow band width $(300 \mathrm{~Hz}$ ) and are subject to repetitive Lorentz-force detuning (few $100 \mathrm{~Hz}$ ) and random pulse-to-pulse microphonic detuning $(10 \mathrm{~Hz})$. For the warm machine, the structure temperatures will be regulated to $0.5^{\circ} \mathrm{C}$, which will limit the reduction in energy gain to $<0.1 \%$ from beam-to-RF phase slippage within the structures.

Both groups have well developed strategies for equalizing the bunch energy gains. In each machine, the beam current profile will be measured in the damping rings prior to each RF pulse and this information will be used in a feed-forward manner to adjust the RF waveforms to compensate for any variation in beam loading. For the cold machine, this will mainly be used to stabilize the first part of the bunch train (few $\mu \mathrm{s}$ ), after which feedback loops that monitor the phase and amplitude of the RF in the cavities (in 30 unit sums) will regulate the cavity stored energy for the remainder of the pulse. Lorentz-force detuning will be mainly compensated by piezo movers that vary in a fixed pattern pulse to pulse: any residual tuning errors will be corrected by the feedback system. In the warm machine, the pulse length is too short to do intra-train feedback and only pulse-to-pulse feedback will be used. It will be based on measurements of the phase and amplitude of the transmitted RF from the eight structures in each RF unit.

At test facilities for each machine, linear-collider-like bunch trains have been accelerated over several meters and the bunch-to-bunch energy gains have been regulated to the required levels. For the cold test, vector sum control is expected to be easier with 30 cavities (instead of the 16 at TTF) due to better averaging of microphonic induced errors. Five modules will be controlled with same system in TTF in 2004. Piezo mover compensation of Lorentz-force tuning has also been demonstrated. In addition, a dedicated test in the $3 \mathrm{~km}$ S-band SLAC linac showed that NLC/GLC-like beam loading (20\%) could be compensated to better than $0.3 \% \mathrm{rms}$ within a $400 \mathrm{~ns}, 40 \mathrm{GeV}$ bunch train. As for temperature control, the SLAC S-band structures and NLCTA X-band structures are regulated to $<0.5{ }^{\circ} \mathrm{C}$.

\subsubsection{Low emittance transport system design considerations}

To attain the high luminosities needed by the particle physics experiments, the beam emittances and spot sizes at the Interaction Point (IP) must be very small. These low emittance beams are generated in the damping rings. After the beams are damped to the desired emittances, they are extracted and passed through spin rotator and bunch compressor systems. The bunch length must be decreased by a factor of 20 50 to be more closely matched to the linac RF wavelength and to minimize the hourglass effect at the IP. After the bunch compressors, the beams are accelerated in the main linacs to the final energy and then 
focused in the beam delivery system down to the very small spot sizes at the IP.

It is useful to divide the damping ring-to-IP transport into the three logical areas: the bunch compression $(\mathrm{BC})$, which includes the spin rotators, the main linacs (ML), and the beam delivery (BDS). While there are detailed differences in the emittance preservation issues for each region, there are many issues common to the bunch compression regions and beam delivery. The relative importance of the primary sources of emittance dilution for the different regions is given in Table 3.3.4.1.

Table 3.3.4.1: Importance of emittance dilution sources in different regions of the LC. The length of the BC and BDS systems is set by the need to limit synchrotron radiation. Dispersion and wakefields are of medium importance in the cold linacs and of larger importance in the warm linacs.

\begin{tabular}{|c|c|c|c|}
\hline & BC & ML & BDS \\
\hline Synchrotron radiation & Sets length & Negligible & Sets length \\
Betatron matching & Medium & Small & Large \\
Betatron coupling & Large & Small & Medium \\
Dispersion & Large & Medium/Large & Large \\
Nonlinear aberrations & Medium & Small & Large \\
Wakefields & Small & Medium/Large & Small \\
RF phases & Large & Medium & Large \\
\hline
\end{tabular}

In comparing the two designs, the primary differences are in the main linacs but there are slightly different tradeoffs in tolerances in the other regions as well. In the warm linacs, the wakefields are much larger due to the higher RF frequency, and therefore the BNS damping energy spread required to prevent single bunch BBU is also larger. This makes the wakefield and dispersive emittance dilutions more important and different methods are used to control them. In the BDS, the cold design would have somewhat looser tolerances because of a larger IP beta function but this is offset by the increased sensitivity due to the larger IP disruption, and the resulting tolerances are similar. Likewise, the cold bunch compressor might seem easier because it is a single stage system rather than the longer, more complex two-stage system for the warm design. In fact, the energy spread is 2 - 3 times larger in the cold $\mathrm{BC}$ and since many emittance dilutions scale quadratically with energy spread, the resulting tolerance specifications are very similar for the two designs.

In general, aberrations causing emittance dilution in the BC and BDS can be tuned using global knobs as the filamentation through the systems is relatively small. Such knobs are only effective if they are orthogonal, i.e. the dispersion correction cannot introduce betatron coupling, and if the system is sufficiently stable for the tuning to converge. In the linac, the sources of emittance dilution need to be corrected relatively locally as filamentation is important in both warm and cold designs.

In all regions, the emittance dilutions due to installation errors are unacceptably large and emittance tuning or beam-based alignment techniques are needed. Examples of the different methods are listed in Table 3.3.4.2. The tuning techniques differ for each region of the collider and differ for the warm and cold main linacs. Most of the required tuning techniques have been utilized in operating accelerators however they have not been demonstrated at the level that will be required in either the warm or cold linear collider designs. This is at least partly because no other accelerator has operated with the very small beams that will exist in the linear collider. Significant improvements in tuning performance are expected from higher precision diagnostics.

As an example, in the Final Focus Test Beam quad-shunting achieved a beam-based alignment that was less than $7 \mu \mathrm{m}$. The warm linac requires an alignment of $2.5 \mu \mathrm{m}$ which should be achievable with diagnostics that have three times better resolution. The warm linac also has a better optics configuration for the beam-based alignment than in the FFTB beam line. Similarly, ballistic alignment was used in the SLC final focus to achieve an effective alignment that was less than $50 \mu \mathrm{m}$. The cold linac requires an effective alignment of roughly $25 \mu \mathrm{m}$ which it expects to achieve with ballistic alignment using better diagnostics and a more stable beam. 
Table 3.3.4.2: Examples of trajectory and emittance correction techniques for each LC region

\begin{tabular}{|c|c|c|c|}
\hline Bunch Compressor & Beam Delivery & Warm Linac & Cold Linac \\
\hline 1-to-1 steering & 1-to-1 steering & 1-to-1 steering & 1-to-1 steering \\
\hline Quad shunting & Quad shunting & Quad shunting & Ballistic alignment \\
\hline $\begin{array}{c}\text { Global correction of } \\
\eta, \eta^{(2)} \text { and } \beta \text {-coupling }\end{array}$ & $\begin{array}{c}\text { 17 knobs per side } \\
\text { for global aberration tuning }\end{array}$ & $\begin{array}{c}\text { Dispersion Free } \\
\text { Steering }\end{array}$ & $\begin{array}{c}\text { Dispersion Free } \\
\text { Steering }\end{array}$ \\
\hline Beam feedback & Beam feedback & $\begin{array}{c}\text { Global emittance } \\
\text { bumps }\end{array}$ & $\begin{array}{c}\text { Global emittance } \\
\text { bumps }\end{array}$ \\
\hline to lock trajectory & to lock trajectory & Beam feedback & $\begin{array}{c}\text { Beam feedback } \\
\text { to lock trajectory }\end{array}$ \\
\hline
\end{tabular}

For the linear colliders, the emittance dilution budgets have been estimated using simulation programs that model the performance of the tuning algorithms given a set of diagnostics, correction elements, and accelerator errors. The many different linear collider simulation codes have been compared against each other with good agreement and the results from the simulation codes have been compared against operating accelerators, including the SLC, with qualitative agreement [[4], [5]]. It should be noted that, because the emittance dilutions depend on the exact details of the errors, it is very difficult to obtain quantitative agreement between simulations and an operating accelerator except for trivial examples.

Although the simulation programs are quite advanced, there are still many aspects of the tuning process that are not completely modeled. In particular, the time-scale for tuning and the impact of jitter and drifts on the tuning algorithms have not yet been modeled in sufficient detail. It is well known that large beam jitter or significant component drift will degrade the tuning performance. In the linear collider designs, this coupling of the jitter and drift to the emittance budget is not usually included. Instead, relatively tight jitter budgets have been specified and the emittance dilution budgets include some overhead.

The warm and cold designs take slightly different approaches to achieving the tight jitter budgets. In the warm linacs, tight jitter tolerances are specified on the quadrupoles. Measurements have suggested that these are reasonable tolerances, but further measurements are needed on more detailed prototypes. Active stabilization techniques could be used to attain the specified tolerances, if necessary. In the cold linacs, the quadrupoles are located inside the cryostats. Reliable vibration measurements of the quadrupole jitter have not yet been made and there is concern that the motion may be relatively large. Fortunately, the large intra-train bunch spacing in the cold design allows for an intra-train feedback which can be implemented at routine intervals along the linac to reduce the sensitivity to linac quadrupole jitter.

Throughout the low emittance transport, the largest difference between the warm and cold technology is in the linacs because of the larger wakefields in the warm structures. The effect of the transverse wakefield on the beam is best quantified with the energy spread required for BNS damping. The BNS damping energy spread is the equivalent chromatic effect needed to cancel the lowest-order wakefield distortion. It depends on the bunch charge, bunch length and external focusing as well as on the strength of the transverse wakefield. Although the wakefield, convoluted over the bunch, differs by a factor of 1000 between the warm and cold designs, the required BNS damping energy spread differs by only a factor of 5 to 6 . The stronger focusing and smaller bunch charge in the warm design compensate for most of the difference in wakefield strength.

In both the warm and cold linacs, the transverse wakefields must be controlled both short-range (within the bunch) and long-range (between bunches). The long-range wakefield is reduced by using a combination of damping and detuning of the higher-order-modes (HOMs). In the cold design, the HOMs are detuned by roughly $1 \mathrm{MHz}$ and the $Q \mathrm{~s}$ have to be reduced to $10^{4} \sim 10^{5}$ using HOM dampers located at the ends of the 9-cell cavities. In the warm structures, the HOMs are detuned by $2 \mathrm{GHz}$ and the $Q \mathrm{~s}$ are reduced to $\sim 10^{3}$ using "damping manifolds" that couple to each cavity. These techniques are sufficient to eliminate multi-bunch beam breakup (BBU) as a concern for either design. 
Unlike the long-range wakefield, the short-range wakefield cannot be directly reduced by similar techniques, but it can be partially compensated using BNS damping. In the warm linac, the required BNS energy spread is roughly $1 \%$ while it is about 5 times smaller in the cold linac. The BNS damping can prevent single-bunch beam breakup but the accelerator cavities still need to be accurately aligned to prevent dilution of the beam emittance. The BNS energy spread also introduces additional dispersive emittance dilution, requiring the quadrupoles to be well aligned. The tolerances on the warm structures are roughly 50 times tighter than those on the cold cavities for similar levels of emittance dilution. The quadrupole alignment tolerances are also roughly 10 times tighter in the warm linac than in the cold for similar levels of emittance growth, due to the larger BNS energy spread and the larger number of quadrupoles.

Because of the tight tolerances, the warm design has many additional diagnostics and controls. The structures are mounted on girders which have remotely-controlled movers with step size resolution of approximately $300 \mathrm{~nm}$, and yaw/pitch resolution of approximately $100 \mathrm{nrad}$. Rf BPMs which measure the dipole signals from the damping manifolds are used to align the structures to the beam. In principal, this allows the cavities to be aligned to the beam as accurately as it is possible to measure the induced HOM signals. The quadrupoles are also mounted on remotely-controlled movers and are moved to center the beam on the magnetic axis using BPMs which are captured in the bores of the magnets. The quad-shunting technique is used to determine the position of the magnetic center with respect to the electrical center of the BPM.

The situation is rather different for the cold linac because the tolerances are much looser. The cavities are aligned within the cryomodules and further adjustment requires entry into the tunnel. As a result, the alignment requirements for the cold cavities must be satisfied during the ab initio installation. The long term stability requirement, which is at the level of about $300 \mu \mathrm{m}$, also applies over a considerably longer period of time than for the warm linac since the cavities cannot be repositioned remotely. Reliable measurements of cavity position stability across a thermal cycle have only recently been made, and preliminary results are not yet available.

A final source of single-bunch emittance growth is coupling of the structure fundamental mode into the transverse planes. This occurs when a structure has a pitch or yaw angle with respect to the beam trajectory. In the warm linac, this effect can be controlled with the same set of RF BPMs and girder movers used to control the short-range wakefields. In the cold linac, this effect must be controlled through the initial alignment, much as in the case of short range wakefields. The pitch alignment tolerances are included in Table 3.3.4.3.

The linac emittance dilution budgets are based on detailed simulations using best estimates of the component alignment. Many of the alignment errors and simulation results were documented for the warm and cold designs in the report from the 2001 International Linear Collider Technical Review Committee and in subsequent papers [TRC, 6, 7, 8]. Results for one set of simulations using LIAR are listed in Table 3.3.4.3 for both the warm and cold linacs. These results are the average of 100 simulations using different random error seeds; the $90 \%$ confidence level is usually 1.5 to 2 times larger than the average. The simulations listed in Table 3.3.4.3 use DF steering which is sensitive to the RF deflections from tilted cavities. Simulations for the cold linac, described in Ref. [8], arrived at a smaller result of $30 \% \Delta \epsilon_{y} / \epsilon_{y}$ using Ballistic Alignment, however these simulations did not include some important contributions such as quadrupole rolls. The cold linac emittance budget is based on the results from Ref. [8], but include an additional contribution of $10 \%$ $\Delta \epsilon_{y} / \epsilon_{y}$ for quadrupole rolls as listed in Table 3.3.4.3. In this case, simulations for both the warm and cold linacs predict a growth of $40 \% \Delta \epsilon_{y} / \epsilon_{y}$.

Because not all effects are completely represented in the simulations, an additional $10 \%$ has been added to both the warm and cold results to bring the total budget for vertical emittance dilution in the linacs to $50 \%$. Neither set of simulations included final optimization using emittance bumps. Such bumps were used very effectively in the SLC linac and also appear to reduce the emittance dilution by factors of 5 or 6 in simulations of both the warm and cold linacs. One caveat is that emittance bump tuning will be sensitive to drifts of the linac components and thus the real performance is more difficult to predict. For this reason, the improvement from emittance bumps is not included in the emittance budgets, but has been left as a 
Table 3.3.4.3: Emittance and jitter simulation studies for the warm and cold linacs with LIAR

\begin{tabular}{|c|c|c|c|c|c|c|}
\hline & \multicolumn{3}{|c|}{ Warm LC } & \multicolumn{3}{|c|}{ Cold LC } \\
\hline Random Error Term & Value & $\overline{\Delta \Delta y / \sigma_{y}}$ & $\Delta \epsilon_{y} / \epsilon_{y}$ & Value & $\overline{\Delta \Delta y / \sigma_{y}}$ & $\Delta \epsilon_{y} / \epsilon_{y}$ \\
\hline Beam-to-quad offset & $2.0 \mu \mathrm{m}$ & & $14.0 \%$ & $7.8 \mu \mathrm{m}$ & & $2.5 \%$ \\
\hline Beam-to-quad jitter & $15 \mathrm{~nm}$ & $25 \%$ & $7.0 \%$ & $85 \mathrm{~nm}$ & $100 \%$ & \\
\hline Quad Roll & $300 \mu \mathrm{rad}$ & & $7.0 \%$ & $300 \mu \mathrm{rad}$ & & $10.5 \%$ \\
\hline $\mathrm{RF}$ girder offsets & $3 \mu \mathrm{m}$ & & $7.5 \%$ & $200 \mu \mathrm{m}$ & & $22.0 \%$ \\
\hline $\mathrm{RF}$ girder tilts & $4.5 \mu \mathrm{rad}$ & & $0.1 \%$ & $20 \mu \mathrm{rad}$ & & $1.5 \%$ \\
\hline $\mathrm{RF}$ girder bows & $100 \mu \mathrm{m}$ & & $0.1 \%$ & & & \\
\hline RF structure cell offsets & $33 \mu \mathrm{m}$ & & $1.0 \%$ & & & \\
\hline RF structure offsets & $25 \mu \mathrm{m}$ & & $0.0 \%$ & $300 \mu \mathrm{m}$ & & $5.0 \%$ \\
\hline RF structure tilts & $33 \mu \mathrm{rad}$ & & $0.3 \%$ & $300 \mu \mathrm{rad}$ & & $25.5 \%$ \\
\hline RF structure bows & $50 \mu \mathrm{m}$ & & $0.4 \%$ & & & \\
\hline Feedback steering & 1 hour & & $2.5 \%$ & & & \\
\hline BPM drift & $1 \mu \mathrm{m} /$ week & & $1.5 \%$ & & & \\
\hline Total & & $25 \%$ & $41 \%$ & & $100 \%$ & $67 \%$ \\
\hline
\end{tabular}

potential performance enhancement on the operating machine.

Emittance dilution in the bunch compressors or beam delivery systems has not been modeled as extensively as in the main linacs. In the BC systems, the detailed optics decks that exist have been used to estimate the impact of random errors, after trajectory correction but before the use of global tuning knobs, for both the warm and the cold designs. The resulting beam jitter and vertical emittance dilutions are listed in Table 3.3.4.4. These calculations assume similar errors in the warm and cold designs except for the RF cavities. The warm RF cavities are assumed to be aligned using structure movers and RF BPMs mounted in the cavities, similar to the warm linacs, and the cold structures are assumed to be mechanically aligned within the cryostats, similar to the cold linac. With the present designs, the cold BC design has a larger emittance dilution because of the larger energy spread, as shown in Table 3.3.4.4. However, neither design has been optimized to reduce the sensitivities and lattice optimization might change these results.

These calculations can be used to compare the sensitivity to errors but not as tolerances. Properly designed emittance correction procedures should be able to cancel much of these emittance dilutions. For example, simulations of global knobs reduced the vertical emittance dilution from $20,000 \%$ to $10 \%$ for the NLC design. This assumed a stable accelerator [9], and so the actual performance will undoubtedly be worse, limited by a combination of diagnostic resolution and accelerator stability. An estimate of the residual emittance dilution due to the diagnostics alone is about $10 \%$ which is similar to the value found in the NLC simulations. The impact of the beam line stability is harder to estimate but the sensitivity numbers in Table 3.3.4.4 provide an indication. At this time, without better simulations, the dilution budgets for the warm and cold BC systems have been set equal to $10 \%$ and $20 \%$ for the horizontal and vertical emittance and $15 \%$ for the vertical beam jitter. While this is more of a stretch for the cold design because of its higher sensitivity to misalignments, it seems a reasonable starting point.

The emittance dilutions and tolerances are easier to compare in the beam delivery systems because the optical lattices have been chosen to be almost identical. The primary difference is in the incoming matching which is varied to provide an IP beta function for the warm design that is four times smaller than for the cold design. Given the similar dilution budgets in the bunch compressor systems and the main linacs, the incoming beam emittances will also be similar. Because the cold design BDS focuses to a beta function that is $400 \mu \mathrm{m}$ versus $100 \mu \mathrm{m}$ for the warm design, the sensitivities on component alignment and jitter is two times looser to have the same effect relative to the beam size. This is balanced by the higher disruption in the cold design, which makes the system about four times more sensitive to IP offsets or distortions relative to the beam size [10]. As an example, in the warm design, an offset of $1 \mathrm{~nm}$ ( $30 \%$ of the vertical beam size) causes $5 \%$ luminosity loss while, in the cold design, an offset half as large ( $0.5 \mathrm{~nm}$ or $7 \%$ of the vertical beam 
Table 3.3.4.4: Emittance and jitter sensitivities for the warm and cold BC systems

\begin{tabular}{|l|c|c|c|c|c|c|}
\hline & \multicolumn{3}{|c|}{ Warm LC } & \multicolumn{3}{c|}{ Cold LC } \\
\hline \hline Random Error Term & Value & $\Delta y / \sigma_{y}$ & $\Delta \epsilon_{y} / \epsilon_{y}$ & Value & $\Delta y / \sigma_{y}$ & $\Delta \epsilon_{y} / \epsilon_{y}$ \\
\hline Bend roll jitter & $0.1 \mu \mathrm{rad}$ & $7 \%$ & & $0.1 \mu \mathrm{rad}$ & $10 \%$ & \\
Bend roll & $100 \mu \mathrm{rad}$ & & $60 \%$ & $100 \mu \mathrm{rad}$ & & $575 \%$ \\
Quad Y jitter & $15 \mathrm{~nm}$ & $12 \%$ & & $15 \mathrm{~nm}$ & $15 \%$ & \\
Quad Y misalign & $10 \mu \mathrm{m}$ & & $75 \%$ & $10 \mu \mathrm{m}$ & & $80 \%$ \\
Quad Roll & $300 \mu \mathrm{rad}$ & & $153 \%$ & $300 \mu \mathrm{rad}$ & & $30 \%$ \\
Acc Y align & $10 \mu \mathrm{m}$ & & $2 \%$ & $500 \mu \mathrm{m}$ & & $12 \%$ \\
\hline Total & & $\mathbf{1 4 \%}$ & $\mathbf{2 9 0 \%}$ & & $\mathbf{1 8 \%}$ & $\mathbf{6 9 7 \%}$ \\
\hline
\end{tabular}

size) causes a similar luminosity loss.

The higher disruption in the cold design impacts some of the sensitivities but not all. Without more complete simulations including beam-beam effects, it is difficult to estimate the resulting tolerances, but it is clear that some tolerances in the cold will be tighter than in the warm while others will be looser. Based on simulations for the warm design [11], the emittance growth in the vertical plane is estimated to be $30 \%$ for both beam delivery systems.

A summary of the emittance and jitter budgets for both the warm and the cold LC designs is given in Table 3.3.4.5. The budgets are almost identical through the collider, which is to be expected given that the tolerances are similar everywhere but in the main linacs. Even in the main linacs where the RF technology difference has most impact, the predicted emittance dilution is similar because the smaller wakefields of the cold linac are compensated by features of the warm design, including more precise diagnostics and controls. The remotely-controlled movers on all the quadrupoles and RF girders in the warm linac allow these elements to be aligned to the beam to the resolution of the diagnostics. A notable difference between the designs is that the cold linac is more tolerant of beam jitter because of the intra-train feedback at the IP and in the linac.

Table 3.3.4.5: Emittance and jitter budgets for warm and cold LC designs

\begin{tabular}{|c|c|c|c|c|c|c|}
\hline & \multicolumn{3}{|c|}{ Warm LC } & \multicolumn{3}{|c|}{ Cold LC } \\
\hline & $\Delta y / \sigma_{y}$ & $\gamma \epsilon_{x}$ & $\gamma \epsilon_{y}$ & $\Delta y / \sigma_{y}{ }^{a}$ & $\gamma \epsilon_{x}$ & $\gamma \epsilon_{y}$ \\
\hline Damping ring & $10 \%$ & $3.0 \mu \mathrm{m}-\mathrm{rad}$ & 20 nm-rad & $10 \%$ & $8.0 \mu \mathrm{m}-\mathrm{rad}$ & 20 nm-rad \\
\hline Bunch compressor & $15 \%$ & $10 \%$ & $20 \%$ & $15 \%$ & $10 \%$ & $20 \%$ \\
\hline Main linac & $30 \%$ & $5 \%$ & $50 \%$ & $100 \%$ & $5 \%$ & $50 \%$ \\
\hline Beam Delivery & $40 \%$ & $5 \%$ & $30 \%$ & $20 \%$ & $5 \%$ & $30 \%$ \\
\hline IP values & & $3.6 \mu \mathrm{m}-\mathrm{rad}$ & 40 nm-rad & & $9.6 \mu \mathrm{m}-\mathrm{rad}$ & $40 \mathrm{~nm}$-rad \\
\hline $\begin{array}{l}\text { Geometric } \\
\text { Luminosity }\end{array}$ & \multicolumn{3}{|c|}{$1.42 \times 10^{34} \mathrm{~cm}^{-2} \mathrm{~s}^{-1}$} & \multicolumn{3}{|c|}{$1.45 \times 10^{34} \mathrm{~cm}^{-2} \mathrm{~s}^{-1}$} \\
\hline$H_{D}$ & \multicolumn{3}{|c|}{1.42} & \multicolumn{3}{|c|}{1.78} \\
\hline Luminosity & \multicolumn{3}{|c|}{$2.08 \times 10^{34} \mathrm{~cm}^{-2} \mathrm{~s}^{-1}$} & \multicolumn{3}{|c|}{$2.57 \times 10^{34} \mathrm{~cm}^{-2} \mathrm{~s}^{-1}$} \\
\hline
\end{tabular}

${ }^{a}$ The jitter budgets for the cold LC apply in the absence of intratrain feedbacks. Intratrain feedbacks at the IP and in the main linacs will make the cold LC more tolerant of beam jitter.

\subsubsection{Beam delivery system design considerations}

The beam delivery system (BDS) transports the beam from the end of the main linac, focuses the beam to a small beam spot at the IP, and transports the outgoing disrupted beam to the beam dump. Since the beam 
delivery system is, to first order, independent of the details of the accelerating system, it has been possible to produce a common design, which applies to both warm and cold options.

Although the linear collider BDS must focus the beams down to an unprecedented size, many of the basic concepts were demonstrated in the SLC Final Focus and the Final Focus Test Beam (FFTB) at SLAC. This includes the basic optics as well as the tuning techniques, much of the diagnostic equipment, and the other beam line hardware. In particular, the optics at the FFTB demonstrated a larger demagnification than needed for the linear collider and thus, if a beam with the appropriate emittance and energy can be transported to the BDS, the optics should produce the desired spot sizes. The SLC and FFTB BDS are compared with those of the warm and cold linear colliders in Table 3.3.5.1

Table 3.3.5.1: Comparison of SLC, FFTB, and US warm and US cold beam delivery systems

\begin{tabular}{|l|c|c|c|c|}
\hline Parameter & SLC & FFTB & Warm option & Cold option \\
\hline Beam energy $[\mathrm{GeV}]$ & 46 & 47 & 250 & 250 \\
$\beta_{x} / \beta_{y}[\mathrm{~mm}]$ & $3 / 4$ & $10 / 0.1$ & $8 / 0.1$ & $15 / 0.4$ \\
$\gamma \epsilon_{x} / \gamma \epsilon_{y}[\mathrm{~mm}-\mathrm{mrad}]$ & $55 / 10$ & $30 / 3$ & $3.6 / 0.04$ & $9.6 / 0.04$ \\
$\sigma_{x} / \sigma_{y}[\mu \mathrm{m}]$ & $1.4 / 0.7$ & $1.8 / 0.055$ & $0.248 / 0.0030$ & $0.554 / 0.0057$ \\
$N\left[10^{10}\right]$ & 3.6 & 0.7 & 0.75 & 2.0 \\
$N_{b}$ & 1 & 1 & 192 & 2820 \\
Rep Rate $[\mathrm{Hz}]$ & 120 & 30 & 120 & 5 \\
$D_{y}$ & 2 & & 12.8 & 21.9 \\
$H_{d}$ & 2.2 & & 1.46 & 1.77 \\
Beam power $[\mathrm{MW}]$ & 0.035 & 0.002 & 6.9 & 11.3 \\
Solenoid $[\mathrm{T}]$ & 0.6 & & $3.0 \sim 6.0$ & $3.0 \sim 6.0$ \\
Luminosity $\left[\mathrm{cm}^{-2} \mathrm{~s}^{-1}\right]$ & $3 \times 10^{30}$ & & $2.1 \times 10^{34}$ & $2.6 \times 10^{34}$ \\
\hline
\end{tabular}

In both the warm and cold designs, the BDS is designed to be tuned to cancel aberrations to very highorder. This means that most effects due to component misalignments or setting errors can be compensated by tuning; however, it also means that the system is very sensitive to changes. For the tuning procedures to converge, mechanical and thermal stability as well as accurate diagnostics are essential. To avoid significant risk to the LC, it is important that the BDS be constructed with a high level of stability in mind. Active stabilization, feedback, and feed forward techniques can all be used to ease the stability requirements. However, it is sometimes difficult to accurately predict the performance and the interactions of such systems.

In general, the alignment, vacuum, and operational tolerances of most elements of the beam delivery system are similar to those of the main linac. In the cold BDS design, the larger IP beta functions imply that the response to misalignments is roughly two times looser than in the warm LC design; however, the stronger beam-beam disruption in the cold design reduces this difference, so the tolerances for comparable luminosity degradation are similar.

One location where there is a significant difference in tolerances between the BDS and the main linac is on the final focusing magnets at the IP. In the warm and cold BDS designs, effective vibration tolerances of 0.5 $\mathrm{nm}$ and $0.25 \mathrm{~nm}$, respectively, are necessary on this magnet to avoid a significant amount of luminosity loss due to IP beam jitter. In the warm BDS design, this problem must be addressed by active stabilization of the superconducting magnet while, in the cold BDS design, an intra-train feedback should be able to stabilize the beams at the IP. Both approaches will have challenges: direct stabilization of the final superconducting magnet may be complicated by internal vibration modes as well as external modes, while the intra-train feedback will require accurate intra-bunch diagnostics in a region with potentially large backgrounds.

Stabilization of the IP beam jitter illustrates one of the few differences between the warm and cold designs. In the warm design, the goal has been to actively stabilize components as necessary while, in the cold design, the intra-train feedback is used to compensate for motion of any upstream components. Unfortunately, it is difficult (although not impossible) to apply an intra-train feedback to the relatively short bunch train in 
the warm design if the active stabilization systems do not perform as well as desired. Similarly, the low repetition rate in the cold design makes it difficult (although not impossible) to utilize active stabilization techniques if the intra-train feedback does not perform as well as desired.

Both the warm and cold BDS designs have large beam power, in the multi MW range for design conditions. This raises concerns about the disrupted beam spray generating backgrounds in the IR instrumentation, or even quenching the first superconducting elements of the diagnostic line leading to the dump. Commissioning beams, involving single low intensity bunches at design energy, are expected to be less well adjusted in regard to beam trajectory or halo, and as such are also easily capable of destroying machine components or, less catastrophically, of quenching the final focus elements with slightly missteered beam. This is an issue for both warm and cold options.

In order to facilitate beam commissioning, start-up scenarios, and hardware failure modes, the BDS possesses a fast extraction/tune-up line. Using an independent, low power, beam dump, bunches can be steered down this line before the IP to avoid the experimental region. Beam can also be kicked into the same line during a beam abort condition. The design of the tune-up line requires a large acceptance in both energy and emittance.

The small beam spot at the IP at nominal bunch intensities and energies gives rise to significant space charge interactions as the bunches cross each other. The outgoing disrupted beam has a large energy distribution with significant beam tails as low as $25 \%-30 \%$ of the initial energy. Since the beam dump is the only element capable of absorbing the full beam power without damage, extraction line losses are an issue. Transporting the outgoing phase space from the IP to the beam dump without incurring large losses is a challenge for the design of the extraction line.

Although the wakefields in the main linac are not large, any beam halo (produced almost anywhere in the machine) ends up as a problem for the beam delivery system. The whole issue of beam halo, collimation effectiveness, disrupted beam dynamics and experimental backgrounds is easily capable of limiting luminosity. The machine-experiment interaction has been a problem (of varying degrees) at essentially every high-energy facility to date: HERA, RHIC, Tevatron, LEP, and SLC. Since this has proven essentially impossible in the past to understand in sufficient detail to adequately mitigate before building the machine and turning it on, one can well imagine the Linear Collider will be no different. In anticipation of background problems, the beam delivery system has a flexible system of absorbers, collimators, masks and shielding. The present design uses a combination of spoilers that can survive a full bunch train (warm) or a few bunches (cold) to protect against faults that are expected on a more frequent basis and "consumable" spoilers which can be damaged a finite number of times to protect against less frequent errors.

Because the BDS collimators have very small apertures, they can generate wakefields which cause emittance growth. This is essentially independent of technology. The use of strong octupoles to fold the large amplitude beam particles back into the core can permit the collimators to operate at larger distances from the beam and thus mitigate the wakefields to some extent.

In a similar fashion to other systems, a significant fraction of the hardware (mechanical stabilization systems, collimation elements, superconducting final focus magnets, etc) is not prototyped at this time. There is a need for R\&D activity in many areas of the beam delivery systems.

\subsection{Normal-conducting X-Band Linear Collider}

\subsubsection{Introduction}

The NLC collaboration has outlined a design[ZDR, NLC01],[TRC, Chapter 3] for a $500 \mathrm{GeV}$ (c.m.) linear collider, based on a normal conducting X-band linac, with a design luminosity of $2.0 \times 10^{34} \mathrm{~cm}^{-2} \mathrm{~s}^{-1}$, and 
upgradeable to $1 \mathrm{TeV}$ (c.m.). This design has been developed in close cooperation with the JLC (now GLC) collaboration[JLC][ISG] and the designs proposed by both groups are essentially identical. In order to take advantage of this work, the system-level reference design specification for the US-sited X-band linear collider will follow, except as specified below, the design outlined in the recent TRC report[TRC] and in the GLC Roadmap[GLC]. The primary change from the current NLC design is to provide for the possibility of polarized positron production as requested by the American Linear Collider Physics and Detectors scope document[2].

The X-band reference design incorporates the most recent NLC design updates that were adopted by the NLC collaboration in the last year. These include:

- An improved design for the damping rings[12][13].

- An improved design for the positron capture system[14].

- An improved design for the second interaction region to make it compatible with operation up to 1.3 $\mathrm{TeV}$ (c.m.)[15].

- The use of compact superconducting quadrupoles for the final doublets based on the Brookhaven design[16], to improve energy flexibility.

- The use of $60 \mathrm{~cm}$ long X-band structures with an initial group velocity of $3 \%$ and $150^{\circ}$ phase advance per cell, instead of $90 \mathrm{~cm}$ structures.

- The use of a '2 pack' modulator to power a pair of klystrons, instead of one modulator for eight klystrons, to better match the SLED-II pulse compression.

- The use of electromagnetic quadrupoles for the main linacs instead of adjustable permanent magnets. Permanent magnets are still planned for the bypass lines and parts of the injectors[17].

The differences between the X-band reference design and the 2003 NLC design are:

- The use of an undulator-based positron source utilizing the high energy electron beam at $150 \mathrm{GeV}$, instead of a conventional positron source, to allow a future upgrade to polarized positrons.

- At the subsystem and component level, specification changes to facilitate comparison with the cold LC option.

\subsubsection{Overall Parameters}

The overall machine layout for the X-band reference design is illustrated in Fig. 3.4.1.1. The overall parameters are given in Table 3.4.1.1.

The first column in Table 3.4.1.1 represents the reference design for $500 \mathrm{GeV}$, and the second column the design for $1 \mathrm{TeV}$. The beam parameters are identical to those published in the TRC report. In both the $500 \mathrm{GeV}$ and $1 \mathrm{TeV}$ configurations, higher energies can be achieved with the same installed RF power if the current (and luminosity) is reduced. The X-band accelerating structures will be qualified to an unloaded gradient of $65 \mathrm{MV} / \mathrm{m}$, while the loaded gradient at nominal luminosity corresponds to $52 \mathrm{MV} / \mathrm{m}$. Because the cavities are tested to the full $65 \mathrm{MV} / \mathrm{m}$, the X-band collider could operate at an energy roughly $25 \%$ higher than nominal with $30 \%$ of the nominal luminosity by reducing the average beam current. Without modification to the RF system, and with the same site power, the Stage II collider could deliver a luminosity of $9 \times 10^{33} \mathrm{~cm}^{-2} \mathrm{~s}^{-1}$ at a c.m. energy of $1.25 \mathrm{TeV}$. A plot of the luminosity versus energy for Stage II is plotted in Figure 3.4.1.2. In addition, sets of nominal parameters for operation at lower energy are listed in Table 3.4.1.2. 


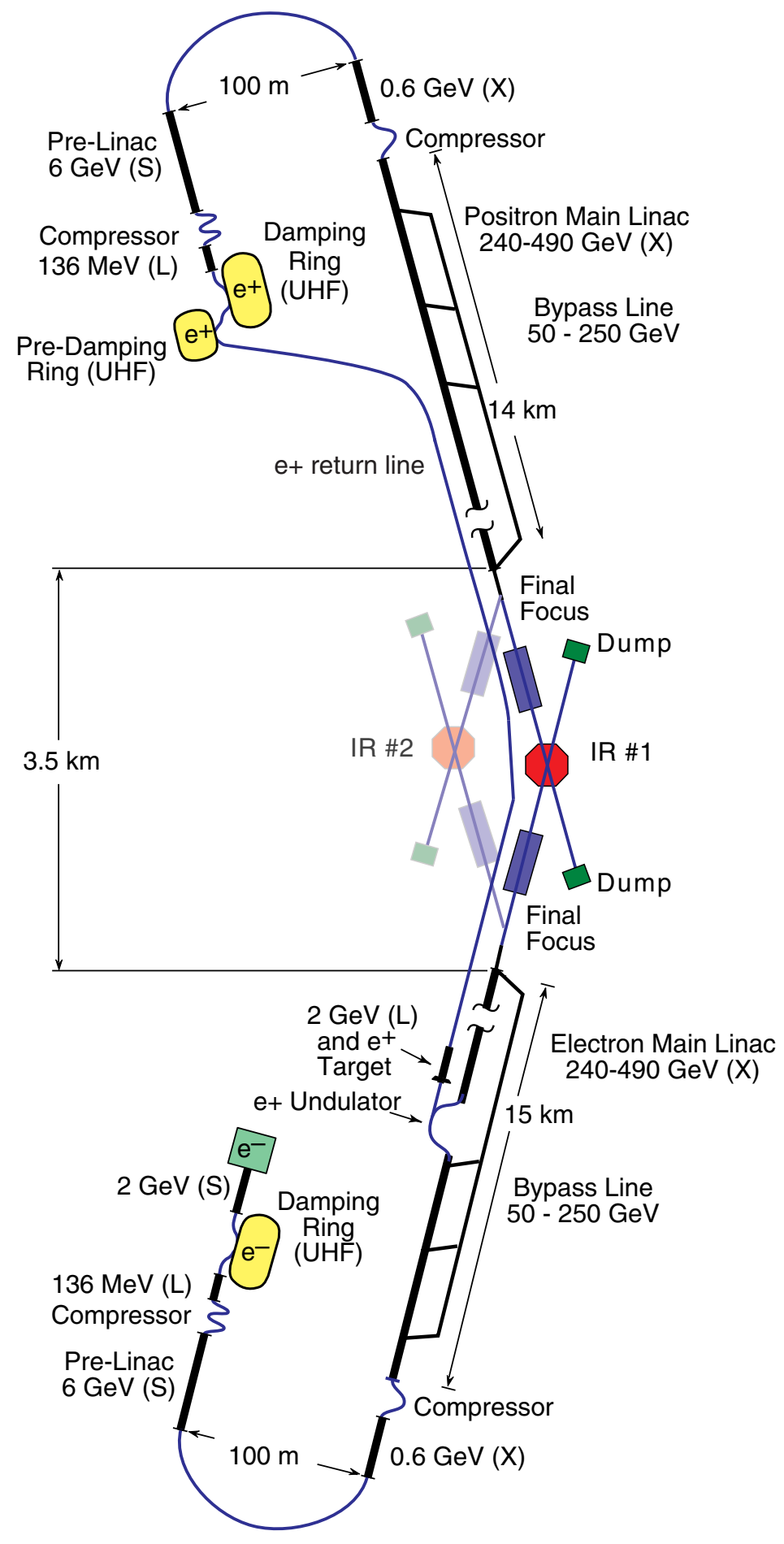

8-2003

8047A611

Figure 3.4.1.1: Overall Machine Layout, $500 \mathrm{GeV}$ c.m. 
Table 3.4.1.1: US X-band Linear Collider: overall parameters

\begin{tabular}{|l|c|c|}
\hline Parameter [Unit] & Reference design & Upgrade \\
\hline Beam Energy [GeV] & 250 & 500 \\
Luminosity $\left[10^{33} \mathrm{~cm}^{-2} \mathrm{~S}^{-1}\right.$ ] & 20.8 & 31.3 \\
Repetition rate $[\mathrm{Hz}]$ & 120 & 120 \\
Luminosity within $1 \%$ of $\mathrm{E}_{c . m .}$ [\%] & 64 & 58 \\
Bunch charge $\left[10^{10}\right]$ & 0.75 & 0.75 \\
Bunches $/ \mathrm{RF}$ pulse & 192 & 192 \\
Bunch separation [ns] & 1.4 & 1.4 \\
Injected $\gamma \epsilon_{x} / \gamma \epsilon_{y}\left[10^{-8} \mathrm{~m} \cdot \mathrm{rad}\right]$ & $300 / 2$ & $300 / 2$ \\
$\gamma \epsilon_{x} / \gamma \epsilon_{y}$ at IP $\left[10^{-8} \mathrm{~m} \cdot \mathrm{rad}\right]$ & $360 / 4$ & $360 / 4$ \\
$\beta_{x} / \beta_{y}$ at IP $[\mathrm{mm}]$ & $8 / 0.11$ & $13 / 0.11$ \\
$\sigma_{x} / \sigma_{y}$ at IP $[\mathrm{nm}]$ & $243 / 3.0$ & $219 / 2.1$ \\
$\sigma_{z}$ at IP $[\mu \mathrm{m}]$ & 110 & 110 \\
Upsilon average & 0.12 & 0.27 \\
Pinch enhancement & 1.46 & 1.41 \\
Beamstrahlung $\delta_{B}[\%]$ & 4.6 & 8.2 \\
Photons per $e^{+} / e^{-}$ & 1.19 & 1.24 \\
Loaded gradient $[\mathrm{MV} / \mathrm{m}]$ & 52 & 52 \\
Linac length $[\mathrm{km}]$ & 6.71 & 13.42 \\
\hline
\end{tabular}

Table 3.4.1.2: Low energy operation parameters

\begin{tabular}{lccc}
\hline Center-of-mass energy $[\mathrm{GeV}]$ & 92 & 250 & 350 \\
\hline \hline Luminosity $\left[10^{33} \mathrm{~cm}^{-2} \mathrm{~s}^{-1}\right]$ & 3.5 & 9.4 & 13.2 \\
Luminosity within $1 \%$ of $\mathrm{E}_{c . m .}[\%]$ & 92 & 75 & 65 \\
Repetition rate $[\mathrm{Hz}]$ & 120 & 120 & 120 \\
Bunch charge $\left[10^{10}\right]$ & 0.75 & 0.75 & 0.75 \\
$\sigma_{x} / \sigma_{y}$ at IP $[\mathrm{nm}]$ & $630 / 6.2$ & $380 / 3.8$ & $320 / 3.2$ \\
Beamstrahlung $\delta_{B}[\%]$ & 0.18 & 1.1 & 2 \\
Photons per $e^{+} / e^{-}$ & 0.49 & 0.79 & 0.92 \\
Polarization loss $[\%]$ & 0.08 & 0.21 & 0.34 \\
\hline \hline
\end{tabular}

\subsubsection{Accelerator System Reference Designs}

With the exception of the positron source, the overall design and specification of the X-band linear collider reference design is identical to the current NLC baseline design. Details of the magnets, power supplies, vacuum systems, diagnostics and utilities are available as part of the NLC 2003 Configuration documentation[NLC03] and will not be reproduced here. In the following sections, each system will be described only briefly. Where newer information is available or there are differences from the 2003 Configuration, the systems will be covered in more detail.

3.4.1.2.1 Electron Source This system will be identical to that described in the NLC 2001 Report[NLC01] and the TRC report[TRC].

3.4.1.2.2 Positron Source This system will use an undulator-based positron source similar to that described in the TESLA TDR[TDR], but in an implementation which covers the entire desired energy range and provides greater stability, higher yield and better reliability. 


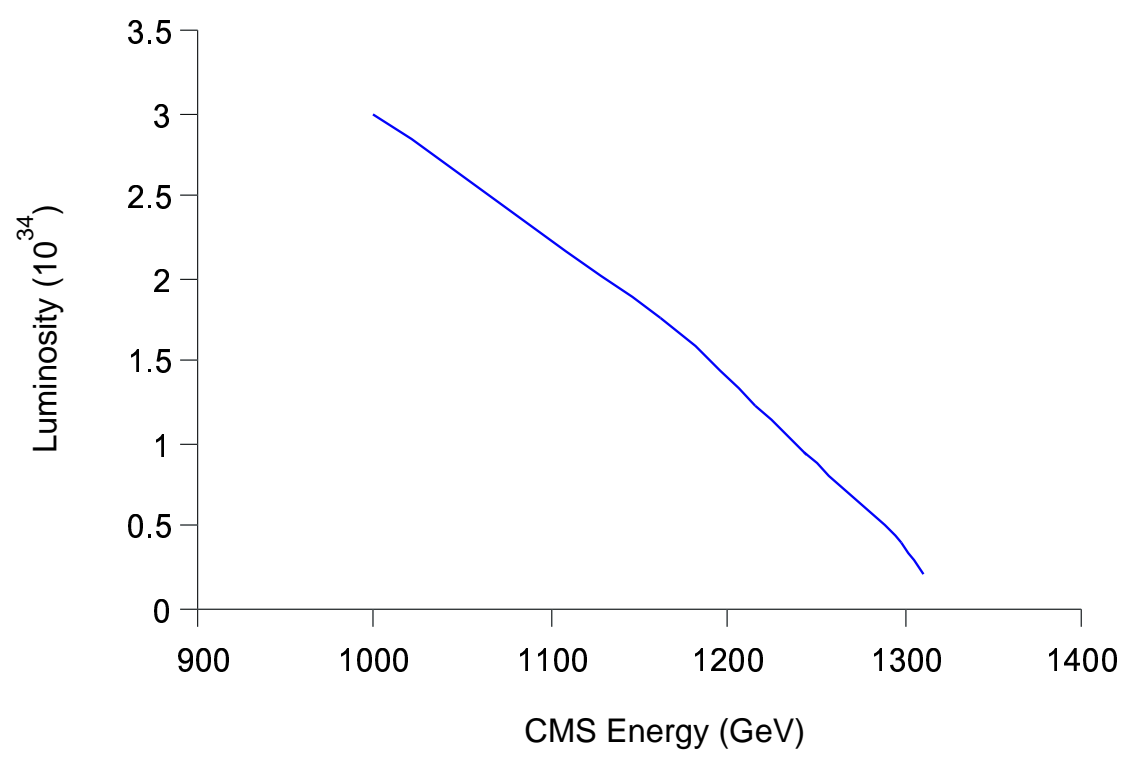

Figure 3.4.1.2: Energy versus luminosity for the Stage II X-band collider.

To provide a flexible range of collision energy, the undulator will be placed at the $\sim 150 \mathrm{GeV}$ point in the electron linac. Downstream from the undulator, the electron beam energy can be controlled by using the additional $\sim 100 \mathrm{GeV}$ of linac as an accelerator or decelerator. In theory, this provides center-of-mass energy variability at the collision point from $\sim 90$ to $500 \mathrm{GeV}$, without the need to retune or otherwise change the positron production system. However the strong wakefields in the X-band design will lead to significant dilutions at the lowest beam energies. To operate with higher quality beam at roughly $90 \mathrm{GeV}$ in the center-of-mass, the bypass line can be used to deliver alternate pulses to the undulator and the IP.

To provide sufficient positron flux, a long $(140 \mathrm{~m})$ helical undulator will be used. Since the beam must be deflected off-axis by about $2.5 \mathrm{~m}$ to pass through the undulator, an insertion of total length about $850 \mathrm{~m}$ will be required to allow the electron beam to be transported into and out of the undulator. This length is sufficient to allow for an upgrade of the undulator to $200 \mathrm{~m}$, which will permit operation with polarized positrons. The capture system will be the improved configuration proposed for the NLC[14].

3.4.1.2.3 Damping Rings and Bunch Compressors The electron and positron main damping rings[12] and positron pre-damping ring[13] will be taken from the recent NLC design. The bunch compressor system will be identical to that described in the NLC 2001 Report[NLC01] and the TRC report[TRC].

3.4.1.2.4 Main Linac The main linac components will be taken from the NLC 2003 Configuration[NLC03]. The basic RF unit contains a pair of 75-MW 1.6- $\mu$ s klystrons driven by a solid-state induction modulator. Each klystron pair powers a dual-mode SLED-II pulse compression system which feeds an RF girder with eight 0.6-m accelerator structures. The structures will use the newest GLC/NLC design, with the damped and detuned configuration developed and tested on earlier long structures but with an initial group velocity of $3 \%$, an a $/ \lambda$ of 0.17 , and a phase advance per cell of $150^{\circ}$.

The linac optics, bypass lines, diagnostic insertions, number of spare RF units, tunnel length and support tunnel configuration will all be as specified in the TRC report[TRC, Chapter 3]. 
3.4.1.2.5 Beam delivery system The beam delivery system will be the current NLC design with improved performance for the second IR, superconducting quadrupoles for the final doublets, and greater energy bandwidth for the tuneup dump line[15].

\subsubsection{Upgrade to $1 \mathrm{TeV}$}

The second column in Table 3.4.1.1 represents the upgrade to $1 \mathrm{TeV}$. The energy limit of the upgraded machine is $1.3 \mathrm{TeV}$ at reduced luminosity. The beam parameters from the sources are identical. The BDS parameters are also the same, except for $\beta_{x}$, which has been increased to reduce $N_{\gamma}$. To implement the upgrade, additional RF systems must be added to the main linacs and the final focus requires minor upgrades to soften the bending radius and lengthen the final quadrupoles.

\subsubsection{Injection Systems}

The injection systems provide the $1.98 \mathrm{GeV}$ electron and positron beams which are delivered to the damping rings. The overall design and specification of the electron source follows the description given in the NLC ZDR[ZDR, Chapter 2]. The positron source is an undulator-based design described in Ref. [18]. This differs from the conventional source in the NLC baseline, and thus is discussed in somewhat more detail.

\subsubsection{Electron Injection System}

The electron source, which is based on the successful SLC polarized source, consists of a polarized highpower laser and a high-voltage dc gun with a semiconductor photocathode. The $80 \mathrm{MeV}$ electron beam is bunched in a $714 \mathrm{MHz}$ RF system and accelerated in an S-band linac to $1.98 \mathrm{GeV}$, the energy of the electron main damping ring. Each beam consists of a bunch train of 192 bunches, with $0.8 \times 10^{10}$ particles, that are spaced by $1.4 \mathrm{~ns}$ (or trains can have half the number of bunches, twice the spacing and double the charge in each bunch). The electrons at the end of the source booster linac have an rms normalized emittance of $100 \times 10^{-6} \mathrm{~m} \cdot \mathrm{rad}$ and have a spin polarization of $80 \%$ or greater. To improve reliability, there are two identical polarized electron sources.

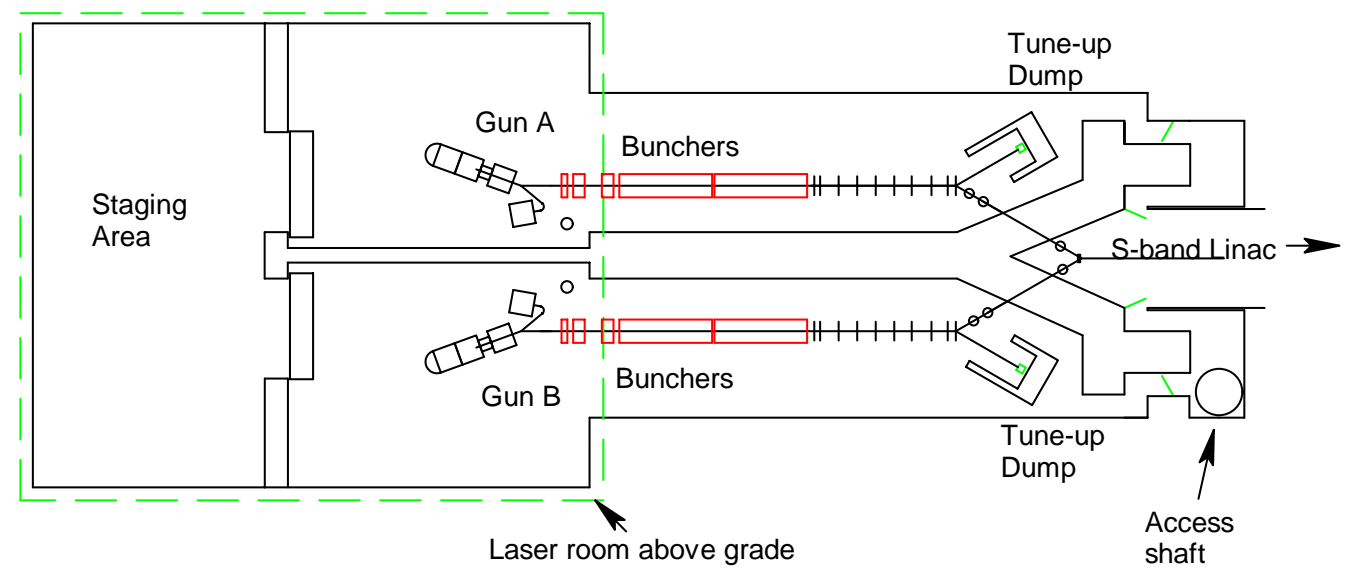

Figure 3.4.2.1: Electron Injection System layout

The electron source, damping ring and pre-linac are located in a separate complex near the low energy end 
of the main electron linac tunnel. The booster linac and pre-linac share a common tunnel with the beams travelling in opposite directions. The polarized source is located off-axis at the beginning of the booster linac. Each source is $35 \mathrm{~m}$ long with a $5 \mathrm{~m}$ long tuneup dump line. The electron injector linac is $173 \mathrm{~m}$ long. A schematic view of the redundant electron sources is provided in Fig. 3.4.2.1.

3.4.2.1.1 Electron Sources The electron production system includes a laser system, a high voltage polarized photocathode electron gun, a $714 \mathrm{MHz}$ sub-harmonic bunching system, an S-band capture and pre-accelerator, and a $1.98 \mathrm{GeV}$ S-band electron booster linac. It delivers a bunch train of 192 (96) bunches spaced at 1.4 (2.8) ns to the electron damping ring system. For better reliability, the design uses two guns, bunching systems and pre-accelerators at the source, and two laser systems which can each be directed at either gun. The pre-accelerators deliver beam at $80 \mathrm{MeV}$ after which the beam is accelerated in a common booster linac. A summary of the design parameters is given in Table 3.4.2.1.1.

Table 3.4.2.1: Beam parameters as delivered by the electron source system to the electron main damping ring system for the $1.4 \mathrm{~ns}$ bunch spacing option.

\begin{tabular}{|l|c|}
\hline Bunch spacing & $1.4 \mathrm{~ns}$ \\
Number of bunches & 192 \\
Particles/bunch & $0.8 \times 10^{10}$ \\
Energy & $1.98 \mathrm{GeV}$ \\
Energy adjustability & $\pm 5 \%$ \\
Bunch energy variation & $1 \%$ Full Width \\
Single bunch energy spread & $1 \%$ Full Width \\
Emittance $\gamma \epsilon_{x, y}$ [rms] & $100 \mu \mathrm{m} \cdot \mathrm{rad}$ \\
Bunch length $\sigma_{z}$ & $<10 \mathrm{~mm}$ \\
Train population uniformity & $1 \% \mathrm{Full} \mathrm{Width}$ \\
Bunch-to-bunch population uniformity & $2 \% \mathrm{rms}$ \\
Repetition rate & $120 \mathrm{~Hz}$ \\
Horizontal beam jitter $\Delta \gamma J_{x}$ & $50 \mu \mathrm{m} \cdot \mathrm{rad}$ \\
Vertical beam jitter $\Delta \gamma J_{y}$ & $50 \mu \mathrm{m} \cdot \mathrm{rad}$ \\
Polarization & $80 \%$ \\
Beam power & $58 \mathrm{~kW}$ \\
\hline
\end{tabular}

The overall optics from the polarized gun through the bunchers, pre-linac, and transfer line into the damping ring is shown in Fig. 3.4.2.2 and Fig. 3.4.2.3.

3.4.2.1.1.1 Laser system The laser system produces a train of light pulses with sufficient intensity to excite the requisite number of electrons from the photocathode. The pulse structure of 192 (96) bunches spaced at $1.4(2.8) \mathrm{ns}$ is produced by the laser, with each laser pulse $0.5 \mathrm{~ns}$ long. The requirements of the electron beam for intensity stability must be met by the laser system. The laser light must be a specific wavelength to match the bandgap of the III-V semiconductor photocathode and must be circularly polarized to create suitably spin polarized electrons. An identical laser system would be used to provide polarized electrons for optional $e^{-}-e^{-}$collisions or for the unpolarized electron source for positron generation if a conventional target scheme were used.

3.4.2.1.1.2 High voltage photocathode gun The high voltage photocathode gun utilizes a III-V semiconductor photocathode excited by the laser system to provide the electron bunches. The photocathode is designed to deliver the requisite instantaneous current with electron polarization greater than $80 \%$. For the NLC, the gun is specified to operate at a high voltage of $175 \mathrm{kV}$ in order to accommodate the charge 


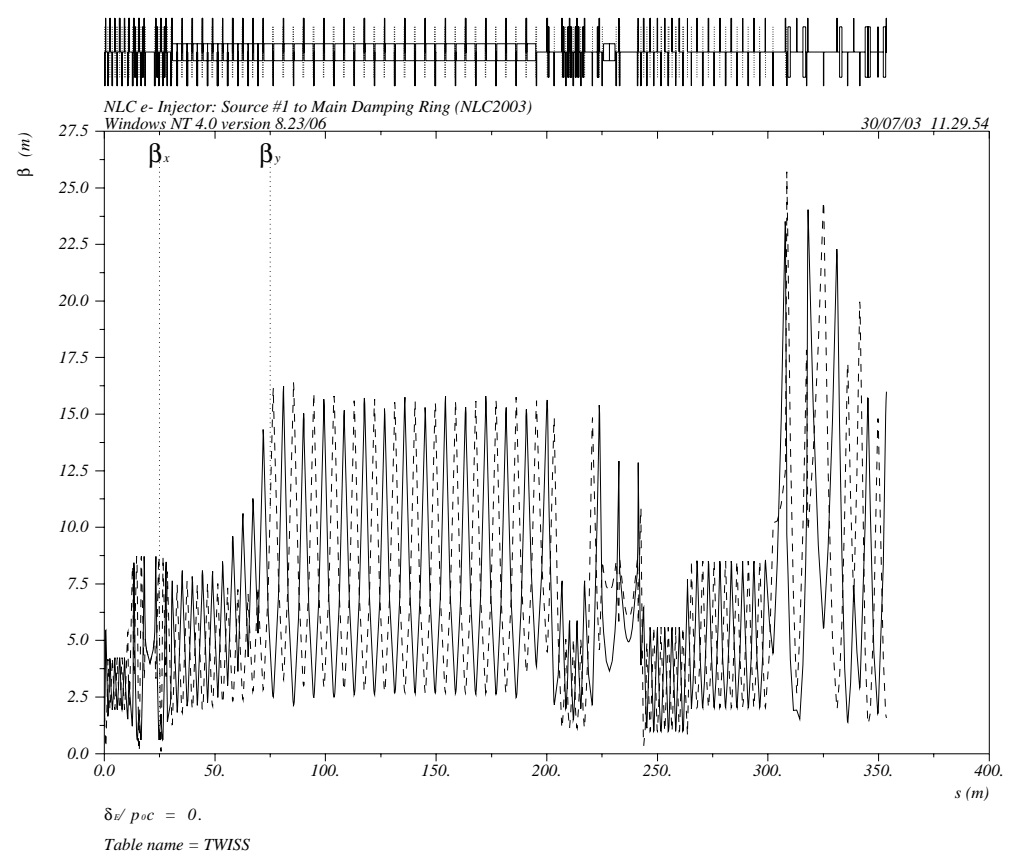

Figure 3.4.2.2: Beta Functions of Electron Injection System from Gun to Damping Ring

Table 3.4.2.2: Laser parameters

\begin{tabular}{|c|c|c|c|c|}
\hline Parameter Name & Symbol & \multicolumn{2}{|c|}{ Source Output } & Units \\
\hline Bunch Spacing & $T_{b}$ & 2.8 & 1.4 & $\mathrm{~ns}$ \\
Wavelength & $\lambda$ & $740-850$ & $740-850$ & $\mathrm{~nm}$ \\
Bandwidth & $\delta \lambda$ & 3 & 3 & $\mathrm{~nm}$ \\
Bunch Length & $\delta t$ & $<0.5$ & 0.5 & $\mathrm{~ns}$ \\
Energy/Bunch & $E_{b}$ & 8.4 & 4.2 & $\mu \mathrm{J}$ \\
Energy Uniformity & $\Delta n_{B} / n_{B}$ & $<0.5$ & $<0.5$ & $\%$ \\
Energy Uniformity along train & & 2 & 2 & $\%$ \\
Number of Bunches & $N_{b}$ & 96 & 192 & $\#$ \\
Repetition Rate & $f$ & 120 & 120 & $\mathrm{~Hz}$ \\
Polarization & $P_{\gamma}$ & 99.9 & 99.9 & $\%$ \\
\hline
\end{tabular}

requirement for the $2.8 \mathrm{~ns}$ bunch spacing. The design of the gun is based on the design of the SLC polarized electron gun, which is a diode gun containing a photocathode biased at $120 \mathrm{kV}$.

The NLC photocathode design is based upon the SLC photocathode, which exhibited a charge limit at currents below the NLC specifications. This charge limit effect has been overcome by using a heavily doped surface layer on the cathode. The E158 experiment at SLAC has used a recently developed (2002) strained layer photocathode which has produced beam polarization measured at $50 \mathrm{GeV}>80 \%$. In separate studies, the total charge extracted in a single $300 \mathrm{~ns}$ pulse far exceeded the NLC total charge requirements. The systematic improvement of photocathode charge limit and polarization is an ongoing R\&D project for the NLC. 


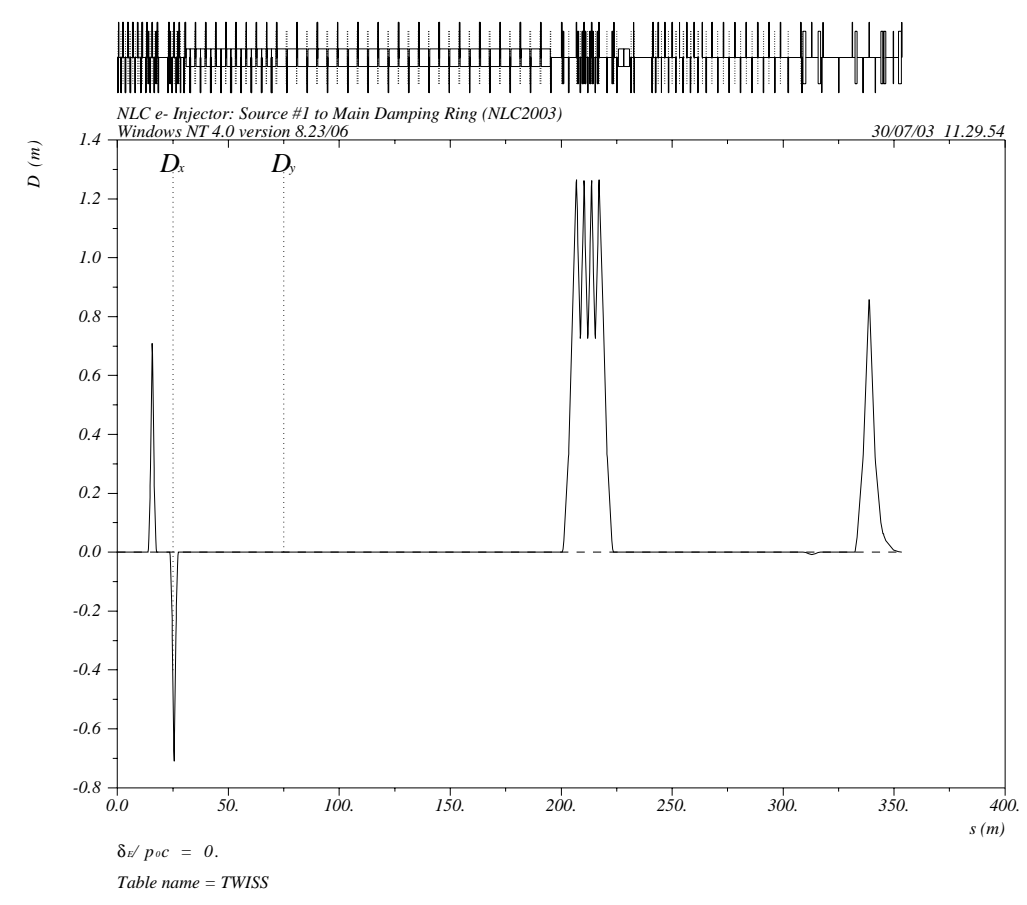

Figure 3.4.2.3: Dispersion Functions of Electron Injection System from Gun to Damping Ring 
Table 3.4.2.3: Gun parameters

\begin{tabular}{|c|c|c|c|c|}
\hline Parameter Name & Symbol & \multicolumn{2}{|c|}{ Source Output } & Units \\
\hline Bunch Spacing & $T_{b}$ & 2.8 & 1.4 & $\mathrm{~ns}$ \\
High Voltage & $V$ & 175 & 175 & $\mathrm{kV}$ \\
Bunch Length & $\delta t$ & 0.5 & 0.5 & $\mathrm{~ns}$ \\
Particles/Bunch & $n_{b}$ & 2.4 & 1.2 & $10^{10}$ particles \\
Population Uniformity & $\Delta n_{B} / n_{B}$ & $<0.5$ & $<0.5$ & $\%$ \\
Population Uniformity along train & & 2 & 2 & $\%$ \\
Number of Bunches & $N_{b}$ & 96 & 192 & $\#$ \\
Repetition Rate & $f$ & 120 & 120 & $\mathrm{~Hz}$ \\
Polarization & $P_{e}$ & $>80$ & $>80$ & $\%$ \\
\hline
\end{tabular}

3.4.2.1.1.3 Beam Bunching System The electrons produced from the gun are longitudinally bunched in a sub-harmonic RF system for injection into the S-band booster linac. The bunchers run at a frequency of $714 \mathrm{MHz}$ for both $1.4 \mathrm{~ns}$ and $2.8 \mathrm{~ns}$ bunch spacing. The major technical components of the bunchers and booster linac are given in Table 3.4.2.1.1.3.

RF system: The sub-harmonic bunching system is two $714 \mathrm{MHz} R F$ amplifiers powering two bunching cavities. This is followed by a 4 cavity S-Band buncher and a 3 meter S-Band acceleration section.

Magnet system: The bunching system is enclosed in a solenoid magnet system. All quadrupoles between the bunching system and the accelerator are electromagnets. There are horizontal and vertical correctors at each quadrupole.

Vacuum system: The photocathode gun is an ultra-high vacuum system with a total pressure $<10^{-11}$ Torr. The gun is pumped by ion and NEG pumps and is monitored by a residual gas analyzer. The bunching system is pumped by a manifold ion pump scheme, with a hot filament gauge and has an operating vacuum pressure of $<10^{-8}$ Torr.

Table 3.4.2.4: Major Technical Components: Electron Bunchers and Injector Linac

\begin{tabular}{|c|c|}
\hline Component & Description \\
\hline Sub-harmonic buncher & Two 714 MHz bunching cavities \\
S-band buncher & One 4 cell TW S-band buncher \\
Focusing system & Solenoid magnets around bunchers \\
Matching & 24 Electromagnet quadrupoles, BPMs, horizontal and vertical dipoles \\
Pre-accelerator & One 3 m S-band accelerator \\
Diagnostics (80 MeV) & 4 wire emittance, energy, energy spread, bunch length measurement \\
Booster linac acceleration & 6 S-band RF modules \\
RF power source & 6 solid-state modulators, 12 50 MW S-band klystrons, 12 SLED-I \\
Structures & 364 m structures @ 17 MV/m (loaded) \\
Focusing system & Two (1st 10 cells), then One, quadrupole per structure \\
Steering system & One $10 \mu$ m BPM, horizontal and vertical corrector per quadrupole \\
Diagnostics (before DR) & 4 wire emittance, energy, energy spread, bunch length measurement \\
Vacuum system & $\sim 340$ pumps \\
\hline
\end{tabular}

3.4.2.1.1.4 S-band Electron Booster Linac The $e^{-}$booster linac accelerates the electrons from 80 $\mathrm{MeV}$ to $1.98 \mathrm{GeV}$. The linac has $6 \mathrm{~S}$-band $(2856 \mathrm{MHz}) \mathrm{RF}$ modules, each consisting of a solid-state modulator powering two klystrons, that feed two SLED assemblies which together power six 4 meter accelerator structures. The combination of two SLED cavities permits vernier control of the RF waveform for beam 
loading compensation, while allowing for constant power delivery to the structures during a machine protection system fault and recovery. The $6 \mathrm{RF}$ modules include one spare RF module for overhead. The loaded gradient of each structure is $17 \mathrm{MV} / \mathrm{m}(21 \mathrm{MV} / \mathrm{m}$ unloaded).

Magnet system: Beam focusing is accomplished with tunable, permanent magnet quadrupoles between each structure, in addition to quadrupoles which wrap around parts of each of the first 5 structures. The beginning and end of $e^{-}$booster has matching sections with electromagnet quadrupoles and steering feedback. Each non-wrap-around quadrupole has a BPM. There is a horizontal and vertical corrector associated with each non-wrap-around quadrupole, while each wrap-around quadrupole has steering trim windings.

Vacuum system: The accelerator vacuum module system is a manifold ion pump scheme, with a hot filament gauge. The operating vacuum pressure is $<10^{-8}$ Torr.

3.4.2.1.1.5 Instrumentation To measure the beam emittance, 4 -wire non-invasive emittance diagnostics are located after the $e^{-}$source (at $80 \mathrm{MeV}$ ) and before injection into the main damping ring. In addition, energy, energy spread, and bunch length diagnostics are located in a chicane at the $80 \mathrm{MeV}$ point and in the $60^{\circ}$ arc before injection into the main damping ring. To preserve electron helicity, the spin must be rotated into the vertical direction prior to injection into the damping ring. The $60^{\circ}$ arc also rotates the polarization vector from the longitudinal direction into the horizontal and a subsequent superconducting solenoid then orients the polarization vertically. To stabilize the trajectory and preserve the emittance, all the quadrupoles have BPMs with $10 \mu \mathrm{m}$ resolution and horizontal or vertical steering correctors depending on the focusing plane.

\subsubsection{Positron Injection System}

The positron source is an undulator-based system similar to that described in the TESLA TDR [TDR]. The particular implementation used is one developed by the NLC collaboration for polarized positron production [18]. This implementation includes several changes made to improve the operation and reliability of the positron source system.

1. The undulator has been located midway along the electron linac at a fixed beam energy of $150 \mathrm{GeV}$ which is used for improved energy flexibility over that of the TDR design.

2. The undulator is assumed to be helical to generate polarized positrons when such an option is desired. To provide for sufficient yield and to ensure reliable operation, the helical undulator is roughly 200 meters in length - this should have a positron yield (number of captured $e^{+}$per incoming $e^{-}$) which is four times that of the system described in the TESLA TDR.

3. Relatively short undulator sections are placed between quadrupole magnets to ease the undulator tolerances and magnet alignment.

4. Two targets and capture sections are installed so that when one system fails, the other can be used without waiting for a time consuming cool-down period in the radioactive environment. In addition, a polarized electron source is installed next to the capture sections to facilitate commissioning and operation with $e^{-}-e^{-}$or $\gamma-\gamma$.

5. The positron beam is immediately accelerated after the capture sections to the nominal damping ring energy of $1.98 \mathrm{GeV}$ and transported to the ring at this energy rather than at $290 \mathrm{MeV}$ as in the TESLA TDR.

6. Additional diagnostics and compensating optics have been explicitly designed into the system to aid commissioning and operation. 
Parameters for the polarized and unpolarized undulator-based positron source are compared with those of the TESLA TDR in Table 3.4.2.5. Table 3.4.2.5 also lists parameters for a conventional positron source as originally foreseen for the NLC. The conventional source is not part of the reference design. The positron source must be capable of producing polarized positrons and thus the enclosure for the full positron system must be excavated. For initial operation only the undulator required to generate the an unpolarized positron beam needs to be installed. It might also be possible to only install the length necessary to obtain a simulated yield of 1 while having the option of installing additional undulator if the operational yield is lower; however this is probably a misplaced efficiency. Alternately, one could install a planar undulator system which is more straightforward to construct. However, with equal peak fields, a planar undulator would have to be twice as long as a helical undulator for the same yield.

Table 3.4.2.5: Warm Option Positron Source Parameters

\begin{tabular}{|c|c|c|c|c|}
\hline & TESLA TDR & Unpolarized & Polarized & Conventional \\
\hline Drive Beam Energy [GeV] & 250 & 153 & 153 & 6.2 \\
\hline Beam Energy Loss [GeV] & 3 & 4.9 & 6.5 & - \\
\hline Beam Energy Spread In [\%] & 0.05 & 0.5 & 0.5 & - \\
\hline Beam Energy Spread Out [\%] & 0.10 & 0.46 & 0.44 & - \\
\hline Additional linac length [m] & 120 & 95 & 126 & 230 \\
\hline Undulator length [m] & 100 & 150 & 200 & - \\
\hline Undulator insertion length $^{\S}[\mathrm{m}]$ & 340 & 790 & 850 & - \\
\hline Positron source length $[\mathrm{m}]$ & 400 & 450 & 450 & 450 \\
\hline Photon energy* [MeV] & 28 & 10.7 & 10.7 & - \\
\hline Undulator type & $=1 ;$ planar & $K=1 ;$ helical & $K=1 ;$ helical & - \\
\hline Undulator field [T] & 0.75 & 1.07 & 1.07 & - \\
\hline Undulator period [cm] & 1.4 & 1 & 1 & - \\
\hline Undulator full gap [mm] & 5 & 6 & 6 & - \\
\hline Pulse energy on target [kJ] & 26.9 & 1.1 & $1.1^{\diamond}$ & $0.5^{\natural}$ \\
\hline Average power on target [kW] & 135 & 136 & $127^{\diamond}$ & $57^{\natural}$ \\
\hline Spot size on target [mm] & 0.75 & 0.75 & 0.75 & 1.6 \\
\hline Target material & Ti-alloy & Ti-alloy & Ti-alloy & $\mathrm{W}_{74} \mathrm{Re}_{26}$ \\
\hline Target thickness [r.l.] & 0.4 & 0.4 & 0.4 & 4.0 \\
\hline Target energy absorption [\%] & 4 & 9 & 8 & 14 \\
\hline Beam polarization [\%] & 0 & 0 & 59 & 0 \\
\hline Positron yield & & & & \\
\hline
\end{tabular}

$\S$ The undulator insertion length includes the length of the undulator and the drift to the target if this increases the system length.

I The source length is defined as the length required to generate, capture, and accelerate the positrons to damping ring energy without including the length of low energy transport line.

* Denotes the photon energy at the first harmonic cutoff.

$\diamond$ Includes intensity reduction due to an angular cut at $\gamma \theta=1.414$.

$\natural$ Drive beam energy and power per target; three targets operating in parallel are required.

$\star$ There are known inconsistencies in the TESLA TDR design where the listed photon beam power does not correspond to the listed undulator parameters.

$\dagger$ Positron yield is defined as the number of positrons captured in the damping ring divided by the number of electrons used to generate the positrons.

$\ddagger$ The positron yield of 2 is listed in the TDR; however, other calculations find values between 1 and 1.5 for the TDR system[14]. 
3.4.2.2.1 Positron Source Layout As mentioned, one of the major changes to the positron system was to move the undulator location to lower energy. In the TESLA TDR design, the source was located at the end of the electron linac and the incoming electron beam energy is roughly one half of the collision energy. Both the undulator photon energy and the undulator photon power scale as the square of the electron beam energy and thus the performance of the positron system will change significantly as the beam energy is varied.

There are two real issues here. First, because the positron source parameters such as the yield, energy, bunch length, and emittance, are all very sensitive to the incoming beam energy, small energy changes would require significant retuning of the positron source system. If the positron source is located at the end of the linac, even energy scans of a few percent would probably require retuning the source. The delicacy of the positron source tuning was evident during the SLC operation where the positron source parameters were tuned more often than any other part of the collider. Because of the very large beam emittances and high backgrounds due to beam loss around the positron source, it is difficult to install reliable beam diagnostics that simplify the tuning process. Thus, it is felt to be quite important to minimize the tuning needed by installing the source at a fixed energy along the collider.

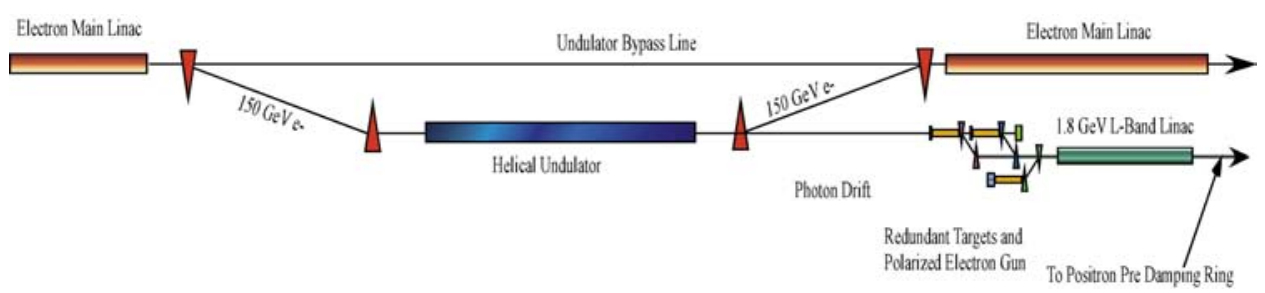

Figure 3.4.2.4: Undulator-based positron generation scheme

Second, the positron yield scales roughly quadratically with beam energy. Thus, if the system is designed to have a an operating yield of 1 at $150 \mathrm{GeV}$, the luminosity drops very rapidly as the beam energy is further decreased. The solution proposed in the TESLA TDR was to use one portion of the linac to accelerate the beam to $150 \mathrm{GeV}$ for positron production and use the remaining $100 \mathrm{GeV}$ of linac (during Stage I) to accelerate beam to the IP. This technique allows center-of-mass energy coverage from $90 \mathrm{GeV}$ up to about $200 \mathrm{GeV}$; however there is still an important gap from $200 \mathrm{GeV}$ until $300 \mathrm{GeV}$ in the center-of-mass where neither positron production technique is sufficient. By placing the undulator partway along the linac, the beam can be either accelerated or decelerated after the undulator, thereby increasing the operating range of the collision energy.

For these two reasons we have chosen a source location at a beam energy of $153 \mathrm{GeV}$ rather than at the end of the electron linac. At this location, 150-meters of undulator, needed for a simulated yield of 1.5, decreases the beam energy by $4.9 \mathrm{GeV}$. Thus, to obtain a full beam energy of $250 \mathrm{GeV}, 101.9 \mathrm{GeV}$ of linac must be installed after the positron source undulator. This means that the stage I electron linac should be able to produce a beam with energies between $46 \mathrm{GeV}$ and $250 \mathrm{GeV}$ while generating a stable positron beam. The only difficulty is that the energy jitter and the emittance dilutions increase as the beam is decelerated. However, since most running at the Z-pole for detector calibration does not require enormous luminosity, these are not thought to be unmanageable liabilities.

If the positron source is installed midway along the linac, the undulator and positron target, capture, and acceleration must be installed in a parallel tunnel. A schematic of the layout for the positron source is illustrated in Fig. 3.4.2.4[18]. The undulator and positron source systems are offset by 2.5 meters transversely from the main linac. The 2.5-meter offset allows for sufficient shielding so that the positron target and capture regions do not irradiate the main linac tunnel. Arcs, which generate small levels of emittance dilution, are used to inject the main electron beam into the undulator and then re-inject it into the main linac tunnel. The photon beam drifts straight ahead for roughly 200 meters after the end of the undulator to the positron 
targets. There are two positron target and capture assemblies in line so that if one fails, the other can be inserted with minimal impact to the run. In addition, there is a polarized electron gun which would be used for $e^{-}-e^{-}$or $\gamma-\gamma$ operation. This electron system would also be used to commission the positron beam lines since the much lower electron beam emittance would make it much easier to diagnose the beam line.

The total length of the undulator insertion and the subsequent positron target, capture, and acceleration system which are all located 2.5 meters offset from the main tunnel is about $1.3 \mathrm{~km}$. The system length is roughly twice as long as the layout in the TESLA TDR due to undulator insertion and undulator length, the more extensive RF system for operational overhead, and the more extensive diagnostics.

3.4.2.2.2 Undulator Insertion The arcs displace the undulator by 2.5 meters from the linac centerline. The arcs are based on sixteen 14-meter 90 degree FODO cells plus dispersion suppressing cells at either end[19]. The incoherent SR growth of the beam is roughly $2.5 \%$ for the LTU (linac-to-undulator) and $2.5 \%$ for the return. Each arc is roughly 250 meters in length. The trajectory is shown in Fig. 3.4.2.5 while the beta functions and the dispersion are shown in Figs. 3.4.2.6 and 3.4.2.7. Finally, the second-order dispersion is canceled with eight sextupoles located in each arc to ensure a bandpass of at least $\pm 3 \%$. The bandpass is plotted in Fig. 3.4.2.8; a reasonable bandpass is needed to prevent damage due to routine energy fluctuations and may be needed for beam-based alignment techniques. Additional sacrificial spoilers will undoubtedly be needed to protect the undulator and the insert from larger energy errors due to machine faults.

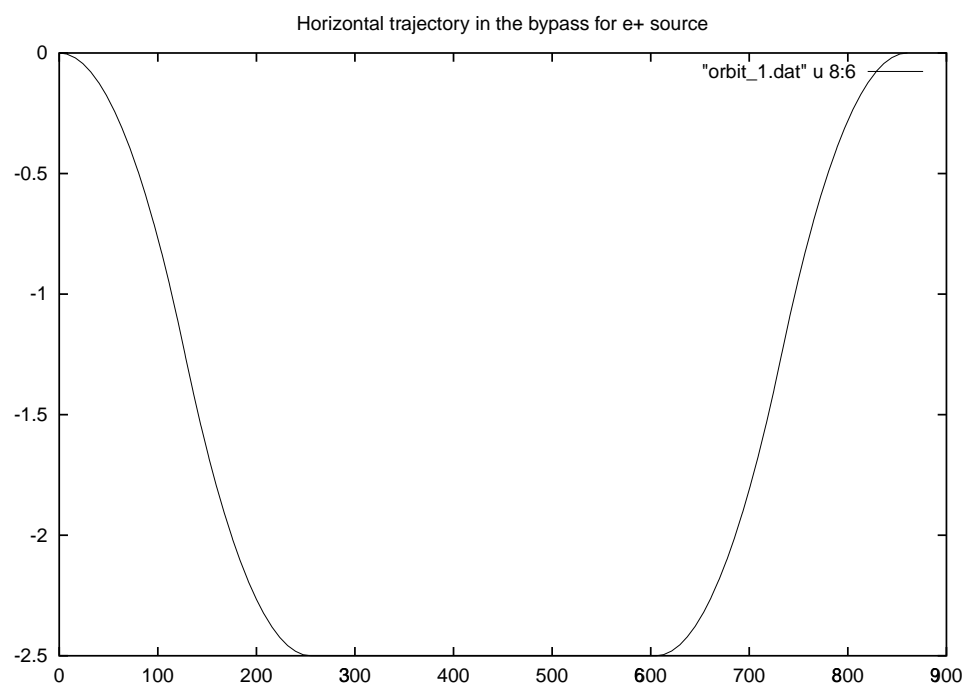

Figure 3.4.2.5: Horizontal orbit through the undulator bypass. Horizontal axis: $s[\mathrm{~m}]$. Vertical axis: Horizontal orbit displacement from linac axis $[\mathrm{m}]$.

The undulator has distributed focusing along its length using ten 24-meter FODO cells. The focusing significantly reduces the tolerances on the undulator fields and alignment. The phase advance of the FODO cells is nominally set to 72 degrees/cell so that many systematic undulator field errors naturally cancel over the length. Additional studies are needed to determine the field error limits and full alignment tolerances for the undulator sections. The external focusing also reduces the sensitivity to incoming energy errors and makes the optical aberrations of the undulator insertion much easier to control. The quadrupoles have to be aligned using beam-based alignment techniques and the nominal alignment tolerance is about $5 \mu \mathrm{m}$ for $2 \%$ vertical emittance dilution.

The undulator section is 240 meters in length, with the undulator filling 200 meters of this, which is sufficient for a simulated yield of 1.5 and an average polarization of 59\%. The installed undulator during initial 


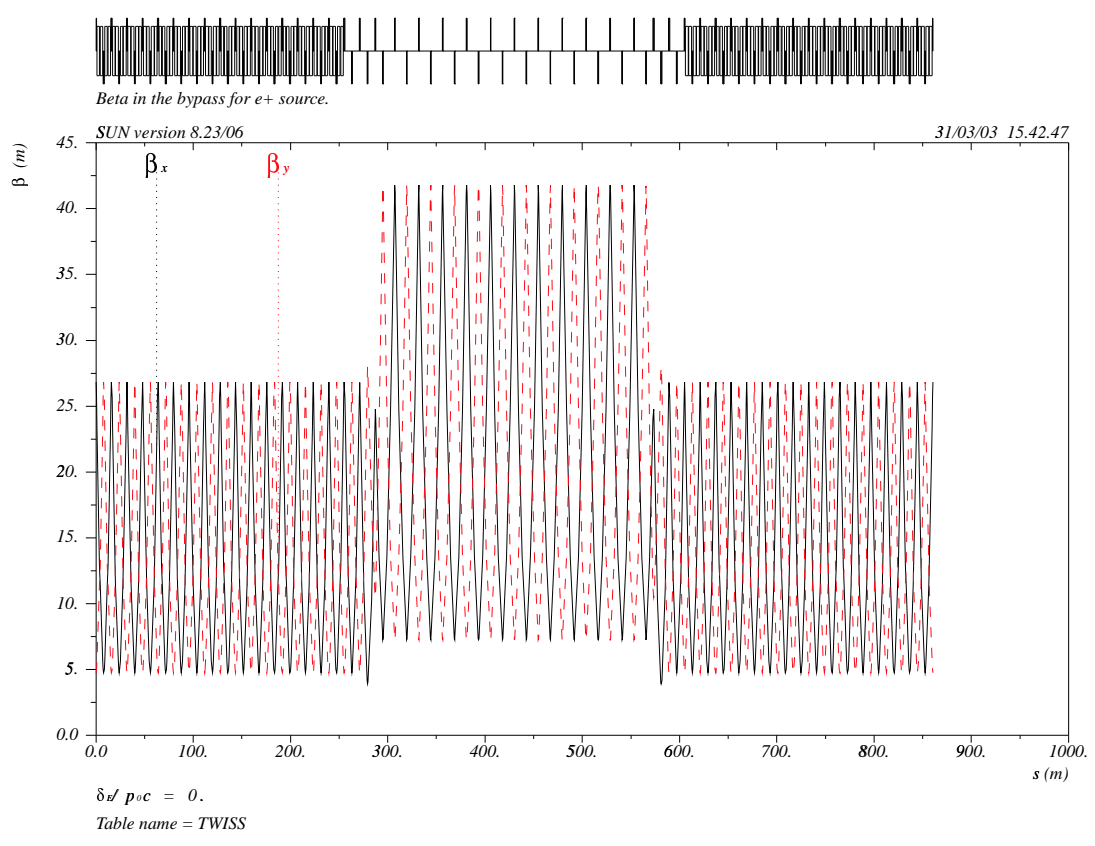

Figure 3.4.2.6: Beta functions through the undulator insertion.

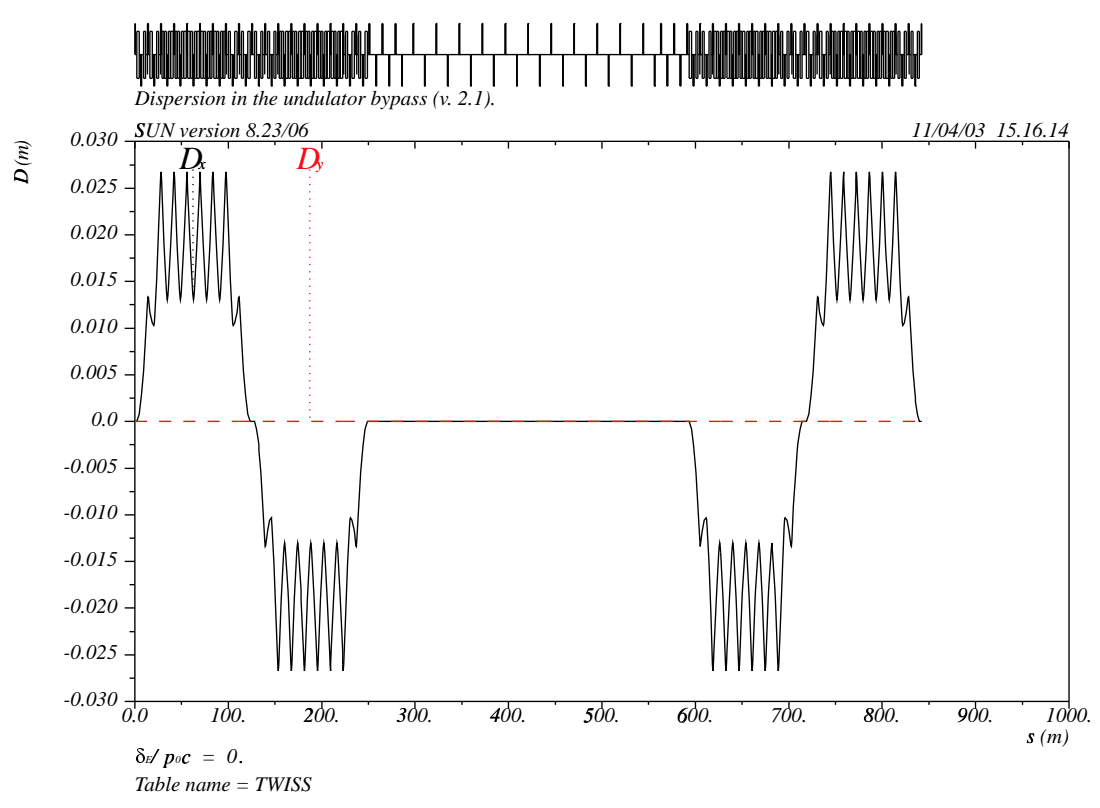

Figure 3.4.2.7: Horizontal dispersion function through the undulator insertion. 


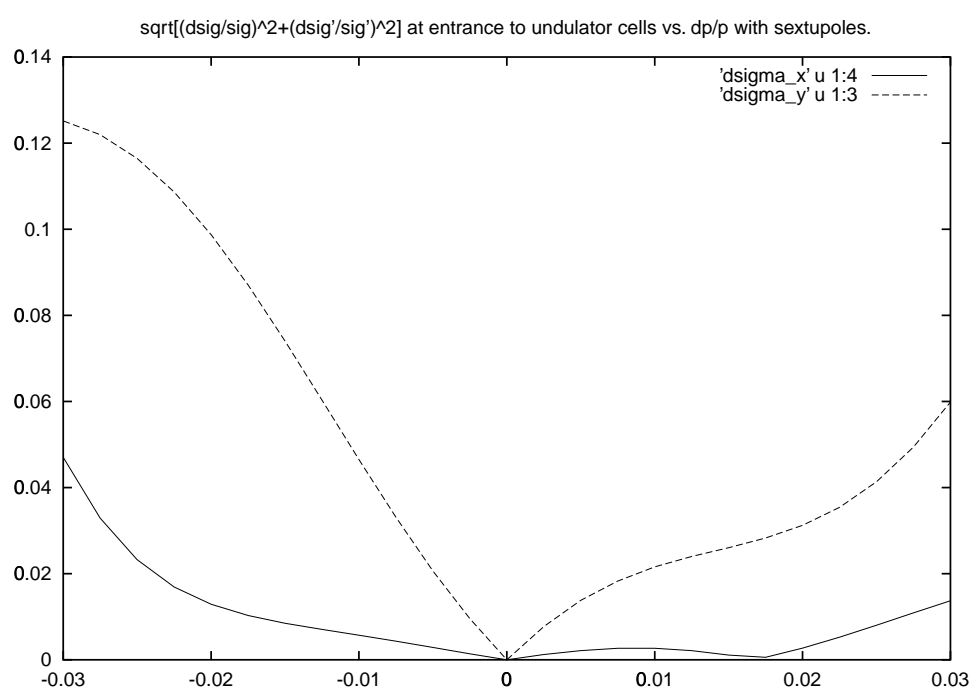

Figure 3.4.2.8: Horizontal and vertical bandpass at the end of the undulation insertion arc. Horizontal axis: $d p / p$. Vertical axis: $\sqrt{\left(\frac{d \sigma}{\sigma}\right)^{2}+\left(\frac{d \sigma^{\prime}}{\sigma^{\prime}}\right)^{2}}$, with $\sigma=$ rms beam size, $\sigma^{\prime}=$ rms beam divergence.

\section{Undulator Lattice Half Cell}

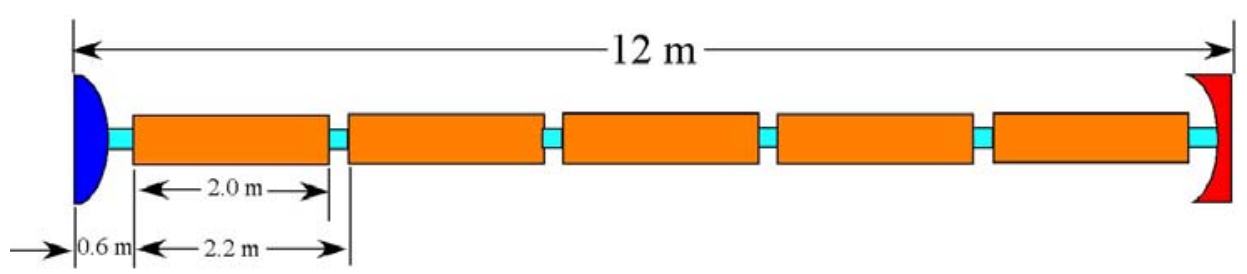

[Undulator Module Parameters: Helical, $\mathrm{K}=1, \lambda_{\mathrm{u}}=1 \mathrm{~cm}\left(\mathrm{~B}_{0}=1.07 \mathrm{~T}\right), \mathrm{ID}=6-7 \mathrm{~mm}, \mathrm{~L}_{\mathrm{u}}=2.0 \mathrm{~m}$ ]

\section{Undulator Lattice, 10 FODO Cells}

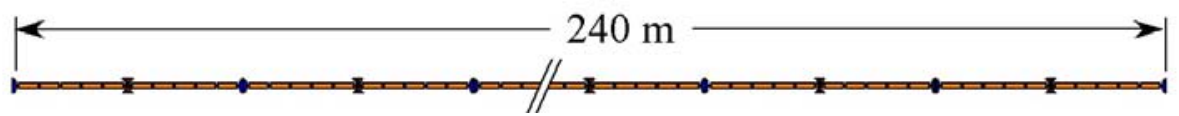

Figure 3.4.2.9: Undulator layout

operation would likely be significantly less but the space exists to install additional sections as needed to obtain an operation yield of 1 . The full undulator necessary for polarized positrons radiates roughly $6 \mathrm{GeV}$ of the beam energy and generates an energy spread of $0.004 \%$. The undulator would be constructed in 2 -meter sections with 5 sections installed between quadrupoles as illustrated in Fig. 3.4.2.9.

There are two 90 degree FODO cells at the undulator entrance for trajectory feedback control and additional diagnostics. The dispersion matching tolerance is roughly $100 \mu \mathrm{m}$ at a beta function of 40 meters to limit the vertical emittance growth to $2 \%$ in the undulator. The entire undulator insert is roughly 850 meters in length and the $e^{+}$target would be placed 200 meters downstream of the end of the undulator, close to where 
the undulator insert beam line reenters the main linac beam line.

The full undulator insert consists of 18 arc cells, 3 matching and diagnostic cells, 10 undulator cells, 3 diag/matching cell, and, finally, 18 arc cells. The full length of the system is 846 meters. At this time, the ends of the insert have not been matched into the main linac lattice; however this is expected to be straightforward and will, at most, require an additional matching cell at either end.

3.4.2.2.3 Undulator The undulator is a helical undulator with $K$ equal to 1 and a peak field of 1.1 Tesla. The advantage of the helical undulator is that it allows for polarized positrons and, for the same peak field, it radiates twice as much energy per unit length as a planar undulator [36]. A disadvantage is that a helical undulator is likely more difficult to design. To achieve the $11 \mathrm{MeV}$ photons from a $153 \mathrm{GeV}$ beam, the period of the undulator must be $1 \mathrm{~cm}$.

As discussed, the undulator is assumed to be constructed from 2-meter segments. At this time, conceptual studies have begun on helical undulator systems. The favored solution is a superconducting bifilar coil wrapped around a $6 \mathrm{~mm}$ radius beam pipe. At this time, a simple prototype has been constructed; however the field, period, and field quality requirements have not yet been attained [37]. Another option is to use a pulsed normal conducting coil [38]. This might perform adequately with the shorter pulse length of the Xband collider but pulsed heating could be a problem for the millisecond pulse length of the superconducting linac. One of the primary concerns with the bi-filar coil concept is the control of the field tolerances which requires very accurate conductor placement. Alternate technologies include permanent magnet systems where the magnet blocks are offset to generate the helicity. Here, the primary difficulty is generation of the required fields.

One disadvantage of the bifilar coil configuration is that it is not possible to make changes to the undulator helicity and hence flip the sign of the positron polarization which is important in understanding the systematic errors at the particle physics detector. Most likely the only way to enable pulse-by-pulse changes to the helicity with the undulator-based scheme is the use of separate beam lines which may introduce their own systematics - this is discussed further in Section 3.4.2.2.9.

3.4.2.2.4 Target For this study, it is assumed that the positron source target is similar to that described in the TESLA TDR, namely, 0.4 radiation lengths of titanium [TDR, Section 4.3.4]. The production rate in a titanium target is about $40 \%$ lower than in tungsten, but the higher heat capacity and the mechanical properties of titanium allow much higher particle densities inside the target. The target is rotated so that successive beam pulses do not overlap. Unlike the TESLA TDR case, a circumferential target speed of only $0.54 \mathrm{~m} / \mathrm{s}$ provides $6 \sigma$ spot size separation between pulses. The target parameters are given in Table 3.4.2.6.

Table 3.4.2.6: Positron Target Parameters

\begin{tabular}{|c|c|c|c|c|}
\hline & TESLA TDR & Unpolarized & Polarized & Conventional \\
\hline Pulse energy on target [kJ] & 26.9 & 1.1 & 1.1 & 0.5 \\
\hline Average power on target [kW] & 135 & 136 & 127 & 57 \\
\hline Spot size on target [mm] & 0.75 & 0.75 & 0.75 & 1.6 \\
\hline Target material & Ti-alloy & Ti-alloy & Ti-alloy & $\mathrm{W}_{74} \mathrm{Re}_{26}$ \\
\hline Target thickness [r.l.] & 0.4 & 0.4 & 0.4 & 4.0 \\
\hline Target energy absorption [\%] & 4 & 9 & 8 & 14 \\
\hline Target Radius [m] & 0.40 & 0.125 & 0.125 & 0.125 \\
\hline Revolution Rate [rpm] & 1200 & 46 & 46 & 46 \\
\hline Pulsed Temperature Rise $\left[{ }^{\circ} \mathrm{C}\right]$ & 420 & 422 & 371 & 189 \\
\hline Number of targets $/ \mathrm{spares}$ & $1 / 0$ & $1 / 1$ & $1 / 1$ & $3 / 1$ \\
\hline
\end{tabular}


Figure 3.4.2.10 [40] is a plot of the temperature profile in the target due to the energy deposited by a $267 \mathrm{~ns}$ photon beam pulse of $722 \mathrm{~J}$ and an average photon energy of $22.1 \mathrm{MeV}$. The peak temperature rise is $233{ }^{\circ} \mathrm{C}$ and peak induced stress is about 0.2 of the material yield strength for undamaged alpha-beta titanium alloy. If one scales these results to a higher total incident energy $(1.1 \mathrm{~kJ})$ and to higher energy absorption due to lower energy photons (10.7 MeV versus $28 \mathrm{MeV}$ for the first harmonic cutoff), the estimated single pulse temperature rise is $422{ }^{\circ} \mathrm{C}$ and the peak induced stress is 0.4 of material yield strength. The target is rotated at a velocity of about $0.6 \mathrm{~m} / \mathrm{s}$ to avoid overlap of successive beam pulses and to provide for uniform heating of the target wheel. For the present parameters, with $11 \mathrm{MeV}$ photons, the energy absorption in the target is calculated with EGS4 to be roughly $9 \%$ of the incident photon energy. Approximately $12 \mathrm{~kW}$ of absorbed power must be removed through water cooling of the target.

One outstanding question regarding the choice of target material is the susceptibility to radiation damage. There is substantial experience with radiation damage in the conventional positron targets such as that in the SLC. In the SLC positron system, a W-26\%Re alloy was selected because of the exceptional physical properties such as high strength and ductility at the anticipated target operating temperatures, between 115 and $200{ }^{\circ} \mathrm{C}$. At $150{ }^{\circ} \mathrm{C}$, ductility of these alloys increases $10 \%$ with no significant decrease in yield strength, maintaining it at $1100 \mathrm{MPa}$. However, the catastrophic changes in mechanical properties of these materials due to irradiation were not anticipated. This led to target failure at stresses that were a factor of two times smaller than expected for the undamaged materials [41]. It is expected that Titanium will exhibit similar or worse behavior after radiation damage [42] - this may be especially true if the incident photon energy is close to $\sim 20 \mathrm{MeV}$ where neutrons are produced through the giant dipole resonance [43]. The rate of radiation damage in Ti requires further study.

3.4.2.2.5 Positron Yield Positron yield is defined as the ratio of the number of captured positrons to the number of electrons transported through the undulator. The number of photons incident on the target is proportional to the number of drive electrons and to the length of the undulator. The photon energy spectrum depends upon the undulator strength parameter, $K$, the undulator period, and the square of the drive beam energy. For the systems under consideration, a strength parameter of $K=1$ and period of $1 \mathrm{~cm}$ combined with a drive beam energy of $150 \mathrm{GeV}$ produce photons in the energy range of 0-12 MeV. The rate of photon production for these parameters is essentially 1 photon per electron, per meter of undulator, for a planar device and about twice this value for a helical undulator.

The number of positrons produced and captured varies with the photon energy, the choice of target material and the acceptance of the downstream systems. In the photon energy range of 10-40 MeV, the pair production cross section rises linearly with energy but at lower energy, it rolls off. A calculation of positron emission from the converter target must include the effect of energy loss and absorption during drift to the surface. For a 0.4 r.l. thick Ti-alloy target material, the emission probability is proportional to photon energy over a range of $5-50 \mathrm{MeV}$. The resulting efficiency varies from $0-11 \%$. Below about $5 \mathrm{MeV}$ incident photon energy, very few positrons emerge from the target. For a downstream system with a phase space acceptance of 0.03 m-rad and a $40 \mathrm{MeV}$ cutoff, the capture probability of emitted positrons is in the range of $25-50 \%$.

A number of codes have been developed to simulate the production of photons in an undulator, the production of positrons in various target materials, and the subsequent capture and acceleration of the positrons generated. The results of these yield simulations are given in Table 3.4.2.5 [20] [14]. For the parameters chosen, the positron-to-electron capture yield is approximately $1 \%$ per meter of undulator for unpolarized positron production and about $0.75 \%$ per meter of undulator for polarized positron production.

3.4.2.2.6 Positron Capture Region and Pre-accelerator The capture region and the positron pre-accelerator (PPA) accelerate the positrons to $250 \mathrm{MeV}$. This system consists of a magnetic matching system (MMS) and initial accelerator structures. The MMS provides a tapered solenoidal field to match the transverse phase space from the target to the accelerator structures. It consists of a dc tapered-field solenoid producing a peak field of $1.2 \mathrm{~T}$ used in combination with a pulsed flux concentrator which acts as the phase 


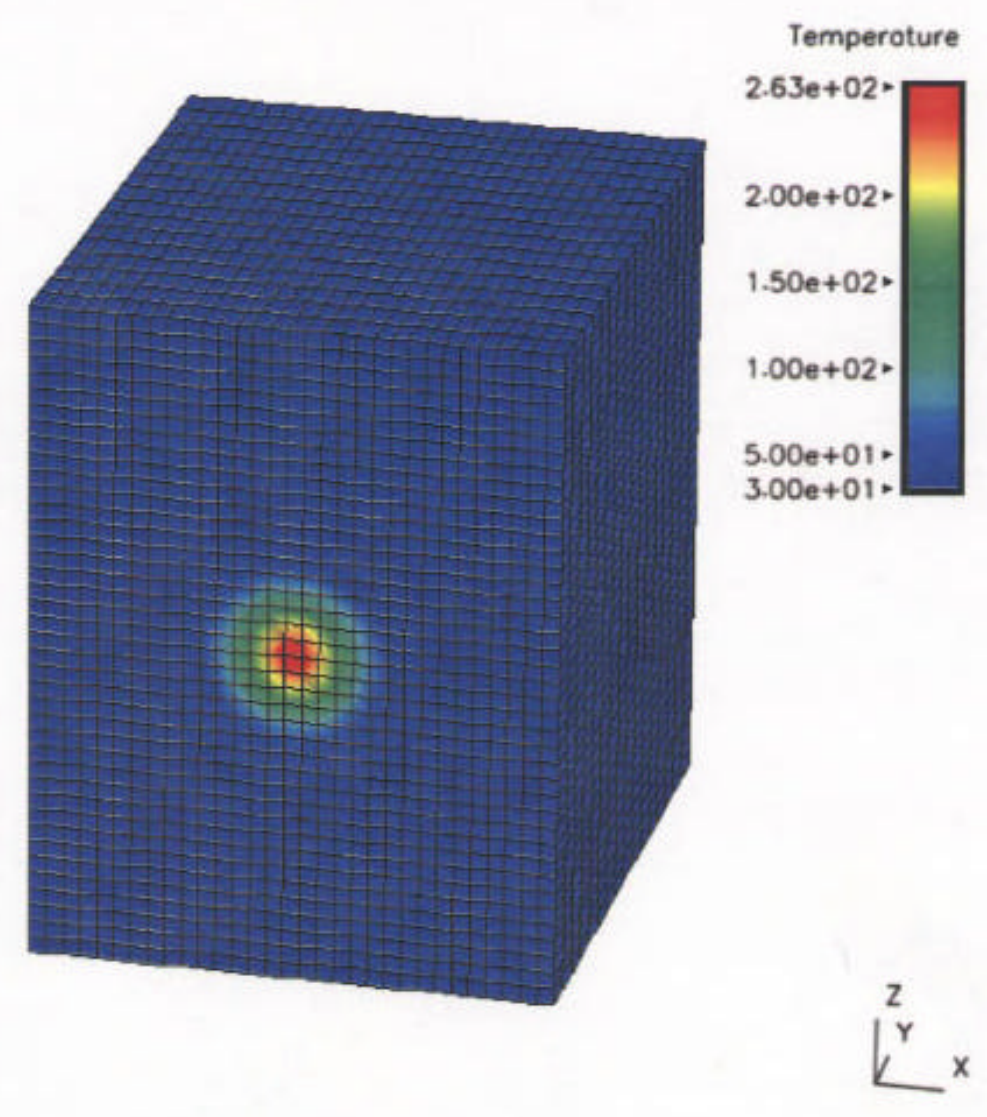

Figure 3.4.2.10: Target heating from a single pulse. Temperatures are in ${ }^{\circ} \mathrm{C}$.

space transformer, in which the magnetic field varies adiabatically from $7 \mathrm{~T}$ to $0.5 \mathrm{~T}$. A $0.5 \mathrm{~T}$ uniform-field solenoid that encloses the accelerating structures is used to provide transverse focusing in the PPA. This design is based on the flux concentrator operating at the SLC and designed for the NLC. The required peak field $(5.8 \mathrm{~T})$ and entrance aperture $(4.5 \mathrm{~mm}$ id) are both larger than in the case of the SLC (5T and $3.5 \mathrm{~mm}$ id). A prototype NLC flux concentrator is being developed.

Following the MMS is the $e^{+}$pre-accelerator which has two $75 \mathrm{MW}$ L-Band klystrons that are driven by a solid-state modulator. The SLED-I outputs of the two klystrons are combined and distributed to two 5-m-long normal conducting L-band (1428 MHz) accelerator structures operating at a loaded gradient of $24 \mathrm{MeV} / \mathrm{m}$. The combination of the two SLED outputs permits vernier control of the RF waveform for beam loading compensation, while allowing for constant power delivery to the structures during a machine protection system fault and recovery.

For redundancy, a second positron target, capture region and pre-accelerator are located immediately downstream of the first and can be activated if the first target fails. Both target plus capture regions are mounted on girders to facilitate removal and replacement. A photon beam dump is located downstream of the second capture region. The combined length of the in-line redundant target, capture and pre-acceleration systems 

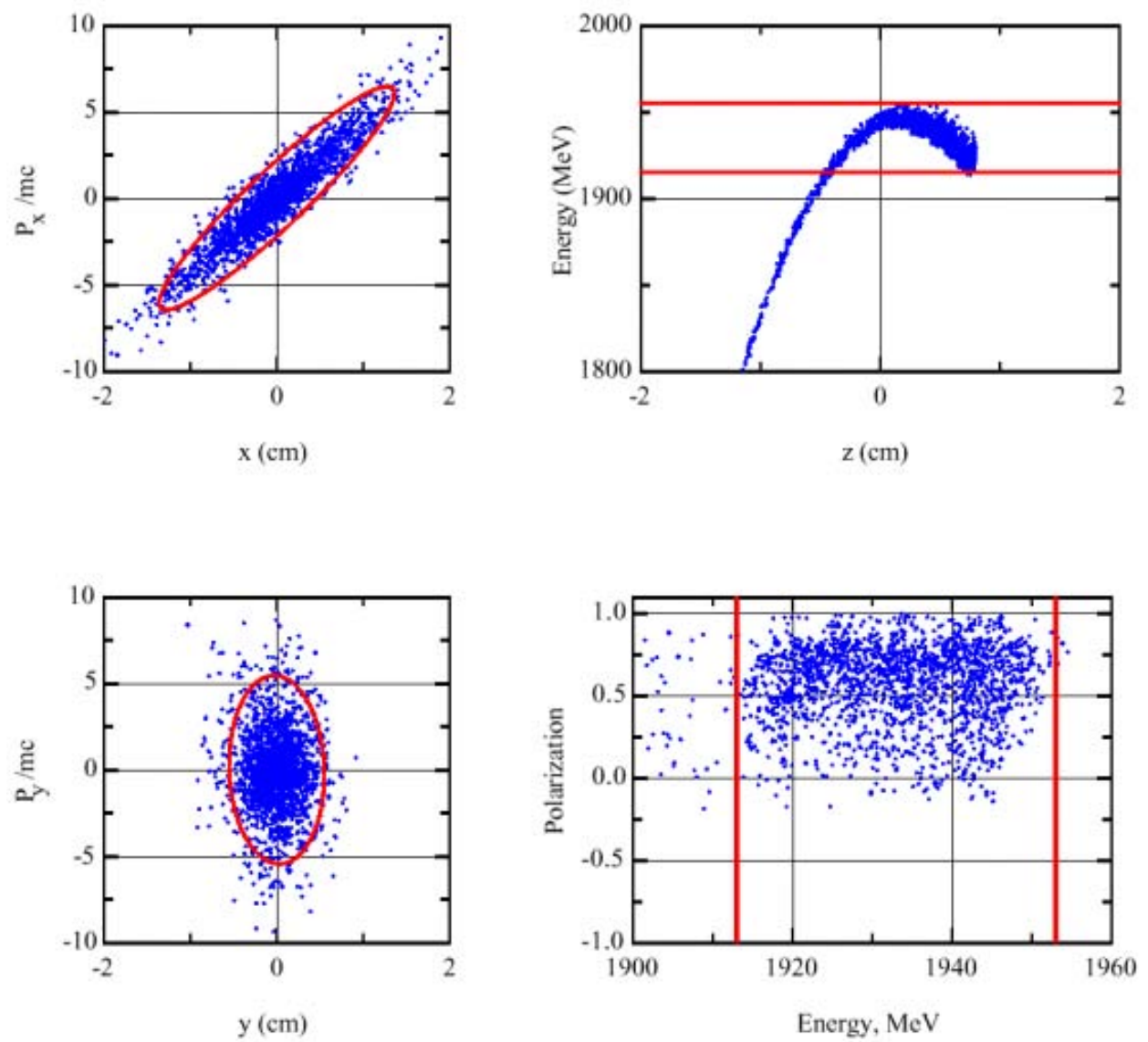

Figure 3.4.2.11: Positron capture phase space

is about 30 meters and requires 475 -MW klystrons. An electron injector is placed parallel to the positron capture sections as illustrated in Fig. 3.4.2.4.

3.4.2.2.7 Positron Booster Linac (PBL) The positron beam is then accelerated in an L-band linac from the $250 \mathrm{MeV}$ point to the damping ring energy of $1.98 \mathrm{GeV}$. The accelerator structures are 5-m-long normal conducting L-band (1428 MHz) TW disk-loaded waveguides, identical to those in the PPA, but operating at a loaded gradient of $13.2 \mathrm{MV} / \mathrm{m}$. Each RF module has two $75 \mathrm{MW}$ L-Band klystrons, driven by a solid-state modulator, whose SLED-I outputs are combined and distributed to six accelerator structures. The combination of two SLED outputs permits vernier control of the RF waveform for beam loading compensation, while allowing for constant power delivery to the structures during a machine protection system fault and recovery. The positron booster linac has $6 \mathrm{RF}$ modules, including one spare RF module for overhead (a total of 6 modulators, 12 75-MW klystrons, 12 SLED-1 assemblies and 36 5-meter accelerating structures.

Beam focusing is accomplished using electromagnet quadrupoles between each structure and one or two wrap around quadrupoles evenly spaced around each structure. Each quadrupole in-between a structure has a BPM. There are horizontal and vertical correctors between each structure and each wrap around quadrupole has corrector trim windings.

Diagnostics in the $e^{+}$booster include eight wire scanners for emittance measurements; four at the beginning and four at the end of the beamline. Also there are two beam charge toroids and 3 profile monitors. Beam 
loss is detected by PLIC.

3.4.2.2.8 High Energy Positron Transfer Line The positron transfer line takes the $1.98 \mathrm{GeV}$ positrons from the booster linac and the $1.98 \mathrm{GeV}$ diagnostic station and deflects them into the main linac tunnel. This portion of the transfer line is roughly 30 meters. After being re-injected into the electron main linac tunnel, the positron beam is transported along the tunnel, through the beam delivery system, into a parallel tunnel that crosses between the two interaction regions, and back along the positron main linac to the positron damping ring. The total length of this transfer line is about $25 \mathrm{~km}$ before the beam is injected into the positron ring. These systems are under design. Although the transfer lines are relatively simple and need to transport a large emittance beam, these systems still require attention because of the high beam power of over $60 \mathrm{~kW}$ that is being transported.

3.4.2.2.9 Polarization control, Spin rotation, Energy compressor and Damping ring injection It is likely that, to control systematic errors, the IP polarization will need to be reversed on a pulse-by-pulse basis. The polarization control system is designed to flip the helicity on a pulse-by-pulse basis while the spin rotation system is required to rotate the polarization into the vertical plane before injection into the damping ring. Likely the simplest solution is to combine these systems where first an arc of roughly 8 degrees is used to rotate the spin from the longitudinal direction into the horizontal direction and then a bipolar pulsed kicker directed the beam to one of two parallel beam lines with solenoids which rotates the spin either up or down from the horizontal plane. The solenoids require an integral field strength of $10 \mathrm{~T}-\mathrm{m}$ to rotate the spin from the horizontal plane into the vertical direction. The parallel beam lines would then be recombined using another bipolar kicker. The systematic differences between the parallel beam lines might be reduced by occasionally reversing the solenoid fields.

One additional concern is that, because the transport from the positron source to the damping ring is quite long and complicated, the precession due to any additional horizontal and vertical bending should be considered and perhaps a more flexible spin rotation system will be needed to optimize the spin before injection into the positron damping ring. This flexible spin rotation system may become relatively long and complicated [49]. Of course, not all of the solenoids need to be installed when operating with unpolarized positrons, but the beam line must be installed with the correct bending angles as changes to the beam line geometry become hard to retrofit.

Finally, an energy compressor may be desired before injection into the positron pre-damping ring. The longitudinal phase space of the positron beam from the source is highly mismatched to the pre-damping ring and the large incoming energy spread can exceed the dynamic aperture. The energy compressor would use the $R_{56}$ of the spin rotation arc to generate a correlation between energy deviation and longitudinal position and then an RF system to reduce the energy spread [50].

\subsubsection{Damping Rings}

In this section, we detail some of the technical specifications for the present designs of the warm LC damping rings. General design issues (including comparison with existing storage rings and the cold LC damping rings) are discussed in Section 3.3.2, and risks associated with the damping rings are evaluated in the risk discussion, Section 8.3.

\subsubsection{Functional and Layout Description}

The warm LC uses two identical Main Damping Rings (MDRs): one for the electron beam and one for the positron beam. In addition, a pre-damping ring (PDR) is needed for the positron beam, because the 
emittance of the incoming positrons is much larger than that of the electrons. The MDRs store three bunch trains, while the PDR stores two. At each machine cycle, a single fully damped bunch train is extracted, while a new bunch train is injected. Thus, the electron bunch trains are damped over three machine cycles, while the positron bunch trains are damped over five machine cycles.

The damping rings systems also include the transport lines that connect the rings with the sources upstream and the first bunch compressor downstream. The transport lines provide functions such as injection/extraction and spin rotation, and must provide appropriate locations for the necessary diagnostics.

Detailed parameters for the MDRs and PDR are given in Table 3.4.3.1.

Table 3.4.3.1: Parameters for main damping rings and the pre-damping ring.

\begin{tabular}{|lcc|}
\hline \hline & MDR & PPDR \\
\hline Circumference $[\mathrm{m}]$ & 299.792 & 230.933 \\
Energy $[\mathrm{GeV}]$ & 1.98 & 1.98 \\
Maximum current $[\mathrm{A}]$ & 0.8 & 0.75 \\
Maximum repetition rate $[\mathrm{Hz}]$ & 120 & 120 \\
Bunch trains $\times$ bunches per train & $3 \times 192$ & $2 \times 192$ \\
Train / bunch separation [ns] & $65 / 1.4$ & $117 / 1.4$ \\
$\nu_{x}, \nu_{y}, \nu_{s}$ & $21.15,10.35,0.0118$ & $12.15,6.19,0.0098$ \\
Injected normalized emittance $[\mu \mathrm{m} \cdot \mathrm{rad}]$ & $150(\mathrm{rms})$ & $45,000(\mathrm{edge})$ \\
Normalized natural emittance $[\mu \mathrm{m} \cdot \mathrm{rad}]$ & 2.37 & 46 \\
$\gamma \epsilon_{y}$ equilib. $[\mu \mathrm{m} \cdot \mathrm{rad}]$ & 0.01 & 4.6 \\
$\sigma_{\Delta E / E}, \sigma_{z}$ & $0.098 \%, 5.5 \mathrm{~mm}$ & $0.089 \%, 5.1 \mathrm{~mm}$ \\
$\xi_{x}$ uncorr., $\xi_{y}$ uncorr. & $-30.7,-28.8$ & $-26.3,-15.6$ \\
$\tau_{x}, \tau_{y}, \tau_{\epsilon}[\mathrm{ms}]$ & $3.63,4.08,2.18$ & $3.5,3.8,2.0$ \\
Energy loss per turn from wigglers $[\mathrm{kV} / \mathrm{turn}]$ & 834 & 519 \\
Total energy loss per turn $U_{s r}[\mathrm{kV} / \mathrm{turn}]$ & 970 & 803 \\
$\alpha_{p}$ & $1.39 \times 10^{-3}$ & $1.69 \times 10^{-3}$ \\
$V_{R F}$ & $2.0 \mathrm{MV}, 714 \mathrm{MHz}$ & $1.7 \mathrm{MV}, 714 \mathrm{MHz}$ \\
RF acceptance & $1.5 \%$ & $1.5 \%$ \\
Lattice & $32 \mathrm{TME}$ cells & $10 \mathrm{DBA}$ cells \\
\hline \hline
\end{tabular}

Although a centralized injector complex could be considered for some potential sites (at an additional cost), the layout chosen for the reference design assumes that the separated electron and positron injectors are built at the low energy ends of the main linacs, separated by $\sim 30 \mathrm{~km}$. The injectors are at the same level as the main linacs. The beams from the sources start in the same direction as the main linac, and are bent back toward the bunch compression systems by the damping rings. The PDR has approximate ten-fold symmetry, with injection and extraction in different straights. The MDRs have two-fold symmetry, with injection and extraction in opposite straights, and these rings therefore reverse the direction of the beam.

3.4.3.1.1 Electron Damping Ring System The electron beams are transported from the S-Band booster to the main damping ring through optics that rotate the spin of the electrons' polarization into the vertical plane, so that the electrons do not depolarize in the ring. This linac-to-ring optics system also reduces the energy spread of the incoming beam, using an S-band compressor structure[50]. The trains are injected into the downstream end of the FODO (non-wiggler) straight using an achromatic kicker system.

In the electron main damping ring (EMDR), the vertical emittance of the beam is reduced by a factor of 3000 and the horizontal emittance is reduced by a factor of 50. The vertical damping time is $4.1 \mathrm{~ms}$. Damping occurs dominantly from radiation produced in $62 \mathrm{~m}$ of wiggler (divided between two opposite straight sections in the lattice), but also in the dipoles in the Theoretical Minimum Emittance cells in the four arc sectors. The wiggler has peak field $2.15 \mathrm{~T}$ and period $0.27 \mathrm{~m}$. There are three bunch trains inside 
the ring at any one time, which allows each train to be damped for three inter-pulse periods (25 ms). The circumference of the main ring is $300 \mathrm{~m}$, with an aperture radius of $20 \mathrm{~mm}$, except in the wiggler section, where the aperture radius is $8 \mathrm{~mm}$. A $1 \mathrm{MW}, 714 \mathrm{MHz}$ RF system using five PEP-II style damped cavities generates total gap voltages of up to $2.5 \mathrm{MV}$ to replace the lost beam energy. Injection and extraction of the trains take place simultaneously to reduce beam-loading effects in the RF cavities for the trains still in the ring. The bunch length at extraction is $5.5 \mathrm{~mm}$. A chicane is incorporated in one of the non-wiggler straights for circumference adjustment up to $\pm 2 \mathrm{~mm}$.

After the EMDR the beam is transported to and passes through the spin rotator, which uses solenoid pairs separated by a horizontal arc to allow an arbitrary orientation of the polarization. The solenoids are optically separated to cancel coupling and avoid increasing the vertical emittance. Figure 3.4.3.1 shows the layout of the electron damping ring complex.

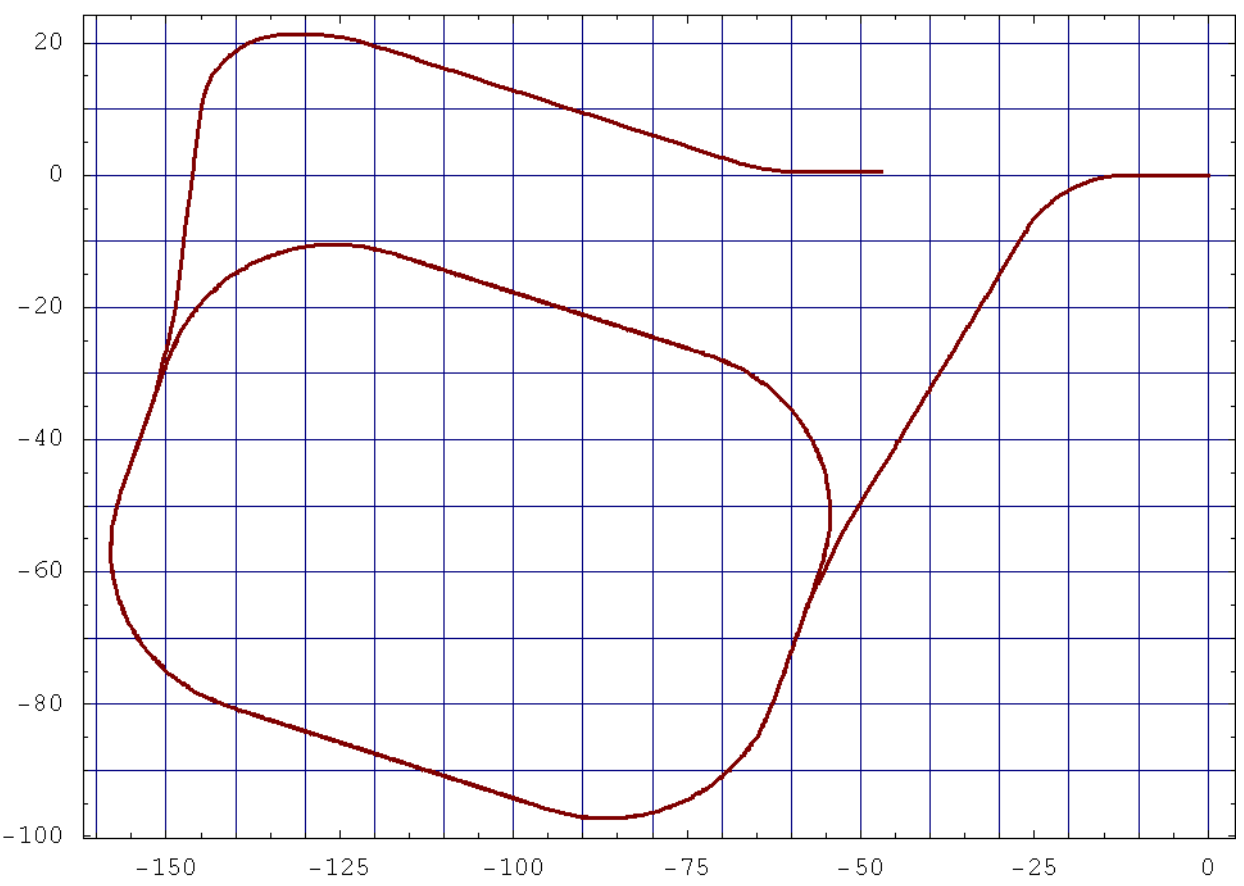

Figure 3.4.3.1: Layout of the electron damping ring complex. Units are meters.

3.4.3.1.2 Positron Damping Ring System The positron beams from the L-Band booster linac are transported to a pre-damping ring through optics that are identical to those of the electrons, except that polarization spin control solenoids and polarimeter are omitted and the energy compressor is L-band. The positron beamline does allow for future installation of spin rotation equipment if needed.

The PPDR (Positron pre-damping ring) is necessary due to the large emittances of the positron beam. There are two beam trains in the PPDR at a time and each train is pre-damped for two machine cycles (16.6 ms). There are $50 \mathrm{~m}$ of wiggler, with peak field $1.4 \mathrm{~T}$ and period $1.0 \mathrm{~m}$. Four $714 \mathrm{~Hz}$ PEP-II style damped RF cavities are utilized in the PPDR to maintain the beam energy. The circumference of the PPDR is $230 \mathrm{~m}$, with an aperture radius of $32 \mathrm{~mm}$. After initial damping in the PPDR, the positrons are extracted, and injected into the positron main damping ring (PMDR).

The PMDR is identical in layout and function to the EMDR, but the input parameters are slightly different due to the fact that the beam has been through the PPDR. After the PMDR the beam is transported to 
the PBC1 beamline (first bunch compressor). The Positron DR System does not incorporate a spin rotator system, but space is left for one to be installed in the future to allow an upgrade to polarized positrons. Figure 3.4.3.2 shows the layout of the positron damping ring complex.

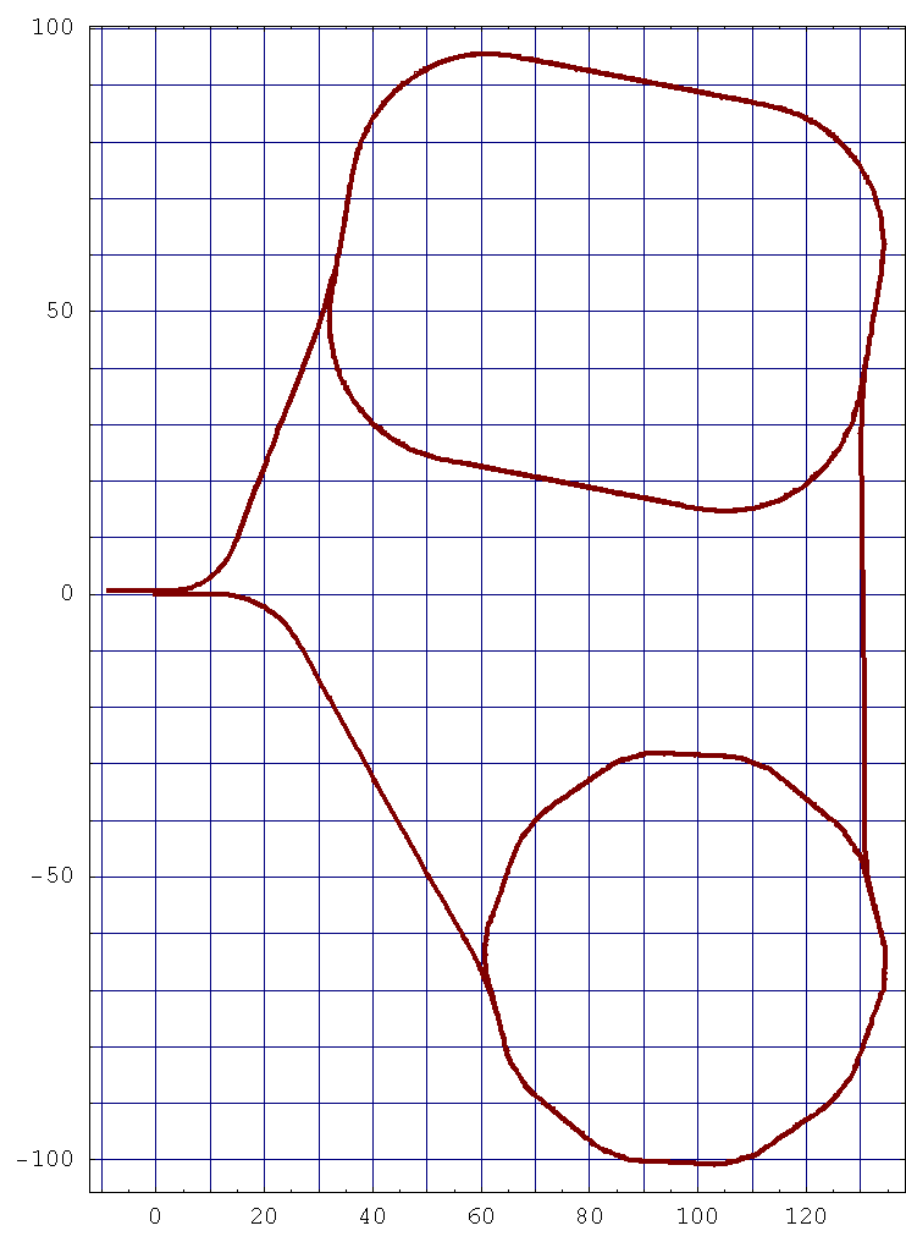

Figure 3.4.3.2: Layout of the positron damping ring complex. Units are meters.

\subsubsection{Main Damping Rings}

3.4.3.2.1 Overview The NLC main damping rings are $300 \mathrm{~m}$ in circumference and they measure roughly $80 \mathrm{~m}$ by $100 \mathrm{~m}$ with a nominal energy of $1.98 \mathrm{GeV}$. The main damping rings are designed to damp beams with injected emittances $\gamma \epsilon_{x, y}=1.5 \times 10^{-4} \mathrm{~m} \cdot \mathrm{rad}$ to give extracted beam emittances of $\gamma \epsilon_{x}=3 \times 10^{-6} \mathrm{~m} \cdot \mathrm{rad}$ and $\gamma \epsilon_{y}=2 \times 10^{-8} \mathrm{~m} \cdot \mathrm{rad}$. The rings operate at $120 \mathrm{~Hz}$. They provide sufficient damping to decrease the injected vertical emittance by four orders of magnitude. The arcs in the main damping ring are based on detuned Theoretical Minimum Emittance (TME) cells, which were chosen because of efficiency in generating low emittance. The chromaticity is corrected with two families of sextupoles and the dynamic aperture is more than sufficient to ensure lossless injection. The damping is dominated by the $62 \mathrm{~m}$ of wiggler.

The dynamic aperture, including effects of errors, is predicted to be close to 15 times the injected beam size. Potential limitations due to the contribution from the wiggler magnet have been studied. Analytical 
expressions of arbitrary three-dimensional wiggler fields have been developed, and tracking including the non-linear components of the wiggler field will be used to determine the minimum pole width requirement for the $2.15 \mathrm{~T}$ hybrid wiggler magnet. Preliminary analysis indicates that the wiggler with a magnet pole width of $11 \mathrm{~cm}$ does not seriously impact the dynamic aperture [25].

The rings operate with three trains of 192 bunches spaced by 1.4 ns or 96 bunches spaced by 2.8 ns. The bunch trains are injected onto and extracted from the closed orbit using pulsed kickers and DC septa. The bunch trains are separated by 65 ns to allow for the rise and fall times of the injection and extraction kickers. To avoid coupled-bunch instabilities the RF cavities use higher-order-mode damping, based on the PEP-II design, and a transverse bunch-by-bunch feedback system. As stated, the rings are designed to operate with maximum bunch charges of $1.6 \times 10^{10}$ particles; this is roughly $10 \%$ more than the maximum needed at the IP with a $2.8 \mathrm{~ns}$ bunch spacing.

Finally, because the rings must generate extremely small beam emittances, there are tight jitter and alignment tolerances. Extensive effort has been made to include cancellations and tuning procedures in the design that will ease the tolerances to reasonable levels. Quadrupoles and sextupoles have independent trim control, and magnet movers are used for orbit and coupling correction. BPMs having $<0.3 \mu \mathrm{m}$ resolution for the stored orbit are located at every quadrupole. In addition, a synchrotron radiation port is used for bunch length and initial beam size measurements and, a laser wire, like the system commissioned at the ATF at KEK, will likely be used to measure the damped beam. There is a 4-wire parasitic emittance measurement as well as energy and energy spread diagnostics located in the extraction line before the spin rotator and first stage bunch compressor. Additional emittance, energy spread and bunch length diagnostics are located in and after the first stage bunch compressor.

3.4.3.2.2 Lattice The lattice is constructed from four arcs with seven full TME (theoretical minimum emittance) cells per arc, with an additional two cells for matching into the dispersion-free straight sections. Two opposite straights contain wiggler sections, and the remaining two straights contain the injection and extraction systems, RF cavities, and circumference correction chicane. The total circumference of the lattice is a little less than $300 \mathrm{~m}$. The footprint is shown in Figure 3.4.3.3. Lattice functions in different parts of the lattice are shown in Figure 3.4.3.4 through Figure 3.4.3.9.

3.4.3.2.2.1 Design margins The challenges in construction and operation of the damping ring make it prudent to include some margin between the specified performance and the ideal performance of a machine constructed and operating precisely as designed. In some cases, the performance specifications already include some margin. For example, the injected beam is assumed to have a normalized emittance of $150 \mu \mathrm{m}$, which allows a $50 \%$ margin for beam jitter on top of the nominal $100 \mu \mathrm{m}$ emittance of the beam coming from the source. Some margins (e.g. in damping time) are easy to quantify, while others (e.g. in dynamic aperture) are not. In Table 3.4.3.2 we attempt to identify those parameters that include some margin, indicate the reason for or benefit of the margin, and quantify the margin where possible.

\subsection{Technical components}

3.4.3.2.3.1 RF System An RF voltage of $2.0 \mathrm{MV}$ is required to achieve a momentum acceptance of $\pm 1.5 \%$. Each cavity is capable of providing up to $0.5 \mathrm{MV}$, so in principle four cavities could be sufficient. However, this allows no overhead, and it might be necessary, for example, to compensate intensity-dependent bunch lengthening effects by increasing the RF voltage. We have therefore designed the lattice to accommodate five cavities. Each cavity requires approximately 1 meter of free space [22]. To avoid transients arising from variations in beam loading during injection/extraction, the cavities must be placed downstream of the injection kicker and upstream of the extraction kicker (in the present design they are positioned just before 


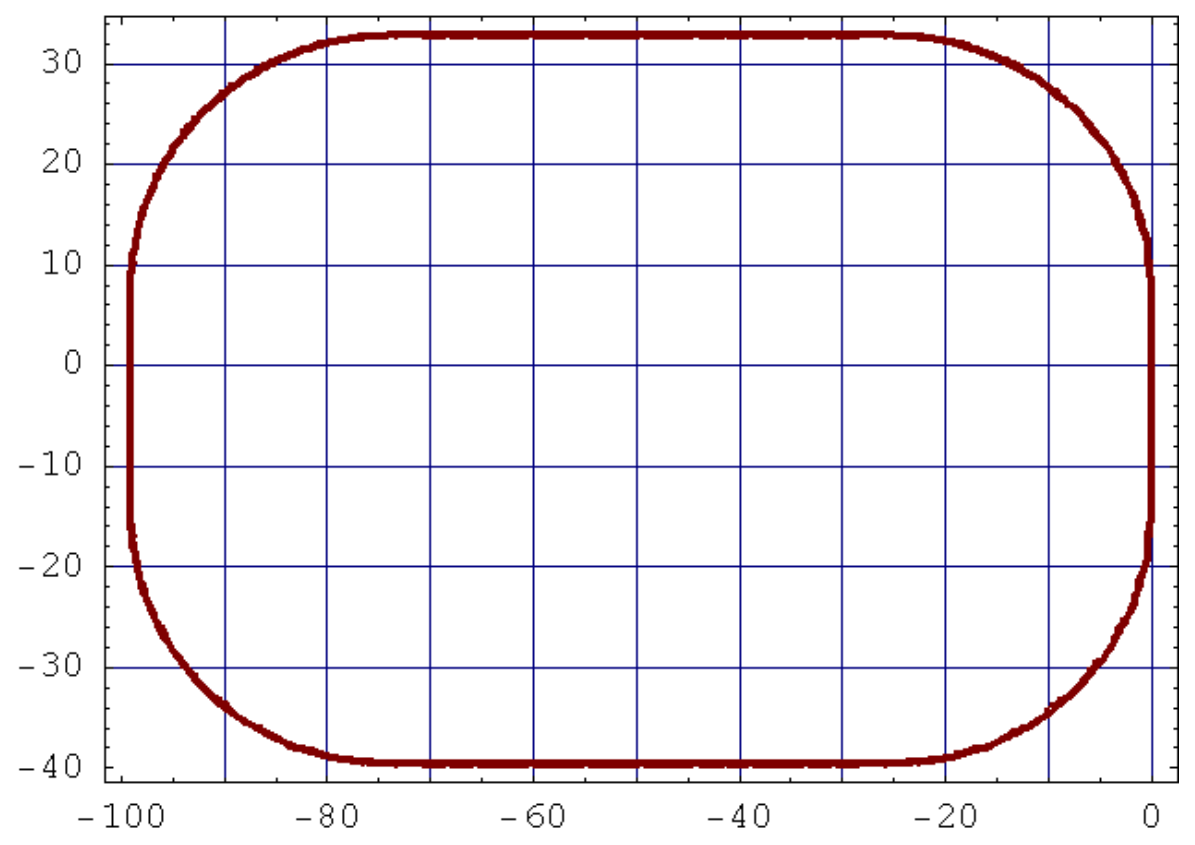

Figure 3.4.3.3: Footprint of the NLC MDR lattice. Units are meters. The long straight sections contain the wiggler, and the shorter sections contain the injection and extraction systems, RF cavities and circumference correction chicane.

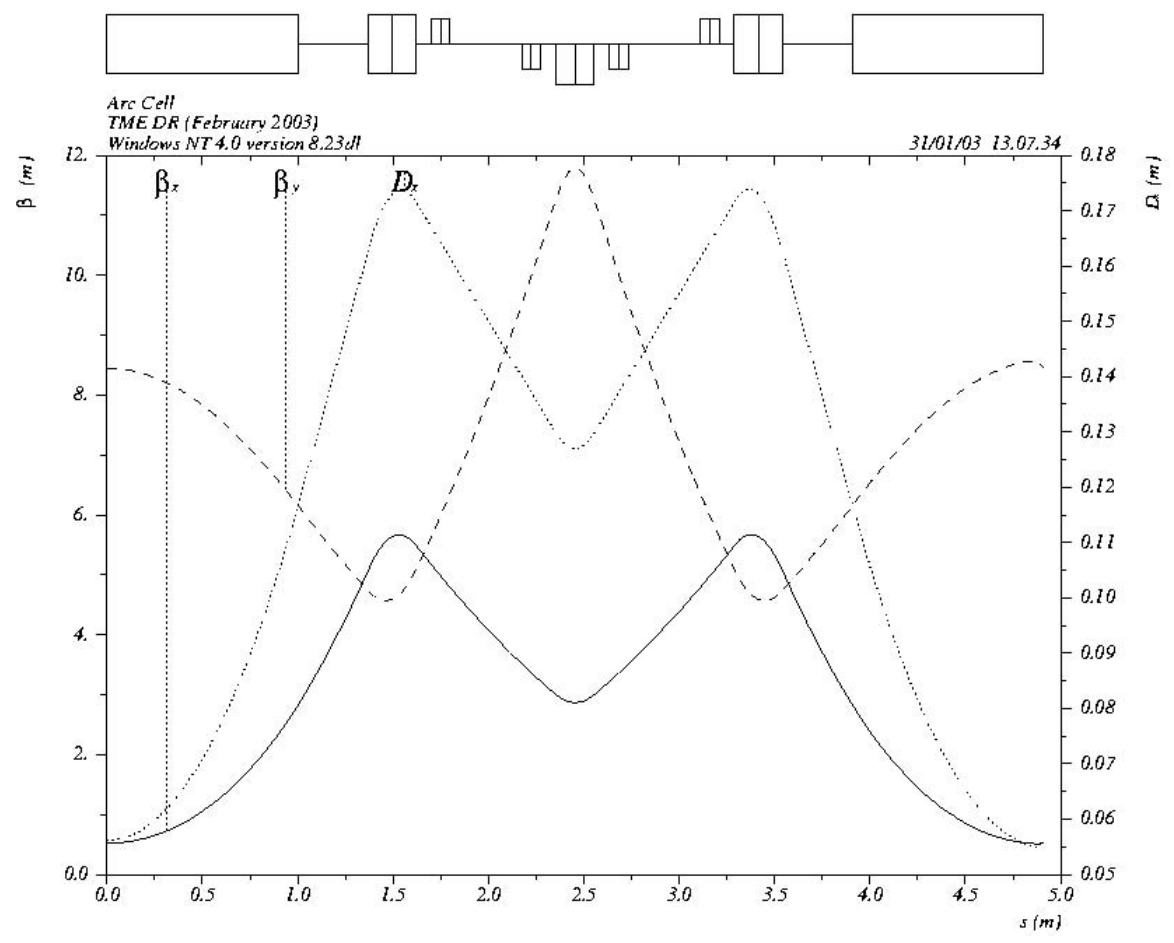

Figure 3.4.3.4: Lattice function in one arc cell. 


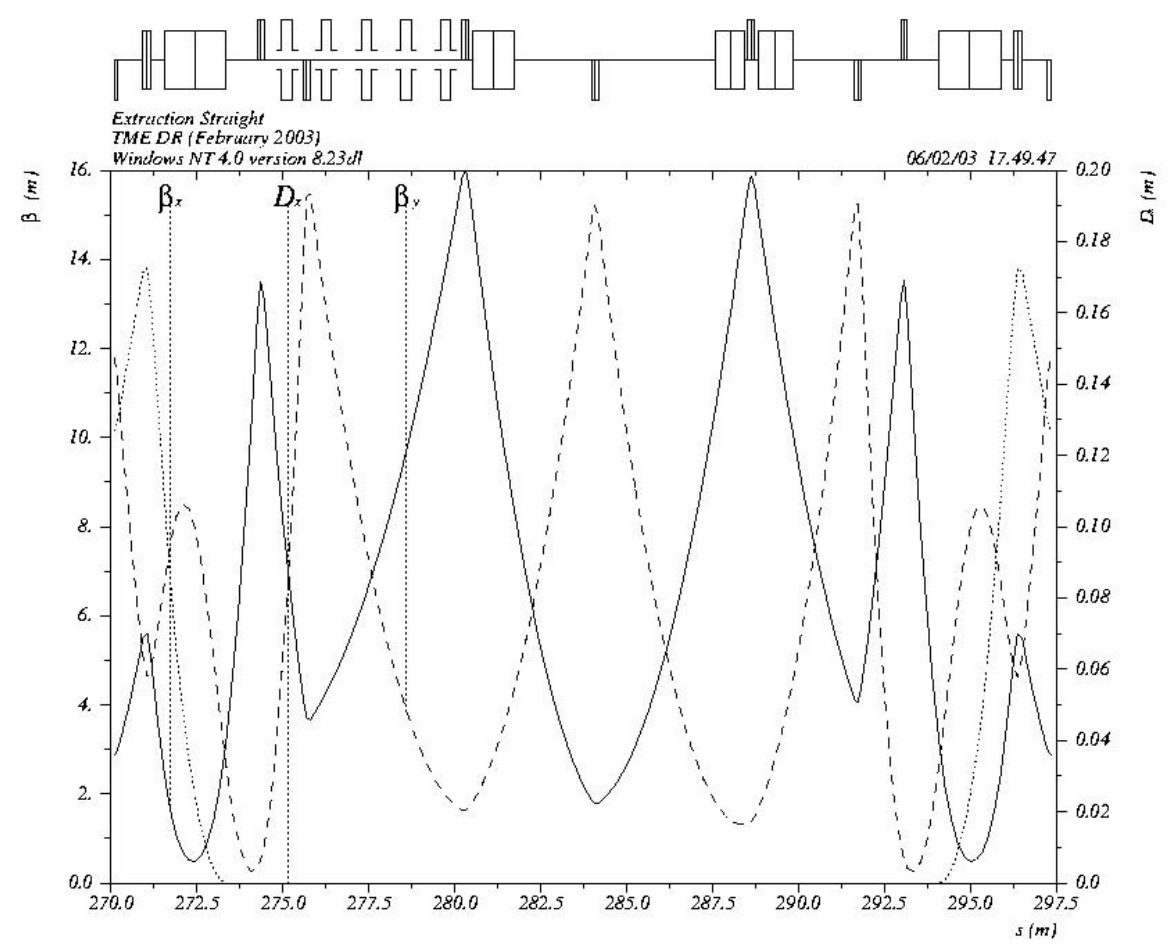

Figure 3.4.3.5: Lattice functions in the RF and extraction straight.

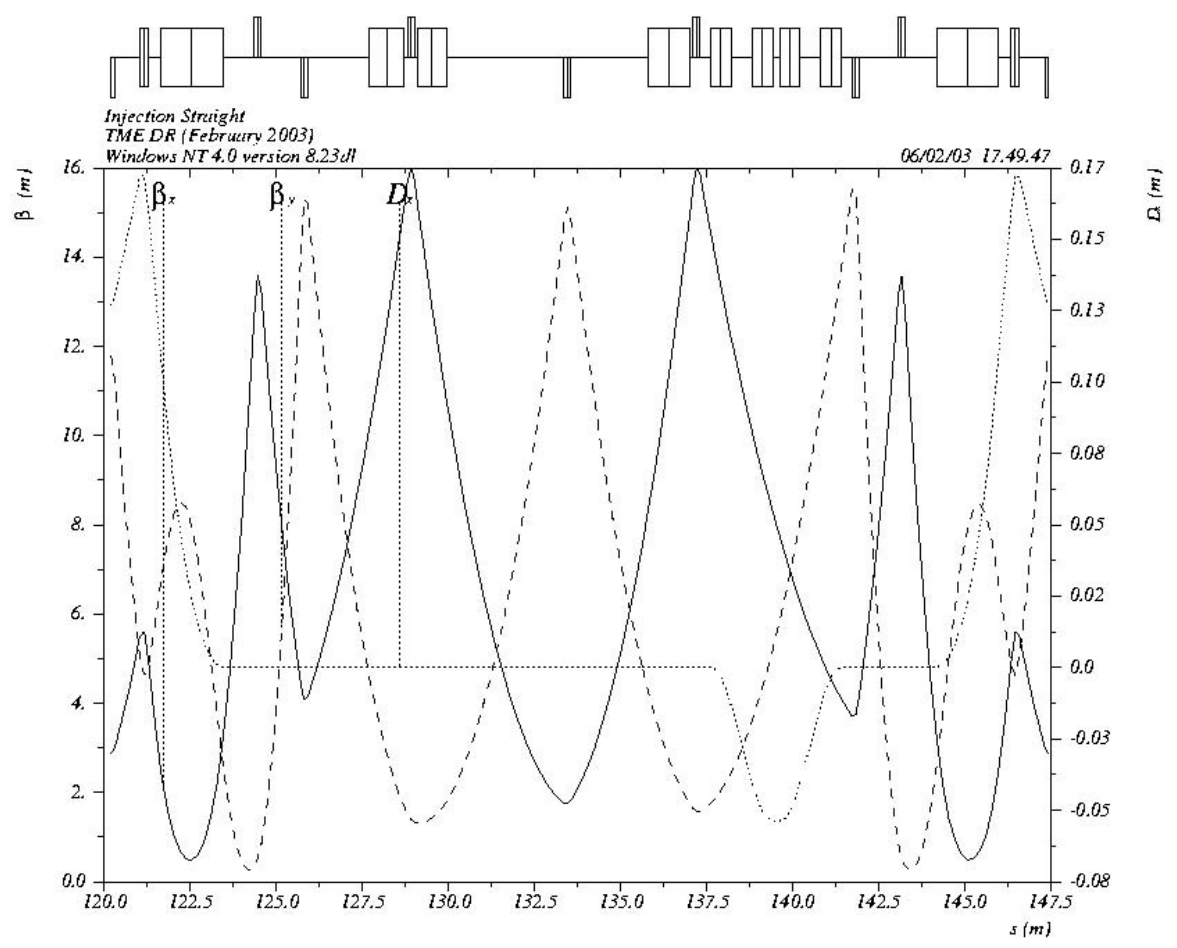

Figure 3.4.3.6: Lattice functions in the injection and chicane straight. 


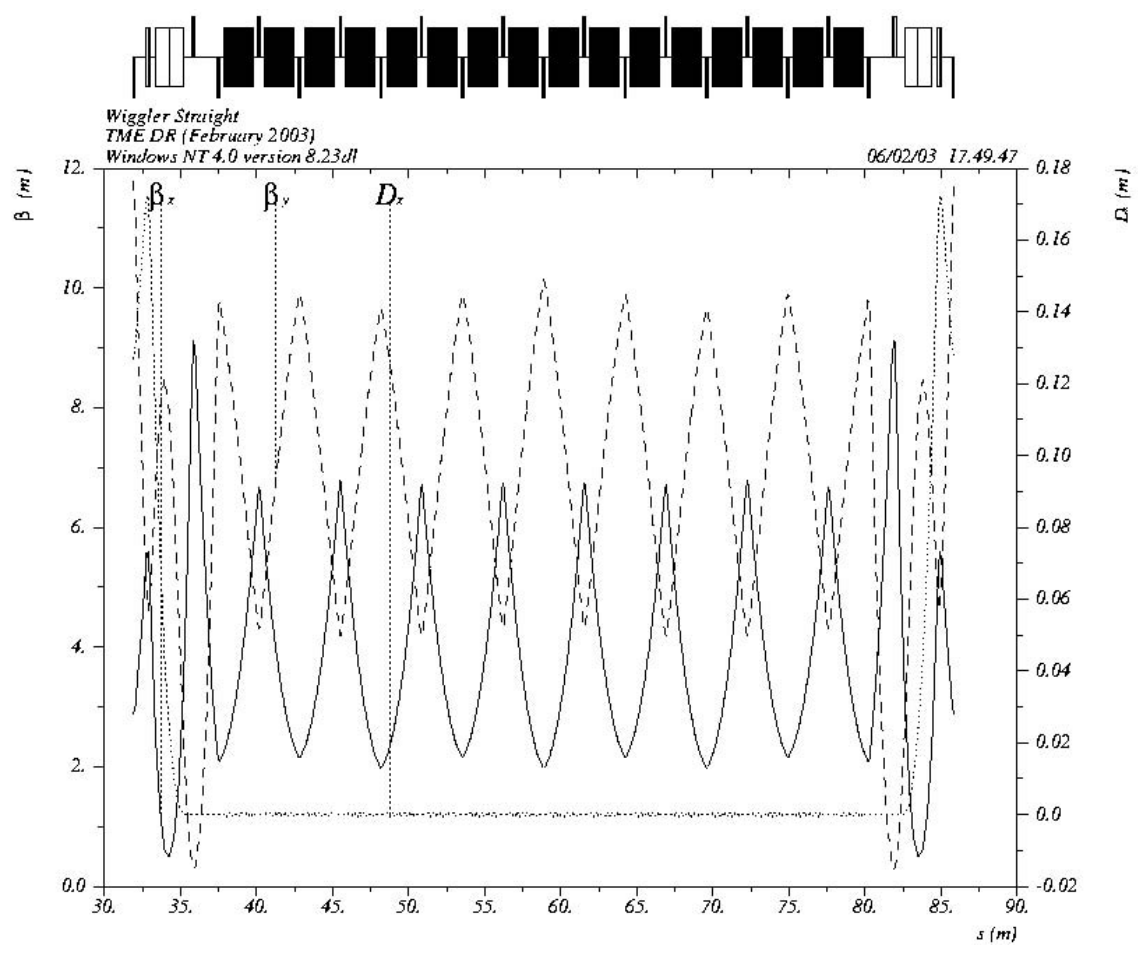

Figure 3.4.3.7: Lattice functions in the wiggler straight.

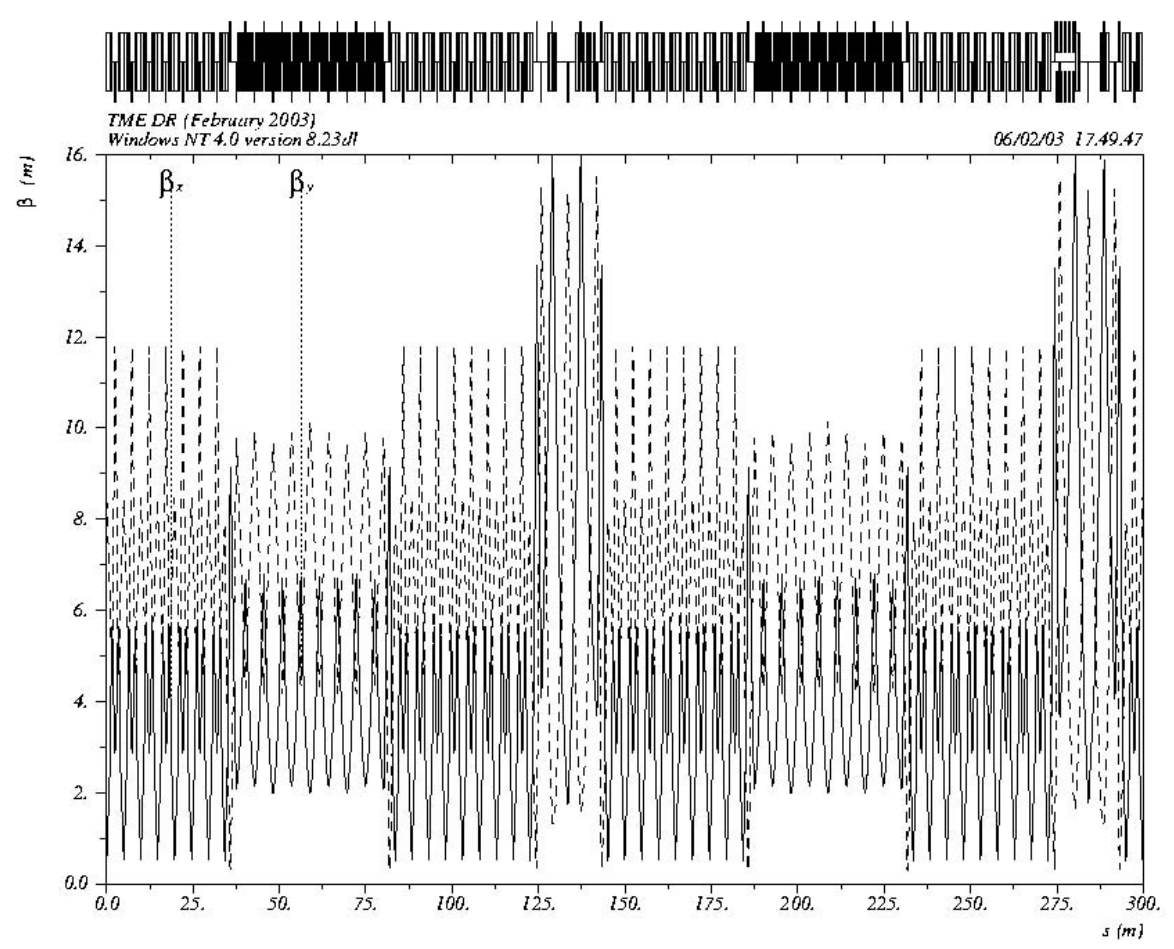

Figure 3.4.3.8: Beta functions in the full lattice. 


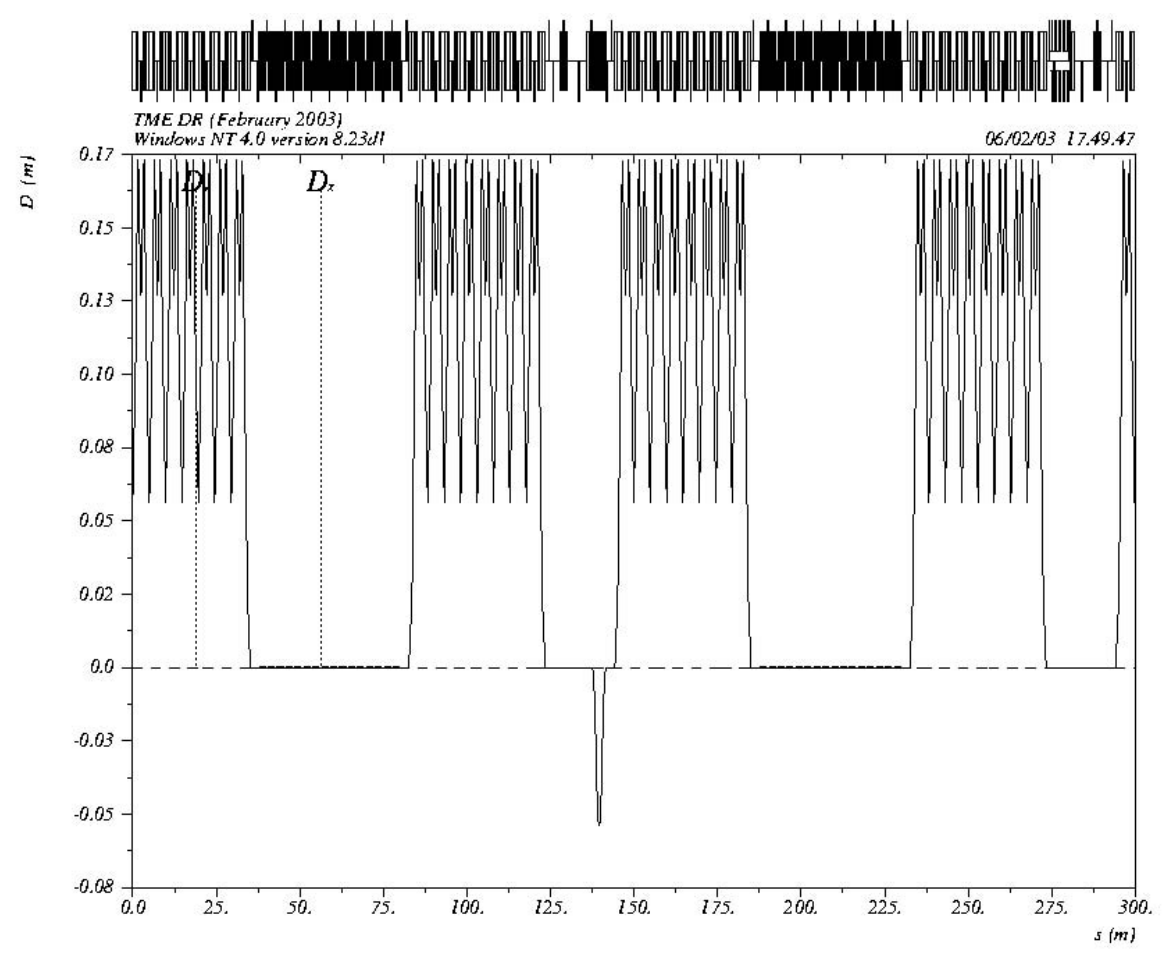

Figure 3.4.3.9: Dispersion function in the full lattice.

Table 3.4.3.2: Summary of margins between nominal and design parameter values.

\begin{tabular}{|l|c|c|c|}
\hline \hline Quantity & $\begin{array}{c}\text { Nominal or } \\
\text { Required Value }\end{array}$ & Design Value & Margin allowed for \\
\hline \hline Injected emittance & $100 \mu \mathrm{m}$ & $150 \mu \mathrm{m}$ & Injection jitter, filamentation \\
\hline Horizontal damping time & $5 \mathrm{~ms}$ & $3.63 \mathrm{~ms}$ & Variation in wiggler field \\
\hline Natural emittance & $3.0 \mu \mathrm{m}$ & $2.36 \mu \mathrm{m}$ & Tuning errors, collective effects \\
\hline Vertical damping time & $5 \mathrm{~ms}$ & $4.08 \mathrm{~ms}$ & Relaxation of coupling requirement \\
\hline $\begin{array}{l}\text { Equilibrium normalized } \\
\text { vertical emittance }\end{array}$ & $0.013 \mu \mathrm{m}$ & $<0.019 \mu \mathrm{m}$ & \begin{tabular}{c} 
Relaxation of alignment tolerances \\
\hline Energy spread
\end{tabular} \\
\hline Bunch length & $<0.1 \%$ & $0.0975 \%$ & $\begin{array}{c}\text { Almost no margin for collective effects } \\
\text { but low impact }\end{array}$ \\
\hline Maximum RF Voltage & $2.0 \mathrm{MV}$ & $2.5 \mathrm{MV}$ & $\begin{array}{c}\text { Increased RF acceptance, } \\
\text { control of bunch length }\end{array}$ \\
\hline Circumference & $298.95 \mathrm{~m}$ & $299.79 \mathrm{~m}$ & Additional 1 ns (!) kicker rise/fall time \\
\hline $\begin{array}{l}\text { Dynamic aperture } \\
\text { (injected beam size) }\end{array}$ & few $\times$ & $15 \times$ & $\begin{array}{c}\text { Magnet errors, tuning errors, } \\
\text { linearity of phase space }\end{array}$ \\
\hline Energy acceptance & $2 \%$ full width & $2 \%$ full width & No margin \\
\hline $\begin{array}{l}\text { Magnet (not wiggler) } \\
\text { pole-tip radius }\end{array}$ & $20 \mathrm{~mm}$ & $24 \mathrm{~mm}$ & \begin{tabular}{c} 
Increase in vacuum pipe aperture \\
\hline \hline
\end{tabular} \\
\hline
\end{tabular}


the extraction kicker). In this case, no transients are induced as long as injection occurs simultaneously with extraction.

At present, the cavities are separated by $2.25 \mathrm{RF}$ wavelengths, that allows pairs of cavities to be powered by a single klystron, with the power divided in a "magic T". Thus, there are several possible configurations, using between three and five klystrons; since it is also possible to power three cavities from a single klystron, it may be possible to use just two klystrons. The optimal configuration will need further study, and we simply note at present that using one klystron for each cavity provides the greatest flexibility, and provides some overhead, allowing the ring to continue operation (albeit with impaired performance) in the case of any one klystron failing.

3.4.3.2.3.2 Magnet System The wiggler sections are all hybrid in design, utilizing vanadium permandur poles and $\mathrm{NdFeB}$ pole magnets, except for the electromagnet trim coils on the end half poles. The parameters of the dipoles, quadrupoles, sextupoles, kickers and septa are given in Table 3.4.3.3. All are tunable. The use of permanent magnet hybrids is being considered. The limiting factors are the radiation level and its effect on rare earth type permanent magnets and the required size of magnet if ferrite is necessary. Thus, the decision is electro- vs. rare earth hybrid vs. ferrite hybrid magnet. The same tradeoffs are made in the transport lines. The dipoles, quadrupoles and sextupoles are powered in strings with one large power supply per string. Each quadrupole and sextupole also has an individual low power trim supply.

Table 3.4.3.3: Parameters of main magnets in the NLC MDR lattice. Quantities are for one ring.

\begin{tabular}{|c|c|c|c|c|}
\hline Dipoles & Quantity & $\begin{array}{l}\text { Length, } \\
\text { mag. (m) }\end{array}$ & $\begin{array}{l}\text { Pole-tip } \\
\text { Field (T) }\end{array}$ & $\begin{array}{c}\text { Pole-tip } \\
\text { Radius }(\mathrm{mm})\end{array}$ \\
\hline Arc & 28 & 2.0 & 0.6575 & 25 \\
\hline Matching & 8 & 1.8 & 0.3653 & 25 \\
\hline Chicane & 4 & 0.6 & 0.9844 & 25 \\
\hline \multicolumn{5}{|l|}{ Quadrupoles } \\
\hline Arc QF & 56 & 0.25 & -0.7353 & 25 \\
\hline Arc QD & 32 & 0.25 & 0.6932 & 25 \\
\hline Arc match & 8 & 0.25 & 0.7599 & 25 \\
\hline Injection straight & 6 & 0.20 & $0.30-0.77$ & 25 \\
\hline Injection straight & 1 & 0.20 & 0.6840 & 60 \\
\hline Extraction straight & 6 & 0.20 & $0.28-0.77$ & 25 \\
\hline Extraction straight & 1 & 0.20 & 0.7217 & 60 \\
\hline Wiggler straight & 20 & 0.15 & $-0.20,0.53$ & 25 \\
\hline Wiggler match & 4 & 0.25 & 0.6739 & 25 \\
\hline Wiggler match & 12 & 0.15 & $-0.20,0.77$ & 25 \\
\hline \multicolumn{5}{|l|}{ Sextupoles } \\
\hline $\mathrm{SF}$ & 64 & 0.25 & 0.3400 & 25 \\
\hline $\mathrm{SD}$ & 64 & 0.25 & -0.3249 & 25 \\
\hline $\begin{array}{l}\text { Injection/ } \\
\text { Extraction }\end{array}$ & & & $\begin{array}{c}\text { Bend } \\
\text { Angle (rad) }\end{array}$ & \\
\hline Kicker & 2 & 1.2 & 0.0025 & \\
\hline Septum & 2 & 0.83 & 0.025 & \\
\hline Septum & 2 & 1.00 & 0.090 & \\
\hline
\end{tabular}

3.4.3.2.3.3 Wiggler The damping wiggler is a hybrid type with nominal peak field $2.15 \mathrm{~T}$ and period $0.27 \mathrm{~m}$. A magnetic design has been produced, and a field map for the periodic sections is available. An 
analysis of the beam dynamics in the wiggler has been performed[12] and the lattice design and parameters include an estimate of the vertical and horizontal focusing, integrated field strength, and octupole component. The wiggler parameters are given in Table 3.4.3.4.

Table 3.4.3.4: Wiggler parameters for main damping rings.

\begin{tabular}{|l|c|}
\hline \hline Nominal peak field & $2.15 \mathrm{~T}$ \\
Period & $0.27 \mathrm{~m}$ \\
Full vertical aperture & $18 \mathrm{~mm}$ \\
Wiggler parameter $\left(K_{w}\right)$ & 38.3 \\
Periods/section + ends & $6.5+2 \times 0.5$ \\
Section length & $1.924 \mathrm{~m}$ \\
Total wiggler length & $61.568 \mathrm{~m}$ \\
\hline \hline
\end{tabular}

3.4.3.2.3.4 Vacuum System The beam pipe has a circular internal cross-section, with radius $21 \mathrm{~mm}$. This allows a $3 \mathrm{~mm}$ minimum wall thickness, and $1 \mathrm{~mm}$ clearance between the chamber and the $25 \mathrm{~mm}$ pole-tip radius magnets. In the wiggler section, the radius reduces to $8 \mathrm{~mm}$. There is an antechamber extending fully round the ring to extract the synchrotron radiation.

In the electron damping ring, the vacuum pressure should be below 1 nTorr, to avoid the fast ion instability. In the positron damping ring, it is possible that a slightly higher vacuum pressure might be tolerated. The vacuum chamber in the positron ring will be coated throughout with a material with low secondary electron yield (e.g. conditioned titanium nitride) to prevent build-up of electron cloud. It is possible that weak solenoids might also be used where space permits.

Most if not all the chambers are aluminum for ease of fabrication, to reduce costs, and for machine protection issues. Antechambers are designed into the chambers to accept the fans of synchrotron radiation in the damping rings, and many photon stops are required to remove the radiated power. The antechamber allows isolation of the desorbed gas load and photoelectrons from the beam aperture, and the isolated cooled photon stops reduce thermal expansion in the vacuum chamber. An ion pump takes the outgassing load at each stop. Additionally, distributed pumping is required along the length of each wiggler section, and Ti sublimation pumps are designed to go into the antechambers along both sides of the wiggler.

3.4.3.2.3.5 Injection and Extraction The injection/extraction components have been positioned according to space constraints and the required phase advances. Each system consists of a kicker $(1.2 \mathrm{~m}$ length with a kick angle of $2.5 \mathrm{mrad})$ and a pair of septa $(0.83 \mathrm{~m}, 25 \mathrm{mrad}$ with a thin blade and $1.0 \mathrm{~m}, 90 \mathrm{mrad}$ with a thick blade). The phase advance between the kicker and the septa is close to 0.25 horizontally, so that the horizontal momentum change from the kicker is converted into a large transverse displacement at the first septum.

The extraction geometry is shown in Figure 3.4.3.10. Assuming a half-aperture in the zero-field region of the first septum of $20 \mathrm{~mm}$ (the same as in the arcs) and a blade thickness of $5 \mathrm{~mm}$, there is a clearance of $2.5 \mathrm{~mm}$ between the trajectory of the injected/extracted beam and the septum blade. This is a rather smaller clearance than desirable. The injected/extracted beam passes through two quadrupoles. At Q4E it passes between $7 \mathrm{~mm}$ and $8 \mathrm{~mm}$ off-axis, receiving a kick of $3.2 \mathrm{mrad}$ in the same direction as that from the kicker. At Q5E, located between the septa in the scheme shown, it passes between 45 and $50 \mathrm{~mm}$ off-axis, receiving a kick of $17.6 \mathrm{mrad}$ in the opposite direction to that from the kicker. This magnet needs a pole-tip radius of around $60 \mathrm{~mm}$, which at its nominal strength would give a pole-tip field of $0.72 \mathrm{~T}$. Overall, the engineering design of the injection/extraction regions (bearing in mind the need for a low impedance vacuum chamber) is likely to present some challenges, though it might be possible to modify the septum lengths and fields to 
make things easier. The present septum parameters are based on those of the operating SLC damping rings, but the NLC versions specify thicker blades and about a factor of two lower fields.

The apertures through the zero-field regions of the septa are assumed to be the same as the aperture in the arcs to avoid the need for transitions that may add to the impedance. Engineering studies are needed to determine the best configuration.

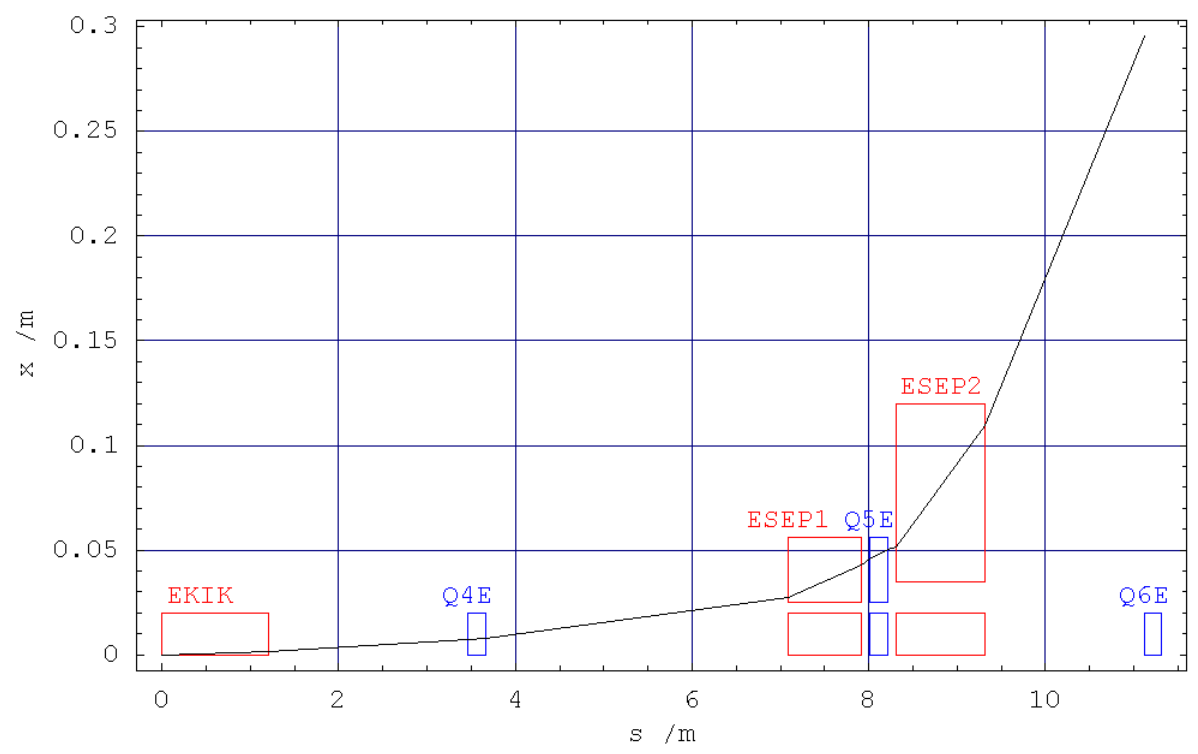

Figure 3.4.3.10: Geometry of the extraction system. The rectangles represent the nominal length and aperture of the quadrupoles, extraction kicker and septa. The first septum applies a 25 mrad kick and has a $5 \mathrm{~mm}$ blade thickness. The second septum applies a $90 \mathrm{mrad}$ kick, and has a $15 \mathrm{~mm}$ blade thickness.

The rise/fall time of the kickers is assumed to be $65 \mathrm{~ns}$, which is slightly less than the gap between bunch trains, though it is to be expected that the following stored train will see some of the falling edge of each kicker.

3.4.3.2.3.6 Circumference Correction Chicane A chicane provides the possibility of making small corrections to the circumference without the need to adjust the RF frequency. The specified correction range, based on observations of existing rings, is $\pm 2 \mathrm{~mm}$, and the chicane included in the present lattice design meets this specification. The length of the chicane is $3.80 \mathrm{~m}$, which allows it to fit in a $4.42 \mathrm{~m}$ drift section of the injection/extraction straight, with a drift of $0.31 \mathrm{~m}$ between the faces of the outside dipoles and the nearest quadrupoles. Each of the four chicane magnets has an effective length of $0.6 \mathrm{~m}$ at the nominal (half maximum) field strength of $0.492 \mathrm{~T}$.

3.4.3.2.3.7 Diagnostics and Correction Tuning studies for the previous version of the Main Damping Ring focused on vertical orbit and coupling correction. Good performance was achieved in simulations using a system specified as follows.

- Every quadrupole and sextupole magnet is supported on a mover, allowing independent positioning control of each magnet.

- The resolution of each mover is $0.1 \mu \mathrm{m}$ (relative positioning). 
- A BPM is located at each quadrupole.

- The required BPM resolution is $<5 \mu \mathrm{m}$. $0.5 \mu \mathrm{m}$ should be achievable with averaging, for dispersion measurement.

The Damping Ring Systems also requires instrumentation to allow measurement of the beam spot size in the rings and extracted emittance.

3.4.3.2.3.8 Installation Magnets, vacuum chambers, pumps, and associated components are to be assembled on girders and aligned outside the tunnel to reduce the installation time inside the tunnel.

\subsubsection{Positron Pre-damping ring}

3.4.3.3.1 Overview The pre-damping ring is $230 \mathrm{~m}$ in circumference and has 10 dispersion-free straight sections for injection, extraction, RF, circumference correction chicanes, and damping wigglers; the latter have a total length of about $50 \mathrm{~m}$. The ring has a radius of 40 meters. It stores two bunch trains which are separated by more than $100 \mathrm{~ns}$ to allow for the rise and fall times of the injection and extraction kickers. These kickers must provide larger deflections than those in the main damping rings. To minimize RF transients during injection and extraction, a new bunch train is injected one half turn after a train is extracted. In addition, the RF cavities are placed downstream of the injection kicker and upstream of the extraction kicker so that the injection/extraction process does not interrupt the beam current seen by the cavities.

The positron pre-damping ring is designed to damp the large emittance beam from the positron source to an emittance of less than $\gamma \epsilon_{x, y}=1.5 \times 10^{-4} \mathrm{~m} \cdot \mathrm{rad}$; the parameters are summarized in Table 3.4.3.1. The extracted positrons are then injected into the main damping ring where they are damped to the desired final emittances. The pre-damping ring allows the large aperture requirements for the incoming positron beams to be decoupled from the final emittance requirements of the linear collider.

The magnets and vacuum systems are designed to provide sufficient aperture to accept a 2-GeV beam with an edge emittance of $\gamma \epsilon_{x, y}=0.03 \mathrm{~m} \cdot \mathrm{rad}$ and momentum spread of $|d p / p|=1.5 \%$ plus betatron-action jitter of $\Delta \gamma J_{x, y}=0.015 \mathrm{~m} \cdot \mathrm{rad}$ for misalignments and missteering; this provides a substantial margin for injection and internal mismatches. In addition, the injector specifications allow significant overhead for injection losses into the pre-damping ring. The pre-damping ring is designed to operate with a maximum bunch charge that is roughly $20 \%$ greater than the maximum required at the IP.

Like the main damping rings, quadrupoles and sextupoles have independent control, and magnet movers are used for orbit correction. BPMs are located at every quadrupole and have a resolution better than $15 \mu \mathrm{m}$ for a single turn and $5 \mu \mathrm{m}$ after averaging the stored orbit. In addition, a synchrotron radiation port is used for bunch length and beam size measurements. There are beam size as well as energy and energy spread diagnostics located in the transfer line to the main damping ring.

3.4.3.3.2 Lattice The lattice uses a 10-fold symmetric double-bend achromat (DBA) structure. This structure allows separation of the different components, so that the damping wiggler, injection/extraction systems, RF cavities and chicane are all placed in separate straights; this greatly assists lattice design, since the different cells can be optimized independently, and should also ease engineering constraints, for example in locating photon stops to absorb the synchrotron radiation from the dipoles and wiggler.

The lattice consists of ten double bend achromats (DBA). The main parameters of the lattice are given in Table 3.4.3.1; The basic double-bend achromat structure of a single cell is shown in Figure 3.4.3.11. The dispersion is matched to zero at either end of the achromat. The wiggler generates only small amounts of residual dispersion. The achromat uses two quadrupole doublets, and three chromatic sextupoles. In addition, two harmonic sextupoles are located outside either end of the achromat. The beta functions are 


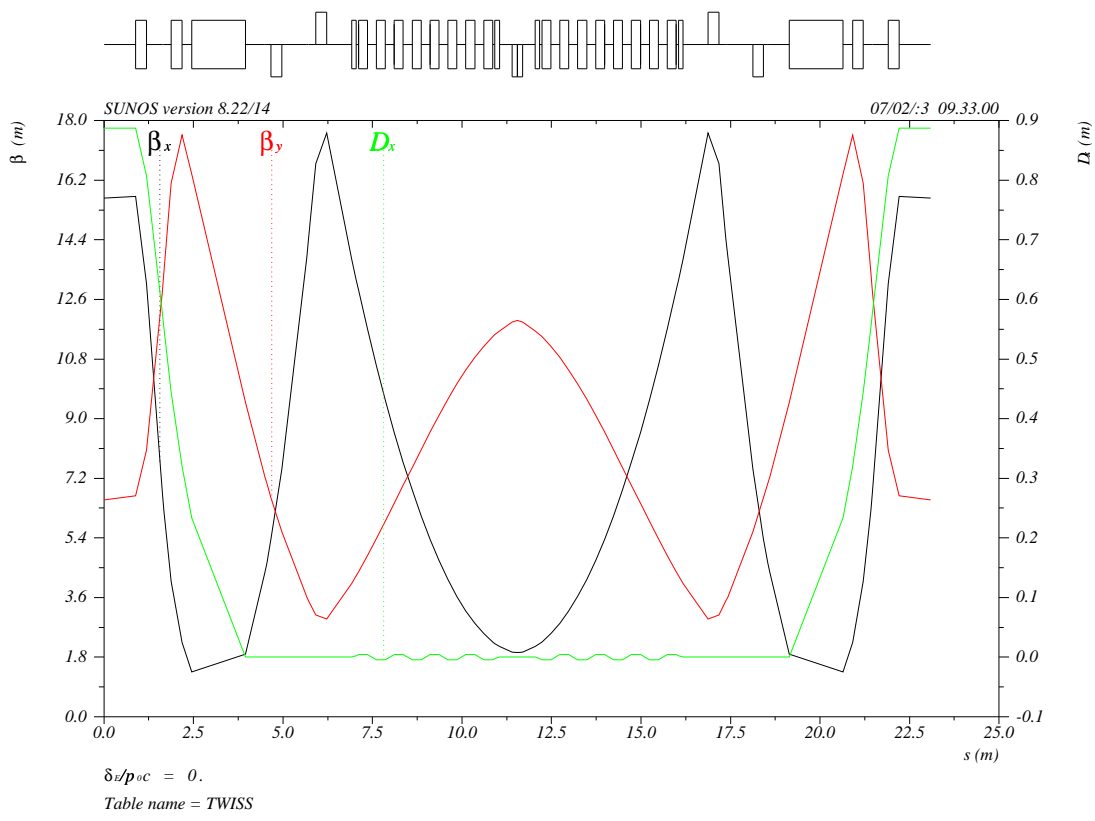

Figure 3.4.3.11: Lattice functions in one wiggler cell.

moderate, below $18 \mathrm{~m}$ horizontally and vertically throughout the cell. All quadrupoles in the achromat are horizontally offset by $1.5 \mathrm{~mm}$.

Other types of cell are constructed for the RF cavities (Figure 3.4.3.12), chicane (Figure 3.4.3.13), injection (Figure 3.4.3.14) and extraction systems (Figure 3.4.3.15). Note that the structure and lattice functions in these different types of cell are very similar. The beta functions are slightly larger than in the wiggler cell, but still below $23 \mathrm{~m}$. The geometry of the lattice is simplified by having the cells the same length, and the spacing of the dipoles the same in each case; thus, the layout is a regular decagon.

3.4.3.3.3 RF cell There is more than sufficient space for four RF cavities. The RF system requires that the distance between the centerlines of the cavities is a whole number plus three-quarters of an RF wavelength. Adjacent cavities are separated by 3.75 wavelengths allowing pairs of cavities to be fed from a single klystron via a "magic T". It is assumed at present that the cavities are the same design as used in the main damping rings [21, 22], being HOM-damped structures based on the PEP-II design. Studies of instabilities driven by modes in the cavities have yet to be carried out for the pre-damping ring, though we note that in the case of the main damping rings, only a few modes transverse and longitudinally are above the damping threshold [23], and growth rates for these modes can readily be dealt with by feedback systems.

3.4.3.3.4 Chicanes The present design uses two separate chicanes, each being identical in design to that used in the main damping ring, and allowing adjustment of the circumference of $\pm 2 \mathrm{~mm}$ each. Thus, the total circumference adjustment range is $\pm 4 \mathrm{~mm}$. The use of the same system in the pre-damping ring as in the main damping ring in itself brings some benefits. Using two chicanes rather than one eases the technical requirements and minimizes the retuning required in the circumference adjustment; it also allows the symmetry of the ring to be maintained.

3.4.3.3.5 Injection/Extraction Systems The injection/extraction kickers and septa are the same design as given in the NLC ZDR [ZDR]; the kickers and septa are each $2 \mathrm{~m}$ long, the kickers providing a deflection of $8 \mathrm{mrad}$ (injection) and $6.6 \mathrm{mrad}$ (extraction), and the septa providing a deflection of $150 \mathrm{mrad}$. 


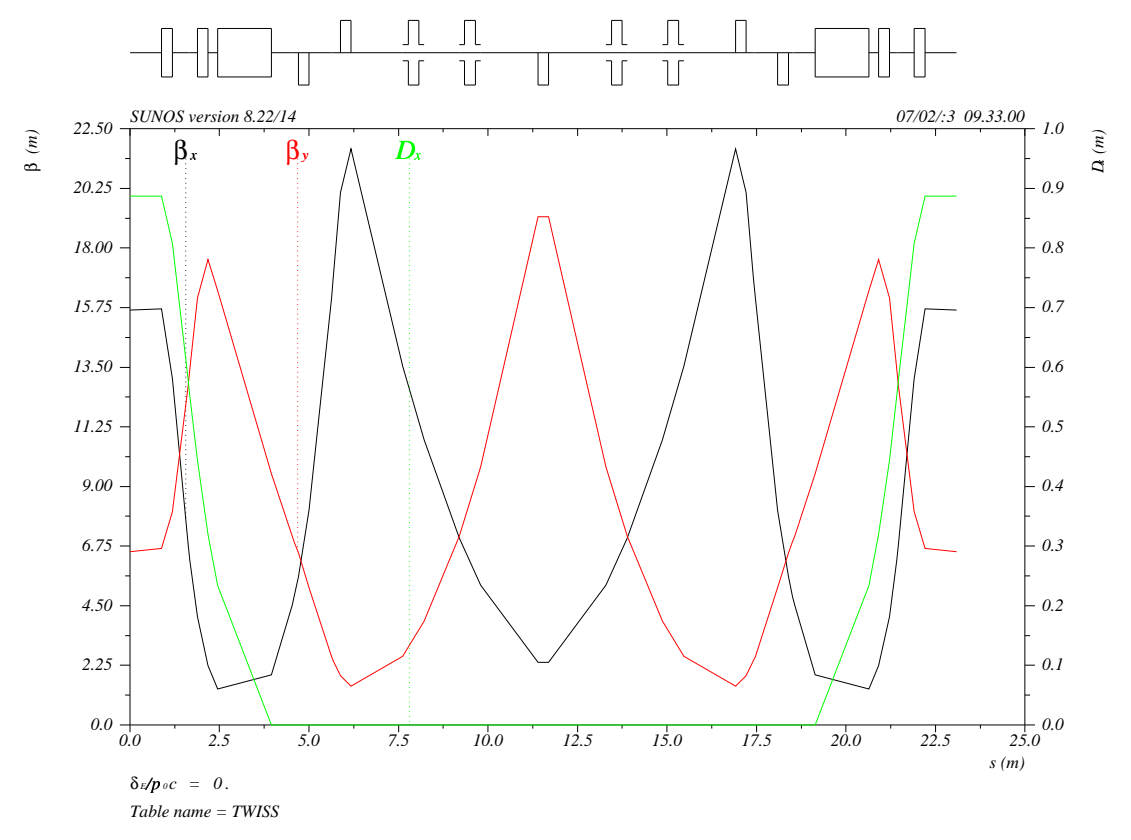

Figure 3.4.3.12: Lattice functions in the RF cell.

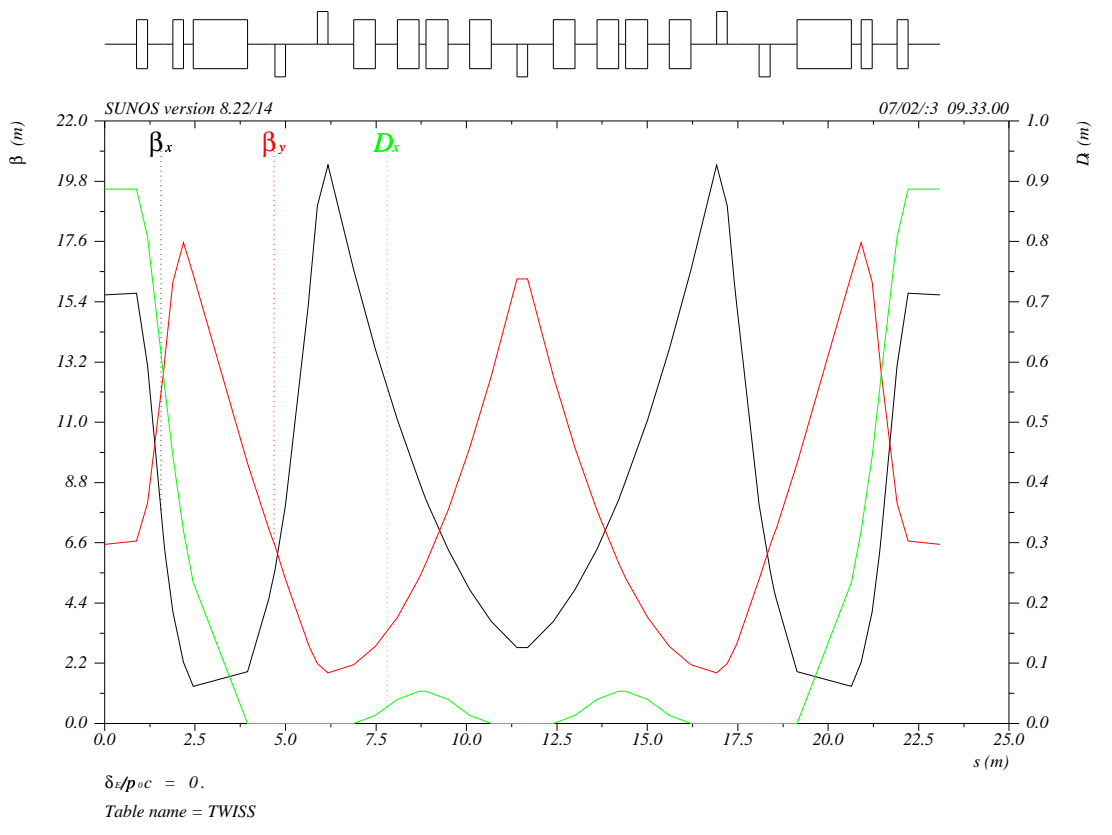

Figure 3.4.3.13: Lattice functions in the chicane cell. 


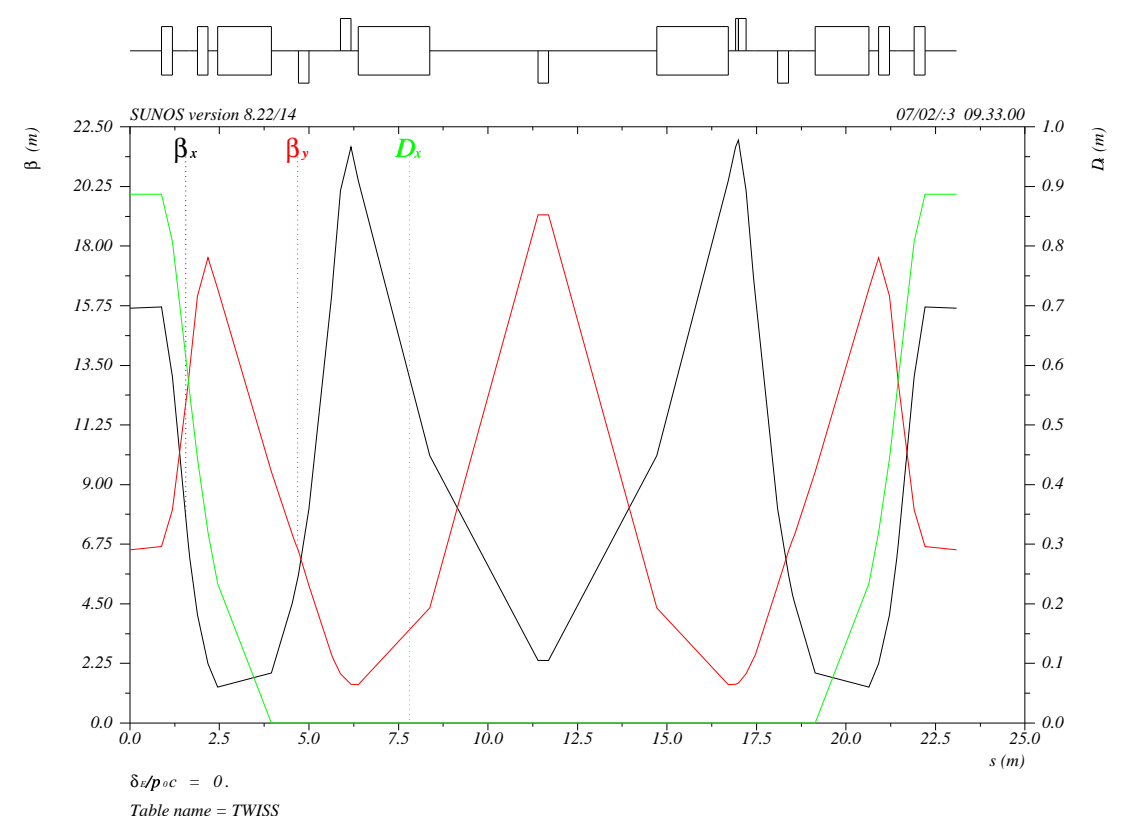

Figure 3.4.3.14: Lattice functions in the injection cell.

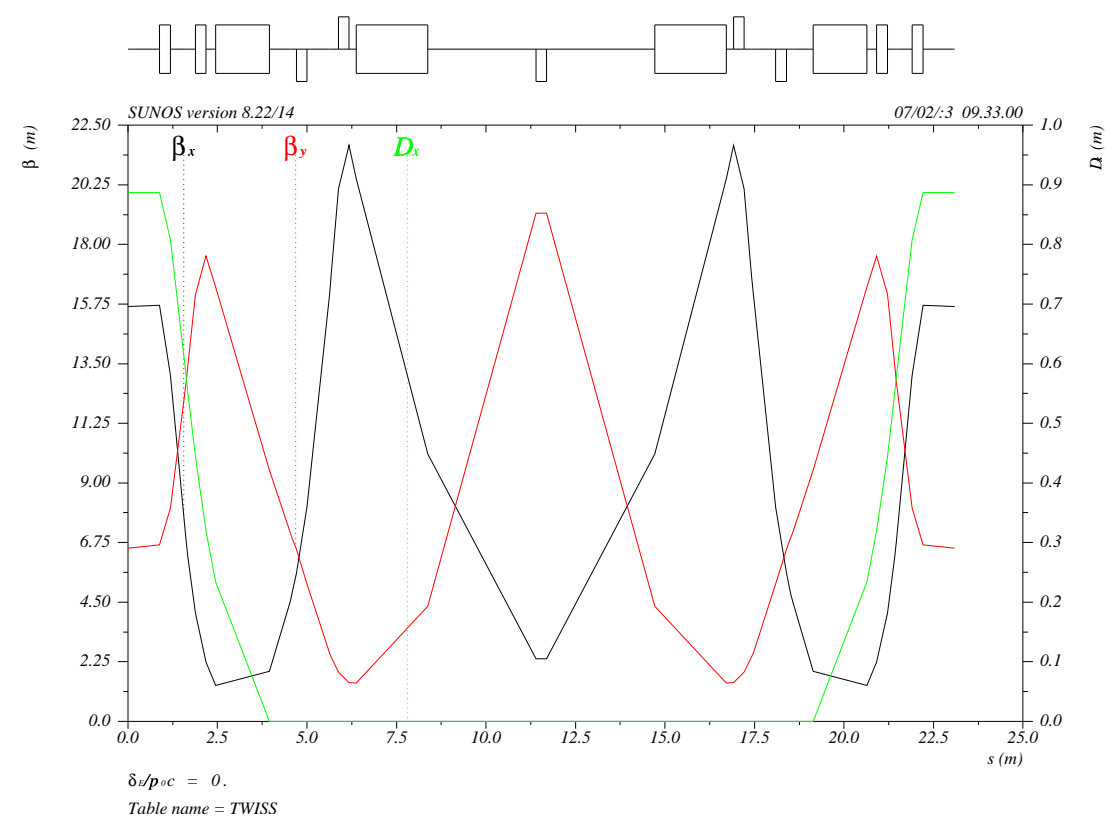

Figure 3.4.3.15: Lattice functions the extraction cell. 


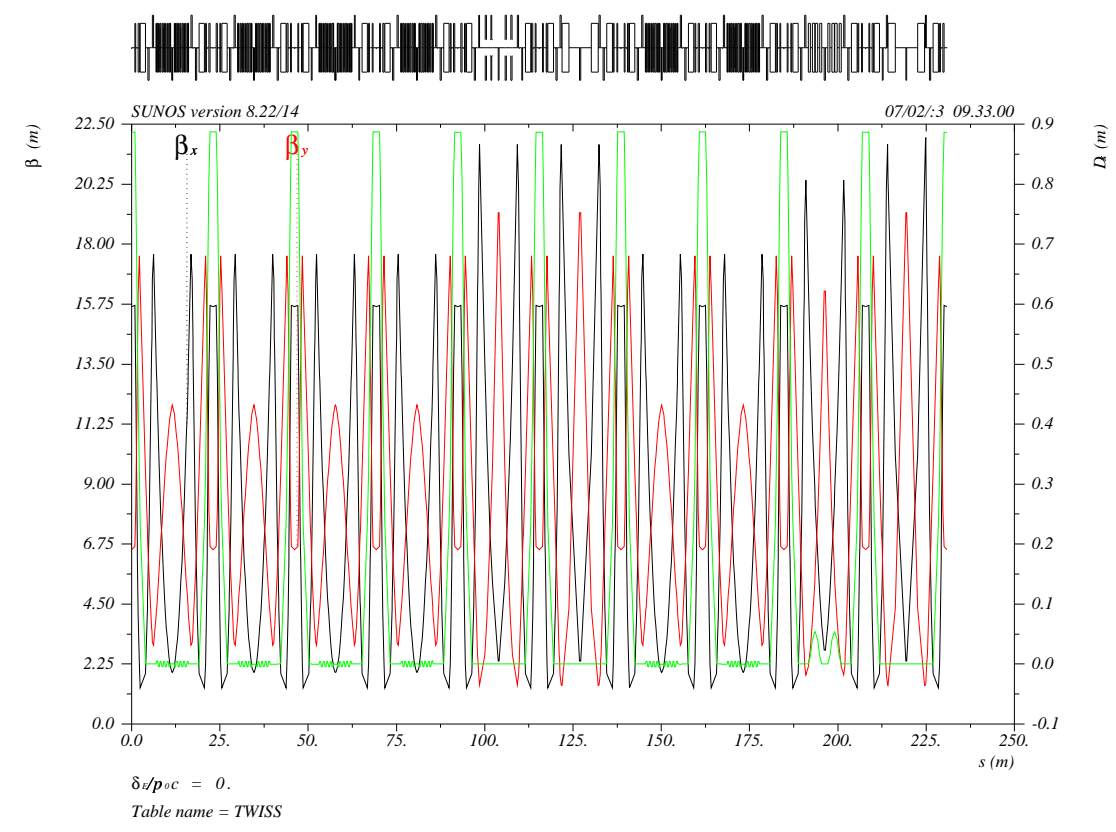

Figure 3.4.3.16: Lattice functions around the whole ring.

The central defocusing quadrupole provides additional bending for the injected/extracted beam. The circumference of the lattice allows for a kicker rise/fall time of $119 \mathrm{~ns}$. With the present design, the kicked beam is $81 \mathrm{~mm}$ off-axis relative to the stored beam at the entrance to the septum (see Fig. 3.4.3.10). At the location of the next quadrupole following the last septum in the direction of the extracted beam, the kicked beam is $298 \mathrm{~mm}$ off-axis, with respect to the stored beam. The technical constraints look reasonable.

\subsection{Technical components}

3.4.3.3.6.1 RF System The RF cavities are intended to be identical for the main and pre-damping rings, with five in each of the MDRs and four in the PPDR. The systems are $714 \mathrm{MHz}$, using PEP-II style damped cavities generating gap voltages of maximum $0.5 \mathrm{MV}$ per cavity, which allows a maximum of 2.0 MV for the four cavities in the PMDR.

3.4.3.3.6.2 Magnet System The magnets for the PPDR are similar to those used in the MDR except the PPDR has a much larger aperture, requiring gaps that are too large for permanent magnets to be feasible, so those magnets are electromagnets. Magnet parameters for the PPDR are summarized in Table 3.4.3.5. The dipoles, quadrupoles and sextupoles are powered in strings with one large power supply per string. Each quadrupole and sextupole also has an individual low power trim supply.

3.4.3.3.6.3 Wiggler A magnetic design and field map for the hybrid technology damping wiggler has been produced [29]. For analysis of the dynamics, we have followed the same approach as in [12], where more details of the technique are given. 
Table 3.4.3.5: PPDR magnet parameters

\begin{tabular}{|llrrrrr|}
\hline Type & Location & $\begin{array}{c}\text { Length } \\
{[\mathrm{m}]}\end{array}$ & $\begin{array}{c}\text { Pole-tip } \\
\text { radius } \\
{[\mathrm{m}]}\end{array}$ & $\begin{array}{c}\text { Pole-tip } \\
\text { field } \\
{[\mathrm{T}]}\end{array}$ & $\begin{array}{c}\text { Quad, Sext } \\
\text { strength }^{a} \\
{\left[\mathrm{~m}^{-2}\right],\left[\mathrm{m}^{-3}\right]}\end{array}$ & Count \\
\hline \hline Dipole & & 1.5 & 0.04 & 1.38 & & 20 \\
Dipole & Chicane & 0.6 & 0.04 & 0.98 & & 4 \\
Dipole & Chicane & 0.6 & 0.04 & -0.98 & & 4 \\
\hline Quadrupole & Achromat $\mathrm{QF}^{b}$ & 0.3 & 0.04 & 0.531 & 2.01 & 20 \\
Quadrupole & Achromat QD & & \\
\hline Quadrupole & Wiggler straight & 0.3 & 0.04 & -0.472 & -1.79 & 20 \\
Quadrupole & Wiggler straight & 0.3 & 0.04 & -0.006 & -0.02 & 12 \\
Quadrupole & Wiggler straight & 0.3 & 0.04 & 0.447 & 1.69 & 12 \\
Quadrupole & Chicane straight & 0.3 & 0.04 & -0.060 & -0.23 & 6 \\
Quadrupole & Chicane straight & 0.3 & 0.04 & 0.467 & -0.43 & 2 \\
Quadrupole & Chicane straight & 0.3 & 0.04 & -0.289 & -1.17 & 2 \\
Quadrupole & Straight & 0.3 & 0.04 & -0.158 & -0.60 & 1 \\
Quadrupole & Straight & 0.3 & 0.04 & 0.488 & 1.84 & 6 \\
Quadrupole & Straight & 0.3 & 0.05 & -0.399 & -1.21 & 3 \\
\hline Sextupole & Achromat SX & 0.05 & 0.04 & 0.417 & 78.9 & 10 \\
Sextupole & Achromat SY & 0.05 & 0.04 & -0.278 & -52.7 & 20 \\
Sextupole & Wiggler straight & 0.05 & 0.04 & -0.528 & -100.0 & 12 \\
Sextupole & Wiggler straight & 0.05 & 0.04 & 0.228 & 43.1 & 12 \\
Sextupole & Straight & 0.05 & 0.04 & -0.449 & -84.8 & 8 \\
Sextupole & Straight & 0.05 & 0.04 & 0.192 & 36.4 & 8 \\
\hline
\end{tabular}

${ }^{a}$ gradient normalized to beam rigidity

${ }^{b}$ horizontally offset by $1.5 \mathrm{~mm}$ 


\subsubsection{Infrastructure}

The main damping rings are $300 \mathrm{~m}$ in circumference and the pre-damping ring is $\sim 230 \mathrm{~m}$ in circumference. They are located in a tunnel with $3 \mathrm{~m}$ inner diameter. This tunnel is part of the separate injector complex. 


\subsubsection{Bunch Compressor and pre-Linac Collimation}

The X-band design uses a two-stage bunch compressor system in which the first stage follows the damping ring and the second stage is at the exit of the S-band pre-linac at a beam energy of $8 \mathrm{GeV}$. Electron and positron bunch compression systems are identical. This scheme was described in detail in the NLC ZDR, Chapter 5. Further studies to optimize cost and performance resulted in the choice of a lower energy (8 $\mathrm{GeV}$ ) for the 2nd stage compression[27][28]. This design is described in Ref.[NLC01] and [TRC].

A schematic of the full bunch compression system from the exit of the damping rings to the linac entrance is shown in Figure 3.4.4.1. Included are the post-DR spin rotation system, 1st bunch compressor, diagnostics and matching section, $6 \mathrm{GeV}$ S-band pre-linac, pre-linac collimation section, 2nd bunch compressor, pre-linac diagnostics, and pre-linac tuneup dump line.

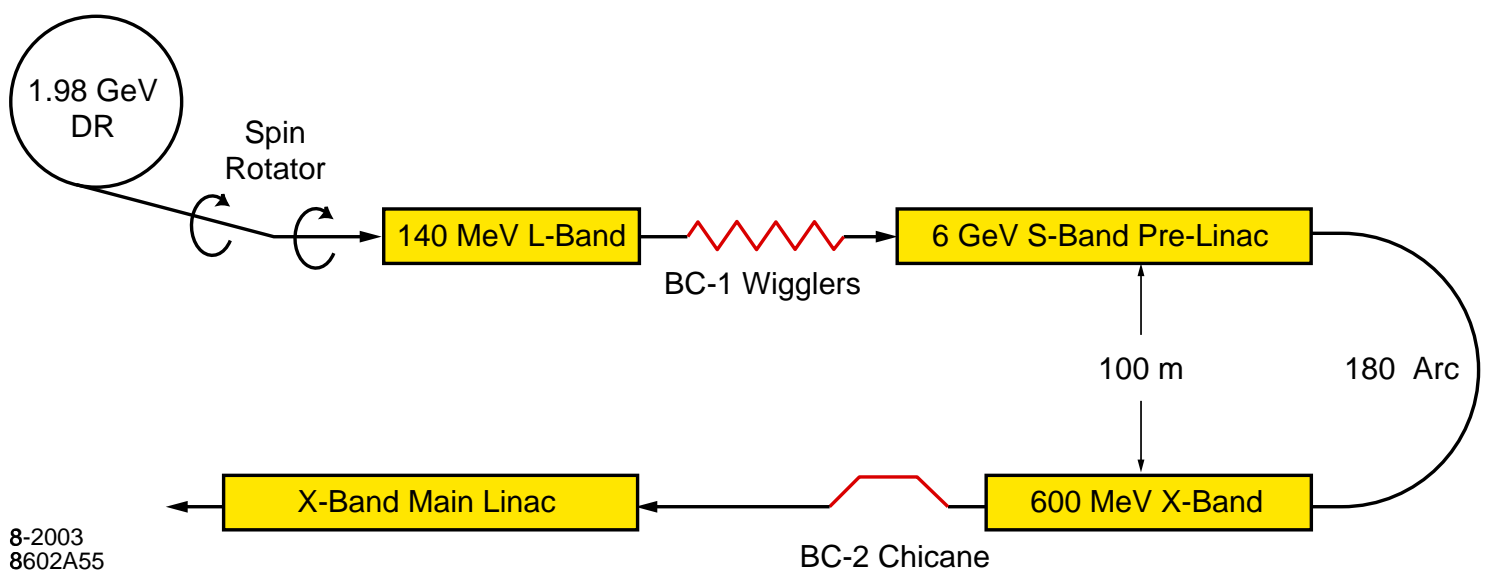

Figure 3.4.4.1: Schematic of two-stage bunch compressor layout.

The optics from the ring through the second bunch compressor with spin rotator solenoids off is shown in Figure 3.4.4.2. The spin rotator section has matching quadrupoles so that the optics looks very similar when the solenoids are tuned to the required settings. Note that the section right at the exit of the damping ring has not yet been updated for the 2003 MDR design. The system lengths are given in Table 3.4.4.

Table 3.4.4.1: Subsystem lengths for the two stage bunch compressor

\begin{tabular}{|lr|}
\hline DRx & $66.015 \mathrm{~m}$ \\
BC1 & $214.568 \mathrm{~m}$ \\
PLIN & $503.191 \mathrm{~m}$ \\
PCOL & $72.736 \mathrm{~m}$ \\
BC2 & $278.686 \mathrm{~m}$ \\
& \\
Total system length & $1135.196 \mathrm{~m}$ \\
\hline
\end{tabular}

\subsubsection{Overview}

The bunch compressors must reduce the $\sim 5 \mathrm{~mm}$ rms length of the bunches extracted from the damping rings to the 90 to $150 \mu \mathrm{m}$ bunch length required for the main linacs and final focus systems. A two-stage compressor system has been designed to meet the following additional goals: 

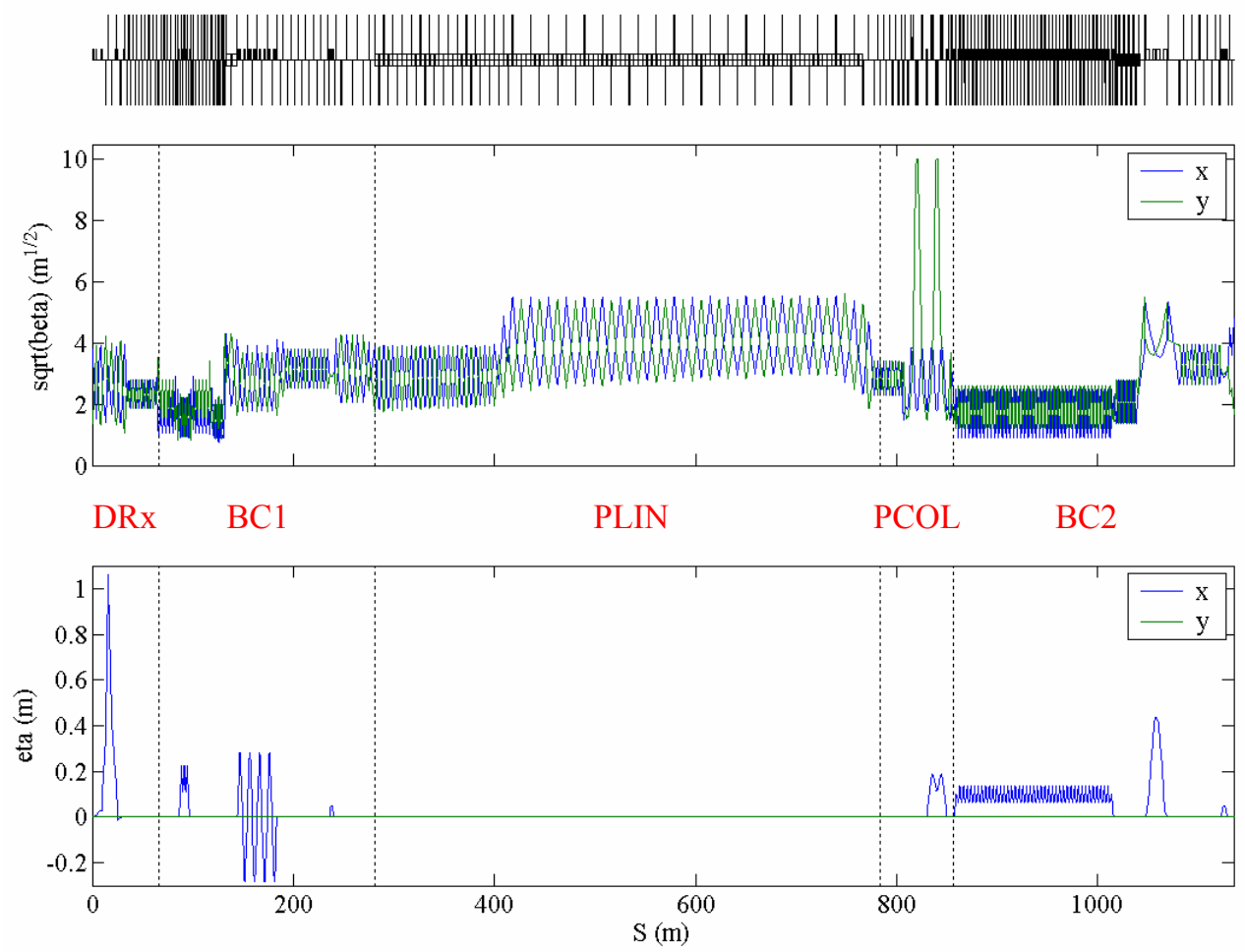

Figure 3.4.4.2: Optics from the ring through the second bunch compressor with spin rotator solenoids off. 
1. Multibunch phase variations in the damping ring of up to $\pm 5 \mathrm{~mm}$ should not produce relative energy variations that are larger than $\pm 0.1 \%$ in the final focus systems.

2. The system should include a $180^{\circ}$ turn-around arc to permit future main linac extensions and to allow beam abort and feedforward systems.

3. The transverse emittances must be preserved to within a reasonable budget with diagnostics and correction elements included in the design.

4. The compression systems should not depolarize the beams.

The two-stage system has a number of advantages over a single-stage compressor. In particular, it keeps the rms energy spread less than about $1.5 \%$ and the bunch length is more naturally matched to the acceleration RF frequency so that energy spread due to the longitudinal wakefields can be cancelled locally. The disadvantage of the two-stage design is that it is more complex and lengthy than a single-stage compressor. The first stage rotates the longitudinal phase by $\frac{\pi}{2}$ while the second stage performs a $2 \pi$ rotation. In this manner, phase errors due to the beam loading in the damping rings and energy errors due to imperfect multibunch energy compensation in the $6 \mathrm{GeV}$ S-band pre-linacs do not affect the beam phase at injection into the main linac.

Assuming an incoming rms energy spread of $\sigma_{\delta}=1 \times 10^{-3}$ and $\mathrm{rms}$ bunch length of $\sigma_{z}=5 \mathrm{~mm}$, the first stage compresses the damping ring beam to a bunch length of about $0.6 \mathrm{~mm}$ and an energy spread of $1 \%$. This stage consists of a $140 \mathrm{MV}$ L-band $(1.4 \mathrm{GHz}) \mathrm{RF}$ section followed by a long period wiggler which generates the momentum compaction needed for the bunch compression. The second bunch compression stage follows the $6 \mathrm{GeV}$ pre-linac. The nominal configuration compresses the beam to a bunch length of $110 \mu \mathrm{m}$. This compressor is a telescope in longitudinal phase space which rotates the phase space by $2 \pi$. It consists of a $180^{\circ}$ arc which is followed by a $600 \mathrm{MeV}$ X-band $(11.4 \mathrm{GHz}) \mathrm{RF}$ section and a chicane. Adjustments to either the low-energy or the high-energy compressors permit control of the final bunch length over the specified range of 150 to $90 \mu \mathrm{m}$.

One of the rationales behind the compressor design has been to utilize naturally achromatic magnetic lattices wherever the beam energy spread is large. In particular, the optics is chosen so that quadrupoles are not placed in regions of large dispersion and thus strong sextupoles are not needed. This choice arises from experience with the second-order achromats in the SLC bunch compressors in which quadrupoles are located in dispersive regions and strong sextupoles are used to cancel the chromatic aberrations. Unfortunately, the SLC design was difficult to operate and tune because of large nonlinearities and sensitivity to multipole errors in the quadrupoles; over the years additional nonlinear elements were added (skew sextupoles and octupoles) to help cancel the residual aberrations but tuning remained problematic. To facilitate tuning, orthogonal tuning controls and diagnostics have been explicitly designed into the NLC system, which should make it relatively straightforward to operate. Details of the diagnostic equipment can be found in the optics decks.

Finally, although the tolerances on components in the bunch compressor systems are not nearly as tight as in the main linacs or the final focus systems, the same methods of beam-based alignment and tuning have been adopted. In particular, to ease the alignment procedures, all of the quadrupoles are mounted on magnet movers and each quadrupole contains a BPM with a resolution of $<2 \mu \mathrm{m}$. Similarly, all of the accelerator structures are instrumented with RF BPMs to measure the induced dipole modes and each RF girder is remotely movable for minimization of wakefields. There are 4-wire parasitic emittance measurement sections and subsequent tune-up dumps after the first-stage bunch compressors, the $6 \mathrm{GeV}$ pre-linacs, and the secondstage bunch compressors before injection into the main linac. There are also synchrotron radiation-based bunch length and energy spread diagnostics in the first-stage wigglers, the second-stage arcs, and the second stage chicanes and there are RF deflector-based bunch length monitors before injection into the main linac. 


\subsubsection{DRx: transfer line from damping ring to $\mathrm{BC} 1$}

The transfer line from the damping ring to the first bunch compressor $\mathrm{BC} 1$ contains first a diagnostic and matching section, and then a section for spin rotation. The spin rotation is initially only implemented on the electron side but space is left for a future upgrade to polarized beams on the positron side. After the spin rotator is a coupling correction section.

3.4.4.2.1 Diagnostic section A wire-array emittance measurement station [56] is located immediately after the damping ring. The section is designed to continuously monitor the matching and emittance of the beam extracted from the ring. The wire-array station is constructed from a series of 4 FODO cells, with a profile monitor (mechanical- or laser-wire scanner) located at each vertically focusing quadrupole.

The optics from the exit of the ring through the diagnostics section is shown in Figure 3.4.4.3. The numbers indicate the location of the key elements. Not shown explicitly are the locations of collimators.

1. MDR extraction kicker

2. MDR extraction septa

3. partial $-I$ transform optics (with "quad scan" emittance wire scanner)

4. compensating bend magnet ("B1")

5. partial $+I$ transform optics

6. compensating kicker

7. feedback section

8. 4-wire $2 \mathrm{D}$ emittance diagnostic section

3.4.4.2.2 Spin rotator The spin rotator is constructed from superconducting solenoids and a normal conducting bend section ( $\mathrm{arc}$ ), located upstream of the bunch compressor. Since the damped beam is flat, the cross-plane coupling induced by the solenoids must be compensated. This is achieved by a spin rotator unit constructed from two identical superconducting solenoids, separated by a short beamline whose (transverse) optics form a $-I$ transformation in $y$ and an $I$ transform in $x$; this effectively cancels the betatron coupling, while the spin rotation of the two solenoids add. A single unit can rotate the spin around the longitudinal axis by up to $90^{\circ}$. The complete spin rotator is constructed from three sections:

- an initial solenoid pair, which rotates the spin around the local longitudinal $(z)$ axis by $90^{\circ}$;

- a normal conducting horizontal arc, which further rotates the spin around the vertical axis by $90^{\circ}$; and

- a final solenoid pair, providing an additional rotation about the $z$-axis by $90^{\circ}$.

With the above combination of rotations, all possible spin orientations can be achieved. The focusing effect of the solenoids is corrected with four matching quadrupoles per paired solenoid section. The matching quadrupoles are positioned between the solenoid sections and the central arc. With the solenoids at maximum field strength, the chromatic emittance growth is $\sim 1 \%\left(\sigma_{\delta_{i}} \approx 0.13 \%\right)$. The parameters of the spin rotator system are given in Table 3.4.4.2.2.

The optics through the spin rotation and coupling correction sections is shown in Figure 3.4.4.4. The numbers indicate the location of the key elements. 


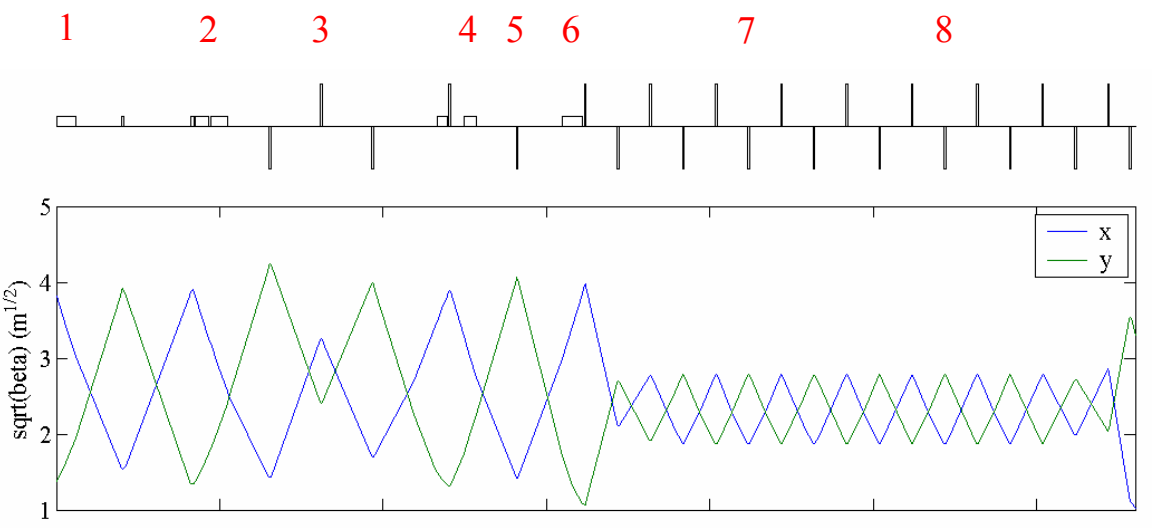

WS WS WS WS WS

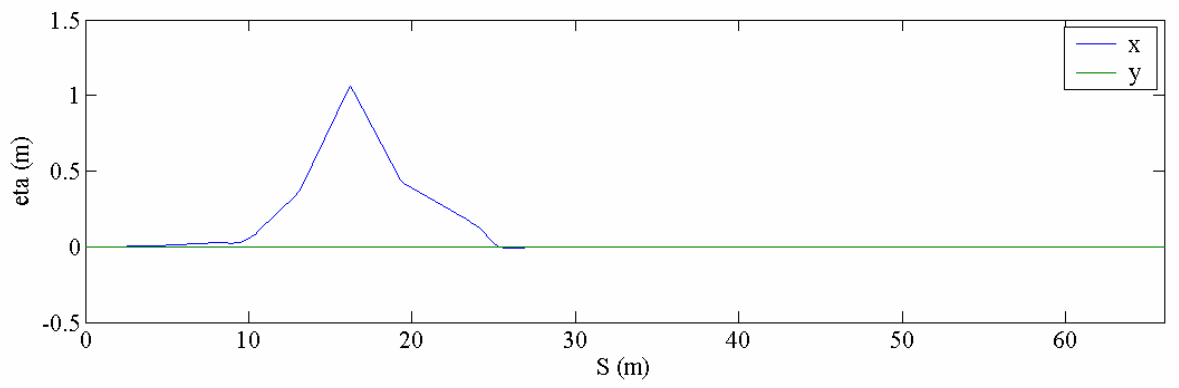

Figure 3.4.4.3: Optics from the ring through the diagnostics section. 
Table 3.4.4.2: Basic spin rotator parameters longitudinal dispersion $R_{56}[\mathrm{~m}] \quad 0.005$ maximum spin rotation in solenoid [degrees] $\quad 90$ total arc bend angle [degrees] 8 total length of rotator system $[\mathrm{m}] \quad 85$ SR induced horizontal emittance growth [\%] 0.02

1. spin rotator solenoid ( $e^{-}$only)

2. horizontal $+I /$ vertical $-I$ transform optics

3. spin rotator solenoid ( $e^{-}$only)

4. $20^{\circ} \operatorname{arc}$

5. spin rotator solenoid ( $e^{-}$only)

6. horizontal $+I$ / vertical $-I$ transform optics

7. spin rotator solenoid ( $e^{-}$only)

8. skew correction section
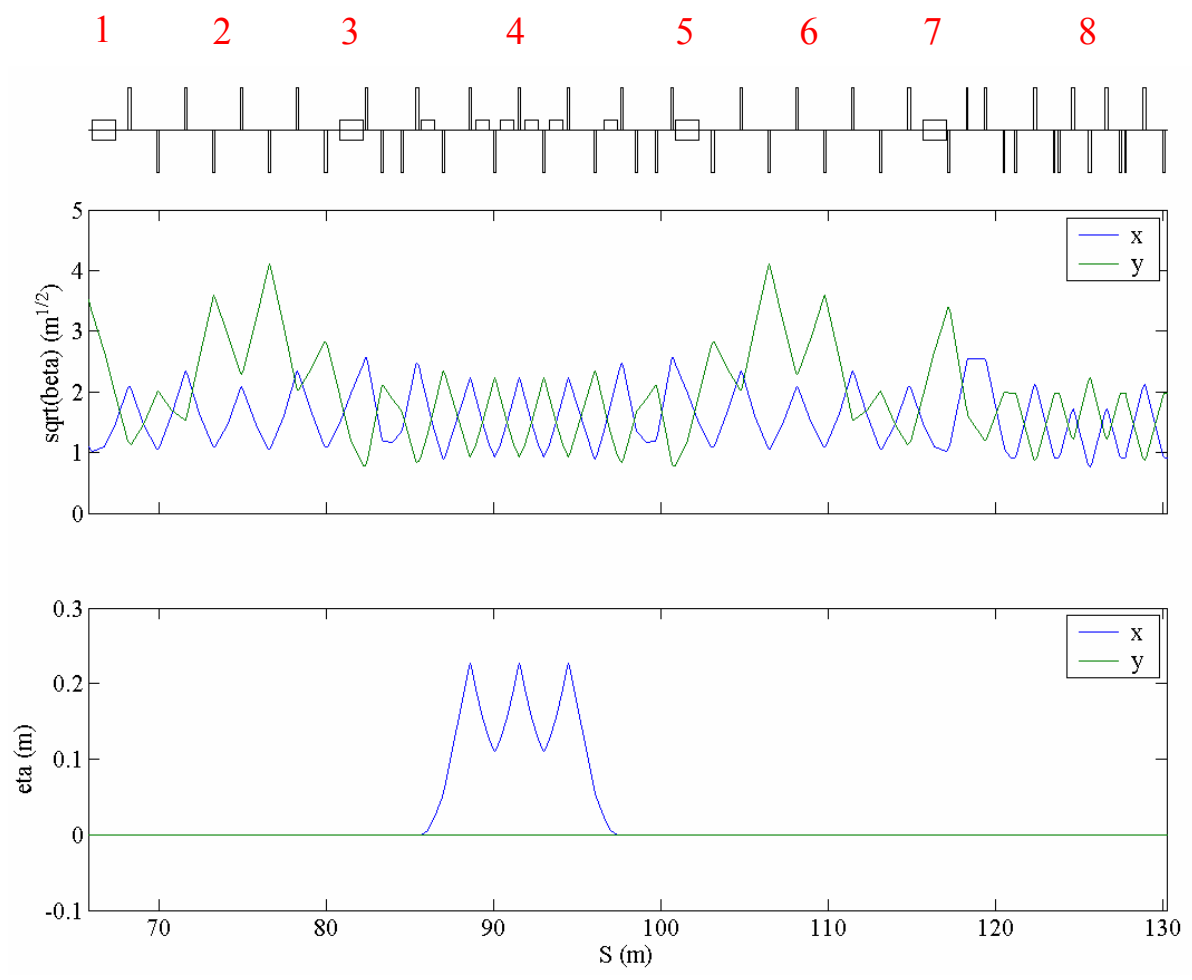

Figure 3.4.4.4: Optics from the spin rotation and coupling correction sections. 
3.4.4.2.3 Coupling correction section In order to empirically correct anomalous cross-plane coupling due to spin rotator errors, a coupling correction section of the type proposed in [56] is included. The system is constructed from four skew-quadrupoles with zero nominal strength. Using skew-quadrupoles with a length of $0.1 \mathrm{~m}, \pm 0.1 \mathrm{~T}$ pole-tip field, and a $0.01 \mathrm{~m}$ pole-tip radius, a factor of about two in emittance increase can be corrected per skew-quadrupole.

\subsubsection{Bunch Compressor BC-1}

The electron and positron first bunch compression systems (BC-1) compress the bunch length of the beams from the respective damping ring system from $5 \mathrm{~mm}$ to $500 \mu \mathrm{m}$, as required for acceptance by the pre-linac systems. The main components of each BC-1 are a $140 \mathrm{MeV}$ L-Band RF section and a 10 dipole magnetic wiggler section. Each BC-1 has a diagnostics region at its end to measure emittance, bunch length and energy spread. There is a pulsed bend tune up dump off the end of the BC-1, for tuning the beams without sending them into the pre-linacs. An additional requirement is that the positron system must be able to deliver electrons to the downstream systems for commissioning. Polarized electrons are also required for $\gamma$ $\gamma$ or $e^{-}-e^{-}$running.

The optics through the first stage bunch compression is shown in Figure 3.4.4.5. The numbers indicate the location of the key elements. Not shown explicitly is the tuneup dump line.

1. L-band compressor RF $(2 \times 5.039 \mathrm{~m}$ structures $)$

2. wiggler section

3. feedback section

4. 4-wire 2D emittance diagnostic section

5. diagnostic chicane

6. PPS section (1 dump kicker, 3 stoppers, 2 shielding blocks, 1 gate)

The diagnostic section downstream of $\mathrm{BC} 1$ will be used to tune the spin rotator system and the $\mathrm{BC} 1$. The diagnostic section consists of 4 laser wire monitors separated by $45^{\circ}$ in betatron phase. The nominal rms beam size at the monitors is $5 \mu \mathrm{m}$ vertically and $70 \mu \mathrm{m}$ horizontally. A correctly matched beam is easily identified since the beam size (for a single plane) is identical at each of the four wire scanners. The crossplane coupling can not be accurately determined using this system: however, by simply minimizing the vertical emittance (flat beam) with the four skew-quadrupoles in the upstream coupling correction section, all coupling can be corrected (with some iteration necessary in extreme cases). The beam parameters before and after the first bunch compressor are given in Table 3.4.4.3.

3.4.4.3.1 Layout and Functional Description The BC-1 functions by having the L-band RF produce an energy-time correlation along the bunch and the wiggler has a energy dependent path length. Specifically the front of the bunch is given higher energy and thus it takes a longer path when bending while the back of the bunch has lower energy and takes a shorter path length. In accelerator physics terms, each bunch is compressed by applying a pseudo $\pi / 2$ rotation in longitudinal phase space.

Optically the BC-1 beamline starts with two 5-meter L-Band acceleration sections and is followed by a dispersion suppression section. Next, there is a 10 dipole magnet wiggler section which is followed by a dispersion suppression section and a matching section. Following the matching section is a 4-laser wire emittance diagnostic section and PPS stopper section. At the end of the BC-1 there is a short tune up dump line, which is reached by a pulsed bend. 

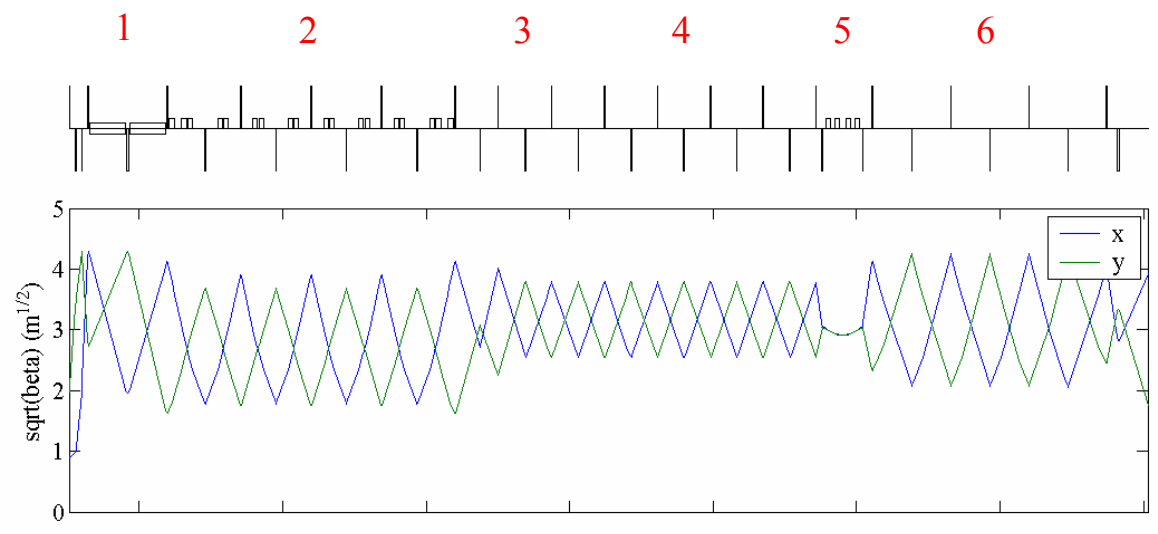

WS WS WS WS

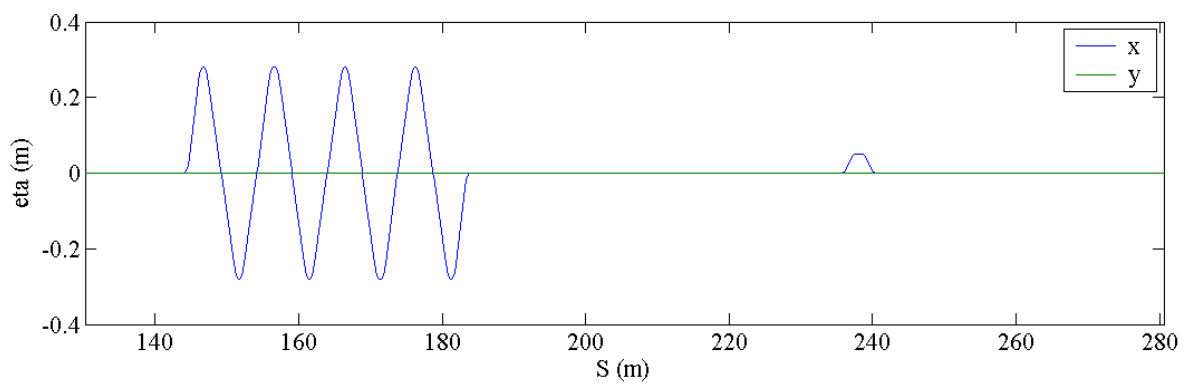

Figure 3.4.4.5: Optics through the first stage bunch compressor, BC1. 
Table 3.4.4.3: Beam parameters before and after the first bunch compressor, BC-1

\begin{tabular}{|c|c|c|c|}
\hline Parameter Name & Symbol & Output & Unit \\
\hline Beam energy & $E$ & 1.98 & $\mathrm{GeV}$ \\
\hline Energy adjustability & $\Delta E / E$ & \pm 5 & $\%$ \\
\hline Bunch energy variation & $\delta E / E$ & 1 & $\%$ Full Width \\
\hline Energy spread & $\sigma_{E} / E$ & 0.1 & $\% \mathrm{rms}$ \\
\hline Horizontal emittance (norm. rms) & $\gamma \varepsilon_{x}$ & 3 & $\mathrm{~mm}$-mrad \\
\hline Vertical emittance (norm. rms) & $\gamma \varepsilon_{y}$ & 0.02 & mm-mrad \\
\hline Horizontal Beam Jitter & $\Delta \gamma J_{x}$ & 0.015 & mm-mrad \\
\hline Vertical Beam Jitter & $\Delta \gamma J_{y}$ & 0.0004 & mm-mrad \\
\hline Bunch length (rms) & $\sigma_{z}$ & 0.5 & $\mathrm{~mm}$ \\
\hline
\end{tabular}

Beam focusing and steering is accomplished using tunable, permanent magnet quadrupoles on movers for most of the BC-1. The final matching section is the exception, with electromagnetic quadrupoles and steering feedback correctors. Each quadrupole has a BPM.

Each BC-1 has one 4-D emittance measurement laser wire area, two bunch length measuring cavities and streak cameras, three laser wires for energy spread measurements, and a laser polarimeter. Beam loss is detected by PLIC $^{1}$ through the entire beamline.

3.4.4.3.2 Magnet system Most quadrupoles are tunable permanent magnets except those in the matching section, which are electromagnets. All quadrupoles are on independent $x, y$ and roll movers and each quadrupole contains a BPM. All the bends are adjustable permanent magnets. The polarities of the magnets in the positron line are reversible to allow $e^{-}$operation.

3.4.4.3.3 Vacuum System The accelerator vacuum module system is a manifold ion pump scheme, with a hot filament gauge. The operating vacuum pressure is $<10^{-8}$ Torr.

3.4.4.3.4 Instrument System Beam emittance and bunch length measurements are made at end of the BC-1 using a laser wire system. BC-1 beam loss is detected by PLIC, which runs its entire length. All quadrupoles are on independent $x, y$ and roll movers and each quadrupole contains a BPM. The beam line instrumentation must function equally well for either positron or electron beams.

3.4.4.3.5 Infrastructure The BC-1 beamline must have one enclosure for the beamline components and local electronics, which cannot be accessed while beams are running and three 40' x 14' support areas, that are accessible during beam operation.

\subsubsection{Pre-linacs}

The electron and positron pre-linacs accelerate the beams received from their respective first bunch compressor systems from 1.98 to $8.0 \mathrm{GeV}$, while maintaining the low emittance produced in the damping rings. Each pre-linac uses 4-meter S-band $(2856 \mathrm{MHz})$ structures for acceleration, each a with loaded energy gradient of $17 \mathrm{MV} / \mathrm{m}$ (21 MV/m unloaded). Transverse focusing is accomplished using quadrupoles on movers between every or every other accelerator structure. In addition, the positron system must be able to deliver electrons to the downstream systems for commissioning. Polarized electrons are required for $\gamma-\gamma$ or $e^{-}-e^{-}$running.

\footnotetext{
${ }^{1}$ ionization chambers
} 
The optics through the pre-linac is shown in Figure 3.4.4.6. The pre-linac is $\sim 450 \mathrm{~m}$ long and contains 108 $4.035 \mathrm{~m}$ RDS S-band structures in $18 \mathrm{RF}$ modules with 6 structures per module including 2 spare modules for reliability. The first $5 \mathrm{RF}$ modules have 1 quad per structure and the next $13 \mathrm{RF}$ modules have 1 quad per 2 structures. This is followed by a feedback section. The beam parameters before and after the pre-linac are given in Table 3.4.4.4.

1

2

3

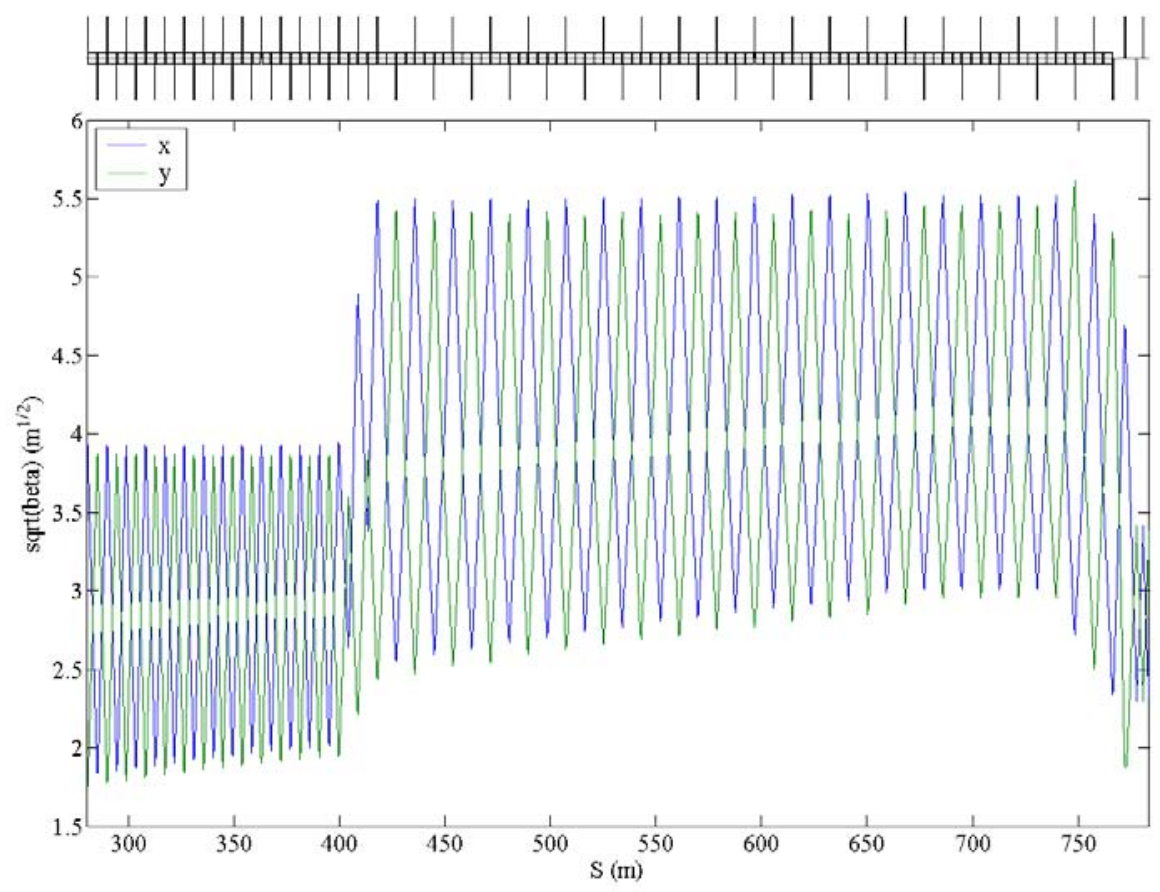

Figure 3.4.4.6: Optics for the pre-linac.

Table 3.4.4.4: Beam parameters before and after the pre-Linac

\begin{tabular}{|llcccl|}
\hline Parameter Name & Symbol & Input & Output & Unit \\
\hline Beam energy & $E$ & 1.98 & & 8 & $\mathrm{GeV}$ \\
Bunch energy variation & $\delta E / E$ & \multicolumn{2}{c}{1} & & $\%$ Full Width \\
Energy spread & $\sigma_{E} / E$ & 1 & \multicolumn{2}{c}{0.25} & $\%$ rms \\
Horizontal emittance (norm. rms) & $\gamma \varepsilon_{x}$ & \multicolumn{2}{c}{3} & & mm-mrad \\
Vertical emittance (norm. rms) & $\gamma \varepsilon_{y}$ & \multicolumn{2}{c}{0.02} & mm-mrad \\
Horizontal Beam Jitter & $\Delta \gamma J_{x}$ & 0.015 & 0.015 & $\mathrm{~mm}-\mathrm{mrad}$ \\
Vertical Beam Jitter & $\Delta \gamma J_{y}$ & 0.0004 & 0.0008 & $\mathrm{~mm}-\mathrm{mrad}$ \\
Bunch length (rms) & $\sigma_{z}$ & \multicolumn{2}{c}{500} & $\mu \mathrm{m}$ \\
\hline \hline
\end{tabular}

3.4.4.4.1 Layout and Functional Description The pre-linacs are part of the second bunch compression scheme, which requires a pseudo $2 \pi$ longitudinal phase space rotation for stability, which can only be achieved by two RF systems (pre-linac, post arc RF) and two magnetic compressors (180 turnaround arc, chicane magnet). The pre-linacs are the first RF system and accelerate the beams from 1.98 to $8 \mathrm{GeV}$, while maintaining the low emittances from the damping rings. 
Each pre-linac has 18 S-band RF modules, each consisting of a modulator powering two klystrons, that feed two SLED assemblies which together power six 4-meter accelerator structures. The $18 \mathrm{RF}$ modules include two spare RF modules of overhead. The loaded gradient of the structures is $17 \mathrm{MV} / \mathrm{m}(21 \mathrm{MV} / \mathrm{m}$ unloaded).

Beam focusing is accomplished using tunable, permanent magnet quadrupoles between each structure for the first 5 modules and between every second structure for the remainder. The end of pre-linac is a matching section with electromagnetic quadrupoles and steering feedbacks. Each quadrupole has a captured BPM. The pre-linac diagnostics include a bunch length measuring cavity, three RF BPM output couplers in each accelerator structure and PLIC to detect beam loss.

3.4.4.4.2 RF system There are $18 \mathrm{RF}$ modules in the pre-linac, each of which has one Solid State Modulator, two S-Band Klystrons, two SLED I cavities, rectangular Waveguide and six 4 meter accelerator structures. RF generation begins with two klystrons that are driven by a solid-state modulator. The SLED output of the two klystrons are combined and distributed to 6 accelerator structures. The combination of two the SLEDs cavities permits vernier control of the RF waveform for beam loading compensation, while allowing for constant power delivery to the structures during a machine protection system fault and recovery. Each RF accelerator structure has three output couplers along its length, each sampled by an RF BPM module. All structures have independent $x, y$ and roll movers.

3.4.4.4.3 Magnet system The quadrupoles are all electromagnets. Each quadrupole contains a BPM. Beam steering is accomplished by independent $x, y$ and roll movers. The polarities of the magnets in the positron line are reversible to allow $e^{-}$operation.

3.4.4.4.4 Vacuum System The accelerator vacuum system is a manifold ion pump design, with a hot filament gauge. The operating vacuum pressure is $<10^{-8}$ Torr.

3.4.4.4.5 Instrument System Pre-Linac beam loss is detected by PLIC, which runs its entire length. All quadrupoles are on independent $x, y$ and roll movers and each quadrupole contains a BPM. Each RF accelerator structure has three output couplers along its length, sampled by an RF BPM module. All structures have independent $x, y$ and roll movers. The beam line instrumentation must function equally well for either positron or electron beams.

3.4.4.4.6 Infrastructure The Pre-Linacs must have two enclosures, one for the beamline components and local electronics, which cannot be accessed while beams are running, and another for the modulator/klystron pairs and other accelerator utilities that need to be accessed while beams are running.

\subsubsection{Pre-collimation}

The electron and positron pre-collimation systems collimate the transverse beam halo of the low emittance beams from the damping rings before these beams are accelerated to full energy in the main linacs. The system consists of three stages: a betatron collimation section immediately downstream of the damping ring, an energy scraper in the high dispersion region of BC1, an energy collimation system after the pre-linac, and an energy scraper in the high dispersion of the BC2 chicane. The three energy collimation systems are designed to collimate the bunch length where tails can easily be deflected out to large transverse amplitudes. At this time, only the pre-linac collimation system has been explicitly designed. The betatron collimation downstream of the damping ring is expected to be straightforward and will look like a scaled version of the post-linac betatron collimation system with a length of approximately 50 meters. The energy scrapers in $\mathrm{BC} 1$ and $\mathrm{BC} 2$ are straightforward. 
The pre-linac collimation system consists of the energy collimation preceded by a beam emittance diagnostic station and followed by a tune up dump, which is reached by a pulsed bend, so that the pre-linacs can be operated and tuned before sending the beam around the $180^{\circ}$ arc of $\mathrm{BC} 2$. In addition, the positron system must be able to deliver electrons to the downstream systems for commissioning. Polarized electrons are required for $\gamma-\gamma$ or $e^{-}-e^{-}$running.

The optics through the pre-linac is shown in Figure 3.4.4.7. There is a 4-wire 2D emittance diagnostic section after the pre-Linac which is followed by the collimation system. The collimation section is designed to remove up to $1 \%$ of the beam without increasing the emittance or the transverse jitter. Not shown explicitly are the locations of the energy scrapers, the dump kicker and the tuneup dump line.

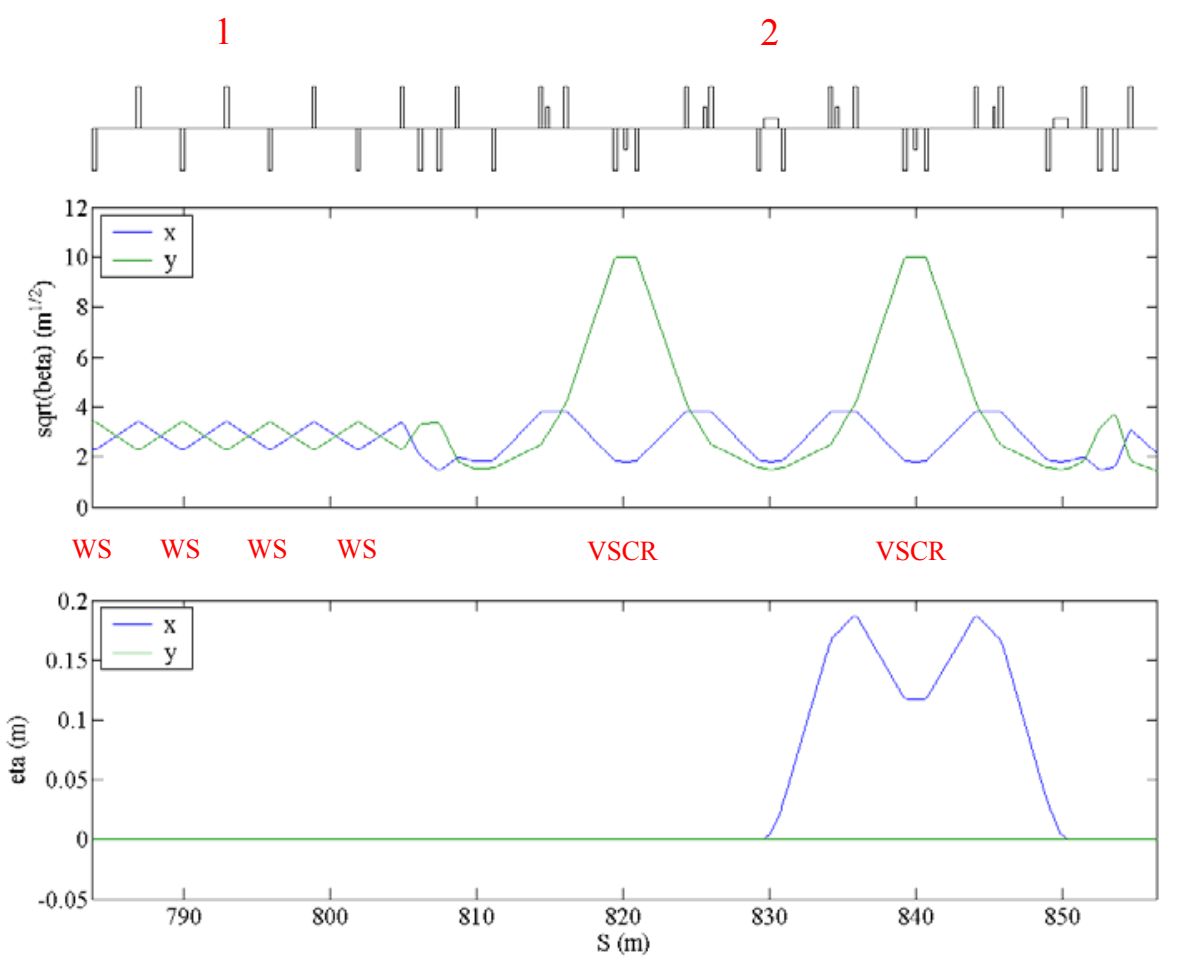

Figure 3.4.4.7: Optics through the pre-Linac collimation.

3.4.4.5.1 Layout and Functional Description Optically, the pre-linac collimation beamline system has a matching section, a collimation section and another matching section, with a 4 laser wire emittance diagnostic section. At the end of the pre-linac collimation beamline there is a short tuneup dump line, which is reached by a pulsed bend. Most beam focusing and chromatic correction is accomplished using tunable, permanent magnet quadrupoles and sextupoles, though the matching sections have electromagnetic quadrupoles and feedback correctors. Each quadrupole has a BPM. Diagnostics in the pre-linac collimation include laser wires for emittance measurements, a streak camera, toroids and PLIC.

The pre-linac collimation beamline must have one enclosure for the beamline components and local electronics, which cannot be accessed while beams are running. One 40' x 14' support area, that is accessible during beam operation, is also needed. 
3.4.4.5.2 Magnet system The quadrupoles are all electromagnets. The sextupoles are tunable permanent magnets. All quadrupoles and sextupoles are on independent $x, y$ and roll movers and each contain a BPM. The polarities of the magnets in the positron line are reversible to allow $e^{-}$operation.

3.4.4.5.3 Vacuum System The vacuum system is a manifold ion pump design with a hot filament gauge. The operating vacuum pressure is $<10^{-8}$ Torr.

3.4.4.5.4 Instrument System At the end of the pre-linac collimation there is a laser wire system, which can measure beam emittance and bunch length. Measurements are made at four separate locations along the beam path. A streak camera also measures the bunch length of the beam. There is a laser wire polarimeter in the dump line at the end of the pre-linac collimation. Pre-linac collimation beam loss is detected by PLIC, which runs its entire length. The beam line instrumentation must function equally well for either positron or electron beams.

\subsubsection{Second bunch compressor - BC-2}

The electron and positron second bunch compression systems (BC-2) compress the bunch length of the beams from the pre-collimation or reverse bend system length of $500 \mu \mathrm{m}$ to between $90-150 \mu \mathrm{m}$, as required for acceptance by the main linac systems. Each BC-2 beamline has an $180^{\circ}$ turnaround arc, a post arc 600 $\mathrm{MeV} \mathrm{X}$-band RF acceleration section and a chicane magnet section. The BC-2 is part of the second stage of compression in the injector, which requires a pseudo $2 \pi$ longitudinal phase space rotation for stability, and is achieved by using two RF systems: the pre-linac and the $\mathrm{BC}-2$ post arc $\mathrm{X}$-Band $\mathrm{RF}$ section, and two magnetic compressors: the $\mathrm{BC}-2180^{\circ}$ turnaround arc and the $\mathrm{BC}-2$ magnet chicane. The $\mathrm{BC}-2$ has multiple diagnostic regions for measuring emittance, bunch length and energy spread. There is a pulsed bend tune up dump off the end of the BC-2, so the beams can be tuned without sending them into the main linacs. In addition, the positron system must be able to deliver electrons to the downstream systems for commissioning. Polarized electrons are required for $\gamma-\gamma$ or $e^{-}-e^{-}$running.

The optics of the first half of the second bunch compressor BC2 is shown in Figure 3.4.4.8. There is an $180^{\circ}$ arc with 4 sextupoles for matching the 2nd order dispersion. Diagnostics include a bunch length monitor and synchrotron light monitor. This is followed by the X-band compressor, a chicane and another diagnostics section as shown in Figure 3.4.4.9. The numbers indicate the location of the key elements. Not shown explicitly are the dump kicker and tuneup dump line.

1. "quad scan" emittance wire scanner

2. X-band compressor RF

3. compressor chicane

4. feedback section

5. 4-wire 2D emittance diagnostic section

6. diagnostic chicane

The beam parameters before and after the 2nd bunch compressor are given in Table 3.4.4.6. 

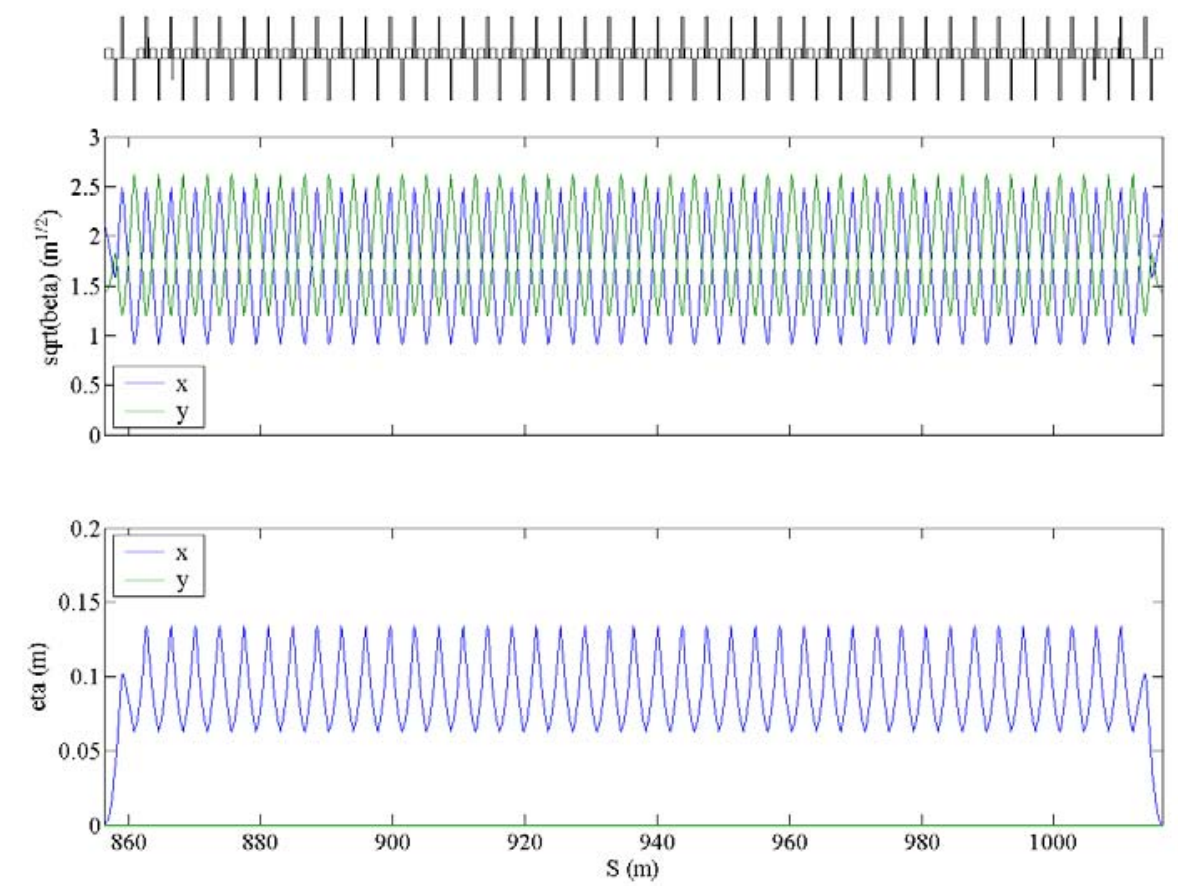

Figure 3.4.4.8: Optics through the $180^{\circ}$ arc of the second bunch compressor BC2. 

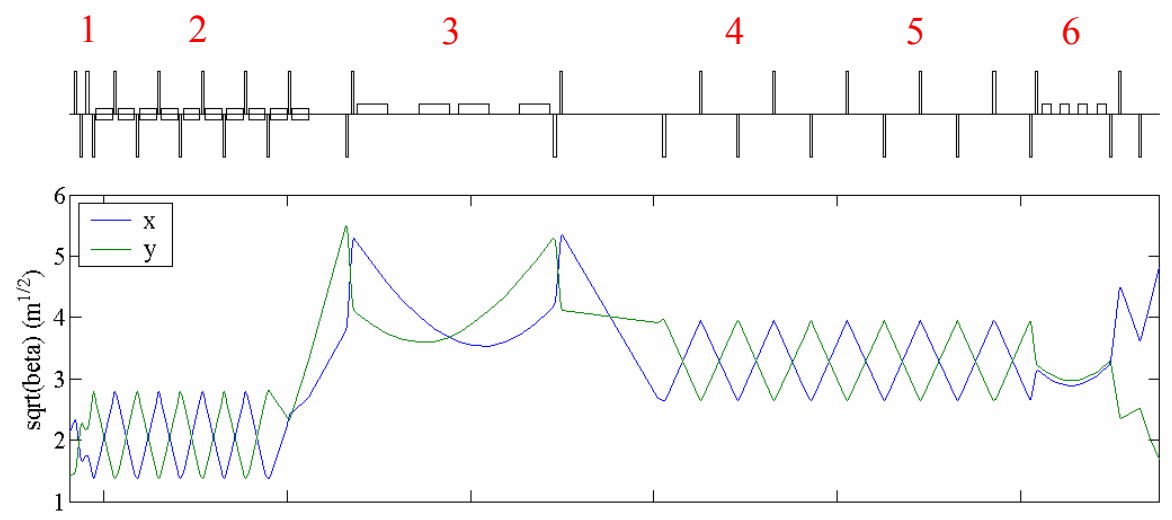

WS WS WS WS WS

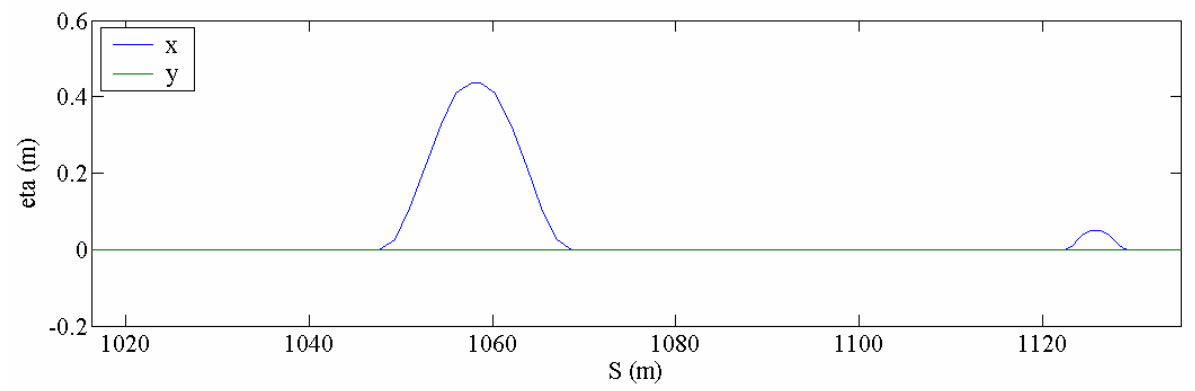

Figure 3.4.4.9: Optics through the second half of BC2, including the X-band compressor, chicane and diagnostics.

Table 3.4.4.5: Beam parameters before and after the second bunch compressor

\begin{tabular}{|c|c|c|c|c|}
\hline Parameter Name & Symbol & Input & Output & Unit \\
\hline Beam energy & $E$ & \multicolumn{2}{|r|}{8} & $\mathrm{GeV}$ \\
\hline Energy adjustability & $\Delta E / E$ & \multicolumn{2}{|r|}{5} & $\%$ \\
\hline Bunch energy variation & $\delta E / E$ & \multicolumn{2}{|r|}{1} & $\%$ Full Width \\
\hline Energy spread & $\sigma_{E} / E$ & 0.25 & 1.5 & $\% \mathrm{rms}$ \\
\hline Horizontal emittance (norm. rms) & $\gamma \varepsilon_{x}$ & \multicolumn{2}{|r|}{3} & mm-mrad \\
\hline Vertical emittance (norm. rms) & $\gamma \varepsilon_{y}$ & \multicolumn{2}{|c|}{0.02} & mm-mrad \\
\hline Horizontal Beam Jitter & $\Delta \gamma J_{x}$ & & 015 & mm-mrad \\
\hline Vertical Beam Jitter & $\Delta \gamma J_{u}$ & \multirow{2}{*}{\multicolumn{2}{|c|}{$\begin{array}{c}0.0008 \\
500 \quad 90-150\end{array}$}} & mm-mrad \\
\hline Bunch length (rms) & $\sigma_{z}$ & & & $\mu \mathrm{m}$ \\
\hline
\end{tabular}

3.4.4.6.1 Layout and Functional Description Optically the BC-2 beamline starts with an $180^{\circ}$ arc, which has dispersion suppression sections on each end. After the arc there is a $600 \mathrm{MeV} \mathrm{X}$-band compressor acceleration section and then a chicane compression section. At the end of the BC-2, there is a matching section that has a 4-laser wire emittance diagnostic followed by a full power beam dump which is reached by a pulsed bend.

Beam focusing and steering is accomplished using electromagnetic quadrupoles on movers for most of the BC-2. Each quadrupole has a BPM. Each BC-2 has one 4-D emittance measurement laser wire area, two bunch length measuring cavities and streak cameras, one laser wire for energy spread measurements, and a 
laser polarimeter. Beam loss is detected by PLIC through the entire beamline.

3.4.4.6.2 Magnet system The quadrupoles are electromagnets. All quadrupoles are on independent $x, y$ and roll movers and each quadrupole contains a BPM. The $180^{\circ}$ arc bending magnets are either fixed field permanent magnets or electromagnets while the chicane has four $1.3 \mathrm{~m}$ electromagnetic bends. The polarities of the magnets in the positron line are reversible to allow $e^{-}$operation.

3.4.4.6.3 Vacuum System The accelerator vacuum module system is a manifold ion pump scheme, with a hot filament gauge. The operating vacuum pressure is $<10^{-8}$ Torr.

3.4.4.6.4 Instrument System Beam emittance measurements are made at the end of the BC-2 using a laser wire system. Two pairs of RF cavities and streak cameras measure the bunch length of the beam, one pair after the $180^{\circ}$ arc and the other at the end of the BC-2. There is a laser wire polarimeter in the dump-line at the end of the BC-2, which measures the polarization and energy spread of the beam. BC-2 beam loss is detected by PLIC, which runs its entire length. All quadrupoles are on independent $x, y$ and roll movers and each quadrupole contains a BPM. The beam line instrumentation must function equally well for either positron or electron beams.

3.4.4.6.5 Infrastructure The BC-2 beamline must have one enclosure for the beamline components and local electronics, which cannot be accessed while beams are running and three 40' x 14' support areas, that are accessible during beam operation.

\subsubsection{Summary of components}

The complete list of magnets and RF modules (and their specifications) for the entire ring-to-linac bunch compressor system is given in Table 3.4.4.7. 
Table 3.4.4.6: Major system components

\begin{tabular}{|c|c|c|c|}
\hline Element & Number & Max. Field & Total length \\
\hline \multicolumn{4}{|c|}{ DRx and BC1 } \\
\hline Bends & 28 & $14.491 \mathrm{kG}$ & $18.801 \mathrm{~m}$ \\
\hline Quadrupoles & 96 & $564.38 \mathrm{kG} / \mathrm{m}$ & $12.167 \mathrm{~m}$ \\
\hline Solenoids & 4 & $\pm 37.5 \mathrm{kG}$ & $1.38 \mathrm{~m}$ \\
\hline Correctors & 32 & & \\
\hline Wiggler dipoles & 10 & $10.2-12.3 \mathrm{kG}$ & $0.8 \mathrm{~m}$ \\
\hline L-band klystrons & 1 & & \\
\hline L-band RF structures & 2 & $14 \mathrm{MV} / \mathrm{m}$ & $5.039 \mathrm{~m}$ each \\
\hline Q BPMs & 96 & & \\
\hline movers $(x, y$, roll $)$ & 96 & & \\
\hline 4-Wire Diagnostic & 2 & & \\
\hline bunch length diagnostics & 2 & & \\
\hline streak camera & 2 & & \\
\hline energy spread diagnostics & 2 & & \\
\hline laser wire polarimeter & 1 & & \\
\hline \multicolumn{4}{|c|}{ PLIN and PCOL } \\
\hline Quadrupoles & 73 & $270.15 \mathrm{kG} / \mathrm{m}$ & $16.800 \mathrm{~m}$ \\
\hline S-band RF modules & 18 & & \\
\hline S-band klystrons & 36 & & \\
\hline S-band structures & 108 & $17 \mathrm{MV} / \mathrm{m}$ & $4.035 \mathrm{~m}$ each \\
\hline Q BPMs & 73 & & \\
\hline S BPMs & 324 & & \\
\hline movers $(x, y$, roll $)$ & 181 & & \\
\hline 4-Wire Diagnostic & 1 & & \\
\hline RF cavity bunch length meas. & 0 & & \\
\hline streak camera & 0 & & \\
\hline energy spread diagnostics & 1 & & \\
\hline laser wire polarimeter & 0 & & \\
\hline \multicolumn{4}{|c|}{$\mathrm{BC} 2$} \\
\hline Bends & 94 & $9.748 \mathrm{kG}$ & $103.33 \mathrm{~m}$ \\
\hline Quadrupoles & 156 & $921.83 \mathrm{kG} / \mathrm{m}$ & $40.560 \mathrm{~m}$ \\
\hline $\mathrm{X}$-band klystrons & 10 & & \\
\hline $\mathrm{X}$-band structures & 30 & $33 \mathrm{MV} / \mathrm{m}$ & $60 \mathrm{~cm}$ each \\
\hline Q BPMs & 156 & & \\
\hline S BPMs & 90 & & \\
\hline movers $(x, y$, roll $)$ & 186 & & \\
\hline 4-Wire Diagnostic & 1 & & \\
\hline RF cavity bunch length meas. & 2 & & \\
\hline streak camera & 2 & & \\
\hline energy spread diagnostics & 1 & & \\
\hline laser wire polarimeter & 1 & & \\
\hline
\end{tabular}




\subsubsection{Main Linac}

\subsubsection{Introduction}

The main linacs accelerate the electron and positron beams from the output of the bunch compressors, at $8 \mathrm{GeV}$, to the collision energy. To support low energy operation, there is a bypass line installed alongside the main beamline with pickoffs at $50 \mathrm{GeV}, 150 \mathrm{GeV}$ and $250 \mathrm{GeV}$. These extraction points are located just downstream of three diagnostic stations. In the initial installation, the second half of each linac tunnel is a drift region which is long enough to allow for $1 \mathrm{TeV}$ center of mass (cms) operation by adding more RF sections of the original design (the operating parameters would also remain the same). A schematic of the positron linac is shown in Figure 3.4.5.1. The electron linac differs in that it includes a $850 \mathrm{~m}$ long area just upstream of the $150 \mathrm{GeV}$ diagnostic region that contains an off-axis undulator. The radiation produced in the undulator by the nominal electron beam impinges on a thin target from which positrons are collected. The positrons are then accelerated to $1.98 \mathrm{GeV}$ in an L-Band linac and transported through the beamline tunnels to the positron pre-damping ring.

The overall design and specification of the main linacs is the same as the current NLC design, which is similar to that presented in the TRC description of JLC-X/NLC[TRC, Chapter 3]. Besides the addition of the undulator, the primary differences from the TRC configuration are:

- The use of $60 \mathrm{~cm}$ long X-band structures with an initial group velocity of $3 \%$ instead of $90 \mathrm{~cm}$ structures with an initial group velocity of $5 \%$ (both designs have a $150^{\circ}$ phase advance per cell).

- The use of a 'two-pack' modulator to power a pair of klystrons instead of one modulator for eight klystrons, to better match the SLED-II pulse compression.

- The use of electromagnetic quadrupoles for the main linacs instead of adjustable permanent magnet quadrupoles. Permanent magnets are still planned for the bypass lines and parts of the injectors[17].

The design $500 \mathrm{GeV}$ cms beam parameters for the main linacs are presented in Table 3.4.5.1. Table 3.4.5.3 and Table 3.4.5.2 give overviews of the main linac components for both $500 \mathrm{GeV}$ and $1 \mathrm{TeV}$ cms.

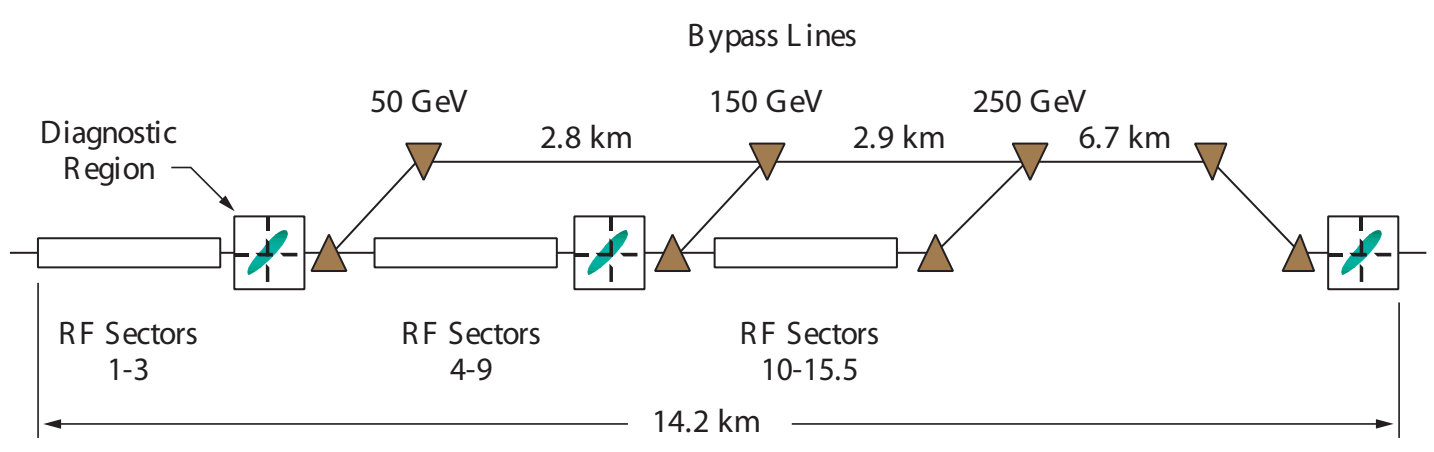

Figure 3.4.5.1: Schematic of the X-band positron linac layout; each RF sector contains $72 \mathrm{RF}$ units, each 6 meters long. 
Table 3.4.5.1: Design linac beam parameters for $500 \mathrm{GeV}$ cms operation.

\begin{tabular}{lc}
\hline Accelerator gradient $[\mathrm{MV} / \mathrm{m}]$ & 52 \\
Injection energy $[\mathrm{GeV}]$ & 8 \\
Final energy $[\mathrm{GeV}]$ & 250.5 \\
Bunch charge $\left[10^{10}\right]$ & 0.75 \\
Bunch spacing $[\mathrm{ns}]$ & 1.4 \\
Bunch length $[\mu \mathrm{m}]$ & 110 \\
Normalized rms emittance at injection $\epsilon_{x}, \epsilon_{y}\left[10^{-6} \mathrm{~m} \cdot \mathrm{rad}\right]$ & $3,0.02$ \\
Budgeted emittance growth in linac $\epsilon_{x}, \epsilon_{y}\left[10^{-6} \mathrm{~m} \cdot \mathrm{rad}\right]$ & $0.3,0.01$ \\
Initial uncorrelated energy spread $\sigma_{E, i} / E[\%]$ & 1.5 \\
Off-crest RF phase $\Phi_{r f}(8$ to $30 / 30$ to $170 / 170$ to $250 \mathrm{GeV})\left[^{\circ}\right]$ & $-10 / 3 / 30$ \\
Energy spread $\sigma_{E, f} / E$ at linac exit $\left[10^{-3}\right]$ & 2.5 \\
\hline \hline
\end{tabular}

Table 3.4.5.2: Overview of components in the positron main linac for $500 \mathrm{GeV}$ and $1 \mathrm{TeV}$ cms.

\begin{tabular}{lcc}
\hline Center of mass energy & $500 \mathrm{GeV}$ & $1 \mathrm{TeV}$ \\
Length of RF sections [m] & 6710 & 13420 \\
Length Diagnostic/Bypass regions [m] & \multicolumn{2}{c}{752} \\
Total non-drift linac length [m] & 7462 & 14172 \\
Total linac tunnel length [m] & \multicolumn{2}{c}{14172} \\
Number of RF feeds & 1116 & 2232 \\
Number of modulators & 1116 & 2232 \\
Number of klystrons & 2232 & 4464 \\
Number of structures & 8928 & 17856 \\
Length of RF feed (with quad) [m] & \multicolumn{2}{c}{6.0} \\
Number of quadrupoles & 765 & 1130 \\
\hline \hline
\end{tabular}

Table 3.4.5.3: Overview of components in the electron main linac for $500 \mathrm{GeV}$ and $1 \mathrm{TeV}$ cms. In comparison to the positron linac, this linac includes an undulator for positron production and additional RF units to make up for the $5.9 \mathrm{GeV}$ beam energy loss in the undulator.

\begin{tabular}{lcc}
\hline Center of Mass energy & $500 \mathrm{GeV}$ & $1 \mathrm{TeV}$ \\
Length of RF sections [m] & 6874 & 13584 \\
Length of Diagnostic/Bypass regions [m] & \multicolumn{2}{c}{752} \\
Length of Undulator insertion [m] & \multicolumn{2}{c}{850} \\
Total non-drift linac length [m] & \multicolumn{2}{c}{1576} \\
Total linac tunnel length [m] & \multicolumn{2}{c}{15186} \\
Number of RF feeds & 1144 & 2260 \\
Number of modulators & 1144 & 2260 \\
Number of klystrons & 2288 & 4520 \\
Number of structures & 9152 & 18080 \\
Length of RF feed (with quad) [m] & \multicolumn{2}{c}{6.0} \\
Number of quadrupoles & 928 & 1293 \\
\hline \hline
\end{tabular}




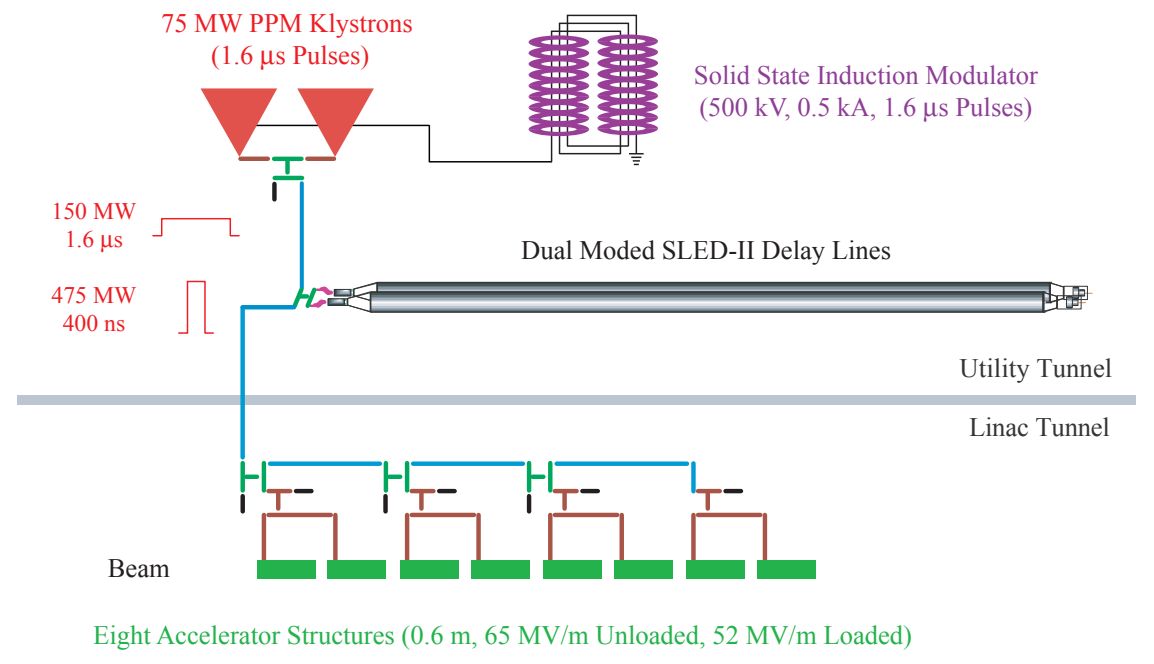

Figure 3.4.5.2: Schematic of an X-band linac RF unit.

\subsubsection{RF System Overview}

The X-band RF system design uses 11.424-GHz components similar in function to those in the 2.856-GHz SLAC linac. As at SLAC, electrical energy is transformed in several stages: modulators convert AC power to high-voltage pulsed DC; klystrons transform the pulsed DC to high-power RF; pulse compressors transform the long klystron pulses to a shorter, higher peak pulses that are more optimal for the structures, and finally, the RF power fed to the structures accelerates the beam. The basic RF component set that performs these functions is referred to as an RF unit.

\subsubsection{RF units}

The baseline RF unit is illustrated in Figure 3.4.5.2. The positron (electron) linac contains 1116 (1144) such RF units for $500 \mathrm{GeV}$ cms operation. Each RF unit contains a solid-state induction modulator that drives two 75-MW, 1.6- $\mu \mathrm{s}$, PPM klystrons. Each klystron pair powers a dual-moded SLED-II compression system that generates shorter (times four) but higher peak power (times three) pulses. The resulting $400 \mathrm{~ns}, 475$ MW pulses feed eight, 0.6-m long accelerator structures, producing a $65 \mathrm{MV} / \mathrm{m}$ unloaded gradient in each structure.

The linac beam-line enclosure contains the accelerator structures while the modulators and klystrons are housed in a separate utility tunnel that runs parallel to the beam line ( $6 \mathrm{~m}$ horizontal offset). This configuration simplifies access and maintenance which is essential to ensure the desired reliability and collider availability. The SLED-II delay lines are placed in the utility tunnel to allow easy access for upgrades and maintenance. There is a penetration between the two tunnels every 12 meters to route the SLED-II output power to the structures. During construction, the installation of the RF units would start from the lowenergy end of the tunnel to allow maximum flexibility in choosing the appropriate energy upgrade steps to match physics interests and funding profiles.

To reduce both construction and operating costs, much effort has been focused on maximizing the efficiency of the conversion and transfer of energy at every stage of the RF system. The pulse compression system chosen for the baseline design is dual-moded SLED-II, however, both the JLC and NLC design teams have been investigating alternate systems with higher efficiency, in particular, the Delay Line Distribution System 
(DLDS). This system is discussed as a variant option in Section 7.4.

The parameters of the X-band major RF subsystems (klystrons, modulators, RF distribution, and accelerator structures) are listed in Table 3.4.5.4 for the positron linac. The table shows changes between the present NLC 2003 configuration and the JLC-X/NLC design as published in the TRC report. The unloaded gradient $\left(G_{U}\right)$ of $65 \mathrm{MV} / \mathrm{m}$ is close to optimal in the tradeoff between energy-related costs (e.g., modulators and klystrons), which scale roughly as $G_{U}$, and length-related costs (e.g., structures and beam-line tunnel), which scale roughly as $1 / G_{U}$. However, the overall linac cost has a fairly weak dependence on unloaded gradient in the range of interest for the collider (50 to $100 \mathrm{MV} / \mathrm{m}$ ). (See Fig. 5.3.2.1). The beam parameters were chosen as a tradeoff between increasing RF-to-beam efficiency and easing tolerances related to both short-range and long-range transverse wakefields effects.

The following subsections describe the RF components. More detailed information can be found in the JLC-X/NLC description in the TRC report[TRC, Chapter 3] and in the JLC roadmap[GLC].

3.4.5.3.1 Modulators and Klystrons The solid-state induction modulator is designed to power two klystrons, rather than eight as was optimal in an earlier design that used DLDS pulse compression. The two-pack modulator contains two stacks of five cores that are made of Metglass (an amorphous ferromagnetic alloy), and are configured to inductively sum 20 low voltage sources. Each core is driven by two $4 \mathrm{kV}$ sources (capacitor banks) that are each switched by a Insulated Gate Bipolar Transistor (IGBT) to produce $3 \mathrm{kA}$ of primary current. A 12-turn secondary loop winds through the two stacks, producing an output pulse of $500 \mathrm{kV}, 500$ Amps. The timing of the IGBT switches is adjusted to yield a flat (to 1\%), $1.6 \mu$ s long pulse. The design rise and fall times of the pulse (10\% to $90 \%$ ) is $200 \mathrm{~ns}$, and the overall efficiency (AC to usable flattop pulse) is $70 \%$. The design is based on the experience gained from the successful operation of four $50 \mathrm{MW}$ klystrons with a 3-turn solid-state induction modulator as part of the Eight-Pack system test being conducted at NLCTA.

Klystrons are being developed that efficiently amplify pulsed X-band RF to high power using a velocitymodulated electron beam vacuum tube, much like the S-band klystrons in the SLAC Linac. The beam in the X-band tubes, however, is focused by periodic permanent magnets (PPMs) instead of electromagnetic solenoids, which halves the average power consumption. The X-band design requires a klystron that produces $75 \mathrm{MW}$ of power in a $1.6 \mu \mathrm{s}$ pulse at a $120 \mathrm{~Hz}$ repetition rate with $55 \%$ efficiency. The JLC group in collaboration with Toshiba has produced a tube that meets the peak power requirements, but the tube was only operated at $25 \mathrm{~Hz}$ due to modulator limitations. Recently, a SLAC-built klystron has operated at full power, full pulse length and the full $120 \mathrm{~Hz}$ repetition rate.

3.4.5.3.2 RF Distribution System The RF distribution system transports the klystron output power to the accelerator structures. This task is complicated by the fact that the klystron pulse length and peak power, which were chosen to minimize klystron costs, are not optimal for powering the structures. To minimize the overall RF system cost, a pulse compression system is included to shorten the klystron output pulse while increasing the peak power.

Several compression methods have been considered during the past decade, but the most practical experience has come from the development of SLED-II, which is a delay line version of the SLAC Linac Energy Doubler (SLED). In its implementation in NLCTA, a 'magic-T' $3 \mathrm{~dB}$ hybrid splits the klystron power equally to fill two 40-m long, $12.1 \mathrm{~cm}$ diameter delay lines (circular waveguides). These lines are shorted at the far ends and have irises at the near ends that partially reflect the RF. During operation, the lines are resonantly filled during the first $5 / 6$ of the $1.5 \mu$ s long klystron pulse, and then effectively discharged through the remaining hybrid port by a 180 degree reversal of the klystron phase during the last $1 / 6$ of the pulse. This yields a shorter ( $1 / 6$ as long), higher power pulse that is used to power NLCTA accelerator structures. Although three SLED-II systems have worked well at NLCTA, with over 20 thousand hours of operation, they are not particularly efficient. Only about $65 \%$ of the input power ends up in the compressed pulse, so the power 
Table 3.4.5.4: Comparison of NLC 2003 positron main linac parameters with TRC values.

\begin{tabular}{|c|c|c|c|}
\hline Configuration & & NLC 2003 & TRC \\
\hline & Units & Values & Values \\
\hline \multicolumn{4}{|l|}{ Beam Parameters } \\
\hline Nominal CMS Energy & $\mathrm{TeV}$ & 0.5 & 0.5 \\
\hline Initial Beam Energy & $\mathrm{GeV}$ & 8 & 8 \\
\hline Final Beam Energy & $\mathrm{GeV}$ & 250.5 & 247.1 \\
\hline Linac Pulse Rate & $\mathrm{Hz}$ & 120 & 120 \\
\hline Number of Bunches per Pulse & & 192 & 192 \\
\hline Number of Particles per Bunch & $10^{10}$ & 0.75 & 0.75 \\
\hline Bunch Separation & ns & 1.40 & 1.40 \\
\hline Beam Current & $\mathrm{A}$ & 0.86 & 0.86 \\
\hline \multicolumn{4}{|l|}{ RF System } \\
\hline RF Units (2 Klystrons / SLED II) per Sector & & 72 & 72 \\
\hline Sectors with RF per Linac & & 15.5 & 14 \\
\hline Sector Length & $\mathrm{m}$ & 432.9 & 472.6 \\
\hline AC Power for Modulators per Linac & MW & 81.4 & 65.5 \\
\hline AC Power for Other RF + Cooling RF System per Linac & MW & 8.6 & 7.5 \\
\hline Total AC Power Related to RF per Linac & MW & 90.0 & 73.0 \\
\hline Beam Power per Linac & MW & 6.7 & 6.6 \\
\hline AC -to- Beam Power Efficiency & & $7.5 \%$ & $9.1 \%$ \\
\hline \multicolumn{4}{|l|}{ Modulators } \\
\hline Modulator Type & & 2 Pack & 8 Pack \\
\hline Modulator Efficiency & & $70 \%$ & $80 \%$ \\
\hline Number of RF Modulators per RF Unit & & 1 & 0.25 \\
\hline \multicolumn{4}{|l|}{ Klystrons } \\
\hline Klystron Type & & PPM & PPM \\
\hline Output Power & MW & 75.0 & 75.0 \\
\hline Number of Klystrons per RF Unit & & 2 & 2 \\
\hline Klystron Pulse Length & ns & 1559 & 1587 \\
\hline Klystron Efficiency & & $55 \%$ & $55 \%$ \\
\hline \multicolumn{4}{|l|}{ Pulse Compression } \\
\hline Type & & SLED II & SLED II \\
\hline Time Compression Factor & & 4 & 4 \\
\hline Compression Efficiency & & $74.6 \%$ & $75.0 \%$ \\
\hline Number of Pulse Compression Systems per RF Unit & & 1 & 1 \\
\hline Switching Time & ns & 10 & 8 \\
\hline RF Pulse Length per Feed & ns & 390 & 397 \\
\hline
\end{tabular}


Table 3.4.5.5: Comparison of NLC 2003 positron main linac parameters with TRC values (Part II).

\begin{tabular}{|l|c|c|c|}
\hline Configuration & & NLC 2003 & TRC \\
\hline & Units & Values & Values \\
\hline Structures & & H60VG3S17 & H90VG5S18 \\
Structure Type & $\mathrm{m}$ & 0.602 & 0.908 \\
Structure Length & $\mathrm{ns}$ & 111 & 6 \\
Number of Structures per Unit & $\mathrm{MW}$ & 64.5 & 120 \\
Fill Time & $(\mathrm{MV} / \mathrm{m}) / \mathrm{A}$ & 15.3 & 87.5 \\
Input Power for Unloaded Gradient of $70 \mathrm{MV} / \mathrm{m}$ & $\mathrm{MW}$ & 56.0 & 75.1 \\
Beam Loading per Amp of Current & $\mathrm{MV} / \mathrm{m}$ & 65.2 & 64.8 \\
Peak RF Power into Structure & $\mathrm{MV} / \mathrm{m}$ & 13.1 & 14.7 \\
Unloaded Accelerator Gradient & $\mathrm{MV} / \mathrm{m}$ & 0.38 & 0.34 \\
Multibunch Loading & $\mathrm{MV} / \mathrm{m}$ & 51.7 & 49.8 \\
Single Bunch Loading & $\mathrm{degrees}$ & 12.3 & 11.0 \\
Loaded Accelerator Gradient & $\%$ & $8 \%$ & $8 \%$ \\
Average RF phase & $\mathrm{MV} / \mathrm{m}$ & 45.1 & 43.5 \\
RF Overhead (3\% BNS + 3\% Failed + 2\% FB/Trips) & & 0.801 & 0.830 \\
Effective Gradient (Not Including Packing Fraction) & & \\
Packing Fraction (Active to Actual Length Ratio) & & \\
\hline
\end{tabular}

gain is about four.

The X-band reference design uses SLED-II pulse compression, but modified from the NLCTA design to lower cost and improve efficiency and power handling capability. The changes include larger diameter $(17.1 \mathrm{~cm})$ delay lines to lower power loss, dual-moded (TE01 and TE02) delay line transport to halve the delay line length, a lower compression ratio (4 instead of 6) to improve efficiency, and the use of over-height planar components for the $3 \mathrm{~dB}$ hybrid to lower surface fields. The design goal is to deliver $450 \mathrm{MW}, 400 \mathrm{~ns}$ pulses to the structures (including transport losses), which corresponds to an overall efficiency of $75 \%$. A prototype of such a system has been built. In December, 2003, it was tested successfully at the NLC 8-Pack, producing a peak power output of $580 \mathrm{MW}$ and a pulse length of $400 \mathrm{~ns}$.

3.4.5.3.3 X-Band Structures The linacs each contain about nine thousand, 0.6-m long X-band accelerator structures to increase the beam energy from the $8 \mathrm{GeV}$ at injection to $250 \mathrm{GeV}$ for collisions at the IP. There are four basic requirements on the structure design: it must transfer the RF energy to the beam efficiently to keep the machine cost low; it must be optimized to reduce the short-range wakefields which depend on the average iris radius; it must suppress the long-range transverse wakefields to prevent multibunch beam breakup; and it must operate reliably at the design gradient. A photo of an X-band structure in shown on the left in Figure 3.4.5.3.

In early 1.8-m long prototype structures, it was demonstrated that the required long-range wakefield suppression could be achieved using a combination of dipole damping and detuning. The right side of Figure 3.4.5.3 shows an example of a wakefield measurement where a hundred-fold reduction of the field was achieved by the time of the next bunch (1.4 ns) as is required for the X-band collider. The manifolds used in these structures to damp the dipole modes also serve well as 'tunable' beam position monitors: that is, the position at a particular location along the structure can be selected for measurement by frequency filtering the manifold signals. Tests using such signals to center the SLC beam in a structure achieved $11 \mu \mathrm{m}$ rms alignment, limited by beam quality (the requirement is $5 \mu \mathrm{m} \mathrm{rms}$ ).

High gradient tests of the 1.8-m structures, however, showed that they incurred breakdown related damage 

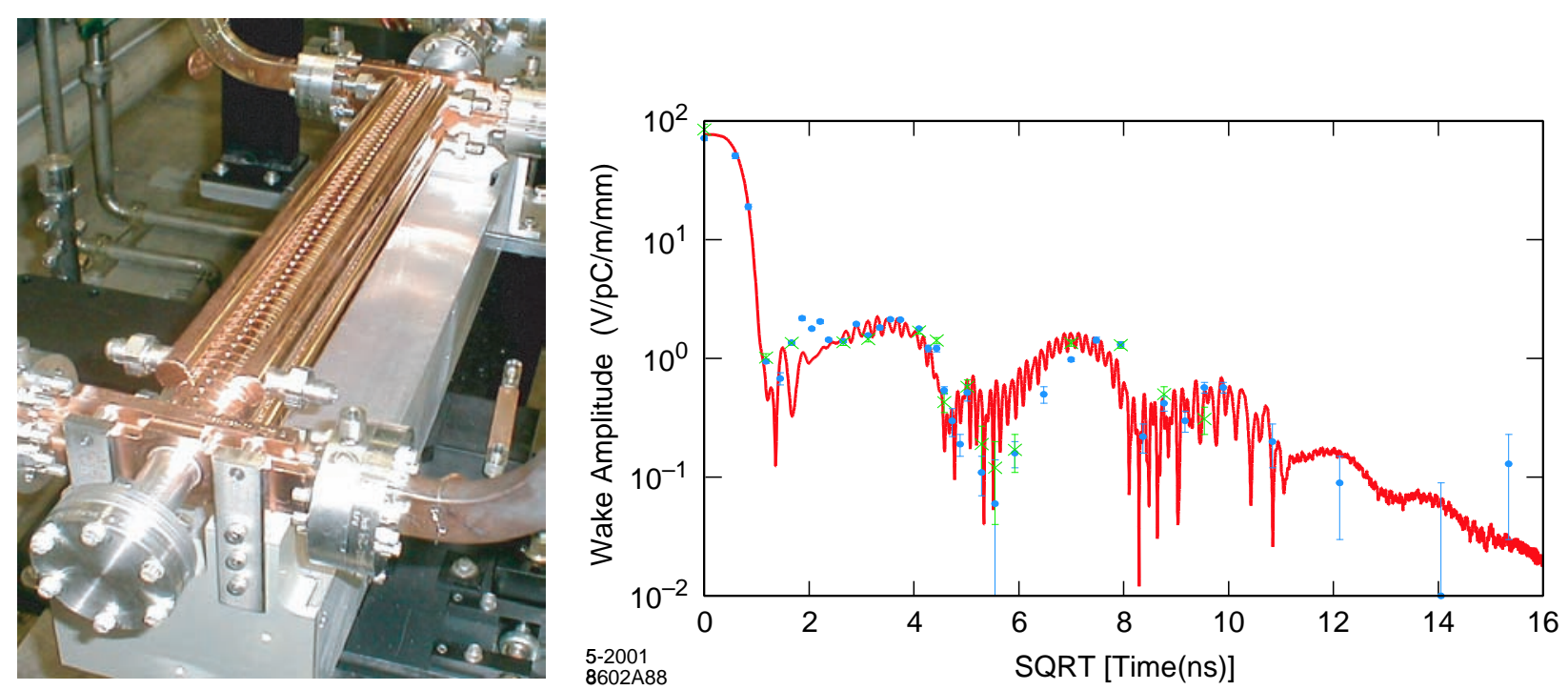

Figure 3.4.5.3: Experimental accelerator structure and measured performance. Left: Photograph of a test structure that is $53 \mathrm{~cm}$ long and has a 120 degree phase advance per cell and $a / \lambda=0.13$. Right: Comparison of the measured and predicted wakefield for the RDDS1 structure.

at gradients above about $50 \mathrm{MV} / \mathrm{m}$. During the last three years, shorter, lower group velocity structures have been developed that operate at higher gradients with little damage (up to $90 \mathrm{MV} / \mathrm{m}$ has been achieved). A recently tested structure with all essential linear-collider features basically met performance requirements at $65 \mathrm{MV} / \mathrm{m}$ (at $60 \mathrm{MV} / \mathrm{m}$, the breakdown rates were well within spec). A lower-power, lower surface field version has been recently adopted that should prove more robust. Like recent designs, it is $60 \mathrm{~cm}$ long and has a $150^{\circ}$ phase advance per cell. However, the average iris size is somewhat smaller $(a / \lambda$ of 0.17 instead of 0.18 ), which increases the transverse wakefield by a modest amount (20\%). Tests of these structures began in late 2003, and by Spring 2004, eight such structures will be operated together at NLCTA.

As noted above, eight structures are fed by one RF unit. However, the eight structures are supported on two girders, four on each, to decrease the sensitivity to temperature changes and to achieve more accurate beam-to-structure alignment.

\subsubsection{Optics}

The linac transport optics were chosen to minimize the dispersive and wakefield-related beam emittance growth. Quadrupole magnets, in a FODO configuration, are located after every (one, two, or three) RF girders at the (beginning, middle, or end) of each linac. The quadrupoles in the RF regions have 12.7-mmdiameter apertures and vary in length from $0.32 \mathrm{~m}$ to $0.96 \mathrm{~m}$. The RF girders and quadrupoles are supported on movers that can be remotely adjusted during beam operation based on signals from the structure manifolds and beam position monitors (BPMs) in the quadrupole magnets. The layout of girders and quadrupoles for the three sections of the machine is shown in Figure 3.4.5.4. The positron linac optical functions for 500 $\mathrm{GeV}$ cms and lower energies are shown in Figure 3.4.5.5 to Figure 3.4.5.7. The energy spread profile along the linac for $500 \mathrm{GeV}$ cms operation is shown in Figure 3.4.5.8. 

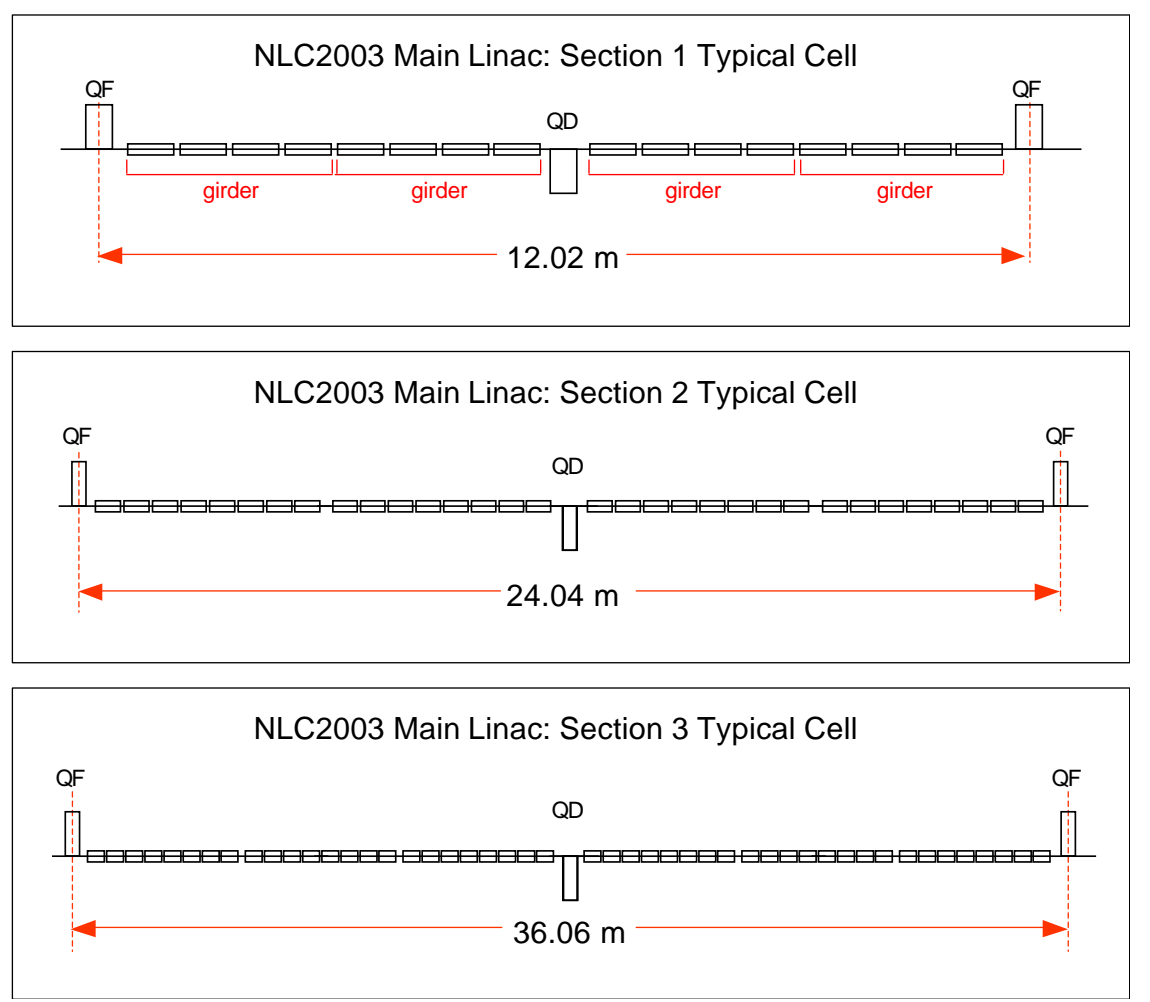

Figure 3.4.5.4: Schematic of the quadrupole layout for the main linac

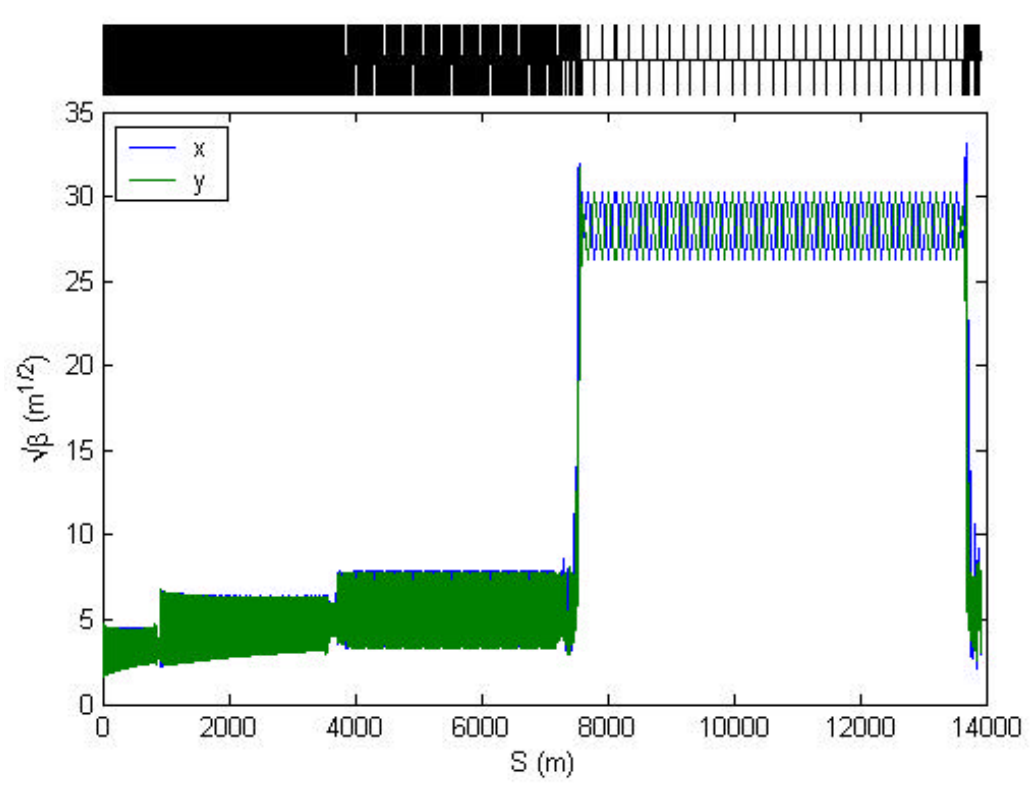

Figure 3.4.5.5: Main linac optics at $500 \mathrm{GeV}$ cms 


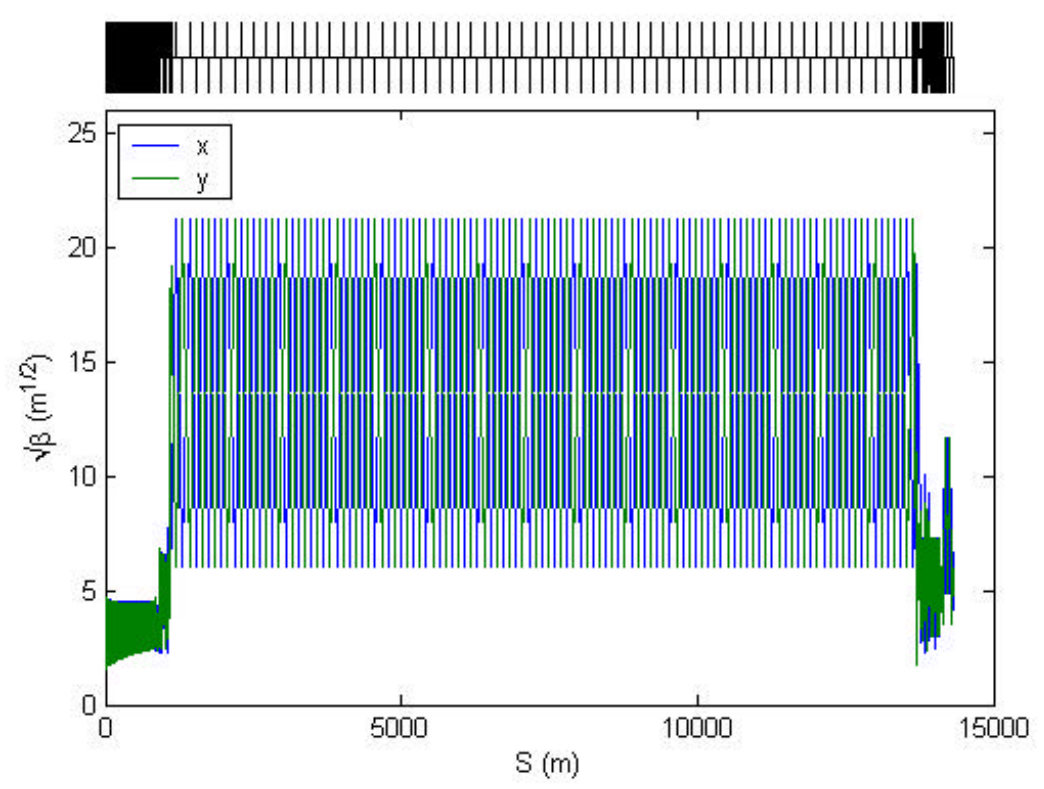

Figure 3.4.5.6: Main linac optics at $100 \mathrm{GeV}$ cms, including bypass line.

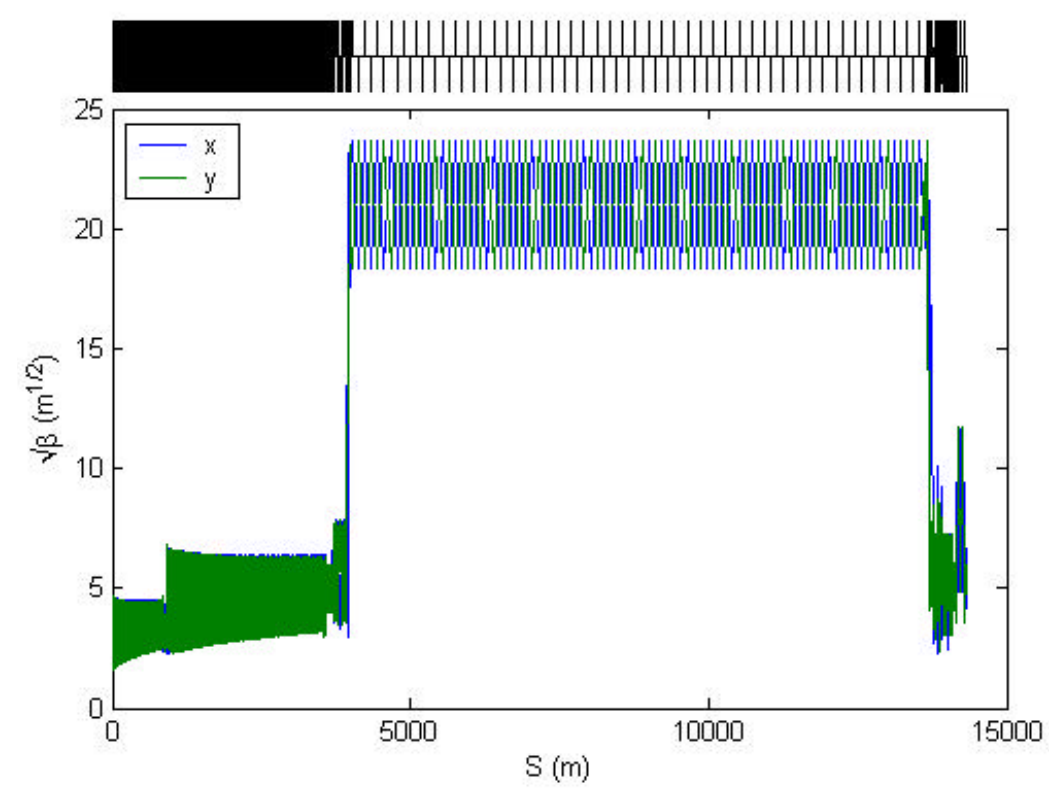

Figure 3.4.5.7: Main linac optics at $300 \mathrm{GeV}$ cms, including bypass line. 


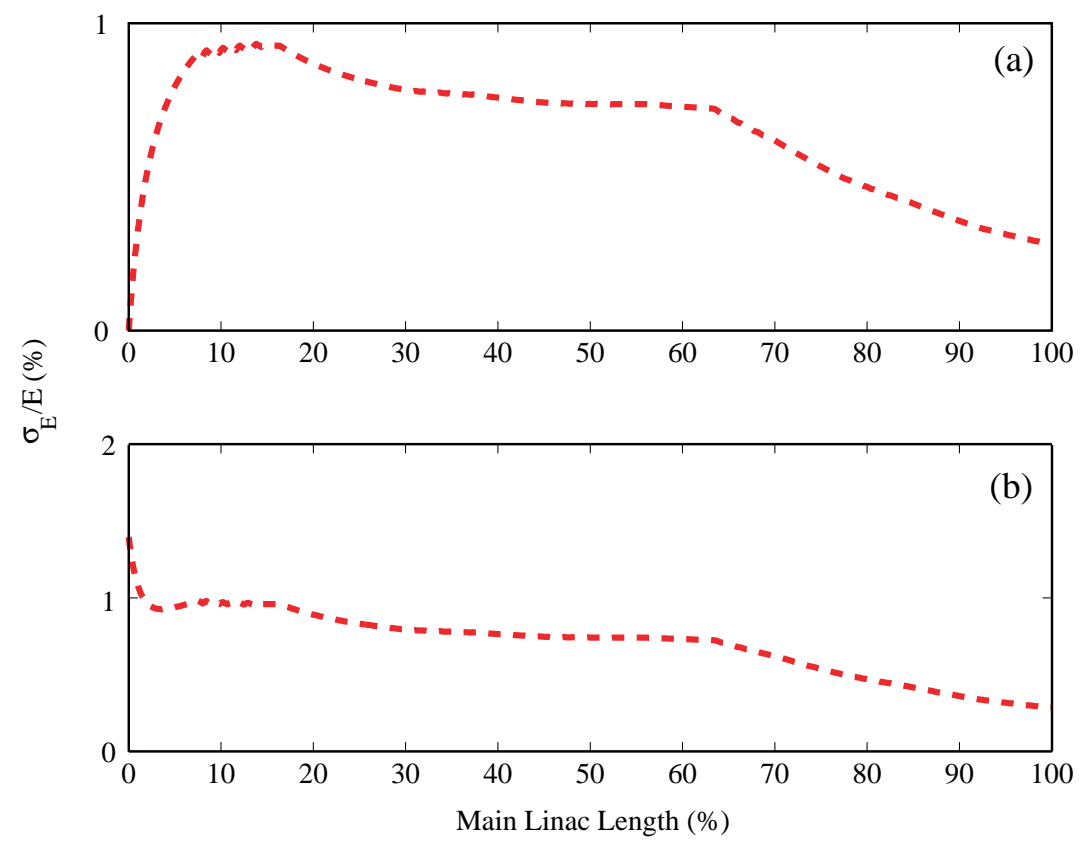

Figure 3.4.5.8: Correlated ("BNS") component (a) and total (b) energy spread profile along the main linac for $500 \mathrm{GeV}$ cms operation.

\subsubsection{Instrumentation and feedback}

Extensive effort has been made to ensure that the small beam emittances will be preserved in the linacs. The signals from the structure manifolds are used to directly align the accelerator structures to the beam as discussed in Section 3.4.5.3.3. BPMs are located in the quadrupole magnets and used in a beam-based procedure to align these magnets, which reduces dispersive emittance growth. Although the required quadrupole alignment is about 3 times smaller than achieved at FFTB, BPMs with 3 times better resolution are specified $(0.3 \mu \mathrm{m}$, which has been demonstrated in prototype RF BPMs). In addition, the dispersion-free steering technique pioneered at the SLC can be applied, and if necessary, emittance correction bumps used.

To monitor the beam energy, energy spread, and emittance, there are four diagnostic regions along the length of the linac where these parameters can be measured parasitically. In addition to being needed for the beam-based alignment and emittance correction techniques, continuous, non-invasive monitoring was found to be essential during the SLC operation because it facilitates rapid diagnosis of faults and makes it possible to correlate disparate effects.

The movers are similar to those used at the Final Focus Test Beam as shown in Figure 3.4.5.9. The step size required is a factor of 6 smaller than achieved at FFTB with microstepping technology. Similar types of movers have been used at the Swiss Light Source.

\subsubsection{Bypass lines}

Because of the transverse wakefields of the accelerator structures, it is undesirable to transport the beam through a large number of unpowered structures. In order to maximize luminosity at lower energy, a nonaccelerating "bypass" line is provided to bring the low-energy beams to the end of the linac. The bypass 

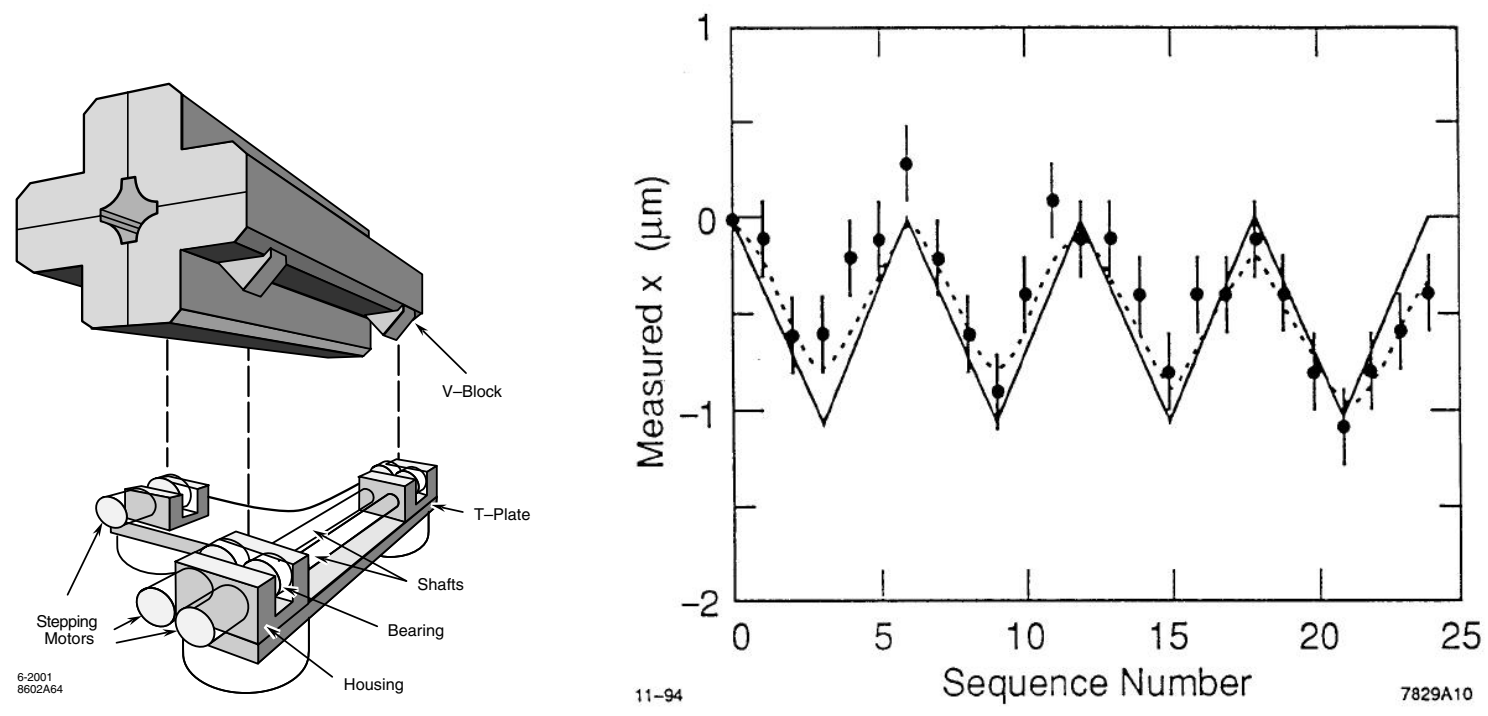

Figure 3.4.5.9: Final Focus Test Beam magnet mover schematic and performance. Left: Schematic. The mover had horizontal, vertical, and roll degrees of freedom, and could move magnets by $0.3 \mu \mathrm{m}$ steps over a range of $3 \mathrm{~mm}$. Right: Single Step performance test of mover - expected (solid) laser interferometer (dashed).

line shares the main-linac tunnel, and is installed at the same elevation as the main beamline. The design includes three transfer points where the beam can be diverted into the bypass line at 50, 150 and $250 \mathrm{GeV}$, and a return at the end of the linac to bring the beam back into the collimation section. These are sufficient to support a continuous variation of beam energy over the whole range. The first two transfer points are located just downstream of a diagnostic section on the linac and the reinjection is just before the end of linac diagnostic section. Optics layouts for the three transfer points and reinjection are shown in Fig. 3.4.5.10 to Fig. 3.4.5.11.
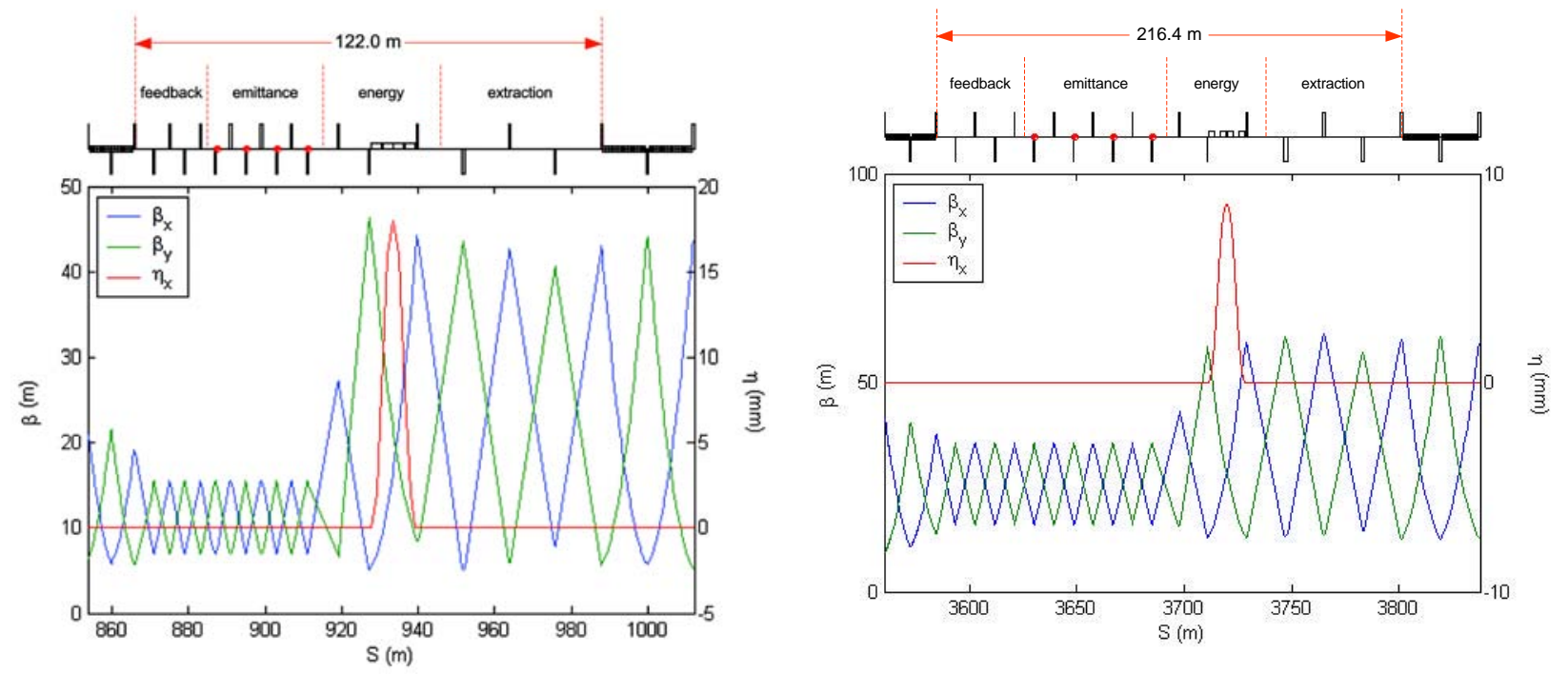

Figure 3.4.5.10: Main linac diagnostic/extraction section layout. Left: section 1 at $50 \mathrm{GeV}$ beam energy. Right: section 2 at $150 \mathrm{GeV}$ beam energy. 

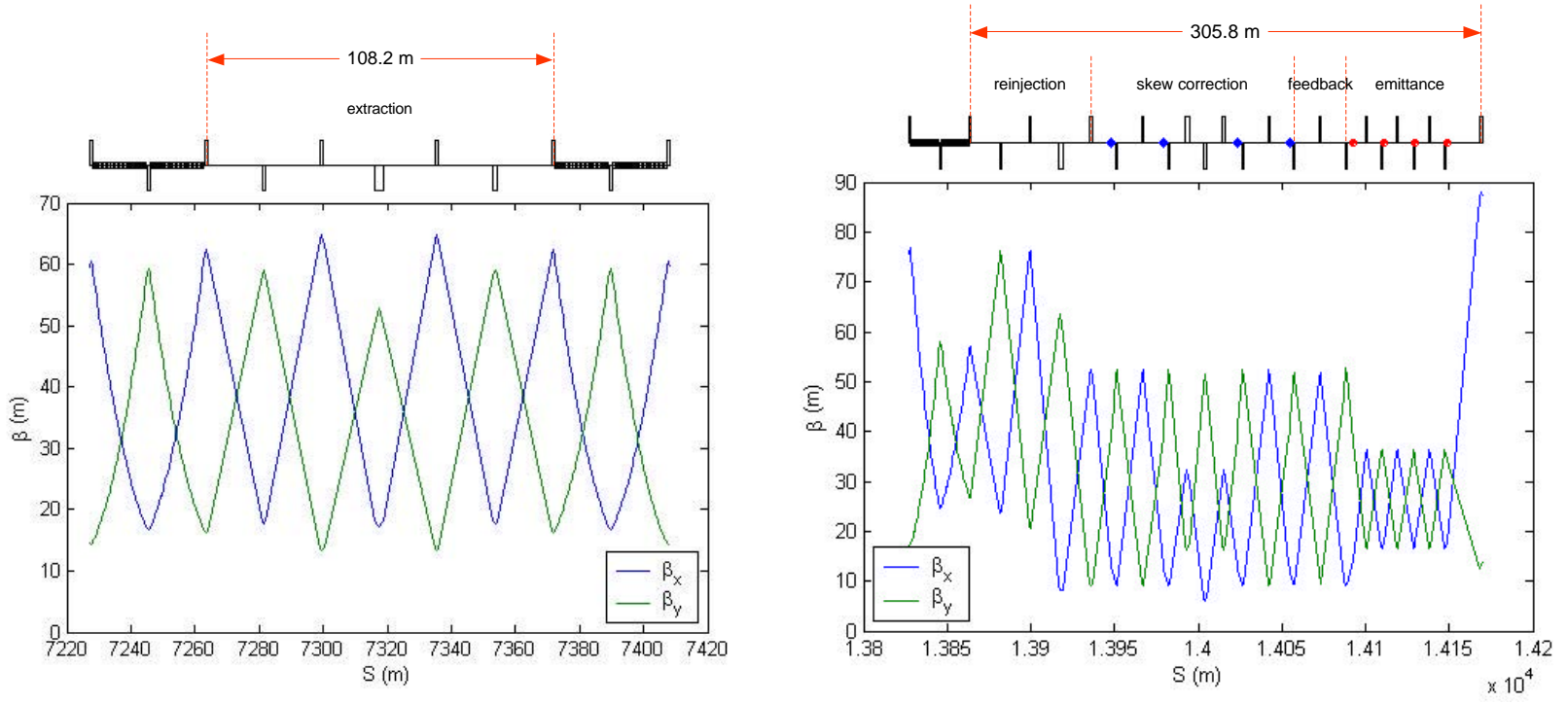

Figure 3.4.5.11: Main linac extraction and reinjection section layout. Left: extraction section 3 at $250 \mathrm{GeV}$ beam energy. Right: reinjection and BSY layout at $250 \mathrm{GeV}$ beam energy.

\subsubsection{Overheads}

For a large RF system, such as in the main linacs, some RF units will be out of service at any time, either awaiting repair or recovering from a brief fault. Additional spare RF units must be included to maintain the energy fixed, given the expected failure and fault rates, and repair or recovery times. For the X-band linac, off-crest operation for BNS damping requires 3\% additional energy. For klystron or other RF component failures, $3 \%$ spares are allocated. An additional $2 \%$ overhead is allocated to cover faults or breakdown events in the structures, klystrons or RF distribution. The assumed overheads are listed in Table 3.4.5.6.

A variety of scenarios were studied to verify that that $3 \%$ repair overhead was adequate with a conservative assumption of 20,000 hr klystron MTBF. The scenarios included both start-up and nominal operation, with a variety of worst case assumptions. The overhead available was adequate for all nominal operation scenarios and was exceeded only in the most extreme startup cases. Given an assumed recovery time of 10 sec after a fault, the required the Mean Time Between Faults for each component is computed to be such that the indicated overhead would be depleted only once a year on average due to that type of fault (in such cases, one would have to wait some fraction of the recovery time to be able to resume operation at full energy). Acceptable fault rates for structures are $<1$ per 4.6 hours, for klystrons $<1$ fault per 1.2 hours, for SLED-II $<1.7$ faults per hour.

Table 3.4.5.6: Overheads assumed

\begin{tabular}{l|l} 
BNS damping & $3 \%$ \\
Klystron failures & $3 \%$ \\
n/SLED faults including feedback & $2 \%$
\end{tabular}

\subsubsection{Beam Delivery Systems}

The beam delivery system (BDS) for the X-band collider is the same as the current NLC design. It includes recent improvements to extend the energy reach of the second Interaction Region (IR) and the use of 
superconducting magnets for the final doublets to provide energy flexibility. The Final Focus optics is the design proposed by Raimondi and Seryi in 2001 that places the chromatic correction locally in the final doublet itself [32]. Because it is more compact and has better performance, this final focus has been adopted by the other linear collider designs, including CLIC and the US superconducting linear collider reference design. The collimation and final focus are designed as an integrated system, with additional tail-folding octupoles. With the $20 \mathrm{mrad}$ crossing angle (30 mrad for the second IR), the extraction line optics allows for instrumentation downstream of the IR to monitor the parameters of the collided beams.

\subsubsection{Overview}

The beam delivery system (BDS) must both focus the beams to the sizes required to produce luminosity and remove any particles that are far enough from the beam core to produce unacceptable detector backgrounds. In addition, the BDS must provide protection for the detector and beamline components against missteered beams emerging from the main linacs, and must safely transport the collided beams to water dumps which can absorb the high beam power density without damage. Finally, the BDS must provide instrumentation that can monitor the parameters of the collided beams, such as the energy spread and polarization after collision, which are required by the particle physics experiments.

Although the parameters of the BDS for a $500 \mathrm{GeV}$ linear collider are far beyond anything that has been achieved in a storage ring, the SLC demonstrated the viability of a fully integrated linear collider beam delivery system with millimeter-sized betatron functions and routine collision of beams with rms sizes of under $1 \mu \mathrm{m}$. The FFTB at SLAC was a single-beam demonstration of a linear collider beam delivery system with IP betatron functions comparable to those in the next generation colliders. The BDS design is based upon experience from these two facilities.

In the final focus systems used at the SLC and the FFTB, which were also the basis of the 1996 NLC ZDR final focus design, the huge chromaticity generated by the strong quadrupole magnets close to the IP was compensated in separate "chromaticity correction" sections upstream of the IP. This design made the BDS extremely long and unfortunately had a very limited energy range over which the beam could be transported without distortion. The new design proposed by Raimondi and Seryi in 2001 places the chromatic correction locally in the final doublet itself [32]. This design greatly reduces the length required for the BDS and has much better performance over a wide range of energies.

\subsubsection{Layout}

The layout of the BDS components is shown in Figure 3.4.6.1. The six main subsystems of the beam delivery, from upstream to downstream, are: the emittance diagnostic and skew correction region, which provides parasitic measurement of the beam emittance and an orthogonal set of four skew quadrupoles to correct all sources of betatron coupling in the beam; the beam switchyard, which separates the beamlines to the two IRs; the collimation system, which provides protection from errant beams and removes particles which might cause backgrounds; the final focus (FF), which focuses the beams down to the small spots; the IR, which provides detector masking and specialized supports for the final doublet quadrupoles of the final focus; and the extraction line, which transports the spent beams to their respective dumps with optics that allows for instrumentation and diagnostics to characterize the IP beam properties such as current, position, energy spread and polarization on a bunch-by-bunch basis. For energy flexibility, compact superconducting magnets are used for the final doublets. In addition, a high-power pulsed beam dump is located in the beam switchyard region to allow the full-power linac beam to be tuned before sending the beam through the final focus.

The beamline for the first IR is $1.8 \mathrm{~km}$ long. This distance includes a $1.4 \mathrm{~km}$ long collimation and final focus region, a 150 meter region where the beamlines for the two IRs diverge, and a 200 meter emittance diagnostic and skew correction section. The beams intersect with a $20 \mathrm{mrad}$ horizontal crossing angle defined 


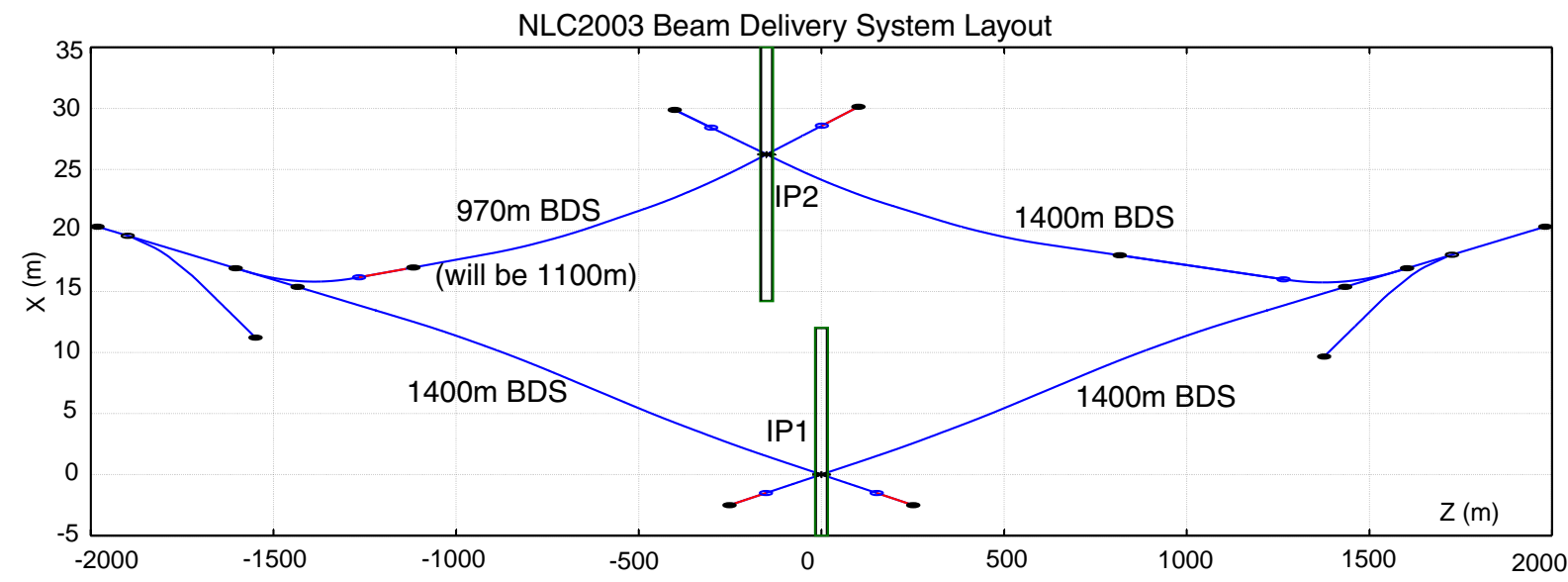

Figure 3.4.6.1: Beam delivery system layout with two interaction regions separated by $150 \mathrm{~m}$ longitudinally. The final focus optics for the $2^{\text {nd }}$ IR has been lengthened and uses a new "one-way" bending design to maximize the energy reach.

by the orientation of the main linacs. The second IR beamline splits off at the end of diagnostic region and includes arcs that bend the beam by about $17 \mathrm{mrad}$ followed by a collimation region and final focus that bend the beam in the same direction by $8 \mathrm{mrad}$. In the second IR, the beams intersect with a $30 \mathrm{mrad}$ horizontal crossing angle; this large crossing angle is compatible with gamma-gamma collisions. The two IRs are separated by about $35 \mathrm{~m}$ transversely and $150 \mathrm{~m}$ longitudinally to provide vibration isolation and shielding so either IR hall may be accessed while the other is in operation.

\subsubsection{Magnet Lattice, Optics and Subsystems}

The current NLC BDS has an integrated design for the final focus and collimation as shown in Figure 3.4.6.2. The BDS is based on the new design with local chromaticity correction, which has three clear advantages: first, the system requires many fewer magnets and is conceptually simpler. Second, it addresses a limitation of the earlier designs where the energy loss from synchrotron radiation between the last sextupole and the IP had to be minimized to avoid causing a breakdown of the chromaticity correction. Because of this requirement the bending magnets in the conventional final focus were weak, and the systems were correspondingly long. The present NLC configuration is much shorter than previous final-focus systems: less than $0.4 \mathrm{~km}$ is required for $750-\mathrm{GeV}$ beams as compared to $1.8 \mathrm{~km}$ in the NLC ZDR design and the present length of $0.7 \mathrm{~km}$ will handle 2500-GeV beams; the energy reach of the final focus is shown in Figure 3.4.6.3. Third, in the new design, off-energy particles tend to have small amplitudes in the final doublet magnet, whereas nonlinearities in the traditional final-focus systems tended to drive off-energy particles to very large amplitudes in the final doublet. The effect of the nonlinear amplitudes has not been considered in the past but the new design simplifies the beam collimation requirements significantly.

The elements of the transport lines from the end of the main linac to the IRs are given in Table 3.4.6.3. IR1 numbers are identical for either $e^{+}$or $e^{-}$beamlines. IR2 numbers are for either $e^{+}$or $e^{-}$beamlines, except as noted. 
Table 3.4.6.1: Beam delivery system elements. Numbers are for a single beam line. Parentheses indicate differences between IR1 and IR2, or between IR2 $e^{-}$and $e^{+}$lines. The indicated strengths are for $250 \mathrm{GeV}$ beam energy.

\begin{tabular}{|c|c|c|c|c|c|c|c|}
\hline \multirow[t]{2}{*}{ Element } & \multicolumn{2}{|c|}{ Number } & \multirow{2}{*}{$\begin{array}{l}\text { Z Position } \\
{[\mathrm{m}]}\end{array}$} & \multirow{2}{*}{$\begin{array}{l}\text { Diameter } \\
{[\mathrm{cm}]}\end{array}$} & \multirow{2}{*}{$\begin{array}{c}\text { Pole-Tip } \\
{[\mathrm{kG}]}\end{array}$} & \multirow{2}{*}{$\begin{array}{c}\text { Length } \\
{[\mathrm{cm}]}\end{array}$} & \multirow[t]{2}{*}{ Comment } \\
\hline & IR1 & IR2 & & & & & \\
\hline \multirow[t]{3}{*}{ IR1 Dipoles } & & & $738-1134$ & 2.00 & 0.081 & $360 \mathrm{~m}$ & \\
\hline & & & $389-507$ & 2.50 & 0.084 & $120 \mathrm{~m}$ & \\
\hline & & & $96-300$ & 6.00 & $0.02-0.07$ & $204 \mathrm{~m}$ & \\
\hline \multirow[t]{3}{*}{ IR2 Dipoles } & & & $1266-1583$ & 1.27 & $0.54-0.58$ & $208 \mathrm{~m}$ & \\
\hline & & & $86-607$ & 2.00 & $0.157-0.222$ & $343 \mathrm{~m}$ & \\
\hline & & & $24-55$ & 6.00 & $0.154-0.174$ & $152 \mathrm{~m}$ & \\
\hline \multirow[t]{11}{*}{ Quads } & 4 & 4 & $1862-1968$ & 1.20 & 0.000 & 50 & Skew quad \\
\hline & 26 & 20 & $1448-1980$ & 1.20 & $1.76-4.76$ & 100 & \\
\hline & 8 & 7 & $1437-1923$ & 1.20 & $0.087-3.19$ & 200 & \\
\hline & 1 & 1 & 1687 & 2.00 & 1.980 & 150 & \\
\hline & 26 & $55(67)$ & $520-1493$ & 2.00 & $0.033-4.78$ & 200 & \\
\hline & 7 & 11 & $356-774$ & 2.50 & $0.90-4.13$ & 200 & \\
\hline & 7 & 4 & $658-731$ & 4.00 & $0.039-5.72$ & 200 & \\
\hline & 3 & 0 & $165-219$ & 6.00 & $0.51-3.13$ & 200 & \\
\hline & 1 & 1 & 11.3 & 2.00 & 0.000 & 30 & Skew, in FD cryostat \\
\hline & 1 & 1 & 8.81 & 2.00 & $8.03(8.23)$ & 200 & $\mathrm{QF} 1, \mathrm{SC}$, in FD cryostat \\
\hline & 1 & 1 & 4.61 & 2.00 & $14.16(14.27)$ & 220 & QD0, SC, in FD cryostat \\
\hline \multirow[t]{4}{*}{ Sextupoles } & 1 & 3 & 522 & 2.00 & $0.27-1.49$ & 100 & \\
\hline & 2 & 0 & $358-373$ & 2.50 & $0.28-2.14$ & 30 & \\
\hline & 1 & 1 & 10.1 & 2.00 & $2.99(3.00)$ & 30 & $\mathrm{SC}$, in FD cryostat \\
\hline & 1 & 1 & 6.2 & 2.00 & $3.22(2.89)$ & 60 & $\mathrm{SC}$, in $\mathrm{FD}$ cryostat \\
\hline \multirow[t]{6}{*}{ Octupoles } & 2 & 2 & $638-646$ & 1.40 & & 300 & \\
\hline & 2 & 2 & $594-599$ & 1.40 & & 200 & \\
\hline & 1 & 1 & 556 & 1.40 & & 30 & \\
\hline & 1 & 1 & 538 & 1.40 & & 100 & \\
\hline & 1 & 1 & 10.7 & 2.00 & & 30 & $\mathrm{SC}$, in FD cryostat \\
\hline & 1 & 1 & 5.81 & 2.00 & & 10 & $\mathrm{SC}$, in FD cryostat \\
\hline
\end{tabular}




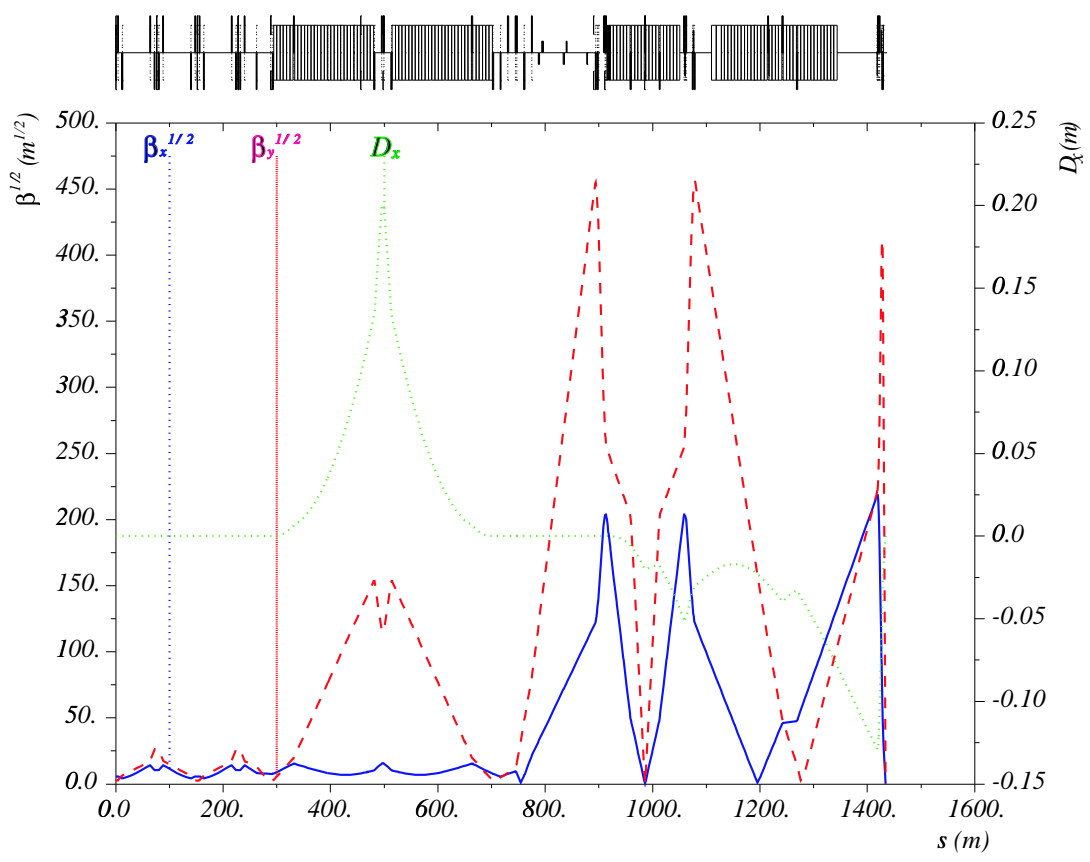

Figure 3.4.6.2: Optics of the NLC collimation and final focus systems for the first IR.

3.4.6.3.1 Second IR Design The location of the second IR is chosen to satisfy several considerations. The two IRs should be well separated to provide vibration isolation and sufficient shielding that one hall may be accessed while there is beam in the other. Since the beam dumps are potential sources of vibration, they should also be well separated from either IR. Finally, the path length difference for beams arriving at the two IRs, should be exactly equal to one damping ring circumference. To satisfy these constraints, the IRs are separated by about $35 \mathrm{~m}$ transversely and $150 \mathrm{~m}$ longitudinally.

Since the main linacs are angled to point directly to the $1^{\text {st }}$ IR, the beamlines for the $2^{\text {nd }}$ IR must have a bend of about $25 \mathrm{mrad}$. This bend causes emittance growth due to synchrotron radiation and inevitably limits the luminosity achievable at the highest energies. The newest optics design minimizes the required bending and increases the length available for the BDS. The beamline for the $2^{\text {nd }}$ IR splits off from the main beamline at the end of the post-linac diagnostic region, first into a "big-bend" that bends the beam by about $17 \mathrm{mrad}$ and then into the collimation region and final focus. The $2^{\text {nd }}$ IR has a "one-way bending" configuration where the bends in the energy collimation and in the final focus are in the same direction. (In the standard configuration the two bends are of opposite sign and nearly cancel.) This allows the BDS to provide about $8 \mathrm{mrad}$ of the total $25 \mathrm{mrad}$ net bending required for the $2^{\text {nd }} \mathrm{IR}$, significantly shortening the length required for the big bend. In this configuration, the BDS for the $2^{\text {nd }}$ IR is almost as long as for the $1^{\text {st }}$ IR (the available space for $2^{\text {nd }}$ IR BDS is $1390 \mathrm{~m}$ for $e^{+}$and $1090 \mathrm{~m}$ for $e^{-}$). This makes the performance of the two IRs very similar up to $1300 \mathrm{GeV}$ c.m., as shown in Figure 3.4.6.3. At still higher energies, the performance of the $2^{\text {nd }}$ IR will be limited by the big bend, while the first IR can accommodate c.m. energies well above $1500 \mathrm{GeV}$.

3.4.6.3.2 Beam Switchyard The beam switchyard region is shown in Figure 3.4.6.4. It includes the post-linac diagnostic section, tuneup dump line and big bend arc which takes off to the second IR. The optics of the end of linac diagnostic section with tuneup dump extraction are shown in Figure 3.4.6.5. The fast DC kicker, located at the end of the diagnostic section, can send beam onto a tuneup dump when it is not desirable to have beam through the IR. This mode is used for machine protection trips and will be 


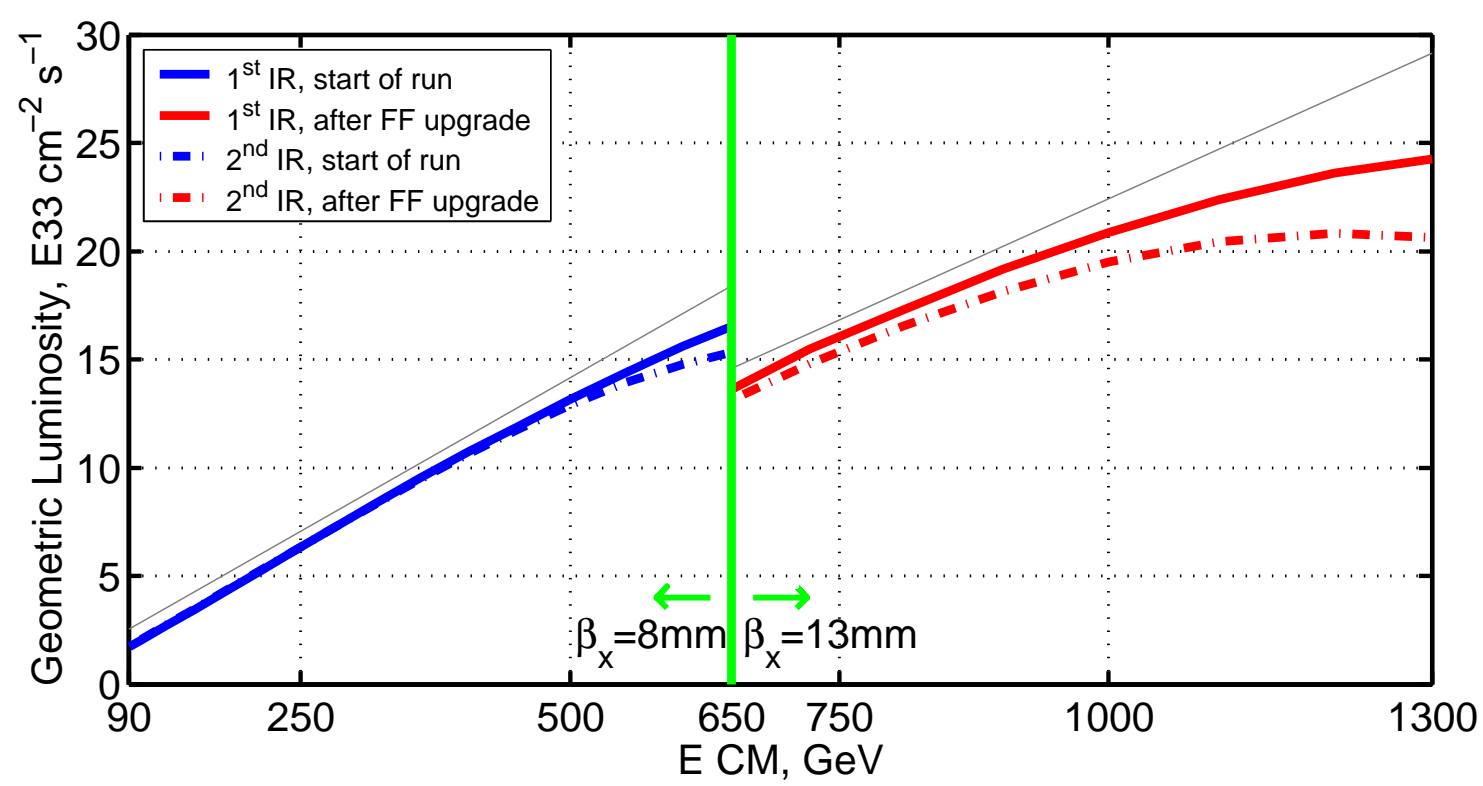

Figure 3.4.6.3: Energy reach of the NLC Beam Delivery for the first and second IR. Here L is the geometric luminosity without the pinch enhancement which includes synchrotron radiation and aberrations but does not include the beam-beam luminosity enhancement. Same normalized emittances assumed for the entire range (emittance growth in big bend of $2^{\text {nd }}$ IR, which amounts to about $30 \%$ at highest energy, is not taken into account). For the upgrade, bending angles are reduced and the FD is lengthened.

especially useful during commissioning.

3.4.6.3.3 Tuneup Dump Line The elements of the tuneup dump lines at the end of each linac are given in Table 3.4.6.3.2. The kicked beam passes off-axis though the first matching quadrupole (defocusing) which adds to the beam deflection. After this, the beam separation is sufficient to install addition bending magnets which deflect the beam horizontally and vertically. The beam dump is separated from the BDS line by $2.5 \mathrm{~m}$ horizontally. Important considerations in the design of the tuneup dump line are that the beam size on the dump is large enough to prevent damage to the dump window and that the energy bandwidth of the line is large enough to accept beams which are significantly off energy. To achieve a large bandwidth, it is essential that dispersion at the front face of the dump is brought to zero to both first and second order. Figure 3.4.6.6 shows the second order dispersion function for the dump line. Figure 3.4.6.7 shows the beam

Table 3.4.6.2: Tuneup dump line elements. The indicated strengths are for $250 \mathrm{GeV}$ beam energy.

\begin{tabular}{|c|c|c|c|c|c|}
\hline Element & Number & $\begin{array}{c}\text { Diameter } \\
{[\mathrm{cm}]}\end{array}$ & $\begin{array}{c}\text { Pole-Tip } \\
{[\mathrm{kG}]}\end{array}$ & $\begin{array}{c}\text { Length } \\
{[\mathrm{cm}]}\end{array}$ & Comment \\
\hline Dipoles & 5 & 1.2 & 2.8 & 300 & Pulsed horizontal bends \\
& 13 & 4 & $2.8-3.6$ & 300 & Horizontal bends \\
& 10 & 8 & 3.6 & 300 & Vertical bends \\
Quads & 11 & 8 & $2.3-3.7$ & 300 & \\
Sextupoles & 4 & 8 & $1.5-2.3$ & 100 & Rotated sextupoles \\
& 4 & 8 & 3.9 & 100 & \\
Octupoles & 7 & 8 & $0.3-4.0$ & 100 & \multicolumn{3}{|c|}{} \\
\hline
\end{tabular}




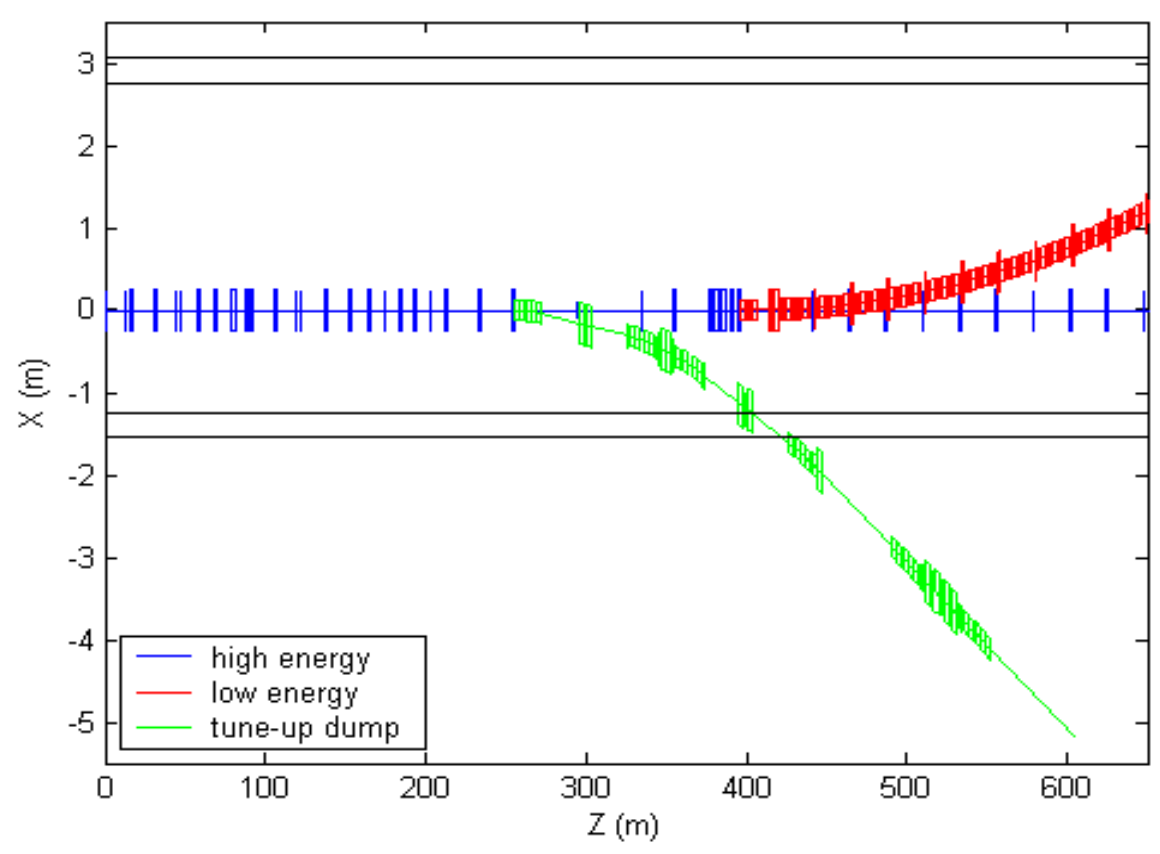

Figure 3.4.6.4: Beam switchyard layout

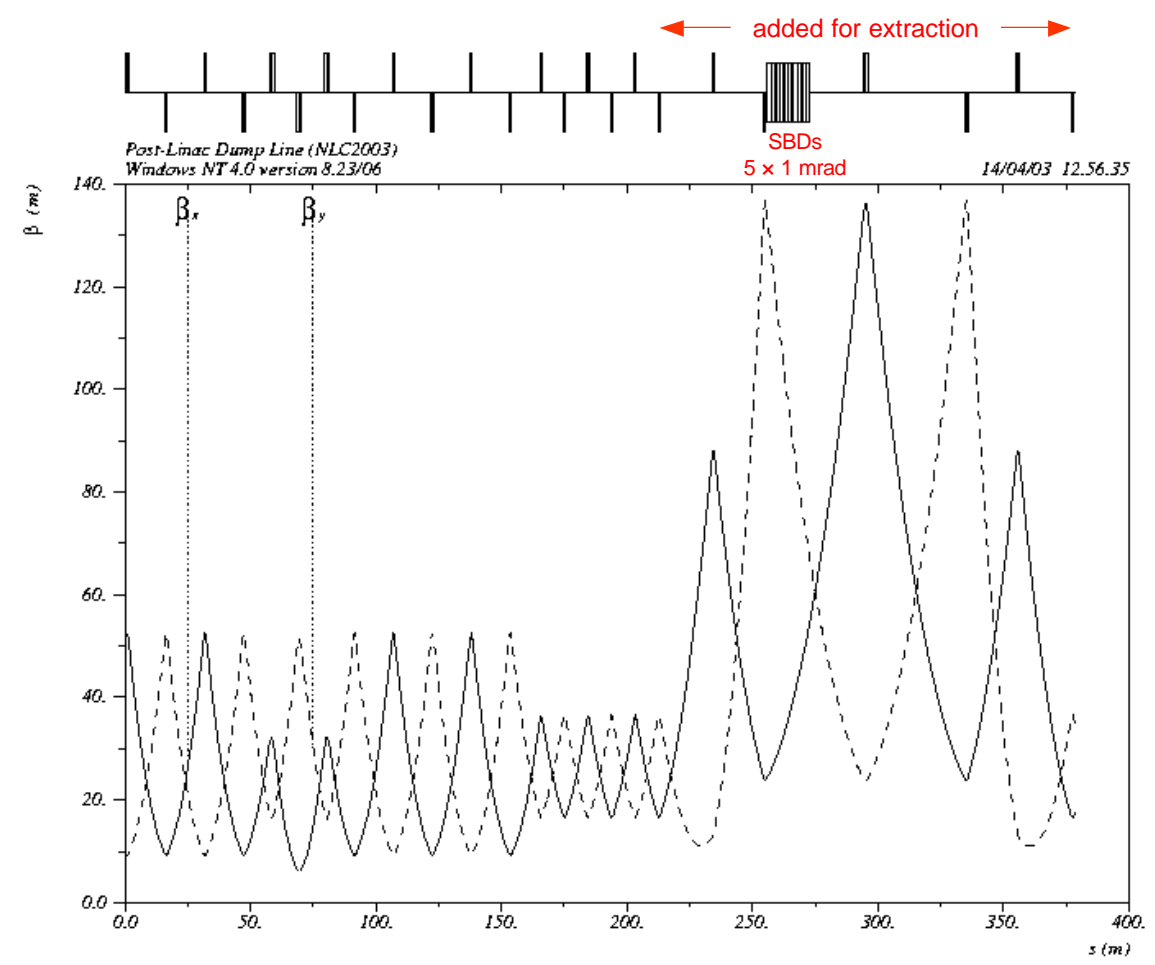

Figure 3.4.6.5: Optics for end of linac diagnostic section and tuneup line. 


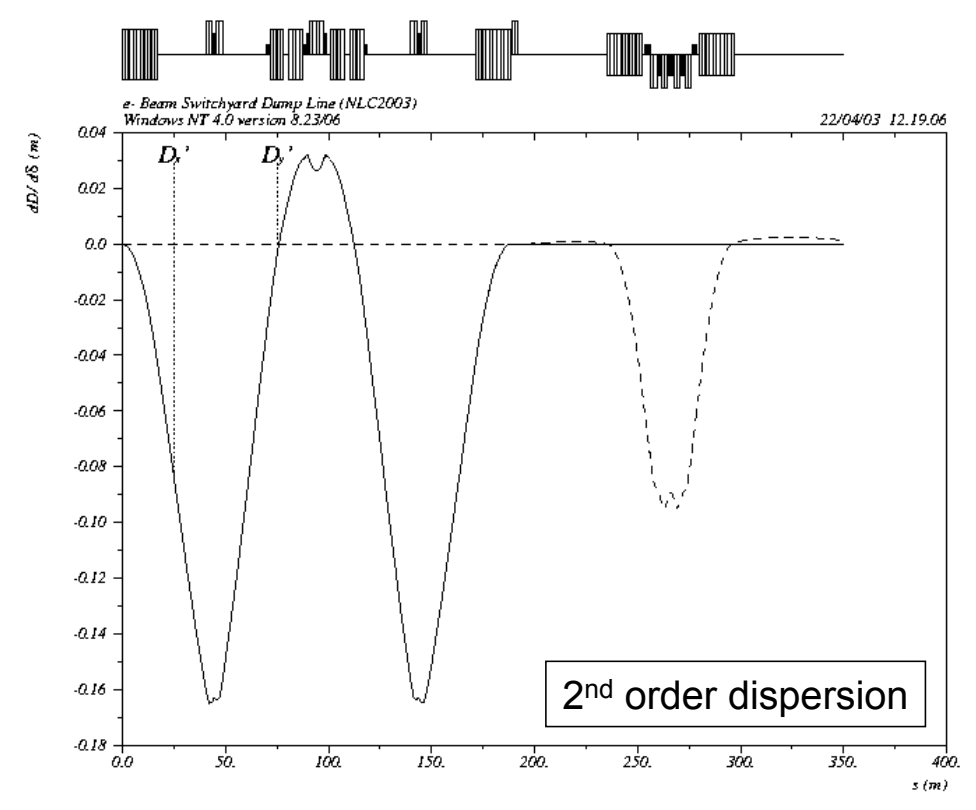

Figure 3.4.6.6: Second order dispersion in the tuneup line, which must be carefully zeroed on the dump to achieve a large energy bandwidth.

size through the dump line. The size on the window is large and well constrained over a wide range of energies.

3.4.6.3.4 Big Bend for IR2 The layout of the big bend arc for the electron side is shown on the left in Figure 3.4.6.8. The positron layout is similar. The critical design criteria for the big bend is that it provide the required crossing angle at the second IR $(30 \mathrm{mrad})$ while limiting the synchrotron radiation emittance growth to less than $30 \%$ up to a beam energy of $650 \mathrm{GeV}$. The optics consists of combined function FODO cells with $L_{\text {cell }}=23 \mathrm{~m}$ as shown on the right in Figure 3.4.6.8.

\subsubsection{Collimation and Background}

The collimation system must remove particles in the beam tails that can generate backgrounds in the detector and it must protect the final focus and detector from errant beams. As is well known, the population and distribution of the beam tails can be very hard to calculate and, because the backgrounds can severely limit the luminosity recorded by the detector, the NLC collimation system has been designed quite conservatively. Beam collimation was one of the limiting factors in the SLC operation. Although predictions for the amount of beam halo in a high energy LC are $\sim 10^{-6}$ of the total beam intensity, the NLC collimation system has been designed to deal with $10^{-3}$ of the total beam intensity. (A $10^{-3}$ beam loss corresponds to $6.9 \mathrm{~kW}$ in the X-band design).

Collimator systems for high energy LC's consist of a combination of thick absorbers ( $\gg 1$ radiation length, typically copper) and thin spoilers ( $<1$ radiation length). The post-linac collimation systems of TESLA, NLC, and CLIC have been assessed and compared by evaluating the efficiency in beam halo and synchrotron radiation collimation. Realistic simulations have been run to compare equitably the collimator system designs and overall efficiency [57]. The performance of these systems is summarized in Figure 3.4.6.9, where the scale factor $K$ defines the window dimension. For $K=1$, the window size corresponds to the nominal design 

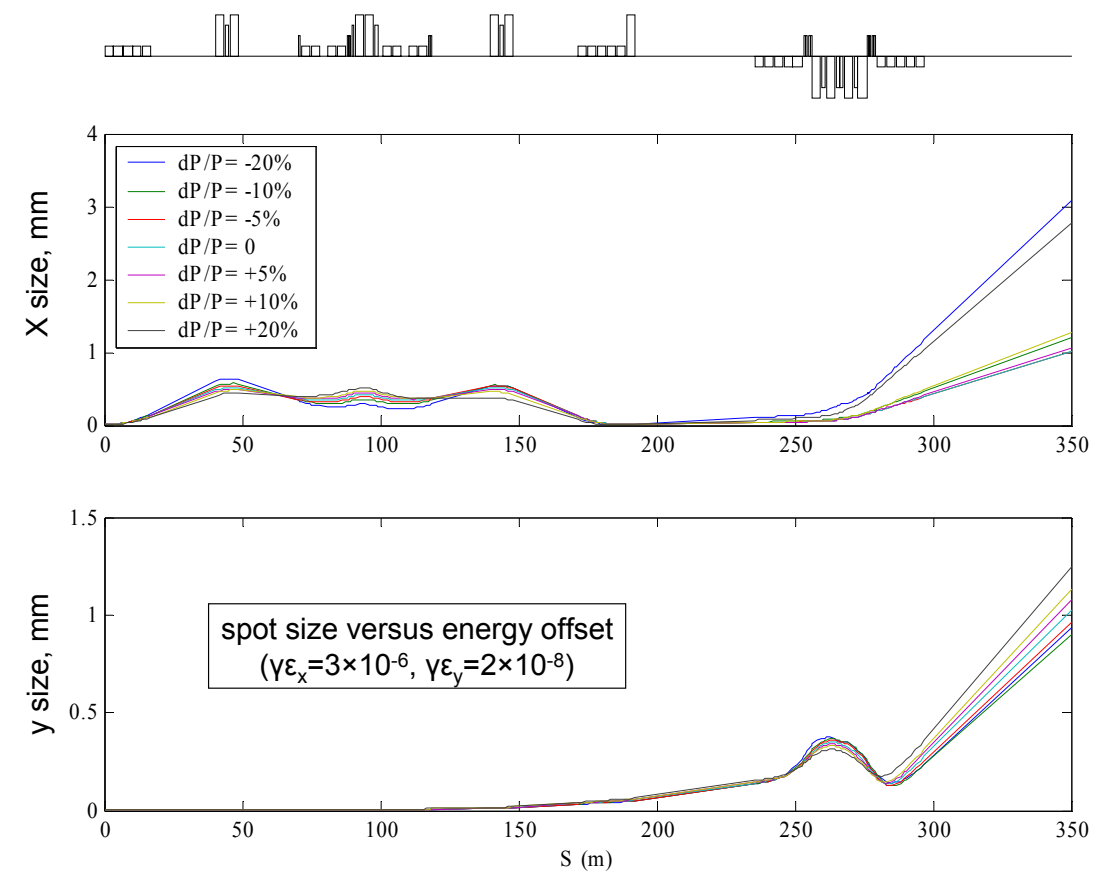

Figure 3.4.6.7: Tuneup dump beam size versus energy offset.
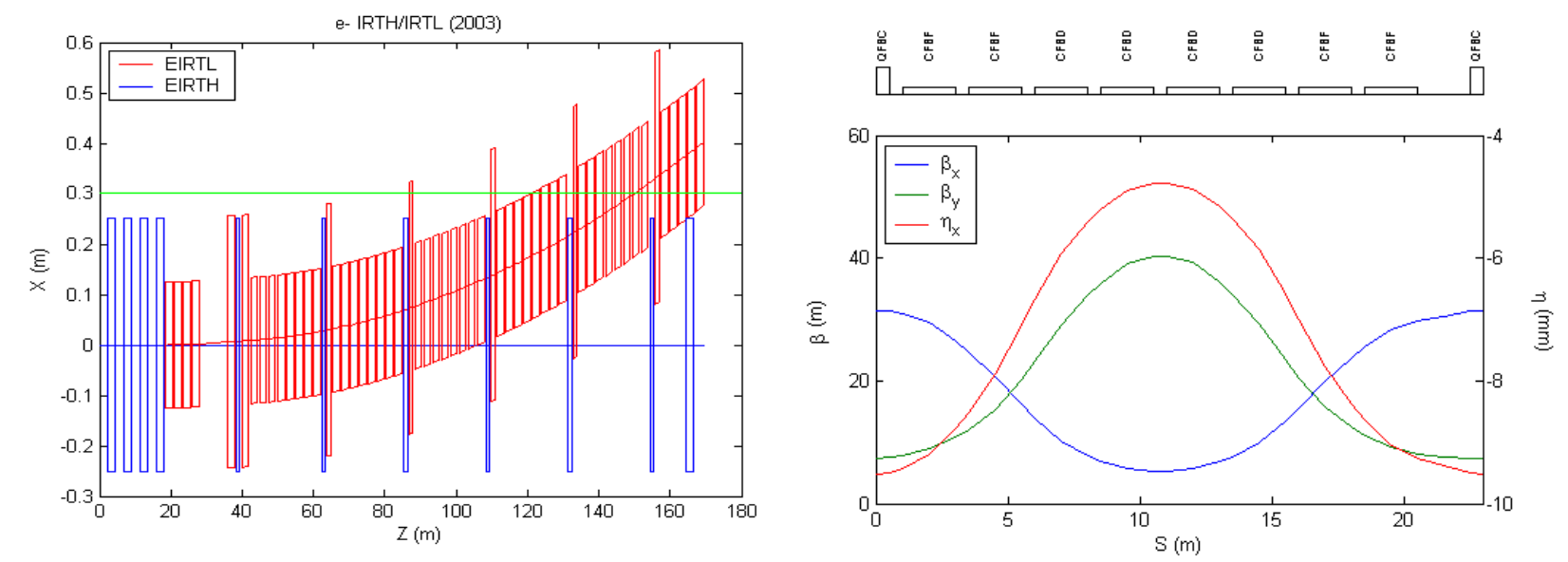

Figure 3.4.6.8: Optics for the big bend region. Left: Layout of the beginning of big bend to 2nd IR on electron side. Right: Combined function FODO cell for big bend line to IR2. 


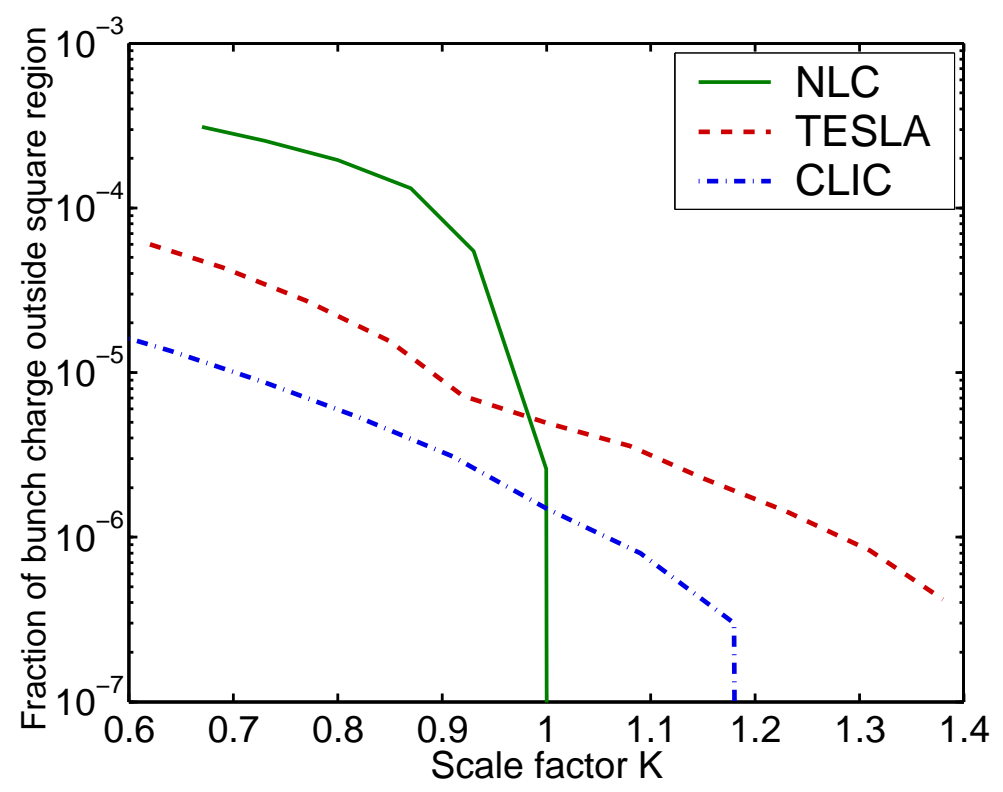

Figure 3.4.6.9: Collimation-system performance as evaluated by the TRC Collimation Task Force. Number of charged-halo particles per bunch, normalized to the nominal bunch charge, in a rectangular $x-y$ window at the entrance to the final doublet, as a function of the collimation depth (assuming an incident fractional halo of $\left.10^{-3}\right)$.

collimation depth required to shadow the vertex detector from synchrotron radiation. Since the NLC design achieves the desired performance, the NLC collimation system has been chosen for both the X-band and superconducting reference designs. The inclusion of 2 pairs of octupole doublets for tail folding further improves the performance and relaxes the collimation requirements. The results with octupoles on and the collimators opened by a factor of three are shown in Figure 3.4.6.10. The performance is improved in spite of the much looser collimation.

3.4.6.4.1 Design considerations The collimation system must remove all primary beam particles which could be lost near the detector. However, tighter constraints arise due to the synchrotron radiation produced by the large amplitude particles in the final doublet focusing magnets. Because of the high beam energy, the photons emitted as the beam is focused in the final doublet have energies that are too high to be able to shield the detector with masking in the IR. Ray tracing shows that to prevent any synchrotron radiation photons from hitting in the IR, the angular divergence of the beam at the IP cannot exceed a rectangular aperture of $570 \mu \mathrm{rad}$ horizontally by $1400 \mu \mathrm{rad}$ vertically. This restriction on the beam tails is significantly tighter than that to prevent primary particles from hitting the vacuum apertures.

Another important consideration is the muons produced by the collimators when the high energy tails are removed. Simulation studies have shown that as many as $10^{9}$ primary electrons or positrons per train can be removed by a collimation system located well upstream of the final focus without producing an unacceptable muon flux in the detector, although this number depends somewhat upon the exact configuration of the beamline. The number of primary particles that can be stopped within the final focus without unacceptable muon production is only $10^{4}$.

Because of the muon generation, the NLC collimation system is designed in four stages. There are two stages of collimation before the main linacs, a transverse collimation section immediately after the damping rings at $1.98 \mathrm{GeV}$ and a longitudinal phase space collimation after the pre-linacs at $8 \mathrm{GeV}$. There are also two stages 


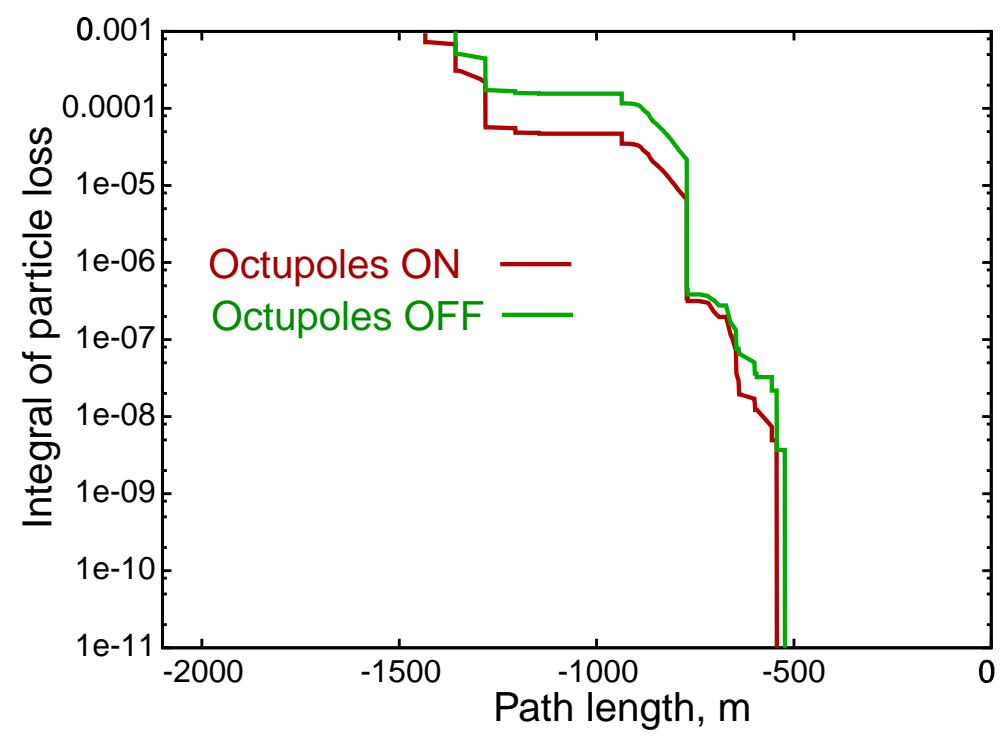

Figure 3.4.6.10: Comparison of NLC collimation-system performance without octupoles and with octupoles and looser collimation. Fractional loss of charged-halo particles, integrating back, starting at the IP (assuming an incident fractional halo of $10^{-3}$ ). The horizontal scale shows the distance from the IP.

of collimation after the linacs. The primary system located at the end of the main linac collimates both the transverse and longitudinal phase spaces with an efficiency of $\sim 10^{-5}$. For $10^{9}$ incident tail particles, only $10^{4}$ will pass through the system. A secondary system collimates both the longitudinal and transverse phase spaces in the final focus itself. This is necessary to remove particles that escape the primary collimation system as well as additional particles scattered by the residual gas downstream of the collimation section; the latter is estimated to be less than $10^{3}$ particles per bunch train.

All of these systems have dual purposes: they must collimate the beam tails and they form an integral part of the machine protection system (MPS). Because the particle beams have such high charge densities, a single bunch at the end of the linac or a few bunches at the linac entrance will damage almost any material unless the beam size is increased to very large values. Because of the risk of damage from errant beams, the collimation uses thin spoilers (0.25-0.5 radiation length) to scrape the halo and enlarge the beam which is then stopped on thick (20 radiation length) absorbers.

The betatron collimation system scrapes the beam halo and provides machine protection against infrequent orbit disruption of on-energy beams. The NLC design uses "consumable spoilers" which are cylindrical spoilers or scrapers that can be rotated to present a clean surface to the beam if damaged by an errant pulse. Their circumference is such that approximately 1000 damaging pulses can be permitted before replacement is necessary. A prototype of such a spoiler has been built at SLAC and is illustrated in Figure 3.4.6.11.

In contrast, the energy collimators are designed to be capable of surviving hits from a full bunch train because klystron trips causing off-energy beams may be relatively frequent events and can occur with only microseconds of warning. As seen in Figure 3.4.6.2, the system combines a large horizontal dispersion and a large vertical betatron function to ensure that the transverse size of beam pulses at the spoilers is large enough that the charge density is below the damage threshold. Multiple coulomb scattering in the spoiler further increases the beam size before the spoiled bunch train is stopped in an absorber downstream.

Finally, a recent development in the collimation system is the use of octupole doublets which permit the beam halo in one betatron phase to be reduced in amplitude, while leaving the beam core nearly unaffected. A pair of these doublets, located in the beta match section at the beginning of the final focus, has been 

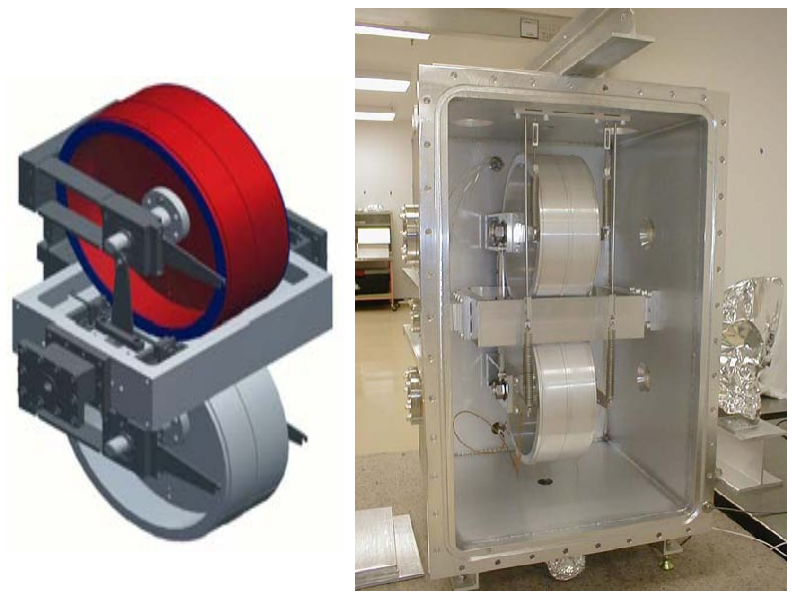

Figure 3.4.6.11: Consumable spoiler mechanical prototype. Left is a mechanical drawing of the collimator. Right is a photograph of the prototype constructed at SLAC.

shown to reduce the halo in the critical final doublet betatron phase by a factor of 3 , which in turn would permit equivalently larger collimator apertures in that phase. This would also dramatically decrease the impact of collimator wakefields, as the wakefields are believed to scale with the inverse square of the gap size.

\subsubsection{Luminosity Performance and Stability}

In order to achieve the luminosity goals, the beam delivery system must preserve the very small emittances from the end of the main linacs, and must collide beams with very small transverse sizes. The alignment tolerances can only be achieved through the use of beam-based diagnostics and corrections, and for this reason, all critical components have position monitors to measure the misalignments and precision remotelycontrolled movers. Both static and dynamic component alignment are important. Motion on fast time scales can cause the beams to miss each other, while motion on slower time scales causes beam size growth. Beambased alignment algorithms are needed to establish a well-aligned beamline, and global correction knobs to cancel residual optical aberrations. Trajectory feedback must compensate for slow drifts due to ground motion. Collision feedback is required to maintain collisions on a train-to-train time scale. Component vibration must be minimized and in some cases, such as the final doublets, active stabilization techniques may be required.

3.4.6.5.1 IP Collision Feedback IP collision feedback is absolutely essential to the performance of a high-energy LC, as the luminosity is extremely sensitive to relatively small offsets of the beams in position and crossing angle. Figure 3.4.6.12 shows the luminosity as a function of vertical offset between the beams for the X-band design. The drop off in luminosity would be even sharper without the strong beam-beam attraction. At an offset of $10 \mathrm{~nm}$, the luminosity would be negligible for rigid beams, while in practice it is more than $50 \%$. The collision feedback uses the strong beam-beam kick experienced by the beams when they do not collide head-on as an error signal, and correction is provided by fast kickers. With the $120 \mathrm{~Hz}$ repetition rate of the $\mathrm{X}$-band collider, the feedback can correct relative motion up to frequencies of a few $\mathrm{Hz}$.

Intra-train feedback system designs have also been developed for TESLA and for the NLC. While the train length and inter-bunch spacing is much shorter for the X-band machine, a very low latency system has been 


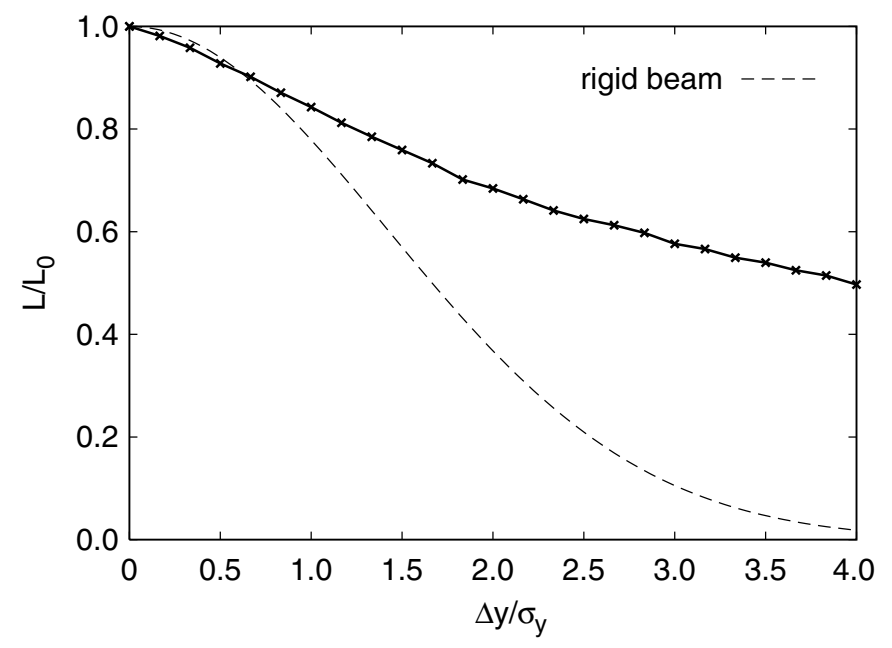

Figure 3.4.6.12: Luminosity versus vertical offset between beams at the IP. Dashed line is for rigid beams. Solid line includes beam-beam interaction.

prototyped and tested in the NLCTA [34]. With a latency on the order of $40 \mathrm{~ns}$, such a feedback could correct small residual displacements for most of the $\sim 270 \mathrm{~ns}$ train. This can potentially ease the requirements on the final focus doublet mechanical stabilization.

3.4.6.5.2 Instrumentation and Correction The final focus of the X-band linear collider, like the main linacs, is designed with powerful diagnostic capabilities and robust correction devices. Every quadrupole and sextupole is on a remotely controlled magnet mover, similar to those in the main linac. Each quadrupole is paired with a BPM with submicron resolution, and in some critical locations ultra-high resolution cavity BPMs with resolutions better than $100 \mathrm{~nm}$ are also specified. Laser-based beam-size monitors are installed at critical locations. All sextupoles, bends, and quads except for the final doublet are iron-dominated electromagnets, with high-precision power supplies. In addition, the final focus has two powerful diagnostics not available at other locations, the luminosity and the beam-beam deflection, each of which is measured on every pulse in order to provide signals for feedback systems.

While the tolerances for the final focus components are small, feedback systems and other non-invasive correction algorithms can be used to stabilize accelerator performance. Understanding the real performance of the final focus requires simulation studies that include the planned diagnostic and correction systems, and their algorithms. As an example, one of the most serious potential sources of emittance dilution is beamline magnet misalignments driven by diffusive ground motion. Figure 3.4.6.13 is the result of a simulation that misaligns the elements of the $1-\mathrm{TeV}$ BDS configuration according to the ATL law ${ }^{2}$ with $A=5 \times$ $10^{-7} \mu \mathrm{m}^{2} / \mathrm{m} / \mathrm{s}$. The curves show that luminosity would degrade under ATL motion in approximately 2 minutes if only the beam-beam deflection collision stabilization feedback was present. If, in addition, trajectory feedback is allowed to steer the beam through the centers of critical quadrupole and sextupole magnets, then the time for luminosity degradation increases to approximately 1 day. Finally, if direct optimization of the main aberrations via global knobs is added to the system, then the luminosity lifetime increases to several months, after which a disruptive realignment procedure would be required.

Experience with the Stanford Linear Collider (SLC) showed that submicron beams could be routinely main-

\footnotetext{
${ }^{2}$ This law describes slow diffusive ground motion, in which the mean square transverse displacement between two points separated by a longitudinal distance $L$, after a time $T$, is given by $\sigma^{2}=A T L$, in which $A$ is a site-dependent constant.
} 


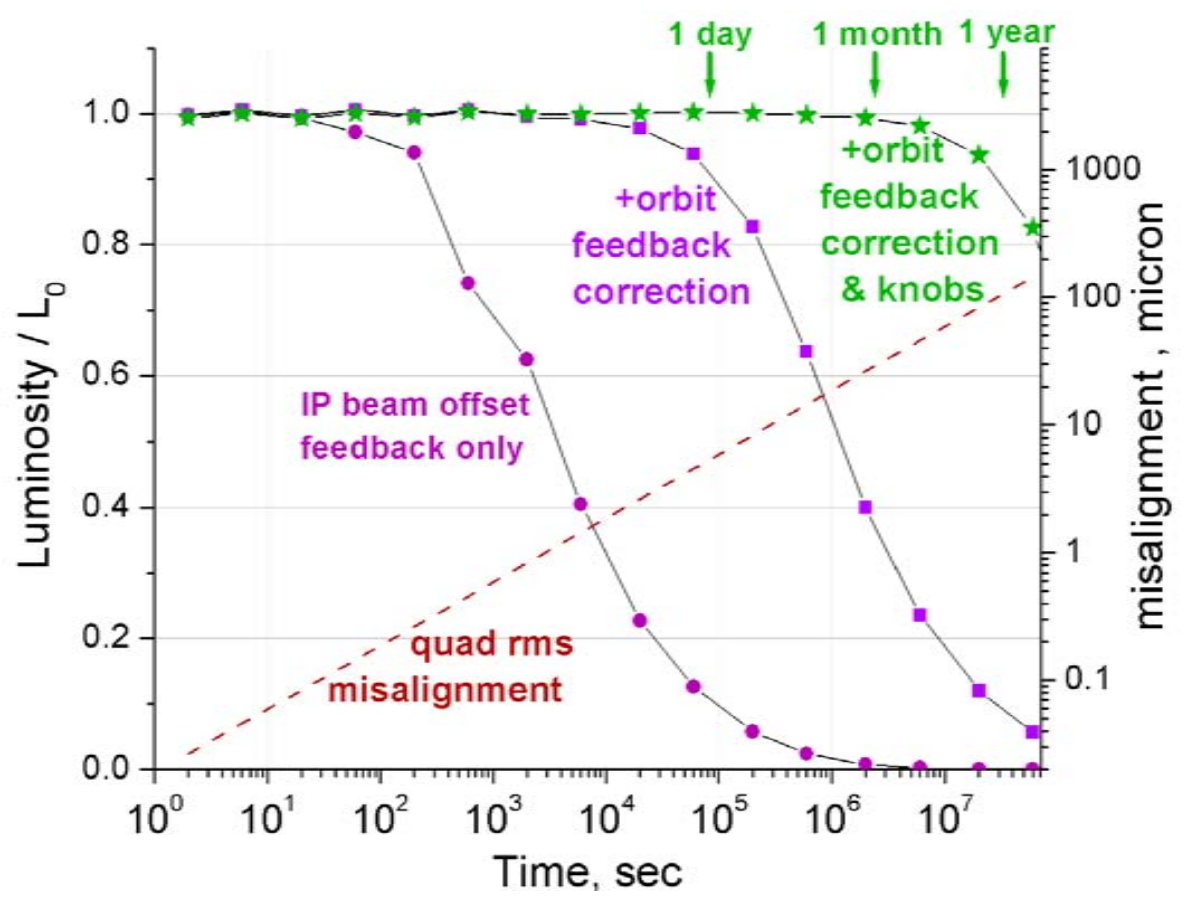

Figure 3.4.6.13: Degradation of alignment under ATL ground motion with IP beam-beam deflection based feedback only, with orbit feedback added and finally, with direct luminosity optimization.

tained in collision with stable control of the emittance and beam size at the IP. The FFTB demonstrated many aspects of the beam-based alignment tools and techniques while the SLC demonstrated the integrated operation of all components of a linear collider. Figure 3.4.6.14 shows the reconstructed vertical position of $\mathrm{Z}^{0} \rightarrow$ hadron events recorded by the SLD vertex detector over a period of approximately 10 days in 1997 . The $\mathrm{Z}^{0}$ events are binned in groups of 30 and the mean $y$ for the group plotted versus time of day. The luminous region for the SLC was approximately $0.7 \mu \mathrm{m}$ vertically, but this figure shows that, while the

vertical IP position varied by up to 40 times this size over the course of 1 day, the collision feedback was able to keep the beams centered on each other. The collision feedback together with other feedback systems to control the trajectory and optimize the luminosity was able to maintain a steady $\mathrm{Z}$ production rate over the entire period.

\subsubsection{Machine Detector Interface}

There are several areas where detector requirements influence machine component design. Detector backgrounds arise from many mechanisms. Principally, these are electrons from beamstrahlung photons, synchrotron radiation from the final focus quadrupoles, and muons produced by interactions in the upstream collimators. Muon flux into the detector is minimized by a series of magnetized iron spoilers downstream of the collimation system. The detector is shielded from low energy electrons with tungsten masks starting 50 $\mathrm{cm}$ from the IP, and defining a $100 \mathrm{mrad}$ ID cone, followed by a $10 \mathrm{~cm}$ thick tungsten pipe.

The experimental solenoid, assumed to have a $<6 \mathrm{~T}$ field, together with the $10 \mathrm{mrad}$ off-axis beam, results in non-negligible dispersion and coupling. Dipole and skew quadrupole correctors in the final focus must compensate these effects. Other issues arising from the detector solenoid field, such as synchrotron radiation and field stability, are benign. The final focus quadrupoles are stabilized to the nanometer level by a 

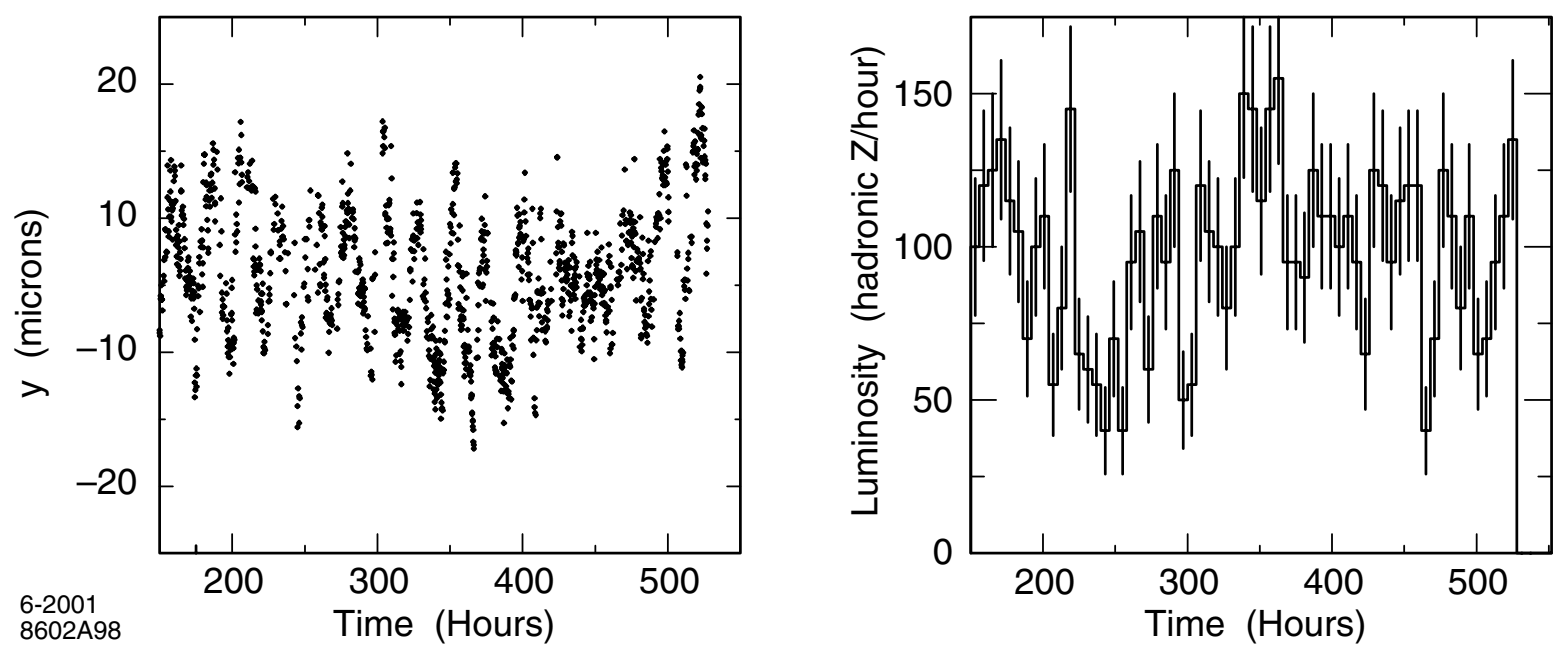

Figure 3.4.6.14: Average reconstructed vertical position of $\mathrm{Z}^{0} \rightarrow$ hadron events in the SLD and the measured SLC luminosity over a 10-day period.

mechanical feedback system based on a either inertial sensors or a laser interferometer, a so-called optical anchor. Since the quadrupoles are located well inside the detector, the interferometer solution would require two optical paths through the detector calorimetry, referenced to the external walls.

3.4.6.6.1 Superconducting Doublets Compact superconducting magnets, consisting of quadrupoles, sextupoles, octupoles and correction dipoles are used in the final focus doublets. They fit within a cryostat having a $57 \mathrm{~mm}$ outer radius. (See Fig. 3.4.6.15).

The conceptual magnet layout is shown in Figure 3.4.6.16. Each corrector package contains skew-quadrupole, sextupole and octupole multilayer windings as well as horizontal and vertical dipole corrector windings, next to each main quadrupole. The superconducting magnets share a common cryostat that is passively and actively stabilized against vibrational motion. The transverse centering of QD0 and QF0 is independently adjustable within the cryostat, with each corrector package rigidly connected to each main quadrupole. Also, the QD0 and QF0 longitudinal magnetic centers are independently fixed in the cryostat in order to minimize coil movement due to thermal contraction during cool down.

The QD0 gradient of $144 \mathrm{~T} / \mathrm{m}$ at $250 \mathrm{GeV}$ is attained with a multi-layer coil structure, as shown in Figure 3.4.6.15, that has two independently powered, concentric quadrupole windings, with helium cooling between and outside the coil windings. For the upgrade to $500 \mathrm{GeV}$ beam energy operation, the final doublet magnets will be replaced with longer versions, rather than simply scaling the gradient, in order to avoid complications from increased synchrotron radiation.

With the QF0 gradient being significantly smaller in magnitude than that of QD0, either the inner or the outer QD0 coil structure is adequate for operation; so QF0 can be powered with a single power supply. For the dual powered QD0 both power supplies must be shut down if either the inner or outer coil begins to quench.

In order to achieve the demanding vibrational tolerances, the outer cryostat of the superconducting magnets is stabilized with a combination of inertial stabilization and laser interferometers (see Section 3.4.6.6), and by eliminating rigid connections to potential external sources of vibration such as cryogenic and electrical feeds. Construction and active damping internal to the cryostat if necessary minimizes internal vibrations. 


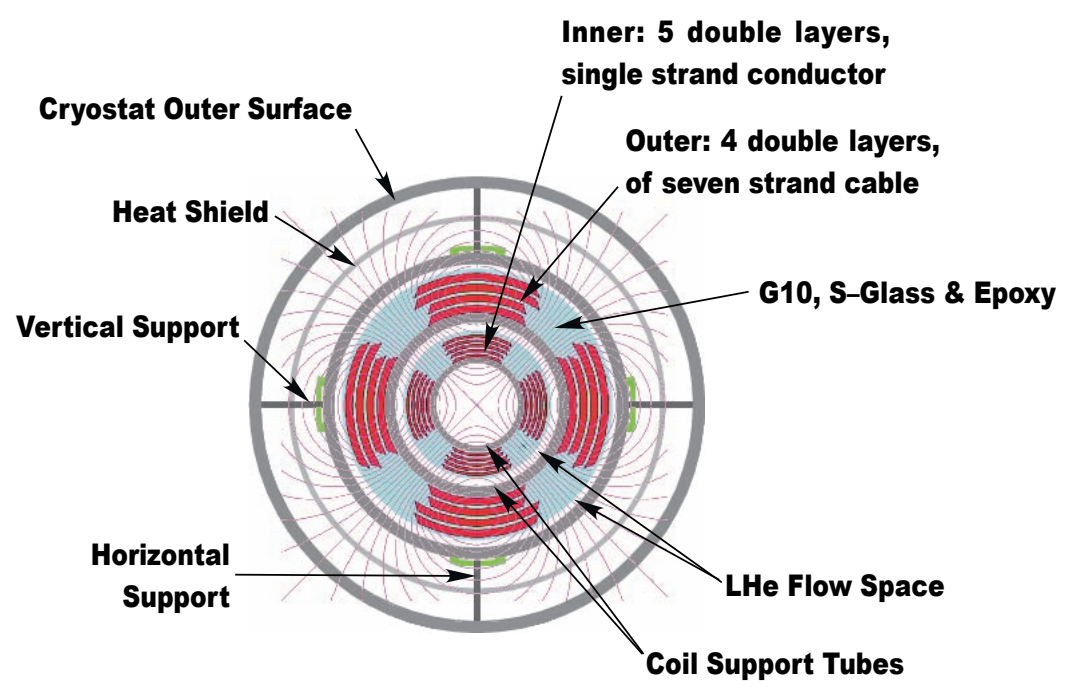

Figure 3.4.6.15: Concept of the QD0 compact SC quad which is being prototyped by BNL. Dimensions: inner beam tube diameter is $20 \mathrm{~mm}$; outer cryostat tube diameter is $114 \mathrm{~mm}$. Magnetic parameters: inner coil contribution $63 \mathrm{~T} / \mathrm{m}$, outer coil contribution $81 \mathrm{~T} / \mathrm{m}$, total $144 \mathrm{~T} / \mathrm{m}$. The beam tube is cold.

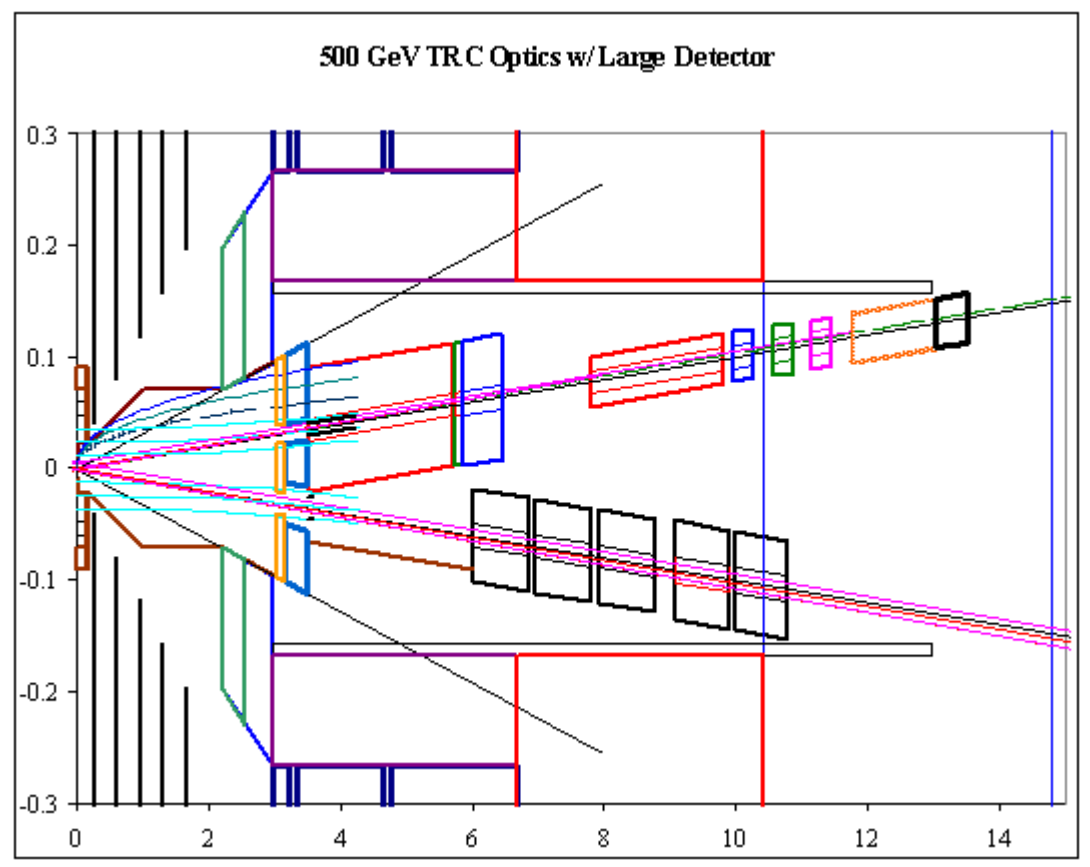

Figure 3.4.6.16: Superconducting Final Focus Magnet Layout Schematic 


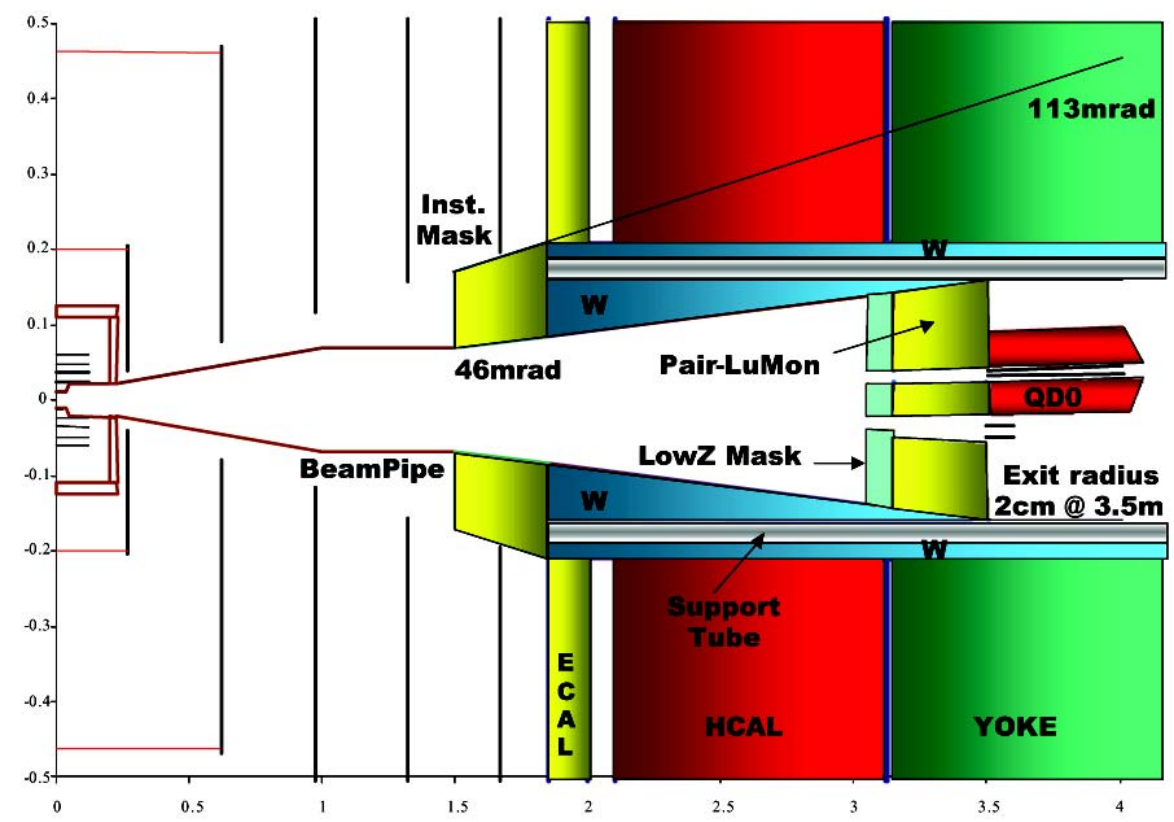

Figure 3.4.6.17: Beamline masking, forward tracking and forward calorimeters for the Silicon Detector.

3.4.6.6.2 Crab Cavities The potential luminosity reduction due to the $20 \mathrm{mrad}$ crossing angle is compensated by the use of crab cavities in each beam which provide a transverse displacement within the bunch that varies with longitudinal distance. The cavities are located in the incoming beamlines on each side of the IP behind the final focus quadrupoles, $\sim 15 \mathrm{~m}$ from the IP. They are S-band to provide the necessary aperture. Differential phase jitter between the cavities is the most demanding tolerance and is minimized by driving both cavities from a single common klystron. The crab cavities are mounted on a movable stage with an adjustable roll angle to allow the introduction of vertical crabbing for luminosity tuning.

3.4.6.6.3 Forward Masking Figure 3.4.6.17 shows the current concept of the beamline masking, forward tracking and forward calorimeters for the Silicon Detector. The cylindrical tungsten mask that protects the calorimeter from particles striking the vacuum pipe of QD0 is located outside the support tube and is mechanically part of the detector, traveling with the door when it opens. The remaining conic section of the mask, with its instrumented tip, extends to $1.5 \mathrm{~m}$ from the IP, $1.65 \mathrm{~m}$ from the front face of the PairLuminosity Monitor. Because the detector supports the weight of all the masking, it is possible to provide more protection for the detector's central tracking systems, without compromising the performance of the vibration reducing systems supporting the final quad. The support tube is at a radius of $17-20 \mathrm{~cm}$ and extends to $1.85 \mathrm{~m}$.

3.4.6.6.4 Spent Beam Transport The spent beam extraction line must safely guide the disrupted beam to the beam dump with minimal losses, while providing locations for energy and polarization diagnostics downstream of the collision point. The NLC extraction line design provides a minimum aperture of $10 \sigma$ of the disrupted beam and an image of the interaction point for diagnostic purposes. The optics of the extraction line from the IP to the dump is shown in Figure 3.4.6.18. The elements of the spent beam transport line from the IP to the beam dump are given in Table 3.4.6.6.4. 


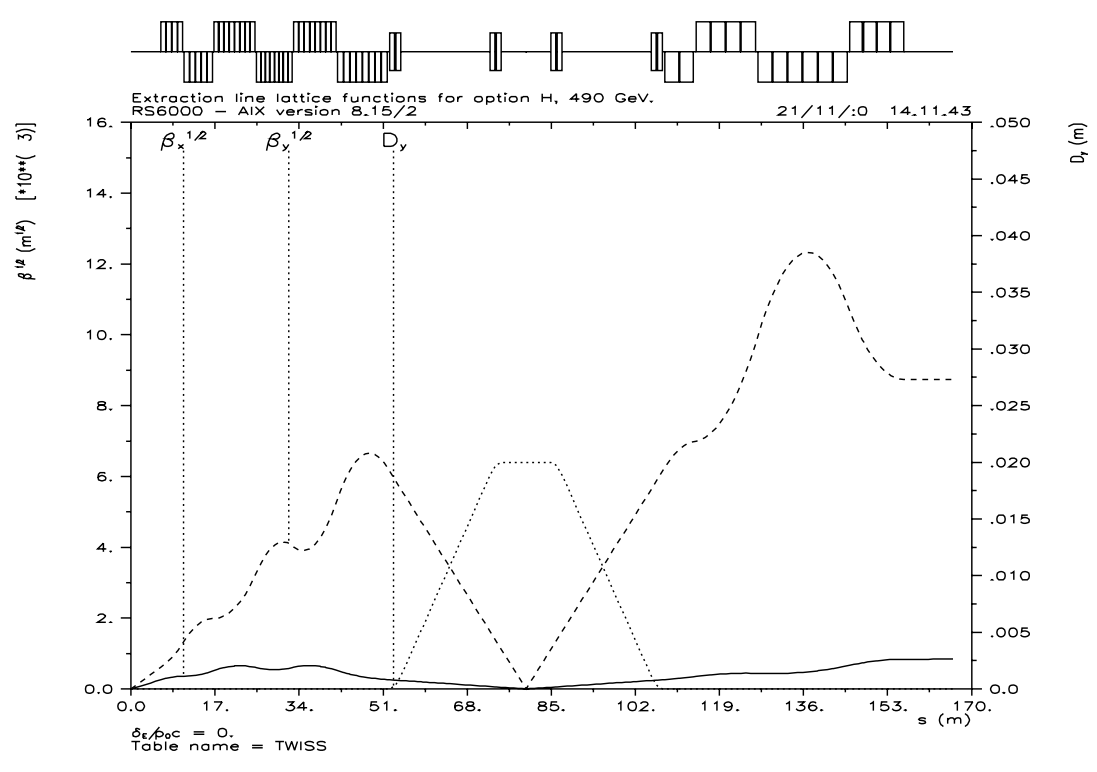

Figure 3.4.6.18: Optics for the spent beam extraction line

Table 3.4.6.3: Spent beam transport line elements. The indicated strengths are for $250 \mathrm{GeV}$ beam energy.

\begin{tabular}{|c|c|c|c|c|c|c|}
\hline Element & Number & $\begin{array}{c}\text { Z Position } \\
{[\mathrm{m}]}\end{array}$ & $\begin{array}{c}\text { Diameter } \\
{[\mathrm{cm}]}\end{array}$ & $\begin{array}{c}\text { Pole-Tip } \\
{[\mathrm{kG}]}\end{array}$ & $\begin{array}{c}\text { Length } \\
{[\mathrm{cm}]}\end{array}$ & Comment \\
\hline Dipoles & 8 & $32-86$ & 17.4 & 4.11 & 100 & Chicane dipoles \\
Quads & 3 & $6.4-8.3$ & 2.0 & 6.00 & 86 & SC, in common cryostat \\
& 4 & $9.5-12.2$ & $2.2-2.6$ & $5.89-6.00$ & 81 & \\
& 4 & $13.4-16.9$ & $3.2-3.6$ & $5.87-6.00$ & 104 & \\
& 6 & $18.2-24.4$ & $4.2-5.0$ & $5.76-6.00$ & 114 & \\
& 5 & $25.9-30.7$ & $5.6-6.4$ & $5.72-6.00$ & 110 & \\
& 2 & $88.9-92.1$ & 18.8 & 5.000 & 300 & \\
& 3 & $95.9-103.2$ & 21.0 & 5.000 & 350 & \\
& 5 & $107-119.8$ & 24.4 & 5.000 & 300 & \\
Sextupoles & 3 & $123.4-130$ & 26.4 & 4.930 & 310 & \\
& 5 & $35.7-43.2$ & $10.8-24.0$ & & 140 & \\
& 2 & $76.9-78.7$ & $18.4-21.6$ & & 150 & \\
& 1 & 81 & 17.20 & & 120 & \\
\hline
\end{tabular}


3.4.6.6.5 Beam Dumps The main beam dump is a water dump as described in the NLC ZDR. The dump is capable of absorbing full beam power.

3.4.6.6.6 Vacuum System The pressure requirements in the BDS section are set by tolerable detector background levels. In the NLC ZDR, this is determined to be 10 nTorr for the majority of the BDS section. In the $\sim 300 \mathrm{~m}$ closest to the interaction point, a much lower pressure of $1 \mathrm{nTorr}$ is required.

\subsection{Superconducting L-band Linear Collider}

\subsubsection{Introduction}

The TESLA collaboration has outlined a design[TDR] for a $500 \mathrm{GeV}$ (c.m.) linear collider, based on a superconducting linac, with a design luminosity of $3.4 \times 10^{34} \mathrm{~cm}^{-2} \mathrm{~s}^{-1}$, and upgradeable to $800 \mathrm{GeV}$ (c.m.). In order to take advantage of this work, the system-level reference design specification for the US-sited superconducting linear collider will follow, except as specified below, the design outlined in the TESLA TDR[TDR].

The major changes to be made to the TESLA design are:

- An increase in the upgrade energy to $1 \mathrm{TeV}$ (c.m.), with a tunnel of sufficient length to accommodate this in the initial reference design, assuming a gradient of $35 \mathrm{MV} / \mathrm{m}$.

- Use of the same injector beam parameters for the $1 \mathrm{TeV}$ (c.m.) upgrade as for $500 \mathrm{GeV}$ (c.m.) operation

- The choice of $28 \mathrm{MV} / \mathrm{m}$ as the initial main linac design gradient for the $500 \mathrm{GeV}$ (c.m.) machine.

- The use of a two-tunnel architecture for the linac facilities.

- An expansion of the spares allocation in the main linac.

- A re-positioning of the positron source undulator to make use of the $\sim 150 \mathrm{GeV}$ electron beam, facilitating operation over a wide range of collision energies from 91 to $500 \mathrm{GeV}$

- The adoption of an NLC-style beam delivery system with superconducting final focus quadrupoles, which accommodates both a crossing angle and collision energy variation.

- At the subsystem and component level, specification changes to facilitate comparison with the warm LC option.

\subsubsection{Overall Parameters}

The overall parameters under consideration are given in Table 3.5.1.1. The overall machine layout is illustrated in Fig. 3.5.1.1. 


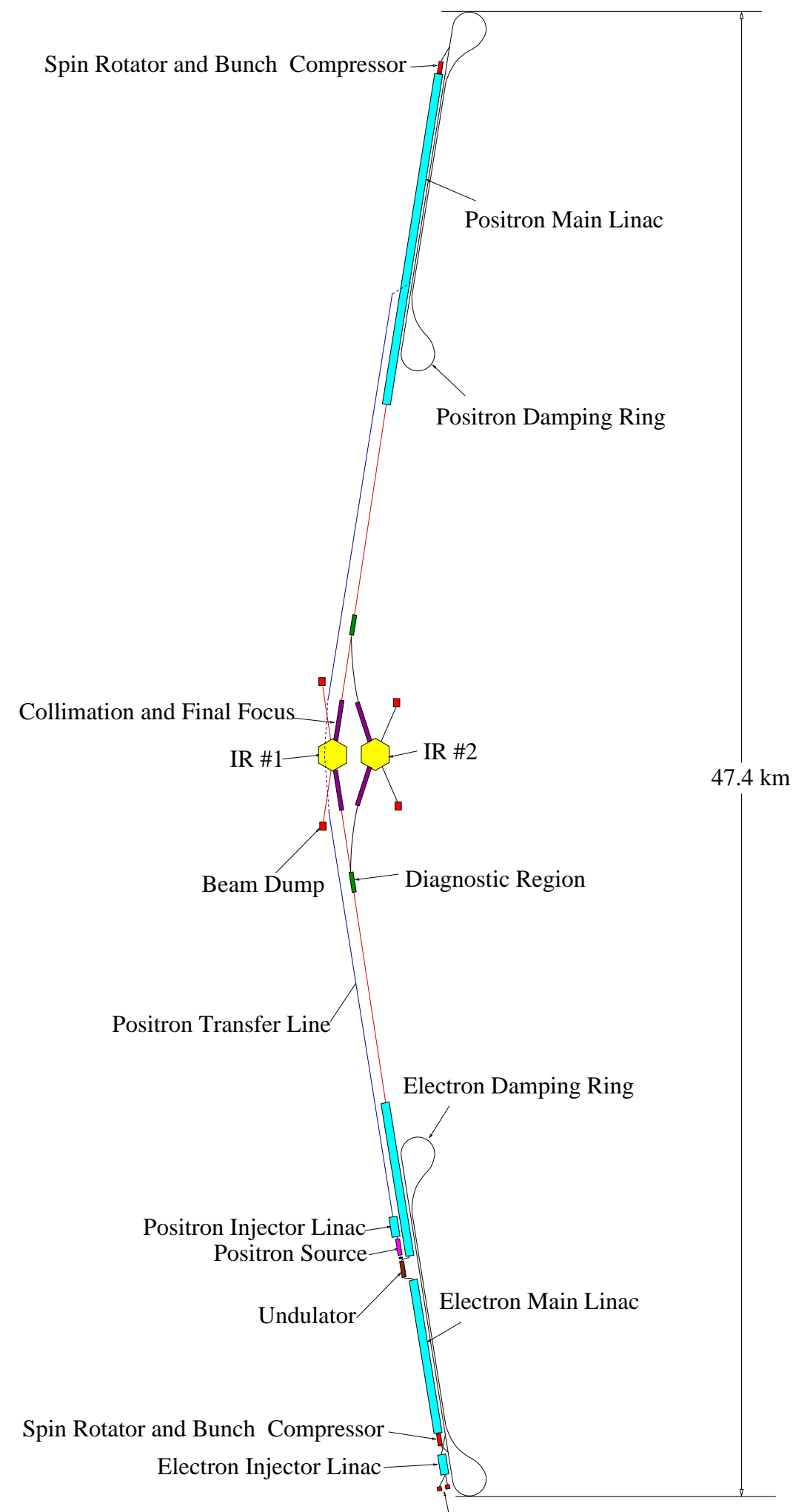

Polarized and Unpolarized Electron Sources

Figure 3.5.1.1: Overall Machine Layout, $500 \mathrm{GeV}$ c.m. 
Table 3.5.1.1: US Superconducting Linear Collider: overall parameters

\begin{tabular}{|c|c|c|c|}
\hline Parameter & Unit & Reference design & Upgrade \\
\hline Beam Energy & $\mathrm{GeV}$ & 250 & 500 \\
\hline RF gradient & $\mathrm{MV} / \mathrm{m}$ & 28 & 35 \\
\hline Two-Linac total length ${ }^{a}$ & $\mathrm{~km}$ & 27.00 & 42.54 \\
\hline Bunches/pulse & & 2820 & 2820 \\
\hline Electrons/bunch & $10^{10}$ & 2 & 2 \\
\hline Pulse/s & $\mathrm{Hz}$ & 5 & 5 \\
\hline$\gamma \varepsilon_{x}(\mathrm{IP})$ & $\mu \mathrm{m}-\mathrm{rad}$ & 9.6 & 9.6 \\
\hline$\gamma \varepsilon_{y}(\mathrm{IP})$ & $\mu \mathrm{m}-\mathrm{rad}$ & 0.04 & 0.04 \\
\hline$\beta_{x}(\mathrm{IP})$ & $\mathrm{mm}$ & 15 & 24.4 \\
\hline$\beta_{y}(\mathrm{IP})$ & $\mathrm{mm}$ & 0.4 & 0.4 \\
\hline$\sigma_{x}(\mathrm{IP})$ & $\mathrm{nm}$ & 543 & 489 \\
\hline$\sigma_{y}(\mathrm{IP})$ & $\mathrm{nm}$ & 5.7 & 4.0 \\
\hline$\sigma_{z}(\mathrm{IP})$ & $\mathrm{mm}$ & 0.3 & 0.3 \\
\hline$D_{y}$ & & 22.0 & 17.3 \\
\hline$H_{D}^{b}$ & & 1.77 & 1.68 \\
\hline $\mathcal{L}$ & $10^{33} \mathrm{~cm}^{-2} \mathrm{~s}^{-1}$ & 25.6 & 38.1 \\
\hline$N_{\gamma}$ & & 1.48 & 1.58 \\
\hline$\delta_{E}$ & $\%$ & 3.0 & 5.9 \\
\hline Average power per beam & MW & 11.3 & 22.6 \\
\hline Peak beam current during pulse & $\mathrm{mA}$ & 9.51 & 9.51 \\
\hline Beam pulse length & $\mu \mathrm{s}$ & 950 & 950 \\
\hline$Q_{e x t}($ matched $)$ & $10^{6}$ & 2.95 & 3.69 \\
\hline Cavity filling time & $\mu \mathrm{s}$ & 501 & 626 \\
\hline External bandwidth (matched) & $\mathrm{Hz}$ & 440 & 352 \\
\hline Total number of klystrons & & 603 & 1211 \\
\hline Peak beam power per klystron & MW & 8.3 & 8.3 \\
\hline Total number of cavities & & 18096 & 29064 \\
\hline Peak beam power per cavity & $\mathrm{kW}$ & 276 & 345 \\
\hline Total AC power for $\mathrm{RF}^{c}$ & MW & 87.3 & 184.3 \\
\hline Total AC power for cryogenics ${ }^{c}$ & MW & 21.3 & 73.7 \\
\hline Total AC power ${ }^{d}$ & MW & 108.6 & 258.0 \\
\hline Overall $\mathrm{AC}^{d}$ to beam efficiency & $\%$ & 20.8 & 17.5 \\
\hline
\end{tabular}

\footnotetext{
${ }^{a}$ including overheads and insertions

${ }^{b}$ Vertical waist assumed to be at the IP

${ }^{c}$ Main linacs only

${ }^{d}$ Main linac RF and cryogenics
}

The first column in Table 3.5.1.1 represents the reference design for $500 \mathrm{GeV}$, with the cavity gradient at 28 $\mathrm{MV} / \mathrm{m}$. The beam parameters are essentially those of the TDR, except that

- The vertical emittance at the IP is $40 \mathrm{~nm}$-rad, vs. $30 \mathrm{~nm}$-rad in the TDR. This change reflects a respecification of the vertical emittance growth budget, for the low emittance transport system (damping ring to IP), to $100 \%$, rather than the $50 \%$ specified in the TDR. Recent simulations both in Europe and the U.S., discussed in Section 3.3.4, indicate that the emittance growth in the cold main linacs is larger than originally anticipated. Since the emittance growth is comparable throughout the low emittance transport system, for both the warm and cold designs, the the emittance budgets have been set equal. Emittance bumps could reduce the growth for both machines, but were kept in reserve since real-life tuning rarely performs as well as simulation. 
- The vertical waist is assumed to be at the IP. This change was made to facilitate a more direct comparison with the warm option.

These two changes reduce the luminosity from the TDR value to $2.56 \times 10^{34} \mathrm{~cm}^{-2} \mathrm{~s}^{-1}$.

The choice of $28 \mathrm{MV} / \mathrm{m}$ for the initial cavity gradient is motivated by a desire to equalize the energy reach of the cold and warm LC options, for fixed RF power (and cryogenic capacity, for the cold option). (See Section 3.2).

\subsubsection{Accelerator System Reference Designs}

3.5.1.2.1 Electron Source This system will be the same as in the TDR, except that two polarized sources are provided, to enhance reliability. Components and sub-systems which are common with the warm option will be made identical if possible.

3.5.1.2.2 Positron Source This system will be the similar to that in the TDR. Again, components which are common with the warm option will be made identical if possible.

To provide a flexible range of collision energy, the undulator will be placed at the $\sim 150 \mathrm{GeV}$ point in the electron linac. Downstream from the undulator, the electron beam energy can be controlled by using the additional $\sim 100 \mathrm{GeV}$ of superconducting linac as an accelerator or decelerator. This provides center-of-mass energy variability at the collision point from $\sim 100$ to $500 \mathrm{GeV}$, without the need to retune or otherwise change the positron production system.

To provide sufficient positron flux, a long $(150 \mathrm{~m})$ helical undulator will be used. Since the beam must be deflected off-axis by about $2.5 \mathrm{~m}$ to pass through the undulator, an insertion of total length about $850 \mathrm{~m}$ will be required to allow the electron beam to be transported into and out of the undulator. This length is sufficient to allow for an upgrade of the undulator to $200 \mathrm{~m}$, which will permit operation with polarized positrons.

3.5.1.2.3 Damping Rings and Bunch Compressors The damping rings will be those specified in the TDR, but with the following changes, some of which were recommended by the recent ILC-TRC study [TRC]:

- the pole width of the positron and electron damping ring wigglers has been increased from 40 to 60 $\mathrm{mm}$, to reduce nonlinear fields associated with the wiggler. In addition, the wigglers will need to be carefully measured and tuned to meet the field specifications.

- the electron damping ring vacuum system pressure has been re-specified to $10^{-10}$ Torr in the straight sections, and $10^{-9}$ Torr in the arcs and wigglers, to reduce the effects of ions.

- the positron damping ring vacuum system pressure has been re-specified to $10^{-9}$ Torr in the arcs and wigglers, to limit the number of primary electrons generated by gas ionization.

- a clearing gap of $600 \mathrm{~ns}$ (about 30 bunches) has been added to the electron damping ring, to suppress the formation of ions.

- low secondary emission coatings have been added to the positron damping ring vacuum chamber to suppress the formation of an electron cloud.

- the supports for the magnet systems have been upgraded, in view of the tight alignment sensitivities discussed in Section 3.3.2. 
Both damping ring straight sections will be placed in the same tunnel as the main linac cryomodules.

The bunch compressors will be identical to those in the TDR, except for additional correction systems and diagnostics. The vertical emittance dilution budget for the bunch compressors has been specified to be $20 \%$.

The spin rotator will be modified to incorporate a performance improvement identified during the ILC-TRC study [TRC].

3.5.1.2.4 Main Linac The main linac components will be taken from the TDR, with cavity operation at $28 \mathrm{MV} / \mathrm{m}$, using matched couplers. Relative to cavity operation at $23.4 \mathrm{MV} / \mathrm{m}$, there is an increase in the matched external $Q$, resulting in a longer cavity fill time, hence less efficiency, and a smaller bandwidth, hence more sensitivity to microphonics and to a failure of piezoelectric tuners required to control Lorentz detuning. To compensate for the latter problem, two tuners will be used in each cavity. The use of the superstructures is not assumed.

Additional changes from the TDR are:

- Two parallel main linac tunnels will be assumed, one housing the cryomodules and damping ring straight sections, the other housing the bulk of the main linac RF system (klystrons, modulators, DC power supplies, and interconnecting cables).

- The number of installed cavities which act as spares for klystron faults and cavity trips will be increased from that specified in the TDR, to a total of $5 \%$.

- The main linac tunnel length will be based on the $1 \mathrm{TeV}$ requirement at $35 \mathrm{MV} / \mathrm{m}$. At $500 \mathrm{GeV}$, the extra length of tunnel will be filled with beam transport line to bring the beam to the beam delivery system.

- Diagnostic insertions similar to those in the NLC design will be placed at $\sim 50 \mathrm{GeV}$, in the undulator insertion at $\sim 150 \mathrm{GeV}$, and at the entrance to the beam delivery system.

- The BPM's located at the main linac quadrupoles will be specified for an rms resolution of $3 \mu \mathrm{m}$.

- Several intratrain feedback systems will be used to control potential sources of beam jitter from the main linac components.

- The vertical emittance dilution budget for the main linac has been specified to be $50 \%$.

- The linac beamline will be laser-straight.

- The cavity dipole mode frequencies will have a controlled variation of about $0.9 \mathrm{MHz}$, introduced during the manufacturing process, to inhibit resonant wakefield effects.

3.5.1.2.5 Beam delivery system The NLC beam delivery system (including the 20 mrad crossing angle) will be "grafted" onto the TESLA linac optics. Superconducting final focus quadrupoles will be used to achieve the IP lattice functions specified in Table 3.5.1.1. A fast extraction line will be added, as in the TDR. There will be two IR's.

The vertical emittance dilution budget for the beam delivery system has been specified to be $30 \%$.

\subsubsection{Upgrade to $1 \mathrm{TeV}$}

The second column in Table 3.5.1.1 represents the upgrade to $1 \mathrm{TeV}$. Since the gradient in this case is taken to be $35 \mathrm{MV} / \mathrm{m}$, the maximum for which the cavities are assumed to be qualified, the energy limit 
of the upgraded machine is $1 \mathrm{TeV}$. The beam parameters from the source are taken to be the same as in column 1; the increase in luminosity comes entirely from additional adiabatic damping in the linac. The BDS parameters are also the same, except for $\beta_{x}$, which has been increased to reduce $N_{\gamma}$.

To implement the upgrade, additional cryomodules and RF systems must be added to the main linacs. The extra piece of linac includes a diagnostic station at $\sim 375 \mathrm{GeV}$. In addition, extra cryogenic capacity must be added. The increase in gradient results in an increase in the energy of the electron beam used for positron production to $\sim 192 \mathrm{GeV}$. The final focus also requires minor upgrades to soften the bending radius and lengthen the final quadrupoles.

\subsubsection{Injection Systems}

The injection systems provide the $5 \mathrm{GeV}$ electron and positron beams which are injected into the damping rings. The overall design and specification of the injection systems generally follows the description given in the TESLA TDR, Chapter 4. In this section, we will briefly describe the injection systems, noting those parts which are identical to the TDR, and in these cases providing simply a summary of the major technical components. For aspects of the design which differ from the TDR, a detailed discussion is provided, together with a summary of the major technical components.

\subsubsection{Electron Injection System}

The electron injection system is composed of two identical polarized electron sources, and one unpolarized source, which provide polarized and unpolarized electron beams at 76 and $20 \mathrm{MeV}$, respectively. Each beam is followed by a superconducting pre-accelerator, which brings the beam to $500 \mathrm{MeV}$. Either of these 500 $\mathrm{MeV}$ electron beams may be delivered to the electron injector linac. The electron injector linac is a $1.3 \mathrm{GHz}$ superconducting accelerator which accelerates the beam to $5 \mathrm{GeV}$, after which it is injected into the electron damping ring.

The polarized sources are located at one end of the main electron linac tunnel, in-line with the electron injector linac, which itself is in-line with the electron main linac. The unpolarized source is located in a separate tunnel, at the same horizontal depth as the polarized sources and the main linac, with a connection to the main linac tunnel. Each source with its pre-accelerator is roughly $50 \mathrm{~m} \mathrm{long}$. The electron injector linac is about $300 \mathrm{~m}$ long. A schematic view is provided in Fig. 3.5.2.1.

3.5.2.1.1 Electron Sources Two polarized electron sources will be available. A polarized electron source is required as the primary source for the collider, and an additional source is provided for improved reliability. In addition, an unpolarized source will also be available for commissioning, machine studies, and for unpolarized luminosity operation.

3.5.2.1.1.1 Unpolarized source and pre-accelerator The unpolarized source is a laser-driven RF gun, followed by a single-cell superconducting "booster" cavity to bring the beam to $20 \mathrm{MeV}$. The source is very similar to the TESLA TTF gun. The beam from the unpolarized source is accelerated from $20 \mathrm{MeV}$ to $500 \mathrm{MeV}$ using three $17 \mathrm{~m}$ long standard main linac cryomodules, which are driven by a single 10-MW klystron. The unpolarized source is not in-line with the injector linac; after the pre-accelerator, a dipole bends the $500 \mathrm{MeV}$ beam so it can be switched into the electron injector linac. The unpolarized source and pre-accelerator are described in the TDR[TDR, Section 4.2.1].

The major technical components are given in Table 3.5.2.1.1.1. The performance requirements are given in Table 3.5.2.1.1.1. 


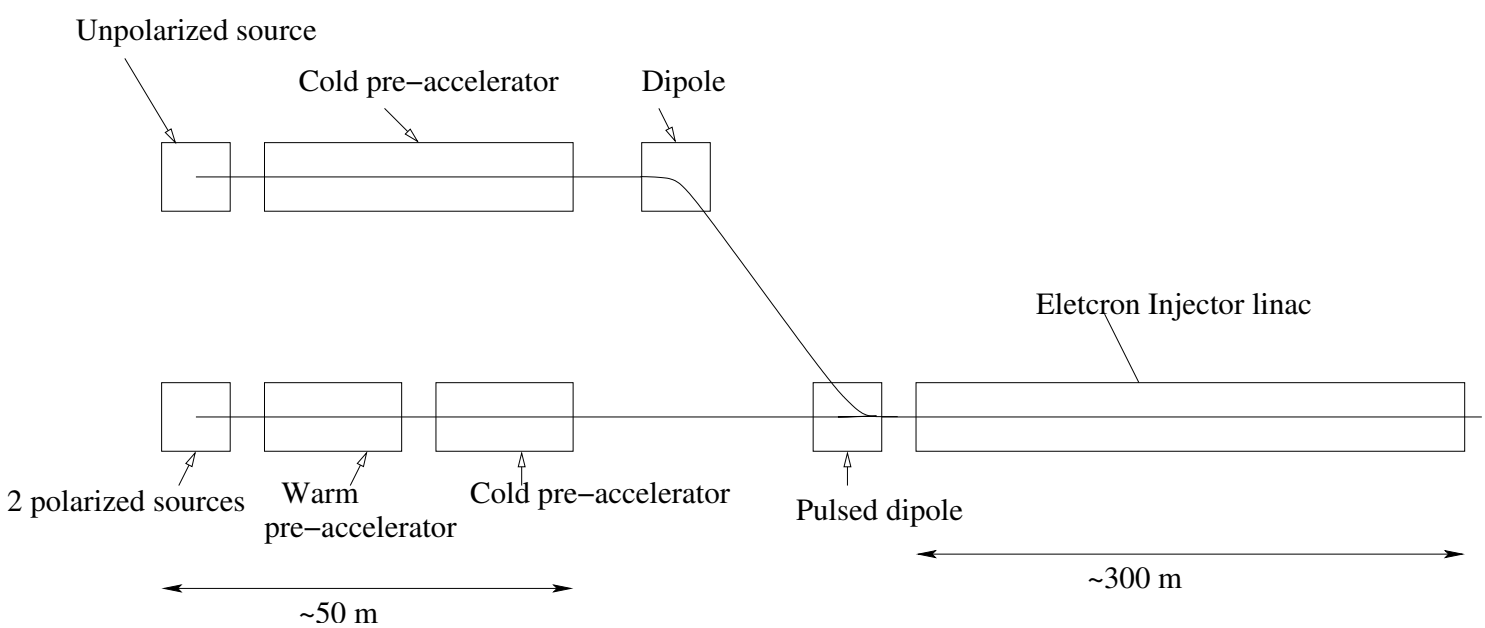

Figure 3.5.2.1: Electron Injection System layout

Table 3.5.2.1: Major Technical Components: Unpolarized Electron Source and Pre-Accelerator

\begin{tabular}{|c|c|}
\hline Component & Description \\
\hline Gun RF cavity & 1.5 cell NCRF L-band TM T10 mode cavity @ $35 \mathrm{MV} / \mathrm{m}$ \\
Gun klystron & $4.5 \mathrm{MW}$ L-band klystron \\
Gun photocathode, vacuum system & Cs 2 Te photocathode \\
Gun laser system & $270 \mathrm{~nm}$ UV laser, with $1 \mathrm{~ms}$ pulse \\
Booster cavity & 9 cell L-band SC cavity @ $16 \mathrm{MV}$ \\
Booster klystron & $200 \mathrm{~kW} \mathrm{L-band} \mathrm{klystron}$ \\
Diagnostic system & dipole spectrometer \\
Cold pre-accelerator cavities & Three $17 \mathrm{~m}$ long cryomodules @ $15 \mathrm{MV} / \mathrm{m}$ \\
Cold pre-accelerator power source & $10 \mathrm{MW} \mathrm{L-band} \mathrm{klystron}$ \\
Transfer magnet to injector linac & Dipole \\
\hline
\end{tabular}

${ }^{a}$ See TDR Table 4.2.1. Only "TESLA 500" parameters are required

Table 3.5.2.2: Performance specifications: Unpolarized Electron Source and Pre-Accelerator

\begin{tabular}{|c|c|c|}
\hline Performance parameter & Unit & Value \\
\hline Electrons/bunch & $10^{10}$ & 2.8 \\
Bunch number & & 2820 \\
Bunch spacing & $\mathrm{ns}$ & 337 \\
Output beam energy & $\mathrm{MeV}$ & 500 \\
Rms bunch length & $\mathrm{mm}$ & 3 \\
Rms energy spread & $\%$ & 1 \\
Normalized rms transverse emittance & mm-mrad & 20 \\
Bunch rms intensity stability & $\%$ & $<5$ \\
Repetition rate & $\mathrm{Hz}$ & 5 \\
\hline
\end{tabular}

3.5.2.1.1.2 Polarized source and pre-accelerator The polarized dc electron gun will use a strained GaAs cathode, illuminated by a tunable $\sim 780 \mathrm{~nm}$ laser, similar in concept to the warm option polarized gun. However, the bunch time structure (bunch length, bunch charge, bunch spacing, and number of bunches) 
and the repetition rate will differ. The major impact of these differences is on the polarized gun laser system, which requires additional R\&D. The polarized gun and laser are described in the TDR[TDR, Section 4.2.2].

The $2 \mathrm{~ns}$ long, $120 \mathrm{kV}$ electron bunch from the gun must be bunch compressed and accelerated to $500 \mathrm{MeV}$. The prebunching is done with two subharmonic NCRF prebuncher cavities, at $108 \mathrm{MHz}$ and $433 \mathrm{MHZ}$. The output beam from the prebuncher has a bunch length of $50 \mathrm{ps} \mathrm{rms}$, and is then accelerated to $12 \mathrm{MeV}$ in a pair of $1.5 \mathrm{GHz} 5$-cell NCRF standing wave buncher cavities. Each cavity dissipates $\sim 4 \mathrm{MW}$ and the pair is driven by a $10 \mathrm{MW}$ klystron. A solenoid surrounding the pre-buncher and buncher provides transverse matching and focusing.

The $12 \mathrm{MeV}$ beam has a geometric emittance which is too large for injection into a standard cryomodule. Hence, the beam is accelerated to $76 \mathrm{MeV}$, after which its geometric emittance is sufficiently small to fit in a cryomodule. The acceleration from 12 to $76 \mathrm{MeV}$ is accomplished using two pairs of $1.5 \mathrm{GHz}$ 17-cell NCRF standing wave cavities. Each pair is powered by a $10 \mathrm{MW}$ klystron. A triplet is required between the cavity pairs for focusing.

After it reaches $76 \mathrm{MeV}$, the beam is matched by another pair of triplets into the superconducting part of the pre-accelerator. This part comprises two standard $17 \mathrm{~m}$ long main linac modules, powered by a $10 \mathrm{MW}$ klystron. It accelerates the polarized beam to $500 \mathrm{MeV}$. The polarized source is in-line with the electron injector linac.

The major technical components are given in Table 3.5.2.1.1.2. The performance requirements are given in Table 3.5.2.1.1.2.

Table 3.5.2.3: Major Technical Components: Polarized Electron Source and pre-Accelerator

\begin{tabular}{|c|c|}
\hline Component & Description \\
\hline Gun DC high voltage supply & $120 \mathrm{kV}$ \\
Gun photocathode & Strained GaAs photocathode \\
Gun laser system & $\sim 780 \mathrm{~nm}$ tunable polarized laser ${ }^{a}$ \\
Prebuncher cavity 1 & $108 \mathrm{MHz}$ NCRF SW cavity @ $40 \mathrm{kV}$ \\
Prebuncher cavity 2 & $433 \mathrm{MHz}$ NCRF SW cavity @ $44 \mathrm{kV}$ \\
Buncher cavities & 2 L-band 5 -cell NCRF SW cavities @ $12 \mathrm{MV} / \mathrm{m}$ \\
Buncher power source & $10 \mathrm{MW} \mathrm{L-band} \mathrm{klystron}$ \\
Buncher focusing & Solenoid, 500 G field, $1.5 \mathrm{~m}$ long \\
Warm pre-accelerator cavities & 4 L-band 17-cell NCRF SW cavities @ $8.5 \mathrm{MV} / \mathrm{m}$ \\
Warm pre-accelerator power source & $210 \mathrm{MW} \mathrm{L-band} \mathrm{klystrons}$ \\
Warm pre-accelerator focusing & Three quadrupole triplets \\
Diagnostic system & dipole spectrometer \\
Cold Pre-accelerator cavities & $217 \mathrm{~m}$ long cryomodules @ $17.8 \mathrm{MV} / \mathrm{m}$ \\
Cold pre-accelerator power source & $10 \mathrm{MW}$ L-band klystron \\
\hline
\end{tabular}

${ }^{a}$ See TDR Table 4.2.3. Only "TESLA 500" parameters are required

3.5.2.1.2 Electron injector linac The electron injector[TDR, Section 4.2.4] accelerates either the polarized or unpolarized beam from $500 \mathrm{MeV}$ to $5 \mathrm{GeV}$, prior to injection into the damping ring. The energy gain of $4.5 \mathrm{GeV}$ is provided by 18 standard $17 \mathrm{~m}$ long main linac modules, powered by $610 \mathrm{MW}$ klystrons. Focusing is provided by one quadrupole in every module, providing a phase advance of $45^{\circ}$. A pulsed dipole at the injection end is used to switch in the unpolarized beam if required.

The major technical components are given in Table 3.5.2.1.2. The performance requirements are given in Table 3.5.2.1.2. 
Table 3.5.2.4: Performance specifications: Polarized Electron Source and Pre-Accelerator

\begin{tabular}{|c|c|c|}
\hline Performance parameter & Unit & Value \\
\hline Beam longitudinal polarization & $\%$ & $>80$ \\
Electrons/bunch & $10^{10}$ & 2.3 \\
Bunch number & & 2820 \\
Bunch spacing & $\mathrm{ns}$ & 337 \\
Output beam energy & $\mathrm{MeV}$ & 500 \\
Rms bunch length & $\mathrm{mm}$ & 3.4 \\
Rms energy spread & $\%$ & 1 \\
Normalized rms transverse emittance & $\mathrm{mm}-\mathrm{mrad}$ & 40 \\
Bunch rms intensity stability & $\%$ & $<5$ \\
Repetition rate & $\mathrm{Hz}$ & 5 \\
\hline \multicolumn{2}{|c}{} \\
\hline
\end{tabular}

Table 3.5.2.5: Major Technical Components: Electron Injector Linac

\begin{tabular}{|c|c|}
\hline Component & Description \\
\hline Transfer magnet & Pulsed dipole \\
Accelerator cavities & $1817 \mathrm{~m}$ long cryomodules @ 20 MV/m \\
Focusing system & One quadrupole per module \\
Rf power source & Six 10 MW L-band klystrons \\
\hline
\end{tabular}

Table 3.5.2.6: Performance specifications: Electron Injector Linac

\begin{tabular}{|c|c|c|}
\hline Performance parameter & Unit & Value \\
\hline Beam longitudinal polarization & $\%$ & $>80$ \\
Electrons/bunch & $10^{10}$ & 2.3 \\
Bunch number & & 2820 \\
Bunch spacing & $\mathrm{ns}$ & 337 \\
Output beam energy & $\mathrm{GeV}$ & 5 \\
Rms bunch length & $\mathrm{mm}$ & 3.4 \\
Rms energy spread & $\%$ & 1 \\
Normalized rms transverse emittance & $\mathrm{mm}-\mathrm{mrad}$ & 40 \\
Bunch rms intensity stability & $\%$ & $<5$ \\
Repetition rate & $\mathrm{Hz}$ & 5 \\
\hline
\end{tabular}

\subsubsection{Positron Injection System}

The positron source is an undulator-based system very similar to that described in the TESLA TDR[TDR]. The particular implementation used is one developed by the NLC collaboration for polarized positron production [18]. This implementation includes several changes made to improve the operation and reliability of the positron source system.

1. The undulator has been located midway along the electron linac at a fixed beam energy of $150 \mathrm{GeV}$ which is used for improved energy flexibility over that of the TDR design.

2. The undulator is assumed to be helical to generate polarized positrons when such an option is desired. To provide for sufficient yield and to ensure reliable operation, the helical undulator is roughly 200 meters in length - this should have a positron yield (number of captured $e^{+}$per incoming $e^{-}$) which is four times that of the system described in the TESLA TDR. 
3. Relatively short undulator sections are placed between quadrupole magnets to ease the undulator tolerances and magnet alignment.

4. Two targets and capture sections are installed so that when one system fails, the other can be used without waiting for a time consuming cool-down period in the radioactive environment. In addition, a polarized electron source is installed next to the capture sections to facilitate commissioning and operation with $e^{-}-e^{-}$or $\gamma-\gamma$.

5. The positron beam is immediately accelerated after the capture sections to the nominal damping ring energy of $5 \mathrm{GeV}$ and transported to the ring at this energy rather than at $290 \mathrm{MeV}$ as in the TESLA TDR.

6. Additional diagnostics and compensating optics has been explicitly designed into the system to aid commissioning and operation.

Parameters for the polarized and unpolarized undulator-based positron source are compared with those of the TESLA TDR in Table 3.5.2.7. Table 3.5.2.7 also lists parameters of a possible conventional positron source[35] which is not part of the reference design. The positron source must be capable of producing polarized positrons and thus the enclosure for the full positron system must be excavated. However, for initial operation, only the undulator required to generate the an unpolarized positron beam needs to be installed. It might also be possible to only install the length necessary to obtain a simulated yield of 1 while having the option of installing additional undulator if the operational yield is lower; however this is probably a misplaced efficiency. Alternately, one could install a planar undulator system which is more straightforward to construct. However, with equal peak fields, a planar undulator would have to be twice as long as a helical undulator for the same yield.

3.5.2.2.1 Positron Source Layout As mentioned, one of the major changes to the TESLA TDR positron system was the change in undulator location. In the TESLA TDR design, the source was located at the end of the electron linac and the incoming electron beam energy is roughly one half of the collision energy. Both the undulator photon energy and the undulator photon power scale as the square of the electron beam energy and thus the performance of the positron system will change dramatically as the beam energy is varied.

There are two real issues here. First, because the positron source parameters such as the yield, energy, bunch length, and emittance, are all very sensitive to the incoming beam energy, small energy changes will require significant retuning of the positron source system. If the positron source is located at the end of the linac, even energy scans of a few percent will probably require retuning the source. The delicacy of the positron source tuning was evident during the SLC operation where the positron source parameters were tuned more often than any other part of the collider. Because of the very large beam emittances and the unfriendly environment around the positron source, it is difficult to install reliable beam diagnostics that simplify the tuning process. Thus, it is felt to be quite important to minimize the tuning that will be needed which can be done by installing the source a fixed energy along the collider.

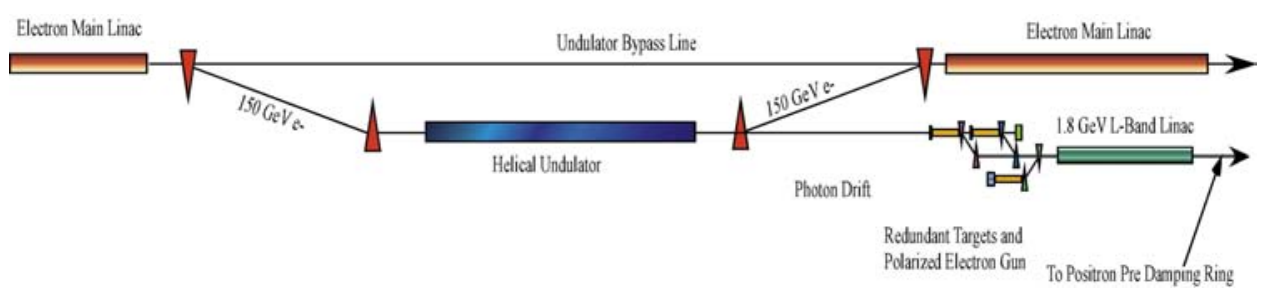

Figure 3.5.2.2: Undulator-based positron generation scheme 
Table 3.5.2.7: Cold Option Positron Source Parameters

\begin{tabular}{|c|c|c|c|c|}
\hline & TESLA TDR $^{\star}$ & Unpolarized & Polarized & Conventional \\
\hline Drive Beam Energy $[\mathrm{GeV}]$ & 250 & 153 & 153 & 6.2 \\
\hline Beam Energy Loss $[\mathrm{GeV}]$ & 3 & 4.9 & 6.5 & - \\
\hline Beam Energy Spread In [\%] & 0.05 & 0.5 & 0.5 & - \\
\hline Beam Energy Spread Out [\%] & 0.10 & 0.46 & 0.44 & - \\
\hline Additional linac length $[\mathrm{m}]$ & 120 & 170 & 240 & 230 \\
\hline Undulator length $[\mathrm{m}]$ & 100 & 150 & 200 & - \\
\hline Undulator insertion length $^{\S}[\mathrm{m}]$ & 340 & 790 & 850 & - \\
\hline Positron source length $[\mathrm{m}]$ & 400 & 450 & 450 & 450 \\
\hline Photon energy* $[\mathrm{MeV}]$ & 28 & 10.7 & 10.7 & - \\
\hline Undulator type & $K=1 ;$ planar & $K=1 ;$ helical & $K=1$; helical & - \\
\hline Undulator field $[\mathrm{T}]$ & 0.75 & 1.07 & 1.07 & - \\
\hline Undulator period $[\mathrm{cm}]$ & 1.4 & 1 & 1 & - \\
\hline Undulator full gap [mm] & 5 & 6 & 6 & - \\
\hline Pulse energy on target $[\mathrm{kJ}]$ & 26.9 & 44 & $41.0^{\diamond}$ & $28.0^{\natural}$ \\
\hline Average power on target $[\mathrm{kW}]$ & 135 & 222 & $207^{\diamond}$ & $140^{\natural}$ \\
\hline Spot size on target $[\mathrm{mm}]$ & 0.75 & 0.75 & 0.75 & 2.5 \\
\hline Target material & Ti-alloy & Ti-alloy & Ti-alloy & $\mathrm{W}_{75} \mathrm{Re}_{25}$ \\
\hline Target thickness [r.l.] & 0.4 & 0.4 & 0.4 & 4.0 \\
\hline Target energy absorption [\%] & 4 & 8 & 8 & 14 \\
\hline Beam polarization [\%] & 0 & 0 & 59 & 0 \\
\hline Positron yield ${ }^{\dagger}$ & $2.0^{\ddagger}$ & 1.5 & 1.5 & 1.5 \\
\hline
\end{tabular}

$\S$ The undulator insertion length includes the length of the undulator and the drift to the target if this increases the system length.

I The source length is defined as the length required to generate, capture, and accelerate the positrons to damping ring energy without including the length of low energy transport line.

$*$ Denotes the photon energy at the first harmonic cutoff.

$\diamond$ Includes intensity reduction due to an angular cut at $\gamma \theta=1.414$.

$\natural$ Drive beam energy and power per target; two targets operating in parallel are required.

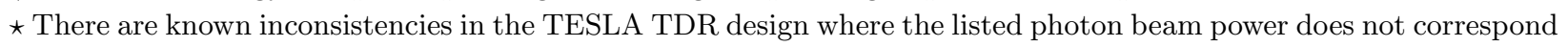
to the listed undulator parameters.

$\dagger$ Positron yield is defined as the number of positrons captured in the damping ring divided by the number of electrons used to generate the positrons.

$\ddagger$ The positron yield of 2 is listed in the TDR; however, other calculations find values between 1 and 1.5 for the TDR system.

Second, the positron yield scales roughly quadratically with beam energy. Thus, if the system is designed to have a an operating yield of 1 at $150 \mathrm{GeV}$, the luminosity will drop very rapidly as the beam energy is further decreased. The solution proposed in the TESLA TDR was to use one portion of the linac to accelerate the beam to $150 \mathrm{GeV}$ for positron production and use the remaining $100 \mathrm{GeV}$ of linac (during Stage I) to accelerate beam to the IP. This technique allows coverage from $90 \mathrm{GeV}$ up to about $200 \mathrm{GeV}$; however there is still an important gap from $200 \mathrm{GeV}$ until $300 \mathrm{GeV}$ in the center-of-mass where neither positron production technique is sufficient. By placing the undulator partway along the linac, the beam can be either accelerated or decelerated after the undulator, thereby increasing the operating range of the collision energy.

For these two reasons we have chosen a source location at a beam energy of $153 \mathrm{GeV}$ rather than the end of the electron linac. At this location, 150-meters of undulator, needed for a simulated yield of 1.5, will decrease 
the beam energy by $4.9 \mathrm{GeV}$. Thus, to obtain a full beam energy of $250 \mathrm{GeV}, 101.4 \mathrm{GeV}$ of linac must be installed after the positron source undulator. This means that the stage I electron linac should be able to produce a beam with energies between $45 \mathrm{GeV}$ and $250 \mathrm{GeV}$ while generating a stable positron beam. The only difficulty with the scheme is the energy jitter and the emittance dilutions will increase as the beam is decelerated. However, since most running at the Z-pole for detector calibration does not require enormous luminosity, these are not thought to be unmanagable liabilities.

If the positron source is to be installed midway along the linac, the undulator and positron target, capture, and acceleration must be installed in a parallel tunnel. A schematic of the layout for the positron source is illustrated in Fig. 3.5.2.2. The undulator and positron source systems are offset by 2.5 meters transversely from the main linac. The 2.5-meter offset will allow for sufficient shielding so that the positron target and capture regions will not irradiate the main linac tunnel. Arcs, which generate small levels of emittance dilution, will be used to inject the main electron beam into the undulator and then re-inject it into the main linac tunnel. The photon beam will drift straight ahead for roughly 200 meters after the end of the undulator to the positron targets. There will be two positron target and capture assemblies in line so that if one fails, the other can be inserted with minimal impact to the run. In addition, it is suggested that there be a polarized electron gun which would be used for $e^{-}-e^{-}$or $\gamma-\gamma$ operation. This electron system would likely be used to commission the positron beam lines since the much lower electron beam emittance will make it much easier to diagnose the beam line.

Finally, the configuration that is proposed here has the undulator parallel to the main linac. It is likely possible to achieve similar separation of the positron target by placing the undulator at an angle to the main linac and this may reduce the total bending that is required. This option has not yet been investigated. The total length of the undulator insertion and the subsequent positron target, capture, and acceleration system which are all located 2.5 meters offset from the main tunnel is about $1.3 \mathrm{~km}$. The system length is roughly twice as long as the layout in the TESLA TDR due to undulator insertion and undulator length, the more extensive RF system for operational overhead, and the more extensive diagnostics.

3.5.2.2.2 Undulator Insertion The arcs displace the undulator by 2.5 meters from the linac centerline. The arcs are based on sixteen 14-meter 90 degree FODO cells plus dispersion suppressing cells at either end. The incoherent SR growth of the TESLA beam is roughly $1 \%$ for the LTU (linac-to-undulator) and 1\% for the return. Each arc is roughly 250 meters in length. The trajectory is shown in Fig. 3.5.2.3 while the beta functions and the dispersion are shown in Figs. 3.5.2.4 and 3.5.2.5. Finally, the second-order dispersion is canceled with eight sextupoles located in each arc to ensure a bandpass of at least $\pm 3 \%$. The bandpass is plotted in Fig. 3.5.2.6; a reasonable bandpass is needed to prevent damage due to routine energy fluctuations and may be needed for beam-based alignment techniques. Additional sacrificial spoilers will undoubtedly be needed to protect the undulator and the insert from larger energy errors due to machine faults.

The undulator has distributed focusing along its length using ten 24-meter FODO cells. The focusing significantly reduces the tolerances on the undulator fields and alignment. The phase advance of the FODO cells is nominally set to 72 degrees/cell so that many systematic undulator field errors naturally cancel over the length. Additional studies are needed to determine the field error limits and full alignment tolerances for the undulator sections. The external focusing also reduces the sensitivity to incoming energy errors and makes the optical aberrations of the undulator insertion much easier to control. The quadrupoles will have to be aligned using beam-based alignment techniques and the nominal alignment tolerance is about $5 \mu \mathrm{m}$ for $2 \%$ vertical emittance dilution.

The undulator section is about 240 meters in length, with the undulator filling 200 meters of this, which is sufficient for a simulated yield of 1.5 and an average polarization of $59 \%$. The installed undulator during initial operation would likely be significantly less but the space exists to install additional sections as needed to obtain an operation yield of 1 . The full undulator necessary for polarized positrons radiates roughly 6 $\mathrm{GeV}$ of the beam energy and generates an energy spread of $0.004 \%$. The undulator would be constructed in 2-meter sections with 5 sections installed between quadrupoles as illustrated in Fig. 3.5.2.7. 


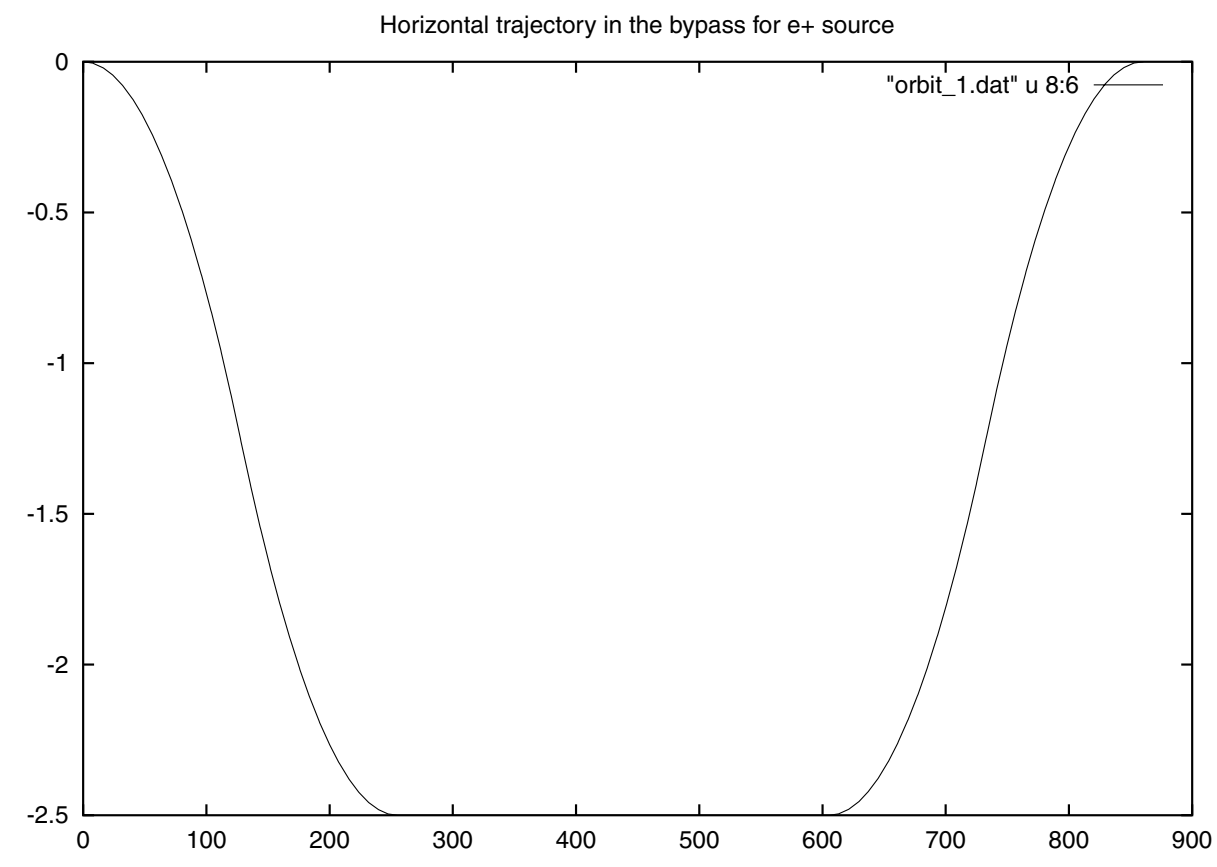

Figure 3.5.2.3: Horizontal orbit through the undulator bypass. Horizontal axis: $s[\mathrm{~m}]$. Vertical axis: Horizontal orbit displacement from linac axis $[\mathrm{m}]$.

There are two 90 degree FODO cells at the undulator entrance for trajectory feedback control and additional diagnostics. The dispersion matching tolerance is roughly $100 \mu \mathrm{m}$ at a beta function of 40 meters to limit the vertical emittance growth to $2 \%$ in the undulator. The entire undulator insert is roughly 850 meters in length and the $e^{+}$target would be placed 200 meters downstream of the end of the undulator, close to where the undulator insert beam line rejoins the main linac beam line.

The full undulator insert consists of 18 arc cells, 3 matching and diagnostic cells, 10 undulator cells, 3 $\mathrm{diag} / \mathrm{matching}$ cell, and, finally, 18 arc cells. The full length of the system is 846 meters. At this time, the ends of the insert have not been matched into the superconducting main linac lattice; however this is expected to be straightforward and will, at most, require an additional matching cell at either end.

3.5.2.2.3 Undulator The undulator is a helical undulator with $K$ equal to 1 and a peak field of 1.1 Tesla. The advantage of the helical undulator is that it allows for polarized positrons and, for the same peak field, it produces twice as many photons per unit length as a planar undulator [36]. The disadvantage is that a helical undulator is more technically more difficult to design. To achieve the $11 \mathrm{MeV}$ photons from a $153 \mathrm{GeV}$ beam, the period of the undulator must be $1 \mathrm{~cm}$.

As discussed, the undulator is assumed to be constructed from 2-meter segments. At this time, conceptual studies have begun on helical undulator systems. The favored solution is a superconducting bifilar coil wrapped around a $6 \mathrm{~mm}$ radius beam pipe. At this time, a simple prototype has been constructed; however the field, period, and field quality requirements have not yet been attained [37]. Another option is to use a pulsed normal conducting coil [38] although, for the millisecond pulse length of the superconducting linac, pulsed heating may become a problem. One of the primary concerns with the bi-filar coil concept is the control of the field tolerances which will require very accurate conductor placement. Alternate technologies include permanent magnet systems where the magnet blocks are offset to generate the helicity. Here, the 


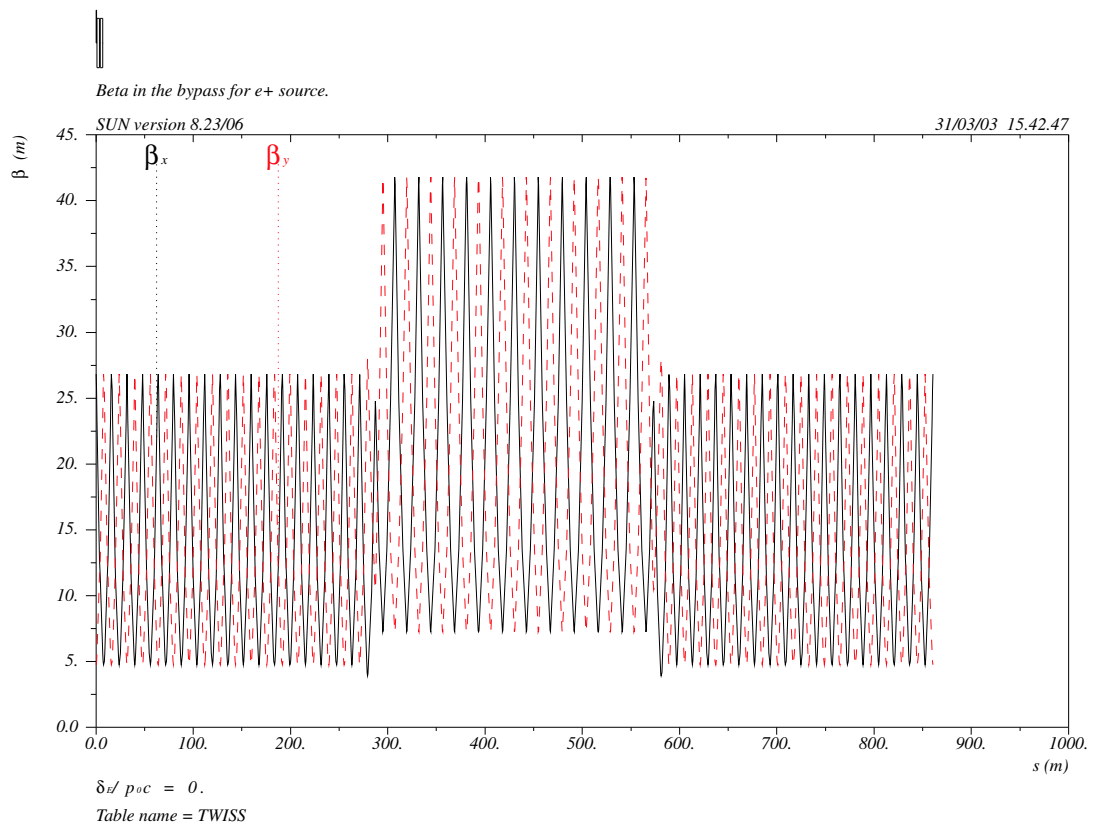

Figure 3.5.2.4: Beta functions through the undulator insertion

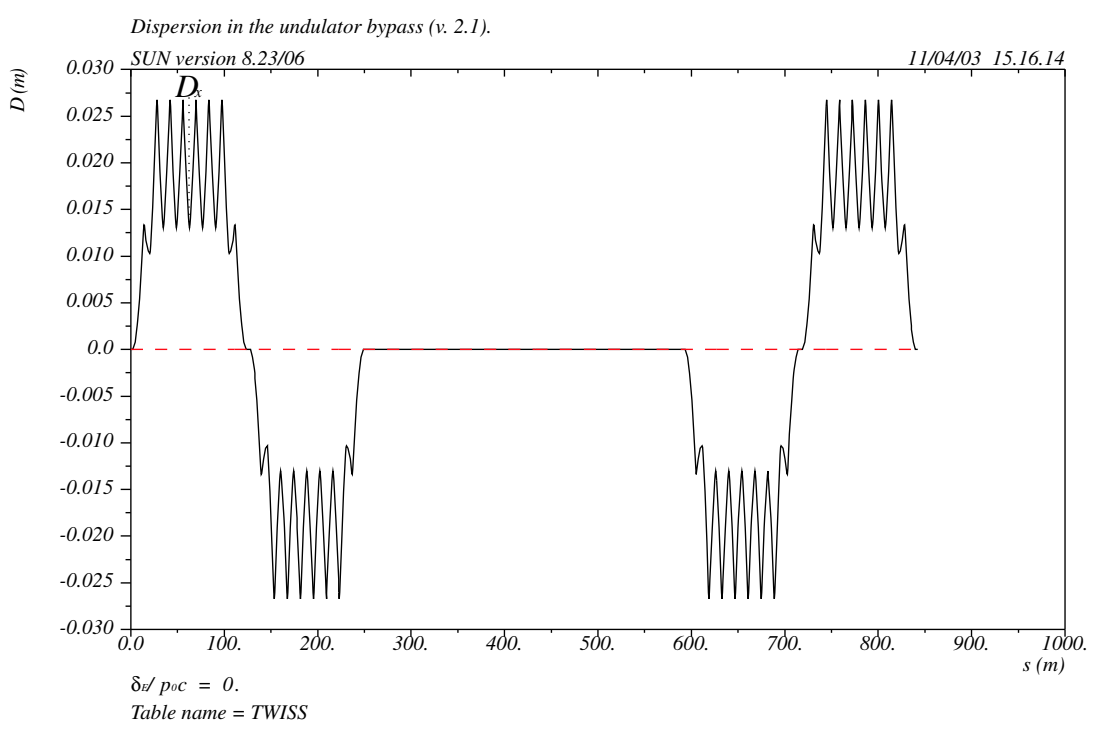

Figure 3.5.2.5: Horizontal dispersion function through the undulator insertion. 


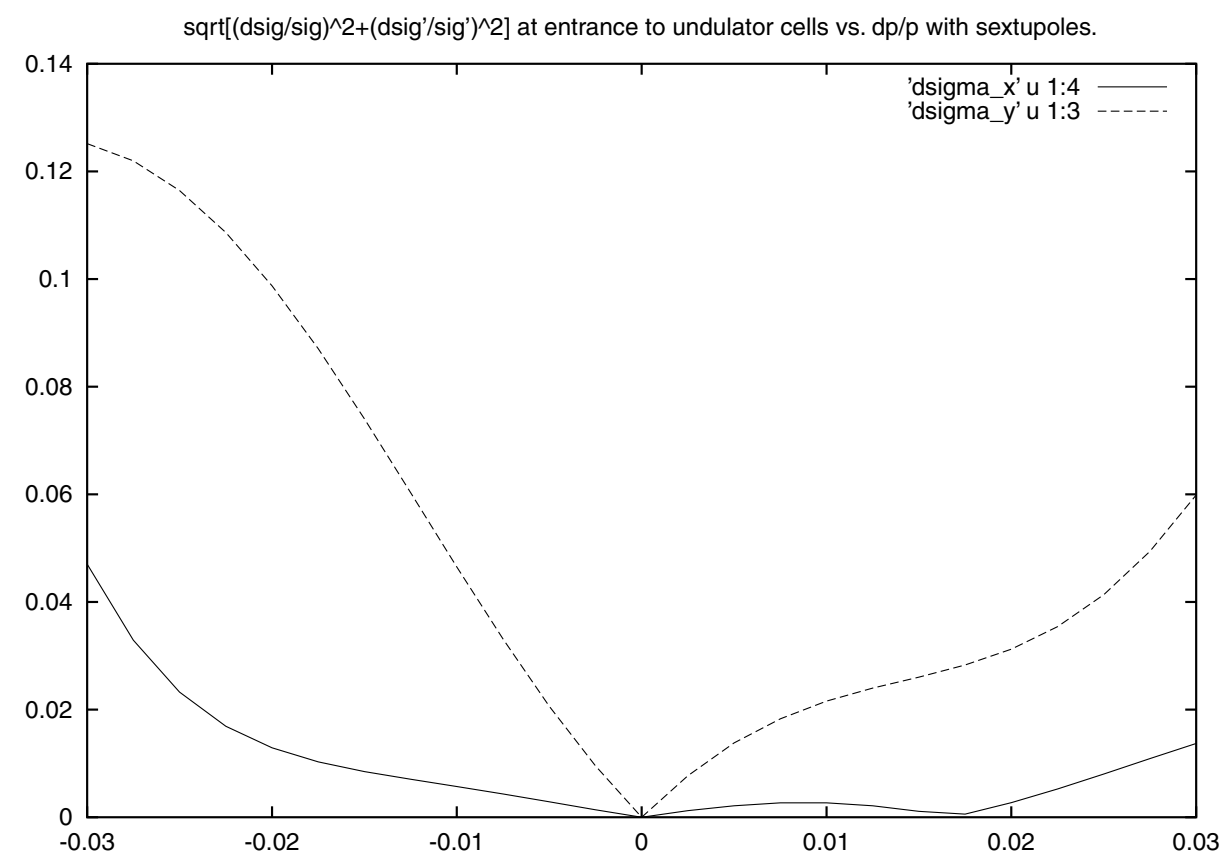

Figure 3.5.2.6: Horizontal and vertical bandpass at the end of the undulation insertion arc. Horizontal axis: $d p / p$. Vertical axis: $\sqrt{\left(\frac{d \sigma}{\sigma}\right)^{2}+\left(\frac{d \sigma^{\prime}}{\sigma^{\prime}}\right)^{2}}$, with $\sigma=$ rms beam size, $\sigma^{\prime}=$ rms beam divergence.

\section{Undulator Lattice Half Cell}

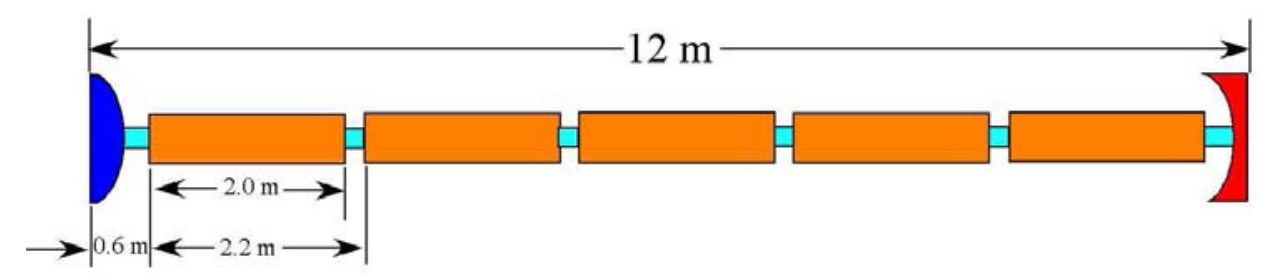

[Undulator Module Parameters: Helical, $\mathrm{K}=1, \lambda_{\mathrm{u}}=1 \mathrm{~cm}\left(\mathrm{~B}_{0}=1.07 \mathrm{~T}\right), \mathrm{ID}=6-7 \mathrm{~mm}, \mathrm{~L}_{\mathrm{u}}=2.0 \mathrm{~m}$ ]

\section{Undulator Lattice, 10 FODO Cells}

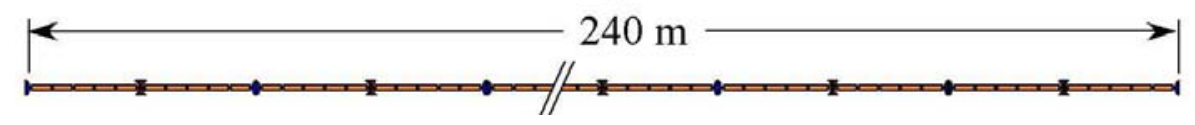

Figure 3.5.2.7: Undulator layout

primary difficulty is generation of the required fields.

One disadvantage of the bifilar coil configuration is that it is difficult to make fast changes to the undulator helicity which is important to be able to control the systematic errors at the particle physics detector. Most likely the only way to enable pulse-by-pulse changes to the helicity with the undulator-based scheme is the 
use of separate beam lines which may introduce their own systematics - this is discussed further in Section 3.5.2.2.9.

3.5.2.2.4 Target For this study, it is assumed that the positron source target is similar to that described in the TESLA TDR, namely, 0.4 radiation lengths of titanium [TDR, Section 4.3.4]. The production rate in a titanium target is about $40 \%$ lower than in tungsten, but the higher heat capacity and the mechanical properties of titanium allow much higher particle densities inside the target. The target has to rotate with a high velocity in order to avoid an overlapping of all bunches within one RF pulse. A velocity of about 50 $\mathrm{m} / \mathrm{s}$ at the circumference of the target will spread out a single bunch train over a distance of $5 \mathrm{~cm}$. The target parameters are given in Table 3.5.2.8.

Table 3.5.2.8: Positron Target Parameters

\begin{tabular}{|c|c|c|c|c|}
\hline & TESLA TDR & Unpolarized & Polarized & Conventional \\
\hline Pulse energy on target [kJ] & 27 & 44 & 41 & 28 \\
\hline Average power on target [kW] & 135 & 222 & 207 & 140 \\
\hline Spot size on target [mm] & 0.75 & 0.75 & 0.75 & 2.5 \\
\hline Target material & Ti-alloy & Ti-alloy & Ti-alloy & $\mathrm{W}_{75} \mathrm{Re}_{25}$ \\
\hline Target thickness [r.l.] & 0.4 & 0.4 & 0.4 & 4.0 \\
\hline Target energy absorption [\%] & 4 & 8 & 8 & 14 \\
\hline Target Radius [m] & 0.40 & 0.80 & 0.80 & 1.0 \\
\hline Revolution Rate [rpm] & 1200 & 1200 & 1200 & 1200 \\
\hline Pulsed Temperature Rise [ $\left.{ }^{\circ} \mathrm{C}\right]$ & 420 & 410 & 361 & 256 \\
\hline Number of targets/spares & $1 / 0$ & $1 / 1$ & $1 / 1$ & $2 / 3$ \\
\hline
\end{tabular}

Figure 3.5.2.8 [39] is a plot of the temperature in the target after the TESLA TDR format photon beam where the peak temperature rise is roughly $420{ }^{\circ} \mathrm{C}$. This is more than a factor of two below the stress limit for undamaged Titanium. The TDR assumes a velocity at the circumference of the target of $50 \mathrm{~m} / \mathrm{s}$ and thus this maximum heat load corresponds to about 100 bunches overlapping at the same location. In the TDR, the incident photon beam power is $135 \mathrm{~kW}$ and the absorption is stated to be $4 \%$. The target diameter of $80 \mathrm{~cm}$ was chosen to evenly distribute the heat load over the entire circumference of the target over many 5 $\mathrm{Hz}$ pulses where the average heat load amounts to $5 \mathrm{~kW}$. Cooling by radiation might be sufficient in the case of the large target wheel, but this needs further investigation. Cooling with water may be possible using a vacuum feed-through based on differential pumping.

For the present parameters, with $11 \mathrm{MeV}$ photons, the energy absorption in the target is calculated with EGS4 to be roughly $9 \%$ of the incident photon energy. With the incident photon beam power of $222 \mathrm{~kW}$, this would require a velocity at the target edge that is roughly $100 \mathrm{~m} / \mathrm{s}$ to maintain a similar pulsed temperature rise. Either the revolution frequency could be maintained and the target diameter doubled or the revolution rate could be doubled. Here, we assume that the target diameter is doubled so that the possibility of radiation cooling has not changed and the target lifetime, to be discussed, is increased. In this case, the pulsed temperature rise is calculated to be $410{ }^{\circ} \mathrm{C}$, and the stress is still a factor of two below the limit for undamaged titanium.

One outstanding question regarding the choice of target material is the susceptibility to radiation damage. There is substantial experience with radiation damage in the conventional positron targets such as that in the SLC. In the SLC positron system, a $\mathrm{W}_{26}$ Re alloy was selected because of the exceptional physical properties such as high strength and ductility at the anticipated target operating temperatures, between 115 and $200{ }^{\circ} \mathrm{C}$. At $150{ }^{\circ} \mathrm{C}$, ductility of these alloys increases $10 \%$ with no significant decrease in yield strength, maintaining it at $1100 \mathrm{MPa}$. However, the catastrophic changes in mechanical properties of these materials due to irradiation were not anticipated. This led to target failure at stresses that were a factor of two times smaller than expected for the undamaged materials [41]. It is expected that Titanium will exhibit similar 
or worse behavior after radiation damage [42] - this may be especially true if the incident photon energy is close to $\sim 20 \mathrm{MeV}$ where neutrons are produced through the giant dipole resonance [43]. The rate of radiation damage in $\mathrm{Ti}$ requires further study.

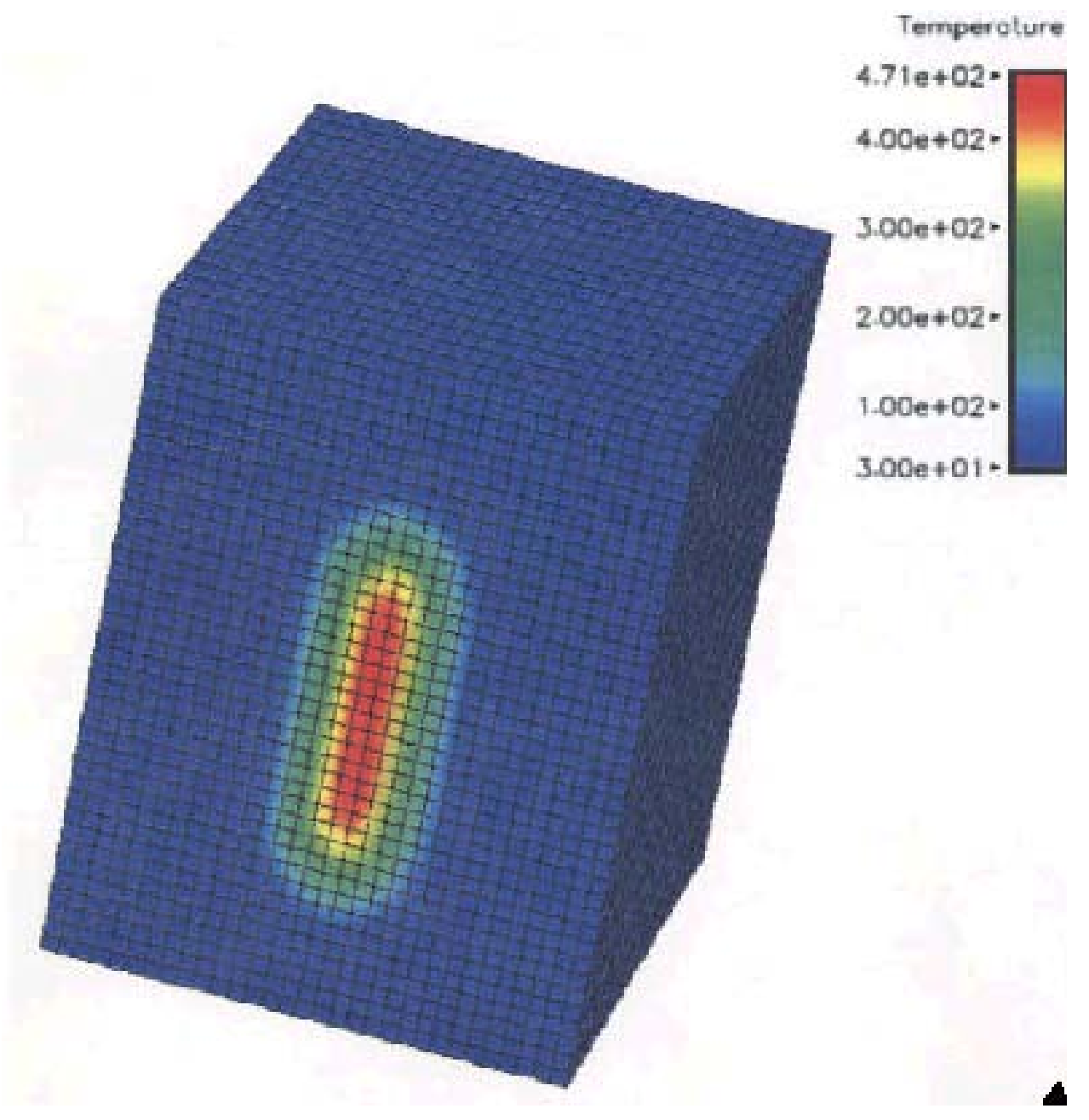

Figure 3.5.2.8: Target temperature distribution after $0.2 \mathrm{~ms}$. Temperatures are in ${ }^{\circ} \mathrm{C}$.

3.5.2.2.5 Positron Yield Positron yield is defined as the ratio of the number of captured positrons to the number of electrons transported through the undulator. The number of photons incident on the target is proportional to the number of drive electrons and to the length of the undulator. The photon energy spectrum depends upon the undulator strength parameter, $K$, the undulator period, and the square of the drive beam energy. For the systems under consideration, a strength parameter of $K=1$ and period of $1 \mathrm{~cm}$ combined with a drive beam energy of $150 \mathrm{GeV}$ produce photons in the energy range of 0-12 MeV. The rate of photon production for these parameters is essentially 1 photon per electron, per meter of undulator, for a planar device and about twice this value for a helical undulator.

The number of positrons produced and captured varies with the photon energy, the choice of target material and the acceptance of the downstream systems. In the photon energy range of $10-40 \mathrm{MeV}$, the pair production cross section rises linearly with energy but at lower energy, it rolls off. A calculation of positron emission from the converter target must include the effect of energy loss and absorption during drift to the surface. 

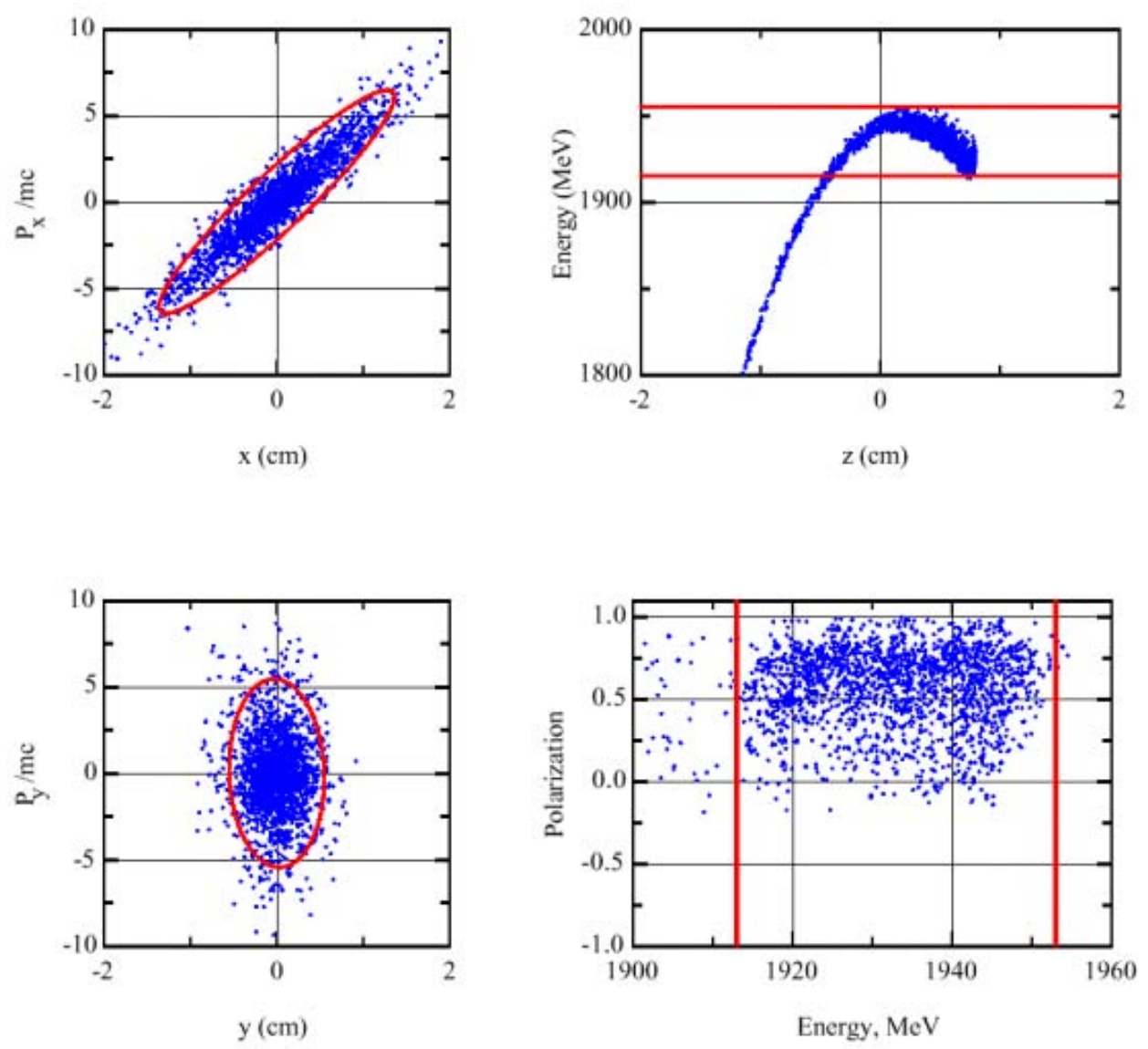

Figure 3.5.2.9: Positron capture phase space

For a 0.4 r.l. thick Ti-alloy target material, the emission probability is proportional to photon energy over a range of 5-50 MeV. The resulting efficiency varies from 0-11\%. Below about $5 \mathrm{MeV}$ incident photon energy, very few positrons emerge from the target. For a downstream system with a phase space acceptance of 0.03 m-rad and a $40 \mathrm{MeV}$ cutoff, the capture probability of emitted positrons is in the range of $25-50 \%$.

A number of codes have been developed to simulate the production of photons in an undulator, the production of positrons in various target materials, and the subsequent capture and acceleration of the positrons generated. The results of these yield simulations are given in Table 3.5.2.7 [20] [14]. For the parameters chosen, the positron-to-electron capture yield is approximately $1 \%$ per meter of undulator for unpolarized positron production and about $0.75 \%$ per meter of undulator for polarized positron production.

3.5.2.2.6 Positron Capture Region and Pre-accelerator The capture region and the positron preaccelerator (PPA) will accelerate the positrons to roughly $250 \mathrm{MeV}$. This system consists of the Adiabatic Matching Device (AMD) and the first set of accelerator structures. The AMD provides a tapered solenoidal field to match the transverse phase space from the target to the accelerator structures. The TDR description assumes a peak field of 6 Tesla which is tapered to 0.16 Tesla [TDR, Section 4.3.5]. This is similar to the flux concentrator operating at the SLC and those designed for the JLC and NLC. However, the pulse length must be roughly 1 millisecond instead of the $5 \mu$ s for the SLC device, and pulsed heating would make the SLC technology inapplicable. Alternate technologies include superconducting solenoids although the high 
radiation environment may makes these difficult. Further R\&D is required.

The accelerator structures following the AMD will be normal conducting structures because of the high radiation environment combined with large beams losses. However, the long pulse length makes cooling the high gradient normal conducting structures difficult. This system will likely be similar to that proposed in the TESLA TDR [TDR, Section 4.4.1]. The gradient of the first two capture sections is $14.5 \mathrm{MV} / \mathrm{m}$ to maximize the positron yield and then the gradient of the subsequent structures in the PPA would be $8.5 \mathrm{MV} / \mathrm{m}$. Each capture structure is roughly 1.1 meters in length and is fed by a 10-MW klystron. The following seven structures are each roughly 4 meters in length and are each fed by 10-MW klystrons.

The capture region, which consists of the first two high gradient structures and the following two lower gradient structures, is focused with solenoidal focusing. The capture region is followed by a dogleg to separate the positrons from the electrons and photons. For redundancy, a second positron target and capture region would be placed immediately downstream of the first and could be activated should the first target fail. Both capture regions would be mounted on girders to allow for quick removal and insertion. The photon beam dump would be placed after the second capture region. The total length of the capture and PPA is about 70 meters and requires 13 10-MW klystrons. Finally, the polarized electron injector could be placed parallel to the positron capture sections as illustrated in Fig. 3.5.2.2.

The TESLA TDR capture and PPA sections are based on the CDS structures [45]. These structures are standing wave $\pi$-mode structures with magnetic coupling slots. These structures were developed to operate with very high average heat loads in a cw linac. Operation at gradients of $14.5 \mathrm{MV} / \mathrm{m}$ correspond to an average heat load of about $30 \mathrm{~kW} / \mathrm{m}$ which is well below the $\sim 200 \mathrm{~kW} / \mathrm{m}$ for which the structures were designed. Further numerical studies have verified that this average thermal stress is not a limitation at gradients of up to $15 \mathrm{MV} / \mathrm{m}$ [46] however the pulsed stresses have not yet been evaluated [47]. The largest concern will be the pulsed temperature rise around the magnetic coupling slots and this needs further study. Studies of pulsed temperature rises in $\mathrm{Cu}$ structures have observed significant damage to the surface with pulsed temperature rises of $\sim 100^{\circ} \mathrm{C}[48]$.

3.5.2.2.7 Positron Injector Linac (PIL) The positron beam will be accelerated in a superconducting linac from the $250 \mathrm{MeV}$ point to the damping ring energy of $5 \mathrm{GeV}$. The linac accelerator structures can be assumed to be identical to the superconducting main linac described elsewhere in this document. The structures will be grouped into two different type of modules: CM-1 and CM-2 as described in the TESLA TDR [TDR, Section 4.4.4]. The linac will consist of 6 CM-1 modules followed by 18 CM-2 modules. Each group of three modules will be powered by one 10-MW klystron for a total of eight klystrons in the PIL. The total length of the PIL is 320 meters. This layout leaves one klystron as a spare should one of the other seven fail.

The only difference between this layout and that described in the TDR is that the gradient is increased from $24 \mathrm{MV} / \mathrm{m}$ to $35 \mathrm{MV} / \mathrm{m}$ and linac is located immediately after the normal conducting PPA. In the TDR, this linac was placed after the low energy beam was transported across the IP. This location was chosen because there are no cryogenic facilities to support the booster linac along the beam delivery system until the beam is adjacent to the positron main linac after crossing the IP. In the present case, cryogenic facilities for the electron main linac exist in the parallel tunnel. Furthermore, there is concern regarding space if the main linac superconducting accelerator and the positron booster superconducting accelerator are both placed in the same tunnel.

3.5.2.2.8 High energy positron Transfer line The positron transfer line takes the $5 \mathrm{GeV}$ positrons from the booster linac and the $5 \mathrm{GeV}$ diagnostic station and deflects them into the main linac tunnel. This portion of the transfer line is roughly 30 meters. After being re-injected into the electron main linac tunnel, the positron beam is transported along the tunnel, through the beam delivery system, into a parallel tunnel that crosses between the two interaction regions, and back along the positron main linac to the positron 
damping ring. The total length of this transfer line is about $30 \mathrm{~km}$ before the beam is injected into the positron ring. These systems are under design. Although the transfer lines are relatively simple and need to transport a large emittance beam, these systems still require attention because of the very high beam power of over $200 \mathrm{~kW}$ that is being transported. Concerns due to time-dependent stray fields from the pulsed klystrons must also be studied.

3.5.2.2.9 Polarization control, Spin rotation, Energy compressor and Damping ring injection It is likely that, to control systematic errors, the IP polarization will need to be reversed on a pulse-by-pulse basis. The polarization control system is designed to flip the helicity on a pulse-by-pulse basis while the spin rotation system is required to rotate the polarization into the vertical plane before injection into the damping ring. Likely the simplest solution is to combine these systems where first an arc of roughly 8 degrees is used to rotate the spin from the longitudinal direction into the horizontal direction and then a bipolar pulsed kicker directed the beam to one of two parallel beam lines with solenoids which rotates the spin either up or down from the horizontal plane. The solenoids will require an integral field strength of $26 \mathrm{~T}$-m to rotate the spin from the horizontal plane into the vertical direction. The parallel beam lines would then be recombined using another bipolar kicker. The systematic differences between the parallel beam lines might be reduced by occasionally reversing the solenoid fields.

One additional concern is that, because the transport from the positron source to the damping ring is quite long and complicated, the precession due to any additional horizontal and vertical bending should be considered and perhaps a more flexible spin rotation system will be needed to optimize the spin before injection into the positron damping ring. This flexible spin rotation system may become relatively long and complicated [49]. Of course, not all of the solenoids need to be installed when operating with unpolarized positrons, but the beam line must be installed with the correct bending angles as changes to the beam line geometry become hard to retrofit.

Finally, an energy compressor may be desired before injection into the positron damping ring. The longitudinal phase space of the positron beam from the source is highly mismatched to the positron damping ring and the large incoming energy spread can exceed the dynamic aperture of the positron ring. The energy compressor would use the $R_{56}$ of the spin rotation arc to generate a correlation between energy deviation and longitudinal position and then an RF system to reduce the energy spread [50]. At this point, none of these systems have been designed.

\subsubsection{Damping Rings}

In this section, we detail some of the technical specifications for the present designs of the cold LC damping rings. General design issues (including comparison with existing storage rings and the cold LC damping rings) are discussed in Section 3.3.2, and risks associated with the damping rings are evaluated in the risk discussion, Section 8.3.

\subsubsection{Functional and Layout Description}

The electron and positron damping rings must accommodate the long beam pulse of 2820 bunches. To do so while keeping a reasonable circumference, the bunch train must be folded on itself with a bunch spacing much smaller than in the rest of the accelerator. The bunches must be injected and extracted individually by very fast kicker magnets. The minimum bunch spacing is set primarily by the rise- and fall-time of the injection and extraction kickers. A $20 \mathrm{~ns}$ bunch spacing may be technologically feasible, leading to a damping ring circumference of $17 \mathrm{~km}$. To avoid the expense of building two additional $17 \mathrm{~km}$ tunnels for the damping rings, most of each damping ring is a straight section that shares the linac tunnel. Relatively short return arcs require their own tunnels. Figure 3.5.3.1 shows the "dog-bone" layout of one damping ring. 


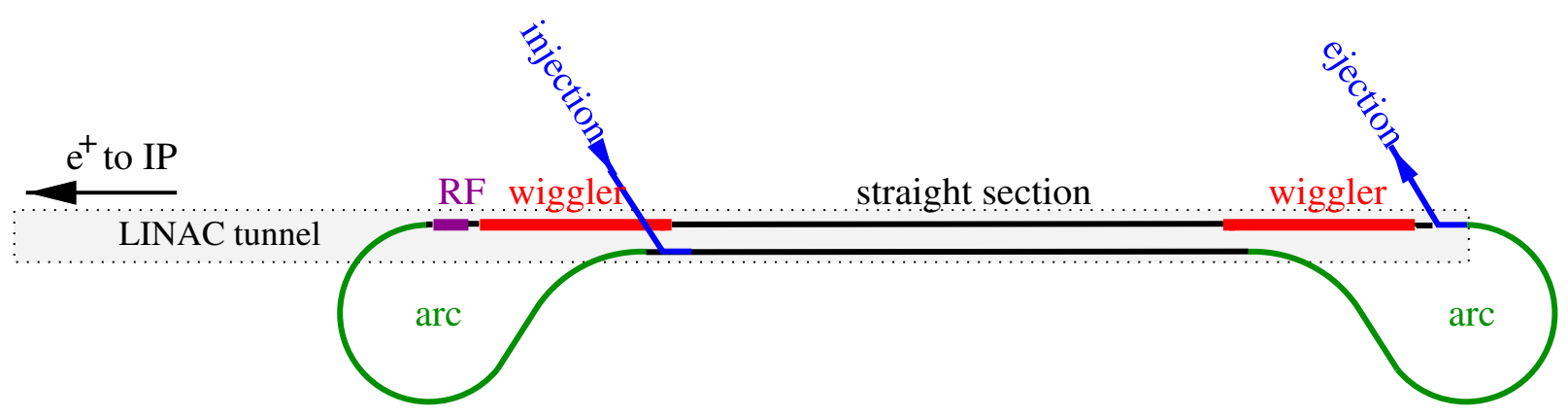

Figure 3.5.3.1: Conceptual layout of the positron damping ring. The electron ring is similar with the exception that the injection point is located close to the indicated ejection position at the beginning of the linac.

Most of the damping occurs in long damping wigglers in the straight sections of each damping ring. The vertical space charge tune shift would be unacceptable for flat beams with the specified extracted vertical emittance, so the part of the straight section without wigglers has a closed coupling bump which produces a round beam within the straight, resulting in a space charge tune shift which is expected to be acceptable. The ring energy of $5 \mathrm{GeV}$ is a compromise between space charge effects and wiggler costs (which favor higher energy) and RF power needs and arc lattice design (which favor lower energy).

The overall parameters of the damping rings are listed in Table 3.5.3.1. The damping rings described here are those of the TESLA TDR [TDR], with the following differences:

1. the pole width of the positron and electron damping ring wigglers has been increased from 40 to 60 $\mathrm{mm}$, to reduce nonlinear fields associated with the wiggler. In addition, the wigglers will need to be carefully measured and tuned to meet the field specifications.

2. the electron damping ring vacuum system pressure has been re-specified to $10^{-10}$ Torr in the straight sections, and $10^{-9}$ Torr in the arcs and wigglers, to reduce the effects of ions.

3. the positron damping ring vacuum system pressure has been re-specified to $10^{-9}$ Torr in the arcs and wigglers, to limit the number of primary electrons generated by gas ionization.

4. a clearing gap of $600 \mathrm{~ns}$ (about 30 bunches) has been added to the electron damping ring, to suppress the formation of ions.

5. low secondary emission coatings have been added to the positron damping ring vacuum chamber to suppress the formation of an electron cloud.

6. the supports for the magnet systems have been upgraded, in view of the tight alignment sensitivities discussed in Section 3.3.2.

Many of the specific details (component drawings and component counts) are taken from the technical layout reports produced by INFN-LNF and Ansaldo [51]. 
Table 3.5.3.1: Damping ring parameters.

\begin{tabular}{|l|c|c|}
\hline \hline Energy & $E$ & $5 \mathrm{GeV}$ \\
\hline Circumference & $C$ & $17 \mathrm{~km}$ \\
\hline Horizontal extracted emittance $\left(e^{+}\right.$and $\left.e^{-}\right)$ & $\gamma \epsilon_{x}$ & $8 \times 10^{-6} \mathrm{~m}-\mathrm{rad}$ \\
\hline Vertical extracted emittance $\left(e^{+}\right.$and $\left.e^{-}\right)$ & $\gamma \epsilon_{y}$ & $0.02 \times 10^{-6} \mathrm{~m}-\mathrm{rad}$ \\
\hline Injected $e^{+}$emittance (horiz. and vert.) & $\gamma \epsilon_{x, y}$ & $0.01 \mathrm{~m}-\mathrm{rad}$ \\
\hline Injected $e^{-}$emittance (horiz. and vert.) & $\gamma \epsilon_{x, y}$ & $10^{-5} \mathrm{~m}-\mathrm{rad}$ \\
\hline Number of damping times, $e^{+}$ & $n_{\tau}$ & 7.2 \\
\hline Number of damping times, $e^{-}$ & $n_{\tau}$ & 4.0 \\
\hline Cycle time & $T_{c}$ & $0.2 \mathrm{~s}$ \\
\hline Damping time, $e^{+}$ & $\tau_{d}$ & $28 \mathrm{~ms}$ \\
\hline Damping time, $e^{-}$ & $\tau_{d}$ & $44 \mathrm{~ms}$ \\
\hline Number of bunches & $n_{b}$ & 2820 \\
\hline Bunch spacing & $\Delta t_{b}$ & $20 \mathrm{~ns}$ \\
\hline Number of particles per bunch & $N_{e}$ & $2.0 \times 10^{10}$ \\
\hline Current & $I$ & $160 \mathrm{~mA}$ \\
\hline Energy loss/turn, $e^{+}$ & $U_{0}$ & $21 \mathrm{MeV}$ \\
\hline Energy loss/turn, $e^{-}$ & $U_{0}$ & $12 \mathrm{MeV}$ \\
\hline Total radiated power, $e^{+}$ & $P$ & $3.2 \mathrm{MW}$ \\
\hline Total radiated power, $e^{-}$ & $P$ & $1.8 \mathrm{MW}$ \\
\hline Tunes & $Q_{x}, Q_{y}$ & $76.31,41.18$ \\
\hline Natural chromaticities & $\xi_{x}, \xi_{y}$ & $-125,-62$ \\
\hline Momentum compaction & $\alpha_{c}$ & $0.12 \times 10^{-3}$ \\
\hline Equilibrium bunch length & $\sigma_{z}$ & $6 \mathrm{~mm}$ \\
\hline Equilibrium momentum spread, $e^{+}$ & $\sigma_{p} / P_{0}$ & $0.13 \%$ \\
\hline Equilibrium momentum spread, $e^{-}$ & $\sigma_{p} / P_{0}$ & $0.10 \%$ \\
\hline Transverse acceptance, $e^{+}$(horiz. and vert.) & $A_{x, y}$ & $0.05 \mathrm{~m}$ \\
\hline Transverse acceptance, $e^{-}$(horiz. and vert.) & $A_{x, y}$ & $0.012 \mathrm{~m}$ \\
\hline Momentum acceptance, $e^{+}$ & $A_{p}$ & $1 \%$ \\
\hline Momentum acceptance, $e^{-}$ & $A_{p}$ & $0.5 \%$ \\
\hline \hline & & \\
\hline
\end{tabular}




\subsubsection{Lattice and Magnet Systems}

3.5.3.2.1 Lattice The damping ring lattice has three major regions: the arcs; the wiggler straight sections; and the long straight sections.

3.5.3.2.1.1 Arc lattice The arc lattice is a theoretical minimum emittance lattice with separated function magnets. Each $6^{\circ}$ dipole magnet is flanked by quadrupole doublets. Sextupoles are located between the quadrupoles in each doublet and at the end of each cell. Figure 3.5.3.2 shows an arc cell.
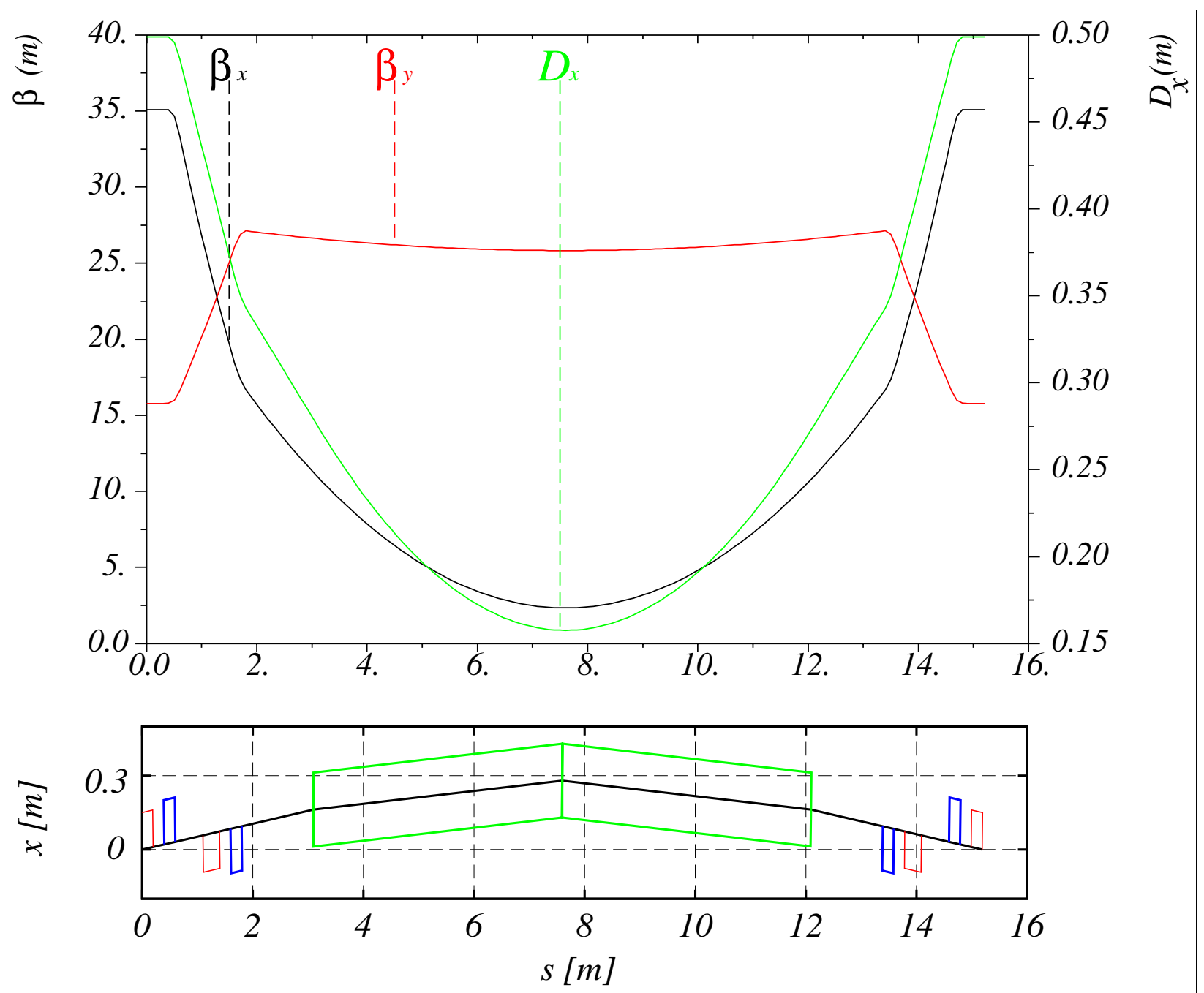

Figure 3.5.3.2: Arc cell lattice functions. Upper plot: horizontal (black) and vertical (red) $\beta$ functions and horizontal dispersion (green). Lower plot: arc cell geometry showing the location of dipoles (green), quadrupoles (blue) and sextupoles (red).

3.5.3.2.1.2 Wiggler sections Wigglers are necessary to provide the necessary damping rate. The baseline design uses permanent magnet wigglers. The wigglers are embedded in a FODO lattice with a $12.2 \mathrm{~m}$ cell length. 
3.5.3.2.1.3 Long straight sections The long straight section consists of FODO cells of approximately $100 \mathrm{~m}$ length with a phase advance of $\mu_{x, y} / 2 \pi=0.125$. The choice of both length and phase advance is a compromise between the need to keep the magnet apertures small (smaller $\beta$ functions), and a small contribution to the overall chromaticity of the ring (larger $\beta$ functions).

\subsection{Magnets and power supplies}

3.5.3.2.2.1 Magnets The damping rings use conventional water cooled magnets. Magnet parameters and quantities are listed in Tables 3.5.3.2 and 3.5.3.3. Dipole, quadrupole, and sextupole magnets use laminated yokes. Bending dipoles and steering magnets have C-shaped cross-sections, while multipole magnets can be split in half for assembly of the vacuum chamber. Current densities in the coils have been kept below $2.5 \mathrm{~A} / \mathrm{mm}^{2}$. Magnets, vacuum chambers, pumps, and associated components are to be assembled on girders and aligned outside the tunnel to reduce the installation time inside the tunnel. Each quadrupole will have an associated BPM.

Table 3.5.3.2: Damping ring magnets (Part 1)

\begin{tabular}{|l|c|c|c|c|c|}
\hline \hline Dipoles & $\begin{array}{c}\text { Quantity } \\
e^{+} \text {ring }\end{array}$ & $\begin{array}{c}\text { Quantity } \\
e^{-} \text {ring }\end{array}$ & $\begin{array}{c}\text { Length, } \\
\text { mag. }(\mathrm{m})\end{array}$ & $\begin{array}{c}\text { Field } \\
(\mathrm{T})\end{array}$ & $\begin{array}{c}\text { Gap } \\
(\mathrm{mm})\end{array}$ \\
\hline \hline Quadrupoles & 208 & 208 & 4.5 & 0.194 & 40 \\
\hline Quantity & $e^{+}$ring & $\begin{array}{c}\text { Quantity } \\
e^{-} \text {ring }\end{array}$ & $\begin{array}{c}\text { Length, } \\
\text { mag., }(\mathrm{m})\end{array}$ & $\begin{array}{c}\text { Gradient } \\
(\mathrm{max}, \mathrm{T} / \mathrm{m})\end{array}$ & $\begin{array}{c}\text { Bore rad. } \\
(\mathrm{mm})\end{array}$ \\
\hline Arc & 4 & 4 & 0.1 & 7.01 & 24 \\
\hline Wiggler & 458 & 458 & 0.2 & 21.2 & 24 \\
\hline Wiggler & 8 & 8 & 0.1 & 17.3 & 28 \\
\hline Wiggler & 132 & 86 & 0.2 & 20.5 & 18 \\
\hline Wiggler & 8 & 8 & 0.3 & 14.1 & 28 \\
\hline Long straight & 280 & 282 & 0.2 & 7.06 & 28 \\
\hline Long straight & 12 & 12 & 0.4 & 3.07 & 52 \\
\hline \hline Sextupoles & $\begin{array}{c}\text { Quantity } \\
e^{+} \text {ring }\end{array}$ & $\begin{array}{c}\text { Quantity } \\
e^{-} \text {ring }\end{array}$ & $\begin{array}{c}\text { Length, } \\
\text { mag., }(\mathrm{m})\end{array}$ & $\begin{array}{c}\text { Gradient } \\
\left(\text { max., } \mathrm{T} / \mathrm{m}^{2}\right)\end{array}$ & $\begin{array}{c}\text { Bore rad. } \\
(\mathrm{mm})\end{array}$ \\
\hline S1M/P & 200 & 200 & 0.3 & 85.4 & 24 \\
\hline S2ML/PL & 96 & 96 & 0.4 & 106 & 24 \\
\hline S2M/P & 8 & 8 & 0.2 & 106 & 24 \\
\hline \hline Steering mag. & Quantity & Quantity & $\begin{array}{c}\text { Length, } \\
\text { mag. }(\mathrm{m})\end{array}$ & $\begin{array}{c}\text { Field } \\
(\text { nom., } \mathrm{T})\end{array}$ & $\begin{array}{c}\text { Gap } \\
(\mathrm{mm})\end{array}$ \\
\hline Arc, wiggler & 654 & 608 & 0.1 & 0.08 & 120 \\
\hline Long straight & 292 & 294 & 0.1 & 0.003 & 105 \\
\hline \hline
\end{tabular}

3.5.3.2.2.2 Magnet power supplies Magnet power supply requirements and quantities are listed in Table 3.5.3.4.

3.5.3.2.3 Wigglers Parameters for the permanent magnet wigglers are listed in Table 3.5.3.5. Note that the pole width will be $60 \mathrm{~mm}$. 
Table 3.5.3.3: Damping ring magnets (Part 2).

\begin{tabular}{|c|c|c|c|c|}
\hline Dipoles & $\begin{array}{c}\text { Ampere-turns } \\
\text { (A) }\end{array}$ & $\begin{array}{c}\text { Weight of windings } \\
(\mathrm{kg})\end{array}$ & $\begin{array}{l}\text { Weight of yoke } \\
(\mathrm{kg})\end{array}$ & $\begin{array}{c}\text { Power } \\
\text { (W/mag.) }\end{array}$ \\
\hline & 6176 & 208 & 2385 & 2557 \\
\hline Quadrupoles & $\begin{array}{l}\text { Ampere-turns } \\
\text { per pole }(\mathrm{A})\end{array}$ & $\begin{array}{c}\text { Weight of windings } \\
(\mathrm{kg})\end{array}$ & $\begin{array}{c}\text { Weight of yoke } \\
(\mathrm{kg})\end{array}$ & $\begin{array}{c}\text { Power } \\
\text { (W/mag.) }\end{array}$ \\
\hline Arc & 1606 & 22 & 50 & 197 \\
\hline Arc & 4858 & 66 & 101 & 504 \\
\hline Wiggler & 5396 & 73 & 54 & 585 \\
\hline Wiggler & 2643 & 36 & 87 & 261 \\
\hline Wiggler & 4398 & 59 & 163 & 442 \\
\hline Wiggler & 2826 & 38 & 218 & 276 \\
\hline Long straight & 7617 & 103 & 148 & 1007 \\
\hline Long straight & 3303 & 45 & 297 & 318 \\
\hline Sextupoles & $\begin{array}{c}\text { Ampere-turns } \\
\text { per pole }(\mathrm{A})\end{array}$ & $\begin{array}{c}\text { Weight of windings } \\
(\mathrm{kg})\end{array}$ & $\begin{array}{c}\text { Weight of yoke } \\
(\mathrm{kg})\end{array}$ & $\begin{array}{c}\text { Power } \\
\text { (W/mag.) }\end{array}$ \\
\hline $\mathrm{S} 1 \mathrm{M} / \mathrm{P}$ & 187 & 2.0 & 12.1 & 47 \\
\hline $\mathrm{S} 2 \mathrm{ML} / \mathrm{PL}$ & 239 & 3.4 & 16.5 & 80 \\
\hline $\mathrm{S} 2 \mathrm{M} / \mathrm{P}$ & 239 & 1.7 & 8.1 & 40 \\
\hline Steering mag. & $\begin{array}{c}\text { Ampere-turns } \\
\text { (A) }\end{array}$ & $\begin{array}{c}\text { Weight of windings } \\
(\mathrm{kg})\end{array}$ & $\begin{array}{c}\text { Weight of yoke } \\
(\mathrm{kg})\end{array}$ & $\begin{array}{c}\text { Power } \\
\text { (W/mag.) }\end{array}$ \\
\hline Arc, wiggler & 8620 & 7 & 7.8 & 190 \\
\hline Long straight & 288 & 0.8 & 4.0 & 2 \\
\hline
\end{tabular}

Table 3.5.3.4: Magnet power supplies (excluding steering magnets).

\begin{tabular}{|l|c|c|c|l|l|}
\hline \hline Magnets powered & $\begin{array}{c}\text { Quantity } \\
e^{+} \text {ring }\end{array}$ & $\begin{array}{c}\text { Quantity } \\
e^{-} \text {ring }\end{array}$ & $\begin{array}{c}\text { Voltage } \\
(\max .)\end{array}$ & $\begin{array}{l}\text { Current } \\
(\max .)\end{array}$ & Type \\
\hline \hline Dipoles & 2 & 2 & 20 & 775 & Simple bridge thyristor \\
\hline Dipoles & 2 & 2 & 340 & 775 & Double bridge thyristor \\
\hline Quadrupoles & 41 & 41 & 30 & 140 & Switching double resonant \\
\hline Quadrupoles & 2 & 2 & 470 & 140 & Simple bridge thyristor \\
\hline Quadrupoles & 2 & 2 & 655 & 225 & Double bridge thyristor \\
\hline Sextupoles & 6 & 6 & 100 & 50 & Switching double resonant \\
\hline \hline
\end{tabular}

Table 3.5.3.5: Wiggler magnets.

\begin{tabular}{|c|c|c|c|c|c|c|c|}
\hline \hline Type & $\begin{array}{c}\text { Quantity } \\
e^{+} \text {Ring }\end{array}$ & $\begin{array}{c}\text { Quantity } \\
e^{-} \text {Ring }\end{array}$ & $\begin{array}{c}\text { Period } \\
\text { length }(\mathrm{mm})\end{array}$ & $\begin{array}{c}\text { Number of } \\
\text { periods }\end{array}$ & $\begin{array}{c}\text { Field } \\
(\text { max., } \mathrm{T})\end{array}$ & $\begin{array}{c}\frac{1}{L} \int B^{2} d l \\
\left(\mathrm{~T}^{2}\right)\end{array}$ & $\begin{array}{c}\text { Gap } \\
(\mathrm{mm})\end{array}$ \\
\hline Hybrid & 108 & 62 & 400 & 10 & 1.67 & 1.37 & 25 \\
\hline \hline
\end{tabular}

\subsubsection{Injection and Extraction Systems}

The damping ring injection/extraction scheme requires a kicker system that can selectively deflect a single bunch in a train with $20 \mathrm{~ns}$ spacing. The kick strength for both injection and ejection is $\theta \approx 0.6 \mathrm{mrad}$ or $\int B d l \approx 0.01 \mathrm{~T}-\mathrm{m}$ at $5 \mathrm{GeV}$ and $\beta_{k i c k e r}=50 \mathrm{~m}$. To ensure an ejected beam stability of $<0.1 \sigma_{x}$, the 
amplitude stability of the kicker system has to be $7 \times 10^{-6} \mathrm{~T}-\mathrm{m}$ for both the maximum deflection and the remaining ripple after the kicker pulse; this corresponds to a relative stability of $7 \times 10^{-4}$ at maximum deflection. Reduction of the relative stability requirement cannot be achieved using (for example) an orbit bump at the ejection septum, since the full aperture of the machine is required for the undamped bunches.

A traveling wave kicker showing the required time response has been built and successfully bench tested using a MOSFET pulser [52]. Depending on the chosen kicker/pulser technology, 20 to 40 kickers are required per ring.

\subsubsection{RF Systems}

The RF system makes use of 12 superconducting (SC) cavities of the same type developed for CESR [53] and KEK-B[54]. The SC cavities are single cell $\mathrm{Nb} 500 \mathrm{MHz}$ resonators, each housed in its own liquid helium cryostat. Large diameter beam tubes permit the higher order modes (HOMs) to propagate out of the cavities, where they are damped using dissipative material applied to the inner surface of both beam tubes just outside the cryostat at room temperature, as in CESR and KEK-B.

Reaching the required gradient requires high residual resistivity ratio (RRR) Niobium, improved cavity heat treatment and operation at a temperature of $2 \mathrm{~K}$. Twelve cavities would provide up to $54 \mathrm{MV}$. At $2 \mathrm{~K}$, the cavity $Q_{0}$ can be close to $10^{10}$. With $R / Q=45 \Omega$ per cell $\left(R=V^{2} / 2 P\right)$, the power loss per cell will be $\leq 30 \mathrm{~W}$ at $15 \mathrm{MV} / \mathrm{m}$. The CESR cryostat design, which operates at $4.2 \mathrm{~K}$, has to be re-designed for $2 \mathrm{~K}$ operation, where static heat losses of $30 \mathrm{~W}$ should be achievable. The total cooling power of $720 \mathrm{~W}$ at $2 \mathrm{~K}$ will be provided by the main linac refrigerators. The $3.2 \mathrm{MW}$ required for the beam will be shared among the twelve cavities, and the input RF power per coupler is therefore $270 \mathrm{~kW}$; this is below the power handling capability (in traveling wave mode) of RF windows already developed and tested at other laboratories. Powerful klystrons (1.2 $\mathrm{MW} \mathrm{CW}$ at $500 \mathrm{MHz})$ are produced by industry and operate routinely at KEK. With a klystron efficiency $\eta \approx 60 \%$, the total power required by the RF is $6.5 \mathrm{MW}$.

The heat load for the conventional cooling is mainly the klystron tubes, and is about $2.5 \mathrm{MW}$. Each cavity cryostat is equipped with a pair of $300 \mathrm{l} / \mathrm{s}$ vacuum ion pumps. Two additional $60 \mathrm{l} / \mathrm{s}$ pumps are connected to the waveguide power coupler. Table 3.5.3.6 lists the RF system parameters and Fig. 3.5.3.3 shows a block diagram of the high-level RF system.

Table 3.5.3.6: RF system parameters.

\begin{tabular}{|l|c|c|}
\hline \hline RF frequency & & $500 \mathrm{MHz}$ \\
\hline RF peak voltage & & $50 \mathrm{MV}$ \\
\hline Current & $I$ & $160 \mathrm{~mA}$ \\
\hline Energy loss/turn, $e^{+}$ & $U_{0}$ & $21 \mathrm{MeV}$ \\
\hline Energy loss/turn, $e^{-}$ & $U_{0}$ & $12 \mathrm{MeV}$ \\
\hline Total radiated power, $e^{+}$ & $P$ & $3.2 \mathrm{MW}$ \\
\hline Total radiated power, $e^{-}$ & $P$ & $1.8 \mathrm{MW}$ \\
\hline \hline
\end{tabular}

\subsubsection{Vacuum Systems}

The positron vacuum system has been designed to achieve an average pressure inside the vacuum chamber of $\sim 10^{-9}$ Torr throughout the machine. The electron vacuum system must achieve the the same average pressure of $\sim 10^{-9}$ in the arcs. An even lower average pressure of $\sim 10^{-10}$ Torr is required in the electron ring's long straight sections, to avoid a large ion-produced tune shift. To realize this, the electron damping ring has pumps at smaller intervals in the long straight sections. 


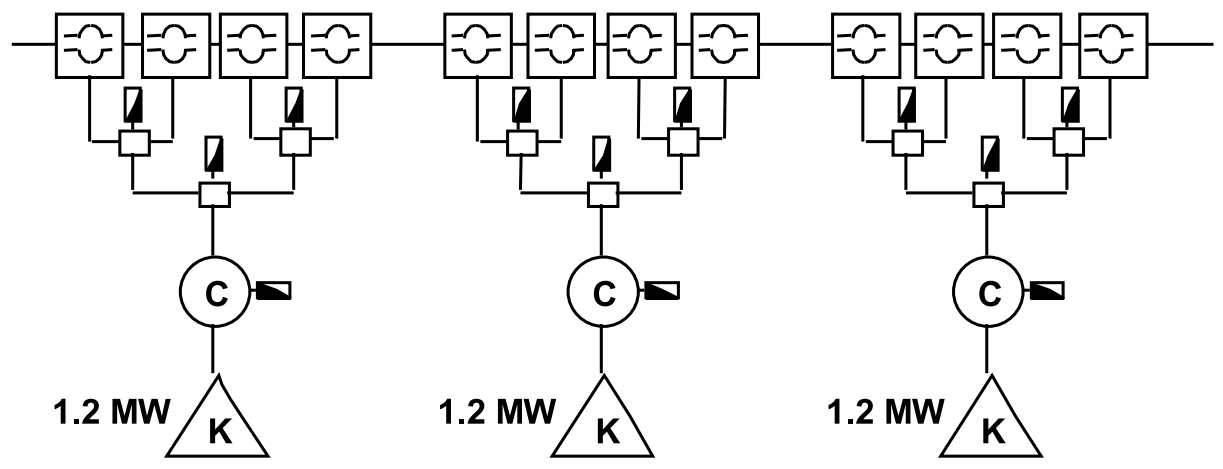

Figure 3.5.3.3: RF system.

The vacuum chambers are all constructed from an aluminum alloy. The chambers in the arcs and wigglers in both rings will need antechambers to extract the synchrotron radiation. They will be similar to those of the Advanced Photon Source, which have similar requirements. The positron damping ring vacuum chamber is subject to the electron cloud instability and requires a low secondary emission yield coating such as TiN to suppress formation of the electron cloud.

In the two $7 \mathrm{~km}$ long straight sections in both rings, the vacuum chamber is essentially a pipe of $100 \mathrm{~mm}$ outside diameter and $2 \mathrm{~mm}$ thickness, and no cooling is needed. The arc vacuum chamber and straight sections require bellows with sliding RF contacts.

Table 3.5.3.7 lists the quantities of vacuum chambers required for a single ring. Tables 3.5.3.8 and 3.5.3.9 list the quantities of vacuum chambers required for the positron and electron rings, respectively. Tables 3.5.3.10, 3.5.3.11, and 3.5.3.12 list the valves, vacuum instrumentation, and bellows needed for each ring.

Table 3.5.3.7: Vacuum chamber assemblies.

\begin{tabular}{|l|l|c|}
\hline \hline Type or Location & $\begin{array}{l}\text { Total length } \\
e^{+} \text {ring }(\mathrm{m})\end{array}$ & $\begin{array}{l}\text { Total length } \\
e^{-} \text {ring }(\mathrm{m})\end{array}$ \\
\hline \hline Arc dipole & 936 & 936 \\
\hline Arc & 644 & 644 \\
\hline Long straights & 13950 & 14180 \\
\hline Wiggler section quad & 120 & 70 \\
\hline Wiggler & 420 & 240 \\
\hline Matching/other & 930 & 930 \\
\hline \hline
\end{tabular}

\subsubsection{Infrastructure}

The damping ring arc of nearly $1 \mathrm{~km}$ length is located in a tunnel with $3 \mathrm{~m}$ inner diameter. This tunnel is connected at two positions to the main linac tunnel. Infrastructure in the arcs consists of: a monorail transportation system capable of transporting material, components and people; a compressed air distribution line; fire safety systems; normal and emergency lighting; an optical video line; and a ventilation and air extraction system.

Electrical power needs and cooling requirements are summarized in Tables 3.5.3.13 and 3.5.3.14. The main linac refrigerators will supply the cooling capacity of $720 \mathrm{~W}$ at $2 \mathrm{~K}$ for the superconducting $\mathrm{RF}$ cavities. 
Table 3.5.3.8: Pumps (positron ring).

\begin{tabular}{|l|l|}
\hline \hline Quantity & Item \\
\hline \hline 208 & distributed sputter ion pumps, $192 \mathrm{l} / \mathrm{s}$ \\
\hline 1876 & lumped sputter ion pumps, $50 \mathrm{l} / \mathrm{s}$ \\
\hline 368 & lumped sputter ion pumps, $150 \mathrm{l} / \mathrm{s}$ \\
\hline 192 & lumped sputter ion pumps, $300 \mathrm{l} / \mathrm{s}$ \\
\hline 1876 & Ti sublimation pumps, $1000 \mathrm{l} / \mathrm{s}$ \\
\hline 208 & vacuum HV feedthroughs \\
\hline 1422 & dual power supplies \\
\hline 626 & single power supplies (controller) \\
\hline 1422 & cables for dual power supplies \\
\hline 1876 & cables for single power supplies \\
\hline 1876 & cables with TSP connector and 3+1 welding wires \\
\hline 1876 & automatic 6-throw switches for TiSP filaments \\
\hline 3 & portable units with roughing and turbo pump, gauges, RGA, leak detector \\
\hline \hline
\end{tabular}

Table 3.5.3.9: Pumps (electron ring).

\begin{tabular}{|l|l|}
\hline \hline Quantity & Item \\
\hline \hline 208 & distributed sputter ion pumps, $192 \mathrm{l} / \mathrm{s}$ \\
\hline 1876 & lumped sputter ion pumps, $50 \mathrm{l} / \mathrm{s}$ \\
\hline 368 & lumped sputter ion pumps, $150 \mathrm{l} / \mathrm{s}$ \\
\hline 192 & lumped sputter ion pumps, $300 \mathrm{l} / \mathrm{s}$ \\
\hline 3752 & Ti sublimation pumps, $1000 \mathrm{l} / \mathrm{s}$ \\
\hline 208 & vacuum HV feedthroughs \\
\hline 1422 & dual power supplies \\
\hline 626 & single power supplies (controller) \\
\hline 1422 & cables for dual power supplies \\
\hline 1876 & cables for single power supplies \\
\hline 3752 & cables with TSP connector and $3+1$ welding wires \\
\hline 3752 & automatic 6-throw switches for TiSP filaments \\
\hline 3 & portable units with roughing and turbo pump, gauges, RGA, leak detector \\
\hline \hline
\end{tabular}

Table 3.5.3.10: Valves. Quantities are for one ring.

\begin{tabular}{|l|l|}
\hline \hline Quantity & Item \\
\hline \hline 27 & electro-pneumatic gate valves DN 63 with RF contact \\
\hline 92 & electro-pneumatic gate valves DN 100 with RF contact \\
\hline 6 & electro-pneumatic gate valves DN 200 with RF contact \\
\hline 125 & manual angle valve DN40 \\
\hline 125 & manual angle valve DN63 \\
\hline 125 & mini power supplies for electro-pneumatic gate valves \\
\hline 250 & $\begin{array}{l}\text { cables, } 24 \mathrm{~V} \text { compressed air solenoid valves, tubing, etc. } \\
\text { for electro-pneumatic gate valves }\end{array}$ \\
\hline \hline
\end{tabular}


Table 3.5.3.11: Vacuum instrumentation. Quantities are for one ring.

\begin{tabular}{|l|l|}
\hline \hline Quantity & Item \\
\hline \hline 248 & ionization gauges \\
\hline 248 & controllers for ionization gauges \\
\hline 248 & cables for ionization gauges \\
\hline 248 & cold cathode gauges \\
\hline 248 & controllers for cold cathode gauges \\
\hline 248 & cables for cold cathode gauges \\
\hline 248 & Pirani gauges \\
\hline 248 & controllers for Pirani gauges \\
\hline 248 & cables for Pirani gauges \\
\hline
\end{tabular}

Table 3.5.3.12: Bellows. Quantities are for one ring.

\begin{tabular}{|l|l|}
\hline \hline Quantity & Type \\
\hline \hline 662 & Arc, $43 \mathrm{~mm}$ ID \\
\hline 1876 & Long straight, 96 mm ID \\
\hline \hline
\end{tabular}

Table 3.5.3.13: Electrical power requirements of damping ring systems.

\begin{tabular}{|l|c|c|}
\hline \hline System & Positron ring & Electron ring \\
\hline \hline RF klystrons & $6.5 \mathrm{MW}$ & $4.3 \mathrm{MW}$ \\
\hline Magnet power supplies & $2.0 \mathrm{MW}$ & $2.0 \mathrm{MW}$ \\
\hline Other systems & $0.5 \mathrm{MW}$ & $0.5 \mathrm{MW}$ \\
\hline \hline Total & $9.0 \mathrm{MW}$ & $6.8 \mathrm{MW}$ \\
\hline \hline
\end{tabular}

Table 3.5.3.14: Cooling requirements for damping rings.

\begin{tabular}{|l|c|c|}
\hline \hline & Positron ring & Electron ring \\
\hline \hline RF klystrons & $3.3 \mathrm{MW}$ & $2.2 \mathrm{MW}$ \\
\hline Synchrotron radiation & $3.2 \mathrm{MW}$ & $2.1 \mathrm{MW}$ \\
\hline Magnets and power supplies & $2.0 \mathrm{MW}$ & $2.0 \mathrm{MW}$ \\
\hline \hline Total & $8.5 \mathrm{MW}$ & $6.3 \mathrm{MW}$ \\
\hline \hline
\end{tabular}

\subsubsection{Bunch Compressor and Transfer to Main Linac}

The damping ring arc and spin-rotator/bunch compressor are shown, approximately to scale, in Fig. 3.5.4.1.

\subsubsection{Introduction}

The equilibrium bunch length in the damping ring is $6 \mathrm{~mm}$, too long by an order of magnitude for optimum collider performance $\left(\sigma_{z}=0.3 \mathrm{~mm}\right)$. Hence the bunch must be compressed longitudinally by a factor of $\sim 20$ before being injected into the main linac. 


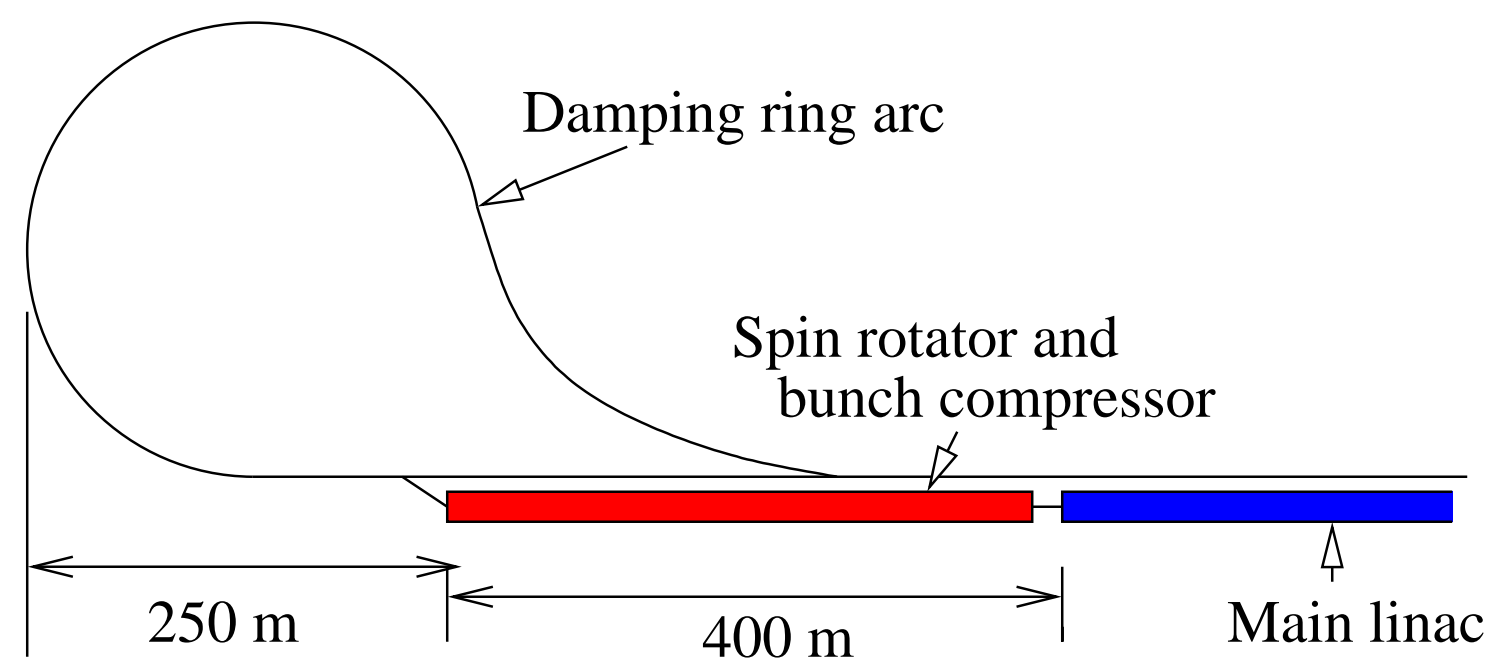

Figure 3.5.4.1: Damping ring arc, spin rotator and bunch compressor layout

Compression is achieved by introducing an energy-position correlation along the bunch length using an RF section, followed by a dispersive beamline with an energy dependent path length: in this way, the tail of the bunch can be made to follow a shorter path than the head, and the bunch becomes shorter. For the dispersive (compressing) beamline, a simple wiggler chicane will be used.

In addition to the bunch compressor, the ring-to-linac transfer line also includes the following sections:

- Spin rotator. The particle spin orientation in the damping ring must be vertical to preserve the polarization. To adjust the spin vector to the required orientation at the collision point, a so-called spin rotator is required. The system consists of a combination of dipole and superconducting solenoid magnets.

- Coupling correction section. The large horizontal to vertical emittance ratio requires a high degree of betatron coupling control. A section containing skew-quadrupoles provides a sufficient empirical correction of the cross-plane coupling.

- Diagnostic and collimation section. The transfer line includes a diagnostic section which allows continuous monitoring of the beam phase space. The diagnostics serve as a quality control for the complete injection system. For commissioning and tune up a beam dump downstream of the diagnostic section is foreseen. The section also contains the collimators which remove the beam halo before injection into the main linac, decreasing the particle flux at the post-linac collimation system in the beam delivery section, and protecting the accelerating structures in the linac.

\subsubsection{Bunch Compressor}

A detailed description of the bunch compressor can be found in [55]. Here only a brief overview is given. The first stage of bunch compression is to introduce a longitudinally correlated energy spread in the bunch; this is accomplished by injecting the bunch into an accelerating section at a phase close to the zero-crossing of the $\mathrm{RF}$ waveform. The bunch is then compressed by transporting it through a dispersive section, constructed from a series of magnetic chicanes, which provides the necessary path length dependence on energy. The beam parameters before and after the bunch compressor are given in Table 3.5.4.2. 
Table 3.5.4.1: Beam parameters before and after the bunch compressor

\begin{tabular}{lc}
\hline rms horizontal emittance $\gamma \varepsilon_{x}[\mathrm{~mm}-\mathrm{mrad}]$ & 8 \\
rms vertical emittance $\gamma \varepsilon_{y}[\mathrm{~mm}-\mathrm{mrad}]$ & 0.02 \\
initial rms bunch length $\sigma_{z_{i}}[\mathrm{~mm}]$ & 6 \\
initial energy spread $\sigma_{\delta_{i}}[\%]$ & 0.13 \\
beam energy $E_{0}[\mathrm{GeV}]$ & 5 \\
\hline final rms bunch length $\sigma_{z_{f}}[\mathrm{~mm}]$ & 0.3 \\
final energy spread $\sigma_{\delta_{f}}[\%]$ & 2.7 \\
\hline \hline
\end{tabular}

The magnetic wiggler system for the compressor consists of bending magnet chicanes (wiggler type) embedded in a FODO structure. No additional optical elements are included between the bending magnets of each individual wiggler section. As a result, the dispersion is zero at every quadrupole, and no higher-order dispersion is generated. The wiggler layout is shown in Fig. 3.5.4.2.

The parameters for the wiggler-based compressor are presented in Table 3.5.4.2. The required peak voltage of the RF before the wiggler is $890 \mathrm{MV}$ at a phase of $113^{\circ}$; this voltage will be provided by three TESLA accelerating modules with an average gradient of $23.8 \mathrm{MV} / \mathrm{m}$. The average energy loss is $0.4 \mathrm{GeV}$ which has to be compensated by two additional accelerating modules. The total length of the bunch compressor (including three accelerating modules) is $\sim 140 \mathrm{~m}$. The maximum deviation from the central axis is $0.3 \mathrm{~m}$, which should fit easily into the main linac tunnel.

Table 3.5.4.2: Parameters of the wiggler bunch compressor

\begin{tabular}{lc}
\hline longitudinal dispersion $R_{56}[\mathrm{~m}]$ & 0.215 \\
final rms energy spread [\%] & 2.8 \\
total RF voltage [MV] & 890 \\
RF phase angle [degrees] & 113 \\
total chicane length [m] & 86.4 \\
SR induced horizontal emittance growth [\%] & 0.02 \\
\hline
\end{tabular}

\subsubsection{Spin rotator}

The spin rotation is constructed from superconducting solenoids and a normal conducting bend section (arc), located upstream of the bunch compressor. Since the damped beam is flat, the cross-plane coupling induced by the solenoids must be compensated. This is achieved by a spin rotator unit constructed from two identical superconducting solenoids, separated by a short beamline whose (transverse) optics form a $-I$ transformation; this effectively cancels the betatron coupling, while the spin rotation of the two solenoids add. A single unit can rotate the spin around the longitudinal axis by up to $90^{\circ}$. The complete spin rotator is constructed from three sections:

- an initial solenoid pair, which rotates the spin around the local longitudinal (z) axis by $90^{\circ}$;

- a normal conducting horizontal arc, which further rotates the spin around the vertical axis by $90^{\circ}$; and

- a final solenoid pair, providing an additional rotation about the $z$-axis by $90^{\circ}$.

With the above combination of rotations, all possible spin orientations can be achieved. The focusing effect of the solenoids is corrected with four matching quadrupoles per paired solenoid section. The matching quadrupoles are positioned between the solenoid sections and the central arc (see figure 6.5.1). With the 


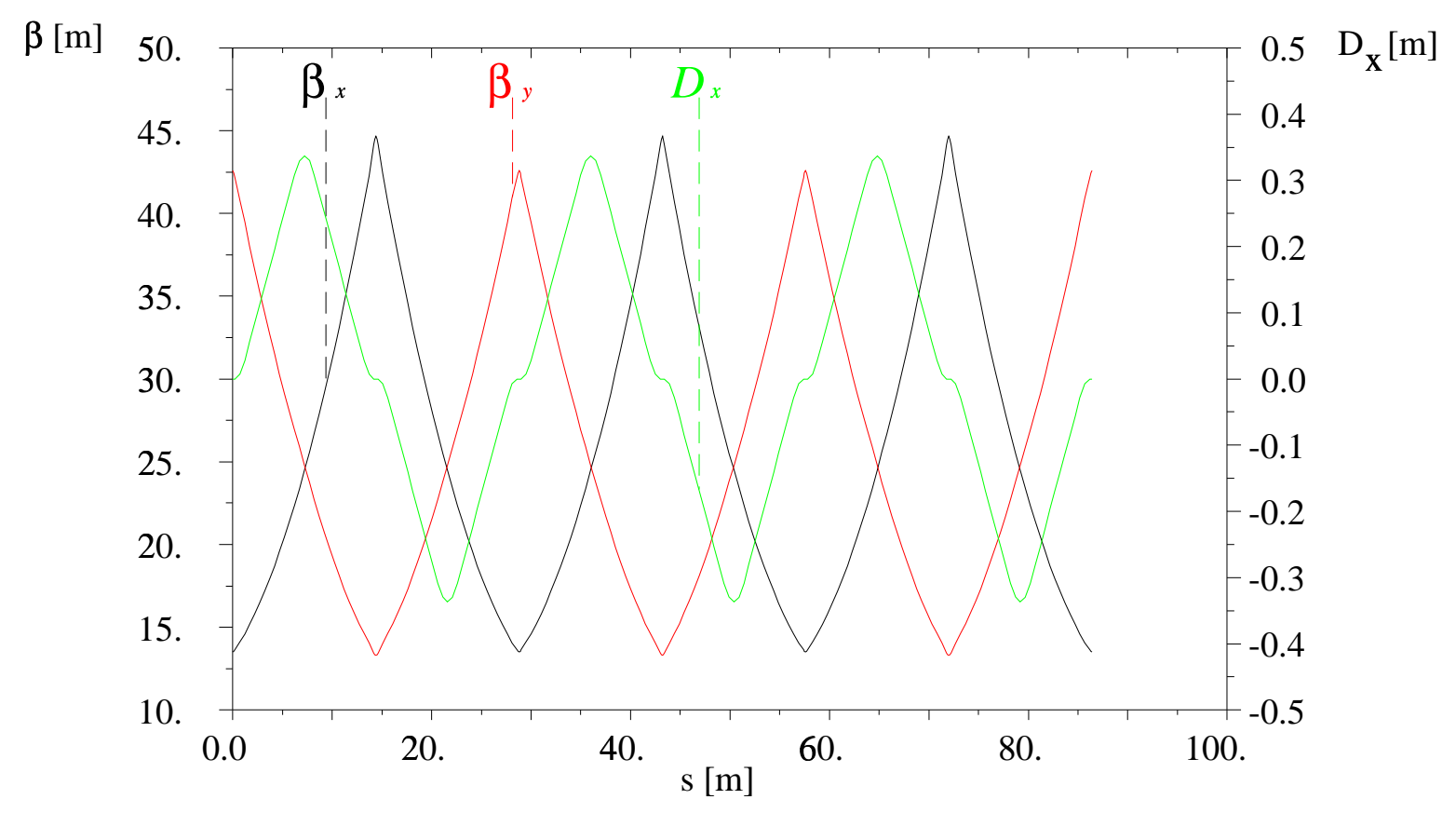

$\mathrm{x}[\mathrm{m}]$

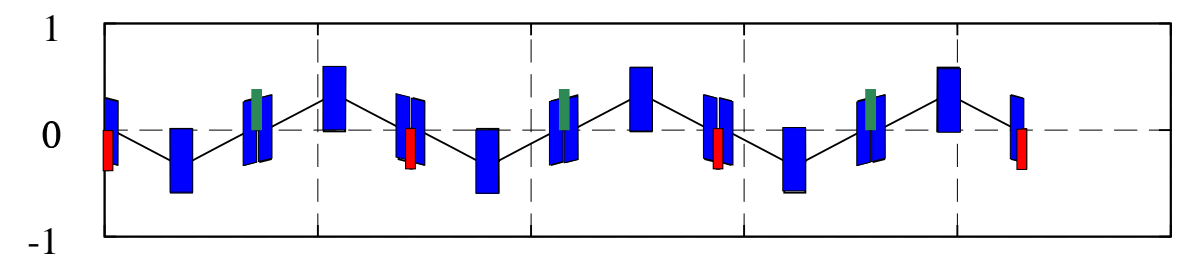

: Bending Magnets I, I]: Focusing, Defocusing Quadrupole Magnets

Figure 3.5.4.2: Optical functions and floor plan of the wiggler bunch compressor: horizontal (black) and vertical (red) betatron function and horizontal dispersion (green).

solenoids at maximum field strength, the chromatic emittance growth is $\sim 1 \%\left(\sigma_{\delta_{i}} \approx 0.13 \%\right)$. The parameters of the spin rotator system are given in Table 3.5.4.3.

\subsubsection{Coupling correction section}

In order to empirically correct anomalous cross-plane coupling due to either damping ring extraction or spin rotator errors, a coupling correction section of the type proposed in [56] is included. The system is 
Table 3.5.4.3: Basic spin rotator parameters

\begin{tabular}{lc}
\hline longitudinal dispersion $R_{56}[\mathrm{~m}]$ & 0.005 \\
maximum spin rotation in solenoid [degrees] & 90 \\
total arc bend angle [degrees] & 8 \\
total length of rotator system [m] & 85 \\
SR induced horizontal emittance growth [\%] & 0.02 \\
\hline
\end{tabular}

constructed from four skew-quadrupoles with zero nominal strength. Using skew-quadrupoles with a length of $0.1 \mathrm{~m}, \pm 0.1 \mathrm{~T}$ pole-tip field, and a $0.01 \mathrm{~m}$ pole-tip radius, a factor of about two in emittance increase can be corrected per skew-quadrupole.

\subsubsection{Diagnostic and collimation section}

A wire-array emittance measurement station [56] is located downstream of the wiggler section. The section is designed to continuously monitor the matching and emittance of the beam before being injected into the linac. The wire-array station is constructed from a series of 4 FODO cells, with a profile monitor (mechanicalor laser-wire scanner) located at each vertically focusing quadrupole.

The nominal rms beam size at the monitors is $5 \mu \mathrm{m}$ vertically and $70 \mu \mathrm{m}$ horizontally. A correctly matched beam is easily identified since the beam size (for a single plane) is identical at each of the four wire scanners. The cross-plane coupling can not be accurately determined using this system: however, by simply minimizing the vertical emittance (flat beam) with the four skew-quadrupoles in the upstream coupling correction section, all coupling can be corrected (with some iteration necessary in extreme cases).

Transverse phase space collimation is obtained by placing four mechanical spoiler pairs in a FODO lattice with appropriate phase advance. Energy collimation will also be included in this section.

\subsubsection{Beamline Geometry}

Figure 3.5.4.3 shows the floor plan of the entire tunnel in the electron damping ring extraction and injection region. The floor plan starts at the ' $500 \mathrm{MeV}$ point', where the various pre-accelerator linacs deliver the bunch trains for collider operation to the $5 \mathrm{GeV}$ electron injector linac (Section 3.5.2.1.2).

At the exit of the injector linac, a vertical transfer line horizontally injects the beam into the damping ring. The damping ring is situated about two meters above the main linac axis. After $200 \mathrm{~ms}$ the beam is horizontally ejected from the damping ring, and deflected down to the level of the main linac, where it is injected into the spin rotator and the bunch compressor. This beamline runs parallel to the injector linac.

After passing though the bunch compressor, coupling correction, and diagnostics and collimation sections, the beam is injected into the main linac. The positron system is essentially the same as that described above. The geometry is simplified, however, since the positron injection occurs at the opposite end of the damping ring.

\subsubsection{Summary of components}

The complete list of magnets and RF modules (and their specifications) for the entire ring-to-linac transfer line is given in Table 3.5.4.7. 
Table 3.5.4.4: Major system components

\begin{tabular}{|c|c|c|c|c|c|}
\hline $\begin{array}{c}\text { Bending } \\
\text { Magnets }\end{array}$ & Quantity & $\begin{array}{c}\text { Mag. Length } \\
{[\mathrm{m}]}\end{array}$ & $\begin{array}{c}\text { Defl. Angle } \\
{[\mathrm{deg}]}\end{array}$ & $\begin{array}{c}\text { MagField } \\
{[\mathrm{T}]}\end{array}$ & $\begin{array}{c}\text { Gap } \\
{[\mathrm{mm}]}\end{array}$ \\
\hline horizontal dogleg & 4 & 1.0 & 2 & 0.582 & 20 \\
vertical dogleg & 4 & 1.25 & 2.5 & 0.582 & 20 \\
spin rotator & 4 & 0.75 & 2 & 0.776 & 20 \\
& 4 & 1.5 & 4 & 0.776 & 20 \\
bunch compressor & 12 & 1.075 & 3.2 & 0.785 & 20 \\
& 6 & 2.15 & 6.4 & 0.785 & 20 \\
\hline
\end{tabular}

\begin{tabular}{|c|c|c|c|c|}
\hline Quadrupoles & Quantity & $\begin{array}{c}\text { Mag. Length } \\
{[\mathrm{m}]}\end{array}$ & $\begin{array}{c}\text { Max. Gradient } \\
{[\mathrm{T} / \mathrm{m}]}\end{array}$ & $\begin{array}{c}\text { Bore } \\
{[\mathrm{mm}]}\end{array}$ \\
\hline & 140 & $0.2-0.4$ & 25.0 & 20 \\
\hline
\end{tabular}

\begin{tabular}{|c|c|c|c|c|}
\hline Solenoids & Quantity & $\begin{array}{c}\text { Mag. Length } \\
{[\mathrm{m}]}\end{array}$ & $\begin{array}{c}\text { Nom. Field } \\
{[\mathrm{T}]}\end{array}$ & $\begin{array}{c}\text { Bore } \\
{[\mathrm{mm}]}\end{array}$ \\
\hline spin rotator & 4 & 3.55 & 3.8 & 20 \\
\hline
\end{tabular}

\begin{tabular}{|c|c|c|c|}
\hline RF-Modules & Quantity & $\begin{array}{c}\text { Number of } \\
\text { Cavities }\end{array}$ & $\begin{array}{c}\text { Max. Gradient } \\
{[\mathrm{MV} / \mathrm{m}]}\end{array}$ \\
\hline bunch compressor & 3 & 12 & 23.8 \\
\hline
\end{tabular}

\begin{tabular}{|c|c|c|c|c|c|}
\hline Correctors & Quantity & $\begin{array}{c}\text { Mag. Length } \\
{[\mathrm{m}]}\end{array}$ & $\begin{array}{c}\text { Max. Defl. } \\
{[\mathrm{deg}]}\end{array}$ & $\begin{array}{c}\text { Max. Field } \\
{[\mathrm{T}]}\end{array}$ & $\begin{array}{c}\text { Gap } \\
{[\mathrm{mm}]}\end{array}$ \\
\hline & 120 & 0.1 & 0.009 & 0.025 & 20 \\
\hline
\end{tabular}




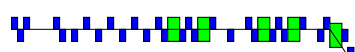

Side View

Damping ring extraction

Dan

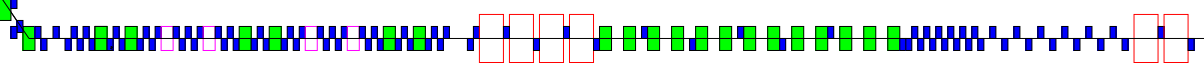

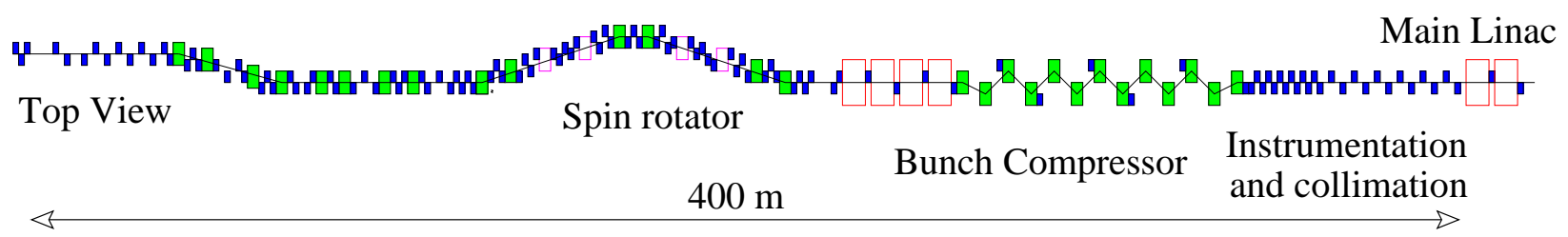

Figure 3.5.4.3: Floor plan for the complete bunch compressor beamline. In the floor plan, bending magnets are green, quadrupoles blue, $\mathrm{RF}$ cavities red and solenoid magnets magenta.

\subsubsection{Main Linac}

\subsubsection{Introduction}

The main linacs accelerate the electron and positron beams from the output of the bunch compressors, at 5 $\mathrm{GeV}$, to the collision energy, $250 \mathrm{GeV}$. The overall design and specification of the main linacs is similar to that presented in the TDR[TDR, Sections 2 and 3].

The cavities to be installed in the main linacs will be qualified to a maximum gradient of $35 \mathrm{MV} / \mathrm{m}$. Recent results from DESY for an electropolished cavity, "fully dressed" with input coupler, tested in a horizontal cryostat, showed a $Q_{0}$ value above $7 \times 10^{9}$ at $35 \mathrm{MV} / \mathrm{m}$. (See Fig. 3.5.5.1). A maximum gradient of 37 $\mathrm{MV} / \mathrm{m}$ was measured in stable operation for one hour. The quench limit was $37-38 \mathrm{MV} / \mathrm{m}$, and operation at the $34-36 \mathrm{MV} / \mathrm{m}$ level was sustained for more than 1000 hours without a cavity trip. Another fully equipped unit is under test and has reached $35 \mathrm{MV} / \mathrm{m}$ at a $Q_{0}$ of $6 \times 10^{9}$.

The design beam parameters for the main linac are presented in Table 3.5.5.1. Table 3.5.5.2 and Table 3.5.5.3 give overviews of the main linac components.

Table 3.5.5.1: Design beam parameters for the main linac

\begin{tabular}{lc}
\hline Accelerating gradient $[\mathrm{MV} / \mathrm{m}]$ & 28 \\
Injection energy $[\mathrm{GeV}]$ & 5 \\
Final energy [GeV] & 250 \\
Bunch charge $\left[10^{10}\right]$ & 2 \\
Bunch spacing $[\mathrm{ns}]$ & 337 \\
Bunch length $[\mu \mathrm{m}]$ & 300 \\
Normalized rms emittance at injection $\epsilon_{x}, \epsilon_{y}\left[10^{-6} \mathrm{~m} \cdot \mathrm{rad}\right]$ & $8,0.02$ \\
Normalized rms emittance at IP $\epsilon_{x}, \epsilon_{y}\left[10^{-6} \mathrm{~m} \cdot \mathrm{rad}\right]$ & $10,0.03$ \\
Beam size at injection $\sigma_{x, i}, \sigma_{y, i}[\mu \mathrm{m}]$ & 320,16 \\
Beam size at linac exit $\sigma_{x, f}, \sigma_{y, f}[\mu \mathrm{m}]$ & 60,3 \\
Initial uncorr. energy spread $\sigma_{E, i} / E[\%]$ & 2.5 \\
Off-crest RF phase $\Phi_{r f}(5$ to $25 / 25$ to $250 \mathrm{GeV})\left[^{\circ}\right]$ & $-27 / 5$ \\
Energy spread $\sigma_{E, f} / E$ at linac exit $\left[10^{-4}\right]$ & 7 \\
\hline \hline
\end{tabular}




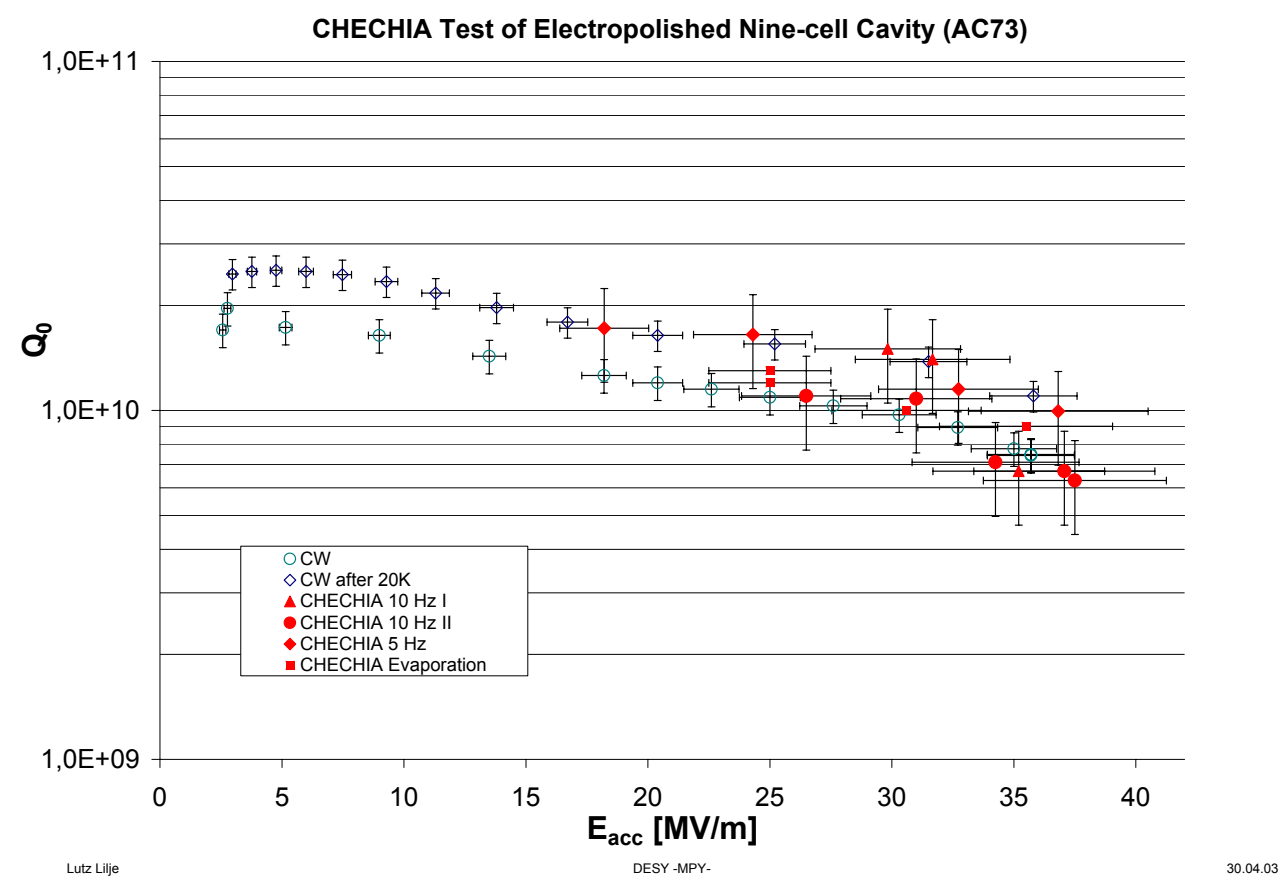

Figure 3.5.5.1: $Q_{0}$ vs. gradient for an electropolished 9-cell cavity. Pulsed operation at $5 \mathrm{~Hz}$ in a horizontal cryostat, with input coupler attached. (Courtesy Lutz Lilje, DESY)

\subsubsection{Cryomodules}

In this section, we describe the cryomodules and their arrangement in the main linacs, with an emphasis on the differences from the TDR.

3.5.5.2.1 Superconducting RF Cavities The parameters of the 9-cell cavity, operating at $28 \mathrm{MV} / \mathrm{m}$, with a beam current of $9.5 \mathrm{~mA}$, are given in Table 3.5.5.4. The increased gradient, at fixed beam current, results in a larger value for the matched $Q_{e x t}$ than in the TDR. This, in turn, reduces the cavity bandwidth and increases the cavity fill time. The quality factor is taken to be $10^{10}$ at $28 \mathrm{MV} / \mathrm{m}$. The 9-cell cavity is shown in Fig. 3.5.5.2.

The cavities will be made from pure niobium, and processed as described in the TDR[TDR, Sections 2.1.42.1.6], including electropolishing and low-temperature bake-out, to reach the design gradient. Each cavity is equipped with a helium tank; a tuning system driven by a stepping motor; a coaxial RF power coupler; a pickup probe; two higher-order mode (HOM) couplers; and two piezo-electric fast tuners for Lorentz force detuning compensation. Two piezo-tuners are used to provide redundancy.

The superstructure cavity configuration[TDR, Section 2.1.8] will not be adopted for the reference design in this study. However, this configuration is considered as a design variant in Section 7.5.

3.5.5.2.2 Cryostat As in the TDR[TDR, Section 3.3.1], twelve 9-cell cavities will be assembled into a cryomodule, which is housed in a cryostat to provide the thermal insulation necessary to allow the cavities to 
Table 3.5.5.2: Overview of components in the electron main linac. The overheads which have been assumed are indicated in Table 3.5.5.11.

\begin{tabular}{lc}
\hline Injection energy [GeV] & 5 \\
Undulator energy loss [GeV] & 5.922 \\
Final energy [GeV] & 250.125 \\
Linac length (cryomodules) [m] & 12698.8 \\
Diagnostic insertion @ $5 \mathrm{GeV}[\mathrm{m}]$ & 66.8 \\
Diagnostic insertion @ $55 \mathrm{GeV}[\mathrm{m}]$ & 66.8 \\
Undulator insertion @ $155 \mathrm{GeV}[\mathrm{m}]$ & 850 \\
Diagnostic insertion @ $155 \mathrm{GeV}[\mathrm{m}]$ & 195.68 \\
Diagnostic insertion at BDS entrance [m] & 195.68 \\
Total length, including insertions [km] & 14.074 \\
Active length [km] & 9.504 \\
Real estate gradient [MV/m] & 19.303 \\
Number of cavities per module & 12 \\
Number of modules & 763 \\
Number of cavities & 9156 \\
Module length (with quad) [m] & $15.9(16.8)$ \\
Number of quadrupoles & 316 \\
Number of klystrons & 306 \\
Number of cavities per klystron & 30 \\
\hline \hline
\end{tabular}

Table 3.5.5.3: Overview of components in the positron main linac. The overheads which have been assumed are indicated in Table 3.5.5.11.

\begin{tabular}{lc}
\hline Injection energy [GeV] & 5 \\
Final energy [GeV] & 250.125 \\
Linac length (cryomodules) [m] & 12400.38 \\
Diagnostic insertion @ 5 GeV [m] & 66.8 \\
Diagnostic insertion @ $50 \mathrm{GeV}[\mathrm{m}]$ & 66.8 \\
Diagnostic insertion @ $152 \mathrm{GeV}[\mathrm{m}]$ & 195.68 \\
Diagnostic insertion at BDS entrance [m] & 195.68 \\
Total length, including insertions [km] & 12.925 \\
Active length [km] & 9.28 \\
Real estate gradient [MV/m] & 19.767 \\
Number of cavities per module & 12 \\
Number of modules & 745 \\
Number of cavities & 8940 \\
Module length (with quad) [m] & $15.9(16.8)$ \\
Number of quadrupoles & 310 \\
Number of klystrons & 298 \\
Number of cavities per klystron & 30 \\
\hline \hline
\end{tabular}

be cooled to $2 \mathrm{~K}$. Within the cryostat is contained the helium distribution system need to cool the cavities. A cross-sectional view of the cryomodule is shown in Fig. 3.5.5.3.

The details of the mechanical design of the cryostat, and the insulating vacuum, are given in TDR[TDR, Section 3.3.1]. The heat loads for a cryomodule with quadrupole are given in Table 3.5.5.5. This table is based on the TDR[TDR, Table 3.3.1], with the following modification. The dynamic heat loads due to the 


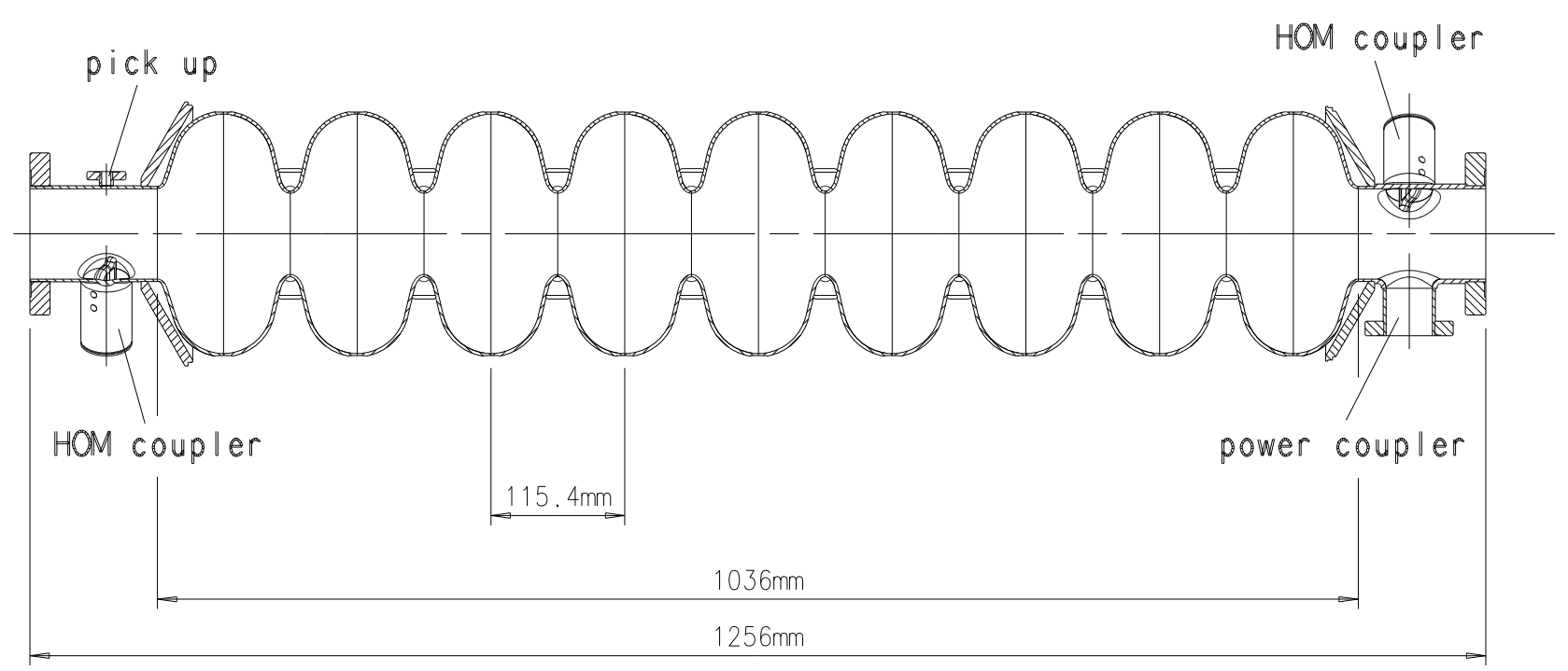

Figure 3.5.5.2: Nine-cell superconducting cavity

Table 3.5.5.4: Parameters of the 9-cell cavity (note that we adopt here the definition of shunt impedance by the relation $R=V^{2} / P$, where $P$ is the dissipated power and $V$ the peak voltage in the equivalent parallel LCR circuit).

\begin{tabular}{lc}
\hline Type of accelerator structure & standing wave \\
Accelerating mode & $\mathrm{TM}_{010}, \pi$-mode \\
Fundamental frequency & $1300 \mathrm{MHz}$ \\
Nominal gradient $E_{a c c}$ & $28 \mathrm{MV} / \mathrm{m}$ \\
Quality factor $Q_{0}$ & $>10^{10}$ \\
Active length $L$ & $1.038 \mathrm{~m}$ \\
Cell-to-cell coupling $k_{c c}$ & $1.87 \%$ \\
Iris diameter & $70 \mathrm{~mm}$ \\
$R / Q$ & $1036 \Omega$ \\
$E_{\text {peak }} / E_{\text {acc }}$ & 2.0 \\
$B_{\text {peak }} / E_{\text {acc }}$ & $4.26 \mathrm{mT} /(\mathrm{MV} / \mathrm{m})$ \\
Tuning range & $\pm 300 \mathrm{kHz}$ \\
$\Delta f / \Delta L$ & $315 \mathrm{kHz} / \mathrm{mm}$ \\
Lorentz force detuning constant $K_{L o r}$ & $\sim 1 \mathrm{~Hz} /(\mathrm{MV} / \mathrm{m})^{2}$ \\
$Q_{\text {ext }}$ of input coupler & $2.95 \times 10^{6}$ \\
Cavity bandwidth at $Q_{\text {ext }}=2.95 \times 10^{6}$ & $440 \mathrm{~Hz}$ \\
Fill time & $501 \mu \mathrm{s}$ \\
Number of HOM couplers & 2 \\
\hline \hline
\end{tabular}

$\mathrm{RF}$ have been scaled up by the following product: the square of the gradient ratio,

$$
\left(\frac{28}{23.4}\right)^{2}=1.432
$$

and the ratio of the RF pulse lengths,

$$
\left(\frac{1.45}{1.37}\right)=1.059
$$




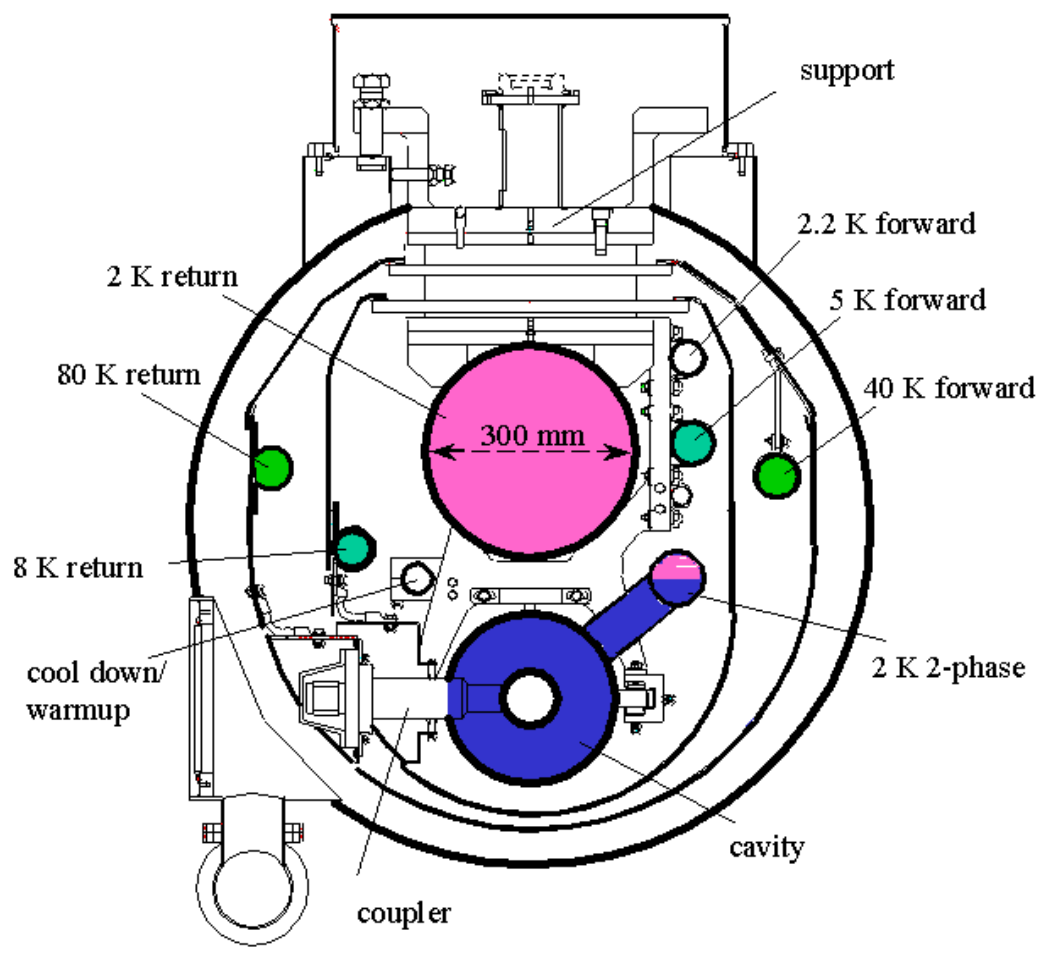

Figure 3.5.5.3: Cross section of a cryomodule

giving an overall factor of $1.432 \times 1.059=1.517$.

Table 3.5.5.5: Calculated heat loads for $17 \mathrm{~m}$ long cryomodule with quadrupole.

\begin{tabular}{|c|c|c|c|c|}
\hline Temperature & Static $[\mathrm{W}]$ & Dynamic(RF) $[\mathrm{W}]$ & Dynamic(HOM) $[\mathrm{W}]$ & Total $[\mathrm{W}]$ \\
\hline $2 \mathrm{~K}$ & 1.74 & 7.72 & 2.21 & 11.67 \\
$5-8 \mathrm{~K}$ & 11.32 & 1.80 & 3.43 & 16.55 \\
$40-80 \mathrm{~K}$ & 90.13 & 90.09 & 28.5 & 208.72 \\
\hline
\end{tabular}

3.5.5.2.3 Quadrupoles and Correction Magnets The cryomodules for the main linac come in two varieties: the standard variety is $15.927 \mathrm{~m}$ long, and contains 12 9-cell cavities, with associated helium distribution system. The other type is $16.728 \mathrm{~m}$ long, and also includes a quadrupole, steering coils, and a beam position monitor. The quadrupoles and correction magnets are detailed in the TDR[TDR, Section 3.3.1]. 
3.5.5.2.4 Cryomodule layout As outlined in the TDR[TDR, Section 3.2.1], the main linac FODO lattice will be established using the quadrupoles in the cryomodules. From 5 to about $125 \mathrm{GeV}$, four cryomodules, two standard and two with quadrupoles, form a $66.83 \mathrm{~m}$ long FODO cell. This section of each main linac contains 185 standard cryomodules and 185 cryomodules with quadrupoles. From 125 to $250 \mathrm{GeV}$, six cryomodules, four standard and two with quadrupoles, form a $97.842 \mathrm{~m}$ long FODO cell. This section of the positron (electron) main linac contains 250 (262) standard cryomodules and 125 (131) cryomodules with quadrupoles. The additional cryomodules in the electron main linac provide the energy required to restore that lost in the undulator $(5.92 \mathrm{GeV})$.

The two main linacs will require a total of 1508 cryomodules. Of these, 882 are of the standard variety, and 626 are cryomodules with a quadrupole.

As outlined in the TDR[TDR, Section 8.7.0.1], the cryomodules are grouped into cryogenic "strings" of 10 cryomodules each. One cryomodule at the end of each string will be equipped with valves, tubing, flow meters and controllers, and instrumentation. The cryogenic strings will be combined into cryounits. Each cryounit will be supplied through a distribution box with cryogens from a refrigerator located in an above-ground service hall.

The segmentation of the cryogenic strings into cryounits has been chosen to accommodate the need for warm sections in the linac, required for diagnostics and for positron production. Table 3.5.5.2.4 and Table 3.5.5.2.4 show the details of the cryogenic segmentation in the main linacs. At the start of the main linac, a warm insertion equal to one cell length $(66.8 \mathrm{~m})$ is provided for the implementation of energy and emittance diagnostics. Another such insertion is provided after cryounit 1, at about $66 \mathrm{GeV}$. After cryounit 2 in the electron main linac, at about $155 \mathrm{GeV}$, a warm insertion equal to $850 \mathrm{~m}$ is provided to allow the beam to be diverted through an undulator for positron production, and a two-cell diagnostic insertion (195.684 $\mathrm{m})$ is provided for energy and emittance diagnostics. In the positron main linac, a warm insertion equal to two cell lengths, at about $152 \mathrm{GeV}$, is provided for energy and emittance diagnostics. In both main linacs, two cell lengths are provided for final energy and emittance diagnostics, at the end of the main linac tunnel, just before the start of the beam delivery system.

The electron main linac layout is shown in Fig. 3.5.5.4, and the positron main linac layout in Fig. 3.5.5.5.

Table 3.5.5.6: Segmentation of the electron main linac, $250 \mathrm{GeV}$ beam energy

\begin{tabular}{|c|c|c|c|}
\hline Linac segment & $\begin{array}{c}\text { Number of } \\
\text { cryomodules }\end{array}$ & $\begin{array}{c}\text { Length of } \\
\text { segment }(\mathrm{km})\end{array}$ & $\begin{array}{c}\text { Beam energy at } \\
\text { end of segment }(\mathrm{GeV})\end{array}$ \\
\hline Diagnostic section 1 & Warm & 0.0668 & 5.0 \\
Cryounit 1 & 158 & 2.640 & 56.99 \\
Diagnostic section 2 & Warm & 0.0668 & 56.99 \\
Cryounit 2 & 158 & 2.648 & 108.972 \\
Cryounit 3 & 141 & 2.337 & 155.36 \\
Undulator & Warm & 0.850 & 149.442 \\
Diagnostic section 3 & Warm & 0.1957 & 149.442 \\
Cryounit 4 & 143 & 2.370 & 196.493 \\
Cryounit 5 & 163 & 2.702 & 250.125 \\
Diagnostic section 5 & Warm & 0.1957 & 250.125 \\
\hline
\end{tabular}

\subsubsection{Cryogenics}

The cryogenic requirements of the main linacs are presented in Table 3.5.5.3. These numbers have been computed from the cryomodule heat loads given in Table 3.5.5.5, together with the cryounit module counts given in Table 3.5.5.2.4 and Table 3.5.5.2.4. The cryogenics will be supplied by six refrigerators located in 
Table 3.5.5.7: Segmentation of the positron main linac, $250 \mathrm{GeV}$ beam energy

\begin{tabular}{|c|c|c|c|}
\hline Linac segment & $\begin{array}{c}\text { Number of } \\
\text { cryomodules }\end{array}$ & $\begin{array}{c}\text { Length of } \\
\text { segment }(\mathrm{km})\end{array}$ & $\begin{array}{c}\text { Beam energy at } \\
\text { end of segment }(\mathrm{GeV})\end{array}$ \\
\hline Diagnostic section 10 & Warm & 0.0668 & 5.0 \\
Cryounit 16 & 149 & 2.49 & 54.025 \\
Diagnostic section 9 & Warm & 0.0668 & 54.025 \\
Cryounit 15 & 149 & 2.49 & 103.05 \\
Cryounit 14 & 149 & 2.49 & 152.075 \\
Diagnostic section 8 & Warm & 0.1957 & 152.075 \\
Cryounit 13 & 149 & 2.49 & 201.10 \\
Cryounit 12 & 149 & 2.49 & 250.125 \\
Diagnostic section 6 & Warm & 0.1957 & 250.125 \\
\hline
\end{tabular}

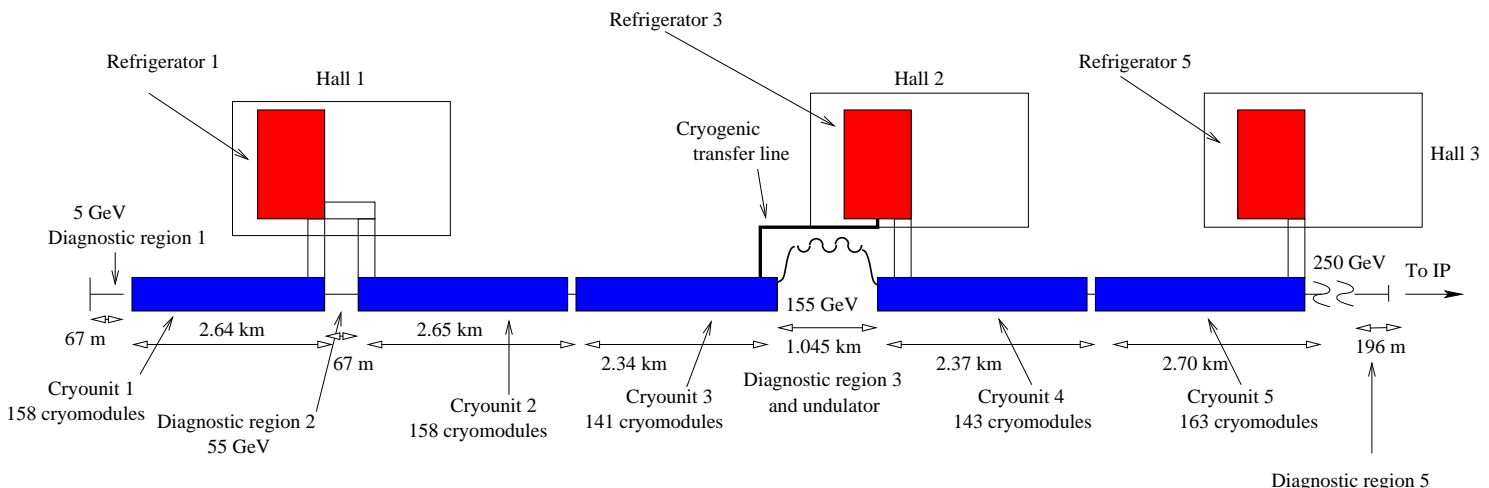

Figure 3.5.5.4: Electron main linac layout, $250 \mathrm{GeV}$ beam energy

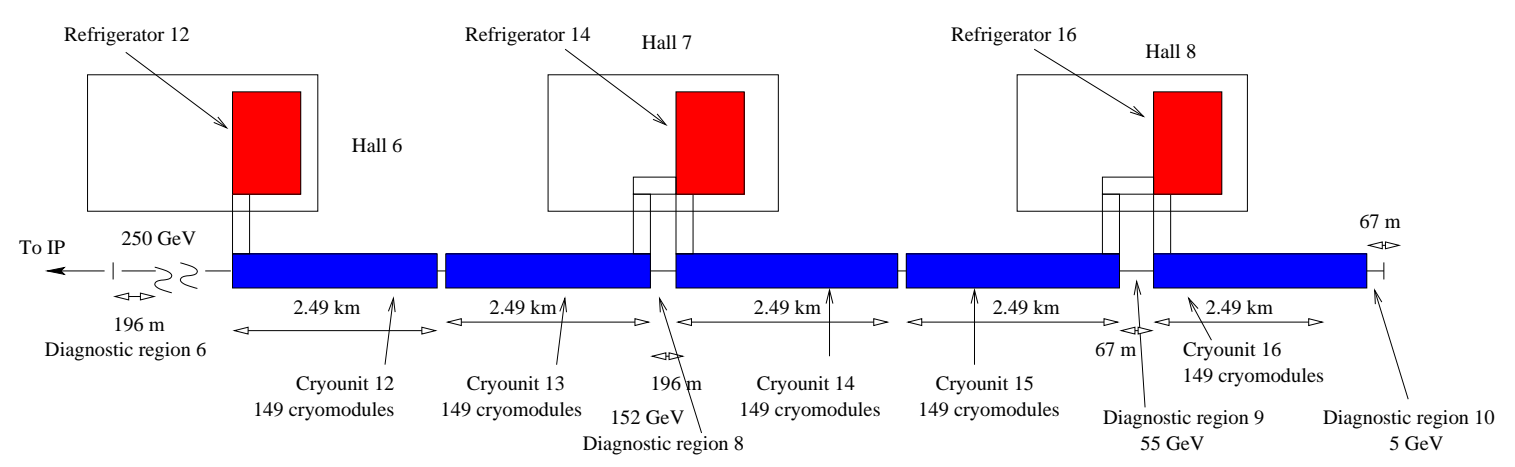

Figure 3.5.5.5: Positron main linac layout, $250 \mathrm{GeV}$ beam energy

the six above-ground service halls shown in Fig. 3.5.5.4 and Fig. 3.5.5.5.

The AC cryogenic power requirements of the main linacs are given in Table 3.5.5.9. To compute the AC power requirements, the following COP's have been used (from the TDR[TDR, Table 8.7.5]): for $2 \mathrm{~K} 588$ $\mathrm{W} / \mathrm{W}$; for 5-8 K, $168 \mathrm{~W} / \mathrm{W}$; and for 40-80 K, $17 \mathrm{~W} / \mathrm{W}$. Cold compressor losses have also been included. 
Table 3.5.5.8: Cryogenic requirements of the main linacs

\begin{tabular}{|c|c|c|c|c|}
\hline Cryounit & Temperature $[\mathrm{K}]$ & Static load $[\mathrm{kW}]$ & Dynamic load $[\mathrm{kW}]$ & Total $[\mathrm{kW}]$ \\
\hline \hline 1,2 & 2 & 0.27 & 1.57 & 1.84 \\
& $5-8$ & 1.79 & 0.83 & 2.62 \\
& $40-80$ & 14.24 & 18.74 & 32.98 \\
\hline 3 & 2 & 0.25 & 1.40 & 1.65 \\
& $5-8$ & 1.60 & 0.74 & 2.33 \\
& $40-80$ & 12.71 & 16.72 & 29.43 \\
\hline 4 & 2 & 0.25 & 1.42 & 1.67 \\
& $5-8$ & 1.62 & 0.75 & 2.37 \\
& $40-80$ & 12.89 & 16.96 & 29.85 \\
\hline 5 & 2 & 0.28 & 1.62 & 1.90 \\
& $5-8$ & 1.5 & 0.85 & 2.70 \\
& $40-80$ & 14.69 & 19.33 & 34.02 \\
\hline $12-16$ & 2 & 0.26 & 1.48 & 1.74 \\
& $5-8$ & 1.69 & 0.78 & 2.47 \\
& $40-80$ & 13.43 & 17.67 & 31.10 \\
\hline
\end{tabular}

Table 3.5.5.9: Total cryogenic operating power requirements, $250 \mathrm{GeV}$ beam energy

\begin{tabular}{lc}
\hline AC power cryogenics at $2 \mathrm{~K}$, Cryounits $1-5$ and $12-16[\mathrm{MW}]$ & 11.07 \\
$\mathrm{AC}$ power cryogenics at $5-8 \mathrm{~K}$, Cryounits $1-5$ and $12-16[\mathrm{MW}]$ & 4.48 \\
$\mathrm{AC}$ power cryogenics at $40-80 \mathrm{~K}$, Cryounits $1-5$ and $12-16[\mathrm{MW}]$ & 5.73 \\
Total AC power for cryogenics [MW] & 21.28 \\
\hline \hline
\end{tabular}

\subsubsection{RF system}

In a superconducting linac, during the beam pulse essentially all of the power delivered by the RF system goes into the beam. Since the beam power in this design is the same as in the TDR, the RF system is essentially the same. There is a small increase in the total required AC power for RF, because the RF pulse length is about $6 \%$ longer than in the TDR (due to the increased external $Q$ ).

The RF system consists of 298 (305) stations per positron (electron) main linac, each of which provides power at $1.3 \mathrm{GHz}$ to a total of 30 accelerating cavities (two and a half cryomodules). The number of RF stations per linac includes an $6 \%$ overhead, as indicated in Table 3.5.5.11. Each RF station consists of a klystron and a modulator. The number of cavities per station has been reduced relative to the TDR (in which it is 36) in order to allow the use of the same $10 \mathrm{MW}$ klystrons as specified in the TDR[TDR, Section 3.4.2], at the increased gradient. The peak RF power needed for one superconducting cavity at full gradient and maximum beam current $(28 \mathrm{MV} / \mathrm{m}$ and $9.5 \mathrm{~mA})$ is $276 \mathrm{~kW}$; the nominal peak power needed for 30 cavities is $8.28 \mathrm{MW}$. Taking into account a regulation reserve of $10 \%$ for phase and amplitude control, and another $10.2 \%$ for circulator and waveguide losses, a total of $9.96 \mathrm{MW}$ is required. The RF pulse length is $1.45 \mathrm{~ms}$, which includes the beam pulse length of $0.95 \mathrm{~ms}$, and the cavity fill time of $0.50 \mathrm{~ms}$. The repetition rate is $5 \mathrm{~Hz}$.

The overall efficiency and power requirements of the RF systems are given in Table 3.5.5.10.

3.5.5.4.1 Klystrons and modulators The klystron will be a $10 \mathrm{MW}$ multi-beam device, with a 1.5 ms output pulse, a design efficiency of $65 \%$, and a gain of $48 \mathrm{~dB}$. The klystron will be driven by solidstate amplifier. A detailed description and set of design specifications, and the measured performance of a prototype, is given in the TDR[TDR, Section 3.4.2]. 
Table 3.5.5.10: Efficiencies and power requirements for the RF system

\begin{tabular}{lc}
\hline RF station peak power [MW] & 9.96 \\
Duty cycle [\%] & 0.73 \\
RF station average power [kW] & 72.2 \\
Klystron efficiency [\%] & 65 \\
Modulator efficiency [\%] & 85 \\
AC power per RF station [kW] & 130.8 \\
AC power for auxiliaries per RF station [kW] & 14 \\
Number of stations in electron linac & 305 \\
Electron linac AC wall plug power for RF [MW] & 44.18 \\
Number of stations in positron linac & 298 \\
Positron linac AC wall plug power for RF [MW] & 43.14 \\
Total AC wall plug power for RF [MW] & 87.32 \\
Beam power [MW] & 22.38 \\
AC to beam efficiency for RF [\%] & 25.6 \\
\hline \hline
\end{tabular}

The modulator provides the $120 \mathrm{kV}$ voltage pulse applied to the klystron cathode. It consists of high voltage power supply, a high voltage pulser unit, and a pulse transformer. Details of the modulator design and specification are provided in TDR[TDR, Section 3.4.3].

3.5.5.4.2 Waveguide distribution system The klystrons and modulators will be placed in a separate tunnel. There will be cross-connects between the two tunnels every $34 \mathrm{~m}$, the length of two cryomodules. Waveguides will deliver the RF from the klystrons to the tunnel containing the linac. The separation between the centers of the two tunnels will be $11.4 \mathrm{~m}$ (See Fig. 3.5.7.1).

For initial operation at $28 \mathrm{MV} / \mathrm{m}$, klystrons will be installed at four out of every five tunnel cross-connects. These klystrons will feed 30 cavities, using an arrangement shown in Fig. 3.5.5.6. With this arrangement, the average distance from klystron to RF cavity will be about $22 \mathrm{~m}$, plus the length of the cross tunnel. The total average distance from klystron to RF cavity is then $11.4 \mathrm{~m}+22 \mathrm{~m}=33.4 \mathrm{~m}$. Using WR770 waveguide, with an attenuation factor of $-0.011 \mathrm{~dB} / \mathrm{m}$, the power loss in the waveguide is $8.2 \%$. According to the TDR[TDR, Section 3.4.6], there is an additional $2 \%$ loss in the circulator, giving a total loss of $10.2 \%$.

3.5.5.4.3 Low level RF The low level RF system will be similar to that described in the TDR[TDR, Section 3.4.7]. A significant difference is that only 30, rather than 36 , cavities are driven by a single klystron.

\subsubsection{Overheads}

The assumed overheads are listed in Table 3.5.5.11. The overhead for BNS damping and longitudinal wakefield compensation, for operation at $250 \mathrm{GeV}$ beam energy and a gradient of $28 \mathrm{MV} / \mathrm{m}$, is $2.44 \mathrm{GeV}$ (about 1\%). A $2 \%$ allocation of spares for klystron failures has been made. The cavities associated with these spares are assumed to be mechanically detuned. In addition, the design includes a $2 \%$ allocation of spares for cavity faults. If the cavity fault rate is 1 trip every 30 hours, and the trip recovery time is $4 \mathrm{sec}$, then on average the $2 \%$ cavity fault spares will be depleted only once per year. A tuned, unpowered spare cavity will decelerate the beam by roughly $35 \mathrm{MV} / \mathrm{m}$, so additional powered cavities must be provided as compensation. Assuming that half of the spares will be tuned at any given time, an additional $1 \%$ for the compensating cavities has been allocated, bringing the total spares needed for cavity fault control to $3 \%$. 


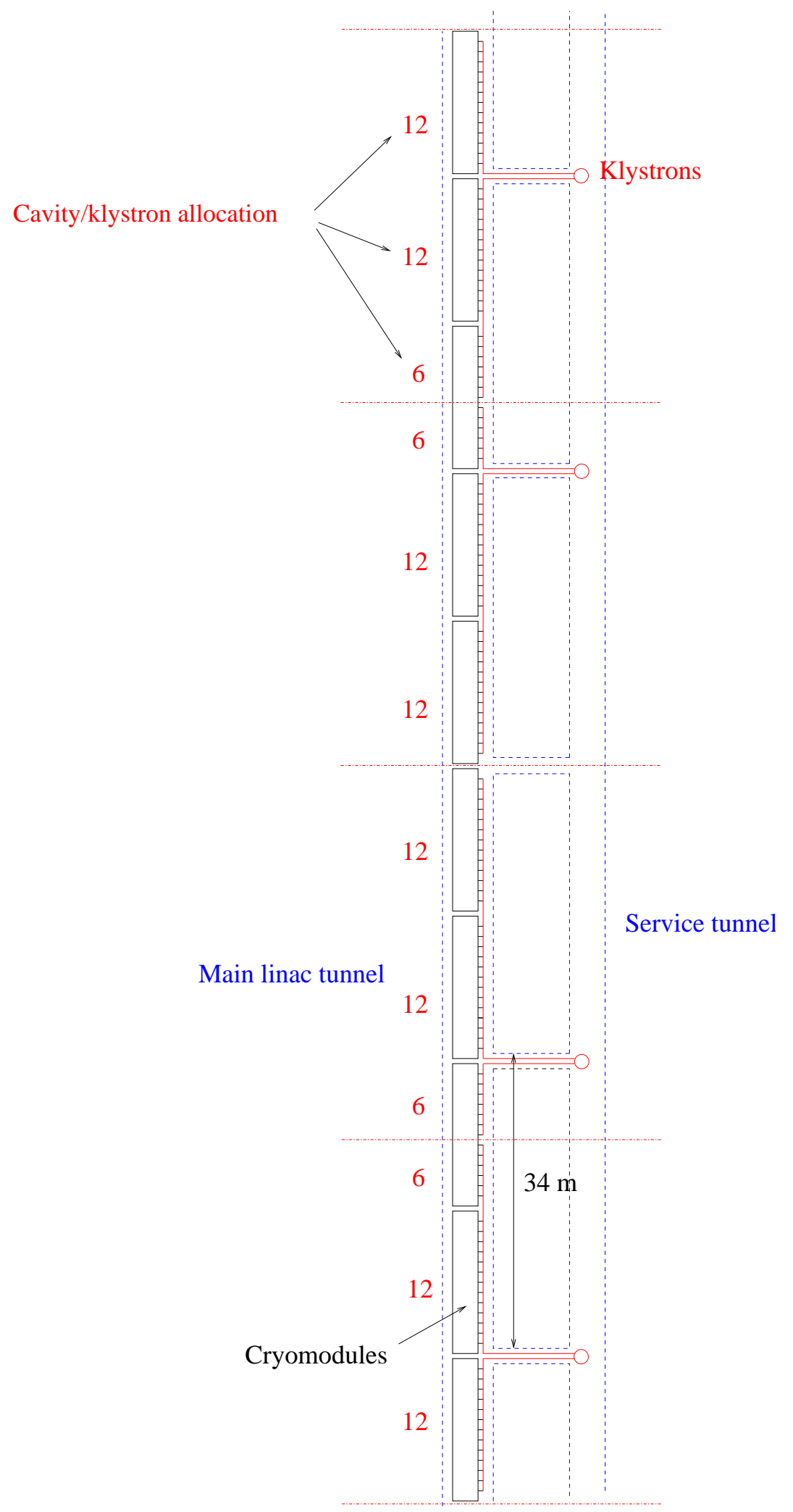

Figure 3.5.5.6: Waveguide distribution, two tunnels, $250 \mathrm{GeV}$ beam energy 
Table 3.5.5.11: Overheads assumed, in \%

\begin{tabular}{c|c}
\hline BNS overhead & 1 \\
Klystron failure overhead $^{a}$ & 2 \\
Cavity faults overhead $^{b}$ & 3 \\
\hline
\end{tabular}

\footnotetext{
${ }^{a}$ Assumes spare cavities are detuned

${ }^{b}$ including loading
}

\subsubsection{Instrumentation and feedback}

The beam position monitors for the linac cryomodules are described in the TDR[TDR, Section 3.3.2.5]. The information from the BPM's, in conjunction with instrumentation located in the diagnostic insertions described above, will be used to perform the beam-based alignment of the linacs to the level required for the specified emittance preservation. The BPM's will also be used for slow feedback to maintain alignment in the face of slow ground motion. The BPM rms resolution required for beam-based alignment is $3 \mu \mathrm{m}$.

This reference design also includes the provision for several bunch-by-bunch feedback systems in the main linac. These intratrain feedback systems are similar to the systems which stabilize the beam position and angle at the IP. The main linac systems will serve to stabilize the main linac beam against high frequency noise introduced by the motion of the ground, or the motion of components, such as the main linac quadrupoles. The BPM's used for fast feedback will have an rms resolution capability of $0.6 \mu \mathrm{m}$.

\subsubsection{Upgrade to a beam energy of $500 \mathrm{GeV}$}

The length of the tunnels for the main linac will be sufficient to allow the machine to be upgraded to $1 \mathrm{TeV}$, using cavities with an accelerating gradient of $35 \mathrm{MV} / \mathrm{m}$, without additional underground civil construction. The design beam parameters for the main linac at $500 \mathrm{GeV}$ are presented in Table 3.5.5.12. Table 3.5.5.13 and Table 3.5.5.14 give overviews of the main linac components.

Table 3.5.5.12: Design beam parameters for the main linac at $500 \mathrm{GeV}$ beam energy

\begin{tabular}{lc}
\hline Accelerating gradient $[\mathrm{MV} / \mathrm{m}]$ & 35 \\
Injection energy $[\mathrm{GeV}]$ & 5 \\
Final energy $[\mathrm{GeV}]$ & 500 \\
Bunch charge $\left[10^{10}\right]$ & 2 \\
Bunch spacing $[\mathrm{ns}]$ & 337 \\
Bunch length $[\mu \mathrm{m}]$ & 300 \\
Normalized rms emittance at injection $\epsilon_{x}, \epsilon_{y}\left[10^{-6} \mathrm{~m} \cdot \mathrm{rad}\right]$ & $8,0.02$ \\
Normalized rms emittance at IP $\epsilon_{x}, \epsilon_{y}\left[10^{-6} \mathrm{~m} \cdot \mathrm{rad}\right]$ & $10,0.03$ \\
Beam size at injection $\sigma_{x, i}, \sigma_{y, i}[\mu \mathrm{m}]$ & 320,16 \\
Beam size at linac exit $\sigma_{x, f}, \sigma_{y, f}[\mu \mathrm{m}]$ & 42,2 \\
Initial uncorr. energy spread $\sigma_{E, i} / E[\%]$ & 2.5 \\
Off-crest RF phase $\Phi_{r f}(5$ to $25 / 25$ to $500 \mathrm{GeV})\left[^{\circ}\right]$ & $-27 / 5$ \\
Energy spread $\sigma_{E, f} / E$ at linac exit $\left[10^{-4}\right]$ & 3.5 \\
\hline \hline
\end{tabular}

3.5.5.7.1 Cryomodules In this section, we describe the cryomodules and their arrangement in the main linacs for $500 \mathrm{GeV}$ beam energy The parameters of the 9-cell cavity, operating at $35 \mathrm{MV} / \mathrm{m}$, with a beam current of $9.5 \mathrm{~mA}$, are given in Table 3.5.5.15. The increased gradient, at fixed beam current, results in a 
Table 3.5.5.13: Overview of components in the electron main linac for $500 \mathrm{GeV}$ beam energy. The overheads which have been assumed are indicated in Table 3.5.5.11.

\begin{tabular}{lc}
\hline Injection energy [GeV] & 5 \\
Undulator energy loss [GeV] & 5.922 \\
Final energy [GeV] & 500.02 \\
Linac length (cryomodules) [m] & 20242.0 \\
Diagnostic insertion @ 5 GeV [m] & 66.8 \\
Diagnostic insertion @ $55 \mathrm{GeV}[\mathrm{m}]$ & 66.8 \\
Undulator insertion @ $192 \mathrm{GeV}[\mathrm{m}]$ & 850 \\
Diagnostic insertion @ $192 \mathrm{GeV}[\mathrm{m}]$ & 195.68 \\
Diagnostic insertion @ $375 \mathrm{GeV}[\mathrm{m}]$ & 195.68 \\
Diagnostic insertion at BDS entrance [m] & 195.68 \\
Total length, including insertions [km] & 21.813 \\
Active length [km] & 15.171 \\
Real estate gradient [MV/m] & 24.455 \\
Number of cavities per module & 12 \\
Number of modules & 1218 \\
Number of cavities & 14616 \\
Module length (with quad) [m] & $15.9(16.8)$ \\
Number of quadrupoles & 468 \\
Number of klystrons & 609 \\
Number of cavities per klystron & 24 \\
\hline \hline
\end{tabular}

Table 3.5.5.14: Overview of components in the positron main linac for $500 \mathrm{GeV}$ beam energy. The overheads which have been assumed are indicated in Table 3.5.5.11.

\begin{tabular}{lc}
\hline Injection energy [GeV] & 5 \\
Final energy [GeV] & 500.185 \\
Linac length (cryomodules) [m] & 20009.91 \\
Diagnostic insertion @ 5 GeV [m] & 66.8 \\
Diagnostic insertion @ $50 \mathrm{GeV}[\mathrm{m}]$ & 66.8 \\
Diagnostic insertion @ $152 \mathrm{GeV}[\mathrm{m}]$ & 195.68 \\
Diagnostic insertion @ $375 \mathrm{GeV}[\mathrm{m}]$ & 195.68 \\
Diagnostic insertion at BDS entrance [m] & 195.68 \\
Total length, including insertions [km] & 20.731 \\
Active length [km] & 14.997 \\
Real estate gradient [MV/m] & 24.747 \\
Number of cavities per module & 12 \\
Number of modules & 1204 \\
Number of cavities & 14448 \\
Module length (with quad) [m] & $15.9(16.8)$ \\
Number of quadrupoles & 463 \\
Number of klystrons & 602 \\
Number of cavities per klystron & 24 \\
\hline \hline
\end{tabular}

even larger value for the matched $Q_{\text {ext }}$ than in the TDR, than for $250 \mathrm{GeV}$ beam energy. This, in turn, further reduces the cavity bandwidth and increases the cavity fill time. The quality factor is taken to be $5 \times 10^{9}$ at $35 \mathrm{MV} / \mathrm{m}$. 
Table 3.5.5.15: Parameters of the 9-cell cavity for use at $500 \mathrm{GeV}$ beam energy, (note that we adopt here the definition of shunt impedance by the relation $R=V^{2} / P$, where $P$ is the dissipated power and $V$ the peak voltage in the equivalent parallel LCR circuit).

\begin{tabular}{lc}
\hline Type of accelerator structure & standing wave \\
Accelerating mode & $\mathrm{TM}_{010}, \pi$-mode \\
Fundamental frequency & $1300 \mathrm{MHz}$ \\
Nominal gradient $E_{a c c}$ & $35 \mathrm{MV} / \mathrm{m}$ \\
Quality factor $Q_{0}$ & $>5 \times 10^{9}$ \\
Active length $L$ & $1.038 \mathrm{~m}$ \\
Cell-to-cell coupling $k_{c c}$ & $1.87 \%$ \\
Iris diameter & $70 \mathrm{~mm}$ \\
$R / Q$ & $1036 \Omega$ \\
$E_{\text {peak }} / E_{\text {acc }}$ & 2.0 \\
$B_{\text {peak }} / E_{\text {acc }}$ & $4.26 \mathrm{mT} /(\mathrm{MV} / \mathrm{m})$ \\
Tuning range & $\pm 300 \mathrm{kHz}$ \\
$\Delta f / \Delta L$ & $315 \mathrm{kHz} / \mathrm{mm}$ \\
Lorentz force detuning constant $K_{\text {Lor }}$ & $\sim 1 \mathrm{~Hz} /(\mathrm{MV} / \mathrm{m})^{2}$ \\
$Q_{\text {ext }}$ of input coupler & $3.69 \times 10^{6}$ \\
Cavity bandwidth at $Q_{\text {ext }}=3.69 \times 10^{6}$ & $352 \mathrm{~Hz}$ \\
Fill time & $626 \mu \mathrm{s}$ \\
Number of HOM couplers & 2 \\
\hline \hline
\end{tabular}

3.5.5.7.1.1 Cryostat The heat loads for a cryomodule with quadrupole are given in Table 3.5.5.16. This table is based on the TDR[TDR, Section 3.3.1], with the following modification. The dynamic heat loads due to the RF have been scaled up by the following product: the square of the gradient ratio,

$$
\left(\frac{35}{23.4}\right)^{2}=2.237
$$

the ratio of the unloaded $Q, 2$, and the ratio of the $\mathrm{RF}$ pulse lengths,

$$
\left(\frac{1.58}{1.37}\right)=1.151
$$

giving an overall factor of 5.149 .

Table 3.5.5.16: Calculated heat loads for $17 \mathrm{~m}$ long cryomodule with quadrupole, at $35 \mathrm{MV} / \mathrm{m}$ gradient.

\begin{tabular}{|c|c|c|c|c|}
\hline Temperature & Static $[\mathrm{W}]$ & Dynamic(RF) $[\mathrm{W}]$ & Dynamic(HOM) $[\mathrm{W}]$ & Total $[\mathrm{W}]$ \\
\hline $2 \mathrm{~K}$ & 1.74 & 26.21 & 2.21 & 30.16 \\
$5-8 \mathrm{~K}$ & 11.32 & 6.13 & 3.43 & 20.88 \\
$40-80 \mathrm{~K}$ & 90.13 & 305.84 & 28.5 & 424.47 \\
\hline
\end{tabular}

3.5.5.7.1.2 Cryomodule layout The layout for the first five cryounits is described in the Section 3.5.5.2.4. The increase in the gradient implies that the $66.83 \mathrm{~m}$ FODO cells now extend from $5 \mathrm{GeV}$ to $157 \mathrm{GeV}$. For the electron linac, the beam energy incident on the undulator increases to about $192 \mathrm{GeV}$. The beam energy at the end of the first five cryounits is about $312 \mathrm{GeV}$ for both linacs.

The linac extensions required to bring the beam energy to $500 \mathrm{GeV}$ will use $97.842 \mathrm{~m}$ long FODO cells. The complete $500 \mathrm{GeV}$ beam energy linacs will require a total of total of 2422 cryomodules. Of these, 1491 are of the standard variety, and 931 are cryomodules with a quadrupole. 
The additional cryomodules in the linac extensions will form an additional three cryounits, containing roughly 150 cryomodules each. There will be a two-cell diagnostic insertion at about $375 \mathrm{GeV}$ in each linac. Table 3.5.5.7.1.2 and Table 3.5.5.7.1.2 show the details of the cryogenic segmentation for the complete main linacs.

The electron main linac layout is shown in Fig. 3.5.5.4, and the positron main linac layout in Fig. 3.5.5.5.

Table 3.5.5.17: Segmentation of the electron main linac at $500 \mathrm{GeV}$ beam energy

\begin{tabular}{|c|c|c|c|}
\hline Linac segment & $\begin{array}{c}\text { Number of } \\
\text { cryomodules }\end{array}$ & $\begin{array}{c}\text { Length of } \\
\text { segment }(\mathrm{km})\end{array}$ & $\begin{array}{c}\text { Beam energy at } \\
\text { end of segment }(\mathrm{GeV})\end{array}$ \\
\hline Diagnostic section 1 & Warm & 0.0668 & 5.0 \\
Cryounit 1 & 158 & 2.640 & 69.98 \\
Diagnostic section 2 & Warm & 0.0668 & 69.98 \\
Cryounit 2 & 158 & 2.647 & 134.965 \\
Cryounit 3 & 141 & 2.337 & 192.956 \\
Undulator & Warm & 0.850 & 187.034 \\
Diagnostic section 3 & Warm & 0.1957 & 187.034 \\
Cryounit 4 & 143 & 2.370 & 245.847 \\
Cryounit 5 & 163 & 2.702 & 312.886 \\
Cryounit 6 & 153 & 2.536 & 375.813 \\
Diagnostic section 4 & Warm & 0.1957 & 375.813 \\
Cryounit 7 & 151 & 2.503 & 437.917 \\
Cryounit 8 & 151 & 2.503 & 500.020 \\
Diagnostic section 5 & Warm & 0.1957 & 500.020 \\
\hline
\end{tabular}

Table 3.5.5.18: Segmentation of the positron main linac at $500 \mathrm{GeV}$ beam energy

\begin{tabular}{|c|c|c|c|}
\hline Linac segment & $\begin{array}{c}\text { Number of } \\
\text { cryomodules }\end{array}$ & $\begin{array}{c}\text { Length of } \\
\text { segment }(\mathrm{km})\end{array}$ & $\begin{array}{c}\text { Beam energy at } \\
\text { end of segment }(\mathrm{GeV})\end{array}$ \\
\hline Diagnostic section 10 & Warm & 0.0668 & 5.0 \\
Cryounit 16 & 149 & 2.49 & 66.281 \\
Diagnostic section 9 & Warm & 0.0668 & 66.281 \\
Cryounit 15 & 149 & 2.49 & 127.562 \\
Cryounit 14 & 149 & 2.49 & 188.843 \\
Diagnostic section 8 & Warm & 0.1957 & 188.843 \\
Cryounit 13 & 149 & 2.49 & 250.125 \\
Cryounit 12 & 149 & 2.49 & 311.406 \\
Cryounit 11 & 153 & 2.54 & 374.332 \\
Diagnostic section 7 & Warm & 0.1957 & 374.332 \\
Cryounit 10 & 153 & 2.54 & 437.258 \\
Cryounit 9 & 153 & 2.54 & 500.185 \\
Diagnostic section 6 & Warm & 0.1957 & 500.185 \\
\hline
\end{tabular}

3.5.5.7.2 Cryogenics The cryogenic requirements of the main linacs are presented in Table 3.5.5.7.2. These numbers have been computed from the cryomodule heat loads given in Table 3.5.5.16, together with the cryounit module counts given in Table 3.5.5.7.1.2 and Table 3.5.5.7.1.2. The cryogenics will be supplied by 16 refrigerators located in the eight above-ground service halls shown in Fig. 3.5.5.7 and Fig. 3.5.5.8.

The AC cryogenic power requirements of the main linacs are given in Table 3.5.5.9. To compute the AC power requirements, the following COP's have been used (from the TDR[TDR, Table 8.7.5]): for 2 K 588 


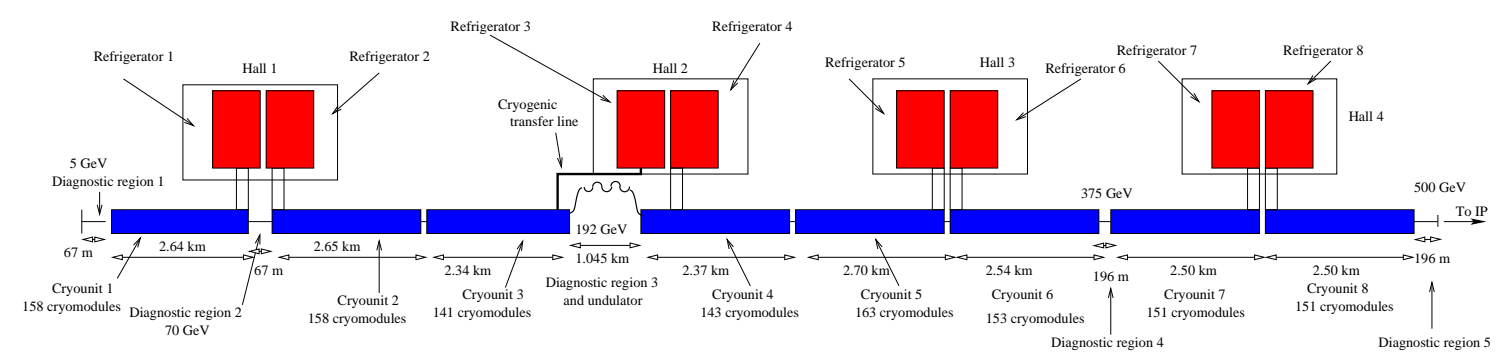

Figure 3.5.5.7: Electron main linac layout, $500 \mathrm{GeV}$ beam energy

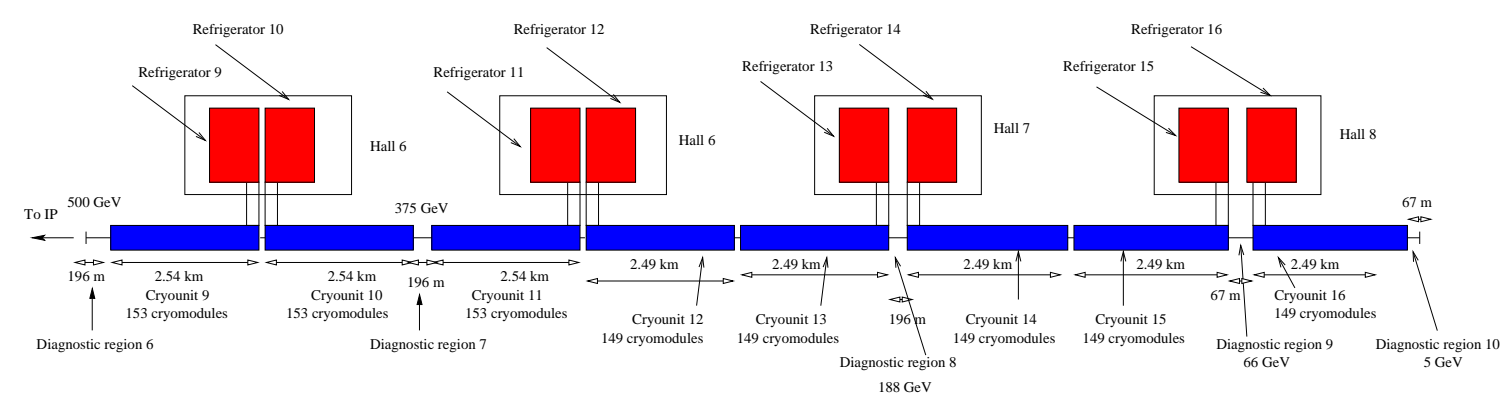

Figure 3.5.5.8: Positron main linac layout, $500 \mathrm{GeV}$ beam energy

W/W; for 5-8 K, $168 \mathrm{~W} / \mathrm{W}$; and for 40-80 K, $17 \mathrm{~W} / \mathrm{W}$. Cold compressor losses have also been included.

Table 3.5.5.19: Cryogenic requirements of the main linacs at $500 \mathrm{GeV}$ beam energy

\begin{tabular}{|c|c|c|c|c|}
\hline Cryounit & Temperature $[\mathrm{K}]$ & Static load $[\mathrm{kW}]$ & Dynamic load $[\mathrm{kW}]$ & Total $[\mathrm{kW}]$ \\
\hline \hline 1,2 & 2 & 0.27 & 4.49 & 4.76 \\
& $5-8$ & 1.79 & 1.51 & 3.30 \\
& $40-80$ & 14.24 & 52.83 & 67.07 \\
\hline 3 & 2 & 0.25 & 4.07 & 4.25 \\
& $5-8$ & 1.60 & 1.35 & 2.94 \\
& $40-80$ & 12.71 & 47.14 & 59.85 \\
\hline 4 & 2 & 0.25 & 4.06 & 4.31 \\
& $5-8$ & 1.62 & 1.37 & 2.99 \\
& $40-80$ & 12.89 & 47.81 & 60.70 \\
\hline 5 & 2 & 0.28 & 4.63 & 4.92 \\
& $5-8$ & 1.85 & 1.56 & 3.40 \\
& $40-80$ & 14.69 & 54.50 & 69.19 \\
\hline $6,9-11$ & 2 & 0.27 & 4.35 & 4.61 \\
& $5-8$ & 1.73 & 1.46 & 3.19 \\
& $40-80$ & 13.79 & 51.15 & 64.94 \\
\hline 7,8 & 2 & 0.26 & 4.29 & 4.55 \\
& $5-8$ & 1.71 & 1.44 & 3.19 \\
& $40-80$ & 13.61 & 50.49 & 64.94 \\
\hline $12-16$ & 2 & 0.26 & 4.23 & 4.49 \\
& $5-8$ & 1.69 & 1.42 & 3.11 \\
& $40-80$ & 13.43 & 49.82 & 63.25 \\
\hline
\end{tabular}


Table 3.5.5.20: Total cryogenic operating power requirements, $500 \mathrm{GeV}$ beam energy

\begin{tabular}{lc}
\hline AC power cryogenics at 2 K, Cryounits 1-16 [MW] & 45.95 \\
AC power cryogenics at 5-8 K, Cryounits 1-16 [MW] & 9.09 \\
AC power cryogenics at 40-80 K, Cryounits 1-16 [MW] & 18.70 \\
Total AC power for cryogenics [MW] & 73.74 \\
\hline \hline
\end{tabular}

3.5.5.7.3 RF system The RF system for $500 \mathrm{GeV}$ beam energy consists of 602 (609) stations per positron (electron) main linac, each of which provides power at $1.3 \mathrm{GHz}$ to a total of 24 accelerating cavities (two cryomodules). The number of RF stations per linac includes an $6 \%$ overhead, as indicated in Table 3.5.5.11. Each RF station consists of a klystron and a modulator. The peak RF power needed for one superconducting cavity at full gradient and maximum beam current $(35 \mathrm{MV} / \mathrm{m}$ and $9.5 \mathrm{~mA})$ is $345 \mathrm{~kW}$; the nominal peak power needed for 30 cavities is $8.28 \mathrm{MW}$. Taking into account a regulation reserve of $10 \%$ for phase and amplitude control, and another $6.9 \%$ for circulator and waveguide losses, a total of $9.68 \mathrm{MW}$ is required. The RF pulse length is $1.58 \mathrm{~ms}$, which includes the beam pulse length of $0.95 \mathrm{~ms}$, and the cavity fill time of $0.63 \mathrm{~ms}$. The repetition rate is $5 \mathrm{~Hz}$.

The overall efficiency and power requirements of the RF systems are given in Table 3.5.5.21.

Table 3.5.5.21: Efficiencies and power requirements for the RF system at $500 \mathrm{GeV}$ beam energy

\begin{tabular}{lc}
\hline RF station peak power [MW] & 9.68 \\
Duty cycle [\%] & 0.79 \\
RF station average power [kW] & 76.3 \\
Klystron efficiency [\%] & 65 \\
Modulator efficiency [\%] & 85 \\
AC power per RF station [kW] & 138.1 \\
AC power for auxiliaries per RF station [kW] & 14 \\
Number of stations in electron linac & 609 \\
Electron linac AC wall plug power for RF [MW] & 92.66 \\
Number of stations in positron linac & 602 \\
Positron linac AC wall plug power for RF [MW] & 91.59 \\
Total AC wall plug power for RF [MW] & 184.25 \\
Beam power [MW] & 44.95 \\
AC to beam efficiency for RF [\%] & 24.4 \\
\hline \hline
\end{tabular}

3.5.5.7.4 Waveguide distribution system For operation at $35 \mathrm{MV} / \mathrm{m}$, klystrons will be installed at every tunnel cross-connect. These klystrons will feed 24 cavities, using the arrangement shown in Fig. 3.5.5.9. With this arrangement, the average distance from klystron to RF cavity will be about $8.5 \mathrm{~m}$, plus the length of the cross tunnel. The total average distance from klystron to RF cavity is then $11.4 \mathrm{~m}+8.5 \mathrm{~m}=19.9$ m. Using WR770 waveguide, with an attenuation factor of $-0.011 \mathrm{~dB} / \mathrm{m}$, the power loss in the waveguide is $4.9 \%$. According to the TDR[TDR, Section 3.4.6], there is an additional $2 \%$ loss in the circulator, giving a total loss of $6.9 \%$. 


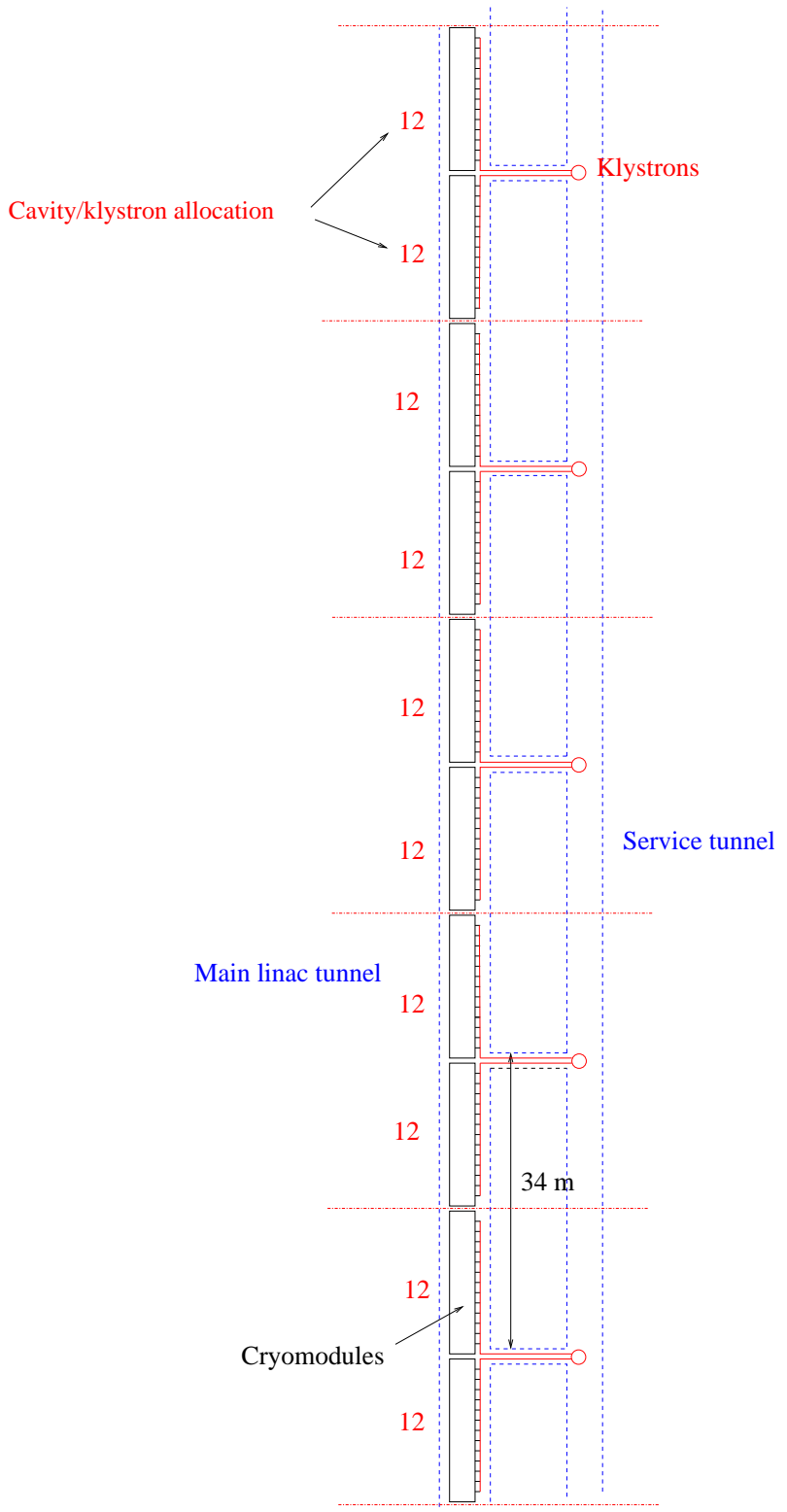

Figure 3.5.5.9: Waveguide distribution, two tunnels, $500 \mathrm{GeV}$ beam energy 


\subsubsection{Beam Delivery Systems}

\subsubsection{Overview}

The Beam Delivery System (BDS) transports the beam from the end of the Linac to the Interaction Point (IP) and from there to the beam dump. The BDS also focuses the beam to a small spot size to maximize luminosity with full chromatic correction. The cold Linac is matched into an "NLC-like" BDS to meet the desired crossing-angle requirement and in addition uses a superconducting final focus to facilitate collision energy and optics variability. These technical choices for the BDS design permit an essentially common approach between the warm and cold options. As for the warm option, two interaction regions are provided.

The first portion of the BDS is an instrumentation section to characterize the beam parameters such as energy spread and transverse emittance exiting the Linac. This is followed by a fast extraction/tune-up section which is a separate beam line to an independent beam dump. This system protects both the detector and final focus region from missteered or off-energy beam with a fast abort system. It also allows a Linac tune-up mode with DC operation of the same line. A switch section that selects one of the two experimental areas precedes the main BDS optics section. The optics section provides the main beam transport to the final focus, and introduces dispersion for chromatic correction. Throughout the first portion of the main transport section there is collimation system consisting of both spoilers and absorbers. This system serves to limit beam halo and synchrotron light from the IP as well as providing protection from off-energy beam pulses. Tail-folding octupoles are also present in the main transport section. The final focus section produces the small beam spot at the IP as well as chromatic corrections. The beams intersect with a 20 mrad horizontal crossing angle defined by the orientation of the main linacs. Crab cavities are used to prevent any loss of luminosity arising from the crossing angle. Following the IP an out-going extraction line transports the disrupted beam to a beam dump with optics that allows for instrumentation and diagnostics to characterize the IP beam properties such as current, position, energy spread and polarization on a bunch-by-bunch basis. The beam dump is capable of absorbing up to $23 \mathrm{MW}$ of DC beam power.

The beam delivery system is $\sim 3.6 \mathrm{~km}$ in length and uses superconducting magnets for the final focus doublets and conventional ones in other places. As a consequence of the very small beam size at the IP, the alignment and vibration tolerances are especially demanding challenges. The final focus magnets may be stabilized with mechanical feedback and slow magnetic field drifts are compensated via beam steering feedback. Fast errors are compensated by an intra-train feedback system, as in the TESLA TDR.

\subsubsection{Magnet Lattice and Optics}

In place of the head-on TESLA design for the BDS magnet lattice, an NLC-like BDS is be used to provide a $20 \mathrm{mrad}$ crossing angle. In order to produce the TESLA lattice functions at the IP, an additional section will be used to match from the cold linac optics to the unmodified NLC BDS magnet lattice as shown in Fig. 3.5.6.1. The matching section will be $250 \mathrm{~m}$ long and will have four independently powered quadrupoles that when combined with the rest of the NLC BDS will obtain the TESLA $\left(\beta_{x}^{*}, \beta_{y}^{*}\right)$ of $(15 \mathrm{~mm}, 0.4 \mathrm{~mm})$ at the IP. The matching section will also contain additional dipoles and kicker magnets, as described in Section 3.5.6.4, for the Fast Extraction Line. In particular the space between the first and second matching quadrupole will be used to make a $12 \mathrm{~mm}$ fixed dogleg bump. Beam position offset, as seen by BPM's at the $12 \mathrm{~mm}$ dispersion point, will indicate an energy offset of the incoming beam and will be used to provide a fast trigger signal for the Fast Extraction System. Chromatic corrections, collimation optics and final focus magnet parameters are identical between both technical options. Immediately downstream of the main linac and before the matching section is a diagnostic region consisting of four linac half-cells without accelerating structures. The elements of the transport line from the end of the main linac to the IP are given in Table 3.5.6.2. 


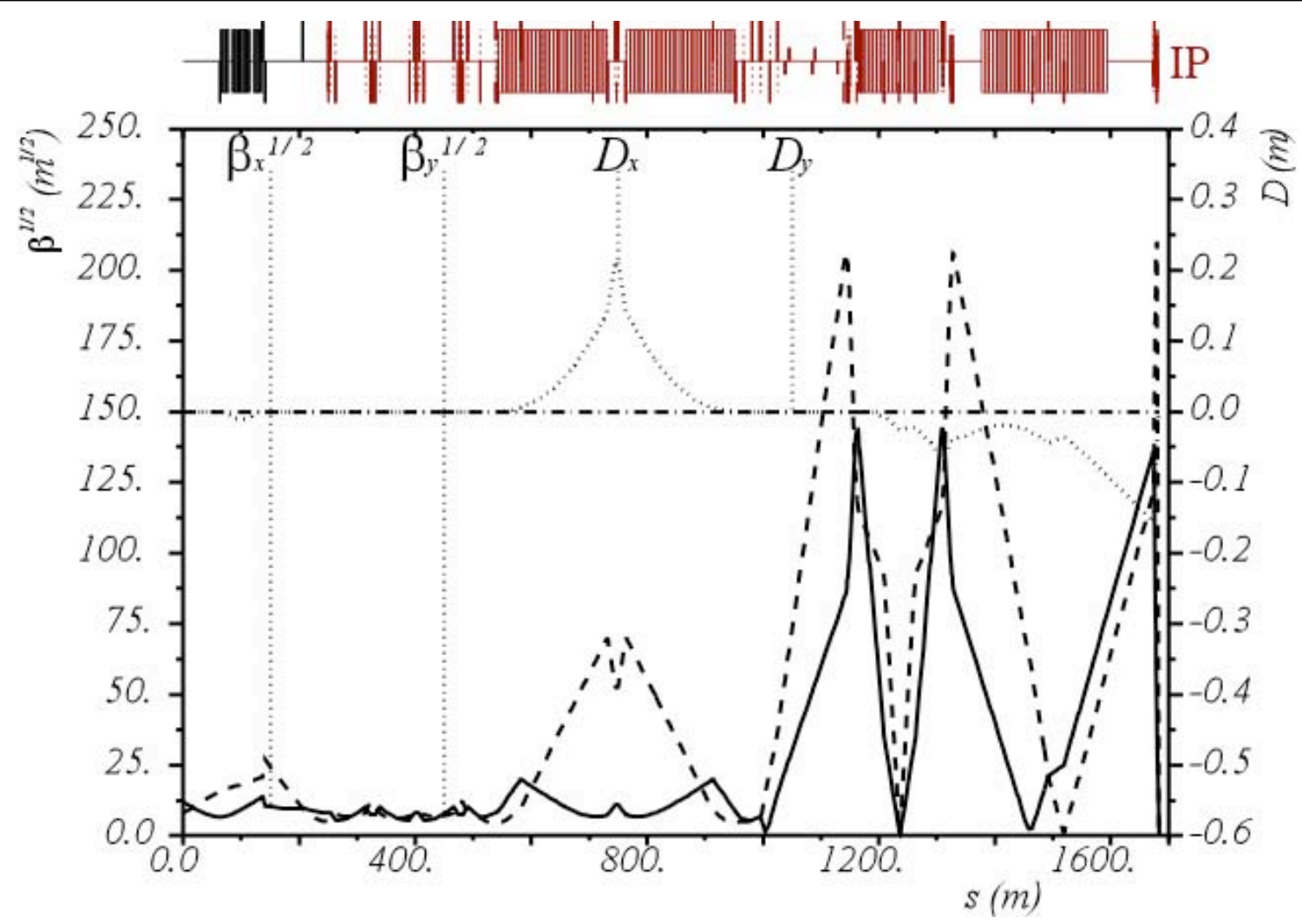

Figure 3.5.6.1: NLC-TESLA Hybrid Optics

\subsubsection{Collimation Systems}

The general requirements for the collimation system are to:

- limit the beam halo in the final doublet so that synchrotron radiation passes freely through the IP and the dump line beyond;

- limit the halo loss throughout the final focus system (FFS) to keep the muon flux in the detector tolerable;

- protect the beam line from off-energy beam pulses from the linac.

To fulfill these requirements the general design calls for:

- a primary collimation system upstream from the FFS;

- a secondary clean-up collimation system within the FFS.

NLC, TESLA and CLIC have produced designs for collimation systems that, though differing in implementation design, share the overall layout and functionality. Although predictions for the amount of beam halo in a high energy LC are $\sim 10^{-6}$ of the total beam intensity, collimation systems are typically designed to deal with $10^{-3}$ of the total beam intensity on the basis of operational experience at the SLC. (A $10^{-3}$ beam loss corresponds to $11.3 \mathrm{~kW}$ in Tesla, $6.9 \mathrm{~kW}$ in the NLC and $4.9 \mathrm{~kW}$ in CLIC). 
Table 3.5.6.1: Major Technical Components: Electron Beam Delivery Line (IR1). The strengths quoted are for $250 \mathrm{GeV} /$ beam operation.

\begin{tabular}{|c|c|c|c|c|c|c|}
\hline Element & $\#$ & $\begin{array}{c}\text { Z Position } \\
{[\mathrm{m}]}\end{array}$ & Type & $\begin{array}{c}L_{m a g} \\
{[\mathrm{~m}]}\end{array}$ & $\begin{array}{c}\text { Strength } \\
\mathrm{B}, \mathrm{G} \\
{[\mathrm{T}, \mathrm{T} / \mathrm{m}]}\end{array}$ & $\begin{array}{c}\text { Aperture } \\
\mathrm{H} \times \mathrm{V}, \mathrm{ID} \\
{[\mathrm{mm}]}\end{array}$ \\
\hline QX1-4 & 4 & $64-206$ & F/D quads & 2.0 & 20 & 12 \\
QB0-3 & 16 & $254-542$ & F/D quads & 2.0 & 7 & 20 \\
XCORFB & 6 & $250-1440$ & feedback/steering dipoles & 0.5 & & 20 \\
YCORFB & 6 & $250-1440$ & feedback/steering dipoles & 0.5 & & 20 \\
BS1 & 30 & $550-951$ & H-dipoles & 12.0 & 0.008 & $20 \times 144$ \\
QS1-3 & 6 & $582-918$ & F/D quads & 2.0 & 20 & 20 \\
QM11-16 & 7 & $954-1026$ & F/D quads & 2.0 & 7 & 40 \\
OC7,10 & 5 & $1038-1146$ & tail folding octupoles & 1.5 & & 40 \\
QD2-10 & 2 & $1144-1520$ & D Quads & 2.0 & 3 & 60 \\
QF3-9 & 2 & $1160-1493$ & F Quads & $1.0-2.0$ & 3 & 60 \\
B5 & 10 & $1178-1307$ & H-Dipoles & 12.0 & 0.008 & $25 \times 328$ \\
B2 & 12 & $1378-1518$ & H-Dipoles & 12.0 & 0.007 & $60 \times 232$ \\
B1 & 6 & $1526-1594$ & H-Dipoles & 12.0 & 0.002 & $60 \times 232$ \\
SF1-6, SD0-4 & 3 & $1161-1677$ & chromatic sextupoles & 0.5 & & \\
Crab Cavity & 1 & & 1672 & 0.5 & & \\
SQ3 & 1 & 1672 & skew quadrupole & 0.2 & $\leqslant 20$ & 12 \\
QF1 & 1 & 1675 & final focus F quad package & 2.0 & 144 & 20 \\
QD0 & 1 & 1679 & final focus F quad package & 2.0 & 144 & 20 \\
IP & & 1684 & Interaction Point & & & \\
\hline
\end{tabular}

Collimator systems for high energy LC's consist of a combination of thick absorbers ( $\gg 1$ radiation length, typically copper) and thin spoilers ( $<1$ radiation length). The post-linac collimation systems of TESLA, NLC, and CLIC have been assessed and compared by evaluating the efficiency in beam halo and synchrotron radiation collimation. Halo needs to be removed to a collimation depth generally set by the synchrotron radiation generated by the halo particles in the last few magnets close to the IP. The collimator depth at the spoilers is respectively $13 \sigma_{x}$ and $80 \sigma_{y}$ for TESLA, $15 \sigma_{x}$ and $31 \sigma_{y}$ for NLC and $11 \sigma_{x}$ and $100 \sigma_{y}$ for CLIC. Realistic simulations have been run to compare equitably the collimator system designs and overall efficiency[57].

The overall existence of satisfactory solutions for the different designs insures that a specific solution for the cold option can be found, taking as a basis the NLC collimation system design. The inclusion of 2 pairs of octupole doublets for halo tail folding to reduce the halo transverse size in final doublet is part of the collimation system.

\subsubsection{Tuneup and Fast Extraction Beam Line}

Separate beam lines that terminate in beam dumps will be provided for both incoming beams. Beams will be dispatched to these dumps according to two operational modes. The first will be Fast Extraction (FEX) mode in which fast kicker magnets are energized to send beam to the dump and thus protect downstream BDS components during beam upset conditions. The second mode will be Tuneup (TU) mode in which DC magnets are energized to automatically send beam on to the same dump when it is not desirable to send beam through the IR. TU mode will be especially useful during commissioning. The elements of the FEX/TU are given in Table 3.5.6.4 
Table 3.5.6.2: FEX/TU line elements. The indicated strengths are for $250 \mathrm{GeV}$ beam energy.

\begin{tabular}{|c|c|c|c|c|c|c|}
\hline Element & $\#$ & $\begin{array}{c}\text { Z Position } \\
\text { [m] }\end{array}$ & Type & $\begin{array}{c}L_{m a g} \\
{[\mathrm{~m}]}\end{array}$ & $\begin{array}{c}\text { Strength } \\
\mathrm{B}, \mathrm{G} \\
{[\mathrm{T}, \mathrm{T} / \mathrm{m}]}\end{array}$ & $\begin{array}{c}\text { Aperture } \\
\mathrm{H} \times \mathrm{V}, \mathrm{ID} \\
{[\mathrm{mm}]}\end{array}$ \\
\hline BTU/FEK & 24 & 33 to 62 & H-Dipole/Kicker & 1.0 & 0.033 & $32 \times 32$ \\
BFE & 20 & 103 to 175 & H-Dipole & 2.8 & 0.033 & $56 \times 34$ \\
QFEX & 1 & 154 to 157 & F-Quadrupole & 2.4 & 5.4 & 80 \\
BPAI & 10 & 175 to 205 & V-Kicker & 2.6 & 0.002 & $72 \times 24$ \\
Absorber & 1 & $1000 \mathrm{~m}$ & & & & \\
\hline
\end{tabular}

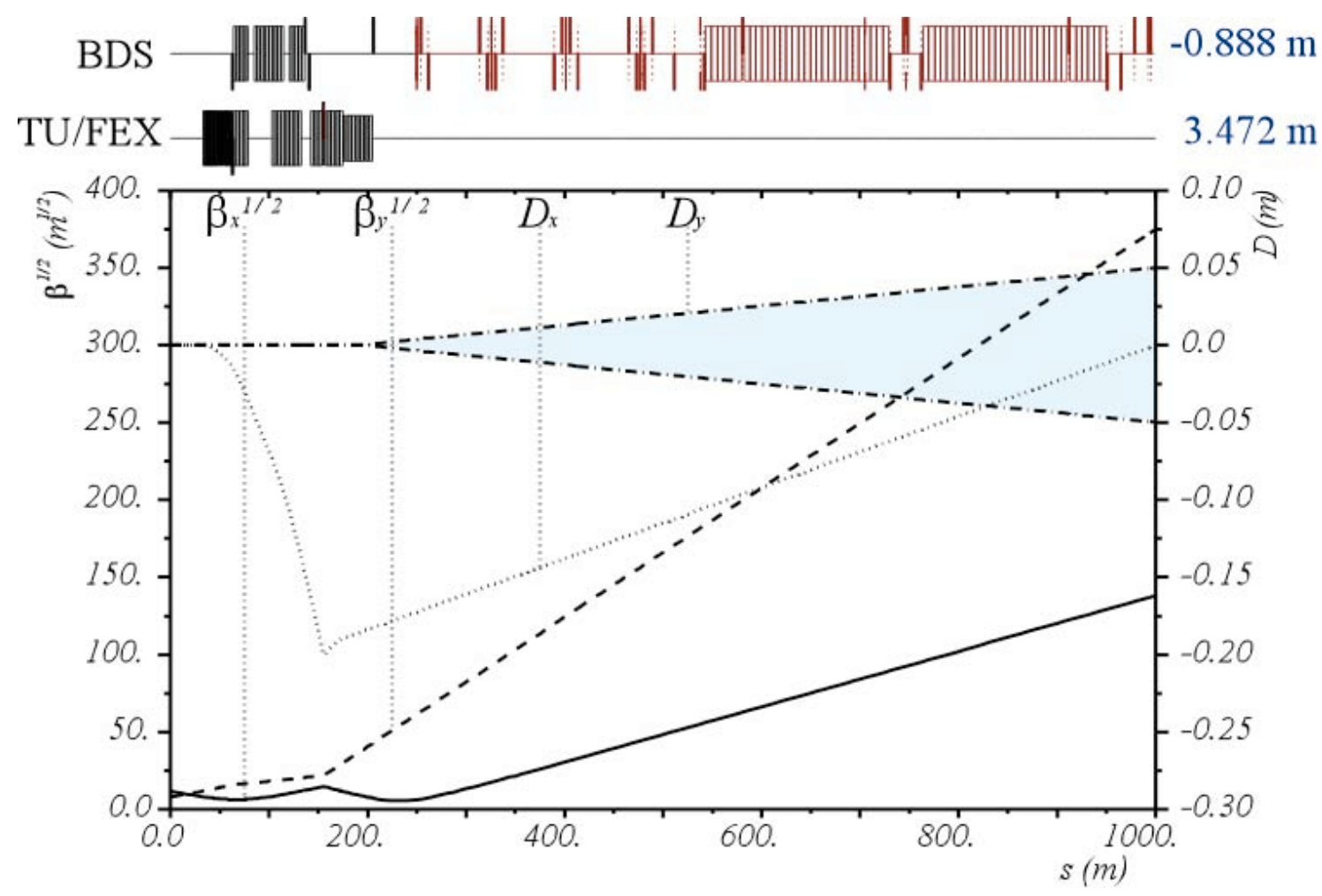

Figure 3.5.6.2: Fast Extraction and Tuneup Optics 
3.5.6.4.1 Fast Extraction The FEX lattice is shown in Figure 3.5.6.2 along with the beginning of the BDS lattice. Signals from BPM's near the $12 \mathrm{~mm}$ dispersion in the BDS dogleg and signals from upstream BPM's in dispersion free sections will be processed to decide if the beam should be sent to a dump rather than risking damage of downstream BDS components or the experimental detector. If so, a set of 24 independently powered horizontal kicker magnets will be energized, sending subsequent bunches down the FEX beam line. With 24 units, misfire of a single unit leads to $\sim 4 \%$ total kick error which is accommodated within FEX beam line apertures. Kicker firing will be synchronized to occur during the $337 \mathrm{~ns}$ bunch spacing and the kicker power supplies produce a rise time of less than $150 \mathrm{~ns}$.

The kicked beam will pass off-axis though the first matching quadrupole (defocusing) that adds to the beam deflection. By the time FEX beam reaches the central dogleg dipoles the beam separation is sufficient for the FEX beam to pass outside the dipole yoke. The FEX beam dump is separated from the BDS line by 4.4 $\mathrm{m}$. Dispersion at the front face of the dump will be brought to zero.

The spot size at the dump is $610 \times 93 \mu \mathrm{m}(1 \sigma)$. In order to reduce localized radiation damage of the absorber entrance window and to avoid locally overheating the cooling water, the beam will be painted vertically at the dump using oscillating-field dipole magnets in the FEX beam line.

3.5.6.4.2 Tuneup The TU beam line is the FEX line, i.e. same magnets, vacuum chamber, absorber etc., except that in TU mode all 24 kicker magnets are energized by a common 8-turn DC winding so that the beam is continuously steered to the dump. Note that for TU mode, switches at the ends of the 24 magnet string are closed in order to be able to energize the DC windings.

\subsubsection{Final Focus}

3.5.6.5.1 Superconducting Doublets Compact superconducting magnets, consisting of quadrupoles, sextupoles, octupoles and correction dipoles will be used in the final focus doublets. They will fit within a cryostat having a $57 \mathrm{~mm}$ outer radius.

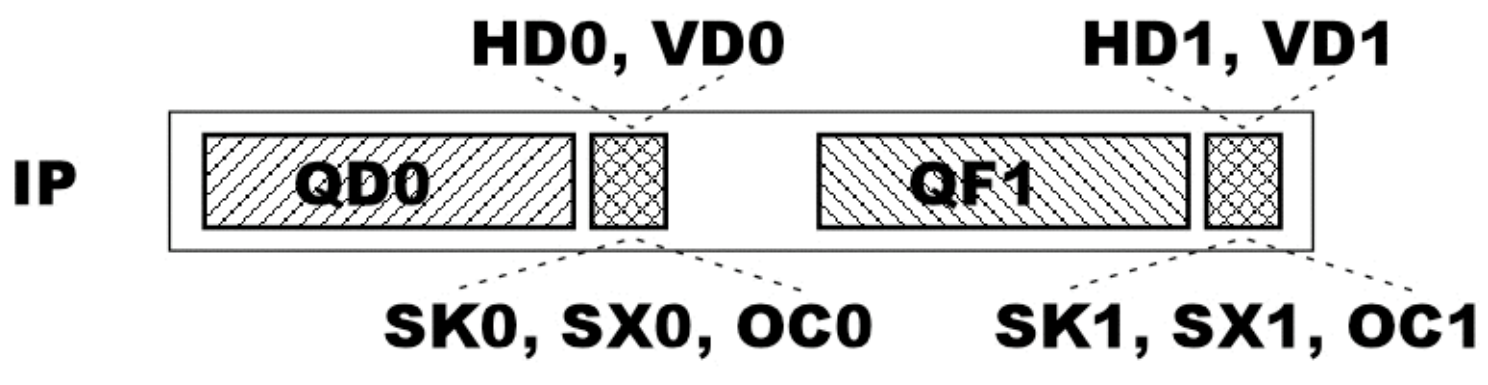

Figure 3.5.6.3: Superconducting Final Focus Magnet Layout Schematic

The conceptual magnet layout is shown in Fig. 3.5.6.3. Each corrector package contains skew-quadrupole, sextupole and octupole multilayer windings as well as horizontal and vertical dipole corrector windings, next to each main quadrupole. The superconducting magnets will share a common cryostat that is passively and actively stabilized against vibrational motion. The transverse centering of QD0 and QF0 will be independently adjustable within the cryostat, with each corrector package rigidly connected to each main quadrupole. Also, the QD0 and QF0 longitudinal magnetic centers will be independently fixed in the cryostat in order to minimize coil movement due to thermal contraction during cool down.

The QD0 gradient of $144 \mathrm{~T} / \mathrm{m}$ at $250 \mathrm{GeV}$ will be attained with a multi-layer coil structure, as shown in Fig. 3.5.6.4, that will have two independently powered, concentric quadrupole windings, with helium 


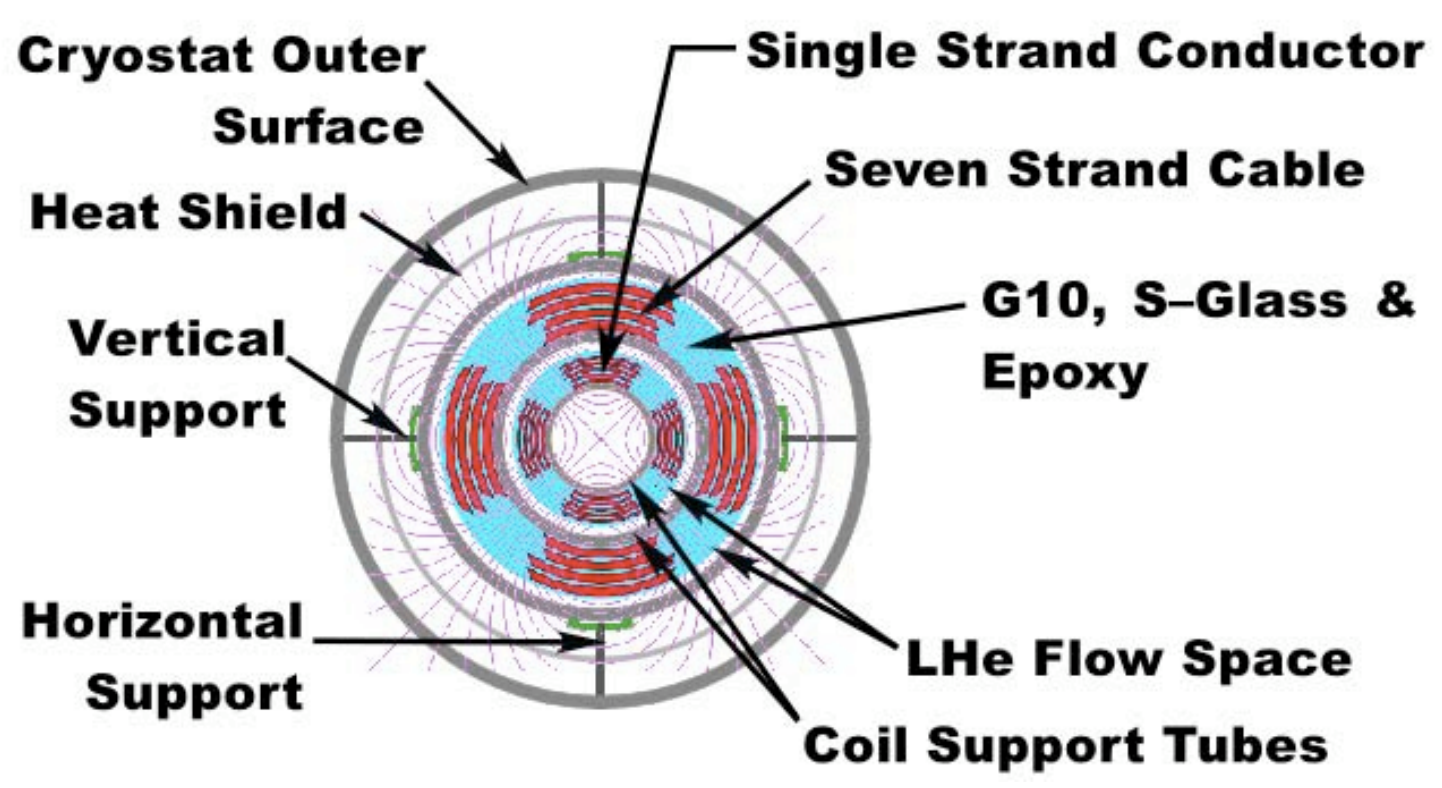

Figure 3.5.6.4: Superconducting QD0 Design Schematic

cooling between and outside the coil windings. For the upgrade to $500 \mathrm{GeV}$ beam energy operation, the final doublet magnets will be replaced with longer versions, rather than simply scaling the gradient, in order to avoid complications from increased synchrotron radiation.

With the QF0 gradient being significantly smaller in magnitude than that of QD0, either the inner or the outer QD0 coil structure will be adequate for operation; so QF0 can be powered with a single power supply. For the dual powered QD0 both power supplies must be shut down if either the inner or outer coil begins to quench.

If required, the outer cryostat of the superconducting magnets will be stabilized with an optical anchor (see Section 3.5.6.5.3), and by eliminating rigid connections to potential external sources of vibration such as cryogenic and electrical feeds. Construction and active damping internal to the cryostat if necessary will minimize internal vibrations.

3.5.6.5.2 Crab Cavities The potential luminosity reduction due to the $20 \mathrm{mrad}$ crossing angle is compensated by the use of crab cavities in each beam which provide a transverse displacement within the bunch that varies with longitudinal distance. The cavities are located in the incoming beam-lines on each side of the IP behind the final focus quadrupoles, $\sim 15 \mathrm{~m}$ from the IP. They are of similar construction to the Main Linac structures, but since the maximum required voltage kick is less than $10 \mathrm{MV}$, they will be considerably shorter. Alignment, voltage, and phase tolerances are similar to the main linac. Differential phase jitter between the cavities is the most demanding tolerance and will be minimized by driving each cavity from a single common klystron. Voltage and phase control will be performed using similar controls to those employed on the Main Linac.

The crab cavities will be mounted on a movable stage with an adjustable roll angle, to allow the introduction of vertical crabbing for luminosity tuning.

3.5.6.5.3 Detector Interface There are several areas where detector requirements influence machine component design. Detector backgrounds arise from many mechanisms. Principally, these are electrons 
from beamstrahlung photons, synchrotron radiation from the final focus quadrupoles, and muons produced by interactions in the upstream collimators. Muon flux into the detector will be minimized by a series of magnetized iron spoilers downstream of the collimation system. The detector will be shielded from low energy electrons with tungsten masks starting $50 \mathrm{~cm}$ from the IP, and defining a $100 \mathrm{mrad}$ ID cone, followed by a $10 \mathrm{~cm}$ thick tungsten pipe. A copper RF shield will protect the inner detector components from image currents and tungsten shielding will also protect the front face of the final focus quads.

The experimental solenoid, assumed to have a $3 \mathrm{~T}$ field, together with the $10 \mathrm{mrad}$ off-axis beam, results in non-negligible dispersion and coupling. Dipole and skew quadrupole correctors in the final focus must compensate these effects. Other issues arising from the detector solenoidal field, such as synchrotron radiation and field stability, are benign. If required, the final focus quadrupoles will be stabilized to the nanometer level by a mechanical feedback system based on a laser interferometer, a so-called optical anchor. Since the quadrupoles are located well inside the detector, there will need to be two optical paths through the detector calorimetry, referenced to the external walls.

\subsubsection{IP Feedback Systems}

An intra-train IP collision feedback is a key factor in the performance of a high-energy LC, as the luminosity is extremely sensitive to relative small offsets of the beams in position and crossing angle. The feedback signal is derived directly from the strong beam-beam kick experienced by the beams when they do not collide head-on, and correction is provided by fast kickers.

Intra-train feedback system designs have been developed for TESLA and the NLC. The key factors in the intra-train IP feedback performance are bunch spacing and length of the train. A large bunch spacing (337 ns) eases the feedback time response requirements for the system. Realistic performance studies for the TESLA IP position feedback system predict that an initial $100 \sigma_{y}$ offset can be reduced by 3 orders of magnitude within 90 bunches, or $3 \%$ of the bunch train. The response time to correct a $\sim 10 \sigma_{y^{\prime}}$ signal is $\sim 10$ bunches[57].

The TESLA-like bunch structure and bunch trains for the cold option will make it possible to integrate an intra-train IP feedback system into a NLC-like beam delivery system. This will ease considerably the requirements on the final focus doublet mechanical stabilization.

\subsubsection{Spent Beam Transport}

The purpose of the dump line is to provide an image of the interaction point for diagnostic purposes, before safely guiding the spent beam to the beam dump. Though beam sizes differ significantly from those of the NLC, the NLC extraction line design provides a minimum aperture of $10 \sigma$ and is therefore also considered sufficient for the cold option, especially since beam disruption at the IP leads to sharp edges of the transverse distribution rather than long tails.

The elements of the spent beam transport line from the IP to the beam dump are given in Table 3.5.6.7.

\subsubsection{Beam Dumps}

The beam dump will be the same or very similar to the one described in the TESLA TDR, namely a water dump. The dump will be capable of absorbing $22 \mathrm{MW}$ of DC beam power. 
Table 3.5.6.3: Spent beam transport line elements. The indicated strengths are for $250 \mathrm{GeV}$ beam energy.

\begin{tabular}{|c|c|c|c|c|c|c|}
\hline Element & $\#$ & $\begin{array}{c}\text { Z Position } \\
{[\mathrm{m}]}\end{array}$ & $\begin{array}{c}L_{\text {mag }} \\
{[\mathrm{m}]}\end{array}$ & $\begin{array}{c}\text { Ttrength } \\
{[\mathrm{T}, \mathrm{T} / \mathrm{m}]}\end{array}$ & $\begin{array}{c}\text { Aperture } \\
{[\mathrm{mm}]}\end{array}$ \\
\hline QF1 & 3 & 6 to 9 & F-Quadrupole & 0.9 & 120 & $20 \mathrm{ID}$ \\
QD2 & 3 & 9 to 13 & D-Quadrupole & 0.8 & $110 \rightarrow 90$ & $22 \rightarrow 26$ ID \\
QF3 & 4 & 13 to 18 & F-Quadrupole & 1.0 & $75 \rightarrow 66$ & $32 \rightarrow 36$ ID \\
QD4 & 6 & 19 to 25 & D-Quadrupole & 1.1 & $57 \rightarrow 48$ & $42 \rightarrow 50$ ID \\
QF5 & 5 & 26 to 31 & F-Quadrupole & 1.1 & $43 \rightarrow 37$ & $56 \rightarrow 64$ ID \\
BD1 & 2 & 32 to 34 & V-Dipole & 1.0 & 0.82 & $300 \times 200$ \\
BD2 & 2 & 52 to 54 & V-Dipole & 1.0 & 0.82 & $300 \times 200$ \\
IP Image & & 59 & Diagnostic Point & & & \\
BD3 & 2 & 65 to 67 & V-Dipole & 1.0 & 0.82 & $300 \times 200$ \\
BD4 & 2 & 85 to 87 & V-Dipole & 1.0 & 0.82 & $300 \times 200$ \\
QD6 & 2 & 90 to 94 & D-Quadrupole & 3.0 & 11 & 188 ID \\
QF7 & 3 & 97 to 105 & F-Quadrupole & 3.5 & 9.5 & 210 ID \\
QD8 & 5 & 108 to 121 & D-Quadrupole & 3.0 & 8.2 & 244 ID \\
QF9 & 3 & 125 to 131 & F-Quadrupole & 3.1 & 7.5 & 264 ID \\
Absorber & 1 & 152 & r & & & \\
\hline
\end{tabular}

\subsubsection{Vacuum System}

The pressure requirements in the BDS section are set by tolerable detector background levels. In the NLC $\mathrm{ZDR}$, this is determined to be $50 \mathrm{nTorr}$ for the majority of the BDS section. In the $\sim 300 \mathrm{~m}$ closest to the interaction point, a much lower pressure of $1 \mathrm{nTorr}$ is required.

\subsubsection{Infrastructure and Auxiliary Systems}

\subsubsection{Site layout and civil construction}

3.5.7.1.1 Overall layout A tabulation of the overall length of the technical components is presented in Table 3.5.7.1.1, for a cavity gradient of $28 \mathrm{MV} / \mathrm{m}$ for $250 \mathrm{GeV}$ beam energy, and a cavity gradient of 35 $\mathrm{MV} / \mathrm{m}$ for $500 \mathrm{GeV}$ beam energy. The overall estimate for $500 \mathrm{GeV}$ beam energy is $47.4 \mathrm{~km}$.

Table 3.5.7.1: Tabulation of technical component lengths. All lengths are in km.

\begin{tabular}{|c|c|c|}
\hline Length & $250 \mathrm{GeV}$ beam energy & $500 \mathrm{GeV}$ beam energy \\
\hline Electron Linac cryomodules & 12.699 & 20.242 \\
Electron Linac insertions & 1.375 & 1.571 \\
Positron Linac cryomodules & 12.400 & 20.010 \\
Positron Linac insertions & 0.525 & 0.721 \\
\hline Overall two-linacs with insertions & 26.999 & 42.544 \\
BDS length (total) & 3.6 & 3.6 \\
Spin rotator/bunch compressor length (total) & 0.80 & 0.80 \\
DR ends length (total) & 0.5 & 0.5 \\
Total technical component length & 31.899 & 47.444 \\
\hline
\end{tabular}


3.5.7.1.2 Underground construction The injectors, damping rings, main linacs, and beam delivery systems will be housed in underground tunnels. Vertical access shafts are required for the construction of the tunnels, and to provide a link for LCW, power, and cryogenics from the surface facilities, and to allow for installation of the technical components in the underground enclosures. Details of the underground tunnels and shafts are provided in Sections 6.3.1 and 6.3.2 on civil construction and siting.

3.5.7.1.2.1 Main Linac tunnel layout The cryomodules for the main linac and the beam lines for the straight sections of the damping rings are installed in a $4.1 \mathrm{~m}$ diameter tunnel. The RF components for the main linac (klystrons, pulse transformers, modulators, and modulator high-voltage supplies) are installed in a parallel, $4.2 \mathrm{~m}$ diameter tunnel. The tunnel centers are separated by $11.4 \mathrm{~m}$. There will be crossconnects between the two tunnels every $34 \mathrm{~m}$, to allow the RF power from the klystrons to be distributed to the cryomodules. (See Section 3.5.5.4.2). AC power to the service tunnel will be provided from the above-ground buildings through the access shafts. A cross section for the two-tunnel layout is shown in Fig. 3.5.7.1.

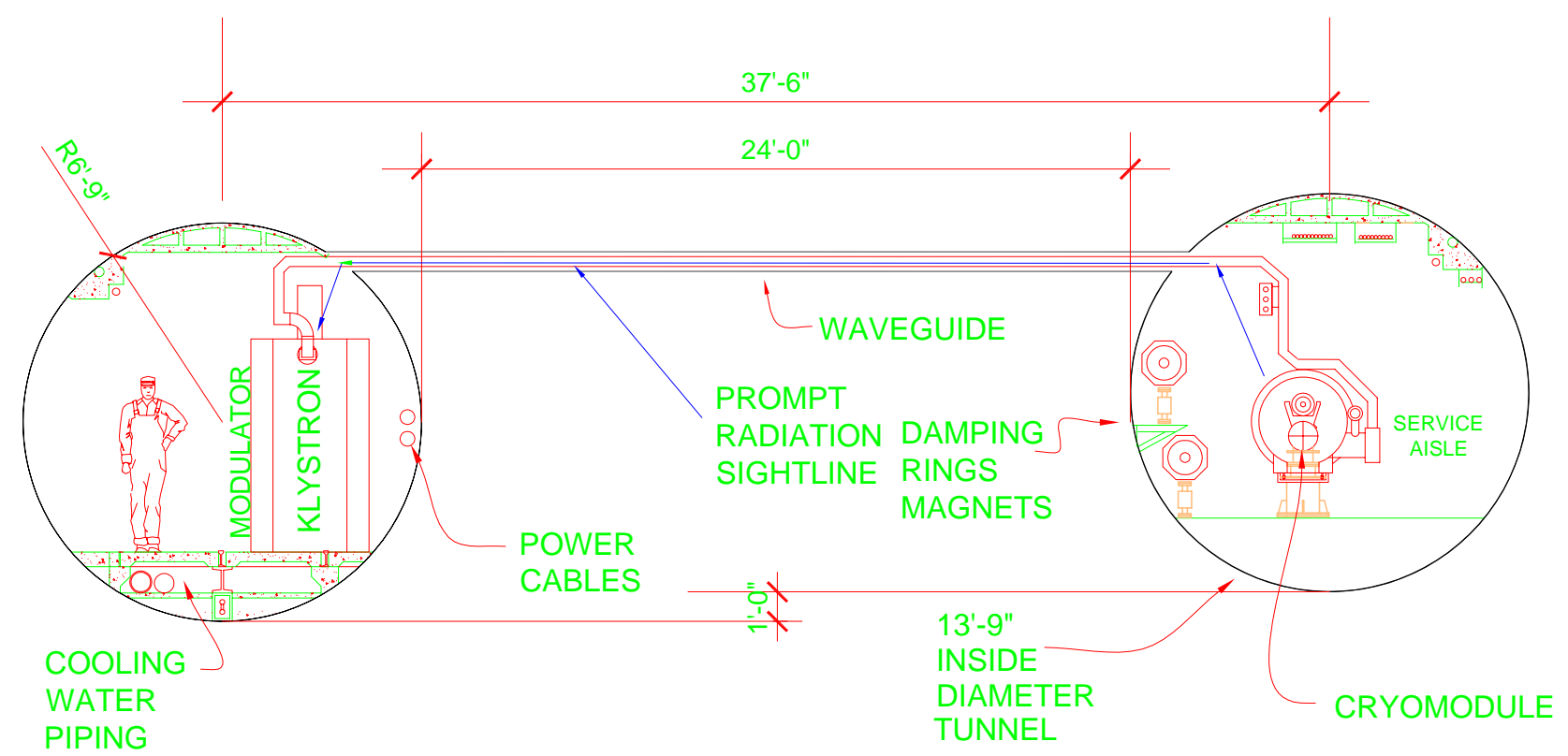

Figure 3.5.7.1: Representative two-tunnel cross section 
3.5.7.1.3 Surface buildings There will be above grade service buildings located at each shaft, to house the service facilities for the accelerators and detectors. Details of the surface buildings are provided in Sections 6.3 .1 and 6.3 .2 on civil construction and siting.

\subsubsection{Radiation safety}

Radiation safety requirements are outlined in the TDR[TDR, Section 8.3]. The design of the shielding between the main linac tunnel and service tunnel will allow full-time occupation of the service tunnel during machine operation, for repair and maintenance of components.

\subsubsection{AC power requirements}

The overall AC power requirements are given in Sections 6.3.1 and 6.3.2 on civil construction and siting.

\subsubsection{Water and HVAC}

The heat rejection systems required for the machine are described in Sections 6.3.1 and 6.3.2 on civil construction and siting.

\subsubsection{Survey and alignment}

Survey and alignment techniques are covered in the TDR[TDR, Section 8.6].

\subsubsection{Cryogenics}

\subsection{Cryogenic heat loads and cryogenic plant requirements}

3.5.7.6.1.1 $500 \mathrm{GeV} \mathbf{~ c m}$ operation In Table 3.5.7.6.1.1, static and dynamic (RF on) cryogenic heat loads for a $500 \mathrm{GeV}$ cm collider are presented for each of the temperature levels. These heat loads have been taken from the requirements given in Section 3.5.5.3. In addition, heat loads required for the electron and positron injectors as well as the electron and positron damping ring RF systems are also given. These have been taken from Section 3.5.2 and Section 3.5.3.4.

For a $500 \mathrm{GeV}$ cm collider, three cryogenic plants are required for each linac. Each plant provides refrigeration to one or two cryounits of $\sim 2.6 \mathrm{~km}$ long. The requirements on the plants are shown in Table 3.5.7.6.1.1. Cryogenic plant 1 provides refrigeration for the electron injector linac, as well as linac cryounits 1 and 2. Plant 3 supports the cryogenic needs of the positron injector linac and the electron damping ring RF, as well as linac cryounits 3 and 4 . In addition, linac cryounit 3 of cryogenic plant 3 contains a 1,045 m long cryogenic bypass to make room for diagnostic region 3 and the undulator. This bypass will need to have a large size low pressure return line in order to not significantly contribute to the overall cryounit GRP pressure drop. This is necessary in order to maintain the similar GRP pressure drop across the $3.4 \mathrm{~km}$ cryounit 3 . Plants 5 and 12 supply linac cryounits 5 and 12, respectively. Plant 14 feeds the positron damping ring RF, as well as linac cryounits 13 and 14 . Plant 16 supplies linac cryounits 15 and 16.

3.5.7.6.1.2 $1 \mathrm{TeV}$ cm operation Table 3.5.7.6.1.2 and Table 3.5.7.6.1.2 presents heat load requirements for a $1 \mathrm{TeV}$ upgrade of the collider. It should be noted that, in this case, the $2 \mathrm{~K}$ dynamic heat load represents $94 \%$ of the total $2 \mathrm{~K}$ load. This presents a challenge to the control of the cryogenic system during RF turn 
Table 3.5.7.2: Cryounit Heat Loads for a $500 \mathrm{GeV}$ Collider

\begin{tabular}{|c|c|c|c|c|}
\hline Linac Segment & $\begin{array}{c}\text { Temperature } \\
{[\mathrm{K}]}\end{array}$ & $\begin{array}{c}\text { Static Load } \\
\mathrm{kW} \\
\end{array}$ & $\begin{array}{c}\text { Dynamic Load } \\
\mathrm{kW}\end{array}$ & $\begin{array}{c}\text { Total Load } \\
\mathrm{kW} \\
\end{array}$ \\
\hline \multirow{3}{*}{$\begin{array}{c}\text { Cryounit } 1 \\
158 \text { Cryomodules }\end{array}$} & 2 & 0.27 & 1.57 & 1.84 \\
\hline & $5-8$ & 1.79 & 0.83 & 2.62 \\
\hline & $40-80$ & 14.24 & 18.74 & 32.98 \\
\hline \multirow{3}{*}{$\begin{array}{l}\text { Electron Injector Linac } \\
23 \text { Cryomodules }\end{array}$} & 2 & 0.04 & 0.14 & 0.18 \\
\hline & $5-8$ & 0.26 & 0.10 & 0.36 \\
\hline & $40-80$ & 2.07 & 1.66 & 3.73 \\
\hline \multirow{3}{*}{$\begin{array}{c}\text { Cryounit } 2 \\
158 \text { Cryomodules }\end{array}$} & 2 & 0.27 & 1.57 & 1.84 \\
\hline & $5-8$ & 1.79 & 0.83 & 2.62 \\
\hline & $40-80$ & 14.24 & 18.74 & 32.98 \\
\hline \multirow{3}{*}{$\begin{array}{c}\text { Cryounit } 3 \\
141 \text { Cryomodules }\end{array}$} & 2 & 0.25 & 1.40 & 1.65 \\
\hline & $5-8$ & 1.60 & 0.74 & 2.33 \\
\hline & $40-80$ & 12.71 & 16.72 & 29.43 \\
\hline \multirow{3}{*}{$\begin{array}{l}\text { Electron Damping Ring } \\
12 \text { Cryomodules }\end{array}$} & 2 & 0.36 & 0.36 & 0.72 \\
\hline & $5-8$ & 0.13 & 0.13 & 0.25 \\
\hline & $40-80$ & 2.55 & 2.55 & 5.10 \\
\hline \multirow{3}{*}{$\begin{array}{c}\text { Cryogenic Bypass } \\
1045 \mathrm{~m}\end{array}$} & 2 & 0.21 & 0 & 0.209 \\
\hline & $5-8$ & 0.63 & 0 & 0.627 \\
\hline & $40-80$ & 4.18 & 0 & 4.18 \\
\hline \multirow{3}{*}{$\begin{array}{c}\text { Cryounit } 4 \\
143 \text { Cryomodules }\end{array}$} & 2 & 0.25 & 1.42 & 1.67 \\
\hline & $5-8$ & 1.62 & 0.75 & 2.37 \\
\hline & $40-80$ & 12.89 & 16.96 & 29.85 \\
\hline \multirow{3}{*}{$\begin{array}{l}\text { Positron Injector Linac } \\
20 \text { Cryomodules }\end{array}$} & 2 & 0.03 & 0.09 & 0.12 \\
\hline & $5-8$ & 0.23 & 0.07 & 0.30 \\
\hline & $40-80$ & 1.80 & 1.06 & 2.86 \\
\hline \multirow{3}{*}{$\begin{array}{c}\text { Cryounit } 5 \\
163 \text { Cryomodules }\end{array}$} & 2 & 0.28 & 1.62 & 1.90 \\
\hline & $5-8$ & 1.85 & 0.85 & 2.70 \\
\hline & $40-80$ & 14.69 & 19.33 & 34.02 \\
\hline \multirow{3}{*}{$\begin{array}{c}\text { Cryounit } 12 \\
149 \text { Cryomodules }\end{array}$} & 2 & 0.26 & 1.48 & 1.74 \\
\hline & $5-8$ & 1.69 & 0.78 & 2.47 \\
\hline & $40-80$ & 13.43 & 17.67 & 31.10 \\
\hline \multirow{3}{*}{$\begin{array}{c}\text { Cryounit } 13 \\
149 \text { Cryomodules }\end{array}$} & 2 & 0.26 & 1.48 & 1.74 \\
\hline & $5-8$ & 1.69 & 0.78 & 2.47 \\
\hline & $40-80$ & 13.43 & 17.67 & 31.10 \\
\hline \multirow{3}{*}{$\begin{array}{c}\text { Cryounit } 14 \\
149 \text { Cryomodules }\end{array}$} & 2 & 0.26 & 1.48 & 1.74 \\
\hline & $5-8$ & 1.69 & 0.78 & 2.47 \\
\hline & $40-80$ & 13.43 & 17.67 & 31.10 \\
\hline \multirow{3}{*}{$\begin{array}{c}\text { Positron Damping Ring } \\
12 \text { Cryomodules }\end{array}$} & 2 & 0.36 & 0.36 & 0.72 \\
\hline & $5-8$ & 0.13 & 0.13 & 0.25 \\
\hline & $40-80$ & 2.55 & 2.55 & 5.10 \\
\hline \multirow{3}{*}{$\begin{array}{c}\text { Cryounit } 15 \\
149 \text { Cryomodules }\end{array}$} & 2 & 0.26 & 1.48 & 1.74 \\
\hline & $5-8$ & 1.69 & 0.78 & 2.47 \\
\hline & $40-80$ & 13.43 & 17.67 & 31.10 \\
\hline \multirow{3}{*}{$\begin{array}{c}\text { Cryounit } 16 \\
149 \text { Cryomodules }\end{array}$} & 2 & 0.26 & 1.48 & 1.74 \\
\hline & $5-8$ & 1.69 & 0.78 & 2.47 \\
\hline & $40-80$ & 13.43 & 17.67 & 31.10 \\
\hline
\end{tabular}


Table 3.5.7.3: $500 \mathrm{GeV}$ operation cryogenic plant requirements

\begin{tabular}{|c|c|c|c|c|}
\hline Plant: & $\begin{array}{c}\text { Temperature } \\
\text { Load }\end{array}$ & $\begin{array}{c}\text { Static Load } \\
\mathrm{kW}\end{array}$ & $\begin{array}{c}\text { Dynamic Load } \\
\mathrm{kW}\end{array}$ & $\begin{array}{c}\text { Total Load } \\
\mathrm{kW}\end{array}$ \\
\hline \hline $1:$ & 2 & 0.59 & 3.27 & 3.86 \\
Linac Cryounits 1+2 & $5-8$ & 3.84 & 1.75 & 5.59 \\
+Elec. Inj. Linac & $40-80$ & 30.55 & 39.13 & 69.68 \\
\hline $3:$ & 2 & 1.10 & 3.27 & 4.37 \\
Linac Cryounits 3+4 & $5-8$ & 4.21 & 1.68 & 5.89 \\
+Pos. Inj. Linac+Electron DR & $40-80$ & 32.66 & 38.76 & 71.42 \\
\hline $5:$ & 2 & 0.28 & 1.62 & 1.90 \\
Cryounit 5 & $5-8$ & 1.85 & 0.85 & 2.70 \\
& $40-80$ & 14.69 & 19.33 & 34.02 \\
\hline $12:$ & 2 & 0.26 & 1.48 & 1.74 \\
Cryounit 12 & $5-8$ & 1.69 & 0.78 & 2.47 \\
& $40-80$ & 13.43 & 17.67 & 31.10 \\
\hline $14:$ & 2 & 0.88 & 3.32 & 4.20 \\
Cryounit $13+14$ & $5-8$ & 3.51 & 1.67 & 5.18 \\
+Positron DR & $40-80$ & 27.94 & 39.36 & 67.30 \\
\hline $16:$ & 2 & 0.52 & 2.96 & 3.48 \\
Cryounit $15+16$ & $5-8$ & 3.37 & 1.56 & 4.93 \\
& $40-80$ & 26.86 & 35.34 & 62.20 \\
\hline
\end{tabular}

on/off transitions. To minimize operational costs during extended RF off periods, installation of a small cryogenic plant should be considered, to satisfy the static heat load requirements of the load.

For a $1 \mathrm{TeV}$ cm collider, eight cryogenic plants are required for each linac. Each plant provides refrigeration to one cryounit of $\sim 2.6 \mathrm{~km}$ long, plus the auxiliary injector and damping ring systems noted above. The requirements on the plants are shown in Table 3.5.7.6.1.2.

3.5.7.6.2 Cryogenic plant design specifications The cryogenic plant requirements and design specifications for the $500 \mathrm{GeV}$ collider are given in Table 3.5.7.6.2. The requirements are taken from Table 3.5.7.6.1.1. The cryogenic loads at the three different temperatures have been converted into a net equivalent load at $4.5 \mathrm{~K}$.

To calculate the $4.5 \mathrm{~K}$ equivalent refrigeration, one first calculates the ideal power required to produce this refrigeration. For a non-isothermal load, this depends on helium properties and the load mass flow rate, and is given as:

$$
P_{\text {ideal }}=\dot{m}\left[T_{\text {ref }}\left(s_{\text {out }}-s_{\text {in }}\right)-\left(h_{\text {out }}-h_{\text {in }}\right)\right]
$$

One has to be careful with the $2 \mathrm{~K}$ load. It is not just a matter of converting the $2 \mathrm{~K}$ isothermal load to a $4.5 \mathrm{~K}$ equivalent using the ratio of coefficients of Carnot. For the $2 \mathrm{~K} \mathrm{load}$, there is an additional load imposed by the cold compressors in generating the usable $2 \mathrm{~K}$ load. This process is spelled out in LHC Project Report 391[60]. Process parameters must be chosen and the cycle calculated in order to determine this additional load. For a $2 \mathrm{~K}$ cold compressor cycle, an additional $40 \%$ to $50 \%$ of $4.5 \mathrm{~K}$ equivalent load is required to compensate cold compression. This particular number is a function of the cycle selected. The cycle for this reference design study requires an additional load of $5 \%$ compared to the TESLA cycle due to the deep tunnel installation.

For the process cycle used in this reference design (see below, Section 3.5.7.6.3), the equivalent 4.5 K power may be found using the relation

$$
P_{4.5}=f_{T} P_{T}
$$


Table 3.5.7.4: Cryounit Heat Loads for a $1 \mathrm{TeV}$ Collider, electron linac side

\begin{tabular}{|c|c|c|c|c|}
\hline Linac Segment & $\begin{array}{c}\text { Temperature } \\
{[\mathrm{K}]}\end{array}$ & $\begin{array}{c}\text { Static Load } \\
\mathrm{kW}\end{array}$ & $\begin{array}{c}\text { Dynamic Load } \\
\mathrm{kW}\end{array}$ & $\begin{array}{l}\text { Total Load } \\
\text { kW }\end{array}$ \\
\hline "Cryounit 1 & $\overline{22}$ & $\overline{0.27}$ & $\overline{4.49}$ & "4.76 \\
\hline \multirow{2}{*}{158 Cryomodules } & $5-8$ & 1.79 & 1.51 & 3.30 \\
\hline & $40-80$ & 14.24 & 52.83 & 67.07 \\
\hline \multirow{3}{*}{$\begin{array}{l}\text { Electron Injector Linac } \\
23 \text { Cryomodules }\end{array}$} & 2 & 0.04 & 0.14 & 0.18 \\
\hline & $5-8$ & 0.26 & 0.10 & 0.36 \\
\hline & $40-80$ & 2.07 & 1.66 & 3.73 \\
\hline \multirow{3}{*}{$\begin{array}{c}\text { Cryounit } 2 \\
158 \text { Cryomodules }\end{array}$} & 2 & 0.27 & 4.49 & 4.76 \\
\hline & $5-8$ & 1.79 & 1.51 & 3.30 \\
\hline & $40-80$ & 14.24 & 52.83 & 67.07 \\
\hline \multirow{3}{*}{$\begin{array}{c}\text { Cryounit } 3 \\
141 \text { Cryomodules }\end{array}$} & 2 & 0.25 & 4.01 & 4.25 \\
\hline & $5-8$ & 1.60 & 1.35 & 2.94 \\
\hline & $40-80$ & 12.71 & 47.14 & 59.85 \\
\hline \multirow{3}{*}{$\begin{array}{l}\text { Electron Damping Ring } \\
12 \text { Cryomodules }\end{array}$} & 2 & 0.36 & 0.36 & 0.72 \\
\hline & $5-8$ & 0.13 & 0.13 & 0.25 \\
\hline & $40-80$ & 2.55 & 2.55 & 5.10 \\
\hline \multirow{3}{*}{$\begin{array}{c}\text { Cryogenic Bypass } \\
1045 \mathrm{~m}\end{array}$} & 2 & 0.21 & 0 & 0.209 \\
\hline & $5-8$ & 0.63 & 0 & 0.627 \\
\hline & $40-80$ & 4.18 & 0 & 4.18 \\
\hline \multirow{3}{*}{$\begin{array}{c}\text { Cryounit } 4 \\
143 \text { Cryomodules }\end{array}$} & 2 & 0.25 & 4.06 & 4.31 \\
\hline & $5-8$ & 1.62 & 1.37 & 2.99 \\
\hline & $40-80$ & 12.89 & 47.81 & 60.70 \\
\hline \multirow{3}{*}{$\begin{array}{l}\text { Positron Injector Linac } \\
20 \text { Cryomodules }\end{array}$} & 2 & 0.03 & 0.09 & 0.12 \\
\hline & $5-8$ & 0.23 & 0.07 & 0.30 \\
\hline & $40-80$ & 1.80 & 1.06 & 2.86 \\
\hline \multirow{3}{*}{$\begin{array}{c}\text { Cryounit } 5 \\
163 \text { Cryomodules }\end{array}$} & 2 & 0.28 & 4.63 & 4.92 \\
\hline & $5-8$ & 1.85 & 1.56 & 3.40 \\
\hline & $40-80$ & 14.69 & 54.50 & 69.19 \\
\hline \multirow{3}{*}{$\begin{array}{c}\text { Cryounit } 6 \\
153 \text { Cryomodules }\end{array}$} & 2 & 0.27 & 4.35 & 4.61 \\
\hline & $5-8$ & 1.73 & 1.46 & 3.19 \\
\hline & $40-80$ & 13.79 & 51.15 & 64.94 \\
\hline \multirow{3}{*}{$\begin{array}{c}\text { Cryounit } 7 \\
151 \text { Cryomodules }\end{array}$} & 2 & 0.26 & 4.29 & 4.59 \\
\hline & $5-8$ & 1.71 & 1.44 & 3.15 \\
\hline & $40-80$ & 13.61 & 50.49 & 64.09 \\
\hline \multirow{3}{*}{$\begin{array}{c}\text { Cryounit } 8 \\
151 \text { Cryomodules }\end{array}$} & 2 & 0.26 & 4.29 & 4.55 \\
\hline & $5-8$ & 1.71 & 1.44 & 3.15 \\
\hline & $40-80$ & 13.61 & 50.49 & 64.09 \\
\hline
\end{tabular}

in which the conversion factors are $f_{2}=3.3, f_{5-8}=0.683$, and $f_{40-80}=0.07$. The design specification of 30 $\mathrm{kW} @ 4.5 \mathrm{~K}$ corresponds to a margin of about 30\% for the largest plant (plant 3). This provides a $50 \%$ overcapacity for static heat loads and a $25 \%$ overcapacity for dynamic heat loads at each temperature level. It allows operation at gradients up to $35 \mathrm{MV} / \mathrm{m}$, with reduced current and cycle rate, which allows an energy reach of up to about $625 \mathrm{GeV}$ at reduced luminosity. Plants 1, 14, and 16 are also designed for $30 \mathrm{~kW} @ 4.5$ $\mathrm{K}$, and will have a somewhat greater margin. Plants 5 and 12, which feed only a single linac cryounit, could be half-sized, but are taken to be $30 \mathrm{~kW} @ 4.5 \mathrm{~K}$ also as they will be required to be this size for the $1 \mathrm{TeV}$ upgrade.

The cryogenic plant requirements and design specifications for 1 Tev collider operation are given in Ta- 
Table 3.5.7.5: Cryounit Heat Loads for a $1 \mathrm{TeV}$ Collider, positron linac side

\begin{tabular}{|c|c|c|c|c|}
\hline Linac Segment & $\begin{array}{c}\text { Temperature } \\
{[\mathrm{K}]}\end{array}$ & $\begin{array}{c}\text { Static Load } \\
\mathrm{kW}\end{array}$ & $\begin{array}{c}\text { Dynamic Load } \\
\mathrm{kW}\end{array}$ & $\begin{array}{c}\text { Total Load } \\
\mathrm{kW}\end{array}$ \\
\hline \hline Cryounit 9 & 2 & 0.27 & 4.35 & 4.61 \\
153 Cryomodules & $5-8$ & 1.73 & 1.46 & 3.19 \\
& $40-80$ & 13.79 & 51.15 & 64.94 \\
\hline Cryounit 10 & 2 & 0.27 & 4.35 & 4.61 \\
153 Cryomodules & $5-8$ & 1.73 & 1.46 & 3.19 \\
& $40-80$ & 13.79 & 51.15 & 64.94 \\
\hline Cryounit 11 & 2 & 0.27 & 4.35 & 4.61 \\
153 Cryomodules & $5-8$ & 1.73 & 1.46 & 3.19 \\
& $40-80$ & 13.79 & 51.15 & 64.94 \\
\hline Cryounit 12 & 2 & 0.26 & 4.23 & 4.49 \\
149 Cryomodules & $5-8$ & 1.69 & 1.42 & 3.11 \\
& $40-80$ & 13.43 & 49.82 & 63.25 \\
\hline Cryounit 13 & 2 & 0.26 & 4.23 & 4.49 \\
149 Cryomodules & $5-8$ & 1.69 & 1.42 & 3.11 \\
& $40-80$ & 13.43 & 49.82 & 63.25 \\
\hline Cryounit 14 & 2 & 0.26 & 4.23 & 4.49 \\
149 Cryomodules & $5-8$ & 1.69 & 1.42 & 3.11 \\
& $40-80$ & 13.43 & 49.82 & 63.25 \\
\hline Cryounit 15 & 2 & 0.26 & 4.23 & 4.49 \\
149 Cryomodules & $5-8$ & 1.69 & 1.42 & 3.11 \\
& $40-80$ & 13.43 & 49.82 & 63.25 \\
\hline Cryounit 16 & 2 & 0.26 & 4.23 & 4.49 \\
149 Cryomodules & $5-8$ & 1.69 & 1.42 & 3.11 \\
& $40-80$ & 13.43 & 49.82 & 63.25 \\
\hline Positron Damping Ring & 2 & 0.36 & 0.36 & 0.72 \\
12 Cryomodules & $5-8$ & 0.13 & 0.13 & 0.25 \\
& $40-80$ & 2.55 & 2.55 & 5.10 \\
\hline
\end{tabular}

ble 3.5.7.6.2. The requirements are taken from Table 3.5.7.6.1.2. The plant design specifications have all been taken to be the same as that needed for $500 \mathrm{GeV}$ operation, which provides roughly a $30 \%$ or greater margin in all cases.

The AC power requirements for the plants, when operating at nominal load, as well as the installed power, are also given in Tables 3.5.7.6.2 and 3.5.7.6.2. The power requirements presented represent the purchased power, including motor efficiency, for the motors associated with the compressors, pumps and fans required for the cryogenic plants. To compute the AC power requirements, the following COP's have been used (from the TDR[Table 8.7.5][TDR]): for $2 \mathrm{~K} 588 \mathrm{~W} / \mathrm{W}$; for 5-8 K, $168 \mathrm{~W} / \mathrm{W}$; and for 40-80 K, $17 \mathrm{~W} / \mathrm{W}$. The cold compressor heat loads have also been included.

For $500 \mathrm{GeV}$ c.m. operation, the cryogenic AC power required is $23.2 \mathrm{MW}$, and for $1 \mathrm{TeV}$ c.m. operation, it is $75.7 \mathrm{MW}$. 
Table 3.5.7.6: $1 \mathrm{TeV}$ operation cryogenic plant requirements

\begin{tabular}{|c|c|c|c|c|}
\hline $\begin{array}{c}\text { Plants: } \\
\text { Load }\end{array}$ & $\begin{array}{c}\text { Temperature } \\
{[\mathrm{K}]}\end{array}$ & $\begin{array}{c}\text { Static Load } \\
\mathrm{kW}\end{array}$ & $\begin{array}{c}\text { Dynamic Load } \\
\mathrm{kW}\end{array}$ & $\begin{array}{c}\text { Total Load } \\
\text { kW }\end{array}$ \\
\hline 1: & 2 & 0.31 & 4.63 & 4.94 \\
\hline Linac Cryounit 1 & $5-8$ & 2.05 & 1.61 & 3.66 \\
\hline +Elec. Inj. Linac & $40-80$ & 16.31 & 54.48 & 70.79 \\
\hline $2:$ & 2 & 0.27 & 4.49 & 4.76 \\
\hline Linac Cryounit 2 & $5-8$ & 1.79 & 1.51 & 3.30 \\
\hline & $40-80$ & 14.24 & 52.83 & 67.07 \\
\hline 3: & 2 & 0.85 & 4.45 & 5.30 \\
\hline Linac Cryounit 3 & $5-8$ & 2.59 & 1.54 & 4.13 \\
\hline +Pos. Inj. Linac+Electron DR & $40-80$ & 19.77 & 52.22 & 71.99 \\
\hline $4:$ & 2 & 0.25 & 4.06 & 4.31 \\
\hline Linac Cryounit 4 & $5-8$ & 1.62 & 1.37 & 2.99 \\
\hline & $40-80$ & 12.89 & 47.81 & 60.70 \\
\hline 5: & 2 & 0.28 & 4.63 & 4.92 \\
\hline Linac Cryounit 5 & $5-8$ & 1.85 & 1.56 & 3.40 \\
\hline & $40-80$ & 14.69 & 54.50 & 69.19 \\
\hline 6: & 2 & 0.27 & 4.35 & 4.61 \\
\hline Linac Cryounit 6 & $5-8$ & 1.73 & 1.46 & 3.19 \\
\hline & $40-80$ & 13.79 & 51.15 & 64.94 \\
\hline $7:$ & 2 & 0.26 & 4.29 & 4.55 \\
\hline Linac Cryounit 7 & $5-8$ & 1.71 & 1.44 & 3.15 \\
\hline & $40-80$ & 13.61 & 50.49 & 64.09 \\
\hline 8: & 2 & 0.26 & 4.29 & 4.61 \\
\hline Linac Cryounit 8 & $5-8$ & 1.71 & 1.44 & 3.19 \\
\hline & $40-80$ & 13.61 & 50.49 & 64.94 \\
\hline 9: & 2 & 0.27 & 4.35 & 4.61 \\
\hline Linac Cryounit 9 & $5-8$ & 1.73 & 1.46 & 3.19 \\
\hline & $40-80$ & 13.79 & 51.15 & 64.94 \\
\hline 10: & 2 & 0.27 & 4.35 & 4.61 \\
\hline Linac Cryounit 10 & $5-8$ & 1.73 & 1.46 & 3.19 \\
\hline & $40-80$ & 13.79 & 51.15 & 64.94 \\
\hline 11: & 2 & 0.27 & 4.35 & 4.61 \\
\hline Linac Cryounit 11 & $5-8$ & 1.73 & 1.46 & 3.19 \\
\hline & $40-80$ & 13.79 & 51.15 & 64.94 \\
\hline 12: & 2 & 0.26 & 4.23 & 4.49 \\
\hline Linac Cryounit 12 & $5-8$ & 1.69 & 1.42 & 3.11 \\
\hline & $40-80$ & 13.43 & 49.82 & 63.25 \\
\hline 13: & 2 & 0.26 & 4.23 & 4.49 \\
\hline Linac Cryounit 13 & $5-8$ & 1.69 & 1.42 & 3.11 \\
\hline & $40-80$ & 13.43 & 49.82 & 63.25 \\
\hline 14: & 2 & 0.62 & 4.59 & 5.21 \\
\hline Linac Cryounit 14 & $5-8$ & 1.83 & 1.53 & 3.36 \\
\hline +Positron DR & $40-80$ & 14.51 & 53.84 & 68.35 \\
\hline 15: & 2 & 0.26 & 4.23 & 4.49 \\
\hline Linac Cryounit 15 & $5-8$ & 1.69 & 1.42 & 3.11 \\
\hline & $40-80$ & 13.43 & 49.82 & 63.25 \\
\hline 16: & 2 & 0.26 & 4.23 & 4.49 \\
\hline Linac Cryounit 16 & $5-8$ & 1.69 & 1.42 & 3.11 \\
\hline & $40-80$ & 13.43 & 49.82 & 63.25 \\
\hline
\end{tabular}


Table 3.5.7.7: Cryogenic Plant Nominal and Design, $500 \mathrm{GeV}$

\begin{tabular}{|c|c|c|c|c|c|c|}
\hline $\begin{array}{c}\text { Hall } \\
\#\end{array}$ & $\begin{array}{c}\text { Plant } \\
\#\end{array}$ & $\begin{array}{c}\text { Nominal } \\
{[\mathrm{kW}] @ 4.5 \mathrm{~K}}\end{array}$ & $\begin{array}{c}\text { Design } \\
{[\mathrm{kW}] \text { @ 4.5 K }}\end{array}$ & $\begin{array}{c}\text { Margin } \\
{[\%]}\end{array}$ & $\begin{array}{c}\text { AC Power at Nominal } \\
{[\mathrm{MW}]}\end{array}$ & $\begin{array}{c}\text { Installed AC power } \\
{[\mathrm{MW}]}\end{array}$ \\
\hline \hline 1 & 1 & 21.5 & 30.3 & 41 & 4.70 & 6.63 \\
2 & 3 & 23.4 & 30.3 & 29 & 5.10 & 6.63 \\
3 & 5 & 10.5 & 30.3 & 189 & 2.30 & 6.63 \\
6 & 12 & 9.6 & 30.3 & 216 & 2.10 & 6.63 \\
7 & 14 & 22.1 & 30.3 & 37 & 4.80 & 6.63 \\
8 & 16 & 19.2 & 30.3 & 58 & 4.21 & 6.63 \\
\hline Total & & 106.3 & 182 & 71 & 23.2 & 39.76 \\
\hline
\end{tabular}

Table 3.5.7.8: Cryogenic Plant Nominal and Design, $1 \mathrm{TeV}$

\begin{tabular}{|c|c|c|c|c|c|c|}
\hline $\begin{array}{c}\text { Hall } \\
\#\end{array}$ & $\begin{array}{c}\text { Plant } \\
\#\end{array}$ & $\begin{array}{c}\text { Nominal } \\
{[\mathrm{kW}] @ \text { @ 4.5 K }}\end{array}$ & $\begin{array}{c}\text { Design } \\
{[\mathrm{kW}] @ \text { @ 4.5 K }}\end{array}$ & $\begin{array}{c}\text { Margin } \\
{[\%]}\end{array}$ & $\begin{array}{c}\text { AC Power at Nominal } \\
{[\mathrm{MW}]}\end{array}$ & $\begin{array}{c}\text { Installed AC power } \\
{[\mathrm{MW}]}\end{array}$ \\
\hline \hline 1 & $1+2$ & 46.4 & 59.7 & 29 & 9.86 & 12.8 \\
2 & $3+4$ & 45.9 & 59.7 & 30 & 9.74 & 12.8 \\
3 & $5+6$ & 45.3 & 59.7 & 32 & 9.62 & 12.8 \\
4 & $7+8$ & 43.6 & 59.7 & 37 & 9.26 & 12.8 \\
5 & $9+10$ & 43.9 & 59.7 & 36 & 9.32 & 12.8 \\
6 & $11+12$ & 43.3 & 59.7 & 38 & 9.20 & 12.8 \\
7 & $13+14$ & 45.7 & 59.7 & 31 & 9.66 & 12.8 \\
8 & $15+16$ & 42.8 & 59.7 & 40 & 9.07 & 102.7 \\
\hline Total & & 356.9 & 478 & 34 & 75.7 & \\
\hline
\end{tabular}


3.5.7.6.3 Cryogenic plant optimization Various ways to optimize the cryogenic configuration for the this reference design study have been considered. The parameters to optimize and the imposed constraints are given as:

- Parameters to optimize:

1. Balance the heat loads between refrigerators in order to utilize a single cryogenic plant design.

2. Minimize the number of refrigerator locations in order to lower civil construction costs (surface infrastructure, vertical shafts, caverns, etc.).

3. Optimize capital and operating costs by splitting the cryogenic plant into above ground and tunnel level sections in order to compensate for helium head effects.

- Technical Constraints:

A At the heat loads of the TESLA $800^{3}$, the size of the $300 \mathrm{~mm}$ OD gas return pipe (GRP) limits the cryounit length to about $2.5 \mathrm{~km}$ at a mass flow rate of $256 \mathrm{~g} / \mathrm{s}$.

- Non-Technical Constraints:

A Cavern construction cost uncertainty has been realized in the LHC project through cost overruns.

B LHC studies with cryogenic industry has shown that a $24 \mathrm{~kW}$ (4.5K equivalent) sized plant is the largest for which the LHC Project Report 317[58] design methodology is applicable.

Technical constraint A was investigated by DESY for TESLA 800. Their conclusion that the pressure drop in the GRP limits the cryounit length to about $2.5 \mathrm{~km}$ also holds for this reference design study. Due to manpower constraints, we do not intend to investigate or duplicate this work. Simplified calculations have been done to investigate segmentation of the cryogenic strings for this reference design study.

According to TESLA Report 2001-37[59] for TESLA $500^{4}$, a $2.5 \mathrm{~km}$ cryounit has a mass flow rate of $68 \mathrm{~g} / \mathrm{s}$ and a resulting pressure drop in the $300 \mathrm{~mm}$ GRP of $0.33 \mathrm{mbar}$. For TESLA 800, it has been stated that the cryogenic capacity will need to be doubled[TDR]. It is further assumed that $30 \%$ of the cryogenic plant overcapacity would be used to supply the load. These assumptions lead to a TESLA 800 Cryounit mass flow a factor of 2.6 higher than TESLA 500 (2 x 1.3), corresponding to a mass flow rate of $177 \mathrm{~g} / \mathrm{s}$.

For this study, two flow rate conditions are considered: nominal and design. The nominal flow rate is based on the expected heat load and includes no contingency. The design flow rate is based on the maximum plant flow rate considering overcapacity to cover uncertainties. Based on information from DESY, it is assumed that a mass flow rate of $265 \mathrm{~g} / \mathrm{s}$ is the limit for the GRP. The nominal flow rate of $241 \mathrm{~g} / \mathrm{s}$ for the $1 \mathrm{TeV}$ option is within the GRP limit. However, utilizing the cryogenic plant at its design point, corresponding to an overhead of approximately $30 \%$, would result in a design flow rate of $304 \mathrm{~g} / \mathrm{s}$, which exceeds the GRP limit. Thus, operation at $1 \mathrm{TeV}$ which utilizes the reserve capacity has the potential of either operating the cryomodules at warmer temperatures, or running at the limit of the cryogenic pumping, which will affect operating cost.

Figure 3.5.7.2 shows the temperature profiles across the longest cryounit for this study and for TESLA at the design flow rates. At the nominal flow rate for $1 \mathrm{TeV}$, the pressure drop is approximately 4.0 mbar across the longest cryounit. This equates to a temperature gradient along the Cryounit of $46 \mathrm{mK}$. For the design flow rates of $138 \mathrm{~g} / \mathrm{s}$ for $500 \mathrm{GeV}$ or $304 \mathrm{~g} / \mathrm{s}$ for $1 \mathrm{TeV}$ in this study, the resulting cryounit pressure drops are 1.31 mbar and 6.4 mbar respectively, which equate to temperature gradients of $14 \mathrm{mK}$ and $75 \mathrm{mK}$, respectively. As shown in Figure 3.5.7.2, the maximum temperature difference between TESLA 800 and this study is $53 \mathrm{mK}$. For this study, it is planned to operate the cryogenic plant $53 \mathrm{mK}$ colder. This results in a $1 \mathrm{TeV}$ suction pressure at the distribution box of 2,489 Pa, versus 3,097 $\mathrm{Pa}$ for TESLA 500, or 2,930 Pa for

\footnotetext{
${ }^{3}$ This refers to the $800 \mathrm{GeV}$ (c.m.) upgrade described in the TESLA TDR.

${ }^{4}$ This refers to the baseline TESLA TDR.
} 
TESLA 800. For $500 \mathrm{GeV}$ c.m. operation in this reference design, the suction pressure at the distribution box will be $2,998 \mathrm{~Pa}$.

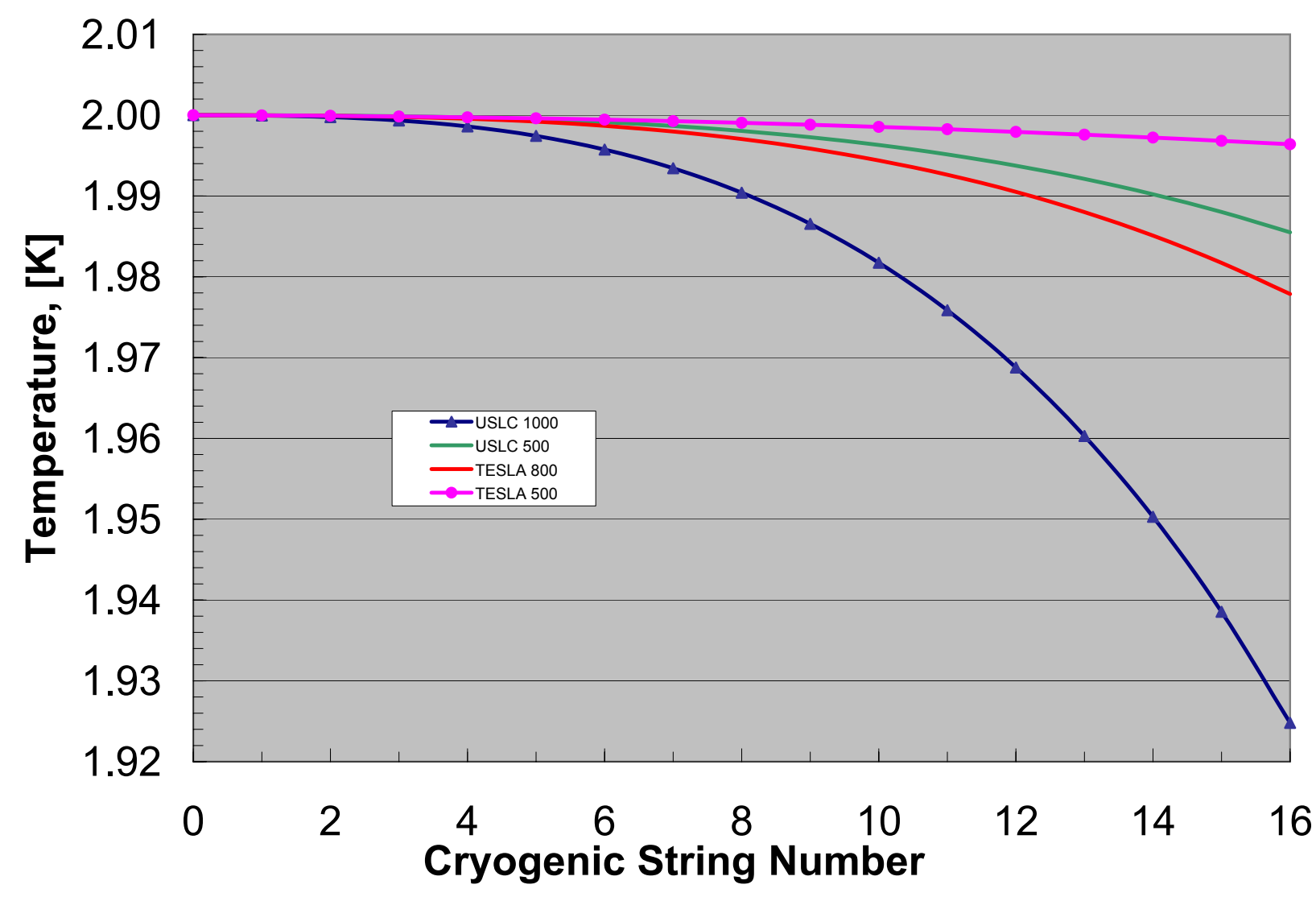

Figure 3.5.7.2: Cryounit gas return pipe temperature profile

In addition there is a pressure drop associated with the deep site installation. As opposed to the TESLA proposed depth, the nominal depth of 100 meters for this reference design study imposes additional energy penalties to the cryogenic cycle. The penalties are an increased enthalpy due the hydrostatic head compression of the subcooled supply helium, and additional heat load and hydrostatic pressure drop of the vertical gas return pipe.

Splitting the cold box at about the $20 \mathrm{~K}$ temperature level can minimize the penalties. This would require having above ground and tunnel level cold boxes. The tunnel level would house the cold compressors, turbine expanders, heat exchangers and associated systems below $20 \mathrm{~K}$.

In order to understand the magnitude of the penalties, a model of the cryogenic system was developed. A simplified schematic of the model is shown in Figure 3.5.7.3. Two options were analyzed and are presented with the change in elevation located at A-A and B-B in Figure 3.5.7.3. With the change of elevation located at A-A, all of the cryogenic plant will be located above ground and is connected to the load low pressure heat exchanger via a large vertical transfer line. This transfer line contains a 16" low pressure gas return pipe, subcooled $4.5 \mathrm{~K}$ supply, 5-8 K shield supply and return lines and 40-80 K shield supply and return lines. 


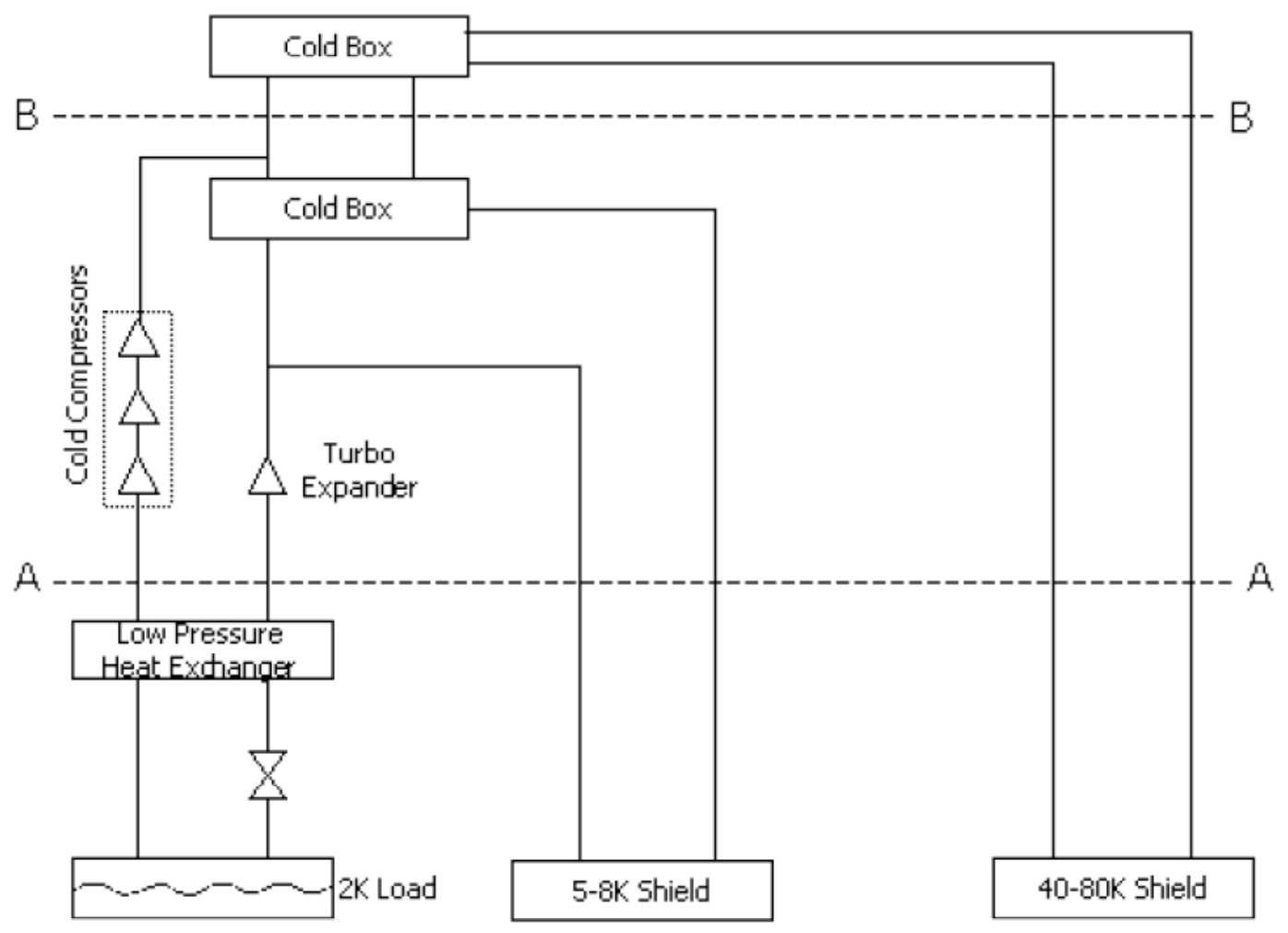

Figure 3.5.7.3: Simplified cryogenic plant schematic

With the change in elevation located at B-B, all cold compressors, turbine expanders and heat exchangers below about $20 \mathrm{~K}$ would be located at the tunnel level. A simpler vertical transfer line would be required to connect to the above ground cold box. It would consist of a $20 \mathrm{~K}$ supply and return and a $40-80 \mathrm{~K}$ shield supply and return. The tunnel level cryogenic equipment would require a significant cavern located in proximity to the accelerator. In addition, personnel will need to have the ability to access the area during machine operation.

The model assumed the supply to the low pressure heat exchanger was at $4.5 \mathrm{~K}$ and 1.5 bar. Three stages of cold compression were considered with an overall pressure ratio of $\sim 27$, relative to the GRP pressure in the tunnel distribution box, and a fixed cold compressor train outlet pressure of $687 \mathrm{mbar}$. The isentropic efficiency of the cold compressors was assumed to be $75 \%$.

The efficiency penalty of the model is considered to be a minimum since second order effects such as the heat leak of the vertical transfer line and changes in cold compressor power were ignored. The model predicts a minimum of $3.6 \%$ operating efficiency penalty for a totally above ground cryogenic plant due to a $100 \mathrm{~m}$ elevation change.

In order to proceed with a conceptual design and cost for this reference design study, it was decided to consider a totally above ground cryogenic plant, with a modest tunnel level cavern of $30^{\prime}$ x $35^{\prime}$ x $40^{\prime}$ for the cryogenic distribution and associated equipment. Longer term, a detailed analysis is warranted to best 
compare the capital costs of larger tunnel caverns associated with splitting the cold box versus the associated operating cost savings. The accuracy to which the cavern constructions costs can be estimated poses the greatest uncertainty in developing the detailed analysis. Experience at CERN in the LHC project has shown that actual cavern construction costs were considerably higher than estimated.

The cryogenic layout of the linear collider is shown in Figure 3.5.7.4. The cryogenic layout was devised to distribute total loads as evenly as possible between plants, to minimize the number of penetrations by establishing the equivalent cryounit lengths near the maximum of $2.5 \mathrm{~km}$, and to have the $250 \mathrm{GeV}$ point between plants.

\section{GeV@28 MV/m USLC Cryogenic Configuration}

August 29, 2003
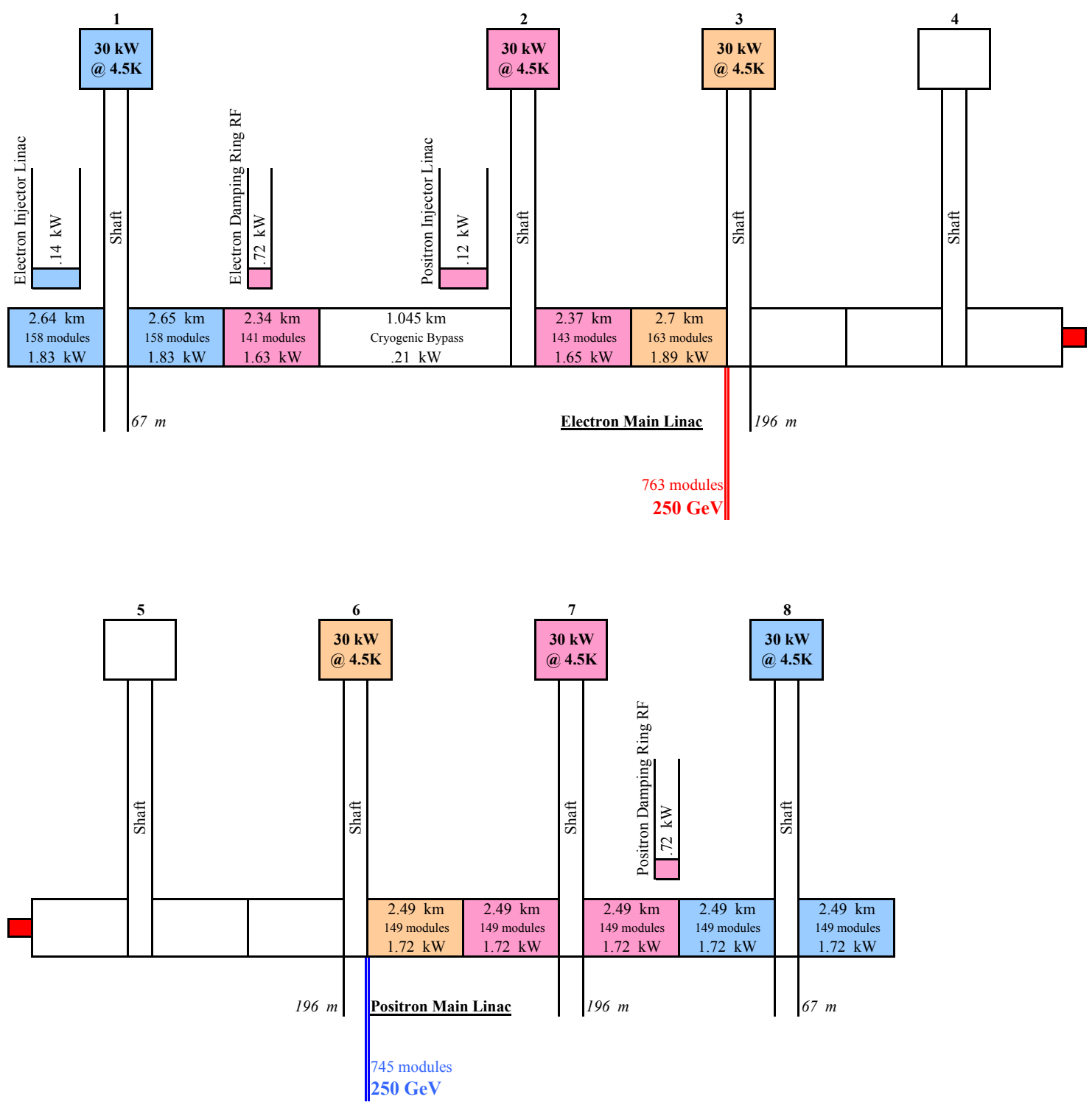

Figure 3.5.7.4: Cryogenics layout 
The required cryogenic heat load for a $500 \mathrm{GeV}$ collider is presented in Table 3.5.7.6.1.1. Table 3.5.7.6.1.2 presents the heat load requirements for a $1000 \mathrm{GeV}$ upgrade of the $500 \mathrm{GeV}$ collider. It should be noted that the $2 \mathrm{~K}$ dynamic heat load represents $94 \%$ of the total $2 \mathrm{~K}$ load in the $1000 \mathrm{GeV}$ design. This presents a challenge to the control of the cryogenic system during RF turn on/off transitions. To minimize operational costs during extended RF off periods, it should be considered to install a small cryogenic plant to satisfy the static heat load requirements of the load.

Capacity and power requirements for the cryogenic locations are shown in Table 3.5.7.6.2 for a $500 \mathrm{GeV}$ collider, and in Table 3.5.7.6.2 for a $1 \mathrm{TeV}$ collider. The variation in required capacity is small enough to warrant procuring the same sized plants for each location. The largest refrigeration requirements are at plant 3 in hall 2, which has the additional cryogenic loads associated with the positron injector linac and electron damping ring. The model plant for $500 \mathrm{GeV}$ operation $(30 \mathrm{~kW}$ of $4.5 \mathrm{~K}$ equivalent refrigeration, requiring a minimum of $7 \mathrm{MW}$ installed wall power) is based on the maximum load requirements of plant 3 and the pumping requirement of the longest cryounit (5). It should be noted that the refrigeration requirement at a single location is $25 \%$ larger than any helium refrigeration plant built to date. As a result, further studies are warranted to determine the optimum split of the plant into two systems, considering various operating modes. For the purpose of this study, we will consider halls 1-3 and 6-8 to have identical $30 \mathrm{~kW}$ at $4.5 \mathrm{~K}$ systems. For a $500 \mathrm{GeV}$ collider, only one of the plants would be required at hall 3 and hall 6 .

The upgrade to $1000 \mathrm{GeV}$ would require an incremental plant at each hall. The incremental plant has a considerably smaller $5-8 \mathrm{~K}$ shield capacity. This is dictated by the $5-8 \mathrm{~K}$ load being dominated by static heat load, resulting in the total load not scaling at the same rate with RF gradient as the other loads. This forces the requirement of having two different plants for a $1000 \mathrm{GeV}$ upgrade from a $500 \mathrm{GeV}$ initial configuration. As shown in Table 3.5.7.6.3, the $1000 \mathrm{GeV}$ upgrade plant has a considerably smaller 5-8 K shield capacity.

The model plant has a cryogenic cycle with a capability of $2 \mathrm{~K}$ capacity turndown to about a factor of two (2) with a minimum sacrifice in efficiency. Turndown is required for two reasons: the actual operating load will be below the design operating load and operation at lower gradients or repetition rates will require reduction in cryogenic plant's capacity. This is accomplished using a combination of cold compressors and warm vacuum pumps. Manipulating the pressure ratio between the cold compressor train and warm vacuum pumps varies the $2 \mathrm{~K}$ capacity.

A comparison of the TESLA 500, TESLA 800 and the reference design cryogenic plant cycles is given in Table 3.5.7.6.3. The proposed cryogenic cycle of the reference design is based on the TESLA Design Report[TDR]. The table shows the major design parameters for the $2 \mathrm{~K}$ primary load, $5-8 \mathrm{~K}$ shield load and the $40-80 \mathrm{~K}$ shield load. The load temperature at the distribution box has been adjusted to accommodated a common maximum cryomodule temperature for all the collider configurations. The maximum cryomodule temperature is assumed to be $2.00 \mathrm{~K}$.

It should be noted that nominal mass flow rate corresponds to the load capacity, where design flow rate corresponds to the design capacity. TESLA 500 cryogenic parameters are the baseline for the TESLA 800 and for the cryogenic systems in this reference design study. For the TESLA 800 upgrade the $2 \mathrm{~K}$ cryogenic plant design mass flow rate equals twice the design mass flow rate of the TESLA 500 plant. The nominal 2 K flow rate more than doubles, because of a lower overcapacity margin utilized for the TESLA 800 upgrade.

\subsubsection{Accelerator module test facility}

A test facility for the accelerator modules will be required, as described in the TDR[TDR, Section 8.8].

\subsubsection{Global control system}

The global control system for the accelerator complex is described in the TDR[TDR, Section 8.9]. 
Table 3.5.7.9: Cryogenic cycle comparisons. The $500 \mathrm{GeV}$ reference design plant corresponds to plants 1,3,5,12,14,16 in Table 3.5.7.6.2. The $1 \mathrm{TeV}$ reference design plant corresponds to the plants added for the $1 \mathrm{TeV}$ upgrade, which are plants 2,4,6,7,8,9,10,11,13, and 15, in Table 3.5.7.6.2.

\begin{tabular}{|c|c|c|c|c|c|}
\hline Parameter & Unit & TESLA 500 & TESLA 800 & $\begin{array}{l}\text { Reference } \\
\text { design } \\
1 \text { TeV c.m. }\end{array}$ & $\begin{array}{c}\text { Reference } \\
\text { design } \\
500 \mathrm{GeV} \text { c.m. }\end{array}$ \\
\hline \multicolumn{6}{|l|}{$2 \mathrm{~K}$} \\
\hline Elevation change & [m] & 10 & 10 & 100 & 100 \\
\hline Supply Temperature & {$[\mathrm{K}]$} & 4.50 & 4.50 & 4.50 & 4.50 \\
\hline Supply Pressure & {$[\mathrm{Pa}]$} & 150,000 & 150,000 & 150,000 & 150,000 \\
\hline Supply Pressure Head & {$[\mathrm{Pa}]$} & 11,826 & 11,826 & 118,260 & 118,260 \\
\hline Vapor Quality & {$[-]$} & 0.122 & 0.120 & 0.143 & 0.150 \\
\hline Load Pressure Drop & {$[\mathrm{Pa}]$} & 33.0 & 221.0 & 658.8 & 135.1 \\
\hline Nominal Mass Flow & {$[\mathrm{kg} / \mathrm{sec}]$} & 0.136 & 0.352 & 0.263 & 0.218 \\
\hline Design Mass Flow & {$[\mathrm{kg} / \mathrm{sec}]$} & 0.204 & 0.408 & 0.333 & 0.275 \\
\hline Load Capacity & {$[\mathrm{W}]$} & 2,794 & 7,248 & 5,271 & 4,329 \\
\hline Design Capacity & {$[\mathrm{W}]$} & 4,190 & 8,401 & 6,666 & 5,474 \\
\hline Return Pressure Head & {$[\mathrm{Pa}]$} & 44.8 & 42.1 & 355.5 & 428.1 \\
\hline CC Suction Temperature & {$[\mathrm{K}]$} & 3.288 & 3.278 & 3.123 & 3.150 \\
\hline CC Suction Pressure & {$[\mathrm{Pa}]$} & 3,051 & 2,866 & 2,115 & 2,566 \\
\hline CC Train Discharge Temperature & {$[\mathrm{K}]$} & 20.689 & 21.124 & 22.762 & 21.332 \\
\hline CC Train Discharge Pressure & {$[\mathrm{Pa}]$} & 68,694 & 68,694 & 68,694 & 68,694 \\
\hline Ideal Power Load & {$[\mathrm{kW}]$} & 873 & 1,759 & 1,470 & 1,190 \\
\hline Ideal CC & {$[\mathrm{kW}]$} & 88 & 179 & 155 & 124 \\
\hline $4.5 \mathrm{~K}$ equivalent load & {$[\mathrm{kW}]$} & 13.3 & 26.8 & 22.4 & 18.1 \\
\hline $4.5 \mathrm{~K}$ equivalent $\mathrm{CC}$ & {$[\mathrm{kW}]$} & 1.3 & 2.7 & 2.4 & 1.9 \\
\hline CC pressure ratio & {$[\mathrm{Pa} / \mathrm{Pa}]$} & 2.80 & 2.80 & 2.80 & 2.80 \\
\hline Number of CC & & 3 & 3 & 3 & 3 \\
\hline $\mathrm{CC} \eta$ & {$[\%]$} & 75 & 75 & 75 & 75 \\
\hline System Pressure Ratio & {$[\mathrm{Pa} / \mathrm{Pa}]$} & 22.51 & 23.97 & 32.48 & 26.77 \\
\hline $\mathrm{r}$ & {$[\mathrm{kW} / \mathrm{kW}]$} & 3.17 & 3.19 & 3.36 & 3.31 \\
\hline $\mathrm{r}^{*} \mathrm{Q}$ & {$[\mathrm{kW}]$} & 13.3 & 26.8 & 22.4 & 18.1 \\
\hline \multicolumn{6}{|l|}{$5-8 \mathrm{~K}$} \\
\hline Temperature in & {$[\mathrm{K}]$} & 5.16 & 5.16 & 5.16 & 5.16 \\
\hline Temperature out & {$[\mathrm{K}]$} & 8.2 & 8.2 & 8.2 & 8.2 \\
\hline Pressure in & {$[\mathrm{Pa}]$} & 550,000 & 550,000 & 550,000 & 550,000 \\
\hline Pressure out & {$[\mathrm{Pa}]$} & 500,000 & 500,000 & 500,000 & 500,000 \\
\hline Design Mass Flow & {$[\mathrm{kg} / \mathrm{sec}]$} & 0.250 & 0.500 & 0.056 & 0.273 \\
\hline Load Capacity & {$[\mathrm{W}]$} & 4,977 & 12,882 & 1,209 & 5,889 \\
\hline Design Capacity & {$[\mathrm{W}]$} & 7,466 & 14,932 & 1,675 & 8,162 \\
\hline $4.5 \mathrm{~K}$ equivalent load & {$[\mathrm{kW}]$} & 5.10 & 10.20 & 1.14 & 5.58 \\
\hline \multicolumn{6}{|l|}{$40-80 \mathrm{~K}$} \\
\hline Temperature in & {$[\mathrm{K}]$} & 40 & 40 & 40 & 40 \\
\hline Temperature out & {$[\mathrm{K}]$} & 80 & 80 & 80 & 80 \\
\hline Pressure in & {$[\mathrm{Pa}]$} & $1,600,000$ & $1,600,000$ & $1,600,000$ & $1,600,000$ \\
\hline Pressure out & {$[\mathrm{Pa}]$} & $1,400,000$ & $1,400,000$ & $1,400,000$ & $1,400,000$ \\
\hline Design Mass Flow & {$[\mathrm{kg} / \mathrm{sec}]$} & 0.383 & 0.767 & 0.402 & 0.442 \\
\hline Load Capacity & {$[\mathrm{W}]$} & 53,851 & 139,379 & 65,008 & 71,475 \\
\hline Design Capacity & {$[\mathrm{W}]$} & 80,777 & 161,553 & 84,746 & 93,178 \\
\hline $4.5 \mathrm{~K}$ equivalent load & {$[\mathrm{kW}]$} & 5.68 & 11.36 & 5.96 & 6.55 \\
\hline
\end{tabular}




\section{Chapter 4}

\section{Availability Design}

\subsection{Introduction}

As part of the Technology Comparison exercise, an availability task force was formed to assess the impact of various technology or configuration choices on overall machine performance. The approach taken was to set an overall availability goal for any linear collider and then use that to develop an unavailability budget, which apportioned the down time among the different systems of the accelerator. The required availability specifications were compared to the performance of existing accelerators to see how much the reliability of individual components must be improved. This level of improvement could then be used to determine the added costs needed to achieve the budgeted availability and to assess the risks of not achieving the necessary improvements.

Note that the goal was not to determine the availability of each proposed option or variant and declare it a bad design if it was down too much. Rather, the assumption is that, with proper design, either of the options or any of the variants can be made available enough; it is only a matter of how much it will cost, and the risk of failure if components must be made too much more reliable than the present state-of-the-art.

Given the limited time available, this study is of necessity only a first crude step. Nevertheless, it has produced a very useful tool to evaluate the overall availability of an accelerator. While the tool has not been benchmarked against an existing accelerator and overall predicted downtimes could change with different assumptions, we believe the comparisons between different accelerator variants (warm/cold, 1 vs. 2 tunnel, conventional vs. undulator $e^{+}$source) are quite significant.

Before proceeding, it is worthwhile to give a few definitions.

availability : the fraction of time the accelerator hardware is up enough to be providing useful beams to the detector. For this exercise, substandard performance while tuning emittance or backgrounds, or recovering from MPS trips is still considered to be available. The luminosity loss due to these items must be accounted separately.

unavailability : just one minus the availability. For this exercise, time spent tuning the accelerator to recover after a repair downtime is considered as unavailable.

reliability : sort of a synonym for availability, not used in a precise manner.

MTBF : the Mean Time Between Failures is the average time between failures of a component assuming normal preventive maintenance has been done. It is very important to realize that if a component is specified as having an MTBF of 50 million hours that does not mean it must run for that long 
without being touched by human hands. Fans, bearings, or even the whole device can be replaced on a regular basis. Only breakages of the device which occur outside our control count toward the MTBF. The MTBF as used here does not include infant mortality as it is the steady state running of the accelerator that is being considered; hence the devices have been burned in. MTBF is not not the lifetime of a device; it can be much longer than that since one can use preventive maintenance to replace the wearing part or the whole device before it wears out. It is only if one neglects to do this preventive maintenance that the MTBF will be less than the lifetime.

MTTR : the Mean Time To Repair a component. This is the time from when one has access to the component until it is repaired. It includes travel time, but not time for the accelerator to cool down before an access starts, nor time to turn on and tune up the accelerator.

region : a major part of the accelerator such as the $e^{-}$injector, $e^{+}$damping ring, or $e^{-}$linac.

MD : Machine Development or time devoted to accelerator physics experiments, including trying out possible improvements.

opportunistic MD : Machine Development done in a region of the accelerator while some other region(s) are undergoing repairs or are still recovering from a repair.

\subsection{Method}

A detailed list of components was compiled for two major regions of the collider, the linacs and damping rings (DRs). These regions were chosen for detailed analysis both because of their size and complexity, and because they were the areas that change most significantly depending on the main linac technology. The component list included items identified as potential sources of failure from experience with existing facilities, such as RF components, magnets, magnet power supplies, power supply controllers, vacuum pumps, pump power supplies, movers, diagnostics, etc., with counts for each item.

Each component was then assigned a starting MTBF and MTTR. Where possible, the numbers were based on data from repair statistics at SLAC, Fermilab or other labs. In a few cases (e.g. magnet power supplies), the linear collider proposals had already engineered a level of redundancy to improve the MTBF. Here, the starting numbers assumed the designed redundancy and those cases are explicitly mentioned in the results section. In some cases, no good data was available and the MTBF used was just a reasonable guess (typically 100,000 hours). It is worth noting that even for the most common devices, MTBFs can vary by a factor of 10 depending on the exact design and application of the device.

Due to lack of time, the other regions of the accelerator were not modeled in detail. Rather, the sources and beam delivery systems (which are very similar in all designs) and bunch compressors were treated as individual elements with an MTBF and MTTR for the whole region.

To develop a more quantitative evaluation, a simulation was written which used all of this input data to estimate the availability of the whole LC. A brief description of this simulation is given in the next section, and more complete details are available in LCC note 127.

The simulation assumes the accelerator has reached a steady state after several years of operation. It does not use a bathtub curve which would give poorer MTBFs in the early years due to infant mortality and in later years due to devices wearing out. A special run was made with all parameters degraded to simulate what operation might be like in the early years.

The results of the simulation gave the total unavailability and how each device type contributed to it. As expected, the availability of the LC was too poor and it was necessary to improve the MTBFs of some devices to attain the goal availability. Typically, the devices which were contributing more than most to 
the unavailability were identified and improved. The rationale was that it would be cheaper to concentrate efforts on a few critical devices rather than trying to improve all devices by the same factor.

There is clearly flexibility in which devices to improve in order to achieve the goal availability. Ideally the improvements chosen would be those which minimize cost increases. While there was not time to do this quantitatively, this report provides a reasonable first iteration. As a design progresses more, these tools should clearly continue to be used to optimize the design.

The final result is a table of the MTBFs of devices that are needed to achieve the desired availability with an indication of how much these must be improved over the present state of the art. There are also comparisons of how the availability would change when going from 2 tunnels to 1 tunnel or from an undulator positron source to a conventional source.

\subsection{Brief description of the simulation}

Many accelerators have estimated their availabilities during the design phase with a spreadsheet. (Examples from SNS and APT were reviewed.) There are formulas to combine the availabilities of the components to get the availability of the whole. There are also reliability software packages that help do this. The approach taken here was to write a simulation which could allow several complexities to be handled that would have been nearly impossible in a spreadsheet and quite difficult in the commercial software packages. These complexities include the recovery and tuning time needed after a downtime, the complex redundancies built into the LC designs, the way in which accelerator physics experiments (Machine Development or MD) can be done when only part of the accelerator is down, and the way in which many devices are typically repaired during an access by a limited number of people. By writing a simulation tailored to the task, it was possible to incorporate the decades of experience in running real accelerators accumulated by the people on the task force. The following paragraphs give a brief description of these aspects of the simulation. LCC note 127 gives details for those who are interested.

\subsubsection{Handling Complex Redundancies}

If all the devices in the linac had to be working at the same time, the LC would never be up. All designs have a built in energy overhead so that some energy producing components can be broken and the LC still functions because an energy feedback loop keeps the energy constant. Many components such as the klystrons, modulators, RF structures, AC power for the RF, water pumps, and tuners will degrade the energy when they malfunction. Some of these can be repaired while the accelerator is running. Others must await an access. Each degrades the energy by a certain number of $\mathrm{MeV}$. The simulation starts with a perfect accelerator with the energy overhead given in the design. Each time a component breaks, it decreases the energy overhead by the corresponding amount and schedules it for repair (either immediately if it can be done "hot" or at the next downtime). When the repair is completed, the energy overhead is increased. If the energy overhead gets down to zero, the accelerator is declared broken and many accumulated repairs get done.

The two linac $\left(e^{+}\right.$and $\left.e^{-}\right)$energy overheads are individually tracked. Each warm DR RF system is also redundant (with one more cavity or RF station than needed ) and is handled in a similar fashion. Also the cold DR's injection and extraction kickers are redundant (21 kicker systems where only 20 are needed).

Things also break which can degrade the luminosity without turning off the machine. Examples are diagnostics such as BPMs and laser wires which make tuning more difficult and hence reduce the luminosity. For other devices such as such as correctors or movers in both warm and cold LCs or linac quadrupoles in the cold LC, it is assumed that after some downtime for retuning, the machine can continue to run with the broken device. The simulation makes each of these broken devices degrade the luminosity a bit $(0.1 \%$ 
per BPM and $5 \%$ per wire). If the luminosity gets down to half its design value, the accelerator is declared broken and many accumulated repairs get done.

If an item that simply degrades a parameter can be repaired while the accelerator is running, the simulation does so, as long as sufficient repair people are available. These are called hot repairs. The repair of klystrons and modulators are the most important examples of this.

Of course, some components are not redundant and when they fail, the accelerator is considered broken and repairs must be scheduled immediately. Most magnets other than correctors, together with their power supplies and controllers, are typical examples. In the cold linac, however, the quadrupoles are treated as a special case. The focusing lattice is weak enough that the simulation assumes that one can tune around the problem in a couple of hours to recover $99 \%$ of the luminosity. The failed component is then repaired at the next convenient time (typically during the long down for the magnet which is in a cryostat). Other devices handled in a similar fashion are movers in the warm linac and DR, trim windings and correctors in both DRs, and tuners and couplers in the cold linac.

\subsubsection{Downtime planning}

Without doubt the downtime planning is the most complicated part of the simulation. This should come as no surprise to anyone who has participated in the planning of a repair day. It is even harder in the simulation because computers don't get a gestalt of the situation like humans do. Briefly, it figures out what parameter (e.g. $e^{-}$linac energy overhead or $e^{+}$DR extraction kicker strength or luminosity) got degraded too much, and plans to fix things that degrade that parameter. Having figured out how long the downtime must be to fix items which simply have to be repaired, it then schedules other items for repair allowing them to extend the downtime by 50 to $100 \%$. Some other issues must also be taken into account:

- If an access to the accelerator tunnel is required, 1 hour is allowed for prompt radiation to decay before entry. 1 hour is also allowed for locking up, turning on and standardizing power supplies. (This cooldown time is what is typically required at SLAC to minimize exposure of employees to residual radiation.)

- The devices chosen for repair are those that give the most bang for the buck (most improvement in the parameter per hour of repair time).

- The number of people in the accelerator tunnel is limited to 50 (to minimize the chaos of PPS control). This number is consistent with practice at existing facilities. The number of people doing other repairs is limited to 100 to reflect the finite resources available. All repair people are considered equal and specialties are not tracked. It will be shown later that the number of personnel could be limited still further without degrading the availability.

- There are no regularly scheduled maintenance shutdowns, except yearly. Interventions occur only when the accelerator is broken, which is what happens at most operating accelerators. In real life, maintenance might be planned when the energy overhead was getting low without waiting to actually run out of energy. However, since the simulation does not add any penalty for unplanned or off-hours downtimes, this becomes a subtlety which does not really impact the results.

- Things which break during the downtime are just ignored (assumed they are immediately fixed). The long recovery time which is described in the next section is intended to account for this.

\subsubsection{Recovery times}

The simulation assumes that all repairs get done on schedule. It seemed an unnecessary complication to throw random numbers to distribute the repair times around the MTTR as the simulation is run for long 
enough to average out such variations anyway.

Recovery of the beam is modeled in a crude fashion which matches the qualitative experience on many accelerators. This common experience is that it takes time to recover good beams after a downtime. In fact, the longer the down, the longer the recovery time. Contributions to the recovery come from myriad factors such as

Hardware failures - devices such as pumps and power supplies which break because they were turned off during the shutdown or devices which just happen to break while the accelerator was down and were not detected

Environmental factors - temperature changes caused by the access or ground motion over a few hour period which can be significant enough to require retuning

Human error - mistakes made in doing the repairs (valves left closed, cables left disconnected...) or failure to restore settings after hardware or software tests

Parameter drifts - multiple parameters which are continuously tracked and optimized during normal operation which all need to be identified and retuned

Commissioning - hardware or software improvements made during the shutdown which need to be tested, calibrated, etc.

$\cdots$

Rather than trying to model recovery procedures in detail, the simulation simply assumes that the time it takes to get good beam out of a region of the accelerator is proportional to the time that region was without beam. Table 4.3.3.1 shows the constants of proportionality used for each region (typically $10 \%$, except for the DRs and IR, which are given 20\%). In real operation, the beam quality recovers gradually as each region is tuned up in succession, and the luminosity gradually ramps up to nominal. The simulation simplifies this by assuming that the machine goes from no beam at the end of a region to perfect beam at the end of the recovery time. Similarly, the luminosity jumps from zero up to the design value immediately at the end of the recovery/tuning time. While this is certainly an oversimplification, if the recovery time used in the simulation is considered to be the time it takes to get back to half the design luminosity, then the overall effect is reasonably well reproduced.

\subsubsection{Lumped Systems}

As mentioned in Section 4.2, some systems were not modeled in detail due to lack of time, but rather modeled as one piece. The details are given in Table 4.3.4.1. Each such system was considered to have two types of failures: one which required access to the accelerator tunnel (flagged by a "1" in the "access needed?" column) and one which did not require access. A nominal downtime was chosen for each system for each access state, typically $0.2 \%$. The warm bunch compressors have two stages and are more complex so they were given $0.3 \%$, and the somewhat simpler IP regions and cold bunch compressors were given $0.15 \%$. These downtimes were then converted into effective MTBFs. The output of the simulation shows a downtime for these systems which is roughly double the nominal downtimes in the table due to the modeled recovery time. Together these lumped systems required two-thirds of the unavailability budget and the rest was allocated to the linacs and DRs.

\subsubsection{Machine Development}

Machine Development is an essential tax on the operating efficiency of any accelerator. It is time used to better characterize the machine, develop new tuning procedures, and test possible future improvements. The 
Table 4.3.3.1: Regions of the machine with allocated tuning time and MD. The first column gives the names of all the regions. The second gives the ratio of the time needed to recover beam to the time beam has been absent from the region. The third column gives the percent of scheduled running time that is needed to do MD for the warm design. Column 4 gives the same for the cold design. Note that the MD time includes the time to recover from the MD work. Hence the useful time to do actual MD measurements will be somewhat less than the times given in the table.

\begin{tabular}{|l|c|c|c|}
\hline Region name & Tune time fraction & \% MD time warm & \% MD time cold \\
\hline \hline$e^{-}$injector & 0.1 & 1 & 1 \\
\hline$e^{-}$DR & 0.2 & 2 & 2 \\
\hline$e^{-}$compressor & 0.1 & 1 & 1 \\
\hline$e^{-}$linac & 0.1 & 1 & 1 \\
\hline$e^{-}$BDS & 0.1 & 1 & 1 \\
\hline$e^{+}$source & 0.1 & 1 & 1 \\
\hline$e^{+}$PDR & 0.2 & 1 & 0 \\
\hline$e^{+}$DR & 0.2 & 2 & 2 \\
\hline$e^{+}$compressor & 0.1 & 1 & 1 \\
\hline$e^{+}$linac & 0.1 & 1 & 1 \\
\hline$e^{+}$BDS & 0.1 & 1 & 1 \\
\hline IP & 0.2 & 1 & 1 \\
\hline
\end{tabular}

Table 4.3.4.1: Details on how the lumped systems were modeled.

\begin{tabular}{|l|c|c|c|c|r|r|}
\hline System & $\begin{array}{c}\text { access } \\
\text { needed? }\end{array}$ & $\begin{array}{c}\text { MTTR } \\
(\mathrm{hrs})\end{array}$ & $\begin{array}{c}\text { warm nominal } \\
\text { \% downtime }\end{array}$ & $\begin{array}{c}\text { cold nominal } \\
\text { \% downtime }\end{array}$ & $\begin{array}{c}\text { warm MTBF } \\
\text { (hrs) }\end{array}$ & $\begin{array}{c}\text { cold MTBF } \\
\text { (hrs })\end{array}$ \\
\hline \hline$e^{-}$injector & 1 & 8 & 0.2 & 0.2 & 4000 & 4000 \\
\hline$e^{-}$injector & 0 & 1 & 0.2 & 0.2 & 500 & 500 \\
\hline$e^{-}$compressor & 1 & 8 & 0.3 & 0.15 & 2667 & 5333 \\
\hline$e^{-}$compressor & 0 & 1 & 0.3 & 0.15 & 333 & 667 \\
\hline$e^{-}$BDS & 1 & 8 & 0.2 & 0.2 & 4000 & 4000 \\
\hline$e^{-}$BDS & 0 & 1 & 0.2 & 0.2 & 500 & 500 \\
\hline$e^{+}$source & 1 & 8 & 0.2 & 0.2 & 4000 & 4000 \\
\hline$e^{+}$source & 0 & 1 & 0.2 & 0.2 & 500 & 500 \\
\hline$e^{+}$PDR & 1 & 8 & 0.2 & $1 \times 10^{-40}$ & 4000 & $8 \times 10^{42}$ \\
\hline$e^{+}$PDR & 0 & 1 & 0.2 & $1 \times 10^{-40}$ & 500 & $1 \times 10^{42}$ \\
\hline$e^{+}$compressor & 1 & 8 & 0.3 & 0.15 & 2667 & 5333 \\
\hline$e^{+}$compressor & 0 & 1 & 0.3 & 0.15 & 333 & 667 \\
\hline$e^{+}$BDS & 1 & 8 & 0.2 & 0.2 & 4000 & 4000 \\
\hline$e^{+}$BDS & 0 & 1 & 0.2 & 0.2 & 500 & 500 \\
\hline IP & 1 & 8 & 0.15 & 0.15 & 5333 & 5333 \\
\hline IP & 0 & 1 & 0.15 & 0.15 & 667 & 667 \\
\hline Cryo plants & 0 & 10 & $1 \times 10^{-40}$ & 1 & $1 \times 10^{43}$ & 1000 \\
\hline site power & 0 & 10 & 0.5 & 0.5 & 2000 & 2000 \\
\hline global controls & 0 & 5 & 0.2 & 0.2 & 2500 & 2500 \\
\hline
\end{tabular}

amount of time spent on MD varies through the life of a project, with more MD required in the early stages or after a major upgrade. For this simulation, the LC is assumed to have operated for a few years and to have settled into a nominal schedule of MD, which would occupy approximately $10 \%$ of the time. As with the recovery time, the MD was allocated to the individual regions of the machine. Each region was allocated $1 \% \mathrm{MD}$ with the exception of the DRs, which were given $2 \%$ because of the complexity of the invasive tuning anticipated. 
To more fully mirror the complexity of operation of a real machine, the simulation assumed that some of the required MD could be done on an opportunistic basis. Typically repairs may be completed in some regions earlier than others. As an example, there could be 16 hours of repair work needed in the $e^{-}$linac, 2 hours in the $e^{-}$injector and none anywhere else. In this case, there will be time after the $e^{-}$injector is repaired when beam has been tuned up into the $e^{-}$DR while repairs are still going on in the $e^{-}$linac. If this is more than two hours, the simulation assumes useful $\mathrm{MD}$ can be done in the $e^{-}$injector and $\mathrm{DR}$. If there is a conventional positron target, MD can also be done anywhere in the $e^{+}$system. This opportunistic MD time is tracked by the simulation. It then assumes that sometime during the running period enough scheduled MD is done in each region to bring the total of opportunistic plus scheduled MD up to the desired levels given in Table 4.3.3.1. Note that the MD times in the table include recovery from the MD. During scheduled MD the LC is up, but not producing luminosity for the detector.

\subsubsection{Important assumptions that effect availability}

There are several assumptions incorporated into the simulation that are important and are not explicitly stated in the description of the accelerators. If the final LC designers are foolhardy enough to not meet these design requirements, they should be sure to repeat the simulation to see the effects of their less reliable design.

1. Klystrons located in the support tunnel can be replaced while the accelerator is running. This requires a valve or extra window in the high power waveguide, which is not implemented in the existing test facilities, TTF or NLCTA.

2. All electronics modules and power supplies are accessible in the support tunnel including vacuum pump power supplies and BPM processors. Electronics modules are hot swappable; that is, they can be replaced without turning off other electronics.

3. There is a tune-up dump at the end of each PPS zone so that beam can be run in an upstream region while people are in the tunnel performing repairs downstream.

\subsubsection{The need for bench marking (and why it is hard)}

During the development of this simulation, progress reports were given to several groups. While most people agree that the technique looks good, someone always asks, "Shouldn't you benchmark this against an existing accelerator to make sure it is working properly." The answer is "Yes, but." Yes, it should be done, but it is a lot of work and there was not enough time to do it. Hopefully, future work with the simulation will include benchmarking it against a real accelerator. This is a nontrivial task for several reasons:

- Getting together the list of components is real work

- MTBFs and MTTRs for that actual accelerator should be used if one wants an accurate comparison. Again, it is real work to get these from whatever trouble reporting database the accelerator keeps. For the cases studied (SLC and Fermilab), there were often more errors than correct entries in the trouble database and it was necessary to go through thousands of errors by hand and correct the entries.

- Most labs record the recovery from a downtime as "tuning", not as downtime attributed to the device which originally broke. One would either have to modify the simulation to reproduce what the lab did (trivial, but misleading), or go back through a few years of logbooks and try to correct the data for the accelerator.

- Most labs try to schedule repairs before they completely bring the accelerator down (e.g. a water leak that hasn't yet caused a magnet ground fault or the repair of a water pump whose bearing is screeching) 
at least a day in advance. They then account this as scheduled downtime (no different than a scheduled Christmas shutdown or 3 month shutdown) rather than unscheduled downtime. Whether it is scheduled or unscheduled, no luminosity is made, so the simulation just calls it downtime. The data from a real accelerator would have to be corrected for this effect.

\subsection{Results}

The task was to create an unavailability budget for several variants of the LC. It was decided to allow $25 \%$ downtime due to hardware problems and the recovery from them. This is comparable to what present HEP accelerators accomplish after several years of effort. The LC is much more complex, so it will be more difficult to achieve. Of the $25 \%$ downtime, only $15 \%$ was explicitly budgeted to specific devices. The other $10 \%$ was held as contingency for devices that were not included or for major design mistakes that cause poor reliability and that remain a problem after a few years of operation. To make it crystal clear what is and is not included in the $25 \%$, it is worthwhile to list all of the factors used to turn peak luminosity into integrated luminosity.

HA is the Hardware Availability. This includes the recovery from a hardware problem. This is the only thing has been studied in detail with the simulation. A $25 \%$ downtime is allowed or $75 \%$ uptime.

MD is the fraction of time spent doing scheduled Machine Development. If there were no opportunistic MD done, then scheduled MD would be about $7 \%$ for the conventional $e^{+}$source and $12 \%$ for the undulator source. The actual number varies from $2 \%$ to $11 \%$ depending on the accelerator variant. This variation is due to the use of opportunistic MD and to the possibility of doing MD in two parts of the accelerator at once. The simulation produces an estimate of MD, but for this example, an average of $10 \%$ will be used.

SD is the fraction of time in the long Shut Down. A 3 month shutdown once every year gives $25 \%$ for this.

SU is the fraction of time Starting Up and Recovering from the long shutdown. Typically the luminosity ramps up gradually to the nominal value. Consider SU to be the fraction of a running year to get to half the nominal luminosity and then if the ramp were linear (which it usually isn't) then the fractional loss in luminosity is simply SU. For this example a 1.5 month recovery from the 3 month shutdown will be used giving $\mathrm{SU}=1.5 / 9=16.7 \%$

MPS is the fraction of time lost to MPS trips (and recovery from them) and other similar very short outages. For this example $5 \%$ will be used.

DT is the De-Tuning factor. It is the fraction by which the average non-zero luminosity is lower than the peak luminosity. Contributors to this are non-optimum tuning due either to mistakes or to the accelerator drifting away faster than feedbacks and operators can tune. For this example $10 \%$ will be used.

Taking all this into account then gives:

Integrated Luminosity per year

$$
\begin{aligned}
& =\text { Peak Luminosity } \times \text { Seconds } / \text { year } \times \mathrm{HA} \times(1-\mathrm{MD}) \times(1-\mathrm{SD}) \times(1-\mathrm{SU}) \times(1-\mathrm{MPS}) \times(1-\mathrm{DT}) \\
& =2 \times 10^{34} \mathrm{~cm}^{-2} \mathrm{~s}^{-1} \times 3.14 \times 10^{7} \mathrm{~s} \times 0.75 \times 0.90 \times 0.75 \times 0.833 \times 0.95 \times 0.90 \\
& =2.26 \times 10^{41} \mathrm{~cm}^{-2}=226 \text { inverse femtobarns per year }
\end{aligned}
$$




\subsubsection{Simulations of the warm and cold reference designs, the cold 1 tunnel variant, and the conventional $\mathrm{e}^{+}$variant}

Table 4.4.1.1 shows the results of simulation runs for the warm and cold reference designs, the cold 1 tunnel variant, and the conventional $\mathrm{e}^{+}$variant. Each run is given a unique name such as Warm1 or Cold3 in the first column and this name is used here to identify the run as each is described in turn.

Runs Warm1 and Cold1 simulate the main warm and cold LC options which have 2 tunnels (one for the accelerator and a second for support equipment) and an undulator based positron source. Nominal MTBFs (taken from operating accelerators or educated guesses when that data was unavailable) were used with the following exceptions:

1. All magnet supplies for both warm and cold use an MTBF of 200,000 hours instead of the 40-50 thousand hours experienced at SLAC and Fermilab. This comes from a design done for the TESLA TDR which has redundant regulators (which are the most common component to fail in a power supply) and hence an MTBF that depends mostly on the low power components and bulk supply which were not made redundant.

2. The power coupler interlock electronics and sensors for the cold LC were given an MTBF of 1 million hours instead of the nominal 100,000 hour MTBF that is used for most electronic modules and sensors. This was done because it is planned to build redundancy into the circuits and sensors.

3. The cold design calls for the cavity tuner stepping motors to be inside the cryostat. Using a nominal MTBF of 500,000 hours (taken from SLAC mover experience) resulted in so many tuner failures that a multi-month access was needed to perform repairs. To keep this from skewing the results, 1 million hours was used. It will become evident later that it will be necessary to make these components redundant or to bring them outside the cryostat.

4. The cryo plant for the cold linac is assumed to have a $99 \%$ uptime. As it is made of six systems each with a capacity of $30 \mathrm{~kW}$ at $4 \mathrm{~K}$, each system must have an uptime of $99.84 \%$. Each system is comparable in size to the Fermilab central helium liquefier which after recent major improvements is up about $99.6 \%$ of the time (not counting outages due to utilities like power and cooling water). CERN's LEP refrigerators were about half that capacity and were up about $99.83 \%$ of the time (again not including utility outages). Including utility outages tends to double or triple the downtime. Depending on what you compare to and whether outages due to utilities are included, the cryo plant's MTBF is a factor of 1 to 6 better than present experience.

5. The MTBF of present SLAC modulators is 30,000 hours with many of the failures coming from the thyratrons. The LC modulator designs all use IGBTs instead and hence an MTBF of 50,000 hours was used for them. In addition, the modulator design for the warm linac has built in redundancy as there are extra IGBT boards which allow the modulator to deliver full performance even with failed boards. For this reason it was assigned an MTBF of 100,000 hours.

A description of all the devices and the MTBFs and MTTRs used for them may be found in the appendix. The actual input and results spreadsheets and the simulation code used in all the runs may be found on the website: www.slac.stanford.edu/ tmh/availability/. With only the above exceptions to nominal present day MTBFs, the downtimes of the warm and cold LCs end up being 28 and $31 \%$ respectively (taken from the number in the third column of the table). These are remarkably similar, and remarkably good given how complex the LCs are. However, it will not be trivial to achieve the goal of $15 \%$ down. The regions not modeled in detail (injector, bunch compressors, BDS, site power, cryo plant, global control system) were given fixed downtime budgets to cause a downtime of about $10 \%$. Hence the linac and DRs which are modeled in detail cause about $30-10=20 \%$ downtime with the nominal MTBFs. This must be reduced to $5 \%$ or a factor of about 4 to fit into the budget. 


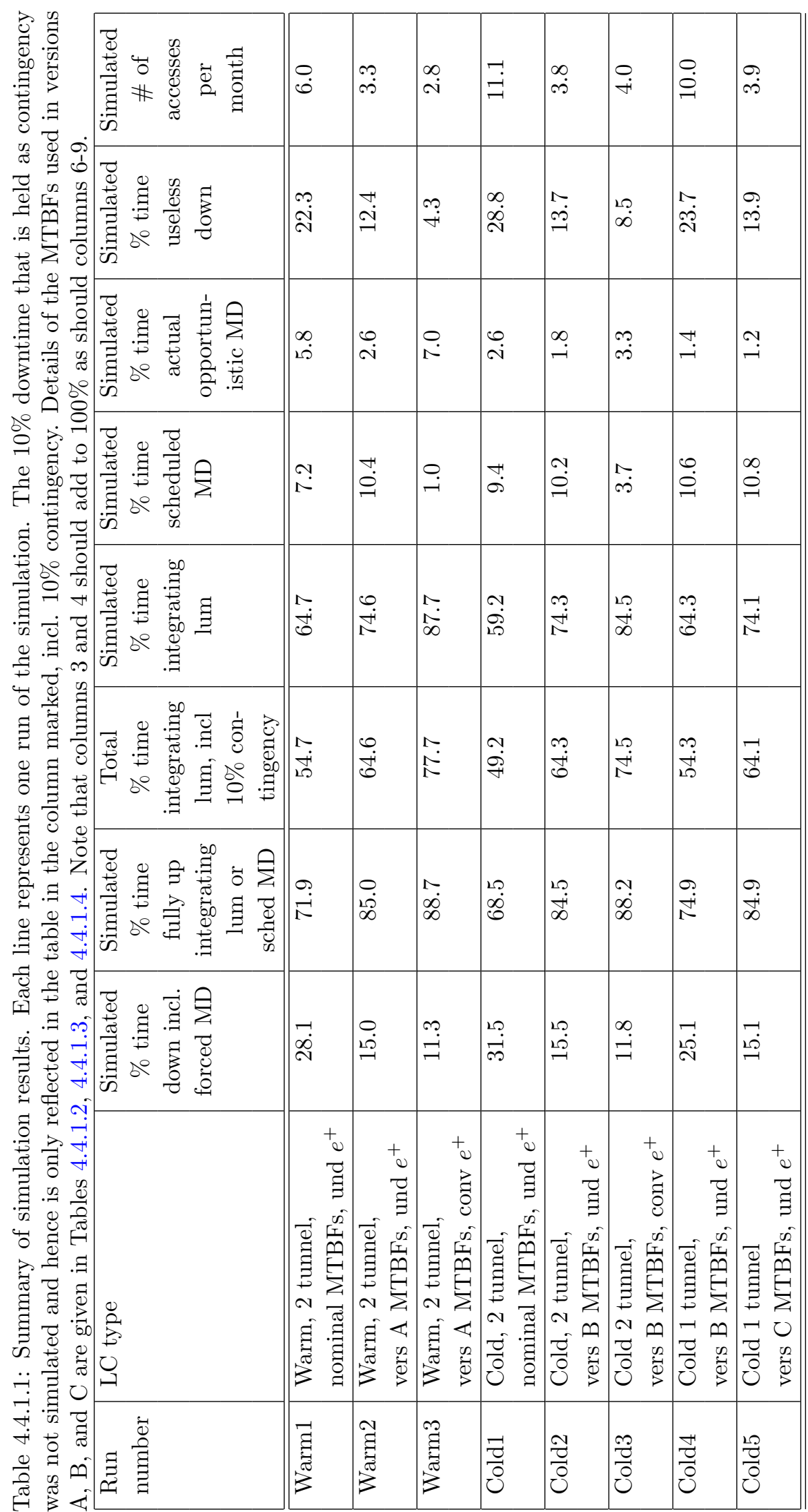


CHAPTER 4. AVAILABILITY DESIGN

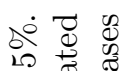

대욜

:

言

要

造

苟苟

$\approx \stackrel{2}{2}$

$\therefore \mp .5$

递总

0
0
0

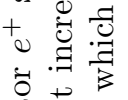

总落菦

$\exists$

$\exists \in$

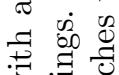

$B$ :

의

寻宅

ㄱ. 클

믈

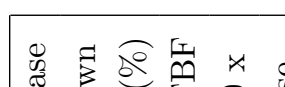

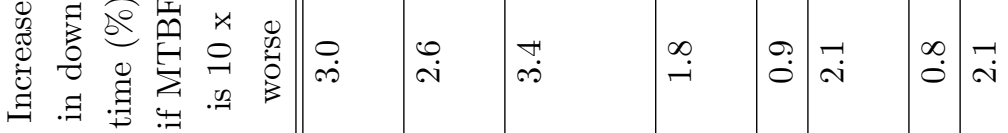

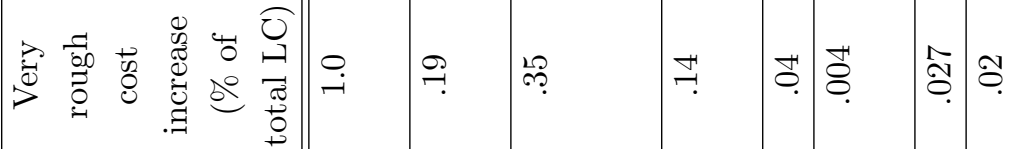

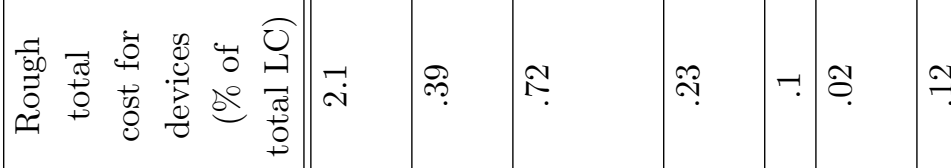

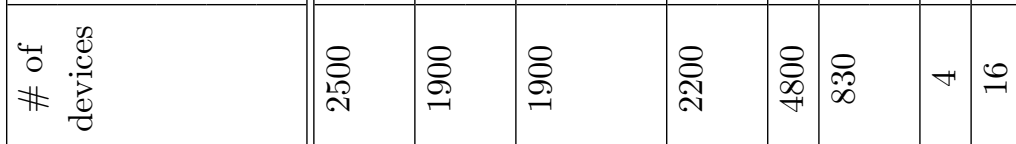

莽

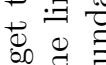

$\therefore+\frac{10}{0}$

过

\begin{tabular}{|l|l|l|l|}
\hline & & $\infty$ \\
\hline
\end{tabular}

(2)

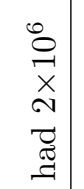

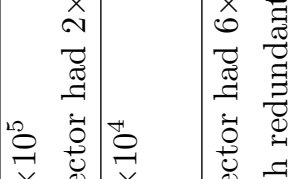

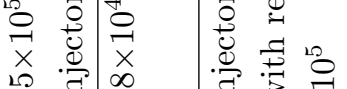

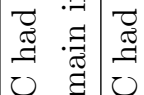

O

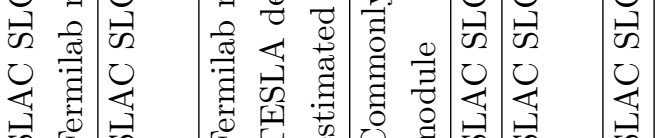

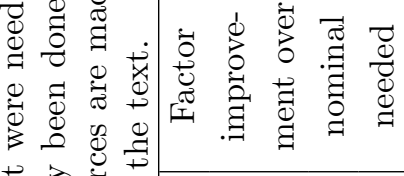

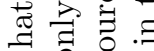

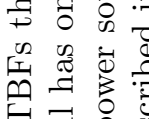

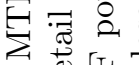

$\varangle$ 远

형

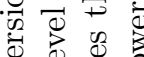

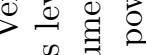

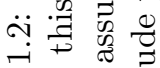

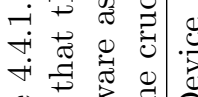

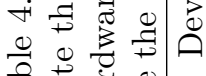

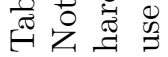

\begin{tabular}{|c|c|c|c|c|c|c|c|c|c|}
\hline 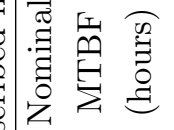 & $\| \stackrel{\vec{x}}{x}$ & 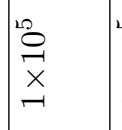 & 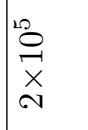 & 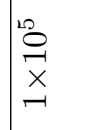 & 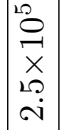 & $\begin{array}{l}n \\
0 \\
x \\
0 \\
0 \\
\dot{20}\end{array}$ & 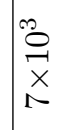 & 总 & \\
\hline 苋 & 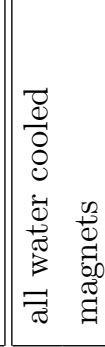 & 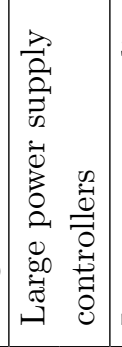 & 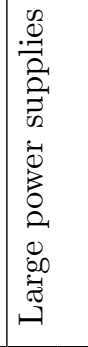 & 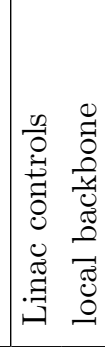 & 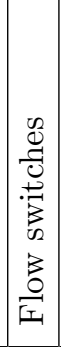 & 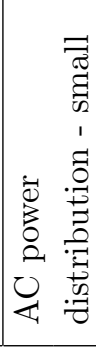 & 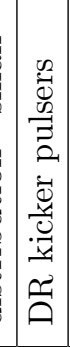 & 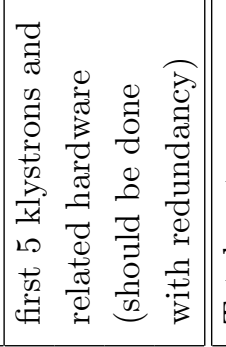 & 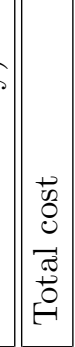 \\
\hline
\end{tabular}




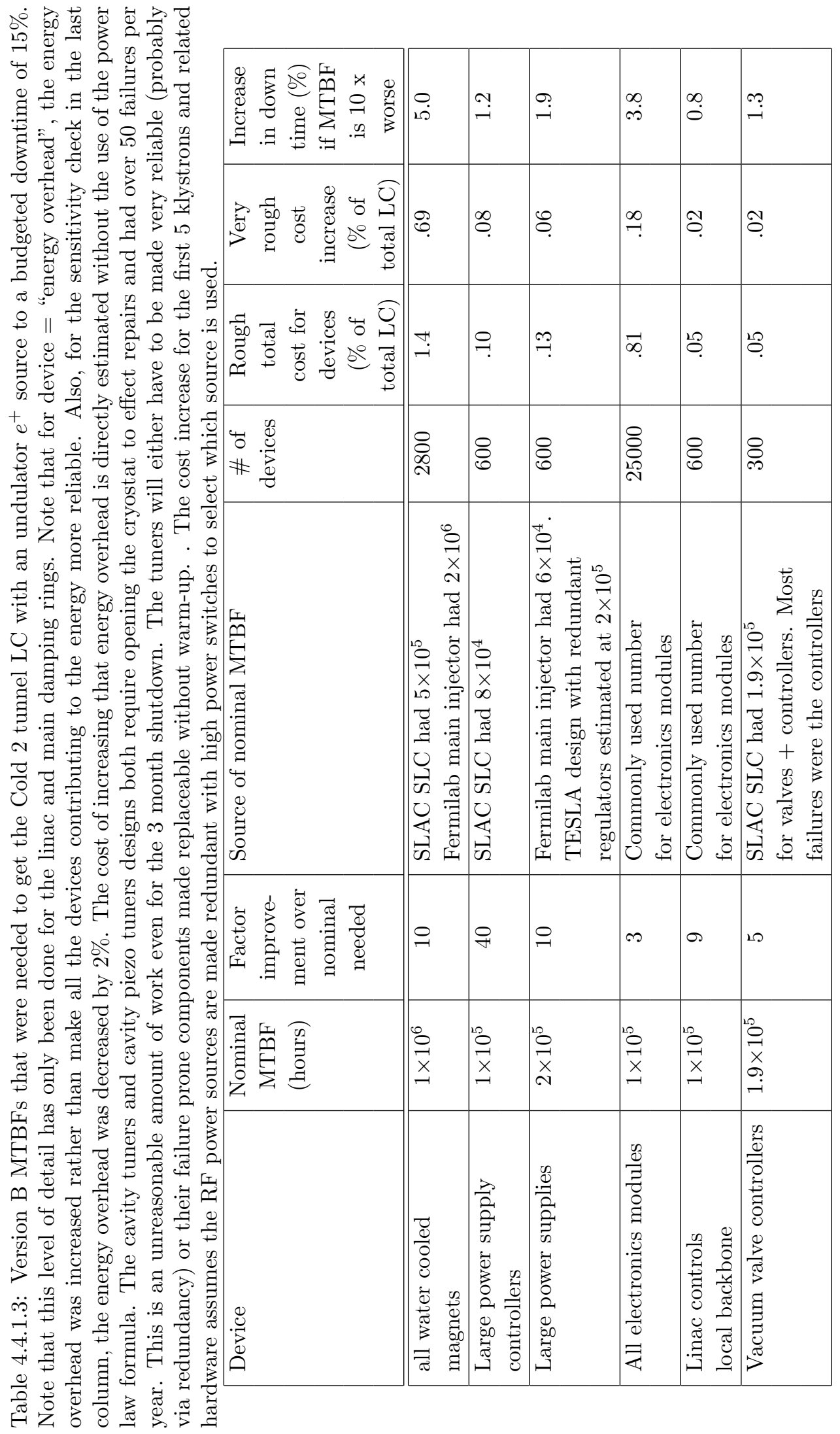




\begin{tabular}{|c|c|c|c|c|c|c|c|c|c|}
\hline & 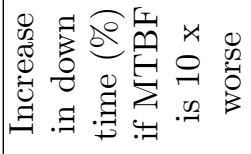 & $\infty$ & ־ & ت. & $\stackrel{+}{0}$ & $\ddot{0}$ & $\stackrel{0}{0}$ & $\begin{array}{c}0 \\
2 \dot{0}\end{array}$ & \\
\hline & 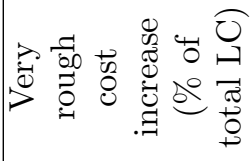 & $\stackrel{0}{0}$ & $\ddot{8}$ & $\stackrel{\infty}{\infty}$ & $\stackrel{\infty}{\infty}$ & ְి & b! & 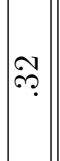 & $\vec{A}$ \\
\hline & 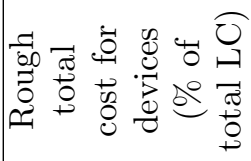 & $\dddot{0}$ & $\tilde{\delta}$ & $\delta$ & & ְె. & 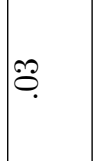 & & \\
\hline & 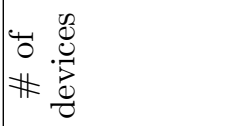 & $\begin{array}{c}8 \\
\mathbb{1} \\
-1\end{array}$ & 品 & $\stackrel{8}{R}$ & $\stackrel{ }{-1}$ & $\underset{8}{8}$ & 10 & & \\
\hline 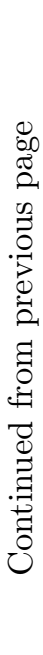 & 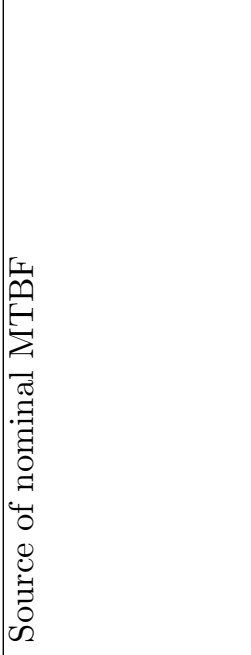 & 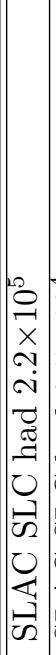 & 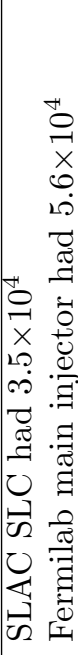 & 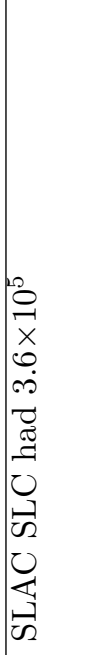 & & 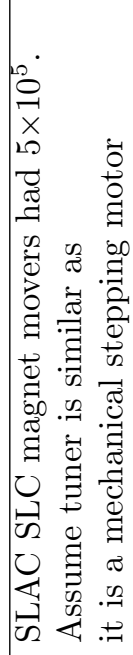 & $\begin{array}{l}0 \\
0 \\
b \\
5 \\
50\end{array}$ & 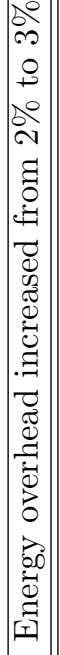 & \\
\hline & 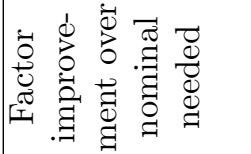 & $\stackrel{0}{-1}$ & $\infty$ & 으 & 尺ి & D. & 0 & $\begin{array}{l}2 \\
-1\end{array}$ & \\
\hline & 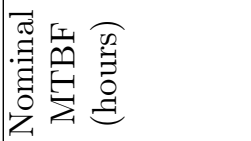 & $\left|\begin{array}{l}n \\
0 \\
\hdashline \\
x \\
2 o \\
\sim \\
\sim\end{array}\right|$ & $\begin{array}{l}\vec{H} \\
\overrightarrow{0} \\
\vec{x} \\
x \\
0\end{array}$ & $\begin{array}{l}\infty \\
0 \\
0 \\
x \\
0 \\
i \infty \\
0\end{array}$ & 总 & $\begin{array}{l}\stackrel{8}{\circ} \\
\vec{x} \\
-1\end{array}$ & $\begin{array}{l}20 \\
\stackrel{0}{1} \\
x \\
-1 \\
\end{array}$ & $\begin{array}{l}\stackrel{0}{\circ} \\
\stackrel{2}{*}\end{array}$ & \\
\hline & 岂 & 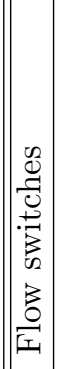 & 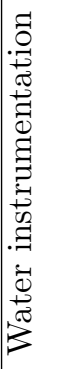 & 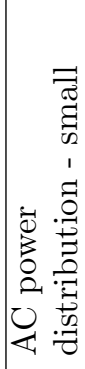 & 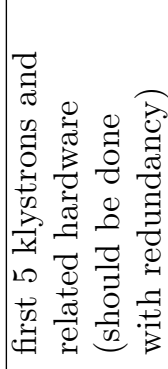 & 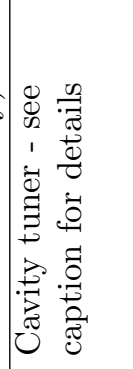 & 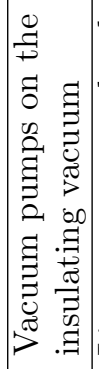 & 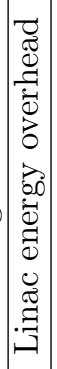 & 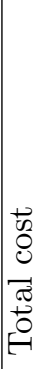 \\
\hline
\end{tabular}




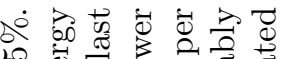

10 可

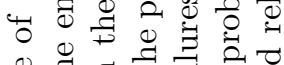

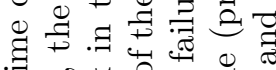

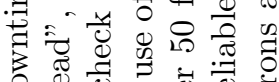

总过

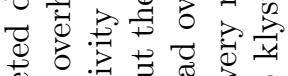

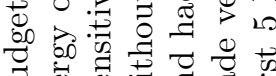

范

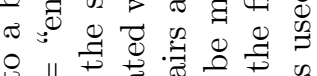

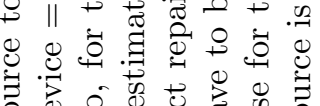

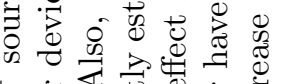

+

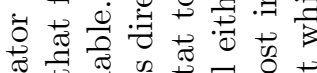

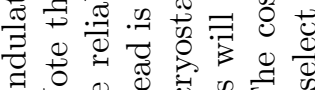

\begin{tabular}{|c|c|c|c|c|c|c|c|c|c|c|}
\hline 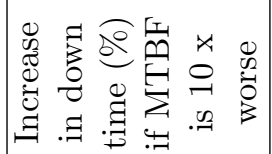 & $\|$ & 我 & $\stackrel{a}{a}$ & تُ & $\tilde{y}$ & 20 & $\dddot{m}$ & $\stackrel{0}{0}$ & $\ddot{0}$ & $=$ \\
\hline 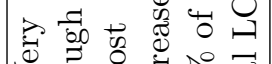 & $\infty$ & $\exists$ & 8 & \% & $\%$ & $\stackrel{\infty}{-1}$ & 8 & $\stackrel{\infty}{\infty}$ & $\stackrel{\infty}{\varrho}$ & \\
\hline
\end{tabular}

乙 010

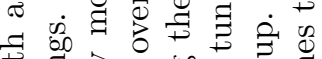

菅苛

U.

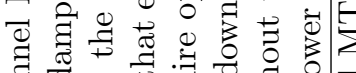

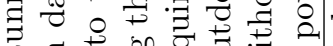

$\neg . \exists$.

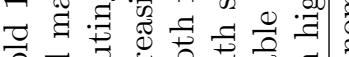

ర

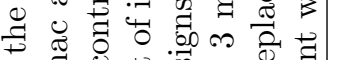

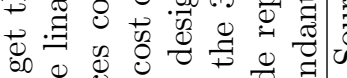

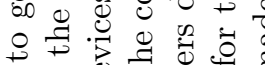

๘

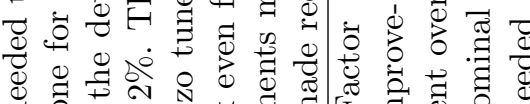

จ

वี

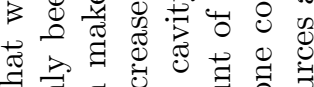

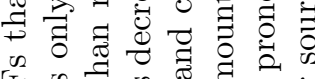

项

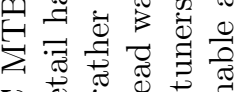

$\checkmark$ 讨

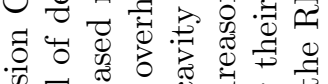

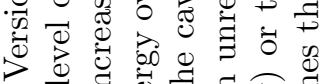

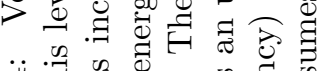

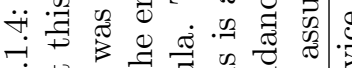

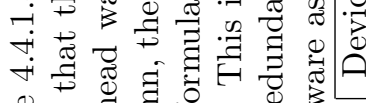

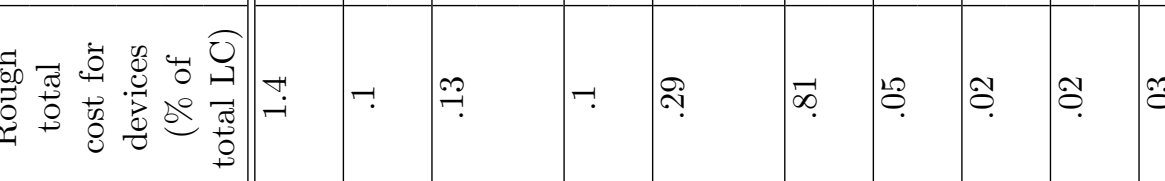

० 0 의

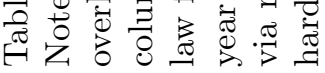

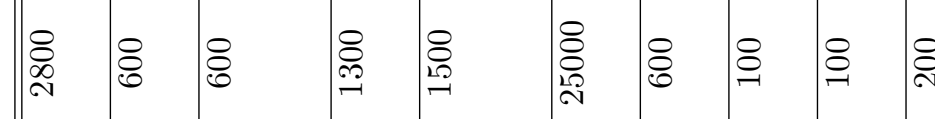

\begin{tabular}{|c|c|c|c|c|c|c|c|c|c|c|}
\hline 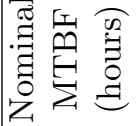 & 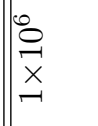 & 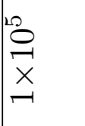 & 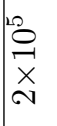 & $\begin{array}{l}\stackrel{8}{\circ} \\
\vec{x} \\
x\end{array}$ & $\stackrel{20}{\stackrel{1}{x}}$ & 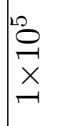 & 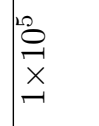 & $\begin{array}{l}\stackrel{2}{0} \\
\stackrel{x}{x} \\
-1\end{array}$ & 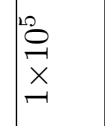 & $\begin{array}{l}28 \\
\stackrel{1}{x} \\
-1 \\
-1\end{array}$ \\
\hline 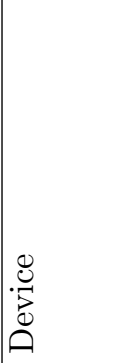 & 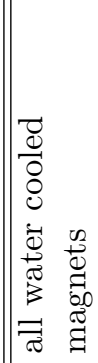 & 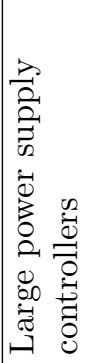 & 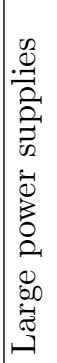 & 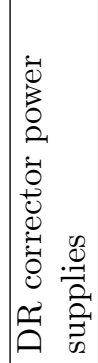 & 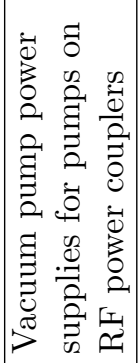 & 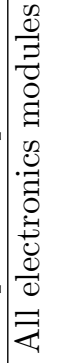 & 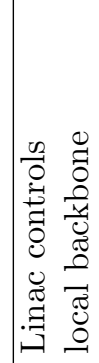 & 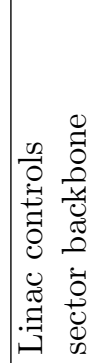 & 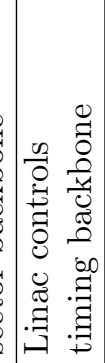 & 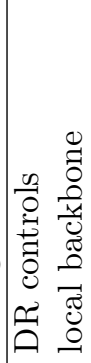 \\
\hline
\end{tabular}


CHAPTER 4. AVAILABILITY DESIGN

\begin{tabular}{|c|c|c|c|c|c|c|c|c|c|}
\hline 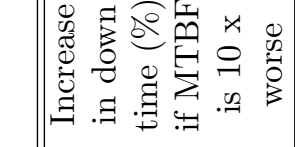 & $\stackrel{20}{\circ}$ & تُ & N & - & $\grave{o}$ & $\begin{array}{l}\infty \\
\infty \\
\infty\end{array}$ & 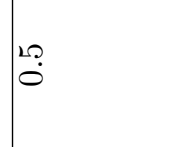 & $\stackrel{?}{\rightarrow}$ & $\stackrel{\overbrace{}}{-}$ \\
\hline 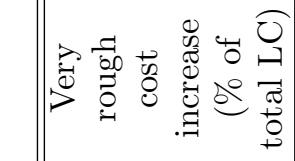 & $\dddot{g}$ & 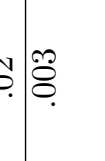 & ڤ̊. & 28 & ఝి & $\because-4$ & 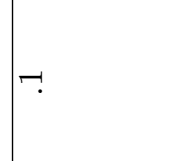 & 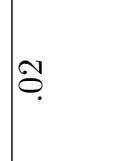 & 28 \\
\hline 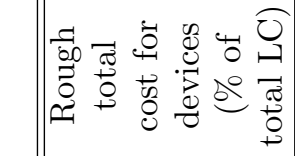 & $\stackrel{0}{2}$ & 8 & 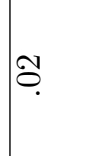 & $\stackrel{0}{\infty}$ & $\Gamma$ & 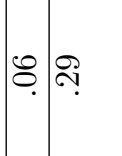 & ণ̣ & $\approx$. & $\stackrel{\overbrace{}}{\oplus}$ \\
\hline \# & ठ్లి & 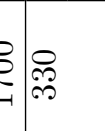 & 还 & $\stackrel{ }{\circ}$ & 0 & 他 & 兽 & ஓి & 㴜 \\
\hline 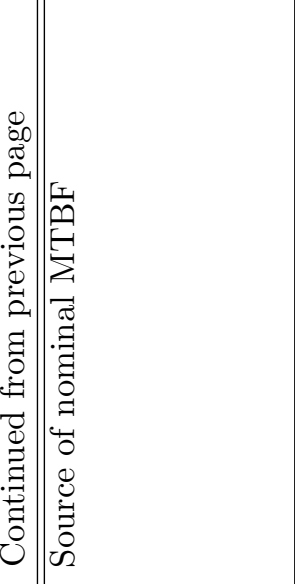 & 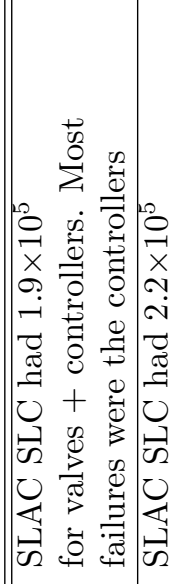 & 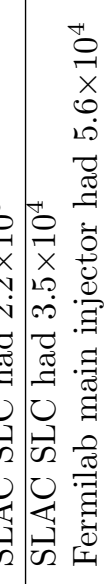 & 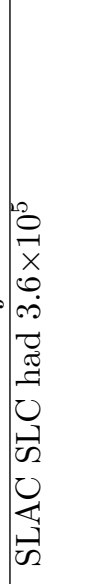 & & & 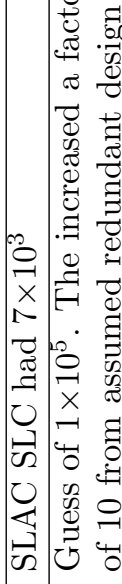 & 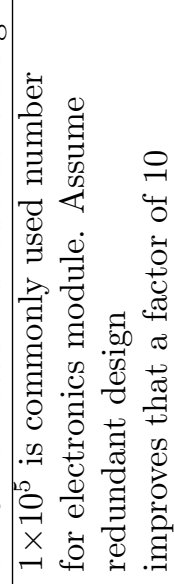 & 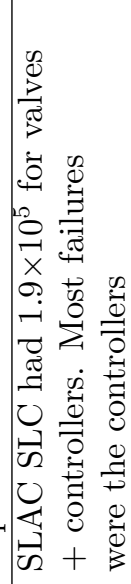 & 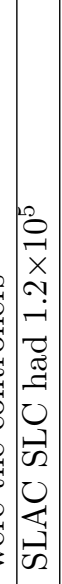 \\
\hline 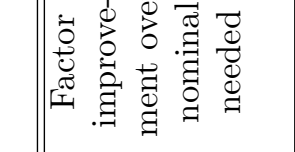 & $\ddot{m}$ & $\theta \infty$ & 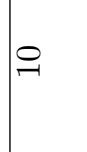 & নิ & 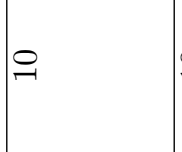 & 010 & 10 & $\infty$ & $\infty$ \\
\hline 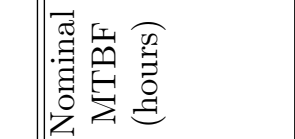 & 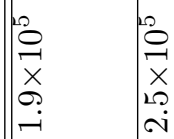 & 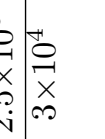 & $\begin{array}{l}0 \\
0 \\
x \\
0 \\
0 \\
0 \\
0\end{array}$ & 节 & 营 & 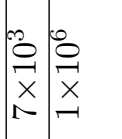 & 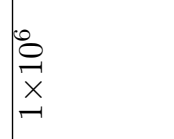 & 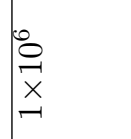 & 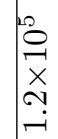 \\
\hline 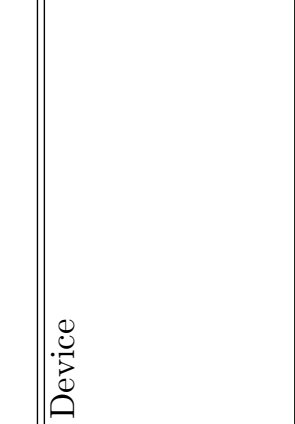 & 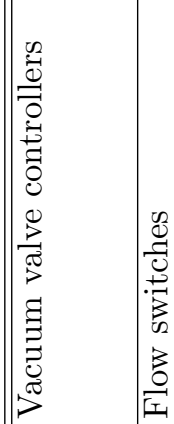 & 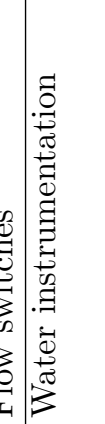 & 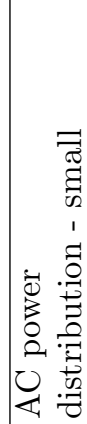 & 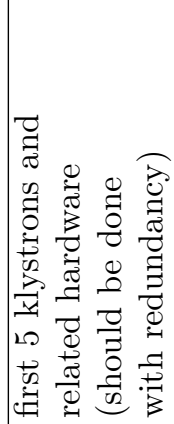 & 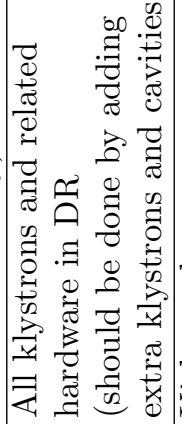 & 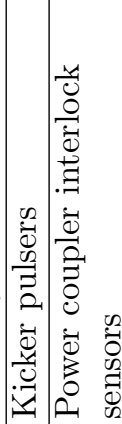 & 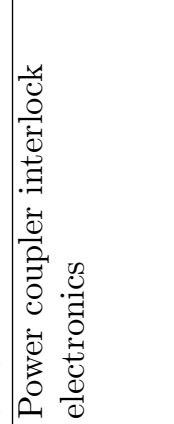 & 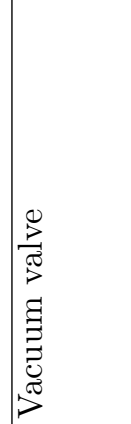 & 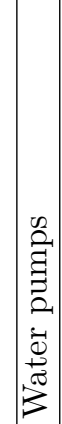 \\
\hline
\end{tabular}




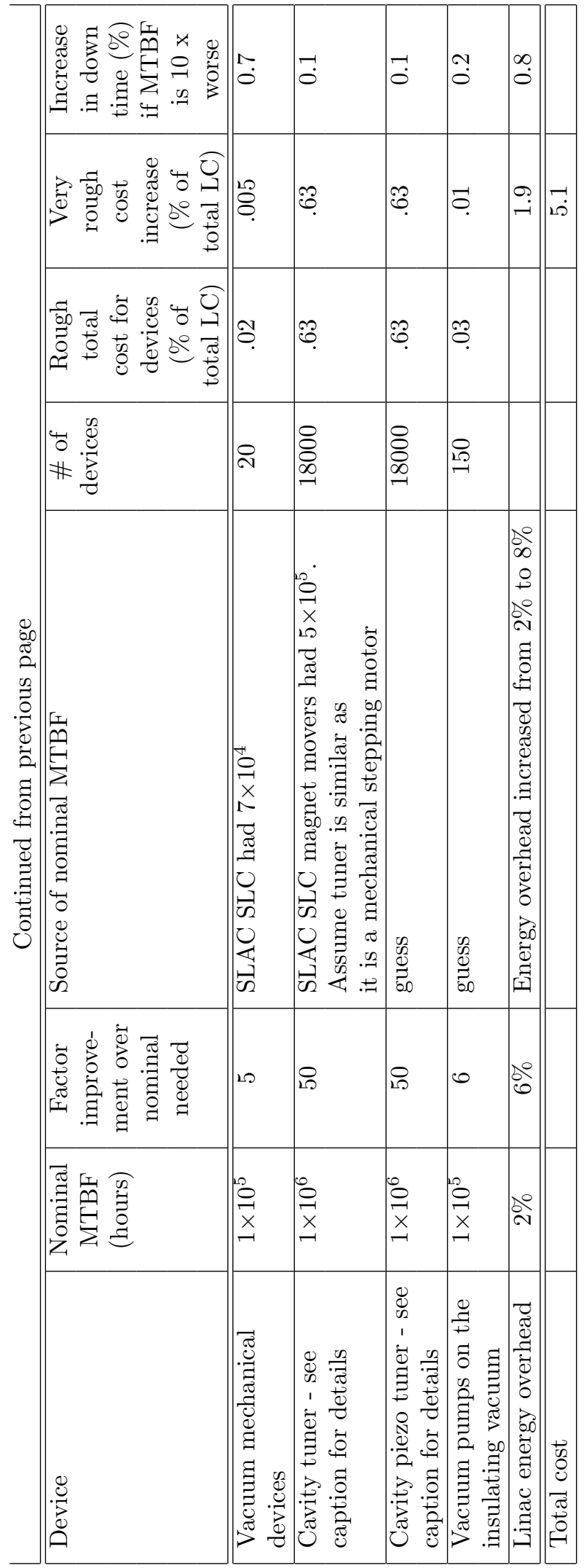


Runs Warm2 and Cold2 start from the conditions of runs Warm1 and Cold1 and increase some MTBFs in order to achieve the goal 15\% downtime. This could be done by increasing them all by a factor of 4 . Rather than this, devices which contributed more than most to the downtime were improved by larger factors, leaving the MTBFs of most of the devices at their nominal values. It is presumably cheaper to redesign or make redundant a small fraction of the devices than to do that for all of them. The goal is to get the desired downtime for as little additional expense as possible. As the design of the LC progresses, it will be necessary to do real engineering to estimate the cost of the improvements to MTBFs and modify the goal values arrived at here in order to minimize the cost. For simplicity here, all devices which contributed more than about $0.2 \%$ to $0.3 \%$ to the downtime had their MTBFs improved sufficiently to get below that level. An exception was those components whose failure simply reduced the energy gain in the main linac. If too much downtime was caused by these components, then the energy overhead was increased instead of increasing the MTBFs, as that few percent increase in linac length would probably be cheaper than improving so many components.

While what has been improved in the simulation is MTBFs, one could get some of the gain by decreasing the MTTRs. This optimization will be left to a later stage of the design. It should be noted that decreasing the MTTR by a factor of two is not as effective as increasing the MTBF by a factor of two for devices in the accelerator tunnel because of the overhead for access and the possibility of overlapping multiple repairs.

While many MTBFs are left unimproved compared to present practice, engineers must remain vigilant. The LC is enormously complex and it will not take many design errors to make it too unreliable.

Tables 4.4.1.2 and 4.4.1.3 show the MTBFs that were adjusted to achieve the goal downtime of $15 \%$ for the warm and cold LCs (neglecting the additional 10\% downtime reserved for contingency). To allow easy comparison to present state of the art, achieved numbers from some present accelerators are also given. While some of the MTBFs are rather large, this should not cause immediate panic. Assigning a 50 million hour MTBF does not mean the device must continue working for 571 years without being touched. It means that with proper preventive maintenance, which can include completely replacing the device before it wears out or replacing redundant components after they have failed, there will be an average of one failure per 571 device-years of running.

It is difficult to estimate the MTBF of a device accurately, even using historical data, as the answer varies widely with the person who did the analysis and with the running period that was considered. Industry tables of MTBFs show a variation of over a factor of 10 for the same type of device. For these reasons, simulations were done to study the effect on overall LC availability if the actual MTBF of a device were significantly worse than specified. The last column in the three tables above shows the effect on overall downtime if the particular device type had an MTBF a factor of ten worse than the budget allocated (the first times the second column). Typically the downtime increases a few percent, which indicates that failure to meet one or two of the MTBF budgets would not be a disaster. This provides some flexibility as in a project this size some mistakes will inevitably be made. The $10 \%$ availability contingency was intended to cover such problems.

The next-to-last column in the tables gives an incredibly crude estimate of the extra cost to reach the desired MTBF. Better estimates would require real engineering studies of the details of each system, analyzing the failure modes and devising remedies. This simply was not within the scope of this task force, but will be required as the LC heads towards construction. In some cases, the extra cost is only in the engineering design, which is amortized over a large number of components. In other cases, it may be necessary to provide redundancy which could significantly increase the device cost.

The crude estimates were based on the following logic:

- Since most of the devices to be improved are those that traditionally cause downtime in existing accelerators, many years of effort have already gone into making them reliable. Hence, there is likely no cheap solution to get a large improvement (like adding an extra cooling fan to a power supply). 
- When a factor of 10 improvement is needed, it is assumed to be accomplished by making the most troublesome half of the device redundant, leaving about $10 \%$ remaining single point failures. In this model, a factor of 10 improvement increases the cost by $50 \%$.

- A factor of 2 improvement might be gained by better quality control, burn-in, shake testing etc. This factor of 2 might add $13 \%$ to the cost.

- These two cost increases can be described by a power law. Each extra factor of 10 multiplies the cost by 1.5. Conveniently, the cost increase chosen to give a factor of 2 improvement lies on this power curve so we have

- The factor by which the cost increases $=(\text { MTBFdesired } / \text { MTBFnominal })^{(\ln (1.5) / \ln (10))}$

This is obviously not universally correct, and is more likely to apply to power supplies and interlocks than to magnets and vacuum valves.

Run Warm3 (Cold3) is intended to show the difference between using an undulator $e^{+}$source and a conventional $e^{+}$source. Otherwise, it is identical to Warm2 (Cold2). Since the positron source was not modeled in detail and the same overall MTBF was used for both systems, the difference is not due to the specific components of the two sources. The significant change is that the undulator source requires high energy electrons before positrons can be produced, while the conventional source does not. This one difference changes the downtime from $15 \%$ to about $11 \%$ because of the shorter recovery time when both systems can be restored independently. More importantly, the amount of time spent integrating luminosity increases from about $75 \%$ to over $85 \%$ in the simulation, or from $65 \%$ to $75 \%$ including the $10 \%$ downtime contingency. This increase is due to the reduced downtime mentioned above, and to a decrease in the time spent in scheduled MD (1-4\%) because more MD can be done opportunistically. If differences between the actual reliabilities of the sources are ignored, the choice of an undulator source in order to potentially produce polarized positrons reduces the integrated luminosity by more than $15 \%$.

This large reduction in luminosity with the undulator positron source comes from three effects, all due to the need for high energy electrons in order to produce positrons. The first effect has nothing to do with reliability. It is the simple fact that with a conventional $e^{+}$source one can do MD simultaneously in for example the $e^{-} \mathrm{DR}$ and the $e^{+}$linac, which is not possible with the undulator. The second effect is that recovery from a downtime is slower as one cannot start tuning the $e^{+}$system until beam is recovered in the $e^{-}$linac. The third effect is that with a conventional $e^{+}$source, but not with the undulator source, one can do opportunistic MD in the $e^{+}$system when something in the $e^{-}$system is down or recovering.

This problem might be ameliorated by using a conventional $e^{+}$source to provide positrons to the $e^{+}$DR when no undulator $e^{+}$were available, but this case has not been simulated. The impact would depend on the switchover time between the two $e^{+}$sources and on whether the conventional source was full power or only low power.

Run Cold4 examines the one tunnel variant of the cold LC with everything else the same as Cold2. With one tunnel, devices like linac klystrons and magnet power supplies are located in the accelerator tunnel and require access to fix. The modulators remain accessible and there are long pulsed cables connecting them to the klystrons. With the same MTBFs, the $15 \%$ downtime for Cold2 becomes 25\%. Since the lumped components are causing about $10 \%$ of this, the linac and DR components must be improved by another factor of 3 on average, a total of a factor of 12 over nominal. Run Cold5 includes the MTBF improvements shown in Table 4.4.1.4, which were designed to meet this tougher demand. Not surprisingly, the list in Table 4.4.1.4 is much longer and the numbers are much higher than for the two tunnel cold version (Cold2) in Table 4.4.1.3. The difference in the total cost given in the two tables gives an idea of the incremental cost to achieve the same availability with one tunnel as with two. This difference is about $3 \%$ of the total project cost. This should be balanced against the savings in other costs such as tunneling to evaluate the cost effectiveness of a single tunnel. It is worth mentioning that there are other issues with a single tunnel, such as the extra difficulty involved in debugging problems that involve beam without access to the electronics. 
Figures 4.4.1.1-4.4.1.3 illustrate the downtime budgets for the warm and cold reference design options, and for the cold one-tunnel variant. Roughly a third of the downtime is caused by the linac and DRs that have been modeled in detail. The other two-thirds is caused by site-wide systems (power, global controls, and cryogenic plants) and by the regions of the accelerator not modeled in detail. For the warm case, the linacs cause more downtime than the damping rings while that situation is reversed for the cold case. This is reasonable given the relative complexity of the damping rings. In all cases, the electron regions cause more downtime than the corresponding positron regions. This is not due to the intrinsic reliability of the components (which are the same for the $e^{+}$and $e^{-}$systems). Rather, with the use of the undulator $e^{+}$ source, it takes longer to recover from an outage of an electron region than from the corresponding positron region as beam must be recovered first through the electron region and then through all the positron regions. 

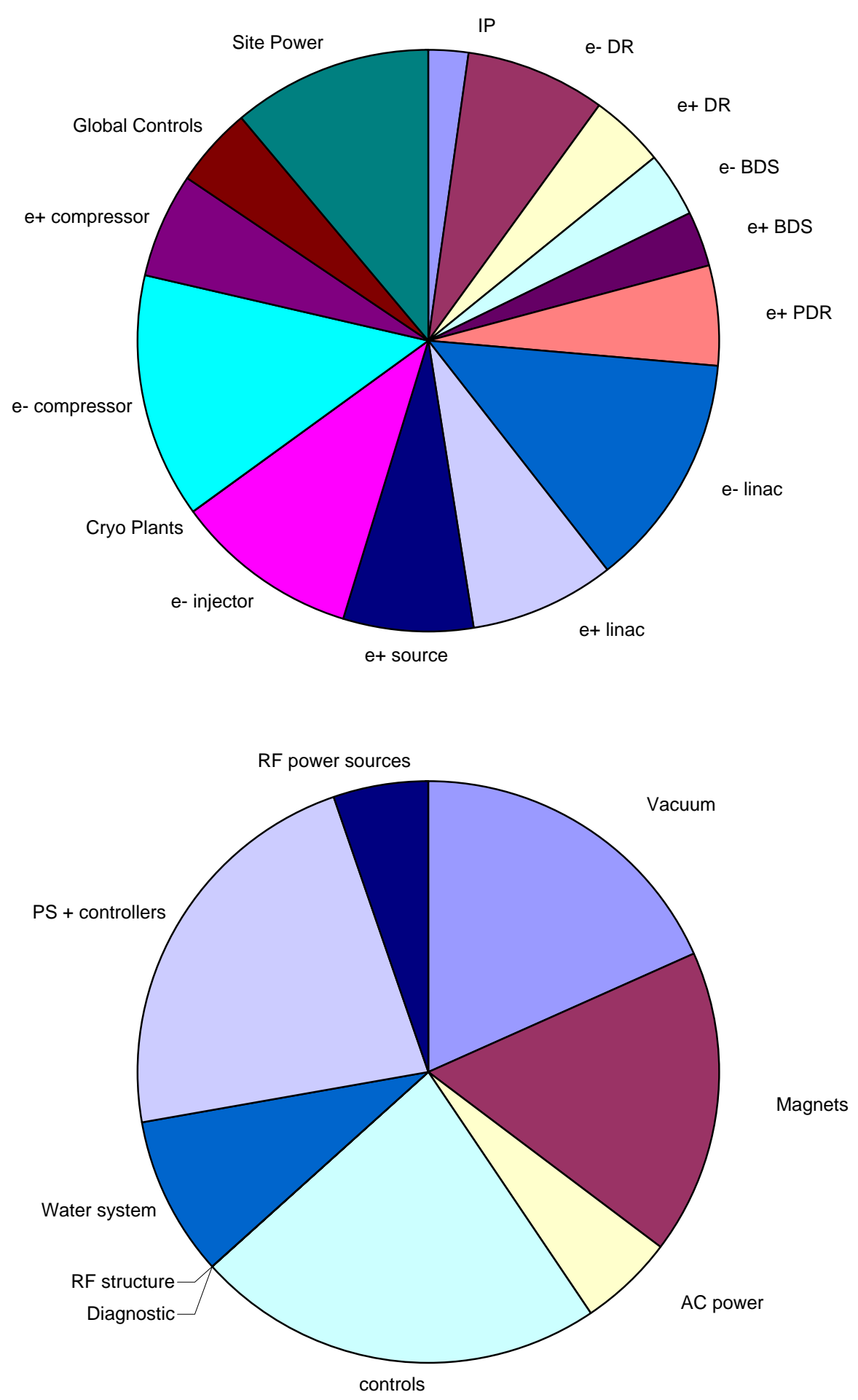

Figure 4.4.1.1: Downtime summary for run Warm2 (2 tunnel, undulator $e^{+}$source, version A MTBFs). The top chart divides it by region while the bottom chart divides the linac and DR downtimes by system. 

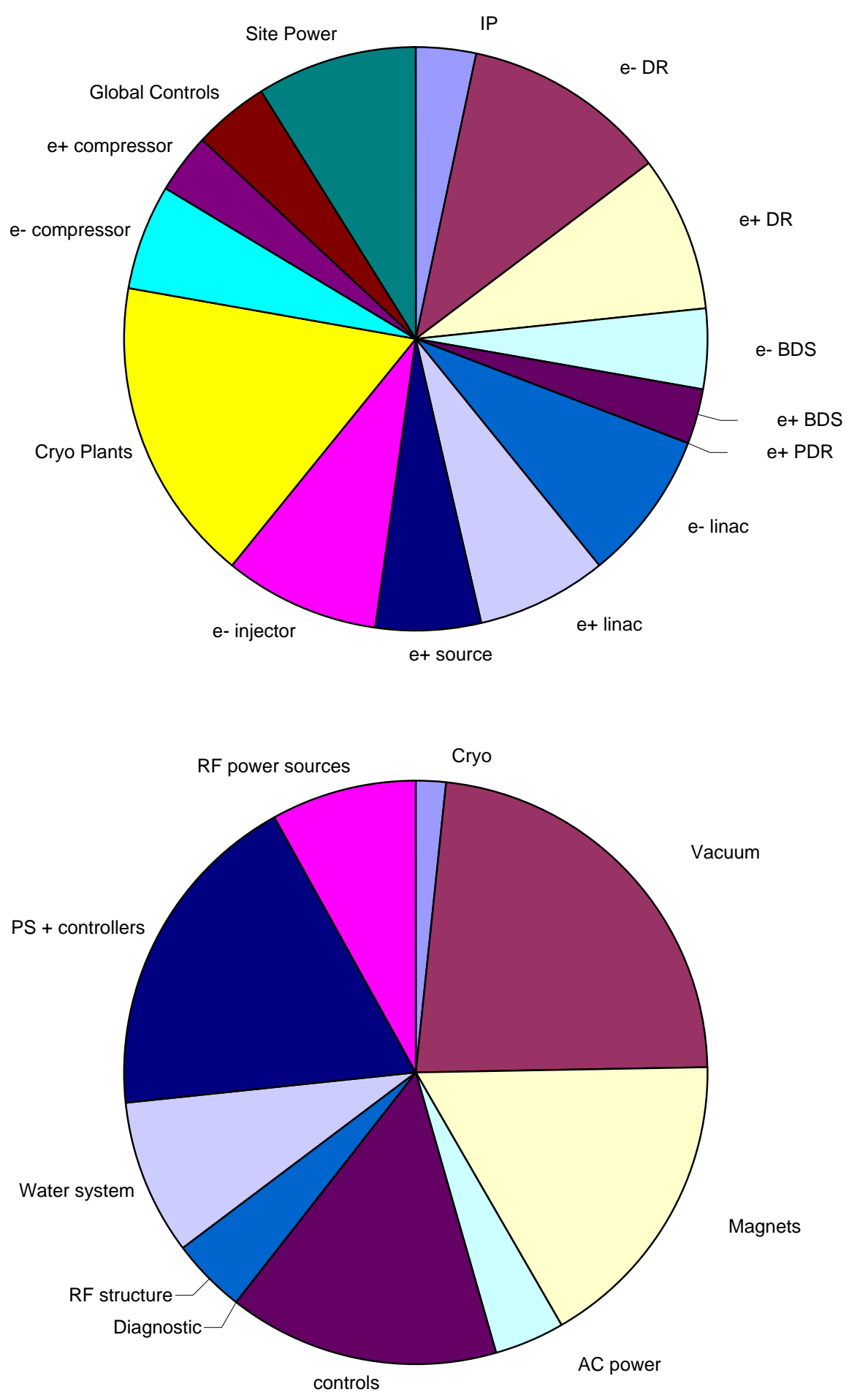

Figure 4.4.1.2: Downtime summary for run Cold2 (2 tunnel, undulator $e^{+}$source, version B MTBFs). The top chart divides it by region while the bottom chart divides the linac and DR downtimes by system. 

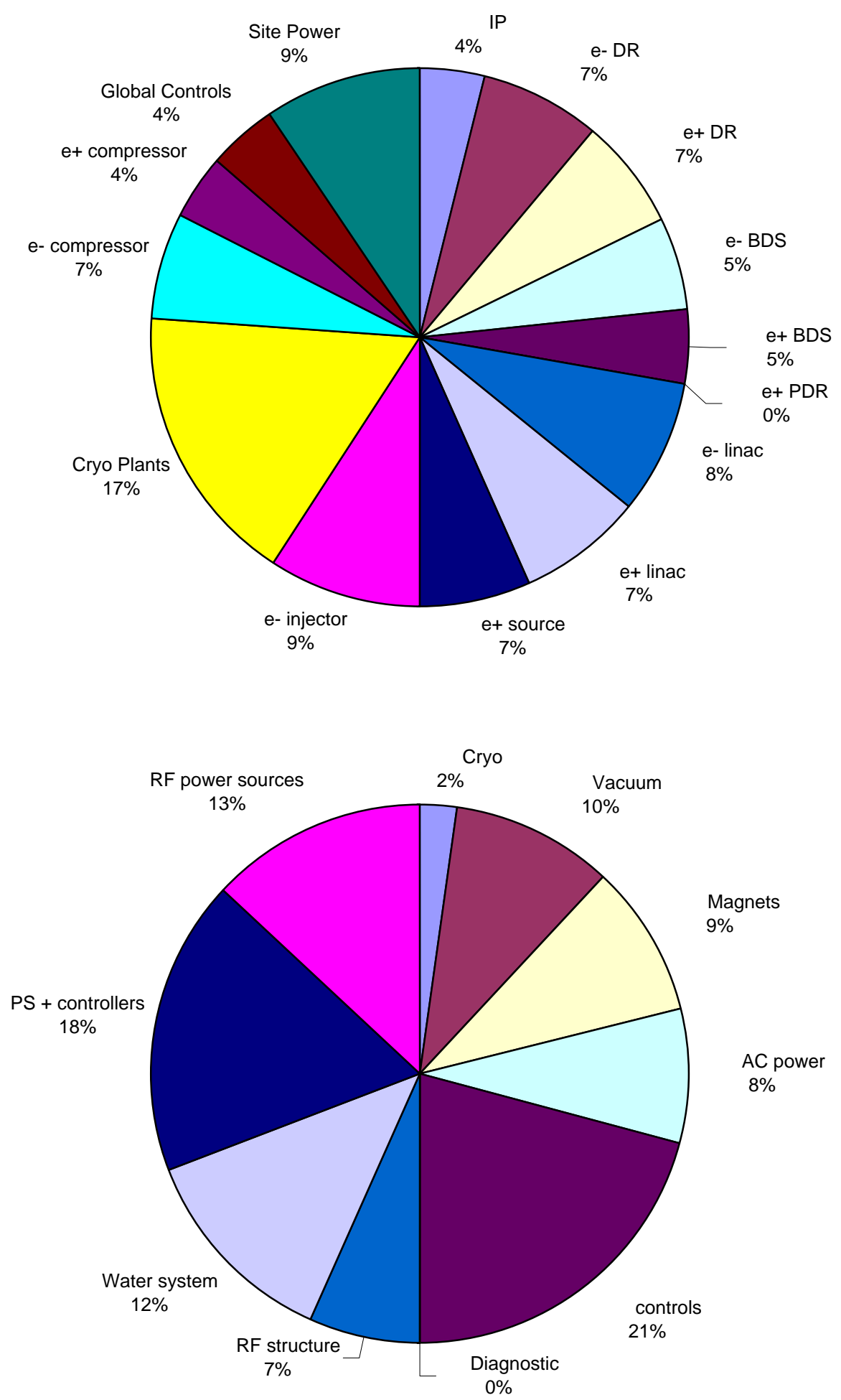

Figure 4.4.1.3: Downtime summary for run Cold5 (1 tunnel, undulator $e^{+}$source, version C MTBFs). The top chart divides it by region while the bottom chart divides the linac and DR downtimes by system. 


\subsubsection{Sensitivity studies}

The simulations described above have assumed conditions corresponding to steady state operation of the accelerator after most of the bugs have been ironed out and it is running fairly well. This will hopefully be the case after a couple of years of operation. To investigate what the availability might be during the commissioning period before things are running quite so smoothly, the simulations were repeated with three simple changes:

\section{All the MTBFs were halved.}

2. Twice as much time was spent on MD.

3. The recovery time from outages was doubled.

The results of these simulations are shown in Table 4.4.2.1. Run Warm4 (Cold6) is the same as Warm2 (Cold2) except with these "commissioning" features. It represents the base design with an undulator $e^{+}$ source. The downtime (column 3) has gone from 15\% to $46-48 \%$ and the time spent integrating luminosity (column 5) has dropped precipitously from about $75 \%$ to $31 \%$, or from $65 \%$ to $21 \%$ if the $10 \%$ downtime reserved for contingency is also included. This low an efficiency effectively means the detector gets no useful luminosity. Changing from an undulator to a conventional $e^{+}$source makes a dramatic difference. Run Warm5 (Cold7) depicts the LC with a conventional positron source during the commissioning period. The fraction of time spent integrating luminosity improves considerably to over $64 \%$ instead of $31 \%$ ( $54 \%$ including contingency).

This commissioning scenario demonstrates that an LC (warm or cold) should start with a conventional positron source and only switch to an undulator source as an upgrade after the accelerator has been running for several years. Otherwise, the loss in integrated luminosity is at least a factor of two.

The next three sensitivity studies were only done for the warm design as the results were expected to be very similar for the cold design. Run Warm6 explores the sensitivity of the simulation (and presumably the accelerator) to the recovery time. It is the same as Warm2 but the recovery and tuning after a downtime take half as long. The downtime decreased from $15 \%$ to $10 \%$ which is a significant change. Achieving a short recovery time is worth considerable effort. This effort could include: controlling temperatures so they are the same during running and during access; automated setup and tuning procedures and feedbacks; careful checks and crosschecks and procedures for work that is done during an access; diagnostics to find what is broken without beam; and magnets which are covered so they can remain on during access.

Run Warm7 checks the effect of increasing the cooldown time from 1 hour to 3 hours. Otherwise, it is the same as Warm2. This change may be necessary if the radioactive air from the tunnel is going to be exhausted into a populated area (as assumed in the TESLA TDR). The downtime increased from $15 \%$ to $16 \%$ indicating that this is a small but noticeable effect, probably worth further study.

Run Warm 8 checks the effect of decreasing the MTTRs by a factor of two. The downtime goes from $15 \%$ to $9 \%$ indicating that decreasing the mean time to repair is another very worthwhile effort.

Run Cold8 documents the effect of putting the cold damping ring in its own tunnel (the warm DR is always in its own tunnel). It has the same run conditions as Cold2 except that the DR is in its own PPS zone, which makes it possible to have beam in the DR while people are in the linac tunnel. Downtime decreased by only $0.5 \%$. This is a surprisingly small effect and merits further study to make sure nothing important has been missed. 


\begin{tabular}{|c|c|c|c|c|c|c|c|c|c|}
\hline 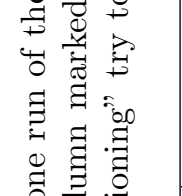 & 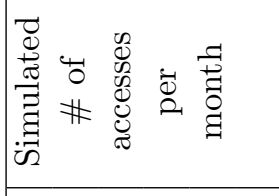 & 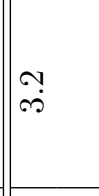 & $\stackrel{\overrightarrow{+}}{\longrightarrow}$ & 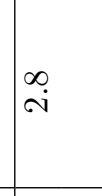 & $\vec{i}$ & $\underset{\dot{\theta}}{\dot{O}}$ & $\stackrel{\sim}{+}$ & $\begin{array}{l}\tilde{b} \\
\dot{b}\end{array}$ & $\begin{array}{l}\infty \\
\infty \\
\dot{\infty}\end{array}$ \\
\hline 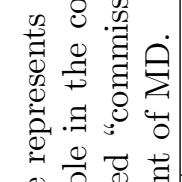 & 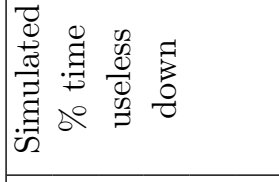 & $\stackrel{\stackrel{a}{\mathcal{H}}}{\stackrel{H}{H}}$ & $\begin{array}{l}L \rho \\
\stackrel{0}{0}\end{array}$ & $\stackrel{r}{r}$ & $\stackrel{0}{\dot{\rho}}$ & $\stackrel{20}{\Gamma}$ & $\begin{array}{l}0 \\
\dot{S} \\
\dot{q}\end{array}$ & ָ̃ & $\begin{array}{l}0 \\
\stackrel{I}{I}\end{array}$ \\
\hline 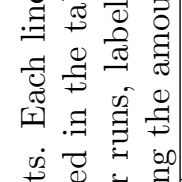 & 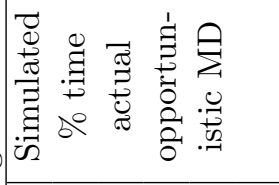 & $\mid \begin{array}{l}0 \\
\dot{\infty}\end{array}$ & 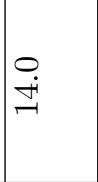 & م. & $\ddot{\infty}$ & $\stackrel{10}{-10}$ & $\stackrel{\tilde{N}}{\sim}$ & $\stackrel{0}{0}$ & $\stackrel{\vec{H}}{\text { ì }}$ \\
\hline 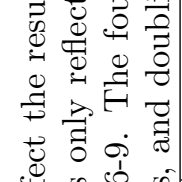 & 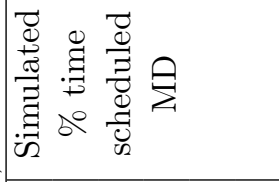 & $\underset{\stackrel{H}{H}}{\stackrel{H}{N}}$ & 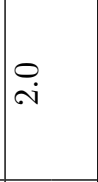 & $\stackrel{0}{\circ}$ & $\hat{\sigma}$ & $\stackrel{10}{=}$ & $\stackrel{\Re}{\stackrel{\sim}{*}}$ & $\stackrel{\vec{H}}{+}$ & $\stackrel{0}{0}$ \\
\hline 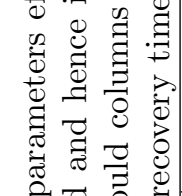 & 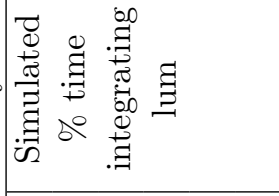 & $\stackrel{\leftrightarrow}{-\infty}$ & $\mid \begin{array}{l}10 \\
10\end{array}$ & 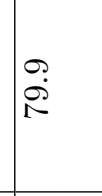 & $\underset{I}{\stackrel{0}{+}}$ & $\begin{array}{l}10 \\
0 \\
0\end{array}$ & $\frac{\dot{m}}{\dot{m}}$ & $\begin{array}{l}\infty \\
\dot{0}\end{array}$ & $\frac{10}{10}$ \\
\hline 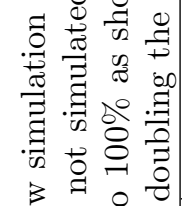 & 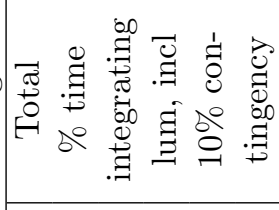 & $\| \stackrel{\Re}{\vec{A}}$ & 先 & $\begin{array}{l}\dot{\theta} \\
\dot{\theta}\end{array}$ & $\underset{\dot{+}}{0}$ & $\begin{array}{l}10 \\
80\end{array}$ & $\stackrel{0}{\stackrel{\dot{A}}{N}}$ & $\begin{array}{l}\infty \\
\dot{0} \\
\text { i. }\end{array}$ & $\stackrel{4}{4}$ \\
\hline 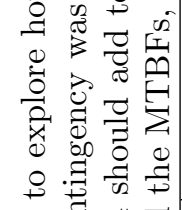 & 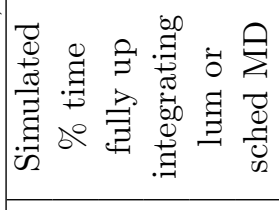 & $\mid \begin{array}{l}\infty \\
\text { in } \\
\text { i. }\end{array}$ & $\begin{array}{l}20 \\
80 \\
08\end{array}$ & $\begin{array}{l}\dot{\rho} \\
\dot{\infty}\end{array}$ & $\begin{array}{l}\tilde{\infty} \\
\ddot{\infty}\end{array}$ & $\frac{0}{\dot{\sigma}}$ & 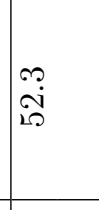 & $\begin{array}{l}\tilde{y} \\
\infty \\
0\end{array}$ & $\begin{array}{l}0 \\
18 \\
\infty\end{array}$ \\
\hline 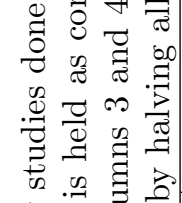 & 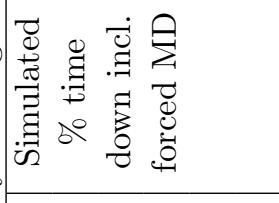 & $\mid \begin{array}{l}\mathfrak{o} \\
\dot{f}\end{array}$ & مُ & $\stackrel{-1}{\circ}$ & $\begin{array}{l}\mathscr{\theta} \\
\mathscr{\varphi}\end{array}$ & $\stackrel{0}{0}$ & \begin{tabular}{r}
$\sim$ \\
\multirow{r}{*}{}
\end{tabular} & $\infty$ & $\begin{array}{l}0 \\
10 \\
-10\end{array}$ \\
\hline 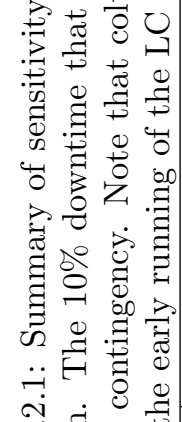 & 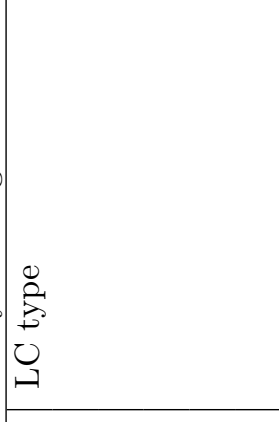 & 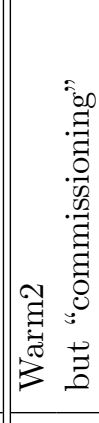 & 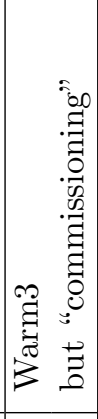 & 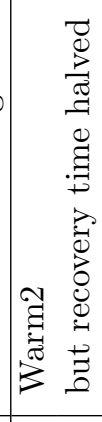 & 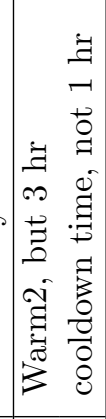 & 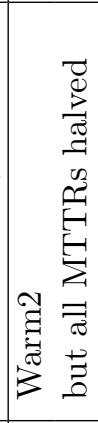 & 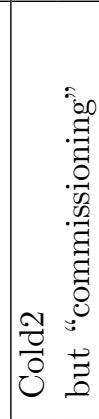 & 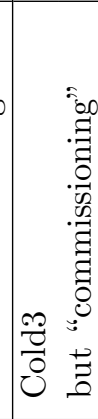 & 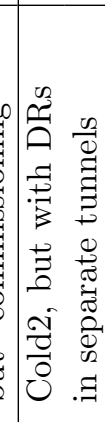 \\
\hline 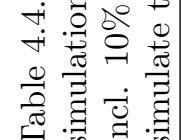 & 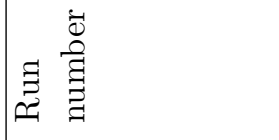 & 节 & 赵 & : & בै: & $\underset{\infty}{\infty}$ & $\frac{\pi}{0}$ & ن & ঠo \\
\hline
\end{tabular}




\subsubsection{Estimates of repair personnel}

The simulations also explored how many people are necessary for accelerator repairs. Three classes of people were included:

1. Those who go into the accelerator enclosure to make repairs. This was limited to 50 in all the simulations.

2. Those who make repairs to devices outside the accelerator enclosure that can only be done when the accelerator is down. This was limited to 100 in all the simulations.

3. Those who make repairs to devices that are hot swappable. This was limited to 50 in all the simulations.

These numbers are for personnel required at the time of the repairs. The number of available people was chosen to be large for the simulations so that limited manpower would not degrade the availability. To see how many people are actually needed, the simulation has been run repeatedly for different numbers of staff people. This was done individually for each of the three classes of people. Figure 4.4.3.1 shows these curves for people who go into the accelerator tunnel for each of the three baseline accelerators. Table 4.4.3.1 summarizes the number of staff needed on shift by giving how many people of each class are needed to keep the downtime only $1 \%$ above its minimum value (when there are a very large number of people). Very few people of class 2 are needed as most devices outside the accelerator tunnel are hot swappable and most of the remaining devices completely break the accelerator (which means they get repaired immediately and hence are not queued up for repair when something else breaks).

The numbers from Table 4.4.3.1 cannot be directly used to determine the required staffing level at the future LC laboratory. Firstly, the simulation assumes the people are available 24/7. That requires five employees for each one on shift. Secondly, the people who repair hot swappable devices could probably be used to perform the downtime tasks. Thirdly, there are many activities that are not simulated which occur on a down day which also require people. Examples include minor upgrades, preventive maintenance, and minor repairs which don't degrade the accelerator performance but should be fixed (e.g. a small water leak).

Table 4.4.3.1: The number of each class of person that must be available 24 hours a day to avoid degrading the downtime by one percent.

\begin{tabular}{|l|c|c|c|}
\hline Class of people & Warm 2 tunnel & Cold 2 tunnel & Cold 1 tunnel \\
\hline \hline In accelerator tunnel & 4 & 6 & 25 \\
\hline Outside accelerator tunnel & 4 & 4 & 4 \\
\hline Repair hot swappable devices & 6 & 2 & Not simulated \\
\hline
\end{tabular}




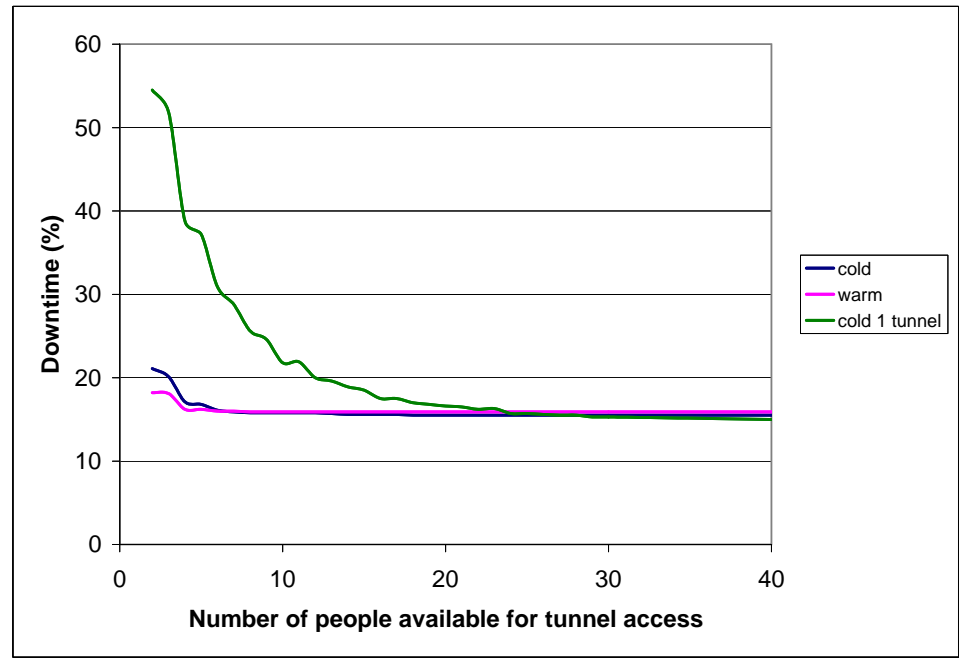

Figure 4.4.3.1: Downtime as a function of the number of maintenance people available to enter the accelerator tunnel to perform repairs.

\subsection{Summary}

A simulation has been developed which calculates the overall downtime of an accelerator given the reliability characteristics of its parts. This has been used to develop a detailed availability budget for the linac and damping rings and a less detailed budget for the other regions of the LC.

This budget is very much a first iteration. The allocation of the unavailability is not fully optimized. However, the budget does give an indication of the level of difficulty that will be involved to make the LC sufficiently reliable. The tools developed are very useful and should continue to be used in parallel with actual reliability engineering to refine this first iteration. It would also be useful to benchmark the results against a real accelerator.

The power of the simulation really shows up when comparing different versions of the accelerator. These comparisons shouldn't depend heavily on the details of the availability budget. The comparisons reveal:

1. The fact that an undulator positron source requires well tuned high energy electrons before positrons can be produced significantly reduces the integrated luminosity of a LC. For example in the warm LC after a few years of running, the luminosity integrated in a year would be $18 \%$ less for an undulator positron source than for a conventional one.

2. During commissioning, it would be far worse; a factor of two less luminosity would be integrated.

3. There is not a great difference between warm and cold 2 tunnel designs. Both are very large and complex accelerators where significant effort and expense will be needed to make them reliable enough.

4. If all components had the same reliability, the cold 1 tunnel design would have a downtime of $25 \%$ instead of the $15 \%$ of the 2 tunnel design. The required improvements to Mean Time To Failures (MTBFs) of many components and the necessary $5 \%$ increase in the energy overhead needed to recover the $15 \%$ availability are costly. This cost should be compared to the amount saved in building one tunnel instead of two to see if it is truly worthwhile. 
5. The effects of Mean Time To Repair (MTTR) and the speed of recovery from a downtime are quite significant. It will be worth considerable design effort to keep these small. Success at this could reduce the requirements on the MTBFs

\subsection{Addendum: Component descriptions}

The linacs and damping rings were divided into many components. Below is a description of these components and the nominal MTBFs and MTTRs (in hours) that were used as starting values in the simulation.

Magnets - includes the magnet itself, interlocks, water connections and cables from power supply to the magnet, but not the power supply or its controller. All magnets require access to repair and most failures stop operation. Exceptions are correctors or trim windings where it is assumed one can tune around scattered failures. It is also assumed that one can match around quadrupoles in the cold main linac, which has relatively weak focusing. The luminosity degradation due to a matched around quad or corrector is $1 \%$, and $0.1 \%$ for a trim.

General magnet - MTBF: $1 \times 10^{6}$ (Water cooled) $1 \times 10^{7}$ (superconducting), $1 \times 10^{7}$ (permanent magnet)

General magnet - MTTR: 8(repair) 2(quad retune) 472(repair SC quad later)

Correctors \& trims - MTBF: $1 \times 10^{7}$ MTTR: 0.5 (retune) 2 (later repair)

Kickers - in the cold damping rings, injection or extraction requires 20 out of 21 kickers so two failures are required before stopping operation. Kickers for tuneup dumps are considered part of diagnostics.

Kicker magnet - MTBF: $1 \times 10^{5}$ MTTR: 8

Kicker pulser - MTBF: $1 \times 10^{4}$ MTTR: 2

PS + controllers - power supplies and their controllers are generally installed in the support housing and do not require access for repair, except for the one tunnel variant of the cold machine where most are inaccessible during operation. Their impact depends on the type of magnet controlled as discussed under magnets. Since these are a well identified reliability risk, redundancy is already incorporated in the designs. Cold linac and DR magnets have 4 of 5 redundancy. Most warm linac and DR magnets have 1 of 2 redundancy. This raises the estimated MTBF to $2 \times 10^{5}$ from $5 \times 10^{4}$ experienced at the labs.

Power supply - MTBF: $2 \times 10^{5}$ (redundant) MTTR: 2 (normal) 4 (large)

Controller - MTBF: $1 \times 10^{5}$ MTTR: 1

Movers - movers are installed on the beamline and require access to repair but it is assumed one can tune around failed movers. The luminosity degradation due to a matched around mover is $0.1 \%$. Mover controllers are 16 channels, accessible and hot swappable.

Mover - MTBF: $5 \times 10^{5}$ MTTR: 0.5 (retune) 2 (repair later)

Controller - MTBF: $1 \times 10^{5}$ MTTR: 1

Magnet flow switches - water cooling systems for electromagnets require flow switches. For the warm linac and cold DR straights, there is assumed to be a flow switch every $100 \mathrm{~m}$. For the warm DR and cold DR arcs, there is a flow switch every 2 girders or approximately $10 \mathrm{~m}$. These are in the tunnel and require access for repair. 
Water flow switch - MTBF: $2.5 \times 10^{5}$ MTTR:1

Vacuum mechanical device - this covers any device in vacuum which moves into the beamline such as stoppers or profile monitors. Since they are on the beamline, access is required for repair.

Vacuum device - MTBF: $1 \times 10^{5}$ MTTR: 8

Vacuum pumps and PS - The beamlines have vacuum pumps about every $100 \mathrm{~m}$ in the warm regions and and one per 9 cryomodules in the cold linacs. (During the study, one pump per module was used in error. This has little effect on the simulation results). The pumps are in the tunnel and require access to repair; the power supplies are in the support housing (except for 1 tunnel variant). Since the systems have a certain amount of redundant pumping, the luminosity is not degraded when a pump fails.

Vacuum pump - MTBF: $1 \times 10^{7}$ MTTR: 4

Vacuum pump power supply - MTBF: $1 \times 10^{5}$ MTTR: 1

Vacuum valves and controllers - There are assumed to be vacuum valves every $200 \mathrm{~m}$ in all regions. This number was chosen as a reasonable guess as the published numbers for the cold linac were too small to be reasonable and for the cold DR too large. A valve failure is assumed to interrupt operation (a valve stuck out would likely not be noticed). Valves require access to repair, controllers are in the support housing (except 1 tunnel).

Vacuum valve - MTBF: $1 \times 10^{6}$ MTTR: 4

Vacuum valve controller - MTBF: $1.9 \times 10^{5}$ MTTR: 2

Beamline water systems - These systems supply water to beamline components. Each pump has associated instrumentation and a flow switch. All systems are assumed to be accessible for repair (except 1 tunnel). In the DRs and cold linacs, water pump failures turn off the machine. In the warm linacs, most of the pumps supply water to the structures and hence only reduce the energy overhead and can be replaced during operation.

Water pump - MTBF: $1.2 \times 10^{5}$ MTTR: 4

Water pump instrumentation - MTBF: $3 \times 10^{4}$ MTTR: 2

Water flow switch - MTBF: $2.5 \times 10^{5}$ MTTR: 1

Beam position monitors - Failure of the physical cavity or stripline monitor are extremely rare and ignored. Only readout module failures are included. These are in the support housing (except 1 tunnel) and hot swappable during operation. Luminosity degradation is assumed to be $0.1 \%$ per failed unit.

BPM - MTBF: $1 \times 10^{5}$ MTTR: 1

Wire scanners (laser and conventional) - Failures are assumed to be in the instrumentation or in the laser itself, all accessible during operation. Luminosity degradation for laser wires is $5 \%$ as they are critical for tuning. Loss of conventional wires does not degrade luminosity.

Wire scanner - MTBF: $1 \times 10^{5}$ MTTR: 2 
Tuneup Kickers - Like laser wires, these are important for efficient tuning. The kickers are in the tunnel and require access, the pulsers are in the support housing. Luminosity degradation is taken to be $5 \%$.

Kicker - MTBF: $1 \times 10^{5}$ MTTR: 8

Kicker pulser - MTBF: $1 \times 10^{4}$ MTTR: 2

RF power sources - The RF power sources for DRs and linac structures and cavities are klystrons powered by a modulator for the linacs or by a power supply for the DRs. Each klystron has a pre-amplifier, vacuum gauge/controller, vacuum pump and pump power supply. All of these are accessible for repair and hot swappable, except for the 1 tunnel cold machine where the linac klystrons and all associated devices are installed in the tunnel. While MTBFs for klystrons and modulators vary slightly, the other components are similar so they are described here only once. When a klystron vacuum pump fails, the klystron must be replaced, so the MTTR is long.

Klystron - MTBF: $3 \times 10^{4}$ (DRs) $2.5 \times 10^{4}$ (warm linac) $4 \times 10^{4}$ (cold linac) MTTR: 8

Modulator - MTBF: $5 \times 10^{4}$ (cold linac) $1 \times 10^{5}$ (warm linac) MTTR: 4

DR Klystron power supply - MTBF: $5 \times 10^{4}$ MTTR: 4

LLRF controls - MTBF: $1 \times 10^{5}$ MTTR: 1

Klystron pre-amp - MTBF: $1 \times 10^{5}$ MTTR: 1

Klystron vacuum gauge - MTBF: $1 \times 10^{5}$ MTTR: 1

Klystron vacuum pump - MTBF: $1 \times 10^{7}$ MTTR: 8

Klystron pump power supply - MTBF: $1 \times 10^{5}$ MTTR: 1

RF cavities (warm DR) - There are 5 normal conducting cavities in each DR of the warm machine, of which only 4 are required for operation. Each cavity has LLRF controls. The cavity is on the beamline and requires access to repair. The controls are accessible and hot swappable. Each DR cavity is powered by a klystron. Loss of the power source turns off the associated cavity, but does not interrupt operation unless there are two failures at once.

Cavity - MTBF: $1 \times 10^{8}$ MTTR: 24

RF cavities (cold DR) - There are 12 superconducting cavities in each DR of the cold machine, of which only 11 are required for operation. Each cavity has LLRF controls. Four cavities are in a single cryomodule, fed from a single power coupler which has interlock sensors, coupler interlock controls and two vacuum pumps with two power supplies, all of which are required for cavity operation. The cavity, coupler and pumps are on the beamline and require access to repair. The controls and pump supply are accessible (except for 1 tunnel). The cryo system has a cryo vacuum enclosure which may develop minor leaks or more serious problems. Each DR 4-cavity cryomodule is powered by a klystron. Loss of a power source turns off the associated 4 cavities, which interrupts operation.

Cavity - MTBF: $1 \times 10^{8}$ MTTR: 72

Coupler - MTBF: $1 \times 10^{7}$ MTTR: 16

Coupler interlock sensors - MTBF: $1 \times 10^{5}$ MTTR: 1

Coupler interlock controls - MTBF: $1 \times 10^{5}$ MTTR: 1

Coupler vacuum pump - MTBF: $1 \times 10^{7}$ MTTR: 4

Coupler pump power supply - MTBF: $1 \times 10^{5}$ MTTR: 1

Cryo vacuum leak - MTBF: $1 \times 10^{5}$ MTTR: 8

Cryo vacuum failure - MTBF: $3 \times 10^{5}$ MTTR: 8 
Water system for DR klystrons - The DR klystron cooling water system has a water pump and instrumentation. There is one system per ring for the warm machine and one per 4-cavity cryomodule for the cold machine. There are two flow switches per pump on the cold machine and one per klystron or cavity on the warm. The pumps and instrumentation are accessible as are the flow switches. A water pump outage removes enough energy to interrupt operation, but the warm machine only loses one klystron/cavity pair when a flow switch fails.

Water pump - MTBF: $1.2 \times 10^{5}$ MTTR: 4

Water pump instrumentation - MTBF: $3 \times 10^{4}$ MTTR: 2

Water flow switch - MTBF: $2.5 \times 10^{5}$ MTTR: 1

RF power sources and structures (warm linac) - In the warm main linac, an RF unit consists of a pair of klystrons fed from a single modulator with a single LLRF controller. Each RF unit feeds two SLED systems, which power 16 accelerator structures. Each klystron has a pre-amplifier, flow switch, vacuum gauge/controller, vacuum pump and pump power supply. The two SLED systems together have about 100 vacuum pumps driven by 5 20-channel pump controller chassis. Cooling water is supplied by one water pump with instrumentation per $16 \mathrm{RF}$ units. All parts are accessible and hot swappable, except the structures.

SLEDs - MTBF: $1 \times 10^{5}$ MTTR: 4

SLED vacuum pump - MTBF: $1 \times 10^{7}$ MTTR: 4

SLED vacuum pump chassis - MTBF: $1 \times 10^{5}$ MTTR: 1

Structure - MTBF: $1 \times 10^{8}$ MTTR: 168 (1 week)

RF power sources (cold linac) - In the cold main linac, an RF unit consists of a modulator powering a klystron. Each RF unit feeds 2.5 cryomodules, each containing 12 9-cell cavities. Each klystron has a pre-amplifier, flow switch, vacuum gauge/controller, vacuum pump and pump power supply. Cooling water is supplied by one water pump with instrumentation per $2 \mathrm{RF}$ units. In the two tunnel layout, all parts are accessible and hot swappable, except the cryomodules. In the single tunnel layout, only the modulator is accessible. Everything else is located in the accelerator housing and requires access for repair. In addition, the klystron in the single tunnel is connected to the modulator by high power pulsed cables that are up to $2.5 \mathrm{~km}$ long, with a pulse transformer located next to the klystron. The MTBF is taken from experience with electrical power distribution cables (unpulsed but similar voltage). While there are spare cables, they must be routed to the correct RF unit so the MTTR is also long.

Pulsed cables (1 tunnel) - MTBF: $2 \times 10^{5}$ MTTR: 8

Pulse transformer (1 tunnel) - MTBF: $2 \times 10^{5}$ MTTR: 4

RF cavities (cold linac) - In the cold main linac, there are 12 9-cell cavities per cryomodule, and a total of 30 cavities per klystron/modulator RF unit. Since the cavities, tuners and couplers are in cryomodules, repairs require warmup of the cryo string and hence are only feasible during the yearly shutdown. Rather than turn off the entire RF unit when one cavity has a problem, there are a number of possible softer failure modes. Because of the complexity of the possible responses, many of these failure modes have been modelled individually.

Cavity gradient - If the cavity trips at full gradient, the response is to lower the gradient for just that cavity up to $10 \mathrm{MV} / \mathrm{m}$ by a combination of detuning and adjustments to the 3 stub tuner. If the cavity cannot stay on at lower gradient, the RF unit is turned off until the next access when the individual cavity can be disconnected. 
Cavity gradient degraded (average loss $8 \mathrm{MeV}$ ) - MTBF: $1 \times 10^{8}$ MTTR: 672 (4 wks)

Cavity broken/disconnected (loss $29 \mathrm{MeV}$ ) - MTBF: $1 \times 10^{8}$ MTTR: 672 (4 wks)

Cavity tuner and drive - The tuner is required to keep the cavity on resonance and compensate for pressure and thermal changes. It is in the cryomodule so repair is long. Failures might not affect performance for a while but eventually the cavity would have to be disconnected to prevent errant behavior.

Cavity tuner and drive (loss $29 \mathrm{MeV})-\mathrm{MTBF}$ : $1 \times 10^{6}$ MTTR: 672 (4 wks)

Piezo tuner - The piezo tuner is required to compensate for Lorentz force detuning at gradients above 24 $\mathrm{MV} / \mathrm{m}$. For redundancy, two tuners per cavity are planned. Failures require lowering gradient to 24 $\mathrm{MV} / \mathrm{m}$. At a nominal gradient of $28 \mathrm{MV} / \mathrm{m}$, this can be done for a single cavity. For operation at $35 \mathrm{MV} / \mathrm{m}$, either all cavities would need to operate at somewhat lower gradient or the coupler for the failed cavity would have to be disconnected.

Cavity piezo tuner (loss $5 \mathrm{MeV}$ ) - MTBF: $5 \times 10^{6}$ MTTR: 672 (4 wks)

LLRF - Complex LLRF algorithms are required to protect the cavity. Failure means that cavity must be detuned.

LLRF (loss $29 \mathrm{MeV}$ ) - MTBF: $1 \times 10^{5}$ MTTR: 1

Coupler breakdown - If the coupler shows breakdown activity, the gradient must be lowered. A temporary fix without access is to lower the gradient for the entire RF unit. If the problem is not too serious, lowering to $20 \mathrm{MV} / \mathrm{m}$ should be adequate. For serious failures, the entire RF unit must be turned off. During the next access, the single cavity can be disconnected and full power RF restored to remaining cavities. The coupler is in the cryomodule so repairs require a long shutdown.

Coupler problem (minor) (average loss $240 \mathrm{MeV}$ ) - MTBF: $1 \times 10^{7}$ MTTR: 2 (disconnect)

Coupler problem (major) (loss $872 \mathrm{MeV}$ ) - MTBF: $1 \times 10^{7}$ MTTR: 2 (disconnect)

Coupler repair - MTTR: $672(4 \mathrm{wks})$

Coupler interlocks - Both the input and HOM couplers are instrumented with a variety of sensors and interlocks. Some sensors will be redundant and some cross checks with other sensors may be used to backup a failed sensor. If there is no workaround for the failed sensors, the klystron must be turned off. The sensors are on the cryomodule and require access but not warm-up for repair, the electronic readout for the sensors is in the support tunnel, except for 1 tunnel variant. Long MTBFs are used to account for the redundancy that is assumed.

Coupler interlock sensors (loss $872 \mathrm{MeV}$ ) - MTBF: $1 \times 10^{6}$ MTTR: 1

Coupler interlock electronics (loss $872 \mathrm{MeV}$ ) - MTBF: $1 \times 10^{6}$ MTTR: 1

Coupler vacuum pump - There is a vacuum manifold for each cryomodule to distribute vacuum to all the couplers. Only a single pump is required but two pumps and power supplies are installed for redundancy. Failures require the entire RF unit to be turned off. The pumps are in the accelerator housing and require access to repair. The pump power supplies are in the support tunnel (except with 1 tunnel). The redundant pump/supply MTBFs are assumed to be 10 times nominal. 
Coupler vacuum pump (loss $872 \mathrm{MeV}$ ) - MTBF: $1 \times 10^{8}$ MTTR: 4

Coupler pump power supply (loss $872 \mathrm{MeV}$ ) - MTBF: $1 \times 10^{6}$ MTTR: 1

Linac cryomodules (cold linac) - In the cold main linac, each cryo plant feeds two cryo units (each about $2.5 \mathrm{~km}$ long). Insulating vacuum is divided into 5 sections per cryo unit (each about $500 \mathrm{~m} \mathrm{long}$ ). These sections are separated by vacuum barriers. Small leaks to the insulating vacuum may be overcome by hooking up a local turbo pump during an access. Major problems require warmup. 10 cryomodules are grouped into a cryostring with valves and controls at the ends of the string, in particular a JT valve. Repairs to any of these systems require access. Failures require that the entire cryo system affected be turned off. The JT valve has a relatively short MTTR as it is assumed there will be some workaround short of replacement.

Cryo vacuum enclosure (no luminosity) - MTBF: $3 \times 10^{5}$ MTTR: 8

Cryo insulating vacuum (loss $3488 \mathrm{MeV}$ ) - MTBF: $1 \times 10^{5}$ MTTR: 8

Cryo JT valve (loss $3488 \mathrm{MeV}$ ) - MTBF: $3 \times 10^{5}$ MTTR: 2 (workaround)

Electrical distribution - A simple model was used to develop a rough count of breakers in the electrical distribution system. High power breakers (>0.5 MW) were assigned for the RF units. Lower power breakers $(50 \mathrm{KW}-0.5 \mathrm{MW})$ were assigned for magnets, pumps, and controls. In the DRs, there is a high power circuit to drive each klystron and a low power circuit per klystron for controls. In the warm linac, there is a high power circuit per four klystrons and one per two klystrons in the cold linac. There are also an equal number of control circuits. Low power control circuits were assigned for every 10 magnets, for each structure water pump in the warm linac, and for each controls sector.

High power electrical circuit - MTBF: $3.6 \times 10^{5}$ MTTR: 4

Low power electrical circuit - MTBF: $3.6 \times 10^{5}$ MTTR: 2

Controls, MPS and PPS - A simple model was used to develop a rough count of units for the control system, Machine Protection and Personnel Protection. First, failures of these systems which affect the entire complex were assigned an overall downtime of $0.2 \%$. This downtime was treated explicitly like site power or cryogenics, rather than assigned to a particular region. The controls system was assumed to have a communications backbone every $200 \mathrm{~m}$, designated a "sector". In the linacs, each sector also has a timing distribution associated with it. Each sector is then subdivided into local backbone units (logically crates). These are assigned 1 per klystron in the linacs, and 1 per 10 magnets in the DRs. Individual controllers for magnets, movers and LLRF have been included earlier. In addition, each klystron is assumed to have a local timing distribution and 3 more controls modules associated with it. For Personnel Protection, there is assumed to be a circuit for each entry point, 5 in the linacs, 1 for the warm DR, 2 for the 2 arcs of the cold DR. Global communications within a region such as Machine Protection, Fast Feedforward on beam current in the linacs, and fast feedback in the DRs were counted as two network circuits per region. Failures at the backbone level or higher interrupt operation in that region. Klystron control failures only take the affected units offline. All of these controls are accessible for repair without access, except for the single tunnel linac where the sector and local controls are in the accelerator tunnel.

Sitewide controls, MPS, PPS - MTBF: $2.5 \times 10^{3}$ MTTR: 5

Sector controls backbone - MTBF: $1 \times 10^{6}$ MTTR: 2

Sector timing distribution - MTBF: $1 \times 10^{5}$ MTTR: 1

Local controls backbone - MTBF: $1 \times 10^{5}$ MTTR: 1

Local timing distribution - MTBF: $1 \times 10^{5}$ MTTR: 1 
Local klystron controls - MTBF: $1 \times 10^{5}$ MTTR: 1

PPS controls - MTBF: $1 \times 10^{5}$ MTTR: 1

MPS, Fast Feedforward, Fast Feedback networks - MTBF: $5 \times 10^{3}$ MTTR:1 


\section{Chapter 5}

\section{Cost and Schedule}

\subsection{Goals, Strategy, and Overview}

We report a comparison of expected costs and schedules for completion of a collider based on the warm and cold U.S. reference designs. This is a report of a "study", not new work. We did not set out to make new estimates for either technology, but to provide a level comparison of the two options. We have relied on the large body of work done by the TESLA[TDR] and GLC/NLC Collaborations[NLC01, GLC] on cost estimation and project planning. We worked closely with these collaborations to make certain we have understood and used the available information properly, but we did not verify the cost estimates presented by these groups. At the request of Fermilab Director Michael Witherell, a team of engineers and scientists from major U.S. laboratories recently reviewed the TESLA project[66]; we have extensively used the results and conclusions of that work. Information from other accelerator projects in High Energy Physics and Basic Energy Sciences has also been used when appropriate.

Many components and systems of a linear collider do not depend on the choice of main linac technology. We have used identical technical models and cost estimates for as many of these common elements as possible. These include such items as unit costs for vacuum components, instrumentation and controls, electrical power systems, water systems, and civil construction. We have also used identical cost estimates for those parts of the machine that do not depend on the main linac technology choice - particularly the injectors (but not the damping rings) and beam delivery systems. More generally, the work and cost breakdown for the R\&D, engineering and design, fabrication, installation, and commissioning of a collider is quite similar for the two technology options. We have used a common work breakdown structure (WBS) to capture these similarities and simplify comparisons. This includes adoption of identical models for items like project management and oversight, business services and support, site management, commissioning and pre-operations, and labor rates and regulations.

Our study is based on a Total Project Cost (TPC) using U.S. Department of Energy (DOE) project accounting standards[67]. The WBS used for these studies was previously reviewed by a committee[68] convened by the DOE and found to include all items necessary in a TPC. However, costs for land acquisition, experimental detectors, escalation, and contingency were omitted from this exercise.

It is known from previous studies that the cost breakdown for a collider will consist very roughly of equal parts for purchase of technical components from industrial vendors, conventional civil construction and supporting infrastructure, and "everything else" taken together. The difference in the costs for the two technology options is primarily in three factors: purchase of the components of the main linac (including power and cooling), civil construction of the greater length of housings needed for the cold main linac, and components for the larger damping rings needed to support the longer bunch train of the cold machine. We 
have reviewed and adopted the estimates supplied by the TESLA and GLC/NLC Collaborations for the prices expected to be charged by industrial suppliers for the components of the main linac (in the required quantities). All other costs associated with the main linac (e.g., R\&D, engineering and design, component handling, installation, and quality control) are treated on an equal footing for the warm and cold options. Costs for civil construction and infrastructure have been estimated by us for both warm and cold machines built in the U.S. according to U.S. codes and restrictions. This includes the refrigeration plant and cryogenic distribution external to the cryomodules for the cold option. Estimates were made for reference sites in Illinois and California, and averages taken to produce realistic, but site independent, estimates for each technology. We found it necessary to re-estimate the cost of the damping rings for the cold technology choice to account for differences in the technical specifications of the U.S. cold reference design from those in the TESLA TDR.

We do not present absolute cost estimates in this report. Our results are presented in two forms. Relative fractions of the total project cost are given for various break-outs of the cost for each technology option. This serves to provide both an internal picture of the cost structure and a check on biases between the cost estimates for the two technology choices. We also provide comparison of the relative costs for the two technology choices - for this we choose to normalize to the estimated total for the warm technology.

\subsection{Technical Models and Cost Estimation}

\subsubsection{Work Breakdown Structure}

\subsubsection{General Considerations}

The first step in developing estimates for both colliders was establishing a common work breakdown structure (WBS). A WBS is a list of all the activities required to construct the collider, including the management, planning, R\&D, and design activities necessary to support the design, manufacture, installation, and commissioning functions. The existing WBS structures for the TESLA and NLC designs were compared, and a structure that would maximize the use of the available information was selected.

The second step was to populate the common elements of the WBS for both machines with the identical estimates for parts and labor wherever possible. The approach taken was that equal estimates were used unless a clean and crisp difference could be established. There are a number of cases where the choice of main linac technology will create other technical differences that have insignificant implications for cost. The IP beam feedback is an example. We have ignored these cost differences in our model.

The third step was to select a software tool for the construction of the WBS lists. MS Excel while ubiquitous requires the model logic to be constructed along with the data so it is labor-intensive and prone to contain errors. WBS software for actual projects is designed to be very detailed and powerful but not flexible, dynamic, and nimble. We selected a very simple, no-frills, easy-to-use, and robust piece of Java software that had been written at SLAC just for the purpose of aggregating WBS costs. The database is kept under password control.

\subsubsection{Structuring the WBS}

For estimating, the colliders were broken up into four major functional machine areas. The Injector Systems create the bunch train and condition it so that it is ready for the Main Linacs. This includes the beam sources, damping rings, and bunch compressors, and extends to the $5 \mathrm{GeV}$ point for the cold LC, and 8 $\mathrm{GeV}$ for the warm. The Main Linacs accelerate this beam to the physical point that it would be passed to the Beam Delivery system in a $1 \mathrm{TeV}$ design (in the $500 \mathrm{GeV}$ version the second half of the Main Linacs 
is just a transport line). The Beam Delivery has no active RF other than the crab cavities, and does the de-magnification and collimation necessary to get the bunch trains through the Interaction Points and into the beam dumps. The fourth machine area is Global Controls, which also contains the machine and personnel protection systems, but which does not include local process controls. The Civil Construction and Conventional Facilities associated with each of the above areas, excluding Global Controls, is integrated into the hardware costs for each area, so that "all" of the estimated costs associated with each major machine area can be seen.

There remain some Civil/Conventional Facility costs that cannot be meaningfully allocated to the machine areas-examples would be surface roads and Campus Buildings. These are collected in a separate WBS section. In addition, the Cryogenic System costs for the cold LC are also collected into a separate standalone WBS section since it was done that way in the TDR, and it is one of the fundamental warm/cold differences.

The balance of the WBS is ad hoc functions that are created just for the collider project. They are not components of the collider, and they do not remain around once the project is over. These functions are Project Management, Accelerator Physics, Pre-Operations, and centralized planning and support functions for design, logistics management, and installation.

Land acquisition, experimental detectors, escalation, and contingency were omitted from this exercise.

The format of the WBS is an indented set of numbered levels. A top-level view is shown in Fig. 5.2.1.1. The same WBS architecture and numbering was used for both the warm and cold options wherever possible, including putting in placeholders for missing elements so that the balance of the WBS numbering would remain identical. For example, the warm collider has a Cryogenic Systems placeholder to match the large system needed for the cold collider, and the cold collider has a placeholder for warm magnets even though the magnets are buried in the cryomodules in the cold design.

Finally, since there was an interest in establishing the cost of upgrading the colliders to $1 \mathrm{TeV}$, this incremental cost was built into the same software file alongside the WBS for the $500 \mathrm{GeV}$ colliders. By doing this, configuration control between the $500 \mathrm{GeV}$ and $1 \mathrm{TeV}$ versions is maintained.

\subsubsection{Populating the WBS}

The most recent cost estimates for cold and warm linear colliders came from the TESLA TDR and from internal NLC cost estimates and data respectively. These two sources are in different states of release. The TDR estimates have been published formally, so they are readily available, but they are aggregated at a high level-in some cases they are in $\$ 1 \mathrm{~B}$-sized lumps. The warm collider cost estimate has not been published so it lacks a TDR-equivalent "official" stamp, but it is available at a very detailed level.

More detailed information for the TDR design was requested from DESY, and was made available to us, but it is not possible to make this information public. Due to this, and the fact that the designs selected for this exercise do not match either the TDR or NLC designs (and the fact that the collider designs are really in a pre-conceptual design stage), it was decided to make only relative comparisons on cost and avoid any reference to absolute costs. However, absolute cost estimates for both colliders were compiled as the basis for the relative cost comparison.

For the estimates that were imported directly from the TESLA TDR, a ratio of 1:1 between the U.S. dollar and E.U. euro was used. This is an average of the exchange rate over the recent past.

Cost estimates for the 2003 version of the NLC were readily available. This was used as the basis for the warm collider costs, but these had to be adjusted for the difference between that design and the design being used in this exercise. The most obvious example is the inclusion of an undulator-based positron source instead of one based on conventional SLC-like technology. 


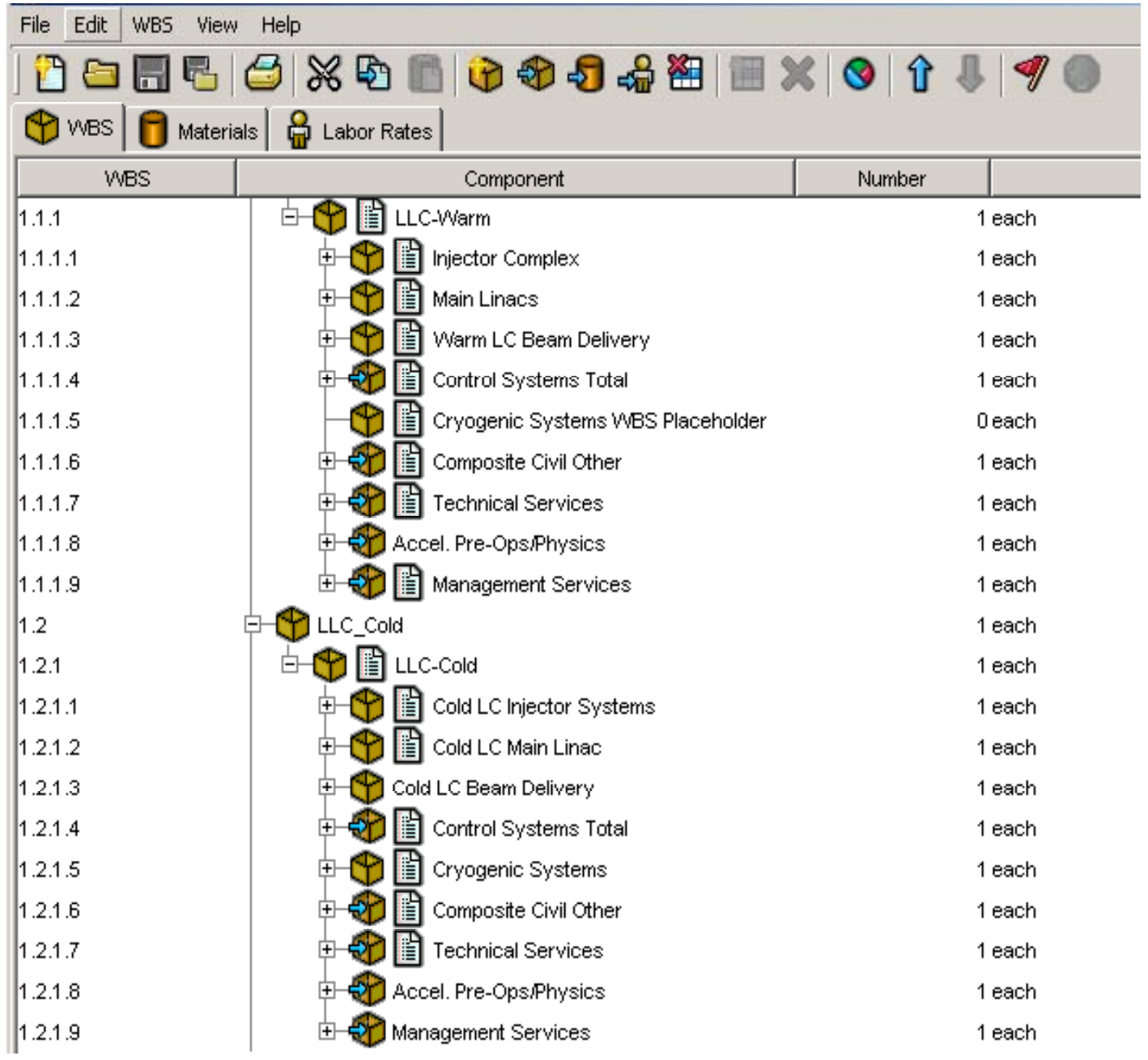

Figure 5.2.1.1: Top-level view of the WBS. There are approximately 3,400 line items in the WBS if it is displayed to its lowest level (11 levels).

Because the desire for commonality, costs for project-type activities, such as project management and accelerator physics, were taken from the warm collider estimate and applied identically (dollar for dollar) to the cold estimate. The cost necessary to design and engineer the items that end up on the parts list for the collider, termed EDIA in the U.S., were not fully included in the TDR as expensed costs. On a case-by-case (machine area by machine area) basis, either an independent estimate was utilized or the warm collider cost was utilized for these cost elements for the cold collider. If the warm cost was used, it was used dollar for dollar (not scaled for size or scope) with the exception of Conventional Facilities, where the EDIA costs were budgeted as a percentage of the cost of the work being performed to build the collider. If these costs were higher due to longer tunnels, then the EDIA costs were commensurately higher also. In cases in which an independent estimate was used (the best example is the cold Damping Ring estimate by LBNL), the EDIA estimate made by LBNL was utilized. We did not want the project costs to feature in any cost difference between the two technologies, and by using this methodology they have not.

A third category of costs is the labor and material costs associated with constructing, installing, and testing the systems that go into the collider. These costs were also not sufficiently detailed in the TDR. On a case- 
by-case basis, either the same basis as the warm machine was used (e.g., a percentage of the hardware costs), the same dollar value was used (e.g., Beam Delivery where the costs are identical for both technologies), or the information contained in the Fermilab study[66] of the TDR cost estimate was utilized (primarily in the Main Linac).

Common Source and Pre-Linac/Bunch Compressor costs were used wherever possible (such as polarized photocathode or thermionic guns); the pre-accelerators for the two options necessarily are different. The Damping Ring costs for the cold LC are not those of the TDR, but are the result of an independent estimate that we have made. Since the Cryogenic System differed significantly from that of the TDR, we made an independent estimate of its cost.

For the Main Linac, the TESLA TDR estimated costs were used for the cryomodules and the RF stations, which represent the total hardware cost for the linacs. Unfortunately, these costs are aggregated at a very high level, and no cost detail is visible. An alternate source[66] was used to expand the costs into the lower WBS levels. While the details of the TDR estimate were not shown in the Fermilab report[66], the cost details for the TESLA Test Facility (TTF) were shown. These TTF cost details were extrapolated downwards so that in total they matched the TDR estimates while still preserving the lower-level detail. Obviously this methodology is a problem from the perspective of any one specific part, but in the aggregate the costs are correct.

Technical differences in the Beam Delivery and Global Control systems needed for the two linac options produce insignificant cost differences, so we have used identical cost estimates for the two options.

Conventional Facilities Other (roads, campus buildings, etc.) were also set equal. For the Tunnel Boring Machine (TBM) cost for the Main Linac and Beam Delivery tunnels, and the road-header excavations in other underground areas, the cost per unit length or volume were set to the same value. Care was taken to eliminate any possible cost advantage or handicap due to the representative sites that were chosen for this study.

The present compilation of estimates is for a representative U.S. site.

\subsubsection{Project Activities and Costs}

The Linear Collider WBS includes some project-wide elements that are not uniquely allocated to the injector, main linacs, beam delivery, or control systems, or to the conventional facilities that appear in the WBS in each machine area. These include: Management Services, Technical Services, Accelerator Physics and Pre-operations. There are also costs associated with site-wide civil construction and infrastructure. They represent the investment in infrastructure needed to execute the project. Common estimates for all of these costs have been used for both the warm and cold technology options.

\subsubsection{Management Services}

These functions include the project directorate, managers and staff, plus all the necessary administrative support functions. These include public affairs, internal audit, affirmative action, planning, reporting, costschedule control system administration, legal, procurement, human resources, communications, ES\&H, and the requisite computing support. This WBS element also includes the Management and Operating (M\&O) contract fee. 


\subsubsection{Technical Services}

These activities include the continued R\&D necessary to develop industrial component designs and manufacturing processes, conceptual design work required to specify the collider, detailed design to convert that conceptual design into drawings and specifications that can be bid and contracted, value engineering, the purchase of first articles, the purchase of engineering studies or product testing on reliability, maintainability, and/or MTBF, the oversight to make sure that what is procured and built meets the requirements, and supervision of the checkout activities to make sure everything works as planned. It also includes system engineering to make sure that all the interfaces between components, sub-systems, systems, and machine areas are clearly defined, well-managed, and configuration-controlled.

Included in these activities are:

Engineering Support Services Engineering Services includes all of the engineers and designers needed to develop the specifications and drawings for the collider, including their servers, software, and workstations.

\section{Project Systems Engineering}

Engineering Team Management

\section{Central Design Services}

\section{Engineering Tools and Support for Data Management and CAD}

Manufacturing Support Services, Facilities and Administration Manufacturing Support Services includes all of the on-site facilities to perform assembly and testing functions. This includes clean room assembly areas, cleaning and plating facilities, magnet measurement facilities, Metrology and CMM functions, electronic rack factories, test and burn-in facilities, laser labs, and installation kitting and set-up facilities. It also includes the equipment and tooling necessary to store and move components, including warehouse space, storage racks, forklifts, overhead cranes, trucks, buses, conveyors, docks, and waste disposal functions.

Assembly and Testing Facilities This includes engineering, equipment and overhead for clean room assembly, girder assembly and testing, beam line assembly, laser assembly, RF assembly and testing, electronic rack assembly, electromagnetic measurements, permanent magnet assembly, metrology, cavity testing, and waveguide testing.

Manufacturing Logistics Support This includes engineering, equipment and overhead for tooling, waste disposal, warehousing, receiving and conveying.

Installation Support Services and Administration This includes the specialized equipment associated with installation and testing, including that needed for polarized and thermionic sources, positron target handling equipment, specialized moving equipment for modulators, klystrons, and cryomodules/girders for the Injector areas and the Main Linacs, moving equipment for collimators and other specialized Beam Delivery systems, vacuum equipment, magnet moving equipment, rack moving equipment, and a host of general-purpose equipment such as welders, lighting, portable clean rooms, small tools, communication equipment, supplies, etc

\section{Installation Systems Engineering}

Installation Logistics Support This includes engineering, equipment and overhead for installing the accelerator components. It also includes the logistics and installation planning to make sure that arriving and produced components and assemblies can be stored, transported, kitted, and installed at the right place at the right time with the right equipment and tooling on hand. Quality Assurance must be planned in from the very beginning, from working with suppliers on how parts will be dimensioned 
and measured, to procurement policy with respect to ISO 9000 and the oversight of outside and inside manufacturing, assembly, testing, and installation.

\subsubsection{Composite Site-Wide Civil Construction}

This covers the Civil Construction and Facilities work that is not attributable to a specific area of the collider (e.g., Main Linacs). It includes the initial grading and surveying of the site, roads, sewers, communications, electrical main substations, and the various campus buildings not associated with an area of the machine. (Cryogenic buildings and access shafts are included in the Main Linac section, not here.)

\subsubsection{Accelerator Physics and Pre-Operations}

Costs for accelerator physics support and machine pre-operations are collected in one location in the WBS rather than separately allocated to the different accelerator systems.

Accelerator Physics includes all physicist support for the collider through all phases of its R\&D, design, construction, and commissioning.

Accelerator Pre-operations refers to the preoperational commissioning of the accelerator systems as they become complete, through the transition to accelerator operations for further machine development and high energy physics. Pre-operations includes staff labor, electrical power, klystron repairs/replacements, and other materials and services.

Our model for preoperational commissioning of the accelerator systems as they become complete is illustrated in Section 5.4. The final year of accelerator pre-operations is one part of the transition from construction project to full facility operations. The other part consists of an incremental and cost-efficient administrative transition from construction project to operating laboratory, facilitated (perhaps) by the use of a common management entity for both construction and operations.

\subsubsection{Injector Complex - Sources}

Sources used common cost estimates wherever possible. Most of the cold collider acceleration is done with SC cavities and the warm with normal conducting, and so the estimates for the warm and cold options differ. Areas with common cost estimates encompass the polarized and un-polarized electron guns, electronic racks, and installation, and the complete positron production system excluding acceleration beyond the $240 \mathrm{MeV}$ point. The transfer lines to the Damping Rings are common except for the length differences between the cold and warm colliders.

\subsubsection{Beam Delivery}

Cost estimates for this area are completely common between the warm and cold collider. Both machines have two Interaction Regions and the same lengths and functionalities along the beamlines. Technical differences that arise in designs due to the differing bunch train formats and beam charges create insignificant cost differences. So identical cost estimates were used for the two main linac technologies, including conventional construction. 


\subsubsection{Damping Rings}

Several key technical specifications for the US cold LC damping rings are different from those used for the TESLA TDR. In particular, there have been changes to the number of magnets, the specification of the damping wiggler, and the vacuum system. These changes are described in Section 3.5.3 of this report. To account for these differences we have made a new estimate of the cost.

Our new cost estimate for the damping rings is based on a breakdown into the following subsystems:

- Magnets, excluding damping wigglers

- Magnet power supplies

- Damping wigglers

- Vacuum system

- RF system

- Diagnostics and controls

- Support and alignment hardware

Here, we describe the basis for the estimate of the hardware costs, and the costs of assembly and installation. EDIA and management costs are added as a percentage of the total hardware, assembly and installation costs. We aim to use a common basis for the costs of the damping rings for the warm and cold LCs as far as possible. In compiling the present estimate, we used data from a number of sources, including: previous cost estimates of the NLC damping rings; previous cost estimates[51] of the TESLA damping rings (compiled by INFN for the TESLA TDR); and costs of comparable systems and components in existing facilities. The particular source for individual subsystems is indicated in the following sections, where relevant.

Although the overall structures of the warm and cold LC damping rings are very different, there are many common components for which common unit costs can be used. These components include:

- Quadrupole and sextupole magnets

- Magnet power supplies where specifications are similar between warm and cold LC damping rings

- Diagnostics and controls

- Magnet supports (excluding movers in the warm LC damping rings)

These components are common to many existing storage rings, including third generation synchrotron light sources, and cost estimates therefore have a significant experience base. In the following subsections, we discuss in detail only those components that are specialty items, or differ significantly in specification between warm and cold LC.

Figure 5.2.5.1 shows the contribution of each subsystem to the hardware costs of the warm LC damping rings. Assembly and installation is approximately $13 \%$ of the hardware costs.

Figure 5.2.5.2 shows the contribution of each subsystem to the cost of the hardware for the cold LC damping rings. Assembly and installation is roughly $30 \%$ of the hardware cost, and is dominated by the assembly and installation in the arcs and wiggler sections. Note that we use the same cost per meter for assembly and installation of the arcs and wigglers of the cold LC damping rings, as for all sections of the warm LC damping rings. The difference in the assembly and installation as a fraction of the hardware cost arises from the fact that the average hardware costs per meter are much lower in the cold LC damping rings than in the warm LC damping rings. 


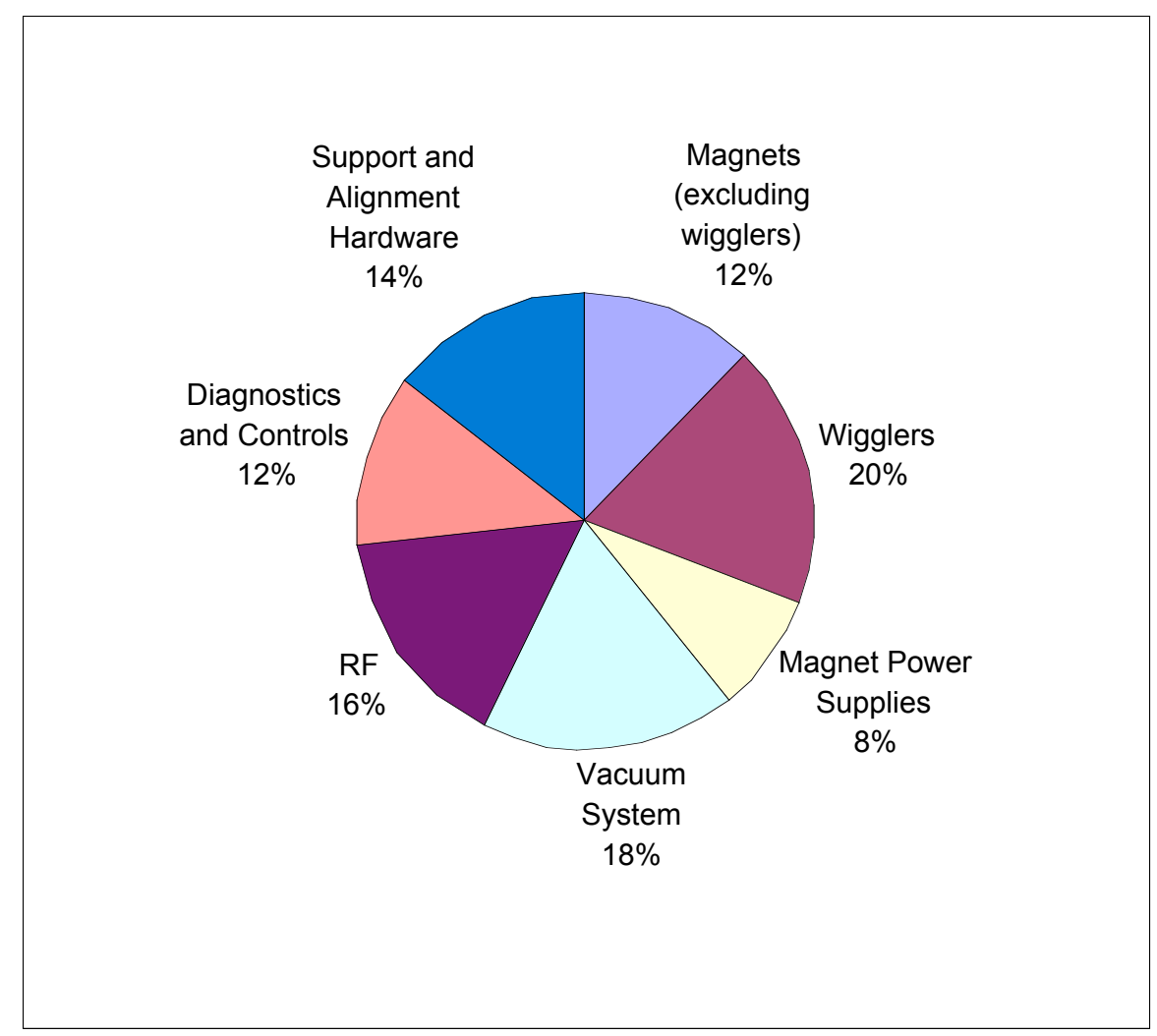

Figure 5.2.5.1: Contribution of each subsystem to the cost of hardware in the warm LC damping rings.

\subsubsection{Magnets (excluding wigglers)}

The arc dipoles in the cold LC have a low field $(0.194 \mathrm{~T})$ and large magnetic length $(4.5 \mathrm{~m})$ compared to the warm LC damping ring dipoles, and the dipoles in many storage rings. For the cost of the cold LC damping ring dipoles, we used the estimate by INFN[51] produced for the TESLA TDR. The warm LC damping ring dipoles are comparable to those used in modern synchrotron light sources.

Other multipole magnets (quadrupoles and sextupoles) are standard items, and the same unit costs were used for the warm and cold LC damping rings. It is possible that some reduction in unit cost may be expected from the larger numbers of magnets required for the cold LC damping rings, but this will not significantly affect the overall costs of the systems.

The kickers for both warm and cold LC damping rings are specialty items that will require R\&D to meet the specifications. It is therefore unclear how to cost these devices. However, it is unlikely that their cost will be significant compared to the total hardware cost for the damping rings.

\subsubsection{Magnet Power Supplies}

The power supplies are standard items. Note that the warm LC damping rings actually specify more magnet power supplies than the cold LC damping rings, since each quadrupole will be separately powered to provide tuning flexibility. In the cold LC damping rings, families of magnets are powered by a single supply wherever 


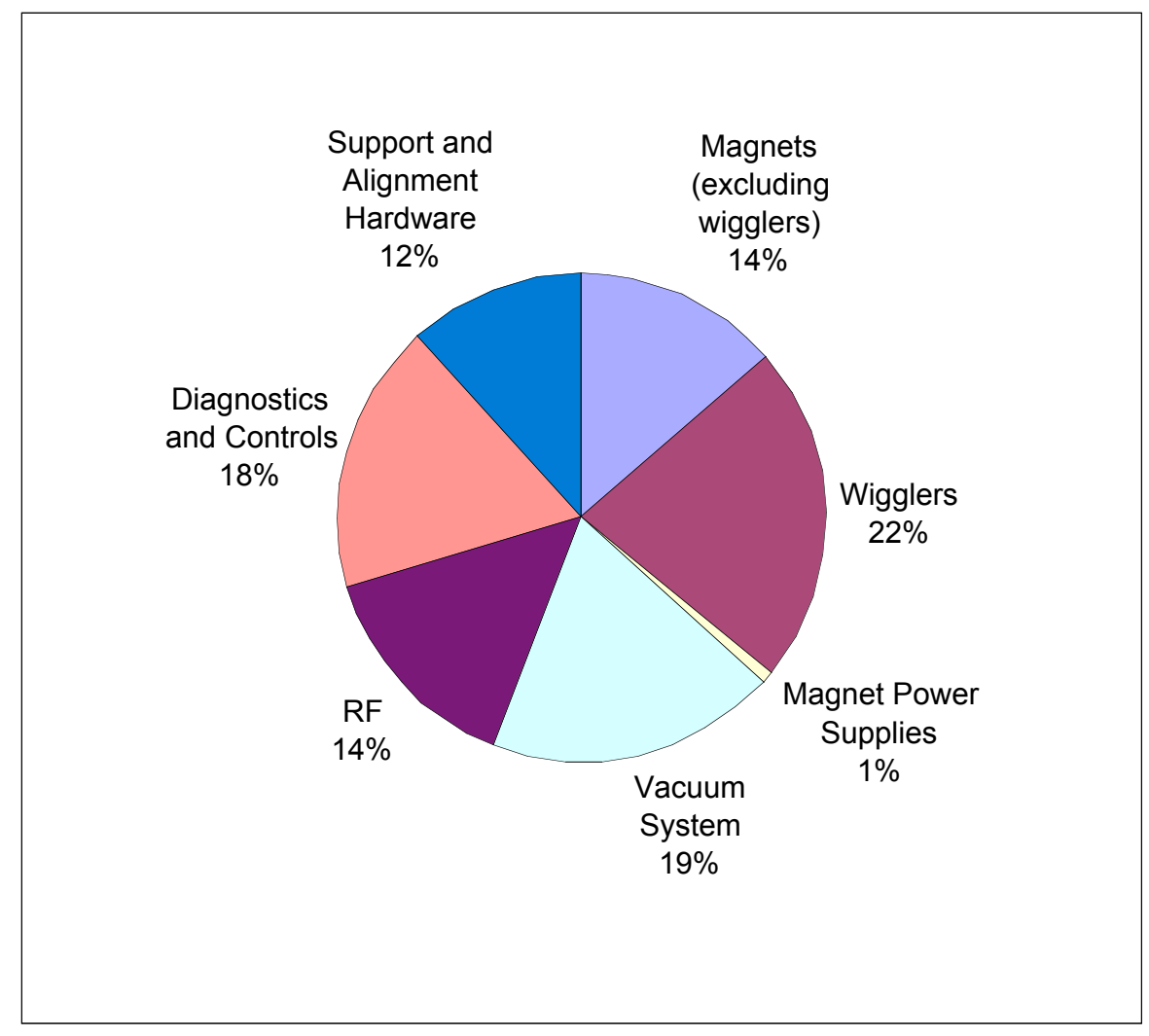

Figure 5.2.5.2: Contribution of each subsystem to the cost of hardware in the cold LC damping rings.

possible.

\subsubsection{Damping wigglers}

The damping wigglers for both warm and cold LC damping rings are constructed from permanent magnet material driving flux through iron poles. They are simpler than comparable devices used in third generation light sources, in that they do not require a variable gap. On the other hand, the requirements for field quality are demanding.

For the cold LC damping rings, considerable changes in the underlying technology and specifications exist between the present wiggler magnet and the wiggler assumed in previous estimates. The present configuration employs a permanent magnet wiggler rather than electromagnetic, which was costed by INFN. In addition, dynamical sensitivities identified by the ILC-TRC have required significantly more stringent magnetic specifications on the permanent magnet wiggler. Previous estimates did not include the level of field adjustment and tuning that will be necessary to achieve the required magnetic field specifications. For the present estimate, we used benchmark information from industry engaged in the design, fabrication, testing and field adjustment of wigglers and undulators. With permanent magnet wigglers of the required period and field specification, it is often the case that the cost of integration, testing and tuning of the device is equal to that of the hardware materials alone. Previous information reported only the hardware costs and did not include this necessary testing and tuning. 
For the cold LC damping rings, the length of the damping wiggler is the only significant difference between the positron (432 $\mathrm{m}$ wiggler) and electron (248 $\mathrm{m}$ wiggler) damping rings.

Although the specific parameters of the warm LC damping wigglers are a little different than those in the cold LC, the estimated materials and tuning costs per unit length are comparable.

\subsubsection{Vacuum System}

The vacuum system in the cold LC damping ring arcs is comparable to that in the Advanced Photon Source (APS). The cost of the APS vacuum system is detailed in the 1987 APS Conceptual Design Report[69]. Although there were technical problems in the construction of the APS vacuum system (arising from corrosion of the cooling-water pipes, and electrical problems in the NEG bake-out circuit) these problems could have been avoided without additional cost, if recognized in advance. The vacuum system in the cold DR arcs is therefore costed at the same rate (per meter) as the APS vacuum system, with allowance made for inflation since 1987 .

In the straights of the cold LC damping rings, the cost of the vacuum system will be dominated by the cost of the vacuum pumps, which are standard items.

For the warm LC damping rings, the vacuum system is more complex because of the higher synchrotron radiation loads from the dipoles, and the lower bending radius of the dipoles which will make it difficult to use an extruded chamber. The costs of the vacuum system were therefore based on the costs for the ALS, which uses a machined aluminum vacuum chamber, and achieves a vacuum pressure with beam comparable to that needed in the warm LC damping rings. This leads to a significantly higher cost per meter for the vacuum system for the warm LC damping rings than for the cold LC damping rings.

\subsubsection{RF System}

The RF system in the cold LC damping rings uses superconducting cavities, and calls for a relatively high voltage (50 MV in the positron damping ring). The cost was estimated by INFN[51] for the TESLA TDR, and we use the same costs in the present estimate.

The RF system in the warm LC damping rings is based on normal conducting RF cavities, and has parameters comparable to those of RF systems used in existing third generation synchrotron light sources.

\subsubsection{Diagnostics and Controls}

Since the major components of the diagnostics and controls (BPM's, control of quadrupole current for tuning and beam based alignment, control of steering magnets or movers, etc.) are associated with the quadrupole magnets, we have assumed the same cost per quadrupole for the diagnostics and controls in the warm and cold LC damping rings.

\subsubsection{Assembly and Installation}

The arcs of the cold LC damping rings are comparable in complexity to the warm LC damping rings, and the same cost per meter is therefore used for both. The straights of the cold LC damping rings are much simpler, consisting essentially of straight beam pipe with vacuum pumps every few meters, and quadrupoles every $100 \mathrm{~m}$. From this point of view, they are comparable to the main linac bypass lines in the warm LC, and we have therefore used the same cost per meter for assembly and installation as for this system. 


\subsubsection{Main Linac}

In this section we summarize the information we received from the TESLA and NLC/GLC Collaborations on the industrial models used to derive their cost estimates. This is our understanding of the information provided to us, and in some cases is augmented with our own experiences. We have tried to keep the two sources of information clear, but any misrepresentation is the fault of the authors of this report.

Part of our study of the main linac was to compile a summary of the sources of the components used for the R\&D facilities constructed to date, and the production models used in estimating the component costs for the warm and cold options. A summary of this compilation is given in Table 5.2.6.1, Table 5.2.6.2, Table 5.2.6.3, and Table 5.2.6.4, at the end of this section.

\subsubsection{RF Sources (Including Distribution)}

5.2.6.1.1 Low Level RF (LLRF) System This system has a well-defined scope for both warm and cold colliders, and functional prototypes have been built for both. The number of systems and their functional requirements are well established. System designs are in place and procurement estimates are based on specific commercial components. Labor estimates are developed from current experience creating similar software for existing facilities.

5.2.6.1.2 Klystron Thales provided the cost estimate for the multi-beam klystron planned for the superconducting collider, in quantities of all the tubes, half the tubes, and even smaller increments, assuming single fixed-price procurement, i.e. no explicit opportunity for mid-course price reductions based on real production performance. They have built three klystrons for DESY so far, one prototype and two tubes, incorporating some improvements and changes with respect to their standard design approach.

The study of the multi-beam klystron included an analysis of the cost drivers for the tube, and an estimate of all costs needed to meet performance specifications, including the development of the tube and required upgrades to the factory. The study also provided an estimate of the cost of performing tube repairs. About $10 \%$ of the cost is for the facility improvements required, and the cost of the average tube for the full quantity (750 tubes) is about $40 \%$ of the cost of purchasing only 1-3 tubes. Thales also made an estimate of the tube life (30,000 to 40,000 hours), which is driven by the life of the cathode and is based on experience with similar cathodes. Two other competent klystron manufacturers are now building prototype tubes, and the back-up option is to use two five-megawatt tubes (existing product) instead of the single $10 \mathrm{MW}$ tube. While the estimate provided by Thales assumed two sources, the attractiveness of this business to the microwave tube industry should bring out a larger number of competitors.

Klystrons similar to those used in the warm collider are normally built at the rate of one per month or less, therefore the cost estimate was based on the assumption of a dedicated facility sized both to build the initial complement of tubes and to provide a steady stream of repairs and replacements (mostly repairs). A financial model of a stand-alone klystron factory was constructed with assumptions for initial cost of parts and labor hours required for manufacturing operations, for learning curves ${ }^{1}$ of these factors over time, for the amount of equipment and facility required (based on the number of tubes per year), and then for a variety of financial factors necessary to enable a financial model to work. For example, the factory is financially liquidated at some arbitrary point in time instead of just truncating the stream of activity. The resulting

\footnotetext{
${ }^{1}$ Learning curves are based on the concept that the resources required to produce each additional unit decline as the total number of units produced increases. The major premise of learning curves is that each time the number of units doubles, the cost per unit will be reduced by a fixed percentage of the prior unit cost. This percentage is referred to as the learning curve slope. If $C(P)$ is the unit cost of the $P^{\text {th }}$ unit, and $b$ is the learning curve slope, then $C(P)=C(1) P^{\log (b) / \log (2)}$. For example, for a learning curve with a $90 \%$ slope, the unit cost will be reduced by $10 \%$ for every factor of 2 increase in the number of units produced.
} 
prices for tubes per year are then averaged over the full quantity needed (two sources are assumed), and this price was used in the cost estimate for the warm LC.

5.2.6.1.3 Klystron Drivers For the superconducting collider, each klystron requires about $160 \mathrm{~W}$ of drive power. At the operating frequency of $1.3 \mathrm{GHz}$, solid-state drivers have the best life-cycle costs. Acceptable prototypes are already in operation at the TTF, and there is no reason why multiple, reliable suppliers for this component in volume production would not be available. The cost estimate for this item was provided by SSB Technologies, a volume producer of solid-state power amplifiers (SSPA) for the communications, military, and instrumentation markets.

For the normal conducting collider, the klystrons must be driven with approximately $1 \mathrm{~kW}$ of X-Band power. The peak power demand dictates a traveling wave tube amplifier (TWTA), which can easily handle the average power and bandwidth requirements. Functional prototypes are operating in the NLCTA. Anticipated lifetime is on the order of 100,000 hours, which removes this item from the list of consumables. The current cost estimate is based on budgetary estimates from the supplier of the existing prototypes. Relative to other applications for TWTAs, the requirements of the collider are modest.

5.2.6.1.4 Modulator The estimate of superconducting collider modulator cost was based on a detailed study by PPT (Puls-Plasmatechnik, GmbH), and provides an average cost for the full quantity of modulators. Prior to the study PPT had built one modulator - a copy of a Fermilab design - as of now they have built a total of five. These modulators were quickly inspected and found to be manufactured using solid, professional, and appropriate high voltage power supply techniques, even though these represent the first production units. The PPT study showed in detail the staffing required, the cost for parts (almost all the parts are standard components), and the investment in equipment required. Some comments were made in the study about areas where further savings may be possible. No comparison with the current cost of a modulator has been made.

For the warm collider, the cost estimate is based on a parts list of 32 items costed at the projected cost in volume production. A $94 \%$ learning curve is used, which is a flat learning curve typical of parts that have already been manufactured in significant quantities so that additional learning is difficult. Thirteen assembly steps are costed based on a $90 \%$ learning curve and labor rates typical for production. An allowance for profit is also included. An industrial company has already built their own version of an IGBT-switched modulator that drives two $75 \mathrm{MW}$ klystrons. Many other commercial power supply companies exist, and while this design is unusual in terms of its combination of peak voltage and average power, the fundamental principles and processes associated with the construction of such supplies do exist and should make it relatively easy to establish a supplier base.

\subsubsection{RF Distribution and Cavities}

5.2.6.2.1 RF Distribution Since the RF distribution system of the superconducting collider is made of standard L-Band microwave waveguide components, suppliers of these components were asked to provide costs in a range of quantities. Several companies did, although one indicated that their catalog prices should be used. For costing purposes, the parts contain bolted flanges, with no attempt yet made to go to a more cost-effective connection design. The costs scale with quantity so that they decline by $22 \%$ for each factor of 10 increase in quantity. ${ }^{2}$

The RF distribution system for the normal-conducting collider has additional functionality in that it must compress the RF pulses to increase their peak amplitude and reduce their duration. To do this the system uses passive but sophisticated microwave components with internal geometries that are either rectangular

\footnotetext{
${ }^{2}$ This corresponds to about a $93 \%$ learning curve.
} 
or round, with in some cases internal posts, protrusions, irises, and bends to accomplish the RF conversions needed. This system is composed of four types of components. The first kind is standard vacuum pumps and gauges, and these were estimated just using the current catalog prices. Assuming that these components are quite mature, a 95\% learning curve was used to scale the small catalog quantity pricing to the large quantities needed in the collider. The second type of component is standard microwave components in the size of WR90, which is the commonly accepted designation for X-Band. Due to the predominance of military radars at X-Band, these components have been and are widely available. Reflecting this, only a $91 \%$ learning curve was used, on the basis that while much learning has already occurred, the high quantities demanded by the LC will occasion additional savings. The third category of parts is functionally-common microwave components (like bends) that are in an unusual size. The fourth category is unusual microwave parts in atypical sizes. These latter two categories were given an $88 \%$ learning curve.

All of these parts, while appearing unusual, can be made on standard high precision milling machines using standard tools. All are made from high purity $\mathrm{OFE}^{3}$ copper in two pieces that are then brazed together in a standard hydrogen furnace. In the case of the WR90 parts and the special components, all of these parts either have been made or are in the process of being made for an R\&D project at SLAC (the Eight-Pack Project). The Chief Engineer estimated the cost of the first production part for all of these components, and then the learning curves mentioned earlier were respectively applied.

\subsection{Accelerator Modules}

5.2.6.2.2.1 Accelerator Structure Metal The estimate for the cost of niobium for the superconducting linacs was obtained through a study by three of the principal suppliers. As a final check, the authors of the TESLA TDR estimated the cost of setting up a niobium refinery and producing the material for the project in a dedicated facility. The estimated cost of niobium under this scenario matched that generated in the industrial study.

The warm collider uses high purity OFE copper as the raw material for the accelerator cells and couplers, as well as almost all the surfaces that are in contact with high power microwaves. This material is basically a commodity whose price is well known. Many different mills throughout the world make this material, and obtaining adequate sources of OFE copper at competitive prices should not be an issue.

5.2.6.2.2.2 Accelerator Structure Cavities The cost estimate for cavity fabrication for the superconducting linacs is based on experience gained in building the TTF, and on studies by companies familiar with the process. The study took the list of cavity assembly activities developed at various laboratories for the TTF, and built a bottom-up cost estimate based on a model of an industrial approach. The production model assumed outsourcing, multiple production lines, improved production equipment (multi-chambered electron beam welders and multi-part fixturing), and revised parts flow to eliminate wasted motion and delays.

The cavity fabrication process for the warm collider is based on the structures made by Fermilab, SLAC, and KEK. Those familiar with the design or production process of coupled-cavity traveling wave tubes will recognize the warm cavity assembly as an all-copper coupled-cavity body assembly with a coupler on both ends and all the lossy materials missing.

The warm-cavity cost estimate took an aggregate of the current experience described in the preceding paragraph and adjusted it both for the higher quantity and rate and the anticipated differences in the manufacturing process. A parts list for the cavity assembly was developed and costed. The final cost of manufacturing individual copper cells, since they are a significant cost, was developed in substantial detail based on process times and the cost of equipment for each process. These final costs were then run backwards

\footnotetext{
${ }^{3}$ old designation OFHC
} 
up learning curves that are representative of the processes involved to come up with an average cost of all units. This analysis resulted in a cost estimate for the first production unit which is just about equal to the current $\mathrm{R} \& \mathrm{D}$ costs for these cells.

5.2.6.2.2.3 Accelerator Structures Couplers The cost estimate for fundamental power couplers for the superconducting linacs predicts a lower unit cost for couplers than for cavities. Some differential between estimated costs of cavities and couplers is expected to come from the fact that the production of the latter requires a smaller infrastructure investment. In addition, changes have been proposed in cryomodule design, which it is hoped will relax coupler specifications enough to produce significant simplifications and cost reductions.

For the warm collider, the couplers are an integral part (brazed together) of the cavity assembly. These couplers do not have windows, are not coaxial (not TEM), and are rectangular machined geometry (TE mode in waveguide) that symmetrically transitions to a periodic disk-loaded structure (TM mode). Today's cost in R\&D for this item is about equal to the cost estimate for the last units in the model production quantity. Their cost is contained in the accelerator structure cavity cost estimate described above.

5.2.6.2.2.4 Cavity String Assembly To assist in the preparation of a cost estimate for the superconducting linacs, industrial studies were commissioned with Noell/Krantz and ACCEL. Each of the industrial consultants was provided with detailed information about the specific tasks and required materials and independently developed detailed manufacturing plans that were separately loaded with labor and costs. The two studies came to similar conclusions on the need for multiple production lines and greater use of dedicated fixtures and facilities to maximize throughput. The principal difference was on the assumed facility and labor utilization efficiency. The cost estimators adopted the infrastructure investment requirement for the lower facility utilization model, and selected an intermediate value of labor efficiency. The cost estimate assumes an absence of process failures, but also low facility utilization with time for preventative maintenance. The cost estimate takes no credit for potential future improvements or simplifications to the process.

There is no equivalent step in the warm collider.

5.2.6.2.2.5 Cryomodule/Girder Assembly The cost estimate for this element of the superconducting linacs is based on an industrial study by E. Zanon. The key to economical construction will be the development of effective tooling and component design that minimizes the amount of hand fitting required. These are standard manufacturing engineering problems.

This last level of integration for the warm linac accelerator structures includes assembly of the accelerator structures, power loads, vacuum pumps, and water cooling lines on the girder that provides alignment and stability. The cost estimates for these items were based on the average unit cost for one-third of the quantity now being used. The technical complexity of these items is very low.

\subsubsection{Cryogenic Systems}

Models for the cost of cryogenic refrigeration systems have been developed and updated over the years[61, $62,63,64,65,58]$. These models were based on the actual costs to purchase cryogenic refrigeration systems operating between $4.2 \mathrm{~K}$ and $80 \mathrm{~K}$. In order to fairly compare these systems, actual costs were adjusted to a reference refrigeration system. The reference system attempts to eliminate variations in the systems, such as storage, controls, load related systems, etc. As a result, the costing model reflects the common components of a basic cryogenic refrigeration system: the cold box with expanders and the compressor systems.

Helium refrigeration systems operating below $4.2 \mathrm{~K}$ require additional hardware that is not reflected in previous costing models. The large scale requirement for superfluid helium refrigeration systems in the LHC 
project motivated CERN personnel to develop a cost model which takes the additional hardware and cycle considerations into account. The model[60] begins with a previous model for $4.5 \mathrm{~K}$ equivalent refrigeration and adds two additional models to take into account the $2 \mathrm{~K}$ cold box with its associated warm subatmospheric compressors and stages of cold helium compressors. This LHC model[60] was used to estimate the cost of the cryogenic plant.

The potential cost increase due to the splitting the cold box into smaller sizes was not considered. A study on how to split the cold boxes, considering reliability, redundancy and various operational scenarios, needs to be performed.

The costing model was verified with the recent purchase of the $1.8 \mathrm{~K}$ refrigeration system for the Spallation Neutron Source (SNS). The predicted costs of the $4.5 \mathrm{~K}$ cold box, warm compressors, $2 \mathrm{~K}$ cold box and cold compressors was within $2 \%$ of the actual purchase order costs.

Quantity discount is not considered in the cost estimate except for process control software development. Many of the cryogenic system costs are not considered sensitive to quantity discounts due to the labor intensive nature of the work. Some cost saving can be expected on some items due to the redundant plant design or quantity discount of standard industrial components such as piping, compressors, controls hardware, etc.

There is a minimal-to-medium technical risk in the procurement of the cryogenic system. The plants under consideration are well within the capabilities of the existing cryogenic industry. However, there is a potential for an extra cost due to the limited number of cryogenic equipment vendors. This extra cost would be associated with maintaining cryogenic supply from industry by splitting contracts over multiple vendors. Failure to award to multiple vendors carries a high risk of resulting in a monopolistic environment within the cryogenic industry, potentially creating problems for future projects.

The controls for the cryogenic plants consist of digital control system hardware and software. It is assumed that the plant's manufacturer supplies logic and algorithms for the plant control. It is assumed that the interface to the accelerator controls and load process controls software will be developed by laboratory labor. The cost of the refrigeration system controls was calculated as $15 \%$ of a refrigerator cost for the first plant, and $5 \%$ for subsequent plants.

Cost estimates for the warm gas and liquid helium storage were obtained from recent purchases for the SNS project. LHC experience estimates that the cost of the piping necessary to connect all of the warm subsystems, such as warm compressors, cold boxes, upper bayonet can, inventory storage and so on, is well approximated as $10 \%$ of the cryogenic plant cost. Also based on LHC experience, $2 \%$ of the cryogenic plant cost has been added for miscellaneous items such as liquid nitrogen storage tanks, ODH systems, instrument air, inventory controls, etc. 


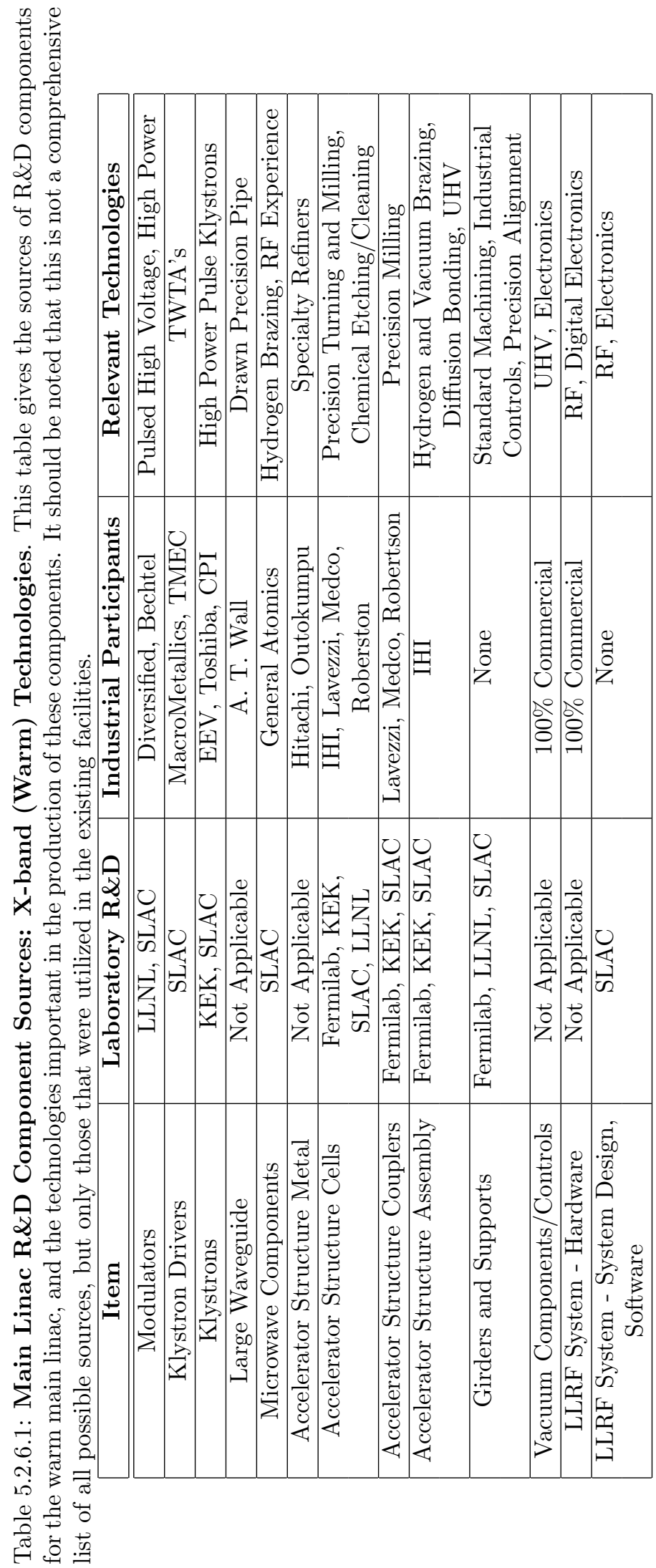




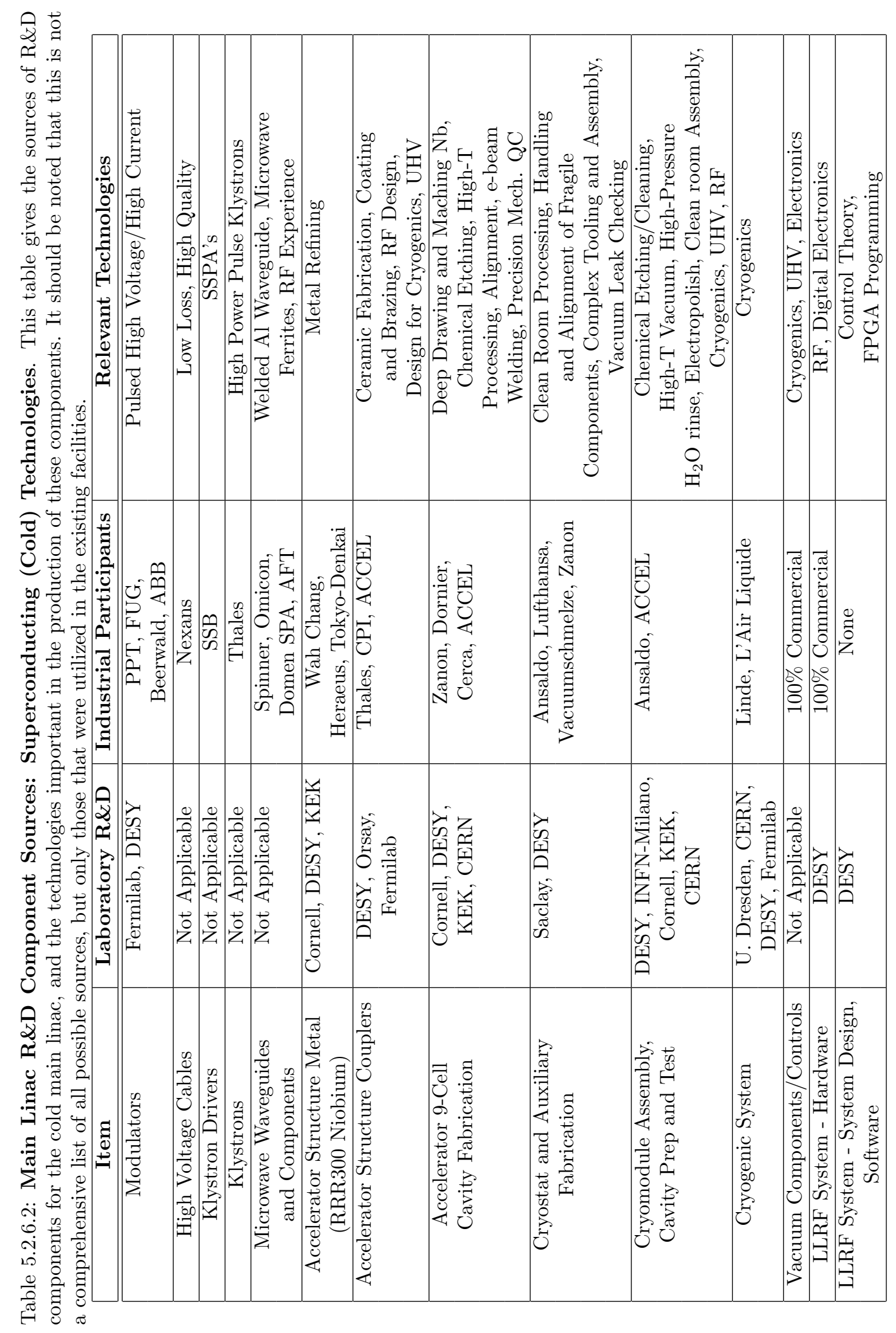




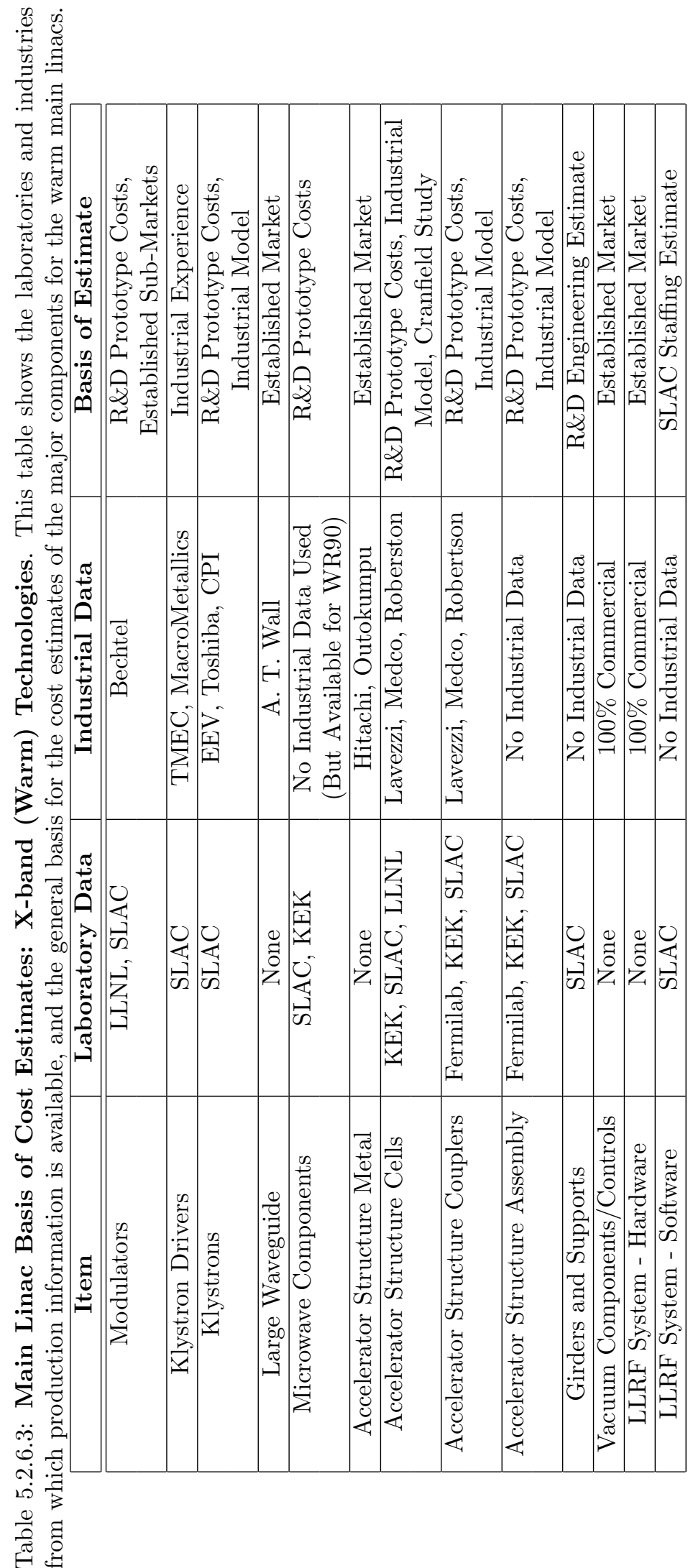




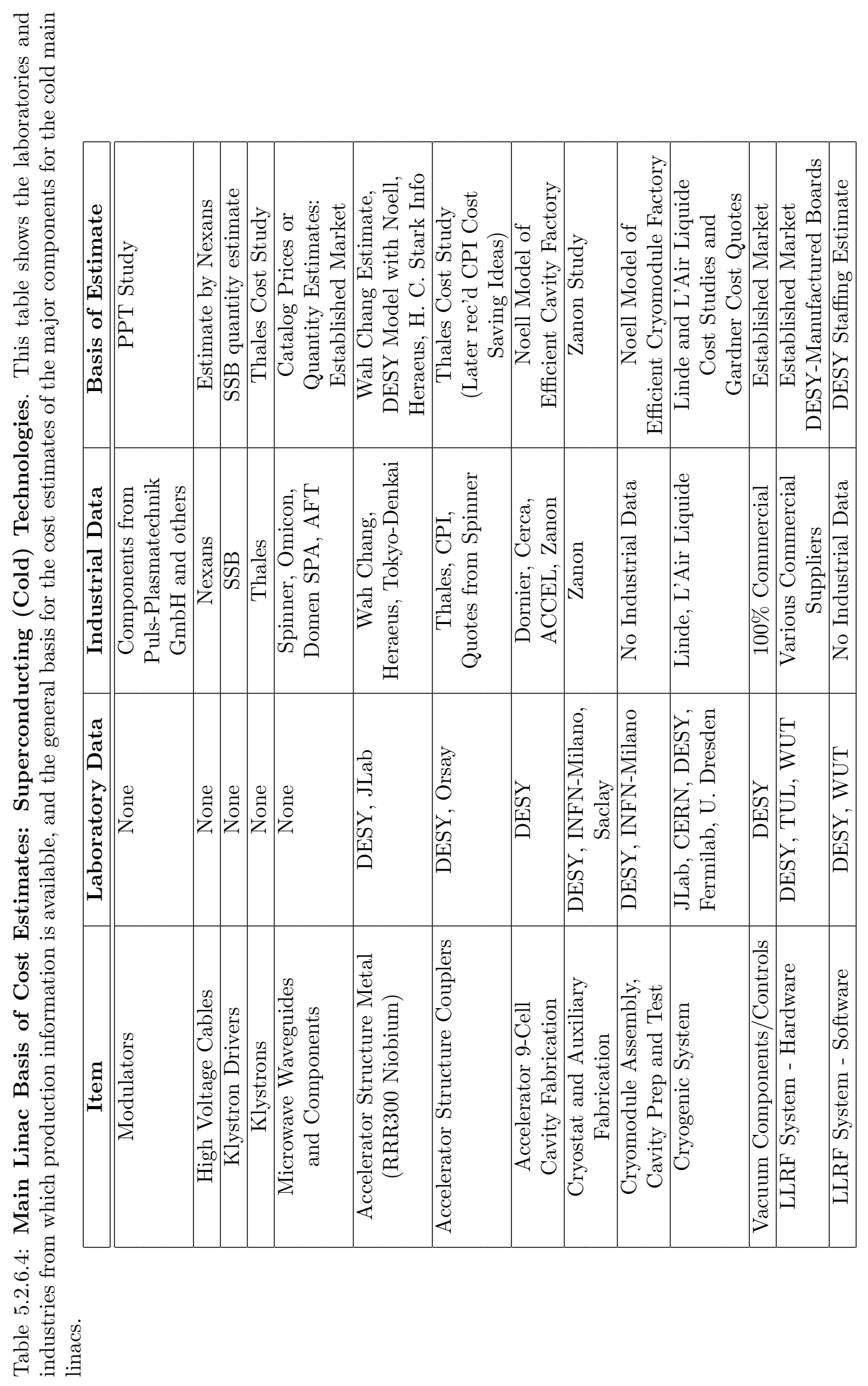




\subsubsection{Conventional Construction}

The cost and schedule information contained in this chapter has been generated using the four design solutions for a linear collider (normal conducting and superconducting in both California and Illinois) described in Chapter 6. They are the result of several design iterations that have explored a wide range of representative siting configurations and construction methods. Unit costs have been provided for inclusion into the overall project cost estimate.

With the exception of the unit costs derived for tunnel and shaft construction, all other costs provided for conventional facilities are based on prevailing industry standards and previous experience gained from construction projects at existing high-energy physics laboratories. The unit costs for tunnel and shaft construction were generated from a comprehensive examination of existing tunneling projects in both dolomite and sandstone/claystone rock formations, which are common to the representative sites reviewed in Illinois and California. Existing information was normalized to a single unit cost for tunnel and shaft construction using a consistent model for all available information. This method has thus provided a unit cost that is site neutral and therefore representative of general tunneling and shaft costs.

With respect to the schedule for the completion of the conventional facilities and enclosures, each of the major components of the project was reviewed and a generic schedule for completion was developed. A single scheme for the completion of all the work was generated using the normal conducting machine as a baseline for overall scope. It should be noted that this schedule has not been optimized for any specific parameter, but is meant to demonstrate a plausible method for the construction of this project. Other constraints, beyond the control of the construction process, may have a significant effect on the overall schedule. Nevertheless it serves as a demonstration for the constructability of the project. In addition, since the tunnels required for the superconducting machine are longer due to machine parameters, an additional study was done to demonstrate that the longer tunnels could be constructed in a similar timeframe to that of the normal conducting machine. This study showed that the overall scope of the project provides ample opportunity for parallel activities, without a premium in cost, such that the superconducting machine could be constructed in approximately the same timeframe as a normal conducting machine. Therefore the schedule for the construction of the conventional facilities for this project should be considered a neutral component to the eventual technology decision process.

\subsection{Cost Analysis and Discussion}

\subsubsection{Analysis of Cost Compilation}

In order to analyze the cost estimates of the warm and cold reference designs for consistency and fairness, the WBS software tool (Sec. 5.2.1) was enhanced to allow each line item of the WBS to be "tagged" by an arbitrary number of descriptive variables. The variables and their values were chosen based on our experience with questions typically raised at project cost reviews. Using the tags, the total project cost of each design could be examined in terms of the tag values, regardless of the structure chosen for the WBS. The three tags used in this report are AREA, TSET, and Cost Type.

AREA values are injector (sources, pre-linacs and bunch compressors), damping rings, main linac (including bypasses, diagnostic regions and positron-production related housings), bds (or beam delivery systems, comprising all housings and equipment from the end of the linacs to the main beam dumps, including the interaction region halls and full power linac tune-up dumps), and common (which contains all area-nonspecific costs such as management, pre-operations and accelerator physics, the control system backbone, and common technical (fabrication, warehouse \& QC) services.) 
TSET refers to the technical subsystem whose expertise is required. TSET values are structures (accelerating structures with girders, movers and in the case of the cold design, cryostats), $R F$ (modulators, klystrons and pulse compression systems), magnets (including supports, movers and bpms), vacuum (enclosures, pumps and instrumentation), controls (global readout, racks, electronics and software), instrumentation (all specialized equipment not called out separately, including electron guns, wire scanners, beam stoppers, collimators, dumps and diagnostic regions), cryo (refrigerators, storage and distribution of cryogens, power under $34.5 \mathrm{kV}$ and cryo cooling tower utilities to support compressors), $c f$ (conventional facilities), installation (installation and in-tunnel final testing), operations (personnel and electric power required for preoperations) and finally systems eng (which encompasses the standing army of managers, scientists, engineers and support staff required for production, testing, $R \& D$ and that are not assignable to other more specific TSET groups).

Cost Type variables are hardware (QCd components ready for installation), $c f$ (mechanical, electrical and civil engineering and construction costs), edi\&̋a (captures engineering, design, inspection and acquisition for all TSET's, including $c f$ and installation), installation (component installation and in-tunnel final tests), preops (personnel and power required for pre-ops) and management (project directorate, public affairs, internal auditing, planning, business services and ES\&H).

Figures 5.3.1.1, 5.3.1.3 and 5.3.1.5 below show the total project cost of the warm design as a broken down in each of the three variables described above. Figures 5.3.1.2, 5.3.1.4, and 5.3.1.6 are the equivalent plots for the cold design. In each case the percentages add up to 100\%. The difference in the total project cost of the two designs is discussed in the next section (Sec.5.3.2).

Figures 5.3.1.1 and 5.3.1.2 show the TPC as a function of technical subsystem. Comparison shows that the technical components of each project that are independent of the technology choice are equal to within 1\%. For example, cf costs comprise $31 \%$ of each design; systems engineering (8-9\%), installation (6-7\%), magnets $(6 \%)$, vacuum (3-4\%), controls (4-5\%), operations (4\%), and instrumentation (2\%) are essentially identical. Most of these costs (i.e., those not in the common or bds areas) were independently estimated for each design. While the costs for structures, RF, and cryo, which comprise the core technologies of each design, necessarily differ, their sum is $34 \%$ in both cases.

The cost comparison in terms of Cost Type in Figures 5.3.1.3 and 5.3.1.4 leads to a similar result: the cost of each design is split among hardware (49\%), cf (24-25\%), edi\&a (15-16\%), installation (4-5\%), pre-operations (4\%) and management (2-3\%) categories to within a percent.

Figures 5.3.1.5 and 5.3.1.6, which compare cost fractions as a function of machine region, are perhaps best considered after glancing at Figure 5.3.2.3, which has each cost normalized to the warm design TPC. In Figure 5.3.2.3, it is clear that the $b d s$ and common elements have been put in at numerically identical values. The net cost difference arises due to the main linac, the damping rings and the rest of the injector region. The percentages in Figures 5.3.1.5 and 5.3.1.6, each totaling 100\%, primarily reflect the fact that the cold linac and damping rings, due to the technology, have a larger fraction of their respective TPCs than the warm design.

The conclusion is that the cost estimates have been compiled using the same "rules of the game" with as much commonality as could be applied. Any resulting difference in the cost estimate between the two designs is attributable to the technology choice. 


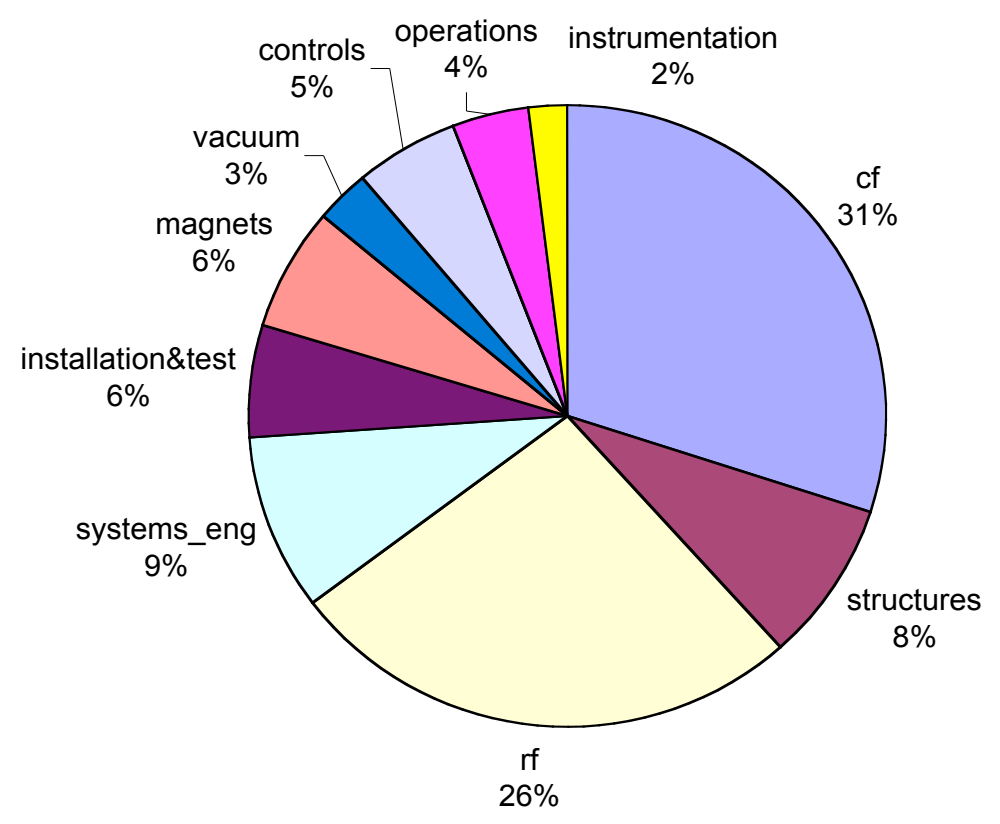

Figure 5.3.1.1: The Total Project Cost of the Warm LC as a function of Technical Expertise

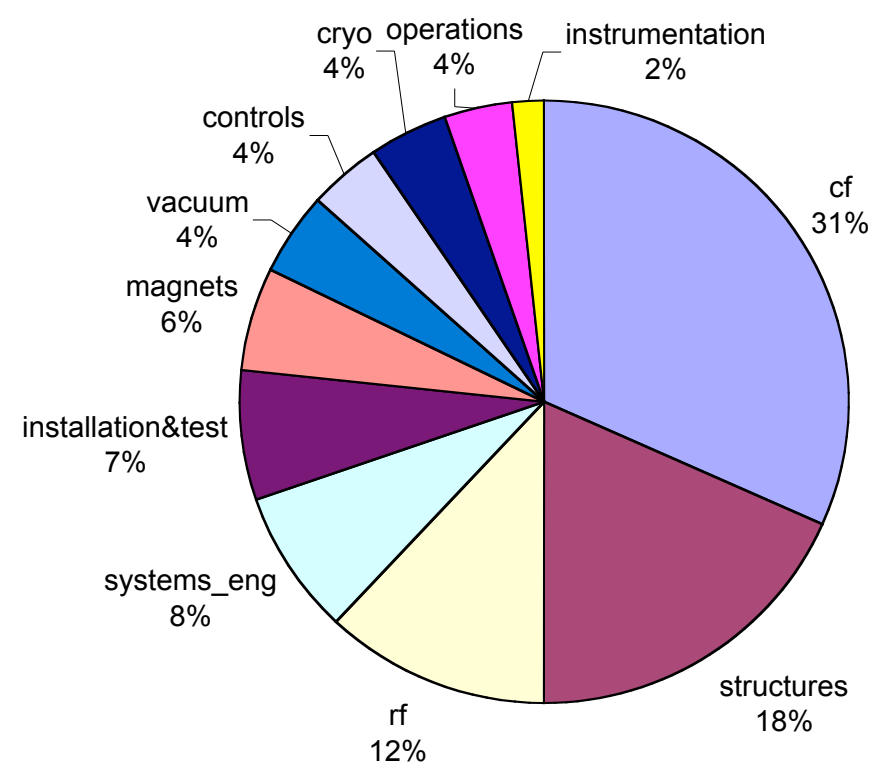

Figure 5.3.1.2: The Total Project Cost of the Cold LC as a function of Technical Expertise 


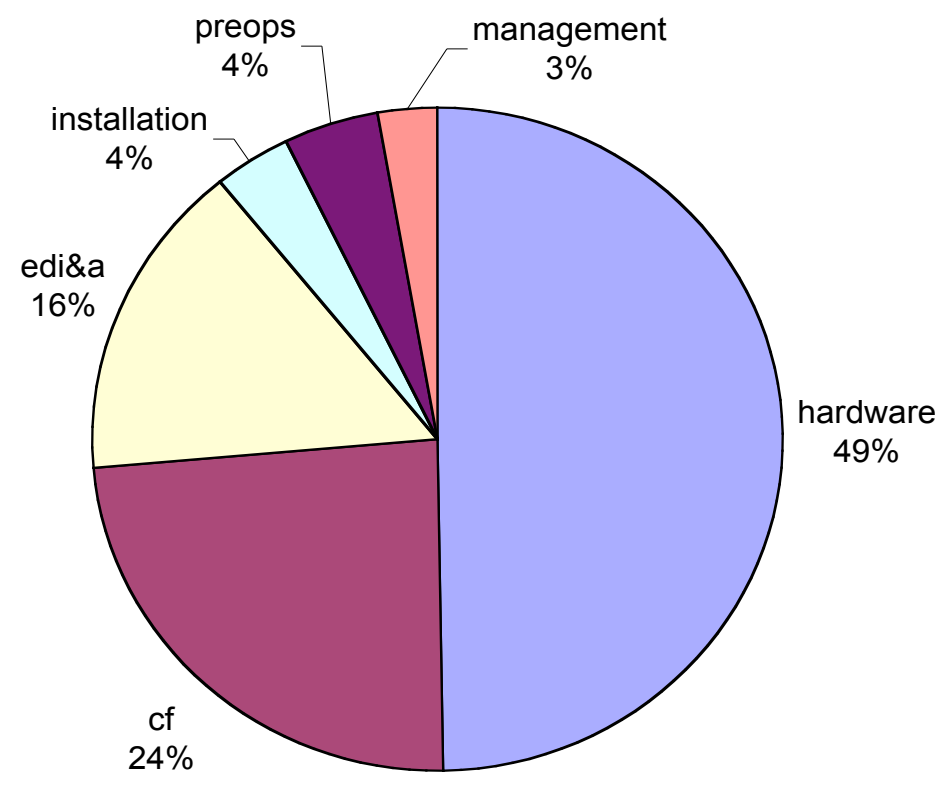

Figure 5.3.1.3: The Total Project Cost of the Warm LC broken down by the type of cost.

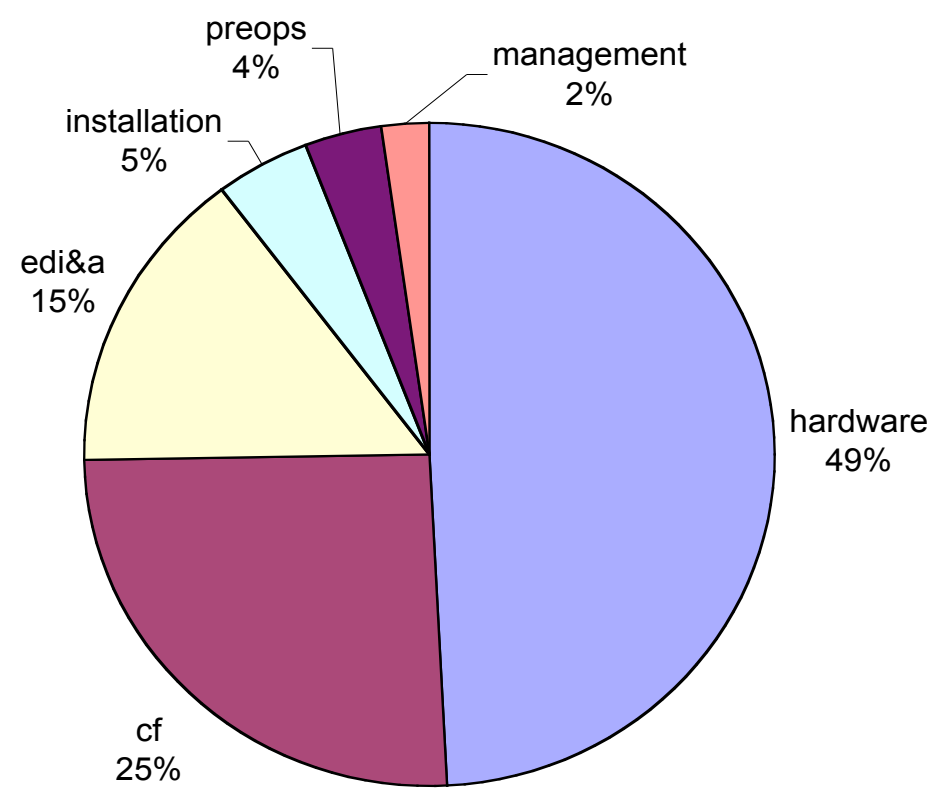

Figure 5.3.1.4: The Total Project Cost of the Cold LC broken down by the type of cost. 


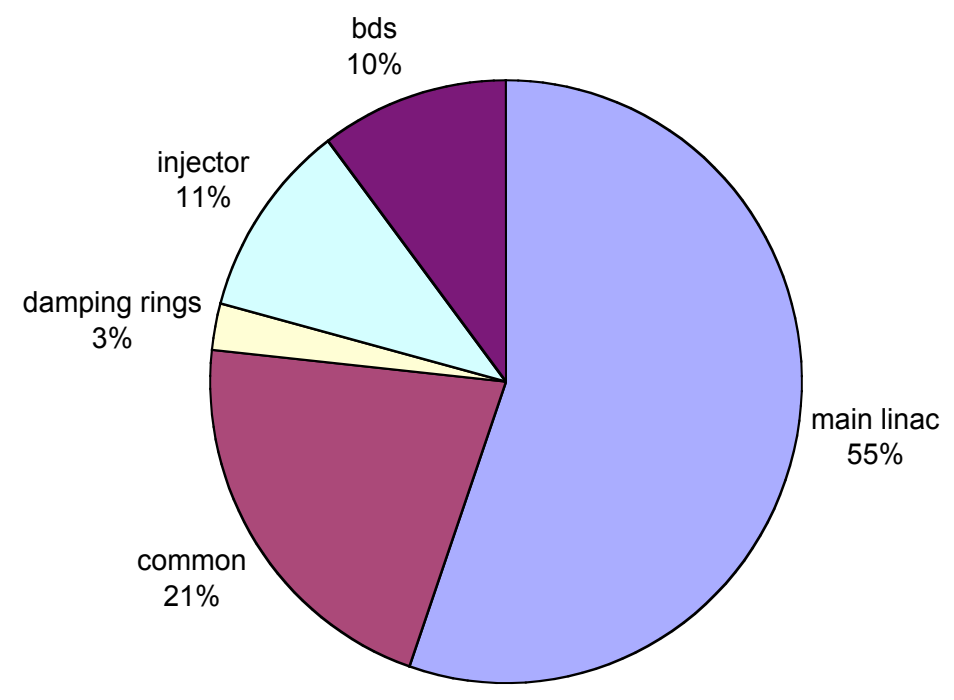

Figure 5.3.1.5: The Total Project Cost of the Warm LC broken down by area

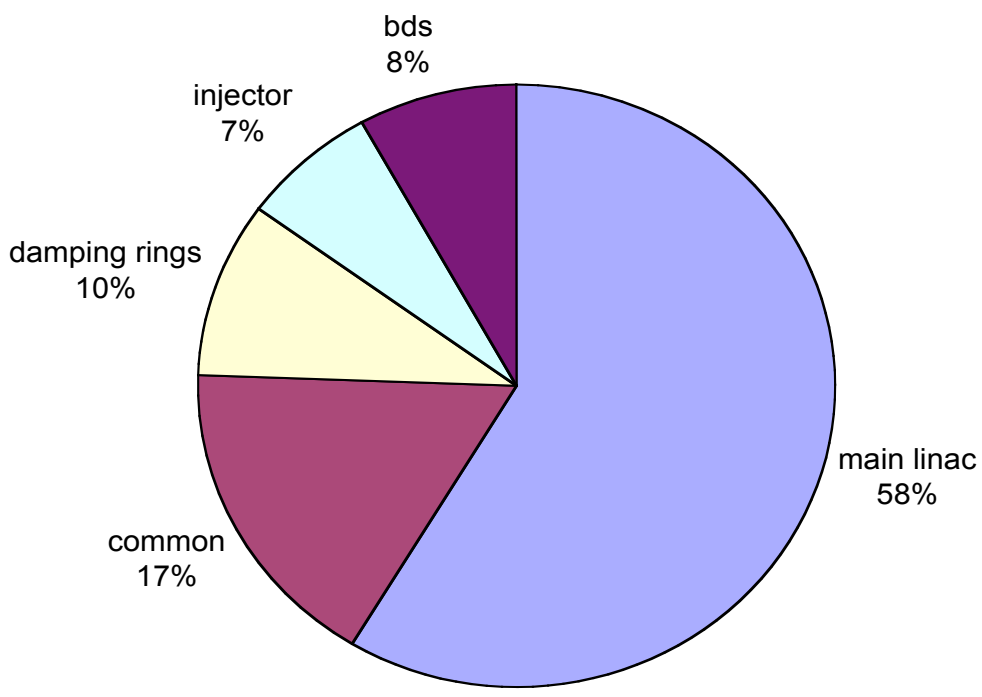

Figure 5.3.1.6: The Total Project Cost of the Cold LC broken down by area. 


\subsubsection{Discussion}

Major high energy accelerators have been built in many locations, and the international scientific community has accumulated a solid base of experience in estimation and management of costs and schedules for these construction projects. These facilities include those built for high energy physics research, and also modern storage rings used in research with synchrotron light. More directly, major test facilities have been built over the last decade as part of the preparatory $\mathrm{R} \& \mathrm{D}$ for both the warm and cold collider technologies. These dedicated test facilities, and the components built for them, provide information about acquisition of the critical components of a collider - technical requirements for their manufacture as well as first indications of costs. The available cost estimates for both the warm and cold technologies are based on this substantial experience with existing accelerator facilities, and on the technology-specific R\&D prototypes and test facilities.

The cost of the components of the main linac will be a substantial portion (though less than half) of the total collider project cost. The accelerator consists of repetitive units each containing a relatively small variety of components that must be produced and assembled in large quantities ranging from thousands of cryostats to a million individual copper cells. The industrial technologies and experience to produce the components and systems of each machine technology choice are well-defined and available in worldwide industries (Tables 5.2.6.1 and 5.2.6.2). These industries have been involved with fabrication of R\&D prototypes, but the industrial capacity to build the quantities of components ultimately needed for the collider do not exist today for either technology. There will be large extrapolated reductions from presentday R\&D unit costs to those incurred in building the collider. The estimates of these extrapolations provided by the TESLA and NLC/JLC Collaborations range up to factors of three to five for high-volume components of the cold cryomodules, and as high as six for some of the larger quantities of warm copper components. The overall extrapolation is somewhat larger for the warm technology than the cold because of the larger number of smaller repetitive components involved. Industrial models of large-scale production have been used to estimate the costs of components in the necessary quantities (Tables 5.2.6.3 and 5.2.6.4). These models are grounded in extensive experience with similar production of other products (including production of large quantities of components for other accelerator projects), but these extrapolations represent the greatest source of risk in the cost estimates.

The overall cost of the main linac as a function of increasing accelerating gradient is a trade between the rising costs of cryogenic systems and sources of microwave power on one hand, and the falling costs of the beamline components and supporting infrastructure on the other (Figure 5.3.2.1). At the reference gradients of $28 \mathrm{MV} / \mathrm{m}$ (loaded) and $65 \mathrm{MV} / \mathrm{m}$ (unloaded), the cold and warm technologies are close to the minimum in this balance. This is the result of the extensive R\&D done on both technologies.

The major differences in the estimated costs between colliders built with the cold and warm technologies can be traced to: (i) The lower gradient of the cold accelerator technology which results in linacs that are twice as long as those needed with the warm technology at $500 \mathrm{GeV}$ cms. (ii) The tunnel needed to accommodate the $1 \mathrm{TeV}$ upgrade energy with the cold technology which is $50 \%$ longer than needed with the warm technology. (iii) The longer bunch train used with the cold technology which requires a more extensive damping ring.

Projections of the relative costs are given in Figures 5.3.2.2 and 5.3.2.3 by technical expertise and by machine area. More than two thirds of the warm costs are independent of the accelerator technology, and the corresponding cold costs are the same. The cold-warm difference arises because the costs that are specific to the cold technology choice are estimated to be about twice as large as those that are specific to the warm option. The differences in the specific costs result in an estimated total project cost for the cold machine that is $25 \% \pm 10 \%$ greater than for the warm machine.

The quoted uncertainty assumes a $\pm 15 \%$ (rms) uncertainty in the specific costs, and ignores correlations between the warm and cold cost estimates. We have not done a complete analysis of the uncertainties in these costs, which would have to include any correlations. The presence of correlations would lower the uncertainty. 

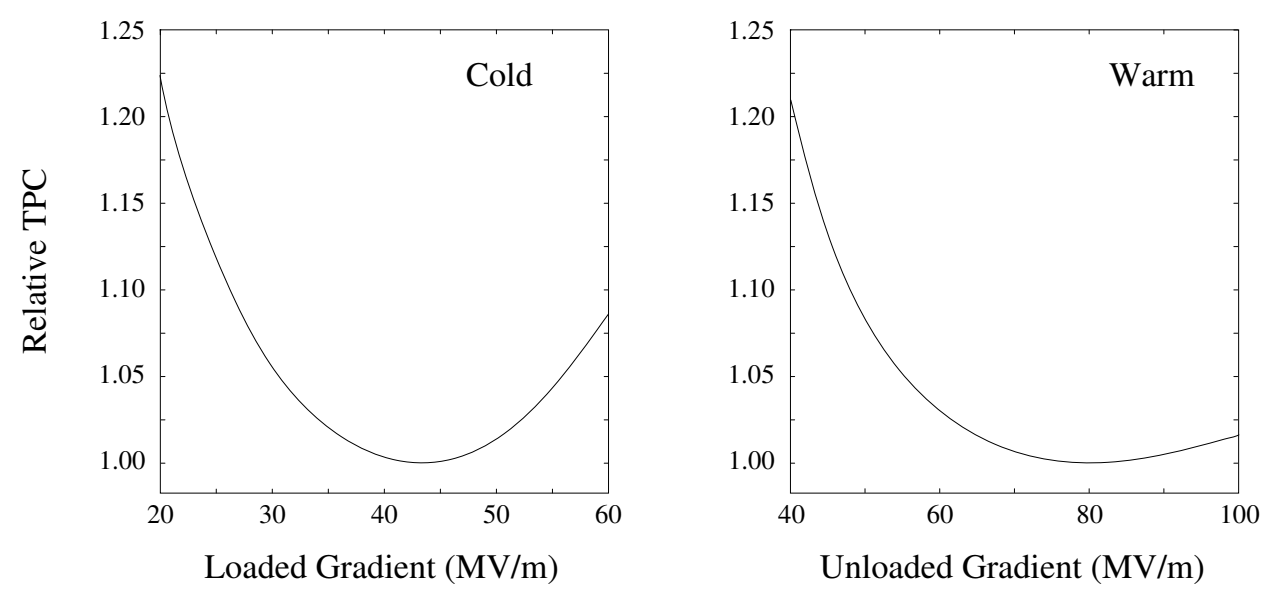

Figure 5.3.2.1: The estimated total cost of $500 \mathrm{GeV}$ c.m. linear colliders, built according to the U.S. cold and warm reference designs, as a function of the main linac cold loaded and warm unloaded gradients. The cost includes both that of the main linac components and power sources, and that of fully outfitted tunnel housings of the appropriate length sufficient to achieve $500 \mathrm{GeV}$ c.m.; it does not include the cost of portions of unoccupied tunnel needed for the $1 \mathrm{TeV}$ upgrade. The vertical scale is normalized to the costs at the minimum of the curve for each option. The curves are plotted without taking into account technology limitations. 


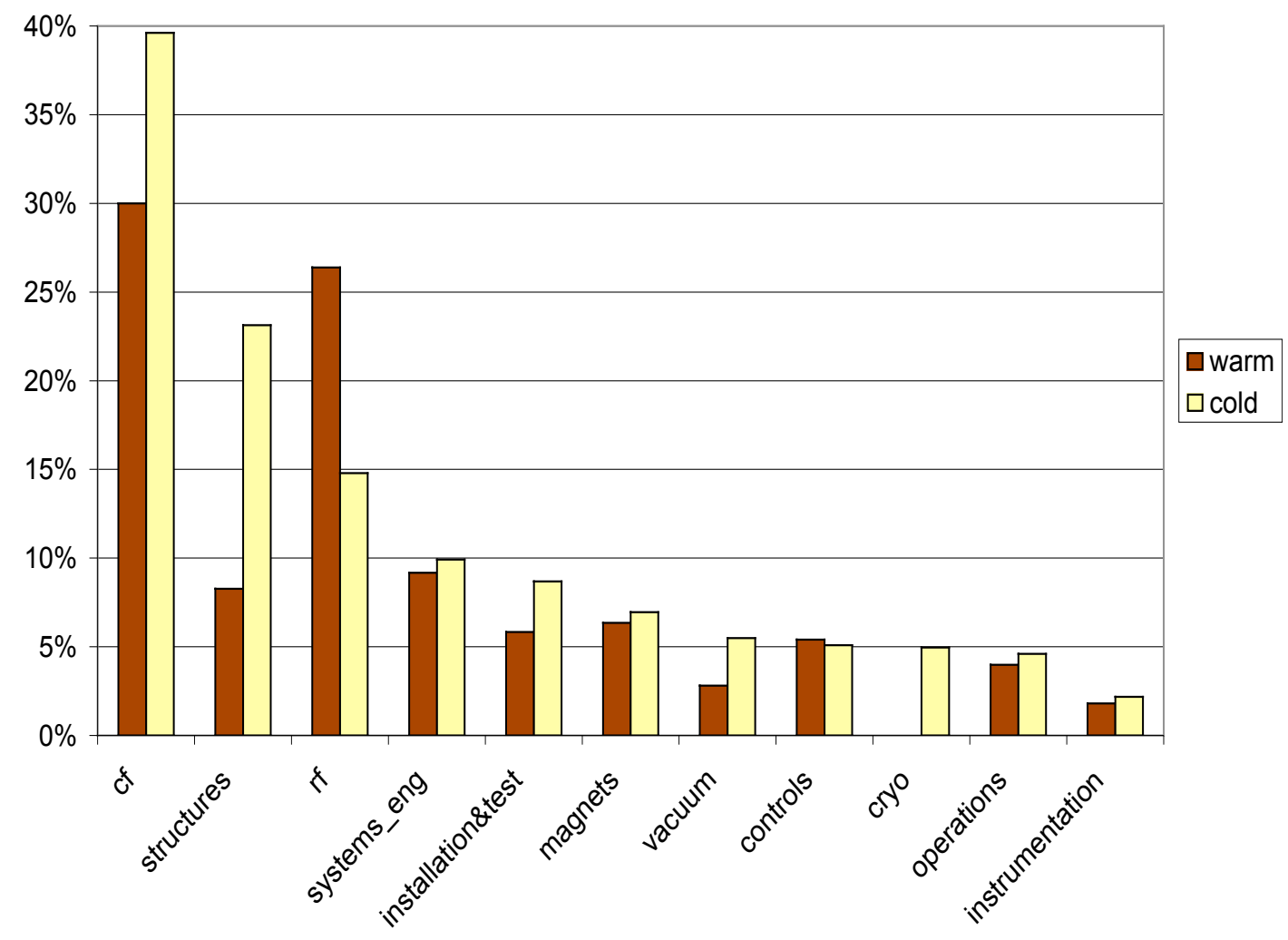

Figure 5.3.2.2: Costs for each technology option normalized to the total of the warm option shown by technical subsystem. 


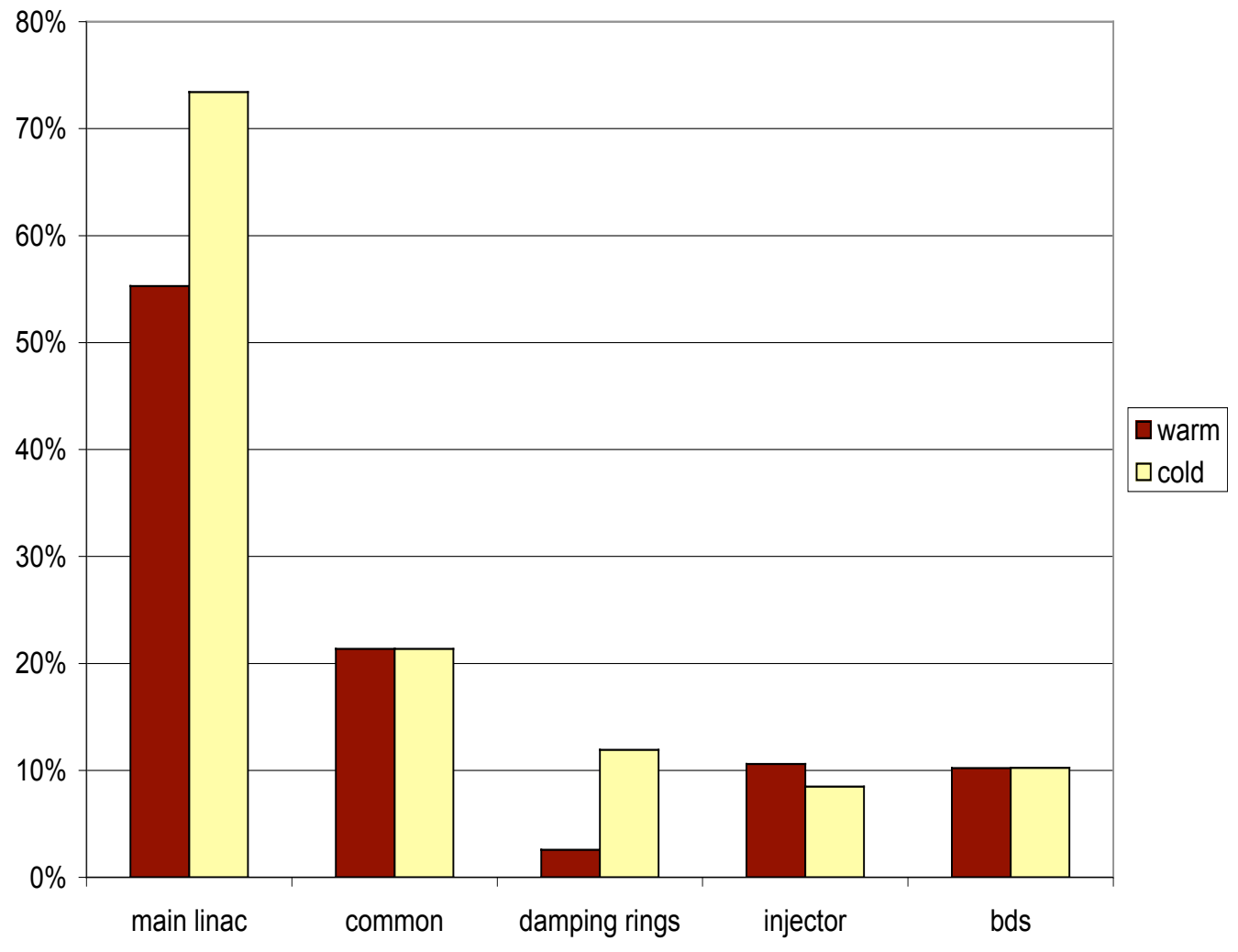

Figure 5.3.2.3: Costs for each technology option normalized to the total of the warm option shown by machine region 


\subsection{Project Models and Schedules}

The goals of schedule analysis at this early stage of the linear collider project are to identify and assess the scheduling consequences of the different technology options and configuration variants. Potentially significant risks related to those scheduling consequences may impact the project's scope, cost or schedule. These goals require identifying the sequence and interrelationship of project activities such as $\mathrm{R} \& \mathrm{D}$, engineering design, civil construction, component production, installation and commissioning for the different systems of the linear collider.

\subsubsection{Preconstruction Activities}

There is considerable uncertainty regarding the near-term actions that launch the project, and even regarding the definition of project initiation under the separate project management processes of the different potential co-sponsors. For modeling the project schedule, it is assumed that there will be a coordinated, globally collaborative effort of research, development, and engineering design based on the linac technology choice recommended by ICFA in 2004. During the years 2005-2007, this international effort will include concept definition, system and component optimization, prototyping, manufacturing $\mathrm{R} \& \mathrm{D}$, technology transfer, systems engineering tests (at test facilities), and engineering design for site-independent procurement packages. The process initially will be organized under laboratory-to-laboratory agreements, and will commence in 2005 with international review and approval of the conceptual design and cost range defining the scope of the project.

Completion of the international, site-independent engineering design in 2007 will establish a baseline for the scope, cost and schedule for the remaining detailed design, construction, installation and testing. Concurrent with these international site-independent design activities, it is assumed that the U.S. design team will select at least one candidate site in the U.S. and assess its environmental, technical, cost and schedule impact to support a competitive national bid to host the construction project, should the U.S. government decide to take such action. For the preferred candidate site, site characterization, subsurface geotechnical investigation and site-specific civil engineering design will be carried out in 2006-2008 to enable on-site construction to start in 2009. Similar efforts world-wide will be conducted by the proponent of each site candidate.

In 2007, a U.S. Government interagency task force will negotiate, with the other partners internationally, the formalities of the international site selection process and the structure of the legal entity that will execute the project. In 2008, the international partners are expected to have chosen the site, agreed on the roles and responsibilities for construction, and established the legal entity of the project. The President's budget request for FY 2009, submitted to Congress in January or February 2008, will reflect the role and responsibilities the U.S. has negotiated in the international construction project.

The warm-versus-cold choice of linac technology should have little impact on the above chronology of events because the $R \& D$ on both options is comparably mature and because the events that will drive the schedule are managerial and political, not technical.

The level of project definition, $\mathrm{R} \& \mathrm{D}$, and engineering design through 2008 should be comparable to the technical basis for the ITER Final Design Report[70] that supports the international preparations for construction of ITER (the International Thermonuclear Experimental Reactor) ${ }^{4}$ an international fusion energy project of the same scale as the linear collider project. Approximately 15-20\% of the estimated cost of ITER has been spent in preparation for the site decision milestone and start of construction.

\footnotetext{
${ }^{4}$ The ITER Project Website is http://www.iter.org/. The participant teams negotiating the ITER site and construction agreement are from China, Europe, Japan, Korea, Russia and the U.S. (DOE).
} 


\subsubsection{Construction Activities}

For schedule modeling, it is assumed that the first construction funds will be appropriated in U.S. Fiscal Year 2009, with the possible exception of longer-lead procurement of plant and equipment for manufacturing and testing the Main Linac components.

The construction and commissioning schedule depends on the technology and configuration option chosen. Schedules have been developed for two fundamental technology options:

- W. Warm Main Linac and Undulator-driven Positron Source,

- C. Cold Main Linac and Undulator-driven Positron Source,

and for one additional configuration variant:

- W-1. Option-W variant with a conventional positron source.

We have also considered the scheduling issues for the single-tunnel cold-option variant, in which the dampingring straight sections, the main linac, the klystrons and their power supplies share the same tunnel. This is referred to below as "Variant C-1".

The logic for scheduling the construction and commissioning activities depends on the option or variant chosen. Model schedules for the options and Variant W-1 are shown in Figures 5.4.2.1 to 5.4.2.3. The activity durations assumed in the model schedules are summarized in Table 5.4.2.1 and Table 5.4.2.2. To facilitate comparative evaluation of the differences, some significant milestone dates from the schedules are summarized in Table 5.4.2.3.

Civil construction for the entire project is planned within a five-year time frame and is anticipated to commence with excavating the Interaction Region (IR) Halls, to allow the longest possible time for the elevation of the floor under the detectors to rebound and settle. Excavation of the injector vaults probably will begin during excavation of the IR. The beam line and equipment tunnels for the Main Linac and Beam Delivery systems will be created by a combination of boring and/or mining operations launched upstream from the IR, downstream from the injector vault, or from points in between. The site and its topography will determine how to coordinate the launches most advantageously with the collider's specified shaft locations. The average rate of advance will be approximately 16 meters per day for each tunneling operation. ${ }^{5}$ Assuming that two such operations are employed simultaneously to create each beam line tunnel and each equipment tunnel for each Main Linac (eight operations in play simultaneously), the net duration for construction of the two 14-km-long housings of the warm collider (Option W or Variant W-1) will be about two years. For this study, we assume that the two 21-km-long housings for the cold collider (Option C or Variant C-1) also may be created in the same two-year duration by mobilizing additional tunnel boring or mining operations. For either the warm or the cold option, several possible scenarios will exist for mobilizing combinations of boring and/or mining operations capable of creating the Main Linac tunnels and permitting beneficial occupancy within a two-year time frame. One illustrative sample scenario for each option is shown schematically below in Figure 5.4.2.4.

Site and campus facilities such as critical elements of the electrical and cooling systems, the control room facility and the core of the accelerator control system are scheduled in time for accelerator commissioning. Later, fabrication and assembly facilities will be needed in time for construction of the experimental detectors. Later still, other campus facilities will be needed for laboratory and experimental operations.

Installation of each Main Linac is scheduled to take 30 months under the assumption that five installation crews will install preassembled units of the warm linac (for Option W or Variant W-1) at a rate of about 94

\footnotetext{
${ }^{5}$ The actual rate will depend greatly on site-specific geology and hydrology.
} 


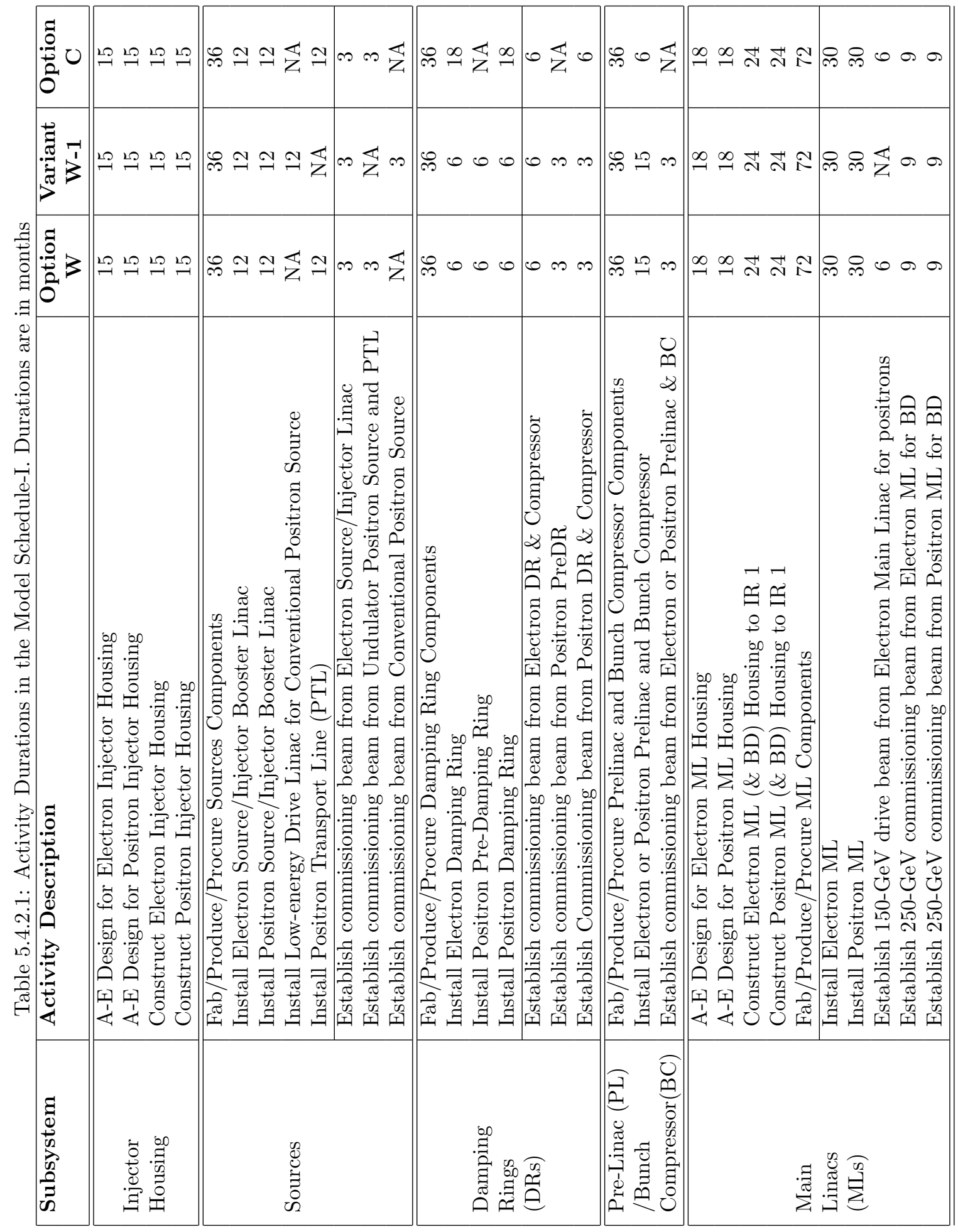




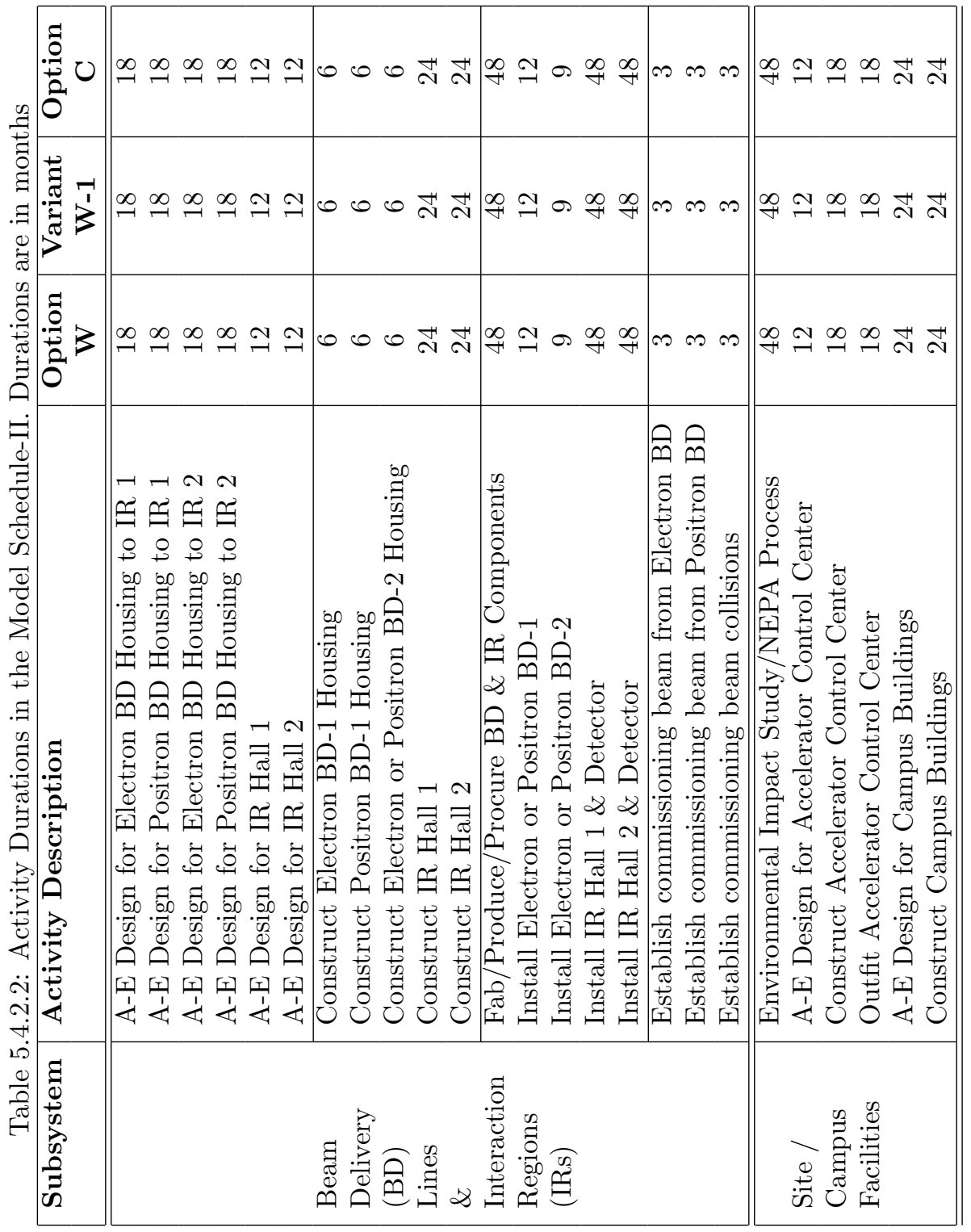


Table 5.4.2.3: Milestone Dates in the Model Schedules

\begin{tabular}{|c|c|c|c|}
\hline Milestone & $\begin{array}{l}\text { Option } \\
\text { W }\end{array}$ & $\begin{array}{l}\text { Variant } \\
\text { W-1 }\end{array}$ & $\begin{array}{c}\text { Option } \\
\mathrm{C}\end{array}$ \\
\hline $\begin{array}{l}\text { Approve Conceptual Design } \\
\text { and Cost Range }\end{array}$ & \multicolumn{3}{|c|}{ FY2005 } \\
\hline $\begin{array}{l}\text { Establish Baseline for Scope, } \\
\text { Cost and Schedule }\end{array}$ & \multicolumn{3}{|c|}{ FY2007 } \\
\hline $\begin{array}{l}\text { Choose Site, Establish Roles } \\
\text { and Responsibilities }\end{array}$ & \multicolumn{3}{|c|}{ FY2008 } \\
\hline Start Construction & \multicolumn{3}{|c|}{ FY2009 } \\
\hline $\begin{array}{l}\text { Start Commissioning } \\
\text { Electron Main Linac }\end{array}$ & $\begin{array}{c}\text { FY2013 Q2 } \\
\text { (After } \\
\text { installing } 150 \\
\text { GeV of } \\
\text { Electron ML) }\end{array}$ & $\begin{array}{c}\text { FY2014 Q2 } \\
\text { (After } \\
\text { installing } \\
\text { Electron ML) }\end{array}$ & $\begin{array}{c}\text { FY2013 Q3 } \\
\text { (After installing } 150 \mathrm{GeV} \text { of } \\
\text { Electron ML and } \\
\text { commissioning Electron DR) }\end{array}$ \\
\hline $\begin{array}{l}\text { Start Commissioning } \\
\text { Positron Main Linac }\end{array}$ & $\begin{array}{c}\text { FY2014 Q4 } \\
\text { (After } 150 \mathrm{GeV} \\
\text { electrons, } \\
\text { Positron } \\
\text { Source and } \\
\text { PTL) }\end{array}$ & $\begin{array}{l}\text { FY2014 Q3 } \\
\text { (After } \\
\text { separate 6- } \\
\text { GeV Driver) }\end{array}$ & $\begin{array}{c}\text { FY2014 Q4 } \\
\text { (After } 150 \mathrm{GeV} \text { electrons, } \\
\text { Positron Source, PTL, and } \\
\text { Positron DR) }\end{array}$ \\
\hline $\begin{array}{c}\text { Start Commissioning } \\
\text { Colliding Beams }\end{array}$ & FY2016 Q4 & FY2015 Q3 & FY2016 Q4 \\
\hline
\end{tabular}


meters of beam line per month per crew, or that they will install preassembled units of the cold linac (for Option C) at a rate of 140 meters of beam line per month per crew. The difference is based primarily on the relative length and number of X-band girders in the warm linacs compared to cryomodules in the cold linacs.

The duration of Damping Ring installation is much shorter for the conventional rings, in Option W or Variant $\mathrm{W}-1$, than for the dog-bone shaped rings in Option C or Variant C-1. Installation of each ring is estimated to advance at a rate of 50-100 meters of beam line per month per installation crew, using preassembled rafts or girders, in line with experience from the PEP-II and SPEAR-III rings at SLAC. At that rate, each conventional ring, in Option W or Variant W-1 (with 300-meter circumference), can be installed by a single crew working for 6 months at an average advance rate of 50 meters per month.

A faster rate of advance is justified for installation of the dog-bone rings because $80 \%$ of the installable length of each ring is composed of simpler straight sections. Installing each of the dog-bone rings (with 7500 meters of double-chamber straight sections plus 2000 meters of arc sections) is estimated to take as much as 18 months for five crews, or 30 months for three crews, working simultaneously at an average advance rate of $100 \mathrm{~m} / \mathrm{mo}$ with separate entry points into the housing. In Option C and Variant $\mathrm{C}-1$, installation of each dog-bone ring is scheduled concurrently with installation of the Main Linac because each dog-bone ring shares the Main Linac's tunnel. There is risk that interference between the different installation crews working in the same area will cause critical schedule delay. That risk is even greater for Variant C-1 (without a separate equipment tunnel) because the klystrons, power supplies and other equipment, in addition to the Main Linac and the dog-bone ring, would all share the same tunnel.

Installation of the Beam Delivery systems, which will be among the last systems commissioned, is scheduled late in the project in order to benefit from as much $R \& D$ as possible before making critical design choices, and to level the overall funding and resource profiles by delaying production and installation of the beam delivery components.

\subsubsection{Commissioning Activities}

Preoperational commissioning is a critical component of the project that can, and should, begin long before the completion of construction. It should not be deferred until all parts of the collider have been installed because the flexibility of choices available for solving problems generally decreases, and the cost of solutions increases, as the project progresses toward completion. Done sequentially as systems become complete, commissioning can and should drive learning and reduce risk for subsequent efforts on other systems. It will also provide stakeholders with timely reassurance of the success of the project, sooner rather than later.

The commissioning strategy has a major impact on the scheduling of installation tasks and on the project completion milestone for the different options, as summarized below and in Table 5.4.2.3.

In Option $\mathrm{W}$ or $\mathrm{C}$, or Variant C-1 (all with undulator-driven positron sources), positron commissioning will be delayed until $150-\mathrm{GeV}$ electrons are available to drive the undulator, and until the Positron Transport Line is available to deliver the positrons from the undulator to the Positron Damping Ring(s). The use of an auxiliary positron source, as proposed in the TDR[TDR, Section 4.3.6], or an auxiliary electron source as in the reference designs, would help to speed up commissioning.

In Option C or Variant C-1 (with undulator-driven positron source and dog-bone shaped damping rings), Damping Ring (DR) commissioning may delay installation of part of the Main Linac (ML) because much of each DR and ML share the same housing. The schedule for Option C shows a year's hiatus in the installation of the Electron ML to accommodate commissioning of the DR and $150 \mathrm{GeV}$ of the ML (rather than delay DR and ML commissioning until the entire ML is installed), and another year's hiatus in the installation of both MLs, the Beam Delivery Lines and IR Hall 1 in order to accommodate commissioning the positron source and transport line, the positron DR, and part of the positron ML. Installation of the balance of each 
ML, Beam Delivery and IR Hall 1 resumes afterwards, when the Positron Transport Line is not in use.

The model schedules for Options $\mathrm{W}$ and $\mathrm{C}$ start colliding-beam commissioning approximately at the same time (late FY2016). Variant W-1 has the potential to achieve colliding beams sooner-by 15 months in the model schedule - because the conventional positron source is driven by a dedicated 6 - GeV linac separate from the Electron Main Linac.

These differences may affect the scope of commissioning and the initial operational performance of the new collider for physics. For each option/variant, Figure 5.4.3.1 shows the cumulative build-up of commissioning time (from the model schedules) for electron and positron beams separately in the DRs, MLs and Beam Delivery (BD) systems. Options $\mathrm{W}$ and $\mathrm{C}$ are comparable. Variant $\mathrm{W}-1$ accumulates the most Beam Delivery commissioning time.

\subsubsection{Summary of Schedule-related Risks}

Several potentially significant schedule-related risks have been identified above, including:

1. Injector commissioning may be delayed by site electrical power or cooling systems.

2. Injector commissioning may be delayed by the control center or control system.

3. Main Linac, Beam Delivery or IR installation may be delayed by commissioning operations involving the Positron Transport Line that carries the positron beam from the undulator-based source in the Electron Main Linac all the way to the beginning of the Positron Main Linac. (This risk does not apply to Variant W-1 because the conventional positron source has no Positron Transport Line.)

4. Initial operational performance may be limited by inadequate commissioning time.

5. Main Linac or Damping Ring installation may be delayed by problems mobilizing sufficient underground construction resources, a greater risk for the cold option because more resources must be mobilized to build the longer housing in the same time.

6. Sharing of the same housing by the dog-bone damping ring and the main linac in the cold technology option (C) will increase the risk of a critical schedule delay during installation and/or commissioning. The increased complexity of installation caused by the sharing of a single tunnel by the damping ring, main linac accelerator components, and main linac power components in the single-tunnel design variant for the cold technology option $(\mathrm{C}-1)$ will further increase the risk of a critical schedule delay during installation and/or commissioning. 


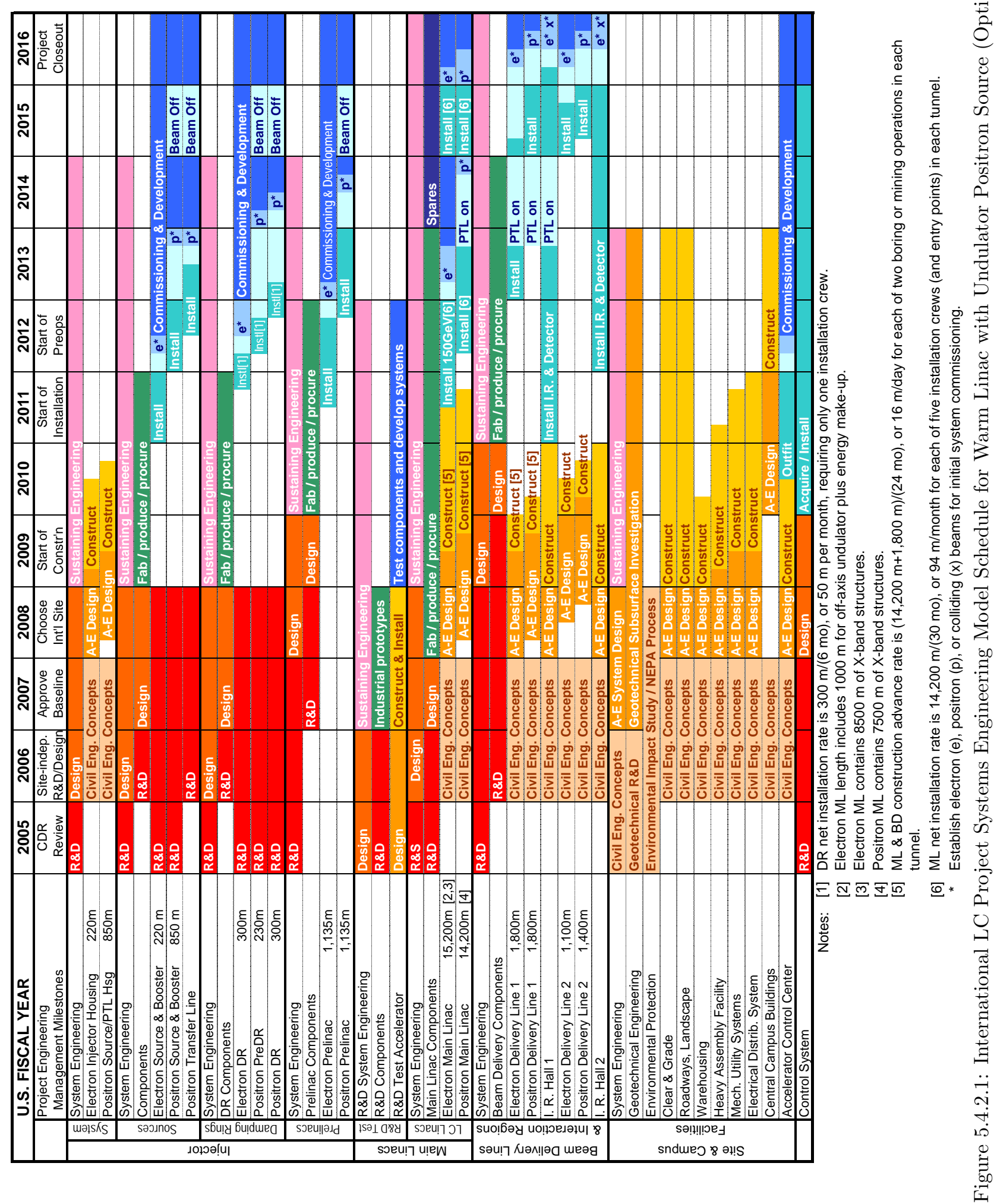




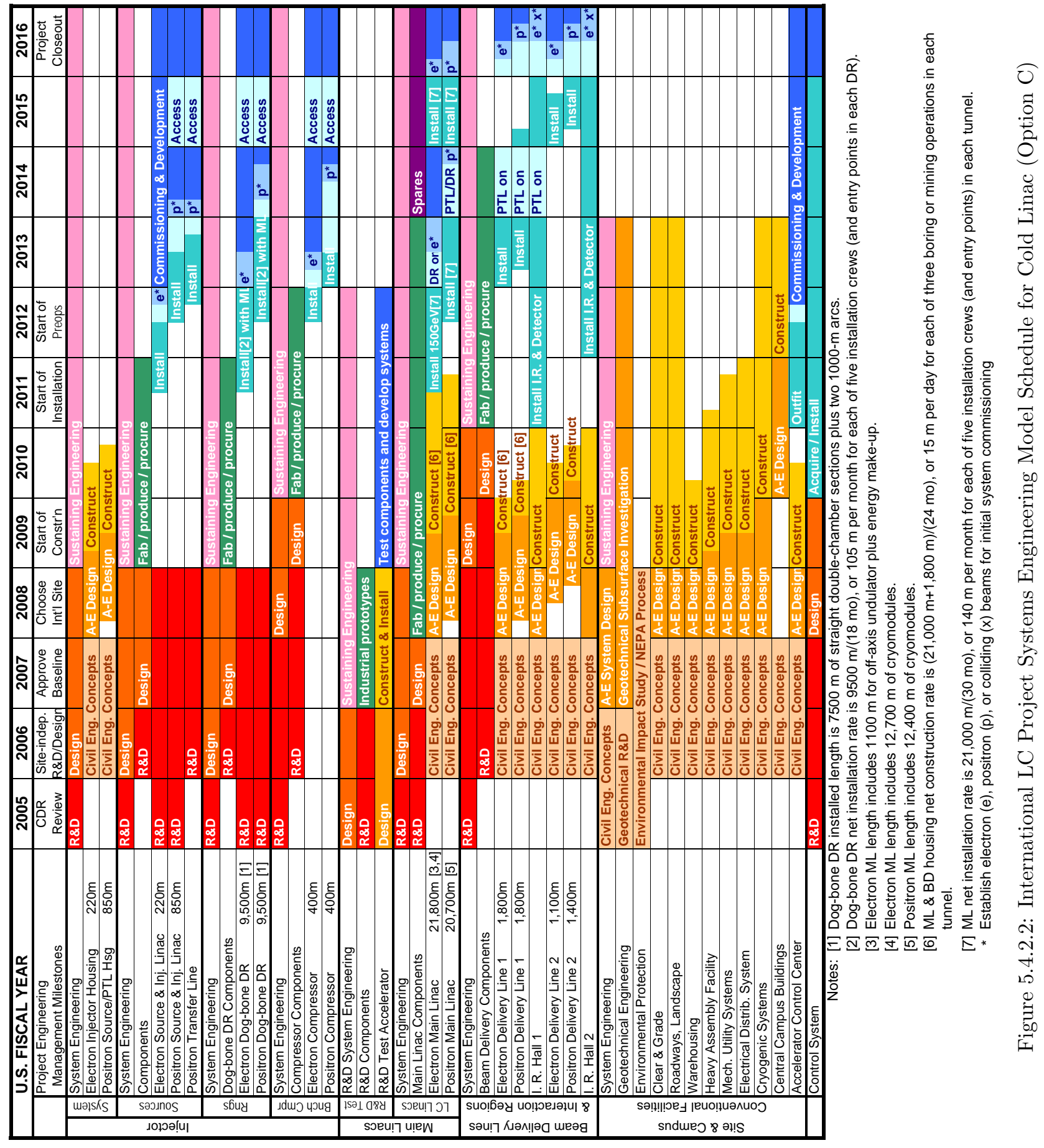




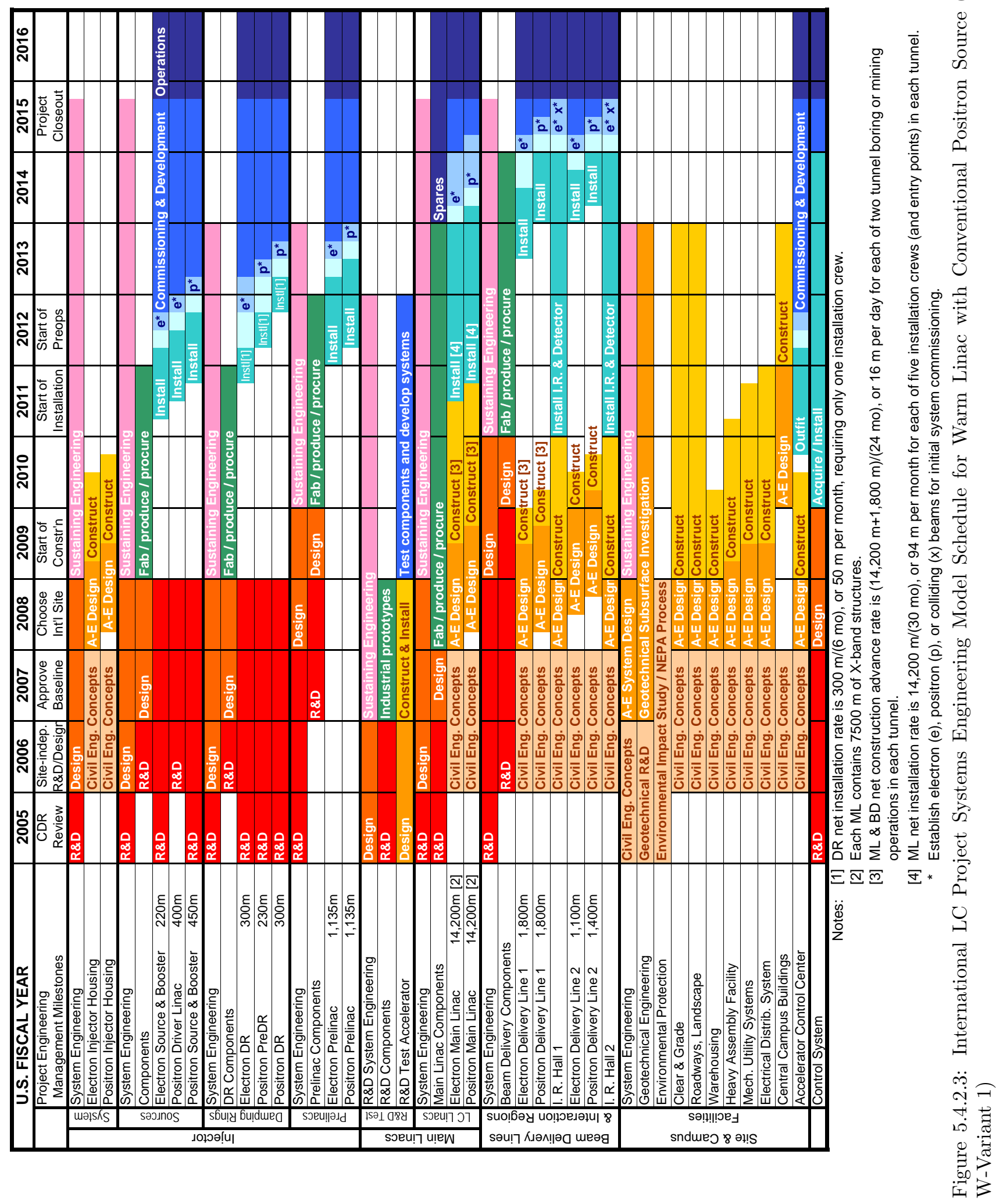


(a) Warm Option Scenario: Tunneling Operations for the Beam and Equipment Tunnels

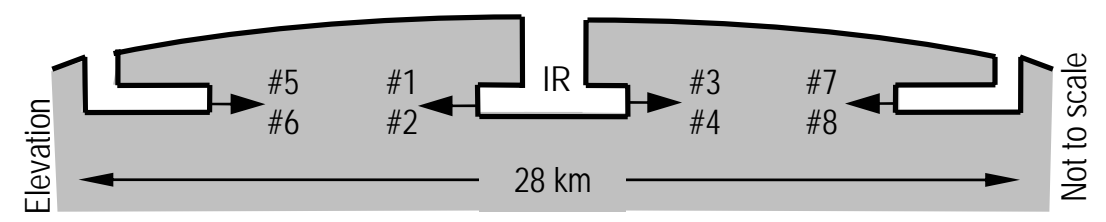

(b) Cold Option Scenario: Tunneling Operations for the Beam and Equipment Tunnels

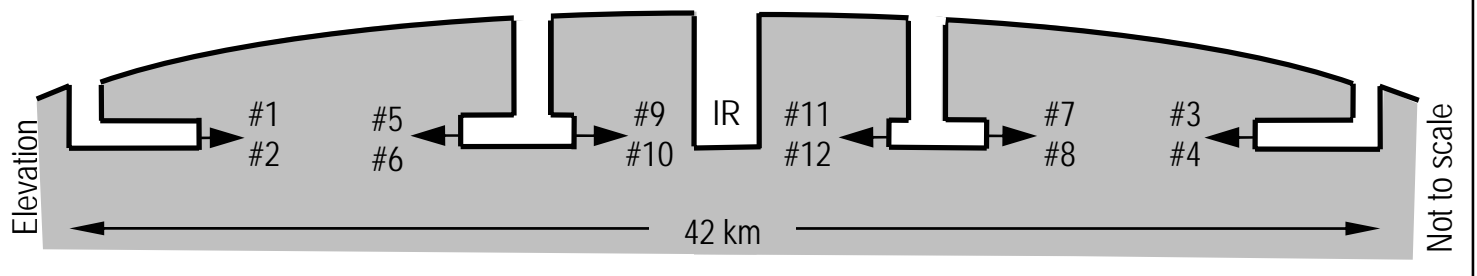

Figure 5.4.2.4: Illustrative sample tunneling scenarios 


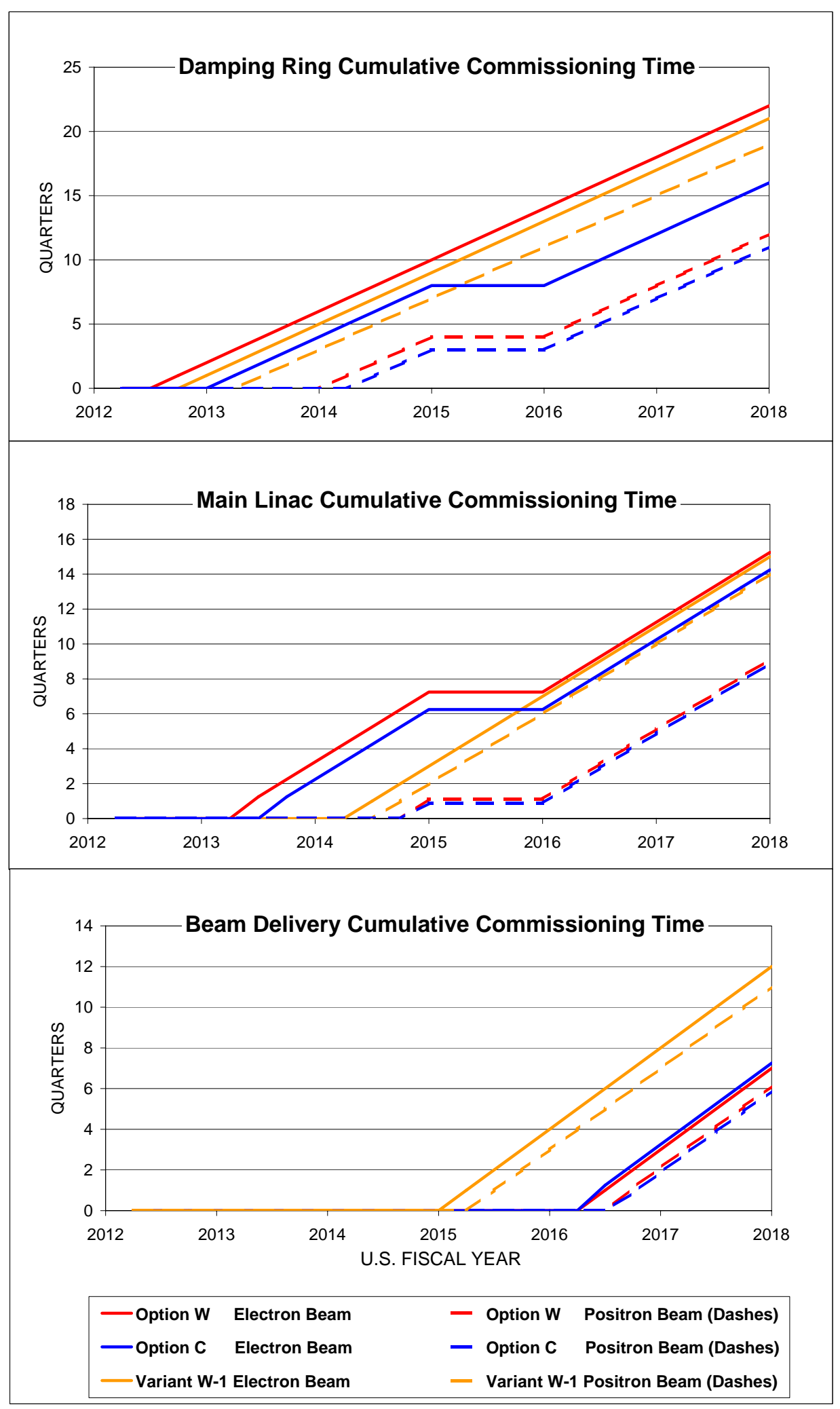

Figure 5.4.3.1: Cumulative Build-up of Commissioning Time 


\subsection{Cost and Schedule for Upgrade and Variants}

\subsubsection{Upgrade to $1 \mathrm{TeV}$}

The warm and cold reference designs include sufficient tunnel lengths to allow the colliders to reach 1 TeV when filled with accelerators operating at loaded gradients of $52 \mathrm{MV} / \mathrm{m}$ and $35 \mathrm{MV} / \mathrm{m}$ respectively. The tunnels are only partially filled with components for the $500 \mathrm{GeV}$ stage, and the unfilled parts of the tunnels include only what is necessary to transport the beams to the Beam Delivery Section. The upgrade to 1 TeV requires not only fabrication and installation of the remaining accelerator components, but also completion of the power, water, and cryogenic systems.

The initial gradient used in the $500 \mathrm{GeV}$ warm collider is the same as required for the upgraded $1 \mathrm{TeV}$ machine, so the extension is done by replicating essentially all that is installed in the tunnel in the first stage. The $500 \mathrm{GeV}$ cold collider uses a lower gradient than the upgraded machine, so more than half of the tunnel is initially filled with cryomodules. On the other hand, the cryogenic system for the initial configuration must be upgraded along with extending the length of the linacs.

Our estimate is that it will cost approximately the same amount to upgrade either machine. This is a consequence of the trade-off in the length and gradient seen in Figure 5.3.2.1.

\subsubsection{One Tunnel for the Cold Option}

The option to build a collider with the cold linac technology in one 5-meter diameter tunnel rather than two 4-meter diameter tunnels was considered. The reduction in total length of tunnel is partially offset by the increased volume of the single tunnel. We estimate that the tunnel and infrastructure costs will be reduced by $5.1 \%$ of the total project cost. The expected hardware availability of a machine built with a single tunnel has been estimated to be significantly less than a machine with two tunnels. A rough estimate of $3 \%$ has been made for the costs needed to increase component reliability to meet overall availability requirements. The net is a reduction of the total project cost of about $2 \%$ if the collider is built in a single tunnel.

The increased complexity of installation, caused by the sharing of a single tunnel by the damping ring, main linac accelerator components, and main linac power components, increases the risk of delay in the completion of the construction and commissioning of the collider.

\subsubsection{Initial Gradient of $35 \mathrm{MV} / \mathrm{m}$ for Cold Option}

The overall cost of the main linac as a function of increasing accelerating gradient is a trade between the rising costs of cryogenic systems and sources of microwave power on one hand, and the falling costs of the beamline components and supporting infrastructure on the other (Figure 5.3.2.1). The cold machine reaches this minimum at $40-45 \mathrm{MV} / \mathrm{m}$, and we estimate the reduction in total project cost at $35 \mathrm{MV} / \mathrm{m}$ to be about $3 \%$ relative to our reference design at $28 \mathrm{MV} / \mathrm{m}$.

\subsubsection{Superstructure for Cold Option}

It may be possible to use a single power coupler to provide RF to a pair of 9-cell cavities in the cold cryomodules. This would reduce the number of couplers required for the machine by a factor of two, and the increased packing fraction of the pairs of cavities would reduce the length of the civil construction by about $5 \%$. We estimate the combination would reduce the total project cost by about $3 \%$. 


\subsubsection{DLDS for Warm Option}

The DLDS pulse compression scheme for the warm collider more efficiently uses the power produced by the klystrons because all of the energy reaches structures in phase with the beam. (The SLED-II system inherently transfers about $25 \%$ of the energy from the klystrons to the structures at the wrong time.) If it proves possible to develop klystrons with twice the $1.6 \mu \mathrm{s}$ of the warm reference design, and the DLDS system proves able to handle the increased energy, then the number of modulators and klystrons can be reduced by a factor of two. The number of pulse compression components is also reduced. The potential reduction in the total project cost is about $8 \%$.

\subsubsection{Conventional Positron Source}

Use of a conventional positron source is an option for the warm machine, and may be also an option for the cold. If either machine were built with a conventional positron source instead of the undulator source, then an unpolarized electron gun, a $6 \mathrm{GeV}$ electron linac, multiple targets, and a pre-linac with associated civil support facilities would need to be substituted for the linac space and components needed for the undulator positron source and electron energy make-up, positron pre-linac, and tunnel and components of the positron transfer line. The cost would be reduced by approximately $2 \%$, but later upgrade to include a polarized positron source would be difficult. If the initial configuration includes the space required in the linac to later install the components of the undulator-based source, the energy make-up in the linac, and the tunnel needed for the positron transfer line, then the cost would be approximately the same as starting with an undulator source with no conventional source.

Use of a conventional positron source can be expected to have a major impact on commissioning the collider. The reference undulator positron source couples operations of the entire positron system to operation of the electron source, damping ring, and first $150 \mathrm{GeV}$ of the electron linac. While it may be possible to work around these difficulties, the use of a conventional positron source could result in a reduction of as much as a year in the construction and commissioning of the collider.

The option to build the collider with a conventional positron source and the vacant space needed to later implement a polarized undulator source should be more vigorously considered.

Table 5.5.6.1: Cost of Variants. This table contains a summary of the impacts of the Variants to the warm and cold US reference designs that we have studied. Some Variants may lengthen the duration of the project, and others may shorten the project schedule. We have indicated this in the last column.

\begin{tabular}{|c|c|c|c|}
\hline Variant & Technology & $\begin{array}{c}\text { Cost Impact } \\
\text { (\% of TPC) }\end{array}$ & Schedule Impact \\
\hline Single Tunnel & Cold & Reduction of $2 \%$ & Lengthen \\
$35 \mathrm{MV} / \mathrm{m}$ & Cold & Reduction of $3 \%$ & None \\
Superstructure & Cold & Reduction of $3 \%$ & None \\
DLDS & Warm & Reduction of $8 \%$ & None \\
Conventional $\mathrm{e}^{+}$ & Both & None & Shorten \\
\hline
\end{tabular}

\subsection{Cost and Schedule Conclusions}

- The available cost estimates for both technologies are based on experience with existing accelerator facilities, technology specific R\&D prototypes and test facilities, and industrial models of large-scale production. 
- The maturity and confidence in the cost estimates for both technologies are appropriate for a major U.S. project in the conceptual design stage. Extrapolated reductions in unit costs from present-day R\&D to the high-volumes needed to build the collider are a source of risk in the cost estimates. Extrapolations of up to factors of three to five were made for the components of the cold crymodules, and as high as six for some of the warm copper components. The overall extrapolation is somewhat larger for the warm technology because of the larger number of small repetitive components involved.

- More than two thirds of the warm costs are independent of the accelerator technology, and the corresponding cold costs are the same. The cold-warm difference arises because the costs that are specific to the cold technology choice are estimated to be about twice as large as those that are specific to the warm option. The differences in the specific costs result in an estimated total project cost for the cold machine that is $25 \% \pm 10 \%$ greater than for the warm machine.

- The quoted uncertainty assumes a $\pm 15 \%$ (rms) uncertainty in the specific costs, and ignores correlations between the warm and cold cost estimates. We have not done a complete analysis of the uncertainties in these costs, which would have to include any correlations. The presence of correlations would lower the uncertainty.

- We found it necessary to re-estimate the cost of the damping rings for the cold technology to account for differences in the technical specifications from those in the TESLA TDR.

- The industrial technologies and experience needed to produce the components and systems of each machine choice are well-defined and available in world-wide industries. Industrial capacities needed to produce the quantities of the components for the main linac do not exist today for either the warm or cold technology.

- Both technologies are ready for substantial value engineering (VE) and design-for-manufacture (DFM). We expect the cost and confidence in the acquisition of the components needed for each technology to respond favorably to continued design analysis, technology R\&D, and VE and DFM.

- Provided appropriate funding during construction, the time needed to build and commission a collider can be independent of the choice of linac technology.

- With the initial configurations that we have assumed, the estimated cost to upgrade the $500 \mathrm{GeV}$ reference design to $1 \mathrm{TeV}$ is approximately the same for each technology. The cost to upgrade will depend significantly on when and how it is done. 


\section{Chapter 6}

\section{Civil Construction and Siting}

\subsection{Introduction and overview}

This chapter describes civil design studies that have been completed for two representative U.S. sites, one situated in Illinois and the other in California. For each site, two designs are outlined-one based on a L-band, superconducting (cold) linac, the other on an X-band normal conducting (warm) linac. Civil design criteria in all instances are based on the requirements described in Chapter 3 of this document.

The primary distinguishing feature between the cold and warm designs are the length of the linac enclosure and the need to provide cryogenic infrastructure in the case of the cold machine. The requirement of accommodating an eventual upgrade to $1 \mathrm{TeV}$, coupled with the assumed accelerating gradients, leads to facility footprints that are roughly $47 \mathrm{~km}$ and $33 \mathrm{~km}$ respectively for the cold and warm machines. Both designs are based on two parallel tunnels bored through hard rock. One tunnel accommodates the linac itself and the second houses support equipment. This is a major departure from the TESLA design and ramifications of a single tunnel design are discussed in Section 7.2. This configuration leads to minimal surface presence over the extended length of the linac in the two machinesperiodic access shafts in both cases and cryogenic facilities spaced by $5 \mathrm{~km}$ in the case of the cold machine. Two interaction regions are provided and in both cases a campus with support buildings.

The civil design descriptions can be found in Sections 6.2 (normal conducting) and 6.3 (superconducting). Included are design concepts for the tunnel enclosures, interaction region halls, enclosure access, site power distribution, site cooling water distribution and heat dissipation, and campus facilities. Descriptions are provided separately for both California and Illinois representative sites. The majority of design features for the California and Illinois sites are identical. Primary distinguishing features between California and Illinois designs include tunnel access (vertical in Illinois, horizontal in California) and heat dissipation methods. Complete drawing sets have been developed for both sets of sites and both sets of linac technologies. A high level subset of these drawings is appended to the design descriptions in Section 6.2 and 6.3.

In parallel with the design preliminary cost estimates and construction schedules were developed. These are described in Chapter 5. 


\subsection{Conventional Facilities Design Summaries and Drawings for the Normal-conducting X-band option}

\subsubsection{Conventional Facilities Reference Design Summary for a Normal-conducting X-band Linear Collider at Logan Ridge, California ${ }^{1}$}

Table 6.2.1.1: Normal-conducting Reference Design Summary Parameters

\begin{tabular}{|c|c|}
\hline Item & Baseline \\
\hline $\begin{array}{l}\text { Housing } \\
\text { Radiation } \\
\text { Containment }\end{array}$ & $\begin{array}{l}\text { For two parallel bored tunnels of } 4.5 \text { meters inside diameter, a minimum of } 8 \text { feet of } \\
\text { concrete or } 24 \text { feet of earth \& rock shielding is required for general radiation con- } \\
\text { tainment. Tunnel-to-tunnel penetrations must adequately collimate and attenuate } \\
\text { prompt line-of-sight radiation between tunnel housings and include from source to } \\
\text { receiver at least } 90^{\circ} \text { of angular offsets to the direct penetration sight line. Beam } \\
\text { absorbers (dumps), positron sources and damping rings have greater requirements } \\
\text { and are shielded as required by location. All beam tunnel housing and support } \\
\text { tunnel housing drain water is collected, pumped, batched, held, and tested before } \\
\text { release to surface drains. }\end{array}$ \\
\hline $\begin{array}{l}\text { Special } \\
\text { Beam } \\
\text { Housing } \\
\text { Stability }\end{array}$ & $\begin{array}{l}\text { Water cooling circuits in the beam housings are to have a maximum flow velocity } \\
\text { of } 9 \text { feet per second and are to be entirely plumbed with flexible hoses. Except for } \\
\text { drainage sump motors, no utility motors are permitted inside the beam housings. }\end{array}$ \\
\hline Cooling & $\begin{array}{l}\text { For steady-state full power operations at } 120 \mathrm{pps} \text {, the low conductivity cooling water } \\
\text { for klystrons, modulators, electro-magnets, and totally enclosed electronics racks is } \\
\text { to be } 94{ }^{\circ} \mathrm{F} \text { supply, } \pm 5{ }^{\circ} \mathrm{F}, 65^{\circ} \mathrm{F} \text { rise for klystrons, } 15{ }^{\circ} \mathrm{F} \text { rise (max) for magnets } \\
\text { and } 5{ }^{\circ} \mathrm{F} \text { rise for I\&C racks. Low conductivity cooling water for accelerator sections } \\
\& \text { SLED-II RF pulse compression distribution is to be } 82^{\circ} \mathrm{F} \text { supply, } \pm 0.3^{\circ} \mathrm{F}, 12 \\
{ }^{\circ} \mathrm{F} \text { rise, } 9 \mathrm{fps} \text { maximum velocity in flexible hoses. Heat exchangers for ACS and } \\
\mathrm{KCS} \text { are in series. The cooling supply water is fed to the ACS heat exchanger at } \\
80^{\circ} \mathrm{F} \text { and then to the } \mathrm{KCS} \text { heat exchanger at } 91^{\circ} \mathrm{F} \text {. The support tunnel housing air } \\
\text { temperature is } 85^{\circ} \mathrm{F}, \pm 10^{\circ} \mathrm{F} \text { maximum with access \& season and } 88 \mathrm{fpm} \text { minimum } \\
\text { velocity. Beam housing air is } 95^{\circ} \mathrm{F}, \pm 5^{\circ} \mathrm{F} \text { with access \& season, } 0 \text { fpm velocity } \\
\text { with beam and } 88 \mathrm{fpm} \text { velocity with personnel access. }\end{array}$ \\
\hline Power & $\begin{array}{l}\text { Two } 230 \mathrm{kV} \text { substations, four of six existing } 230 \mathrm{kV} \text { dual feed high availability } \\
\text { transmission sources. Site distribution: } 34.5 / 19.9 \mathrm{kV}, 3 \mathrm{ph}, 60 \mathrm{hz} \text {, grounded wye, } \\
100 \text { amp, } 150 \mathrm{kV} \text { bil, w/ } 220 \mathrm{mil} \text { XLP cable w/ } 27 \mathrm{kV} \text { arrestors \& ESNA termi- } \\
\text { nations. Load type is: } 70 \% \mathrm{RF} \& 15 \% \text { cooling, \& } 15 \% \text { other loads. Phase locked } \\
60 \mathrm{hz} \text { variable frequency motor drives are required site-wide. Rotating equipment } \\
\text { shall be isolated on } 3 \text { hertz skids. }\end{array}$ \\
\hline $\begin{array}{l}\text { Special } \\
\text { Maintenance } \\
\text { Access }\end{array}$ & $\begin{array}{l}\text { Klystrons, modulators and SLED-II are to be available for maintenance by person- } \\
\text { nel } 24 \text { hours a day. They are to be located as close as possible to beam accelerator } \\
\text { sections to reduce RF distribution losses between the klystrons and the beam ac- } \\
\text { celerator sections. }\end{array}$ \\
\hline
\end{tabular}

\subsubsection{Configuration and Design Features}

6.2.1.1.1 Purpose This section describes the conventional construction needed to build the U.S. sited normal-conducting X-band linear collider, which is referred to in this section as the "XLC". The accelerator

\footnotetext{
${ }^{1}$ Near Stanford Linear Accelerator Center, Lawrence Livermore National Laboratory, and Lawrence Berkeley National Laboratory
} 
must meet stringent requirements for sustained luminosity and machine stability and should be located close to existing physics laboratories. The solution presented is only one possible option which can be used to determine the impact that a given site might have on an accelerator and its related design.

Criteria for the conventional construction are given in Section 3.4, and references [NLC01] and [71]. Design solutions were developed with input from a combination of engineers, consultants and physicists at SLAC, FNAL and other laboratories.

The location of the representative site chosen is in close proximity to existing physics laboratories at Stanford Linear Accelerator Center (SLAC), Lawrence Berkeley National Laboratory (LBNL), Lawrence Livermore National Laboratory (LLNL) and surrounding communities. It is roughly 120 miles north of SLAC and 90 miles north of LBNL. The site is supported by the San Francisco Bay's entire regional infrastructure.

6.2.1.1.2 Configuration The representative site has a north-south alignment in the bedrock of the foothills located along the west edge of California's Central Valley. End-to-end, it is approximately 35 kilometers in length. The design explores the construction of two parallel below grade tunnels bored through stable well-cemented bedrock that consists of Upper Cretaceous (91 to 73 million year old) sedimentary rocks commonly known as the Great Valley Group. Locally, the formation consists primarily of thinly bedded sandstones, with small quantities of siltstones and claystones.

Ramps and some utility shafts provide access between the surface and the below grade housings through which air, cooling water and electric power are routed. Ramps at the injector, main linacs and the interaction region will be the major access points for equipment as well as a conduit for utilities and services. The dimensions of the ramps and shafts will generally not exceed the diameter of the beamline or support tunnels with some exceptions. All ramps will serve as an egress corridor from the tunnels below. A minimum of 5 ramps with utility shafts and 8 additional utility shafts will be required along the entire length of the machine. Cross tunnel passageways will connect the beam housings to the support housings at every ramp access location and also between those locations at periodic intervals not to exceed 8,200 feet. These will include adequate offsets and shielding to attenuate housing-to-housing radiation exposure down to safe levels for personnel working in the support housings.

Necessary project infrastructure that already exists at the nearby physics laboratories will be used for the support of the XLC. This minimizes the required local campus space while providing support for long-term operations, maintenance and world wide connectivity.

The site has a nearly north-south orientation parallel to the US Interstate 5 (I-5) corridor, which is approximately $8 \mathrm{~km}$ east of the representative XLC site. Six $230 \mathrm{kV}$ power transmission lines run parallel to the machine and to I-5. The California aqueduct, a primary source of cooling and potable water, also runs parallel to the I-5 corridor and the XLC alignment. The location was chosen to satisfy several important criteria:

1. Avoid populated areas. This reduces cultural noise and simplifies land acquisition.

2. Allow all of the housings to be constructed in bedrock in the Great Valley Group formation.

3. Provide reasonable access to existing transportation infrastructure.

4. Locate near existing high voltage power and water distribution.

More studies to evaluate property needs for the project will help to minimize the impact to the public and surrounding communities and optimize land use. We anticipate that the U.S. DoE would acquire a corridor of property rights and easements. It would be as much as 500 feet wide in some locations. We estimate that as many as 1000 acres of various property rights and easements will be required for all of the XLC, including land to construct the local campus. 
6.2.1.1.3 Design Features The accelerator is placed in surface bedrock that crops out in the low lying hills along the west side of Northern California's Central Valley east of Antelope Valley. The bedrock of the area consists of the Great Valley Group, made primarily of well-cemented, interbedded sandstones. This places the depth of the tunnels in a range from 30 feet to 340 feet below the surface, but allows for shallow ramp access to the machine at depths of 30 feet to occasionally 150 feet above the tunnels via east-west running valleys and other low-lying areas of Northern California's Central Valley. Key features of the design are:

1. Two parallel tunnel enclosures providing mutual life safety support.

2. The use of normal-conducting X-band RF main linacs.

3. Shielding naturally provided by the rock mass between and above the tunnel enclosures.

4. Two substations providing electrical power, each located mid-linac, and providing $34.5 \mathrm{kV}$ site wide high voltage distribution.

5. Egress via labyrinth type access ways from tunnel enclosures.

Development of a local XLC campus supported by existing SLAC, LBNL \& LLNL facilities nearby is assumed.

\subsubsection{Major Components}

6.2.1.2.1 Local Campus A single local campus is planned for the XLC. This campus will be located approximately $8 \mathrm{~km}$ west of the existing I- 5 corridor. The campus area will include spaces and facilities to operate and maintain the XLC.

The local site campus will primarily support specific operations and maintenance space. Other space needs will be met at the nearby LLNL, SLAC and LBNL laboratories. Table 6.2.1.2 outlines assumptions for construction of local campus building space.

6.2.1.2.2 Injectors The configuration of the injector complex will place the electron injector at the south end of the machine. The remote injection configuration places a significant portion of the XLC complex well away from the interaction region, which is especially sensitive to cultural noise and vibration.

6.2.1.2.3 Main Linac The electron and positron main linacs will be in tunnels below but near the surface. The positron main linac tunnel is approximately $14 \mathrm{~km}$ in length. Using an undulator based positron source, the electron main linac tunnel will be approximately $15 \mathrm{~km}$ in length. Klystrons and modulators will be housed in the support tunnel, parallel and adjacent to the main linac beam tunnel.

6.2.1.2.4 Beam Delivery At the ends of the main linacs is a region that provides final focusing and steering of the beams prior to reaching the Interaction Regions.

6.2.1.2.5 Interaction Regions The Interaction Regions will consist of two near surface underground halls of equal size. They will house the IR1 and IR2 detector facilities. The detectors will be self shielded. Initially just one interaction region will be outfitted for use. These halls will each have access ramps to the surface. The halls will allow for final detector assembly and be supported with bridge cranes. 
Table 6.2.1.2: Local Campus Space

\begin{tabular}{|l|c|l|}
\hline $\begin{array}{l}\text { Local Campus } \\
\text { Support Space }\end{array}$ & $\begin{array}{c}\text { Area } \\
\text { (sq. ft.) }\end{array}$ & Description \\
\hline \hline $\begin{array}{l}\text { Experimental } \\
\text { Campus } \\
\text { Laboratory } \\
\text { Buildings }\end{array}$ & 60,000 & $\begin{array}{l}\text { Laboratory buildings will contain permanent office space and re- } \\
\text { lated support for roughly 150 persons distributed over three eight } \\
\text { hour shifts each day. }\end{array}$ \\
\hline $\begin{array}{l}\text { Experimental } \\
\text { Campus } \\
\text { Heavy } \\
\text { Assembly } \\
\text { Buildings }\end{array}$ & 80,000 & $\begin{array}{l}\text { Heavy assembly buildings will provide low bay and high bay in- } \\
\text { dustrial space, serviced by overhead cranes, for assembly of larger } \\
\text { accelerator and detector components. }\end{array}$ \\
\hline $\begin{array}{l}\text { Experimental } \\
\text { Campus } \\
\text { Shop } \\
\text { Buildings }\end{array}$ & 27,000 & $\begin{array}{l}\text { Three shop buildings will house various clean room spaces and } \\
\text { shops. Clean room space includes the optics maintenance facility, } \\
\text { buildings include shops such as cryogenic, vacuum, machine, weld- } \\
\text { ing, sheet metal, piping, and carpentry shops. Ten and five-ton } \\
\text { overhead cranes will service these buildings. }\end{array}$ \\
\hline $\begin{array}{l}\text { Experimental } \\
\text { Campus } \\
\text { Support } \\
\text { Buildings }\end{array}$ & 109,600 & $\begin{array}{l}\text { Support buildings, primarily warehousing, provide for receiving, } \\
\text { security and emergency services, cafeteria, vehicle servicing and } \\
\text { miscellaneous functions. }\end{array}$ \\
\hline $\begin{array}{l}\text { Experimental } \\
\text { Control } \\
\text { Rooms }\end{array}$ & 30,000 & $\begin{array}{l}\text { Accelerator and detector control room space with adjacent world } \\
\text { wide communications and networking. }\end{array}$ \\
\hline \hline Total & $\mathbf{3 0 6 , 6 0 0}$ & \\
\hline
\end{tabular}

6.2.1.2.6 Conventional Utilities Site water and waste water systems include the treatment, storage and distribution facilities for water used in experimental cooling systems, for potable water, and for water needed for fire suppression. Also included are waste water systems, such as sewers and sewage treatment plants, and facilities needed for solid waste, such as holding and transfer facilities.

The facilities included with the cooling systems include raw water systems, distribution piping, storage tanks and low conductivity water systems. Vibration isolation and attenuation will be installed to minimize transmission of utility vibration to beam line components from mechanical pumping equipment. In addition mechanical equipment will be separated from beam line components by as much distance as is practical depending on location.

\subsubsection{Electrical Power: Availability, Configuration and Demand}

At least six existing $230 \mathrm{kV}$ power transmission lines run parallel to the XLC in a North-South alignment. The use of four of these transmission corridor sources will optimize XLC availability. At $0.5 \mathrm{TeV}$ c.m., the $\mathrm{XLC}$ will consume about $260 \mathrm{MW}$ of power when it is running. At $0.5 \mathrm{TeV} \mathrm{cm}$, a primary capacity of no less than 265 MVA, combined, at 0.98 power factor, is planned. This will come from two primary substations, one located near the north positron main linac midpoint and one located near the south electron main linac midpoint. Machine area loads are described in Table 6.2.1.3. 
Table 6.2.1.3: Machine Area Power Load Summary in Megawatts, X-band collider, CA site. This table lists megawatt loads by machine area. The main linac is shown in more detail as it uses the most power.

\begin{tabular}{|c|r|r|}
\hline Machine Area & $\mathbf{0 . 5}$ TeV c.m. & 1.0 TeV c.m. \\
\hline \hline$e^{+} e^{-}$Injector \& acceleration to 2 GeV & 1.6 & 1.5 \\
\hline$e^{-}$Damping Ring (1) - 2 GeV 360 Arcs & 3.5 & 3.5 \\
\hline$e^{+}$Damping Rings (2) - 2 GeV 720 $0^{\circ}$ Arcs & 11.6 & 11.6 \\
\hline$e^{+} e^{-}$Pre-Linacs \& BC2 (2) - 6 GeV 180 Arcs & 11.1 & 11.1 \\
\hline$e^{+} e^{-}$Main Linacs (2) subtotal of below & $\mathbf{2 0 7 . 6}$ & $\mathbf{3 8 9 . 9}$ \\
\hline modulator input power & 164.8 & 327.6 \\
klystron filament supplies & 3.1 & 6.2 \\
magnet power supplies & 2.0 & 3.5 \\
other rack power & 6.8 & 13.5 \\
accelerator structure water pumps & 3.6 & 7.3 \\
klystron cooling water pumps & 7.5 & 12.0 \\
cooling towers with pumps and fans & 14.1 & 14.1 \\
lightiling, drains, fire protection, ... & 3.0 & 3.0 \\
\hline$e^{+} e^{-}$IR Transport \& Dumps - HE \& LE & & 2.7 \\
\hline$e^{+} e^{-}$Interaction Halls (2) & 4.6 & 4.6 \\
\hline Losses in power distribution and motor efficiencies & 2.6 & 2.6 \\
\hline \hline Total & $\mathbf{2 5 9 . 9}$ & $\mathbf{4 5 4 . 3}$ \\
\hline
\end{tabular}

\subsubsection{Heat Rejection}

6.2.1.4.1 Overall System Description An estimated $230 \mathrm{MW}$ of low conductivity water (LCW) heat load will need to be rejected to the environment during machine operation. Using evaporative cooling towers, 7.25 acre-feet of water will be consumed every 24 hours at $0.5 \mathrm{TeV}$ c.m. Heat transfer occurs between two primary LCW closed loops and the support tunnel secondary cooling water system using small plate heat exchangers which are located within packaged equipment skids. The secondary cooling water system carries water in 20 in diameter piping along the access ramps and support tunnel housing and has a temperature rise of $31^{\circ} \mathrm{F}$. The cooling water system will be distributed along the entire length of the collider. The secondary cooling water return piping system will be fully insulated to reduce the transfer of return water piping heat into the air of the support tunnel.

6.2.1.4.2 LCW for Klystrons, Modulators, Magnets and Racks Klystron-modulator 2-packs and totally enclosed electronics racks located in the support tunnel, and electro-magnets located in the beam tunnel, are all cooled with $94^{\circ} \mathrm{F}$ LCW via a primary closed loop circuit running between the support tunnel and the beam tunnel through tunnel-tunnel penetrations. The klystron-modulator 2-packs will operate with an LCW temperature rise of $65^{\circ} \mathrm{F}$, controlled to $\pm 5^{\circ} \mathrm{F}$. The $\mathrm{LCW}$ is pumped from the same equipment skid location in the support tunnel that provides the LCW for accelerator structures and the pulse compression.

6.2.1.4.3 LCW for Accelerator Sections and RF Pulse Compression The X-band accelerator structure sections located in the beam tunnel, and the SLED-II pulse compression distribution located in the support tunnel, are both cooled with $82{ }^{\circ} \mathrm{F}$ LCW via a primary closed loop circuit running between the support tunnel and the beam tunnel through tunnel-tunnel penetrations. They will operate with an LCW temperature rise of $12{ }^{\circ} \mathrm{F}$, controlled to $\pm 0.3^{\circ} \mathrm{F}$. The LCW is pumped from the same equipment skid location in the support tunnel that provides the LCW for klystrons, modulators, magnets and racks. The cooling water piping and hoses are supported and sectionalized with flexible seismic hangers-supports to 
reduce mechanical resonance and to isolate the piping systems from the tunnel structures.

6.2.1.4.4 Beam Tunnel Operating States The low conductivity cooling system will cool the beam line RF components during normal steady-state full load operations. These normal operations include occasional brief periods where a klystron 2-pack will switch off and then will later switch back on. The beam tunnel LCW system will maintain the RF components associated with the cycling klystron 2-pack at a stable operating temperature throughout these excursions. In addition the low conductivity water system in the beam housing will control the temperature of RF components in the housing during beam off periods, both with and without RF, and also throughout summer alignment smoothing periods.

6.2.1.4.5 Beam Tunnel Ventilation During beam-off conditions with personnel access, the beam tunnel will be supplied with filtered outside surface air at every supply ramp or shaft, and exhausted through every exhaust ramp or shaft. Supply and exhaust systems will be combined and located every 2 miles. During beam operation, the ventilation of the beam tunnel will be turned off. During smoke and fire control emergency conditions, this same ventilation system will be used for fire control.

6.2.1.4.6 Support Tunnel Ventilation Support tunnel ventilation will be similar to the beam tunnel ventilation except that personnel will have continuous access to the support tunnel and continuous ventilation regardless of the beam tunnel beam status. The water cooled SLED-II system in the support tunnel with a very large surface area will act to stabilize the support housing air temperature.

The air speed for both beam and support tunnels during personnel access will be no less than 88 feet per minute. Both tunnels will have smoke detection and heat detection systems, as well as fire suppression water sprinklers.

6.2.1.4.7 Tunnel Drainage At the base of one sidewall of both the beam and support tunnel housings, there is a gutter to collect water. Beam housing and support housing drain water is pumped, batched, held, and tested before release to gravity fed surface drains. Water from inside the housings must be controlled as it is potentially radioactive and requires evaluation for metals as well as chemical properties such as $\mathrm{pH}$, total dissolved solids and specific conductivity.

The anticipated total influx of ground water to beam and support housings is expected to be very small as the surrounding sandstone rock formations are high in elevation and typically very dry.

\subsubsection{Vibration}

6.2.1.5.1 Mechanical Noise A design requirement for the XLC is the maintenance of sub-micron beam line alignment. Vibration sources along the XLC alignment must be consistently configured so as to be benign to the beam. Slow ground motion can be compensated through beam-based feedback. Faster motion up to $100 \mathrm{~Hz}$ is managed by isolation and attenuation of the source relative to the beam support structure. Above $100 \mathrm{~Hz}$ the beam is almost equally sensitive to any frequency, but with increasing frequency the amplitude of vibrations typically decrease so that an integrated amplitude is of relevance.

6.2.1.5.2 Experimental Region Noise and Vibration Isolation The two experimental detectors are located in separate experimental areas identified as IR1 Hall and IR2 Hall. Compared to the other areas of the XLC, these halls are the most sensitive of all to vibration and noise. Every effort must be made to select a specific and very quiet location for these experimental halls. The sites should be free of man-made cultural noise and isolated from the utility equipment necessary to support experimental systems within the halls, 
including, wherever possible, power, process water, heating, ventilation, controls and so forth. The planned location for the experimental halls is over 14 kilometers from the injector system and is in the middle of the full machine alignment. The local experimental support campus is located at the very southern end of the site near the electron injector so as to place campus-related noise far from the detectors. The experimental hall locations have been evaluated by direct field measurement to determine local cultural noise levels. They have been confirmed to be suitable for the XLC in California.

6.2.1.5.3 Near Field Vibration The primary source of vibration that can affect the machine's alignment in the beam tunnel comes from mechanical devices located within the support tunnels. Sources of mechanical noise include pumps, water moving in pipes, and transformers. LCW equipment skids located in the support tunnel are floated and balanced on $3 \mathrm{~Hz}$ counter-wound helical spring pairs to afford isolation of the skids from the support tunnel floor, as well as from the adjacent beam line tunnel floor. Acoustic baffles about the skids will provide additional isolation and attenuation. Similar measures will be employed for other equipment.

6.2.1.5.4 Far Field Vibration Measurements It has been demonstrated that surface noise generated near the Interaction Region is small and the XLC Logan Ridge site is very quiet. Surface vibration measurements were made in October of 2001, at a location within a few hundred meters of the XLC interaction region. Geophones were secured to rock on the undisturbed surface where vibration measurements were recorded during a 5 hour period. Results indicated that there was no cultural noise of consequence and that far field highway traffic noise measured at this location, $8 \mathrm{~km}$ west of Interstate I-5, was not disruptive.

6.2.1.5.5 Alignment Smoothing Interval The planned cycle for beam operation is to run for 9 months, from September 1 to May 30 each year. This coincides with the most efficient operating cycle for scheduling water and power usage. During a 9 month run the beam position is in part controlled with magnet movers located on each magnet pedestal. These movers will position the magnets to account for relative position shifts from all sources including the beam housing floor. Realignment smoothing will take place during the annual 90 day maintenance interval.

\subsubsection{Geology and Tunneling Means and Methods}

6.2.1.6.1 Site A site-specific detailed geological and geotechnical study[72] was conducted by Philip A. Frame, R.G., Consulting Engineering Geologist in 2000. This study was commissioned for the purpose of finding suitable California locations to build the XLC, then called the Next Linear Collider. The study details the geology of an area along a $50 \mathrm{~km}$ alignment parallel to Interstate 5 . The study found that highly favorable conditions existed in the region for building the XLC in competent, well-cemented, interbedded sandstones, with some siltstones, and claystones as well.

The XLC representative site is in low relief hills east of the Antelope Valley and trends north-south along the west edge of Northern California's Central Valley. The hills are dissected with a series of valleys that drain the upper reaches from west to east and allow for optimum access to parallel bored tunnels via side hill ramps. This remote area is used primarily for agriculture and cattle grazing. These hills lie in the rain shadow of the California Coast Ranges and, on average, get 17.3 inches of rain per year. Based on the relatively low average rainfall and high relief, these hills should provide for dry conditions during construction and during operations.

The alignment of the machine is laser straight. The rock cover directly above the tunnel enclosures will vary from less than $30 \mathrm{ft}$ to as much as $340 \mathrm{ft}$ with the existing surface topography. Tunnel depth at the surface access ramps will allow for shallow ramp access to the machine at an average elevation 50 feet above the tunnels via east-west running valleys and other low-lying areas of Northern California's Central Valley. 
Mechanical excavation using a Tunnel Boring Machine (TBM) for the underground tunnel enclosure construction will be very attractive from both a cost and schedule perspective.

The bedrock of the region is characterized as well cemented fine- to coarse-grained sandstone with some interbedded siltstones and claystones that total approximately $7 \mathrm{~km}$ in thickness. These materials were shed off of the ancestral Sierra Nevada Mountains into a shallow sea between 91 and 73 million years ago. The sandstone beds are steeply dipping to the east. This is a structurally sound rock that is considered a softer and less problematic rock to tunnel in relation to other sedimentary, metamorphic or igneous rocks. These properties will allow for a higher than average rate of penetration during tunneling operations. Normal tunnel support methods (i.e., rock bolts and 2 in of shotcrete with wire mesh as used at SLAC) will provide adequate support for the beam and support tunnels.

The representative XLC site lies within a large body of bedrock of the cretaceous sedimentary strata commonly referred to as the Great Valley Group. The site is underlain by structure informally known as the Funks segment of the Great Valley fault, a blind, west-dipping thrust fault which underlies the region at a depth of about $5 \mathrm{~km}$. Published geologic reports indicate that the most recent displacement in the region of the Logan Ridge site occurred more than 10,000 years ago. The slip-rate on this segment is calculated to average $0.1 \mathrm{~mm} / \mathrm{yr}$ and is thought to be capable of generating a 6.4 magnitude earthquake recurring every 6,000 years. (See reference [73])

6.2.1.6.2 Rock Spoils Approximately 2,000,000 cubic yards of material (not bulked) will be generated during excavation. It is anticipated that spoil stockpiles can be placed in many of the canyon valleys at various locations along the machine's alignment.

6.2.1.6.3 Construction Means and Methods Excavation of rock using TBM means and methods for the tunnels has been used for cost estimating purposes. For dry conditions in long tunnels in soft competent sandstone rock, TBM's, driving with high daily advance rates, offer significant cost and schedule benefits. Localized grouting, and invert construction pinned directly to bedrock, will be required along the length of the enclosure. We are anticipating that about $20 \%$ of the tunnel length may require some additional lining. Based on the available data and local experience, this appears to be conservative. The excavated tunnel overbore will need to account for the final reinforced shotcrete application and any additional lining thickness where an added lining appears to be required. More specific geologic and geotechnical exploration programs along the final alignment will support this determination. Only straight ahead tunnels will employ TBM's for excavation. Nearly all other underground excavation will be done with road headers and/or drilling rigs.

\subsubsection{Conventional Facilities Design Summary for an X-band Linear Collider, North-South Illinois Alignment, West of the Fox River, Illinois ${ }^{2}$}

\subsubsection{Configuration and Salient Design Features}

6.2.2.1.1 Purpose This section describes the conventional construction needed to build the U.S. sited normal-conducting X-band linear collider, referred to here as "FY03 NS-NC." The accelerator must meet stringent requirements for sustained luminosity and machine stability.

The purpose of this section is to determine the impacts that a specific site has on the accelerator and the accelerator housings design, as well as the resulting cost and schedule impacts. For the purposes of this presentation a location for the accelerator has been shown on various maps. The location shown represents only one of many possible alternatives. The location could vary by several miles and still be valid for the design solution described in this report. The location of the representative site chosen is in close proximity

\footnotetext{
${ }^{2}$ Near Fermi National Accelerator Laboratory
} 
to existing physics laboratories at Fermi National Accelerator Laboratory. It is roughly 20 miles west of Fermilab. End-to-end, it is approximately 35 kilometers in length.

Criteria for the conventional construction have been derived from discussions with physicists at SLAC and Fermilab, from the reference design described in Section 3.4, and from reference [NLC01]. Design solutions have been developed in consort with engineers at SLAC and Fermilab, and consulting engineers. The designs generated have used the current SLAC and Fermilab accelerators as models for determining criteria for installation, support, servicing and maintenance of the accelerator. Environmental requirements for the tunnel housings are assumed to be consistent with other high-energy physics accelerators.

6.2.2.1.2 Proposed Facilities The conventional facilities will consist of above and below grade structures, utilities and support facilities generally found at high-energy physics accelerator laboratories. The Illinois FY03 NS-NC design iteration considers constructing conventional facilities to support a $500 \mathrm{GeV}$ center-of-mass accelerator, expandable to $1 \mathrm{TeV}$ center-of-mass. The design explores the construction of the tunnels within the Galena Platteville Dolomite, in a north south alignment, on a hypothetical site some 20 miles west of the Fermilab site.

The below grade accelerator is comprised of several major elements: electron source, damping rings, undulator, positron system, main linac, beam transport and final focus system. After accelerating the electrons to $150 \mathrm{GeV}$, the electrons will be extracted to the undulator and transported either to the remaining electron main linac or to the positron target. The positron main linac tunnel is approximately $14 \mathrm{~km}$ in length. Using an undulator-based positron source, the electron main linac tunnel will be approximately $15 \mathrm{~km}$ in length. Klystrons and modulators will be housed in the support tunnel, parallel and adjacent to the main linac beam tunnel. Each main linac (electron and positron) will contain a series of RF cavities that accelerates the beam towards a central interaction region. RF cavities will be installed in the active portion of the main linac tunnel to produce the energies for 250 on $250 \mathrm{GeV}$ collisions. An inactive portion of the main linac tunnel will contain a beam transport line that can be upgraded with additional RF cavities to increase the final energy to 500 on $500 \mathrm{GeV}$. The Beam Delivery at the ends of the main linac is a region that provides final focusing and steering of the beams prior to reaching the Interaction Regions. The Interaction Regions will consist of two underground halls, which house the IR1 and IR2 detector facilities. Initially only one interaction region will be outfitted for use. The halls will allow for final detector assembly and be supported with bridge cranes. There will be a parallel service tunnel that houses beamline support technical equipment for the length of the project.

Shafts are required for construction of the tunnels, as well as to provide a link between the surface and the below grade housings through which LCW, power and elevators will be routed. Shafts at the ends of the linacs will act as the major equipment access for RF cavities and other beamline support equipment, as well as a conduit for the utilities and services. The shafts located at each end of the main linac will be sized to facilitate TBM excavating equipment, and accelerator RF cavities. At the interaction halls, shafts are needed to support each of the IR Halls as well as services for the absorbers and final focusing equipment. All shafts will have elevators and/or stairs that will serve as part of the egress corridor from the tunnels below. Fourteen major shafts are required, one at each end at the damping ring, five utilities shafts to support each of the linacs (10 total), one at the IR2 Hall and one at the IR1 Hall.

Above grade support buildings, located directly above the shaft, are required at each major shaft to support the below grade accelerator and support housings. The Interaction Region area and the central campus facilities will be located on a common site, although separated to reduce and control vibration and noise near the Interaction Regions. The IR2 Hall will be outfitted and the above grade service building constructed to provide life safety and minimal services. The new campus within 20 miles of Fermilab will allow some of the required infrastructure that already exists on the Fermilab site to be used for the support of this accelerator. 
6.2.2.1.3 Siting This report considers a site with a nearly north south orientation, roughly centered on interstate I- 88 approximately 12 miles east of DeKalb, IL. The preliminary alignment was chosen to optimize the following site criteria:

1. Avoid populated areas. This reduces cultural noise and simplifies land acquisition.

2. Allow all of the housings to be constructed in Galena Platteville dolomite formation.

3. Provide reasonable access to transportation infrastructure, both to Fermilab and area airports.

4. Locate near existing high voltage power distribution.

We anticipate that an estimated 500-foot wide corridor of property rights and easements would be acquired by the U.S. DoE. We estimate that as much as 1350 acres of underground property rights and easements will be required for the enclosure corridor, and another 3000 acres of land acquisition will be required to construct the campus and satellite buildings and to provide for future expansion.

\subsection{Salient Design Features Salient design features of this study include:}

- Locating the length of the laser straight accelerator in the Galena Platteville rock formations places the depth of the tunnels in a range from 120 feet to 400 feet below the surface.

- Distributed electrical Sub-Stations: new $345 \mathrm{kV} / 34.5 \mathrm{kV}$ sub-stations with electrical distribution will be located at each main linac shaft.

- Two parallel enclosures. Provisions for life safety are modeled from transportation tunnels. Shielding is naturally provided by rock between the enclosures. Egress is via shielded ramp access ways between the enclosures.

- Development of a Central Campus supported by existing Fermilab facilities is assumed.

\subsubsection{Description of Major Components}

6.2.2.2.1 Site Development $\quad$ Most of the current land use along the proposed accelerator is agriculture. Except at the Service Building Campuses and the Central Campus, we are not expecting to change the current land use. Conventional utilities (domestic water, natural gas, sanitary sewer) do not exist and are anticipated to be constructed with this project or adapted from the local municipal systems as required.

\subsection{Surface Buildings}

6.2.2.2.1 Central Campus A central campus is planned for this project. This campus will be located near Interstate I-88. The campus areas will include office spaces and facilities to build, operate, service, and maintain the project. Although specific functions, and facilities will need to be located at the project site, current communication technologies will allow for many of the project's personnel needs to be met at remote locations such as Fermilab and other high-energy laboratories. Table 6.2.2.1 outlines our assumptions for construction of new building spaces. In addition, facilities that will be required for the Interaction Halls at the surface will be located within the boundaries of the Central Campus site. 
Table 6.2.2.1: Fermilab FY03 NS-NC Orientation Central Campus Facilities Assumptions

\begin{tabular}{|l|r|}
\hline Description of Building & sq. ft. \\
\hline \hline New Office Space & 30,000 \\
New Computing Space & 20,000 \\
New Light Lab Space & 5,000 \\
New Cafeteria & 5,000 \\
New Large Heavy Assembly Buildings & 40,000 \\
New Small Heavy Assembly Buildings & 40,000 \\
New Light Shop Space & 27,000 \\
New Warehousing & 40,000 \\
New Gas \& Storage & 6,000 \\
New Security Buildings & 1,600 \\
New Emergency Response & 5,000 \\
New Medical \& ES\&H & 4,000 \\
New Heavy \& Light Equipment Maintenance & 13,000 \\
New Campus Utility Plant Building & 40,000 \\
New Operations Control Room & 15,000 \\
New Operations Annex Wing & 10,000 \\
New Communications Center & 5,000 \\
\hline Total & 306,600 \\
\hline
\end{tabular}

6.2.2.2.2 LCW Surface Plant Buildings Located at each shaft is a LCW Surface plant. Power, low conductivity cooling water (LCW), sprinkler water (ICW), potable water (DWS), sewer, pump discharge, and HVAC are processed and routed to/from the tunnel in these buildings. These buildings function as staging areas for material access to the tunnel as well as personnel egress from the tunnels.

\subsection{Underground Structures}

6.2.2.2.3.1 Injectors A combined Positron / Electron injector facility will be constructed at the south end of the accelerator complex. Downstream from the electron sources is an undulator followed by the positron source. A beamline transports the positrons past the IR region to the damping ring located on the north end of the accelerator. A support enclosure is required to support the injectors. The injector will be excavated using drill and blast methods.

6.2.2.3.2 Damping Rings Damping rings are located at each end of the accelerator complex. The damping rings will be excavated using drill and blast methods.

6.2.2.2.3.3 Main Linac The two main linacs will involve the construction of $29 \mathrm{~km}$ of parallel twin bored tunnels, with 13.5 feet inside diameter. The beamline enclosure will primarily house main linac RF cavities and beamline components. The support enclosure will be a parallel tunnel that houses klystrons, modulators, power supplies, instrumentation, and controls. The support enclosure will allow personnel to access critical components during beamline operation for maintenance and operations purposes. The klystrons distribute RF to the cryostats through "duct-like" wave-guides that pass between the two tunnels in two-foot diameter transverse penetrations spaced at 12 meters along the main linac.

6.2.2.2.3 Beam Delivery Starting at the ends of the main linac is a region that provides final focusing and steering of the beams prior to reaching the Interaction Regions. 
6.2.2.3.5 Interaction Regions Each Interaction Region will consist of an above grade assembly building and below grade collision hall. Two such detector facilities are planned, IR1 Hall and IR2 Hall. Each IR region will require collision halls below grade sized at $110 \mathrm{ft}$ wide by $250 \mathrm{ft}$ long by $100 \mathrm{ft}$ high. The grade-level assembly area will be for detector subassembly and will include a heavy crane. Mezzanines will provide areas for computer, control room and office facilities. Other utility areas will house process water systems, electrical power services and air handling equipment. The assembly area will likely be constructed of conventional steel and reinforced concrete construction.

\subsubsection{Electrical Power}

6.2.2.3.1 Availability A $345 \mathrm{kV}$ power line exists along an east west alignment near the proposed central campus area. A second $345 \mathrm{kV}$ power line source is roughly 5 miles southwest of the central campus. Each of these $345 \mathrm{kV}$ power lines currently has the capacity to supply the power required by the Linear Collider.

6.2.2.3.2 Description Both sources of electric power delivered from ComEd will be extended on common poles in a north south direction along the length of the Linear Collider. The transmission line service voltage is $345 \mathrm{kV}$. Feeder taps from the two overhead lines feed 13 separate areas along the length the LC. Two 40 MVA step-down transformers at each LCW building will supply $34.5 \mathrm{kV}$ for distribution to the surface and underground loads. These transformers provide power to two separate loads: 1) Modulator/Power Supply loads and tunnel conventional power (underground), 2) Surface Conventional and LCW loads. Power to Central region loads (Central Campus and IR Halls) will be from a single 40 MVA transformer (backed up by a 40 MVA installed spare transformer) and will be distributed, via circuit breakers at a substationhouse, to underground feeders. Each location will be constructed with a concrete pad and switchgear for one additional transformer. The components at each substation will be standardized. LCW and building power will be from ten (on average) $1500 \mathrm{kV}(34.5 \mathrm{kV}$ to $480 \mathrm{~V})$ transformers.

Power to Modulator/Power Supply loads will be via a three phase $34.5 \mathrm{kV}$ transmission line directly down each shaft and distributed along the length of the active linac, connecting to the modulator/conventional transformers, in series, via two internal fused disconnects. Part of this load is a series of $15 \mathrm{kVA}$ transformers (spaced 25.9 meters apart) inside the service tunnel, stretching out each direction away from the vertical shaft. This is for all conventional electrical loads (lighting, pumps, and HVAC units). Each feeder cable is sized at $35 \mathrm{kV} 750 \mathrm{MCM}$ and is routed to the tunnel via 6 inch metal conduits inside the vertical shaft.

Each substation will be monitored and controlled at a prefabricated control room. Lower voltage lines after the substation will be monitored and controlled locally. Backup emergency power will be available at each Substation. Control Power for each substation will be via a battery bank. A Kirk Key system will be established to safeguard against parallel feeds.

Lighting design luminance for the tunnel, corridors and hallways will be 20 foot-candles. Welding receptacles, 60 A $480 \mathrm{~V}$ outlets, will be provided at every 800 feet. $120 \mathrm{~V} / 208 \mathrm{~V}$ receptacles will be provided at every 120 ft. Emergency lighting will be provided for general safety.

Power distribution system redundancy has been maximized while isolation devices have been kept to a minimum at the various levels. Life safety equipment such as emergency lighting and fire detection will be powered from uninterruptible power supplies and critical systems will be connected to emergency generators via automated transfer switches.

6.2.2.3.3 Configuration and Demand At $0.5 \mathrm{TeV}$ c.m., the collider will consume about $260 \mathrm{MW}$ of power when it is running. At $0.5 \mathrm{TeV} \mathrm{cm}$, a primary capacity of no less than $265 \mathrm{MVA}$, combined, at 0.98 power factor, is planned. This will come from two primary substations, one located near the north positron 
main linac midpoint and one located near the south electron main linac midpoint. Machine area loads are described in Table 6.2.2.2.

Table 6.2.2.2: Machine Area Power Load Summary in Megawatts, X-band collider, IL site. This table lists megawatt loads by machine area. The main linac is shown in more detail as it uses the most power.

\begin{tabular}{|r|r|r|}
\hline Machine Area & $\mathbf{0 . 5}$ TeV c.m. & $\mathbf{1 . 0}$ TeV c.m. \\
\hline \hline$e^{+} e^{-}$Injector \& acceleration to 2 GeV & 1.6 & 1.5 \\
\hline$e^{-}$Damping Ring (1) - 2 GeV 360 Arcs & 3.5 & 3.5 \\
\hline$e^{+}$Damping Rings (2) - 2 GeV 720 $0^{\circ}$ Arcs & 11.6 & 11.6 \\
\hline$e^{+} e^{-}$Pre-Linacs \& BC2 (2) - 6 GeV 180 Arcs & 11.1 & 11.1 \\
\hline$e^{+} e^{-}$Main Linacs (2) subtotal of below & $\mathbf{2 0 7 . 6}$ & $\mathbf{3 8 9 . 9}$ \\
\hline modulator input power & 164.8 & 327.6 \\
klystron filament supplies & 3.1 & 6.2 \\
magnet power supplies & 2.0 & 3.5 \\
other rack power & 6.8 & 13.5 \\
accelerator structure water pumps & 3.6 & 7.3 \\
klystron cooling water pumps & 7.5 & 12.0 \\
cooling towers with pumps and fans & 14.1 & 14.1 \\
ventilation supply, exhaust, chillers & 3.0 & 3.0 \\
lighting, drains, fire protection, .. & 2.7 & 2.7 \\
\hline$e^{+} e^{-}$IR Transport \& Dumps - HE \& LE & 4.6 & 4.6 \\
\hline$e^{+} e^{-}$Interaction Halls (2) & 2.6 & 2.6 \\
\hline Losses in power distribution and motor efficiencies & 17.3 & 29.4 \\
\hline \hline Total & $\mathbf{2 5 9 . 9}$ & $\mathbf{4 5 4 . 3}$ \\
\hline
\end{tabular}

\subsubsection{Heat Rejection}

6.2.2.4.1 Project Requirements The estimated 230 MW low conductivity water (LCW) heat load will be rejected to wet type cooling towers located at each LCW cooling plant (total 13), approximately $2.7 \mathrm{~km}$ apart. The LCW/HVAC surface plant is estimated to be $230 \mathrm{ft} \times 75 \mathrm{ft}$. The LCW cooling system arrangement is based on non-summer months operation, or beam operation for 9 months, from September to May each year. Details are presented in Table 6.2.2.3.

6.2.2.4 LCW (Low Conductivity Water) System Each surface LCW Cooling Plant facility will have approximately four (2-cell) wet cooling towers, indoor water tank, three tower pumps, two heat exchangers, three secondary pumps, water treatment system, filtration system and controls. Sizing of the cooling tower is based on $15{ }^{\circ} \mathrm{F} \Delta T$ at $71^{\circ} \mathrm{F}$ wet bulb overall. The LCW cooling system will consist of primary-secondary-tertiary cooling loops. The primary loop is a $15^{\circ} \mathrm{F} \Delta T$ cooling tower system (at $78^{\circ} \mathrm{F}$ $/ 93^{\circ} \mathrm{F}$ ) that includes all the cooling towers, tower water pumps and industrial grade heat exchangers. The secondary loop (at $80^{\circ} \mathrm{F}$ supply / $111^{\circ} \mathrm{F}$ return) is a non-deionized cooling water loop that will distribute cooling water from heat exchangers at the surface level to the individual skids in the service tunnel, up to approximately $1.35 \mathrm{~km}$ each way, and return to the heat exchangers on the surface. All the piping, valves, fittings and other components in the secondary system will be designed and selected for use at higher pressure rating due to the length of the system and elevations involved. The return piping will be insulated to minimize heat transfer to the surroundings. The tertiary loop includes a modular heat exchanger/pump skid and uses deionized LCW to feed the accelerator structures (at $82^{\circ} \mathrm{F}$ supply / $94{ }^{\circ} \mathrm{F}$ return, controlled to $\pm 0.3^{\circ} \mathrm{F}$ ), and Modulators/Klystrons/Racks (at $94^{\circ} \mathrm{F}$ supply / $159^{\circ} \mathrm{F}$ return, controlled to $\pm 0.5^{\circ} \mathrm{F}$ ). The cooling skid for the third loop will be located in the service tunnel. 


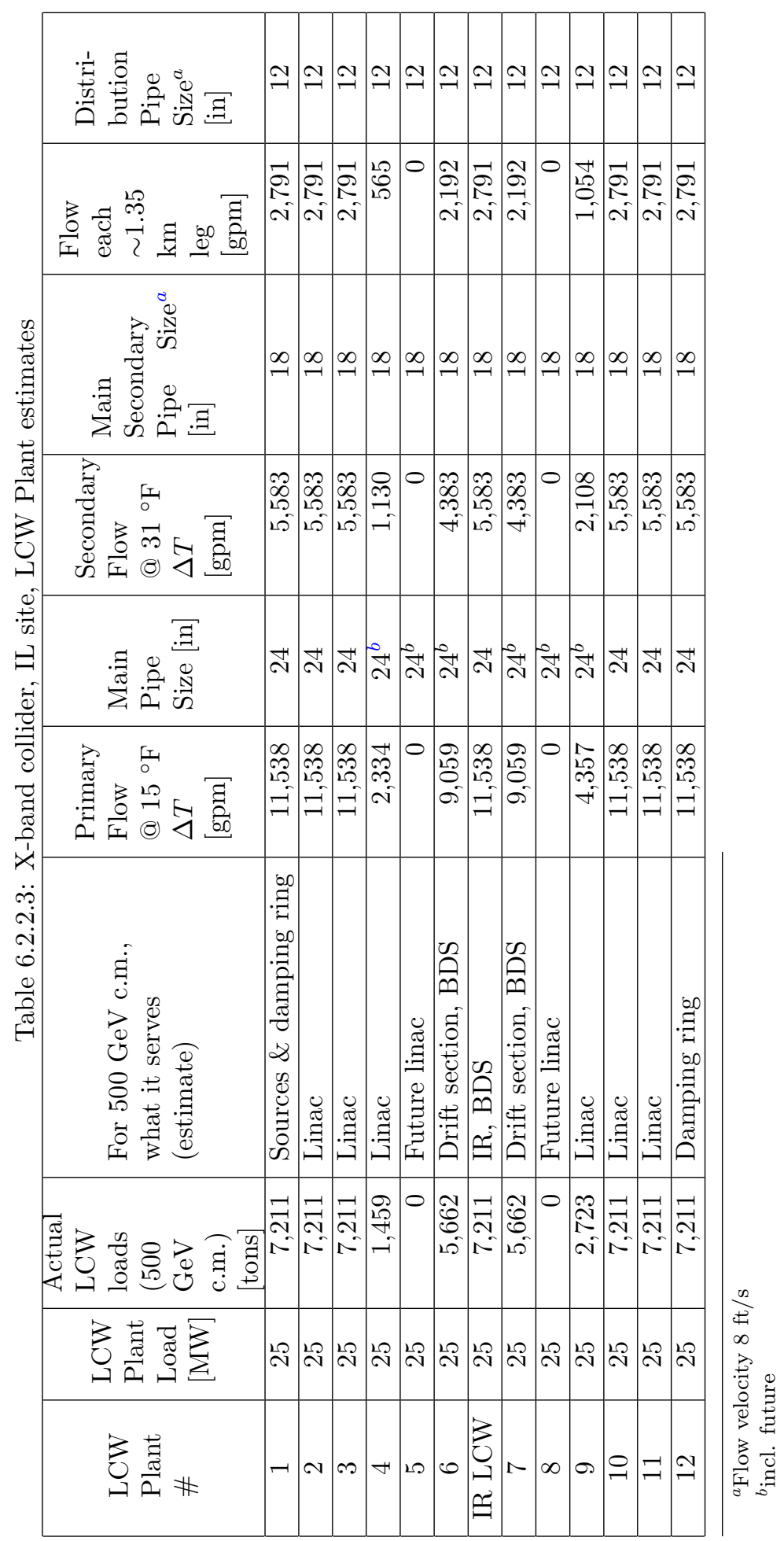


The system design for primary and secondary loops will include redundancy considerations using multiple pumps and heat exchangers to prevent accelerator shutdowns due to equipment failures. Each skid on the tertiary loop will be provided with one heat exchanger/one pump and no backup. All of the tower water and primary heat exchanger systems will be located in the surface facilities to simplify maintenance.

6.2.2.4.3 Design variations Various design options were considered during the conceptual design stage to optimize the system design. Examples of these design options are a Dry Cooler system in combination with the wet towers, cooling ponds, and a higher $\left(32^{\circ} \mathrm{F}\right) \Delta T$ cooling tower system.

6.2.2.4.4 Ventilation (Beam Tunnel) During beam-off condition and personnel access, the beam tunnel will be supplied with $100 \%$ filtered/conditioned/dehumidified air at every supply shaft, and exhausted through every exhaust shaft. Supply and exhaust shafts will be alternately located every approximately 2.7 $\mathrm{km}$. During beam operation, the ventilation of the beam tunnel will be turned off. During smoke and fire control emergency condition, this same ventilation system will be used and airflow will be diverted to the proper spaces.

6.2.2.4.5 Ventilation (Service Tunnel) Each of the service tunnel sections between the LCW plants will be operated independently and each will be supplied with $100 \%$ filtered/conditioned /dehumidified outside air. The system will be capable of bypassing an LCW hall and feeding the air directly to the next hall. Alternating plant location will provide supply and exhaust flow. During smoke and fire control emergency conditions, this same ventilation system will be used and airflow will be diverted to proper spaces.

The air speed for both tunnels will be $88 \mathrm{fpm}$. Both tunnels will have smoke detection and heat detection systems. There will be various water cooled air conditioners and heat pumps that will condition the klystron/modulator area to maintain the space suitable for workers in the service tunnels. Condenser water to these units will come from the secondary cooling water that feeds the equipment load. To separate these units from the high-pressure secondary water system, HX/pump skids will be provided to feed multiple units.

6.2.2.4.6 Drainage The water from beam tunnel walls and the fan coil drains will drain by gravity towards the shaft sump pit at every shaft location. The inflow estimate is $50 \mathrm{gpm}$ per mile per tunnel. The water will be pumped from the sump pit to the ground surface.

\subsubsection{Geology, Siting and Tunneling Means and Methods}

6.2.2.5.1 Site The proposed siting used the geological and geotechnical studies prepared for the Superconducting Super Collider (SSC) studies in the late 1980's by the Illinois Departments of Energy and Natural Resources, State Geological Survey Division[74]. The SSC studies geologically define an area roughly 20 miles north and south of Fermilab, 45 miles to the west and 6 miles to the east of Fermilab. This information is augmented by more recent rock maps and cross-sections provided by the State's Geological Survey. From a siting and construction basis, the Linear Collider is similar to that of the SSC. These studies indicate that favorable conditions exist in the vicinity of the Dekalb / Kane county lines. The conditions exist that will allow the project to optimize the following criteria:

1. Avoid populated areas. This reduces cultural noise and simplifies land acquisition.

2. Allow all of the housings to be constructed in Galena Platteville dolomite formation for its entire length.

3. Minimize the depth of shafts. 
4. Utilize rock formations with a favorable hydrogeologic setting.

The dominant land use in the proposed siting area is currently under farm production, spotted with small to medium size towns. Urban sprawl, now several miles to the east, is an issue that will need to be addressed in detail to determine potential impacts.

The Galena Platteville is characterized as a fine to medium grained dolomite, that is cherty. The Maquoketa shales overlaying the dolomite have a low hydraulic conductivity that will act as a hydrogeologic barrier between upper overburden aquifers and the dolomite. At the proposed siting, the Galena Platteville varies from 300 to 350 feet in thickness, gently rising in datum elevation from the south to the north. The Galena is covered by 50 to 100 feet of shale, which in turn, is overlaid by 50 to 150 feet of overburden. It is expected that features requiring grouting will be encountered. The upper Silurian dolomite found at the Fermilab site is absent, for a significant distance to the east. These geologic conditions should provide a relatively dry tunnel, both during construction and during operations. The Galena is the most structurally sound rock in the area and, in general, should not require any extraordinary rock support methods.

The criteria for the alignment of the machine is a laser straight configuration, thus the cover over the enclosure will vary from 120 to 400 feet. Approximately 150 feet of this depth at the center is a result of the earth's curvature. It is anticipated that mechanical excavation techniques using Tunnel Boring Machine (TBM) for the underground enclosure construction will be used as outlined in the means and methods section below.

6.2.2.5.2 Spoils Approximately 1,750,000 cubic yards of material (not bulked) will be generated during excavation. It is anticipated that spoil stockpiles will be necessary at as many as five locations. The majority of the spoil will be dolomite that may be utilized as structural fill on this and other projects. It is anticipated that the rock spoil will be cost neutral for the project.

6.2.2.5.3 Construction Means and Methods Excavation of rock using a TBM for the tunnels was selected for estimating purposes. For long tunnels, TBM's offer significant cost and schedule benefits. Localized grouting, and invert construction will be required along the length of the enclosure. We are anticipating that about $25 \%$ of the tunnel length may require some lining. Based on the available data this appears to be conservative. The excavated tunnel diameter will need to account for the final lining thickness, if lining appears to be required. This determination will be influenced by the geologic and geotechnical ground exploration program. IR halls, access ramps and waveguide penetrations are all anticipated to be excavated using drill and blast methods.

\subsubsection{Vibration}

6.2.2.6.1 Mechanical Noise as an Issue in site Selection A design requirement for the X-band linear collider is the maintenance of sub-micron beam line alignment. Vibration sources along the collider alignment must be consistently configured so as to be benign to the beam. Slow ground motion can be compensated through beam-based feedback. Faster motions up to $100 \mathrm{~Hz}$ are managed by isolation and attenuation of the source relative to the beam support structure. Above $100 \mathrm{~Hz}$ the beam is almost equally sensitive to any frequency, but with increasing frequency the amplitude of vibrations typically decrease so that integrated amplitude is of relevance.

The two experimental detectors are located in separate experimental areas identified as IR1 Hall and IR2 Hall. Compared to the other areas of the collider, these halls are the most sensitive of all to vibration and noise. Every effort must be made to select or control a specific and very quiet location for these experimental halls. The sites should be free of man-made cultural noise and isolated from the utility equipment necessary to support experimental systems within the halls, including wherever possible, power, process water, heating, ventilation, controls and so forth. The local support campus is located away from but adjacent to the 
detectors so that noise can be controlled. Prairie will be planted around the detectors for a distance (to be determined) to control cultural noise.

The siting of a linear collider along a road or railway right-of-way may be problematic, because of the requirement to maintain, in some cases, sub-micron alignment tolerances. The affects of mechanical vibrations from railways should be a topic for further study. It is being explored in the ongoing studies being performed in the fixed target area and the nearby North Aurora Tunnel. There are three train rails that cross or are near to crossing the proposed alignment. It is not known whether it is a problem or not, and the incorporation of beam-based feedback could bring these effects under acceptable control. Earth noise, which is seismic noise created, for example, by the interaction with wind or sea, is another form of noise that can pose a problem for the machine design. Typically, earth noise lies in frequencies below $1 \mathrm{~Hz}$, while cultural noise lies above $1 \mathrm{~Hz}$. Amplitude of ground motion decreases as $1 / f^{2}$ with increasing frequency.

Seismic noise conditions may affect the final siting decisions, because a plane seismic wave traveling perpendicular to the accelerator has a minimal affect on machine alignment. For example, a site near a shoreline might align the accelerator parallel to the shoreline.

The primary source of vibration that can affect the machine's alignment in the beam tunnel comes from mechanical devices located within the support tunnels. Sources of mechanical noise include pumps, water moving in pipes, and transformers. The LCW equipment skids located in the support tunnel are floated and balanced on $3 \mathrm{~Hz}$ counter-wound helical spring pairs to afford isolation of the skids from the support tunnel floor as well as from the adjacent beam line tunnel floor. Acoustic baffles about the skids will provide additional isolation and attenuation. Similar measures will be employed for other equipment. A siting criteria might include provision for seismic noise to be as small as sources of mechanical noise emanating from within the accelerator tunnel. It's likely, but has not been demonstrated, that surface noise generated above 200 feet of overburden will be small compared to mechanical sources from within the accelerator tunnel.

6.2.2.6.2 Vibration Studies A study for the Illinois SSC proposal investigated vibrations of truck and railroad traffic, which was considered the largest source of vibrations in the region. This investigation monitored truck traffic over an expansion joint of a bridge and the passage of freight trains. Truck traffic showed displacement of 2.9 to $19.3 \mu \mathrm{m}$ on the bridge abutment. However, 65 feet down and 150 feet horizontally to a rock quarry floor, these movements were attenuated 92 to 241 times, down to .03 to .08 $\mu \mathrm{m}$. The train traffic displacements on the ground next to the rail line were 1.6 to $4.06 \mu \mathrm{m}$, but 60 feet down

and 190 feet horizontally to the quarry floor, the displacements were attenuated 62 to 123 times, down to .013 to $.066 \mu \mathrm{m}$.

\subsubsection{Selected Conventional Facilities Design Drawings for the Normal Con- ducting X-band option}




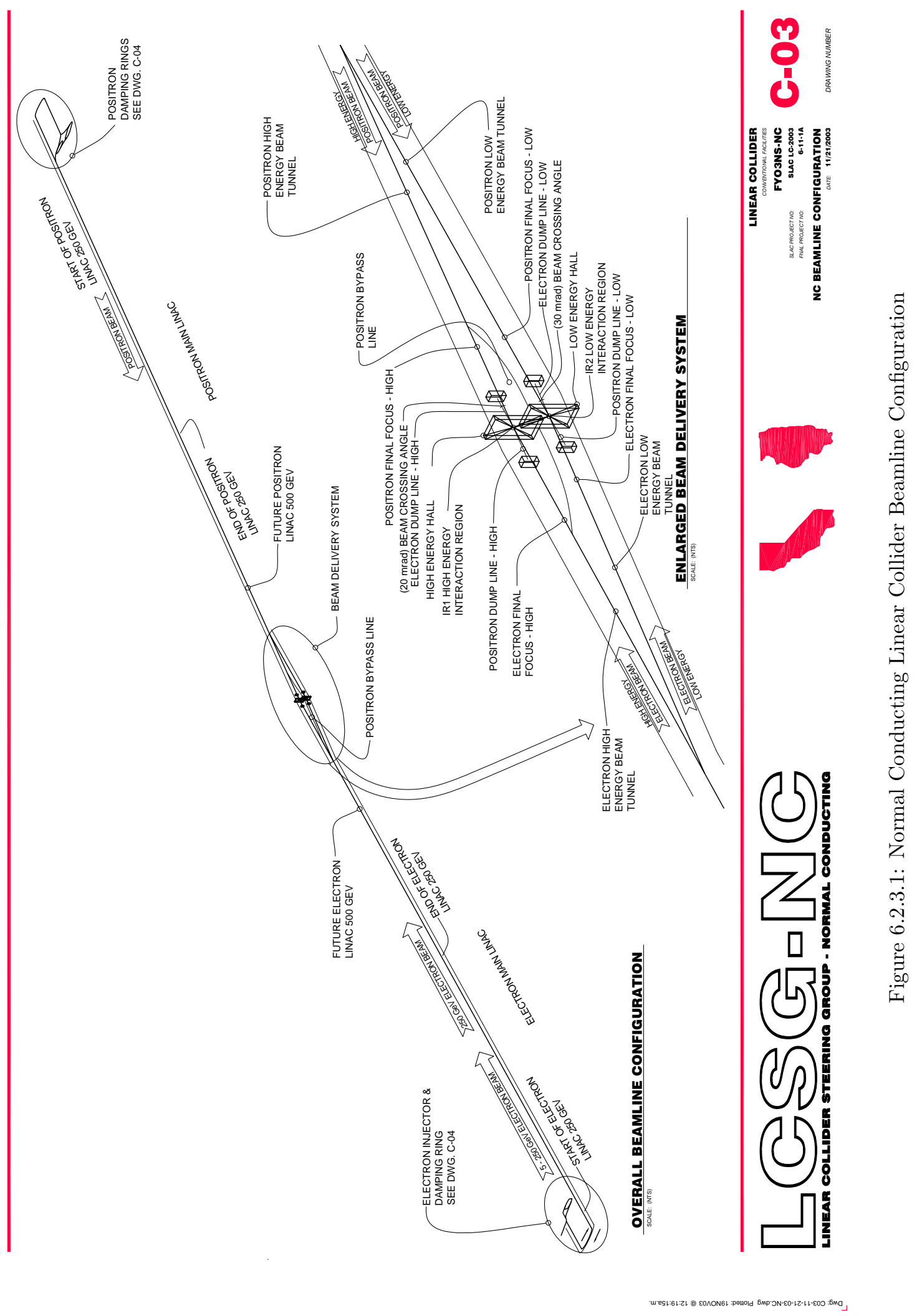




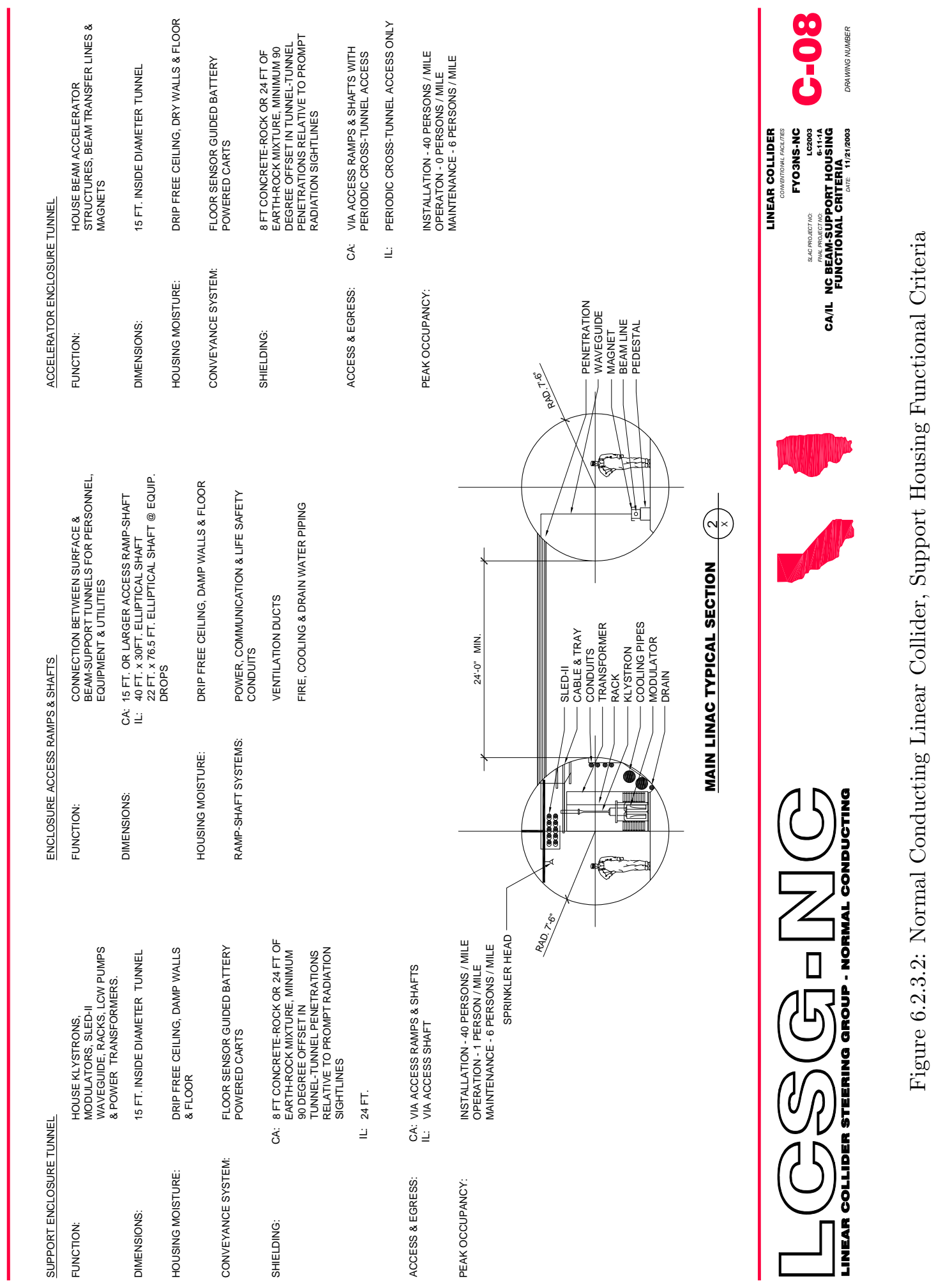




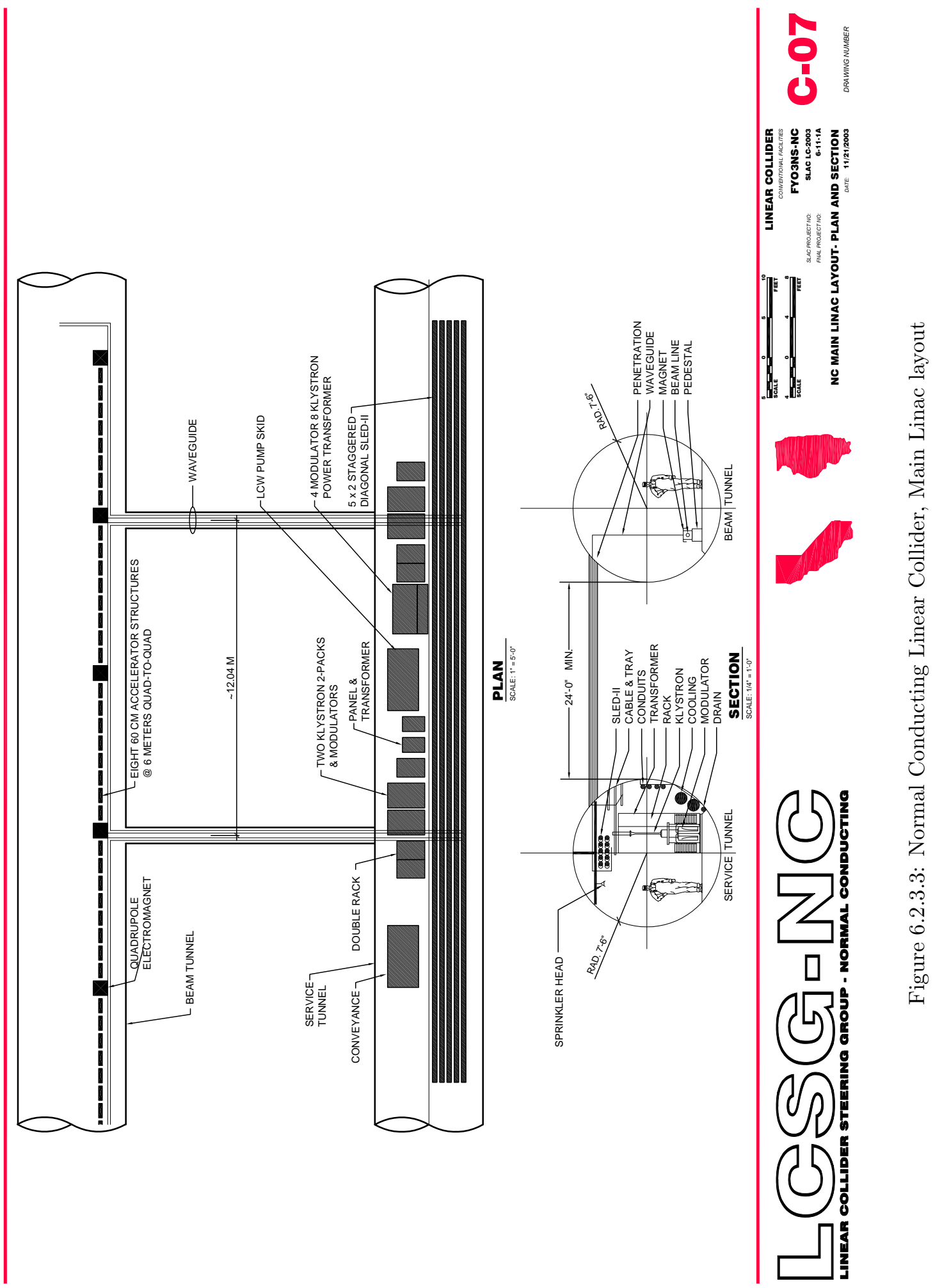



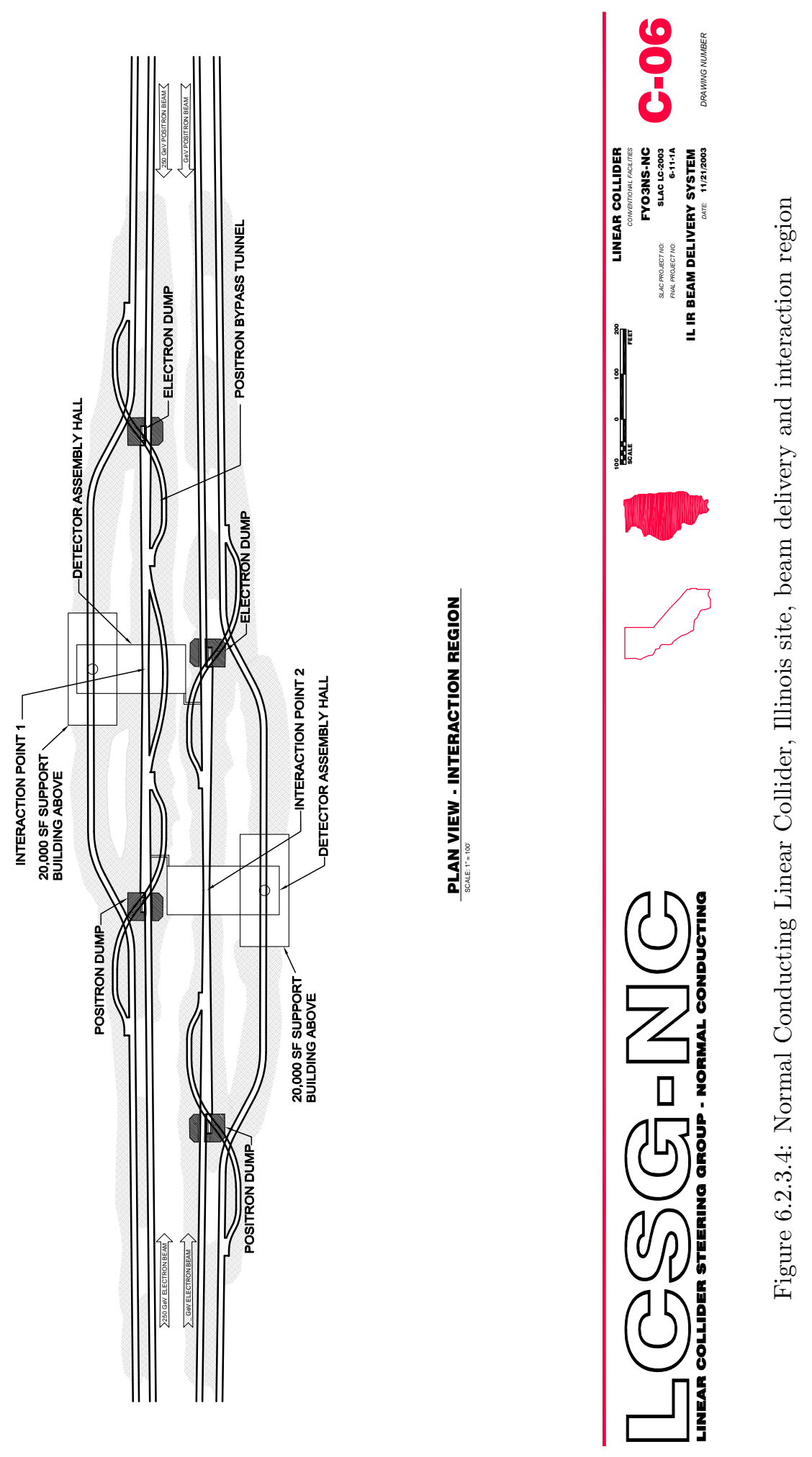


\subsection{Conventional Facilities Design Summaries and Drawings for the Superconducting L-band option}

\subsubsection{Conventional Facilities Reference Design Summary for a Superconducting L-band Linear Collider at Logan Ridge, California ${ }^{3}$}

Table 6.3.1.1: Superconducting Reference Design Summary Parameters

\begin{tabular}{|c|c|}
\hline Item & Baseline \\
\hline $\begin{array}{l}\text { Housing } \\
\text { Radiation } \\
\text { Containment }\end{array}$ & $\begin{array}{l}\text { For two parallel bored tunnels of } 4.5 \text { meters inside diameter, a minimum of } 8 \text { feet of } \\
\text { concrete or } 24 \text { feet of earth \& rock shielding is required for general radiation con- } \\
\text { tainment. Tunnel-to-tunnel penetrations must adequately collimate and attenuate } \\
\text { prompt line-of-sight radiation between tunnel housings and include from source to } \\
\text { receiver at least } 90^{\circ} \text { of angular offsets to the direct penetration sight line. Beam } \\
\text { absorbers (dumps), positron sources and damping rings have greater requirements } \\
\text { and are shielded as required by location. All beam tunnel housing and support } \\
\text { tunnel housing drain water is collected, pumped, batched, held, and tested before } \\
\text { release to surface drains. }\end{array}$ \\
\hline $\begin{array}{l}\text { Special } \\
\text { Beam } \\
\text { Housing } \\
\text { Stability }\end{array}$ & $\begin{array}{l}\text { Cryogenic system cryomodule jitter and long term stability require appropriate } \\
\text { design constraints and mitigation to achieve adequate stability. Except for drainage } \\
\text { sump motors, no utility motors are permitted inside the beam housings. }\end{array}$ \\
\hline Cooling & $\begin{array}{l}\text { For steady-state full power operations at } 5 \mathrm{pps} \text {, the low conductivity cooling water } \\
\text { for the L-band klystrons, modulators, and electromagnets, is to be } 82^{\circ} \mathrm{F} \text { supply, } \\
\pm 5{ }^{\circ} \mathrm{F}, 70^{\circ} \mathrm{F} \text { rise for klystrons, } 15^{\circ} \mathrm{F} \text { rise (max) for magnets. Heat exchangers for } \\
\text { Klystron Cooling Systems (KCS) are located in the support tunnel. The cooling } \\
\text { supply water is fed to the KCS heat exchanger at } 82^{\circ} \mathrm{F} \text {. The support tunnel hous- } \\
\text { ing air temperature is } 85^{\circ} \mathrm{F}, \pm 10^{\circ} \mathrm{F} \text { maximum with access \& season and } 88 \mathrm{fpm} \\
\text { minimum velocity. Beam housing air is } 95^{\circ} \mathrm{F}, \pm 5^{\circ} \mathrm{F} \text { with access \& season, } 0 \text { fpm } \\
\text { velocity with beam and } 88 \mathrm{fpm} \text { velocity with personnel access. }\end{array}$ \\
\hline Power & $\begin{array}{l}\text { Eight } 230 \mathrm{kV} \text { substations, four of six existing } 230 \mathrm{kV} \text { dual feed high availability } \\
\text { transmission sources. Site distribution: } 34.5 / 19.9 \mathrm{kV}, 3 \mathrm{ph}, 60 \mathrm{~Hz} \text {, grounded wye, } \\
100 \mathrm{amp}, 150 \mathrm{kV} \text { bil, w/ } 220 \text { mil XLP cable w/ } 27 \mathrm{kV} \text { arrestors \& ESNA termina- } \\
\text { tions. Load type is: } 55 \% \mathrm{RF}, 20 \% \text { cooling, \& } 25 \% \text { other loads. Phase locked } 60 \mathrm{~Hz} \\
\text { variable frequency motor drives are required site-wide. Rotating equipment shall } \\
\text { be isolated on } 3 \mathrm{~Hz} \text { skids. }\end{array}$ \\
\hline $\begin{array}{l}\text { Special } \\
\text { Maintenance } \\
\text { Access }\end{array}$ & $\begin{array}{l}\text { Klystrons and modulators are to be available for maintenance by personnel } 24 \text { hours } \\
\text { a day. They are to be located as close as possible to beam accelerator sections } \\
\text { to reduce RF distribution losses between the klystrons and the beam accelerator } \\
\text { sections. }\end{array}$ \\
\hline
\end{tabular}

\subsubsection{Configuration and Design Features}

6.3.1.1.1 Purpose This section describes the conventional construction needed to build the U.S. sited superconducting L-band linear collider, which is referred to in this section as the "LLC". The accelerator must meet stringent requirements for sustained luminosity and machine stability and should be located close to existing physics laboratories. The solution presented is only one possible option which can be used to determine the impact that a given site might have on an accelerator and its related design.

\footnotetext{
${ }^{3}$ Near Stanford Linear Accelerator Center, Lawrence Livermore National Laboratory, and Lawrence Berkeley National Laboratory
} 
Criteria for the conventional construction are given Section 3.5, and from references [TDR] and [NLC01]. Design solutions were developed with input from a combination of engineers, consultants and physicists at SLAC, FNAL and other laboratories.

The location of the representative site chosen is in close proximity to existing physics laboratories at Stanford Linear Accelerator Center (SLAC), Lawrence Berkeley National Laboratory (LBNL), Lawrence Livermore National Laboratory (LLNL) and surrounding communities. It is roughly 120 miles north of SLAC and 90 miles north of LBNL. The site is supported by the San Francisco Bay's entire regional infrastructure.

6.3.1.1.2 Configuration The representative site has a north-south alignment in the surface bedrock of the foothills located along the west edge of California's Central Valley. End-to-end, it is approximately 47 kilometers in length. The design describes the construction of two parallel near-surface tunnels bored through stable well-cemented bedrock that consists of Upper Cretaceous (91 to 73 million year old) sedimentary rocks commonly known as the Great Valley Group. Locally, the formation consists primarily of thinly bedded sandstones, with small quantities of siltstones and claystones.

Ramps and some utility shafts provide access between the surface and the below grade housings through which air, cooling water, cryogens and electric power are routed. Ramps at the injector, main linacs and the interaction region will be the major access points for equipment as well as a conduit for utilities and services. The dimensions of the ramps and shafts will generally not exceed the diameter of the beamline or support tunnels with some exceptions. All ramps will serve as an egress corridor from the tunnels below. A minimum of 9 access and utility ramps and 3 additional utility shafts will be required along the entire length of the machine. Cross tunnel passageways will connect the beam housings to the support housings at every ramp access location, and also between those locations, at periodic intervals not to exceed 8,200 feet. These will include adequate offsets and shielding to attenuate housing-to-housing radiation exposure down to safe levels for personnel working in the support housings.

Necessary project infrastructure that already exists at the nearby physics laboratories will be used for the support of the LLC. This minimizes the required local campus space while providing support for long-term operations, maintenance and world wide connectivity.

The site has a nearly north-south orientation parallel to the US Interstate 5 (I-5) corridor which is approximately $8 \mathrm{~km}$ east of the representative LLC site. Six $230 \mathrm{kV}$ power transmission lines run parallel to the machine and to I-5. The California aqueduct, a primary source of cooling and potable water also runs parallel to the I-5 corridor and the LLC alignment. The location was chosen to satisfy several important criteria:

1. Avoid populated areas. This reduces cultural noise and simplifies land acquisition.

2. Allow all of the housings to be constructed in bedrock in the Great Valley Group formation.

3. Provide reasonable access to existing transportation infrastructure.

4. Locate near existing high voltage power and water distribution.

More studies to evaluate property needs for the project will help to minimize the impact to the public and surrounding communities and optimize land use. We anticipate that the U.S. DoE would acquire a corridor of property rights and easements. It would be as much as 500 feet wide in some locations. We estimate that as many as 1800 acres of various property rights and easements will be required for all of the LLC, including land to construct the local campus.

6.3.1.1.3 Design Features The accelerator is placed in surface bedrock that crops out in the low lying hills along the west side of Northern California's Central Valley east of Antelope Valley. The bedrock of the 
area consists of the Great Valley Group, primarily well-cemented interbedded sandstones. This places the depth of the tunnels in a range from 40 feet to 400 feet below the surface but allows for shallow side-hill ramp access to the machine at depths of 40 feet to occasionally 150 feet above the tunnels via east-west running valleys and other low-lying areas of Northern California's Central Valley. Key features of the design are:

1. Two parallel tunnel enclosures providing mutual life safety support.

2. The use of superconducting L-band RF main linacs.

3. Shielding naturally provided by the rock mass between and above the tunnel enclosures.

4. Eight substations providing electrical power, providing $34.5 \mathrm{kV}$ site wide high voltage distribution.

5. Egress via labyrinth type access ways from tunnel enclosures.

Development of a local LLC campus supported by existing SLAC, LBNL \& LLNL facilities nearby is assumed.

\subsubsection{Major Components}

6.3.1.2.1 Local Campus A single local campus is planned for the LLC. This campus will be located approximately $8 \mathrm{~km}$ west of the existing I- 5 corridor. The campus area will include spaces and facilities to operate and maintain the LLC.

The local site campus will primarily support specific operations and maintenance space. Other space needs will be met at the nearby LLNL, SLAC and LBNL laboratories. Table 6.3.1.2 outlines assumptions for construction of local campus building space.

6.3.1.2.2 Injectors The configuration of the injector complex will place the electron injector at the south end of the machine. The remote injection configuration places a significant portion of the LLC complex well away from the interaction region, which is especially sensitive to cultural noise and vibration.

6.3.1.2.3 Main Linac The electron and positron main linacs will be in tunnels below but near the surface. The positron main linac tunnel is approximately $20 \mathrm{~km}$ in length. Using an undulator-based positron source, the electron main linac tunnel will be approximately $21 \mathrm{~km}$ in length. Klystrons and modulators will be housed in the support tunnel, parallel and adjacent to the main linac beam tunnel.

6.3.1.2.4 Beam Delivery At the ends of the main linacs is a region that provides final focusing and steering of the beams prior to reaching the Interaction Regions.

6.3.1.2.5 Interaction Regions The Interaction Regions will consist of two near surface underground halls of equal size. They will house the IR1 and IR2 detector facilities. The detectors will be self shielded. Initially just one interaction region will be outfitted for use. These halls will each have access ramps to the surface. The halls will allow for final detector assembly and be supported with bridge cranes.

6.3.1.2.6 Conventional Utilities Site water and waste water systems include the treatment, storage and distribution facilities for water used in experimental cooling systems, for potable water, and for water needed for fire suppression. Also included are waste water systems, such as sewers and sewage treatment plants, and facilities needed for solid waste, such as holding and transfer facilities. 
Table 6.3.1.2: Local Campus Space

\begin{tabular}{|l|l|l|}
\hline $\begin{array}{l}\text { Local Campus } \\
\text { Support Space }\end{array}$ & $\begin{array}{l}\text { Area } \\
\text { (sq. ft.) }\end{array}$ & Description \\
\hline $\begin{array}{l}\text { Experimental } \\
\text { Campus } \\
\text { Laboratory } \\
\text { Buildings }\end{array}$ & 60,000 & $\begin{array}{l}\text { Laboratory buildings will contain permanent office space and re- } \\
\text { lated support for roughly 150 persons distributed over three eight } \\
\text { hour shifts each day. }\end{array}$ \\
\hline $\begin{array}{l}\text { Experimental } \\
\text { Campus } \\
\text { Heavy } \\
\text { Assembly } \\
\text { Buildings }\end{array}$ & 80,000 & $\begin{array}{l}\text { Heavy assembly buildings will provide low bay and high bay in- } \\
\text { dustrial space, serviced by overhead cranes, for assembly of larger } \\
\text { accelerator and detector components. }\end{array}$ \\
\hline $\begin{array}{l}\text { Experimental } \\
\text { Campus } \\
\text { Shop } \\
\text { Buildings }\end{array}$ & 27,000 & $\begin{array}{l}\text { Three shop buildings will house various clean room spaces and } \\
\text { shops. Clean room space includes the optics maintenance facility, } \\
\text { \& space for assembly of accelerator and detector components. The } \\
\text { buildings include shops such as cryogenic, vacuum, machine, weld- } \\
\text { ing, sheet metal, piping, and carpentry shops. Ten and five-ton } \\
\text { overhead cranes will service these buildings. }\end{array}$ \\
\hline $\begin{array}{l}\text { Experimental } \\
\text { Campus } \\
\text { Support } \\
\text { Buildings }\end{array}$ & 109,600 & $\begin{array}{l}\text { Support buildings, primarily warehousing, provide for receiving, } \\
\text { security and emergency services, cafeteria, vehicle servicing and } \\
\text { miscellaneous functions. }\end{array}$ \\
\hline $\begin{array}{l}\text { Experimental } \\
\text { Control } \\
\text { Rooms }\end{array}$ & 30,000 & $\begin{array}{l}\text { Accelerator and detector control room space with adjacent world } \\
\text { wide communications and networking. }\end{array}$ \\
\hline Total & $\mathbf{3 0 6 , 6 0 0}$ & \\
\hline
\end{tabular}

The facilities included with the cooling systems include raw water systems, distribution piping, storage tanks and low conductivity water systems. Vibration isolation and attenuation will be installed to minimize transmission of utility vibration to beam line components from mechanical pumping equipment. In addition mechanical equipment will be separated from beam line components by as much distance as is practical depending on location.

\subsubsection{Electrical Power: Availability, Configuration and Demand}

At least six existing $230 \mathrm{kV}$ power transmission lines run parallel to the LLC in a North-South alignment. The use of four of these transmission corridor sources will optimize LLC availability. At $0.5 \mathrm{TeV}$ c.m., the LLC will consume about $180 \mathrm{MW}$ of power when it is running. At $0.5 \mathrm{TeV}$ c.m., a primary capacity of no less than 209 MVA, combined, at 0.86 power factor, is planned. This will come from eight primary substations, located at intervals along the alignment. Machine area loads are described in Table 6.3.1.3.

\subsubsection{Heat Rejection}

6.3.1.4.1 Overall System Description An estimated $146 \mathrm{MW}$ of low conductivity water (LCW) heat load will need to be rejected to the environment during machine operation. Using evaporative cooling towers, 4.23 acre-feet of water will be consumed every 24 hours at $0.5 \mathrm{TeV}$ c.m. Heat transfer occurs between the LCW closed loop and the support tunnel secondary cooling water piping system using small plate heat exchangers which are located within packaged equipment skids. The secondary cooling water piping system carries water in 10 in diameter piping in access ramps and shafts, and 8 in diameter distribution branches along the support tunnel housing, and has a temperature rise of $72^{\circ} \mathrm{F}$. The cooling water system will be 
Table 6.3.1.3: Machine Area Power Load Summary in Megawatts, L-band collider, CA site. This table lists megawatt loads by machine area. The main linac is shown in more detail as it uses the most power.

\begin{tabular}{|r|r|r|}
\hline Machine Area & 0.5 TeV c.m. & 1.0 TeV c.m. \\
\hline \hline$e^{+} e^{-}$Injector \& acceleration to 5 GeV & 2.7 & 2.9 \\
\hline$e^{-}$Damping Ring (1) - 5 GeV 720 Arcs & 10.5 & 10.5 \\
\hline$e^{+}$Damping Rings (2) -5 GeV 720 Arcs & 10.5 & 10.5 \\
\hline$e^{+} e^{-}$Main Linacs (2) subtotal of below & $\mathbf{1 3 2 . 7}$ & $\mathbf{2 9 5 . 9}$ \\
\hline modulator input power & 78.8 & 167.2 \\
cryo plants & 23.2 & 75.7 \\
klystron auxiliary power & 8.4 & 17.0 \\
magnet power supplies & 0.2 & 0.4 \\
other rack power & 3.6 & 7.3 \\
cryo cooling towers, pumps, fans, & & \\
auxiliary equipments & 4.1 & 11.0 \\
klystron cooling water pumps & 0.6 & 1.2 \\
cooling towers with pumps and fans & 5.1 & 7.4 \\
ventilation supply, exhaust, chillers & 4.6 & 4.6 \\
lighting, drains, fire protection, .. & 4.1 & 4.1 \\
\hline$e^{+} e^{-}$IR Transport \& Dumps - HE \& LE & & 4.6 \\
\hline$e^{+} e^{-}$Interaction Halls (2) & 2.6 & 2.6 \\
\hline Losses in power distribution and motor efficiencies & 15.9 & 28.7 \\
\hline \hline Total & $\mathbf{1 7 9 . 4}$ & $\mathbf{3 5 5 . 7}$ \\
\hline
\end{tabular}

distributed along the entire length of the collider. The secondary cooling water return piping system will be fully insulated to reduce the transfer of return water piping heat into the air of the support tunnel.

6.3.1.4.2 LCW for Klystrons, Modulators, Magnets and Racks Klystron-modulator assemblies located in the support tunnel, and electro-magnets located in the beam tunnel, are all water cooled with $86{ }^{\circ} \mathrm{F}$ LCW via a primary closed loop circuit running between the support tunnel and the beam tunnel through tunnel-tunnel penetrations. The klystron-modulator will operate with an LCW temperature rise of $70{ }^{\circ} \mathrm{F}$, controlled to $\pm 5^{\circ} \mathrm{F}$.

6.3.1.4.3 Beam Tunnel Operating States The cryogenic cooling system will cool the beam line RF components during normal steady-state full load operations. These normal operations include occasional brief periods where a klystron station will switch off and then will later switch back on. The beam tunnel cryogenic cooling system will maintain the cryogenically cooled RF components associated with the cycling klystron station at a stable operating temperature throughout these excursions. In addition, the cryogenic cooling system in the beam housing will control the temperature of beam line RF components in the housing during beam off periods, both with and without RF, and throughout periodic realignment maintenance including global summer alignment smoothing periods.

6.3.1.4.4 Beam Tunnel Ventilation During beam-off conditions with personnel access, the beam tunnel will be supplied with filtered outside surface air at every supply ramp or shaft, and exhausted through every exhaust ramp or shaft. Supply and exhaust systems will be combined and located every 2 miles. During beam operation, the ventilation of the beam tunnel will be turned off. During smoke and fire control emergency conditions, this same ventilation system will be used for fire control. 
6.3.1.4.5 Support Tunnel Ventilation Support tunnel ventilation will be similar to the beam tunnel ventilation except that personnel will have continuous access to the support tunnel and continuous ventilation regardless of the beam tunnel beam status.

The air speed for both beam and support tunnels during personnel access will be no less than 88 feet per minute. Both tunnels will have smoke detection and heat detection systems, as well as fire suppression water sprinklers.

6.3.1.4.6 Tunnel Drainage At the base of one sidewall of both the beam and support tunnel housings there is a gutter to collect water. Beam housing and support housing drain water is pumped, batched, held, and tested before release to gravity fed surface drains. Water from inside the housings must be controlled as it is potentially radioactive and requires evaluation for metals as well as chemical properties such as $\mathrm{pH}$, total dissolved solids and specific conductivity.

The anticipated total influx of ground water to beam and support housings is expected to be very small as the surrounding sandstone rock formations are high in elevation and typically very dry.

\subsubsection{Vibration}

6.3.1.5.1 Mechanical Noise A design requirement for the LLC is the maintenance of sub-micron beam line alignment. Vibration sources along the LLC alignment must be consistently configured so as to be benign to the beam. Slow drift due to ground motion as well as other motion must be sufficiently slow to avoid frequent disruptive mechanical realignment of the beam line. Faster motion up to $100 \mathrm{~Hz}$ is managed by isolation and attenuation of the source relative to the beam support structure, and by the use of intratrain feedbacks. Internal cryomodule noise as it affects magnets requires mitigation by design.

6.3.1.5.2 Experimental Region Noise and Vibration Isolation The two experimental detectors are located in separate experimental areas identified as IR1 Hall and IR2 Hall. Compared to the other areas of the LLC, these halls are the most sensitive of all to vibration and noise. Every effort must be made to select a specific and very quiet location for these experimental halls. The sites should be free of man-made cultural noise and isolated from the utility equipment necessary to support experimental systems within the halls, including, wherever possible, power, process water, heating, ventilation, controls and so forth. The planned location for the experimental halls is over 20 kilometers from the injector system and is in the middle of the full machine alignment. The local experimental support campus is located at the very southern end of the site near the electron injector so as to place campus-related cultural noise far from the detectors. The experimental hall locations have been evaluated by direct field measurement to determine local cultural noise levels. They have been confirmed to be suitable for the LLC in California.

6.3.1.5.3 Near Field Vibration The primary source of vibration that can affect the machine's alignment in the beam tunnel comes from mechanical and cryogenic devices located within the support tunnels and connected to the beam tunnel cryomodules. Sources of mechanical noise include pumps, water moving in pipes, transformers and cryogenic systems. Internal cryomodule noise as it affects magnets requires mitigation by design. LCW equipment skids located in the support tunnel are floated and balanced on $3 \mathrm{~Hz}$ counter-wound helical spring pairs to afford isolation of the skids from the support tunnel floor as well as from the adjacent beam line tunnel floor. Acoustic baffles about the skids will provide additional isolation and attenuation. Similar measures must be employed for other equipment.

6.3.1.5.4 Far Field Vibration Measurements It has been demonstrated that surface noise generated near the Interaction Region is small and that the LLC Logan Ridge site is very quiet. Surface vibration 
measurements were made in October of 2001 at a location not far from the middle of the LLC interaction region. Geophones were secured to rock on the undisturbed surface where vibration measurements were recorded during a 5 hour period. Results indicated that there was no cultural noise of consequence and that far field highway traffic noise measured at this location, $8 \mathrm{~km}$ west of Interstate I-5, was not disruptive.

6.3.1.5.5 Alignment Smoothing Interval The planned cycle for beam operation is to run for 9 months, from September 1 to May 30 each year. This coincides with the most efficient operating cycle for scheduling water and power usage. Global realignment will take place during the annual 90 day maintenance interval.

\subsubsection{Geology and Tunneling Means and Methods}

6.3.1.6.1 Site A site-specific detailed geological and geotechnical study[72] was conducted by Philip A. Frame, R.G., Consulting Engineering Geologist in 2000. This study was commissioned for the purpose of finding suitable California locations to build the LLC, then called the Next Linear Collider. The study details the geology of an area along a $50 \mathrm{~km}$ alignment parallel to Interstate 5 . The study found that highly favorable conditions existed in the region for building the LLC in competent, well-cemented, interbedded sandstones, with some siltstones and claystones as well.

The LLC representative site is in low relief hills east of the Antelope Valley and trends north-south along the west edge of Northern California's Central Valley. The hills are dissected with a series of valleys that drain the upper reaches from west to east and allow for optimum access to parallel bored tunnels via side hill ramps. This remote area is used primarily for agriculture and cattle grazing. These hills lie in the rain shadow of the California Coast Ranges and, on average, get only 17.3 inches of rain per year. Based on the relatively low average rainfall and high relief, these hills should provide for dry conditions during construction and during operations.

The alignment of the machine is laser straight. The natural rock cover directly above the tunnel enclosures will vary from less than $40 \mathrm{ft}$ to as much as $400 \mathrm{ft}$ with the existing surface topography. Tunnel depth at the surface access ramps will allow for shallow ramp access to the machine at an average elevation 50 feet above the tunnels via east-west running valleys and other low-lying areas of Northern California's Central Valley.

Mechanical excavation using a Tunnel Boring Machine (TBM) for the underground tunnel enclosure construction will be very attractive from both a cost and schedule perspective.

The bedrock of the region is characterized as well cemented fine- to coarse-grained sandstone with some interbedded siltstones and claystones that total approximately $7 \mathrm{~km}$ in thickness. These materials were shed off of the ancestral Sierra Nevada Mountains into a shallow sea between 91 and 73 million years ago. The sandstone beds are steeply dipping to the east. This is a structurally sound rock that is considered a softer and less problematic rock to tunnel in relation to other sedimentary, metamorphic or igneous rocks. These properties will allow for a higher than average rate of penetration during tunneling operations. Normal tunnel support methods (i.e., rock bolts and 2 in of shotcrete with wire mesh as used at SLAC) will provide adequate support for the beam and support tunnels.

The representative LLC site lies within a large body of bedrock of the cretaceous sedimentary strata commonly referred to as the Great Valley Group. The site is underlain by structure informally known as the Funks segment of the Great Valley fault, a blind, west-dipping thrust fault which underlies the region at a depth of about $5 \mathrm{~km}$. Published geologic reports indicate that the most recent displacement in the region of the Logan Ridge site occurred more than 10,000 years ago. The slip-rate on this segment is calculated to average $0.1 \mathrm{~mm} / \mathrm{yr}$ and is thought to be capable of generating a 6.4 magnitude earthquake recurring every 6,000 years. (See reference [73].) 
6.3.1.6.2 Rock Spoils Approximately 2,000,000 cubic yards of material (not bulked) will be generated during excavation. It is anticipated that spoil stockpiles can be placed in many of the canyon valleys at various locations along the machine's alignment.

6.3.1.6.3 Construction Means and Methods Excavation of rock using TBM means and methods for the tunnels has been used for cost estimating purposes. For dry conditions in long tunnels in soft competent sandstone rock, TBM's, driving with high daily advance rates, offer significant cost and schedule benefits. Localized grouting, and invert construction pinned directly to bedrock will be required along the length of the enclosure. We are anticipating that about $20 \%$ of the tunnel length may require some additional lining. Based on the available data and local experience, this appears to be conservative. The excavated tunnel overbore will need to account for the final reinforced shotcrete application and any additional lining thickness where an added lining appears to be required. More specific geologic and geotechnical exploration programs along the final alignment will support this determination. Only straight ahead tunnels will employ TBMs for excavation. Nearly all other underground excavation will be done with road headers and/or drilling rigs.

\subsubsection{Conventional Facilities Design Summary for a Superconducting Linear Collider, North-South Illinois Alignment, West of the Fox River, Illinois ${ }^{4}$}

\subsubsection{Configuration and Salient Design Features}

6.3.2.1.1 Purpose This section describes the conventional facilities required to construct and house the U.S. sited superconducting L-band linear collider, referred to here as "FY03 NS-SC." This accelerator will be utilized by the high-energy physics community to explore the sub-atomic building blocks of nature and the origins of the universe by observing collisions of electrons and positrons.

The purpose of this section is to determine the impacts that a distinct site has on the accelerator and the accelerator housing design, as well as the resulting cost and schedule impacts. For the purposes of this presentation a location for the accelerator has been shown on various maps. The location shown represents only one of many possible alternatives. The location could vary by several miles and still be valid for the design solution described in this report.

Criteria for the conventional construction have been derived from discussions with physicists at SLAC and Fermilab, from the reference design described in Section 3.5, and from references [TDR] and [NLC01]. Design solutions have been developed in consort with engineers at SLAC and Fermilab, and consulting engineers. The designs generated have used the current SLAC and Fermilab accelerators as models for determining criteria for installation, support, servicing and maintenance of the accelerator. Environmental requirements for the tunnel housings are assumed to be consistent with other high-energy physics accelerators.

6.3.2.1.2 Proposed Facilities The conventional facilities will consist of above and below grade structures, utilities and support facilities generally found at high-energy physics accelerator laboratories. The Illinois FY03 NS-SC design iteration considers constructing conventional facilities to support a $500 \mathrm{GeV}$ center of mass accelerator, expandable to $1 \mathrm{TeV}$ center of mass. The design explores the construction of the tunnels within the Galena Platteville Dolomite, in a north south alignment, on a hypothetical site some 20 miles west of the Fermilab site.

The below grade accelerator is comprised of several major elements: electron sources, damping rings, undulator, positron system, main linac, beam transport and final focus. Two electron sources will generate either polarized or unpolarized electrons. The injectors will be located in separate, adjacent tunnels. After accelerating the electrons to $150 \mathrm{GeV}$, the electrons will be extracted to the undulator and transported either

\footnotetext{
${ }^{4}$ Near Fermi National Accelerator Laboratory
} 
to the remaining electron main linac or to the positron target. The main linac tunnels, each nearly $20 \mathrm{~km}$ long, will contain a series of RF cavities that accelerates the beams towards a central interaction region. RF cavities will be installed in the first portion of the main linac tunnel. This active portion of the main linac will produce energies for 250 on $250 \mathrm{GeV}$ collisions. An inactive portion of the main linac tunnel will contain a beam transport line that could be upgraded with additional RF cavities to increase the final energy to 500 on $500 \mathrm{GeV}$. A beam delivery system will be required between the Interaction area and the end of the two main linacs. There will be a parallel service tunnel that houses beamline support technical equipment.

Shafts will be required for construction of the tunnels, as well as to provide a link between the surface and the below grade housings through which LCW, power, cryo piping, and elevators will be routed. Shafts at the extreme ends of the of the linacs will act as the major equipment access for cryostats and other beamline support equipment as well as a conduit for the utilities and services for the injectors and damping rings. The main linac and cavities will be supported by facilities spaced roughly $2.5 \mathrm{~km}$ from each end and every $5 \mathrm{~km}$ along the length of the main linac. The shafts located $12.5 \mathrm{~km}$ from each end will be sized similar to the end shafts to facilitate TBM excavating equipment, and accelerator cryostats. At each of these locations there will be a shaft to service the enclosures below from service buildings on the surface. At the interaction halls, shafts will be needed to support each of the IR Halls as well as services for the absorbers and final focusing equipment. All shafts will have elevators and/or stairs that will serve as part of the egress corridor from the tunnels below. Twelve major shafts are required, including one at each end of the linacs, 8 cryo and utility shafts, one at the IR2 Hall and one at the IR1 Hall. Additional smaller, drilled shafts will be required for services at special locations such as the damping rings.

Above grade support buildings are required at each major shaft to support the below grade accelerator and support housings. Each shaft will have a building located directly above the shaft. The IR2 Hall will be outfitted and the above grade service building constructed to provide only life safety and minimal services.

The siting of a new campus within 20 miles of Fermilab will allow some of the required infrastructure that currently exists on the Fermilab site to be used for the support of this accelerator.

6.3.2.1.3 Siting The preliminary alignment was chosen to optimize the following site criteria:

1. Avoid populated areas. This reduces cultural noise and simplifies land acquisition.

2. Allow all of the housings to be constructed in Galena Platteville dolomite formation.

3. Provide reasonable access to transportation infrastructure, both to Fermilab and area airports.

4. Locate near existing high voltage power distribution with adequate capacity and redundancies.

This report considers a nearly north-south orientation, roughly centered on Interstate I- 88 approximately 12 miles east of DeKalb, IL. A $345 \mathrm{kV}$ Commonwealth Edison power distribution line is currently located running in an east west alignment close to the central campus area. An additional $345 \mathrm{kV}$ power source is located roughly 5 miles to the southwest of the Central Campus.

We anticipate that an estimated 500-foot wide corridor of property rights and easements would be acquired by the U.S. DoE. We estimate that as much as 2000 acres of underground property rights and easements will be required for the enclosure corridor and another 3000 acres of land acquisition will be required to construct the campus and satellite buildings and to provide for future expansion.

\subsection{Salient Design Features Salient design features of this study include:}

- Locating the length of the laser straight accelerator in the Galena Platteville rock formations. This places the depth of the tunnels in a range from 120 feet to 400 feet below the surface. 
- The use of superconducting Main Linacs is the primary change from previous design iterations. The primary focus region and the Interaction Regions are consistent with the designs associated with the normal-conducting linac option.

- Distributed electrical Sub-Stations with new $345 \mathrm{kV}$ Sub-stations located at each Cryo/LCW buildings with electrical distribution via an above grade distribution pole line.

- Two parallel enclosures. Provision for life safety is modeled from transportation tunnels. Shielding is naturally provided by rock between the enclosures. Egress is via shielding ramp access ways between the enclosures.

- Development of a Central Campus supported by existing Fermilab facilities.

\subsubsection{Description of Major Components}

6.3.2.2.1 Site Development Most of the current land use along the proposed accelerator is agricultural. No change in land use is expected, except at the Cryo Service Building Campuses and the Central Campus. Conventional utilities (domestic water, natural gas, sanitary sewer) do not currently exist and will be constructed with this project or adapted from the local municipal systems as required.

\subsection{Surface Buildings}

6.3.2.2.2.1 Central Campus A central campus is planned for this project. This campus will be located near Interstate I-88. The campus areas will include office spaces and facilities to build, operate, service, and maintain the project. Although some facilities will need to be located at the project site, current communication technologies will allow for many of the projects needs to be met remotely at locations such as Fermilab and other high-energy laboratories. Table 6.3.2.1 outlines our assumptions for construction of new building spaces. In addition, facilities that will be required for the Interaction Halls at the surface will be located within the boundaries of the Central Campus site.

6.3.2.2.2 Cryo/LCW Surface Plant Buildings Located at each shaft and supporting roughly a 5 $\mathrm{km}$ portion of the accelerator is a Cryo/LCW Surface plant. Power, Low conductivity cooling water (LCW), sprinkler water (ICW), potable water (DWS), sewer, pump discharge, HVAC, and cryogenic (cryo) systems are processed and routed to and from the tunnel in these buildings. These buildings function as staging areas for material access to the tunnel as well as egress from the tunnels.

\subsection{Underground Structures}

6.3.2.2.3.1 Injectors A combined positron / electron injector facility will be constructed at the south end of the accelerator complex. At the extreme south end of the accelerator, polarized and unpolarized electron sources will be located in separate tunnels. Downstream from the electron sources is an undulator followed by the positron source. A beamline will transport the positrons past the IR region to the damping ring located on the north end of the accelerator. A short linac section will accelerate the beam to $5 \mathrm{GeV}$ prior to transport to the damping ring.

6.3.2.2.3.2 Damping Rings Damping rings are located at each end of the accelerator complex. A portion of the damping rings are located in the same tunnel as the Main Linac with hoops at each end of the damping rings. The electrons and positrons are then transfered to the main linac. 
Table 6.3.2.1: Fermilab FY03 NS-SC Orientation Central Campus Facilities Assumptions

\begin{tabular}{|l|r|}
\hline Description of Building & sq. ft. \\
\hline \hline New Office Space & 30,000 \\
New Computing Space & 20,000 \\
New Light Lab Space & 5,000 \\
New Cafeteria & 5,000 \\
New Large Heavy Assembly Buildings & 40,000 \\
New Small Heavy Assembly Buildings & 40,000 \\
New Light Shop Space & 27,000 \\
New Warehousing & 40,000 \\
New Gas \& Cryo Storage & 6,000 \\
New Security Buildings & 1,600 \\
New Emergency Response & 5,000 \\
New Medical \& ES\&H & 4,000 \\
New Heavy \& Light Equipment Maintenance & 13,000 \\
New Campus Utility Plant Building & 40,000 \\
New Operations Control Room & 15,000 \\
New Operations Annex Wing & 10,000 \\
New Communications Center & 5,000 \\
\hline Total & 306,600 \\
\hline
\end{tabular}

6.3.2.2.3.3 Main Linac Each of the main linacs will involve the construction of nearly $20 \mathrm{~km}$ of parallel twin bored tunnels, with an inside diameter of 13.5 feet. The beamline enclosure will house main linac cryostats and beamline components. The support enclosure will be a parallel tunnel that houses klystrons, modulators, power supplies, instrumentation, and controls. The support enclosure will allow personnel to access critical components during beamline operation for maintenance and operations purposes. The klystrons distribute RF to the cryostats through "duct-like" wave-guides that pass between the two tunnels in two-foot diameter penetrations spaced at 34 meters along the main linac. For $500 \mathrm{GeV}$ center-of-mass operation, three cryogenic halls are placed along the length of each main linac (for a total of six) to provide liquid helium to the superconducting cryostats.

6.3.2.2.3.4 Beam Delivery Starting at the ends of the main linac is a region that provides final focusing and steering of the beams prior to reaching the Interaction Regions.

6.3.2. 2.3.5 Interaction Regions Each Interaction Region will consist of an above grade assembly building and below grade collision hall. Two such detector facilities are planned, IR1 Hall and IR2 Hall. Each IR region will require collision halls below grade sized at $110 \mathrm{ft}$ wide by $250 \mathrm{ft}$ long by $100 \mathrm{ft}$ high. The grade-level assembly area will be for detector subassembly and will include a heavy crane. Mezzanines will provide areas for computer, control room and office facilities. Other utility areas will house process water systems, electrical power services and air handling equipment. The assembly area will likely be constructed of conventional steel and reinforced concrete construction.

\subsubsection{Electrical Power}

6.3.2.3.1 Availability A $345 \mathrm{kV}$ power line exists along an east west alignment near the proposed central campus area. A second $345 \mathrm{kV}$ power line source is roughly 5 miles southwest of the central campus. Each of these $345 \mathrm{kV}$ power lines currently has the capacity to supply the power required by the Linear Collider. 
6.3.2.3.2 Description Both sources of electric power delivered from ComEd will be extended on common poles in a north south direction along the length of the Linear Collider active Linac (From Cryo/LCW building 1 to Cryo/LCW building 8). The transmission line service voltage is $345 \mathrm{kV}$. Feeder taps from the two overhead lines feed 9 separate areas along the length the LC. Two 40 MVA step-down transformers at each Cryo / LCW building will supply $34.5 \mathrm{kV}$ for distribution to the surface and underground loads. These transformers provide power to two separate loads: 1) Modulator/Power Supply loads (underground), 2) Conventional, LCW and Cryo loads. Power to Central region loads (Central Campus and IR Halls) will be from a single 40 MVA transformer (backed up by a 40 MVA installed spare transformer) and will be distributed via circuit breakers at a substation-house to underground feeders. Each location will be constructed with a concrete pad and switchgear for one additional transformer. The components at each substation will be standardized.

Power to Modulator/Power Supply loads will be via a three phase $34.5 \mathrm{kV}$ transmission line directly down each Cryo shaft and distributed along the length of the active linac, connecting to the modulator transformers, in series, via two internal fused disconnects. The modulator transformers are $225 \mathrm{KVA}$, are spaced 34 meters apart (powered in a pattern of 4 out of 5 cells), and are located inside the service tunnel.

Each substation will be monitored and controlled at a prefabricated control room. Lower voltage lines after the substation will be monitored and controlled locally. Backup emergency power will be available at each Substation. Control Power for each substation will be via a battery bank. A Kirk Key system will be established to safeguard against parallel feeds.

Cryo, LCW, building and conventional power will be from ten (on average) $1500 \mathrm{kV}$ (34.5 kV to $4160 \mathrm{~V}$ or $480 \mathrm{~V}$ ) transformers. Part of this load is a series of 15 KVA transformers (spaced 34 meters apart) inside the service tunnel stretching out $2.5 \mathrm{~km}$ each direction away from the vertical shaft. This is for all conventional electrical loads (lighting, pumps, and HVAC units). Each feeder cable is sized at $35 \mathrm{kV} 750 \mathrm{MCM}$ and is routed to the tunnel via 6 inch metal conduit inside the vertical shaft.

Lighting design luminance for the tunnel, corridors and hallways will be 20 foot-candles. Welding receptacles 60 A $480 \mathrm{~V}$ outlets will be provided at every 800 feet. $120 \mathrm{~V} / 208 \mathrm{~V}$ receptacles will be provided at every 120 ft. Emergency lighting will be provided for general safety.

Power distribution system redundancy has been maximized while isolation devices have been kept to a minimum at the various levels. Life safety equipment such as emergency lighting and fire detection will be powered from uninterruptible power supplies and critical systems will be connected to emergency generators via automated transfer switches.

6.3.2.3.3 Configuration and Demand At $0.5 \mathrm{TeV} \mathrm{cm}$, the LLC will consume about $180 \mathrm{MW}$ of power when it is running. At $0.5 \mathrm{TeV} \mathrm{cm}$, a primary capacity of no less than $209 \mathrm{MVA}$, combined, at 0.86 power factor, is planned. This will come from eight primary substations, located at intervals along the alignment. Machine area loads are described in Table 6.3.2.2.

\subsubsection{Heat Rejection}

6.3.2.4.1 Project Requirements The estimated 146 MW low conductivity water (LCW) heat load will be rejected to wet type cooling towers located at each LCW cooling plant (total nine), approximately 5 $\mathrm{km}$ apart, and located adjacent to each cryogenic plant on the surface level. The LCW/HVAC surface plant is estimated to be $230 \mathrm{ft} \times 75 \mathrm{ft}$. The LCW cooling system arrangement is based on non-summer months operation, or beam operation for 9 months, from September to May each year, thereby eliminating the need for chillers to provide the required klystron supply water temperature of $86{ }^{\circ} \mathrm{F}$. Details are presented in Table 6.3.2.3. 
Table 6.3.2.2: Machine Area Power Load Summary in Megawatts, L-band collider, IL site. This table lists megawatt loads by machine area. The main linac is shown in more detail as it uses the most power.

\begin{tabular}{|r|r|r|}
\hline Machine Area & 0.5 TeV c.m. & 1.0 TeV c.m. \\
\hline \hline$e^{+} e^{-}$Injector \& acceleration to 5 GeV & 2.7 & 2.9 \\
\hline$e^{-}$Damping Ring (1) - 5 GeV 720 Arcs & 10.5 & 10.5 \\
\hline$e^{+}$Damping Rings (2) -5 GeV 720 Arcs & 10.5 & 10.5 \\
\hline$e^{+} e^{-}$Main Linacs (2) subtotal of below & $\mathbf{1 3 2 . 7}$ & $\mathbf{2 9 5 . 9}$ \\
\hline modulator input power & 78.8 & 167.2 \\
cryo plants & 23.2 & 75.7 \\
klystron auxiliary power & 8.4 & 17.0 \\
magnet power supplies & 0.2 & 0.4 \\
other rack power & 3.6 & 7.3 \\
cryo cooling towers, pumps, fans, & & \\
auxiliary equipments & 4.1 & 11.0 \\
klystron cooling water pumps & 0.6 & 1.2 \\
cooling towers with pumps and fans & 5.1 & 7.4 \\
ventilation supply, exhaust, chillers & 4.6 & 4.6 \\
lighting, drains, fire protection, .. & 4.1 & 4.1 \\
\hline$e^{+} e^{-}$IR Transport \& Dumps - HE \& LE & 4.6 & 4.6 \\
\hline$e^{+} e^{-}$Interaction Halls (2) & 2.6 & 2.6 \\
\hline Losses in power distribution and motor efficiencies & 15.9 & 28.7 \\
\hline \hline Total & $\mathbf{1 7 9 . 4}$ & $\mathbf{3 5 5 . 7}$ \\
\hline
\end{tabular}

6.3.2.4.2 LCW (Low Conductivity Water) System Each surface LCW Cooling Plant facility will have approximately four (2-cell) wet cooling towers, indoor water tank, three tower pumps, two heat exchangers, three secondary pumps, water treatment system, filtration system and controls. Additional equipment will be required at the IR LCW plant. Sizing of the cooling tower is based on $15^{\circ} \mathrm{F} \Delta T$ at $71^{\circ} \mathrm{F}$ wet bulb overall. The LCW cooling system will consist of primary-secondary-tertiary cooling loops. The primary loop is a $15^{\circ} \mathrm{F} \Delta T$ cooling tower system (at $78^{\circ} \mathrm{F} / 93^{\circ} \mathrm{F}$ ) that includes all the cooling towers, tower water pumps and industrial grade heat exchangers. The secondary loop (at $80^{\circ} \mathrm{F}$ supply / $154{ }^{\circ} \mathrm{F}$ return) is a non-deionized cooling water loop that will distribute cooling water from heat exchangers at the surface level to the individual skids in the service tunnel, up to approximately $2.5 \mathrm{~km}$ each way, and return water to the heat exchangers on the surface. All the piping, valves, fittings and other components in the secondary system will be designed and selected for use at higher pressure rating due to the length of the system and elevations involved. The tertiary loop includes modular heat exchanger/pump skid and uses deionized LCW to feed the Modulators/Klystrons/Racks (at $86^{\circ} \mathrm{F}$ supply / $156^{\circ} \mathrm{F}$ return). The cooling skid for the third loop will be located in the service tunnel.

The system design for primary and secondary loops will include redundancy considerations using multiple pumps and heat exchangers to prevent accelerator shutdowns due to equipment failures. Each skid on the tertiary loop will be provided with one heat exchanger/one pump and no backup. All of the tower water and primary heat exchanger systems will be located in the surface facilities to simplify maintenance.

6.3.2.4.3 Design variations Various design options were considered during the conceptual design stage to optimize the system design. Examples of these design options are a Dry Cooler system in combination with the wet towers, cooling ponds, and a higher $\left(32^{\circ} \mathrm{F}\right) \Delta T$ cooling tower system.

6.3.2.4.4 Ventilation (Beam Tunnel) During beam-off condition and personnel access, the beam tunnel will be supplied with $100 \%$ filtered/conditioned/dehumidified air at every supply shaft, and exhausted 


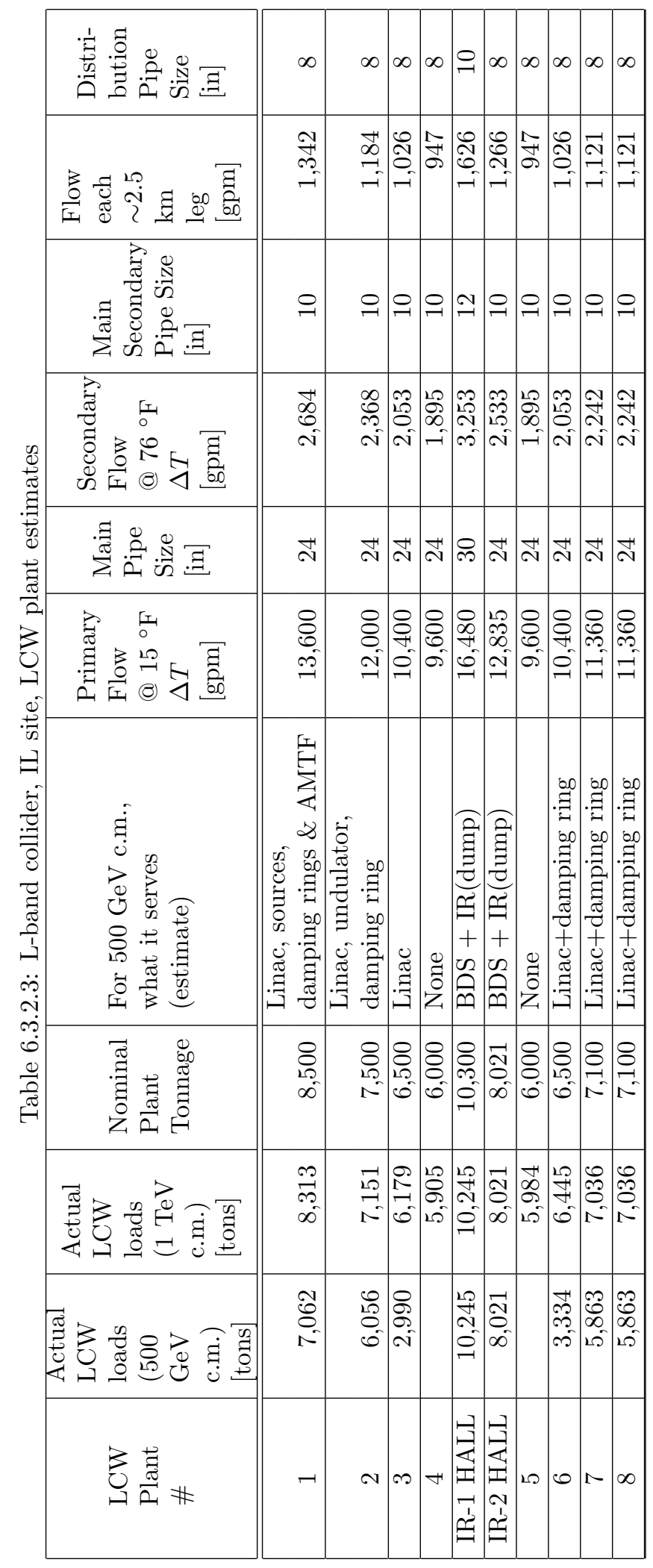


through every exhaust shaft. Supply and exhaust shafts will be alternately located every approximately 5 $\mathrm{km}$. During beam operation, the ventilation of the beam tunnel will be turned off. During smoke and fire control emergency condition, this same ventilation system will be used and airflow will be diverted to the proper spaces.

6.3.2.4.5 Ventilation (Service Tunnel) Each of the service tunnel sections between the LCW plants will be operated independently and each will be supplied with $100 \%$ filtered/conditioned /dehumidified outside air. The system will be capable of bypassing an LCW hall and feeding the air directly to the next hall. Alternating plant location will provide supply and exhaust flow. During smoke and fire control emergency conditions, this same ventilation system will be used and airflow will be diverted to proper spaces.

The air speed for both tunnels will be $88 \mathrm{fpm}$. Both tunnels will have smoke detection and heat detection systems. There will be various water cooled air conditioners and heat pumps that will condition the klystron/modulator area to maintain the space suitable for workers in the service tunnels. Condenser water to these units will come from the secondary cooling water that feeds the equipment load. To separate these units from the high-pressure secondary water system, HX/pump skids will be provided to feed multiple units.

6.3.2.4.6 Drainage The water from beam tunnel walls and the fan coil drains will drain by gravity towards the shaft sump pit at every shaft location. The inflow estimate is $50 \mathrm{gpm}$ per mile per tunnel. The water will be pumped from the sump pit to the ground surface.

6.3.2.4.7 Cryogenic System Housing For a $0.5 \mathrm{TeV}$ center-of-mass Linear Collider, three refrigerator plants (cryogenic hall) per Linac, approximately $5 \mathrm{~km}$ apart, will be used for a total of six cryogenic halls. Each plant provides refrigeration for approximately $2.5 \mathrm{~km}$ for an upstream and downstream string of superconducting RF modules. Each cryogenic surface building is estimated at $230 \mathrm{ft} \times 262 \mathrm{ft}$. In addition to the surface building, the cryogenic system requires a dedicated space of $40 \mathrm{ft} \times 30 \mathrm{ft} \times 35 \mathrm{ft}$ located near the accelerator tunnel for housing the technical distribution box. All other cryogenic components, including the cooling tower dedicated to the cryogenic system, and indoor electrical switchgear for technical cryo equipment, are part of the technical components and are not included in the civil work.

\subsubsection{Geology, Siting and Tunneling Means and Methods}

6.3.2.5.1 Site The proposed siting used the geological and geotechnical studies prepared for the Superconducting Super Collider (SSC) studies in the late 1980s by the Illinois Departments of Energy and Natural Resources, State Geological Survey Division[74]. The SSC studies geologically define an area roughly 20 miles north and south of Fermilab, 45 miles to the west and 6 miles to the east of Fermilab. This information is augmented by more recent rock maps and cross-sections provided by the State's Geological Survey. From a siting and construction basis, the Linear Collider is similar to that of the SSC. These studies indicate that favorable conditions exist in the vicinity of the Dekalb / Kane county lines. The conditions exist that will allow the project to optimize the following criteria:

1. Avoid populated areas. This reduces cultural noise and simplifies land acquisition.

2. Allow all of the housings to be constructed in Galena Platteville dolomite formation for its entire length.

3. Minimize the depth of shafts.

4. Utilize rock formations with a favorable hydrogeologic setting. 
The dominant land use in the proposed siting area is currently agricultural, dotted with small-to medium sized towns. Urban expansion towards our siting, currently several miles to the east, is an issue that will need to be addressed in detail to determine potential impacts.

The Galena Platteville is characterized as a fine to medium grained dolomite, that is cherty. The Maquoketa shales overlaying the dolomite have a low hydraulic conductivity that will act as a hydrogeologic barrier between upper overburden aquifers and the dolomite. At the proposed siting the Galena Platteville varies from 300 to 350 feet in thickness, gently rising in datum elevation from the south to the north. The Galena is covered by 50 to 100 feet of shale, which in turn, is overlaid by 50 to 150 feet of overburden. It is expected that features requiring grouting will be encountered. The upper Silurian dolomite found at the Fermilab site is absent, for a significant distance to the east. These geologic conditions should provide a relatively dry tunnel, both during construction and during operations. The Galena is the most structurally sound rock in the area and, in general, should not require any extra ordinary rock support methods.

The criteria for the alignment of the machine is a laser straight configuration, thus the cover over the enclosure will vary from 120 to 400 feet. Approximately 150 feet of this depth at the center is a result of the earth's curvature. It is anticipated that mechanical excavation techniques using Tunnel Boring Machine (TBM) for the underground enclosure construction will be used as outlined in the means and methods section below.

6.3.2.5.2 Spoils Approximately 2,000,000 cubic yards of material (not bulked) will be generated during excavation. It is anticipated that spoil stockpiles will be necessary at as many as five locations. The majority of the spoil will be dolomite that may be utilized as structural fill on this and other projects. It is anticipated that the rock spoil will be cost neutral for the project.

6.3.2.5.3 Construction Means and Methods Excavation of rock using a TBM for the tunnels was selected for estimating purposes. For long tunnels, TBM's offer significant cost and schedule benefits. Localized grouting, and invert construction will be required along the length of the enclosure. We are anticipating that about $25 \%$ of the tunnel length may require some lining. Based on the available data this appears to be conservative. The excavated tunnel diameter will need to account for the final lining thickness if lining appears to be required. This determination will be influenced by the geologic and geotechnical ground exploration program. IR halls, access ramps and waveguide penetrations are all anticipated to be excavated using drill and blast methods.

\subsubsection{Vibration}

6.3.2.6.1 Mechanical Noise as an Issue in site Selection A design requirement for the superconducting linear collider is the maintenance of sub-micron beam line alignment. Vibration sources along the colliders alignment must be consistently configured so as to be benign to the beam. Slow ground motion can be compensated through beam-based feedback. Faster motions up to $100 \mathrm{~Hz}$ are managed by isolation and attenuation of the source relative to the beam support structure, and by the use of intratrain feedbacks. Above $100 \mathrm{~Hz}$, the beam is almost equally sensitive to any frequency, but with increasing frequency the amplitude of vibrations typically decrease so that integrated amplitude is of relevance.

The two experimental detectors are located in separate experimental areas identified as IR1 Hall and IR2 Hall. Compared to the other areas of the collider, these halls are the most sensitive of all to vibration and noise. Every effort must be made to select or control a specific and very quiet location for these experimental halls. The sites should be free of man-made cultural noise and isolated from the utility equipment necessary to support experimental systems within the halls, including wherever possible, power, process water, heating, ventilation, controls and so forth. The local support campus is located away from but adjacent to the detectors so that noise can be controlled. Prairie will be planted around the detectors for a distance (to be determined) to control cultural noise. 
The siting of a linear collider along a road or railway right-of-way may be problematic, because of the requirement to maintain, in some cases, sub-micron alignment tolerances. The affects of mechanical vibrations from railways should be a topic for further study. It is being explored in the ongoing studies being performed in the fixed target area and the nearby North Aurora Tunnel. There are three train rails that cross or are near to crossing the proposed alignment. It is not known whether it is a problem or not, and the incorporation of beam based feedback could bring these effects under acceptable control. Earth noise, which is seismic noise created, for example, by the interaction with wind or sea, is another form of noise that can pose a problem for the machine design. Typically, earth noise lies in frequencies below $1 \mathrm{~Hz}$, while cultural noise lies above $1 \mathrm{~Hz}$. Amplitude of ground motion decreases as $1 / f^{2}$ with increasing frequency.

Seismic noise conditions may affect the final siting decisions, because a plane seismic wave traveling perpendicular to the accelerator has a minimal affect on machine alignment. For example, a site near a shoreline might align the accelerator parallel to the shoreline.

The primary source of vibration that can affect the machines alignment in the beam tunnel comes from mechanical devices located within the support tunnels. Sources of mechanical noise include pumps, water moving in pipes, and transformers. The LCW equipment skids located in the support tunnel are floated and balanced on $3 \mathrm{~Hz}$ counter-wound helical spring pairs to afford isolation of the skids from the support tunnel floor as well as from the adjacent beam line tunnel floor. Acoustic baffles about the skids will provide additional isolation and attenuation. Similar measures will be employed for other equipment. A siting criteria might include provision for seismic noise to be as small as sources of mechanical noise emanating from within the accelerator tunnel. It's likely, but has not been demonstrated, that surface noise generated above 200 to 400 feet of overburden will be small compared to mechanical sources from within the accelerator tunnel.

6.3.2.6.2 Vibration Studies A study for the Illinois SSC proposal investigated vibrations of truck and railroad traffic, which was considered the largest source of vibrations in the region. This investigation monitored truck traffic over an expansion joint of a bridge and the passage of freight trains. Truck traffic showed displacement of 2.9 to $19.3 \mu \mathrm{m}$ on the bridge abutment. However, 65 feet down and 150 feet horizontally to a rock quarry floor these movements were attenuated 92 to 241 times, down to .03 to $.08 \mu \mathrm{m}$. The train traffic displacements on the ground next to the rail line were 1.6 to $4.06 \mu \mathrm{m}$, but 60 feet down and 190 feet horizontally to the quarry floor, the displacements were attenuated 62 to 123 times, down to .013 to $.066 \mu \mathrm{m}$.

\subsubsection{Selected Conventional Facilities Design Drawings for the Superconducting L-band option}




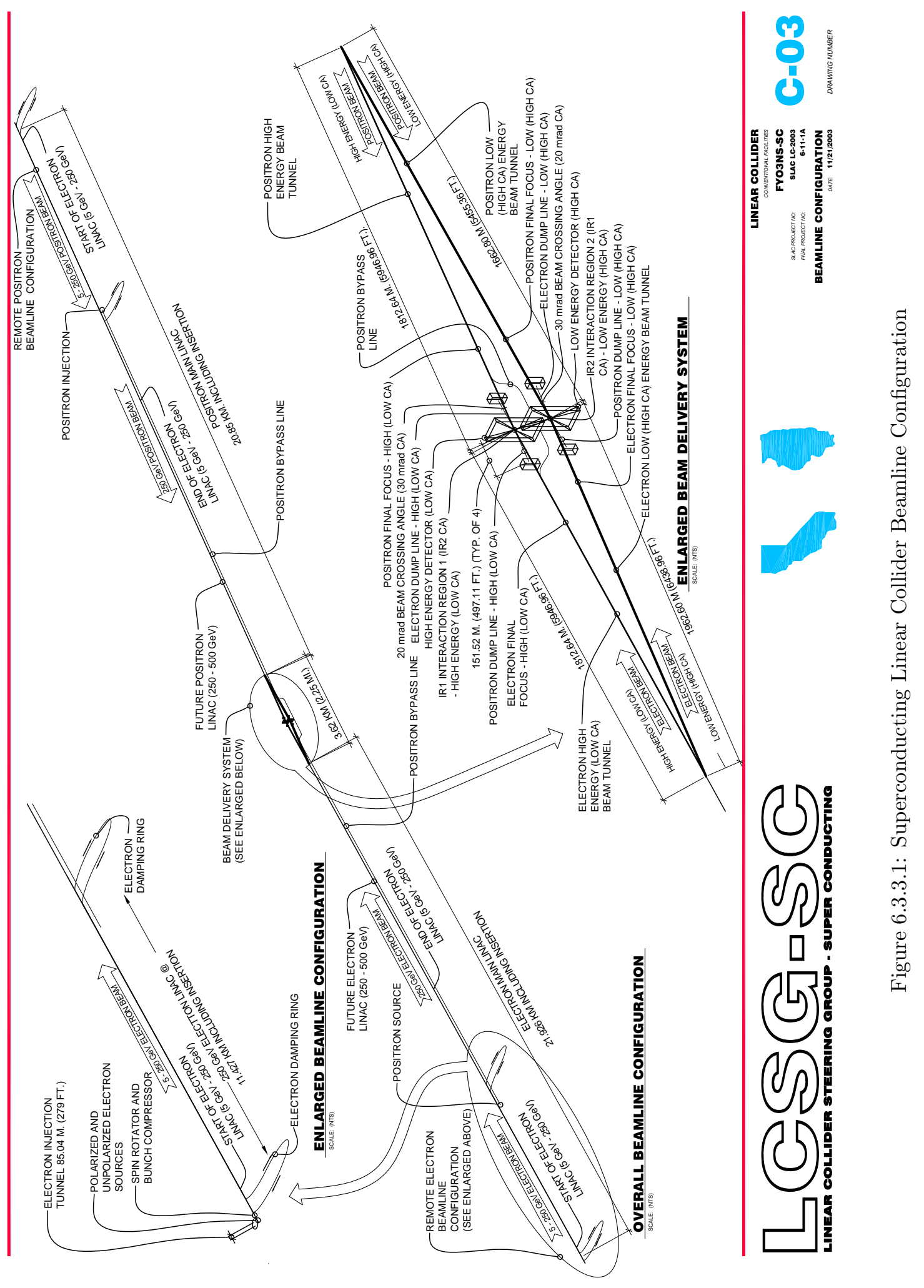




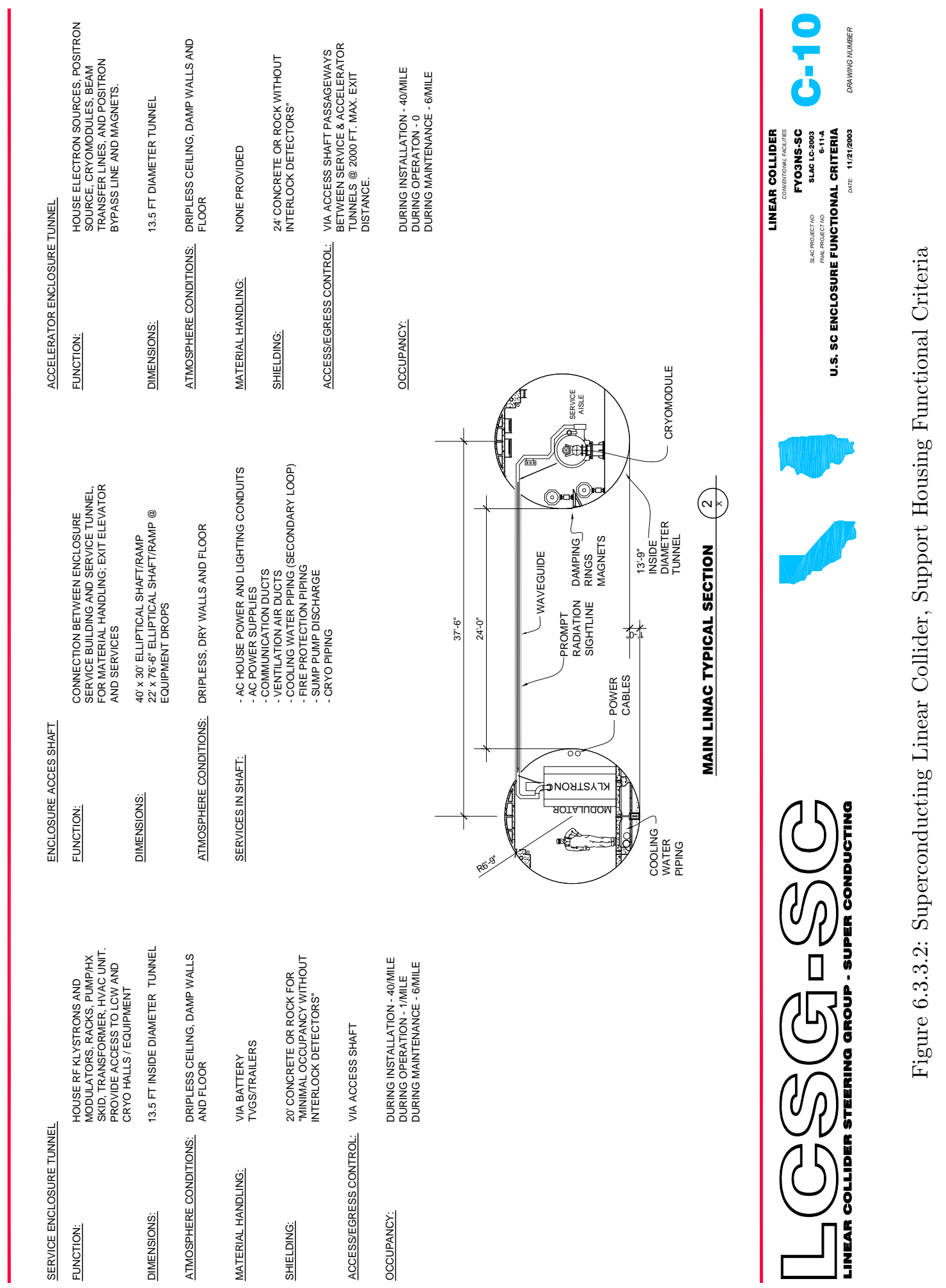



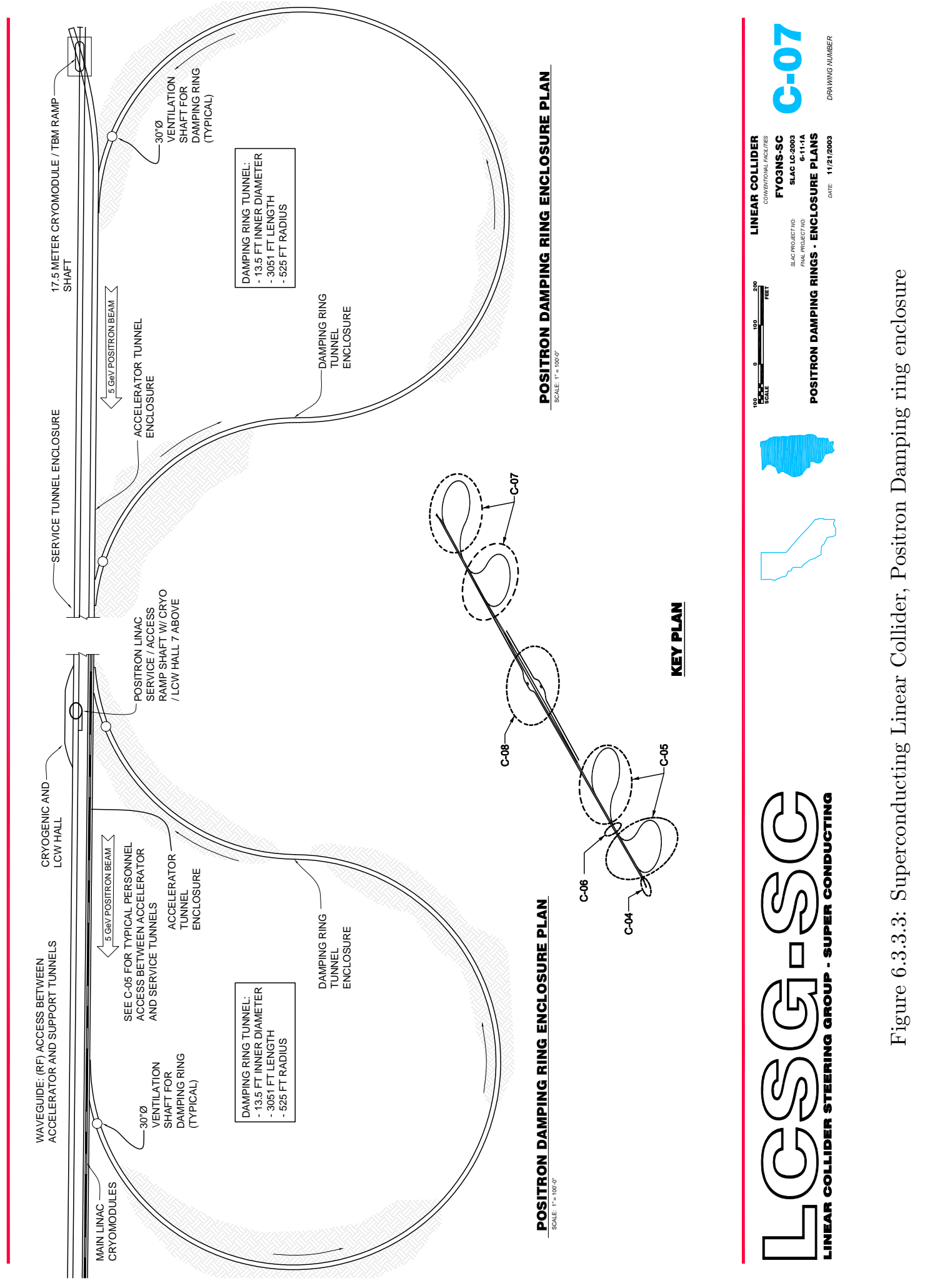


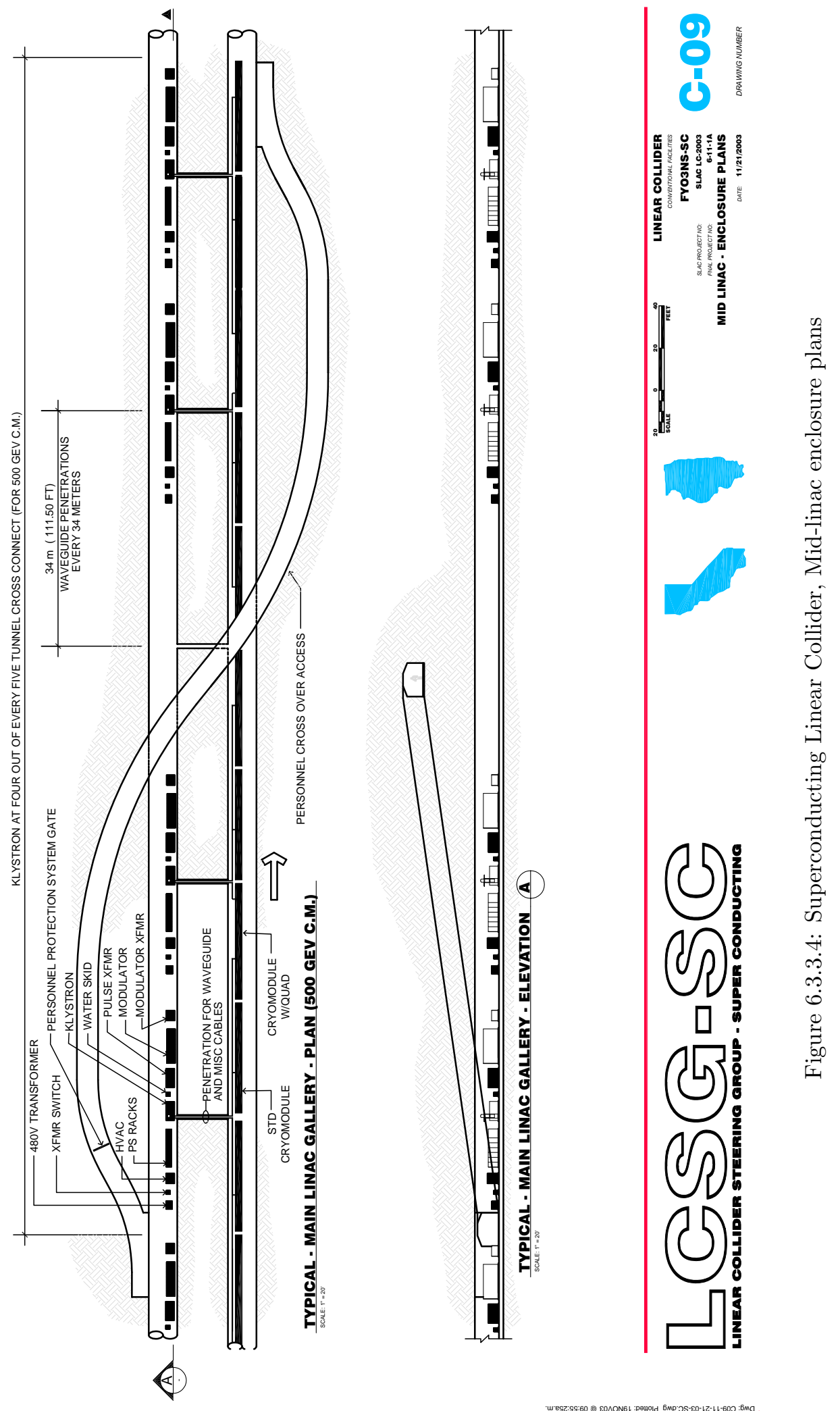



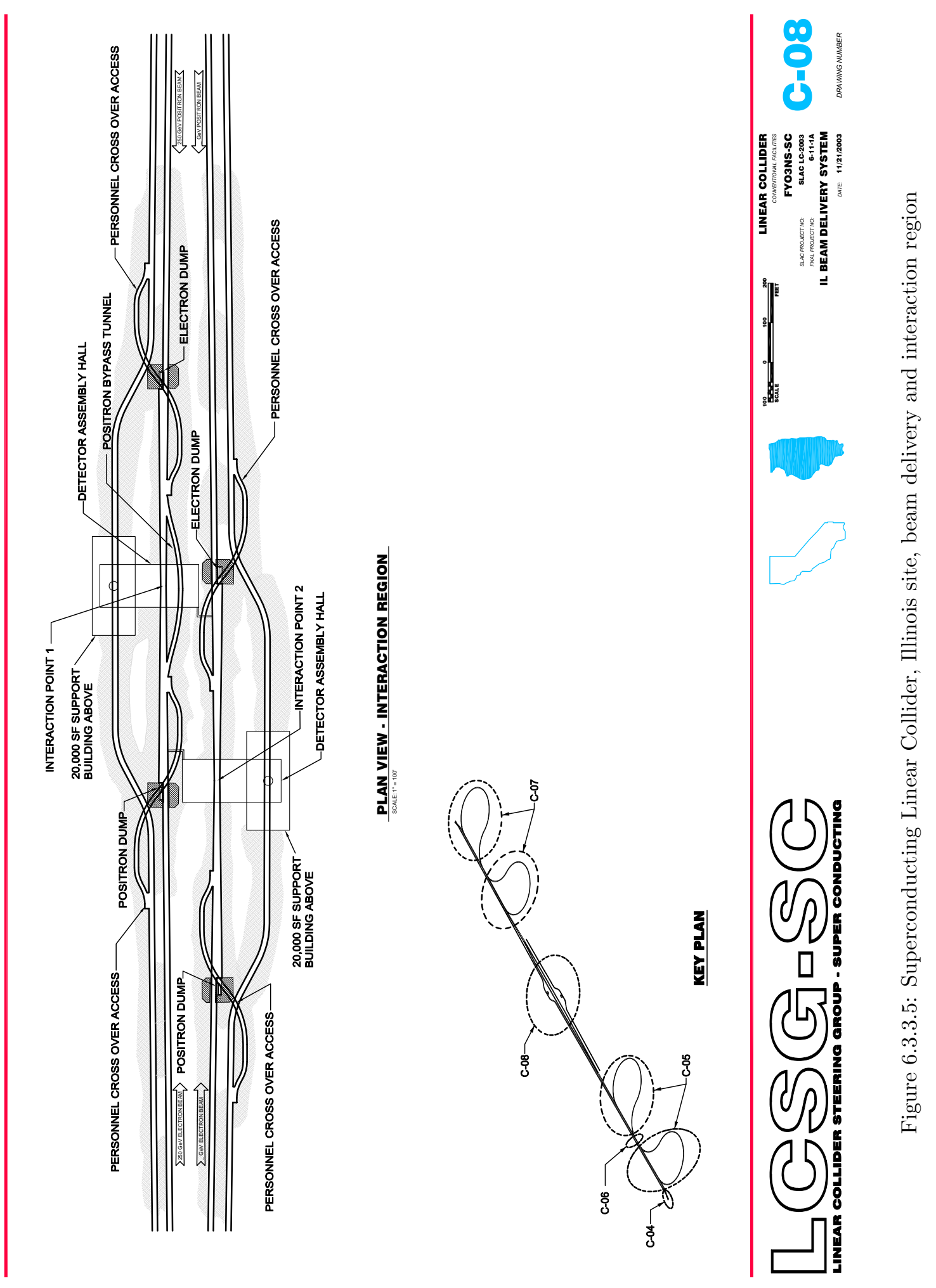

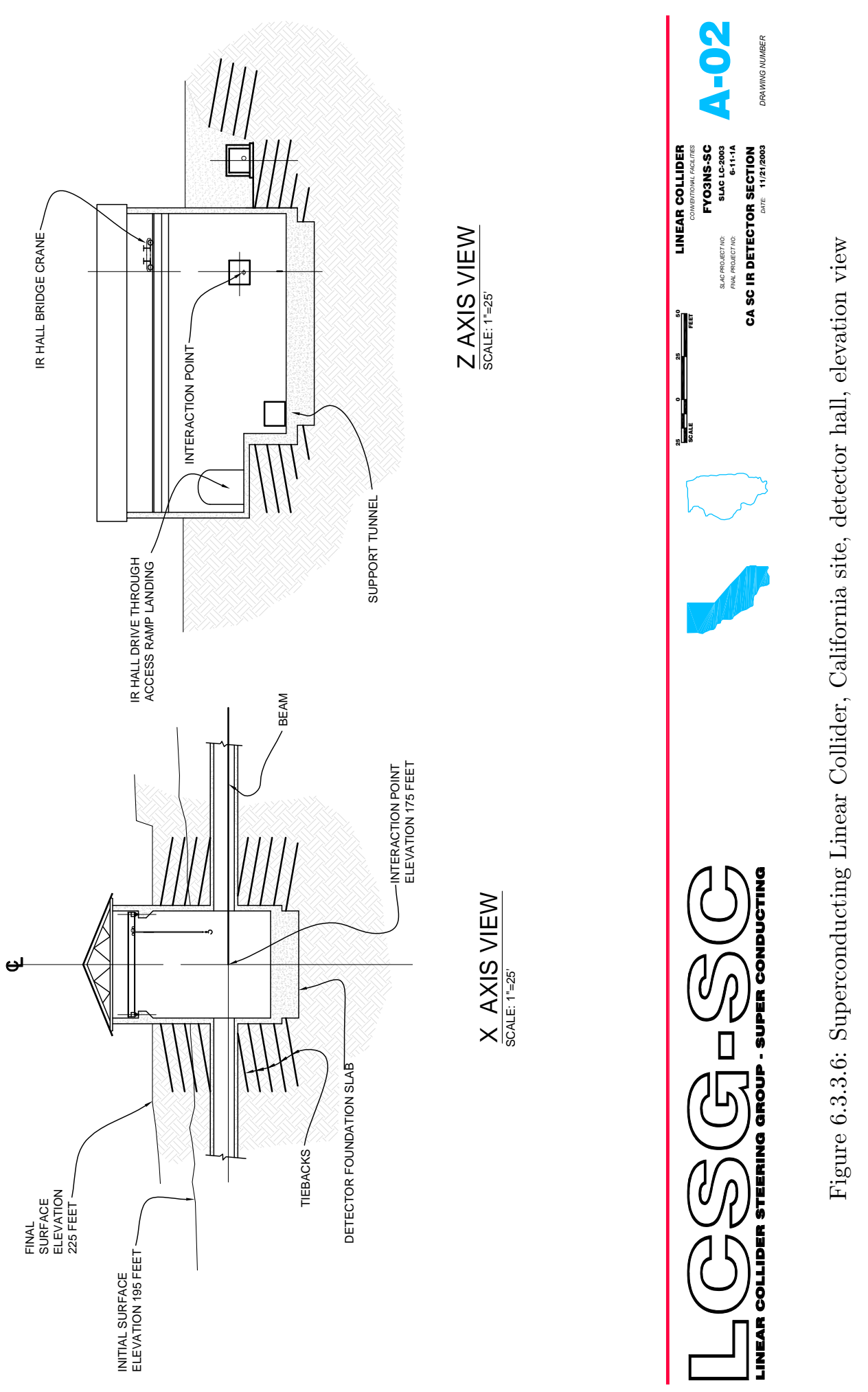


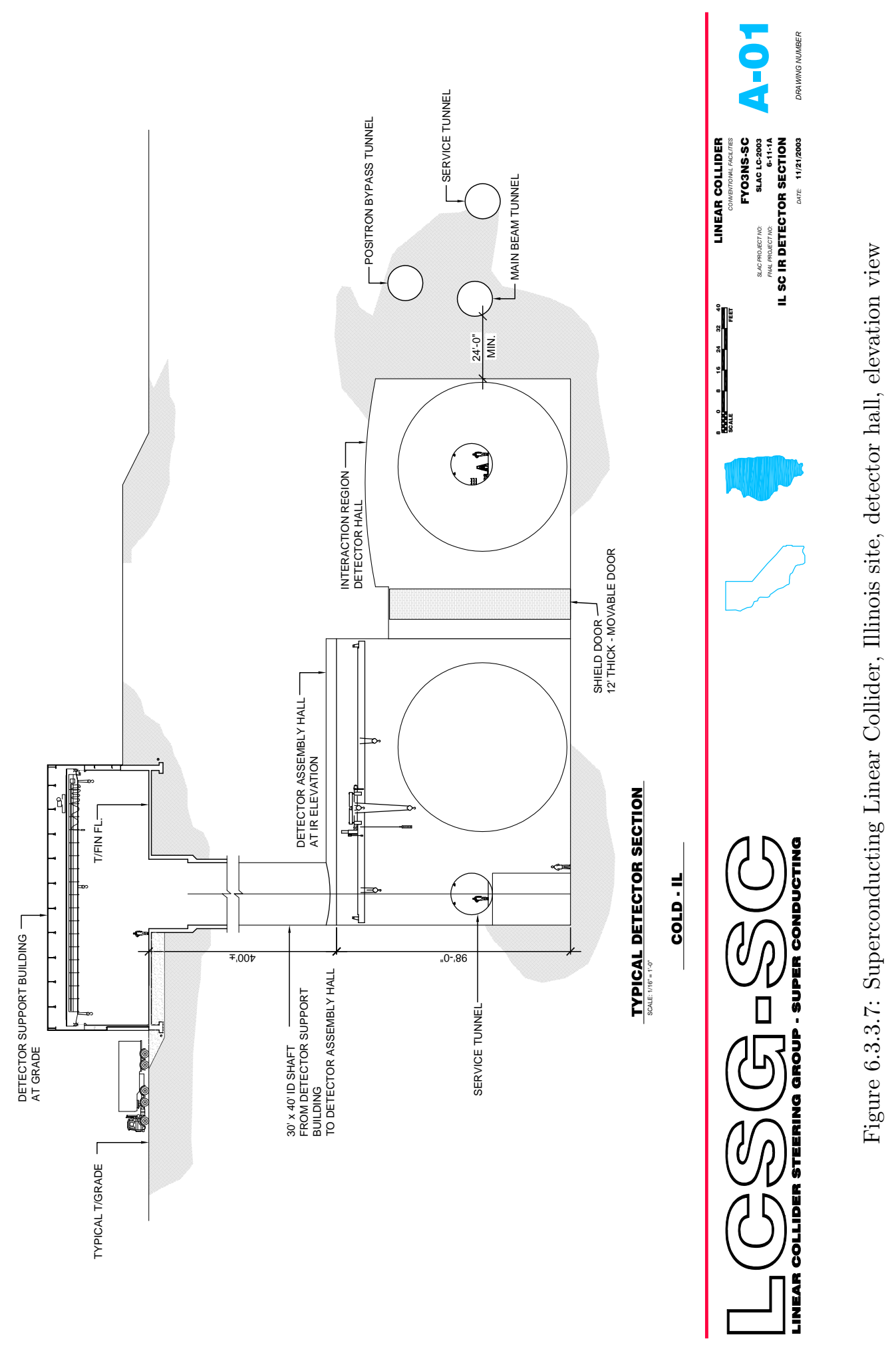




\subsection{Discussion and Conclusions}

The four design solutions for a linear collider described in this chapter (normal conducting and superconducting in both California and Illinois) are the result of several design iterations that have explored a wide range of configurations and construction methods. This previous work has been based on a design solution utilizing two parallel tunnels, one housing the accelerator components, and one housing the related support equipment for the enclosures required to house and operate this machine. The option of a single tunnel solution has not at this time been formally pursued, however a discussion of this option is contained in Section 7.2 of this report. Uniform, competent, dry rock strata has been determined to be the best material for tunnel construction. All of the representative locations investigated have met necessary stability requirements with respect to ground motion for a linear collider and are suitable for tunnel construction regardless of the linear collider technology selected.

Several aspects of the design solutions presented are very similar in nature, but there are some differences. General site services and utility support are similar for either technology. Electrical power requirements are driven by the technology used, but in both cases, a substantial power source is needed. Heat rejection and cooling are also technology driven. The normal conducting machine requires the entire heat rejection load to be accommodated by conventional mechanical means such as cooling towers and/or ponds configured to optimize local climate and weather conditions. In the case of the superconducting machine, a portion of that heat rejection load is removed by way of the cryogenic cooling system. While this will reduce the overall size of the conventional mechanical systems required for heat rejection, that fact is offset by the need for a complete cryogenic cooling system with cooling towers contained elsewhere in the project. The lengths of the tunnel enclosures for the superconducting machine are necessarily longer than for those of the normal conducting machine due to difference between the accelerating gradients for the normal conducting and superconducting structures. The superconducting and the normal conducting machines require comparable access ramps and/or shafts. Damping ring configurations are different for the two machines, but neither configuration poses a substantial impact on the overall conventional construction effort. The electron and positron source configuration, beam delivery enclosures and interaction regions are the same regardless of the technology selected. The main campus configuration and required building square footage is the same, by definition, for either technology and although the superconducting machine requires fewer grade level service buildings, they are larger in area to accommodate the cryogenic equipment.

The work that has been completed to date on the conventional construction aspects of this project points toward two fundamental conclusions. First, although the scope of this project is large, the construction process itself is fairly straightforward. Shaft and tunnel construction is a well documented and understood process. Successful tunneling projects have been completed in all parts of the world. While the process of tunneling requires a certain appreciation for contingency, this contingency is almost always based on the potential for unexpected ground or rock conditions, and not uncertainties in the tunneling process itself. From the standpoint of feasibility, the facilities required for either of these technologies can be successfully constructed with currently employed and understood construction methods.

A second conclusion can also be drawn. Although there are distinctions in the requirements for specific aspects of the project that are salient with respect to the normal conducting and superconducting technologies, none of these distinctions are sufficiently compelling to suggest that one technology is more appropriate than the other with respect to the conventional construction process. Both technologies use similar construction techniques and similar tunnel and shaft configurations. Support buildings are very similar in nature. Utility needs, from the standpoint of conventional construction, are very similar, with the only qualification being that the efficiency of heat rejection through mechanical cooling will be necessarily dependent on local climate and weather conditions. The conventional construction should be considered a neutral component to the eventual technology decision process. 


\section{Chapter 7}

\section{Design Configuration Variants}

\subsection{Introduction}

The chapter describes the design variants. The design variants which have been considered are:

- Section 7.2: A single main linac tunnel architecture for the cold option.

- Section 7.3: The use of $35 \mathrm{MV} / \mathrm{m}$ as the initial design gradient (i.e., at $500 \mathrm{GeV}$ c.m.) for the cold option.

- Section 7.4: The use of a DLDS pulse compression system for the warm option

- Section 7.5: The use of superstructures for the cold option.

- Section 7.6: The use of a conventional positron source for both options.

For each design variant, this document specifies the differences from the reference design. This description was used by the cost and schedule, siting, and availability design task forces to evaluate differences in these respective areas from the reference design. These differences are also described here.

\subsection{Cold Option: Single tunnel architecture for the main linac}

This section describes the use of a single main linac tunnel architecture for the cold option.

\subsubsection{Introduction}

The single tunnel cold option linear collider is similar to the design described in the TESLA Technical Design Report [TDR]. The klystrons, their transformers, controls and power converters are located in the accelerator housing. The klystron modulators are located in accessible surface buildings along with the cryogen plants.

Single tunnel architecture is a viable variant for the cold option because of the $1 \mathrm{~ms}$ long RF pulse. In the cold option, losses in the long, multi-km cables are low and the klystron transformer primary side voltage is low. The primary justification for this variant is the reduction in civil construction cost. In this section, we describe key design features of a single tunnel cold option design, with particular focus on those aspects that have a significant impact on the cost. 
In an attempt to compare the salient design features of one tunnel and two tunnel superconducting linear collider scenarios, a partial civil conventional design was prepared for the one tunnel solution. This partial one tunnel design solution was then compared to the previously prepared Illinois superconducting linear collider two tunnel design solution to better understand the inherent differences.

This section has subsections with: the design considerations, a description of the cost difference per unit length, an estimate of the total cost difference, and a discussion of some of the technical issues, not fully costed as part of this evaluation.

\subsubsection{Main Linac tunnel layout and discussion of assumptions}

The partial one tunnel design solution relies heavily on the design depicted in the TESLA TDR, including cross section and machine layout. A machine configuration of $500 \mathrm{GeV} \mathrm{CM}$ within the active main linac regions was considered for this exercise. Since the one tunnel and two tunnel solutions have similar lengths, power requirements and heat rejection requirements, we assumed that the substantial difference in cost exists in the construction and outfitting of the main linacs and damping rings, with only small differences in the utility plant costs.

Life safety and egress is one of the most important criteria to develop in early conceptual work for such a machine. A twin parallel tunnel design, with fire stopped interconnecting passageways, is a proven and accepted solution to safe egress for the two tunnel solution. We believe an appropriate conceptual solution to emergency egress of a one tunnel scenario lies in constructing enclosed egress alcoves spaced at approximately every mile. This concept is based on the accepted plan for egress in the Superconducting Super Collider (SSC). The SSC solution also included a vehicle designed to rescue individuals located at the egress alcoves in emergency situations. Since the LC designs do not include such a vehicle, we have included the cost of a 5' diameter rescue shaft to the surface at each egress alcove.

Because the one tunnel solution will need to house more technical components, including klystrons and cabling, we have assumed a 20' excavated diameter (17' finished with local lining). The two tunnel solution is anticipated to require a 16' excavated diameter (13.5' finished with local linings).

We have concluded that construction, configuration and spacing of access shafts would be very similar between one and two tunnel scenarios. Furthermore, ramps utilized for installing the cryomodules would not be significantly different for either one or two tunnel scenarios. Outfitting costs for cryogenics piping, and LCW systems would also be similar.

The primary technical difference of the one and two tunnel design solutions is location of the modulators and length of waveguide. Modulators and klystrons are located in the service tunnel approximately 30' away from the beamline tunnel in the two tunnel scenario. Waveguides are anticipated to travel through small excavated passageways between the two tunnels at each klystron location. The one tunnel solution is anticipated to include modulators located at the surface adjacent to the shaft head house and klystrons located within the beamline tunnel similar to the TESLA design. More than 400 high voltage cables would travel down the access shaft and up to $2.5 \mathrm{~km}$ in each direction along the beamline tunnel to supply DC power to the klystrons in the one tunnel solution.

Figures 7.2.2.1 and 7.2.2.2 show a comparison between one and two tunnel main linac cross-sections. The designs are based on the Illinois site layout (Section 6.3.2). In that design, the main linac tunnel is situated in a thick layer of dolomite about 300 feet below grade. This is much deeper than the tunnel described in the TESLA TDR and the design is changed accordingly. The cross section shown in Figure 7.2.2.1 is aligned with the cold box access shaft (7 in each linac). Because of the depth of the tunnels in the Illinois design, housing level access through a large shaft is required for operation and maintenance of the cold box. The shafts are wholly accessible during operation. The shaft head house is somewhat larger in the one tunnel variant. 
Figure 7.2.2.2 shows a close up cross section of the accelerator housing tunnel in the one and two tunnel design solution. The support tunnel is omitted from the schematic of 2 tunnel layout, shown at right in the figure. The subfloor space is used to house the long cables that bring the high voltage pulse $(1 \mathrm{~ms}, \sim 20 \mathrm{KV})$ from the modulators located in surface buildings to the klystrons in the tunnel. Since it is believed that the design in the TDR is an optimized engineering solution, and that small changes would not have a large impact on the cost, we left the component placement unchanged.

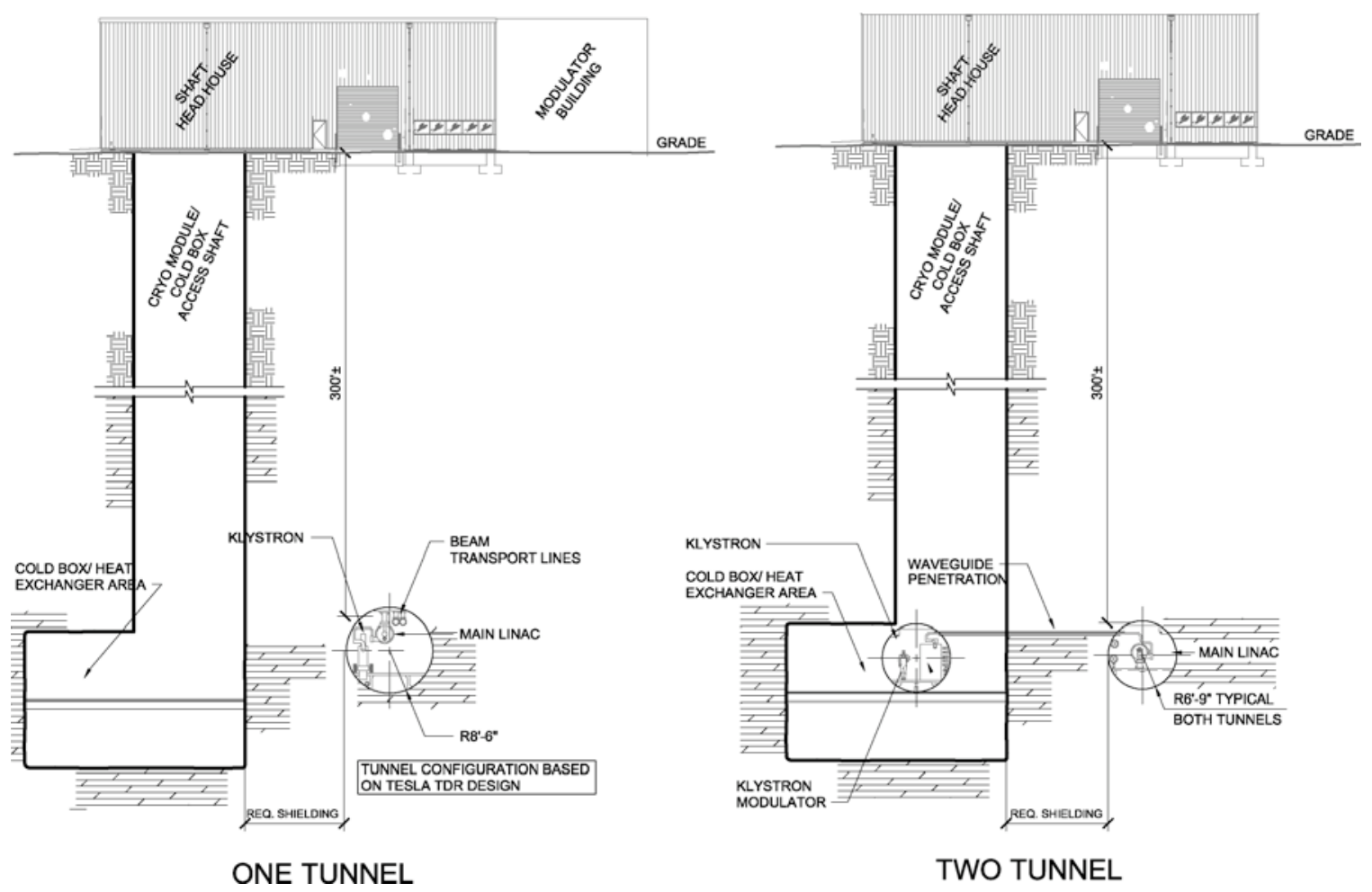

Figure 7.2.2.1: Site cross-section comparison for the one and two tunnel SC LC, Illinois site

\subsubsection{Civil construction cost comparison}

The issues outlined in the discussions of assumptions provide the basis for understanding much of the differences in cost of a one tunnel and two tunnel scenario. The costs discussed in this section are purely construction in nature, and do not include escalation, indirects, EDIA or management reserve. The paragraphs below provide additional discussion of these particular elements of work.

\section{Excavation}

The most obvious difference between the one tunnel and two tunnel solutions is the tunnel excavation. Based on a recent study of tunnel bored excavation costs in Illinois, we anticipate a 20' diameter excavation required for the one tunnel construction, including locally required grouting and linings, would cost $41.8 \%$ of the total one tunnel variant linac tunnel and outfitting cost. Similarly, the smaller 16' diameter excavations for the two tunnel design are anticipated to cost $59.6 \%$ of the respective two tunnel reference machine linac tunnel and outfitting cost. The cost difference due to the excavation of the second tunnel is offset somewhat by the additional cost of high voltage distribution system needed for the one tunnel variant. The cost difference from excavation alone is 1.2 times the final main linac tunnel and outfitting difference. 

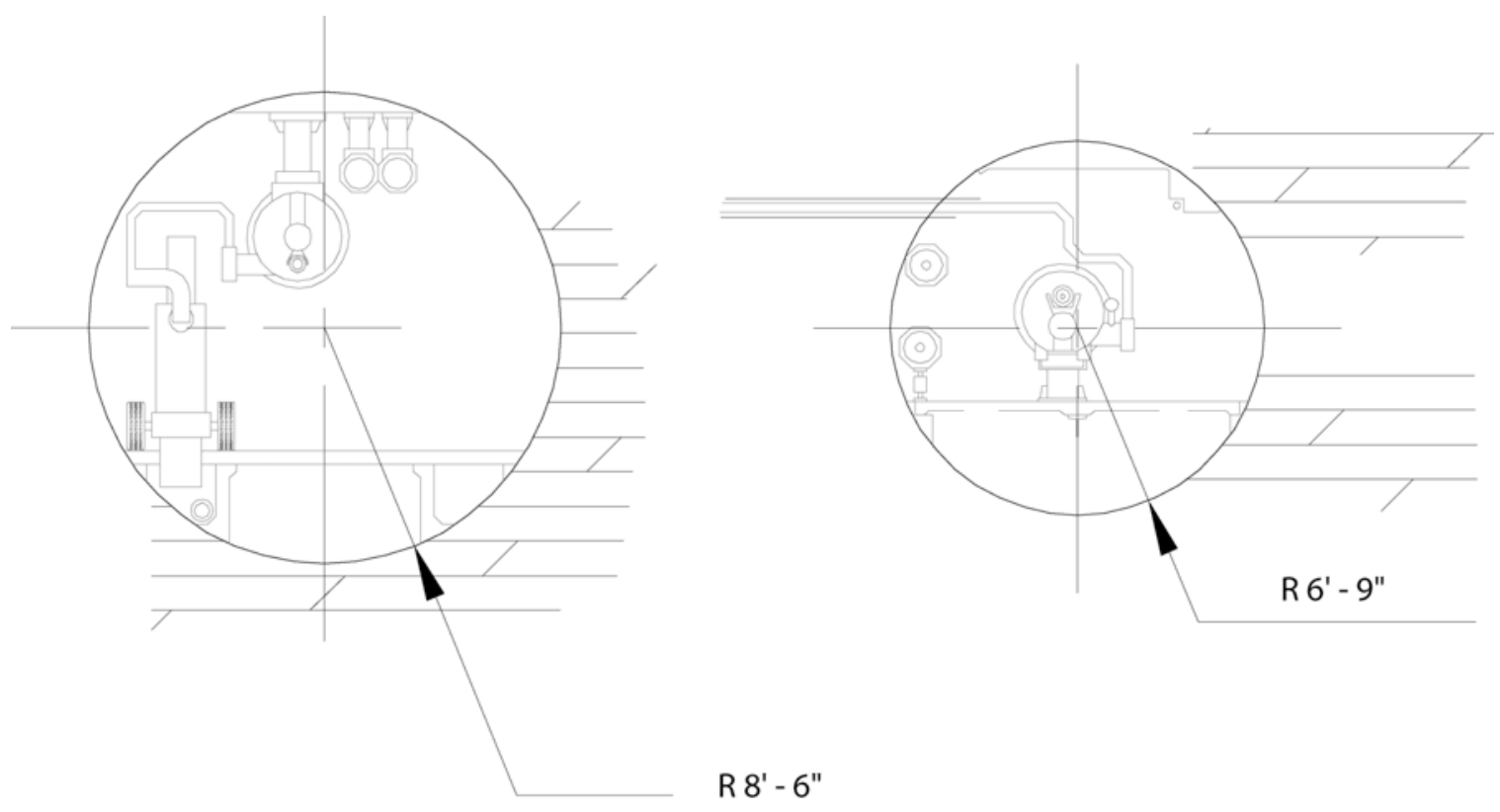

Figure 7.2.2.2: Close up cross section of the one (left) and two tunnel (right) accelerator enclosure tunnel

\section{Drainage and Water Treatment}

Drainage costs involve the anticipated construction of ground water collection and discharge systems including trenches, pipes, and pump stations. Water treatment costs include the treatment necessary to extract pollutants, such as suspended solids and oils from ground water during construction. The recent Neutrinos at Main Injector Project (NuMI) is the basis for our estimates of the water treatment costs. The added water treatment costs for the two tunnel reference machine contribute $2.2 \%$ to the difference.

\section{Concrete Invert Construction}

We utilized the recent study of tunnel bored excavation costs in Illinois to estimate this cost. We anticipate the invert construction of the two tunnel solution would contribute $12.1 \%$ of the difference.

Egress Alcove(One Tunnel)/ Egress Crossover (Two Tunnel) As was discussed above, we anticipate emergency egress and access to be much different between the one and two tunnel solutions. The one tunnel solution would include alcoves with rescue shafts at one mile spacing and the two tunnel concept includes drill and blast cross overs at 200' centers, contributing $3.5 \%$ to the cost difference.

Power Distribution, lighting, and HV Cables (One tunnel) While power distribution for conventional systems such as lighting and receptacles is very similar between the one and two tunnel concepts, there is a significant difference in technical power distribution. The one tunnel solution relies on modulators located at the surface connected to tunnel housed klystrons via high voltage cables. We anticipate more than 800 such cables would be fed down the access shaft and be distributed in either direction into the beam line tunnel. We estimate that four HV pulse cables would be needed for each modulator. Additional cost is needed for a cable support tray system.

On the other hand the two tunnel solution houses klystrons, modulators, and transformers in the support tunnel. High voltage AC feeders would be required within the support tunnel to power the transformers, but their cost does not offset the cost of the one tunnel high voltage cables. Thus, the one tunnel variant costs for this subsystem are much more than those for the reference machine. The high voltage pulse cable feeder costs are $35.9 \%$ of the difference. 
Modulator Surface Building (One tunnel) Additional space at the surface near the access shaft would be required for the one tunnel solution to house the modulators and related equipment. We assumed $6400 \mathrm{ft}^{2}$ of space at each shaft is necessary. This cost distributed along the applicable portion of the one tunnel active main linac contributes $8.7 \%$ to the difference.

Net Cost Variance The one tunnel variant cost, detailed in Table 7.2.3.1, results in a savings of $23 \%$ of the reference design (two-tunnel, Table 7.2.3.2) linac tunnel and outfitting cost.

Table 7.2.3.1: Single tunnel civil construction and utility cost percentage breakdown summary

\begin{tabular}{|l|r|r|}
\hline Tunnel Baseline Cost & $\mathbf{5 6 . 4 \%}$ \\
Excavation, 20 ft dia TBM & 1.5 & \\
Drainage (includes piping, drains and sumps) & 0.5 & \\
Water treatment (during construction) & 9.8 & \\
Concrete Invert (floor) & 2.6 & \\
Egress shafts and small alcove 4 ft diameter every mile & & $\mathbf{4 3 . 7 \%}$ \\
\hline Tunnel outfitting cost & Same & \\
Cryo Piping & 15.5 & \\
LCW piping, pumps, heat exchangers, tertiary and secondary & 21.7 & \\
HV cables with tray or duct & 1.0 & \\
Fire detection & 0.4 & \\
Fire protection (local at alcoves) & 0.6 & \\
Lighting & 2.6 & \\
Modulator building at shaft head house $\left(6400 \mathrm{ft}^{2}\right)$ & & $\mathbf{1 0 0 \%}$ \\
\hline Utility power & & \\
\hline Total comparative cost & & \\
\hline
\end{tabular}

Summary Table 7.2.3.3 summarizes the cost savings related to civil construction for this variant. It includes the difference for the active part of the linac tunnel, outfitted according to the estimates detailed in Tables 7.2.3.1 and 7.2.3.2, and the difference for the drift section of the linac tunnel, assuming that the high voltage cables are not installed in this section, that the surface buildings are not sized for the modulators needed for the upgrade, and that the utility power in the two tunnel design drift section costs $60 \%$ of the power distribution cost for the active part of the linac tunnel. The table includes the cost reduction associated with the removal of the beam delivery/interaction region bypass tunnel bot both interaction regions. Finally, the table also includes the civil construction EDI\&A costs, at the nominal rate of $25.5 \%$. The expected savings is $4.5 \%$ of the total reference machine cost.

\subsubsection{Availability Considerations}

Since the klystrons are located within in the beamline enclosure in the single tunnel variant, rather than in the accessible support tunnel, some machine downtime is incurred in order to service failed klystrons. In order to minimize the downtime, klystron repair entries should be spaced at intervals so that many exchanges are done at once and take a relatively short time.

As described in detail in Chapter 4, the availability of the one tunnel variant of the cold LC was also studied. With the same set of component MTBFs which give the two-tunnel reference design an unavailability of $15 \%$, the one tunnel variant has an unavailability of $25 \%$. To regain the required $15 \%$ unavailability, it was necessary to require improvement factors of 3-80 over nominal for the MTBF's of 26 general categories of components, and the linac energy overhead also had to be increased from $3 \%$ to $8 \%$. A very crude estimate of the required cost increase associated with these component reliability improvements, beyond those required to achieve $15 \%$ unavailability for the two tunnel cold reference design, is roughly $3 \%$ of the total project cost. 
Table 7.2.3.2: Double tunnel civil construction and utility cost percentage breakdown

\begin{tabular}{|l|r|r|}
\hline Accelerator Enclosure & & \\
\hline Tunnel Baseline Cost & 29.8 & $\mathbf{3 6 . 1 \%}$ \\
Excavation, 20 ft dia TBM & 0.6 & \\
Drainage (includes piping, drains and sumps) & 0.5 & \\
Water treatment (during construction) & 5.2 & \\
Concrete Invert (floor) & & $\mathbf{1 . 3 \%}$ \\
\hline Tunnel outfitting cost & Same & \\
Cryo Piping & 0.8 & \\
Fire detection & 0.5 & \\
Lighting & & $\mathbf{3 8 . 9 \%}$ \\
\hline Comparative cost & & \\
\hline \hline Service Tunnel & & $\mathbf{3 8 . 9 \%}$ \\
\hline Tunnel Baseline Cost & 29.8 & \\
Excavation & 0.6 & \\
Drainage & 0.5 & \\
Water treatment & 5.2 & \\
Concrete Invert & 2.8 & \\
Egress Crossover and waveguide penetrations & & $\mathbf{2 3 . 8 \%}$ \\
\hline Tunnel outfitting cost & Same & \\
Cryo Piping & 11.9 & \\
LCW piping, pumps, heat exchangers & 0.8 & \\
Fire detection & 0.6 & \\
Fire protection & 0.5 & \\
Lighting & 9.9 & \\
Power distribution, conventional and technical & & $\mathbf{6 2 . 6 \%}$ \\
\hline Comparative cost & & $\mathbf{1 0 0 \%}$ \\
\hline Total two tunnel comparative cost &
\end{tabular}

Table 7.2.3.3: Percent cost difference due to civil construction - One / Two tunnel Cold IL LC, at $500 \mathrm{GeV}$ c.m.

\begin{tabular}{|c|c|c|}
\hline & Length $[\mathrm{m}]$ & Percent of total cost \\
\hline $\mathrm{e}^{+}$active linac & 12922 & 0.80 \\
$\mathrm{e}^{-}$active linac & 14070 & 0.87 \\
Drift tunnel, both linacs & 15757 & 1.51 \\
BD/IR & 3621 & 0.86 \\
\hline Construction total & & 4.04 \\
EDI\&A & & 1.03 \\
\hline Overall Total & & $\mathbf{5 . 1}$ \\
\hline
\end{tabular}

This estimate of the cost of the required reliability improvements is crude, but it does indicate that they could be substantial, and comparable to the savings associated with the reduced civil costs of the single tunnel.

\subsubsection{Main Linac infrastructure engineering}

The single tunnel variant requires an additional initial engineering investment in order to offset the inaccessibility and more crowded placement of critical accelerator systems. In order to maintain equivalent availability, more design effort must be invested into: 1) redundancy, 2) radiation exposure, 3) engineering 
test and development, 4) commissioning and 5) mitigation of electrical interference.

The cost associated with mitigating radiation exposure effects is more difficult to estimate. Controls equipment has been successfully installed and maintained inside storage ring enclosures, as at HERA, but the amount of shielding required in the higher power linear collider and the likelihood of digital memory corruption caused by radiation events, as seen at PEP-II, is greater. It was not possible, in the time allowed for this study, to evaluate the cost associated with control of radiation exposure to controls electronics, but it is clearly a risk.

In addition to increased component redundancy, we expect to apply more strict engineering practice controls for equipment to be installed inside the single tunnel enclosure. Stricter controls and more thorough development testing is needed because of limited access during beam commissioning and limited access for repair. We anticipate that electrical-magnetic interference will be more difficult to control in the one tunnel variant because of the greater density of high power components. Since we expect to be able to operate the cold machine klystron systems without evacuating personnel from the tunnel (by disconnecting the structure feed waveguides), field testing under near-realistic conditions will be possible.

\subsubsection{Schedule implications}

The implications of the one tunnel variant on the project schedule were studied as part of the work described in Section 5.4. It was found that the increased complexity of installation, caused by the sharing of a single tunnel by the damping ring, main linac accelerator components, and main linac power components, increases the risk of delay in the completion of the construction and commissioning of the collider.

\subsubsection{Conclusions}

A partial civil construction design and cost estimate were produced for the evaluation of the single tunnel variant. The resulting cost savings is $5.1 \%$ of the total project cost, somewhat less than expected, in part because the depth of the tunnel in the Illinois civil construction model requires additional shafts and longer high voltage pulse cables. Additional costs of about 3\% associated with availability (estimated in Chapter 4) reduce the savings substantially. Costs associated with radiation exposure mitigation, additional engineering and commissioning were not estimated.

\subsection{Cold Option: Initial Operation at $35 \mathrm{MV} / \mathrm{m}$}

\subsubsection{Introduction}

This chapter describes the use of $35 \mathrm{MV} / \mathrm{m}$ as the initial design gradient (i.e., at $500 \mathrm{GeV}$ c.m.) for the cold option. In this case, the installed linac is operating at its maximum gradient, so this variant will limit the energy reach of the cold option reference design to $500 \mathrm{GeV}$, unless more of the tunnel is initially filled with accelerator components, at higher cost.

The main linacs accelerate the electron and positron beams from the output of the bunch compressors, at 5 $\mathrm{GeV}$, to the collision energy, $250 \mathrm{GeV}$. The overall design and specification of the main linacs is similar to that presented in the TDR.

The design beam parameters for the main linac are presented in Table 7.3.1.1. Table 7.3.1.2 and Table 7.3.1.3 give overviews of the main linac components. 
Table 7.3.1.1: Design beam parameters for the main linac

\begin{tabular}{lc}
\hline Accelerating gradient $[\mathrm{MV} / \mathrm{m}]$ & 35 \\
Injection energy $[\mathrm{GeV}]$ & 5 \\
Final energy [GeV] & 250 \\
Bunch charge $\left[10^{10}\right]$ & 2 \\
Bunch spacing $[\mathrm{ns}]$ & 337 \\
Bunch length $[\mu \mathrm{m}]$ & 300 \\
Normalized rms emittance at injection $\epsilon_{x}, \epsilon_{y}\left[10^{-6} \mathrm{~m} \cdot \mathrm{rad}\right]$ & $8,0.02$ \\
Normalized rms emittance at IP $\epsilon_{x}, \epsilon_{y}\left[10^{-6} \mathrm{~m} \cdot \mathrm{rad}\right]$ & $10,0.03$ \\
Beam size at injection $\sigma_{x, i}, \sigma_{y, i}[\mu \mathrm{m}]$ & 320,16 \\
Beam size at linac exit $\sigma_{x, f}, \sigma_{y, f}[\mu \mathrm{m}]$ & 60,3 \\
Initial uncorr. energy spread $\sigma_{E, i} / E[\%]$ & 2.5 \\
Off-crest RF phase $\Phi_{r f}(5$ to $25 / 25$ to $250 \mathrm{GeV})\left[^{\circ}\right]$ & $-27 / 5$ \\
Energy spread $\sigma_{E, f} / E$ at linac exit $\left[10^{-4}\right]$ & 7 \\
\hline \hline
\end{tabular}

Table 7.3.1.2: Overview of components in the electron main linac. The overheads which have been assumed are indicated in Table 7.3.7.1.

\begin{tabular}{lc}
\hline Injection energy [GeV] & 5 \\
Undulator energy loss [GeV] & 5.758 \\
Final energy [GeV] & 250.125 \\
Linac length (cryomodules) [m] & 10152.935 \\
Diagnostic insertion @ 5 GeV [m] & 66.8 \\
Diagnostic insertion @ $50 \mathrm{GeV}[\mathrm{m}]$ & 66.8 \\
Undulator insertion @ $147.5 \mathrm{GeV}[\mathrm{m}]$ & 850 \\
Diagnostic insertion @ $147.5 \mathrm{GeV}[\mathrm{m}]$ & 195.68 \\
Diagnostic insertion at BDS entrance [m] & 195.68 \\
Total length, including insertions [km] & 11.528 \\
Active length [km] & 7.598 \\
Real estate gradient [MV/m] & 24.143 \\
Number of cavities per module & 12 \\
Number of modules & 610 \\
Number of cavities & 7320 \\
Module length (with quad) [m] & $15.9(16.8)$ \\
Number of quadrupoles & 253 \\
Number of klystrons & 305 \\
Number of cavities per klystron & 24 \\
\hline \hline
\end{tabular}

\subsubsection{Cryomodules}

In this section, we describe the cryomodules and their arrangement in the main linacs, with an emphasis on the differences from the TDR.

\subsubsection{Superconducting RF Cavities}

The parameters of the 9-cell cavity, operating at $35 \mathrm{MV} / \mathrm{m}$, with a beam current of $9.5 \mathrm{~mA}$, are given in Table 7.3.3.1. The increased gradient, at fixed beam current, results in a larger value for the matched $Q_{e x t}$ than in the TDR. This, in turn, reduces the cavity bandwidth and increases the cavity fill time. The quality 
Table 7.3.1.3: Overview of components in the positron main linac. The overheads which have been assumed are indicated in Table 7.3.7.1.

\begin{tabular}{lc}
\hline Injection energy [GeV] & 5 \\
Final energy [GeV] & 250.125 \\
Linac length (cryomodules) [m] & 9920.84 \\
Diagnostic insertion @ 5 GeV [m] & 66.8 \\
Diagnostic insertion @ $50 \mathrm{GeV}[\mathrm{m}]$ & 66.8 \\
Diagnostic insertion @ $147.5 \mathrm{GeV}[\mathrm{m}]$ & 195.68 \\
Diagnostic insertion at BDS entrance [m] & 195.68 \\
Total length, including insertions [km] & 10.446 \\
Active length [km] & 7.424 \\
Real estate gradient [MV/m] & 24.708 \\
Number of cavities per module & 12 \\
Number of modules & 596 \\
Number of cavities & 7152 \\
Module length (with quad) [m] & $15.9(16.8)$ \\
Number of quadrupoles & 249 \\
Number of klystrons & 298 \\
Number of cavities per klystron & 24 \\
\hline \hline
\end{tabular}

factor is taken to be $5 \times 10^{9}$ at $35 \mathrm{MV} / \mathrm{m}$.

Table 7.3.3.1: Parameters of the 9-cell cavity (note that we adopt here the definition of shunt impedance by the relation $R=V^{2} / P$, where $P$ is the dissipated power and $V$ the peak voltage in the equivalent parallel LCR circuit).

\begin{tabular}{lc}
\hline Type of accelerator structure & standing wave \\
Accelerating mode & $\mathrm{TM}_{010}, \pi$-mode \\
Fundamental frequency & $1300 \mathrm{MHz}$ \\
Nominal gradient $E_{a c c}$ & $35 \mathrm{MV} / \mathrm{m}$ \\
Quality factor $Q_{0}$ & $>5 \times 10^{9}$ \\
Active length $L$ & $1.038 \mathrm{~m}$ \\
Cell-to-cell coupling $k_{c c}$ & $1.87 \%$ \\
Iris diameter & $70 \mathrm{~mm}$ \\
$R / Q$ & $1036 \Omega$ \\
$E_{\text {peak }} / E_{\text {acc }}$ & 2.0 \\
$B_{\text {peak }} / E_{\text {acc }}$ & $4.26 \mathrm{mT} /(\mathrm{MV} / \mathrm{m})$ \\
Tuning range & $\pm 300 \mathrm{kHz}$ \\
$\Delta f / \Delta L$ & $315 \mathrm{kHz} / \mathrm{mm}$ \\
Lorentz force detuning constant $K_{\text {Lor }}$ & $\sim 1 \mathrm{~Hz} /(\mathrm{MV} / \mathrm{m})^{2}$ \\
$Q_{\text {ext }}$ of input coupler & $3.69 \times 10^{6}$ \\
Cavity bandwidth at $Q_{e x t}=3.69 \times 10^{6}$ & $352 \mathrm{~Hz}$ \\
Fill time & $626 \mu \mathrm{s}$ \\
Number of HOM couplers & 2 \\
\hline \hline
\end{tabular}




\subsubsection{Cryostat}

As in the TDR[TDR, Section 3.3.1], twelve 9-cell cavities will be assembled into a cryomodule, which is housed in a cryostat to provide the thermal insulation necessary to allow the cavities to be cooled to $2 \mathrm{~K}$. Within the cryostat is contained the helium distribution system need to cool the cavities.

The details of the mechanical design of the cryostat, and the insulating vacuum, are given in TDR[TDR, Section 3.3.1]. The heat loads for a cryomodule with quadrupole are given in Table 7.3.4.1. This table is based on the TDR[TDR, Table 3.3.1], with the following modification. The dynamic heat loads due to the $\mathrm{RF}$ have been scaled up by the following product: the square of the gradient ratio,

$$
\left(\frac{35}{23.4}\right)^{2}=2.237,
$$

the ratio of the cavity $Q$-values, 2, and the ratio of the RF pulse lengths,

$$
\left(\frac{1.58}{1.37}\right)=1.151,
$$

giving an overall factor of $2.237 \times 2 \times 1.151=5.149$.

Table 7.3.4.1: Calculated heat loads for $17 \mathrm{~m}$ long cryomodule with quadrupole.

\begin{tabular}{|c|c|c|c|c|}
\hline Temperature & Static $[\mathrm{W}]$ & Dynamic(RF) $[\mathrm{W}]$ & Dynamic(HOM) $[\mathrm{W}]$ & Total $[\mathrm{W}]$ \\
\hline $2 \mathrm{~K}$ & 1.74 & 26.21 & 2.21 & 30.16 \\
$5-8 \mathrm{~K}$ & 11.32 & 6.13 & 3.43 & 20.88 \\
$40-80 \mathrm{~K}$ & 90.13 & 305.84 & 28.5 & 424.47 \\
\hline
\end{tabular}

\subsubsection{Cryomodule layout}

As outlined in the TDR[TDR, Section 3.2.1], the main linac FODO lattice will be established using the quadrupoles in the cryomodules. From 5 to about $125 \mathrm{GeV}$, four cryomodules, two standard and two with quadrupoles, form a $66.83 \mathrm{~m}$ long FODO cell. This section of each main linac contains 150 standard cryomodules and 150 cryomodules with quadrupoles. From 125 to $250 \mathrm{GeV}$, six cryomodules, four standard and two with quadrupoles, form a $97.842 \mathrm{~m}$ long FODO cell. This section of the positron (electron) main linac contains 198 (207) standard cryomodules and 99 (104) cryomodules with quadrupoles. The additional cryomodules in the electron main linac provide the energy required to restore that lost in the undulator (5.75 $\mathrm{GeV})$.

The two main linacs will require a total of 1207 cryomodules. Of these, 705 are of the standard variety, and 502 are cryomodules with a quadrupole.

As outlined in TDR[TDR, Section 8.7.0.1], the cryomodules are grouped into cryogenic "strings" of 10 cryomodules each. One cryomodule at the end of each string will be equipped with valves, tubing, flow meters and controllers, and instrumentation. The cryogenic strings will be combined into cryounits. Each cryounit will be supplied through a distribution box with cryogens from a refrigerator located in an aboveground service hall.

The segmentation of the cryogenic strings into cryounits has been chosen to accommodate the need for warm sections in the linac, required for diagnostics and for positron production. Table 7.3.4.1 and Table 7.3.4.1 show the details of the cryogenic segmentation in the main linacs. At the start of the main linac, a warm insertion equal to one cell length $(66.8 \mathrm{~m})$ is provided for the implementation of energy and emittance diagnostics. Another such insertion is provided after cryounit 1, at about $66 \mathrm{GeV}$. After cryounit 2 in the 
electron main linac, at about $147.3 \mathrm{GeV}$, a warm insertion equal to $850 \mathrm{~m}$ is provided to allow the beam to be diverted through an undulator for positron production, and a two-cell diagnostic insertion (195.684 $\mathrm{m})$ is provided for energy and emittance diagnostics. In the positron main linac, a warm insertion equal to two cell lengths, at about $189 \mathrm{GeV}$, is provided for energy and emittance diagnostics. In both main linacs, two cell lengths are provided for final energy and emittance diagnostics, at the end of the main linac tunnel, just before the start of the beam delivery system.

The electron main linac layout is shown in Fig. 7.3.4.1, and the positron main linac layout in Fig. 7.3.4.2.

Table 7.3.4.2: Segmentation of the electron main linac

\begin{tabular}{|c|c|c|c|}
\hline Linac segment & $\begin{array}{c}\text { Number of } \\
\text { cryomodules }\end{array}$ & $\begin{array}{c}\text { Length of } \\
\text { segment }(\mathrm{km})\end{array}$ & $\begin{array}{c}\text { Beam energy at } \\
\text { end of segment }(\mathrm{GeV})\end{array}$ \\
\hline Diagnostic section 1 & Warm & 0.0668 & 5.0 \\
Cryounit 1 & 153 & 2.557 & 67.93 \\
Diagnostic section 2 & Warm & 0.0668 & 67.93 \\
Cryounit 2 & 153 & 2.556 & 130.85 \\
Cryounit 3b & 40 & 0.663 & 147.3 \\
Undulator & Warm & 0.850 & 141.55 \\
Diagnostic section 3 & Warm & 0.1957 & 141.55 \\
Cryounit 3a & 111 & 1.840 & 187.2 \\
Cryounit 4 & 153 & 2.536 & 250.125 \\
Diagnostic section 6 & Warm & 0.1957 & 250.125 \\
\hline
\end{tabular}

Table 7.3.4.3: Segmentation of the positron main linac

\begin{tabular}{|c|c|c|c|}
\hline Linac segment & $\begin{array}{c}\text { Number of } \\
\text { cryomodules }\end{array}$ & $\begin{array}{c}\text { Length of } \\
\text { segment }(\mathrm{km})\end{array}$ & $\begin{array}{c}\text { Beam energy at } \\
\text { end of segment }(\mathrm{GeV})\end{array}$ \\
\hline Diagnostic section 12 & Warm & 0.0668 & 5.0 \\
Cryounit 16 & 149 & 2.49 & 66.28 \\
Diagnostic section 11 & Warm & 0.0668 & 66.28 \\
Cryounit 15 & 149 & 2.49 & 127.56 \\
Cryounit 14 & 149 & 2.49 & 188.84 \\
Diagnostic section 10 & Warm & 0.1957 & 188.84 \\
Cryounit 13 & 149 & 2.49 & 250.125 \\
Diagnostic section 7 & Warm & 0.1957 & 250.125 \\
\hline
\end{tabular}

\subsubsection{Cryogenics}

The cryogenic requirements of the main linacs are presented in Table 7.3.5. These numbers have been computed from the cryomodule heat loads given in Table 7.3.4.1, together with the cryounit module counts given in Table 7.3.4.1 and Table 7.3.4.1. The cryogenics will be supplied by eight refrigerators located in the four above-ground service halls shown in Fig. 7.3.4.1 and Fig. 7.3.4.2.

The AC cryogenic power requirements of the main linacs are given in Table 7.3.5.2. To compute the AC power requirements, the following COP's have been used (from the TDR[TDR, Table 8.7.5]): for $2 \mathrm{~K} 588$ $\mathrm{W} / \mathrm{W}$; for 5-8 K, $168 \mathrm{~W} / \mathrm{W}$; and for 40-80 K, $17 \mathrm{~W} / \mathrm{W}$. Cold compressor losses have also been included. 


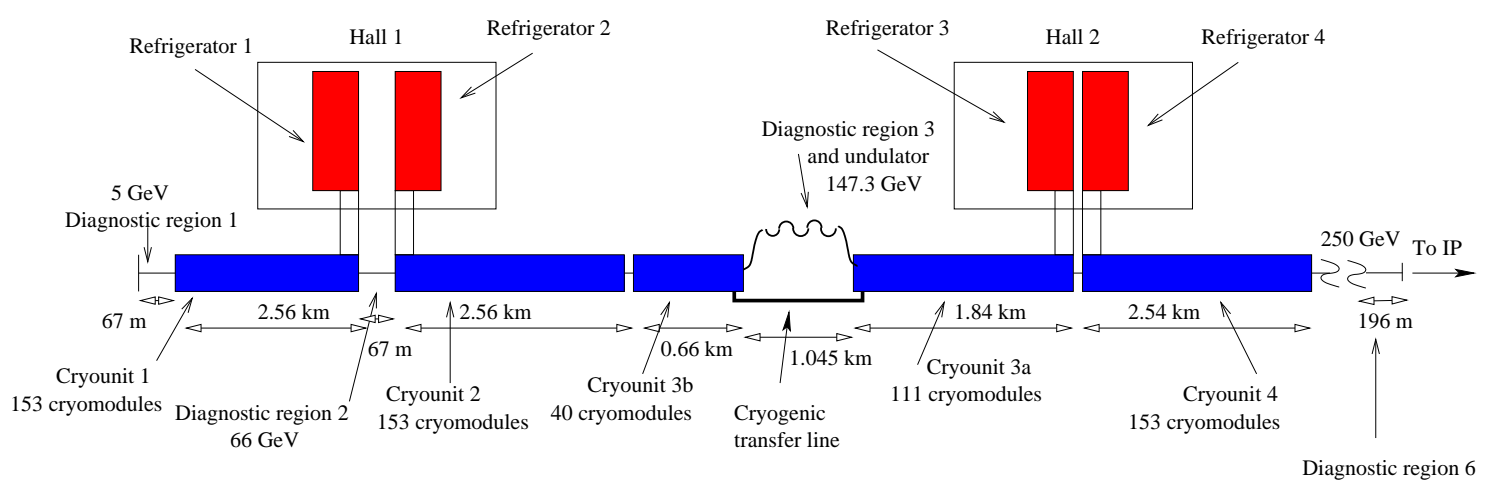

Figure 7.3.4.1: Electron main linac layout

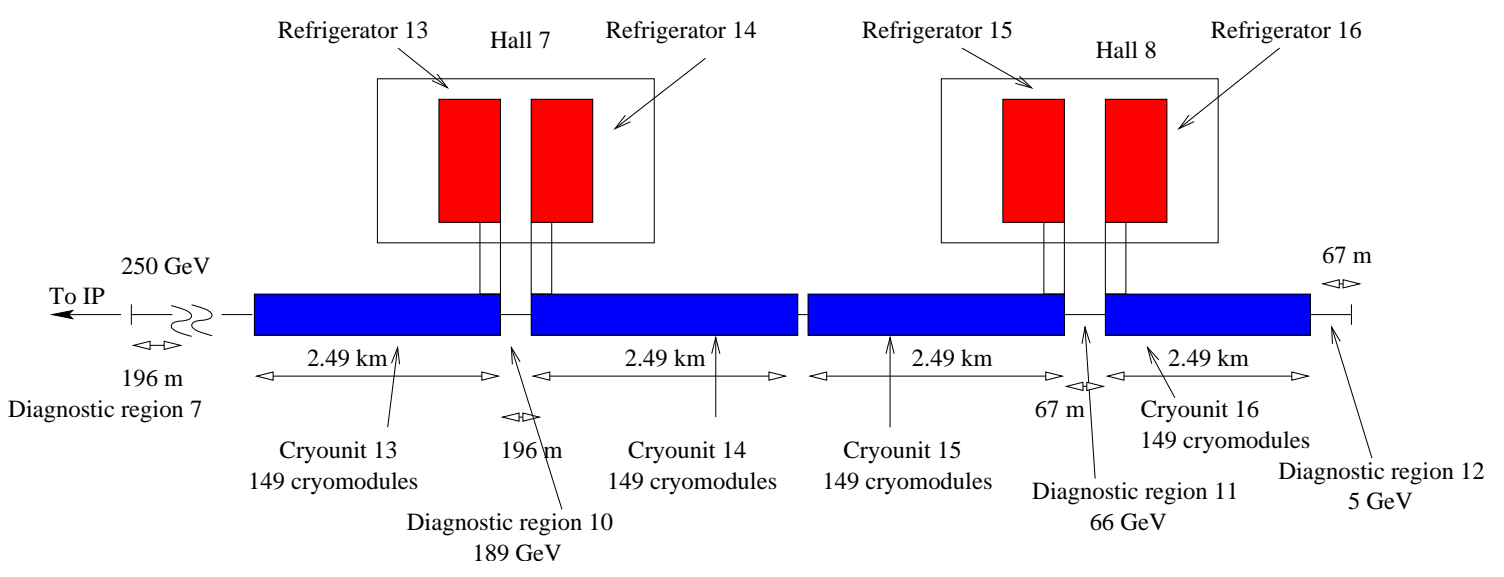

Figure 7.3.4.2: Positron main linac layout 
Table 7.3.5.1: Cryogenic requirements of the main linacs

\begin{tabular}{|c|c|c|c|c|}
\hline Cryounit & Temperature $[\mathrm{K}]$ & Static load $[\mathrm{kW}]$ & Dynamic load $[\mathrm{kW}]$ & Total $[\mathrm{kW}]$ \\
\hline \hline $1,2,4$ & 2 & 0.27 & 4.35 & 4.61 \\
& $5-8$ & 1.73 & 1.46 & 3.19 \\
& $40-80$ & 13.79 & 51.15 & 64.94 \\
\hline 3b & 2 & 0.07 & 1.14 & 1.21 \\
& $5-8$ & 0.45 & 0.38 & 0.84 \\
& $40-80$ & 3.61 & 13.37 & 16.98 \\
\hline 3a & 2 & 0.19 & 3.15 & 3.35 \\
& $5-8$ & 1.26 & 1.06 & 2.32 \\
& $40-80$ & 10.00 & 37.11 & 47.12 \\
\hline $13,14,15,16$ & 2 & 0.26 & 4.23 & 4.49 \\
& $5-8$ & 1.69 & 1.42 & 3.11 \\
& $40-80$ & 13.43 & 49.82 & 63.25 \\
\hline
\end{tabular}

Table 7.3.5.2: Total cryogenic operating power requirements, $250 \mathrm{GeV}$ beam energy

\begin{tabular}{lc}
\hline AC power cryogenics at $2 \mathrm{~K}$, Cryounits $1-4$ and $13-16[\mathrm{MW}]$ & 22.88 \\
AC power cryogenics at $5-8 \mathrm{~K}$, Cryounits $1-4$ and $13-16[\mathrm{MW}]$ & 4.53 \\
AC power cryogenics at $40-80 \mathrm{~K}$, Cryounits $1-4$ and $13-16[\mathrm{MW}]$ & 9.31 \\
Total AC power for cryogenics [MW] & 36.72 \\
\hline \hline
\end{tabular}

\subsubsection{RF system}

In a superconducting linac, during the beam pulse essentially all of the power delivered by the RF system goes into the beam. Since the beam power in this design is the same as in the TDR, the RF system is essentially the same. There is a small increase in the total required AC power for RF, because the RF pulse length is about $15 \%$ longer than in the TDR (due to the increased external $Q$ ).

The RF system consists of 298 (305) stations per positron (electron) main linac, each of which provides power at $1.3 \mathrm{GHz}$ to a total of 24 accelerating cavities (two cryomodules). The number of RF stations per linac includes an $6 \%$ overhead, as indicated in Table 7.3.7.1. Each RF station consists of a klystron and a modulator. The number of cavities per station has been reduced relative to the TDR (in which it is 36) in order to allow the use of the same $10 \mathrm{MW}$ klystrons as specified in the TDR, at the increased gradient. The peak RF power needed for one superconducting cavity at full gradient and maximum beam current $(35 \mathrm{MV} / \mathrm{m}$ and $9.5 \mathrm{~mA})$ is $345 \mathrm{~kW}$; the nominal peak power needed for 24 cavities is $8.28 \mathrm{~kW}$. Taking into account a regulation reserve of $10 \%$ for phase and amplitude control, and another $7.4 \%$ for circulator and waveguide losses, a total of $9.7 \mathrm{MW}$ is required. The RF pulse length is $1.58 \mathrm{~ms}$, which includes the beam pulse length of $0.95 \mathrm{~ms}$, and the cavity fill time of $0.63 \mathrm{~ms}$. The repetition rate is $5 \mathrm{~Hz}$.

The overall efficiency and power requirements of the RF systems are given in Table 7.3.6.1.

\subsubsection{Waveguide distribution system}

For operation at $35 \mathrm{MV} / \mathrm{m}$, klystrons will be installed at every tunnel cross-connect. These klystrons will feed 24 cavities, using the arrangement shown in Fig. 7.3.6.1. With this arrangement, the average distance from klystron to RF cavity will be about $8.5 \mathrm{~m}$, plus the length of the cross tunnel. The cross tunnel length is $11.4 \mathrm{~m}$, so the total average distance from klystron to RF cavity is then $11.4 \mathrm{~m}+8.5 \mathrm{~m}=19.9 \mathrm{~m}$. Using WR770 waveguide, with an attenuation factor of $-0.011 \mathrm{~dB} / \mathrm{m}$, the power loss in the waveguide is $4.9 \%$. 
Table 7.3.6.1: Efficiencies and power requirements for the RF system

\begin{tabular}{lc}
\hline RF station peak power [MW] & 9.68 \\
Duty cycle [\%] & 0.79 \\
RF station average power [kW] & 76.3 \\
Klystron efficiency [\%] & 65 \\
Modulator efficiency [\%] & 85 \\
AC power per RF station [kW] & 138.1 \\
AC power for auxiliaries per RF station [kW] & 14 \\
Number of stations in electron linac & 305 \\
Electron linac AC wall plug power for RF [MW] & 46.41 \\
Number of stations in positron linac & 298 \\
Positron linac AC wall plug power for RF [MW] & 45.34 \\
Total AC wall plug power for RF [MW] & 91.74 \\
Beam power [MW] & 22.38 \\
AC to beam efficiency for RF [\%] & 24.4 \\
\hline \hline
\end{tabular}

According to the TDR[TDR, Section 3.4.6], there is an additional $2 \%$ loss in the circulator, giving a total loss of $6.9 \%$.

\subsubsection{Low level RF}

The low level RF system will be similar to that described in the TDR[TDR, Section 3.4.7]. A significant difference is that only 24 , rather than 36 , cavities are driven by a single klystron.

\subsubsection{Overheads}

The assumed overheads are listed in Table 7.3.7.1. The overhead for BNS damping and longitudinal wakefield compensation, for operation at $250 \mathrm{GeV}$ beam energy and a gradient of $35 \mathrm{MV} / \mathrm{m}$, is $2.44 \mathrm{GeV}$ (about $1 \%$ ). A $2 \%$ allocation of spares for klystron failures has been made. The cavities associated with these spares are assumed to be mechanically detuned. In addition, the design includes a $2 \%$ allocation of spares for cavity faults. If the cavity fault rate is 1 trip every 30 hours, and the trip recovery time is $4 \mathrm{sec}$, then on average the $2 \%$ cavity fault spares will be depleted only once per year. A tuned, unpowered spare cavity will decelerate the beam by roughly $35 \mathrm{MV} / \mathrm{m}$, so additional powered cavities must be provided as compensation. Assuming that half of the spares will be tuned at any given time, an additional $1 \%$ for the compensating cavities has been allocated, bringing the total spares needed for cavity fault control to $3 \%$.

Table 7.3.7.1: Overheads assumed, in \%

\begin{tabular}{c|c}
\hline BNS overhead & 1 \\
Klystron failure overhead $^{a}$ & 2 \\
Cavity faults overhead $^{b}$ & 3 \\
\hline
\end{tabular}

\footnotetext{
${ }^{a}$ Assumes spare cavities are detuned

${ }^{b}$ including loading
} 


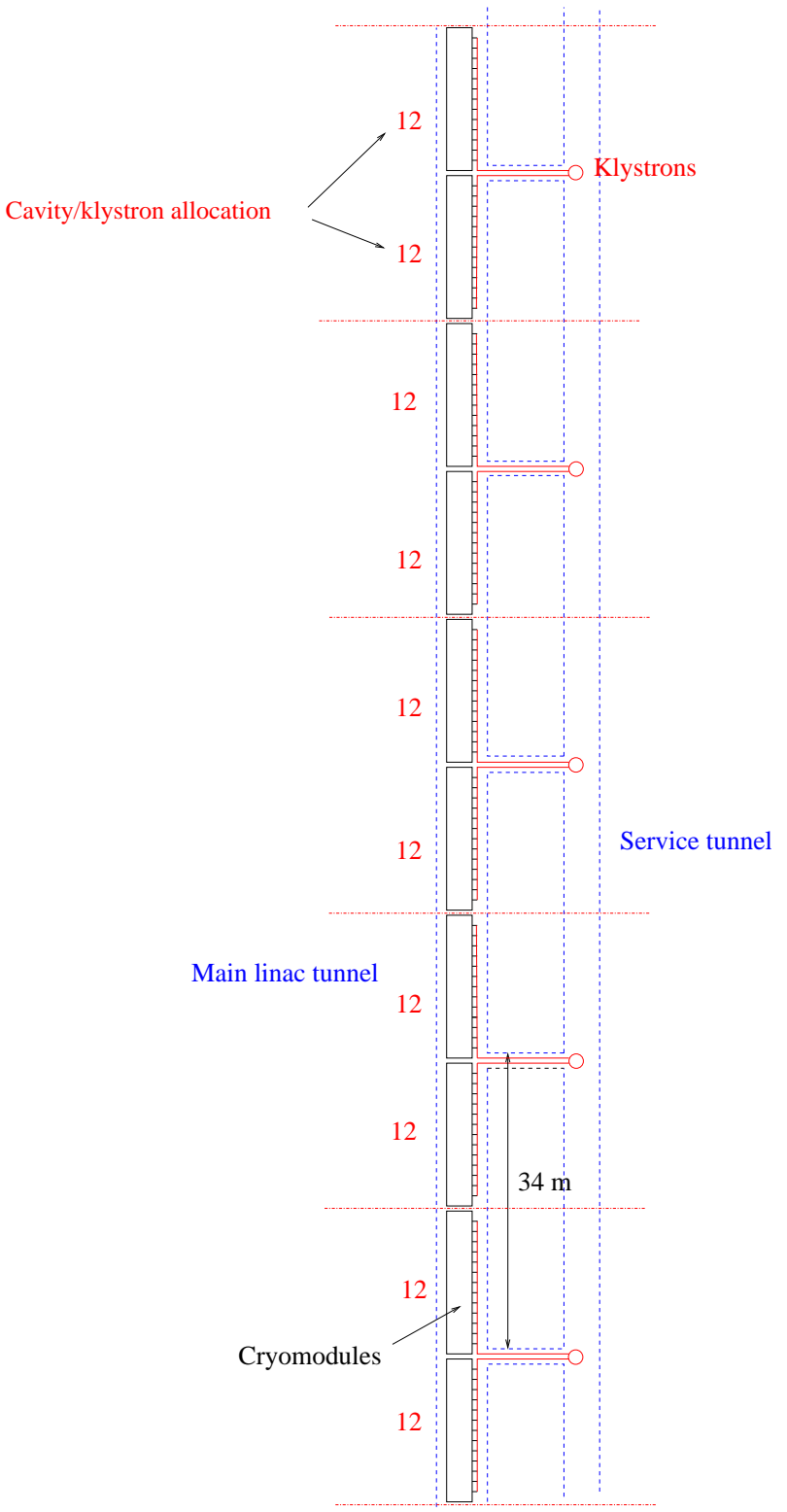

Figure 7.3.6.1: Waveguide distribution, two tunnels 


\subsubsection{Overall layout}

A tabulation of the overall length of the technical components is presented in Table 7.3.8.1, for a cavity gradient of $35 \mathrm{MV} / \mathrm{m}$. The overall estimate is $47.3 \mathrm{~km}$.

Table 7.3.8.1: Tabulation of technical component lengths. All lengths are in km.

\begin{tabular}{|c|c|c|}
\hline Length & $250 \mathrm{GeV}$ beam energy & $500 \mathrm{GeV}$ beam energy \\
\hline Electron Linac cryomodules & 10.152 & 20.166 \\
Electron Linac insertions & 1.375 & 1.571 \\
Positron Linac cryomodules & 9.921 & 19.934 \\
Positron Linac insertions & 0.525 & 0.721 \\
\hline Overall two-linacs with insertions & 21.974 & 42.392 \\
BDS length (total) & 3.6 & 3.6 \\
Spin rotator/bunch compressor length (total) & 0.80 & 0.80 \\
DR ends length (total) & 0.5 & 0.5 \\
Total technical component length & 26.874 & 47.292 \\
\hline
\end{tabular}

\subsubsection{Cryogenics}

\subsubsection{Cryogenic heat loads and cryogenic plant requirements}

In Table 7.3.9.1, static and dynamic (RF on) cryogenic heat loads for a $500 \mathrm{GeV}$ cm collider are presented for each of the temperature levels. These heat loads have been taken from the requirements given in Section 7.3.5. In addition, heat loads required for the electron and positron injectors as well as the electron and positron damping ring RF systems are also given. These have been taken from the cold option reference design.

For a $500 \mathrm{GeV}$ cm collider, four cryogenic plants are required for each linac. Each plant provides refrigeration to one cryounits of $\sim 2.6 \mathrm{~km}$ long. The requirements on the plants are shown in Table 7.3.9.1. Cryogenic plant 1 provides refrigeration for the electron injector linac, as well as linac cryounits 1 . Plant 2 supports linac cryounit 2. Plant 3 supports the cryogenic needs of the positron injector linac and the electron damping ring $\mathrm{RF}$, as well as linac cryounit 3 . In addition, linac cryounit 3 of cryogenic plant 3 contains a 1,045 m long cryogenic bypass to make room for diagnostic region 3 and the undulator. This bypass will need to have a large size low pressure return line in order to not significantly contribute to the overall cryounit GRP pressure drop. This is necessary in order to maintain the similar GRP pressure drop across the 3.4 $\mathrm{km}$ cryounit 3. Plants 13, 15 and 16 supply linac cryounits 13, 15 and 16, respectively. Plant 14 feeds the positron damping ring RF, as well as linac cryounit 14 .

\subsubsection{Cryogenic plant design specifications}

The cryogenic plant requirements and design specifications for the $500 \mathrm{GeV}$ collider are given in Table 7.3.9.2. The requirements are taken from Table 7.3.9.1. The cryogenic loads at the three different temperatures have been converted into a net equivalent load at $4.5 \mathrm{~K}$ using the relation

$$
P_{4.5}=f_{T} P_{T}
$$

in which the conversion factors are $f_{2}=3.3, f_{5-8}=0.683$, and $f_{40-80}=0.07$. The design specification of 30.3 $\mathrm{kW} @ 4.5 \mathrm{~K}$ was chosen by requiring a margin of about $20 \%$ for the largest plant (plant 3 ). 
Table 7.3.9.1: Cryounit Heat Loads for a $500 \mathrm{GeV}$ Collider

\begin{tabular}{|c|c|c|c|c|}
\hline Linac Segment & $\begin{array}{c}\text { Temperature } \\
{[\mathrm{K}]}\end{array}$ & $\begin{array}{c}\text { Static Load } \\
\text { kW }\end{array}$ & $\begin{array}{c}\text { Dynamic Load } \\
\mathrm{kW}\end{array}$ & $\begin{array}{l}\text { Total Load } \\
\text { kW }\end{array}$ \\
\hline " Cryounit 1 & $\overline{\overline{2}}$ & "0.27 & (4.35 & (4.61 \\
\hline \multirow{2}{*}{153 Cryomodules } & $5-8$ & 1.73 & 1.46 & 3.19 \\
\hline & $40-80$ & 13.79 & 51.15 & 64.94 \\
\hline \multirow{3}{*}{$\begin{array}{l}\text { Electron Injector Linac } \\
23 \text { Cryomodules }\end{array}$} & 2 & 0.04 & 0.14 & 0.18 \\
\hline & $5-8$ & 0.26 & 0.1 & 0.36 \\
\hline & $40-80$ & 2.07 & 1.66 & 3.73 \\
\hline \multirow{3}{*}{$\begin{array}{c}\text { Cryounit } 2 \\
153 \text { Cryomodules }\end{array}$} & 2 & 0.27 & 4.35 & 4.61 \\
\hline & $5-8$ & 1.73 & 1.46 & 3.19 \\
\hline & $40-80$ & 13.79 & 51.15 & 64.94 \\
\hline \multirow{3}{*}{$\begin{array}{c}\text { Cryounit 3b } \\
40 \text { Cryomodules }\end{array}$} & 2 & 0.07 & 1.14 & 1.21 \\
\hline & $5-8$ & 0.45 & 0.38 & 0.84 \\
\hline & $40-80$ & 3.61 & 13.37 & 16.98 \\
\hline \multirow{3}{*}{$\begin{array}{l}\text { Electron Damping Ring } \\
12 \text { Cryomodules }\end{array}$} & 2 & 0.36 & 0.36 & 0.72 \\
\hline & $5-8$ & 0.13 & 0.13 & 0.25 \\
\hline & $40-80$ & 2.55 & 2.55 & 5.10 \\
\hline \multirow{3}{*}{$\begin{array}{c}\text { Cryogenic Bypass } \\
1045 \mathrm{~m}\end{array}$} & 2 & 0.21 & 0 & 0.209 \\
\hline & $5-8$ & 0.63 & 0 & 0.627 \\
\hline & $40-80$ & 4.18 & 0 & 4.18 \\
\hline \multirow{3}{*}{$\begin{array}{c}\text { Cryounit 3a } \\
111 \text { Cryomodules }\end{array}$} & 2 & 0.19 & 3.15 & 3.35 \\
\hline & $5-8$ & 1.26 & 1.06 & 2.32 \\
\hline & $40-80$ & 10.00 & 37.11 & 47.12 \\
\hline \multirow{3}{*}{$\begin{array}{c}\text { Cryounit } 4 \\
153 \text { Cryomodules }\end{array}$} & 2 & 0.27 & 4.35 & 4.61 \\
\hline & $5-8$ & 1.73 & 1.46 & 3.19 \\
\hline & $40-80$ & 13.79 & 51.15 & 64.94 \\
\hline \multirow{3}{*}{$\begin{array}{l}\text { Positron Injector Linac } \\
20 \text { Cryomodules }\end{array}$} & 2 & 0.03 & 0.09 & 0.12 \\
\hline & $5-8$ & 0.23 & 0.07 & 0.30 \\
\hline & $40-80$ & 1.80 & 1.06 & 2.86 \\
\hline \multirow{3}{*}{$\begin{array}{c}\text { Cryounit } 13 \\
149 \text { Cryomodules }\end{array}$} & 2 & 0.26 & 4.23 & 4.49 \\
\hline & $5-8$ & 1.69 & 1.42 & 3.11 \\
\hline & $40-80$ & 13.43 & 49.82 & 63.25 \\
\hline \multirow{3}{*}{$\begin{array}{c}\text { Cryounit } 14 \\
149 \text { Cryomodules }\end{array}$} & 2 & 0.26 & 4.23 & 4.49 \\
\hline & $5-8$ & 1.69 & 1.42 & 3.11 \\
\hline & $40-80$ & 13.43 & 49.82 & 63.25 \\
\hline \multirow{3}{*}{$\begin{array}{c}\text { Cryounit } 15 \\
149 \text { Cryomodules }\end{array}$} & 2 & 0.26 & 4.23 & 4.49 \\
\hline & $5-8$ & 1.69 & 1.42 & 3.11 \\
\hline & $40-80$ & 13.43 & 49.82 & 63.25 \\
\hline \multirow{3}{*}{$\begin{array}{c}\text { Cryounit } 16 \\
149 \text { Cryomodules }\end{array}$} & 2 & 0.26 & 4.23 & 4.49 \\
\hline & $5-8$ & 1.69 & 1.42 & 3.11 \\
\hline & $40-80$ & 13.43 & 49.82 & 63.25 \\
\hline \multirow{3}{*}{$\begin{array}{l}\text { Positron Damping Ring } \\
12 \text { Cryomodules }\end{array}$} & 2 & 0.36 & 0.36 & 0.72 \\
\hline & $5-8$ & 0.13 & 0.13 & 0.25 \\
\hline & $40-80$ & 2.55 & 2.55 & 5.10 \\
\hline
\end{tabular}

The AC power requirements for the plants, when operating at nominal load, as well as the installed power, are also given in Table 7.3.9.2. The power requirements presented represent the purchased power, including motor efficiency and power factor, for the motors associated with the compressors, pumps and fans required 
Table 7.3.9.2: $500 \mathrm{GeV}$ operation cryogenic plant requirements

\begin{tabular}{|c|c|c|c|c|}
\hline Plant: & $\begin{array}{c}\text { Temperature } \\
{[\mathrm{K}]}\end{array}$ & $\begin{array}{c}\text { Static Load } \\
\mathrm{kW}\end{array}$ & $\begin{array}{c}\text { Dynamic Load } \\
\mathrm{kW}\end{array}$ & $\begin{array}{c}\text { Total Load } \\
\mathrm{kW}\end{array}$ \\
\hline \hline $1:$ & 2 & 0.31 & 4.48 & 4.79 \\
Linac Cryounit 1 & $5-8$ & 1.99 & 1.56 & 3.55 \\
+Elec. Inj. Linac & $40-80$ & 15.86 & 52.81 & 68.67 \\
\hline $2:$ & 2 & 0.27 & 4.35 & 4.61 \\
Linac Cryounit 2 & $5-8$ & 1.73 & 1.46 & 3.19 \\
& $40-80$ & 13.79 & 51.15 & 64.94 \\
\hline $3:$ & 2 & 0.87 & 4.74 & 5.60 \\
Linac Cryounit 3 & $5-8$ & 2.71 & 1.63 & 4.34 \\
+Pos. Inj. Linac+Electron DR & $40-80$ & 20.67 & 55.56 & 76.24 \\
\hline $4:$ & 2 & 0.27 & 4.35 & 4.61 \\
Linac Cryounit 4 & $5-8$ & 1.73 & 1.46 & 3.19 \\
& $40-80$ & 13.79 & 51.15 & 64.94 \\
\hline $13:$ & 2 & 0.26 & 4.23 & 4.49 \\
Linac Cryounit 13 & $5-8$ & 1.69 & 1.42 & 3.11 \\
& $40-80$ & 13.43 & 49.82 & 63.25 \\
\hline $14:$ & 2 & 0.62 & 4.59 & 5.21 \\
Linac Cryounit 14 & $5-8$ & 1.83 & 1.53 & 3.36 \\
+Positron DR & $40-80$ & 14.51 & 53.84 & 68.35 \\
\hline 15: & 2 & 0.26 & 4.23 & 4.49 \\
Linac Cryounit 15 & $5-8$ & 1.69 & 1.42 & 3.11 \\
& $40-80$ & 13.43 & 49.82 & 63.25 \\
\hline $16:$ & 2 & 0.26 & 4.23 & 4.49 \\
Linac Cryounit 16 & $5-8$ & 1.69 & 1.42 & 3.11 \\
& $40-80$ & 13.43 & 49.82 & 63.25 \\
\hline
\end{tabular}

Table 7.3.9.3: Cryogenic Plant Nominal and Design, $500 \mathrm{GeV}$

\begin{tabular}{|c|c|c|c|c|}
\hline $\begin{array}{c}\text { Plant } \\
\#\end{array}$ & $\begin{array}{c}\text { Nominal } \\
{[\mathrm{kW}] @ \text { @ } .5 \mathrm{~K}}\end{array}$ & $\begin{array}{c}\text { Design } \\
{[\mathrm{kW}] @ \text { 4.5 K }}\end{array}$ & $\begin{array}{c}\text { Margin } \\
{[\%]}\end{array}$ & $\begin{array}{c}\text { AC Power at Nominal } \\
{[\mathrm{MW}]}\end{array}$ \\
\hline \hline 1 & 23.0 & 30.3 & 31 & 4.90 \\
2 & 22.0 & 30.3 & 38 & 4.66 \\
3 & 25.4 & 30.3 & 19 & 5.69 \\
4 & 22.0 & 30.3 & 38 & 4.66 \\
13 & 21.4 & 30.3 & 42 & 4.54 \\
14 & 24.3 & 30.3 & 25 & 5.13 \\
15 & 21.4 & 30.3 & 42 & 4.54 \\
16 & 21.4 & 30.3 & 42 & 4.54 \\
\hline Total & 180.75 & 242.4 & 34 & 38.66 \\
\hline
\end{tabular}

for the cryogenic plants. To compute the AC power requirements, the following COP's have been used (from the TDR[TDR, Table 8.7.5]): for $2 \mathrm{~K} 588 \mathrm{~W} / \mathrm{W}$; for 5-8 K, $168 \mathrm{~W} / \mathrm{W}$; and for 40-80 K, $17 \mathrm{~W} / \mathrm{W}$. The cold compressor heat loads have also been included. 


\subsubsection{Cost implications}

The reduction in the number of cryomodules results in a costs reduction, which is partially cancelled by the additional two cryogenic plants needed. The net savings is about $3 \%$ of total project cost.

\subsection{Warm Option: Delay Line Distribution System}

This section describes the use of a DLDS pulse compression system for the warm option.

For the warm machine, the klystron pulse length and peak power were chosen to minimize klystron costs, and are not optimal for powering the structures. To transform the relatively long, low power klystron pulses to the short, high power pulses required for the structures, several methods were considered during the past decade. They are generally referred to as pulse compression systems, since the pulse length is shortened in order to increase peak power. Most practical experience with pulse compression has come from the development of SLED-II, which is a delay line version of the SLAC Linac Energy Doubler (SLED). This compression system was selected for the baseline design because of this experience, and because such a system is much easier to demonstrate than the alternatives. As noted in the warm reference design description, the version of SLED II that was chosen has been designed to have a higher efficiency and a higher power handling capability than the systems that have operated successfully in the NLCTA. However, as with any SLED system, this new design has intrinsic power losses due to the RF transmitted from the delay lines as they are being resonantly filled.

As an alternative, a more efficient scheme of pulse compression called the Delay Line Distribution System (DLDS) is proposed. It eliminates the need for resonant RF storage and utilizes the time-of-flight of the beams to reduce the delay line length. In this latter respect, it is superior to an early proposal called Binary Pulse Compression (BPC), which also had high intrinsic efficiency. As initially conceived, power in the DLDS delay lines is transported in a single mode. To further reduce the amount of waveguide, multi-moded DLDS was introduced, with two modes transported in each delay line.

Fig. 7.4.0.1 shows a schematic of a linac RF unit incorporating dual-moded DLDS. During operation, the $3.2 \mu \mathrm{s}$ pulses from the eight $75 \mathrm{MW}$ klystrons are combined and then sent upstream (opposite the beam direction) in eight sequential, 396 ns long pulses. The power routing in the "launcher" is controlled via the phase of the RF drive to the individual klystrons. The 396-ns pulses are transported in two circular waveguide modes $\left(\mathrm{TE}_{01}\right.$ and $\left.\mathrm{TE}_{12}\right)$ in the delay lines. In each line, the $\mathrm{TE}_{12}$ pulse is extracted to feed a nearby set of structures. The $\mathrm{TE}_{01}$ pulse passes through the extractor to feed a set further upstream. At the end of each feed, the power is split evenly among eight, 0.6-meter structures. The feeds are spaced so that the same beam-to-RF arrival time is achieved in each set of structures. Nine such RF units would be interleaved to power a contiguous set of structures in each RF sector of the linacs.

This particular DLDS configuration was chosen because PPM klystrons have operated with pulse lengths up to $3.2 \mu \mathrm{s}$. Since a $396 \mathrm{~ns}$ pulse is required for the accelerator structures, this DLDS was designed to route the combined klystron power in eight time slices, which requires a minimum of eight sources. The overall transport efficiency is expected to be $85 \%$ for the times-eight compression, to be compared to $75 \%$ for the times-four compression of SLED II in the reference design. More significantly, only half of the number of klystrons are required because of the longer pulse length (such a high compression ratio would impractical with SLED II because of the increased loss of efficiency). In addition, the modulator for this system would likely be designed to power eight klystrons instead of two. The number of RF distribution components would be roughly the same, although the total delay length would double.

The Eight-Pack project at NLCTA was originally conceived to test the essential components of this eightklystron DLDS configuration. The modulator that was built for the project was designed to drive eight 75 MW PPM-focused klystrons with $3.2 \mu$ s pulses. The initial goal was to power a SLED II system with two 


\section{DLDS-Based Linac RF Unit}

Induction Modulator

Low Level RF System

One 490 kV 3-Turn Induction Modulator

Eight 2 KW TWT Klystron Drivers (not shown)

Eight 75 MW PPM Klystrons

Delay Line Distribution System (2 Mode, 4 Lines)

Eight Accelerator Structure Octets

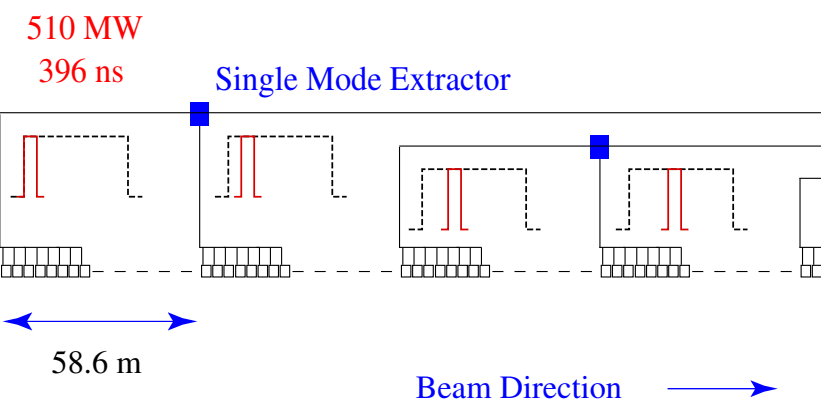

Klystron RF Pulse

$75 \mathrm{MW}, 3168 \mathrm{~ns}$

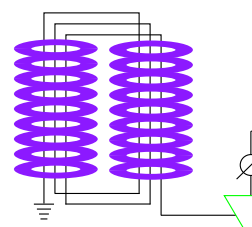

11.4 GHz RF Source

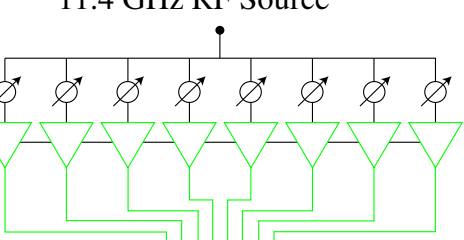

$\leftarrow$

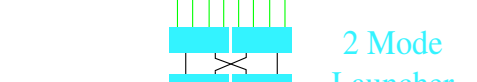

Launcher

Figure 7.4.0.1: A schematic of a linac RF unit incorporating dual-moded DLDS

klystrons to demonstrate the power handling capabilities of DLDS-like components (concerns about the cost and schedule of testing a full-scale DLDS eventually led to the adoption of SLED II with $1.6 \mu$ s pulses as the NLC/GLC baseline choice, and made the SLED II test the primary project goal). In addition, the waveguide system upstream and downstream of SLED II was designed to allow dual-moded transport as required for DLDS. Specifically, both $\mathrm{TE}_{01}$ and $\mathrm{TE}_{11}$ modes ( $\mathrm{TE}_{11}$ would be converted to $\mathrm{TE}_{12}$ in an actual DLDS) can be generated in circular waveguide with the proper choice of klystron phases. The $\mathrm{TE}_{01}$ mode powers the SLED II delay lines, while the $\mathrm{TE}_{11}$ mode bypasses the SLED II hybrid. The latter mode will be generated to demonstrate that a second mode can be efficiently transported and separately extracted in a common waveguide system. High power tests with each mode were carried out successfully in late 2003.

\subsubsection{Cost implications}

The cost savings associated with the use of the more efficient DLDS and the longer klystron pulse for the warm option is about $8 \%$ of total project cost.

\subsection{Cold Option: Superstructures}

This section describes the use of superstructures for the cold option.

\subsubsection{Introduction}

To maximize the active length of the linac, it is desirable to use structures with as many cells as possible. However, the number of cells per cavity is limited by the requirements of field homogeneity and the need 
to avoid trapped modes. These limitations can be circumvented by joining several multicell cavities to form a so-called superstructure, using a short, large diameter connecting tube. Such a configuration is shown in Fig. 7.5.1.1. The cell-to-cell coupling in this structure is much larger than the coupling between the two

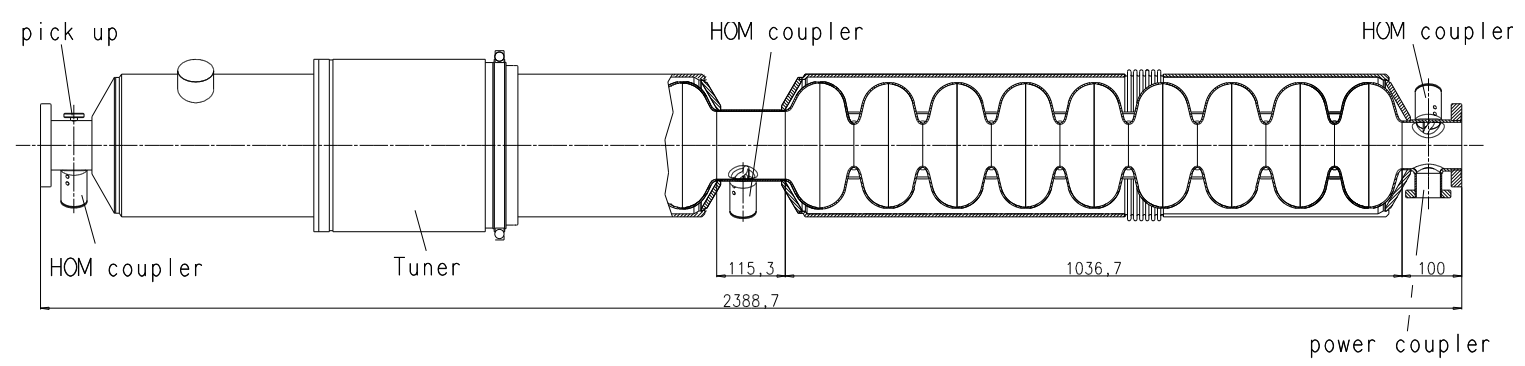

Figure 7.5.1.1: Superstructure consisting of two 9-cell resonators joined by a $114 \mathrm{~mm}$ diameter beam pipe.

adjacent cavities. The comparatively weak inter-cavity coupling makes the issues of field homogeneity and HOM damping much less of a problem than in a single long cavity with 18 cells. The RF parameters of the $2 \times 9$ superstructure are given in Table 7.5.1.1. Note that the two cavities are fed by a single power coupler.

Power flow through the superstructure has been studied using HOMDYN and MAFIA. The energy flow was found to be sufficient to refill the cells in the time interval between two adjacent bunches. The HOM modes have also been studied, and although the dipole mode impedances differ from those of a single 9-cell cavity, the overall effect on the beam dynamics is similar.

Table 7.5.1.1: Parameters of the $2 \times 9$-cell superstructure (note that we adopt here the definition of shunt impedance by the relation $R=V^{2} / P$, where $P$ is the dissipated power and $V$ the peak voltage in the equivalent parallel LCR circuit).

\begin{tabular}{lc}
\hline Type of accelerator structure & standing wave \\
Accelerating mode & $\mathrm{TM}_{010}, \pi$-mode \\
Fundamental frequency & $1300 \mathrm{MHz}$ \\
Nominal gradient $E_{a c c}$ & $28 \mathrm{MV} / \mathrm{m}$ \\
Quality factor $Q_{0}$ & $>10^{10}$ \\
Active length $L$ & $2.08 \mathrm{~m}$ \\
Cell-to-cell coupling $k_{c c}$ & $1.9 \%$ \\
Iris diameter & $70 \mathrm{~mm}$ \\
$R / Q$ & $1972 \Omega$ \\
$E_{\text {peak }} / E_{a c c}$ & 2.0 \\
$B_{\text {peak }} / E_{a c c}$ & $4.18 \mathrm{mT} /(\mathrm{MV} / \mathrm{m})$ \\
$Q_{\text {ext }}$ of input coupler & $3.10 \times 10^{6}$ \\
Cavity bandwidth at $Q_{\text {ext }}=3.10 \times 10^{6}$ & $419 \mathrm{~Hz}$ \\
Fill time & $527 \mu \mathrm{s}$ \\
Number of HOM couplers & 3 \\
\hline \hline
\end{tabular}

\subsubsection{Cryomodules and couplers}

A cryomodule would contain six $2 \times 9$-cell superstructures. The effective interconnection length for the superstructure is $0.283 \mathrm{~m}+0.1153 \mathrm{~m}=0.3983 \mathrm{~m}$, vs. $0.283 \mathrm{~m}$ for the standard 9-cell cavity. Thus, a use of 
the superstructure allows the cryomodule length to be reduced by

$$
12 \times 0.283 \mathrm{~m}-6 \times 0.3983 \mathrm{~m}=1.006 \mathrm{~m} .
$$

A standard cryomodule is reduced in length from $15.927 \mathrm{~m}$ to $14.921 \mathrm{~m}(6.3 \%)$, and a cryomodule with a quadrupole is reduced in length from $16.728 \mathrm{~m}$ to $15.722 \mathrm{~m}(6.0 \%)$. The total number of cryomodules, and the energy gain per cryomodule, do not change from the reference design values.

The number of input couplers per cryomodule is reduced from 12 to 6. (See Fig. 7.5.2.1.) The number of all auxiliary components needed to distribute the RF power, like waveguides, bends, circulators, 3 -stub transformers, loads etc., will also be reduced. In addition, the layout reduces the amount of electronics controlling phase and amplitude of cavities (low level RF) in the linac, and simplifies the design of cryomodules due to less openings for the input couplers.

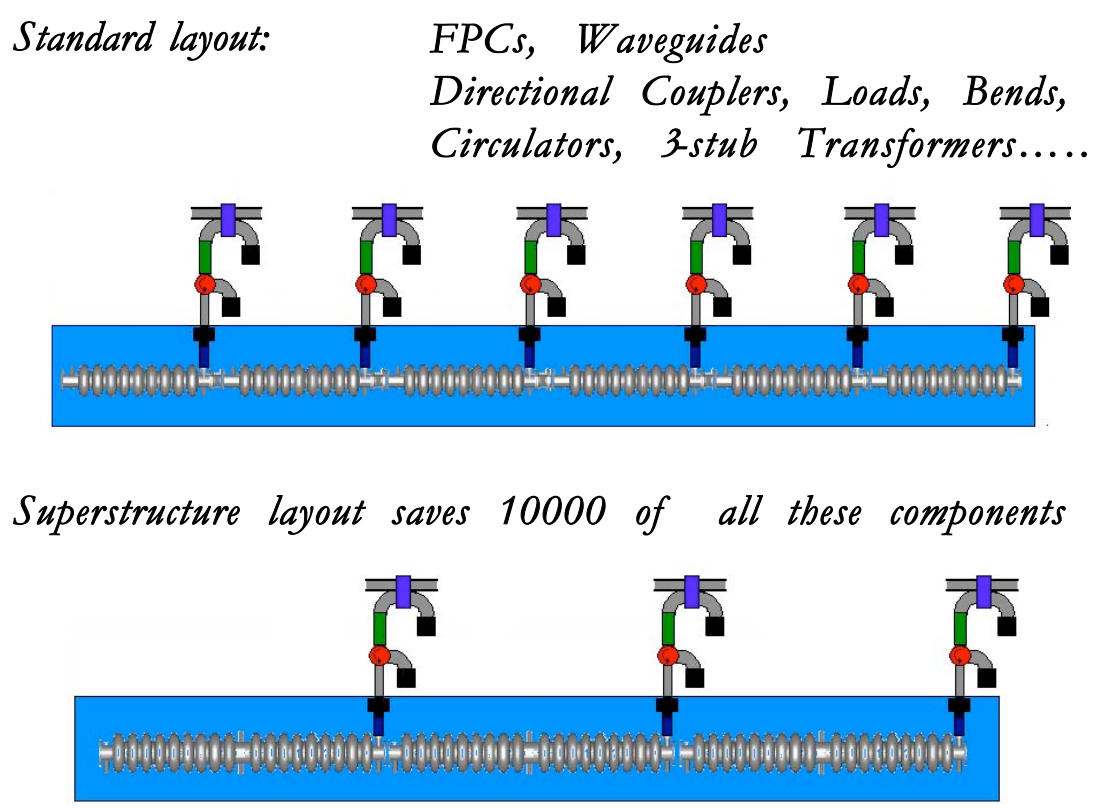

Figure 7.5.2.1: Cavity RF feed layouts with and without superstructures

Each coupler must transmit $552 \mathrm{~kW}$ of power at $28 \mathrm{MV} / \mathrm{m}$, compared with $276 \mathrm{~kW}$ in the reference design. The corresponding numbers at $35 \mathrm{MV} / \mathrm{m}$ are $690 \mathrm{~kW}$ for the superstructure, vs. $345 \mathrm{~kW}$ for the standard 9-cell cavity.

There are small increases in the required average RF power and cryogenic power $(\sim 1 \%)$, because of the slightly increased $R F$ fill time associated with the smaller $R / Q$ value (per unit length) of the superstructure, shown in Table 7.5.1.1.

\subsubsection{Linac and site lengths}

The reduction in cryomodule length leads to a reduction in the lengths of all the cryostrings, and in the overall length of the linacs. The lengths of the electron and positron linacs for the reference design energy 
of $500 \mathrm{GeV}$ c.m, with a gradient of $28 \mathrm{MV} / \mathrm{m}$, are given in Tables 7.5.3.1 and 7.5.3.2.

Table 7.5.3.1: Electron main linac, using superstructures, $250 \mathrm{GeV}$ beam energy

\begin{tabular}{lc}
\hline Injection energy [GeV] & 5 \\
Undulator energy loss [GeV] & 5.922 \\
Final energy [GeV] & 250.125 \\
Linac length (cryomodules) [m] & 11931.1 \\
Diagnostic insertion @ $5 \mathrm{GeV}[\mathrm{m}]$ & 62.8 \\
Diagnostic insertion @ $55 \mathrm{GeV}[\mathrm{m}]$ & 62.8 \\
Undulator insertion @ $155 \mathrm{GeV}[\mathrm{m}]$ & 850 \\
Diagnostic insertion @ $155 \mathrm{GeV}[\mathrm{m}]$ & 183.61 \\
Diagnostic insertion at BDS entrance [m] & 183.61 \\
Total length, including insertions [km] & 13.274 \\
Active length [km] & 9.504 \\
Real estate gradient [MV $/ \mathrm{m}]$ & 20.545 \\
\hline \hline
\end{tabular}

Table 7.5.3.2: Positron main linac, using superstructures, $250 \mathrm{GeV}$ beam energy

\begin{tabular}{lc}
\hline Injection energy [GeV] & 5 \\
Final energy [GeV] & 250.125 \\
Linac length (cryomodules) [m] & 11650.76 \\
Diagnostic insertion @ 5 GeV [m] & 62.8 \\
Diagnostic insertion @ 50 GeV [m] & 62.8 \\
Diagnostic insertion @ $152 \mathrm{GeV}[\mathrm{m}]$ & 183.61 \\
Diagnostic insertion at BDS entrance [m] & 183.61 \\
Total length, including insertions [km] & 12.144 \\
Active length [km] & 9.28 \\
Real estate gradient [MV $/ \mathrm{m}]$ & 21.039 \\
\hline \hline
\end{tabular}

The lengths of the electron and positron linacs for the upgrade energy of $1 \mathrm{TeV}$ c.m., and cavity gradient of $35 \mathrm{MV} / \mathrm{m}$, are given in Tables 7.5.3.3 and 7.5.3.4.

Table 7.5.3.3: Electron main linac, using superstructures, $500 \mathrm{GeV}$ beam energy

\begin{tabular}{lc}
\hline Injection energy [GeV] & 5 \\
Undulator energy loss [GeV] & 5.922 \\
Final energy [GeV] & 500.02 \\
Linac length (cryomodules) [m] & 19016.5 \\
Diagnostic insertion @ $5 \mathrm{GeV}[\mathrm{m}]$ & 62.8 \\
Diagnostic insertion @ $55 \mathrm{GeV}[\mathrm{m}]$ & 62.8 \\
Undulator insertion @ $192 \mathrm{GeV}[\mathrm{m}]$ & 850 \\
Diagnostic insertion @ $192 \mathrm{GeV}[\mathrm{m}]$ & 183.61 \\
Diagnostic insertion @ $375 \mathrm{GeV}[\mathrm{m}]$ & 183.61 \\
Diagnostic insertion at BDS entrance [m] & 183.61 \\
Total length, including insertions [km] & 20.543 \\
Active length [km] & 15.171 \\
Real estate gradient [MV/m] & 26.031 \\
\hline \hline
\end{tabular}


Table 7.5.3.4: Positron main linac, using superstructures, $500 \mathrm{GeV}$ beam energy

\begin{tabular}{lc}
\hline Injection energy [GeV] & 5 \\
Final energy [GeV] & 500.185 \\
Linac length (cryomodules) [m] & 18798.4 \\
Diagnostic insertion @ 5 GeV [m] & 62.8 \\
Diagnostic insertion @ $50 \mathrm{GeV}[\mathrm{m}]$ & 62.8 \\
Diagnostic insertion @ $152 \mathrm{GeV}[\mathrm{m}]$ & 183.61 \\
Diagnostic insertion @ $375 \mathrm{GeV}[\mathrm{m}]$ & 183.61 \\
Diagnostic insertion at BDS entrance [m] & 183.61 \\
Total length, including insertions [km] & 19.475 \\
Active length [km] & 14.997 \\
Real estate gradient [MV/m] & 26.342 \\
\hline \hline
\end{tabular}

A tabulation of the overall length of the technical components is presented in Table 7.5.3.5, for a cavity gradient of $28 \mathrm{MV} / \mathrm{m}$ for $250 \mathrm{GeV}$ beam energy, and a cavity gradient of $35 \mathrm{MV} / \mathrm{m}$ for $500 \mathrm{GeV}$ beam energy. The overall length of the footprint for $500 \mathrm{GeV}$ beam energy is $44.9 \mathrm{~km}$.

Table 7.5.3.5: Tabulation of technical component lengths. All lengths are in km.

\begin{tabular}{|c|c|c|}
\hline Length & $250 \mathrm{GeV}$ beam energy & $500 \mathrm{GeV}$ beam energy \\
\hline Electron Linac cryomodules & 11.931 & 19.016 \\
Electron Linac insertions & 1.343 & 1.527 \\
Positron Linac cryomodules & 11.651 & 18.798 \\
Positron Linac insertions & 0.493 & 0.676 \\
\hline Overall two-linacs with insertions & 25.418 & 40.017 \\
BDS length (total) & 3.6 & 3.6 \\
Spin rotator/bunch compressor length (total) & 0.80 & 0.80 \\
DR ends length (total) & 0.5 & 0.5 \\
Total technical component length & 30.318 & 44.917 \\
\hline
\end{tabular}

\subsubsection{Superstructure R\&D}

Beam tests of two prototype $2 \times 7$ superstructures were carried out at TTF[80] in 2002. The energy stability, the HOM damping, the frequency and the field adjustment methods were tested and results confirmed expectations of the superstructure's performance. The measured energy stability was $<2 \times 10^{-4}$, which is less than the reference design requirement of $0.05 \%$. The HOM properties (damping of dipole modes) was also better than required for the collider.

In these tests, the superstructures were operated at a relatively low gradient of $15 \mathrm{MV} / \mathrm{m}$. The principal concern with increased gradient is the relatively high levels of power transmitted through the input coupler. In a collider built with superstructures, the couplers must reliably transmit power levels about twice that of the reference design, up to $\sim 700 \mathrm{~kW}$ at $35 \mathrm{MV} / \mathrm{m}$. Although couplers at TTF have been tested up to 1500 $\mathrm{kW}$, additional R\&D is needed to establish the reliability metrics of input couplers at these power levels. 


\subsubsection{Cost reduction}

The cost reduction associated with the superstructure variant is due to the reduction of a factor of two in the number of couplers (although with an increase in the power requirement, which will increase the unit cost of the coupler), and a reduction by about $2.5 \mathrm{~km}(\sim 6 \%)$ in the length of the linac tunnels. The overall cost reduction is estimated to be $3 \%$ of the total project cost.

\subsection{Conventional Positron Source}

This section describes the use of a conventional positron source for the cold and warm options.

\subsubsection{Warm option}

In the NLC design, unpolarized positrons are produced by colliding $6.2 \mathrm{GeV}$ electrons with a high $Z$ material target, capturing the resulting positrons, and accelerating them to the $1.98 \mathrm{GeV}$ energy of the pre-damping ring system. Multiple positron targets are required to keep the energy deposited in each target below the threshold for material damage. The electrons are split by an RF separator and directed onto 3 out of 4 multiplexed targets and positron capture sections. The bunches are then recombined into the desired bunch train format before acceleration to $1.98 \mathrm{GeV}$. Because of the large emittance of the captured positrons, largeaperture L-band RF is used for acceleration and a pre-damping ring is required to reduce the emittance of the positrons before injection into the main damping ring. As required by the pre-damping ring acceptance, the positrons have an edge emittance of $0.03 \mathrm{~m} \cdot \mathrm{rad}$ and a transverse jitter that is less than $0.015 \mathrm{~m} \cdot \mathrm{rad}$; this jitter corresponds to about a $7 \mathrm{~mm}$ oscillation at the damping ring entrance.

The design of the positron system is based on the system used for the SLC, which demonstrated excellent reliability over many years of operation. The total number of positrons required for the NLC bunch train is almost two orders of magnitude greater than the number of positrons in the single SLC bunch. The design goal is to build a target system which is expected to survive a 9 month run $(120 \mathrm{~Hz}, 24$ hours per day, 7 days per week, with no scheduled outages for maintenance). Targets can be replaced/repaired annually in a scheduled 3 month maintenance period.

Positrons are produced by targeting a $6.2 \mathrm{GeV}$ electron beam onto a WRe target to create an electromagnetic shower. The positrons produced in the shower are collected using a 5.8 Tesla magnetic flux concentrator, accelerated to $250 \mathrm{MeV}$ in L-band structures encased in a 0.5 Tesla solenoidal magnetic field, and then injected into an L-band linac and accelerated to $1.98 \mathrm{GeV}$. The average deposited power is handled by rotating the target and removing the excess heat through water cooling. Of critical concern for target damage is the instantaneous energy deposition per unit volume.

After approximately 1000 days of operation ( $~ 5$ calendar years), the SLC positron system failed. Upon examination it was found that a water-to-vacuum leak had occurred in one of the target cooling tubes. In addition, cracking and material ejection were found on the exit face of the target. The peak energy deposition in the SLC target was about $50 \mathrm{~J} / \mathrm{g}$ under the conditions at which the target failed. This level produces an instantaneous mechanical shock in the $W R e$ target material which is about a factor of 2 below the expected ultimate tensile strength of pristine material. However, material hardening of a factor of about 2 from target entrance to target exit was measured along the beam path. The calculated radiation damage to the material is in excess of 3 dislocations per atom (dpa) and the target embrittlement and subsequent loss of material integrity are consistent with the calculated exposure level.

Because of the consistency of the observed damage with expectations from the simulations, it has been decided to limit the shock in the targets to that of the SLC system. In particular, the peak energy deposition and irradiation fluences are kept by design to less than $50 \mathrm{~J} / \mathrm{g}$ and $1 \mathrm{dpa}$. Investigations into the connection 


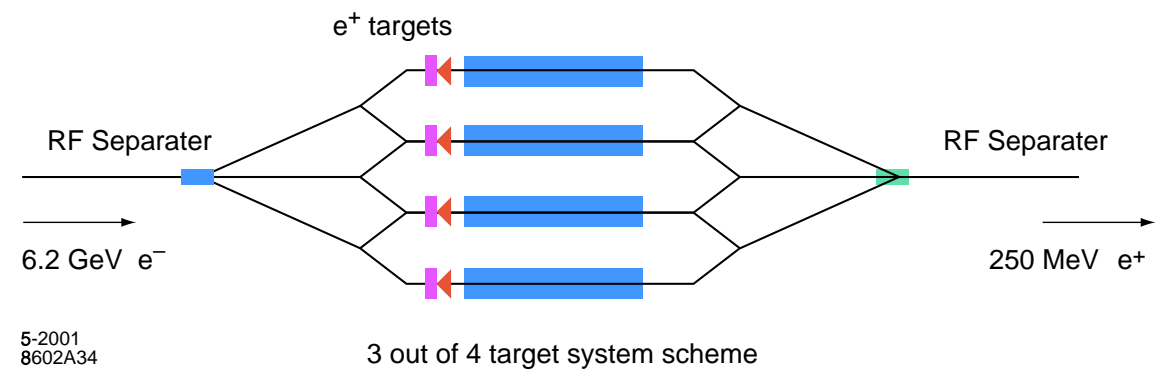

Figure 7.6.1.1: Schematic of the conventional $e^{+}$production system.

between radiation damage due to electrons with that from neutron/proton exposure are continuing. It is useful to tap into the data on material property degradation due to neutron/proton damage since the database of electron induced damage is comparatively limited. Further beam damage tests of candidate target materials may be warranted.

In order to keep the peak shock stress in the target below the threshold for damage, three $e^{+}$targets operating in parallel are planned to produce the LC beam. To assure overall system availability, a layout has been adopted where there are 4 target/capture modules, 3 of which are operating at any one time as illustrated in Figure 7.6.1.1. The bunches are separated using an RF separator and then directed to the desired targets using dc bending magnets. Access is possible to the fourth target/capture module for maintenance and repair while the other 3 modules are in operation.

The $6.2 \mathrm{GeV}$ electron drive beam, which is used to create the positrons, is accelerated using S-band technology. Because of the need to use three quasi-independent target/capture sections for positron production, the electrons are generated using a photocathode based source. Fine tuning of the individual electron bunch populations within the drive train is possible through bunch-to-bunch intensity adjustments at the source laser. The unpolarized electron source system is essentially identical to the polarized electron source with the exception that shorter laser wavelengths and photocathodes with higher quantum yields are used.

Positron yield is defined as the number of positrons captured in the pre-damping ring divided by the number of electrons incident on the target. The NLC has adopted the use of L-band $(1.4 \mathrm{GHz})$ for both the initial $250 \mathrm{MeV}$ capture and $1.73 \mathrm{GeV}$ booster linacs. The larger aperture and longer wavelength of the L-band affords a factor of about 30 increase in acceptance over an S-band system. Yield into the pre-damping ring acceptance is calculated based on the initial $e^{+}$distribution, generated using EGS4. The calculated yield is about 1.5, but experience with the SLC shows that this yield can be rapidly degraded by alignment and optical errors in the transport between the $e^{+}$source and the damping rings. It is believed that a $50 \%$ margin in the yield should be sufficient. If necessary, the population of the drive $e^{-}$beam can be adjusted to produce the desired number of $e^{+}$.

To measure the beam emittance, 4-wire non-invasive emittance diagnostics are located after the $250 \mathrm{MeV}$ point in the $e^{+}$beam line and before injection into the pre-damping ring. In addition, energy, energy spread, and bunch length diagnostics are located in a chicane at the $250 \mathrm{MeV}$ point and in the $60^{\circ}$ arc before injection into the pre-damping ring. To stabilize the trajectory and preserve the emittance all of the quadrupoles have BPMs with $10 \mu \mathrm{m}$ resolution and horizontal or vertical steering correctors depending on the focusing plane.

\subsubsection{Cold option}

Preliminary studies indicate the possibility of developing a conventional positron system for the cold option LC design[35]. This design is based on the NLC design for the production of unpolarized positrons but has been modified to accommodate the differences in the beam pulse format and the higher energy of the cold 
option positron damping ring. Because of the preliminary nature of the cold option conventional positron source design, more optimization studies are required than in the case for the warm option conventional source. Initial required studies include an improved calculation of the yield of positrons captured into the damping ring acceptance, a simulation of the mechanical stresses in the target, and target station engineering.

Unpolarized positrons are produced by colliding $6.2 \mathrm{GeV}$ electrons into two separate high $Z$ material targets. Captured positrons are accelerated to the $5.0 \mathrm{GeV}$ energy of the positron damping ring system. A pair of positron targets are required to keep the energy deposited in each target below the threshold for material damage. The electron bunches are split by an RF separator and directed onto 2 out of 3 multiplexed targets and capture sections. The positron bunches are then recombined into the desired bunch train format before acceleration to $5.0 \mathrm{GeV}$. The capture system and booster linac are the same as in the case of an undulator based source but with the caveat that the emittance of the initial positron distribution off the targets is a factor of 3-4 greater for the conventional system. Because of the larger emittance of the initial positrons, additional studies of the capture into the damping ring are required.

The design of the positron system for the cold LC is very similar to that for the warm LC, which in turn was based on the SLC positron system. The primary difference is the use of superconducting L-band technology for acceleration of both the drive beam and the positron beam. Because of the different bunch format, only two targets are required rather than three. Otherwise the two designs are nearly identical in layout, have the same diagnostics and feedback systems, and produce the same yield of positrons into the damping ring, all as described in the previous section.

The total number of positrons required for the cold option bunch train is more than three orders of magnitude greater than the number of positrons in the single SLC bunch and the average positron production rate is larger by a factor of about 60 . The design goal is to build a target system which is expected to survive a 9 month run ( $5 \mathrm{~Hz}, 24$ hours per day, 7 days per week, with no scheduled outages for maintenance). Targets can be replaced/repaired annually in a scheduled 3 month maintenance period.

Positrons are produced by targeting a $6.2 \mathrm{GeV}$ electron beam onto a WRe target to create an electromagnetic shower. The positrons produced in the shower are collected using a tapered field magnetic solenoid, accelerated to $250 \mathrm{MeV}$ in L-band structures encased in a uniform field magnetic solenoid, and then injected into an L-band linac and accelerated to $5.0 \mathrm{GeV}$. The average deposited power is handled by rotating the target and removing the excess heat through water cooling. Of critical concern for target damage is the instantaneous energy deposition per unit volume.

In order to keep the peak shock stress in the target below the damage threshold, the target must be rotated at a high angular velocity. If the tangential target velocity is $125 \mathrm{~m} / \mathrm{s}$, two $e^{+}$targets operating in parallel are necessary to handle the energy of the drive beam. To assure overall system availability, a layout has been adopted where there are 3 target/capture modules, 2 of which are operating at any one time. This is very similar to the $3 \times 4$ target scheme for the warm option conventional positron sources that is illustrated in Figure 7.6.1.1. The bunches are separated using an RF separator and then directed to the desired targets using dc bending magnets. Access is possible to the third target/capture module for maintenance and repair while the other 2 modules are in operation. If a target design can be developed for $250 \mathrm{~m} / \mathrm{s}$ tangential velocity, it is possible that a single target is can handle the energy of the drive beam. In the case of a single target, a redundant target station will be placed in a $1 \times 2$ parallel layout.

Acceleration of the $6.2 \mathrm{GeV}$ electron drive beam, which is used to create the positrons, is based on superconducting L-band technology. Because of the need to use two quasi-independent target/capture sections for positron production, the electrons are generated using a photocathode based source. Fine tuning of the individual electron bunch populations within the drive train is possible through bunch-to-bunch intensity adjustments at the source laser. The unpolarized electron source system is based on the RF-gun system developed for the TESLA FEL.

Both the double and single target schemes rely upon a high target velocity so as to spread out the initial energy deposition of the drive electron beam. This is possible because of the $337 \mathrm{~ns}$ bunch spacing of the 
cold option. Thermal hydrodynamic simulations of the induced material stresses, that take into account improved yield estimates for the larger emittance beams, are needed to improve confidence in the notion of the conventional target scheme. In addition, engineering studies are required to better define which target station issues require prototyping.

\subsubsection{Cost implications}

If either machine were built with a conventional positron source instead of the undulator source, then an unpolarized electron gun, a $6 \mathrm{GeV}$ electron linac, multiple targets, and a pre-linac with associated civil support facilities would need to be substituted for the linac space and components needed for the undulator positron source and electron energy make-up, positron pre-linac, and tunnel and components of the positron transfer line. The cost would be reduced by approximately $2 \%$, but a later upgrade to include a polarized positron source would be difficult. If the initial configuration includes the space required in the linac to later install the components of the undulator-based source, the energy make-up in the linac, and the tunnel needed for the positron transfer line, then the cost would be approximately the same as starting with an undulator source with no conventional source.

\subsubsection{Availability considerations}

As detailed in Chapter 4, availability comparisons were made between an undulator $e^{+}$source and a conventional $e^{+}$source. The positron source was not modeled in detail and the same overall MTBF was used for both systems. The significant difference is that the undulator source requires high energy electrons before positrons can be produced, while the conventional source does not. This one difference changes the downtime from $15 \%$, for the undulator $e^{+}$source, to about $11 \%$ for the conventional $e^{+}$source, because of the shorter recovery time when both systems can be restored independently. More importantly, the amount of time spent integrating luminosity increases from about $75 \%$ to over $85 \%$. This increase is due to the reduced downtime mentioned above, and to a decrease in the time spent in scheduled MD to only $1-4 \%$. Since the conventional $e^{+}$source does not require high energy electrons before positrons can be produced, much more downtime can be spent as opportunistic MD, and the scheduled MD time drops.

Changing from an undulator to a conventional $e^{+}$source makes an even more dramatic difference during commissioning, when MTBF's are shorter, recovery times longer, and more machine development time is needed. The simulated fraction of time spent integrating luminosity improves considerably to over $64 \%$ for the conventional source, instead of $31 \%$ for the reference design. This commissioning scenario demonstrates that an LC (warm or cold) should start with a conventional positron source and only switch to an undulator source as an upgrade after the accelerator has been running for several years. Otherwise, the LC delivers at least a factor of two less luminosity.

\subsubsection{Schedule implications}

The implications of a conventional positron source on the project schedule were studied as part of the work described in Section 5.4. It was found that the use of a conventional positron source reduces the complexity of the construction, installation, and initial commissioning of the injectors and damping rings, and could result in up to a year's reduction in the duration of the construction phase of the project. 


\subsubsection{Conclusions}

In contrast to the undulator-based source in the reference design, a conventional positron source will not allow the production of polarized positrons. However, the potential benefits of the use of a conventional positron source are sufficiently great that more aggressive consideration should be made of this variant for each collider technology. 


\section{Chapter 8}

\section{Risk Assessments}

\subsection{Introduction}

There is international consensus on the initial physics mission of the linear collider. The primary goals of this mission are:

- Construction of a collider with initial center of mass collision energy $500 \mathrm{GeV}$ that can be upgraded later to $1 \mathrm{TeV}$ (or higher).

- Delivery of $500 \mathrm{fb}^{-1}$ of integrated luminosity within the first 4 years of physics running.

It is considered important that the linear collider run concurrently with the LHC to provide the strongest synergism and feedback between the physics research programs. We have taken as a mission goal to begin operations for physics research by the middle of the next decade (2015).

We have carried out an assessment of the risks posed to successful completion of this mission. This assessment has been done at the highest levels of function of the collider - major technical systems and machine areas. Risks are assessed against the threat they pose to the highest performance parameters - beam collision energy and integrated luminosity within the time frame specified in the project mission. We assess risk as it stands today, based on the existing body of work on accelerator design and technology R\&D. We understand that such an evaluation will change as the linear collider project progresses through R\&D, engineering and design, industrial development, fabrication and construction, installation, and ultimately commissioning. Re-evaluation of risk and development of mitigation strategies and plans are expected to be technical management activities throughout the course of the project.

We have identified potential failures that could prevent the project from meeting its mission goals. We have considered four factors that make up our definition of the risk of a potential failure:

- The source or reason for a potential failure (Table 8.1.0.1).

- The severity of the failure as characterized by its impact on the project mission goals (Table 8.1.0.2).

- When in the course of the linear collider project the failure will occur or become apparent (Table 8.1.0.3).

- The consequence of the failure characterized by what would have to be done to overcome it (Table 8.1.0.4). 
For each of these factors we established a description and numerical ranking to characterize each identified potential failure. These are given in Tables 8.1.0.1 to 8.1.0.4. It is important that the descriptions were created before systematic identification of potential failures and assessment of their risks. During the assessment process each risk was assigned a ranking according to the best description option. A product was formed of the numerical rankings for each risk. It is important to emphasis that this product does not have any direct interpretation such as a "probability of failure". But the distribution of risk products provides a useful comparison and priority ranking method for mitigation efforts. The goal was to flag the large outstanding issues. Given the time constraints, the result is undoubtedly incomplete and the results are qualitatively reasonable but not quantitatively precise.

We have found no "show-stoppers" to the successful completion of the physics mission by a collider based on either the warm or cold accelerator technology. We have identified a number of possible failures that pose sufficient risk to be included in our assessment. All but a few of these risks exist for both technologies, but most are more or less severe for one technology or the other. We summarize our findings at the end of this Chapter.

Table 8.1.0.1: Table of Reasons

\begin{tabular}{|l|c|l|}
\hline Reason & Ranking & Description \\
\hline Beam Physics & 5 & No Theoretical Model and No Data \\
& 4 & Theoretically Understood Data Indicates Problem \\
& 3 & Poor or Ambiguous Data Indicate a Problem \\
& 2 & Best Theory Indicates Problem, No Data to the Contrary \\
& 1 & Understood Theory and Data Indicate No Problem \\
\hline Engineering/Design & 5 & Beyond Current Engineering Solutions \\
& 4 & Feasibility of Engineering Solution is Uncertain \\
& 3 & Engineering Feasible, but Untested Design \\
& 2 & Tested R\&D Design \\
& 1 & Tested Industrial Design or Similar Design in Hand \\
\hline Technology & 5 & Beyond State of the Art \\
& 4 & State of the Art - Should be Able to Do It but No Proof \\
& 3 & R\&D Prototypes, but Extrapolation Remains \\
& 2 & Available, but a Specialty Item \\
& 1 & Commercially Available Off The Shelf \\
\hline
\end{tabular}

Table 8.1.0.2: Severity Table

\begin{tabular}{|l|c|l|}
\hline Severity & Ranking & Description \\
\hline Limiting & 5 & Effect on Parameter is a limit less than design. \\
Steep & 4 & Effect on Parameter is quadratic or steeper. \\
Linear & 3 & Effect on Parameter is linear. \\
Marginal & 2 & Effect on Parameter is less than linear. \\
Contributing & 1 & Parameter dominated by other effects. \\
\hline
\end{tabular}

Table 8.1.0.3: Detection Table

\begin{tabular}{|l|c|l|}
\hline Detection & Ranking & Description \\
\hline PreOps & 3 & Not detected until facility preoperations. \\
PE\&D & 2 & Not detected until project engineering and design. \\
R\&D & 1 & Detected by R\&D. \\
\hline
\end{tabular}


Table 8.1.0.4: Table of Consequences

\begin{tabular}{|l|c|l|}
\hline Consequence & Ranking & Description \\
\hline Impossible & 5 & Would be impossible or too expensive to fix. \\
R\&D & 4 & More R\&D would be needed. \\
Major & 3 & Possible, but would require major redesign or rework. \\
Minor & 2 & Alternate design available, would need new plan or minor rework. \\
Ops & 1 & Alternate operating point will meet mission goals. \\
\hline
\end{tabular}

\subsection{Electron and Positron Sources}

\subsubsection{Beam loading in the electron capture region limits available electron charge}

For the case of the warm LC bunch format, significant beam loading in the subharmonic bunchers must be compensated to prevent degradation of the bunching along the $267 \mathrm{~ns}$ bunch train. A design study in the ZDR indicates that compensation is a tractable problem. Because of the larger bunch spacing in the cold design (337 ns between successive bunches), beam loading is significantly lower. While not ultimately expected to be a limiting problem, significant engineering is required prior to design completion.

Reason for Concern:

Warm and Cold LC: Engineering/Design - Engineering Feasible, but Untested Design: 3

Severity:

Warm and Cold LC: Steep: Effect on Parameter is quadratic or steeper: 4

Detection:

Warm and Cold LC: R\&D: Detected by R\&D: 1

$\mathrm{R} \& \mathrm{D}$ can address this issue but has not been initiated.

Consequence:

Warm and Cold LC: Ops: Alternate operating point will meet mission goals: 1

\subsubsection{Source laser stability problems feed directly into electron beam instabil- ities}

Source lasers for both the cold and warm LC designs require significant engineering and prototyping. Of primary concern are the issues of amplitude, pointing, wavelength, and polarization stability and of maintainability. These issues were solved in the case of the double bunch SLC source but require additional attention due to the multibunch requirements of both the warm and cold designs. Source lasers are not commercially available and will require several years of development prior to commencement of full collider operation. Complete stabilization to within specifications are likely to take several years of attention after the sources are turned on. The different bunch formats of the warm and cold LC designs require different laser systems each of which will have their own specific problems and remedies. Because of the development of lasers for the TTF and TFEL projects, some of the solutions for the cold LC are presently being addressed and tested.

Reason for Concern:

Warm and Cold LC: Engineering/Design - Engineering Feasible, but Untested Design: 3

Severity:

Warm and Cold LC: Linear: Effect on Parameter is linear: 3 
Detection:

Warm and Cold LC: PE\&D: Not detected until project engineering and design: 2

Consequence:

Warm and Cold LC: Minor: Alternate design available, would need new plan or minor rework: 2

This would be a major laser rework, but a minor project rework.

\subsubsection{A reduced strength undulator results in reduced positron intensity for a fixed length undulator}

Undulator prototypes for both the warm or cold design options, polarized or unpolarized, presently do not exist. The most likely failure scenario is a reduction in the undulator strength parameter for a given period and aperture. This of course will be known at the time of installation but care needs to be taken so that sufficient margin is included in the design. As prototype undulators are developed and tested, the uncertainties can be reduced along with required civil construction margins.

Reason for Concern:

Warm and Cold LC: Engineering/Design - Feasibility of Engineering Solution is Uncertain: 4

Severity:

Warm and Cold LC: Linear: Effect on Parameter is linear: 3

Detection:

Warm and Cold LC: R\&D: Detected by R\&D: 1

On-going R\&D may address this issue.

Consequence:

Warm and Cold LC: Minor: Alternate design available, would need new plan or minor rework: 2

The amount of rework can be reduced be leaving extra length in the undulator line to permit installation of additional undulator sections.

\subsubsection{Low AMD performance will result in reduced positron capture efficiency}

In the case of the warm design, the positron system adiabatic matching device (AMD) is a flux concentrator and is very similar in field, pulse length, and aperture to that successfully developed and operated for the SLC. A prototype warm design AMD is under development at BINP and will be tested at VEPP5. This development is expected to be successful. Prototyping of a flux concentrator for the cold design is also under development at BINP, the success of which is not presently assured. A failure of the development of an AMD for the ms long pulse of the cold design will reduce the positron capture efficiency by a factor of about 2 .

\section{Reason for Concern:}

Cold LC: Engineering/Design - Engineering Feasible, but Untested Design: 3

This is not a problem for the warm design; it is likely to be okay in the case of the cold design.

\section{Severity:}

Cold LC: Marginal: Effect on Parameter is less than linear: 2

The difference in positron capture yield is about a factor of two between AMD on/off.

Detection:

Cold LC: R\&D: Detected by R\&D: 1

On going $R \& D$ is likely to find a solution. 
Consequence:

Cold LC: Minor: Alternate design available, would need new plan or minor rework: 2

The worst case is to increase the length of the undulator by up to a factor of two. This requires an increase in the tunnel length. The increase can be less if a reduction in polarization is allowed and the $50 \%$ design yield overhead is used.

\subsubsection{Failure to achieve RF gradients in the positron capture sections will re- duce positron yield}

Both the warm and cold designs incorporate normal conducting, L-band structures for the initial positron acceleration and capture. The warm design utilizes a relatively high gradient $(25 \mathrm{MV} / \mathrm{m})$ while the cold design has a relatively high average power dissipation $(\sim 30 \mathrm{~kW} / \mathrm{m})$. In both cases, failure to achieve these performances may necessitate a reduction in RF gradient and subsequent yield. Additional study is required to better understand the effect on positron yield if such a reduction is required. The consequence of operating at a lower capture gradient is possibly more severe in the cold design than in the warm design.

Reason for Concern:

Warm and Cold LC: Engineering/Design - Engineering Feasible, but Untested Design: 3

Severity:

Warm and Cold LC: Linear: Effect on Parameter is linear: 3

Detection:

Warm and Cold LC: R\&D: Detected by R\&D: 1

For the warm LC, a development project is not presently underway. For the cold LC, there is an ongoing development project. Prototypes need to be developed and tested.

Consequence:

Warm LC: Ops: Alternate operating point will meet mission goals: 1

Cold LC: Minor: Alternate design available, would need new plan or minor rework: 2

If yield decrease is modest, it can be compensated with extra undulator length. We need more simulations to better understand the extent of the consequence.

\subsubsection{Positron target system engineering issues}

Engineering development and prototyping is required to fully realize the positron target systems for both the warm and cold LC designs. The cold design relies upon a rapidly spinning (1200 rpm), large diameter $(\sim 1.6 \mathrm{~m})$, water cooled Ti-alloy annulus. The warm design relies on a slowly rotating but similarly large diameter, water cooled Ti-alloy annulus. The incident power, absorbed energy, and ambient radiation in the cold design is a factor of 1.6 times greater than for the warm design. Both designs are presently based on the high strength properties of Ti-alloy for which little information is available regarding the effect of radiation on the mechanical properties of the material. If the Ti-alloys are not sufficiently robust, considerable redesign of the target systems may be required. Additional engineering studies and radiation tests are required to alleviate these concerns.

Reason for Concern:

Warm and Cold LC: Engineering/Design - Feasibility of Engineering Solution is Uncertain: 4 The uncertainty can be reduced through additional engineering studies.

Severity:

Warm and Cold LC: Linear: Effect on Parameter is linear: 3 
Detection:

Warm and Cold LC: R\&D: Detected by R\&D: 1

Consequence:

Warm and Cold LC: R\&D: More R\&D would be needed: 4

\subsection{Damping Rings}

\subsubsection{Beam acceptance limitations cause poor injection efficiency}

The high injected beam power (around $60 \mathrm{~kW}$ in the warm LC, $225 \mathrm{~kW}$ in the cold) means that close to $100 \%$ injection efficiency must be achieved in the damping rings to avoid radiation damage. Acceptance limitations can be physical or dynamic, and the associated problems are likely to be more severe in the positron damping ring (cold LC) and predamping ring (warm LC) because of the large size of the positron beam coming directly from the source. Dynamic aperture limitations come from systematic and random nonlinearities in the multipole and wiggler fields and are more difficult to characterize and quantify than physical aperture limitations.

Achieving large transverse and longitudinal dynamic aperture is a significant challenge in any low emittance storage ring design; third generation synchrotron light sources have broadly comparable requirements to the damping rings, though the need for large aperture is driven by beam lifetime rather than injection issues. The dynamical effects of the wiggler are difficult to model, and a number of approximations are needed that lead to some uncertainty in the simulation results and lack of confidence in the design of systems that may be used to correct the effects. Although the damping wigglers can be designed and constructed with very small nonlinear field components, the length of the wigglers (420 $\mathrm{m}$ in the cold LC positron damping ring) means that even residual nonlinearities can have significant effects.

Reason for Concern:

Warm and Cold LC: Beam Physics - Best Theory Indicates Problem, No Data to the Contrary: 2

Present lattice designs for the cold LC positron ring, and possibly the warm LC positron predamping ring, do not have sufficient dynamic aperture. Although experience at operating storage rings will be useful, the fact that the damping ring lattice and wiggler designs are unique means that data on the important effects will not be available until commissioning.

Severity:

Warm and Cold LC: Linear: Effect on Parameter is linear: 3

Limited injection efficiency would mean that either the injected current must be reduced, or additional collimation (in the injection line, or more likely, in the ring itself) must be installed. In either case, the effect is potentially a significant loss of positron current. Assuming that the electron current does not also have to be reduced, there will be a linear reduction in luminosity with current.

\section{Detection:}

Warm LC: PE\&D: Not detected until project engineering and design: 2 Cold LC: PreOps: Not detected until facility preoperations: 3

Although the designs can be improved, uncertainties in the wiggler modeling and lack of knowledge of the actual construction errors mean that a problem may not be apparent until commissioning. The scale of the warm LC damping rings means that once the wiggler design is finalized, tests can be performed using prototypes at existing facilities (e.g. the KEK ATF) that will provide relevant data. On the other hand, it is unlikely that any test facility could be constructed that will operate in the same regime as the cold LC damping rings. 


\section{Consequence:}

Warm and Cold LC: Minor: Alternate design available, would need new plan or minor rework: 2

There are a number of correction strategies that might be applied once detailed data on the injection limitations are available. These include nonlinear magnet correctors (as used at SPEAR2 BL11), and collimation. In either case, it should be possible to implement the corrections by making reasonable additions or modifications to existing structures and components.

\subsubsection{The coupling correction fails to achieve the specified vertical emittance}

Magnet misalignments in the damping rings will generate vertical emittance that must be reduced to below the specified value by beam based alignment and coupling correction methods. Typically, the sensitivities are such that once the emittance is corrected to the specified value, relative motion between the beam and the sextupoles (for example) of a few tens of $\mu$ m will double the vertical emittance. Table 3.3.2.2 in Section 3.3.2 indicates the scale of sensitivity to the principal sources of coupling, i.e. quadrupole rotations and vertical beam offsets in the sextupoles. The sensitivities are a function of the lattice design, the required vertical emittance, and the number of magnets in the lattice. Although the alignment sensitivity is a consideration in the lattice design, other design requirements limit what can be done in practice to make the vertical emittance less sensitive to magnet misalignments.

\section{Reason for Concern: \\ Warm LC: Engineering/Design - Tested R\&D Design: 2}

\section{Cold LC: Engineering/Design - Engineering Feasible, but Untested Design: 3}

The sensitivity of the emittance to magnet motion is based on theory that is understood. The calculated sensitivities must be put in context by comparison with existing facilities. We find that the damping rings for the warm LC will operate in regimes (sensitivity and vertical emittance value) that have already been achieved, for example at the KEK ATF and the ALS at LBNL. The requirements on the damping rings for the cold LC are significantly tighter, and the specified vertical emittance has not yet been achieved in any existing storage ring. Simulations of emittance tuning including most of the significant expected effects have been performed for both the warm and cold LC damping rings, and show that the specified vertical emittance should be achievable in each case [78].

Severity:

Warm and Cold LC: Marginal: Effect on Parameter is less than linear: 2

A variety of effects between the damping rings and the IP dilute the vertical emittance. In the low emittance regime that any linear collider will operate in, these effects tend to be additive, and will increase the emittance extracted from the damping ring by something of the order $100 \%$. Furthermore, the luminosity varies as the square root of the vertical emittance. Thus, missing the vertical emittance by a factor of 2 (for example) in both damping rings, will lead to a loss in luminosity less than a factor of 2 .

\section{Detection:}

Warm and Cold LC: PreOps: Not detected until facility preoperations: 3

Difficulties with achieving the required vertical emittance may not be fully understood (or even realized) until well into commissioning.

\section{Consequence:}

Warm and Cold LC: Minor: Alternate design available, would need new plan or minor rework: 2

The most likely problems that would prevent the specified vertical emittance being achieved, are poor performance of the diagnostics or correction system, which could be addressed through some hardware upgrades. 


\subsubsection{The extracted vertical beam has large jitter}

Vertical beam jitter can be the result of vertical vibration of the quadrupoles, beam instability driven by long-range wakefields, or jitter from the extraction kickers and septa acting in the vertical plane through some rotation of the magnet around the beam axis. For both warm and cold LC technology, the specification on vertical beam jitter on extraction from the damping ring is that the jitter should be less than $10 \%$ of the vertical beam size ( Section 3.3.2).

The vertical jitter can be suppressed by careful design and construction of the main lattice magnets and their supports; design of the vacuum chamber to reduce long-range wakefields; use of fast orbit feedback within the damping ring; careful alignment of the extraction kickers and septa. In addition, for the warm option, there is the possibility of using fast feedback acting across an appropriate part of the beamline between the damping ring and main linac.

Reason for Concern:

Warm LC: Engineering/Design - Tested R\&D Design: 2

Cold LC: Engineering/Design - Engineering Feasible, but Untested Design: 3

The SLS has demonstrated (using a fast orbit feedback system) orbit stability at close to the level required for the warm LC damping rings [79]. The vertical size of the beam extracted from the cold LC damping rings is roughly half that extracted from the warm LC damping rings. In addition, the cold LC damping rings are more sensitive to quadrupole jitter than the warm LC damping rings. Thus, the cold LC damping rings need significant extrapolation from proven systems.

Severity:

Warm and Cold LC: Marginal: Effect on Parameter is less than linear: 2

Beam jitter will lead to an increase in emittance. The direct effect (filamentation) is likely to be small compared to the difficulties in tuning the emittance that result from the jitter. This makes it difficult to quantify the impact of beam jitter from the damping rings, but the effect on the luminosity will likely be less than linear since the damping ring jitter is one part of a jitter budget that includes the downstream systems, and the luminosity dependence on the emittance of each beam is less than linear.

Detection:

Warm and Cold LC: PreOps: Not detected until facility preoperations: 3

A problem with the beam jitter would not be detected until commissioning.

Consequence:

Warm and Cold LC: Minor: Alternate design available, would need new plan or minor rework: 2

The emittance increase could likely be addressed by some improvement in the magnet supports and feedback systems.

\subsubsection{The kickers fail to meet the specified performance}

For the warm LC, the main damping ring kickers are specified to have a field integral of $0.017 \mathrm{~T}-\mathrm{m}, 65 \mathrm{~ns}$ rise/fall time, and a flat-top (to $0.05 \%$ ) of 270 ns. The most challenging parameter looks to be the stability of the flat-top, though most of the variation is likely to be systematic, which allows the possibility of feed forward correction to be used. The effect of some residual field from the trailing edge of the kicker pulse acting on the following bunch train will be mitigated by the fact that these bunches will remain in the ring for some 4.6 damping times (in the horizontal plane) before themselves being extracted.

In the cold LC, bunches are injected and extracted individually, with the last bunch being extracted 17 turns later than the first bunch. The rise/fall time of $20 \mathrm{~ns}$ determines the bunch spacing in the damping ring. The field integral is $0.01 \mathrm{~T}-\mathrm{m}$, and the specified pulse-to-pulse stability is $0.07 \%$. The variation between pulses 
is likely to be more random than systematic. Since the bunch following each kicker pulse is extracted some number of turns later, any bunch that sees a residual kick from the extraction of the immediately preceding bunch will have an emittance growth resulting from beam filamentation.

Reason for Concern:

Warm and Cold LC: Engineering/Design - Engineering Feasible, but Untested Design: 3

For both the warm and cold LC, the kicker technology requires some development beyond present capabilities.

Severity:

Warm and Cold LC: Marginal: Effect on Parameter is less than linear: 2

Limitations on the performance will lead to either an increased horizontal jitter in the extracted beam, or a limitation on the number of bunches that can be stored in the ring. A limitation on the number of bunches might be compensated to some extent by increasing the bunch charge (subject to source limitations). An increased jitter will lead to some increased horizontal emittance through beam filamentation.

Detection:

Warm and Cold LC: R\&D: Detected by R\&D: 1

Consequence:

Warm and Cold LC: R\&D: More R\&D would be needed: 4

\subsubsection{Stray magnetic fields cause emittance growth}

The cold LC damping ring is sensitive to stray fields because the large beta functions in the straight sections make the beam sensitive to the steering effects of fields of only a few $\mu \mathrm{T}$. The dispersion generated by these weak fields can lead to an increase in the vertical emittance of the extracted beam. The fields may come from a number of sources, including the main linac klystrons. Magnetic shielding is ineffective for $\mu \mathrm{T}$ fields, and it is likely to be difficult to control all the sources of stray field over the $7.5 \mathrm{~km}$ of tunnel the straight sections will share with other systems. The warm LC is less likely to suffer from this effect because of the different lattice design, and the fact that the damping rings are separated from pulsed power systems.

Reason for Concern:

Warm LC: Beam Physics - Understood Theory and Data Indicate No Problem: 1

Cold LC: Beam Physics - Poor or Ambiguous Data Indicate a Problem: 3

The warm LC damping rings are not sensitive to $\mu$ T fields. Pulsed magnetic fields of the order of a few 10s of $\mu \mathrm{T}$ will affect the emittance of the beam extracted from the damping rings, but data are not presently available that will allow confident predictions of the characteristics of the time-varying fields in the cold LC damping rings.

Severity:

Warm and Cold LC: Marginal: Effect on Parameter is less than linear: 2

The impact of emittance growth in the damping ring is mitigated by dilution effects in downstream systems, and by the fact that the luminosity has a less than linear dependence on the emittance of each beam at the IP.

\section{Detection:}

Warm and Cold LC: PreOps: Not detected until facility preoperations: 3

It will be difficult to predict the presence and effects of the small time-dependent fields until construction is substantially complete, and sources of the fields are turned on.

\section{Consequence:}

Warm and Cold LC: Minor: Alternate design available, would need new plan or minor rework: 2

The emittance increase could likely be compensated by feed-forward orbit correction once the sources of the 
stray fields and their characteristics are known. The system will need to be adaptive to respond to variations in the field sources over time.

\subsubsection{Electron cloud causes beam instability in the positron damping rings}

Electrons are generated by photoemission or ionization of residual gas, and can be accelerated by the positron beam to hit the wall with enough energy to release a significant number of secondary electrons. Under certain circumstances, the density of electrons in the chamber can increase rapidly, and the resulting electron cloud drives instabilities in the positron beam. Electron cloud effects have been observed in proton machines, and in the B-factories. The B-factories have successfully tackled electron cloud effects by using solenoids to trap the electrons near the wall of the chamber where they are reabsorbed before they can be accelerated by the positron beam.

The important parameters for the build-up of the electron cloud are the bunch charge and bunch spacing, the peak secondary electron yield (SEY) of the chamber wall, and the chamber aperture. Bunches in the warm LC damping rings have a much shorter separation than in the cold LC damping rings, and the problem is expected to be more severe in the warm LC.

Electrons can be trapped in magnetic fields, and disperse more slowly between bunches than in field-free regions; this effect may lead to a build-up of electron cloud in the long wiggler of the cold LC positron damping ring. Recent simulations suggest that the reduction in SEY required to eliminate the electron cloud in the cold LC positron damping wiggler (which is $420 \mathrm{~m}$ in length) is comparable to that required to eliminate the cloud in the warm LC positron damping rings.

\section{Reason for Concern:}

Warm and Cold LC: Beam Physics - Poor or Ambiguous Data Indicate a Problem: 3

Simulation codes indicate that both warm and cold LC positron damping rings could suffer from electron cloud effects. Although the results of codes used to simulate build-up of the cloud are in agreement with some of the limited data available, the predicted effects are sometimes not the ones observed. In at least one case $(\mathrm{DA} \Phi \mathrm{NE})$, electron cloud has not been observed at all, despite predictions that it should be a strong effect.

\section{Severity:}

Warm LC: Steep: Effect on Parameter is quadratic or steeper: 4

Cold LC: Linear: Effect on Parameter is linear: 3

The result could be a significant growth in emittance, and/or limitation of the current that can be injected into the ring, both of which will impact the luminosity. The much shorter bunch separation in the warm LC damping ring (1.4 ns) compared to the cold LC damping ring (20 ns) means that any electron cloud effects are likely to be more severe in the warm LC damping rings. Although the effect in the wiggler still needs to be studied in detail, it may be easier to stay below threshold of electron cloud effects in the cold $\mathrm{LC}$ damping ring by a modest reduction in beam current.

\section{Detection:}

Warm and Cold LC: R\&D: Detected by R\&D: 1

Studies are focusing on developing low SEY coatings for the chamber walls, since solenoids will not be effective in regions where there are wiggler or multipole fields, where the most significant problems are expected. Results so far are encouraging, but more research is needed to develop a coating technique that will reliably reduce the SEY to the required level. The simulation codes need to be developed to the point where confident predictions may be made of both the build-up of the electron cloud and its effect on the beam dynamics.

\section{Consequence:}

Warm and Cold LC: R\&D: More R\&D would be needed: 4 
An R\&D program has already begun, that is likely to lead to a solution if the effects are as predicted. In the event that the proposed solution is not sufficient, further research will be needed to develop an effective solution.

\subsubsection{Ion effects in the electron damping rings cause beam instabilities}

Ion trapping in the electron damping rings can be avoided by including a clearing gap in the stored bunch train. The injection/extraction gaps in the warm LC electron damping ring are effective for this purpose, and a gap can be incorporated in the cold LC electron damping ring without significant difficulty (the principal impacts will be a small increase in the ring circumference, and gaps in the extracted bunch train corresponding to the missing bunches in the clearing gap in the ring).

Even with a clearing gap, ions can still accumulate during the passage of a single bunch train. In the straight sections of the cold LC electron damping ring, simulations suggest that at a vacuum pressure of $10^{-9}$ Torr ions will cause a tune shift of 0.28 by the end of the bunch train, which is unacceptable; a vacuum pressure as low as $10^{-10}$ Torr may be needed in the straights, and the design in this report is specified for this. Ions in both the warm and cold LC electron damping rings can lead to coherent oscillations along the bunch train (a fast ion instability) with growth times of the order $100 \mu \mathrm{s}$ at pressures of $10^{-9}$ Torr. It may be possible to suppress these oscillations using a fast feedback system, but further studies are needed.

\section{Reason for Concern:}

Warm and Cold LC: Beam Physics - Best Theory Indicates Problem, No Data to the Contrary: 2

The uncertainties inherent in the simulations of this very complex effect make it difficult to predict with certainty whether instability will occur, and how severe it may be. Present storage rings operate in regimes where only weak effects from the fast ion instability are expected, and more data is needed to verify the simulation results.

\section{Severity:}

Warm and Cold LC: Marginal: Effect on Parameter is less than linear: 2

The result could be a significant growth in emittance, and/or limitation on the current that can be injected into the ring. The impact on luminosity is mitigated, since downstream systems dilute the emittance extracted from the damping rings, and the luminosity in any case has a less than linear dependence on the emittance.

\section{Detection:}

Warm and Cold LC: PreOps: Not detected until facility preoperations: 3

\section{Consequence:}

Warm and Cold LC: Minor: Alternate design available, would need new plan or minor rework: 2

A growth of coherent oscillations along the bunch train may perhaps be dealt with by an upgrade of the feedback system, which would be a relatively minor task. Otherwise, a more significant upgrade to the vacuum system would be needed.

\subsubsection{The vacuum chamber impedance drives beam instabilities}

Microwave instability can result from a vacuum chamber impedance that is too large. If this instability appears as a bursting mode, with large fluctuations in bunch dimensions along a bunch train or between pulses, machine tuning and operation can be made very difficult. Present estimates of the impedance (based on modeling of individual components) and microwave instability threshold (calculated using the Boussard criterion) for both warm and cold LC damping rings are shown in Table 8.3.8.1. Measurements of bunch 
lengthening in KEK-B indicate impedance values $Z / n$ of a little over $70 \mathrm{~m} \Omega$ in each ring.

Table 8.3.8.1: Estimated impedance and instability thresholds in the warm and cold LC damping rings

\begin{tabular}{|c|c|c|}
\hline & $\begin{array}{c}\text { Warm LC } \\
\text { Main Damping Ring }\end{array}$ & $\begin{array}{c}\text { Cold LC } \\
\text { Damping Ring }\end{array}$ \\
\hline Estimated $Z / n[\mathrm{~m} \Omega]$ & 25 (resistive) & 25 (resistive) \\
& & 28 (inductive) \\
\hline Instability threshold $Z / n[\mathrm{~m} \Omega]$ & 600 & 60 \\
\hline
\end{tabular}

Reason for Concern:

Warm LC: Engineering/Design - Tested Industrial Design or Similar Design in Hand: 1 Cold LC: Engineering/Design - Tested R\&D Design: 2

Existing storage rings have achieved impedance values significantly below the microwave threshold for the warm LC damping rings. The structure of the cold LC damping rings drives the threshold about an order of magnitude lower, to a more challenging regime.

Severity:

Warm and Cold LC: Steep: Effect on Parameter is quadratic or steeper: 4

A larger than expected impedance will require a proportional reduction in current in order to stay below the instability threshold. Electron and positron damping rings are likely to be equally affected.

\section{Detection:}

Warm and Cold LC: PreOps: Not detected until facility preoperations: 3

There is often some difference between the design and achieved values of the impedance. For example, the impedance in KEK-B is roughly five times the design value. It is likely that the discrepancy between design and achieved values in many machines comes from coupling between nearby components, which is difficult to include in the impedance model. It is possible to include some margin in the design to allow for this, but the real impedance of the damping rings will not be known for certain until they are commissioned with beam.

\section{Consequence:}

Warm and Cold LC: Minor: Alternate design available, would need new plan or minor rework: 2

If an instability does occur, it may be possible to identify particular components that are making a large contribution to the impedance, and re-design these to reduce their effects. If this correction is not sufficient, then a more significant upgrade of the vacuum chamber would be needed. Major modification of the cold damping ring chamber would be a much larger task than modification of the warm damping ring chambers.

\subsection{Compressors and Main Linacs: Emittance growth and beam jitter}

\subsubsection{There is excessive emittance growth in the bunch compressor/spin rotator systems.}

The bunch length emerging from the damping rings in the warm (cold) option is $5 \mathrm{~mm}(6 \mathrm{~mm})$, while the bunch length at the collisions is $0.1(0.3) \mathrm{mm}$. A bunch compressor system is required to reduce the bunch length by a factor of 50 (20). A spin rotation is also required, to transform the transverse polarization of the beam in the damping rings to the longitudinal polarization required at the collision point.

The warm option uses a two-stage bunch compressor system, consisting of an L-band RF system and a wiggler, followed by the $6 \mathrm{GeV}$ pre-linac, a $180^{\circ}$ arc, and a section of X-band linac. The use of a two-stage 
system, although more complex than a single-stage one, has the advantage that the peak energy spread is limited to about $1.5 \%$. The cold option bunch compressor is a single-stage system, which utilizes a wiggler and an L-band superconducting RF system. The output energy spread is $2.7 \%$.

It is critical that the bunch compressor systems limit the amount of emittance growth which they introduce. Since the number of RF systems is relatively few, the principal source of emittance growth will be dispersive. The potential for a problem is significant, because the energy spread is inevitably large in these systems $(2.7 \%$ in the cold option, $1.5 \%$ in the warm option). Moreover, there are large numbers of dipoles in the wigglers and arcs (30 in the cold option, 122 in the warm option), which can introduce vertical dispersion through roll errors. Correction of the cross-plane coupling introduced by the spin rotator solenoids is also essential. Coherent synchrotron radiation in these systems has been estimated to generate small and tolerable levels of horizontal emittance growth.

The emittance growth resulting from vertical dispersion and coupling from $a b$ initio installation errors (200 $\mu \mathrm{rad}$ dipole rolls, $300 \mu \mathrm{rad}$ quadrupole rolls) is very large ( $\sim 500 \%$ for the warm option, $\sim 2000 \%$ for the cold option.) To reduce this growth, correction systems have been incorporated into the design of the bunch compressors. These systems can be used, in connection with emittance measurements at the bunch compressor exit, to perform beam-based tuning. A study of the tuning of the warm option compressor in 1998 was able to reduce the emittance growth to about 10\%, but did not include dynamic effects. The emittance growth budgets for both the warm and cold option bunch compressors have been taken to be $20 \%$.

Reason for concern:

Warm and Cold LC: Engineering/Design - Engineering Feasible, but Untested Design: 3 Achieving the budgeted level of emittance growth in the bunch compressors will be challenging for both options. The primary limitation is expected to be the drift of components. The cold option has a larger energy spread, and so the challenge will be greater here; however, being a one-stage system, it is a considerably smaller and less complex than the warm option system, and consequently could be easier to tune.

Severity:

Warm and Cold LC: Marginal: Effect on Parameter is less than linear: 2

Emittance growth in the bunch compressor systems has a less than linear effect on the collider luminosity.

\section{Detection:}

Warm and Cold LC: PE\&D: Not detected until project engineering and design: 2

Full confidence that the bunch compressors systems will achieve the required performance in terms of emittance growth will not be possible without substantial component and subsystem engineering and testing efforts.

\section{Consequence:}

Warm and Cold LC: Minor: Alternate design available, would need new plan or minor rework: 2

In the event of a problem, redesign of the optics, the development of feedback and direct stabilization systems, re-engineering of the component support systems, and improvements in the correction systems and/or diagnostics, may be necessary.

\subsubsection{There is excessive emittance growth in the main linacs}

Control of emittance growth in the main linacs is a key challenge for any linear collider. Emittance growth is controlled through a combination of precise alignment of the quadrupoles and RF structures to the beam, and control of wakefields arising from the RF structures.

An initially constructed linac, or one which has not been recently "tuned", will have misalignments much greater than what is required to meet the specified emittance budgets. A step-by-step process of beam position and size measurements, followed by trajectory corrections, must be carried out. This process, 
generally referred to as "quasi-static tuning," follows one or more of a number of algorithms, designed to establish how to place the beam through the centers of the quadrupoles and the RF structures to within the required tolerances. The algorithms used for the procedure must be sufficiently stable and free from susceptibility to systematic errors, that they can operate in the real-world environment.

The random errors[TRC] corresponding to $1 \mathrm{~nm}$-rad of vertical emittance growth are summarized in Table 8.4.2.1. The tighter sensitivities for the beam-to-structure offsets in the warm option are due to the stronger X-band wakefields. The sensitivities of the quadrupole-to-beam alignment are set by the requirement to limit dispersive emittance growth, which is driven by beam energy spread and the strength of the focusing lattice. The warm option has tighter tolerances here also, because the stronger X-band wakefields require a larger correlated energy spread for BNS damping and a stronger focusing lattice. The structure tilt tolerance arises not from wakefields, but from the transverse field introduced by tilting the cavity's strong longitudinal field by even a small angle. This is why the tilt tolerance is similar for the two options.

In what follows, a number of potential sources of the risk of excessive emittance dilution are discussed.

Table 8.4.2.1: Rms alignment sensitivities corresponding to $1 \mathrm{~nm}$-rad of vertical emittance growth in the main linacs. Note that the total budgeted allowance for vertical emittance growth in the main linacs is 10 nm-rad, for both options.

\begin{tabular}{|c|c|c|}
\hline Quantity & Cold option & Warm option \\
\hline Quadrupole offsets (quad to beam) $[\mu \mathrm{m}]$ & 11 & 1.3 \\
Structure offsets (structure to beam) $[\mu \mathrm{m}]$ & 300 & 5 \\
Structure tilts (structure to beam) $[\mu \mathrm{rad}]$ & 240 & 135 \\
\hline
\end{tabular}

\subsubsection{Tuning procedures}

Quasi-static tuning refers to the procedure by which the main linacs and beam delivery systems are aligned using the beam. Several different algorithms have been proposed for use in quasi-static tuning, and some of them have been tested in practice at the SLC and at other accelerators, such as LEP and HERA. The technique of quad shunting involves varying the current in selected quadrupoles, measuring the resulting change in the orbit, and deducing the relative quadrupole-BPM offsets from this information. Dispersionfree steering (DFS) attempts to measure and simultaneously correct the orbit and the dispersion as well, which is the fundamental quantity responsible for dispersive emittance growth. To do this, the effective beam energy must be changed, either by changing quadrupole strengths or accelerating gradients. Techniques in which the quadrupole field is changed, such as quad shunting or DFS, rely on knowing how the quadrupole center shifts with excitation. If the quadrupoles are turned off, then this effect is not present, although in this case the effects of stray and remnant fields become important. This modification to the DFS scheme is called ballistic alignment (BA), as the beam is ballistic in the region in which the quadrupoles are turned off. Particularly for the cold option, the transverse fields associated with RF cavity tilts must be corrected. To measure these fields, the RF cavities must also be turned off.

In addition to these algorithms, further suppression of emittance growth can be achieved through the use of "emittance bumps". These are localized modifications of the orbit or the structure alignment, constructed at the appropriate phase specifically to cancel dispersive or wakefield-induced emittance growth. Tuning these bumps is done by direct measurement of the emittance or the luminosity. They can provide additional reduction factors in emittance growth.

Quad shunting has been used routinely at many facilities, and at the FFTB it was able to achieve an alignment precision of $7 \mu \mathrm{m}$, close to that required for the LC. DFS has been used in LEP to reduce the emittance by $50 \%$, although the relevance of this experience for the linear collider is open to question. Attempts to use DFS at the SLC met with repeated difficulties, as the procedure can be subject to numerous systematic errors. The only successful application at the SLC involved the use of co-accelerated electron and positron 
beams, which will not be possible in the warm or cold options.

Ballistic alignment was used at the SLC and elsewhere, but only for relatively small sections of beam line. There is no operational experience with this technique on the scale of kilometers. It is expected to be more sensitive to stray fields than the other techniques, and relies on accurate knowledge of the persistent or remnant fields in the de-excited quadrupoles. These fields must be known and controlled at the level of a Gauss.

Emittance bumps were used successfully at the SLC.

A number of different computer simulation codes (LIAR/MATLAB, PLACET, MERLIN) for quasi-static tuning have been written and used to model the process, for both the warm and the cold option. The simulations attempt to include a full optics model, with all the relevant physics, and include the effects of the finite resolution of the diagnostic systems, as well as the detailed implementation procedures for the beam-based alignment algorithms. Confidence that the emittance growth budgets for the warm and cold options (10 nm-rad in the main linac) can be met are based on the predictions of these simulations, using several of the algorithms mentioned above. Recent studies using LIAR/MATLAB and MERLIN/PLACET[8] indicate that quad shunting followed by DFS for the warm option or a combination of DFS and BA for the cold option can reduce the overall emittance dilution to about $8 \mathrm{~nm}$-rad in either case.

The use of emittance bumps would provide additional emittance reduction, according to the simulations, but this is held in reserve to cope with unexpected difficulties. Emittance bumps are expected to be more effective in emittance control for the cold option than for the warm option.

\section{Reason for concern:}

Warm and Cold LC: Technology - State of the Art - Should be Able to Do It but No Proof: 4 The different simulation codes have agreed in several test runs, after care has been taken to be sure that precisely the same algorithm and input assumptions were used. Nevertheless, there are a number of features of the simulations which are less than fully realistic. The impact on the tuning process of errors in the main linac energy profile ${ }^{1}$, and of systematic lattice errors, must be considered. Perhaps most serious issue is the potential sensitivity of the beam-based alignment procedures to jitter. It is to be expected that significant jitter of the input beam, and of the components, will degrade the convergence of beam-based alignment. The studies to date have looked at the effects of jitter in a relatively well-tuned machine, and the tuning of a jitter-free machine. The impact of jitter on an untuned machine may be much greater than on a tuned machine, so that, even in the presence of a level of jitter adequate for operation of a tuned machine, beam-based alignment of the untuned machine could be difficult.

\section{Severity:}

Warm and Cold LC: Marginal: Effect on Parameter is less than linear: 2

Emittance growth in the main linacs has a less than linear effect on the collider luminosity.

\section{Detection:}

Warm and Cold LC: PE\&D: Not detected until project engineering and design: 2

Some information on the application of the quasi-static tuning procedures to a large-scale system will be provided during operation of the prototype main linac in the project engineering and design phase.

\section{Consequence:}

Warm and Cold LC: Major: Possible, but would require major redesign or rework: 3

Development of adaptations to the tuning algorithms, to address problems encountered in operation, should be relatively straightforward. A potential worry is that the levels of beam jitter which meet the requirements for operation nevertheless substantially compromise the quasi-static tuning process. This would require a major rework to reduce component jitter levels throughout the linacs.

\footnotetext{
${ }^{1}$ primarily at low energies
} 


\subsubsection{Diagnostics}

The diagnostic systems, particularly in the main linac and the beam delivery system, are crucial to achieving the required beam-based alignment. There are two principal elements in these systems. The quadrupole BPM system consists of beam position pickups at each quadrupole, which measure the position of the beam relative to the pickup. This system is present in both the warm and cold machines. For the warm machine, there is a second system, the structure BPM system, which utilizes the higher-order mode power generated by the beam in the RF structures to determine the position of the beam relative to the structures. In addition to these beam position monitor systems, emittance measuring stations are provided at several locations in the main linacs to allow the beam profile to be measured.

The precision required for beam-based alignment places stringent requirements on the position resolution of the quadrupole and structure BPM systems. Maintenance of the "gold orbit" during the time interval between invasive tuning events determines the requirements on the long term stability of the BPM centers. The emittance measuring systems must measure the relatively small beam size with sufficient precision to determine the emittance accurately. These systems are used to characterize and monitor the beam. They are also used for first tuning of beam bumps, which would later be optimized directly on the luminosity. The requirements on BPM position resolution and stability, and on emittance measurement system beam size resolution, are presented in Table 8.4.2.2. This table also gives the numbers of BPMs in the main linac, to indicate the scale of the system.

Table 8.4.2.2: Diagnostic system requirements

\begin{tabular}{|c|c|c|}
\hline Quantity & Cold option & Warm option \\
\hline Quadrupole BPM rms resolution required for fast feedback $[\mu \mathrm{m}]$ & 0.6 & 0.3 \\
\hline Quadrupole BPM rms resolution required for beam-based alignment $[\mu \mathrm{m}]$ & 3 & 1 \\
\hline Long-term quadrupole BPM rms position stability $[\mu \mathrm{m}]$ & 11 & 1.3 \\
\hline Number of quadrupole BPM's & 614 & 1700 \\
\hline Structure BPM rms resolution ${ }^{a}[\mu \mathrm{m}]$ & N.A. & 5 \\
\hline Long-term structure BPM rms position stability $[\mu \mathrm{m}]$ & N.A. & 5 \\
\hline Number of structure BPM's & N.A. & 18000 \\
\hline Emittance measuring system, beam size resolution $[\mu \mathrm{m}]$ & 1 & 1 \\
\hline
\end{tabular}

${ }^{a}$ Single-bunch resolution

For the warm option, the quadrupole BPMs are planned to be RF cavity BPM's[75], operating at 11.4 GHz. The cavity diameter is $32 \mathrm{~mm}$. The cold option will also use RF BPM's, operating at $900 \mathrm{MHz}$, with a diameter of $78 \mathrm{~mm}$. The ratio of required resolution, for beam-based alignment, to cavity diameter, is about $3 \times 10^{-5}$ for the warm option, and about $4 \times 10^{-5}$ for the cold option. For both options, the emittance measurement systems utilize laser wires.

Stripline BPM's in the FFTB have demonstrated a resolution of $1 \mu \mathrm{m}$. A high resolution, single cavity RF BPM, operating at C-band, also in the FFTB, has demonstrated a resolution of $0.025 \mu \mathrm{m}$. Prototype cold option BPM's at TTF have demonstrated a resolution of $10 \mu \mathrm{m}$. Structure BPM's have demonstrated a position accuracy of better than $11 \mu \mathrm{m}$ and a sub-micron resolution. Laser wire emittance measurement systems have demonstrated a beam size resolution of $1 \mu \mathrm{m}$.

The long term stability of the electrical centers of quadrupole BPM's in the FFTB was measured to be about $3 \mu \mathrm{m}$ over 1 week. Measurements of BPM stability at the SLS indicate an upper limit of about $1 \mu \mathrm{m}$ per day. The stability required for the cold option, about $10 \mu \mathrm{m}$, is comparable to what was achieved for the LEP BPM system.

Reason for concern:

Warm and Cold LC: Engineering/Design - Engineering Feasible, but Untested Design: 3

Although several prototype BPM's have demonstrated the required performance in terms of resolution and 
stability, the substantial scale of the system needed for main linacs and beam delivery systems is a cause for concern. The complexity of the design and engineering of this system will be a significant challenge. In addition, a significant fraction of the whole system must be functional at all times that luminosity is required, which, because of the large numbers involved, puts a high premium on the reliability of individual components.

For the quadrupole BPM's, these comments apply to both options, since the scales of the system are similar. The structure BPM system is a large scale, complex system, unique to the warm option. The requirements of emittance measuring systems are similar for the two options, but since these systems involve only a few stations, the issue of system scale and complexity is much less severe.

Severity:

Warm and Cold LC: Marginal: Effect on Parameter is less than linear: 2

Emittance growth in the main linacs has a less than linear effect on the collider luminosity.

\section{Detection:}

Warm and Cold LC: PE\&D: Not detected until project engineering and design: 2

Since the principal concern involves the scale and complexity of the overall system, the full extent of a problem, should it exist, will not be encountered except in a large-scale system. During the engineering and design phase of the project, it is planned to fabricate, and commission with beam, a prototype main linac, having a few percent of the length of the ultimate linac for the collider. Although this is still small compared to the final system, it nevertheless is a big step beyond the R\&D phase of a few prototypes, and would provide a valuable opportunity to uncover problems in the diagnostic systems related to size and complexity.

\section{Consequence:}

Warm LC: Major: Possible, but would require major redesign or rework: 3 Cold LC: Minor: Alternate design available, would need new plan or minor rework: 2

If the system failure is due to problems in individual BPM units (the pickups or the electronics), due to problems not uncovered in the R\&D phase, recovery could be difficult, as the number of components to be reworked is large. This is particularly the case for the warm option, which has more quadrupole BPM's than the cold option, and has the structure BPM system, which is not needed in the cold option. For the cold option, the smaller scale of the system makes the potential impact of a problem less serious.

\subsubsection{Main linac structures}

The RF structures in the main linac must be designed and operated so that the transverse wakefields experienced by the beam are controlled. This control must be sufficient to limit the wakefield-induced emittance growth to within the budgeted amount.

Wakefield-induced emittance growth can arise from head-tail oscillations within a single bunch, due to shortrange wakefields. Single bunch dispersive emittance growth can also result from deflections due to tilted RF cavities. Multi-bunch emittance growth results from transverse long-range wakefields. ${ }^{2}$ For wakefieldgenerated emittance growth to occur, the beam must be traveling off-axis through the structures. Off-axis travel can result from a betatron oscillation of the beam, through a perfectly aligned linac, or from travel of an on-axis beam through a misaligned structure.

Emittance growth from short-range wakes in a perfectly aligned linac can be controlled by BNS damping, which effectively establishes a tune difference between the head and tail of the bunch, thereby preventing resonant driving of the tail by the head via the wakefield coupling. This is accomplished by phasing the beam off-crest in the linac, such that the resulting energy spread across the bunch, together with the chromaticity of the linac lattice, is sufficient to establish the required head-tail tune difference. The warm option, with

\footnotetext{
${ }^{2}$ Such effects are nearly static, and to some extent can be corrected by feedback systems which apply a correction which varies along the train, but is the same for each train.
} 
its stronger wakes, requires a correlated BNS energy spread of about $0.8 \%$ The cold option requires much milder BNS damping (correlated energy spread about $0.2 \%$ ) due to the substantially smaller wakefields. One of the disadvantages of BNS damping is that the correlated energy spread increases dispersive emittance growth, requiring tighter tolerances on the quadrupole-beam alignment. ${ }^{3}$ BNS damping has been used quite successfully at the SLAC linac during SLC operation to control head-tail emittance growth.

For the normal-conducting structure, suppression of the long-range wakes is achieved by establishing a controlled variation of the cell dipole mode frequencies (which are in the range of 14-16 GHz) along the length of the structure. This is called "detuning". In addition, to prevent re-coherence of the wakes, the energy in the higher-order dipole modes excited by the beam is coupled out from the beam cavity via a series of damping slots in each cell, and transported along the structure in damping manifolds to the ends. In order for the detuning to be effective, the cell-to-cell dipole mode frequency variation must be controlled at the level of $3 \mathrm{MHz} \mathrm{rms}$. In addition, the structure must be held straight to a tolerance of about $100 \mu \mathrm{m}$ (peak-to-peak), and the pitch of the structure must be aligned with the beam to within about $50 \mu \mathrm{rad}$ rms.

In prototype structures built to date, these requirements have been met. For example, prototype structures have been fabricated for which the cell dipole frequency variation was $<1 \mathrm{MHz}$. Excellent agreement has been found between wakefield predictions and measurements. This provides confidence that the prescription for fabricating the structures will yield the desired performance. During the actual fabrication of the cells, it is only necessary to control the fundamental and first dipole mode frequencies, not necessarily a detailed shape for the cell. A method for controlling this during the automated cell fabrication process has been worked out. The straightness and pitch tolerances must be held during the process of brazing the cells together to form the structure, and this has been accomplished in prototypes. Measurements on these prototypes have indicated that structure bowing due to thermal transients expected during operation $\left(\sim 20^{\circ} \mathrm{F}\right)$ is limited to $<20 \mu \mathrm{m}$.

In the cold option, HOM dampers are placed at each end of the $1 \mathrm{~m}$ cavities, and the HOM power is absorbed in these dampers, thus suppressing the long-range wakes. In a large system, it is required that there be some variation in the cavity dipole mode frequencies, to avoid resonant effects. An rms variation of $1 \mathrm{MHz}$ is required, which will be realized through a combination of $0.1 \mathrm{MHz}$ random fabrication errors, together with a controlled variation, introduced into the manufacturing process, of about $1 \mathrm{MHz}$.

The RF structures will be initially installed[TRC] to the tolerances given in Table 8.4.2.3. For the warm option, the structures are mounted on girders which have remotely-controlled movers with a stepsize resolution of $\sim 300 \mathrm{~nm}$. Alignment of the RF structures to the beam will be accomplished using the RF BPM's and beam-based alignment, as discussed in Section 8.4.2.2.

For the cold option, the situation is rather different, because the cavities are housed within cryostats, which are not movable without entry into the tunnel. Consequently, the alignment requirements for the cold option cavities, the scale of which is indicated in Table 8.4.2.1, must be satisfied during the ab initio alignment. Measurements of the tilts of cavities installed in prototype cryomodules are comparable to the tilt installation alignment requirement given in Table 8.4.2.3.

Moreover, the long-term stability requirement, which is at the level of about $300 \mu \mathrm{m}$, applies over a considerably longer period of time (months) than for the warm option, since the cavities cannot be re-positioned remotely. Reliable measurements of cavity position stability across a thermal cycle have only recently been made, and preliminary results will be available in the near future.

\section{- Main linac structures: Wakefield control demonstration}

Reason for concern:

Warm LC: Technology - State of the Art - Should be Able to Do It but No Proof: 4

\footnotetext{
${ }^{3}$ The incoherent energy spread, which also causes dispersive emittance growth, is $2.7 \%$ at injection into the cold linac, while the incoherent energy spread is about $1.5 \%$ at injection into the warm linac. Although this energy spread rapidly damps, the first $10 \%$ of the linac presents the primary challenge for control of dispersive emittance growth in the cold option.
} 
Table 8.4.2.3: Rms initial alignment tolerances for main linac RF cavities and girders/ cryomodules

\begin{tabular}{|c|c|c|c|}
\hline System & Reference & Cold option & Warm option \\
\hline Cavity offset $[\mu \mathrm{m}]$ & Cryomodule/Girder & 300 & 25 \\
Cavity tilt $[\mu \mathrm{rad}]$ & Cryomodule/Girder & 300 & 33 \\
Cryomodule/Girder offset $[\mu \mathrm{m}]$ & Survey Line & 200 & 50 \\
Cryomodule/Girder tilt $[\mu \mathrm{rad}]$ & Survey Line & 20 & 15 \\
\hline
\end{tabular}

Cold LC: Technology - R\&D Prototypes, but Extrapolation Remains: 3

The large wakefields in the RF structures of the warm linac are of general concern. Much work has been done to reduce the impact of these fields, through the development of the BNS damping technique to control single-bunch growth, and the invention of damping and detuning techniques to reduce longrange wakefields. Nevertheless, at the present time, a structure design, which meets all the gradient and breakdown requirements, and is also able to meet the long-range wakefield requirements, has not yet been demonstrated.

For the cold option, the relatively weak wakefields should not be difficult to control. Of greatest concern are the alignment (tilt) tolerances and long-term position stability of the cavities within the cryostats. For the cold option, an R\&D cavity meeting the gradient and wakefield requirements has been demonstrated, and the required tilt tolerances have been met in prototype cryostats. The question of long-term stability of the cavity position remains open.

\section{Severity:}

Warm and Cold LC: Marginal: Effect on Parameter is less than linear: 2

Emittance growth in the main linacs has a less than linear effect on the collider luminosity.

\section{Detection:}

Warm and Cold LC: R\&D: Detected by R\&D: 1

R\&D currently underway should resolve any of the technology issues noted above.

\section{Consequence:}

Warm and Cold LC: Major: Possible, but would require major redesign or rework: 3

Recovery from excessive long-range wakefields could require a major re-design of the structures. Recovery from the inability to meet the cavity tilt or stability requirements could require re-engineering of the cavity support systems within the cryostat. If this fails, it could require the implementation of remotely movable mounts for the cryostats, and the development of methods to determine the cavity-beam relative position using the HOM power signals.

\section{- Main linac structures: Wakefield control in production}

\section{Reason for concern:}

Warm and Cold LC: Engineering/Design - Engineering Feasible, but Untested Design: 3 For the warm option, fabrication of the large number of structures with the required precision, using cost-effective techniques, will be challenging. This is primarily a manufacturing engineering design issue. For the cold option, there is very little margin between what has been measured in prototypes and what is required in practice for the whole linac, both in terms of tilt tolerance and position stability. The issue is primarily one of engineering design, as well as manufacturing and assembly design and implementation.

\section{Severity:}

Warm and Cold LC: Marginal: Effect on Parameter is less than linear: 2

Emittance growth in the main linacs has a less than linear effect on the collider luminosity.

Detection:

Warm and Cold LC: PE\&D: Not detected until project engineering and design: 2 Problems of the kind described above should be uncovered during the construction and test of the 
prototype main linac, in the project engineering and design phase. The number of systems required for the prototype should be enough to allow design, engineering, and assembly issues to be worked out.

\section{Consequence:}

Warm and Cold LC: Major: Possible, but would require major redesign or rework: 3

Failure of the system to meet the wakefield control and/or alignment requirements would involve a major rework of the manufacturing engineering design and assembly procedures.

\subsubsection{Magnet systems}

The main linac quadrupoles will be installed[TRC] with an initial alignment of their centers to the survey line of about $50 \mu \mathrm{m}$ rms for the warm option, and $360 \mu \mathrm{m}$ rms for the cold option. More precise alignment is then carried out using the beam-based techniques discussed above. For the warm option, the quadrupole alignment will be done using translation stages with remotely-controlled stepper motors having $50 \mathrm{~nm}$ stepsize resolution. At the FFTB, translation stages with $300 \mathrm{~nm}$ stepsize have been demonstrated and, using microstepping technology, the NLC group demonstrated a stepsize of $50 \mathrm{~nm}$.. Because of this translation stage system, the initial alignment requirements for the warm option quadrupoles are not critical.

All of the beam-based alignment procedures depend on knowing accurately the dependence of the quadrupole center on excitation, or the remnant or persistent current fields, or static stray fields, present when the quadrupole is turned off. Stray or remnant fields at the level of a Gauss are expected to be important. Typical quad center variations measured for resistive quadrupoles are a center shift of $1 \mu \mathrm{m}$ for a $20-30 \%$ excitation change. No similar measurements are currently available for the superconducting quadrupoles.

In addition, maintenance of the gold orbit between invasive tuning periods requires stability of the quadrupole centers at the same level as that required for the BPM centers (i.e., $1.3 \mu \mathrm{m}$ for the warm option, $11 \mu \mathrm{m}$ for the cold option). Measurements of electromagnetic quads have indicated a center stability of $<1 \mu \mathrm{m}$ of 2.5 days. Again, data is not currently available for the long-term center stability of superconducting quads. It should be noted that, although it is not in the reference design, it is possible to design the support post for the cold option superconducting quadrupole in such a way that movers external to the cryostat could be used.

\section{- Magnet systems: demonstration}

Reason for concern:

Warm LC: Technology - R\&D Prototypes, but Extrapolation Remains: 3 Cold LC: Technology - State of the Art - Should be Able to Do It but No Proof: 4

For the warm option, additional $R \& D$ is needed to demonstrate translation stages with the required stepsize. The absence of any information currently on the cold option quadrupole center stability or systematic center variation with excitation is a cause for concern, requiring R\&D. These systematic errors should be able to be limited to tolerable levels by proper design of the magnets.

Severity:

Warm and Cold LC: Marginal: Effect on Parameter is less than linear: 2

Emittance growth in the main linacs has a less than linear effect on the collider luminosity.

\section{Detection:}

Warm and Cold LC: R\&D: Detected by R\&D: 1

These issues should be settled during the $R \& D$ phase.

Consequence:

Warm and Cold LC: Minor: Alternate design available, would need new plan or minor rework: 2

The issues noted should be able to be resolved with relatively minor rework.

- Magnet systems: production 


\section{Reason for concern:}

Warm and Cold LC: Engineering/Design - Engineering Feasible, but Untested Design: 3 The required initial alignment tolerances are typical of what has been achieved at other accelerators, but the scale of this effort is significantly larger. For the warm option, the complete mover system is large and complex, and there is concern about the scale-up: this is an engineering and design issue. An additional concern for both options is that the initial quadrupole-center variation may not be stable with time. In this case, the systematic errors in the beam-based alignment process, and consequently the level of alignment achieved, could grow with time.

Severity:

Warm and Cold LC: Marginal: Effect on Parameter is less than linear: 2

Emittance growth in the main linacs has a less than linear effect on the collider luminosity.

\section{Detection:}

Warm and Cold LC: PE\&D: Not detected until project engineering and design: 2

It is likely that any anomalous drift in the quadrupole-center dependence on excitation, or field at zero excitation, would be uncovered during tests of the beam-based alignment systems with the prototype main linac. Issues with achieving installation tolerances, and problems with magnet mover systems, would most likely be uncovered during fabrication and commissioning of the prototype main linac. Stray static magnetic fields due to other accelerator systems may also be uncovered with the prototype main linac.

\section{Consequence:}

Warm and Cold LC: Minor: Alternate design available, would need new plan or minor rework: 2

A retrofit of the main linac magnet systems in the PE\&D phase could be accomplished without major impact.

\subsubsection{Main linac dark current wakes}

Other sources of current in the linac structures, such as dark current, must be limited so that their wakes do not substantially affect the beam and cause emittance growth. Dark current is due to field emission, which grows exponentially with gradient, and is a strong function of the cleanliness of the cavity surface. The design gradient for the X-band structures is roughly equal to the dark current capture gradient for these structures, while the design gradient for the L-band superconducting structures is roughly five times the dark current capture gradient.

Captured dark current in the warm X-band structures, at typical operating gradients, has been measured at about $1 \mathrm{~mA}$. This is to be compared with the beam current of about $900 \mathrm{~mA}$, which produces $20 \%$ beam loading. The dark current has been shown not to excite the lowest band of dipole modes. However, the strength of the transverse fields generated directly by the dark current is not known. Simulations of dark current generation and transport are planned.

At TTF, measurements have been made of dark currents in two TTF sections, with 8 cavities each, operating at gradients around $21 \mathrm{MV} / \mathrm{m}$. In both cases, the bulk of the dark current was produced by a single cavity, up to $100 \mathrm{nA}$. Detuning the bad cavity reduced its dark current, and the total dark current from the remaining seven cavities was around $30 \mathrm{nA}$. This may be compared with the expected beam current, which is about 10 $\mathrm{mA}$. Field emission sites can be eliminated to some extent by high power and He processing. The recently tested electropolished cavity, which exceeded the design gradient of $35 \mathrm{MV} / \mathrm{m}$, showed no signs of field emission below $32 \mathrm{MV} / \mathrm{m}$. Some simulations of dark current generation and transport in the cold option cryomodules have been undertaken, but additional effects need to be included.

Reason for concern:

Warm and Cold LC: Beam Physics - Poor or Ambiguous Data Indicate a Problem: 3

The experimental understanding of dark current needs to be improved, especially for the cold option case 
at the operating gradient of $35 \mathrm{MV} / \mathrm{m}$. Measurements of the spatial distribution of the current need to be made for both options, so that the size of the resulting deflecting fields can be estimated. Simulations need to be done to estimate the effects on the beam for both options.

Severity:

Warm and Cold LC: Marginal: Effect on Parameter is less than linear: 2

Emittance growth in the main linacs has a less than linear effect on the collider luminosity.

Detection:

Warm and Cold LC: R\&D: Detected by R\&D: 1

This beam physics $R \& D$ program should be able to be carried out during the $R \& D$ phase, or near the start of the engineering and design phase of the project. It is likely that the operation of the prototype linac will allow the discovery of any remaining problems not uncovered during R\&D.

\section{Consequence:}

Warm and Cold LC: Major: Possible, but would require major redesign or rework: 3

To resolve the problems, modifications to the cavity fabrication, preparation, and processing would be required.

\subsubsection{Site characterization and civil engineering}

The most fundamental, and unavoidable, source of noise is the natural motion of the ground. At low frequencies, ground motion is expected to follow an ATL-like law ${ }^{4}$. In addition, there may be more complex slow ground motion effects related to settling after major excavation. If not controlled, this motion will result in component misalignment and emittance growth. Slow orbit feedback in the main linacs will be needed to maintain the gold orbit over periods of days, in the presence of this slow ground motion. These feedback systems utilize the BPM signals and rely upon the long term stability of the BPM centroids (see Section 8.4.2.2). For both the warm and cold options, the gold orbit is maintained using dipole correctors on the seconds time scale, and, for the warm option, also the magnet movers on the few-hour time scale.

Reason for concern: Warm and Cold LC: Engineering/Design - Engineering Feasible, but Untested Design: 3

An appropriate level of natural ground motion must be present over the entire extent of both main linacs. The chosen site needs to be well-characterized in terms of slow ground motion, and underground environments can sometimes produce surprises. Slow ground motion related to relaxation and settling after excavation may be particularly difficult to predict.

Severity:

Warm and Cold LC: Marginal: Effect on Parameter is less than linear: 2

Emittance growth in the main linacs has a less than linear effect on the collider luminosity.

Detection:

Warm and Cold LC: PE\&D: Not detected until project engineering and design: 2

During the site selection and characterization phase of the project, extensive testing of the slow motion characteristics of the site will be undertaken. It is expected that any anomalies in the site ground motion characteristics would be uncovered during these studies.

Consequence:

Warm LC: Major: Possible, but would require major redesign or rework: 3

Cold LC: Minor: Alternate design available, would need new plan or minor rework: 2

The warm option is generally more sensitive to alignment tolerances than the cold option, as discussed in the previous sections. Consequently, the impact of excessive slow natural ground motion would be expected

\footnotetext{
${ }^{4}$ This law describes slow diffusive ground motion, in which the mean square transverse displacement between two points separated by a longitudinal distance $L$, after a time $T$, is given by $\sigma^{2}=A T L$, in which $A$ a site-dependent constant.
} 
to be larger in this case, and would require more extensive remediation measures, such as more frequent component realignment, or more extensive slow feedback systems.

\subsubsection{There is excessive beam jitter in the main linacs}

Beam jitter in the main linacs will be transmitted to the beam delivery system, where it can limit the performance of the BDS trajectory feedback systems and can be amplified by collimator and spoiler wakefields, and also result in emittance growth due to those wakefields. The need to limit the emittance growth sets requirements on the level of uncorrelated beam jitter at the exit of the main linac, at frequencies of 3-5 $\mathrm{Hz}$ or greater, at the level of about $0.3 \sigma_{y}(12 \mathrm{~nm})$ for the warm option, and less than $1 \sigma_{y}(85 \mathrm{~nm})$ for the cold option. This problem can be somewhat reduced by the use of tail-folding octupoles in the beam delivery system, which allow the collimators to be placed further from the beam centroid, thereby reducing the wakefields. However, beam jitter in the main linacs, when coupled with the RF cavity wakefields, also results in the development of vertical-longitudinal correlations in the emittance distribution, which can be detrimental to collisions with high disruption parameters, as is the case for the cold option. This may require levels of beam jitter below $85 \mathrm{~nm}$ in the cold main linacs.

The cold main linacs will have a system of several intratrain feedbacks, which will provide some control of fast beam jitter due to linac components.

\subsubsection{Cultural and technical noise}

Jitter may be transmitted to the beam via the mechanical motion of the quadrupoles in the main linacs, and via high frequency electromagnetic fields which act directly on the beam, either as stray fields or as ripple in the main focusing fields that determine the beam's position. Uncorrelated noise at frequencies of 3-5 $\mathrm{Hz}$ or higher is of most concern. The natural tolerance of the beam to lower frequency noise is higher, provided (as is the case, for example, for natural ground motion) the noise is correlated over length scales of order the betatron wavelength.

At suitable sites, the uncorrelated natural ground motion at frequencies above $3-5 \mathrm{~Hz}$ is about $1 \mathrm{~nm}$, well below the tolerance limits. There can be cultural ${ }^{5}$ sources of fast jitter. Cultural noise can be significant at sites close to urbanized areas, resulting in an increase to $10 \mathrm{~nm}$ or more in the uncorrelated noise above 3-5 $\mathrm{Hz}$.

Technical systems within the accelerator can also introduce noise: examples are the water systems of the warm option, and the cryogenic fluids or RF-generated microphonic noise within the cold option cryostat. There have been studies of the noise generated by technical systems to be used for the warm option. Fast vibration of the main linac quadrupoles, due to coupling from the on-girder RF structure water cooling systems, has been measured to be a few nm.

Measurements are underway to determine the vibration levels of the quadrupoles within the cryostats of the cold option, but results are not yet available. Measurements of cavity microphonics indicates cavity length changes of less than $30 \mathrm{~nm}$, but the implications of this for transverse vibrations of the quadrupoles is not clear. Measurements of vertical motion in cold low-beta quadrupoles at RHIC[76] and Fermilab[77] indicate vertical motions in the range of 50-200 nm. It seems likely that if proper attention is given to damping of mechanical modes, then vibrations within the cryomodule should be able to be kept considerably below 100 nm.

The cold option will have a system of intratrain feedbacks in the main linacs to control high frequency component jitter, so that even if component jitter is significant, the effective jitter at the output of the main

\footnotetext{
${ }^{5}$ Human-generated, but external to the accelerator complex
} 
linacs should be able to be kept well below $85 \mathrm{~nm}$, and the development of significant emittance correlations due to jitter should be avoided.

Reason for concern:

Warm and Cold LC: Engineering/Design - Engineering Feasible, but Untested Design: 3

Control of sources of component vibration must be comprehensive across the full extent of the main linacs. Unexpected cultural noise sources (e.g., surface-level trains above the tunnel) are also possible. There are a large number of potential technical noise sources, both mechanical and electromagnetic, and both in the accelerator and the support tunnels, which must be subjected to a rigorous "vibration budget" discipline. The principal issues are in the areas of proper design, and civil, mechanical, and electrical engineering.

Severity:

Warm and Cold LC: Marginal: Effect on Parameter is less than linear: 2

Emittance growth arising from jitter in the main linacs has a less than linear effect on the collider luminosity.

\section{Detection:}

Warm and Cold LC: PreOps: Not detected until facility preoperations: 3

The effects of excessive beam jitter due to the full spectrum of possible cultural and technical noise sources will not be detected until the pre-operation phase of the project.

\section{Consequence:}

Warm LC: Major: Possible, but would require major redesign or rework: 3 Cold LC: Minor: Alternate design available, would need new plan or minor rework: 2

In the case of the warm option, excessive beam jitter would require, for example, the use of active stabilization on all the main linac components, which could be a major re-work. If this risk and the effort of remediation is perceived to be too high, active stabilization elements could be included with the quadrupole installation for minimal additional cost. For the cold option, it should be possible to cope with unexpectedly large beam jitter through an upgrade or extension of the intratrain feedback systems in the main linac, which would be a more minor re-work.

\subsubsection{Cold option main linac intratrain feedback systems}

Feedback systems, operating on several different time scales, are required to correct dynamic sources of error in the main linacs. These errors arise from the noise sources discussed in the previous section, and, if uncorrected, will result in emittance growth.

In the warm option, a train-by-train feedback system is crucial to the control of low frequency noise. In both options, slow feedback systems will be needed to limit component misalignment due to slow ground motion. Such feedback systems were used successfully and extensively at the SLC, and considerable experience in their design and operation is therefore available. The issue with these systems is primarily one of managing the complexity and reliability issues through sound design and engineering. They are considered relatively low-risk, and are not analyzed here.

As noted in the previous section, for control of fast jitter in the cold main linacs, several intratrain feedback systems are stipulated in the reference design. These feedback systems operate on a bunch-by-bunch basis. A system bandwidth of a few hundred $\mathrm{kHz}$ is sufficient to provide effective jitter reduction over the majority of the train. It should be noted that these linac feedback systems have not been tested, nor has their performance, including possible interaction with the IP intratrain feedback, been simulated.

\section{Reason for concern:}

Cold LC: Engineering/Design - Engineering Feasible, but Untested Design: 3

These intratrain feedback systems have demanding requirements. High gain and bandwidth are required, as well as precise measurements of the beam position. A prototype of this system, intended for IP feedback is, at present, in the $R \& D$ phase. 
Severity:

Cold LC: Marginal: Effect on Parameter is less than linear: 2

Emittance growth in the main linacs has a less than linear effect on the collider luminosity.

Detection:

Cold LC: PE\&D: Not detected until project engineering and design: 2

The significant scale of the feedback systems, their complexity, and the critical role they play in collider operation, motivates concern. The intratrain feedbacks in the linac can only be tested adequately during the operation of the prototype main linac.

\section{Consequence:}

Cold LC: Minor: Alternate design available, would need new plan or minor rework: 2

The background issues related to detecting deflections of the disrupted beam, which are a major concern for the IP feedback systems, are not present with the main linac systems. Thus, problems with the main linac intratrain feedback systems would most likely be recoverable through relatively minor re-engineering. It is unlikely that more R\&D would be required.

\subsection{Main Linacs: RF technology}

The RF power source requirements of the warm and the cold linacs are quite different as a result of the differences in technology, RF frequency, RF pulse length and structure fill time. The $1.3 \mathrm{GHz}$ cold machine requires long $(1.5 \mathrm{~ms})$, low peak power pulses $(\sim 275 \mathrm{~kW} / \mathrm{m}$ for $500 \mathrm{GeV}$ and $345 \mathrm{~kW} / \mathrm{m}$ for one TeV) while the $11.4 \mathrm{GHz}$ warm machine requires short (400 ns), high peak power pulses ( 100 MW/m).

The linacs of the machines are composed of hundreds of RF units that independently convert wall plug power to beam power via RF acceleration. For the cold machine, an RF unit consists of a modulator that produces $110 \mathrm{kV}, 1.5 \mathrm{~ms}$ pulses at $5 \mathrm{~Hz}$, a multi-beam klystron that generates $10 \mathrm{MW}$ RF pulses, a non-evacuated waveguide network that divides and distributes this power to 30,1-m long, SC cavities, which accelerate the beam $(9.5 \mathrm{~mA}$ average current during the bunch train). For the warm machine, it consists of a modulator that produces $500 \mathrm{kV}, 1.6 \mu$ s pulses at $120 \mathrm{~Hz}$, two PPM klystrons that each generate $75 \mathrm{MW}$ RF pulses; a SLED II system that compresses (by 4 in time for a times 3 power gain) and distributes the combined klystron power; and, finally 8, 0.6-m long, TW structures that accelerate the beam (890 mA).

There are 600 (2232) such units altogether for the cold (warm) linacs for $500 \mathrm{GeV}$ c.m. operation. These quantities includes a $2 \%$ overhead of "hot-swappable" units to allow for faults (i.e., malfunctions that require only a brief period for recovery, such as RF breakdown), and a $3 \%$ overhead to allow for failures (i.e., malfunctions that require many hours to repair, such a vacuum leak in a klystron that requires it to be replaced). The failure and fault rates for the various RF components are specified such that they would rarely (once a year) deplete the pool of spares assuming realistic repair and recovery periods. None of the required availabilities is greatly out-of-line with what has been achieved for these types of components in existing machines. However, the versions being developed for a linear collider are generally required to operate at significantly higher peak powers, energies and gradients than those in current machines, and few have reached the development stage that would warrant long-term reliability testing.

\section{Modulators}

For the warm machine, solid state induction modulators are being developed to lower cost and to obtain higher efficiency $(70 \%)$ and reliability compared with the PFN-style modulators traditionally used. A fullscale prototype (4-pack) has generated $500 \mathrm{kV}, 300 \mathrm{MW}$ pulses when driving a water load, and $400 \mathrm{kV}$, $500 \mathrm{MW}$ pulses when driving four $50 \mathrm{MW}$ klystrons (in each case with $1.5 \mu$ s pulse widths). However, this modulator has only run a few hundred hours at a low repetition rate $(30 \mathrm{~Hz})$. Current R\&D is focused on improving its performance (local heating problems are limiting higher rate operation) and developing a smaller version (a "Two-Pack") that will drive two $75 \mathrm{MW}$ klystrons (500 kV, $270 \mathrm{MW}$ ). 
Having met the major technical challenges of operating at high voltage and delivering high currents without damaging the modulator or klystrons, the risk of a major setback in solid state induction approach is small. The main tasks that remain are to demonstrate reliable, nominal repetition rate operation and good efficiency.

The cold machine will use a more conventional style modulator to deliver the $120 \mathrm{kV}, 15 \mathrm{MW}$ pulses to each of the $10 \mathrm{MW}$ klystrons. The design being considered has solid state switching and a bouncer-type circuit to compensate to the voltage droop on the storage capacitors. Fermilab has built three modulators and industry has built five units (five modulator/transformer/klystron systems have been operated). Two of the Fermilab modulators are IGBT switched and have operated about 13,000 hours each at $1 \mathrm{~Hz}$.

The basic modulator design has been proved viable. There will be more engineering evaluation and design evolution. In particular, the switch technology is evolving rapidly and packaging for the most efficient layout will need development. The design of a control/interlock module for the modulator is evolving to include more information and remote diagnostics. The next phase of development aims for a modular layout to further enhance reliability and maintainability.

\subsubsection{Klystron Lifetime}

For the klystrons, both machines employ start-of-the-art designs with relatively low perveances to improve efficiency. For the warm machine, periodic permanent magnets (PPM) are used for focusing instead of solenoidal electromagnets to halve the average power consumption. Several PPM klystrons have been made by KEK/Toshiba and SLAC. One of SLAC tubes has met the full specifications $(75 \mathrm{MW}, 1.6 \mu \mathrm{s}, 120 \mathrm{~Hz}$ pulses have produced $55 \%$ efficiency). Another tube at KEK has met all peak power specs, but at a $50 \mathrm{~Hz}$ rep rate. Nonetheless, the cathode current density is fairly high $\left(7.2 \mathrm{~A} / \mathrm{cm}^{2}\right)$ and the tubes will likely be modified in the engineering and design phase of the project to reduce it.

For the cold machine, multiple (7) beam klystrons (MBKs) are used to achieve both a low perveance and low voltage. Prototypes have been built with industry that meet pulse power requirements. Thales has produced three klystrons, and five are on order to be delivered in 2004. All three Thales klystrons met their acceptance specifications, but after 2 to 3 months of operation, developed gun arc problems or symptoms. Thales is conducting a study to isolate the problem. The first rebuild is expected early in 2004. Both CPI and Toshiba are working on prototype $10 \mathrm{MW}$ multi-beam tubes as well (Toshiba is also designing a $5 \mathrm{MW}$ version). These tubes have lower cathode emission requirements than the Thales tube and should be less prone to arcing. The CPI tube should be ready at the beginning of next year and the Toshiba tube by fall of 2004 . The Toshiba efficiency goal is $70 \%$.

\section{Reason for concern:}

Warm and Cold LC: Engineering/Design - Engineering Feasible, but Untested Design: 3

No significant lifetime limits have been established at nominal power operation for the klystrons. Of particular concern are the high $\left(>5 \mathrm{~A} / \mathrm{cm}^{2}\right)$ cathode current densities used in both the current warm and cold designs. Such densities will likely limit the tube lifetime below that required due to evaporation of the cathode material (this could be the source of the cold klystron failures).

\section{Severity:}

Warm and Cold LC: Linear: Effect on Parameter is linear: 3

Shorter than expected lifetime will limit maximum energy.

\section{Detection:}

Warm and Cold LC: PE\&D: Not detected until project engineering and design: 2

The cathode related lifetime will be evaluated in the $R \& D$ phase. Lower current density tubes are already being built for the cold machine. But a several year running period is needed to evaluate all potential problems.

\section{Consequence:}


Warm and Cold LC: Major: Possible, but would require major redesign or rework: 3

As problems are discovered, one could modify existing klystrons during downtimes and change the design of replacements. If lowering power is helpful, one could run with a higher SLED II compression ratio in the warm machine. For the cold machine, two $5 \mathrm{MW}$ klystrons could be used in place of a $10 \mathrm{MW}$ klystron (commercial, single-beam, $5 \mathrm{MW}, 1.5 \mathrm{~ms}$ tubes currently exist, although with $35 \%$ lower efficiency, and Toshiba is developing a $5 \mathrm{MW}$ klystron with $70 \%$ efficiency). Lowering the modulator voltage would be more problematic in either machine since the klystrons would have to be redesigned to maintain a high efficiency.

\subsubsection{RF distribution systems}

SLED-II systems have generated $240 \mathrm{~ns}, 250 \mathrm{MW}$ pulses at the NLCTA and $150 \mathrm{~ns}$, $485 \mathrm{MW}$ pulses in an experimental test facility. A new concept of a dual-moded delay line is being developed to reduce the overall length of the pulse compressor lines and to improve power handling capability (475 MW, $400 \mathrm{~ns}$ pulses are required). Assembly of the first prototype is complete and testing will begin by the end of 2003. There is no industrial experience in building this type of system, which requires precision machining (few tens of $\mu \mathrm{m}$ ) and careful brazing to avoid changing the over-moded waveguide transmission characteristics.

The SLED II delay lines in the support tunnel will be temperature stabilized to $1^{\circ} \mathrm{C}$ using water cooling tubes and insulation. Keeping the SLED II system phase stable is not considered a major challenge as the SLED systems at NLCTA, which are subject to larger temperature variations (several ${ }^{\circ} \mathrm{C}$ ), have stable phases on a short time scale $(<1$ hour) and the long time scale variations are corrected with simple feedback systems (e.g., moving the adjustable delay line shorts to keep the input-to-output RF phase difference constant at the SLED hybrid).

The RF distribution for the cold machine is much simpler since the power and surface field levels are low and no pulse compression is required. At TTF, RF distribution systems are used that are similar to those required for the cold machine (e.g., with motor driven 3-stub tuners and circulators). The power from a single klystron, which is delivered in two waveguide arms, has been distributed to as many as 16 cavities (nominally 30 will be powered in the cold machine). Individual components have been tested at nominal operating power and higher: circulators to $400 \mathrm{KW}$, couplers and hybrids to $5 \mathrm{MW}$, and 3 stub tuners to 1.5 MW. However, there has been no test of an integrated system at full power, because fewer than the nominal number of cavities have been powered. No significant R\&D is planned for the distribution system, which is built from standard components. However, DESY is working with industry to develop integrated waveguide systems to reduce cost and the installation/testing effort.

\section{- SLED II Demonstration:}

Reason for concern:

Warm LC: Technology - State of the Art - Should be Able to Do It but No Proof: 4

The dual mode SLED-II pulse compression system is a new design with many first of a kind microwave components. The system has not been demonstrated at the power levels (475 MW) needed to reach $65 \mathrm{MV} / \mathrm{m}$ at $400 \mathrm{~ns}$. The system will also need to be tested at the higher power levels $(550 \mathrm{MW})$ that would be used to do in-situ conditioning of structures to $70 \mathrm{MV} / \mathrm{m}$ to reach acceptable breakdown rates at $65 \mathrm{MV} / \mathrm{m}$. Both klystron and modulator pulse lengths will have to be increased to $2.0 \mu \mathrm{s}$ (from $1.6 \mu \mathrm{s}$ ) to produce the higher compression.

\section{Severity:}

Warm LC: Linear: Effect on Parameter is linear: 3

Higher than specified fault rate will limit the maximum energy. The high energy pulses transported by the SLED II system could produce significant surface damage during RF breakdown. If such damage produces further breakdowns, the system may not be able to operate stably unless the power is reduced.

\section{Detection:}


Warm LC: R\&D: Detected by R\&D: 1

The SLED-II systems will be tested by the end of 2003 .

\section{Consequence:}

Warm LC: R\&D: More R\&D would be needed: 4

If necessary, one could change the design to a two-feed Delay Line Distribution System with one local feed and one delayed (400 ns) feed. In this case, the power level would be below that already proven, and the klystron and modulator pulse lengths would be a factor of two shorter.

\section{- SLED II Production:}

Reason for concern:

Warm LC: Engineering/Design - Engineering Feasible, but Untested Design: 3

Even if the R\&D systems work, the industrially produced versions could fail to meet performance requirements.

Severity:

Warm LC: Linear: Effect on Parameter is linear: 3

Higher than specified fault rate will limit the maximum energy.

Detection:

Warm LC: PE\&D: Not detected until project engineering and design: 2

Consequence:

Warm LC: Major: Possible, but would require major redesign or rework: 3

\subsubsection{Accelerating Structures}

\section{Warm structures}

For the warm structures, there has been an aggressive R\&D program to reach the unloaded gradient goal of $65 \mathrm{MV} / \mathrm{m}$ with $400 \mathrm{~ns}$ pulses. During the last few years, 25 prototype structures were tested (>15,000 hours operation at $60 \mathrm{~Hz}$ ), with the conclusion that low group velocity (3\%-4\% c at the upstream end), 60 $\mathrm{cm}$ long, high phase advance $\left(150^{\circ}\right)$, traveling-wave structures are the best candidates for the linear collider. Eight of the structures have the appropriate iris radii for acceptable short-range wakefields. Some of these structures include slots in the cells for long-range wakefield damping: all are detuned to suppress the longrange wakefield on the scale of the bunch spacing $(1.4 \mathrm{~ns})$. Of the eight structures, the best performance achieved was a breakdown rate of 0.2 per hour, close to the requirement of $<0.1$ per hour (at the $60 \mathrm{~Hz}$ test rate). To improve the performance, a design with a somewhat smaller iris has been adopted. It has $10 \%$ lower surface fields and requires $10 \%$ less power. Testing of a structure of this design with slots began in November, 2003.

\section{Cold cavities}

The cryomodules consist of twelve 9-cell niobium cavities that must run at gradients of $28 \mathrm{MV} / \mathrm{m}$ for $500 \mathrm{GeV}$ c.m. operation. The cavity $Q$ 's must be $>10^{10}$ to limit cryogenic loading, and the couplers must handle 275 $\mathrm{kW}$ of RF input power. In addition, the cavities must also be capable (at installation) of reaching gradients of $35 \mathrm{MV} / \mathrm{m}$ with $Q$ 's $>5 \times 10^{9}$ and input powers of $345 \mathrm{~kW}$ to allow an upgrade for $1 \mathrm{TeV}$ c.m. operation.

About seventy 9-cell structures have been produced by a standard chemical etching method. Most reached $25 \mathrm{MV} / \mathrm{m}$ and higher in $\mathrm{CW}$ testing. The use of electropolishing and mild baking $\left(100{ }^{\circ} \mathrm{C}\right)$ techniques for preparing the cavities has yielded $\mathrm{CW}$ gradients between $35-37 \mathrm{MV} / \mathrm{m}$ in four units. One of these cavities was fully equipped with tuner, input and HOM couplers and powered with a klystron in a one-cavity cryomodule. It operated stably for more than 1100 hours at $35 \mathrm{MV} / \mathrm{m}$ at a $Q$ value of $7 \times 10^{9}$. Operation was without quenches or trips originating from the cavity-coupler system. The same cavity ran for 57 hours at $36 \mathrm{MV} / \mathrm{m}$. A few (10-20) quench and coupler trips were initiated by power jumps in the klystron and preamp system. These did not degrade the gradient or $Q$ performance of the cavity or coupler. The complete 
$\mathrm{RF} /$ module system ran uninterrupted for a period of 110 hours. Another fully equipped unit is under test and has reached $35 \mathrm{MV} / \mathrm{m}$ at a $Q$ of $6 \times 10^{9}$.

Five 8-cavity modules have been completed, installed and tested in TTF-I and TTF-II. In two modules recently tested, 9 cavities reached $30 \mathrm{MV} / \mathrm{m}$. Three modules have been tested with beam in TTF-I. The best performing module accelerated beam at $22.7 \mathrm{MV} / \mathrm{m}$. Among the beam-tested modules, eleven cavities performed between $25-29 \mathrm{MV} / \mathrm{m}$ with beam. In total, 13,000 hours of beam time have been logged, mostly at $1 \mathrm{~Hz}$.

\section{- Gradient Demonstration:}

Reason for concern:

Warm and Cold LC: Technology - R\&D Prototypes, but Extrapolation Remains: 3

For the warm option, what's needed is a proof-of-principle operation of eight fully-featured structures at $65 \mathrm{MV} / \mathrm{m}$ with acceptable trip rates in the presence of beam. The best operating statistics are from a 720 hour run of a single structure ( 6 slotted cells) at $65 \mathrm{MV} / \mathrm{m}$. The trip rate was 0.2 an hour, twice as large as desired. Thus far the tests have been conducted at a $60 \mathrm{~Hz}$ repetition rate, lower than the nominal $120 \mathrm{~Hz}$.

For the cold option, there has not been a full system test of a cryomodule with cavities operating at 35 $\mathrm{MV} / \mathrm{m}$ with acceptable trip rates in the presence of beam. The best operating statistics are from an 1100 hour run at $5 \mathrm{~Hz}$ of a single 9 cell cavity at $35 \mathrm{MV} / \mathrm{m}$. It incurred no unforced trips, which puts an upper limit on trip rate well within spec. However, there is still concern about the rate of events which would require beam shut-off.

Severity:

Warm and Cold LC: Linear: Effect on Parameter is linear: 3

Higher than specified fault rate will limit the maximum energy.

Detection:

Warm and Cold LC: R\&D: Detected by R\&D: 1

"String tests" are planned by mid 2004 (warm option) and early 2005 (cold option).

Consequence:

Warm and Cold LC: R\&D: More R\&D would be needed: 4

One could also lower the required gradient with a modest cost increase.

\section{- Structure/Cavity Production:}

Reason for concern:

Warm and Cold LC: Engineering/Design - Engineering Feasible, but Untested Design: 3 The concern is that industrially produced structures/cavities fail to meet the performance requirements. For the cold machine, this includes exceeding the dark current limits, as discussed in the cryogenic heat load section below.

Severity:

Warm and Cold LC: Linear: Effect on Parameter is linear: 3

Higher than specified fault rate or dark currents will limit maximum energy.

Detection:

Warm and Cold LC: PE\&D: Not detected until project engineering and design: 2

Consequence:

Warm and Cold LC: Major: Possible, but would require major redesign or rework: 3

\subsubsection{Cold coupler production}

The cavity coupler in the cold machine is designed to transition between warm and cold temperatures, and between atmospheric pressure and vacuum via two RF windows. Also, the inner conductor of its coaxial 
geometry is movable to adjust the cavity coupling coefficient. Couplers of this type are not new and have been used successfully in the past; for example, 300 coaxial couplers $(50 \mathrm{~kW} \mathrm{CW})$ with similar features were used in LEP-II.

Considerable coupler R\&D has been done for the cold machine. This has included the use of computational tools for analysis of multipacting, and has resulted in the implementation of a dc bias between the antenna and the outer coax. Also, design changes have been made to improve vacuum pumping and minimize the warm surface area exposed to RF. About 60 coaxial-style couplers have been fabricated during four design iterations. All couplers have been tested to one MW (some higher) with $1.3 \mathrm{~ms}$ pulses in a warm, high power test stand. The most recent (Style III) couplers (22 built, 40 under construction) have performed the best. Two TESLA couplers have recently been tested at $35 \mathrm{MV} / \mathrm{m}$ in CHECHIA at DESY, with trip rates of less than 1 per 1000 hours of operation.

Couplers need to be reprocessed after installation in the cavity. Conditioning times for couplers are a few days, and drop to one day for couplers pre-conditioned with high power and baked before cool down. Present processing techniques and installation procedures need to be improved to speed up processing time. Likely improvements will come from the baking of cavities and couplers to remove water.

Applying a bias voltage to the center conductor suppresses multipacting and reduces coupler trips during operation. Couplers showing higher trip rates can be re-conditioned with power, or be cycled to $20 \mathrm{~K}$. One effect that takes time ( year) to develop is the desorption of gases from the warm RF window area and re-condensation in the cold window area, which then can cause electron emission or coupler breakdowns. In the recent run at TTF, coupler events were not a problem (few if any events were recorded), but the coupler operated somewhat below nominal power. Continued tests of modules at TTF-II will provide better reliability statistics.

Reason for concern:

Cold LC: Engineering/Design - Tested R\&D Design: 2

The concern is that industrially produced couplers fail to meet performance requirements.

Severity:

Cold LC: Linear: Effect on Parameter is linear: 3

Higher than specified fault rate will limit maximum energy.

\section{Detection:}

Cold LC: PE\&D: Not detected until project engineering and design: 2

\section{Consequence:}

Cold LC: Major: Possible, but would require major redesign or rework: 3

\subsubsection{Cryogenic Heat Load capacity exceeded}

For the $2 \mathrm{~K}$ cryogenic system of the cold machine, $0.1 \mathrm{~W} / \mathrm{m}$ is budgeted for heating from dark currents, which requires that the average current per cavity be $<50 \mathrm{nA}$ in each direction. This limit is based on simulations of dark current transport through the cryomodules and intervening quadrupole magnets. These simulations show that the quadrupole magnets limit the continued build up of dark current along the linac by dispersing the electrons, and that $40 \%$ of the of dark current energy is deposited in the $2 \mathrm{~K}$ cryogenic system.

In a recent test of two cryomodules at TTF, 11 cavities had an average dark current of $38 \mathrm{nA}$ per direction at a gradient of $25 \mathrm{MV} / \mathrm{m}$. For the two cavities operated at $35 \mathrm{MV} / \mathrm{m}$, there was no detectable X-ray activity up to $35 \mathrm{MV} / \mathrm{m}$ in the first, and up to $34 \mathrm{MV} / \mathrm{m}$ in the second (no direct dark current measurements were made for either cavity). More monitoring of the dark currents will occur as further cavities and cryomodules are commissioned for TTF-II. The most revealing measurement will be of the cryogenic load of 
eight electropolished cavities operating at $35 \mathrm{MV} / \mathrm{m}$, which is planned for 2005 .

During the operation of the linear collider, there is a concern that the dark currents may increase in some cavities, for example during recovery periods from vents. High power RF processing has been shown to successfully reduce dark currents. Also, if field emission is high in a few cavities due to accidental contamination, lowering the fields in these cavities by $1-2 \mathrm{MV} / \mathrm{m}$ will greatly reduce the currents since they depend exponentially on surface gradient. For $500 \mathrm{GeV}$ operation, some of the refrigeration overhead capacity may be available for dealing with local regions of higher dark current. For example, if the entire refrigeration overhead were available, it could deal with twice the budgeted dark current in the whole linac.

An additional cryogenic load will come from beam halo losses during normal operation. Such losses are hard to predict because of the multi-step processes likely involved. If the beam loss measured in the SLAC Linac during SLC operation is any indicator, then about $3 \mathrm{~W} / \mathrm{m}$ is expected when scaled to the cold machine maximum beam power. However, the cold design cavity aperture is about three times larger than the SLAC S-band structure iris aperture, so the corresponding loss in the cold machine would likely be considerably smaller, with all other things being equal.

Reason for concern:

Cold LC: Technology - R\&D Prototypes, but Extrapolation Remains: 3

Dark currents are close to the limit at $25 \mathrm{MV} / \mathrm{m}$, and no quantitative dark current data are available at 35 $\mathrm{MV} / \mathrm{m}$. Beam halo losses are unknown.

\section{Severity:}

Cold LC: Marginal: Effect on Parameter is less than linear: 2

Higher than specified heating will limit maximum energy.

\section{Detection:}

Cold LC: R\&D: Detected by R\&D: 1

The heat load during $35 \mathrm{MV} / \mathrm{m}$ operation of a cryomodule will be measured in early 2005 .

\section{Consequence:}

Cold LC: R\&D: More R\&D would be needed: 4

One could also lower the design gradient.

\subsubsection{Loss of Vacuum Integrity}

The cold machine has three vacuum systems: the cavity beamline system, the input coupler system, and the cryostat insulating vacuum (the waveguide between the klystron windows and input couplers is at atmospheric pressure). The coupler vacuum volume lies between the inner RF window, which is evacuated on the cavity side, and the outer RF window, which has dust-free, dry nitrogen gas on the waveguide side. The warm machine has one vacuum system, which extends from the klystron windows through the SLED II system and into the structures and other beamline components. In both machines, fast valves in the beamline would limit sudden pressure rises to $\sim 100-\mathrm{m}$ long regions. In the cold machine, the cryogenic system is divided in $2.5 \mathrm{~km}$ sections, the insulating vacuum system is divided in $0.5 \mathrm{~km}$ sections and the coupler vacuum system is divided by cryomodule.

Leaks that develop in the vacuum flanges are a concern for both machines. The number of flanges would be significantly larger in the warm machine than the cold if the current $R \& D$ prototype components were used. In the engineering and design phase of the warm option, the goal would be to reduce this number by at least an order of magnitude for cost and reliability reasons. For the cold machine, it is the flanges at warm transitions that are of most concern, since the ones between the beamline and insulation vacuum (which constitute most of the flanges) would not necessary require immediate repair if a leak occurred.

Of greater concern are failures of both RF windows in a coupler, a helium vessel to beam vacuum rupture 
or a leak in the insulation vacuum system that caused large heat loss. Leaks of the insulating vacuum, if not too severe, can be mitigated by connecting additional turbo-pumps, or by reducing RF power so that the cryosystem can handle the increased thermal load.

Although the reliability of the warm or cold vacuum systems are difficult to evaluate at this stage, there are some notable differences in the severity of a failure. In the cold machine, the consequences can be severe because of the sensitivity of the cavities to contamination. Also, a failure would have a larger impact on up-time in the cold machine, because it would take about a month to start RF operation again if a $2.5 \mathrm{~km}$ section has to be warmed, compared to about a day to allow beam operation in the warm machine.

If the failure is in the coupler outside of the inner window, the warm coupler part can be replaced in the tunnel without venting the cavities, but it requires warming up and opening the insulating vacuum volume. In the case of a helium leak into the beamline vacuum, even though the differential pressure between the helium and vacuum is small, helium will enter some cavities and liquify. Furthermore, during the warm-up that follows, warm gas may carry dust to adjoining modules. For some vacuum failures, there can be recovery or partial recovery from gas condensation by a partial warm-up to $20 \mathrm{~K}$.

In both machines, some RF processing will be required to re-establish acceptable high gradient performance for the 100 or so cavities or structures exposed during a beamline vent. Experience at NLCTA shows the recovery can take a few days or less and would involve processing at higher than the nominal unloaded gradients $(70 \mathrm{MV} / \mathrm{m}$ compared to $65 \mathrm{MV} / \mathrm{m})$. This could probably be done during normal machine running by operating the structures out-of-time with respect to the beam. For the cold machine, the high power RF processing procedure could also used to condition the cavities, depending on the number and size of the contaminants. Larger particles $(>100 \mu \mathrm{m})$ may bond to the surface and cause quenching at the normal gradient, which would require lowering the gradient. The RF conditioning could in principle be done in the cold machine during beam operation, but quenches induced by the high beam loading may prevent this. However, if the cavities were detuned, at least the couplers could be processed with the beam on.

\section{Reason for concern:}

Warm and Cold LC: Engineering/Design - Tested Industrial Design or Similar Design in Hand: 1

The main experience with large-scale cold RF systems (with windows) has come from two machines. At LEP-II there were 500 meters of cold cavities with 300 couplers that operated for 3 years without a vacuum incident. CEBAF has operated $200 \mathrm{~m}$ of cold linac for 7 years without a serious vacuum incident. Existing large $2 \mathrm{~K}$ magnet systems (few $\mathrm{km}$ ) at HERA and RHIC also have high reliability.

The SLAC Linac has shown it is possible to have good vacuum system reliability in a $3-\mathrm{km}$ warm RF system over a 30 year period.

\section{Severity:}

Warm LC: Contributing: Parameter dominated by other effects: 1;

Cold LC: Linear: Effect on Parameter is linear: 3

For the cold option, the effect on the (integrated) luminosity is linear. For the warm option, the integrated luminosity is expected to be dominated by other effects.

Detection:

Warm and Cold LC: PreOps: Not detected until facility preoperations: 3

Consequence:

Warm and Cold LC: Minor: Alternate design available, would need new plan or minor rework: 2 


\subsubsection{Pulse-to-Pulse and Bunch-to-Bunch Energy Variations too large}

During acceleration of the beams in either machine, the bunch energy gains must be tightly regulated both within a train and between trains to limit the loss in luminosity due to dispersion. The bunch-to-bunch and pulse-to-pulse energy variations are required to be $<0.05 \% \mathrm{rms}$ in the cold machine, and $<0.3 \% \mathrm{rms}$ in the warm machine. The regulation is tighter in the cold machine to allow $300 \mu \mathrm{m}$ quadrupole magnet alignment tolerances in the cryomodules, typical for cold cryostats. (In the warm machine, the larger intra-bunch energy spread required for BNS damping does permit such a tradeoff).

The main factors that affect the beam energy are the RF amplitude, RF phase, beam current and cavity tuning. The higher beam loading in the cold machine makes it more sensitive to current variations. For example, a $1 \%$ change in the average current would change the gradient by $1 \%$ in the cold machine if the $\mathrm{RF}$ is not compensated, while the change in the warm machine would be $0.25 \%$. Also, the higher loaded $Q$ 's of the cavities in the cold machine makes their tuning much more critical than in the warm machine (the effective warm structure $Q$ 's are several orders of magnitude smaller). Specifically, the cold cavities have a narrow band width $(300 \mathrm{~Hz})$ and are subject to repetitive Lorentz-force detuning (few $100 \mathrm{~Hz}$ ) and random pulse-to-pulse microphonic detuning $(10 \mathrm{~Hz})$. For the warm machine, the structure temperatures will be regulated to $0.5{ }^{\circ} \mathrm{C}$, which will limit the reduction in energy gain to $<0.1 \%$ from beam-to-RF phase slippage within the structures.

Both options have well developed strategies for equalizing the bunch energy gains. In each machine, the beam current profile will be measured in the damping rings prior to each RF pulse and this information will be used in a feed-forward manner to adjust the RF waveforms to compensate for any variation in beam loading. For the cold machine, this will mainly be used to stabilize the first part of the bunch train (few $\mu \mathrm{s}$ ), after which feedback loops that monitor the phase and amplitude of the RF in the cavities (in 30 unit sums) will regulate the cavity stored energy for the remainder of the pulse. Lorentz-force detuning will be mainly compensated by piezo-movers that vary in a fixed pattern pulse to pulse: any residual tuning errors will corrected by the feedback system. In the warm machine, the pulse length is too short to do intra-train feedback, and only pulse-to-pulse feedback will be used. It will be based on measurements of the phase and amplitude of the transmitted RF from the eight structures in each RF unit.

At test facilities for each machine, linear-collider-like bunch trains have been accelerated over several meters and the bunch-to-bunch energy gains have been regulated to the required levels. For the cold test, vector sum control is expected to be easier with 30 cavities (instead of the 16 at TTF) due to better averaging of microphonics induced errors. Five modules will be controlled with same system in TTF in 2004. Piezo-mover compensation of Lorentz-force tuning has also been demonstrated. In addition, a dedicated test in the $3 \mathrm{~km}$ S-band SLAC linac showed that NLC/GLC-like beam loading (20\%) could be compensated to better than $0.3 \% \mathrm{rms}$ within a $400 \mathrm{~ns}, 40 \mathrm{GeV}$ bunch train. As for temperature control, the SLAC S-band structures and NLCTA X-band structures are regulated to $<0.5^{\circ} \mathrm{C}$.

One concern that remains for both machines is how these energy stabilization techniques will work on a larger scale, where systematic errors will likely dominate (i.e., those common to all feedback systems such the linearity of the amplitude measurements) and where few direct measurements of the bunch energies will be available. The tighter energy tolerances and higher energy sensitivities would likely make such errors more problematic for the cold machine.

Reason for concern:

Warm LC: Technology - Available, but a Specialty Item: 2; Cold LC: Technology - R\&D Prototypes, but Extrapolation Remains: 3

The warm option ranking is based on single and multi-bunch operation in SLAC Linac and the NLCTA four-structure results. The cold option ranking is based on tests with two cryomodules at TTF.

Severity:

Warm and Cold LC: Marginal: Effect on Parameter is less than linear: 2 
The effect of energy variations on the luminosity is less than linear.

Detection:

Warm and Cold LC: PE\&D: Not detected until project engineering and design: 2

Consequence:

Warm and Cold LC: Minor: Alternate design available, would need new plan or minor rework: 2

\subsection{Beam Delivery System}

Issues that have not been experimentally verified in the linear collider BDS are those related to: (1) the smaller beam emittances which lead to tighter alignment and jitter tolerances and more difficult beam size measurements, (2) the multi-bunch beams with much higher beam power and a greater sensitivity to collective effects, (3) the very high fields in the beam-beam interaction which leads to large disruption, beamstrahlung, and $e^{+} / e^{-}$pairs, (4) the strong solenoid field at the IP which will be many times stronger than at the SLC, and (5) the crab cavities.

The respective risks to the $\mathrm{LC}$ project will be discussed in the following sections. In general, the tolerances and requirements of the warm and cold BDS differ by less than a factor of two and the risks are similar. For the most part, the BDS components, magnets, vacuum, and instrumentation, are not state-of-the-art. Elements which are more novel such as the superconducting final focusing magnets, the beam collimators, the vibration suppression systems, and the fast feedback systems will require prototyping during the $R \& D$ stage of construction to reduce the risk to the LC project. However, there remains significant risk because the performance of many systems cannot be verified until commissioning. This is especially true of systems that rely on sensitive measurements in areas of potentially large backgrounds; historically, prediction of backgrounds in particle physics detectors or in beam instrumentation has been poor.

\subsubsection{Jitter}

High frequency motion of the final quadrupoles will cause beam motion at the IP. In the warm design, the IP feedback cannot suppress motion at frequencies higher than $\sim 10 \mathrm{~Hz}$. The tolerance on this high frequency motion is $0.5 \mathrm{~nm}$. Achieving this level of stability will require active stabilization. Prototype systems have been developed and have stabilized rigid objects at the desired level for frequencies greater than a few Hertz. New sensors, presently being developed, should further improve this performance. However this stability has not been demonstrated with a superconducting magnet and cryostat as will be required. This must be demonstrated during the R\&D phase of the LC construction. If this active system is not sufficient, an intra-train feedback system, such as the Feedback on Nanosecond Timescales (FONT) system which has been demonstrated at the NLC Test Accelerator (NLCTA), could be used to further reduce the IP beam jitter.

In the cold LC design, the motion of the final magnets should be adequately handled with the intra-train IP feedback provided that this system performs as expected. Active stabilization can be used to further reduce the pulse-to-pulse fluctuations at the IP.

Reason for concern:

Warm LC: Engineering/Design - Engineering Feasible, but Untested Design: 3

Prototype systems have been developed and have stabilized rigid objects at the desired level at frequencies greater than a few Hertz. However this stability has not been demonstrated with a superconducting magnet and cryostat as will be required. This must be demonstrated during the R\&D phase of the LC construction.

\section{Severity:}


Warm LC: Steep: Effect on Parameter is quadratic or steeper: 4

While the luminosity loss is linear or less than linear in the incoming jitter, the ability to tune the collider luminosity depends crucially on the stability of the collisions.

Detection:

Warm LC: PreOps: Not detected until facility preoperations: 3

There is an active program aimed at demonstration of the active stabilization of a superconducting quadrupole similar to that needed for the final magnet. Nevertheless, the real performance limitations will not be encountered until testing in the actual IR environment, with the complete system, including the experimental detector, in place.

\section{Consequence:}

Warm LC: Minor: Alternate design available, would need new plan or minor rework: 2

Poor stabilization of the final magnet can either be addressed by improving the active system or by an intra-train feedback such as the FONT system which has already been demonstrated.

\subsubsection{IP position feedback}

The IP beam position jitter must be stabilized to prevent luminosity loss. Both the warm and cold designs use IP deflection feedbacks to stabilize the relative position of the two beams. The warm design IP deflection feedback is based on the collider repetition rate of $120 \mathrm{~Hz}$ and the cold design would use an intra-train feedback. IP deflection feedbacks have been used to stabilize the collisions in many colliding beam facilities for example, the warm collider IP feedback system is similar to that successfully operated in the SLC and, more recently, IP position feedbacks have been used in the SLAC and KEK B-factories with partial success.

Despite the past success, the IP stabilization requirements and the IR environment in the LC will be very different from that in previous experiments. The stabilization requirements are at the nanometer level and are hundreds of times smaller than that achieved in the SLC and the SLAC and KEK B-factories. In addition, potential background sources which can impact the BPMs, essential for the deflection feedback, are thousands of times higher than in the past examples. Although simulation studies have been made of the deflection feedback performance, there remains significant uncertainty due to the extrapolation in required performance.

The primary differences between the IP feedback in the warm and cold IP designs are due to the larger beam-beam disruption and the higher sampling rate in the cold design. The stabilization requirement on the residual offsets between the beams in both position and angle is roughly 2 times tighter in the cold design than in the warm design because of the larger beam disruption. Residual motion of the beams at the IP can arise from incoming IP angle jitter confusing the deflection BPMs, coupling of the horizontal motion into the vertical by the strong IP solenoid, or noise or backgrounds on the BPMs used to measure the outgoing beams in the feedback system. In the cold design, the IP angle jitter is planned to be removed with an intra-train IP angle feedback for each incoming beam; in the TDR these are located about 850 meters upstream of the IP and would likely have a similar location in the cold LC design. As stated, the sources of backgrounds are many times higher than in previous experiments. These backgrounds, which are very sensitive to the details of the beam trajectory or the beam-beam deflection, may cause errors in the BPM readings used by the feedback system. Another possible error source in the BPMs is RF noise generated by the bunch train.

Reason for concern:

Warm LC: Engineering/Design - Tested R\&D Design: 2

Cold LC: Engineering/Design - Engineering Feasible, but Untested Design: 3

The IP feedback is essential to maintain collisions for times longer than a few seconds. While IP feedbacks are frequently used in colliding beam facilities, the level of control required and the environment in the LC IR will be different from previous examples. In general, the resolution and precision requirements on the cold LC IP feedback are twice as difficult as those in the warm LC design due to the higher disruption. The 
cold LC has two additional complications in that it must have high frequency response and requires the use of both position and angle feedbacks. Recent simulations indicate significant luminosity loss[81].

\section{Severity:}

Warm and Cold LC: Steep: Effect on Parameter is quadratic or steeper: 4

While the luminosity loss due to the beam jitter is linear or less than linear in the feedback performance, the ability to tune the collider luminosity depends crucially on the stability of the collisions and thus the impact is much greater than linear.

\section{Detection:}

Warm and Cold LC: PreOps: Not detected until facility preoperations: 3

While modeling of the IR environment and prototyping of the feedback components will be useful, the real performance limitations in either the warm or the cold LC design will not be encountered until the system is commissioned with colliding beams.

\section{Consequence:}

Warm and Cold LC: Minor: Alternate design available, would need new plan or minor rework: 2

The IP position feedback is essential and there are no known alternate solutions. The system must be made operational which might require additional hardware installation, alternate feedback algorithms, or new electronic modules. Adding masking or changing BPMs would likely involve rebuilding the IR.

\subsubsection{Aberration tuning (luminosity diagnostics)}

The luminosity must continually be optimized with global feedbacks to compensate for component drifts. The optimization feedbacks will control roughly 17 different knobs per side of the IP which include items such as the $x$ and $y$ waist locations, the IP dispersion, and the coupling as well as more subtle aberrations such as 3rd-order dispersion and high-order geometric aberrations. The tuning rate will differ for the different aberrations however it is expected that the low-order aberrations (roughly 5 to 6 knobs per side) will need to be tuned every few minutes. Because of the larger IP beta-functions, many of the tolerances in the cold BDS will be roughly 2 times looser than in the warm for similar impact on the beam. However, the larger IP disruption in the cold design may invert this relation in many cases. Without detailed simulation, we cannot evaluate whether the warm or cold BDS will be more difficult to tune.

Experience has shown that the most effective way to tune these aberrations is with a dither feedback based on luminosity signals. The luminosity monitor must be able to provide an accurate luminosity signal in the presence of background sources, beam jitter and emittance fluctuations. Because of the large number of aberrations that must be tuned, small errors in the luminosity measurement are compounded.

\section{Reason for concern:}

Warm and Cold LC: Engineering/Design - Engineering Feasible, but Untested Design: 3

The fast luminosity signal is needed for the dither feedbacks which optimize the 34 (17 per beam) global BDS tuning knobs. Due to the large number of aberrations to be tuned, small errors in the luminosity measurement can lead to a significant luminosity loss.

\section{Severity:}

Warm and Cold LC: Linear: Effect on Parameter is linear: 3

The luminosity tuning is linear in the performance of the luminosity diagnostic.

\section{Detection:}

Warm and Cold LC: PreOps: Not detected until facility preoperations: 3

Consequence:

Warm and Cold LC: Minor: Alternate design available, would need new plan or minor rework: 
2

If the fast luminosity monitor has too much noise, different signals proportional to the luminosity will need to be investigated. Fortunately, the time-scales for the aberration tuning are rather large $(\sim$ seconds $)$ and the integrated luminosity over this time will be a large signal.

\subsubsection{BDS component motion (IP jitter and aberration tuning)}

The beam delivery system components must be stabilized against both high frequency motion (jitter) and slower motion (drift). Jitter of the BDS quadrupoles will cause beam position jitter at the IP. Drift of the magnetic positions or strengths will cause a reduction in the luminosity. The most severe tolerance is on effects that change the beam position at the chromatic correction sextupoles. This must be stabilized at the $\mu \mathrm{m}$-level.

In the warm LC design, the vibration tolerance on most quadrupole magnets is equal to that of the main linac quadrupoles $(14 \mathrm{~nm})$. The ground motion and environmental noise sources of quadrupole jitter have been measured to be smaller than the required tolerances; this problem should be easier to deal with in the BDS because there are fewer environmental sources such as the cooling water flow through the accelerator structures. However if the magnet jitter is still too large, the sources of jitter will have to be identified and suppressed through improved engineering, passive isolation, or active vibration damping. All of these techniques have been demonstrated in small systems but never at the scale required for the LC.

In the cold design, the beam position jitter can be removed by the intra-train IP deflection feedback and the tolerance is much looser.

In both the warm and the cold design, the drift tolerances are tight and are at the level of $\sim 200 \mathrm{~nm}$ over time-scales of a few seconds to minutes. There are two stabilization techniques that will be used: first, the position will be stabilized using trajectory feedback and, second, the luminosity will be tuned using global optimization feedback. For the trajectory feedback to be effective, the incoming beam jitter at the IP angle phase must be sufficiently small. This is discussed in Section 8.4.3. For the global luminosity feedbacks to be effective, the component drift rates must be low, an accurate luminosity diagnostic is necessary (discussed in Section 8.6.3), and the incoming beam parameters must be stable. To avoid significant risk to the LC, it is important that the BDS be constructed with a high level of mechanical and thermal stability.

\section{Reason for concern:}

Warm and Cold LC: Engineering/Design - Engineering Feasible, but Untested Design: 3

The BDS component jitter will degrade the performance of the IP deflection feedback and will degrade the performance of the BDS drift feedbacks. Large BDS component drifts could exceed the capability of the drift feedbacks and will impact the luminosity aberration tuning. The component drift tolerances must be attained through mechanical and thermal stabilization.

Severity:

Warm and Cold LC: Linear: Effect on Parameter is linear: 3

Large drift rates will degrade the performance of the aberration tuning.

\section{Detection:}

Warm and Cold LC: PreOps: Not detected until facility preoperations: 3

\section{Consequence:}

Warm and Cold LC: Minor: Alternate design available, would need new plan or minor rework: 2

In the warm LC design, the jitter sources will have to be traced and suppressed. Adequate suppression techniques have been demonstrated. In the cold LC, the IP jitter can be removed with an intra-train feedback system. In both designs, if the drift rate exceeds the luminosity feedback response, either the luminosity feedback will need to be improved or the sources of component drift will need to be corrected. 
The sources will be difficult to diagnose.

\subsubsection{High Power beams (collective/beam physics)}

The beam delivery systems are long beam lines with large beta functions and delicate cancellations of aberrations. This makes them very sensitive to collective effects that can impact the longitudinal or transverse phase space. This includes the electron cloud and ion instabilities and the banana effect due to the very strong beam-beam interaction as well as more standard single and multi-bunch wakefields.

For the most part, these effects are known and are calculable. However, small details, which are difficult to represent in the simulations, can have significant impact and will be extremely difficult to trace. Collective effects may have less impact on the emittance in the cold BDS design due to the larger IP beta functions and the larger bunch spacing. However, the stronger beam-beam interaction will probably make the impact in the warm and cold designs similar.

Reason for concern:

Warm and Cold LC: Beam Physics - Understood Theory and Data Indicate No Problem: 1

Most of the major effects are known and are calculable, although it is difficult to fully calculate all effects that can impact the beam phase space. Because of the sensitivity of the beam delivery system, very small effects can have a large impact.

Severity:

Warm and Cold LC: Linear: Effect on Parameter is linear: 3

Different effects will have different impacts. However, because of the increased sensitivity due to the strong beam-beam interaction, the impact of small correlations on the beam will be magnified.

Detection:

Warm and Cold LC: PreOps: Not detected until facility preoperations: 3

Consequence:

Warm and Cold LC: Minor: Alternate design available, would need new plan or minor rework: 2

Collective aberrations may be observed but the source would likely be quite difficult to diagnose. The solution would probably require new vacuum components.

\subsubsection{High Power beams (collimation, equipment damage survival and machine protection)}

The very high power beams of a future LC are such that a single beam pulse can cause significant damage to the machine. The most sensitive elements will be the beam collimators which must be placed very close to the beams. Other sensitive components are the superconducting magnets, and the beam and detector instrumentation. Damage possibilities are mitigated by the use of a machine protection system and beam spoilers. Unfortunately, because most of these issues require the operating collider before they can be fully evaluated they carry some risk to the LC project.

The beam collimators must be designed to withstand occasional errant pulses of these high power beams. The warm and cold designs differ slightly in that, in the cold design, most of the bunch train can be directed to a beam dump after the passage of a few bunches which is not possible in the warm design. Although this provides some advantage it is difficult to fully utilize because even a single low-emittance bunch can do significant damage to a collimator or other accelerator component.

Although designs exist for the collimation system based on extensive tracking and computer simulation and a prototype of a consumable spoiler has been constructed, there are still large uncertainties in the performance 
of the system. Additional material damage studies, further prototyping of the consumable spoiler, detailed collimator wakefield calculations and measurements, and a complete model for the possible failure modes in the LC are all required along with a detailed engineering design for the beam line. Fortunately, most of these issues can be addressed during the R\&D stage of the LC construction. However, it should be noted that a failure of the collimation system, due to faulty design or incomplete estimation of the possible failure modes, might lead to extensive detector backgrounds or extensive machine damage and a failure of the vacuum system.

The machine protection system will detect errant beam conditions based on many different fault conditions including abnormal beam positions, hardware failure, and the direct observation of beam losses. Fail-safe designs have been quite effective in current operating facilities in ensuring the integrity of the machine protection.

There are two types of BDS magnet system faults: kicker magnets (fast) or conventional / superconducting magnets (slow). There are 24 kicker magnets in the tune-up/fast extraction line and either an erroneous discharge or a fail-to-fire condition results in either a $4 \%$ kick or a $96 \%$ one. In both cases there is, by design, sufficient available aperture in either the straight-ahead condition or the fast extraction line that the missteered beam will arrive at one of the beam dumps. In either the warm or cold design the machine protection system will inhibit the acceleration of subsequent bunches until the fault condition is cleared. The collimator/spoiler elements are physically closest to the beam but are designed to function under an occasional higher than normal beam pulse loss condition. A partially kicked beam will be a design condition.

A failure of a magnet power supply will result in a decay of the magnetic field with a time constant of several seconds. The machine protection system will then inhibit the subsequent bunch. The worst situation occurs when a failure takes place immediately before the first bunch in a train. In the warm linac with a 300 ns bunch train there will be minimal effect on the beam in progress. With the cold linac, the bunch train is also short enough $(\sim 1 \mathrm{~ms})$ that the impact on the beam is small. In this latter case, the response of the machine protection system is fast enough that the option to trigger the fast extraction system also exists. A direct magnet short to ground can produce a much more rapid collapse of the magnetic field than a power supply failure. It will be important to ensure that the MPS system can detect magnet failures of all kinds as quickly as possible. Fortunately catastrophic magnet failures are relatively rare occurrences and are only critical if they occur during a beam pulse itself.

\section{Reason for concern:}

Warm and Cold LC: Engineering/Design - Tested R\&D Design: 2

Collimator/Spoiler engineering designs are feasible but will require beam tests for damage and wakefields. A comprehensive failure mode and effect analysis for the whole complex is difficult. Many effects can result in beam impinging on the collimation elements.

\section{Severity:}

Warm and Cold LC: Steep: Effect on Parameter is quadratic or steeper: 4 Frequent machine component damage can cause significant downtime.

\section{Detection:}

Warm and Cold LC: PreOps: Not detected until facility preoperations: 3

\section{Consequence:}

Warm and Cold LC: Minor: Alternate design available, would need new plan or minor rework: 2

A moderate rework of the protection systems would be required. 


\subsubsection{High power beams (disrupted beam)}

The small spot sizes at the IP needed to produce the desired luminosity result in very high charge densities which cause significant deflections to the opposing beam during the collision process. This results in both the production of synchrotron radiation and large phase space distortions to the outgoing disrupted beam. The extraction line design and magnet aperture are such that the beamstrahlung photons are transported well away from the detector region and it is envisaged that they will be used as a diagnostic. The increased angular divergence and more importantly the energy loss to the disrupted beam result in unavoidable particle losses in the extraction line arising primarily from the over-focusing of the particles in the extreme low energy tail of the distribution. For $1 \mathrm{TeV}$ collisions this loss is estimated at $0.25 \%$ of all particles corresponding to a total power loss of $\sim 5 \mathrm{~kW}$. These losses are reduced by an order of magnitude at $500 \mathrm{GeV}$ due to the non-linear nature of the disruption mechanism. With a judicious choice of extraction line magnet apertures the first systematic loss point will be $\sim 13 \mathrm{~m}$ downstream of the IP. This is sufficiently far removed physically that both detector backgrounds and the quenching of the superconducting elements in the upstream portion of the extraction line should not be a problem. The beam losses are distributed over 7 major loss points and can reach several hundred $\mathrm{W} / \mathrm{m}$ in the absence of any additional collimation. Losses at this level must be addressed by the creation of well shielded collimation regions but do not pose a fundamental risk to machine operation. It is worth remembering that essentially any problem upstream of the IP during beam operation will result in either emittance dilution or the absence of collisions themselves; i.e., beam disruption is eliminated in the presence of other fault conditions.

Beam losses in the superconducting magnets around the IP must be kept below an energy deposition level into the magnet coils of $\mathrm{mJ} / \mathrm{g}$ to avoid magnet quenching. Maintaining the beam halo of a multi MW electron beam below this level, while in principle straightforward, has not been demonstrated. In addition the beam dynamics of halo production is also complex. This uncertainty is strongly mitigated by the huge effective aperture of the magnets in question $(20 \mathrm{~mm}$, corresponding to thousands of beam sigma), so the risk is that of non-standard processes rather than long tails to the distribution.

Reason for concern:

Warm and Cold LC: Beam Physics - Poor or Ambiguous Data Indicate a Problem: 3

Disrupted beam dynamics are only modeled theoretically with no experimental R\&D possible.

Severity:

Warm and Cold LC: Linear: Effect on Parameter is linear: 3

The risk is significant only at ultimate energies.

\section{Detection:}

Warm and Cold LC: PreOps: Not detected until facility preoperations: 3

The problem would be encountered during operation at $1 \mathrm{TeV}$.

\section{Consequence:}

Warm and Cold LC: Minor: Alternate design available, would need new plan or minor rework: 2

A moderate rework of the IP masks and/or extraction line shielding would be required.

\subsubsection{High power beams (detector backgrounds)}

There will be many possible sources of detector backgrounds in the LC including those from the beam-beam interaction and those from halos about the high-power beams. The collimation system has been specified to collimate $0.1 \%$ of the beam without significant backgrounds in the detector. At present, the expected beam halo in both the warm and cold designs is two to three orders-of-magnitude smaller but halo estimation is difficult at best and this factor of 100 - 1000 is thought to be a reasonable margin. Beam tails in the warm option are likely to be larger due to the stronger wakefields; however, other sources of background may be 
larger in the cold design.

Similarly, the beam-beam backgrounds will be controlled using a strong solenoid to constrain the $e^{+} / e^{-}$ pairs, a large outgoing aperture to permit the beamstrahlung and incoming synchrotron radiation to exit the IR without striking any surfaces, and an extraction line with a very large bandpass to prevent particle losses until far from the IP.

Present simulations show that no beam particles and few synchrotron radiation photons will be lost in the IR and the beam-beam backgrounds can be kept under control. However, given the severity of the detector backgrounds and the difficulty usually associated with designing IRs to limit the backgrounds, these issues will carry significant risk. Excessive detector backgrounds will result in a direct reduction of beam intensity and hence luminosity.

Reason for concern:

Warm and Cold LC: Beam Physics - Poor or Ambiguous Data Indicate a Problem: 3

Detector backgrounds are notoriously difficult to estimate from a theoretical basis. No R\&D is practical.

Severity:

Warm and Cold LC: Linear: Effect on Parameter is linear: 3

Backgrounds are generally linear with beam intensity. They are issue at all existing colliding beam facilities, and a severe problem at some.

\section{Detection:}

Warm and Cold LC: PreOps: Not detected until facility preoperations: 3

Detection will come during the commissioning or early operations phase.

\section{Consequence:}

Warm and Cold LC: Major: Possible, but would require major redesign or rework: 3

Minor rework to the Detector trigger logic would be required, at best; a major rework to the collimation/masking system in and around the IP would be needed, at worst.

\subsubsection{Crossing angle issues}

The crossing angle and multi-Tesla solenoid field will pose some new problems for the LC interaction region. To avoid significant luminosity loss, the beams must be "crabbed" to collide head-on. The crab cavities have very tight tolerances on the relative RF phase across the IP. Other tolerances, such as the absolute $\mathrm{RF}$ phase, are looser and are comparable to those demonstrated elsewhere. The crab cavity tolerances in both the warm and cold design are similar and are thought to be attainable using a common RF source and feedback to adjust the relative path lengths of the two waveguide systems. Provided the IP collisions are sufficiently stable, it will be possible to adjust the relative and absolute phases to maximize the luminosity.

A strong IR solenoid is needed to constrain the $e^{+} / e^{-}$pairs arising from the beam-beam interaction however it also introduces significant complications to the IR design. The coupling from the solenoid must be compensated to collide the very flat beams (100:1 aspect ratio) at the IP. To facilitate this coupling correction, the BDS has a coupling correction system at the upstream end of the beam line and a series of dedicated skew correction elements closer to the IP. The coupling is another aberration that must be monitored and tuned; but, provided the BDS is sufficiently stable, it is not expected to present a significant risk.

Another issue common to both designs and related to the interaction of the solenoid and the crossing angle is the deflection of the trajectory, which will result in a non-zero outgoing trajectory and a vertical crossing angle at the IP. The vertical crossing angle should not require correction for $e^{+} / e^{-}$collisions. Small roll adjustments will be incorporated in the crab cavities to facilitate tuning. To facilitate the extraction line diagnostics, the outgoing trajectory will need to be corrected which complicates the IR design but is not a significant risk. 
Reason for concern:

Warm and Cold LC: Engineering/Design - Engineering Feasible, but Untested Design: 3

Severity:

Warm and Cold LC: Contributing: Parameter dominated by other effects: 1

A moderate luminosity reduction would result. (3\% reduction for $0.3^{\circ}$ crab cavity phase error).

Detection:

Warm and Cold LC: PreOps: Not detected until facility preoperations: 3

Consequence:

Warm and Cold LC: Minor: Alternate design available, would need new plan or minor rework: 2

A minor engineering upgrade would be required.

\subsection{Operations and machine protection}

\subsubsection{The operation of the linear collider requires too much manual, non- automated, intervention to maintain subsystem optimization}

Operations will involve a combination of automated and manual tasks. The tasks will be managed by the control system computers. Automated tasks are those which are scheduled and implemented without direct intervention or monitoring. The list of such tasks is large and includes steering feedback, matching the linac energy gain with the focus magnet profile, and interaction region feedback. Manual tasks are usually not routinely scheduled and may involve testing or calibration of various components such as beam size or position monitors. Some more complex manual tasks may involve the optimization of non-linear sub-systems or sub-systems with inherently difficult optimization procedures, such as collimation. These will require high level tools that simplify the process. Each task will have a user interface in order to summarize its behavior and to control its critical parameters.

This system is well beyond the state of the art. Existing machines do not need to rely on so much control system application software. It is known that there are no fundamental problems extending present day systems, except that a great deal of coordination and foresight is required for integration and convergence. Many applications will require prototyping and testing, along with subsequent redesign and development.

Reason for concern:

Warm and Cold LC: Engineering/Design - Engineering Feasible, but Untested Design: 3

Experience has shown the success of large sets of such tasks to be very strongly dependent on credible performance expectations, carefully managed exception handling and extensive testing. There are many examples of well planned automated tasks that have functioned adequately. In cases where the physical response of the machine and associated instrumentation is not well characterized, several prototyping and testing stages are necessary. The concern is that such a system will demand a very large effort and that analysis and redevelopment will be needed more or less indefinitely.

The risk is somewhat different for the cold machine, where less high level application software may be needed for linac beam control but a great deal more is required to manage the accelerator itself. A good example is the integration of the low level RF vector sum feedback controllers. Experience has shown that a large scale application of multi-loop digital controllers, like that required for the cold linac, must include comprehensive diagnostic and modeling tools that are broadly understood and accessible.

Severity:

Warm and Cold LC: Linear: Effect on Parameter is linear: 3

Each task which lacks adequate controls handling will have to be done manually. When the number of 
such tasks exceeds the available resources and time, the machine operation will be limited to those beam parameters for which the set of such tasks is manageable. Thus if one uses, as a performance parameter, the somewhat artificial count of the number of planned controls tasks which fail and thus require continual manual action, the impact is a limiting one. The tasks can be ranked in order of their impact on luminosity. There will be a few such tasks, such as beam trajectory optimization in the warm machine, which will have a relatively high impact on the luminosity, and these will have a correspondingly higher priority.

Perhaps the best metric to use is the number of high impact tasks which fail in a significant way compared to their performance expectations. It was not possible to enumerate these as part of this study. Consider, as an example, a set of tasks whose result is control of emittance in the warm machine, or control of the beam loading compensation in the cold machine (e.g. the interface to the LLRF vector sum controller). If either fails, it probably fails for both the positron and electron halves of the machine. In this example, the emittance or energy distribution is accordingly larger than specified, both beam sizes are increased in a less than linear fashion but the combined effect on the luminosity is a linear reduction.

We feel that controls failures will limit the machine in a linear way.

\section{Detection:}

Warm and Cold LC: PreOps: Not detected until facility preoperations: 3

It is important to plan on several cycles of prototyping and developing applications software, especially for the more important tasks. Since controls development is an issue of scale, detection is likely only when the scale of the system begins to approach that actually needed.

\section{Consequence:}

Warm and Cold LC: Major: Possible, but would require major redesign or rework: 3 Depending on the severity of failure, rework could require major modifications to the control system software, delaying full operation for an extended period and involving as much as $20 \%$ of the original effort.

\subsubsection{The machine protection system does not allow the production of steady high power beam}

The machine protection system (MPS) is the set of all devices which allow continued, smooth, operation of the machine with a minimal chance of beam-related component damage or the generation of unacceptable levels of residual radioactivity. Integration of MPS also involves allocating redundancy to prevent simple single point faults from stopping the high power beam. Generally, the MPS consists of beamline components, associated sensors, beam diagnostic devices, and an interconnection system that controls beam pulses and beam power. The MPS includes automated fault logging, recovery and some level of self-diagnosis that is used to predict performance at a power higher than the current power.

\section{Reason for concern:}

Warm and Cold LC: Engineering/Design - Engineering Feasible, but Untested Design: 3

The linear collider beam energy density is far beyond that of existing accelerators. The scale of the system and the level of automation required is beyond the state of the art. For the warm and cold design, a single, nominal current and nominal transverse dimension fully damped bunch will seriously damage any metal surface. No existing machine has an MPS capable of protecting against "single-beam-pulse" damage. Existing state of the art machine protection systems require extensive human intervention and are based on a sequence of interlock chains. As a result, although such systems provide effective protection of the machine components, they seriously limit steady beam operation. While design concepts exist, both these and issues of scale must be tested before a satisfactory system can be claimed.

Because so little of the design can be extrapolated from existing low power machines, MPS development will require three stages: 1) understanding and testing the basic interaction between the beam and beamline components, 2) development of mechanical engineering guidelines which result in designs that are optimized 
from an MPS point of view and 3) development of controls strategies that are at once reliable, redundant and flexible. The most practical, cost-effective MPS involves optimizing and integrating stages 2) and 3) so that reasonable, well understood designs can be implemented. Of the three stages, the most serious risk is in stage 1), which involves the greatest stretch from existing state of the art. The primary concern associated with stage 1) is the understanding of what happens when a single damped beam (low emittance), nominal current bunch strikes a vacuum chamber or part of a vacuum chamber.

To address the risk associated with single bunches, testing has started using the SLAC Final Focus Test Beam. These tests show that all metals are punctured by nominal emittance, nominal current bunches. It is not clear how to, or indeed whether to, adapt the design of the accelerator structure, warm or cold, in response to this result. It is clear that in the complex parts of the linear collider, such as transitions from one sub-system to another (as in the beginning or end of the linac), care must be taken so that relatively simple component failures do not result in a vacuum system leak, an irrevocably damaged accelerator iris or a leaking cryomodule cavity.

A key issue is whether the machine is "naturally" protected, so that, for instance, the transverse size of a bunch is increased many fold due to aberrations before it hits the vacuum chamber. Studies done using the NLC main linac lattice indicate that, for certain rather improbable failures (e.g. complete loss of excitation of an individual quadrupole magnet pole - with the other three remaining at full excitation), the bunch dimensions are basically unchanged as it moves from the linac centerline to the full iris radius. The MPS design will rely on models of failed components and estimates of what happens to the beam.

In the warm machine, there is no practical way to stop an aberrant train during the pulse; in contrast, this is easy to do in the cold where the bunch to bunch spacing is about $300 \mathrm{~ns}$. Several machines use the "within the pulse" MPS planned for the cold machine.

The scope of the MPS is illustrated by the following example. Both the warm and cold designs use a midlinac undulator, for the production of positrons, with an axis that is displaced from the main linac axis. The deflection system required to divert the primary electron beam has a much smaller energy bandpass than the linac itself $(3 \%$, or about $10 \times$ narrower), so that off-energy machine pulses are likely to hit those components. The MPS must: 1) include beamline hardware that is not destroyed by simple failures of the upstream RF system, and, since it may be quite expensive to do this completely, 2) it should include designs that limit the likelihood of those failures (such as redundancy) and 3) include a "fast beam permit" system that checks that all devices (especially those that can cause an energy change of more than $2 \%$ ) are in order just before extracting the beam from the damping ring. There are several locations in the collider similar to this one, in addition to systems with special concerns (e.g. the damping ring small aperture permanent magnet wiggler).

It is important to keep in mind that it is not enough for the MPS to simply protect against component damage; it must also keep interruptions to a minimum and provide for fast recovery from each one.

\section{Severity:}

Warm and Cold LC: Limiting: Effect on Parameter is a limit less than design: 5

MPS failure occurs when a component or group of components is improperly designed or protected and the beam power must be accordingly restricted. Since beam is interrupted in a large portion of the complex, the performance of MPS is dictated by its weakest link. The system has failed when the beamline design does not allow simple straightforward controls hardware to provide protection. There were examples of this at SLC, where limited beamline space did not allow effective collimation and, as a direct result, it was not possible to protect the machine from simple failures. A hair trigger, very high gain, ion chamber system was implemented giving a system that provided both poor protection and caused substantial beam operations interruptions. A failed MPS system is considered a limiting failure.

\section{Detection:}

Warm and Cold LC: PreOps: Not detected until facility preoperations: 3

Detection of a failed MPS system can occur at any time during the commissioning of the machine, but the 
system is not properly tested until full power operation has been achieved.

\section{Consequence:}

Warm and Cold LC: Major: Possible, but would require major redesign or rework: 3

Based on experience, rework would include mechanical design and controls architecture redesign and reconstruction. If redundant high power components are needed to protect against simple faults, these must also be added.

\subsection{Summary and conclusions}

\subsubsection{Risk assessment summary}

The numerical rankings for each risk identified in the previous chapters have been multiplied together for each risk, and the products are displayed Fig. 8.8.1.1.

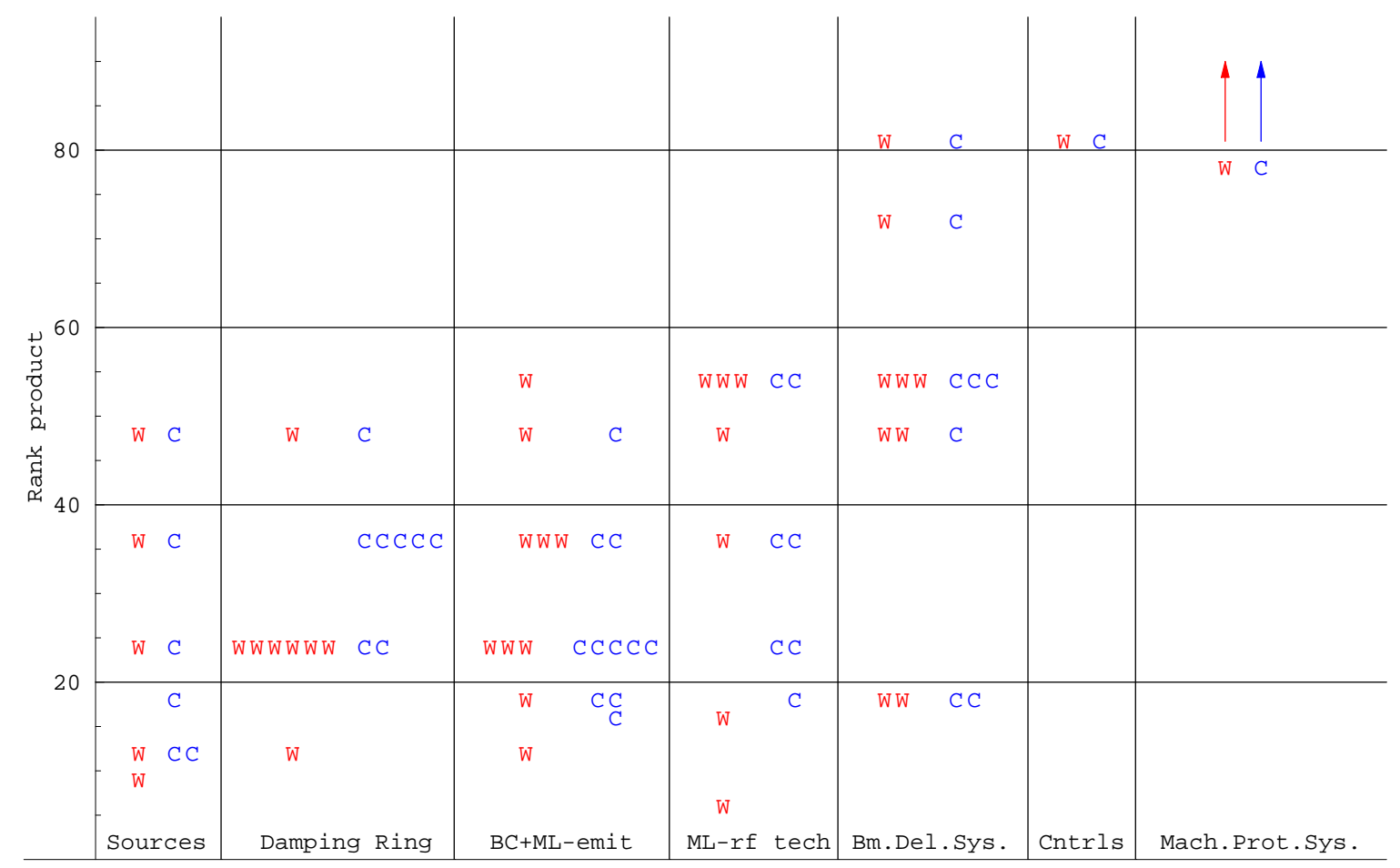

Figure 8.8.1.1: Risk assessment summary plot. The numerical rankings for each risk identified in the previous chapters have been multiplied together to form the rank product. It is important to emphasis that this product does not have any direct interpretation such as a "probability of failure". But the distribution of rank products provides a useful comparison and priority ranking method for mitigation efforts. 


\subsubsection{Conclusions}

Risk assessments for the major collider subsystems have been carried out. In these assessments, the risks have been evaluated in terms of the highest level collider parameters, energy and luminosity, and relative to the primary mission of the collider, as specified by the physics design requirements: the delivery of $500 \mathrm{fb}^{-1}$ of integrated luminosity at $500 \mathrm{GeV}$ in the first 4 years of operation.

The most important risks have been identified for the following major collider subsystems: electron and positron sources; damping rings; bunch compressors and main linacs: emittance preservation and beam jitter; main linacs: RF technology; beam delivery systems; and controls and machine protection. The identified risks were assessed and categorized using the numerical scores in the tables described in the introduction to this section (Section 8.1.) This provides a relative ranking of the risks to the mission, which can be compared across major collider subsystems, and between the two technology options.

As shown in Fig. 8.8.1.1, this analysis revealed that the highest potential risks to the mission for both options are in the areas of the machine protection systems (MPS), the control systems, and the beam delivery systems. The conclusion that the highest potential risks do not lie in the area of high gradient RF cavity development came as a surprise to us. This is due primarily to the intensive past and current technology R\&D programs in this area. The high risks in the beam delivery systems, the controls systems, and the MPS are related to the fact that problems here generally are not encountered until the pre-operations phase of the project, which limits the possible response time.

The other major features shown on this plot are the smaller risks for the cold option than the warm option in the main linac, for both RF technology and emittance preservation; and the substantial difference in the risks for the damping rings, between the two options.

\subsubsection{Machine protection systems and control systems}

The highest risk identified for any collider system, and essentially the same (although details differ) for both technology options, was in the MPS. The MPS is the set of all devices which allow continued, smooth, operation of the machine with a minimal chance of beam-related component damage or the generation of unacceptable levels of residual radioactivity. The risk is that the MPS does not allow the production of steady high power beam.

The linear collider beam energy density is beyond that of existing accelerators. For both technology options, a single, nominal current and nominal transverse dimension fully damped bunch will seriously damage any metal surface. No existing machine has an MPS capable of protecting against single-beam-pulse damage. It is important to keep in mind that it is not enough for the MPS to simply protect against component damage; it must also keep interruptions to a minimum and provide for fast recovery from each one. While design concepts exist, both these and issues of scale must be tested before a satisfactory system can be claimed.

Detection of a failed MPS system can occur at any time during the commissioning of the machine, but the system is not properly tested until full power operation has been achieved. Based on experience, rework, if necessary, would include mechanical design and controls architecture redesign and reconstruction. If redundant high power components are needed to protect against simple faults, these must also be added.

Risks associated with the machine protection systems need further elaboration and study.

Control-system related risks were also identified as being among the most severe. In this case, the risk is that, due to an inadequately specified or implemented control system, the operation of the linear collider requires too much manual, non-automated, intervention to maintain subsystem optimization. Although they have different controls challenges in detail, this risk was deemed to be equal for the two technology options. The controls requirements demand a system which is well beyond the current state of the art. The concern is that such a system will demand a very large effort and that analysis and redevelopment will be needed 
more or less indefinitely. Since controls development is an issue of scale, detection is likely only when the scale of the system begins to approach that actually needed. Depending on the severity of failure, rework could require major modifications to the control system software, delaying full operation for an extended period and involving as much as $20 \%$ of the original effort.

\subsubsection{Beam delivery systems}

The highest risk identified in the beam delivery system is that associated with detector backgrounds due to the high power beams. This risk was seen as being roughly the same for the two technology options. Present simulations show that no beam particles and few synchrotron radiation photons will be lost in the IR, and the beam-beam backgrounds can be kept under control. However, given the potential severity of the detector backgrounds, and the difficulty usually associated with designing IRs to limit the backgrounds, these issues will carry significant risk. The problems would not be detected until the early operations phase of the project, and their resolution could require a major rework to the collimation/masking system in and around the IP.

For the cold option, the second highest risk in the beam delivery system was identified to be the intratrain IP position feedback. Train-to-train IP deflection feedbacks have been used to stabilize the collisions in many colliding beam facilities. Despite the past success, the IP stabilization requirements and the IR environment in the LC will be very different from that in previous experiments. The stabilization requirements are at the nanometer level and are hundreds of times smaller than that previously achieved. In addition, potential background sources which can impact the BPMs, essential for the deflection feedback, are thousands of times higher than in the past examples. The stabilization requirements are tighter (because of the larger beam disruption) and the backgrounds larger (because of the higher beam power) for the cold option. In addition, the cold option uses a high bandwidth intra-train system, with more challenging requirements than for trainto-train systems. While modeling of the IR environment and prototyping of the feedback components will be useful, the real performance limitations will not be encountered until the system is commissioned with colliding beams.

For the warm option, the second highest risk in the beam delivery system was judged to be control of jitter of the final focusing magnets, at the $0.5 \mathrm{~nm}$ level, for frequencies above $10 \mathrm{~Hz}$. Prototype active stabilization systems have stabilized rigid objects at the desired levels, and there is an active program aimed at demonstration of the active stabilization of a superconducting quadrupole similar to that needed for the final magnet. Nevertheless, the real performance limitations will not be encountered until testing in the actual IR environment, with the complete system, including the experimental detector, in place.

\subsubsection{Main linacs}

As exhibited in Fig. 8.8.1.1, the warm option has higher risk than the cold option in the areas of emittance control in the main linacs, and the main linac RF technology.

The three highest risk areas for the warm option main linac RF technology were identified to be engineering and design issues related to klystron lifetime, the production of the SLED-II pulse compression systems, and the production of the RF structures. Two comparable engineering and design risk areas were identified for the cold option: klystron lifetime, and the production of the superconducting RF cavities. In all these areas, the risks were related to the difficulties of scale-up from prototype systems to the mass production of many reliable, high performance units needed for the complete system. Potential scale-up and lifetime issues are assumed to be resolved during the construction of the prototype main linac, which takes place during the project engineering and design phase. The construction and test of this prototype of the main linac is key to mitigating these scale-up risks at an early phase of the project.

The highest risk for the warm option in the area of emittance control in the main linac was judged to be 
potential beam jitter arising from uncontrolled or unanticipated cultural and/or technical noise sources. Control of sources of component vibration must be comprehensive across the full extent of the main linacs. The effects of excessive beam jitter due to the full spectrum of possible cultural and technical noise sources will not be detected until the pre-operation phase of the project. In the case of the warm option, excessive beam jitter would require, for example, the use of active stabilization on all the main linac components, which could be a major re-work. If this risk and the effort of remediation is perceived to be too high, active stabilization elements could be included with the quadrupole installation for minimal additional cost. The cold option design utilizes a series of intra-train feedback systems in the main linacs, which will be able to control high-frequency beam jitter. The issues of backgrounds and stabilization requirements at the sub-nanometer level, which make the IP intratrain-feedback difficult, are not present for these linac systems.

It should be noted that the "quasi-static" tuning algorithms used for beam-based alignment were judged to be a risk almost as severe as the beam jitter risk described in the previous paragraph, and roughly equal for both options. Confidence that the main linac emittance growth budgets for the warm and cold options can be met are based on the predictions of the performance of these algorithms in simulations, and there are a number of features of the simulations which are less than fully realistic. Serious issues are the potential sensitivity of the beam-based alignment procedures to beam jitter, and the scale-up of these procedures to a large system. Information on the application of the quasi-static tuning procedures to a large-scale system will be provided during operation of the prototype main linac in the project engineering and design phase, which is another important reason for this prototype.

\subsubsection{Damping Rings}

The item of most concern in the warm option damping rings was judged to be the risk that the electron cloud causes a beam instability in the positron damping ring. Simulation codes indicate that both warm and cold LC positron damping rings could suffer from electron cloud effects. Although the results of codes used to simulate build-up of the cloud are in agreement with some of the limited data available, the predicted effects are sometimes not the ones observed. The result could be a significant growth in emittance, and/or limitation of the current that can be injected into the ring, both of which will impact the luminosity. The much shorter bunch separation in the warm LC damping ring (1.4 ns) compared to the cold LC damping ring (20 ns) means that any electron cloud effects are likely to be more severe in the warm LC damping rings. Studies are focusing on developing low SEY coatings for the chamber walls, since solenoids will not be effective in regions where there are wiggler or multipole fields, where the most significant problems are expected. An R\&D program has already begun, that is likely to lead to a solution if the effects are as predicted.

The item of most concern in the cold option damping rings was that the vacuum chamber impedance drives microwave beam instabilities. Existing storage rings have achieved impedance values significantly below the microwave threshold for the warm LC damping rings. The structure of the cold LC damping rings drives the threshold about an order of magnitude lower, to a more challenging regime. If necessary, a major modification of the cold damping ring vacuum chamber would be a much larger task than a modification of the warm damping ring vacuum chambers. A larger than expected impedance will require a proportional reduction in current in order to stay below the instability threshold. Electron and positron damping rings are likely to be equally affected. It is possible to include some margin in the design to allow for this, but the real impedance of the damping rings will not be known for certain until they are commissioned with beam.

The two risks discussed in the previous two paragraphs, for the warm and cold options, were judged to be of equal concern. However, an additional four less severe risks were identified, all of which were of more concern for the cold option than the warm option. These were the risks that beam acceptance limitations cause poor injection efficiency, that the coupling correction fails to achieve the specified vertical emittance, that the extracted vertical beam has large jitter, and that stray magnetic fields cause emittance growth.

When all the risks that were identified are considered, the cold option has more risk to achieving the required 
damping ring performance than the warm option. (See Fig. 8.8.1.1.) This is expected given the novel design of the ring. It is possible that the current design of the cold damping ring could be improved, but there are a number of difficult features, related to the bunch format required by the cold RF technology, inherent in the design of any damping ring for the cold option. These features make it more difficult to achieve the same level of risk as for the smaller, more conventional, warm option damping rings.

\subsubsection{Electron and positron sources}

The highest risk area identified for the electron and positron sources was the engineering of the rotating positron production target. The cold design relies upon a rapidly spinning $(1200 \mathrm{rpm})$, large diameter $(\sim 1.6$ $\mathrm{m}$ ), water cooled Ti-alloy annulus. The warm design relies on a slowly rotating but similarly large diameter, water cooled Ti-alloy annulus. The incident power, absorbed energy, and ambient radiation in the cold design is a factor of 1.6 times greater than for the warm design. Both designs are presently based on the high strength properties of Ti-alloy for which little information is available regarding the effect of radiation on the mechanical properties of the material. If the Ti-alloys are not sufficiently robust, considerable redesign of the target systems may be required. Additional engineering studies and radiation tests are required to alleviate these concerns. The risk was judged to be roughly the same for the two technology options.

The second highest risk area was the laser for the polarized electron gun. The lasers for either design are complex systems requiring significant engineering and prototyping prior to design completion. Of primary concern are the issues of amplitude, pointing, wavelength, and polarization stability and of maintainability. Complete stabilization to within specifications are likely to take several years of attention after the sources are turned on. The different bunch formats of the warm and cold LC designs require different laser systems each of which will have their own specific problems and remedies. Because of the development of lasers for the TTF and TFEL projects, some of the solutions for the cold LC are presently being addressed and tested. The risks in this area were judged to be comparable for the two technology options.

\subsubsection{Near-term R\&D challenges}

Both options have significant challenges in near-term beam physics and technology R\&D to limit future risks to the project. A number of potentially high risks were identified in this exercise as being able to be mitigated through R\&D. In Table 8.8.2.1, we present a list of the risks whose "Detection" score was 1, corresponding to R\&D, for the warm option. A similar list is presented in Table 8.8.2.2 for the cold option. The risks are sorted with the most severe risks at the top. The horizontal lines group risks of equal total risk product.

These tables indicate the identified risks which can be addressed through R\&D. The highlights are target engineering for the undulator source, the electron cloud issue in the positron damping ring, and pulse compression and gradient demonstrations in the main linac RF technology programs. Additional important issues are helical undulator development, damping ring kickers, dark current effects in the main linacs, and main linac structure wakes. Many, but not all, of the risks listed in these tables are the subject of currently active $\mathrm{R} \& \mathrm{D}$ programs. It is important for the success of the linear collider project that $\mathrm{R} \& \mathrm{D}$ programs which address all these issues are successful, since deferral of any of these issues to later in the project may substantially increase the risk. 

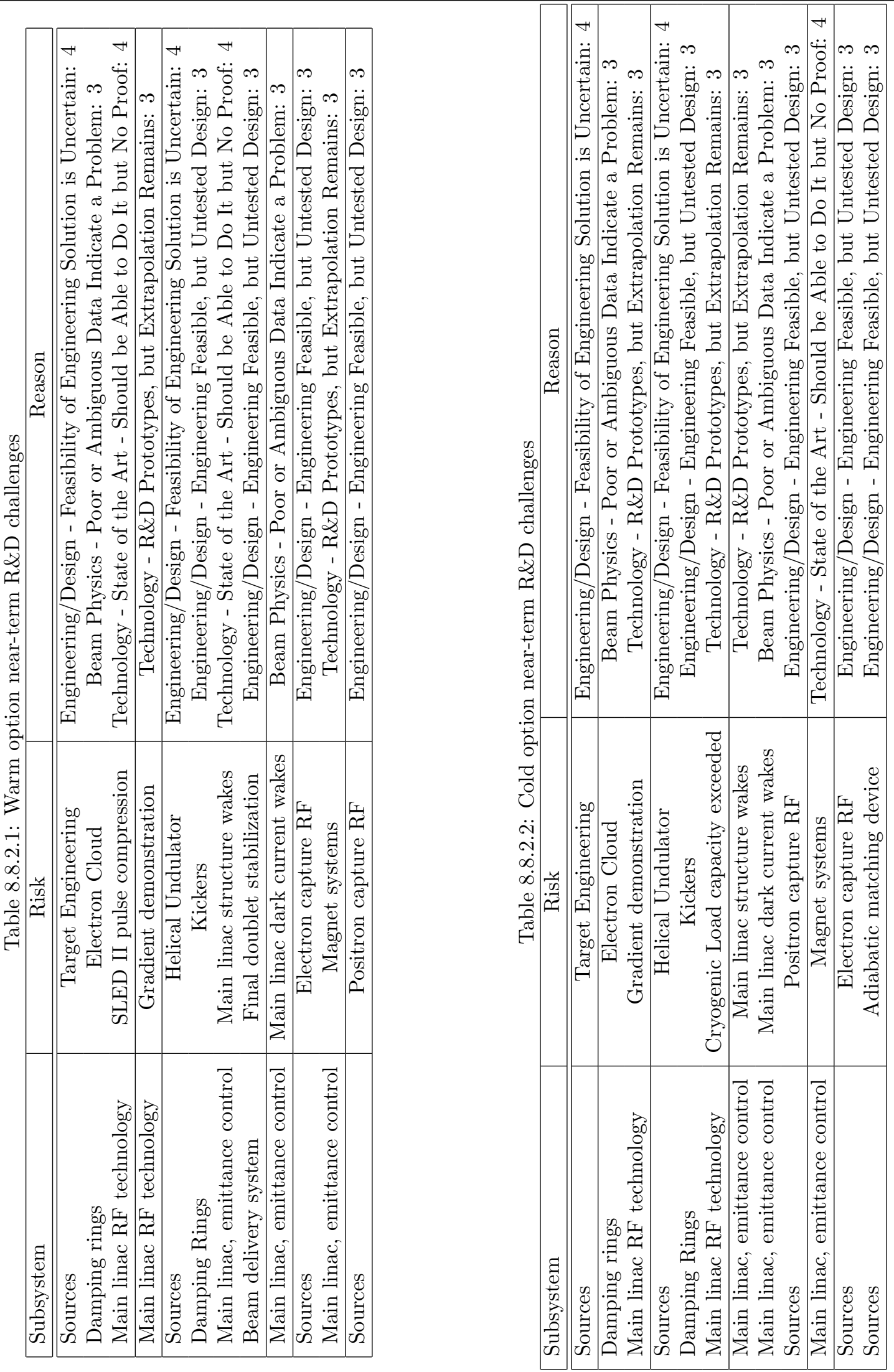


\section{Chapter 9}

\section{Conclusions}

\section{$9.1 \quad$ Overview}

The Accelerator Subcommittee of the U. S. Linear Collider Steering Group (USLCSG) has carried out the evaluation of two options for the siting of a linear collider in the US. The two options were a warm option, following the design of the GLC/NLC Collaboration[ZDR, NLC01],[TRC, Chapter 3], and a cold option, similar to the TESLA design[TDR] at DESY. Both options were developed in concert, using, as much as possible, similar approaches in technical design for similar accelerator systems, and a common approach to cost and schedule estimation, reliability design and evaluation, and to project risk assessments.

To carry out the charge, the Accelerator Subcommittee formed five task forces, to address the following issues:

- Accelerator physics and technology design

- Cost and schedule

- Civil construction and siting

- Availability design

- Risk assessment

The sections below summarize the conclusions of the task forces.

The two technologies examined in this study have different challenges, advantages, and disadvantages, and differ in many details. We found that, within relative factors of $30 \%$ or less, the two approaches would provide similar technical performance at roughly equivalent cost. The two options can ${ }^{1}$ have similar levels of availability, with comparable overall levels of risk, and can be realized on roughly the same schedule.

These two options are at comparable levels of development, and both have the potential to provide a viable route to a linear collider which meets the requirements of the USLCSG.

\footnotetext{
${ }^{1}$ See Section 9.3 .2
} 


\subsection{Design}

In order to facilitate the comparison between the two linear collider technology options, the designs have been crafted with as much commonality as possible. Thus, both designs

- have the same initial stage energy reach;

- are upgradeable to $1 \mathrm{TeV}$ without additional underground construction;

- use an undulator-based positron source, capable of being upgraded to provide polarized positrons;

- have almost identical beam delivery systems and IR configurations;

- require no change to the injector parameters for the upgrade to $1 \mathrm{TeV}$.

\subsection{1 $500 \mathrm{GeV}$ reference designs}

Feasible designs have been established for both technology options which meet the physics mission requirements of the US Linear Collider Steering Group. These designs draw heavily on the work of the GLC/NLC collaboration for the warm technology option, and the work of the TESLA collaboration for the cold technology option.

The physics mission requirements are presented in Section 3.1. To meet these requirements, both the GLC/NLC 2003[ZDR, NLC01],[TRC, Chapter 3] design for the warm technology, and the TESLA TDR[TDR] design for the cold technology, have been modified. In the cold technology case, modifications have also been made to respond to concerns identified during the ILC-TRC [TRC] review. The overall list of design modifications is presented in Section 3.5.1. The modifications from the TESLA TDR for the cold technology are more extensive than the modifications from the GLC/NLC 2003 design for the warm technology.

The cold option has a design luminosity at $500 \mathrm{GeV}$ which is about $25 \%$ higher than that of the warm option. The geometric luminosity (i.e., the luminosity computed in the absence of beam-beam effects) is roughly the same for both options, despite the radically different bunch formats arising from the needs of the different $\mathrm{RF}$ technologies. However, the cold option, with its longer bunch length, has a higher value of the disruption parameter, leading both to a higher value of the luminosity, and to a greater sensitivity of the luminosity to beam offsets at the collision point, and to correlations in the emittance distribution at the IP.

Both $500 \mathrm{GeV}$ reference designs have an energy reach, at reduced luminosity, of up to about $625 \mathrm{GeV}$. The luminosity vs energy for both options, assuming fixed RF power and fixed cryogenic cooling capacity, is shown in Fig. 3.2.1.1. For the warm option, the energy reach is available because the loaded gradient (52 $\mathrm{MV} / \mathrm{m}$ at design beam current) increases as the current is reduced, and the installed cavities will be qualified up to their unloaded gradient of $65 \mathrm{MV} / \mathrm{m}$. For the cold option, the $500 \mathrm{GeV}$ design gradient is $28 \mathrm{MV} / \mathrm{m}$, but the installed cavities will be qualified up to their maximum gradient of $35 \mathrm{MV} / \mathrm{m}$. For operation above $500 \mathrm{GeV}$ with the cold option, the current will be reduced to keep the RF power to the beam constant, and the cycle rate will be reduced as needed to keep within the limits of the installed cryogenic power.

The length of the conventional construction footprint for the cold option is about $42 \%$ greater than for the warm option.

The $\mathrm{AC}$ power required to run the $0.5 \mathrm{TeV}$ cold reference design is about $30 \%$ less than that of the warm reference design. 


\subsubsection{Upgrade features of the reference designs}

Both options use a helical-undulator-based positron source, driven by a $150 \mathrm{GeV}$ electron beam. For the 500 $\mathrm{GeV}$ reference design, a short undulator is used, which provides enough unpolarized positron flux to meet the design requirements. Sufficient length in the lattice is provided for extending the length of the undulator, which allows an adiabatic upgrade path to a polarized positron source.

In order to allow for the possibility of an adiabatic energy upgrade to $1 \mathrm{TeV}$, both technology options have been placed in tunnels which are long enough to accommodate an extension of the linac to $1 \mathrm{TeV}$ at the maximum loaded gradient. At $500 \mathrm{GeV}$, the tunnels will be partially filled with linac structures; the remainder of the tunnel will contain a beam transport line. Consequently, by replacing the beam transport line with linac structures, both options can be upgraded to $1 \mathrm{TeV}$ without additional underground civil construction.

As at $500 \mathrm{GeV}$, the upgraded cold option and warm option have similar geometric luminosities, but because of the higher disruption parameter, the cold option has a design luminosity which is about $25 \%$ higher than that of the warm option at $1 \mathrm{TeV}$.

The upgraded warm option has an energy reach of up to about $1.3 \mathrm{TeV}$, as shown in Fig. 3.2.2.1. As for the $500 \mathrm{GeV}$ case, this energy reach is available by operation with lower current, and increased gradient, up to the unloaded gradient of $65 \mathrm{MV} / \mathrm{m}$. For the cold option, since the cavities must operate at their maximum gradient of $35 \mathrm{MV} / \mathrm{m}$ to reach $1 \mathrm{TeV}$, operation above $1 \mathrm{TeV}$ is not possible.

It should be noted that the upgrade energy is limited by the maximum gradient achievable in the linac structures installed for the upgrade. If, by the time the upgrade is implemented, R\&D has developed structures with higher maximum gradients, an upgrade energy in excess of $1 \mathrm{TeV}$ would be possible.

\subsection{Availability}

The availability task force assessed the impact of various technology or configuration choices on overall machine performance. The approach taken was to set an overall availability goal for any linear collider and then use that to develop an unavailability budget, which apportioned the down time among the different systems of the accelerator. The required availability specifications were compared to the performance of existing accelerators to see how much the reliability of individual components must be improved. This level of improvement could then be used to determine the added costs needed to achieve the budgeted availability and to assess the risks of not achieving the necessary improvements.

Note that the goal was not to determine the availability of each proposed option or variant and declare it a bad design if it was down too much. Rather, the assumption is that, with proper design, either of the options or any of the variants can be made available enough; it is only a matter of how much it will cost, and the risk of failure if components must be made too much more reliable than the present state-of-the-art.

Given the limited time available, this study is of necessity only a first crude step. Nevertheless, it has produced a very useful simulation tool to evaluate the overall availability of an accelerator. While the tool has not been benchmarked against an existing accelerator and overall predicted downtimes could change with different assumptions, we believe the comparisons between different accelerator options and variants (warm/cold, 1 vs. 2 tunnel, conventional vs. undulator $e^{+}$source) are quite significant.

\subsubsection{Method and assumptions}

It was decided to allow $25 \%$ downtime due to hardware problems and the recovery from them. This is comparable to what present HEP accelerators accomplish after several years of effort. The LC is much more 
complex, so it will be more difficult to achieve. Of the $25 \%$ downtime, only $15 \%$ was explicitly budgeted to specific devices. The other $10 \%$ was held as contingency for devices that were not included or for major design mistakes that cause poor reliability and that remain a problem after a few years of operation.

A detailed list of components was compiled for two major regions of the collider, the linacs and damping rings (DRs). These regions were chosen for detailed analysis both because of their size and complexity, and because they were the areas that changed most significantly depending on the main linac technology. Due to lack of time, the other regions of the accelerator (the sources, bunch compressors, beam delivery system (BDS), site power, cryo plant, and global control system) were not modeled in detail. Instead, they were treated as individual elements, with a mean time between failure (MTBF) and mean time to repair (MTTR) for the whole region.

For the main linacs and the DRs, the detailed component list included items identified as potential sources of failure from experience with existing facilities, such as rf components, magnets, magnet power supplies, power supply controllers, vacuum pumps, pump power supplies, movers, diagnostics, etc., with counts for each item. Each component was then assigned a starting MTBF and MTTR. Where possible, the numbers were based on data from repair statistics at SLAC, Fermilab or other labs.

A simulation was written which used all of this input data to estimate the availability of the whole LC. The simulation allows several complexities to be handled that would have been nearly impossible in a spreadsheet and quite difficult in the commercial software packages. These complexities include the recovery and tuning time needed after a downtime, the complex redundancies built into the LC designs, the way in which accelerator physics experiments (Machine Development or MD) can be done when only part of the accelerator is down, and the way in which many devices are typically repaired during an access by a limited number of people.

Recovery of the beam is modeled in a crude fashion which matches the qualitative experience in many accelerators. This common experience is that it takes time to recover good beams after a downtime. In fact, the longer the down, the longer the recovery time. The simulation simply assumes that the time it takes to get good beam out of a region of the accelerator is proportional to the time that region was without beam.

Machine Development is an essential tax on the operating efficiency of any accelerator. It is time used to better characterize the machine, develop new tuning procedures, and test possible future improvements. For this simulation, the LC is assumed to have operated for a few years and to have settled into a nominal schedule of MD, which would occupy approximately $10 \%$ of the time. As with the recovery time, the MD was allocated to the individual regions of the machine.

To more fully mirror the complexity of operation of a real machine, the simulation assumed that some of the required MD could be done on an opportunistic basis. This opportunistic MD time is tracked by the simulation. It then assumes that sometime during the running period enough scheduled MD is done in each region to bring the total of opportunistic + scheduled MD up to the desired levels.

\subsubsection{Results}

The simulation assumes the accelerator has reached a steady state after several years of operation. The results of the simulation gave the total unavailability and how each device type contributed to it. As expected, the availability of the LC was too poor and it was necessary to improve the MTBFs of some devices to attain the goal availability.

Using nominal present day MTBFs, except in a few cases, the downtimes of the warm and cold LCs end up being 28 and $31 \%$, respectively. These are remarkably similar, and remarkably good given how complex the LCs are. However, since the regions not modelled in detail were given fixed downtime budgets that cause a downtime of about $10 \%$, the linac and damping rings cause about $30-10=20 \%$ downtime with the nominal MTBFs. This had to be reduced to $5 \%$, or a factor of about 4 , to fit into the budget. 
Typically, the devices which were contributing more than most to the unavailability were identified and improved. To achieve the required unavailability of $15 \%$, it was necessary to require improvement factors of 3-15 (3-50) over nominal for the MTBF's of 8 (12) general categories of components, for both the warm (cold) reference design options. For the cold option, the linac energy overhead also had to be increased from $2 \%$ to $3 \%$. A very crude estimate of the required cost increase associated with these component reliability improvements was of the order of $2 \%$ of total project cost, for both options. These additional costs have not been included in the cost estimates described in Chapter 5 .

After these improvements, the amount of time integrating luminosity calculated by the simulations for the warm (cold) options was $75 \%(74 \%)$. When the $10 \%$ downtime held as contingency is included, this drops to $65 \%(64 \%)$. In addition to operations $(65 \%)$ and downtime(25\%), roughly $10 \%$ of the time is spent on scheduled MD.

Availability comparisons were made between an undulator $e^{+}$source and a conventional $e^{+}$source. The positron source was not modeled in detail and the same overall MTBF was used for both systems. The significant difference is that the undulator source requires high energy electrons before positrons can be produced, while the conventional source does not. This one difference changes the downtime from $15 \%$ to about $11 \%$ because of the shorter recovery time when both systems can be restored independently. More importantly, the amount of time spent integrating luminosity increases from about $75 \%$ to over $85 \%$. This increase is due to the reduced downtime mentioned above, and to a decrease in the time spent in scheduled MD (1-4\%) because more MD can be done opportunistically. The increase in opportunistic MD comes about because the conventional $e^{+}$source does not require high energy electrons before positrons can be produced. Thus, if differences between the actual reliabilities of the sources are ignored, the choice of an undulator source in order to potentially produce polarized positrons reduces the integrated luminosity by more than $15 \%$.

The availability of the one tunnel variant of the cold LC was also studied. With the same MTBFs, the one tunnel cold option unavailability is $25 \%$. To regain the required $15 \%$ unavailability, the linac and damping ring component MTBF's must be improved by another factor of 3 on average, a total of a factor of 12 over nominal. To achieve this, it was necessary to require improvement factors of 3-80 over nominal for the MTBF's of 26 general categories of components, and the linac energy overhead also had to be increased from $2 \%$ to $8 \%$. A very crude estimate of the required cost increase associated with these component reliability improvements was of the order of $3 \%$ of total project cost, beyond those required to achieve $15 \%$ for the two tunnel cold reference design.

Studies were made of the sensitivity of the results to changes in the recovery time and the assumed MTTR. These were only done for the warm option, but the results are presumably similar for cold. Reducing either the recovery time or the MTTR by a factor of 2 reduced the downtime fraction from $15 \%$ to $10 \%$, which is a significant change. It will be worth considerable design effort to keep the the MTTR and recovery time small. Success at this could reduce the requirements on the MTBFs.

To investigate what the availability might be during the commissioning period, the simulations were repeated with three simple changes:

\section{All the MTBFs were halved.}

2. Twice as much time was spent on MD.

3. The recovery time from outages was doubled.

For the warm or cold reference design with an undulator $e^{+}$source, the downtime increases from $15 \%$ to over $46 \%$, and the simulated time spent integrating luminosity drops precipitously from about $75 \%$ to $31 \%$, even without including the $10 \%$ downtime reserved as contingency. This low an efficiency effectively means the detector gets no useful luminosity. 
Changing from an undulator to a conventional $e^{+}$source makes a dramatic difference. The simulated fraction of time spent integrating luminosity improves considerably to over $64 \%$ instead of $31 \%$. This commissioning scenario demonstrates that an LC (warm or cold) should start with a conventional positron source and only switch to an undulator source as an upgrade after the accelerator has been running for several years. Otherwise, one receives at least a factor of two less luminosity.

Finally, a study was made to estimate how many people would be required to make the repairs included in the simulation. For either warm or cold reference design, only 4 people per shift were needed for repairs in the support tunnel and 4-6 in the accelerator tunnel, but for the cold one-tunnel variant, the number in the tunnel jumped to 25 people per shift. This is in no way a complete personnel estimate as it does not include people required for repairs in other parts of the machine or for things like inspections, preventive maintenance or upgrades.

\subsection{Cost and Schedule}

The available cost estimates for both technologies are based on experience with existing accelerator facilities, technology specific R\&D prototypes and test facilities, and industrial models of large-scale production.

The maturity and confidence in the cost estimates for both technologies are appropriate for a major U.S. project in the conceptual design stage. Extrapolated reductions in unit costs from present-day R\&D to the high-volumes needed to build the collider are a source of risk in the cost estimates. Extrapolations of up to factors of three to five were made for the components of the cold crymodules, and as high as six for some of the warm copper components. The overall extrapolation is somewhat larger for the warm technology because of the larger number of small repetitive components involved.

More than two thirds of the warm costs are independent of the accelerator technology, and the corresponding cold costs are the same. The cold-warm difference arises because the costs that are specific to the cold technology choice are estimated to be about twice as large as those that are specific to the warm option. The differences in the specific costs result in an estimated total project cost for the cold machine that is $25 \% \pm 10 \%$ greater than for the warm machine.

The quoted uncertainty assumes a $\pm 15 \%$ (rms) uncertainty in the specific costs, and ignores correlations between the warm and cold cost estimates. We have not done a complete analysis of the uncertainties in these costs, which would have to include any correlations. The presence of correlations would lower the uncertainty.

We found it necessary to re-estimate the cost of the damping rings for the cold technology to account for differences in the technical specifications from those in the TESLA TDR.

The industrial technologies and experience needed to produce the components and systems of each machine choice are well-defined and available in world-wide industries. Industrial capacities needed to produce the quantities of the components for the main linac do not exist today for either the warm or cold technology.

Both technologies are ready for substantial value engineering (VE) and design-for-manufacture (DFM). We expect the cost and confidence in the acquisition of the components needed for each technology to respond favorably to continued design analysis, technology R\&D, and VE and DFM.

Provided appropriate funding during construction, the time needed to build and commission a collider can be independent of the choice of linac technology.

With the initial configurations that we have assumed, the estimated cost to upgrade the $500 \mathrm{GeV}$ reference design to $1 \mathrm{TeV}$ is approximately the same for each technology. The cost to upgrade will depend significantly on when and how it is done. 


\subsection{Civil construction and siting}

The four design solutions for a linear collider described in this chapter (normal conducting and superconducting in both California and Illinois) are the result of several design iterations that have explored a wide range of configurations and construction methods. This previous work has been based on a design solution utilizing two parallel tunnels, one housing the accelerator components, and one housing the related support equipment for the enclosures required to house and operate this machine. The option of a single tunnel solution has not at this time been formally pursued, however a discussion of this option is contained in Section 7.2 of this report. Uniform, competent, dry rock strata has been determined to be the best material for tunnel construction. All of the representative locations investigated have met necessary stability requirements with respect to ground motion for a linear collider and are suitable for tunnel construction regardless of the linear collider technology selected.

Several aspects of the design solutions presented are very similar in nature, but there are some differences. General site services and utility support are similar for either technology. Electrical power requirements are driven by the technology used, but in both cases, a substantial power source is needed. Heat rejection and cooling are also technology driven. The normal conducting machine requires the entire heat rejection load to be accommodated by conventional mechanical means such as cooling towers and/or ponds configured to optimize local climate and weather conditions. In the case of the superconducting machine, a portion of that heat rejection load is removed by way of the cryogenic cooling system. While this will reduce the overall size of the conventional mechanical systems required for heat rejection, that fact is offset by the need for a complete cryogenic cooling system with cooling towers contained elsewhere in the project. The lengths of the tunnel enclosures for the superconducting machine are necessarily longer than for those of the normal conducting machine due to difference between the accelerating gradients for the normal conducting and superconducting structures. The superconducting and the normal conducting machines require comparable access ramps and/or shafts. Damping ring configurations are different for the two machines, but neither configuration poses a substantial impact on the overall conventional construction effort. The electron and positron source configuration, beam delivery enclosures and interaction regions are the same regardless of the technology selected. The main campus configuration and required building square footage is the same, by definition, for either technology and although the superconducting machine requires fewer grade level service buildings, they are larger in area to accommodate the cryogenic equipment.

The work that has been completed to date on the conventional construction aspects of this project points toward two fundamental conclusions. First, although the scope of this project is large, the construction process itself is fairly straightforward. Shaft and tunnel construction is a well documented and understood process. Successful tunneling projects have been completed in all parts of the world. While the process of tunneling requires a certain appreciation for contingency, this contingency is almost always based on the potential for unexpected ground or rock conditions, and not uncertainties in the tunneling process itself. From the standpoint of feasibility, the facilities required for either of these technologies can be successfully constructed with currently employed and understood construction methods.

A second conclusion can also be drawn. Although there are distinctions in the requirements for specific aspects of the project that are salient with respect to the normal conducting and superconducting technologies, none of these distinctions are sufficiently compelling to suggest that one technology is more appropriate than the other with respect to the conventional construction process. Both technologies use similar construction techniques and similar tunnel and shaft configurations. Support buildings are very similar in nature. Utility needs, from the standpoint of conventional construction, are very similar, with the only qualification being that the efficiency of heat rejection through mechanical cooling will be necessarily dependent on local climate and weather conditions. The conventional construction should be considered a neutral component to the eventual technology decision process. 


\subsection{Design variants}

The design variants refer to modifications to the reference designs that have the potential for cost reduction, performance enhancement, and/or significant positive schedule impact. Five design variants were examined:

- a single tunnel architecture for the cold option main linac, as in the TESLA TDR;

- the use of $35 \mathrm{MV} / \mathrm{m}$ as the initial main linac cavity gradient for the cold option;

- the use of the more efficient superstructure scheme for the cold option cavities;

- the use of the more efficient Delay-Line Distribution System for the warm option pulse compression;

- the use of a conventional positron source for both options.

\subsubsection{One tunnel in cold option}

The single tunnel cold option linear collider is similar to the design described in the TESLA Technical Design Report [TDR]. The klystrons, their transformers, controls and power converters are located in the accelerator housing. The klystron modulators are located in accessible surface buildings along with the cryogen plants.

Single tunnel architecture is a viable variant for the cold option because of the $1 \mathrm{~ms}$ long RF pulse. In the cold option, losses in the multi-km-long cables are low and the klystron transformer primary side voltage is low. The primary justification for this variant is the reduction in civil construction cost. In an attempt to compare the salient design features of one tunnel and two tunnel scenarios, a partial civil conventional design was prepared for the one tunnel solution. This partial one tunnel design solution was then compared to the previously prepared Illinois superconducting linear collider two tunnel design solution to better understand the inherent differences.

The principal issues considered were construction and tunnel outfitting costs, including life safety and egress. Because the one tunnel solution will need to house more technical components, including klystrons and cabling, we have assumed a 20' excavated diameter. The two tunnel solution is anticipated to require a 16' excavated diameter. The primary technical difference of the one and two tunnel design solutions is location of the modulators and length of waveguide. The one tunnel solution is anticipated to include modulators located at the surface adjacent to the shaft head house and klystrons located within the beamline tunnel similar to the TESLA design. More than 400 high voltage cables would travel down the access shaft and up to $2.5 \mathrm{~km}$ in each direction along the beamline tunnel to supply pulsed power to the klystrons in the one tunnel solution.

Including all of the above considerations, the one tunnel variant yields a savings of $23 \%$ of the reference design (two-tunnel) linac tunnel and outfitting cost.

Assuming a construction model that has a $42.7 \mathrm{~km}$ tunnel to house $27 \mathrm{~km}$ of linacs for a $500 \mathrm{GeV}$ center of mass linear collider, the civil construction cost reduction achieved with the single tunnel variant is about $5.1 \%$ of total project cost.

As noted in Section 9.3, the availability of the one tunnel variant was studied. With the same set of component MTBFs which give the two-tunnel reference design an unavailability of $15 \%$, the one tunnel variant has an availability of $25 \%$. To regain the required $15 \%$ unavailability, the MTBF of many linac and DR components must be improved substantially, and the linac energy overhead also must be increased from $3 \%$ to $8 \%$. A very crude estimate of the required cost increase associated with these component reliability improvements was of the order of $3 \%$ of total project cost.

The net is a reduction of the total project cost of about $2 \%$ if the collider is built in a single tunnel. 
The increased complexity of installation, caused by the sharing of a single tunnel by the damping ring, main linac accelerator components, and main linac power components, increases the risk of delay in the completion of the construction and commissioning of the collider.

\subsection{2 $35 \mathrm{MV} / \mathrm{m}$ initial design gradient in cold option}

This variant takes $35 \mathrm{MV} / \mathrm{m}$, the maximum gradient at which the cold option cavities are qualified, as the design gradient for the $500 \mathrm{GeV}$ machine. Since the installed linac is operating at its maximum gradient, this variant will limit the energy reach of the cold option reference design to $500 \mathrm{GeV}$, unless more of the tunnel is initially filled with accelerator components, at higher cost.

In this variant, the number of installed cryomodules is reduced from 1508 to 1207 . The number of klystrons and associated RF hardware is not changed. Each klystron drives 24 cavities, rather than 30, as in the reference design. The peak power delivered through the coupler to each cavity rises from $274 \mathrm{~kW}$ in the reference design, to $345 \mathrm{~kW}$ in this variant.

The increase in the gradient results in an increase in the external $Q$, a reduction in the bandwidth, and an increase in the RF pulse fill time (from 500 to $630 \mu \mathrm{s}$ ). A small increase in the RF power and cryogenic power results from this pulse length increase.

There is a considerably larger increase in the cryogenic power requirements due to the increase in gradient, and the reduction in $Q_{0}$ from $10^{10}$, at $28 \mathrm{MV} / \mathrm{m}$ in the reference design, to $5 \times 10^{9}$, at $35 \mathrm{MV} / \mathrm{m}$. This increase in cryogenic requirements can be satisfied by adding two additional refrigerators, with a $4.5 \mathrm{~K}$ equivalent power of $30 \mathrm{~kW}$, to the six refrigerators in the reference design, bringing the total to 8 (four for each linac).

The reduction in the number of cryomodules results in a cost reduction, which is partially cancelled by the additional two cryogenic plants needed. The net savings is about $3 \%$ of total project cost.

\subsubsection{Superstructure in cold option}

To maximize the active length of the linac, it is desirable to use structures with as many cells as possible. However, the number of cells per cavity is limited by the requirements of field homogeneity and the need to avoid trapped modes. These limitations can be circumvented by joining several multicell cavities to form a so-called superstructure, using a short, large diameter connecting tube. The favored design for a superstructure is two 9-cell resonators joined by a large diameter beam pipe.

The use of such a superstructure in a cryomodule provides more efficient packing of the cavities, allowing the length of a cryomodule to be reduced by about $1 \mathrm{~m}(\sim 6 \%)$. In addition, the number of input couplers per cryomodule is reduced from 12 to 6 . Each coupler must transmit twice the power, however, up to $\sim 700$ $\mathrm{kW}$ at $35 \mathrm{MV} / \mathrm{m}$. The $1 \mathrm{~m}$ reduction in the length of each cryomodule results in an overall reduction in the length of the linac tunnels by about $2.5 \mathrm{~km}$.

Beam tests of two prototype $2 \times 7$ superstructures at TTF have confirmed the performance expectations in terms of energy stability and HOM damping. These tests were performed at gradients of $15 \mathrm{MV} / \mathrm{m}$. The principal concern with increased gradient is the relatively high levels of power transmitted through the input coupler. Additional R\&D is needed to establish the reliability metrics of input couplers at these power levels.

The cost reduction associated with the superstructure variant is due to the reduction in the number of input couplers and the reduction in the length of the linac housings. The overall cost reduction is estimated to be $3 \%$ of the total project cost. 


\subsubsection{DLDS in warm option}

For the warm machine, the klystron pulse length and peak power were chosen to minimize klystron costs, and are not optimal for powering the structures. To transform the relatively long, low power klystron pulses to the short, high power pulses required for the structures, several methods were considered during the past decade. Most practical experience with pulse compression has come from the development of SLED-II, which is a delay line version of the SLAC Linac Energy Doubler (SLED). A dual-mode version of this scheme is used in the reference design.

A more efficient scheme of pulse compression is the multi-moded Delay Line Distribution System (DLDS). It eliminates the need for resonant RF storage, as used in SLED, and utilizes the time-of-flight of the beams to reduce the delay line length, with two modes transported in each delay line. The higher compression permitted in such a system allows a longer $(3.2 \mu \mathrm{s})$ klystron pulse to be used. For such a system, the overall transport efficiency is expected to be $85 \%$, to be compared to $75 \%$ for SLED II in the reference design. More significantly, only half of the number of klystrons are required because of the longer pulse length (such a high compression ratio would impractical with SLED II because of the increased loss of efficiency). In addition, the modulator for this system would likely be designed to power eight klystrons instead of two. The number of RF distribution components would be roughly the same, although the total delay length would double.

The cost savings associated with the use of the more efficient DLDS and the longer klystron pulse for the warm option is about $8 \%$ of total project cost.

Since the DLDS system is not as developed as SLED-II, additional R\&D, including high-power tests of the system, would be required before the DLDS could be qualified as a technically viable alternative to the SLED-II scheme.

\subsubsection{Conventional positron sources in both options}

In contrast to the undulator-based source in the reference design, a conventional positron source will not allow the production of polarized positrons. However, as noted below, there are significant advantages to a conventional positron source.

In the NLC design, unpolarized positrons are produced by colliding $6.2 \mathrm{GeV}$ electrons into three separate high $Z$ material targets, capturing the resulting positrons, and accelerating them to the $1.98 \mathrm{GeV}$ energy of the pre-damping ring system. Multiple positron targets are required to keep the energy deposited in each target below the threshold for material damage. The electrons are split by an RF separator and directed onto 3 out of 4 multiplexed targets and positron capture sections. The bunches are then recombined into the desired bunch train format and accelerated in a $1.98 \mathrm{GeV}$ L-band linac to the positron pre-damping ring.

Preliminary studies indicate the possibility of developing a conventional positron system for the cold option LC design[35]. This design is based on the NLC design for the production of unpolarized positrons but has been modified to accommodate the differences in the beam pulse format and the higher energy of the cold option positron damping ring. Because of the preliminary nature of the cold option conventional positron source design, more optimization studies are required than in the case for the warm option conventional source. Initial required studies include an improved calculation of the yield of positrons captured into the damping ring acceptance, a simulation of the mechanical stresses in the target, and target station engineering.

The capture system and booster linac are the same as in the case of an undulator based source but with the caveat that the emittance of the initial positron distribution off the targets is a factor of 3-4 greater for the conventional system. Because of the larger emittance of the initial positrons, additional studies of the capture into the damping ring are required.

The design of the positron system is based on the system used for the SLC, which demonstrated excellent reliability over many years of operation. The total number of positrons required for the warm option bunch 
train is almost two orders of magnitude greater, and for the cold option, more than three orders of magnitude greater, than the number of positrons in the single SLC bunch. The design goal is to build a target system which is expected to survive a 9 month run $(120 \mathrm{~Hz}, 24$ hours per day, 7 days per week, with no scheduled outages for maintenance). Targets can be replaced/repaired annually in a scheduled 3 month maintenance period.

If either machine were built with a conventional positron source instead of the undulator source, then an unpolarized electron gun, a $6 \mathrm{GeV}$ electron linac, multiple targets, and a pre-linac with associated civil support facilities would need to be substituted for the linac space and components needed for the undulator positron source and electron energy make-up, positron pre-linac, and tunnel and components of the positron transfer line. The cost would be reduced by approximately $2 \%$, but a later upgrade to include a polarized positron source would be difficult. If the initial configuration includes the space required in the linac to later install the components of the undulator-based source, the energy make-up in the linac, and the tunnel needed for the positron transfer line, then the cost would be approximately the same as starting with an undulator source with no conventional source.

Availability comparisons were made between an undulator $\mathrm{e}^{+}$source and a conventional $\mathrm{e}^{+}$source. The positron source was not modeled in detail and the same overall MTBF was used for both systems. The significant difference is that the undulator source requires high energy electrons before positrons can be produced, while the conventional source does not. This one difference changes the downtime from $15 \%$ to about $11 \%$ because of the shorter recovery time when both systems can be restored independently. More importantly, the amount of time spent integrating luminosity increases from about $75 \%$ to over $85 \%$. Changing from an undulator to a conventional $\mathrm{e}^{+}$source makes an even more dramatic difference during commissioning and the first few years of operation of the collider, when MTBF's are shorter, recovery times longer, and more machine development time is needed. The simulated fraction of time spent integrating luminosity improves considerably to over $64 \%$ for the conventional source, instead of $31 \%$ for the reference design.

In addition, the use of a conventional positron source reduces the complexity of the construction, installation, and initial commissioning of the injectors and damping rings, and could result in up to a year's reduction in the construction and commissioning of the collider.

The option to build the collider with a conventional positron source and the vacant space needed to later implement a polarized undulator source should be more vigorously considered.

\subsection{Risk}

Risk assessments for the major collider subsystems have been carried out. In these assessments, the risks have been evaluated in terms of the highest level collider parameters, energy and luminosity, and relative to the primary mission of the collider, as specified by the physics design requirements: the delivery of $500 \mathrm{fb}^{-1}$ of integrated luminosity at $500 \mathrm{GeV}$ in the first 4 years of operation.

Several factors have been included in our assessment of risk. These include the origin and severity of potential modes of failure to carry out the physics mission, the method and time at which such failures will be identified, and the consequences that would occur if such a failure happens. We have not done an iteration of design and planning aimed at mitigation of these risks, but we have identified important areas of R\&D that need to be carried out early in the collider project.

This analysis revealed that the highest potential risks to the mission for both options are in the areas of the beam delivery systems, the controls systems, and the machine protection systems (MPS). The conclusion that the highest potential risks do not lie in the area of high gradient RF cavity development came as a surprise to us. This is due primarily to the intensive past and current technology R\&D programs in this area. 
The high risks in the beam delivery systems, the controls systems, and the MPS are related to the fact that problems here generally are not encountered until the pre-operations phase of the project, which limits the possible response time. The risks associated with the machine protection systems need further elaboration and study.

Larger risks were found for the cold option damping rings than for the warm option damping rings. The analysis also indicated smaller risks for the cold option than the warm option in the main linac, for both emittance preservation and RF technology.

\subsubsection{Machine Protection}

The highest risk identified for any collider system, and essentially the same (although details differ) for both technology options, was in the MPS. The MPS is the set of all devices which allow continued, smooth, operation of the machine with a minimal chance of beam-related component damage or the generation of unacceptable levels of residual radioactivity. The risk is that the MPS does not allow the production of steady high power beam.

The linear collider beam energy density is beyond that of existing accelerators. For both technology options, a single, nominal current and nominal transverse dimension fully damped bunch will seriously damage any metal surface. No existing machine has an MPS capable of protecting against single-beam-pulse damage. It is important to keep in mind that it is not enough for the MPS to simply protect against component damage; it must also keep interruptions to a minimum and provide for fast recovery from each one. While design concepts exist, both these and issues of scale must be tested before a satisfactory system can be claimed.

\subsubsection{Controls}

Control-system related risks were also identified as being among the most severe. The controls requirements demand a system which is well beyond the current state of the art. In this case, the risk is that, due to an inadequately specified or implemented control system, the operation of the linear collider requires too much manual, non-automated, intervention to maintain subsystem optimization. Although they have different controls challenges in detail, this risk was deemed to be equal for the two technology options.

\subsubsection{Beam delivery}

The highest risk identified in the beam delivery system is that associated with detector backgrounds due to the high power beams. This risk was seen as being roughly the same for the two technology options. The problems would not be detected until the early operations phase of the project, and their resolution could require a major rework to the collimation/masking system in and around the IP.

For the cold option, the second highest risk in the beam delivery system was identified to be the intra-train IP position feedback for the cold option. Despite past success with train-to-train feedback, the IP stabilization requirements and the IR environment in the LC will be very different from that in previous experiments. The stabilization requirements are at the nanometer level and are hundreds of times smaller than that previously achieved. These stabilization requirements are tighter (because of the larger beam disruption) and the backgrounds larger (because of the higher beam power) for the cold option. In addition, the cold option uses a high bandwidth intra-train system, with more challenging requirements than for train-to-train systems.

For the warm option, the second highest risk in the beam delivery system was judged to be control of jitter of the final focusing magnets, at the $0.5 \mathrm{~nm}$ level, for frequencies above $\sim 10 \mathrm{~Hz}$. Prototype active stabilization systems have stabilized rigid objects at the desired levels, and there is an active program aimed 
at demonstration of the active stabilization of a superconducting quadrupole similar to that needed for the final magnet. Nevertheless, the real performance limitations will not be encountered until testing in the actual IR environment, with the complete system, including the experimental detector, in place.

\subsubsection{Main linacs}

The three highest risk areas for the warm option main linac RF technology were identified to be engineering and design issues related to klystron lifetime, the production of the SLED-II pulse compression systems ${ }^{2}$, and the production of the RF structures. Two comparable engineering and design risk areas were identified for the cold option: klystron lifetime, and the production of the superconducting RF cavities. In all these areas, the risks were related to the difficulties of scale-up from prototype systems to the mass production of many reliable, high performance units needed for the complete system.

The highest risk for the warm option in the area of emittance control in the main linac was judged to be potential beam jitter arising from uncontrolled or unanticipated cultural and/or technical noise sources. If this risk and the effort of remediation is perceived to be too high, active stabilization elements could be included with the quadrupole installation for minimal additional cost. The cold option design utilizes a series of intra-train feedback systems in the main linacs, which will be able to control high-frequency beam jitter. It should be noted that the "quasi-static" tuning algorithms used for beam-based alignment were judged to be a risk almost as severe as the beam jitter risk, and roughly equal for both options.

\subsubsection{Damping rings}

The item of most concern in the warm option damping rings was judged to be the risk that the electron cloud causes a beam instability in the positron damping ring. Simulation codes indicate that both warm and cold LC positron damping rings could suffer from electron cloud effects. The much shorter bunch separation in the warm LC damping ring (1.4 ns) compared to the cold LC damping ring (20 ns) means that any electron cloud effects are likely to be more severe in the warm LC damping rings.

The item of most concern in the cold option damping rings was that the vacuum chamber impedance drives microwave beam instabilities. Existing storage rings have achieved impedance values significantly below the microwave threshold for the warm LC damping rings. The structure of the cold LC damping rings drives the threshold about an order of magnitude lower, to a more challenging regime. If necessary, a major modification of the cold damping ring vacuum chamber would be a much larger task than a modification of the warm damping ring vacuum chambers.

An additional four less severe risks were identified, all of which were of more concern for the cold option than the warm option. When all the risks that were identified are considered, the cold option has more risk to achieving the required damping ring performance than the warm option. (See Fig. 8.8.1.1.) This is expected given the novel design of the ring. It is possible that the current design of the cold damping ring could be improved, but there are a number of difficult features, related to the bunch format required by the cold RF technology, inherent in the design of any damping ring for the cold option. These features make it more difficult to achieve the same level of risk as for the smaller, more conventional, warm option damping rings.

\subsubsection{Sources}

In the positron source, the item of most risk was judged to be the engineering of the rotating positron production target. Designs for both options are presently based on the high strength properties of Ti-alloy for which little information is available regarding the effect of radiation on the mechanical properties of the

\footnotetext{
${ }^{2}$ A full-power demonstration of the SLED-II pulse compression system was carried out in late 2003.
} 
material. If the Ti-alloys are not sufficiently robust, considerable redesign of the target systems may be required. Additional engineering studies and radiation tests are required to alleviate these concerns. The risk was judged to be roughly the same for the two technology options.

In the electron source, the item of most concern was the laser for the polarized electron gun. The lasers for either design are complex systems requiring significant engineering and prototyping prior to design completion. Complete stabilization to within specifications are likely to take several years of attention after the sources are turned on. The different bunch formats of the warm and cold LC designs require different laser systems each of which will have their own specific problems and remedies. The risks in this area were judged to be comparable for the two technology options.

\subsubsection{Near-term R\&D objectives}

There are important objectives for $R \& D$ that should be addressed early in the project to mitigate risk for each choice of main linac technology. Many of these are not dependent on the linac technology choice at all. The highlights are target engineering for the undulator source, the electron cloud issue in the positron damping ring, and pulse compression and gradient demonstrations in the main linac RF technology programs. Additional important issues are helical undulator development, damping ring kickers, dark current effects in the main linacs, and main linac structure wakes. Many, but not all, of the risks listed in these tables are the subject of currently active $R \& D$ programs. It is important for the success of the linear collider project that R\&D programs which address all these issues are successful, since deferral of any of these issues to later in the project may substantially increase the risk. 


\section{Appendix A}

\section{Comparison of linear collider physics requirements}

This appendix provides a comparison of linear collider physics requirements contained in the Scope Document [2] of the U. S. Linear Collider Steering Group (USLCSG), "Design Considerations for an International Linear Collider", written by the American Linear Collider Physics Group, and those specified by the Parameters Subcommittee[3] of the International Linear Collider Steering Committee (ILCSC). This comparison has been provided by Mark Oreglia, who served as both an editor of "Design Considerations for an International Linear Collider", and also as a member of the Parameters Subcommittee of the ILCSC.

The list of requirements is summarized in Table A.0.0.1, with differences highlighted in italics.

Table A.0.0.1: Comparison of USLCSG and ILCSC linear collider physics requirements

\begin{tabular}{|c|c|c|}
\hline Requirement & USLCSG & ILCSC \\
\hline Baseline energy & $\begin{array}{c}\text { 90-500 GeV } \\
\text { with } \sqrt{s} \text { luminosity scaling } \\
\text { from } 500 \mathrm{GeV}\end{array}$ & $\begin{array}{l}200-500 \mathrm{GeV} \\
\text { with } \sqrt{s} \text { luminosity scaling; } \\
\text { lower luminosity at } 90 \mathrm{GeV} \\
\quad \text { for calibration. }\end{array}$ \\
\hline $\begin{array}{l}\text { Baseline integrated } \\
\text { luminosity }\end{array}$ & $500 \mathrm{fb}^{-1}$ in 4 years & $\begin{array}{c}500 \mathrm{fb}^{-1} \text { in } 4 \text { years, } \\
\text { plus option for another } 500 \mathrm{fb}^{-1} \text { by year } 6\end{array}$ \\
\hline $\begin{array}{l}\text { Baseline energy } \\
\text { quality }\end{array}$ & $\begin{array}{l}\text { beamstrahlung spread } \\
\text { similar to ISR }\end{array}$ & $\begin{array}{l}0.1 \% \text { energy precision } \\
\text { and stability }\end{array}$ \\
\hline Beam polarization & $\begin{array}{c}\text { electrons }>80 \%, \text { and } \\
\text { positrons }>60 \% \text { as upgrade }\end{array}$ & $\begin{array}{c}\text { electrons }>80 \%, \text { and } \\
\text { positrons }>50 \% \text { as upgrade }\end{array}$ \\
\hline \multirow[t]{2}{*}{ IRs } & allow for crossing angle & allow for crossing angle in at least 1 IR \\
\hline & construct 2 IRs & construct 2 IRs and 2 detectors at beginning. \\
\hline $\begin{array}{l}\text { Energy upgrade, } \\
\text { integrated luminosity }\end{array}$ & $\begin{array}{l}\text { approx. } 1 \mathrm{TeV} \\
0.5-2 a b^{-1}\end{array}$ & $\begin{array}{c}\text { approx. } 1 \mathrm{TeV}, \\
1 \text { ab in } 3-4 \text { years } \\
\text { with } \sqrt{s} \text { luminosity scaling at all } \mathrm{E}\end{array}$ \\
\hline$e^{-}-e^{-}$collisions & in baseline & option \\
\hline$\gamma-\gamma, e-\gamma$ collisions & upgrade & option \\
\hline
\end{tabular}

It is evident that there are only three significant differences between the two sets of parameter specifications, namely:

1. the option for luminosity doubling (or learning curve) for years 5-6 in the baseline machine, 
2. the $0.1 \%$ energy precision mandated by the ILCSC, and

3. the $\sqrt{s}$ luminosity scaling from $200-1000 \mathrm{GeV}$ for the upgraded energy machine. 


\section{Bibliography}

[TDR] R. Brinkmann et. al., eds., "TESLA Technical Design Report", Part II, DESY-2001-011, (March, 2001)

[ZDR] T.O. Raubenheimer, ed. [NLC ZDR Design Group], "Zeroth order design report for the Next Linear Collider," SLAC-R-0474, (1996)

[NLC01] N. Phinney, ed. [NLC Collaboration], "2001 report on the Next Linear Collider: A report submitted to Snowmass '01," SLAC-R-571, (2001)

[NLC03] NLC 2003 Configuration

[TRC] G. Loew et. al., "International Linear Collider Technical Review Committee: Second Report", SLAC-R-606, (2003)

[JLC] N. Toge, ed. [JLC Design Study Group], "JLC design study," KEK-REPORT-97-1 (1997).

[ISG] C. Adolphsen, et. al., [International Study Group Collaboration], "International study group progress report on linear collider development," SLAC-R-559, (2000)

[GLC] K. Abe et. al, "GLC Project Report", KEK Report 2003-7, Sep. 2003. Also available at http://lcdev.kek.jp/ProjReport/

[1] DOE/NSF High Energy Physics Advisory Sub-panel on Long Range Planning for U.S. High Energy Physics, (Jan., 2002)

[2] "Design Considerations for an International Linear Collider", the American Linear Collider Physics Group Executive Committee, (2003)

[3] "Parameters for the Linear Collider", Parameters Subcommittee, International Linear Collider Steering Committee, Sept. 30, 2003,

[4] Daniel Schulte, et. al., "Tests of Three Linear Collider Beam Dynamics Simulation Programs", LCC-0091 (2002).

[5] T.O. Raubenheimer, P. Tenenbaum, "Brief Review of Linear Collider Beam-Based Alignment", LCC-0129 (2003).

[6] N. Walker, et. al., "Damping Ring to Interaction Point Beam Transport Issues," p.657, Proceedings of the 2003 Particle Accelerator Conference Portland, OR (2003).

[7] P. Tenenbaum, R. Brinkmann, V. Tsakanov, "Beam-Based Alignment of the TESLA Main Linac," pg. 515, Proceedings of the 2002 European Particle Accelerator Conference, Paris (2002).

[8] D. Schulte, N. Walker, "Simulations of Static Tuning for the TESLA Collider", pg. 2736, Proceedings of the 2003 Particle Accelerator Conference, Portland, OR (2003). 
[9] Paul Emma, [ZDR, Chapter 5] and subsequent undocumented studies in 1998.

[10] C. Sramek, et al., "Beam-Beam Interaction Simulations with Guinea Pig", LCC-0125 (2003).

[11] Y. Nosochkov, et al., "Tuning knobs for the NLC final focus", pg. 476, Proceedings of the 2002 European Particle Accelerator Conference, Paris (2002)

[12] Mark Woodley, Andrzej Wolski, "The NLC Main Damping Ring Lattice," LCC-0113, (February, 2003)

[13] Ina Reichel, Andrzej Wolski, "A Reduced Emittance Lattice for the NLC Positron Predamping Ring," LCC-0114, (February, 2003)

[14] Y. J. Batygin, J. C. Sheppard, "Start-to-End Simulation of Polarized Positron Collection in the NLC", LCC-0117, SLAC (2003).

[15] A. Seryi, talk presented at NLC MAC meeting, (June, 2003).

[16] B. Parker, talk presented at NLC MAC meeting, (November, 2002).

[17] J. Volk, et al., "Adjustable Permanent Quadrupoles for the Next Linear Collider," pg. 217, Proceedings of the 2001 Particle Accelerator Conference, Chicago, IL (2001).

[18] Y. K. Batygin and J. C. Sheppard, "Capture and polarization of positrons in a proposed NLC polarized positron source," SLAC-PUB-9891 and pg. 2751, Proceedings of the 2003 Particle Accelerator Conference, Portland, OR (2003).

[19] Y. Nosochkov, private communication.

[20] Y. Batygin, J. C. Sheppard, "Thermal Stress Analyses for a Thermal Multislug Beam NLC Positron Target," LCC-0090, SLAC (2002).

[21] R. Rimmer, et al., "An RF Cavity for the NLC Damping Rings", pg. 924, Proceedings of the 2001 Particle Accelerator Conference, Chicago, IL (2001).

[22] R.A. Rimmer, D. Atkinson, J.N. Corlett, G. Koehler, D. Li, N. Hartman, J. Rasson, T. Saleh, R. Weidenbach, "RF Cavity R\&D at LBNL for the NLC Damping Rings, FY2000/2001," LCC0072, (Oct., 2001).

[23] S. De Santis, "Coupled Bunch Instabilities in the NLC Damping Rings," LCC0069, (May, 2001).

[24] Tor Raubenheimer, Andrzej Wolski, "Comparison of Alignment Tolerances in the Linear Collider Damping Rings with those in Operating Rings," LCC-0112, (January, 2003).

[25] A. Wolski, private communication.

[26] P. Emma, T. Raubenheimer, "Circumference Correction Chicanes for Damping Rings," LCC-0008, (June, 1998).

[27] Paul Emma, "Cost and Performance Optimization of the NLC Bunch Compressor Systems," LCC0021, (August, 1999).

[28] Paul Emma, "More Options for the NLC Bunch Compressors," LCC-0035, (January, 2000).

[29] S. Marks, private communication.

[30] A. Drozhdin, L. Keller, T. Markiewicz, T. Marayuma, M. Mokhov, T. Raubenheimer, A. Seryi, P. Tenebaum, M. Woodley, "NLC Collimation Update Study," LCC-0118, (May, 2003). 
[31] P. Raimondi and A. Seryi, "A Novel Final Focus Design for Future Linear Colliders," LCC-0039, (May, 2000).

[32] P. Raimondi and A. Seryi, "Novel Final Focus Design for Future Linear Collider", Phys. Rev. Lett. 86, 3779 (2001).

[33] S. Smith, "Design of an NLC Intra-pulse Feedback System", LCC-Note-56, SLAC, March 2001.

[34] P. Burrows, "Feedback on Nanosecond Timescales (FONT): Results from First Beam Tests at the NLCTA at SLAC", pg. 687, Proceedings of the 2001 Particle Accelerator Conference, Chicago, IL (2001)

[35] J. C. Sheppard, "Conventional Positron Target for a Tesla Formatted Beam", LCC-0133, (SLACTN-03-072), (November, 2003).

[36] The radiation spectrum from planar and helical undulators is described in NLC Notes LCC-085 and LCC-095.

[37] T. A. Vsevolozhskaya, A. A. Mikhailichenko, E. A. Perevedentsev, G. I. Silvestrov and A. D. Chernyakin, "Helical Undulator For Conversion System Of The VLEPP Project," SLACTRANS-0225, presented at Int. Conf. on High Energy Accelerators, Novosibirsk, USSR, Aug 7-11, 1986; A. Mikhailichenko, "Short-period SC undulator," CBN-03-4 (2003).

[38] A. Mikhailichenko, "Pulsed undulator for test at SLAC of the polarized positron production," CBN-03-5 (2003).

[39] W. Stein, J. C. Sheppard, "Structural Modeling of the TESLA TDR Positron Target", LCC-0089, SLAC (2002)

[40] W. Stein, J. C. Sheppard, "NLC Polarized Positron Photon Beam Target Thermal Structural Modeling", LCC-0087, SLAC (2002)

[41] V.K. Bharadwaj, et al., "Analysis of Beam-Induced Damage to the SLC Positron Production Target", pg. 2123, Proceedings of the 2001 Particle Accelerator Conference, Chicago, IL (2001).

[42] LCC-note in preparation on Ti damage, (2003).

[43] J. Sheppard, private communication, (2002).

[44] Y. J. Batygin, J. C. Sheppard, "Start-to-End Simulation of Polarized Positron Collection in the NLC", LCC-0117, SLAC (2003).

[45] J.P. Labrie, H. Euteneuer, "Power Handling Capability of Water-cooled CW Linac Structures", Nucl. Instrum. Meth. A247, 281 (1986).

[46] K. Flottmann, V. Paramonov, eds., "Conceptual design of a Positron Pre-Accelerator for the TESLA Linear Collider, "DESY TESLA-99-14, (1999).

[47] K. Flottmann, private communication, (2003).

[48] D. P. Pritzkau and R. H. Siemann, "Experimental study of RF pulsed heating on oxygen free electronic copper," Phys. Rev. ST Accel. Beams 5, 112002 (2002).

[49] For an example of a flexible spin rotation system, see [ZDR, 5.3.3].

[50] For an example of an energy compressor, see [ZDR, 4.3.3].

[51] C. Santelli et. al., "Technical Layout of the TESLA Damping Ring", LNF-01/003(NT) (2001). 
[52] B.I. Grishanov, F.V. Podgorny, J. Ruemmler, V.D. Shiltsev, "Very Fast Kicker for Accelerator Applications", DESY TESLA-96-11, (1996).

[53] E. Chojnacki and J. Sears, "Superconducting RF Cavities and Cryogenics for CESR III Upgrade", Cornell SRF 990716-09, (1999).

[54] T. Tajima, et al., "The Superconducting Cavity System for KEKB", pg. 440, Proceedings of the 1999 Particle Accelerator Conference, New York, NY (1999).

[55] W. Decking, G. Hoffstatter, T. Limberg, "Bunch Compressor for the TESLA Linear Collider", DESY TESLA-00-40, (2000).

[56] M. D. Woodley, P. E. Emma, "Measurement and Correction of Cross Plane Coupling in Transport Lines", pg. 196, Proc. 20th Int. Linac Conference, Monterey, CA, Aug. 2000; SLAC-PUB 8581, (2000).

[57] A. Drozhdin, G. Blair, W. Kozanecki, L. Keller, T. Markiewicz, T. Marayuma, M. Mokhov, O. Napoly, T. Raubenheimer, A. Seryi, D. Schulte, P. Tenenbaum, N. Walker, M. Woodley, F. Zimmerman, "Comparison of the TESLA, NLC and CLIC beam collimation system performance", LCC-0111, SLAC (January, 2003).

[58] S. Claudet, Ph. Gayet, Ph. Lebrun, L. Tavian and U. Wagner, "Economics Of Large Helium Cryogenic Systems: Experience From Recent Projects At CERN", LHC Project Report 317, (December 1999).

[59] S. Wolff, H. Lierl, B. Petersen, H. Quack, "The Tesla Cryogenic Distribution System", TESLA Report $2001-37$.

[60] S. Claudet, Ph. Lebrun, L. Tavian, "Towards Cost-To-Performance Optimization of Large Superfluid Helium Refrigeration Systems ", LHC Project Report 391, (2000).

[61] T. R. Strobridge and D. B. Chelton, "Size and Power Requirements of 4.2 K Refrigerators", Advances in Cryogenic Engineering, 12, (1967).

[62] T. R. Strobridge, "Refrigeration for Superconducting and Cryogenic Systems", IEEE Trans. Nuclear Science, Vol. NS-16, (1969).

[63] T. R. Strobridge, "Cryogenic Refrigerators-An Updated Survey", NBS Technical Note 655, (1974).

[64] M. A. Green, R. A. Byrns and S. J. St. Lorant, "Estimating the Cost of Superconducting Magnets and the Refrigerators Needed to Keep Them Cold", Advances in Cryogenic Engineering 37, pg. $637,(1991)$.

[65] R. A. Byrns and M. A. Green, "An Update On Estimating the Cost of Cryogenic Refrigeration", Advances in Cryogenic Engineering 43, p 1661, (1998).

[66] H. Edwards, P. Garbincius, et al., "Report on the TESLA Engineering Study/Review", Fermilab TM-2179, (2002).

[67] DOE Budget Formulation Handbook, pg. 11.

[68] NLC 1999 DOE Project Review, SLAC, (May, 1999).

[69] "Advanced Photon Source Conceptual Design Report", ANL-87-15, April, 1987.

[70] "ITER Final Design Report", http://www.naka.jaeri.go.jp/ITER/FDR/, July 2001.

[71] Seryi, A., LCD Group Meeting - "Update on NLC Stability Studies", NLC Group Contribution to TRC, October 31, 2002. 
[72] Frame, P., 2000, "Geologic Conditions at Representative Sites for the NLC in California," Prepared for: Stanford Linear Accelerator Center, 30p.

[73] Lettis \& Associates, Inc., Unruh, J., Lettis, W.R., 2002, "Phase II Fault and Seismic Hazards Investigation"-North of Delta Offstream Storage Integrated Storage Investigations, California Department of Water Resources Storage Investigation, August 2002.

[74] Robert A. Bauer and David L. Gross, "Geology of the Greater Fermilab Region", Illinois State Geological Survey.

[75] S. Smith, "Cavity Beam Position Monitor for the Next Linear Collider", LC-02, SLAC, (2002).

[76] C. Montag, private communication.

[77] V. Shiltsev, private communication.

[78] A. Wolski, W. Decking, "Comparison of Emittance Tuning Simulations in the NLC and TESLA Damping Rings," TESLA 2002-10, DESY (2002).

[79] M. Boege, B. Keil, T. Schilcher V. Schlott, "Commissioning of the Fast Orbit Feedback at SLS", pg. 3386, Proceedings of the 2003 Particle Accelerator Conference, Portland, OR (2003).

[80] J. Sekutowicz et. al, "Cold and Beam Tests of the first prototypes of the superstructure for the TESLA Collider", pg. 467, Proceedings of the 2003 Particle Accelerator Conference, Portland, OR (2003); J. Sekutowicz et. al, "Test of two Nb superstructure prototypes", Phys. Rev. ST Accel. Beams 7, 012002 (2004).

[81] N. Walker, D. Schulte, G. White, "Intra-Bunch-Train Luminosity Optimisation for the TESLA Linear Collider", pg. 2733, Proceedings of the 2003 Particle Accelerator Conference, Portland, OR (2003). 